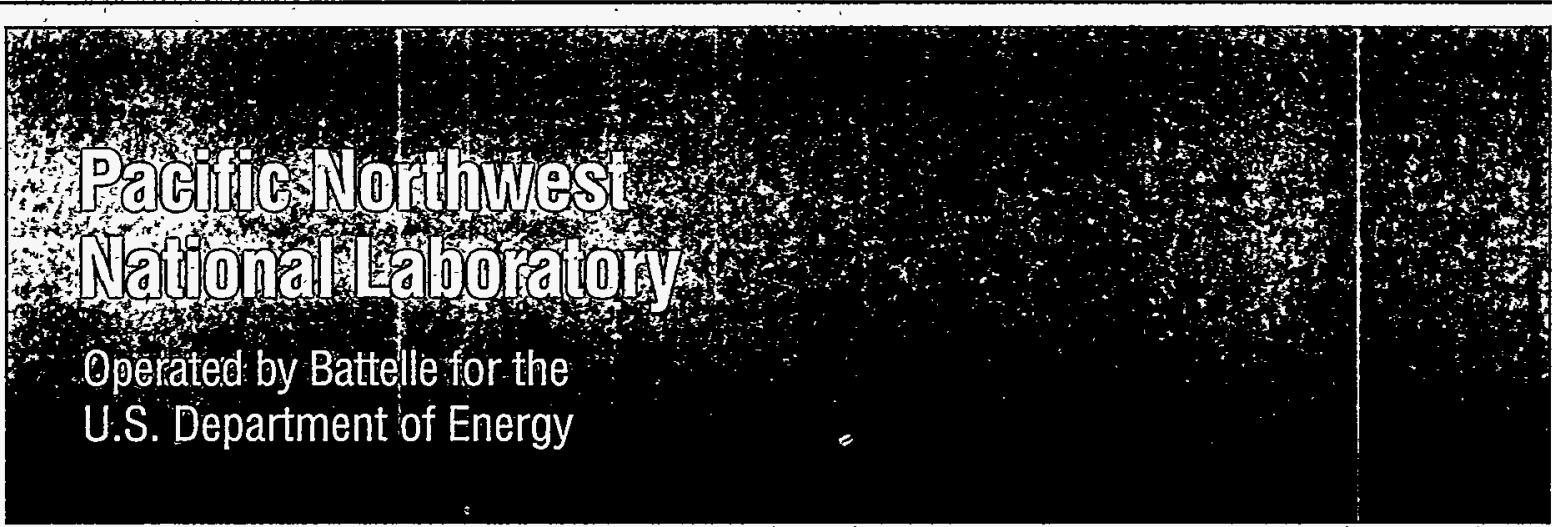

\title{
Composite Analysis for Low-Level Waste Disposal in the 200 Area Plateau of the Hanford Site
}

C.T. Kincaid

M. P. Bergeron

C. R. Cole

M. D. Freshley

N. L. Hassig

V. G. Johnson.

D. I. Kaplan

R. J.Serne

G. P. Streile

D. L. Strenge

P.D. Thorne

L. W. Vail

G. A. Whyatt

S. K. Wurstner

DISTRIBUTION OF THIS DOCUMENT IS UNLIAITED

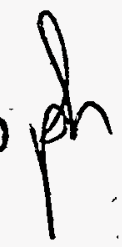

MASTER

March 1998

Prepared for the U.S. Department of Energy under Contract DE-AC06-76RLO 1830 


\section{DİSCLAIMER}

This report was prepared as an account of work sponsored by an agency of the United States Government Neither the United States Government nor any agency thereof, nor Battelle Memorial Institute; nor any of their employees, makes any warranty, express or implied, or assumes any legal liability or responsibility for the accuracy, completeness, or usefulness of any information, apparatus, product, or process disclosed, or represents that its use would not infringe privately owned rights. Reference herein to any specific commercial product, process; or service by trade name trademárk, manufacturer, or otherwise does not necessarily constitute or imply its endorsement recommendation; or favoring by the United States Government or any agency thereof; or Battelle Memorial Institute. The views and opinions of authors expressed herein do not necessarily state or reflect those of the United States Government or any agency thereof:

\section{PACIFIC NORTHWEST NATIONAL LABORATORY

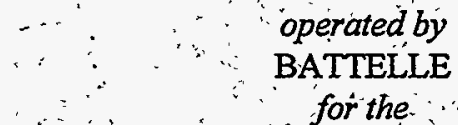 \\ UNTTED STATES DEPARTMENT OF ENERGY under Contract DE-AC06-76RLO 1830}

Printed in the United States of America

Available to DOE and DOE contractors from the

Office of Scientific and Tećhnical Yniformation,P.O. Box 62, Oak Ridge, TN 37831; prices available from (615) $576-8401$.

Available to the public from the National Technical Information Service, US. Department of Commerce, 5285 Port Royal Rd., Springfield, VA 22161 


\section{DISCLAIMER}

Portions of this document may be illegible electronic image products. Images are produced from the best available original document. 
Composite Analysis for Low-Level Waste Disposal in the 200 Area Plateau of the Hanford Site

C. T. Kincaid

M. P. Bergeron

C. R. Cole

M. D. Freshley

N. L. Hassig

V. G. Johnson

D. I. Kaplan

R. J. Serne

G. P. Streile

D. L. Strenge

P. D. Thorne

L. W. Vail

G. A. Whyatt

S. K. Wurstner

March 1998

Prepared for

the U.S. Department of Energy

under Contract DE-AC06-76RLO 1830

Pacific Northwest National Laboratory

Richland, Washington 99352 


\begin{abstract}
This report presents the first iteration of the Composite Analysis for Low-Level Waste Disposal in the 200 Area Plateau of the Hanford Site (Composite Analysis) prepared in response to the U.S. Department of Energy Implementation Plan for the Defense Nuclear Facility Safety Board Recommendation 94-2. The Composite Analysis is a companion document to published analyses of four active or planned lowlevel waste disposal actions: the solid waste burial grounds in the 200 West Area, the solid waste burial grounds in the 200 East Area, the Environmental Restoration Disposal Facility, and the disposal facilities for immobilized low-activity waste. A single Composite Analysis was prepared for the Hanford Site considering only sources on the 200 Area Plateau. The performance objectives prescribed in U.S. Department of Energy guidance for the Composite Analysis were $100 \mathrm{mrem}$ in a year and examination of a lower dose ( $30 \mathrm{mrem}$ in a year) to ensure the "as low as reasonably achievable" concept is followed. The 100 mrem in a year limit was the maximum allowable all-pathways dose for 1000 years following Hanford Site closure, which is assumed to occur in 2050. These performance objectives apply to an accessible environment defined as the area between a buffer zone surrounding an exclusive waste management area on the 200 Area Plateau, and the Columbia River.
\end{abstract}

Estimating doses to hypothetical future members of the public for the Composite Analysis was a multistep process involving the estimation or simulation of inventories; waste release to the environment; migration through the vadose zone, groundwater, and atmospheric pathways; and exposure and dose. Doses were estimated for scenarios based on agriculture, residential, industrial, and recreational land use. The radionuclides included in the vadose zone and groundwater pathway analyses of future releases were carbon-14, chlorine-36, selenium-79, technetium-99, iodine-129, and uranium isotopes. In addition, tritium and strontium- 90 were included because they exist in groundwater plumes. Radionuclides considered in the atmospheric pathway included tritium and carbon-14.

Most of the radionuclide inventory in past-practice liquid discharge and solid waste burial sites on the 200 Area Plateau was projected to be released in the first several hundred years following Hanford Site closure and a significant fraction of the inventory was projected to be released prior to closure. The maximum predicted agricultural dose outside the buffer zone was less than 6 mrem in a year in 2050 and declined thereafter. The maximum doses estimated for the residential, industrial, and recreational scenarios, were $2.2,0.7$, and $0.04 \mathrm{mrem}$ in a year, respectively, and also declined after 2050 . The radiological doses for all of the exposure scenarios outside the buffer zone were well below the performance objectives.

Significant uncertainties exist in the first iteration Composite Analysis, with the largest uncertainty associated with the inventories of key mobile radionuclides. Other sources of uncertainty in the analysis arose from the conceptual and numerical models of contaminant migration and fate in the vadose zone, and assumptions regarding source-term release models and end states. These uncertainties reflect most on the performance of past releases of liquid wastes and past disposals of solid wastes. The review of existing plumes conducted as part of the first iteration Composite Analysis revealed that the exclusive waste management area and buffer zone should be expanded to include the retired Gable Mountain Pond. The Composite Analysis demonstrated a significant separation in time between past-practice discharges 
and disposals, and active and planned disposals of solid waste, environmental restoration waste, and immobilized low-activity waste. The higher integrity disposal facilities and surface covers of these active and planned disposals delay releases, and the delayed releases do not superimpose on the plumes from the near-term past-practice disposals. 


\section{Executive Summary}

This report summarizes the efforts to complete the first iteration of the Composite Analysis for LowLevel Waste Disposal in the 200 Area Plateau of the Hanford Site (Composite Analysis). In this document, the background and performance objectives of the Composite Analysis are described. The methods used, results, and conclusions are summarized. Recommendations are made for work to be undertaken in anticipation of a second analysis.

\section{Introduction}

The Composite Analysis was prepared in response to the U.S. Department of Energy Implementation Plan for the Defense Nuclear Facility Safety Board Recommendation 94-2 and in accordance with U.S. Department of Energy guidance ${ }^{(a)}$. The purpose of the Composite Analysis was to provide an estimate of the cumulative radiological impacts from active and planned low-level radioactive waste disposal actions and other potentially interacting radioactive waste disposal sources that will remain following Hanford Site closure. This Composite Analysis is a companion analysis to published analyses involving four active or planned low-level radioactive waste disposal actions:

- solid waste burial grounds in the 200 West Area ${ }^{(b)}$

- solid waste burial grounds in the 200 East Area $^{(c)}$

- Environmental Restoration Disposal Facility ${ }^{(d)}$

- disposal facilities for immobilized low activity waste. ${ }^{(e)}$

Because these active and planned low-level radioactive waste disposal actions are located within the 200 Area Plateau of the Hanford Site, the U.S. Department of Energy, Richland Operations Office elected

(a) U.S. Department of Energy. 1996. Guidance for a Composite Analysis of the Impact of Interacting Source Terms on the Radiological Protection of the Public from Department of Energy Low-Level Waste Disposal Facilities. U.S. Department of Energy, Washington, D.C.

(b) Wood, M.I., R. Khaleel, P.D. Rittmann, A.H. Lu, S.H. Finfrock, R.J. Serne, K.J. Cantrell, and T.H. DeLorenzo. 1995. Performance Assessment for the Disposal of Low-Level Waste in the 200 West Area Burial Grounds. WHC-ED-0645, Westinghouse Hanford Company, Richland, Washington.

(c) Wood, M.I., R. Khaleel, P.D. Rittmann, S.H. Finfrock, T.H. DeLorenzo, and D.Y. Garbrick. 1996. Performance Assessment for the Disposal of Low-Level Waste in the 200 East Area Burial Grounds. WHC-SD-WM-TI-730, Rev. 0, Westinghouse Hanford Company, Richland, Washington.

(d) U.S. Department of Energy. 1994. Remedial Investigation and Feasibility Study Report for the Environmental Restoration Disposal Facility. DOE/RL-93-99, Rev. 1, U.S. Department of Energy, Richland, Washington.

(e) Mann, F.M.; R.P. Puigh II, C.R. Eiholzer, Y. Chen, N.W. Kline, A.H. Lu, B.P. McGrail, and P.D. Rittmann. Publication planned for March 1998. Hanford Immobilized Low-Activity Tank Waste Performance Assessment. DOE/RL-97-69, Rev. 0, U.S. Department of Energy, Richland, Washington. 
to complete a single Composite Analysis in support of the four disposal actions. The first iteration of the Composite Analysis only considered sources on the 200 Area Plateau because of their proximity to one another on the Plateau and the distance between the Plateau and other contaminated sites located near the shore of the Columbia River at the Hanford Site.

In addition to the active or planned sources, other radioactive sources exist or are planned for placement in the 200 Area Plateau of the Hanford Site. The sources that are the responsibility of U.S. Department of Energy include

- 149 single-shell tanks arrayed in 12 tank farms

- 28 double-shell tanks arrayed in 6 tank farms

- past-practice (pre-1988) solid waste burial grounds

- past-practice (pre-1988) liquid discharges to cribs, ditches, French drains, trenches, ponds, and reverse wells

- graphite cores from surplus production reactors

- canyon buildings and related structures (e.g. B Plant, Plutonium Uranium Extraction facility and tunnels, T-Plant, U-Plant, Reduction Oxidation Facility, and Z-Plant or Plutonium Finishing Plant).

The commercial low-level radioactive waste disposal facility operated by US Ecology, Inc., located immediately southwest of the 200 East Area was included in the Composite Analysis in accordance with guidance on the content and format of the Composite Analysis, and because of its proximity to U.S. Department of Energy operations.

\section{Performance Objectives}

The performance objectives of the Composite Analysis followed U.S. Department of Energy guidance for radiation dose to hypothetical future members of the public. ${ }^{\text {(a) }}$ The U.S. Department of Energy Order 5400.5 (and anticipated 10 CFR 834) set the primary dose limit of 100 mrem in a year, but requires that a lower dose be examined (30 mrem in a year) to ensure the "as low as reasonably achievable" (ALARA) concept is followed. The $100 \mathrm{mrem}$ in a year standard is the maximum allowable projected dose from all pathways to the hypothetical future member of the public. In accordance with U.S. Department of Energy guidance, the regulatory period of performance begins at the time of Hanford Site closure, assumed to be in 2050, and continues for 1000 years. In the Composite Analysis, an options analysis and ALARA assessment were to be prepared if the projected dose exceeded the dose constraint

(a) All doses in the Composite Analysis (except where noted) are in units of mrem effective dose equivalent in a year. 
of $30 \mathrm{mrem}$ in a year. The options and ALARA analyses were to consider alternate actions that would reduce the calculated doses and to provide an assessment of cost and benefit.

At the Hanford Site, the approach adopted to achieve comprehensive environmental management involves a complex process of negotiated decisions among the U.S. Department of Energy, the State of Washington Department of Ecology, and the U.S. Environmental Protection Agency. These negotiations 'govern the U.S. Department of Energy response to Comprehensive Environmental Response, Compensation, and Liability Act (CERCLA) and Resource Conservation and Recovery Act (RCRA) requirements for remedial actions. The selection of alternate remedial actions for study needs to be a joint decision of the three parties. At this time, the U.S. Department of Energy is negotiating the cleanup of past-practice sites on the 200 Area Plateau at the Hanford Site with the regulatory agencies. There was insufficient time and information to determine if alternate remedies are necessary from the results of the Composite Analysis and to identify them through the negotiation process. Accordingly, a single remedial action (i.e., leave in place and cover with surface barrier) was analyzed in the Composite Analysis. Consideration of any additional alternate remediations, if necessary, is deferred to the second iteration of the Composite Analysis.

The point of compliance for exposure and radiological dose predictions to a hypothetical future member of the public in the Composite Analysis was a boundary based on anticipated land use at the Hanford Site. In 1992, the Hanford Future Site Uses Working Group, ${ }^{(a)}$ comprising representatives from government entities (federal, tribal, state, and local) and constituencies (labor, environment, agriculture, economic development, municipal, and public interest groups), defined the concepts of an "exclusive" waste management area within a surrounding buffer zone on the 200 Area Plateau. This area includes the land within and surrounding the 200 East and 200 West Areas of the Hanford Site, the commercial lowlevel radioactive waste disposal facility operated by US Ecology, Inc., and the Environmental Restoration Disposal Facility. The first chapter of this report contains figures that show the relationship between the exclusive waste management area and buffer zone, and the waste sites included in the Composite Analysis. The policy of the U.S. Department of Energy is to control and maintain the land within the exclusive waste management area and buffer zone until it can be released to the public. The U.S. Department of Energy has acknowledged that many low-level radioactive waste disposal facilities may never be suitable for unconditional release to the public, and deed restrictions for use of resources such as groundwater may be necessary. The projected doses to hypothetical future members of the public from the low-level radioactive waste disposal actions and all other sources considered in the Composite Analysis were compared with the dose limit of 100 -mrem in a year and dose constraint of 30 -mrem in a year in the area between the buffer zone and the Columbia River.

\section{Methodology}

The process used in the Composite Analysis is necessarily iterative, adaptive, and flexible in order to respond to the constantly changing technical and decision-maker needs. This document discusses the

(a) Hanford Future Site Uses Working Group. 1992. The Future for Hanford: Uses and Cleanup, the Final Report of the Hanford Future Site Uses Working Group. Hanford Future Site Uses Working Group, Westinghouse Hanford Company, Richland, Washington. 
initial iteration of the Composite Analysis that has resulted in a deterministic baseline. For the first iteration, estimating doses to hypothetical future members of the public for the Composite Analysis was a multistep process.

- The first step involved estimating the inventories of radionuclides for the various sources present or to be placed on the 200 Area Plateau. A complete and accurate inventory of sources of radioactive materials disposed to ground and stored at the Hanford Site does not exist. Consequently, an inventory had to be estimated based on process knowledge and plans for environmental restoration.

- The second step in the analysis involved calculating the radionuclide release from the various sources, based on knowledge of waste form characteristics and long-term performance calculations (recharge characteristics and geochemical behavior).

- The third step involved predicting transport through the vadose zone to the water table under transient flow conditions. The recharge rate in the vadose zone was allowed to vary with the application of different surface treatments and covers (barriers).

- The fourth step involved predicting transport through the unconfined aquifer. The aquifer was modeled as it responded to the cessation of wastewater discharges from Hanford Site operations and its water table declined during the post-closure period.

- The fifth step in the analysis involved calculating dose based on exposure scenarios for hypothetical future members of the public at locations on the present Hanford Site and comparing those doses with the dose limit and constraint standards.

The Data Quality Objectives process was applied to the Composite Analysis for the 200 Area Plateau although no new data were collected. Existing data on source inventories, waste site characteristics, and the vadose zone and groundwater were compiled and used with release and transport models to predict future radionuclide concentrations in environmental media (air, soil, and groundwater) and resulting radiation doses. However, the U.S. Department of Energy guidance for the Composite Analysis specifically prohibited improvement of data through the gathering and analysis of samples for the first iteration analysis.

Four exposure scenarios defined by the Hanford Site Risk Assessment Methodology were used in the Composite Analysis to estimate radiation doses to hypothetical future members of the public. These four scenarios were used to examine the potential variability in future land use. The four Hanford Site Risk Assessment Methodology exposure scenarios, agricultural, residential, industrial, and recreational, were developed for the Hanford Site to facilitate evaluation of risk related to CERCLA remedial investigations and RCRA facility investigations. Groundwater transport was the primary exposure pathway considered in the Composite Analysis for the 200 Area Plateau. However, a limited analysis of exposure and dose from the atmospheric pathway was included in the all-pathways dose assessment.

The waste source inventories at the Hanford Site were screened to select key radionuclides for study in the Composite Analysis. The effort to screen the list of radionuclides benefited from published 
performance assessment and environmental impact analyses and field observations (characterization and monitoring data). Those radionuclides identified as potentially significant contributors to dose in performance assessments for the 200 West and 200 East Area post-1988 burial grounds and the Tank Waste Remediation System immobilized low-activity waste, and the risk assessment for the Environmental Restoration Disposal Facility were assumed to be the key radionuclides in the Composite Analysis. The radionuclides included in the groundwater pathway analysis for future sources included carbon-14, chlorine-36, selenium-79, technetium-99; iodine-129, and uranium isotopes (uranium-233, $-234,-235,-236$, and -238 ). In addition, tritium and strontium- 90 were included because they currently form groundwater plumes at the Hanford Site. Radionuclides considered in the atmospheric pathway included tritium, carbon-14, and radon-222. However, the surplus graphite cores of production reactors were identified as the only potentially significant source for the atmospheric pathway, and they release tritium and carbon-14, but have no appreciable inventory of radon-222 or its parents.

\section{Results}

Prior to conducting the analysis, a review of existing radionuclide contamination in the unconfined aquifer revealed the presence of a strontium- 90 plume beneath the decommissioned Gable Mountain Pond. Strontium-90 is relatively highly sorbed in Hanford groundwater and sediments, (e.g., distribution coefficient of $20 \mathrm{~mL} / \mathrm{g}$ ), and will be reduced relatively soon by decay (i.e., half-life of 28.78 years). Because the contamination is relatively immobile and is in the vadose zone sediment column and the groundwater beneath this retired pond, it is assumed it will not be further remediated. In the following discussion of dose outside the buffer zone, the assumption is made that Gable Mountain Pond is included in the exclusive waste management area and buffer zone.

In the Composite Analysis, most of the radionuclide inventory in past-practice liquid discharge and solid waste burial sites on the 200 Area Plateau was projected to be released within the first several hundred years following Hanford Site closure. A significant fraction of that radionuclide inventory was projected to release prior to Hanford Site closure in 2050, and peak concentrations of key radionuclides in groundwater are predicted to occur before closure in 2050. For the agricultural exposure scenario, which results in the highest predicted doses, the maximum dose from the key radionuclides and all sources considered in the Composite Analysis outside the buffer zone at Hanford Site closure was less than $6 \mathrm{mrem}$ in a year. The maximum dose from the agricultural scenario declined thereafter. The maximum doses estimated for the other scenarios, i.e., residential, industrial, and recreational, were $2.2,0.7$, and $0.04 \mathrm{mrem}$ in a year, respectively, and also declined after 2050. The groundwater plumes from existing and future sources considered in the analysis are predicted to migrate away from the 200 Area Plateau in two primary directions, to the east and southeast following the major existing plumes, and to the north. The groundwater flow paths gradually change from an initial radial pattern from the 200 Area Plateau, to an easterly direction as the water table changes in response to cessation of wastewater disposal.

A brief ALARA assessment showed the cost to society associated with population dose during the 1000 -year period after Hanford Site closure was between $\$ 4$ million and $\$ 40$ million. This estimated range was based on an approximate average dose to an individual of $4 \mathrm{mrem}$ in a year from the agricultural scenario for the 1000-year period, an agricultural community of 1000 people, and a cost to society of between $\$ 1000$ and $\$ 10,000$ per person rem. The dose and community population estimates are 
conservative; therefore, the cost estimate is biased high. Based on this estimated cost to society, a thorough options analysis and ALARA assessment that would provide a detailed investigation of alternate remediations was not justified at this time.

The radiological doses for all of the exposure scenarios outside the buffer zone were well below the dose limit of 100 mrem in a year and the dose constraint of $30 \mathrm{mrem}$ in a year. However, the predicted radionuclide concentrations in groundwater within the exclusion and buffer zones demonstrate the need for continued land use control and monitoring programs at the Hanford Site to meet the primary objective of the long-term protection of human health and safety. This analysis of future radiological dose to the maximally exposed individual on lands outside the buffer zone supports the concept of retiring the Hanford Site boundary to the buffer zone boundary at the time of Hanford Site closure in 2050. However, the conclusions of the Composite Analysis depend on the inclusion of Gable Mountain Pond in the exclusive waste management area, and continued land use controls by the U.S. Department of Energy to prohibit use of resources (groundwater and land) within the exclusive waste management area and buffer zone for the 1000-year period of analysis.

Significant uncertainties exist in the first iteration of the Composite Analysis, with the largest uncertainty associated with the inventories of key radionuclides discharged and disposed at specific facilities by the time of Hanford Site closure. The inventory for post-closure sources at the Hanford Site was assembled from inventories for specific wastes, waste forms, and waste discharge sites. These prior efforts to define specific inventories occurred at different times, under different U.S. Department of Energy programs, and were not coordinated to produce a single and consistent database for wastes that will reside at the Hanford Site after closure. Inventory characterization is also incomplete because of the costs and limitations of characterization efforts to address specific questions. Consequently, the total inventory of key mobile radionuclides examined in the Composite Analysis includes significant uncertainties. A more thorough examination of uncertainty with respect to inventory must follow development of a more consistent inventory where best estimates of both magnitude and final location of radionuclides are included. It would be advantageous to have an inventory model that could generate alternate realizations based on the range of process parameters assigned to Hanford Site facility operations.

Another source of uncertainty in the analysis arose from the conceptual and numerical models of the contaminant migration and fate in the vadose zone. The conceptual model has considered transient recharge rates and spatially varying retardation factors that govern the leaching of waste and its migration. The recharge rates were designed to represent periods of high recharge and leaching prior to the placement of covers and barriers, and periods of low recharge associated with the vegetation of the site or the placement of covers. The model of geochemical mobility has taken into account the character of the waste and the neutralizing effects of contact with soils and sediments. However, the model has not included preferential pathways such as clastic dikes or unsealed well bores. The vadose zone model employed in this first iteration of the Composite Analysis is one-dimensional, and, therefore, was not able to represent multidimensional effects. The model adopted did not consider the potential influence of nonisothermal or high-density fluids on the migration and fate of radionuclides. Currently, the Tank Waste Remediation System Vadose Zone Program is working to gather field data and establish the conceptual models for contaminant migration and fate in the vadose zone beneath tank farms. Because this program 
has just begun and an effort to integrate and coordinate it with other vadose zone studies has just gotten underway, examination of multiple conceptual models of the vadose zone pathway has been deferred until the second iteration Composite Analysis.

Other sources of uncertainty included assumptions regarding source-term release models, and end states for waste sites. These sources of uncertainty are not believed to be as significant as the uncertainties in the inventory estimates or pathway analyses.

\section{Conclusions}

Conduct of the first iteration Composite Analysis has revealed the exclusive waste management area and buffer zone should be extended to include Gable Mountain Pond. This first analysis has also shown a significant separation in time between past-practice discharges and disposals, and active and future disposals at the Hanford Site. Liquid discharge sites including cribs and specific retention trenches, past leaks from single-shell tanks, future sluicing losses from single-shell tanks, and pre-1988 solid waste burial grounds all release to the water table in the coming decades. Significant portions of their inventories release within the next century. Active and planned disposals release much later. When modeled with a high recharge rate prior to the placement of final covers, the post-1988 solid waste burial grounds are shown to begin release of their estimated highly mobile inventories of chlorine-36, selenium-79, and technetium-99 within 200 years from the present. However, a scenario that takes credit for the waste isolation afforded by burial containers showed mean travel times of these mobile radionuclides were between 650 and 1150 years. A mean travel time of approximately 1000 years was associated with burial grounds destined to receive the majority of future solid waste disposals. Neither scenario indicated release of radionuclides exhibiting adsorption in the vadose zone, i.e., carbon-14, iodine-129, or uranium. Neither the Environmental Restoration Disposal Facility nor the Tank Waste Remediation System immobilized low-activity waste were shown to release in the 1000-year analysis period. Thus, the higher integrity disposal facilities and surface covers cause releases from active and planned disposals to occur much later in time and not superimpose on near-term releases that have occurred and will occur from the liquid disposal facilities.

This analysis has also shown that concentrations of radionuclides in the aquifer are much higher during the period from now until Hanford Site closure than they will be after closure. Similarly, doses based on the assumed use of groundwater from now until Hanford Site closure are much higher in the period leading up to site closure than in the period after. Contaminants in the aquifer today are a result of the early discharge of large quantities of liquid waste or direct injection at reverse well sites. Consequently, the resulting plumes had relatively high concentrations, and continue to exhibit relatively high peak values today despite years of groundwater transport, radioactive decay, and dispersal. Past rates of liquid discharge, (e.g., 12,000 curies per year of tritium in $\sim 400$ cubic meters per day of liquid discharge between 1984 and 1986), were orders of magnitude higher than any predicted future release rates to groundwater.

Future releases to the aquifer from the liquid discharge sites, tank leaks, tank losses, and burial grounds will occur, but with a greatly diminished driving force as compared to past releases. This is because natural recharge rates and not large liquid releases will drive future leaching and movement. 
While more curies of specific radionuclides such as technetium-99 will leach into the aquifer in the future than are present today, they will be introduced at lower rates. Because flow in the unconfined aquifer under the 200 Area Plateau will remain relatively constant, these lower projected release rates from sources in the vadose zone will create plumes with lower peak concentrations. Consequently, the Composite Analysis has shown that future doses through time of Hanford Site closure and beyond will be dominated by the existing plumes of tritium and iodine-129. As tritium concentrations are reduced by migration to the Columbia River, dispersion, and decay, the iodine-129, which is less mobile than tritium in Hanford Site sediments, begins to dominate dose projections.

Dose estimates during the post-closure period are low, less than 6 mrem in a year to the maximally exposed individual in the agricultural exposure scenario. The area of the unconfined aquifer predicted to yield estimates of dose above $4 \mathrm{mrem}$ in a year for the agricultural scenario decreases from approximately $40 \mathrm{~km}^{2}$ in 2050 to zero by 2085 . If inventories of the mobile radionuclides assigned to liquid discharge sites, past tank leaks, future tank sluicing losses, and pre-1988 solid waste burial grounds were increased, higher doses could be tolerated before approaching the dose constraint of $30 \mathrm{mrem}$ in a year. If inventories of key mobile nuclides assigned to active disposals were increased beyond the current inventory limits of the facility, protocols require an analysis to ensure the safety of the disposal action prior to waste acceptance. When high concentrations of key radionuclides appear in waste delivered for disposal, the waste acceptance criteria and protocols employed at active solid waste burial grounds reveal their presence. Because of their potential adverse impact on long-term radiological dose, these radionuclides (when in high concentrations) are contained in waste forms or containers that inhibit leaching and release. Thus, greater inventories of key nuclides could be disposed in active or planned disposals only after a thorough analysis of their potential impact and appropriate actions to ensure their safe disposal.

As a companion analysis for the performance and risk assessments associated with current and planned low-level radioactive waste disposal actions at the Hanford Site, the Composite Analysis has shown that the active and planned dry disposals are safe and will not contribute significantly to radiation dose to hypothetical future members of the public for the 1000-year period following Hanford Site closure.

\section{Future Work}

Three key areas where additional data and information will contribute to greater confidence in the second iteration Composite Analysis are

- a fully consistent Hanford Site-wide inventory

- an accepted suite of conceptual models of liquid and dry disposals

- a tested linkage of inventory, release, and vadose zone models sufficient to explain existing plumes.

A Hanford Site-wide inventory model should be created, or an existing model modified, to provide a probabilistic estimate of the magnitude (e.g., mean value and standard deviation) of all key radionuclides 
for all significant disposals at the time of Hanford Site closure. The list of key radionuclides should be reevaluated to consider those found to have greater mobility when disposed with organic chelating agents at liquid discharge sites. The concept of mass conservation should be used to ensure the probabilistic distributions are fully consistent with known quantities generated at or imported to the Hanford Site. The inventory model should include credible estimates of radionuclides lost to the atmosphere, discharged as liquid and disposed as solids. The inventory model must include liquids discharged to facilities (cribs, French drains, reverse wells, and specific retention trenches), leaked from tanks, and forecast to be lost from tanks during waste recovery operations. The model should include estimates of radionuclides retained in canyon buildings, permanent filters, and tunnels. It should include a means of estimating the disposal of key mobile radionuclides to all facilities, and it should address the secondary waste streams coming from future facilities and programs including tank waste recovery operations, chemical separations plants, and plants designed to immobilize both low-activity and high-level wastes.

Efforts are now ongoing to provide greater insight into the present location, and past and present mobility of contaminants from past tank leaks at the Hanford Site. This information and that from the study of past-practice liquid discharge and dry disposal sites will lead to greater understanding of contaminant migration in the vadose zone at the Hanford Site. From this knowledge will come peerreviewed and accepted conceptual models for liquid discharges, tank leaks and losses, and solid waste burials. These conceptual models will identify the applicable recharge rates, geohydrologic formations, dimensionality, and geochemistry of waste-sediment interactions. The potential value of more sophisticated and higher dimensionality vadose zone models to future Composite Analyses should be evaluated. In the second iteration Composite Analysis, the range of conditions defining uncertainty in radionuclide migration should be simulated to capture the associated uncertainty in dose estimates.

Finally, the inventory and conceptual models associated with specific facilities should be tested and evaluated where possible. Each existing vadose zone and groundwater plume is the result of a past waste discharge or disposal. Of particular interest are facilities that received, or are suspected to have received, large inventories of key mobile radionuclides. Of special interest will be those sites with highly uncertain and potentially significant contributions to the composite dose. For such sites, efforts should be made to obtain sound estimates (mean and standard deviation) of the spatial distribution of the mass of contaminants in the vadose zone and unconfined aquifer. Application of inventory, release model, and vadose zone contaminant migration models should yield estimates of mass in the vadose zone and released to the aquifer. These estimates should be in agreement with mass estimates based on field observations. Inventory estimates that can not be supported by reasonable release and vadose zone models when compared to vadose zone and groundwater plume data should be revisited to ensure that overly conservative or bounding estimates of inventory have not been assigned to liquid discharges. If possible, this effort should include facilities that gave rise to plumes in the vadose zone that have not yet reached the water table. Confidence in the present state of contamination in the vadose zone is central to building confidence in projections of future release. Where the results would support the understanding of major contributors to total dose, efforts should be made to sample for and interpret the plume mass of all key mobile radionuclides. 


\section{Acronyms}

ACGIH

ALARA

CERCLA

CFEST

Composite

Analysis

DBBP

DNFSB

DOE

DOE-HQ

DOE-RL

DOH

DQO

DWS

Ecology

EDE

EDTA

EIS

EPA

ERC

ERDA
American Conference of Governmental Industrial Hygienists

as low as reasonably achievable

Comprehensive Environmental Response, Compensation, and Liability Act

Coupled Fluid, Energy, and Solute Transport (code)

Composite Analysis for Low-Level Waste Disposal in the 200 Area Plateau of the Hanford Site

dibutyl butyl phosphate

Defense Nuclear Facility Safety Board

U.S. Department of Energy

U.S. Department of Energy, Headquarters

U.S. Department of Energy, Richland Operations Office

State of Washington Department of Health

data quality objective

drinking water standards

State of Washington Department of Ecology

effective dose equivalent

ethylenediaminetetraacetic acid

environmental impact statement

U.S. Environmental Protection Agency

Environmental Restoration Contractor

U.S. Energy Research and Development Administration 
ERDF

FFTF

GIS

HDW

HEAST

HEDTA

HEPA

HFSUWG

HGWP

HSRAM

HTI

ICRP

ILAW

IRIS

LLW

MEPAS

MMEDE

$\mathrm{NPH}$

NPL

ONWI

ORIGEN2

OU
Environmental Restoration Disposal Facility

Fast Flux Test Facility

geographic information system

Hanford Defined Waste

Health Effects Assessment Summary Tables

$\mathrm{N}$-(2-hydroxyethyl) ethylenediaminetetraacetic acid

high-efficiency particulate air filter

Hanford Future Site Uses Working Group

Hanford Groundwater Project

Hanford Site Risk Assessment Methodology

Hanford Tanks Initiative

International Commission on Radiological Protection

immobilized low-activity waste

Integrated Risk Information System

low-level waste

Multimedia Environmental Pollutant Analysis System

Multimedia Modeling Environmental Database Editor

normal paraffinic hydrocarbon (or kerosene)

National Priorities List

Office of Nuclear Waste Isolation

Oak Ridge Isotope Generation and Depletion (code)

operable unit 


\begin{tabular}{|c|c|}
\hline PCB & polychlorinated biphenyl \\
\hline PFP & Plutonium Finishing Plant \\
\hline PNNL & Pacific Northwest National Laboratory \\
\hline PUREX & Plutonium Uranium Extraction (Plant) \\
\hline RCRA & Resource Conservation and Recovery Act \\
\hline REDOX & Reduction-Oxidation (S Plant) \\
\hline $\mathrm{RfD}$ & Reference Dose \\
\hline RFP & Request for Proposal \\
\hline $\mathrm{RI} / \mathrm{FS}$ & remedial investigation and feasibility study \\
\hline ROD & record of decision \\
\hline SALDS & State-Approved Land Disposal Site \\
\hline SARA & Superfund Amendments and Reauthorization Act \\
\hline SEPA & State Environmental Policy Act \\
\hline SIF & Summary Intake Factors \\
\hline STOMP & Subsurface Transport Over Multiple Phases (code) \\
\hline $\mathrm{S} / \mathrm{V}$ & surface to volume (ratio) \\
\hline SWITS & Solid Waste Information Tracking System \\
\hline TBP & tributyl phosphate \\
\hline TEDF & Treated Effluent Disposal Facility \\
\hline TLV & threshold limit value \\
\hline TRAC & Track Radioactive Components (model) \\
\hline
\end{tabular}

xvii 
Tri-Party

Agreement

TRU

TWRS

UDFs

UTFs

VOC

VOI

WIPP SEIS
Hanford Federal Facility Agreement and Consent Order Between the U.S. Environmental Protection Agency, the U.S. Department of Energy, and the State of Washington Department of Ecology

transuranic

Tank Waste Remediation System

unit dose factors

unit transport factors

volatile organic compounds

Value of Information (analysis)

Waste Isolation Pilot Plant Supplementary Environmental Impact Statement 


\section{Acknowledgments}

The authors wish to thank R. D. Hildebrand, R. M. Ecker, R. M. Smith, and P. D. Meyer for their insightful review of the report and many useful discussions. Thanks also to M. I. Wood, F. M. Mann, P. M. Rogers, R. Khaleel, F. M. Coony, and L. P. Diediker who have generously given their time and provided the Pacific Northwest National Laboratory staff working on the Composite Analysis with information, data, and insight into the Hanford Site's past and future disposals.

The authors also wish to gratefully acknowledge the tireless and timely technical support provided by Dr. Sumant Gupta of CFEST, Inc. His experience with the CFEST96 code and his valuable technical suggestions and assistance were instrumental in successful completion of the groundwater transport simulations of existing contaminant plumes and future releases. 


\section{Contents}

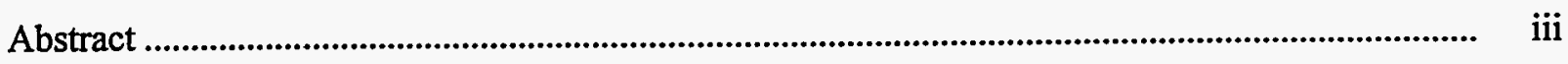

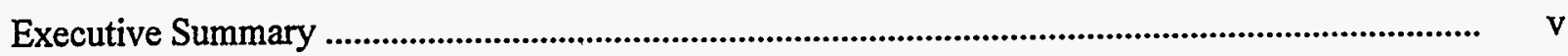

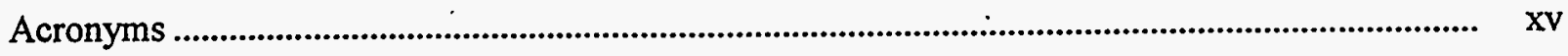

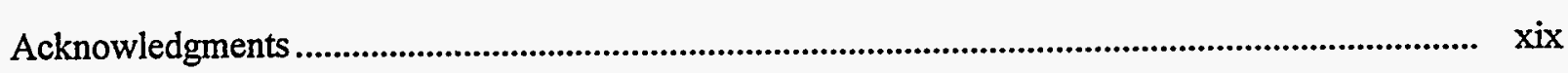

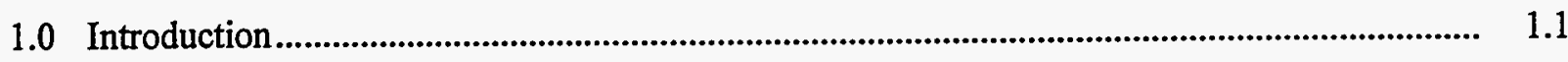

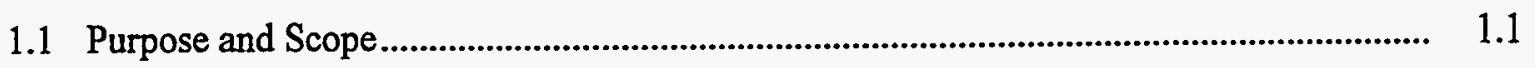

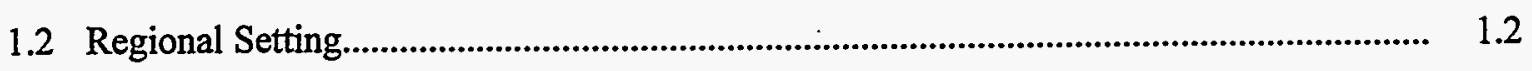

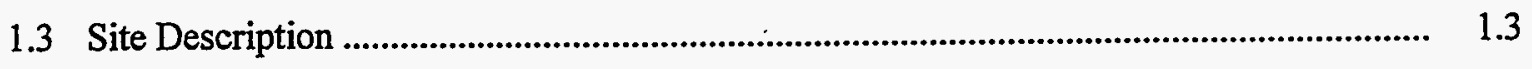

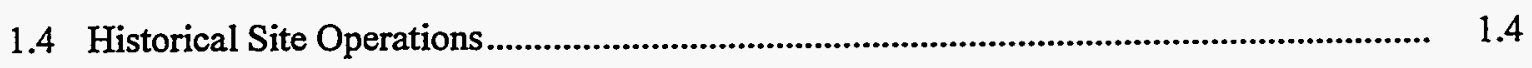

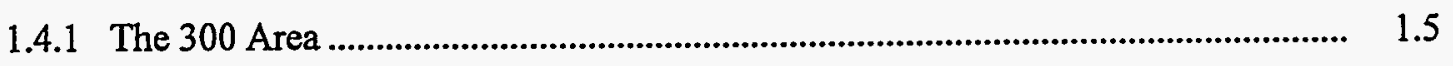

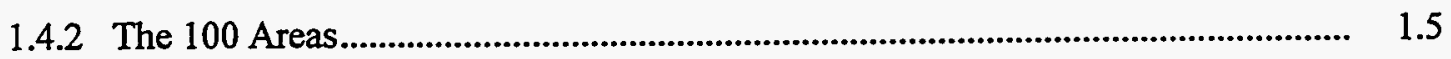

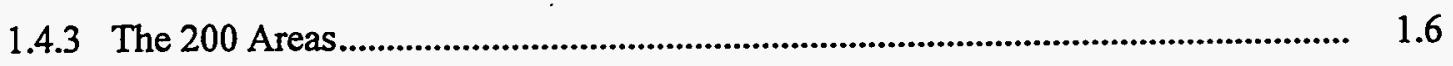

1.5 Low-Level Waste Disposal Facilities at Hanford ......................................................... . 1.7

1.5.1 Active or Planned Disposal Facilities ............................................................... 1.7

1.5.2 Other Sources of Radioactive Contamination ................................................. 1.8

1.6 Operation of Low-Level Waste Disposal Facilities.................................................... 1.9

1.6.1 Active or Planned Disposal Facilities ................................................................. 1.9

1.6.2 Other Sources of Radioactive Contamination .................................................. 1.10

1.7 Waste Management Area Boundary ............................................................................... 1.12

1.8 Performance Objectives of the Composite Analysis ..................................................... 1.13 
2.0 Composite Analysis Process .............................................................................................. 2.1

2.1 Motivation for the Process Used in the Composite Analysis ............................................ 2.1

2.2 Process Flow Diagram ………………………………….....................................................

2.3 The Impact of Various Types of Errors on Uncertainty in Dose ....................................... 2.5

2.4 Decisions Made Throughout the Composite Analysis.................................................... 2.6

2.5 Data Quality Objectives............................................................................................. 2.7

2.5.1 The Standard DQO Approach ............................................................................... 2.7

2.5.2 Modified DQO Process Applied to Model Predictions ........................................... 2.8

2.5.2.1 Steps 1 through 4 .............................................................................. 2.9

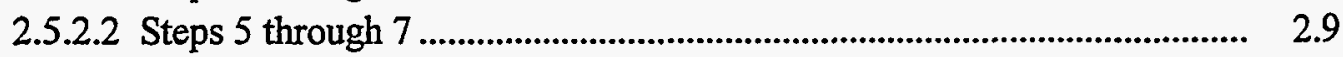

2.6 Initial Iteration of Composite Analysis............................................................................ 2.10

2.7 Subsequent Iterations of the Composite Analysis .......................................................... 2.12

3.0 Source Term Development ……......................................................................................... 3.1

3.1 Sources of Radioactive Material....................................................................................

3.1.1 Solid Waste Burial Grounds ...................................................................................

3.1.2 Environmental Restoration Disposal Facility ........................................................ 3.2

3.1.3 Tank Waste Remediation System Waste ............................................................. 3.3

3.2 Sources that Could Superimpose .........................................................................................

3.3 Sources Excluded..................................................................................................... 3.5

3.3.1 Chemical Separation Plants ............................................................................... 3.5

3.3.2 Atmospheric Pathway …………........................................................................ 3.6

3.4 Estimation of Source Inventory and Release Rate........................................................... 3.7

3.4.1 Selection of Key Radionuclides for Study.......................................................... 3.7

3.4.1.1 Low-Activity Waste from Tanks .............................................................. 3.8 
3.4.1.2 Solid Waste Burial Grounds .................................................................. 3.8

3.4.1.3 Radionuclides Selected by Screenings in Other Analyses........................ 3.9

3.4.1.4 Uranium Daughters.................................................................................. 3.9

3.4.1.5 Radionuclides Included in the Groundwater Pathway............................. 3.10

3.4.2 Solid Waste Burial Grounds ................................................................................ 3.11

3.4.2.1 Suspect Transuranic Waste and Pre-1988 Inventory ................................ 3.12

3.4.2.2 Future Disposal Inventories ................................................................. 3.12

3.4.2.3 Estimation of Non-Reported Radionuclides ............................................. 3.12

3.4.3 Environmental Restoration Disposal Facility ...................................................... 3.14

3.4.4 Hanford Tanks ...................................................................................................... 3.15

3.4.4.1 Immobilized Low-Activity Waste from Tanks......................................... 3.16

3.4.4.2 Single-Shell Tank Farms - Tank Leaks and Slurry Losses ...................... 3.16

3.4.4.3 Single-Shell Tank Farm Residuals ........................................................... 3.18

3.4.4.4 Double-Shell Tank Farm Residuals......................................................... 3.19

3.4.5 CERCLA Sources.......................................................................................... 3.19

3.4.5.1 Description of CERCLA Sources ........................................................... 3.20

3.4.5.2 Assumptions .......................................................................................... 3.20

3.4.5.3 CERCLA Radionuclide Inventories ........................................................ 3.22

3.4.6 US Ecology Commercial LLW Site .................................................................. 3.23

3.4.7 Graphite Cores from Production Reactors............................................................. 3.24

3.5 Inventory Compilation for Composite Analysis .............................................................. 3.25

3.5.1 The Excel ${ }^{\mathrm{TM}}$ Workbook ..................................................................................... 3.25

3.5.2 Multiple Sources of Inventory Data, Inconsistencies in Totals ............................ 3.25

3.5.2.1 Differences in the Kupfer et al. (1997), Agnew et al. (1997), and

Schmittroth et al. (1995) Totals ............................................................. 3.27

3.5.2.2 Carbon-14 .............................................................................................. 3.28

3.5.2.3 Chlorine-36........................................................................................ 3.28

3.5.2.4 Iodine-129............................................................................................ 3.29

3.5.2.5 Selenium-79 ........................................................................................... 3.29

3.5.2.6 Technetium-99.................................................................................... 3.30

3.5.2.7 Uranium-238............................................................................................. 3.31

$4.0 \quad$ Performance Analysis ......................................................................................................... 4.1

4.1 Methodology and Results .......................................................................................... 4.1 
4.1.1 Source Release Models................................................................................ 4.2

4.1.1.1 Background............................................................................................. 4.2

4.1.1.2 Source Term Release Model Implementation ............................................ 4.5

4.1.2 Vadose Zone Model....................................................................................... 4.6

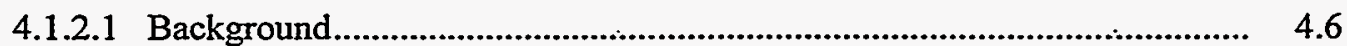

4.1.2.2 Vadose Zone Model Implementation ...................................................... 4.10

4.1.2.3 Vadose Zone Model Results..................................................................... 4.11

4.1.2.4 Vadose Zone Model Sensitivity............................................................... 4.15

4.1.3 Groundwater Flow Model.............................................................................. 4.16

4.1.3.1 Background............................................................................................ 4.17

4.1.3.2 Groundwater Flow Model Selection, Chronology, and
Implementation ................................................................................... 4.19

4.1.3.3 Groundwater Flow Model Results.......................................................... 4.24

4.1.4 Groundwater Transport Model ........................................................................ 4.25

4.1.4.1 Background......................................................................................... 4.25

4.1.4.2 Groundwater Transport Model Implementation ...................................... 4.26

4.1.4.3 Groundwater Transport Model Results................................................... 4.29

4.1.4.4 Groundwater Transport Model Sensitivity ................................................ 4.31

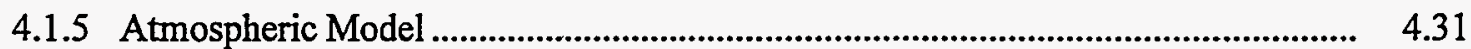

4.1.5.1 Background........................................................................................... 4.32

4.1.5.2 Atmospheric Model Implementation ...................................................... 4.32

4.1.5.3 Atmospheric Model Results ................................................................... 4.32

4.1.6 Exposure and Dose Model................................................................................. 4.33

4.1.6.1 Background.................................................................................................. 4.33

4.1.6.2 Exposure and Dose Model Implementation............................................. 4.34

4.1.6.3 Exposure and Dose Model Results ........................................................... 4.35

4.1.6.4 Exposure and Dose Model Sensitivity....................................................... 4.38

4.2 Comparison with Other 200 Area Modeling Analyses..................................................... 4.38

4.2.1 Hanford Tanks Initiative.................................................................................... 4.39

4.2.2 200 Area Solid Waste Burial Ground Performance Assessments ........................... 4.40

4.2.3 Commercial/Low Level Waste Site Assessment ................................................. 4.41

4.2.4 Remedial Investigation and Feasibility Study for the Environmental Restoration Disposal Facility.............................................................................. 4.41 
4.2.5 Environmental Assessment of Surplus Production Reactors

4.2.6 TWRS ILAW Performance Assessment.............................................................. 4.42

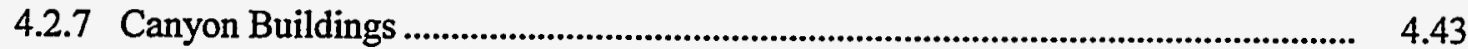

4.3 Model Calibration and Comparisons of Results with Observations ................................. 4.43

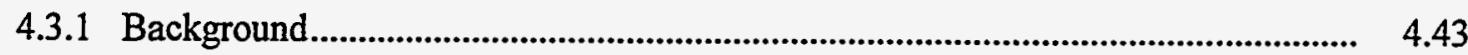

4.3.2 Predicted Contaminant Releases to Groundwater from the Vadose Zone.............. 4.45

4.3.3 Predicted Groundwater Contaminant Concentrations in the Aquifer ..................... 4.48

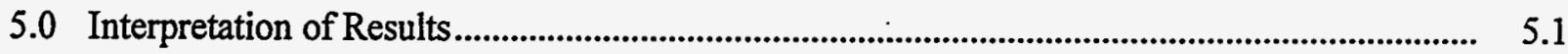

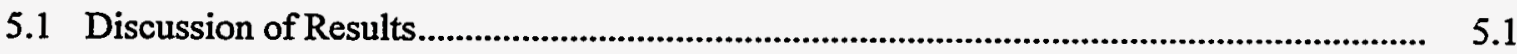

5.1.1 Comparison with the Primary Dose Limit ....................................................... 5.1

5.1.2 The Influence of Uncertain Inventories and Contaminant Mobility....................... 5.3

5.1.2.1 The Influence of an Uncertain Inventory............................................ 5.4

5.1.2.2 Uncertainty in Contaminant Mobility.................................................. 5.8

5.2 Interpretation of Composite Analysis Results ......................................................... 5.11

5.2.1 Consistency with Previous Performance Assessments with the ERDF RI/FS ...... 5.11

5.2.2 Other Sites in the Exclusive Waste Management Area and Buffer Zone............... 5.13

5.2.2.1 Existing Plumes .......................................................................................... 5.13

5.2.2.2 . Liquid Discharge Sites....................................................................... 5.14

5.2.2.3 Past Tank Leaks, Future Tank Losses, and Tank Residuals .................... 5.17

5.2.2.4 Pre-1988 Solid Waste Burial Grounds..................................................... 5.17

5.2.2.5 Graphite Cores..................................................................................... 5.18

5.2.2.6 Chemical Separation Plants and Associated Facilities ............................ 5.18

5.2.2.7 Commercial Low-Level Radioactive Waste Disposal Facility ................ 5.19

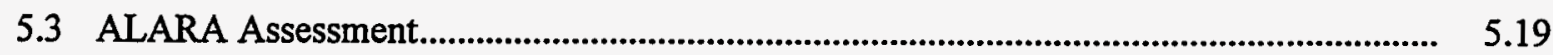

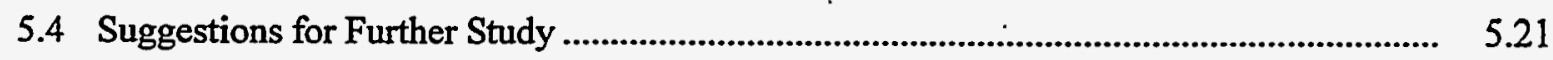

5.4.1 The Inventory .............................................................................................. 5.21

5.4.2 Waste Handling and Engineered Barriers.................................................... 5.23 
5.4.3 Environmental Mobility and Models................................................................. 5.24

5.4.4 Inclusion of Additional Sources ....................................................................... 5.25

5.4.5 Use of Data Quality Objectives ............................................................................ 5.25

5.4.6 Linkage to the Hanford Groundwater Project, the 200 Area Characterization Program, and the TWRS Hanford Tanks Initiative ............................................... 5.26

6.0 References ...................................................................................................................

Appendix A - Solid Waste Inventories........................................................................................ A.1

Appendix B - Environmental Restoration Waste Site Inventories .................................................. B.1

Appendix C - Environmental Restoration Sites Without Inventories .............................................. C.1

Appendix D - Hanford Composite Analysis Source Term Release Models ....................................... D. D.1

Appendix E - Distribution Coefficient Selection for the Composite Analysis................................... E. E.1

Appendix F - Evaluation of Unit Dose Factors ............................................................................... F.1 


\section{Tables}

2.1 DQO Steps 1 Through 4 for the Hanford Site Composite Analysis

3.1 Inventory of Key Radionuclides fơr the Solid Waste Burial Grounds

3.2 Inventory of Key Radionuclides for ERDF

3.3 Inventory of Key Radionuclides for TWRS Low-Activity Waste..

3.4 Inventory of Key Radionuclides for TWRS Single-Shell Tanks

3.5 Inventory of Key Radionuclides for TWRS Double-Shell Tanks.

3.6 Ratios of Cesium-137, Uranium, and Plutonium for Waste Site Groups.

3.7 Inventories of Uranium-238, Technetium-99, and Iodine-129 for Liquid Discharge (216) Sites from the SWITS Database.

3.8 Inventories of Key Radionuclides for CERCLA Sites.

3.9 Inventory of Key Radionuclides for US Ecology

3.10 Inventory of Key Radionuclides for the Decommissioned Reactor Cores

3.11 Summary Table of Inventories Considered in the Composite Analysis

4.1 Summary of Key Assumptions for the Source Release Models

4.2 Description of Worksheets in the Composite Analysis.xls Workbook.

4.3 Source Geometry Data Required for Release Models in the Source Site Worksheet

4.4 Chemical Classification of Waste Sites

4.5 Recharge Rates Applied to Waste Sites

4.6 Geologic Well Logs for the Vadose Zone Model

4.7 Sediment Types and Unsaturated Flow Model Parameters Used in the Composite Analysis ...

4.8 Summary of Key Assumptions for the Vadose Zone Model 
4.9 Summary of Key Assumptions for the Groundwater Flow Model ........................................ 4.87

4.10 Major Hydrogeologic Units Used in the Site-Wide Three-Dimensional Model........................ $\quad 4.89$

4.11 Summary of Key Assumptions for the Groundwater Transport Model ....................................

4.12 Summary of Key Assumptions for the Atmospheric Model .................................................... 4.92

4.13 Summary of Key Assumptions for the Exposure and Dose Model ...................................... 4.93

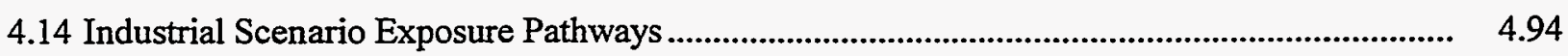

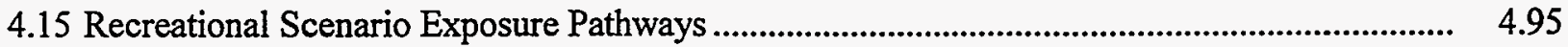

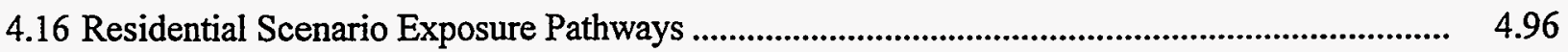

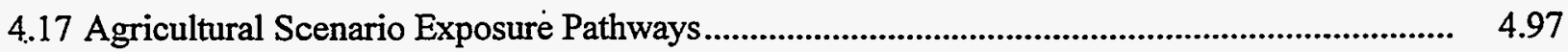

4.18 Unit Dose Factors (UDFs) Used in the Composite Analysis ............................................ 4.98

4.19 Comparison of Unit Dose Factors Between the TWRS Low-Level Tank Waste

Interim Performance Assessment and the Composite Analysis................................................ 4.99 


\section{Figures}

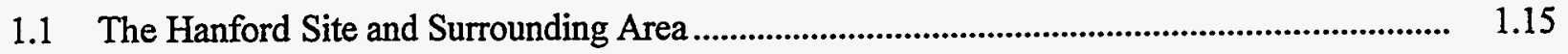

1.2 Waste Storage and Disposal Facilities in the 200 Area .................................................... 1.16

1.3a The Exclusive Waste Management Area and Buffer Zone of the 200 Area Plateau at the Hanford Site.

1.3b Waste Sites of the 200 West Area and Immediate Vicinity Considered in the Composite Analysis

1.3c. Waste Sites of the 200 East Area and Immediate Vicinity Considered in the Composite Analysis .

1.4200 Area Plateau with Exclusive Waste Management Area and Buffer Zone

2.1 Composite Analysis Process Flow Diagram

2.2 Modified Decision Performance Goal Diagram

4.1 Transport and Exposure Pathways Considered in the Composite Analysis

4.2 Relationship Among Software Elements in the Composite Analysis

4.3 Implementation of the Release and Vadose Zone Transport Models in the Excel ${ }^{\mathrm{TM}}$ Spreadsheet..

4.4 Convolution Method for Estimating Cumulative Flux to the Water Table 4.103

4.5a Cumulative Release of Technetium-99 from All Sources to the Water Table from 1940 to 2150

4.5b Cumulative Release of Technetium-99 from All Sources to the Water Table from 1940 to 3000

4.6a Cumulative Release of Iodine-129 from All Sources to the Water Table from 1940 to 2150

4.6b Cumulative Release of Iodine-129 from All Sources to the Water Table from 1940 to 3000 
4.7a Cumulative Release of Carbon-14 from All Sources to the Water Table from 1940 to 2150

4.7b Cumulative Release of Carbon-14 from All Sources to the Water Table from 1940 to 3000

4.8a Cumulative Release of Chlorine-36 from All Sources to the Water Table from 1940 to 2150

4.8b Cumulative Release of Chlorine-36 from All Sources to the Water Table from 1940 to 3000

4.9a Cumulative Release of Selenium-79 from All Sources to the Water Table from 1940 to 2150

4.9b Cumulative Release of Selenium-79 from All Sources to the Water Table from 1940 to 3000

4.10a Cumulative Release of Uranium-238 from All Sources to the Water Table from 1940 to 2150

4.10b Cumulative Release of Uranium-238 from All Sources to the Water Table from 1940 to 3000

4.1 la Cumulative Release of Cobalt-60 from All Sources to the Water Table from 1940 to 2150

4.11b Cumulative Release of Cobalt- 60 from All Sources to the Water Table from 1940 to 3000

4.12a Cumulative Release of Americium-241 from All Sources to the Water Table from 1940 to 2150

4.12b Cumulative Release of Americium-241 from All Sources to the Water Table from 1940 to 3000

4.13a Cumulative Release of Neptunium-237 from All Sources to the Water Table from 1940 to 2150

4.13b Cumulative Release of Neptunium-237 from All Sources to the Water Table from . 1940 to 3000

4.14a Cumulative Release of Technetium-99 to Groundwater from Liquid Discharge Sources for Different Cross Section Area Factors from 1940 to 2040 
4.14b Cumulative Release of Technetium-99 to Groundwater from Liquid Discharge Sources for Different Cross Section Area Factors from 1940 to 3440.

4.15 Cumulative Release of Technetium-99 from Liquid and Solid Waste Sites for Different Initial Soil Moisture Conditions

4.16 Cumulative Release of Technetium-99 from Liquid and Solid Waste Sites for Different Distribution Coefficients

4.17 Sitewide Distribution of Recharge at the Hanford Site Used in the Composite Analysis

4.18 Locations of Hanford Site Wells with Aquifer Test Results Used in the Groundwater Model Calibration

4.19 Finite Element Grid and Boundary Conditions Used in the Groundwater Model of the Unconfined Aquifer for the Composite Analysis

4.20 Water Table Predicted in 2000 with the Three-Dimensional Model

4.21 Water Table Predicted in 2100 with the Three-Dimensional Model 4.121

4.22 Water Table Predicted in 2200 with the Three-Dimensional Model 4.122

4.23 Water Table Predicted in 2350 with the Three-Dimensional Model. 4.123

4.24a Predicted Distribution of Tritium in the Unconfined Aquifer from All Sources in 1997 (Time of Peak Concentration).

4.24b Predicted Distribution of Tritium in the Unconfined Aquifer from All Sources in 2050 4.125

4.25a Distribution of Technetium-99 in the Unconfined Aquifer from Existing Plumes in 1996 (Time of Peak Concentration)

4.25b Predicted Distribution of Technetium-99 in the Unconfined Aquifer from All Sources in 2049

4.26 Predicted Distribution of Technetium-99 in the Unconfined Aquifer from All Sources in 2036 (Time of Secondary Peak Concentration)

4.27a Predicted Distribution of Iodine-129 in the Unconfined Aquifer from All Sources in 2036 (Time of Peak Concentration).

4.27b Predicted Distribution of Iodine-129 in the Unconfined Aquifer from All Sources in 2049 
4.28a Distribution of Strontium-90 in the Unconfined Aquifer from All Sources in 1996 (Time of Peak Concentration).

4.28b Predicted Distribution of Strontium-90 in the Unconfined Aquifer from All Sources in 2049

4.29a Predicted Distribution of Carbon-14 in the Unconfined Aquifer from All Sources in 2027 (Time of Peak Concentration)

4.29b Predicted Distribution of Carbon-14 in the Unconfined Aquifer from All Sources in 2049 .. 4.134

4.30a Predicted Distribution of Chlorine-36 in the Unconfined Aquifer from All Sources in 2019 (Time of Peak Concentration).

4.30b Predicted Distribution of Chlorine-36 in the Unconfined Aquifer from All Sources in 2049

4.31a Predicted Distribution of Selenium-79 in the Unconfined Aquifer from All Sources in 2005 (Time of Peak Concentration).

4.31b Predicted Distribution of Selenium-79 in the Unconfined Aquifer from All Sources in 2049

4.32a Distribution of Uranium (Total) in the Unconfined Aquifer from All Sources at 1996 (Time of Peak Concentration).

4.32b Predicted Distribution of Uranium (Total) in the Unconfined Aquifer from All Sources in 2049

4.33 Results from a Series of Nine Transport Model Location Sensitivity Studies.

4.34 Maximum Dose Versus Time Outside the Buffer Zone for the Agricultural Scenario and the Dose the Various Radionuclides/Sources Contribute.

4.35 Composite Dose Outside the Buffer Zone from All Radionuclides and All Sources Modeled Versus Time for the a) Agricultural, b) Residential, c) Recreational, and d) Industrial Exposure Scenarios

4.36 Predicted Distribution of Composite Dose for the Agricultural Exposure Scenario in 1996 4 4.156

4.37 Predicted Distribution of Composite Dose for the Residential Exposure Scenario in 1996 ... 4.157

4.38 Predicted Distribution of Composite Dose for the Recreational Exposure Scenario in 1996. 4.158 
4.39 Predicted Distribution of Composite Dose for the Industrial Exposure Scenario in 1996..... 4.159

4.40 Predicted Distribution of Composite Dose for the Agricultural Exposure Scenario in 2049

4.41 Predicted Distribution of Composite Dose for the Residential Exposure Scenario in 2049 ... 4.161

4.42 Predicted Distribution of Composite Dose for the Industrial Exposure Scenario in 2049..... 4.162

4.43 Predicted Distribution of Composite Dose for the Agricultural Exposure Scenario in 2159 . 4.163

4.44 Predicted Distribution of Composite Dose for the Residential Exposure Scenario in 2159 ... 4.164

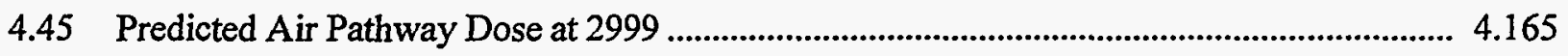

4.46 Comparison of Cumulative Release of Technetium-99 for AX Tank Farm Leaks

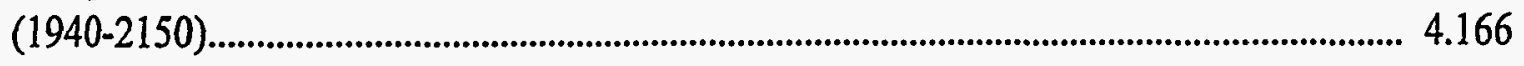

4.47 Comparison of Cumulative Release of Technetium-99 for AX Tank Farm Leaks

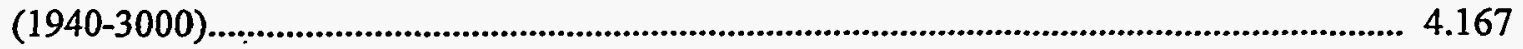

4.48 Cumulative Release of Technetium-99 from All Sources to the Water Table from 1940 to 3440 for Three Inventory/Release Scenarios for the Liquid-Discharge Site ............. 4.168

4.49a Areal Distribution of Tritium Predicted in 2020 Near the SALDS with the High-Resolution, Three-Dimensional, Transport Model

4.49b Areal Distribution of Tritium Predicted in 2045 Near SALDS with the High-Resolution, Three-Dimensional, Transport Mode.

4.50 Areal Distribution of Tritium in 2020 as Predicted with the Three-Dimensional Transport Model.

4.51 Tritium Concentration History for Observation Well 299-E24-2 ....................................... 4.172

4.52 Tritium Concentration History for Observation Well 699-42-12A ...................................... 4.172

4.53 Tritium Concentration Histories for Observation Wells 699-31-31 and 699-34-39A ........... 4.172

4.54 Areal Distribution of Tritium in 1985 as Predicted with the Three-Dimensional Transport Model. 


\subsection{Introduction}

The Composite Analysis for Low-Level Waste Disposal in the 200 Area Plateau of the Hanford Site (Composite Analysis) is a radiological assessment to estimate doses to hypothetical future members of the public from radionuclides from low-level waste (LLW) disposal and all other sources of radioactive contamination at the Hanford Site (Figure 1.1). The first iteration of the Composite Analysis is a companion analysis to the facility-specific risk documentation for the following four active or planned LLW disposal actions:

- post-1988 solid waste burial ground in the 200 West Area (Wood et al. 1995)

- post-1988 solid waste burial ground in the 200 East Area (Wood et al. 1996)

- Environmental Restoration Disposal Facility (ERDF) (DOE 1994b)

- disposal facilities for immobilized low-activity wastes. ${ }^{(a)}$

The Composite Analysis is part of the documentation required for the continued and planned LLW disposal operations at these four facilities at the Hanford Site.

\subsection{Purpose and Scope}

The Implementation Plan for the Defense Nuclear Facility Safety Board (DNFSB) Recommendation 94-2 (DOE 1996e) requires that a Composite Analysis be prepared to accompany the performance assessments for the burial grounds and the planned low-activity tank waste disposal, and the risk assessment for ERDF. This Composite Analysis was prepared to provide an estimate of the cumulative radiological impacts from the active and planned LLW disposal actions and other potentially interacting radioactive sources at the Hanford Site. The calculations for this Composite Analysis were performed with a combination of spreadsheet programs, multidimensional numerical models, and geographic information system software. The U.S. Department of Energy Richland Operations Office (DOE-RL) has elected to complete a single Composite Analysis for wastes disposed in the 200 Area Plateau because multiple LLW disposals will occur at Hanford, and many waste sites are present that may interact with the LLW disposals.

A multistep approach was used to estimate doses in the Composite Analysis.

- The first step was to estimate the inventories of radionuclides for the various sources. A complete and accurate inventory of sources of radioactive materials disposed to ground and stored at the Hanford Site does not exist and had to be estimated based on knowledge of the processes that generated the waste.

(a) Mann, F. M., R. P., Puigh II, C. R. Eiholzer, Y. Chen, N. W. Kline, A. H. Lu, B. P. McGrail, and P. D. Rittman. Publication planned for March 1998. Hanford Immobilized Low-Activity Tank Waste Performance Assessment. DOE/RL-97-69, Rev. 0. U.S. Department of Energy, Richland, Washington. 
- The second step in the analysis was to calculate the radionuclide release from the various sources, based on knowledge of waste form characteristics and long-term performance calculations (e.g., recharge characteristics and geochemical behavior).

- The third step was to predict transport through the vadose zone under transient conditions. The recharge rate in the vadose zone was allowed to vary with different surface conditions and especially surface covers (barriers).

- The fourth step was to predict transport through the unconfined aquifer under transient conditions. Groundwater flow in the unconfined aquifer responded to the cessation of wastewater discharges from Hanford Site operations and declined. Separate analyses of existing contaminant plumes and future releases from the vadose zone were conducted.

- The fifth step in the analysis was to calculate dose based on exposure scenarios for hypothetical future members of the public at locations on the Hanford Site and compare those doses with standards outlined in the Composite Analysis guidance (DOE 1996b). The dose estimates provided represent the effective dose equivalent received over a commitment period of 50 years.

The scope of the first iteration Composite Analysis was to consider all radioactive sources within the 200 Area Plateau of the Hanford Site that could potentially interact with the active and planned LLW disposal actions. The four LLW disposal actions are located on the 200 Area Plateau, located near the center of the Hanford Site.

Chapter 3 describes the Composite Analysis source term. Chapter 4 discusses the release, vadose zone, groundwater, and exposure simulation methods and results. Chapter 5 presents an interpretation of results, a discussion of uncertainties, and suggestions for further study.

An approach for the future reduction of uncertainty and the establishment of greater confidence in subsequent iterations of the Composite Analysis is described in Chapter 2 of this report. The U.S. Department of Energy (DOE) has issued guidance that performance assessments, Comprehensive Environmental Response, Compensation, and Liability Act (CERCLA) risk assessments, and the composite analysis are to be maintained. Significant changes in future land use (e.g., the future DOE property boundary), the inventories, the release models, the environment transport pathways, and exposure and dose scenarios would precipitate another iteration of the composite analysis (DOE 1996b).

\subsection{Regional Setting}

The Hanford Site lies within the semiarid Pasco Basin of the Columbia Plateau in southeastern Washington State (Figure 1.1; Dirkes and Hanf 1997). The site occupies an area of approximately $1,450 \mathrm{~km}^{2}$ (approximately $560 \mathrm{mi}^{2}$ ) located north of the city of Richland, Washington, and the confluence of the Yakima and Columbia Rivers. This large area has restricted public access and provides a buffer for the smaller areas onsite that were used for research, fuel fabrication, fuel irradiation, the production 
of nuclear materials, and the storage and disposal of wastes. Approximately $6 \%$ of the land area has been disturbed and is actively used. The Columbia River flows eastward through the northern part of the Hanford Site and then turns south, forming part of the eastern site boundary. The Yakima River flows near a portion of the southern boundary and joins the Columbia River downstream of the city of Richland.

The cities of Richland, Kennewick, and Pasco (known collectively as the Tri-Cities) constitute the nearest population center and are located southeast of the Hanford Site. Richland and Kennewick are in Benton County, and Pasco is in Franklin County. Land surrounding the Hanford Site is used for urban and industrial development, irrigated and dryland farming, and grazing. In 1995, population totals for Benton and Franklin Counties were estimated at 131,000 and 44,000, respectively (Washington State Office of Financial Management 1995). The estimated 1995 populations of the Tri-Cities were: Richland, 36,270; Pasco, 22,500; and Kennewick, 48,130. The combined populations of three smaller outlying communities of the Tri-Cities (i.e., Benton City, Prosser, and West Richland) totaled 13,320 in 1995.

\subsection{Site Description}

Major operational areas at Hanford are described in the following list.

- The 100 Areas, on the south shore of the Columbia River, are the sites of nine retired plutonium production reactors, including the dual-purpose $\mathrm{N}$ Reactor. The 100 Areas occupy approximately $11 \mathrm{~km}^{2}\left(4 \mathrm{mi}^{2}\right)$.

- The 200 West and 200 East Areas are located on a plateau, approximately 8 and $11 \mathrm{~km}(5$ and $7 \mathrm{mi})$, respectively, south of the Columbia River. Historically, these areas have been dedicated to fuel reprocessing and waste processing management and disposal activities. The 200 Areas cover approximately $16 \mathrm{~km}^{2}\left(6 \mathrm{mi}^{2}\right)$.

- The 300 Area is located just north of the city of Richland. Fuel fabrication facilities were operated in this area, and it is the site of nuclear research and development. This area covers $1.5 \mathrm{~km}^{2}\left(0.6 \mathrm{mi}^{2}\right)$.

- The 400 Area is approximately $8 \mathrm{~km}(5 \mathrm{mi})$ northwest of the 300 Area and is the site of the Fast Flux Test Facility (FFTF), used in the testing of breeder reactor systems. Also included in this area is the Fuels and Materials Examination Facility.

- The 600 Area includes all of the Hanford Site not occupied by the 100,200, 300, and 400 Areas.

- The 1100 and Richland North Areas are located south of the Hanford Site, in the northern portion of the city of Richland. These are support areas that include general stores, transportation maintenance, and the DOE and DOE contractor facilities. 
During 1996, the 3000 Area was cleaned up and vacated by DOE and its contractors. All land and facilities within the area were turned over to the Port of Benton, and the 3000 Area designation was retired (Dirkes and Hanf 1997).

Several areas of the Hanford Site (a total land area of $665 \mathrm{~km}^{2}$ [257 $\left.\mathrm{mi}^{2}\right]$ ) have special designations. These areas include the Fitzner/Eberhardt Arid Lands Ecology Reserve, the U.S. Fish and Wildlife Service Saddle Mountain National Wildlife Refuge, and the Washington State Department of Game Reserve Area (Wahluke Slope Wildlife Recreation Area) (Dirkes and Hanf 1997). Management of the Fitzner/Eberhardt Arid Lands Ecology Reserve was transferred to the U.S. Fish and Wildlife Service in 1997. It is currently part of the National Wildlife Refuge system.

Non-DOE activities on Hanford Site leased land include commercial power production on the land occupied by the Washington Public Power Supply System WNP-2 plant (and partially completed WNP-1 and WNP-4 plants) and operation of a commercial LLW burial site by US Ecology, Inc. Immediately adjacent to the southern boundary of the Hanford Site, Siemens Power Corporation operates a commercial nuclear fuel fabrication facility, and Allied Technology Group Corporation operates an LLW decontamination, supercompaction, and packaging disposal facility. Kaiser Aluminum and Chemical. Corporation leases the 313 Building in the 300 Area to use an extrusion press that was formerly owned by DOE. The National Science Foundation is building the Laser Interferometer Gravitational-Wave Observatory facility between the 200 and 400 Areas.

The Hanford Site description and historical site operation information presented here were taken from the introduction section of the Hanford Site Environmental Report for Calendar Year 1996 (Dirkes and Hanf 1997). More detailed information on the Hanford Site environment is provided by Neitzel (1997).

\subsection{Historical Site Operations}

The Hanford Site was established in 1943 with the mission to produce plutonium for nuclear weapons (Dirkes and Hanf 1997). Hanford operations have resulted in the production of liquid, solid, and gaseous wastes. Most wastes from these operations have a potential to contain radioactive materials. From an operational standpoint, radioactive wastes were originally categorized as "high level," "intermediate level," and "low level," which referred to the level of radioactivity present.

Some high-level solid waste, such as large pieces of machinery and equipment, were placed onto railroad flatcars and stored in underground tunnels. Both intermediate- and low-level solid wastes (e.g., tools, machinery, paper, and wood) were placed into covered trenches at storage and disposal sites known as burial grounds. Beginning in 1970, solid wastes were segregated according to the makeup of the waste material. Solids containing plutonium and other transuranic materials were packaged in special containers and stored in lined trenches covered with soil for possible later retrieval. High-level liquid wastes were stored in large underground tanks in the 200 Areas. 
Intermediate-level liquid waste streams were usually routed to underground structures of various types including cribs, French drains, and specific retention trenches. Occasionally, trenches were filled with the liquid waste and then covered with soil after the waste had soaked into the ground. Low-level liquid waste streams were usually routed to surface impoundments (ditches and ponds). Nonradioactive solid wastes were usually burned in burning grounds. This practice was discontinued in the late 1960s in response to the Clean Air Act, and the materials were buried at sanitary landfill sites instead. These storage and disposal sites, with the exception of high-level waste tanks, are now designated as active or inactive waste sites, depending on whether the site currently receives wastes.

\subsubsection{The 300 Area}

From the early 1940s to the present, most research and development activities at the Hanford Site were carried out in the 300 Area, located just north of Richland. Until 1987, the 300 Area was also the location of nuclear fuel fabrication. Nuclear fuel in the form of pipe-like cylinders (fuel slugs) was fabricated from metallic uranium shipped in from offsite production facilities. Metallic uranium was extruded into the proper shape and encapsulated in aluminum or zirconium cladding.

Substantial amounts of copper, uranium, and other heavy metals were found in 300 Area liquid waste streams. Until the mid-1970s these streams were routed to the 300 Area waste ponds, which were located near the Columbia River shoreline. In more recent times, the low-level liquid wastes were sent to process trenches or shipped to the solar evaporation facility in the 100-H Area (183-H Solar Evaporation Basins). Discharge to process trenches ceased in December 1994.

\subsubsection{The 100 Areas}

The 100 Areas are located on the southern shore of the Columbia River in the northern portion of the Hanford Site, where in the past up to nine nuclear reactors were in operation. The graphite cores of the eight production reactors were the host environment for the conversion of uranium atoms to plutonium atoms. Also produced were radionuclides from the fission and activation processes.

When fresh fuel slugs were pushed into the front face of a reactor's graphite pile, the irradiated fuel slugs were forced out the rear into a deep pool of water called a fuel storage basin. After a brief period of storage, the irradiated fuel was shipped in specially constructed railcars to the 200 Areas for processing.

The N Reactor (the ninth reactor) ran from the early 1970 s to the early 1980 s with the dual missions of electricity and plutonium production. Beginning in 1975, N Reactor irradiated fuel was shipped to the $\mathrm{K}$ East and $\mathrm{K}$ West Fuel Storage Basins for temporary storage where it remains today. This fuel accounts for the majority of the fuel currently stored underwater in the 100-K Area fuel storage basins. The majority of material produced in N Reactor from the early 1980 s until 1987 was processed in the 200 East Area. The remainder is stored in the $\mathrm{K}$ Basins. 


\subsubsection{The 200 Areas}

The 200-East and 200-West Areas are located on a plateau at the center of the Hanford Site. Figure 1.2 shows the areas that housed chemical separation plants that received and dissolved irradiated fuel and then separated out the plutonium (Dirkes and Hanf 1997, Figure 1.0.3). At different times and in different plants, three processes were used to perform the separation. Each of the plutonium production processes began with the dissolution of the aluminum or zirconium cladding material in solutions containing ammonium hydroxide, ammonium nitrate, and ammonium fluoride, followed by the dissolution of the irradiated fuel slugs in nitric acid. This chemical processing step produced large quantities of nitric acid solutions containing high levels of radioactive materials. These wastes were neutralized and stored in large underground tanks. Fumes from the dissolution of cladding and fuel, and from other process steps were discharged to the atmosphere.

The first separation process was the bismuth phosphate precipitation that operated from 1945 until 1956 in B and T Plants. This method was supplanted by a second, more efficient method that involved contacting a methyl isobutyl ketone (hexone) organic phase with an aqueous aluminum nitrate solution of plutonium and uranium from dissolved fuel. This process was run from 1952 until 1966 in the Reduction-Oxidation (REDOX) Plant.

Finally, the REDOX process was replaced by a much-improved solvent extraction based on an organic phase that was a mixture of normal-paraffinic hydrocarbon or kerosene (NPH) and tributyl phosphate (TBP) contacting an aqueous nitric acid solution of plutonium and uranium. The Plutonium Uranium Extraction (PUREX) Plant ran this process from 1956 until 1972 then restarted in 1983 and ran until 1988. Wastes from each of these process steps were neutralized and placed into storage tanks. Some tank wastes were directed to cribs and trenches and disposed in the unsaturated soil profile (Waite 1991).

A uranium recovery campaign was undertaken at U Plant from 1952 until 1956. The bismuth phosphate process did not recover uranium from the process stream, and it was decided to recover uranium from the metal wastes stored in the large underground single-shell tanks. These wastes were sluiced from tanks and a process based on the TBPNPPH solvent extraction was applied. The uranium was purified into uranium oxide powder at the Uranium-TriOxide Plant. Ultimately, because of the volume of waste produced in this process, ferrocyanide was used to scavenge or entrap the cesium-137 in a precipitated sludge, and the supernatant was placed into the cribs or trenches.

The REDOX and PUREX Plants produced uranium nitrate for recycle and plutonium nitrate for weapons. Uranium nitrate was further processed in.the Uranium TriOxide Plant. Plutonium nitrate was transferred to Z Plant (later called the Plutonium Finishing Plant, or PFP) for conversion to plutonium metal. The conversion processes used nitric acid, hydrofluoric acid, carbon tetrachloride, and various oils and degreasers. Varying amounts of all these materials were in the intermediate-level wastes discharged to cribs. Cooling water from the PFP was discharged to U Pond. Solid wastes containing plutonium were segregated and packaged for storage in special earth-covered trenches. 
After separation processing ended at B Plant, the facility was reconfigured. From 1967 until 1976, the reconfigured facility was used to extract strontium from PUREX acid waste and sludges, and cesium from a variety of neutralized supernatants taken from the tanks. The strontium and cesium were concentrated into solid salt materials, melted, and encapsulated. Canisters of encapsulated strontium and cesium are stored today in the Waste Encapsulation Storage Facility.

Evaporators were used to remove excess water and concentrate the tank waste into salt cake and sludge, which remained in the tanks. The evaporated and condensed water contained radioactive tritium and was discharged to cribs.

Large volumes of cooling water and steam condensate discharges to ground have significantly affected the water table by causing the formation of groundwater mounds. Cooling water and steam condensate from B Plant went to B Pond (216-B-3) and those from T Plant went to T Pond (now beneath the 218-W3AE Burial Ground). Cooling water and steam condensates from the U Plant and Uranium Tri-Oxide Plant were routed to U Pond (216-U-10). Cooling water from the REDOX Plant was discharged to the S Ponds (216-S-16 and 216-S-17). Cooling water from the PUREX Plant was discharged to Gable Mountain Pond (216-A-25) and B Pond.

From 1944 to $1988,526,000,000$ gallons $\left(2.0 \times 10^{9} \mathrm{~L}\right)$ of highly radioactive chemical processing waste was placed in single-shell and double-shell tanks at Hanford (Agnew et al. 1997). This amount included metal waste that was reprocessed in U Plant from 1952 until 1956, and PUREX sludge and

supernatants reprocessed in B Plant from 1967 until 1976. Of this total, 63,200,000 gallons $\left(2.4 \times 10^{8} \mathrm{~L}\right)$ were later removed and reprocessed, $129,600,000$ gallons $\left(4.9 \times 10^{8} \mathrm{~L}\right)$ were discharged to cribs and trenches, and $272,400,000$ gallons $\left(1.0 \times 10^{9} \mathrm{~L}\right)$ were removed by evaporation. Approximately $61,000,000$ gallons $\left(2.3 \times 10^{8} \mathrm{~L}\right)$ of waste remain in the tanks. These data reflect conditions on January 1, 1994 as reported by Agnew et al. (1997). The waste volume in tanks will change with time (e.g., waste volume will decline as evaporator campaigns are completed).

\subsection{Low-Level Waste Disposal Facilities at Hanford}

This section identifies the active or planned LLW disposal facilities and other sources of radioactive contamination under consideration in the first iteration of the Composite Analysis.

\subsubsection{Active or Planned Disposal Facilities}

The Composite Analysis provides a first estimate of the potential cumulative impacts to a hypothetical future member of the public from the active or planned LLW disposal facilities at Hanford. The Composite Analysis also includes other sources of radioactive material in the ground that may interact with plumes from the LLW disposal facilities. The four active or planned LLW disposal facilities at Hanford are: 
- post-1988 solid waste burial ground in the 200 West Area

- post-1988 solid waste burial ground in the 200 East Area

- Environmental Restoration Disposal Facility

- disposal facility for immobilized low-activity wastes.

Each of these disposals is located on the central or 200 Area Plateau of the Hanford Site. Figure 1.3 shows the position of these LLW disposals on the 200 Area Plateau.

In accordance with DOE Order 5820.2A (DOE 1988b), performance assessments have been completed for the solid waste burial grounds located in 200 West Area and in 200 East Area. These burial grounds have received solid waste since DOE Order 5820.2A went into effect (September 26, 1988). Burial grounds in the 200 West and East areas were treated separately in performance assessments by Wood et al. (1995) and Wood et al. (1996). Under the CERCLA program, a Remedial Investigation and Feasibility Study was completed for the ERDF (DOE 1994b). The DOE-RL plans to submit a performance assessment ${ }^{(a)}$ for the immobilized low-activity waste (ILAW) from Hanford tanks to U.S. Department of Energy Headquarters (DOE-HQ) in Spring 1998. Pending review and approval by DOE-HQ, the ILAW will be disposed of in a combination of four existing vaults and new facilities that are now in the conceptual design stage.

\subsubsection{Other Sources of Radioactive Contamination}

As is apparent from the description of Hanford Site operations, other radioactive sources are present or will be placed on the 200 Area Plateau of the Hanford Site. These sources may create contaminant plumes in the unconfined aquifer at the same time and in the immediate vicinity of plumes generated by the four LLW disposal facility sources described above. These sources are the responsibility of the DOE and include the following list:

- 149 single-shell tanks arrayed in 12 tank farms (i.e., T, TX, TY, U, S, SX, B, BX, BY, C, A, and AX)

- 28 double-shell tanks arrayed in 6 tank farms (i.e., SY, AP, AN, AZ, AY, and AW)

- past-practice (pre-1988) solid waste burial grounds

- past-practice (pre-1988) liquid discharges to cribs, ditches, French drains, trenches, and ponds

- graphite cores from 9 surplus production reactors

(a) Mann, F. M., R. P., Puigh II, C. R. Eiholzer, Y. Chen, N. W. Kline, A. H. Lu, B. P. McGrail, and P. D. Rittman. Publication planned for March 1998. Hanford Immobilized Low-Activity Tank $\dot{W}$ aste Performance Assessment. DOE/RL-97-69, Rev. 0. U.S. Department of Energy, Richland Operations Office, Richland, Washington. 
- canyon buildings and related structures (e.g., B-Plant, PUREX, T-Plant, U-Plant, REDOX, Z-Plant [PFP], and the PUREX tunnels).

In addition, a commercial low-level radioactive waste disposal facility operated by US Ecology, Inc. is located immediately southwest of the 200 East Area, and was included in this analysis because of its proximity to DOE operations on the plateau. The treatment of each of these facilities was addressed in the analysis.

\subsection{Operation of Low-Level Waste Disposal Facilities}

This section provides a brief description of the facilities and their past, present, and expected future operations.

\subsubsection{Active or Planned Disposal Facilities}

Low-level waste has been disposed in the 200 West and 200 East solid waste burial grounds since nuclear materials production and processing began at Hanford. The initial generators of the majority of disposed waste were the chemical separations plants in each area: T-Plant, U-Plant, REDOX, and PFP, and tank farm operations in the 200 West Area; and PUREX, B-Plant, and tank-farm operations in 200 East Area. Disposals to the 200 West Area LLW facility support both onsite and offsite generators. The U.S. Navy is the only offsite generator contributing to waste disposal in the 200 East Area.

Solid waste disposals have occurred for several decades and as one burial ground filled up, another burial ground was opened. The current method of disposal for LLW is to place waste in an unlined trench about 6 to $7 \mathrm{~m}$ deep and of variable length up to about $500 \mathrm{~m}$. Slopes of trenches are angled at about 45 degrees. Waste packages are stacked to within about $2.5 \mathrm{~m}$ of the surface, and soil is placed over the packages to grade. Some surfaces have been vegetated with grasses to stabilize the cover. In the future, efforts may be made to stabilize the waste in situ to prevent subsidence and to reduce recharge through the waste deposit.

Active burial grounds are defined as those that have received waste since September 26, 1988. Active disposal trenches are found in burial grounds 218-W-3A, 218-W-3AE, 218-W-4C, and 218-W-5 in 200 West Area; and 218-E-10 and 218-E-12B in 200 East Area. Since September 26, 1988, when DOE Order 5820.2A went into effect, 23 trenches have been open and receiving waste in the 200 West Area burial grounds, and 6 trenches have been open and receiving waste in the 200 East Area burial grounds. One additional trench in 218-E-12B, Trench 94, is dedicated to the disposal of defueled ship reactor compartments generated by the U.S. Navy. The performance assessments for the active 200 West and active 200 East solid waste burial grounds stipulate an expected 30 years of operation from the September 1988 start date.

In the summer of 1996, disposal of wastes generated during excavation and remediation of CERCLA past-practice sites on Hanford began. These wastes are disposed in the ERDF trench. This trench is a 
belowgrade excavation that is lined to collect leachate. The excavated material is mounded abovegrade to create a trench of greater disposal volume or capacity. When filled with remediation waste, the trench will be closed with a protective surface barrier. Only remediation wastes originating at Hanford will be disposed in the ERDF. The waste is expected to consist of dangerous and hazardous waste, polychlorinated biphenyl (PCB) and asbestos waste, low-level radioactive waste, and low-level mixed waste containing both dangerous and radioactive waste. The ERDF trench is being developed in stages. Currently it consists of two disposal cells, and approval is now being sought for additional cells. Based on need, it is anticipated the ERDF will be expanded to receive all remediation wastes from Hanford's CERCLA past-practice sites.

Over the last 50 years, radioactive and mixed waste from the production of special nuclear materials has been stored primarily in single- and double-shell tanks in the 200 Areas. Under the Tank Waste Remediation System (TWRS) program, the DOE is proceeding with plans to permanently immobilize and dispose of the low-level portion of this waste onsite in near-surface disposal facilities as outlined in the record of decision (ROD 1997). Wastes will be retrieved from the tanks and pretreated to separate the low-level fraction from other tank waste. The low-level fraction will then be immobilized. Over $200,000 \mathrm{~m}^{3}$ of $L L W$ will be disposed under this program. An initial or interim performance assessment providing initial insight and guidance to the design of disposal facilities has been prepared for this waste form (Mann et al. 1997). A performance assessment is being submitted to DOE-HQ in the spring of 1998 to seek approval for the construction of disposal facilities and the disposal of waste. It is now anticipated the first of the ILAW will be disposed in four existing disposal vaults with the remaining waste disposed in new disposal facilities. Disposals are forecast to begin in 2002. Authorization to close the disposal facilities is expected around 2030.

\subsubsection{Other Sources of Radioactive Contamination}

In addition to the disposal of ILAW, releases to the environment originating from the single-shell tanks must also be considered. Sixty-seven single-shell tanks are known or assumed to have leaked. The Hanford Federal Facility Agreement and Consent Order (also known as the Tri-Party Agreement; Ecology, EPA, and DOE 1989) calls for approximately $99 \%$ of the waste volume in each of the 149 tanks to be removed. At present, sluicing is the method of choice for the removal of these wastes. It is believed that some contaminated liquid could be lost from each single-shell tank during recovery operations. Finally, each of the single- and double-shell tanks will contain some residual after wastes are recovered, separated, and solidified. These residuals will also release radioactive contamination to the surrounding environment in the future. The end-date milestone (Tri-Party Agreement Milestone M-45) for tank waste retrieval is September 2018.

Shallow-land burial of solid waste has occurred at Hanford since the mid-1940s. Burial grounds closed prior to September 26, 1988 are considered among the other sources of radioactive contamination. Prior to 1970, no distinction was made between transuranic (TRU) waste and LLW. In 1970, the Atomic

Energy Commission required that TRU waste be retrievably stored. In the early 1980 s, low-level liquid organic waste was segregated from LLW and placed in retrievable storage underground. Low-level 
waste was further categorized in 1987 when mixed waste (i.e., waste containing both radioactive and hazardous chemicals) disposal in unlined trenches was discontinued. Contact-handled mixed waste is currently stored in aboveground buildings in the Central Waste Complex. Post-1988 LLW in burial grounds exhibits much lower inventories compared to the inventories of pre-1988 burial grounds. The pre-1988 solid waste burial grounds are designated past-practice units, and their remediation, final closure, and end state will be negotiated through the CERCLA process.

Since initial processing of irradiated fuels began in 1944, liquid wastes containing radionuclides have been discharged to the subsurface. These large liquid discharges have resulted in water table rises

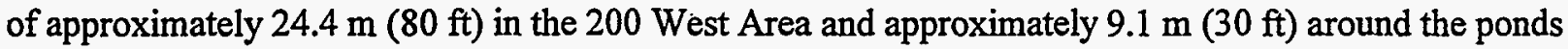
near the 200 East Area (Law et al. 1996). In the past decade this practice has nearly ended; liquid waste discharges continue at only a few sites (e.g., the 200 Area Treated Effluent Disposal Facility [TEDF], the State-Approved Land Disposal Site [SALDS], and the 400 Area discharge ponds). This reduction in liquid disposal will result in the Hanford Site groundwater levels eventually reaching pre-Hanford levels. This will have a significant effect on the routing and movement of contaminants in the aquifer, especially at locations on the Hanford Site where the permeability of the Hanford formation currently dominates the total transmissivity of the system. Past discharges occurred to subsurface facilities including cribs, trenches, French drains, and reverse wells. Large volumes of cooling water and steam condensate generated by chemical separations facilities and evaporators were discharged to surface ponds and ditches. Some of the more significant liquid discharges to the subsurface were the intentional discharge of approximately 120 million gallons $\left(4.5 \times 10^{8} \mathrm{~L}\right)$ of tank waste in various forms, e.g., first-cycle supernatant, second-cycle supernatant, and scavenged uranium recovery wastes. These sites are designated past-practice units and their remediation, final closure, and end states will be addressed through the CERCLA process.

Nine graphite core production reactors were operated at the Hanford Site between 1944 and 1987. Based on the environmental impact statement (EIS) for the eight surplus reactors (DOE 1989), a record of decision (ROD) was issued to follow a safe storage period with one-piece removal of the reactors to the plateau (ROD 1993). Safe storage at their current location along the Columbia River in the 100 Areas would occur for less than 75 years. Then, each reactor block would be transported intact on a tractor-transporter, from its present location to a 200 West Area burial ground for disposal. Since the EIS and ROD were issued, the B Reactor has been declared a national historic monument. Accordingly, it is possible it will be left at its current location along the Columbia River. This reduces the number of reactors affected by the ROD to seven. The $\mathrm{N}$ Reactor was not included in the surplus reactor EIS, and it is probable that it will be removed to the 200 West Area burial ground. Thus, eight reactors are assumed disposed on the 200 Area Plateau in this analysis.

Facilities in which the chemical separations were conducted are long, monolithic, concrete structures. These are known as the canyon buildings and are identified as the 221-B or B Plant, 221-T or $T$ Plant, and other facilities. There are also related nearby structures used in additional process steps, (e.g., the 224-B and 224-T buildings), and storage facilities, (e.g., the two subsurface tunnels at PUREX). Two canyon buildings are in 200 East Area: B-Plant and PUREX. Four canyon buildings are in 
200 West Area: T Plant, U Plant, REDOX, and Z Plant (PFP). In general, these structures contain inventories of mixed fission products and mixed activation products; however, they are in fixed or immobile settings inside metal vessels and piping and contained inside monolithic concrete cells. The end state of these structures and associated facilities is being defined through negotiations with regulators; however, the current baseline assumes canyon facilities will be demolished to the cover block grade with the remaining structure covered with a surface barrier.

The commercial LLW disposal facility opened in 1965 on 100 acres located southwest of the 200 East Area. The LLW that is packaged and shipped for disposal at the facility comes from medical practices, scientific research, industrial processes, and nuclear power plants. Prior to 1993, LLW came from throughout the United States to this site; but today LLW comes only from Washington, Alaska, Hawaii, Idaho, Montana, Oregon, Utah, Wyoming, Colorado, Nevada, and New Mexico. Naturally occurring radioactive materials can still come from all 50 states. The US Ecology Site is regulated by the Washington State Departments of Health and Ecology and is expected to close by 2063.

\subsection{Waste Management Area Boundary}

With regard to offsite exposure to a hypothetical member of the public, the current Hanford Site boundary of greatest interest is the Columbia River. However, the boundary of interest for the Composite Analysis is the future boundary. In 1992 a working group comprising representatives from governmental entities (federal, tribal, state, and local) and constituencies (labor, environment, agricultural, economic development, cities, and public interest groups) vitally interested in possible future uses of Hanford lands and cleanup efforts was formed. Included in the published report of the Hanford Future Site Uses Working Group (HFSUWG 1992) is the concept of an "exclusive" waste management area (Figure 1.4). This area is defined by the squared-off boundaries of the current 200 Areas expanded to include: 1) the land to the east of the 200 East Area (where TWRS privatization facilities are planned), and 2) the land to the south including the commercial LLW disposal site.

Surrounding the exclusive waste management area is a temporary buffer zone composed of the rest of the Central Plateau including the 200 North Area extending north to the base of Gable Butte. The cleanup target for the exclusive waste management area is to reduce risk outside that area and to minimize the size of the buffer zone. The ultimate cleanup target for the buffer zone is to prepare the land for unrestricted use. Thus, in future analyses, the size of the buffer zone may shrink.

However, it is the policy of DOE (1996h) that it "will control and maintain LLW disposal facilities until the disposal facilities can be released." The requirements for release of DOE property are provided in DOE Order 5400.5 (DOE 1993b). The DOE has acknowledged that many LLW disposal facilities may never be suitable for unconditional release to the public. For example, deed restrictions on the future use of the groundwater resource may be necessary.

The collective locations of waste sites within the 200 Area Plateau are illustrated in Figure 1.3a, b, and $c$. The position of the various waste disposals and other sources are shown. Some liberty was taken 
to locate the graphite core disposal in the western portion of the 200 West Area. A specific location has not been chosen yet, so a logical but not precise location has been selected for the purpose of this analysis. Because the land area associated with many of the liquid discharge sites is quite small, they are represented by uniform red dots in the figure. The disposal sites and other contamination sources cover a significant portion of the exclusive waste management area. While both the ERDF trench and commercial low-level radioactive waste disposal facility lie outside the 200 West and 200 East Areas, they lie within the exclusive waste management area.

\subsection{Performance Objectives of the Composite Analysis}

This analysis will estimate the potential cumulative impact to a hypothetical member of the future public from the active and planned LLW disposals and the other sources of radioactive material to remain at Hanford after Site closure. DOE Order 5400.5 (and anticipated 10 CFR 834) sets the DOE primary dose limit ${ }^{\left({ }^{a}\right)}$ of 100 mrem to members of the public in a year and as low as reasonably achievable (ALARA). This is the maximum allowable projected total dose from all pathways to the future member of the public. An options analysis and ALARA assessment are to be prepared if the projected dose exceeds the $100 \mathrm{mrem}$ in a year limit or a significant fraction of the limit (defined to be $30 \mathrm{mrem}$ in a year). The options analysis and ALARA assessment are to consider those actions that could be taken to reduce the calculated dose and their costs. They are to focus on those sources making a significant contribution to dose. If the projected dose is below the significant fraction of the limit, a brief ALARA assessment should still be performed to determine whether or not a quantitative or semi-quantitative options analysis and ALARA assessment are warranted.

If the projected dose is above 100 mrem in a year, DOE uses the options analysis to identify alternatives that reduce projected future doses to tolerable levels, and selects one for implementation. Performing these calculations provides the DOE with information supporting a comprehensive approach to environmental management that will ensure that the 100 mrem in a year primary annual dose limit is not exceeded in the future and that potential doses are maintained at ALARA levels.

At Hanford, the approach adopted to achieve comprehensive environmental management involves a complex process of negotiated decisions among the DOE, the State of Washington Department of Ecology, and the U.S. Environmental Protection Agency. Even the selection of each alternate remedial action for further study needs to be a joint decision of the three parties. At this time, DOE is beginning to negotiate the cleanup of past-practice sites in the exclusive waste management area. Thus, there.has been insufficient time to determine whether alternative remedies are necessary and to identify them through a negotiation process. Accordingly, the options analysis (if necessary) and ALARA assessment will be deferred to the second iteration of the Composite Analysis.

(a) All doses in the Composite Analysis (except where noted) are in units of mrem effective dose equivalent $(\mathrm{EDE})$ in a year. 
DOE guidance for the composite analysis (DOE 1996b) requires the analysis present results for a time period of at least 1000 years. Subsequent guidance for performance assessments (DOE 1996f) that are related to composite analyses, requires the analysis cover a period of 1000 years following closure of a disposal facility. For this analysis, the time period is assumed to begin at the time of Hanford Site closure (assumed to occur in 2050). The latter guidance also notes that analyses beyond 1000 years may be appropriate in the sensitivity/uncertainty analyses. This analysis has considered a time period of 1500 years beginning in 1944 and including the 1000 years following site closure. 


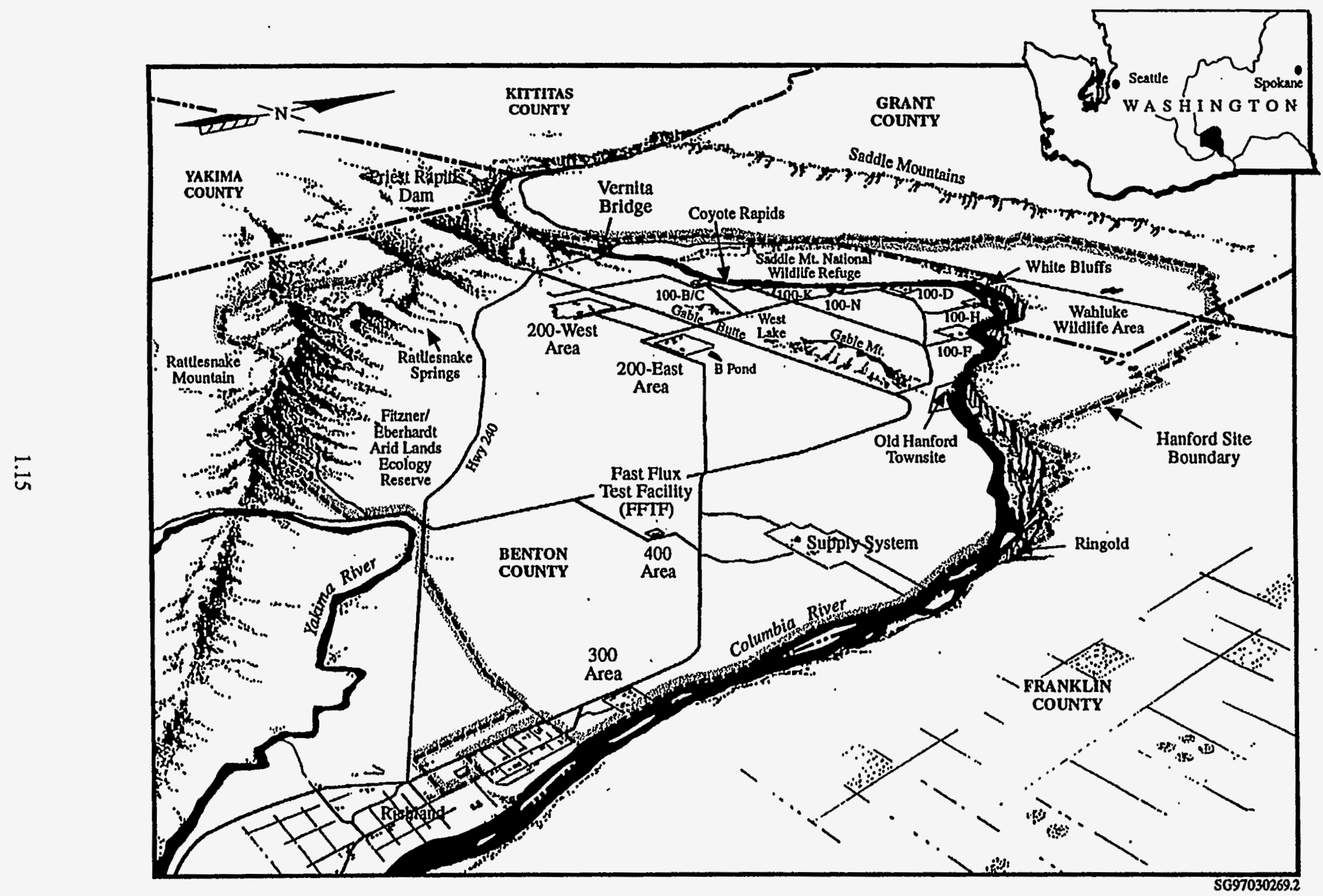

Figure 1.1. The Hanford Site and Surrounding Area 


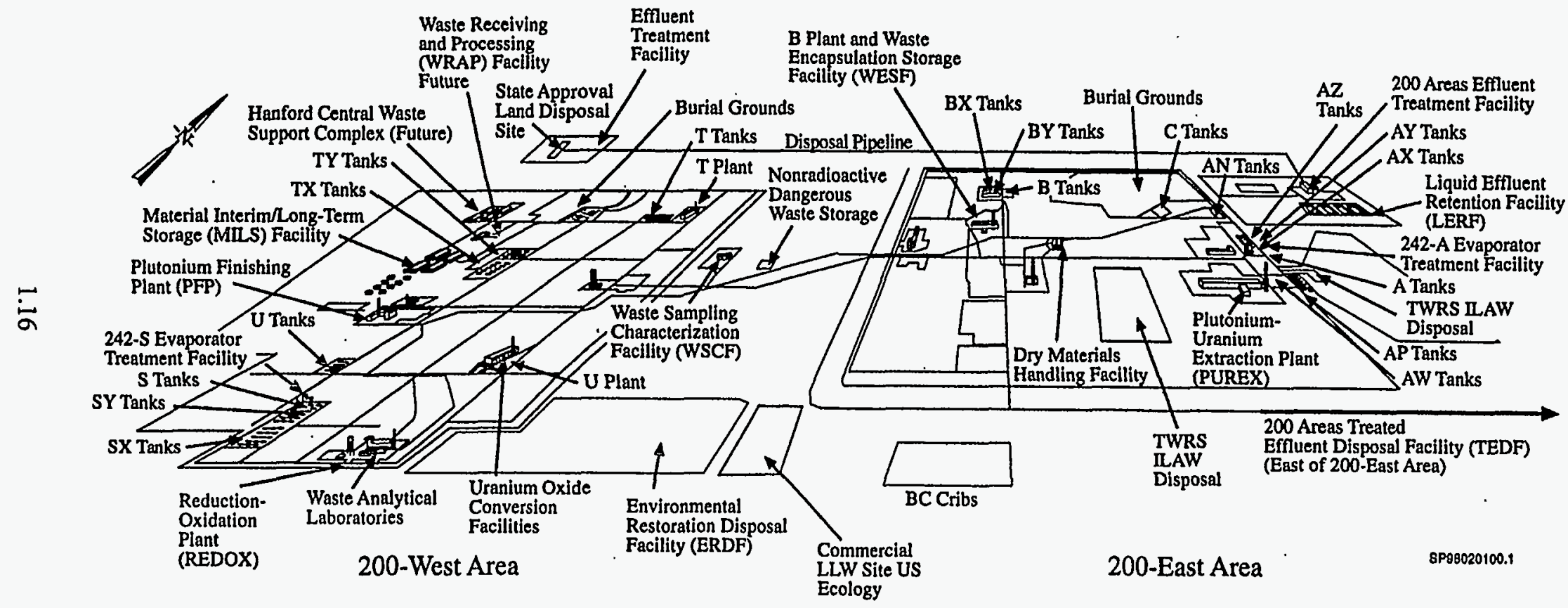

Figure 1.2. Waste Storage and Disposal Facilities in the 200 Area 


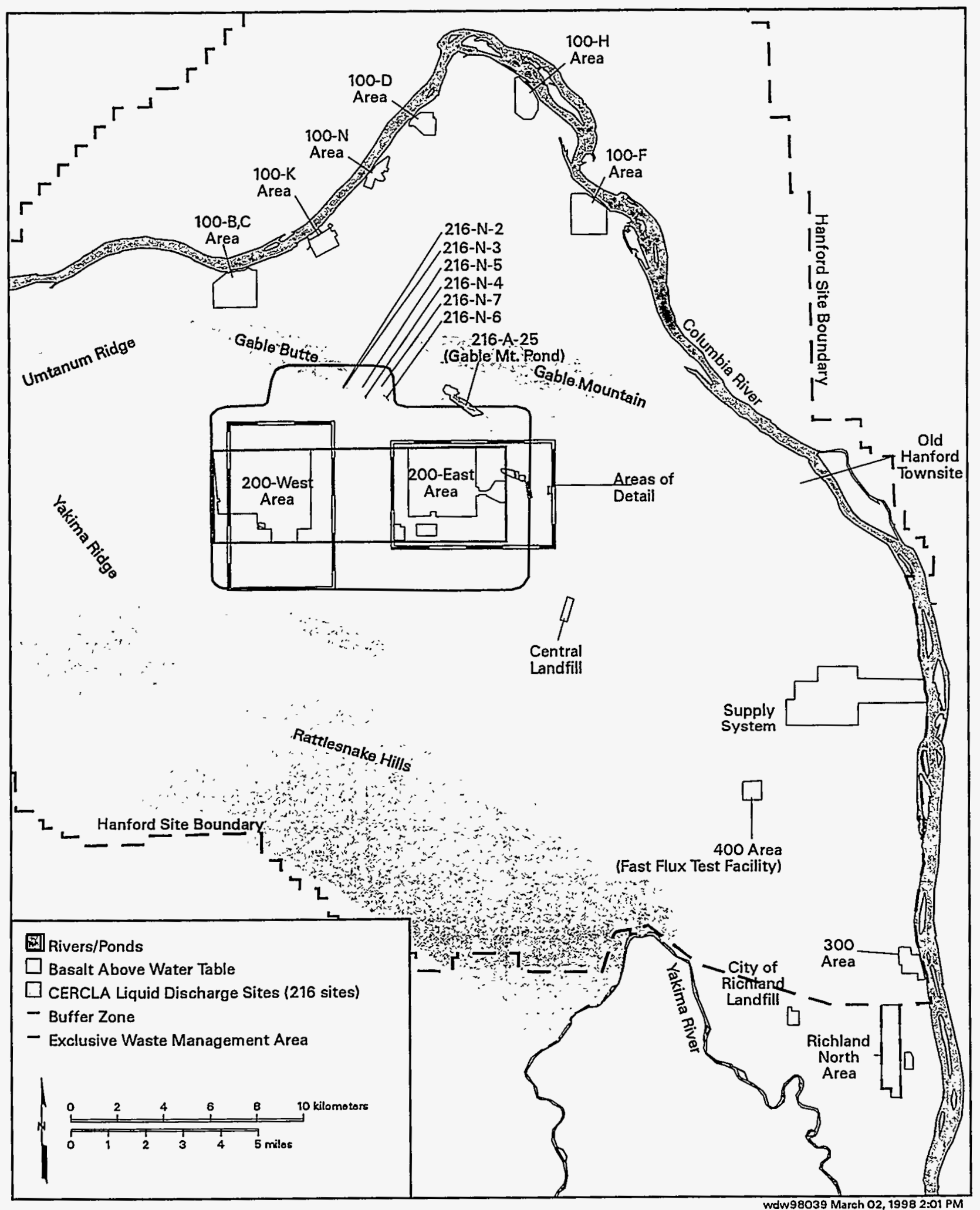

Figure 1.3a. The Exclusive Waste Management Area and Buffer Zone of the 200 Area Plateau at the Hanford Site. 



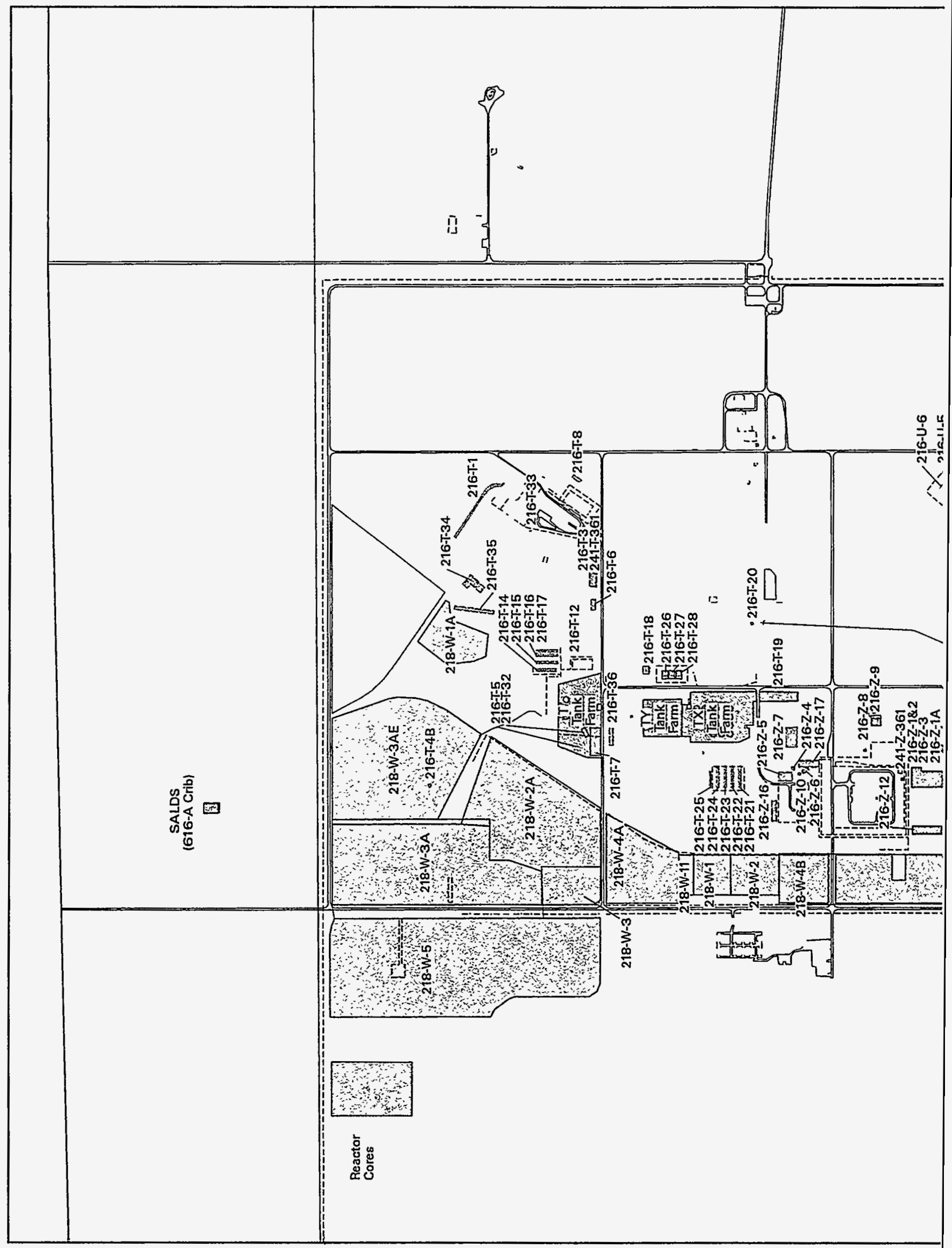

Figure 1.3b. Waste Sites of the 200 West Area and Imm. 


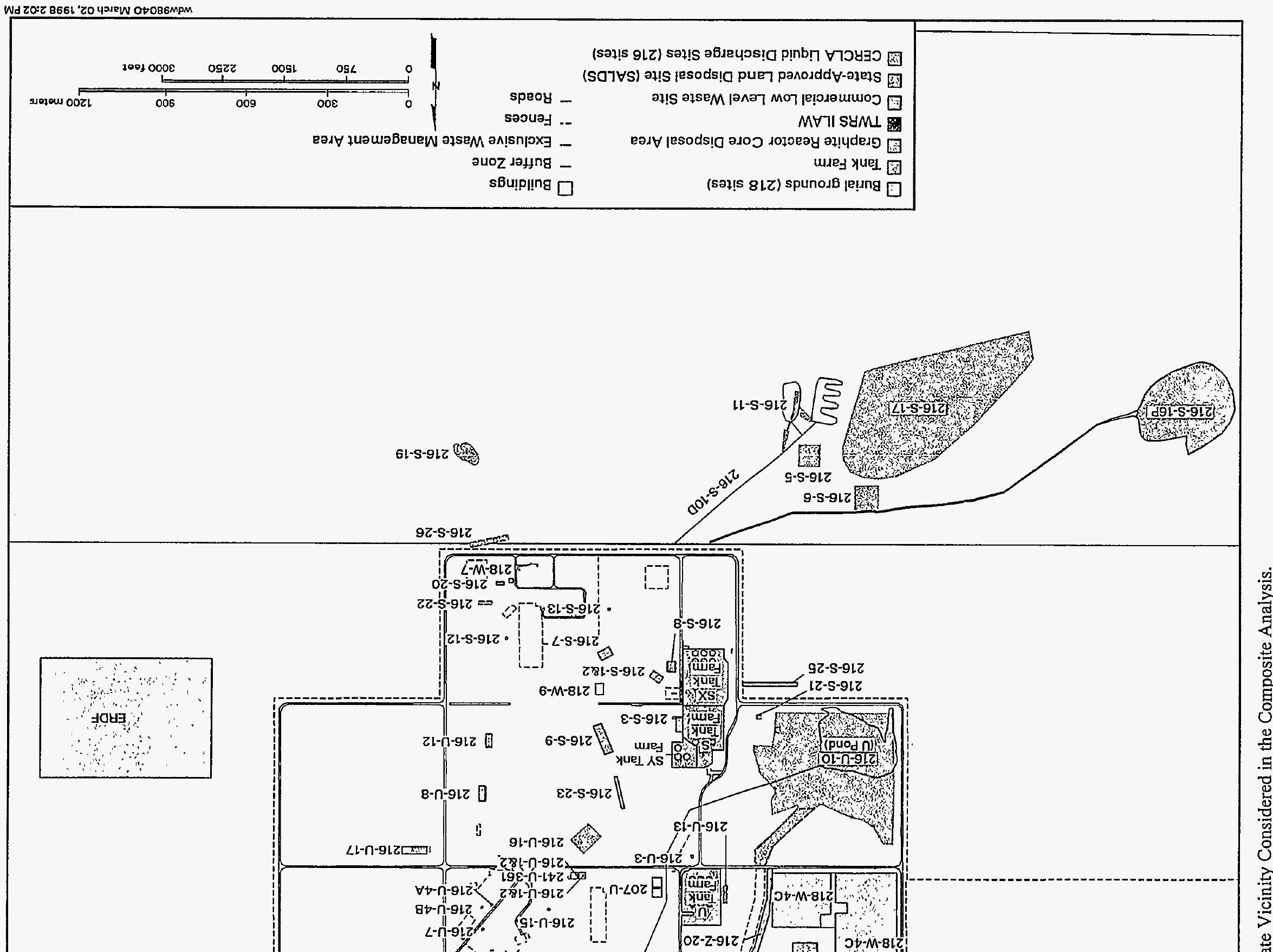




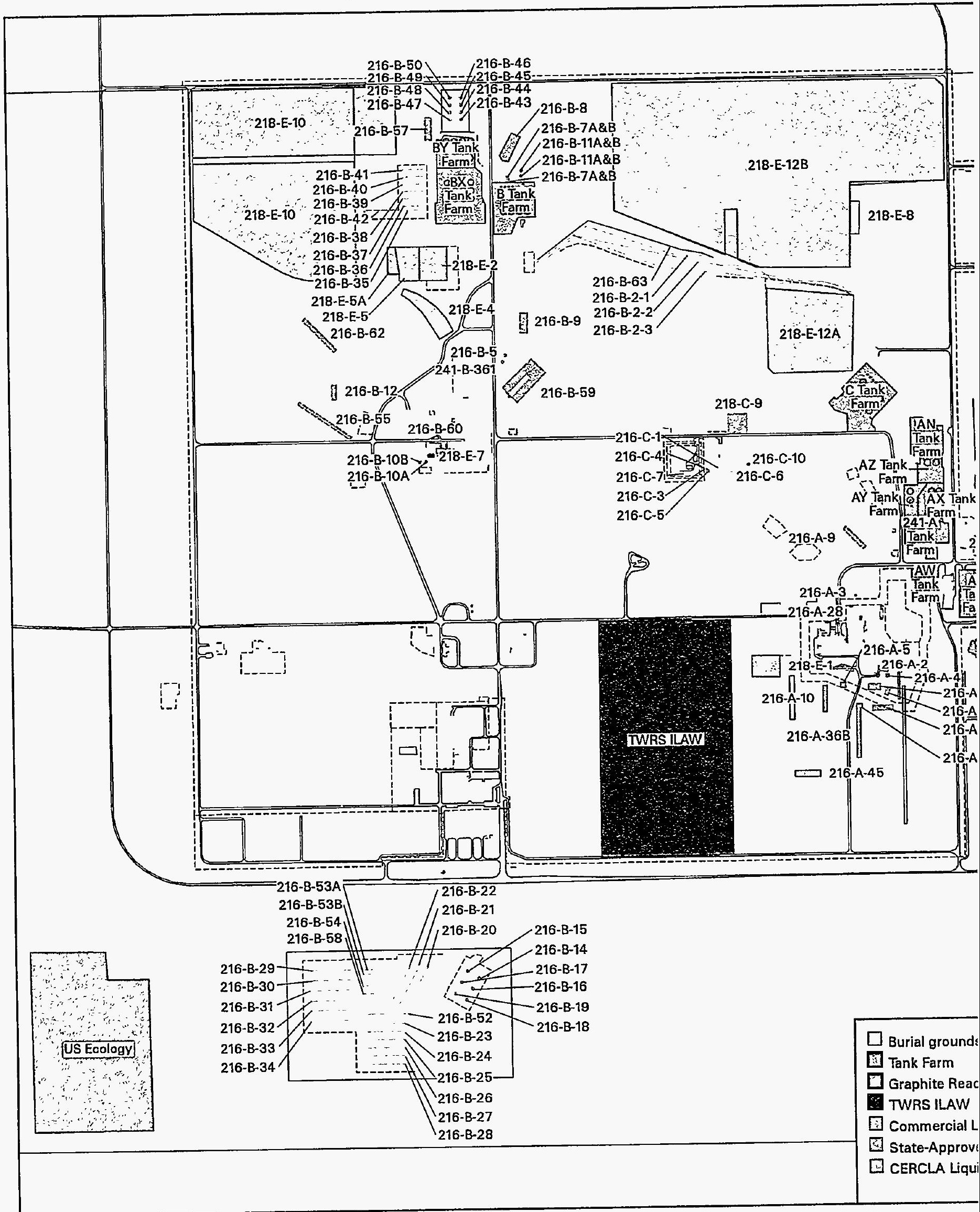

Figure 1.3c. Waste Sites of the 200 East Area and Im! 


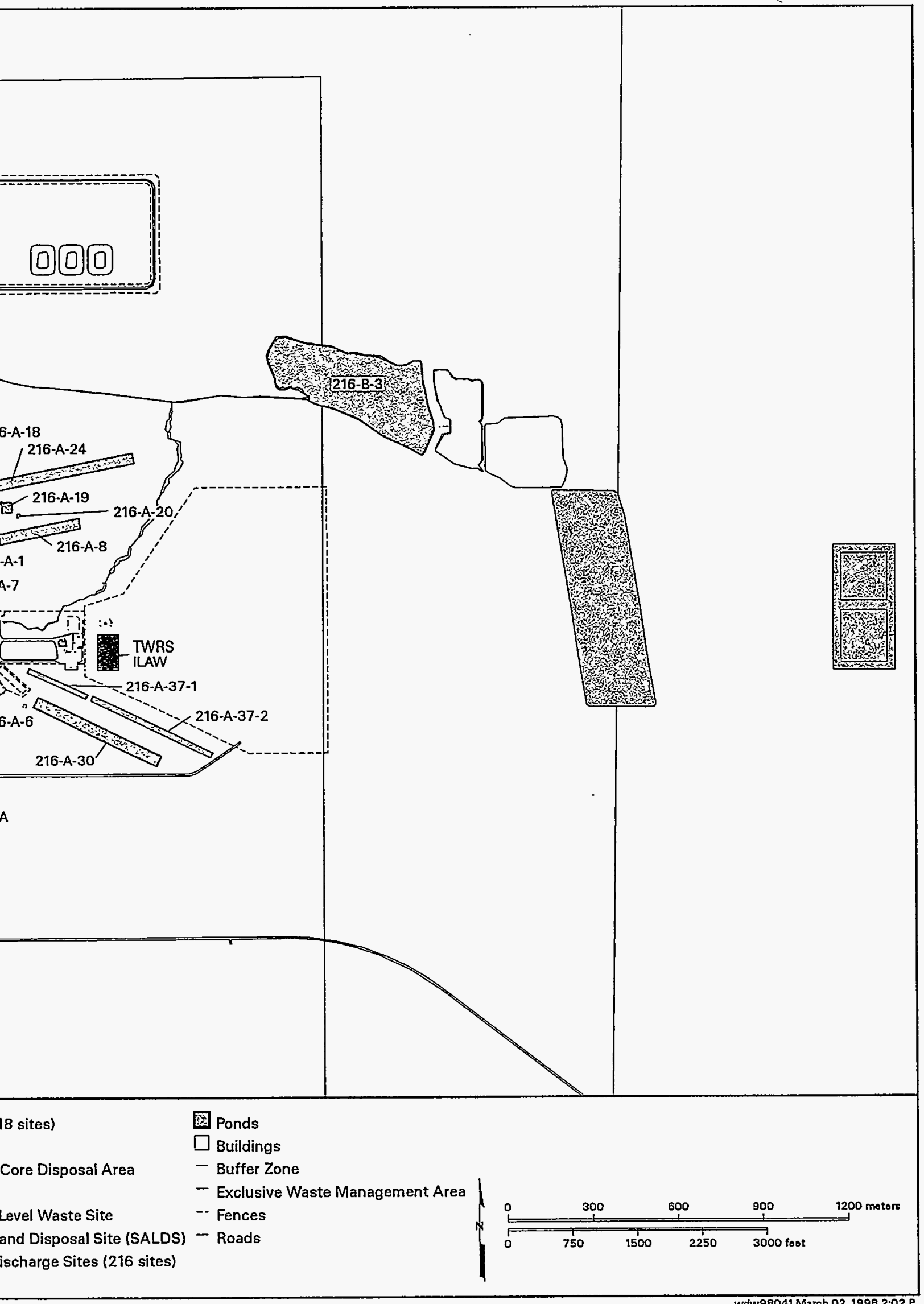

wdw98041 March 02, 1998 2:02 P

liate Vicinity Considered in the Composite Analysis. 


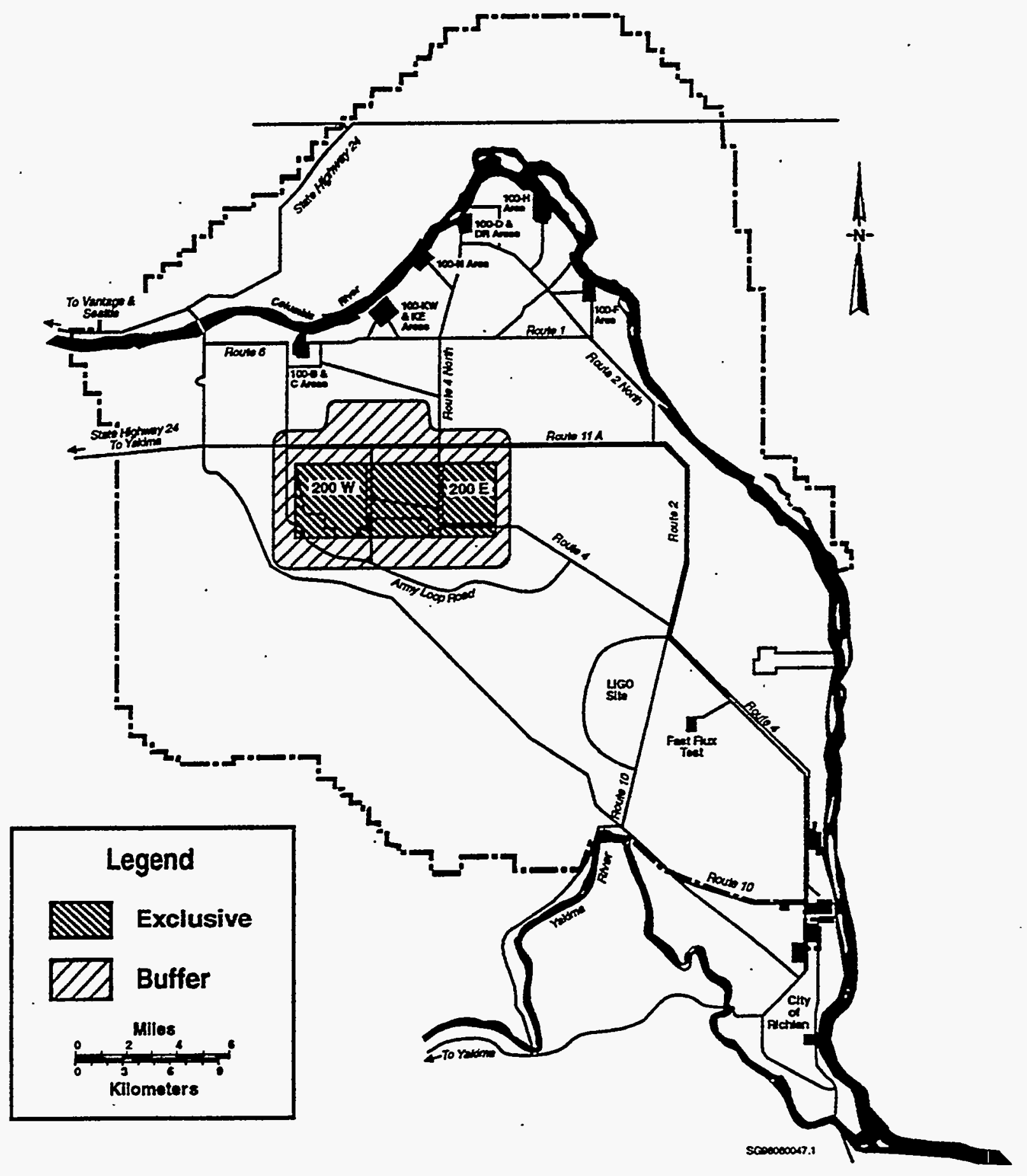

Figure 1.4. 200 Area Plateau with Exclusive Waste Management Area and Buffer Zone 


\subsection{Composite Analysis Process}

This document discusses the initial iteration of the Composite Analysis performed for low-level waste disposed in the 200 Area Plateau of the Hanford Site. In order to respond to the constantly changing technical and decision-maker needs, the process used in the Composite Analysis is necessarily iterative, adaptive, and flexible. This chapter explains the motivation for the process; provides an overview of the process; describes the impact of various types of errors on uncertainty in dose estimates; discusses the decisions made throughout the analysis; describes the data quality objective (DQO) process and how it was adapted for the Composite Analysis; and discusses the process employed in the initial iteration and the process planned for subsequent iterations. The role of DQOs in ensuring that the process adequately reflects the decision needs of the U.S. Department of Energy (DOE) as it seeks to dispose of low-level waste (LLW) at the Hanford Site is described in this chapter. The role of the first iteration Composite Analysis in the sequence of future analyses is also discussed.

\subsection{Motivation for the Process Used in the Composite Analysis}

The DOE directed that a composite analysis of the impact of interacting source terms on the radiological protection of the public from LLW disposal facilities within the Hanford Site be performed. This action was in response to Recommendation 94-2 from the Defense Nuclear Facilities Safety Board (DNFSB) (DOE 1996e). The DNFSB's review of the implementation of DOE Order 5820.2A (DOE 1988b) found that waste disposed prior to 1988 was neglected in evaluating dose impacts. Additionally, the DNFSB found that current guidance allowed evaluators to apply reference dose criteria to disposal facilities individually rather than assessing the composite effects of adjacent burial facilities.

The DNFSB recommended that a complete performance assessment of all active and planned LLW burial sites be performed, based upon the total inventories (past, present, and future) emplaced or planned for burial. In response to these DNFSB recommendations, the DOE decided to continue analyses of individual facilities using performance objectives defined in DOE Order 5820.2A (DOE 1988b) and conduct a Composite Analysis of sources that could commingle, and compare these results to the performance objectives defined in DOE Order 5400.5 (DOE 1993b).

The Composite Analysis process is iterative in order to

- ensure results are available in a timely fashion to assist the ongoing decision-making processes

- ensure analyses are representative of the site as decisions are made, records of decision (RODs) are issued, and new data are gathered and interpreted

- optimize the scope and scheduling of analysis activities.

While the primary objective and purpose of the Composite Analysis are to provide a holistic view of waste disposal and composite dose impacts to the public, each iteration of the Composite Analysis will be 
used to prioritize analysis activities in subsequent iterations. This adaptive analysis process is required, given the significant uncertainty involved in facility end states, inventories of critical contaminants, conceptual models (of contaminant release and transport), computational models (of related release, transport, and exposure), and the assumed future land uses.

A first iteration of the Composite Analysis has been completed. Consistent with the DOE directive for this initial iteration, it established a base case for comparison with the primary dose limit. The DOE directive, acknowledging the iterative process that would be required, stated that no new data should be collected for the first iteration.

The second and subsequent iterations of the Composite Analysis may affect the allocation and scheduling of resources for sitewide LLW management actions (e.g., site remediation) and waste disposal practices (e.g., barriers and waste packaging). Resources could be prioritized toward sites that will result in the greatest reduction in estimated composite dose. Schedules could be altered to favor those sites where delays might result in the greatest adverse impact.

In order for the Composite Analysis to support scheduling and prioritization of sitewide waste management or waste remediation actions, it must clearly articulate the tradeoffs between various objectives. These objectives include: minimizing the risk of underestimating the dose impacts; maximizing the time available before actions must be taken; maximizing the decision maker's confidence that the dose impact assessments are reliable; and minimizing costs. Clearly, these objectives involve tradeoffs. For instance, increasing confidence in dose predictions will result in greater analysis costs. Decision makers formulate the decisions to be made and incorporate the multiple objectives into the decision-making process by working through the seven steps of the DQO process.

The goal of the Composite Analysis is to reduce uncertainty only inasmuch as the reduction in uncertainty will directly affect the actions to be taken. It is not feasible to eliminate uncertainty. Reducing the uncertainty in such complex performance analyses can involve costly laboratory experiments, field experiments, and modeling analyses. The cost of reducing uncertainty is justified, if the reduction in uncertainty would likely alter a waste management or waste remediation decision. The cost of the analysis itself must also be compared to the expected loss from making an incorrect decision. If the analysis itself would cost more than the expected benefit of the analysis (i.e., the benefit to be gained from having improved information on which to base decisions), the analysis should not be performed.

Worst-case analyses are one way to limit the cost of one type of decision error: failure to take action when action is required. However, in a composite analysis, worst-case assumptions can only be applied in a limited manner. Attempting to use worst-case analyses independently for each site is problematic in a composite analysis because this approach neglects the impact of superposition of releases, generally is not able to prioritize actions, and violates sitewide mass conservation. The worst-case dose impact for a specific site is generally defined by the earliest and largest feasible release. However, the worst case for the composite dose from numerous sites is a function of the superposition of each site's plume. Defining. the worst-case scenario for a composite analysis is significantly more difficult than for an individual site's performance assessment. Worst-case assumptions also tend to penalize sites with less information. 
Therefore, prioritization is usually biased towards sites that lack information. Mass conservation is a useful constraint on sitewide inventories in composite analyses. Worst-case analyses for each individual site will violate mass conservation by systematically inflating inventories across the site.

The DQO process is a flexible and adaptive approach that attempts to match the type, quality, and quantity of data collected to the needs of the decision maker for confidence in decisions that will be based on that data. The DOE specifically directed that DQOs be employed in the Composite Analysis process. For a variety of reasons mentioned in Section 2.5, the standard DQO approach has not and can not be directly applied to the dose forecast problem of a composite analysis. Rather than try to "force fit" the DQO process, a slightly modified approach that involves incorporating the concepts of model uncertainty analysis and Value of Information (VOI) analysis is proposed. In the modified DQO process (described further in Section 2.5.2), decision makers use their assessment of the severity of consequences if model predictions are incorrect to justify the cost of any model improvements. In order to completely implement the modified DQO approach, a probabilistic modeling effort and subsequent cost/benefit and VOI analysis will be required.

\subsection{Process Flow Diagram}

Figure 2.1 illustrates the process used in the Composite Analysis. The process iterates until the decision makers have their stated level of required confidence in the dose estimate to support their decisions. The modified DQO process is used throughout the Composite Analysis to ensure that the cost of additional information gathering and model improvements are tied to the decisions to be made and the limits on decision error as specified by the decision makers. Decision makers set limits on the type of decision errors that they can accept, based on the actions identified in the decision rules and their assessment of the severity of the consequences that could result from making incorrect decisions based on model results. The seven activities shown in Figure 2.1 (rectangular boxes numbered 1 through 7 ) are described in detail below.

1. Select models. This step involves the selection of process models and uncertainty models. Examples of process models include release models, vadose zone transport models, groundwater transport models, atmospheric transport models, and exposure/dose models. In subsequent iterations of the Composite Analysis, multiple models may be employed for a single process to help address the issue of uncertainty in the process models. Uncertainty models attempt to define the distribution of errors in process model parameters and inputs, as well as to quantify the uncertainty in the predictions from analyses. In the first iteration of the Composite Analysis, process models were limited to readily available models. These models are discussed in detail in Section 4.1. Since the first iteration provided only a deterministic baseline, uncertainty models were not employed. In all subsequent iterations, uncertainty models will be required. $A$ modified $D Q O$ process will be used to direct the selection of both process models and uncertainty models.

2. Select the type and number of scenarios. This step involves selecting the type and number of scenarios analyzed. Each scenario is described by the manner in which the process models and uncertainty models are combined. Generally, each scenario results in a feasible realization of the estimated composite dose. In some cases, a realization can be eliminated from subsequent 
consideration by comparing its estimate of the current state with observed conditions. In the first iteration of the Composite Analysis, only one scenario for the transport models was considered whereas multiple exposure scenarios were considered. In subsequent iterations, multiple scenarios for the process models will be included. Since additional scenarios will result in additional cost and time to complete that specific iteration, the number of scenarios will be defined through the DQO process.

3. Select sites and radionuclides. This step allows the analysis to limit the number of sites and radionuclides for which detailed analyses are to be performed. Many sites and radionuclides have only a negligible impact on dose, even under worst-case conditions. Lowering the allowable dose to compensate for the combined worst-case dose from these sites and radionuclides can significantly reduce the analysis effort required. Analysis effort can then be focused on the sites and radionuclides most likely to significantly affect the composite dose estimate. In the first iteration of the Composite Analysis, all sites with reported inventories were analyzed. However, only carbon-14, chlorine-36, selenium-79, technetium-99, iodine-129, and uranium isotopes and all their daughters (which were expected to contribute the most to the composite dose) were completely analyzed. In subsequent iterations, the DQO process will be employed to define the amount the allowable dose will be lowered to compensate for the worst-case dose estimates from less significant sites and radionuclides.

4. Conduct performance analysis. This step requires the execution of the process and uncertainty models using the selected scenarios, sites, and radionuclides to assess the performance of the composite waste disposal facilities with respect to the applicable performance objectives. This is generally the most significant element of cost and time in the Composite Analysis process.

5. Perform screening ALARA assessment. If composite dose estimates are less than $\mathbf{3 0}$ mrem in a year, only a screening ALARA (as low as reasonably achievable) assessment is required. In the first iteration of the Composite Analysis, the dose estimate was less than $30 \mathrm{mrem}$ in a year so a screening ALARA was performed. If subsequent analyses continue to result in a dose estimate less than 30 mrem in a year, screening ALARA assessments will be repeated. Since the dose estimates will be probabilistic estimates, the DQO process will need to define the specific manner in which the 30 mrem in a year standard is defined. For example, the standard could be compared to the mean estimated dose or to the upper 95 percentile value.

6. Perform options analysis. If the composite dose estimate exceeds $30 \mathrm{mrem}$ in a year, an options analysis is required. Since the first iteration of the Composite Analysis resulted in doses less than 30 mrem in a year, an options analysis was not performed. If subsequent analyses result in a dose estimate greater than $30 \mathrm{mrem}$ in a year, an options analysis will be performed.

7. Perform ALARA assessment. If the composite dose estimate exceeds 30 mrem in a year, an ALARA assessment is required. Since the first iteration of the Composite Analysis resulted in doses less than $30 \mathrm{mrem}$ in a year, a complete ALARA assessment was not performed. If subsequent analyses result in a dose estimate greater than 30 mrem in a year, a complete ALARA assessment will be performed. 
The purpose of the full options analysis and ALARA assessment would be to pose and analyze alternate actions. These analyses need to be thorough in order to potentially support a DOE decision to change course.

The process proceeds iteratively. Each iteration helps define the optimal steps to improve the confidence in the subsequent analysis. The process terminates when adequate confidence exists in the model predictions and decisions are made to take action based on the model predictions.

\subsection{The Impact of Various Types of Errors on Uncertainty in Dose}

Because of its magnitude, uncertainty in environmental systems cannot be neglected in the decision process. Uncertainty in dose predictions from the models can be attributed to many sources of errors. These errors propagate and compound throughout a composite analysis. The four main types of errors, measurement errors, sampling errors, forecast errors, and model errors, are described below.

- Measurement errors are errors that result from inaccuracies in analytical measurements. The precision and accuracy of analytic measurement equipment and procedures are finite. Measurements of the identical sample will not always yield the same exact value. Measurement errors are readily dealt with using well-established statistical methods.

- Sampling errors are those errors that result from the spatial and/or temporal variability of the items being sampled. For instance, numerous samples are required to develop an understanding of the three-dimensional shape of a groundwater plume. Additionally, samples must be taken over time to characterize the migration and evolution of a plume. Geostatistical methods for estimating spatially variable fields and for estimating the errors in these estimates are currently available.

- Forecast errors are those errors that result from the limited ability to predict future conditions. Future climate, future land use, and exposure scenarios are all examples of processes subject to significant forecast errors. Some forecast errors, climate for instance, can be estimated by assuming the historically observed variability will persist into the future. However, other processes, such as land use and exposure scenarios, have no historical analogs.

- Model errors are those errors that result from the conceptual or numerical formulation of the process models. While many numerical method errors can be readily corrected with improved numerical algorithms, conceptual model errors often require expensive laboratory experiments and/or field measurements in order to validate the model's process formulation. Model errors cannot be dealt with using the statistical and geostatistical methods applied to measurement and sampling errors.

Measurement errors and sampling errors are the easiest to quantify. Unfortunately, in a composite analysis, forecast errors and conceptual model errors contribute significantly more to the overall uncertainty than numerical formulation, sampling or measurement errors. Model forecast errors and conceptual errors are also the most difficult to quantify. Monte Carlo methods have been shown to be useful in evaluating model uncertainty (IAEA 1989; NCRP 1996). Stakeholder acceptance, as well as scientific issues, must be considered in selecting models and future scenarios. 
Challenges to an accepted conceptual model can even require that a whole new set of measurement and sampling methods be developed to measure critical factors that distinguish among alternate conceptualizations. Deciding which of the feasible process models is valid may require expensive experiments. However, it is not essential to determine which exact process model is valid, if the other feasible process models would not result in a different waste management action or waste site remediation decision.

Each iteration of the Composite Analysis will close confidence gaps in the composite dose estimate. This will require the development of a specific set of analytical procedures to quantify performance and uncertainty.

\subsection{Decisions Made Throughout the Composite Analysis}

Decisions made throughout the Composite Analysis process will be directed by the decision makers' answers to three questions. These three questions are:

- Will the dose be greater than a prescribed level?

- How should resources for model development and improvement be allocated?

- What set of possible actions are to be considered in the options analysis, if required?

The primary decisions in the Composite Analysis are associated with the first question; the second and third questions support these primary decisions. Whether the actions identified in the third question are taken depends on the outcome of the answer to the first question and the confidence in this answer. The level of confidence in the predicted dose shapes the second decision on the allocation of resources committed to model development and improvement. The three decisions are tied together, and the methods and techniques for dealing with them show a strong interdependency. However, the focus must remain on the primary decision. It is a decision about some unknown, future state.

Making decisions and taking actions based on model predictions begs the question of "How good must a model be in order to make good decisions?" There is a need to balance the desire for more accurate model predictions against the costs of developing and testing new or revised models. Implicit in the need for better models is the potential cost or loss function associated with making either of the following two types of errors based on inaccurate model predictions:

- Taking actions that are not required (e.g., models predict doses greater than the standard when in fact true doses are less than the standard)

- Not taking actions that are required (e.g., models predict doses less than the standard when in fact true doses are greater than the standard). 
Before extensive resources are expended, answers are needed to the three questions listed below.

- How reliable are the predicted values from the model?

- What level of effort or expenditure of resources is required to get "better" madel results?

- How much "better" does the model need to be?

The answer to the first question depends on the quality of the input data for the predictive models and the reliability of the models themselves. This has not been explicitly addressed in the first iteration of the Composite Analysis. The underlying assumptions to the second question are listed below.

- Better model results can be achieved with greater effort.

- It is possible to quantify the relationship between increased effort and increased probability that the model predictions are correct.

These assumptions have not been explicitly addressed in the first iteration of the Composite Analysis. The answer to the third question requires a DQO approach where all parties that have a stake in the accuracy of model prediction supply input to the decision. The underlying assumption for the third question is that it is possible to quantify the likelihood of making the correct decision when decisions are based on model output. In order to work within this final assumption, it is necessary to take a flexible and somewhat more qualitative approach to the DQO process than the U.S. Environmental Protection Agency (EPA)-sanctioned DQO approach developed for the standard environmental sample collection problem (EPA 1994).

\subsection{Data Quality Objectives}

The DQO process was applied to the Composite Analysis in a different manner than is generally used. The reasons for this are explained in the next two sections. However, the philosophy of the DQO process was incorporated in the first Composite Analysis iteration, and will be incorporated in future iterations. This philosophy is: before extensive effort is expended on collecting data and, in the case of the Composite Analysis, making improvements to models, it is necessary to identify the specific decisions to be made based on the information and the level of confidence in model results required in those decisions. The intended use of data and model predictions, and consequences associated with decision error, drive the type and quality of information needed. Future improvements to the Composite Analysis will be a function of perceived needs to improve the type and quality of information needed to make the necessary decisions.

\subsubsection{The Standard DQO Approach}

The DQO process was developed in response to the need for Comprehensive Environmental Response, Compensation, and Liability Act (CERCLA) and Resource Conservation and Recovery Act (RCRA) investigations to define the quantity and quality of characterization data required to make cleanup decisions. The DQO process normally involves the following seven steps: 
1. Statement of the problem

2. Identification of the decision

3. Identification of inputs to the decision

4. Definition of the boundaries

5. Development of a decision rule

6. Specification of limits on decision errors

7. Optimization of data collection.

The EPA guidance (EPA 1994) provides more detail on the DQO process. For the standard DQO approach, the assumptions, theory, decision-error limits, and relative decision-error consequences combine into a closed-form solution where sample size formulas and equipment quality selection criteria are the outputs of the DQO process. In Step 7, the stakeholders make cost-benefit comparisons that reflect resource constraints and risk versus cost tradeoffs. The result is an "optimized" level of resources to commit to improved sample collection and analysis and hence, improved decision quality. The DQO process does not provide explicit guidance on how to make these tradeoffs, but implicit in Step 7 is the concept that the costs of decision error consequences will be matched against costs of increased sampling, with the assumption that increased sampling will lead to greater confidence in field characterization and fewer decision errors.

\subsubsection{Modified DQO Process Applied to Model Predictions}

Decisions based on model predictions during a future time for a hypothetical maximally exposed individual, rather than on sample data from a current "true" state, require modifications to the later steps of the standard DQO process. However, the objective of the DQO process remains the same: to balance the desire for more and better data (more reliable dose forecasts) against the cost of obtaining more and better data (more reliable dose forecasts). The decision on the amount of data (more reliable dose forecasts) needed is based on the amount of uncertainty that can be tolerated, which, in turn, is related to the consequences of making an incorrect decision.

For the standard CERCLA/RCRA DQO application, collecting a $100 \%$ sample (i.e., complete enumeration) of a current condition results in a $0 \%$ risk of making a decision error on the characterization of that condition, given accurate sample collection and analysis equipment. The metric for decision performance is percent of time a randomly selected sample gives an accurate assessment of the "true" condition if the sampling event is repeated over and over. For a model prediction problem with stochastic variables, there is no single future "true" condition against which to measure accuracy of a model forecast. The analogy of increasing sample sizes to achieve a $0 \%$ risk of making a decision error must be modified before it can be translated into the prediction problem. Increasing expenditure of resources for the modeling problem can decrease uncertainty, but there is no simple linear relationship between increased expenditure and increased confidence in model results. The literature on model uncertainty captures some of this complexity and refers to Type A and Type B uncertainty for the modeling problem (IAEA 1989).

Thus, the standard DQO approach cannot be applied to the dose forecast problem directly. Rather than try to "force fit" the components, it is better to make a slight paradigm shift by using the terminology 
of model uncertainty/model sensitivity analysis coupled with a VOI approach (Morgan and Henrion 1990 ) to making risk/cost/benefit tradeoffs when trying to decide whether model results are good enough for decision making. The result is a modified approach to the DQO process; decision makers use their assessment of the severity of consequences if model predictions are incorrect and balance that with the cost of model confidence or uncertainty reduction. In order to do that, they need a way to assess uncertainty in model output similar to the way statisticians assess the uncertainty in sampling results.

\subsubsection{Steps 1 through 4}

The first four steps of the DQO process can be followed for the modified approach with very few exceptions from the standard approach. Much of the work that is done in the early steps of the DQO process remains the same for the sampling and the dose forecast problem. For the dose forecast problem, the outputs from Steps 1 through 4 translate directly into what is modeled, what parameter inputs are used, and what ranges are considered in selecting parameters and model formulations. The results of Steps 1 through 4 for the initial iteration of the Composite Analysis are discussed in Section 2.6.

\subsubsection{Steps 5 through 7}

Step 5 is usually derived from a statutory or regulatory requirement, along with what form the modeling output takes. In the composite analysis problem, it is the various dose limits established by the DOE (for radionuclides) and the EPA (for chemicals) as protective of human health and the environment that drive the decisions to be made. It is the scope of the modeling effort that drives how detailed the decision rules can be.

In Step 6, the definition and treatment of model prediction uncertainty becomes a critical component of applying the DQO process to the composite analysis problem. In Step 6, decision makers provide the desired or acceptable levels of decision errors they can accommodate based on their assessment of possible consequences of making decision errors. There is no guarantee that these levels can be achieved within budget and practical constraints; but the decision makers must begin the tradeoff process with a decision quality goal in mind.

In Step 7, decision makers are asked to "optimize" the design. This step usually requires the help of experienced statisticians and optimization experts who can design sampling strategies, refine models, and design metrics to measure and assess potential decision errors. For the model prediction problem, this means the decision makers, along with their technical experts, must decide if spending additional resources on improving model input or the model itself is of "value." If the decision makers use their risk-aversion/risk-taking preferences to guide the expenditure of resources, they have complied with the spirit of Step 7 of the DQO process. Expenditure of resources could refer to the resources spent on model improvements; it could also refer to the resources spent on making changes to the LLW projects if the Composite Analysis indicates dose limits will be exceeded. These resource allocations affect the primary decision of whether dose limits have been exceeded and there is sufficient confidence in the model to act. Progress on Steps 5 and 6 for this first iteration of the Composite Analysis is described in Section 2.6. 


\subsection{Initial Iteration of Composite Analysis}

Because the DOE directed that the first iteration of the Composite Analysis use only available information, no field samples were collected for the first iteration. According to the DOE directive, the Composite Analysis should establish a "base case" or "best estimate" for comparison with the primary dose limit. This case should represent a reasonable, yet conservative, forecast of the future state of the Hanford Site, based on current knowledge. It should include expected remedial activities. Where future disposition of a source is not known, a reasonably conservative assumption should be made.

The initial Composite Analysis described in this document represents a deterministic base case. The results of these calculations represent an initial deterministic assessment based on available models and 'best estimates' of most model parameters. The scope of the analyses performed in subsequent iterations of the Composite Analysis will be directed by these preliminary findings and DOE guidance.

Performance analysis calculations were performed for all sites for which data were available. As mentioned earlier, defining a "worst case" in a composite analysis is problematic because of the superposition of plumes. In this analysis, best available estimates were used for nearly all model parameters. However, wherever uncertainty existed in two inventory estimates, the higher value was generally chosen.

In this initial analysis, sensitivity analyses were performed only to calibrate parameters. In order to ensure that results of the Composite Analysis could affect decision making early in the process, the DOE directive providing guidance for the Composite Analysis eliminated any new measurements from the scope of the initial analysis. As described in Chapter 3, attempts were made in the inventory estimate development process to identify errors in sitewide mass conservation for each of the radionuclides considered.

The first four steps of the DQO process were drafted early in the Composite Analysis, which helped define the problem being addressed. The draft addressing the first four steps of the DQO process was presented to a group referred to in later discussions as the DOE representatives, or "decision makers" for each LLW disposal or remediation activity. This group consisted of DOE and contractor representatives from the various Hanford Site programs. The draft was developed without programmatic input to establish a starting point for discussion. The first four steps are summarized in Table 2.1. The initial group did not have major revisions to the drafted Steps 1 through 4; however, they requested the option of revising Steps 1 through 4 once they reviewed the preliminary model results.

In an effort to complete DQO Steps 5 and 6, a meeting was called on December 13, 1996 with the decision makers to determine the acceptable level of uncertainty in the decisions to be made. The following questions were asked:

- Over what spatial area and what time period should the dose be integrated for comparison to a dose limit that would trigger some action? 
- What actions would be taken if doses exceeded limits? This would be broken down into specific actions for specific programs. The representatives may want doses predicted on a smaller scale than currently provided, and integrated over a different range of influence, prior to taking actions that would commit their programs to major redesign expenditures. This is exactly the type of detail, negotiation and discussion that are fostered in the DQO process. Locking in these details prior to seeing the final model runs enables all parties to negotiate upfront and avoids discussions on semantics, assumptions, and meaning once results are final and actions must be taken.

- What dose limits are to be used in the final comparisons? Depending on how the model results deal with and report uncertainty, the operational decision rule may use an upper percentile of the distribution of possible doses to compare to the regulatory limit. A statistician should be involved with this step to make sure double conservatism is not built into the decision making and that uncertainty in model results is accurately compared to the desired limits on uncertainty as provided by the decision makers in Step 6.

At a December 13, 1996 meeting, LLW site representatives provided their best judgements for preliminary limits on decision errors. Specifically, the representatives at the meeting were asked:

"If the model predicts a dose less than the action limit of 30 mrem in a year (and thus no action is required), but the 'true' dose turns out to be $\mathrm{x}$ (values on the $\mathrm{x}$ axis as shown in Figure 2.2), what limit would you want to place on making a decision error?"

The error limits that the LLW site representatives provided were specified over a range of possible outcomes. The responses received from the representatives are shown in Figure 2.2. This figure is a "modified" Decision Performance Goal Diagram and is modeled after the EPA structure for representing user-supplied decision error limits. Shown on the y-axis is the probability of deciding the dose is greater than $30 \mathrm{mrem}$ in a year. Thus, $1-y$ is the probability of making a decision error. This modified diagram shows only one type of decision error: not taking action when it is required.

In this initial iteration of the Composite Analysis, "best estimate" input values and model assumptions are used, with no ranges provided. Therefore, no quantitative measures of the ranges in the dose estimates are possible. Unless decision makers are able to assign a confidence level to input values and model assumptions, there is no way to judge the confidence that should be assigned to model output. A qualitative way to incorporate decision makers' decision error limits into the process is to have the representatives assess their "relative comfort" with the justifications provided that conservative assumptions were used for model input. If the representatives are comfortable with the inputs used and the level of conservatism is consistent with the probabilities provided in Figure 2.2, then on a qualitative basis, the desired decision error limits from the DQO have been achieved and incorporated into the Composite Analysis.

In this initial iteration, maximum predicted doses are about $6 \mathrm{mrem}$ in a year, well below the $30 \mathrm{mrem}$ in a year limit. Only single input values were run through the model. But since there is no way to assess the confidence in the single predicted dose estimate, there is no way to evaluate whether additional model 
improvements are justified. In subsequent iterations, quantitative estimates of confidence will be developed so that model improvements can be addressed directly.

\subsection{Subsequent Iterations of the Composite Analysis}

Several more iterations of the Composite Analysis will be required, before a full probabilistic assessment will be appropriate. Attempting a probabilistic assessment with a conceptual model with which the decision makers have no confidence is not going to increase their confidence. Therefore, uncertainty in the conceptual models will continue to be the dominant concern in early iteration. Monte Carlo methods are not appropriate for assessing unçertainty in the conceptual model, but intercomparisons among alternate conceptual models can bound the impact of model uncertainty. In instances where the impact of conceptual model uncertainty will affect decision making, intercomparisons among alternate conceptual models can suggest laboratory and field experiments that could resolve which conceptual model is appropriate.

One concept in which decision makers have a high degree of confidence is the principle of mass conservation. This is the primary conceptual element of the inventory model/database. While significant uncertainties exist in the exact present and future locations of radionuclides, the total inventory of radionuclides is reasonably well constrained by estimates of the radionuclides produced during reactor operations. Using probabilistic methods, the next iteration of the Composite Analysis will generate multiple equally feasible estimates of inventory for each site that are consistent with the principle of mass conservation. This information is critical to allowing a defensible approach to screening sites and radionuclides from further detailed analysis.

In order to limit the scope of the analyses, sites and radionuclides will be screened by a limited application of worst-case analysis. As discussed earlier, defining a worst-case condition for a composite analysis is considerably more difficult than defining the worst-case conditions for a single site and single radionuclide because of the superposition of plumes. However, by considering just the magnitude of the maximum dose, and not the timing and location of this maximum value, a large number of insignificant contributors to the dose can be placed in a single dose pool. This large number of sites could be simulated as individual releases, and doses could be calculated outside the buffer zone. The maximum dose from each site would be identified, and the sum of all sites accumulated independently of where or when it occurred. The combined dose of this large number of small contributions must be less than the target dose being considered. For instance, if the combined dose of these sites and radionuclides only resulted in a dose of $5 \mathrm{mrem}$ in a year, and $30 \mathrm{mrem}$ in a year was the dose estimate that would result in a different decision, the remaining sites, which would be analyzed in greater detail, would have to equal or exceed $25 \mathrm{mrem}$ in a year before requiring a different decision. Clearly, this approach is biased towards making the decision error of taking actions that are not required. The size of the worst-case reserve, 5 mrem per year in this example, involves a tradeoff between increasing the analysis costs by including more sites and radionuclides in the detailed analysis and decreasing the likelihood of making a decision error of not taking actions that are required. 
Subsequent iterations will also provide a basis for completing Steps 5 through 7 of the DQO process for the primary decision described in Section 2.4. The ways in which the subsequent iterations will affect DQO process Steps 5 through 7 are described as follows.

- Step 5. The decision rules will be modified to address the revised-model output. The decisions rules will address when specific LLW management actions and disposal practices from the options analysis will be undertaken based on model predictions.

- Step 6. Hanford site representatives may want to revisit the inputs provided for the Decision Performance Goal Diagram (Figure 2.2). They will also be asked to provide decision error limits for the second type of error: taking action when none is required.

- Step 7. Once results from the bounding or probabilistic assessment are available, and the conceptual model refined, decision makers can make a qualitative attempt at explicitly incorporating DQO limits on decision error into probabilistic analysis by placing upper confidence bounds on model output and comparing these upper confidence intervals to the dose limits. If the upper bound exceeds the limits, the decision makers are tasked with making resource allocation decisions and tradeoffs. Are model improvements required to reduce uncertainty bounds? Are low-level waste project modifications required to reduce.dose predictions? The decision makers now have the tools and the input required to address these difficult questions posed in Section 2.4. While incorporating decision error limits into a probabilistic analysis and making resource allocations and tradeoffs are challenging, there are examples where such issues have been addressed along with the methods used to address them (Black et al. 1997; Black et al.1994; Freeze et al. 1992; Gilbert, Bittner, and Essington 1995). 
Table 2.1. DQO Steps 1 Through 4 for the Hanford Site Composite Analysis

\begin{tabular}{|c|c|}
\hline Step 1. Define the Problem & $\begin{array}{l}\text { Predict the maximum annual dose to a hypothetical future member of } \\
\text { the public resulting from combined radionuclide releases to } \\
\text { groundwater from multiple sources during the } 1000 \text {-year period } \\
\text { following closure of the Hanford Site. }\end{array}$ \\
\hline Step 2. Define the Decision(s) & $\begin{array}{l}\text { A range of decisions must be made based on the results of the predicted } \\
\text { dose to a hypothetical future member of the public during the } 1000 \text { - } \\
\text { year period following closure of the Hanford Site. } \\
\text { If the maximum predicted dose is greater than } 100 \text { mrem/yr in any } \\
\text { year, then an options analysis and ALARA assessment is performed } \\
\text { and actions (determined by the options analysis) are taken to reduce the } \\
\text { predicted dose below } 100 \text { mrem in a year and ALARA (as determined } \\
\text { by the ALARA assessment). } \\
\text { If the maximum predicted dose is greater than } 30 \text { mrem in a year, but } \\
\text { less than } 100 \text { mrem in a year, then an options analysis and ALARA } \\
\text { assessment are performed to identify the most effective actions that } \\
\text { could be taken to reduce the predicted maximum dose. However, an } \\
\text { alternate action is only recommended if it is feasible and beneficial } \\
\text { considering economic, social-cultural, and ecological-resource factors. } \\
\text { If the maximum predicted dose is less than } 30 \text { mrem in a year, then a } \\
\text { screening-type ALARA assessment that weighs the cost of the options } \\
\text { analysis and the potential benefit of dose reduction is-performed to } \\
\text { determine if a full options analysis and ALARA assessment is } \\
\text { warranted. }\end{array}$ \\
\hline Step 3. Define the Inputs & $\begin{array}{l}\text { The calculated composite dose at locations that are accessible to } \\
\text { hypothetical future members of the public is the information initially } \\
\text { needed to make the decision whether an options analysis and ALARA } \\
\text { assessment is required. These composite doses were calculated by a } \\
\text { series of models that describe the release of radionuclides from waste } \\
\text { sites, transport through the vadose zone, transport through } \\
\text { groundwater, and exposure of individuals. The required inputs are } \\
\text { categorized below: } \\
\text {-Inventory data - total activity of each radionuclide that could } \\
\text { contribute to the calculated composite dose } \\
\text {-Release model assumptions - chemical and physical form of waste, } \\
\text { release mechanism (i.e. dissolution, diffusion, and corrosion) } \\
\text {-Release model parameters - water flux through waste site, dissolution } \\
\text { rates, diffusion coefficients, temperature } \\
\text {-Vadose zone contaminant transport model parameters - depth of } \\
\text { waste, cover type and integrity, recharge rate } \\
\text {-Vadose zone contaminant transport parameters - porosity, unsaturated } \\
\text { flow parameters, moisture content, distribution coefficients }\end{array}$ \\
\hline
\end{tabular}


Table 2.1. (contd)

\begin{tabular}{|c|c|}
\hline & $\begin{array}{l}\text {-Groundwater transport model assumptions - future land use, location } \\
\text { of the boundary where public access is assumed } \\
\text {-Groundwater transport parameters - porosity, saturated hydraulic } \\
\text { conductivity, aquifer thickness, distribution coefficients, recharge rates } \\
\text {-Exposure scenario assumptions and parameters. } \\
\text { If an options analysis and ALARA assessment are required, more } \\
\text { information will be needed regarding treatment and disposal options. } \\
\text { Information is also required to support the ALARA assessment of the } \\
\text { economic, social-cultural, and ecological-resource impacts of alternate } \\
\text { remediation strategies. }\end{array}$ \\
\hline Step 4. Define the Boundaries & $\begin{array}{l}\text { The analysis of exposure and dose to a member of the public applies to } \\
\text { the land area where future members of the public may be exposed to } \\
\text { radionuclides that have migrated from final disposal locations at } \\
\text { Hanford. This area will exclude a waste management area assumed to } \\
\text { remain under DOE control and not be accessible to the public. The } \\
\text { decisions will be based on calculated doses during the first } 1000 \text { years } \\
\text { after Hanford Site closure. However, calculation of doses will be } \\
\text { carried out for longer periods of time to fully understand the migration, } \\
\text { potential, and longer-term fate of the radionuclides. No accident or } \\
\text { intruder scenarios will be considered. } \\
\text { Note: It may be determined that the decision unit is each half acre of } \\
\text { land in a buffer zone near the boundary, and/or that a separate decision } \\
\text { is required for the maximum exposed individual as well as an average } \\
\text { dose. These issues remain to be resolved. }\end{array}$ \\
\hline
\end{tabular}




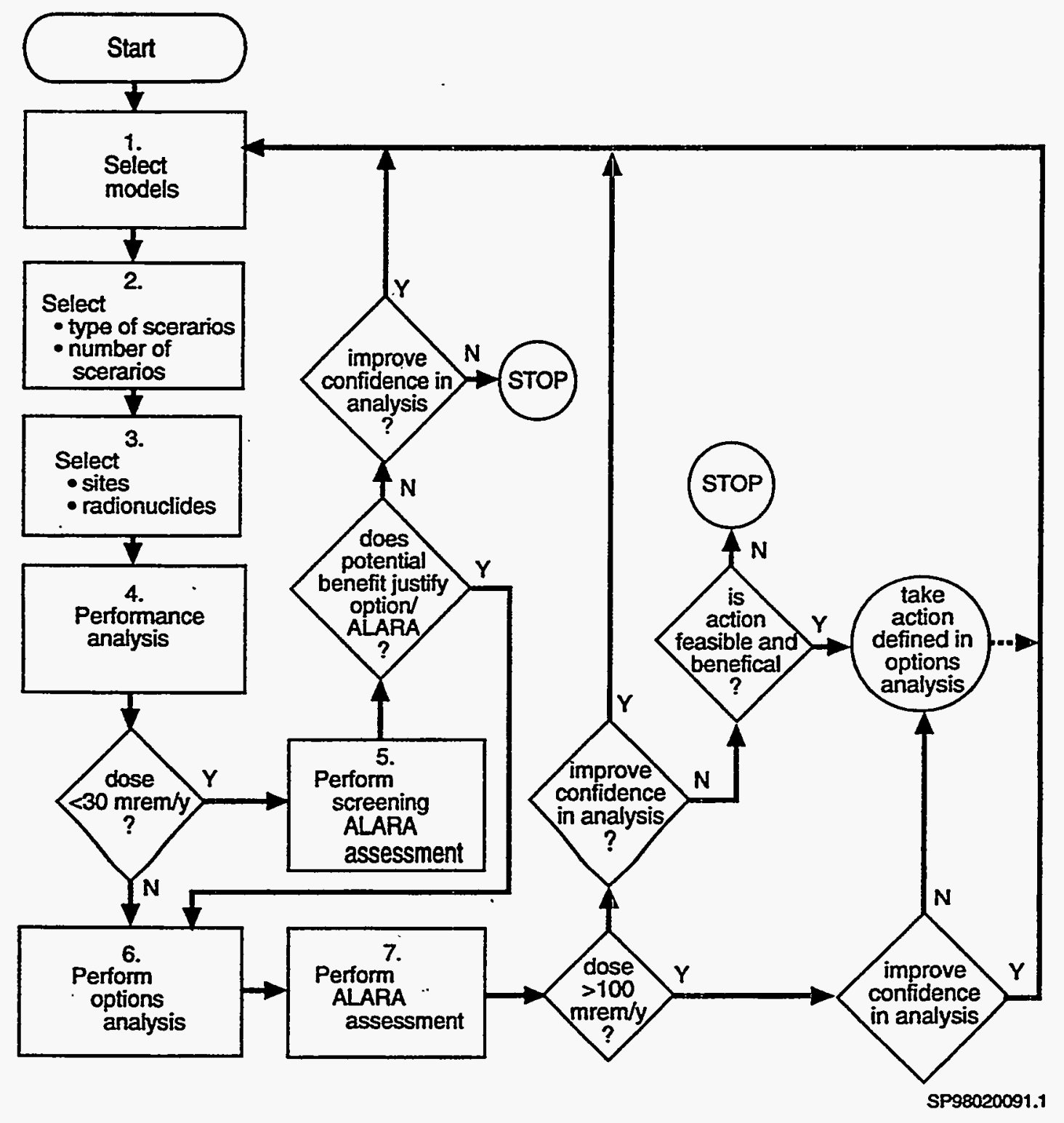

Figure 2.1. Composite Analysis Process Flow Diagram 


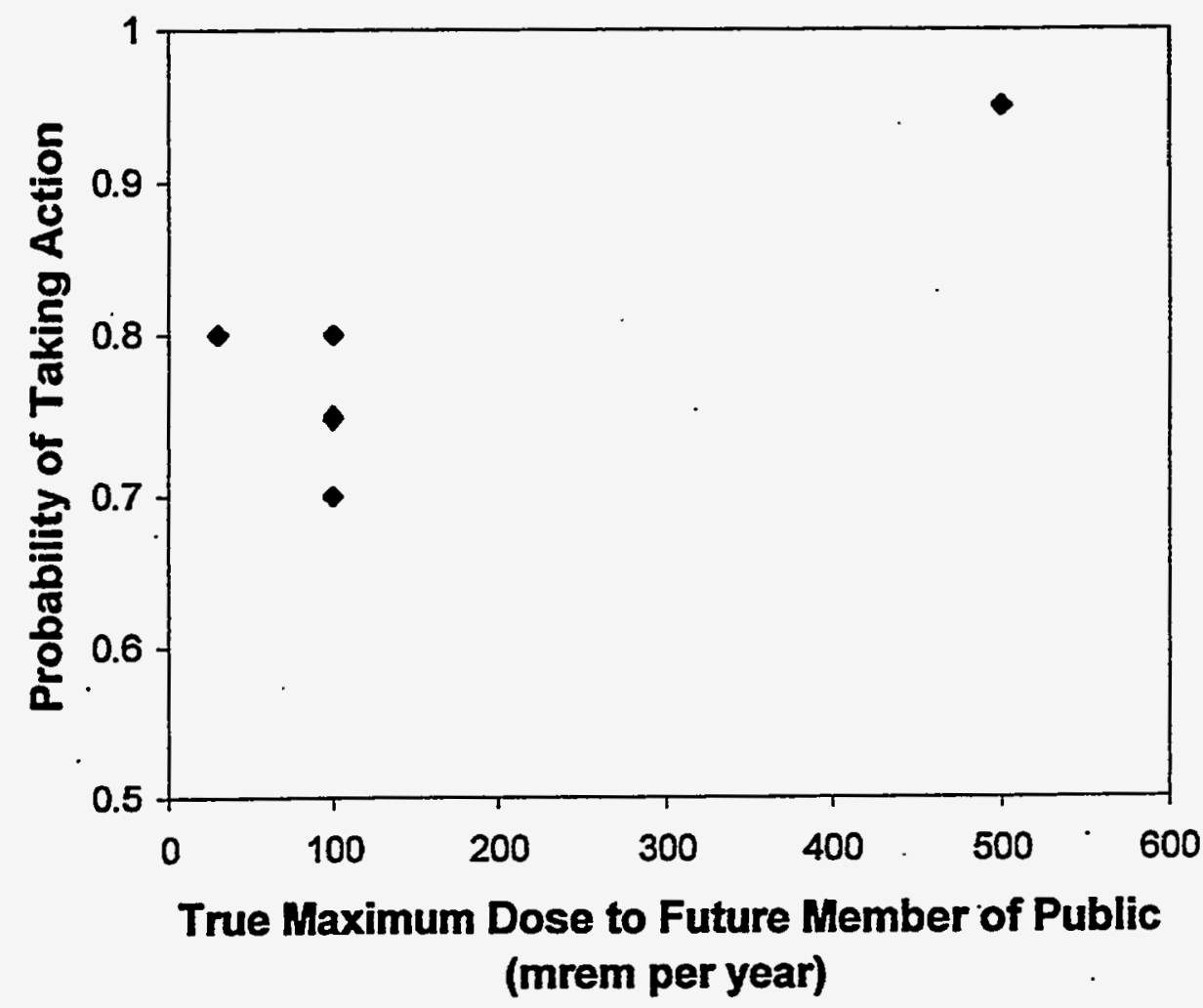

Figure 2.2. Modified Decision Performance Goal Diagram 


\subsection{Source Term Development}

This chapter describes the sources of radioactive material that were considered for the Composite Analysis for Low-Level Waste Disposal in the 200 Area Plateau of the Hanford Site (Composite Analysis). Chapter 3 presents both the rationale for selecting these sources as likely to contribute to the dose from the low-level waste (LLW) disposal facilities received by the hypothetical future member of the public as well as the justification for excluding other sources from the analysis. The basis and justification for estimating the radioactive waste source term, (i.e., inventory) for each source included in the Composite Analysis, and the estimated source terms are also provided.

\subsection{Sources of Radioactive Material}

From 1943 until 1990, the mission of the Hanford Site was to produce special nuclear materials for weapons. After developing the largest site within the U.S. Department of Energy (DOE) complex devoted to production of special nuclear materials, activities at Hanford underwent a series of dramatic changes beginning in 1964. Plutonium production was sharply curtailed in response to the nation's changing defense needs. Eight production reactors were shut down by 1971 . In January 1987, the $\mathrm{N}$ Reactor was placed in stand-down status for an extensive maintenance and safety enhancements program. In February 1988, the N Reactor was placed on cold standby. In July 1991, the DOE decided to cease preservation of the reactor and proceeded with activities leading to the ultimate decommissioning of the facility.

In July 1989, the Hanford Site was listed on the National Priorities List (NPL) under the Comprehensive Environmental Response, Compensation, and Liability Act (CERCLA) of 1980 as amended by the Superfund Amendments and Reauthorization Act (SARA) of 1986. In 1990, the mission of the Hanford Site changed to the safe cleanup and management of the legacy wastes, and the development and deployment of science and technology.

The vast majority of the radioactive waste inventory at Hanford was created during the production mission. There were three distinct steps in the production process: fuel fabrication, fuel irradiation, and chemical separation. During the first decades of production work at Hanford, it was common to locate waste disposal sites relatively close to waste-generating facilities. This practice resulted in numerous and varied disposal sites. The most dangerous radioactive wastes were stored in large single-shell tanks in the 200 Areas (Agnew et al. 1997; Kupfer et al. 1997). Large volumes of solid waste (e.g., contaminated tools and protective clothing) were disposed in burial grounds, and large volumes of liquid waste were discharged to shallow subsurface cribs, French drains, injection (or reverse) wells, and specific retention trenches.

More recently, all fuel fabrication and reactor operation activities ended and cleanup of past-practice units associated with them began in the 300 Area and 100 Areas. Low-level waste from ongoing operations is disposed in specific burial grounds in 200 West and 200 East Areas, and liquid discharges of radioactive wastes are being discontinued. The Tank Waste Remediation System (TWRS) program 
addresses the waste disposal and site cleanup issues for tank wastes and tank farm facilities in the 200 Areas. DOE programs are in place and coordinating with representatives of the State of Washington Department of Ecology (Ecology) and the U.S. Environmental Protection Agency (EPA) to evaluate and decide upon the decontamination, decommissioning, or remediation strategies for reactors, chemical separation plants, and 200 Area past-practice sites (e.g., solid waste burial grounds and liquid discharge sites).

\subsubsection{Solid Waste Burial Grounds}

For the Composite Analysis, active solid waste burial grounds were defined as those open and receiving waste since September 26,1988 . The radionuclide inventories included in previous performance assessments (Wood et al. 1995; Wood et al. 1996) were those disposed since September 26, 1988. The list includes burial grounds $218-\mathrm{W}-3 \mathrm{~A}, 218-\mathrm{W}-3 \mathrm{AE}, 218-\mathrm{W}-4 \mathrm{C}$, and $218-\mathrm{W}-5$ in $200 \mathrm{West}$ Area, and 218-E-10 and 218-E-12B in 200 East Area. These burial grounds continue to receive solid waste (e.g., contaminated tools and clothing) from operations in their respective areas. In addition, some wastes are received from offsite generators within the DOE complex and the U.S. Department of Defense (e.g., U.S. Navy ship reactor compartments in Trench 94 of 218-E-12B).

In the past, wastes from the chemical separations plants were a function of plant operation. Today the wastes that are disposed in solid waste burial grounds at Hanford are from facility deactivation projects. At the end of these projects, the burial grounds will be transitioned to the Environmental Restoration Contractor (ERC). Whatever the source, those wastes containing sufficient inventories of waste that could migrate through the environment and result in potential radiation dose (e.g., technetium-99 and uranium) are stabilized in various grout formulations or disposed in high-integrity containers, or both.

At Hanford, private contractors are becoming involved in the chemical separation of high-level and low-level waste fractions from the tanks, and in the creation of immobilized waste forms (e.g., glass). Secondary low-level waste streams from these private companies were not considered in this analysis. Those secondary waste streams that meet specifications in contracts between the DOE and private companies will be returned to the DOE, and they may be disposed in the solid waste burial grounds at Hanford. Their inventory and volume are unknown at this time, but could include carbon-14, iodine-129, and technetium-99 scrubbed from atmospheric emissions.

\subsubsection{Environmental Restoration Disposal Facility}

The Environmental Restoration Disposal Facility (ERDF) trench receives waste from the remediation of CERCLA past-practice sites. Debris and excavated materials from these sites contain dangerous and hazardous waste, polychlorinated biphenyl (PCB) and asbestos waste, low-level radioactive waste, and low-level mixed waste containing both dangerous and radioactive waste components.

At present, the remediation efforts for CERCLA sites are focused on those nearest the Columbia River, i.e., those in the 300 Area and 100 Areas (Hartman and Dresel 1997). In the 300 Area, the effort is focused on past-practice solid waste disposal sites and liquid discharge sites associated with research conducted in the facilities and fuel fabrication efforts. In the 100 Areas, the effort is focused on similar 
burial ground and liquid discharge sites associated with reactor operation and with the demolition of structures other than of the reactor buildings themselves.

Remediation plans for 200 Area past-practice sites are being developed. These plans require the joint agreement of the DOE, Ecology, and EPA. Facility decommissioning wastes will be disposed in the ERDF trench and not the solid waste burial grounds. The final dispositions of past-practice burial grounds, liquid discharge sites, and canyon facilities are unknown. For example, in the case of canyon buildings, remediation may involve the mounding of facility debris alongside the building prior to placement of a surface barrier or cover designed to limit intrusion and recharge.

\subsubsection{Tank Waste Remediation System Waste}

Since 1944, high-level wastes from the chemical separation plants have been stored in and transferred between large single-shell and double-shell tanks. These wastes are the result of the variety of processes briefly described in Chapter 1. They include waste streams from the dissolution of cladding materials and irradiated fuel slugs, the original bismuth-phosphate precipitation process, the solvent extraction processes used to recover plutonium and uranium, and the evaporators used to concentrate the waste in the tank farms.

As processes used to capture plutonium and uranium from solutions changed, the characteristics of wastes changed. These tank wastes are characterized as concentrated complexed waste, dilute complexed waste, double-shell slurry and double-shell slurry feed, aging waste, and noncomplexed waste (Hanlon 1997). Because carbon steel tanks were used at Hanford, wastes stored in the tanks were neutralized and often have $\mathrm{pH}$ values between 12 and 14 . Wastes containing complexants were segregated from those that do not. The Composite Analysis therefore includes a distinction between complexed and noncomplexed waste regarding their mobility in the subsurface environment.

Sixty-seven of 149 single-shell tanks have leaked or are suspected to have leaked a portion of their inventory into the environment (Hanlon 1997). If sluicing is the method adopted for removal of tank wastes, it is anticipated the single-shell tanks will lose more liquid tank waste to the vadose zone. The TWRS program and private contractors will recover the tank waste, separate it into high-level and lowlevel waste fractions, and immobilize each. The TWRS program has begun the process to have the lowlevel waste fraction that will be disposed onsite declared incident waste, i.e., not high-level waste ${ }^{(a)}$ (Peterson 1996). This low-activity waste fraction from the tanks will become immobilized low-activity waste (ILAW) and will be disposed at the Hanford Site. The high-level waste will be stored until it can be transferred to a national high-level waste repository. The process to declare past tank leaks, future losses, and tank waste residuals incident waste has not begun.

(a) From a letter, dated June 1997, sent by C. J. Papiello, Director of the Office of Nuclear Material Safety and Safeguards, of the U.S. Nuclear Regulatory Commission, to J. Kinzer, Assistant Manager, Office of Tank Waste Remediation System, DOE, "Classification of Hanford Low-Activity Waste Fraction." This letter may be found in Mann et al. (1997). 
The recovery of wastes from both single- and double-shell tanks will not be perfect. The interim retrieval goal in Milestone M-45 of the Hanford Federal Facility Agreement and Consent Order (also known as the Tri-Party Agreement; Ecology, EPA, and DOE 1989) is to leave no more than $360 \mathrm{ft}^{3}$ of waste in each 100-series single-shell tank, and no more than $30 \mathrm{ft}^{3}$ of waste in each 200-series single-shell tank. This corresponds to $1 \%$ of the current single-shell tank waste inventory of 36 million gallons, allocated equally to each of the 149 single-shell tanks in proportion to the cross-sectional area of the tanks. Thus, an estimated $1 \%$ of the waste volume will remain in each tank following completion of recovery operations. For single-shell tanks the waste source types include leaks, losses during recovery operations, and a residual in the tanks after recovery. In the Composite Analysis, double-shell tanks were assumed to maintain their integrity during waste recovery, so only the residual left following recovery operations was considered. In addition to tank waste source types listed above, the TWRS program, specifically the privatization contractors, will produce secondary waste streams during their separations and immobilization steps. These wastes will be returned to DOE for final disposal.

\subsection{Sources that Could Superimpose}

Sources that could superimpose are those likely to contribute to the dose received by hypothetical future members of the public from the four LLW disposal facilities.

Waste disposal at Hanford has been centralized, the vast majority of wastes are to be disposed in the exclusive waste management area. Each of the active or planned LLW disposal facilities is located on the 200 Area Plateau and inside the exclusive waste management area. These wastes are from past operations of the chemical separations plants, from the cleanup and decommissioning of the chemical separation facilities, from the tanks, and from the CERCLA sites (i.e., the 100 Areas and 300 Area) along the Columbia River. The first iteration of the Composite Analysis focused on wastes disposed on the 200 Area Plateau of the Hanford Site because the majority of the low-level radioactive waste disposals at the Hanford Site will reside at this location.

Because the waste disposal sites, liquid discharge sites, chemical separations plants, and tank farms are either within or close to the exclusion area, they were all considered in the Composite Analysis. However, some inventories of radioactive waste are absent, available in insufficient detail to allow simulation, or not of significant magnitude to be included. Thus, many liquid discharge sites and all canyon buildings were omitted from the first iteration of the Composite Analysis. These sites and the reasons for their omission from the analysis are addressed in Section 3.3. While canyon buildings are analyzed, a sensitivity analysis representing the cesium and strontium inventory in a canyon building and filter system is included in Chapter 4.

Wastes in other areas (e.g., 100 Areas, 300 Area, and 400 Area) are located some distance from the 200 Areas. It was assumed that multiple sources within each of the 100 Areas and within the 300 Area will not create significant commingled groundwater plumes with contamination from the 200 Areas. The basis for this assumption is confidence in the CERCLA process to create a safe closure setting for each of these past use areas. If plausible situations are identified where sources from other areas commingle with plumes from 200 Area sources and create a potential threat to human health and safety, they will be analyzed in subsequent iterations of the Composite Analysis. 
With regard to the atmospheric pathway, previously completed environmental impact statements and performance assessments were reviewed. Given the known waste sources and assumed conditions of release, the only waste sources potentially capable of making significant atmospheric pathway contributions to all pathways dose were the graphite cores of the production reactors.

\subsection{Sources Excluded}

This section provides justification for excluding sources from the groundwater or atmospheric pathways for the all-pathways dose estimate in the Composite Analysis.

\subsubsection{Chemical Separation Plants (Canyons)}

Six canyon buildings, designed for the processing of special nuclear materials, are present on the 200 Area Plateau. Two of these plants, B Plant and the Plutonium Uranium Extraction Plant (PUREX), are located inside 200 East Area. Four plants, T Plant, the Plutonium Finishing Plant (PFP), U Plant and Reduction-Oxidation Plant (REDOX), are located inside 200 West Area.

The canyon buildings will be decontaminated and decommissioned under the CERCLA program. However, the various standards (e.g., for levels of contamination) and final disposition of the canyon buildings (e.g., whether cells are to be filled to provide stability and prevent subsidence, canyon buildings are to be demolished to grade, entombed, and covered with surface barriers to reduce infiltration) have not been defined.

In the case of each canyon building, the major radionuclide sources and waste within the retired plant will be removed, reduced, or stabilized. Radiological contamination within the facility will be removed or fixed in place. The canyon buildings are massive concrete structures, and concrete is an excellent waste form for sorbed radionuclides. Whatever structure is left in place will be stabilized (i.e., filled with soil, gravel, or concrete) and all services (such as water) will be disconnected. Retired filters will be isolated and stabilized to ensure a safe condition. It is likely that these areas and especially any remaining structure will be covered with a protective barrier to further isolate contamination from intrusion and recharge. Final disposals will be dry with minimal driving force to mobilize and transport radionuclides from facilities.

In the absence of an inventory including any mobile and long-lived radionuclides, and with the assurance that all contamination will be removed from or entombed in these substantial structures, these facilities are not analyzed in the first Composite Analysis. It appears unlikely that the canyon buildings will be a significant source of groundwater contamination, especially in the next 1000 years. When more is known about their final inventories (e.g., the quantity and radionuclides known to be fixed in place) and physical state (e.g., whether infiltration barriers will be constructed to minimize infiltration), they could be simulated as contaminated concrete monoliths. A sensitivity analysis case was evaluated to determine whether the cesium and strontium inventory in a canyon building and its retired filters could contribute to the composite dose. 
The PUREX storage tunnels (\#1 and \#2) branch off from the PUREX railroad tunnel and extend southward from the east end of the PUREX plant. The tunnels are used for storage of mixed waste (e.g., spent equipment and tank cars) from the PUREX plant and from other onsite sources. The radiological contamination in the tunnels consists primarily of uranium, transuranics, and/or mixed fission products. Currently, each storage tunnel is isolated from the railroad tunnel by a water-filled shielding door. No electrical utilities, water lines, fire detection or suppression systems, radiation monitoring, or communication systems are provided inside the PUREX storage tunnels. Material selected for storage is typically loaded on railcars modified to serve as both transport and storage platforms. Tunnel \#1 is constructed of creosote-treated timber covered by roofing material and $2.4 \mathrm{~m}$ of earthen fill. Tunnel \#2 is constructed of steel and reinforced concrete covered with $2.4 \mathrm{~m}$ of earthen fill.

Final closure of the PUREX storage tunnels will require the evaluation of alternatives. In general, these alternatives will involve either stabilizing the waste in the tunnels, or removing it and then stabilizing the tunnels (DOE 1996c). Alternatives for stabilizing the waste in place include, but are not limited to, backfilling the tunnels, waste, and railcars with gravel, or grout, or a combination of the grout on the bottom and gravel on the top. All means of access to the tunnels would be permanently sealed. Then a final surface barrier that meets Resource Conservation and Recovery Act (RCRA) landfill cover requirements to prevent water from leaching the waste in the tunnels would be constructed. Thus, the tunnels would be left in a stable configuration resistant to consolidation and settlement. The waste would be left in either a grout matrix or a gravel cocoon. Because these options have excellent waste form performance characteristics in the vadose zone when overlain by a surface barrier that significantly limits recharge through the waste emplacement and because of the absence of an inventory including any mobile and long-lived radionuclides, the PUREX tunnels were also excluded from the first iteration of the Composite Analysis.

\subsubsection{Atmospheric Pathway}

The potential for releases of radionuclides to the atmosphere depends on the final configuration of buried waste surface barriers and the radionuclides present in the waste. In order for an atmospheric release to occur, some mechanism is necessary to transport the radionuclides from the waste through the barrier to the surface. Release of radionuclides may occur by diffusion through surface barriers, by erosion of surface barriers followed by wind suspension, by transport to the surface by burrowing animals or plant roots followed by wind suspension, and as the result of disruptive events (e.g., intruder actions or severe natural phenomena).

Atmospheric releases resulting from disruptive events have been covered in previous performance assessments (Wood et al. 1995; Wood et al. 1996; Mann et al. 1997) and are beyond the scope of the present analysis. The previous studies have assumed that institutional controls prevent intrusion into the waste and atmospheric releases for at least 100 years and that passive controls prevent intrusion for 500 years. These studies have also addressed the issue of barrier erosion and concluded erosion is an unlikely mode of release to the atmosphere.

Barriers are expected to effectively inhibit transport to the surface by plant and animal penetration to the waste layer (DOE 1994b). While roots of plants may penetrate below $2.5 \mathrm{~m}$, the quantity of 
radioactive inventory brought to the aboveground plant mass is not expected to be large compared to that for the intrusion scenarios. Material in the aboveground plant mass would not be released to the atmosphere until the plant dies and decays. Barriers designed to limit water infiltration through the use of a capillary break, which reduces the water content below it, would also discourage plant root growth into the waste. Burrowing animals and insects are not expected to penetrate the soil significantly beyond $2 \mathrm{~m}$ (Napier et al. 1988). Previous performance assessment studies for LLW have concluded that plant and animal transport is not a significant route of airborne release.

The previous performance assessments have evaluated diffusion of volatile radionuclides through the barriers as a source of airborne release. The radionuclides considered were tritium, carbon-14, and radon-222. The analyses have required the assumption of unlikely and conservative conditions over time and have resulted in very small estimates of release. Doses calculated to an individual living above the waste have also been small. The production of radon-222 from uranium-238 is small during the first 1000 years after placement, and does not peak until about 100,000 years. Even at the peak release rate, the amount of radon-222 reaching the surface is small because of the delay in diffusion through the soil overburden and the short half-life of radon-222 (about 3.8 days). Prior analysis of the graphite cores from the production reactors (DOE 1989, 1992) produced the only source of small but potentially significant airborne release. In that analysis it was assumed that half of the core inventory was available for release and migration via the atmospheric pathway.

Based on the review of past studies, the only atmospheric releases included in the initial Composite Analysis are the releases of the volatile radionuclides tritium and carbon- 14 from buried graphite reactor cores in the 200 Areas. The graphite cores do not have a source of radon-222 in their inventory.

\subsection{Estimation of Source Inventory and Release Rate}

This section provides the basis and justification for estimating the source term for each source to be included in the Composite Analysis. The estimated radionuclide inventory is included.

\subsubsection{Selection of Key Radionuclides for Study}

The Composite Analysis is the beneficiary of preceding analyses and field observations. It is a companion analysis to the performance assessments for 200 West and 200 East post- 1988 burial grounds (Wood et al. 1995; Wood et al. 1996) and the remedial investigation and feasibility study of ERDF (DOE 1994b). It was also preceded by an analysis of a new waste form, the immobilized low-activity waste from Hanford tank farms (Mann et al. 1997). These and other analyses, (e.g., environmental impact statements) included development of inventory data and application of screening or significance criteria to identify those radionuclides that could be expected to significantly contribute to either the dose or risk calculated in the respective analysis.

Clearly, those radionuclides identified as potentially significant in these published analyses are also expected to be key radionuclides in the Composite Analysis. Older studies were reviewed to identify any radionuclides unique to specific wastes or closed facilities. Of greatest interest were the more recently completed studies including those supporting the disposal of immobilized low-activity radioactive waste 
originating from the single- and double-shell tanks (Mann et al. 1997) and residing in the shallow land burials (Wood et al. 1995; Wood et al. 1996).

\subsubsection{Low-Activity Waste from Tanks}

The activation products, actinides, and fission products generated in the reactors at the Hanford Site are anticipated components of the low-activity radioactive stream coming from Hanford single- and double-shell tanks. The complete list of these isotopes can be found in Schmittroth et al. (1995) and Watrous and Wooten (1997).

The screen applied by Schmittroth et al. (1995) to identify those radionuclides that could be potentially significant contributors to dose in groundwater pathway scenarios yielded twelve potentially important isotopes. In order of their contribution to drinking water dose, a major component to allpathways dose, the twelve isotopes were technetium-99, selenium-79, uranium-233, uranium-234, uranium-238, radium-228, niobium-93m, iodine-129, radium-226, uranium-236, curium-245, and uranium-235. To arrive at this list, Schmittroth et al. (1995) used a simple retardation model, and where distribution coefficient data were absent, made the conservative assumption of no sorption. After reviewing the distribution coefficients, the following values were assigned to several of the elements (Kaplan and Serne 1995; Kaplan et al. 1996): technetium and selenium, $0 \mathrm{~mL} / \mathrm{g}$; uranium, $0.6 \mathrm{~mL} / \mathrm{g}$; radium, $15 \mathrm{~mL} / \mathrm{g}$; niobium, $40 \mathrm{~mL} / \mathrm{g}$; iodine, $3 \mathrm{~mL} / \mathrm{g}$; and curium, $100 \mathrm{~mL} / \mathrm{g}$. The radionuclides that were assigned nonzero distribution coefficient values in the study by Schmittroth et al. (1995) failed the screen as significant contributors to dose via the groundwater pathway. Consequently, those elements (i.e., radium, niobium, and curium) assigned the higher values after the initial screen were also eliminated. Accordingly, only the top eight isotopes contributing to drinking water dose were identified as potential key radionuclides for the Composite Analysis: technetium-99; selenium-79; iodine-129; and uranium-233, $-234,-235,-236,-238$, and their daughters.

\subsubsection{Solid Waste Burial Grounds}

Those radionuclides remaining after the screening process for the 200 East Area burial grounds were long-lived and mobile (Wood et al. 1996). A list of all radionuclides considered in the dose analysis for the 200 East Area burial grounds appears in Wood et al. (1996, Table 4.1). The screening process eliminated all moderately to strongly sorbed radionuclides because they were predicted to have no significant ability to contaminate groundwater in the next 1000 years. Radionuclides passing the screen were tritium, carbon-14, chlorine-36, selenium-79, technetium-99, iodine-129, rhenium-187, and the uranium isotopes. Because of their unique inventory and waste form degradation characteristics, the U.S. Navy ship reactor compartments were treated as a special case. In this special case, the list of radionuclides potentially able to contaminate groundwater is a subset of the above list: carbon-14, chlorine-36, selenium-79, technetium-99, and iodine-129.

One isotope, rhenium-187, that passed the screen was eliminated from further consideration. The screen criteria included potential mobility and decay half-life; however, rhenium-187 is not present at Hanford in sufficient quantity to present a health threat. Rhenium-187 is an activation product of tungsten, and its existence in significant quantities in the DOE radioactive waste would indicate that a 
significant quantity of tungsten had been employed in the fuel or its cladding. This was not the case. Schmittroth et al. (1995) estimated the total production of rhenium-187 at $8.6 \times 10^{-6} \mathrm{Ci}$ using the Oak Ridge Isotope Generation and Depletion (ORIGEN2) code (Croff 1980). Based on its potential contribution to drinking water dose, this quantity will not significantly contribute to dose.

\subsubsection{Radionuclides Selected by Screenings in Other Analyses}

The closure plan for the commercial LLW site operated by US Ecology on the Hanford Site (Grant Environmental, Chase Environmental Group, and US Ecology 1996) presents a total inventory to date and a projection for disposal at the site until its closure in 2063 . The inventory was screened according to two criteria, total activity greater than $1 \mathrm{Ci}$ and decay half-life in excess of 100 years. Of the radionuclides identified for further analysis, several have distribution coefficients at or only slightly greater than zero, including carbon-14, chlorine- 36 , iodine-129, potassium-40, technetium-99, and uranium-238. While all the other radionuclides were identified in prior analyses, potassium- 40 was identified as a contaminant of potential concern. In the review of the inventory for the ERDF trench, potassium- 40 was identified as a potential isotope of concern; however, it was also identified as a radionuclide considered to be derived completely from natural background. Wood et al. (1995) noted that an average background value of $\sim 15 \mathrm{pCi} / \mathrm{g}$ supports this hypothesis. Wood et al. (1995) also noted that potassium-40 is not a known fission product, and consequently, its activity was not considered when calculating the potential dose from DOE wastes such as those in the ERDF. Accordingly, for the purposes of the Composite Analysis, potassium-40 was omitted from the calculation of composite dose from either DOE sites or the commercial LLW disposal facility.

\subsubsection{Uranium Daughters}

During release and migration of radionuclides from the vadose zone to the unconfined aquifer, some radionuclides will decay and produce daughter products. However, radioactive decay involving generation of progeny radionuclides can be difficult to model in systems that allow each chain member to move independently. Physical separation of the chain members is not generally accounted for in decay and environmental transport algorithms. In the Composite Analysis, computational resources did not permit modeling individual progeny, so an alternative treatment was used.

Two options were considered for daughter products in the Composite Analysis: 1) daughter products that do not contribute significantly to dose do not need to be simulated; and 2) decay chain members can be simulated as equally mobile as their parent.

Regarding the first option, the regulatory period of interest is short (1000 years), and may provide insufficient time for significant quantities of uranium daughters to be created. In addition, the decay products in the uranium chains, aside from other uranium isotopes, are radium and thorium. In general, both are more highly sorbed in comparison to the parent uranium. In the aquifer the best estimate distribution coefficient values for uranium, radium and thorium are 3,20 and $1000 \mathrm{~mL} / \mathrm{g}$ respectively (see Appendix E). For only one waste type (i.e., very high salt and very basic tank wastes) are radium and thorium more mobile than uranium in the vadose zone, and this is true only in the sediments immediately below waste tanks. In the lower portion of the vadose zone these wastes are believed to be buffered by 
the vadose zone sediments and soil water, and radium and thorium are again assigned higher distribution coefficients than uranium. For the other five waste types disposed to ground, radium and thorium are always more highly sorbed than uranium. In general, because of their sorption, the radium and thorium daughters will not enter the groundwater from the vadose zone in the 1000-year period. Essentially, radium and thorium found in the aquifer will be a result of uranium entering the aquifer and then undergoing decay to create daughters. The radium and thorium daughters will not move with uranium in the aquifer. Thus, a reasonable treatment for the first 1000 years after Hanford Site closure would be to account for uranium isotopes and uranium daughters, and neglect the radium and thorium daughters in the dose calculation.

Regarding the second option, radioactive chain decay in the subsurface can be separated from the transport calculation if the chain members all travel at the same rate (i.e., without physical separation). If the analysis were conducted with all chain members in the same medium and traveling together, decay could be accounted for based on the elapsed time between initial source definition and the time of interest. In the case of uranium parents, as long as uranium transports as fast or faster than its daughter(s), it is conservative to model the daughter(s) as moving with the parent.

For the Composite Analysis, radioactive chain decay was separated from the transport analysis. To accomplish the separation ORIGEN2 code simulations of irradiated fuels (see Appendix A) were used to define the relative abundance of uranium isotopes in an average Hanford Site waste. The abundance of other uranium isotopes were defined in terms of the uranium-238 level. The grams of uranium isotopes uranium-233, $-234,-235$, and -236 per gram of uranium-238 were assumed as follows: $1.07 \mathrm{E}-08$, $5.65 \mathrm{E}-05,6.70 \mathrm{E}-03$, and $1.46 \mathrm{E}-04$ grams of the isotope per gram of uranium-238. A chain decay calculation was used to determine the relative significance of the uranium progeny contribution to dose when progeny were as mobile as parent. A calculation of the dose resulting from $1 \mathrm{mg} / \mathrm{L}$ of uranium-238, other uranium isotopes, and their progeny, shows that after 1000 years the dose from all progeny in the agriculture scenario was $<10 \%$ of the dose from the uranium parents. The same calculation was performed for the industrial, recreational, and residential scenarios with similar results. Consequently, to be conservative the contribution to composite dose from uranium was based on uranium-238 release and migration, the relative abundance of other uranium isotopes as indicated by ORIGEN2 simulations, and the inclusion of all progeny as though they were as mobile as the parent. This is conservative in light of the greater sorption of the radium and thorium daughters.

\subsubsection{Radionuclides Included in the Groundwater Pathway}

The radionuclides included in the groundwater pathway analysis for future sources were carbon-14; chlorine-36; selenium-79; technetium-99; iodine-129; and uranium-233, -234, -235, -236, -238 and their daughters. This list is the result of merging the two lists from the immobilized low-activity waste from tanks and the solid waste burial grounds. In addition, the remedial investigation and feasibility study (RI/FS) for the ERDF and other environmental impact statements (DOE 1989, 1992, 1994b, and 1996a; DOE and Ecology 1996) were reviewed, and no other radionuclides were identified as potentially significant contributors to groundwater pathway dose. In the first iteration of the Composite Analysis, the 
contribution of uranium and its progeny to dose was estimated by simulating uranium-238, approximating the abundance of other uranium isotopes using a single set of isotopic ratios, and assuming uranium daughter products move with the parent.

Plumes of tritium, strontium-90, technetium-99, and iodine-99 exist in the unconfined aquifer at the Hanford Site. While radionuclides with long half-lives, i.e., technetium-99 and iodine-129, are identified as key nuclides in the Composite Analysis, tritium and strontium-90 are not. Neither tritium nor strontium-90 are included as key mobile radionuclides in the study, but both were included in a recent study of existing plumes (Cole et al. 1997), and the Composite Analysis has included the influence of these existing plumes on future dose projections. Thus, while no effort has been made to assemble inventory data and model release and vadose zone migration of either tritium or strontium- 90 , their effects on dose are included.

Because of its mobility and its disposal to cribs in relatively large volumes of liquid waste, tritium is assumed to be in the aquifer and not significantly retained in the vadose zone. Thus, simulations of the existing plume of tritium and of future disposals of liquid waste at the State-Approved Land Disposal Site have captured the future impact of tritium (Cole et al. 1997). Strontium-90 plumes were simulated by Cole et al. (1997), and those results are also incorporated into the Composite Analysis. Strontium is highly sorbed in the aquifer and does not pose a threat outside the buffer zone when the source is inside the exclusive waste management area and buffer zone. Strontium-90 will be shown to contribute to dose, but only in the immediate vicinity of these releases.

The selection of radionuclides for inclusion in this first iteration Composite Analysis has relied on the results of several disposal studies. Each involved the burial of an essentially dry waste form in the thick vadose zone deposit of the 200 Area Plateau. Future iterations of the Composite Analysis will benefit from ongoing studies of liquid discharge sites and tank leaks. Other radionuclides may be identified in these studies as being sufficiently mobile to reach the aquifer. Their mobility in the vadose zone may be a result of the original waste composition and a lower potential for adsorption to sediments or the precipitation of minerals, or the increased driving force of the liquid discharge. Subsequent iterations of the Composite Analysis will revisit the key radionuclide identification process and take advantage of future findings.

\subsubsection{Solid Waste Burial Grounds}

Inventories of key mobile radionuclides disposed in each of the 200 East and 200 West solid waste burial grounds were estimated for pre-September 1988 and post-September 1988 amounts using an agedfuel-ratio methodology and the record of cesium, uranium, or plutonium disposal (Appendix A). The inventories are stored in the "inventory" worksheet within the Composite Analysis 2.0.XZS Excel" workbook, described in Chapter 4. These inventories of the key mobile radionuclides were estimated using radionuclide inventory information from the Solid Waste Information Tracking System (SWITS) database (Clark 1995). In addition, the ORIGEN2 code (Croff 1980) was used to estimate the abundance of key mobile radionuclides potentially present but not reported in the SWTTS database. 
Activities of cesium-137 and masses of uranium and plutonium disposed were obtained directly from the SWITS database. Two types of SWITS database reports were generated for two periods. The types of reports summarized unsegregated waste and post-1970 non-transuranic (non-TRU) segregated wastes. These reports were generated for startup through September 1988 and startup through December 1996. The inventories of uranium, plutonium, and cesium-137 disposed were totaled between the unsegregated disposal inventory and the segregated non-TRU inventory. This excluded the transuranic (TRU) waste, which was not expected to remain onsite. By subtracting the September 1988 inventory from the December 1996 inventory, an estimate of the post-September 1988 inventory disposed was obtained.

\subsubsection{Suspect Transuranic Waste and Pre-1988 Inventory}

Before 1970, TRU waste at the Hanford Site was not segregated prior to disposal (Wood et al. 1995). After 1970, TRU waste, defined as $>10 \mathrm{nCi} / \mathrm{g}$, was segregated prior to disposal so that it could be retrieved and eventually be disposed offsite. In 1984, the definition of TRU waste was changed from $>10 \mathrm{nCi} / \mathrm{g}$ to $>100 \mathrm{nCi} / \mathrm{g}$. Therefore, a portion of segregated TRU waste disposed between 1970 and 1984 may be reclassified as LLW and be disposed on the Hanford Site. The plans for dealing with this type of waste are being developed. For the Composite Analysis, the suspect TRU waste sites are governed by CERCLA, and, therefore, are associated with the pre-September 1988 inventory. The estimated inventory of pre-September 1988 waste was incremented by the estimated suspect TRU waste inventory that will be reclassified as LLW (see Appendix A, Section A.2.1). This is the pre-1988 solid-waste burial ground inventory applied in the Composite Analysis (included in Table 3.1).

\subsubsection{Future Disposal Inventories}

Future disposal inventories are uncertain. In the Composite Analysis the inventory disposed between September 1988 and December 1996 was extrapolated for the planned 30 years of disposal assuming a constant rate of disposal. The inventory values were compared to projections made in the performance assessments for the 200 East and 200 West Area solid waste burial grounds (Wood et al. 1996; Wood et al. 1995). In cases where the solid waste performance projection values exceeded the linear extrapolation of waste disposal over 30 years, the performance assessment values were used. The differences were the result of having a different and longer record of waste disposed since September 1988 to use as the basis of the future forecast. Table 3.1 includes the future inventory of key radionuclides for the post1988 period of disposal in the solid waste burial grounds. Although key radionuclides in Table 3.1 are listed in association with disposal areas, future waste disposal may not occur in the same locations.

\subsubsection{Estimation of Non-Reported Radionuclides}

While uranium, plutonium, and cesium- 137 are relatively well reported within the SWITS database (Clark 1995), a number of radionuclides may also be present but are not consistently reported. Some of these radionuclides are potentially important to performance assessment calculations, (e.g., carbon-14, chlorine-36, selenium-79, technetium-99, and iodine-129) and were therefore also important to the Composite Analysis. In an effort to estimate inventories of these radionuclides, Version 2.1 of the ORIGEN2 code was used to estimate the relative abundance of other radionuclides that are important but not consistently reported, compared to the major radionuclides that were reported. This method was 
applied to develop inventory for solid waste burial grounds (see Table 3.1) and those liquid discharge sites that did not receive tank waste (see Section 3.4.5 on CERCLA Sources). This section summarizes major points of the estimation method, which is more fully described in Appendix A.

ORIGEN2 calculations were made for single-pass reactor and N-Reactor irradiation to determine radionuclide concentrations in spent fuel and cladding. Impurities in the fuel and cladding were included in the model. The quantities are presented in Appendix A (Tables A.1 through A.6) and are based on Bergsman (1993). A weighted average between the single pass and $\mathrm{N}$-reactor nuclide concentrations was used to estimate the overall average nuclide composition. About $90 \%$ of the fuels processed at Hanford were irradiated in the single-pass reactors.

Inventories of omitted fission products in solid waste were estimated by multiplying the undecayed cesium-137 inventory from SWITS by the ratio of the $\mathrm{Ci} / \mathrm{kg}$ concentration of the radionuclide of interest to that of cesium-137 from the ORIGEN2 calculation. The ratios were developed for a fuel age of 10 years after discharge from the reactor. Estimates based on fuel decayed for 1 year are more conservative for radionuclides with decay half-lives less than that of cesium-137 ( 30 years). The key radionuclides have longer decay half-lives. Estimates based on 10 years of decay prior to disposal were more conservative for radionuclides with longer half-lives. Where the activity of a fission product increased over time beyond 1 year, the maximum activity between 1 and 3000 years was used to calculate the ratio to cesium-137 at 10 years.

The SWITS database reports provide both a mass of uranium disposed, which is not identified by isotope, and quantities of uranium isotopes that are specifically identified. The ORIGEN2 results were used to divide the uranium that was not identified by isotope among the uranium isotopes, and to estimate the quantity of other actinides (except plutonium) that may be present. This was accomplished by multiplying the uranium mass reported in SWITS by the ratio of activity of actinide (or daughter) to uranium mass in discharged fuel. Similar to the fission product case, estimates were provided for fuel with 10 years of decay. As in the case of fission products, the maximum activity between 1 and 3000 years in the ORIGEN2 calculation was used to calculate the ratio to uranium mass. Plutonium reported without isotopic distribution was divided into isotopes based on the relative abundance indicated in the ORIGEN2 results. Quantities of plutonium reported in SWITS as specific isotopes were then added to arrive at total plutonium isotopic values.

Because of its identification as a key mobile radionuclide in the graphite cores, an effort was made to determine the potential significance of chlorine-36 elsewhere in the inventory. Chlorine- 36 is a potentially important radionuclide that may be formed by the irradiation of chlorine impurities in the fuel or cladding. No data on the chlorine-35 impurity levels within metallic uranium fuel were available. Because of the uncertainty in chlorine levels, a calculation was performed assuming a 1-ppm by weight impurity in the fuel. The 1-ppm level is an estimate but is believed to be within an order of magnitude of the actual impurity level. However, it may be a factor of 3 over the impurity level allowed in yellow cake. The chlorine- 36 abundance in waste was calculated according to the reported cesium- 137 content, as was done for other fission products. The purpose of including chlorine- 36 in the inventory is to 
determine if the nuclide is potentially important. If the 1-ppm level is potentially important, a more indepth investigation into chlorine- 36 may be justified, otherwise the additional effort may not be warranted.

The choice of using ORIGEN2 predicted ratios of key mobile radionuclides to cesium-137, i.e., the aged-fuel-ratio method, was based on previous work by Wood et al. (1996) that provided a proposed breakdown of "time after discharge" to be applied to disposals. In their work, Wood et al. (1996) found it was appropriate to use 1-year fuel ratios for waste disposed from 1945 through 1973. However, disposals in more recent years may originate from waste discharged by the reactor several years prior to disposal. After 10 years the cesium- 137 inventory declines by about $20 \%$. As a result, when the inventories of long-lived fission products in wastes were estimated based on cesium-137 content, and using the 10-year fuel age assumption, the values are about $20 \%$ higher than when the inventories are estimated using the 1-year assumption. Overall, the sensitivity to using a 1- or 10-year fuel age assumption was small, relative to the uncertainty caused by using a ratio of other radionuclides to cesium-137. The cesium-137 ratio calculation is based on the assumption that the isotopic ratios in the waste were similar to those in the discharged, irradiated fuel.

\subsubsection{Environmental Restoration Disposal Facility}

A variety of burial grounds and liquid discharge sites in the 300 Area and 100 Areas are undergoing cleanup efforts. The goals are to excavate contaminated soils and clean sites up so that they may support unlimited or unrestricted industrial (300 Area) and residential (100 Areas) use, to control sources of groundwater contamination to protect the Columbia River, and to control future groundwater cleanup costs (DOE 1996g). Wastes from these sites are being disposed in the ERDF trench. The objectives and methods of remediation for 200 Area sites have not yet been negotiated between DOE, Ecology, and the U.S. Environmental Protection Agency (EPA). However, only wastes from CERCLA cleanup activities will be disposed in the ERDF trench.

Two documents describe the environmental consequences of the ERDF disposal facility: the RI/FS report (DOE 1994b) and a performance assessment (Wood et al. 1995). As a result of decisions made by DOE regarding the applicability of DOE Order 5820.2a (DOE 1988b) to the disposal of cleanup wastes from CERCLA sites, the final performance assessment (Wood et al. 1995) was not peer reviewed but was published as a record of work completed and analyses conducted. Based on the RI/FS (DOE 1994b), a record of decision (ROD 1995) was issued January 1995 that authorized the construction and operation of two disposal cells with an expected capacity of $920,000 \mathrm{~m}^{3}\left(1,200,000 \mathrm{yd}^{3}\right)$.

The RI/FS lists the maximum detected concentrations of radionuclides for soils in the waste sites of the 100, 200 and 300 Areas. Overall maximum contaminant concentrations (pCi/g) for soils in all three areas are listed in the RI/FS (DOE 1994b, Table 3.8). Based on the RI/FS, these concentrations of radionuclides were assumed to be disposed in the ERDF. Consequently, in the first iteration of Composite Analysis, these maximum concentrations were assumed to exist in all wastes disposed at the ERDF. 
While the ROD describes the initial construction and operation of two cells, planning is currently underway for the disposal of $3.59 \times 10^{6} \mathrm{~m}^{3}\left(4.7 \times 10^{6} \mathrm{yd}^{3}\right)$ in up to six cells. If approved, extending the disposal pit excavation to the east will create the additional four cells. The volume for a six-cell facility is the current projected waste volume for the cleanup and removal of wastes from all 100 and 300 Area CERCLA sites. The density of these wastes upon delivery to the ERDF is an assumed loose density of $1.66 \times 10^{6} \mathrm{~g} / \mathrm{m}^{3}$ (1.4 tons $\left./ \mathrm{yd}^{3}\right)$. The in-place density compacted to $90 \%$ is $2.02 \times 10^{6} \mathrm{~g} / \mathrm{m}^{3}\left(1.7\right.$ tons $\left./ \mathrm{yd}^{3}\right)$. Therefore, the in-place compacted volume of the disposal will be $2.96 \times 10^{6} \mathrm{~m}^{3}\left(3.87 \times 10^{6} \mathrm{yd}^{3}\right)$. Location details (e.g., Washington State Plane coordinates for the disposal cell corners, bottom elevation of the disposal), for the ERDF were taken from the subgrade survey control drawing, ${ }^{(a)}$ and the eastward projection of the construction was based on personal communications with contractor staff. ${ }^{(b)}$

The maximum contaminant concentrations from the RI/FS (DOE 1994b, Table 3.8) were applied to the estimated $3.59 \times 10^{6} \mathrm{~m}^{3}\left(4.7 \times 10^{6} \mathrm{yd}^{3}\right)$ of loose waste to be delivered to the ERDF to produce the total curies of each radionuclide disposed. This assumption is conservative and likely results in an overestimate of the inventory. These inventory data were stored in the "inventory" worksheet within the Composite Analysis 2.0.XIS Excel ${ }^{\mathrm{TM}}$ workbook (described in Chapter 4). The key radionuclide inventory of the ERDF is shown in Table 3.2.

\subsubsection{Hanford Tanks}

Some waste currently stored in tanks at the Hanford Site will remain at Hanford after closure in one of four forms (DOE and Ecology 1996). The majority will be an ILAW created from the incidental waste fraction recovered from tanks. Some will be in the form of a residual left in the tanks after waste recovery operations. For the Composite Analysis, losses to the surrounding soils during recovery operations were assumed to remain in the soil column as well as past tank leaks (i.e., they will not be removed during remediation). These source inventories, immobilized low-activity waste from tanks, leaks and slurry losses from single-shell tanks, residuals in single-shell tanks, and residuals in doubleshell tanks, are described in the following four sections.

Since the Composite Analysis began, the TWRS program has established standard inventories for chemicals and radionuclides in the tank wastes (Kupfer et al. 1997). The Kupfer et al. (1997) inventory is a best-basis global inventory. A best basis tank-by-tank estimate was also produced. ${ }^{(c)}$ The fourth revision of the Hanford Defined Waste (HDW) model (Agnew et al. 1997) was also issued since the effort to assemble Composite Analysis inventories began. Agnew et al. (1997) is a supporting document to the

(a) U.S. Department of Energy, Drawing No. 0600X-DD-C0033, Rev. 1. Date: 11/18/96. Record number H-6-14624 SHT 1.

(b) Information received by C. T. Kincaid, PNNL, during a meeting on February 4, 1997 with F. V. Roeck and M. A. Casbon, Bechtel Hanford Inc., ERC. The meeting topic was "Composite Analysis/ERC."

(c) From letter FDH-9757750 dated August 29, 1997 from D. J. Washenfelder (Fluor Daniel Hanford) to J. K. McClusky (DOE), "Contract Number DE-AC06-96RL 13200; Completion of Milestone T24-97-158, Contractor Letter to Department of Energy, Richland Operations Office, Reporting Completion of Standard Inventory Estimates for All Tanks." 
more recently published best-basis or standard inventory (Kupfer et al. 1997). While the first iteration of the Composite Analysis was based on data available at the time of the analysis, greater consistency in tank waste inventories will be achieved in future iterations when current editions of the standard or bestbasis inventory for tank wastes are employed.

\subsubsection{Immobilized Low-Activity Waste from Tanks}

The source inventory for the incident waste fraction of waste currently stored in single- and doubleshell tanks is reported in the interim performance assessment for low-level tank waste (Mann et al. 1997, Table 3.1). Following recovery from the tanks, waste will be separated into high-level waste and incident waste fractions. The incident waste fraction will be immobilized and returned to the DOE for disposal as ILAW. The high-level fraction is to be returned to DOE for storage until it also is immobilized. After immobilization, it will be stored until the national high-level waste repository is opened, and then it will be shipped to the repository and disposed.

The inventory that appears in the interim performance assessment is fully documented (Schmittroth et al. 1995) as one among many data packages (Mann 1995) developed in support of the interim performance assessment. This published inventory and the associated release models have been adopted for the first iteration Composite Analysis. Subsequent iterations of the performance assessment for ILAW will rely on the current standard or best-basis inventory (e.g., Kupfer et al. 1997). Plans call for these wastes to be disposed in two locations in four existing vaults and several new disposal vaults. The inventory of ILAW to be disposed in existing facilities is based on the fraction of the waste volume they can contain, and the total inventory reported by Mann et al. (1997). Table 3.3 shows the key radionuclide inventory assumed for each disposal location.

\subsubsection{Single-Shell Tank Farms - Tank Leaks and Slurry Losses}

There are twelve single-shell tank farms containing 149 tanks on the 200 Area Plateau. Six tank farms (S, SX, T, TX, TY, and U) containing 83 tanks are located in the 200 West Area. Six tank farms (A, AX, B, BX, BY, and C) containing 66 tanks are located in the 200 East Area. Three types of releases from single-shell tanks were included in the Composite Analysis. In chronological order of occurrence they are 1) past tank leaks, 2) future losses from tanks during recovery of wastes, and 3) residuals to remain in tanks. Of the 149 single-shell tanks at Hanford, there are 67 tanks confirmed or assumed to have leaked (Hanlon 1996, Appendix H). As noted in Hanlon's monthly reports, volume estimates have been made for these 67 leaking tanks. However, estimates of inventory lost during tank leaks are incomplete. Hanlon (1996) reports only cesium-137 losses for 17 of the 67 leaks. The second and third types of release are the result of the waste recovery operations.

The TWRS program has published the initial retrieval sequence and blending strategy (Penwell, Grenard, and Wittman 1996). The retrieval operation is projected to occur over a 15-year period beginning in 2004 and ending in 2019. Penwell, Grenard, and Wittman (1996) provided detail on the retrieval sequence of each tank and each tank farm. The Composite Analysis simulated losses during the recovery operation time interval for each tank farm as specified in the retrieval sequence document. The TWRS program is committed to revise annually the single-shell tank retrieval sequence, (e.g., Kirkbride 
et al. 1997). Results of this initial Composite Analysis would not differ significantly if the later retrieval sequence had been used. Future Composite Analyses will use the most current retrieval sequence for single-shell tanks.

Using currently available leak detection and mitigation technologies, a tank leak could not be detected before 4000 gallons $\left(15 \mathrm{~m}^{3}\right)$ has been released and not stopped for most tanks before approximately 8000 gallons $\left(30 \mathrm{~m}^{3}\right)$ had been released (WHC 1996). Consequently, the TWRS program assumed an average release volume per single-shell tank of 8000 gallons $\left(30 \mathrm{~m}^{3}\right)$. This is a more current estimate than the 4000 gallons $\left(15 \mathrm{~m}^{3}\right)$ per tank value assumed in the TWRS environmental impact statement (EIS) (DOE and Ecology 1996). Conservative assumptions to establish an upper bound on the amount of leakage from single-shell tank 241-C-106 and its potential impact resulted in a calculated leak volume of 40,000 gallons $\left(150 \mathrm{~m}^{3}\right)$ because of hydraulic sluicing of that tank (Lowe 1993). While an average loss volume of 8000 gallons $\left(30 \mathrm{~m}^{3}\right)$ has been assumed, there are reasons to expect a lower average. For example, some tanks will have better leak detection and mitigation capabilities than others, and tanks that are confirmed leakers ( $\sim 50$ single-shell tanks) are candidates for alternate cleanout technologies that use robotic arms or low-volume liquid methodologies or both.

A significant unknown for both tank leaks and losses during recovery operations is the inventory potentially lost to the subsurface environment. The inventory reported in the TWRS EIS (DOE and Ecology 1996) is a total inventory of radionuclides contained within liquid, sludge, and solid wastes in the tanks. An attempt to use the TWRS EIS inventory data, specifically the average concentration from its total inventory and tank farm volume, combined with Hanlon's (1997) tank leak volumes, failed to qualitatively match the cesium releases noted by Hanlon. This likely resulted from not using an inventory divided among liquid, sludge and solid wastes in the tanks. In other databases and reports (e.g., the Tank Characterization Reports of DiCenso and Simpson [1994] and Winkelman [1996]), liquids are characterized separately from sludge and solids, and they are reported as either supernatant or drainable liquid.

The average concentrations of radionuclides in liquid tank wastes (i.e., including both supernatant and drainable liquid) were calculated using data reported in the Tank Characterization Reports (DiCenso and Simpson 1994; Winkelman 1996). However, insufficient data were found to assemble average values on a tank farm basis. Therefore, average values were assembled for four waste types from data on all singleshell tanks. The four waste types were double-shell-slurry-feed, noncomplexed waste, concentrated complexant waste, and dilute complexant waste. Using Hanlon's (1997) reported volumes and waste types for leaking single-shell tanks, the inventory lost to the subsurface was calculated for each tank farm. Using the estimated loss volume of 8000 gallons $\left(30 \mathrm{~m}^{3}\right)$ per tank, the same concentration data were used to calculate the losses during recovery operations in each tank farm. Because of potentially significant differences in the mobility of complexed as opposed to noncomplexed tank wastes, these inventories lost to the ground were calculated for complexed and noncomplexed waste within each tank farm.

The inventories for the single-shell tank farms are shown in Table 3.4. All of the single-shell tank farm related inventories are reported in this table. 


\subsubsection{Single-Shell Tank Farm Residuals}

Source inventories for the tank wastes were recently compiled and published in the TWRS EIS (DOE and Ecology 1996). The inventory for the no-action alternative of the TWRS EIS (DOE and Ecology 1996, Figure 2.2.2 in Appendix F) was an estimate of the contents of the tanks, and for the Composite Analysis, it was the basis for estimating residuals to be left in the tanks.

Single-shell tanks were originally constructed in tank farms that contained from 4 to 18 tanks each. In the TWRS EIS, single-shell tanks were aggregated into five tank groups that contain tanks from one or more tank farms in physical proximity to one another. For the Composite Analysis, tank waste sources were modeled on the basis of tank farms. The higher resolution of this approach may allow sources and plumes to be associated directly with individual tank farms. In order to be consistent with the inventories reported in the EIS, the Composite Analysis used the same spreadsheet as for the TWRS EIS tank group inventories. This spreadsheet contained inventory data at the tank-farm scale, enabling the Composite Analysis to generate and apply single-shell tank farm inventories consistent with the tank group inventories previously published.

Regarding the residuals remaining after the tank wastes are recovered, the TWRS EIS (DOE and Ecology 1996) states:

"... The amount and type of waste that would remain in the tanks after retrieval is uncertain. The Hanford Federal Facility Agreement and Consent Order (Tri-Party Agreement) (Ecology et al. 1994) set a goal of no more than I percent residuals and the ex situ alternatives have been developed to attempt to achieve that goal. However, achieving this level of tank waste retrieval may require extraordinary efforts and cost and it may not be practical to achieve 99 percent retrieval. Conversely, the contaminants that are not recovered are likely to be those that are insoluble in water since substantial quantities of water would be used in an attempt to dissolve or suspend the waste in water during retrieval. Since neither of these issues can be resolved, a conservative assumption was made to bound the impacts of the residual waste. For purposes of this analysis it was assumed that 99 percent recovery would be achieved but that the residual would contain 1 percent of all the contaminants including the water soluble contaminants."

As in the TWRS EIS, the Composite Analysis estimate of residual was assumed to be $1 \%$ of the original inventory. The original inventory of the no-action alternative was used as the inventory for the Composite Analysis. One percent of each radionuclide was assumed to remain in the tank farms following completion of waste recovery (Table 3.4 or the Inventory worksheet of the Composite Analysis 2.0.xls Excel ${ }^{\mathrm{TM}}$ workbook). As noted above, this assumption is believed conservative because it is likely that the recovery operation will preferentially remove the highly soluble chemical compounds and radionuclides. In general, radionuclides with long decay half-lives and potentially significant geochemical mobility have been shown to contribute significantly to long-term dose. The $1 \%$ residual is believed to overestimate the inventories of these radionuclides (i.e., carbon-14, selenium-79, and technetium-99) that remain in the tanks following Hanford Site closure. 
In the release model for tank residuals, leachate concentrations from residual tank wastes were defined as a function of nitrate dissolution (i.e., a maximum nitrate concentration of $360 \mathrm{~g} / \mathrm{L}$ is maintained) with congruent release of all radionuclides. Thus, the nitrate inventory, water infiltration rate, and solubility of nitrate define the time required for nitrate to be leached from residual wastes. All radionuclides were assumed to linearly release over the same time. As in the TWRS EIS (DOE and Ecology 1996), the Composite Analysis was based on the assumption that the single-shell tank structure and tank farm remediation (e.g., stabilization fill and surface barriers) present a high-integrity barrier to release; consequently, the release was delayed for 500 years.

\subsubsection{Double-Shell Tank Farm Residuals}

There are six double-shell tank farms in the 200 Areas at the Hanford Site. The SY Tank Farm contains 3 tanks and is located in 200 West Area. The AN, AP, AW, AY, and AZ tank farms contain 25 tanks and are all located on the eastern side of 200 East Area. The source inventories for the doubleshell tank wastes were also recently compiled and published in the TWRS EIS (DOE and Ecology 1996). As for the single-shell tanks, the inventory for the no-action alternative (DOE and Ecology 1995, Table F.2.2.2 in Appendix F) was an estimate of double-shell tank contents and is the basis for estimating residuals to be left in these tanks. Because the double-shell tanks provide an ability to detect leaks in the tank annulus, accidental leaks and losses during waste recovery operations were assumed to not occur. As in the case of the single-shell tanks, a $1 \%$ residual was assumed in the double-shell tanks upon completion of waste recovery operations. Therefore, the only assumed release from double-shell tanks in the Composite Analysis was the leaching of a $1 \%$ residual. The TWRS EIS inventory spreadsheet (DOE and Ecology 1996) contained the necessary tank farm data for carbon-14 and technetium-99, and $1 \%$ of the no-action alternative inventory is employed in this release (Table 3.5 or the Inventory worksheet of the Composite Analysis 2.0.xls Excel $^{\text {TM }}$ workbook). Chlorine-36, selenium-79, and uranium-238 inventories were not included for double-shell tanks in the TWRS EIS. Iodine-129 is reported in the published EIS on a tank-farm-group-basis instead of a tank-farm basis, and, therefore, it was omitted from the Composite Analysis. As in the case of the single-shell tanks, the $1 \%$ residual is believed to overestimate the inventories of mobile and long-lived radionuclides in the tanks after completion of waste recovery. As in the case of single-shell tank residuals, nitrate dissolution and congruent release of radionuclides was assumed to occur after the high-integrity structure and remediation delay release for 500 years.

\subsubsection{CERCLA Sources}

The CERCLA source term in the Composite Analysis included past-practice waste sites that are being addressed under the CERCLA process and inactive sites that are being addressed under RCRA. The ERC is responsible for evaluation and remediation of these sites. For administrative purposes, the waste sites have been grouped into Operable Units (OUs) and are designated as either CERCLA past-practice units or RCRA past-practice units. However, the eventual disposition of these sites is similar and in the Composite Analysis, all past-practice waste sites under the jurisdiction of the ERC were grouped as CERCLA sources. The CERCLA source term does not include past-practice waste sites that are under the jurisdiction of tank farm operations or decontamination and decommissioning. 


\subsubsection{Description of CERCLA Sources}

A total of 190 separate CERCLA sources were included in the current iteration of the Composite Analysis. The CERCLA source term includes liquid discharge sites such as cribs, trenches, and ponds. It also includes a few solid waste sites (landfills) and storage tanks. Sites that are not suspected to have received radioactive wastes were eliminated from the Composite Analysis source term. These include septic systems and nonradioactive waste landfills. Although portions of the low-level solid waste burial grounds are considered past-practice units, the source term for pre-1988 solid waste burial grounds were described above (Section 3.4.2.1).

In addition to the 190 CERCLA sites, 151 waste sites and more than 200 "unplanned releases" in the 200 Area Plateau that do not have any documented inventory estimates were identified. These were classified as CERCLA sites, but were not included in this iteration of the Composite Analysis. Most of these waste sites and unplanned releases have very low radionuclide inventories, have already been remediated, or have been included in another source inventory.

\subsubsection{Assumptions}

Only CERCLA sites located on the 200 Area Plateau were included in the source term for the Composite Analysis. It was assumed that past-practice waste sites outside this region, including those in the 100 Areas, 300 Area and 1100 Area, have been or will be remediated to the point where they are not significant sources of cumulative all-pathways dose for interaction with plumes originating from the exclusion zone. Cleanup wastes from CERCLA sites outside the buffer zone will be transported to the ERDF, which is treated as a separate source in the Composite Analysis (Section 3.4.3). The reactor cores from the 100 Areas were also treated as a separate source and are described in Section 3.4.7. Several CERCLA sites that were included in the analysis are outside of the exclusive waste management area. These sites will most likely be remediated, as discussed below. However, for the first iteration of the Composite Analysis, a conservative approach was adopted that treated these sources as being left in place. The sources did not affect the results unless significant levels of contaminants reached the water table within the 1000-year period of analysis. If any of the sources located outside the exclusive waste management area appear as significant contributors to the groundwater pathway, then the assumption that they are left in place will be reexamined in the next iteration of the Composite Analysis.

The assumption that sources outside the central plateau will be remediated and not represent significant sources of radionuclide exposure and dose following site closure was based on goals documented in the Hanford Strategic Plan (DOE 1996d). This document presents goals for seven geographic areas. Goals for the four areas that currently contain wastes sites are described below.

- Reactors on the River. Remove and/or stabilize spent fuel, surplus facilities, and waste sites to protect groundwater and the Columbia River and to ensure protection of people, the environment, and natural/cultural resources. Pending Congressional action on the Wild and Scenic River designation, use will continue to be restricted; sensitive ecological, cultural, and Native American resources will be protected. 
- Central Plateau: The 200 Areas and central plateau will be used for the management of nuclear materials and the collection and disposal of waste materials that remain onsite and for other related and compatible uses. Cleanup levels and disposal standards that are consistent with these long-term uses will be established.

- Central Core. This area will remain in federal ownership consistent with safety analysis boundaries . and continued waste management operations in the 200 Area. These areas will be available for.other federal programs or leased for nonfederal uses, consistent with appropriate recognition of cultural and ecosystem values.

- South 600 Area. The 300 and 400 Area waste sites, materials, and facilities will be remediated to allow industrial and economic diversification opportunities. The federal government will retain ownership of land in and adjacent to the 300 and 400 Areas, but will lease land for private and public uses to support regional industrial and economic development. Excess land in the 1100 and 3000 Areas will be targeted for transition to nonfederal ownership.

These goals are addressed on a site-by-site basis through RODs for CERCLA sites and closure plans for RCRA sites. Although RODs and closure plans are still pending and cleanup actions have not yet been completed at most sites, the Hanford Strategic Plan provides a basis for assuming that no significant sources of radionuclides will remain outside of the central plateau region after site closure.

Some form of remediation was assumed for all significant sources in the 200 Area Plateau. According to DOE (1996i), the strategy for remediation of the 200 Area Plateau is:

"...to cap waste in place for sites with high levels of contamination, to remove contamination at sites that exhibit high levels of spotty contamination or lower levels of persistent contamination over a broad area, and no action at sites where risks are demonstrated to be acceptable or where natural attenuation (e.g., decay of short-lived radionuclides) is an effective remedy. In general, this approach results in placing engineered barriers at sites located within the 200 Area fenceline and removal actions at sites outside the fenceline (i.e., 200 Area buffer zone). Sites that have mobile contaminants deep in the subsurface and have the potential to impact groundwater, may require some level of treatment (preferably in situ)."

Based on this strategy, it was assumed that wastes within the exclusive waste management area zone will remain in place and be capped to limit water infiltration and recharge. It was also assumed that institutional controls within the exclusive waste management area will remain in place as long as necessary to ensure that barriers and waste materials are not excavated or otherwise disturbed.

Additional assumptions were made regarding the inventories of radionuclides, future groundwater recharge conditions, and the timing of remedial activities such as placement of barriers. These assumptions are discussed in the following sections and in Chapter 4. 


\subsubsection{CERCLA Radionuclide Inventories}

There were two primary sources of inventory information for CERCLA site radionuclides, Waste Site Groupings for 200 Areas Soils Investigations (DOE 1997b, Table A.1), and Tank Wastes Discharged Directly to the Soil at the Hanford Site (Waite 1991). When both reports provided an inventory for a specific site, the higher value was used.

In DOE (1997b, Table A.1), there are 23 waste categories based on the type and concentration of both radioactive and chemical contaminants that are likely to be present in the waste. The report lists 662 waste sites located in central plateau area that are under the jurisdiction of the ERC. Of these, 36 are nonradioactive waste burial grounds, 55 are septic tanks or drain fields that are not suspected of having received any radioactive contaminants, and 30 are burial grounds that are already covered under the lowlevel burial grounds source term. Of the remaining 541 potential sources, partial inventory information was listed for 184 sites. However, radionuclides reported were limited to cesium-137, strontium-90, total uranium, total plutonium, and americium-241. A secondary data source was a spreadsheet provided by the ERC (Appendix B). This spreadsheet contained inventories for additional radionuclides at many of the 184 sites, and at 6 additional sites, which brought the total number of sites with inventory information to 190 .

Appendix $\mathrm{C}$ contains a list of those sites without inventory data; the available information on the source, the type of waste (radioactive, chemical, or mixed), the effluent volume, and an evaluation of whether the release constitutes a potentially significant source for the Composite Analysis. It was assumed that sites with some radionuclide information in these data sources were the most significantly contaminated sites and that sites without inventory information were generally less significant sources. However, it is recognized that some sites, particularly those that received waste in the early years of Hanford operations, may have received significant quantities of radionuclides that are not recorded.

The radionuclides most significantly affecting the Composite Analysis results are mobile in the subsurface and have relatively long half-lives. Inventory data for most of these radionuclides are not available for most of the waste sites because they were not commonly measured in waste streams. A strategy based on the use of radionuclide ratios in aged fuel was used to estimate the absent inventories of key mobile nuclides. Thus, the estimated inventories of fission products and actinides are based on inventories of cesium-137, total uranium, and total plutonium, which are usually reported. Some sites were missing the inventory of cesium-137, total uranium, and total plutonium. To calculate the mobile radionuclide inventory, the missing cesium-137, total uranium, and total plutonium inventories were first estimated. This estimate was based on the average ratios of total uranium to cesium-137, total plutonium to cesium-137, or total plutonium to total uranium for other waste sites in the same waste site group defined in DOE (1997b). The average ratios of these species for each waste site group are listed in Table 3.6. The spreadsheet provided by the ERC (see Appendix B) contained reported inventory data for some specific radionuclides in addition to cesium-137, total uranium, and total plutonium for some of the waste sites. To be certain that inventories were not underestimated, the inventories calculated using the methodology described above were compared to the reported inventories listed in the ERC spreadsheet and the maximum values were used. 
Waite (1991) reported the type, quantity, and characteristics of wastes associated with the single-shell storage tanks and discharged intentionally to the subsurface at the Hanford Site. Wodrich (1991) also described these wastes and their inventories in a presentation, including those wastes discharged from the single-shell tanks directly to ground through cascade overflow and by pumping wastes to cribs or specific retention trenches. Being limited to facilities that received different forms of tank waste, these estimates of liquid waste volumes and inventories were generated for relatively few of the CERCLA liquid discharge sites. However, these discharges contain potentially significant radionuclide inventories, e.g., $930 \mathrm{Ci}$ of technetium-99 and $1.8 \mathrm{Ci}$ of iodine-129. Based on the Track Radioactive Components (TRAC) model (Jungfleisch 1980, 1983), inventories were assigned to individual cribs and specific retention trenches (see Table 3.7) ${ }^{\text {(a) }}$ For those sites that received tank waste discharges, the inventories estimated by Coony ${ }^{(b)}$ were applied because they are higher than inventories reported in the Waste Site Groupings report published by the Environmental Restoration program. Inventories of key nuclides for the CERCLA sites are listed in Table 3.8.

\subsubsection{US Ecology Commercial LLW Site}

The inventory for the commercial low-level waste disposal site operated by US Ecology was derived from the recently completed site stabilization and closure plan. The inventory is reported by Grant Environmental, Chase Environmental Group, and US Ecology (1996, in Volume II, Attachment 3 of Attachment D, subsection "Source Term" in section "Pathways Analysis Report"). A detailed accounting of inventory is presented in the same document on page 3.6, Table 3.1 and page 3.12, Table 3.7. The key radionuclides inventory of the commercial disposal site was used in the Composite Analysis; it includes inventories for carbon-14, chlorine-36, technetium-99, iodine-129, and uranium (see Table 3.9). Of the more mobile radionuclides thought to be of concern in DOE wastes at Hanford, selenium-79 was the only one for which no data were available in the detailed inventory.

After receiving the site stabilization and closure plan for the commercial LLW disposal site, the State of Washington Department of Health (DOH) decided to complete a State Environmental Policy Act (SEPA) Environmental Impact Statement (EIS) for the site. The DOH has developed its own inventory for the commercial disposal site ${ }^{(c)}$. Minor differences exist between the DOH and Grant Environmental, Chase Environmental Group, and US Ecology (1996) inventories. One similarity is that selenium-79 is also absent from the DOH inventory. Its absence from the commercial inventory is because it is an inconsequential nuclide in the waste streams accepted at the commercial disposal facility. Where there is

(a) Inventories were developed by F. M. Coony of Waste Management Federal Services of Hanford. Information was received in two electronic mail messages with attached files sent by Coony to C. T. Kincaid: 1) Subject, "Questions on Crib Releases in the 200 Areas," dated November 5, 1997; 2) Subject, "Tc-99 (and I-129)," dated October 29, 1997.

(b) F. M. Coony is the individual responsible for the SWITS database and Hanford input to the complexwide integrated database.

(c) From two electronic mail messages; Subject, "Comments for Composite Analysis": 1) from A. H. Thatcher (DOH, Olympia, Washington) to R. D. Hildebrand (DOE-RL), dated February 2, 1998; 2) from M. Dunkelman (DOH, Olympia, Washington) to R. D. Hildebrand (DOE-RL), dated January 28, 1998. 
a large discrepancy for a key mobile radionuclide, e.g., chlorine-36, the inventory from the stabilization and closure plan is conservative. However, in one instance the $\mathrm{DOH}$ inventory is larger. For carbon-14, which is slightly sorbed, it shows an inventory of $4909 \mathrm{Ci}$ while the stabilization and closure plan inventory shows $3850 \mathrm{Ci}$. While assigning a higher initial inventory, the $\mathrm{DOH}$ assumed $55 \%$ of the carbon-14 was biodegradable and that the entire inventory was released through the gas phase to the atmosphere. In the Composite Analysis, the atmospheric pathway contribution was found to be negligible, and the entire inventory was released through the liquid phase to the soil water and aquifer.

The DOH and Grant Environmental, Chase Environmental Group, and US Ecology (1996) differ in their estimates of future inventory. The $\mathrm{DOH}$ based their projections on recent disposals (i.e., 1994-1996) and included expected inventories from decommissioning of two commercial power stations in the region, (i.e., Trojan and WNP-2). However, because future disposal inventories are small in comparison to past disposals, the total inventories examined in the Composite Analysis were not significantly different than those that will be examined in the SEPA EIS. For example, the DOH cumulative or total inventories for iodine- 129 and technetium- 99 are approximately $4 \%$ and $2 \%$ greater than inventories presented in the stabilization and closure plan.

\subsubsection{Graphite Cores from Production Reactors}

Alternatives for decommissioning the Hanford production reactors were evaluated in a draft EIS (DOE 1989), and its final supplement (DOE 1992). The ROD (1993) states the preferred alternative is for the surplus production reactors to be disposed in the 200 West Area. The EIS evaluated eight of the nine production reactors; omitting the $\mathrm{N}$ Reactor because it was not shutdown when the study was done. The B Reactor was included in the EIS; however, since then, the B Reactor has been declared a national historic monument and may be preserved for future public display at its present location (ROD 1993). Thus, the EIS contains information on seven reactors; C, D, DR, F, H, KE, and KW that will be moved to the plateau when the ROD is implemented.

The source inventories for the seven production reactors were derived from Appendix $A$ of the surplus production reactor EIS (DOE 1989, 1992). Twenty radionuclides were included, including tritium, carbon-14, chlorine-36, technetium-99, and uranium-238. Mobile and long-lived radionuclides of interest in other DOE wastes that were not represented in the graphite cores include selenium-79 and iodine-129. The ERC provided an inventory for the graphite core of the $N$ Reactor. ${ }^{(a)}$ The $N$ Reactor core was assumed to be disposed concurrently with the other seven reactor cores in the 200 West Area. Inventories for each of the reactors are shown in Table 3.10.

(a) The N Reactor inventory was provided by V. G. Edens (from Interoffice Memorandum \#042809; Subject, "105 N and 107 N Hazardous Assessment [Inventories]"; sent by R. S. Day to V. G. Edens of Hanford Environmental Restoration Contractor; February 11, 1997). 


\subsection{Inventory Compilation for Composite Analysis}

The inventories from the different waste sites were compiled in an Excel ${ }^{\mathrm{TM}}$ workbook. These inventory compilations were compared with others that have been made at the Hanford Site to check for inconsistencies.

\subsubsection{The Excel ${ }^{\mathrm{TM}}$ Workbook}

Inventory information was assembled and made available to subsequent Composite Analysis calculations in an Excel ${ }^{\mathrm{TM}}$ workbook called Composite Analysis.xls. This workbook includes Excel ${ }^{\mathrm{TM}}$ macros to extract inventories from a variety of independent workbooks and adjust the inventory estimates to a common date of 2050. These inventory data were stored in the "Inventory" worksheet included in the Composite Analysis.xls workbook. Decay was calculated and did not consider ingrowth in adjusting inventory estimates. The dose contributions of uranium and its progeny were captured in the dose calculation.

Only six elements were explicitly considered in the first iteration of the Composite Analysis (i.e., carbon-14, chlorine-36, selenium-79, technetium -99 , iodine-129, and five uranium isotopes and their daughters). Nitrate inventories were also included in the workbook for tank waste residuals, because the tank-waste-residual-release model is based on nitrate dissolution. Where available, inventories were provided for each of the 241 unique source sites. Twenty-five of the source sites were subdivided to distinguish between waste inventories released in different modes or between wastes disposed of at different times (e.g., past tank leaks, future tank sluicing losses).

\subsubsection{Multiple Sources of Inventory Data, Inconsistencies in Totals}

The inventory for the various sources was assembled from several separate efforts to develop inventories for specific wastes and waste forms. Occurring at different times and under different programs, these separate efforts were not coordinated to provide a single and consistent database for wastes that will reside at the Hanford Site after closure. Consequently, the total inventory examined in the Composite Analysis includes significant inconsistencies. Accordingly, uncertainties with respect to cumulative impact result from the inventory analyzed. Estimates of total inventory and several subtotals are shown in Table 3.11.

Some inventory data are from past TWRS program efforts to define the LLW inventory (Schmittroth et al. 1995). Others were taken from the inventory assembled for the TWRS EIS (DOE and Ecology 1996). Portions were also developed from the Tank Characterization Reports. The TRAC model results, used in the TWRS EIS, were also used as the basis for the Waite (1991) report on tank wastes discharge to the subsurface. Finally, estimates of the abundance of mobile isotopes in some wastes (solids and liquids) were based on the abundance of mobile isotopes in aged fuel, (e.g., fission products were defined by their abundance with respect to cesium in 10-year old fuel). It is apparent that inventory data were developed to satisfy a variety of objectives. Often, conservative estimates were developed for and employed in program specific analyses. 
- The TWRS program is making an effort to define radionuclide and chemical inventories in the singleand double-shell tanks. This includes the effort by Schmittroth et al. (1995) to define the inventory for immobilized low-activity waste. More recently, Kupfer et al. (1997) and Washenfelder ${ }^{(a)}$ have provided a coordinated database for all TWRS and privatization activities including recovery operations, chemical separations, vitrification or immobilization of waste, and disposal. However, not all data are available to fully quantify the chemical separations and immobilization steps that will be undertaken by the privatization vendors.

The Kupfer et al. (1997) and Washenfelder ${ }^{(a)}$ work builds on the Agnew et al. (1997) Hanford Defined Waste (HDW) model and the isotope production estimates produced by Watrous and Wootan (1997). The Watrous and Wooten report is an extension of work documented by Schmittroth et al. (1995). The results presented in this sequence of documents differ primarily in how they split the radionuclides (e.g., between recovered metal and waste streams, between precipitated solids in tanks and liquid waste) during the processing steps that follow the production of isotopes in the reactors. Differences between the earlier and more recent data compilations will be discussed below.

For the Composite Analysis, the inventories for past tank leaks and future tank losses were derived from Tank Characterization Reports. Data on radionuclide concentrations in liquid tank wastes were used to estimate the concentrations of key radionuclides lost to the subsurface during leak and slurry loss events. Because data are sparse for the highly mobile and long-lived key nuclides of greatest interest, the approach adopted in the Composite Analysis was to average the contaminant concentration over all single-shell tanks of similar waste over all time. Thus, the history of tank contents was effectively smoothed over all time because of the absence of data on liquid waste characteristics at specific moments.

Inventories for residual wastes remaining in the tanks after recovery operations were based on the published inventories in the TWRS EIS (DOE and Ecology 1996). These inventories were estimated with TRAC simulations that account for waste stream delivery to tanks, subsequent waste routing among the tanks, and processing steps to concentrate the waste or remove specific radionuclides such as cesium-137 and strontium-90. When the Composite Analysis began, the EIS contained the most recently assembled inventory data on a tank farm basis. For some programs (e.g., TWRS), the TRAC model has been replaced by the HDW model of Agnew et al. (1997). The Kupfer et al. (1997) database supersedes that effort and should be employed in future iterations of the Composite Analysis to increase consistency and better quantify uncertainty.

Inventories of key isotopes in tank waste discharged to ground (e.g., to cribs and specific retention trenches) were taken from Waite (1991). This inventory was based on TRAC simulations and represents only a portion of the liquid discharges to the subsurface. The extent to which process knowledge embedded in TRAC is different than that contained in the more recent HDW model (Agnew et al. 1997)

(a) From letter FDH-9757750 dated August 29, 1997 from D. J. Washenfelder (Fluor Daniel Hanford) to J. K. McClusky (DOE), "Contract Number DE-AC06-96RL 13200; Completion of Milestone T.24-97-158, Contractor Letter to Department of Energy, Richland Operations Office, Reporting Completion of Standard Inventory Estimates for All Tanks." 
needs to be determined. If differences exist, then new and consistent estimates of tank waste inventories discharged to the subsurface are needed to develop and maintain consistency between tank inventories and discharged waste inventories.

For those solid waste burial grounds and liquid discharge sites where the disposal records were limited to the mass of cesium, uranium, and plutonium, an aged-fuel-ratio method was used to estimate the mass of mobile fission products and actinides that might be codisposed with these elements. This method of estimating nonreported isotopes did not take into account process and operational knowledge that could alter the estimate. For example, high cesium and strontium levels in solid waste from B Plant are a result of the separation processes that were used to extract cesium and strontium. These same processes may have also acted to minimize the amount of mobile isotopes in many waste streams. For example, in the B Plant waste, it is expected that the ratio of cesium or strontium to other radionuclides has been increased relative to aged fuel ratios because of the separation process. Thus, high cesium and strontium levels may not imply large inventories of iodine or technetium. Given the significance of B Plant as a source of cesium and strontium activity, estimates of mobile radionuclides in the solid waste burial grounds using the aged-fuel scaling factors may be conservative.

The inventory assigned to the ERDF was based on field data (i.e., maximum measured contamination levels) from environmental restoration sites and the estimated total volume of the ERDF trench. Thus, the ERDF inventory for specific elements such as uranium may be largely independent of reactor operations and chemical separation factors that define the inventory in tanks. However, the ERDF inventories should be related through the Hanford Site inventory to other inventories onsite. For example, the uranium brought onto the Hanford Site for production of special nuclear materials should be accounted for as wastes to remain onsite, as a special nuclear material exported from the Hanford Site, or as a component of high-level or TRU waste to be disposed offsite. Through such a mass balance check the magnitude of the uranium inventory assigned to the ERDF may be called into question, and the decision may be made to improve the ERDF inventory estimate by using more representative estimates of contamination levels in CERCLA cleanup wastes. Certainly, as disposals continue in the ERDF trench, disposal records may provide an alternative inventory for analysis in future iterations of the Composite Analysis.

The inventories for the graphite cores of the production reactors and the commercial LLW disposal site were based on data and models that are substantially independent of the tank waste estimates.

\subsubsection{Differences in the Kupfer et al. (1997), Agnew et al. (1997), and Schmittroth et al. (1995) Totals}

Kupfer et al. (1997) and Agnew et al. (1997) present global estimates of waste inventories in the single- and double-shell tanks. In developing their estimate of the low-level fraction of tank wastes for immobilization and disposal, Schmittroth et al. (1995) present an estimate of total tank wastes in both double- and single-shell tanks. However, significant differences appear in the estimates of key radionuclides carbon-14, technetium-99, and uranium-238 because different split factors were applied in these studies for the chemical processing steps that followed production of isotopes in the reactors. 
In the case of carbon-14, the difference may be related to the assumption in the more recent model (Agnew et al. 1997; Kupfer et al. 1997) that all carbon-14 was routed to the tanks. A portion is suspected to have been lost to the atmosphere during fuel dissolution. Differences with regard to technetium-99 are related the assumed amount exported with uranium to other facilities in the DOE complex. Finally, the amount of uranium-238 is similar in Schmittroth et al. (1995) and Kupfer et al. (1997), 296 and 322 Ci, respectively, but different than in Agnew et al. (1997), $906 \mathrm{Ci}$ in tanks. The apparent overprediction of the HDW model (Agnew et al. 1997) for uranium in the tanks is attributed to the use of a conservative factor for the fraction of uranium metal waste that was not recovered.

\subsubsection{Carbon-14}

By far the greatest inventory of carbon-14 at Hanford $(42,200 \mathrm{Ci})$ is in the graphite cores of the production reactors. Significant inventories of carbon-14 are also associated with the ERDF $(3800 \mathrm{Ci})$ and the commercial LLW disposal facilities (3850 Ci).

Significant differences exist between the Schmittroth et al. (1995) estimate of $769 \mathrm{Ci}$ and those of Agnew et al. (1997) and Kupfer et al. (1997), $4910 \mathrm{Ci}$ and $4808 \mathrm{Ci}$, respectively. Global estimates of carbon-14 by Agnew et al. (1997) and Kupfer et al. (1995) were based on an assumed $100 \%$ delivery of carbon-14 in fuel to the waste tanks. Consequently, their estimates of carbon-14 may be high.

Regardless of the inventory in the tanks, the future location of $99 \%$ of the tank inventory after chemical separation into high-level and low-activity waste streams and immobilization is not clearly identified. One percent (1\%) of the tank inventory is assigned to the ILAW. Ninety-nine percent (99\%) is assigned to the immobilized high-level waste. However, the high-level waste may be a vitrified glass waste form and it may not capture volatile iodine isotopes. Furthermore, the integrated database for spent fuel and radioactive waste (ORNL 1997) shows $4.42 \mathrm{Ci}$ of carbon-14 in $I \mathrm{AW}$ and only $0.0911 \mathrm{Ci}$ in high-level waste glass canisters at the Hanford Site following completion of the chemical separation and immobilization campaigns.

The Composite Analysis accounted for $194 \mathrm{Ci}$ of carbon-14 in the tanks, solid waste burial grounds and liquid discharges of DOE wastes. However, considerably more than that was not accounted for if $99 \%$ if the carbon-14 in the tanks is not retained in the immobilized high-level waste. Ninety-nine percent of the Schmittroth et al. (1995) inventory is $761 \mathrm{Ci}$, a factor of 4 more carbon-14 than modeled as remaining at Hanford. Ninety-nine percent of the Agnew et al. (1997) and Kupfer et al. (1997) inventories are approximately $4760 \mathrm{Ci}$; a factor of 25 more carbon-14 than modeled.

\subsubsection{Chlorine-36}

As with carbon-14, the graphite cores are the dominant source of chlorine-36 at Hanford (302 Ci). In order to investigate the potential significance of chlorine-36 in other Hanford Site wastes, a 1-ppm level of chlorine-35 contamination was introduced in the ORIGEN2 simulations of irradiated fuel. There are no data on the actual chlorine-35 impurity levels in DOE fuel irradiated in the graphite core production 
reactors at Hanford. However, it is believed the 1-ppm level is within an order of magnitude of the true value. This level of impurity has been used to forecast the level of chlorine- 36 in aged fuel. Fuel ratios and the inventory of cesium- 137 were used to build chlorine- 36 inventory into inventories for solid waste burial grounds and liquid discharge sites. If significant impacts from chlorine- 36 are forecast, it is important to remember they may not be real. If such a forecast results, it will be important to determine chlorine impurity levels in DOE fuels and develop a true estimate of its potential contribution to dose.

\subsubsection{Iodine-129}

Total inventory values for iodine-129 are fairly consistent among the past and present TWRS inventories. However, while $\sim 65 \mathrm{Ci}$ were projected to reside in Hanford tanks, fewer than 11 Ci were accounted for in the Composite Analysis as remaining at the Hanford Site after closure. Of this amount, the majority could reside in the ILAW from the tanks. While little of the highly volatile iodine-129 inventory may remain in the ILAW to bound the effect of iodine- 129 dose from this waste form an estimated $10 \%$ of the original tank inventory, or $6.6 \mathrm{Ci}$ of iodine-129, was assigned to ILAW. While $\sim 5 \mathrm{Ci}$ of iodine-129 are distributed among the liquid discharge sites and solid waste burial grounds, it is not clear where $90 \%$ of the original tank contents ( $\sim 58 \mathrm{Ci}$ of iodine-129) reside after chemical separation and immobilization of tank wastes. It is assumed that it will be contained in the immobilized high-level waste.

The total inventory is based on the assumption that all iodine- 129 was routed to the tanks. Such an assumption neglects losses of iodine to the atmosphere, disposals of iodine to solid waste burial grounds and cribs, and the storage of two silver reactors in the second PUREX tunnel. ${ }^{(\mathrm{a})}$ Kupfer et al. (1997) estimated that $71 \%$ of the iodine may have been routed to tanks, and the remainder (i.e., $29 \%$, or $\sim 18 \mathrm{Ci}$ ) to the atmosphere or ground.

The volatile character of iodine implies it will not be captured in a vitrified high-level waste and subsequently exported from the Hanford Site. Some may be identified as leaving the site in TRU waste. With this exception, an upper bound for the final disposal of iodine-129 at Hanford could include the entire inventory generated at the Hanford Site $(\sim 65 \mathrm{Ci})$. This is approximately a factor of 6 more iodine129 than was accounted for in the first iteration of the Composite Analysis.

\subsubsection{Selenium-79}

The global inventories of selenium-79 in the tanks were relatively consistent among the assembled inventories (i.e., Agnew et al. [1997], 773 Ci; Kupfer et al. [1997], 773 Ci; Schmittroth et al. [1995], 1030 Ci). It was assumed the entire selenium-79 inventory in the tanks will be contained in the ILAW (Mann et al. 1997). Fewer than $20 \mathrm{Ci}$ were assigned to the other tank inventories, e.g., tank leaks, solid waste burial grounds, and liquid discharges.

(a) From a letter, dated September 29, 1993, from J. Reddick of Los Alamos Technical Associates, Kennewick, Washington, to D. Washenfelder of Westinghouse Hanford Company, Richland, Washington; Subject: "PUREX and UO3 Plant Inventory Estimates." 
It is anticipated that selenium-79 inventories for the Hanford Site will be reduced by a factor of eight in the near future based on a recent update of the decay half-life of this isotope (Kupfer et al. 1997). The significance of selenium-79 as a contributor to dose should decrease proportionately.

\subsubsection{Technetium-99}

The estimates produced by Schmittroth et al. (1995) for the ILAW disposal were used in this analysis to represent the ILAW. Global estimates of tank waste inventory by Agnew et al. (1997) and Kupfer et al. (1997) were not published when the Composite Analysis inventory was assembled. Schmittroth et al. (1995) estimated a total 27,200 Ci of technetium-99 in the tanks. Of that total, 22,300 Ci are to go into ILAW and the remaining $4900 \mathrm{Ci}$ are to go to high-level waste glass. Agnew et al. (1997) and Kupfer et al. (1997) present global estimates of the amount of technetium-99 produced at the Hanford Site and stored in the single- and double-shell tanks. The Agnew et al. (1997) and Kupfer et al. (1997) estimate of 32,600 Ci technetium-99 in the tanks is higher than the Schmittroth et al. (1995) estimate because they decided to show a bounding inventory value and, therefore, neither took into account the technetium-99 exported from the site. Schmittroth et al. (1995) documented that an estimated $20 \%$ of the technetium- 99 produced at the site was lost from the tank waste. Most of this 5000 - to $6000-\mathrm{Ci}$ inventory was coprocessed with the uranium oxide metal and sent offsite.

While the Schmittroth et al. (1995) estimate shows $~ 5000 \mathrm{Ci}$ of technetium-99 going to high-level waste glass, any technetium-99 produced as a separate waste stream may require special treatment. Privatization contractors that perform the separation and immobilization steps for tank waste may find it advantageous to remove technetium-99 to ensure waste form performance and product acceptance by the DOE. The final disposition of a special waste stream containing technetium-99 is not known. Alternatives include its inclusion in immobilized high-level waste leaving the site, disposal in special packages in solid waste burial grounds, or disposal onsite or offsite as a special waste form.

Based on TRAC model results, it was estimated that liquid discharge sites have received $\sim 930 \mathrm{Ci}$ of technetium-99 (Waite 1991). Based on data in the tank characterization reports for liquid tank wastes, the tanks were estimated to have leaked $460 \mathrm{Ci}$ and to lose $\sim 470 \mathrm{Ci}$ of technetium-99 during retrieval. Based on the TWRS EIS database (DOE and Ecology 1996) and the assumption of $1 \%$ volume remaining following recovery operations, $\sim 320 \mathrm{Ci}$ of technetium- 99 will be in tank residuals. Based on aged-fuel ratios and the inventory of cesium, another $325 \mathrm{Ci}$ of technetium- 99 are assumed to reside in the solid waste burial grounds. These dispositions, which total $\sim 2500 \mathrm{Ci}$, are based on a number of different models. While each method of estimating technetium-99 disposal has been useful, not all are consistent.

Ultimately, aside from the ILAW, the $2500 \mathrm{Ci}$ inventory of technetium-99 lost to or disposed in the subsurface environment at the Hanford Site is less than $10 \%$ of the total technetium- 99 inventory. An effort to generate a fully consistent inventory estimate could yield a lower estimate of losses and disposals. For example, because of its solubility, most of the technetium-99 should be removed from the tanks during the tank waste recovery campaigns, and less than the $320 \mathrm{Ci}$ estimated here should remain in the tank residuals. Similarly, if sluicing methods are used to recover tank wastes, it is likely that contaminant concentrations in sluicing losses from the tanks will be lower than contaminant concentrations in tank wastes. Thus, the estimated $470 \mathrm{Ci}$ of technetium- 99 lost during tank waste recovery 
operations, that was based on tank waste radionuclide concentrations, would decrease. Finally, the Agnew et al. (1997) model provides an estimate of only $107 \mathrm{Ci}$ of technetium-99 lost in past tank leaks compared to the $460 \mathrm{Ci}$ estimated here. Clearly, a lower inventory of loss and disposal could result from a consistent or best-estimate inventory estimate. However, there is also uncertainty in the future technetium- 99 waste streams that private contractors may generate and return to the DOE for disposal.

\subsubsection{Uranium-238}

Kupfer et al. (1997) reconciled the HDW model results for uranium (906 Ci of uranium-238) and tank sample data (322 Ci), and decided in favor of the sample data. The discrepancy among TWRS total inventory estimates of uranium is attributed to the factor used to describe the fraction of metal waste not recovered. However, estimates in Waite (1991) for uranium in tank waste discharges to cribs and specific retention trenches, and estimates provided by Coony ${ }^{(a)}$ are much lower than estimates that appear in Agnew et al. (1997). Coony estimated $47.5 \mathrm{Ci}$ of uranium-238 as compared to $1,310 \mathrm{Ci}$ estimated by Agnew et al. (1997). The Agnew et al. (1997) inventory of uranium-238 sent to ground in liquid discharges may also be an overestimate because it is based on the factor assumed for uranium metal recovery.

A clearly unrealistic and high estimate of uranium-238 is included in the ERDF inventory (i.e., $54,300 \mathrm{Ci}$ ). This inventory estimate is based on maximum observed uranium- 238 concentrations in sediments at CERCLA sites. The composition of uranium in ERDF has the signature of enriched uranium, but this is an artifact of using maximum observed concentrations of uranium isotopes to estimate the total inventory disposed. The commercial LLW disposal facility also contains a considerable inventory of uranium-238 (10,900 Ci).

(a) From an electronic mail message with attached files regarding "Questions on Crib Releases in the 200 Areas." Sent by F. M. Coony of Waste Management Federal Services of Hanford to C. T. Kincaid on November 5, 1997. 
Table 3.1. Inventory of Key Radionuclides for the Solid Waste Burial Grounds

\begin{tabular}{|c|c|c|c|c|c|c|}
\hline & \multicolumn{6}{|c|}{ Radionuclide Inventories ${ }^{*}$ in Curies ${ }^{\star \star}$} \\
\hline Site Name & C-14 & Cl-36 & $\mathrm{I}-129$ & Se-79 & Tc-99 & U-238 \\
\hline $218-E C-9(a)^{+}$ & $2.29 \mathrm{E}-03$ & $1.51 \mathrm{E}-04$ & $1.23 \mathrm{E}-05$ & $1.84 \mathrm{E}-04$ & $6.22 \mathrm{E}-03$ & $0.00 \mathrm{E}+00$ \\
\hline $218-\mathrm{EC}-9(\mathrm{~b})^{4}$ & $2.79 \mathrm{E}-05$ & $1.83 E-06$ & 1.49E-07 & $2.24 \mathrm{E}-06$ & $7.57 \mathrm{E}-05$ & $0.00 \mathrm{E}+00$ \\
\hline $218-\mathrm{E}-1(\mathrm{~b})$ & $1.39 \mathrm{E}-04$ & $9.15 \mathrm{E}-06$ & $7.45 \mathrm{E}-07$ & $1.12 \mathrm{E}-05$ & $3.77 \mathrm{E}-04$ & $1.35 \mathrm{E}-01$ \\
\hline $218-\bar{E}-10(\mathrm{~b})$ & $7.73 E+01$ & $5.08 \mathrm{E}+00$ & $4.14 \mathrm{E}-01$ & $6.19 \mathrm{E}+00$ & $2.10 \mathrm{E}+02$ & 2.69E-01 \\
\hline 218-E-10(a) & $1.15 \mathrm{E}-01$ & $7.58 \mathrm{E}-03$ & $6.17 \mathrm{E}-04$ & $9.23 \bar{E}-03$ & $3.13 \mathrm{E}-01$ & $0.00 \mathrm{E}+00$ \\
\hline $218-E-12 A(b)$ & $1.24 \mathrm{E}-03$ & $8.14 \mathrm{E}-05$ & $6.63 \mathrm{E}-06$ & $9.92 \mathrm{E}-05$ & $3.36 \mathrm{E}-03$ & $3.33 \mathrm{E}-01$ \\
\hline 218-E-12B(b) & $2.03 E+00$ & $1.34 \mathrm{E}-01$ & $1.09 \mathrm{E}-02$ & $1.63 \mathrm{E}-01$ & $5.51 \mathrm{E}+00$ & $6.57 \mathrm{E}-02$ \\
\hline 218-E-12B(a) & $1.73 \mathrm{E}-02$ & $1.14 \mathrm{E}-03$ & $4.14 \mathrm{E}-02$ & $1.38 \mathrm{E}-03$ & $4.69 \mathrm{E}-02$ & $6.68 \mathrm{E}-02$ \\
\hline $218-E-2(b)$ & $3.48 \mathrm{E}-02$ & $2.29 \mathrm{E}-03$ & $1.86 \mathrm{E}-04$ & $2.79 \mathrm{E}-03$ & $9.44 \mathrm{E}-02$ & $1.01 \mathrm{E}-01$ \\
\hline $218-E-4(b)$ & $1.39 \mathrm{E}-05$ & $9.15 \mathrm{E}-07$ & $7.45 \mathrm{E}-08$ & $1.12 \mathrm{E}-06$ & $3.77 \mathrm{E}-05$ & $3.36 \mathrm{E}-04$ \\
\hline 218-E-5(b) & $1.04 \bar{E}-02$ & $6.86 \mathrm{E}-04$ & $5.59 \mathrm{E}-05$ & $8: 36 \mathrm{E}-04$ & $2.8 \overline{3} \mathrm{E}-02$ & $4.04 \mathrm{E}-02$ \\
\hline 218-E-5A(b) & $2.30 \mathrm{E}-02$ & $1.51 \mathrm{E}-03$ & $1.23 \mathrm{E}-04$ & $1.84 \mathrm{E}-03$ & $6.23 \mathrm{E}-02$ & $4.04 \mathrm{E}-02$ \\
\hline $218-\mathrm{E}-8(\mathrm{~b})$ & $1.39 \mathrm{E}-05$ & $9.15 \mathrm{E}-07$ & $7.45 \mathrm{E}-08$ & $1.12 \mathrm{E}-06$ & $3.77 E-05$ & $6.73 \mathrm{E}-04$ \\
\hline $218-W-1(\mathrm{~b})$ & $2.78 \mathrm{E}-04$ & $1.83 \mathrm{E}-05$ & $1.49 \mathrm{E}-06$ & $2.23 \mathrm{E}-05$ & $7.55 \mathrm{E}-04$ & $2.35 \mathrm{E}-01$ \\
\hline $218-W-11(b)$ & $1.39 \mathrm{E}-07$ & $9.15 \mathrm{E}-09$ & $7.45 \mathrm{E}-10$ & $1.12 \mathrm{E}-08$ & $3.77 \mathrm{E}-07$ & $0.00 \mathrm{E}+00$ \\
\hline $218-W-1 A(b)$ & $6.68 \mathrm{E}-02$ & $4.40 \mathrm{E}-03$ & $3.58 \mathrm{E}-04$ & $5.36 \mathrm{E}-03$ & $1.81 \mathrm{E}-01$ & $3.03 \mathrm{E}-01$ \\
\hline $218-W-2(b)$ & $6.96 \mathrm{E}-04$ & $4.58 \mathrm{E}-05$ & $3.72 \mathrm{E}-06$ & $5.58 \mathrm{E}-05$ & $1.89 \mathrm{E}-03$ & 4.71E-01 \\
\hline $218-W-2 A(b)$ & $3.63 \mathrm{E}-01$ & $2.39 \mathrm{E}-02$ & $1.94 \mathrm{E}-03$ & 2.91E-02 & $9.84 \mathrm{E}-01$ & $9.05 \mathrm{E}-01$ \\
\hline $218-W-3(b)$ & $1.25 \mathrm{E}-03$ & $8.24 \mathrm{E}-05$ & $6.70 \mathrm{E}-06$ & $1.00 \mathrm{E}-04$ & $3.40 \mathrm{E}-03$ & $2.35 \mathrm{E}+01$ \\
\hline $218-W-3 A(b)$ & $1.99 \mathrm{E}+01$ & $1.31 \mathrm{E}+00$ & $1.06 \mathrm{E}-01$ & $1.59 \mathrm{E}+00$ & $5.39 \mathrm{E}+01$ & $1.99 \mathrm{E}+01$ \\
\hline $218-W-3 A(a)$ & $6.62 \mathrm{E}-01$ & $4.36 \mathrm{E}-02$ & $3.68 \mathrm{E}-03$ & $5.31 E-02$ & $2.89 E+00$ & $4.23 \mathrm{E}-01$ \\
\hline 218-W-3AE(b) & $8.15 \mathrm{E}-01$ & $5.36 \mathrm{E}-02$ & $4.36 \mathrm{E}-03$ & $6.53 \mathrm{E}-02$ & $2.21 \mathrm{E}+00$ & $8.93 \mathrm{E}+00$ \\
\hline 218-W-3AE(a) & $1.10 \mathrm{E}+01$ & $7.25 \mathrm{E}-01$ & $5.47 \mathrm{E}-02$ & $8.83 E-01$ & $3.58 \mathrm{E}+01$ & $1.87 E+02$ \\
\hline $218-W-4 A(b)$ & $4.61 \mathrm{E}-03$ & $3.03 \mathrm{E}-04$ & $2.47 \mathrm{E}-05$ & $3.70 \mathrm{E}-04$ & $1.25 \mathrm{E}-02$ & $1.33 \mathrm{E}+02$ \\
\hline $218-W-4 B-c(b)$ & $2.35 \mathrm{E}-01$ & $1.55 \mathrm{E}-02$ & $1.26 \mathrm{E}-03$ & $1.88 \mathrm{E}-02$ & $6.37 \mathrm{E}-01$ & $1.00 \mathrm{E}-01$ \\
\hline $218-W-4 B-n(b)$ & $5.13 \mathrm{E}-01$ & $3.37 \mathrm{E}-02$ & $5.00 \mathrm{E}-01$ & $4.11 \mathrm{E}-02$ & $1.39 \mathrm{E}+00$ & $0.00 \mathrm{E}+00$ \\
\hline $218-W-4 B-c(a)$ & $5.68 \mathrm{E}-02$ & $3.74 \mathrm{E}-03$ & $3.04 \mathrm{E}-04$ & $4.55 \mathrm{E}-03$ & $1.54 \mathrm{E}-01$ & $0.00 \mathrm{E}+00$ \\
\hline $218-W-4 C(a)$ & $4.10 \mathrm{E}+00$ & $9.42 \mathrm{E}-03$ & $1.13 \mathrm{E}-02$ & $6.24 \mathrm{E}-02$ & $9.88 \mathrm{E}+00$ & $1.39 \mathrm{E}+02$ \\
\hline $218-W-4 C(b)$ & $2.90 \mathrm{E}+00$ & $1.25 \mathrm{E}-02$ & $1.02 \mathrm{E}-03$ & $1.61 \mathrm{E}-02$ & 6.07E-01 & $7.90 \mathrm{E}-01$ \\
\hline $218-W-5(b)$ & $4.09 E+00$ & $2.73 \mathrm{E}-03$ & $3.00 \mathrm{E}-03$ & $3.33 \mathrm{E}-03$ & $1.13 \mathrm{E}-01$ & $3.99 \mathrm{E}+00$ \\
\hline $218-W-5(a)$ & $1.51 \mathrm{E}+00$ & $5.09 \mathrm{E}-02$ & $1.40 \mathrm{E}-01$ & $6.20 \mathrm{E}-02$ & $2.77 \mathrm{E}+00$ & $1.98 \mathrm{E}+01$ \\
\hline 218-W-7(b) & $5.61 \mathrm{E}-03$ & $3.69 \mathrm{E}-04$ & $3.00 \mathrm{E}-05$ & $4.49 \mathrm{E}-04$ & $1.52 \mathrm{E}-02$ & $2.35 \mathrm{E}-04$ \\
\hline 218-W-8(b) & $1.07 \mathrm{E}-03$ & $7.05 \mathrm{E}-05$ & $5.73 \mathrm{E}-06$ & $8.58 \mathrm{E}-05$ & $2.91 E-03$ & $1.01 \mathrm{E}-04$ \\
\hline $218-W-9(b)$ & $1.39 \mathrm{E}-07$ & $9.15 \mathrm{E}-09$ & $7.45 \mathrm{E}-10$ & $1.12 \mathrm{E}-08$ & $3.77 \mathrm{E}-07$ & $0.00 \mathrm{E}+00$ \\
\hline
\end{tabular}

* See Appendix $A$ for greater detail in the development of solid waste burial ground inventories.

** Inventories are decayed to a common date of 2050.

+ (a) refers to waste disposed after September 30, 1988.

+ (b) refers to waste disposed before September 30, 1988. 
Table 3.2. Inventory of Key Radionuclides for ERDF

\begin{tabular}{|c|c|c|c|c|c|c|}
\hline & \multicolumn{6}{|c|}{ Radionuclide Inventories $^{\star}$ in Curies ${ }^{\star \star}$} \\
\hline Site Name & C-14 & $\mathrm{Cl}-36$ & $\mathrm{I}-129$ & Se-79 & Tc-99 & U-238 \\
\hline ERDF & $3.80 \mathrm{E}+03$ & & & & $6.57 \mathrm{E}+00$ & $5.43 \mathrm{E}+04$ \\
\hline
\end{tabular}

* Total inventory was calculated using waste volumes for a full six-cell ERDF trench and maximum concentrations reported in the ERDF RI/FS (DOE 1994b). Chlorine, iodine, and selenium values were not reported.

** Inventories are decayed to a common date of 2050 .

Table 3.3. Inventory of Key Radionuclides for TWRS Low-Activity Waste

\begin{tabular}{|l|c|c|c|c|c|c|}
\hline & \multicolumn{7}{|c|}{${\text { Radionuclide Inventories }{ }^{*} \text { in Curies }}^{\text {*A }}$} \\
\hline \multicolumn{1}{|c|}{ Site Name } & C-14 & Cl-36 & I-129 & Se-79 & Tc-99 & U-238 \\
\hline $\begin{array}{l}\text { TWRS glass } \\
\text { grout vault }\end{array}$ & $4.54 \mathrm{E}-01$ & & $3.91 \mathrm{E}-01$ & $6.07 \mathrm{E}+01$ & $1.32 \mathrm{E}+03$ & $1.05 \mathrm{E}+00$ \\
$\begin{array}{l}\text { TWRS glass } \\
\text { new site }\end{array}$ & $7.24 \mathrm{E}+00$ & & $6.23 \mathrm{E}+00$ & $9.69 \mathrm{E}+02$ & $2.10 \mathrm{E}+04$ & $1.67 \mathrm{E}+01$ \\
\hline
\end{tabular}

* The waste inventory in each site is based on the fraction of waste volume in each site and the total inventory.

** Inventories are decayed to a common date of 2050. 
Table 3.4. Inventory of Key Radionuclides for TWRS Single-Shell Tanks

\begin{tabular}{|c|c|c|c|c|c|c|}
\hline & \multicolumn{6}{|c|}{ Radionuclide Inventories in Curies } \\
\hline Site Name & C-14 & $\mathrm{Cl}-36$ & $\mathrm{I}-129$ & Se-79 & Tc-99 & U-238 \\
\hline $\mathrm{TK}-\mathrm{A}-\mathrm{S}^{* *}$ & 9.43E-02 & $0.00 \mathrm{E}+00$ & $2.81 \mathrm{E}-02$ & $1.68 \mathrm{E}-02$ & $3.31 \mathrm{E}+01$ & $7.36 \mathrm{E}-04$ \\
\hline$\overline{T K}-\mathrm{A}-\mathrm{L}^{*+\pi}$ & $1.10 \mathrm{E}+00$ & $0.00 \mathrm{E}+00$ & $1.83 \mathrm{E}-01$ & $1.96 \mathrm{E}-01$ & $1.25 \mathrm{E}+02$ & $8.57 \mathrm{E}-03$ \\
\hline $\mathrm{TK}-\mathrm{A}-\mathrm{R}^{+}$ & $2.11 E+00$ & & $1.71 \mathrm{E}-03$ & $8.33 \mathrm{E}-02$ & $1.15 E+00$ & $1.52 \mathrm{E}-01$ \\
\hline TK-AX-S-1 & $8.00 \mathrm{E}-02$ & $0.00 \mathrm{E}+00$ & $0.00 \mathrm{E}+00$ & $0.00 \mathrm{E}+00$ & $1.68 \mathrm{E}+00$ & $0.00 \mathrm{E}+00$ \\
\hline TK-AX-S-2 & $3.14 \mathrm{E}-02$ & $0.00 \mathrm{E}+00$ & $9.38 \mathrm{E}-03$ & $5.60 \mathrm{E}-03$ & $1.10 \mathrm{E}+01$ & $2.45 \mathrm{E}-04$ \\
\hline TK-AX-L-1 & $1.50 \mathrm{E}-02$ & $0.00 \mathrm{E}+00$ & $0.00 \mathrm{E}+00$ & $0.00 \mathrm{E}+00$ & 0.3156146 & $0.00 E+00$ \\
\hline TK-AX-L-2 & $3.14 \mathrm{E}-02$ & $0.00 \mathrm{E}+00$ & $5.14 \mathrm{E}-03$ & $5.60 \mathrm{E}-03$ & $3.43 \mathrm{E}+00$ & $2.45 \mathrm{E}-04$ \\
\hline TK-AX-R-1 & $2.75 \mathrm{E}-02$ & & $3.90 \mathrm{E}-05$ & $1.95 \mathrm{E}-03$ & $2.68 \mathrm{E}-02$ & $2.13 E-03$ \\
\hline TK-AX-R-2 & $1.37 \mathrm{E}-01$ & & $1.95 \mathrm{E}-04$ & $9.74 \mathrm{E}-03$ & $1.34 \mathrm{E}-01$ & $1.07 \mathrm{E}-02$ \\
\hline TK-B-S & $5.03 \mathrm{E}-01$ & $0.00 \mathrm{E}+00$ & $8.23 \mathrm{E}-02$ & $8.96 \mathrm{E}-02$ & $5.48 \mathrm{E}+01$ & $3.92 \mathrm{E}-03$ \\
\hline TK-B-L & $2.12 \mathrm{E}-01$ & $0.00 \mathrm{E}+00$ & $3.46 \mathrm{E}-02$ & $3.77 \mathrm{E}-02$ & $2.31 \mathrm{E}+01$ & $1.65 \mathrm{E}-03$ \\
\hline TK-B-R & $4.98 \mathrm{E}+00$ & & $2.02 \mathrm{E}-02$ & $1.01 \mathrm{E}+00$ & $1.39 \mathrm{E}+01$ & $3.41 \mathrm{E}-01$ \\
\hline TK-BX-S & $3.77 \mathrm{E}-01$ & $0.00 \mathrm{E}+00$ & $6.17 \mathrm{E}-02$ & $6.72 \mathrm{E}-02$ & $4.11 \mathrm{E}+01$ & $2.94 \mathrm{E}-03$ \\
\hline TK-BX-L & $3.79 \mathrm{E}-01$ & $0.00 \mathrm{E}+00$ & $6.20 \mathrm{E}-02$ & $6.75 \mathrm{E}-02$ & $4.13 E+01$ & $2.96 \mathrm{E}-03$ \\
\hline TK-BX-R & $9.18 \mathrm{E}+00$ & & $4.78 \mathrm{E}-02$ & $2.39 E+00$ & $3.28 \mathrm{E}+01$ & $4.87 \mathrm{E}-01$ \\
\hline TK-BY-S & $3.77 \mathrm{E}-01$ & $0.00 \mathrm{E}+00$ & $6.17 \mathrm{E}-02$ & $6.72 \mathrm{E}-02$ & $4.11 \mathrm{E}+01$ & $2.94 \mathrm{E}-03$ \\
\hline TK-BY-L & $1.61 \mathrm{E}-01$ & $0.00 \mathrm{E}+00$ & $2.64 \mathrm{E}-02$ & $2.88 \mathrm{E}-02$ & $1.76 \mathrm{E}+01$ & $1.26 \mathrm{E}-03$ \\
\hline TK-BY-R & $2.18 \mathrm{E}+00$ & & $1.76 \mathrm{E}-02$ & $8.83 \mathrm{E}-01$ & $1.22 \mathrm{E}+01$ & $7.93 \mathrm{E}-01$ \\
\hline TK-C-S-1 & $5.80 \mathrm{E}-02$ & $0.00 \mathrm{E}+00$ & $2.16 \mathrm{E}-03$ & $5.60 \mathrm{E}-03$ & $3.27 \mathrm{E}+00$ & $2.35 \mathrm{E}-04$ \\
\hline TK-C-S-2 & $3.46 \mathrm{E}-01$ & $0.00 \mathrm{E}+00$ & $5.66 \mathrm{E}-02$ & $6.16 \mathrm{E}-02$ & $3.77 \mathrm{E}+01$ & $2.70 \mathrm{E}-03$ \\
\hline TK-C-L-1 & $1.50 \mathrm{E}-03$ & $0.00 \mathrm{E}+00$ & $1.80 \mathrm{E}-04$ & $4.67 \mathrm{E}-04$ & $2.02 E-01$ & $1.96 \mathrm{E}-05$ \\
\hline TK-C-L-2 & $1.07 \mathrm{E}-01$ & $0.00 \mathrm{E}+00$ & $1.75 \mathrm{E}-02$ & $1.91 \mathrm{E}-02$ & $1.17 \mathrm{E}+01$ & $8.35 E-04$ \\
\hline TK-C-R-1 & 9.49E-01 & & $3.53 \mathrm{E}-03$ & $1.68 \mathrm{E}-01$ & $2.32 E+00$ & $3,05 \mathrm{E}-01$ \\
\hline TK-C-R-2 & $8.79 \mathrm{E}-01$ & & $3.27 \mathrm{E}-03$ & $1.55 \mathrm{E}-01$ & $2.15 \mathrm{E}+00$ & $2.83 \mathrm{E}-01$ \\
\hline TK-S-S & $3.14 \mathrm{E}-01$ & $0.00 \mathrm{E}+00$ & $5.99 \mathrm{E}-02$ & $5.60 \mathrm{E}-02$ & $4.95 \mathrm{E}+01$ & $2.45 \mathrm{E}-03$ \\
\hline TK-S-L & $9.43 \mathrm{E}-02$ & $0.00 \mathrm{E}+00$ & $1.54 \mathrm{E}-02$ & $1.68 \mathrm{E}-02$ & $1.03 \mathrm{E}+01$ & $7.36 \mathrm{E}-04$ \\
\hline TK-S-R & $3.82 \mathrm{E}+00$ & & $2.38 \mathrm{E}-02$ & $1.19 \mathrm{E}+00$ & $1.65 \mathrm{E}+01$ & $1.82 \mathrm{E}-01$ \\
\hline TK-SX-S-I & $5.99 \mathrm{E}-03$ & $0.00 \mathrm{E}+00$ & $7.21 \mathrm{E}-04$ & $1.87 \mathrm{E}-03$ & $8.08 \mathrm{E}-01$ & $7.84 \mathrm{E}-05$ \\
\hline TK-SX-S-2 & $3.46 \mathrm{E}-01$ & $0.00 \mathrm{E}+00$ & $6.93 \mathrm{E}-02$ & $6.16 \mathrm{E}-02$ & $6.05 \mathrm{E}+01$ & $2.70 \mathrm{E}-03$ \\
\hline TK-SX-L-2 & $6.30 \mathrm{E}-01$ & $0.00 \mathrm{E}+00$ & $1.06 \mathrm{E}-01$ & $1.12 \mathrm{E}-01$ & $7.45 \mathrm{E}+01$ & $4.92 \mathrm{E}-03$ \\
\hline TK-SX-R-1 & $1.94 \mathrm{E}-01$ & & $2.17 \mathrm{E}-03$ & $1.09 \mathrm{E}-01$ & $1.50 \mathrm{E}+00$ & $1.69 \mathrm{E}-02$ \\
\hline TK-SX-R-2 & $1.68 \mathrm{E}+00$ & & $1.88 \mathrm{E}-02$ & $9.50 \mathrm{E}-01$ & $1.30 \mathrm{E}+01$ & $1.47 \mathrm{E}-01$ \\
\hline TK-T-S & $5.03 \mathrm{E}-01$ & $0.00 \mathrm{E}+00$ & $8.23 \mathrm{E}-02$ & $8.96 \mathrm{E}-02$ & $5.48 \mathrm{E}+01$ & $3.92 \mathrm{E}-03$ \\
\hline TK-T-L & $5.28 \mathrm{E}-01$ & $0.00 \mathrm{E}+00$ & $8.65 \mathrm{E}-02$ & $9.41 \mathrm{E}-02$ & $5.76 \mathrm{E}+01$ & $4.12 \mathrm{E}-03$ \\
\hline TK-T-R & $1.50 \mathrm{E}-01$ & & $5.09 \mathrm{E}-04$ & $2.57 \mathrm{E}-02$ & $3.51 \mathrm{E}-01$ & $8.05 \mathrm{E}-02$ \\
\hline TK-TX-S & $1.89 \mathrm{E}-01$ & $0.00 \mathrm{E}+00$ & $3.09 \mathrm{E}-02$ & $3.36 \mathrm{E}-02$ & $2.06 \mathrm{E}+01$ & $1.47 \mathrm{E}-03$ \\
\hline
\end{tabular}


Table 3.4. (contd)

\begin{tabular}{|l|c|c|c|c|c|c|}
\hline & \multicolumn{7}{|c|}{ Radionuclide Inventories in Curies } \\
\hline \multicolumn{1}{|c|}{ Site Name } & C-14 & Cl-36 & I-129 & Se-79 & Tc-99 & U-238 \\
\hline TK-TX-L & $2.30 \mathrm{E}-01$ & $0.00 \mathrm{E}+00$ & $3.76 \mathrm{E}-02$ & $4.09 \mathrm{E}-02$ & $2.51 \mathrm{E}+01$ & $1.79 \mathrm{E}-03$ \\
\hline TK-TX-R & $2.91 \mathrm{E}+00$ & & $1.35 \mathrm{E}-02$ & $6.76 \mathrm{E}-01$ & $9.34 \mathrm{E}+00$ & $1.56 \mathrm{E}+00$ \\
\hline TK-TY-R & $4.81 \mathrm{E}-01$ & & $5.34 \mathrm{E}-03$ & $2.68 \mathrm{E}-01$ & $3.68 \mathrm{E}+00$ & $7.78 \mathrm{E}-02$ \\
\hline TK-U-S & $4.40 \mathrm{E}-01$ & $0.00 \mathrm{E}+00$ & $8.05 \mathrm{E}-02$ & $7.84 \mathrm{E}-02$ & $6.32 \mathrm{E}+01$ & $3.43 \mathrm{E}-03$ \\
\hline TK-U-L & $3.99 \mathrm{E}-01$ & $0.00 \mathrm{E}+00$ & $6.53 \mathrm{E}-02$ & $7.11 \mathrm{E}-02$ & $4.35 \mathrm{E}+01$ & $3.11 \mathrm{E}-03$ \\
\hline TK-U-R & $1.35 \mathrm{E}-01$ & & $1.32 \mathrm{E}-03$ & $6.52 \mathrm{E}-02$ & $9.08 \mathrm{E}-01$ & $3.10 \mathrm{E}-01$ \\
\hline
\end{tabular}

* Inventories are decayed to a common date of 2050 .

** " $S$ " refers to sluicing losses during recovery of tank wastes. The inventory is based on an 8,000-gallon-per-tank loss and radionuclide concentrations developed from tank characterization reports.

*** " $L$ " refers to past tank leaks as identified in Hanlon (1997). The inventories are based on leak volumes from Hanlon and radionuclide concentrations developed from tank characterization reports.

+ " $R$ " refers to residual wastes remaining in tank after tank waste recovery. Inventories are based on $1 \%$ of tank farm inventory reported in the TWRS EIS (DOE and Ecology 1996). H " 1 " and " 2 " refer to complexed and non-complexed waste, respectively. 
Table 3.5. Inventory of Key Radionuclides for TWRS Double-Shell Tanks

\begin{tabular}{|l|c|c|c|c|c|c|}
\hline & \multicolumn{5}{|c|}{ Radionuclide Inventory in Curies $^{\text {*x }}$} \\
\hline \multicolumn{1}{|c|}{ Site Name } & C-14 & Cl-36 & I-129 $^{\text {*x* }}$ & Se-79 & Tc-99 & U-238 \\
\hline TK-AN-R-1,++ & $8.28 \mathrm{E}+00$ & & & & $5.56 \mathrm{E}+01$ & \\
\hline TK-AN-R-2 & $1.14 \mathrm{E}+01$ & & & & $7.64 \mathrm{E}+01$ & \\
\hline TK-AP-R-1 & $1.00 \mathrm{E}-03$ & & & & $2.63 \mathrm{E}-01$ & \\
\hline TK-AP-R-2 & $2.80 \mathrm{E}-02$ & & & & $7.35 \mathrm{E}+00$ & \\
\hline TK-AW-R & $2.31 \mathrm{E}-02$ & & & & $8.38 \mathrm{E}+00$ & \\
\hline TK-AY-R-1 & $3.57 \mathrm{E}-04$ & & & & $2.77 \mathrm{E}+00$ & \\
\hline TK-AY-R-2 & $3.29 \mathrm{E}-04$ & & & & $2.55 \mathrm{E}+00$ & \\
\hline TK-AZ-R & $3.48 \mathrm{E}+00$ & & & & $2.10 \mathrm{E}+01$ & \\
\hline TK-SY-R-1 & $6.03 \mathrm{E}-03$ & & & & $2.75 \mathrm{E}+01$ & \\
\hline TK-SY-R-2 & $1.98 \mathrm{E}-03$ & & & & $9.05 \mathrm{E}+00$ & \\
\hline
\end{tabular}

* Chlorine-36, selenium-79, and uranium-238 were not reported in the TWRS EIS (DOE and Ecology 1996).

** Inventories decayed to a common date of 2050 .

*** Iodine-129 is reported in the TWRS EIS, but on a tank-farm-group basis, instead of a tank-farm basis. Therefore iodine-129, which has a total inventory of 22.3 $\mathrm{Ci}$ (DOE and Ecology 1996) all in double-shell tanks, is not reported here.

$+\quad$ " $R$ " refers to residual wastes remaining in the tank after the tank waste recovery. Inventories are based on $1 \%$ of the tank farm inventory reported in the TWRS EIS (DOE and Ecology 1996).

+ " 1 " and "2" refer to complexed and noncomplexed waste, respectively. 
Table 3.6. Ratios of Cesium-137, Uranium (Total), and Plutonium (Total) for Waste Site Groups

\begin{tabular}{|l|c|c|c|l|}
\hline \multicolumn{1}{|c|}{$\begin{array}{c}\text { Waste Site } \\
\text { Groups* }\end{array}$} & $\mathbf{U} / \mathbf{P u}$ & $\begin{array}{c}\mathbf{U} / \mathbf{C s}-\mathbf{1 3 7} \\
(\mathbf{g} / \mathbf{C i})\end{array}$ & $\begin{array}{c}\mathbf{P u} / \mathbf{C s}-137 \\
(\mathbf{g} / \mathbf{C i})\end{array}$ & \multicolumn{1}{|c|}{ Notes } \\
\hline Group 2 & 4604 & 2773 & & \\
\hline Groups 3 \& 4 & 5.18 & & 7.19 & \\
\hline Group 5 & & & & $\mathrm{U}, \mathrm{Pu}$, and Cs-137 reported for all sites \\
\hline Group 6 & & & 0.371 & \\
\hline Group 7 & & 348 & 9.89 & \\
\hline Group 8 & & 970 & 31.8 & \\
\hline Group 9 & 400 & 101 & & \\
\hline Group 10 & & & 4.07 & \\
\hline Group 11 & & & & $\mathrm{U}, \mathrm{Pu}$, and Cs-137 reported for all sites \\
\hline Groups 12-16 & & 46,200 & 54.7 & \\
\hline Group 17 & & 66,300 & & \\
\hline Group 18 & & & 138 & \\
\hline Group 19 & 1,000 & & & assumed \\
\hline Groups 21 and 23 & & 21,000 & 6.08 & \\
\hline
\end{tabular}

* Groups 2 through 23 refer to waste site groups defined in DOE (1997b). 
Table 3.7. Inventories of Uranium-238, Technetium-99, and Iodine-129 for Liquid Discharge (216) Sites from the SWITS Database

\begin{tabular}{|c|c|c|c|}
\hline & \multicolumn{3}{|c|}{ Radionuclide Inventories in Curies ${ }^{*}$} \\
\hline Site Name & $\mathrm{U}-238^{\star \star}$ & Tc-99 & I-129 ${ }^{+}$ \\
\hline $216-A-1$ & 5.12E-02 & & \\
\hline $216-A-10$ & $8.09 \mathrm{E}-02$ & & \\
\hline $216-A-18$ & 4.69E-01 & & \\
\hline $216-A-19$ & $1.30 \mathrm{E}+01$ & & \\
\hline $216-A-2$ & $2.60 \mathrm{E}-02$ & & \\
\hline $216-A-20$ & $1.35 \mathrm{E}-01$ & & \\
\hline $216-A-21$ & $6.49 \mathrm{E}-02$ & & \\
\hline $216-A-24$ & $1.66 \mathrm{E}-02$ & & \\
\hline $216-A-25$ & $4.24 \mathrm{E}+00$ & & \\
\hline $216-A-27$ & $2.26 \mathrm{E}-02$ & & \\
\hline $216-A-28$ & $2.11 \mathrm{E}-01$ & & \\
\hline $216-A-3$ & $5.59 \mathrm{E}-01$ & & \\
\hline $216-A-30$ & $9.98 \mathrm{E}-02$ & & \\
\hline $216-A-31$ & $6.99 \mathrm{E}-03$ & & \\
\hline $216-A-36 A$ & $4.83 \mathrm{E}-02$ & & \\
\hline $216-A-36 B$ & $3.99 \mathrm{E}-02$ & & \\
\hline $216-A-37$ & $1.10 \mathrm{E}-02$ & & \\
\hline $216-A-37-2$ & $1.73 \mathrm{E}-02$ & & \\
\hline $216-A-39$ & $0.00 \mathrm{E}+00$ & & \\
\hline $216-A-4$ & $1.33 \mathrm{E}-01$ & & \\
\hline $216-A-40$ & $0.00 \mathrm{E}+00$ & & \\
\hline $216-A-45$ & $2.33 \mathrm{E}-03$ & & \\
\hline $216-A-5$ & $8.75 \mathrm{E}-02$ & & \\
\hline $216-A-6$ & 5.49E-02 & & \\
\hline $216-A-7$ & $2.33 \mathrm{E}-03$ & & \\
\hline $216-A-8$ & $1.23 \mathrm{E}-01$ & & \\
\hline $216-A-9$ & $0.00 \mathrm{E}+00$ & & \\
\hline $216-\mathrm{B}-10$ & $3.00 \mathrm{E}-03$ & & \\
\hline 216-B-10B & $0.00 \mathrm{E}+00$ & & \\
\hline $216-\bar{B}-11$ & $4.66 \mathrm{E}-03$ & & \\
\hline $216-B-12$ & $6.96 \mathrm{E}+00$ & & \\
\hline 216-B-14 & $7.25 \mathrm{E}-02$ & $6.44 \mathrm{E}+00$ & $2.24 \mathrm{E}-02$ \\
\hline $216-B-15$ & 3.49E-02 & $5.20 \mathrm{E}+00$ & $1.81 \mathrm{E}-02$ \\
\hline $216-B-16$ & $1.07 \mathrm{E}-01$ & $1.67 \mathrm{E}+01$ & $5.83 \mathrm{E}-02$ \\
\hline 216-B-17 & $1.18 \mathrm{E}-01$ & $5.65 \mathrm{E}+00$ & $1.97 \mathrm{E}-02$ \\
\hline 216-B-18 & $7.85 \mathrm{E}-02$ & $6.44 \mathrm{E}+00$ & $2.24 \mathrm{E}-02$ \\
\hline $216-B-22$ & $1.39 \mathrm{E}-01$ & $1.19 \mathrm{E}+00$ & $1.88 \mathrm{E}-03$ \\
\hline
\end{tabular}


Table 3.7. (contd)

\begin{tabular}{|c|c|c|c|}
\hline & \multicolumn{3}{|c|}{ Radionuclide Inventories in Curies ${ }^{\star}$} \\
\hline Site Name & $\mathrm{U}-238^{\star \star}$ & Tc- $99^{+}$ & $\mathrm{I}-129^{+}$ \\
\hline $216-\bar{B}-23$ & $5.19 \mathrm{E}-02$ & $2.88 \mathrm{E}+00$ & $4.56 \mathrm{E}-03$ \\
\hline $216-B-24$ & $8.19 \mathrm{E}-02$ & $3.33 \bar{E}+00$ & $5.28 \mathrm{E}-03$ \\
\hline $216-B-25$ & 5.09E-02 & $1.47 \mathrm{E}+00$ & $2.33 \mathrm{E}-03$ \\
\hline $216-B-26$ & $1.96 \mathrm{E}-01$ & $2.48 \mathrm{E}+01$ & $3.92 \mathrm{E}-02$ \\
\hline 216-B-27 & $1.14 \mathrm{E}-01$ & $9.04 \mathrm{E}-01$ & $1.43 \mathrm{E}-03$ \\
\hline $216-B-28$ & $9.98 \mathrm{E}-02$ & $6.22 \mathrm{E}-01$ & $9.84 \mathrm{E}-04$ \\
\hline 216-B-29 & $1.15 \mathrm{E}-01$ & $1.53 \mathrm{E}+00$ & $2.42 \mathrm{E}-03$ \\
\hline $216-B-3$ & $2.10 \mathrm{E}+00$ & & \\
\hline $216-B-30$ & $2.93 \mathrm{E}-02$ & $8.87 \mathrm{E}+01$ & $1.40 \mathrm{E}-01$ \\
\hline 216-B-31 & $4.06 \mathrm{E}-02$ & $7.35 \mathrm{E}-01$ & $1.16 \mathrm{E}-03$ \\
\hline $216-\mathrm{B}-32$ & $3.66 \mathrm{E}-03$ & $3.33 \mathrm{E}+00$ & $5.28 \mathrm{E}-03$ \\
\hline $216-B-33$ & $6.66 \mathrm{E}-03$ & $7.18 \mathrm{E}+00$ & $1.14 \mathrm{E}-02$ \\
\hline 216-B-34 & $2.83 \mathrm{E}-02$ & $4.52 \mathrm{E}-01$ & $7.16 \mathrm{E}-04$ \\
\hline $216-\bar{B}-35$ & $5.66 \mathrm{E}-03$ & $1.05 E+01$ & $1.66 \mathrm{E}-02$ \\
\hline $216-B-36$ & $5.32 \mathrm{E}-03$ & $1.90 \mathrm{E}+01$ & $3.01 E-02$ \\
\hline 216-B-37 & $1.33 \mathrm{E}-03$ & $7.63 \mathrm{E}+01$ & $1.21 \mathrm{E}-01$ \\
\hline $216-\bar{B}-38$ & $1.40 \mathrm{E}-02$ & $1.25 \mathrm{E}+01$ & $1.98 \mathrm{E}-02$ \\
\hline 216-B-39 & $2.00 \mathrm{E}-03$ & $1.09 \mathrm{E}+01$ & $1.72 \mathrm{E}-02$ \\
\hline $216-\bar{B}-40$ & $1.16 \mathrm{E}-02$ & $8.65 \mathrm{E}+00$ & $1.37 \mathrm{E}-02$ \\
\hline $216-B-41$ & $2.66 \mathrm{E}-03$ & $2.18 \mathrm{E}+01$ & $3.45 \mathrm{E}-02$ \\
\hline $216-B-42$ & $2.27 \mathrm{E}-01$ & $2.43 \mathrm{E}+00$ & $3.85 \mathrm{E}-03$ \\
\hline $216-B-43$ & $4.66 \mathrm{E}-03$ & $7.35 \mathrm{E}+00$ & $2.56 \mathrm{E}-02$ \\
\hline 216-B-44 & $6.66 \mathrm{E}-04$ & $1.75 \mathrm{E}+01$ & $6.08 \mathrm{E}-02$ \\
\hline 216-B-45 & $2.33 \mathrm{E}-03$ & $3.76 \mathrm{E}+01$ & $1.31 \mathrm{E}-01$ \\
\hline 216-B-46 & $6.36 \mathrm{E}-02$ & $5.03 \mathrm{E}+00$ & $1.75 \mathrm{E}-02$ \\
\hline 216-B-47 & $2.33 \mathrm{E}-03$ & $3.79 \mathrm{E}+00$ & $1.32 \mathrm{E}-02$ \\
\hline 216-B-48 & $6.66 \mathrm{E}-04$ & $1.13 \mathrm{E}+01$ & $3.94 \mathrm{E}-02$ \\
\hline $216-\bar{B}-49$ & $1.06 \mathrm{E}-01$ & $1.03 \mathrm{E}+01$ & $3.58 \mathrm{E}-02$ \\
\hline $216-\mathrm{B}-5$ & $0.00 \mathrm{E}+00$ & & \\
\hline 216-B-50 & $0.00 \mathrm{E}+00$ & & \\
\hline 216-B-52 & $9.98 \mathrm{E}-03$ & $9.04 \mathrm{E}+00$ & $1.43 \mathrm{E}-02$ \\
\hline $216-B-55$ & $2.66 \mathrm{E}-02$ & & \\
\hline 216-B-57 & $3.33 \mathrm{E}-04$ & & \\
\hline $216-B-58$ & $3.00 \mathrm{E}-03$ & & \\
\hline 216-B-59 & $0.00 \mathrm{E}+00$ & & \\
\hline 216-B-60 & $2.39 \mathrm{E}-01$ & & \\
\hline 216-B-62 & $9.98 \mathrm{E}-03$ & & \\
\hline $216-B-63$ & $1.50 \mathrm{E}-01$ & & \\
\hline
\end{tabular}


Table 3.7. (contd)

\begin{tabular}{|c|c|c|c|}
\hline & \multicolumn{3}{|c|}{ Radionuclide Inventories in Curies } \\
\hline Site Name & $\mathrm{U}-238^{* \star}$ & Tc-99 & $1-129^{+}$ \\
\hline $216-B-7$ & $6.06 \mathrm{E}-02$ & $2.43 \mathrm{E}+00$ & $8.47 \mathrm{E}-03$ \\
\hline $216-\bar{B}-8$ & $1.50 \mathrm{E}-02$ & $1.13 E+00$ & $3.94 \mathrm{E}-03$ \\
\hline 216-B-9 & $1.50 \mathrm{E}-02$ & & \\
\hline $216-\mathrm{C}-1$ & $9.82 \mathrm{E}-02$ & & \\
\hline $216-C-10$ & $0.00 \mathrm{E}+00$ & & \\
\hline $216-C-3$ & $1.50 \mathrm{E}-02$ & & \\
\hline $216-C-4$ & $9.98 \mathrm{E}-04$ & & \\
\hline $216-C-5$ & $1.80 \mathrm{E}-02$ & & " \\
\hline $216-C-6$ & $0.00 \mathrm{E}+00$ & & \\
\hline $216-\mathrm{C}-7$ & $0.00 \mathrm{E}+00$ & & \\
\hline $216-C-9$ & $3.33 \mathrm{E}-04$ & & \\
\hline $216-N-2$ & $0.00 \mathrm{E}+00$ & & \\
\hline $216-N-3$ & $0.00 \mathrm{E}+00$ & & \\
\hline $216-\mathrm{N}-4$ & $1.66 \mathrm{E}-03$ & & \\
\hline $216-N-5$ & $0.00 \mathrm{E}+00$ & & \\
\hline $216-N-6$ & $1.66 \mathrm{E}-03$ & & \\
\hline $216-N-7$ & $0.00 \mathrm{E}+00$ & & \\
\hline $216-S-1 \& 2$ & $7.55 \mathrm{E}-01$ & & \\
\hline $216-S-10$ & $6.72 \mathrm{E}-02$ & & \\
\hline $216-S-11$ & $6.99 \mathrm{E}-03$ & & \\
\hline $216-S-12$ & $1.66 \mathrm{E}-03$ & & \\
\hline $216-S-13$ & $3.03 \mathrm{E}-02$ & & \\
\hline $216-S-16$ & $1.05 \mathrm{E}+00$ & & \\
\hline $216-S-17$ & $4.53 \mathrm{E}-02$ & & \\
\hline $216-\mathrm{S}-19$ & $5.19 \mathrm{E}-02$ & & \\
\hline $216-S-20$ & $1.26 \mathrm{E}-02$ & & \\
\hline $216-S-21$ & $1.33 \mathrm{E}-03$ & & \\
\hline $216-S-3$ & $0.00 \mathrm{E}+00$ & & \\
\hline $216-S-5$ & $9.05 \mathrm{E}-02$ & & \\
\hline $216-S-6$ & $9.05 \mathrm{E}-02$ & & \\
\hline $216-S-7$ & $8.62 \mathrm{E}-01$ & & \\
\hline $216-S-8$ & $6.49 \mathrm{E}-02$ & & \\
\hline $216-S-9$ & $1.13 \mathrm{E}-02$ & & \\
\hline $216-\mathrm{T}-1$ & $1.66 \mathrm{E}-03$ & & \\
\hline $216-\mathrm{T}-12$ & $1.50 \overline{\mathrm{E}}-02$ & & \\
\hline $216-T-14$ & $9.98 \mathrm{E}-03$ & $1.15 \mathrm{E}+01$ & $1.83 \mathrm{E}-02$ \\
\hline $216-T-15$ & $8.99 \mathrm{E}-03$ & $2.54 \mathrm{E}+01$ & $4.03 \mathrm{E}-02$ \\
\hline $216-T-16$ & $7.32 \mathrm{E}-03$ & $1.28 \mathrm{E}+01$ & $2.03 \mathrm{E}-02$ \\
\hline
\end{tabular}


Table 3.7. (contd).

\begin{tabular}{|l|r|r|r|}
\hline & \multicolumn{3}{|c|}{ Radionuclide Inventories in Curies } \\
\hline \multicolumn{1}{|c|}{ Site Name } & U-238 & Tc-99 & I-129 \\
\hline $216-\mathrm{T}-17$ & $6.66 \mathrm{E}-03$ & $9.15 \mathrm{E}+00$ & $1.45 \mathrm{E}-02$ \\
\hline $216-\mathrm{T}-18$ & $8.99 \mathrm{E}-03$ & $1.36 \mathrm{E}+00$ & $4.73 \mathrm{E}-03$ \\
\hline $216-\mathrm{T}-19$ & $3.33 \mathrm{E}-03$ & $9.89 \mathrm{E}+00$ & $3.45 \mathrm{E}-02$ \\
\hline $216-\mathrm{T}-20$ & $1.66 \mathrm{E}-03$ & & \\
\hline $216-\mathrm{T}-21$ & $3.33 \mathrm{E}-04$ & $9.83 \mathrm{E}+00$ & $1.56 \mathrm{E}-02$ \\
\hline $216-\mathrm{T}-22$ & $6.66 \mathrm{E}-04$ & $4.54 \mathrm{E}+01$ & $7.19 \mathrm{E}-02$ \\
\hline $216-\mathrm{T}-23$ & $3.33 \mathrm{E}-04$ & $3.26 \mathrm{E}+01$ & $5.16 \mathrm{E}-02$ \\
\hline $216-\mathrm{T}-24$ & $2.66 \mathrm{E}-03$ & $3.49 \mathrm{E}+01$ & $5.52 \mathrm{E}-02$ \\
\hline $216-\mathrm{T}-25$ & $3.33 \mathrm{E}-04$ & $2.18 \mathrm{E}+02$ & $3.45 \mathrm{E}-01$ \\
\hline $216-\mathrm{T}-26$ & $4.99 \mathrm{E}-02$ & $4.29 \mathrm{E}+00$ & $1.50 \mathrm{E}-02$ \\
\hline $216-\mathrm{T}-27$ & $2.33 \mathrm{E}-03$ & & \\
\hline $216-\mathrm{T}-28$ & $1.30 \mathrm{E}-01$ & $1.09 \mathrm{E}+01$ & $3.80 \mathrm{E}-02$ \\
\hline $216-\mathrm{T}-3$ & $0.00 \mathrm{E}+00$ & & \\
\hline $216-\mathrm{T}-30$ & $1.66 \mathrm{E}-03$ & & \\
\hline $216-\mathrm{T}-32$ & $7.56 \mathrm{E}-01$ & $5.65 \mathrm{E}-01$ & $1.97 \mathrm{E}-03$ \\
\hline $216-\mathrm{T}-33$ & $1.66 \mathrm{E}-03$ & & \\
\hline $216-\mathrm{T}-34$ & $1.33 \mathrm{E}-03$ & & \\
\hline $216-\mathrm{T}-35$ & $1.63 \mathrm{E}-02$ & & \\
\hline $216-\mathrm{T}-36$ & $3.33 \mathrm{E}-04$ & & \\
\hline $216-\mathrm{T}-4$ & $2.32 \mathrm{E}-01$ & & \\
\hline $216-\mathrm{T}-5$ & $1.66 \mathrm{E}-03$ & $1.75 \mathrm{E}+00$ & $2.77 \mathrm{E}-03$ \\
\hline $216-\mathrm{T}-6$ & $7.65 \mathrm{E}-03$ & & \\
\hline $216-\mathrm{U}-10$ & $1.88 \mathrm{E}+00$ & & \\
\hline $216-\mathrm{U}-12$ & $6.77 \mathrm{E}-01$ & & \\
\hline $216-\mathrm{U}-13$ & $0.00 \mathrm{E}+00$ & & \\
\hline $216-\mathrm{U}-15$ & $6.66 \mathrm{E}-04$ & & \\
\hline $216-\mathrm{U}-16$ & $5.99 \mathrm{E}-03$ & & \\
\hline $216-\mathrm{U}-17$ & $3.33 \mathrm{E}-04$ & & \\
\hline $216-\mathrm{U}-3$ & $5.99 \mathrm{E}-03$ & & \\
\hline $216-\mathrm{U}-4 \mathrm{~A}$ & $3.00 \mathrm{E}-03$ & & \\
\hline $216-\mathrm{U}-4 \mathrm{~B}$ & $0.00 \mathrm{E}+00$ & & \\
\hline $216-\mathrm{U}-5$ & $1.21 \mathrm{E}-01$ & & \\
\hline $216-\mathrm{U}-6$ & $1.21 \mathrm{E}-01$ & & \\
\hline $216-\mathrm{U}-8$ & $8.00 \mathrm{E}+00$ & & \\
\hline $216-\mathrm{W}-\mathrm{LWC}$ & $6.66 \mathrm{E}-04$ & & \\
\hline $216-Z-1 \& 2$ & $2.70 \mathrm{E}-02$ & & \\
\hline $216-Z-10$ & $0.00 \mathrm{E}+00$ & & \\
\hline $216-Z-12$ & $0.00 \mathrm{E}+00$ & & \\
\hline & & & \\
\hline
\end{tabular}


Table 3.7. (contd)

\begin{tabular}{|l|r|c|c|}
\hline & \multicolumn{3}{|c|}{ Radionuclide Inventories in Curies } \\
\hline \multicolumn{1}{|c|}{ Site Name } & U-238 $^{\text {** }}$ & Tc-99 & I-129 $^{+}$ \\
\hline $216-Z-16$ & $0.00 \mathrm{E}+00$ & & \\
\hline $216-Z-17$ & $0.00 \mathrm{E}+00$ & & \\
\hline $216-Z-18$ & $0.00 \mathrm{E}+00$ & & \\
\hline $216-Z-1 \mathrm{~A}$ & $0.00 \mathrm{E}+00$ & & \\
\hline $216-Z-1 \mathrm{~A} A$ & $0.00 \mathrm{E}+00$ & & \\
\hline $216-Z-1 \mathrm{~A} B$ & $0.00 \mathrm{E}+00$ & & \\
\hline $216-Z-1 \mathrm{~A} C$ & $0.00 \mathrm{E}+00$ & & \\
\hline $216-Z-20$ & $0.00 \mathrm{E}+00$ & & \\
\hline $216-Z-3$ & $0.00 \mathrm{E}+00$ & & \\
\hline $216-Z-4$ & $0.00 \mathrm{E}+00$ & & \\
\hline $216-Z-5$ & $0.00 \mathrm{E}+00$ & & \\
\hline $216-Z-6$ & $0.00 \mathrm{E}+00$ & & \\
\hline $216-Z-7$ & $1.66 \mathrm{E}-03$ & & \\
\hline $216-Z-8$ & $0.00 \mathrm{E}+00$ & & \\
\hline $216-\mathrm{Z}-9$ & $0.00 \mathrm{E}+00$ & & \\
\hline
\end{tabular}

* Inventories decayed to a common date of 2050 .

** Inventory was developed by F. M. Coony. From an electronic mail message with attached files regarding "Questions on Crib Releases in the 200 Areas." Sent by F. M. Coony of Waste Management Federal Services of Hanford to C. T. Kincaid on November 5, 1997.

+ Inventories were developed by F. M. Coony. From an electronic mail message with attached files regarding Tc-99 (and I-129). Sent by F. M. Coony of Waste Management Federal Services of Hanford to C. T. Kincaid on October 29, 1997. 
Table 3.8. Inventories of Key Radionuclides for CERCLA Sites

\begin{tabular}{|c|c|c|c|c|c|c|}
\hline & \multicolumn{6}{|c|}{ Radionuclide Inventories $^{*}$ in Curies ${ }^{\star \star}$} \\
\hline Site I & C-14 & Cl-36 & $\mathrm{I}-129$ & $\mathrm{Se}-79$ & Tc-99 & \\
\hline $207-U$ & $6.38 \mathrm{E}-05$ & $4.2 \mathrm{E}-06$ & $3.42 \mathrm{E}-07$ & 5.11E-06 & $1.73 \mathrm{E}-04$ & $1.51 \mathrm{IE}-02$ \\
\hline $216-A-1$ & $2.91 \mathrm{E}-06$ & $1.91 \mathrm{E}-07$ & $1.56 \mathrm{E}-08$ & 2.33E-07 & $7.89 \mathrm{E}-06$ & $5.12 \mathrm{E}-02$ \\
\hline $216-\bar{A}-10$ & $5.27 \mathrm{E}-03$ & $3.47 \mathrm{E}-04$ & $1.07 \mathrm{E}-01$ & $4.23 \mathrm{E}-04$ & $1.43 \mathrm{E}-02$ & $8.09 \mathrm{E}-02$ \\
\hline $216-A-18$ & $2.91 \mathrm{E}-06$ & 1.91E-07 & $1.56 \mathrm{E}-08$ & $2.33 \mathrm{E}-07$ & $7.89 \mathrm{E}-06$ & $4.69 \overline{\mathrm{E}-01}$ \\
\hline $216-\mathrm{A}-19$ & $2.91 \mathrm{E}-06$ & $1.91 \mathrm{E}-07$ & $1.56 \mathrm{E}-08$ & $2.33 \mathrm{E}-07$ & $7.89 \mathrm{E}-06$ & $1.30 \mathrm{E}+01$ \\
\hline $216-A-2$ & $9.5 \mathrm{E}-05$ & $6.25 \mathrm{E}-06$ & $5.08 \mathrm{E}-07$ & $7.61 \mathrm{E}-06$ & $2.58 \mathrm{E}-04$ & $2.60 \mathrm{E}-02$ \\
\hline $216-A-20$ & $2.91 \mathrm{E}-06$ & $1.91 \mathrm{E}-07$ & $1.56 \mathrm{E}-08$ & $2.33 \mathrm{E}-07$ & $7.89 \mathrm{E}-06$ & $1.35 \mathrm{E}-01$ \\
\hline $216-A-21$ & $5.14 \mathrm{E}-03$ & $3.38 \mathrm{E}-04$ & $2.75 E-05$ & $4.12 \mathrm{E}-04$ & $1.40 \mathrm{E}-02$ & $6.49 \mathrm{E}-02$ \\
\hline $216-A-24$ & $1.76 \mathrm{E}-02$ & $1.15 \mathrm{E}-03$ & $9.4 \mathrm{E}-05$ & $1.41 \mathrm{E}-03$ & $4.76 \mathrm{E}-02$ & $1.66 \mathrm{E}-02$ \\
\hline $216-A-25$ & $1.34 \mathrm{E}-02$ & 8.79E-04 & $7.15 E-05$ & $1.07 \mathrm{E}-03$ & $3.63 \mathrm{E}-02$ & $4.24 \mathrm{E}+0 \mathrm{C}$ \\
\hline 216-A-27 & $2.12 \mathrm{E}-03$ & $1.40 \mathrm{E}-04$ & $1.14 \mathrm{E}-05$ & $1.70 \mathrm{E}-04$ & $5.76 \mathrm{E}-03$ & $2.26 \mathrm{E}-02$ \\
\hline $216-A-28$ & $1.48 \mathrm{E}-02$ & $9.75 \mathrm{E}-04$ & $7.93 \mathrm{E}-05$ & $1.19 \mathrm{E}-03$ & $4.02 \mathrm{E}-02$ & $2.11 \mathrm{E}-01$ \\
\hline $216-A-3$ & $2.98 \mathrm{E}-06$ & $1.96 \mathrm{E}-07$ & $1.6 \mathrm{E}-08$ & $2.39 \mathrm{E}-07$ & $8.09 \mathrm{E}-06$ & $5.59 \mathrm{E}$ \\
\hline $216-A-30$ & $7.66 \mathrm{E}-03$ & $5.04 \mathrm{E}-04$ & $4.1 \mathrm{E}-05$ & $6.14 \mathrm{E}-04$ & $2.08 \mathrm{E}-02$ & $9.98 \mathrm{E}-02$ \\
\hline $216-A-31$ & $5.37 \mathrm{E}-03$ & $3.53 \mathrm{E}-04$ & $2.88 \mathrm{E}-05$ & 4.31E-04 & $1.46 \mathrm{E}-02$ & $6.99 E-03$ \\
\hline $216-A-36 A / B$ & $7.84 \mathrm{E}-02$ & $5.16 \mathrm{E}-03$ & $4.20 \mathrm{E}-04$ & $6.28 \mathrm{E}-03$ & $2.13 \mathrm{E}-01$ & $8.82 \mathrm{E}-02$ \\
\hline $216-A-37-1$ & $6.2 \mathrm{E}-06$ & $4.08 \mathrm{E}-07$ & $4.26 \mathrm{E}-03$ & $4.97 \mathrm{E}-07$ & $1.68 \mathrm{E}-05$ & $1.10 \overline{\mathrm{E}}-02$ \\
\hline $216-A-37-2$ & $1.34 \mathrm{E}-05$ & $8.79 \mathrm{E}-07$ & $7.15 \mathrm{E}-08$ & $1.07 \mathrm{E}-06$ & $3.63 \mathrm{E}-05$ & $1.73 \mathrm{E}-02$ \\
\hline $216-A-4$ & $4.54 \mathrm{E}-04$ & $2.99 \mathrm{E}-05$ & & $3.64 \mathrm{E}-05$ & $1.23 \mathrm{E}-03$ & $1.33 \overline{\mathrm{E}-01}$ \\
\hline $216-A-45$ & $6.35 \mathrm{E}-07$ & $4.18 \mathrm{E}-08$ & $1.10 \mathrm{E}-02$ & $5.09 \mathrm{E}-08$ & $1.72 \mathrm{E}-06$ & $2.33 \mathrm{E}-03$ \\
\hline $216-A-5$ & $7.93 \mathrm{E}-04$ & $5.21 \mathrm{E}-05$ & $4.24 \mathrm{E}-06$ & $6.35 \mathrm{E}-05$ & $2.15 \mathrm{E}-03$ & $8.75 \mathrm{E}-02$ \\
\hline $216-A-6$ & $6.88 \mathrm{E}-03$ & $4.52 \mathrm{E}-04$ & $3.68 \mathrm{E}-05$ & $5.51 \mathrm{E}-04$ & $1.87 \mathrm{E}-02$ & $5.49 \overline{\mathrm{E}-02}$ \\
\hline $216-A-7$ & $1.51 \mathrm{E}-04$ & $9.95 \mathrm{E}-06$ & $8.1 \mathrm{E}-07$ & $1.21 \mathrm{E}-05$ & $4.11 \mathrm{E}-04$ & $2.33 \mathrm{E}-03$ \\
\hline 216-A-8 & $3.42 \mathrm{E}-02$ & $2.25 \mathrm{E}-03$ & $1.83 \mathrm{E}-04$ & $2.74 \mathrm{E}-03$ & $9.28 \mathrm{E}-02$ & $1.23 \mathrm{E}-01$ \\
\hline $216-A-9$ & $3.05 \mathrm{E}-04$ & $2 \mathrm{E}-05$ & $1.63 \mathrm{E}-06$ & $2.44 \mathrm{E}-05$ & $8.26 \mathrm{E}-04$ & $8 \mathrm{E}-05$ \\
\hline 216-B-10A & $2.63 \mathrm{E}-05$ & $1.73 \mathrm{E}-06$ & $1.41 \mathrm{E}-07$ & $2.11 \mathrm{E}-06$ & $7.13 \mathrm{E}-05$ & $3.00 \mathrm{E}-03$ \\
\hline 216-B-10B & $6.55 \mathrm{E}-09$ & $4.31 \mathrm{E}-10$ & $3.51 \mathrm{E}-11$ & $5.25 \mathrm{E}-10$ & $1.78 \mathrm{E}-08$ & $2.23 \mathrm{E}-06$ \\
\hline 216-B-11A\& & $1.40 \mathrm{E}-03$ & $9.18 \mathrm{E}-05$ & $7.47 \mathrm{E}-06$ & $1.12 \mathrm{E}-04$ & $3.79 \mathrm{E}-03$ & $4.66 \mathrm{E}-03$ \\
\hline $216-B-12$ & $4.69 \mathrm{E}-02$ & $3.09 \mathrm{E}-03$ & $2.51 \mathrm{E}-04$ & $3.76 \mathrm{E}-03$ & $1.27 \mathrm{E}-01$ & $6.96 \mathrm{E} \div 00$ \\
\hline 216-B-14 & $7.47 \mathrm{E}-03$ & $4.91 \mathrm{E}-04$ & $2.24 \mathrm{E}-02$ & $5.99 \mathrm{E}-04$ & $6.44 \mathrm{E}+00$ & $7.25 \mathrm{E}-02$ \\
\hline 216-B-15 & $6.05 \mathrm{E}-03$ & $3.98 \mathrm{E}-04$ & $1.81 \mathrm{E}-02$ & $4.85 \mathrm{E}-04$ & $5.20 \mathrm{E}+00$ & $3.49 \mathrm{E}-02$ \\
\hline $216-B-16$ & $1.94 \mathrm{E}-02$ & $1.28 \mathrm{E}-03$ & $5.83 \mathrm{E}-02$ & $1.55 \mathrm{E}-03$ & $1.67 \mathrm{E}+0 \mathrm{I}$ & $1.07 \mathrm{E}-01$ \\
\hline 216-B-17 & $6.55 \mathrm{E}-03$ & $4.31 \mathrm{E}-04$ & $1.97 \mathrm{E}-02$ & $5.25 \mathrm{E}-04$ & $5.65 \mathrm{E}+00$ & $1.18 \mathrm{E}-01$ \\
\hline 216-B-18 & $7.47 \mathrm{E}-03$ & $4.91 \mathrm{E}-04$ & $2.24 \mathrm{E}-02$ & $5.99 \mathrm{E}-04$ & $6.44 \mathrm{E}+00$ & $7.85 \mathrm{E}-02$ \\
\hline 216-B-19 & $8.25 \mathrm{E}-03$ & $5.43 \mathrm{E}-04$ & $2.48 \mathrm{E}-02$ & $6.62 \mathrm{E}-04$ & $7.12 \mathrm{E}+00$ & $6.06 \mathrm{E}-02$ \\
\hline $216-B-20$ & $4.48 \mathrm{E}-02$ & $2.95 \mathrm{E}-03$ & $6.12 \mathrm{E}-02$ & $3.59 \mathrm{E}-03$ & $3.86 \mathrm{E}+01$ & $1.17 \mathrm{E}-01$ \\
\hline 216-B-21 & $1.11 \mathrm{E}-02$ & $7.28 \mathrm{E}-04$ & $1.51 \mathrm{E}-02$ & $8.87 \mathrm{E}-04$ & $9.55 \mathrm{E}+00$ & $2.25 \mathrm{E}-01$ \\
\hline
\end{tabular}


Table 3.8. (contd)

\begin{tabular}{|c|c|c|c|c|c|c|}
\hline & \multicolumn{6}{|c|}{ Radionuclide Inventories ${ }^{*}$ in Curies ${ }^{\star \star}$} \\
\hline Site Name & C-14 & $\mathrm{Cl}-36$ & I-129 & Se-79 & Tc-99 & U-238 \\
\hline $216-\mathrm{B}-2-1$ & $6.13 \mathrm{E}-03$ & $4.03 \mathrm{E}-04$ & $3.28 \mathrm{E}-05$ & $4.91 \mathrm{E}-04$ & $1.66 \mathrm{E}-02$ & $1.45 \mathrm{E}+00$ \\
\hline $216-\mathrm{B}-2-2$ & $2.06 \mathrm{E}-05$ & $1.35 \mathrm{E}-06$ & $1.1 \mathrm{E}-07$ & $1.65 \mathrm{E}-06$ & $5.58 \mathrm{E}-05$ & $4.88 \mathrm{E}-03$ \\
\hline 216-B-23 & $3.33 \mathrm{E}-03$ & $2.19 \mathrm{E}-04$ & $4.56 \mathrm{E}-03$ & $2.67 \mathrm{E}-04$ & 2.881494 & $5.19 \mathrm{E}-02$ \\
\hline $216-\mathrm{B}-2-3$ & $2.06 \mathrm{E}-05$ & $1.35 \mathrm{E}-06$ & $1.1 \mathrm{E}-07$ & $1.65 \mathrm{E}-06$ & $5.58 \mathrm{E}-05$ & $4.88 \mathrm{E}-03$ \\
\hline $216-B-24$ & $3.84 \mathrm{E}-03$ & $2.53 \mathrm{E}-04$ & $5.28 \mathrm{E}-03$ & $3.08 \mathrm{E}-04$ & $3.33 E+00$ & $8.19 \mathrm{E}-02$ \\
\hline $216-\mathrm{B}-25$ & $1.67 \mathrm{E}-03$ & $1.10 \mathrm{E}-04$ & $2.33 \bar{E}-03$ & $1.34 \mathrm{E}-04$ & $1.47 \mathrm{E}+00$ & $5.09 \mathrm{E}-02$ \\
\hline $216-B-26$ & $2.87 \mathrm{E}-02$ & $1.89 \mathrm{E}-03$ & $3.92 \mathrm{E}-02$ & $2.30 \mathrm{E}-03$ & $2.47 \mathrm{E}+01$ & 1.96E-01 \\
\hline 216-B-27 & $1.04 \mathrm{E}-03$ & $6.81 \mathrm{E}-05$ & $1.43 \mathrm{E}-03$ & $8.3 \mathrm{E}-05$ & $9.04 \mathrm{E}-01$ & $1: 14 \mathrm{E}-01$ \\
\hline $216-B-28$ & $7.01 \mathrm{E}-04$ & $4.61 \mathrm{E}-05$ & $9.84 \mathrm{E}-04$ & $5.62 \mathrm{E}-05$ & $6.21 \mathrm{E}-01$ & $9.98 \mathrm{E}-02$ \\
\hline 216-B-29 & $1.80 \mathrm{E}-03$ & $1.18 \mathrm{E}-04$ & $2.42 \mathrm{E}-03$ & $1.44 \mathrm{E}-04$ & $1.53 \mathrm{E}+00$ & $1.15 \mathrm{E}-01$ \\
\hline $216-B-3$ & $6.13 \mathrm{E}-03$ & $4.03 \mathrm{E}-04$ & $3.28 \mathrm{E}-05$ & $4.91 \mathrm{E}-04$ & $1.66 \mathrm{E}-02$ & $2.10 \mathrm{E}+00$ \\
\hline $216-\bar{B}-30$ & $1.03 \mathrm{E}-01$ & 6.77E-03 & $1.40 \mathrm{E}-01$ & $8.24 \mathrm{E}-03$ & $8.87 \mathrm{E}+01$ & $2.93 \mathrm{E}-02$ \\
\hline $216-B-31$ & $7.80 \mathrm{E}-02$ & $5.13 \mathrm{E}-03$ & $1.16 \mathrm{E}-03$ & $6.25 \mathrm{E}-03$ & $7.34 \mathrm{E}-01$ & $4.06 \mathrm{E}-02$ \\
\hline $216-B-32$ & $3.84 \mathrm{E}-03$ & $2.53 \mathrm{E}-04$ & $5.28 \mathrm{E}-03$ & $3.08 \mathrm{E}-04$ & $3.33 \mathrm{E}+00$ & $3.66 \mathrm{E}-03$ \\
\hline 216-B-33 & $8.32 \mathrm{E}-03$ & $5.47 \mathrm{E}-04$ & $1.14 \mathrm{E}-02$ & $6.67 \mathrm{E}-04$ & $7.18 \mathrm{E}+00$ & $6.66 \mathrm{E}-03$ \\
\hline $216-B-34$ & $5.18 \mathrm{E}-04$ & $3.41 \mathrm{E}-05$ & $7.16 \mathrm{E}-04$ & $4.15 \mathrm{E}-05$ & $4.52 \overline{\mathrm{E}}-01$ & $2.83 \mathrm{E}-02$ \\
\hline $216-B-35$ & $1.21 \mathrm{E}-02$ & $7.97 \mathrm{E}-04$ & $1.66 \mathrm{E}-02$ & $9.71 \mathrm{E}-04$ & $1.05 \mathrm{E}+01$ & $5.66 \mathrm{E}-03$ \\
\hline $216-B-36$ & $2.20 \mathrm{E}-02$ & $1.45 \mathrm{E}-03$ & $3.01 \mathrm{E}-02$ & $1.76 \mathrm{E}-03$ & $1.90 \mathrm{E}+01$ & $5.32 \mathrm{E}-03$ \\
\hline $216-B-37$ & $8.84 \mathrm{E}-02$ & $5.82 \mathrm{E}-03$ & $1.21 \mathrm{E}-01$ & $7.09 \mathrm{E}-03$ & $7.63 \mathrm{E}+01$ & $1.33 \mathrm{E}-03$ \\
\hline 216-B-38 & $1.45 \mathrm{E}-02$ & $9.52 \mathrm{E}-04$ & $1.98 \mathrm{E}-02$ & $1.16 \mathrm{E}-03$ & $1.25 \mathrm{E}+01$ & $1.40 \mathrm{E}-02$ \\
\hline $216-\bar{B}-39$ & $1.26 \mathrm{E}-02$ & $8.27 \mathrm{E}-04$ & $1.72 \mathrm{E}-02$ & $1.01 \mathrm{E}-03$ & $1.08 \mathrm{E}+01$ & $2.00 \mathrm{E}-03$ \\
\hline 216-B-40 & $1.00 \mathrm{E}-02$ & $6.59 \mathrm{E}-04$ & $1.37 \mathrm{E}-02$ & $8.03 \mathrm{E}-04$ & $8.64 \mathrm{E}+00$ & $1.16 \mathrm{E}-02$ \\
\hline $216-B-41$ & $2.53 \mathrm{E}-02$ & $1.66 \mathrm{E}-03$ & $3.45 \mathrm{E}-02$ & $2.03 \mathrm{E}-03$ & $2.18 \mathrm{E}+01$ & $2.66 \mathrm{E}-03$ \\
\hline $216-B-42$ & $2.80 \mathrm{E}-03$ & $1.84 \mathrm{E}-04$ & $3.85 \mathrm{E}-03$ & $2.24 \mathrm{E}-04$ & $2.43 \mathrm{E}+00$ & $2.27 \mathrm{E}-01$ \\
\hline $216-\mathrm{B}-43$ & $8.52 \mathrm{E}-03$ & $5.60 \mathrm{E}-04$ & $2.56 \mathrm{E}-02$ & $6.83 \mathrm{E}-04$ & $7.34 \mathrm{E}+00$ & $4.66 \mathrm{E}-03$ \\
\hline 216-B-44 & $2.02 \mathrm{E}-02$ & $1.33 \mathrm{E}-03$ & $6.08 \mathrm{E}-02$ & $1.62 \mathrm{E}-03$ & $1.75 \mathrm{E}+01$ & $6.66 \mathrm{E}-04$ \\
\hline $216-B-45$ & $4.36 \mathrm{E}-02$ & $2.87 \mathrm{E}-03$ & $1.31 \mathrm{E}-01$ & $3.50 \mathrm{E}-03$ & $3.76 \mathrm{E}+01$ & $2.33 \mathrm{E}-03$ \\
\hline 216-B-46 & $5.82 \mathrm{E}-03$ & $3.83 \mathrm{E}-04$ & $1.75 \mathrm{E}-02$ & $4.67 \mathrm{E}-04$ & $5.03 E+00$ & $6.36 \mathrm{E}-02$ \\
\hline $216-\mathrm{B}-47$ & $4.36 \mathrm{E}-03$ & $2.87 \mathrm{E}-04$ & $1.32 \mathrm{E}-02$ & $3.50 \mathrm{E}-04$ & $3.79 \mathrm{E}+00$ & $2.33 \mathrm{E}-03$ \\
\hline 216-B-48 & $1.31 \mathrm{E}-02$ & $8.62 \mathrm{E}-04$ & $3.94 \mathrm{E}-02$ & $1.05 \mathrm{E}-03$ & $1.13 \mathrm{E}+01$ & $6.66 \mathrm{E}-04$ \\
\hline 216-B-49 & $1.19 \mathrm{E}-02$ & $7.84 \mathrm{E}-04$ & $3.58 \mathrm{E}-02$ & $9.56 \mathrm{E}-04$ & $1.03 \mathrm{E}+01$ & $1.06 \mathrm{E}-01$ \\
\hline $216-B-5$ & $1.91 \mathrm{E}-03$ & $1.26 \mathrm{E}-04$ & $1.02 \mathrm{E}-05$ & $1.53 \mathrm{E}-04$ & $5.19 \mathrm{E}-03$ & $9.52 \mathrm{E}-03$ \\
\hline $216-B-50$ & $3.35 \mathrm{E}-03$ & $2.21 \mathrm{E}-04$ & $1.8 \mathrm{E}-05$ & $2.69 \mathrm{E}-04$ & $9.10 \mathrm{E}-03$ & 1E-04 \\
\hline $216-\mathrm{B}-52$ & $1.05 \mathrm{E}-02$ & $6.89 \mathrm{E}-04$ & $1.43 \mathrm{E}-02$ & $8.40 \mathrm{E}-04$ & $9.04 \mathrm{E}+00$ & $9.98 \mathrm{E}-03$ \\
\hline $216-B-53 A$ & $3.66 \mathrm{E}-06$ & $2.41 \mathrm{E}-07$ & $1.96 \mathrm{E}-08$ & $2.94 \mathrm{E}-07$ & $9.93 \mathrm{E}-06$ & $7.65 \mathrm{E}-03$ \\
\hline $216-\bar{B}-53 B$ & $2.42 \mathrm{E}-04$ & $1.59 \mathrm{E}-05$ & $1.3 \mathrm{E}-06$ & $1.94 \mathrm{E}-05$ & $6.58 \mathrm{E}-04$ & $3.00 \mathrm{E}-03$ \\
\hline $216-B-54$ & $3.58 \mathrm{E}-06$ & $2.36 \mathrm{E}-07$ & $1.92 \mathrm{E}-08$ & $2.87 \mathrm{E}-07$ & $9.72 \mathrm{E}-06$ & $3.00 \mathrm{E}-03$ \\
\hline 216-B-55 & $8.98 \mathrm{E}-04$ & $5.9 \mathrm{E}-05$ & $4.8 \mathrm{E}-06$ & $7.19 \mathrm{E}-05$ & $2.43 \mathrm{E}-03$ & $2.66 \mathrm{E}-02$ \\
\hline
\end{tabular}


Table 3.8. (contd)

\begin{tabular}{|c|c|c|c|c|c|c|}
\hline & \multicolumn{6}{|c|}{ Radionuclide Inventories in Curies ${ }^{\star \star}$} \\
\hline Site Name & C-14 & $\mathrm{Cl}-36$ & I-129 & Se-79 & Tc-99 & U-238 \\
\hline $216-B-57$ & $1.48 \mathrm{E}-02$ & $9.74 \mathrm{E}-04$ & $7.92 \mathrm{E}-05$ & 1.19E-03 & $4.02 \mathrm{E}-02$ & 3.33E-04 \\
\hline $216-\mathrm{B}-58$ & $2.88 \mathrm{E}-04$ & $1.9 \mathrm{E}-05$ & $1.54 \mathrm{E}-06$ & $2.31 \mathrm{E}-05$ & $7.82 \mathrm{E}-04$ & $3.00 \mathrm{E}-03$ \\
\hline $216-\mathrm{B}-59$ & $7.86 \mathrm{E}-07$ & $5.17 \mathrm{E}-08$ & 4.21E-09 & $6.3 \mathrm{E}-08$ & $2.13 \mathrm{E}-06$ & $1.86 \mathrm{E}-04$ \\
\hline $216-\bar{B}-60$ & $1.70 \mathrm{E}-02$ & $1.12 \mathrm{E}-03$ & $9.1 \mathrm{E}-05$ & $1.36 \mathrm{E}-03$ & 4.61E-02 & $2.39 \mathrm{E}-01$ \\
\hline 216-B-62 & $8.84 \mathrm{E}-03$ & $5.82 \mathrm{E}-04$ & $4.73 \mathrm{E}-05$ & $7.09 \mathrm{E}-04$ & $2.40 \mathrm{E}-02$ & $9.98 \mathrm{E}-03$ \\
\hline $216-B-63$ & $4.09 \mathrm{E}-05$ & $2.69 \mathrm{E}-06$ & $2.19 \mathrm{E}-07$ & $3.28 \mathrm{E}-06$ & $1.11 \mathrm{E}-04$ & $1.50 \mathrm{E}-01$ \\
\hline 216-B-7A\&B & $2.83 \mathrm{E}-03$ & $1.86 \mathrm{E}-04$ & 8.47E-03 & $2.27 \mathrm{E}-04$ & $2.43 \mathrm{E}+00$ & $6.06 \mathrm{E}-02$ \\
\hline $216-B-8$ & $1.30 \mathrm{E}-03$ & $8.53 \mathrm{E}-05$ & $3.94 \mathrm{E}-03$ & $1.04 \mathrm{E}-04$ & $1.13 \mathrm{E}+00$ & $1.50 \mathrm{E}-02$ \\
\hline $216-B-9$ & $2.57 \mathrm{E}-04$ & $1.69 \mathrm{E}-05$ & $1.37 \mathrm{E}-06$ & $2.06 \mathrm{E}-05$ & $6.97 \mathrm{E}-04$ & $1.50 \mathrm{E}-02$ \\
\hline $216-C-1$ & $2.98 \overline{\mathrm{E}}-06$ & $1.96 \mathrm{E}-07$ & $1.6 \mathrm{E}-08$ & 2.39E-07 & $8.09 \mathrm{E}-06$ & $9.82 \mathrm{E}-02$ \\
\hline $216-C-10$ & $5.6 \mathrm{E}-06$ & $3.68 \mathrm{E}-07$ & $3 \mathrm{E}-08$ & $4.49 \mathrm{E}-07$ & $1.52 \mathrm{E}-05$ & $1.68 \mathrm{E}-05$ \\
\hline $216-C-3$ & $2.78 \mathrm{E}-06$ & $1.83 \mathrm{E}-07$ & $1.49 \mathrm{E}-08$ & $2.23 \mathrm{E}-07$ & $7.53 \mathrm{E}-06$ & $1.50 \mathrm{E}-02$ \\
\hline $216-C-4$ & $2.84 \mathrm{E}-06$ & $1.87 \mathrm{E}-07$ & $1.52 \mathrm{E}-08$ & $2.27 \mathrm{E}-07$ & $7.69 \mathrm{E}-06$ & $9.98 \mathrm{E}-04$ \\
\hline $216-C-5$ & $2.91 \mathrm{E}-06$ & $1.91 \mathrm{E}-07$ & $1.56 \mathrm{E}-08$ & $2.33 \mathrm{E}-07$ & $7.89 \mathrm{E}-06$ & $1.80 \mathrm{E}-02$ \\
\hline $216-C-6$ & $3.05 \mathrm{E}-06$ & $2 \mathrm{E}-07$ & $1.63 \mathrm{E}-08$ & $2.44 \mathrm{E}-07$ & $8.26 \mathrm{E}-06$ & $1 \mathrm{E}-$ \\
\hline $216-C-7$ & $3.5 \mathrm{E}-06$ & $2.3 \mathrm{E}-07$ & $1.87 \mathrm{E}-08$ & $2.8 \mathrm{E}-07$ & $9.49 \mathrm{E}-06$ & $3.36 \mathrm{E}-06$ \\
\hline $216-C-9$ & $4.61 \mathrm{E}-05$ & $3.03 \mathrm{E}-06$ & $2.46 \mathrm{E}-07$ & $3.69 \mathrm{E}-06$ & $1.25 \mathrm{E}-04$ & $3.33 \mathrm{E}-04$ \\
\hline $216-\mathrm{N}-2$ & $5.14 \mathrm{E}-06$ & $3.38 \mathrm{E}-07$ & $2.75 \mathrm{E}-08$ & $4.12 \mathrm{E}-07$ & $1.4 \mathrm{E}-05$ & $1.22 \mathrm{E}-03$ \\
\hline $216-N-3$ & $5.77 \mathrm{E}-06$ & $3.8 \mathrm{E}-07$ & $3.09 \mathrm{E}-08$ & $4.63 \mathrm{E}-07$ & $1.57 \mathrm{E}-05$ & $1.37 \mathrm{E}-03$ \\
\hline $216-N-4$ & 5.33E-06 & $3.5 \mathrm{E}-07$ & $2.85 \mathrm{E}-08$ & $4.27 \mathrm{E}-07$ & $1.44 \mathrm{E}-05$ & $1.66 \mathrm{E}-03$ \\
\hline $216-N-5$ & $5.77 \mathrm{E}-06$ & $3.8 \mathrm{E}-07$ & $3.09 \mathrm{E}-08$ & $4.63 \mathrm{E}-07$ & $1.57 \mathrm{E}-05$ & $1.37 \mathrm{E}-03$ \\
\hline $216-\mathrm{N}-6$ & $5.33 \mathrm{E}-06$ & $3.5 \mathrm{E}-07$ & $2.85 \mathrm{E}-08$ & $4.27 \mathrm{E}-07$ & $1.44 \mathrm{E}-05$ & $1.66 \mathrm{E}-1$ \\
\hline $216-N-7$ & $5.77 \mathrm{E}-06$ & $3.8 \mathrm{E}-07$ & $3.09 \mathrm{E}-08$ & $4.63 \mathrm{E}-07$ & $1.57 \mathrm{E}-05$ & $1.37 \mathrm{E}-03$ \\
\hline $216-S-1 \& 2$ & $7.21 \mathrm{E}-02$ & $4.74 \mathrm{E}-03$ & $3.86 \mathrm{E}-04$ & $5.78 \mathrm{E}-03$ & $1.95 \mathrm{E}-01$ & $7.55 \mathrm{E}-01$ \\
\hline 216-S-10D & $8.12 \mathrm{E}-05$ & $5.34 \mathrm{E}-06$ & $4.35 \mathrm{E}-07$ & $6.51 \mathrm{E}-06$ & $2.20 \mathrm{E}-04$ & $6.72 \mathrm{E}-0$ \\
\hline $216-S-11$ & $5.37 \mathrm{E}-05$ & $3.53 \mathrm{E}-06$ & $2.88 \mathrm{E}-07$ & $4.31 \mathrm{E}-06$ & $1.46 \mathrm{E}-04$ & $6.99 \mathrm{E}-03$ \\
\hline $216-S-12$ & $2.84 \mathrm{E}-05$ & $1.87 \mathrm{E}-06$ & $1.52 \mathrm{E}-07$ & $2.28 \mathrm{E}-06$ & $7.71 \mathrm{E}-05$ & $1.66 \mathrm{E}-03$ \\
\hline 216-S-13 & $1.81 \mathrm{E}-04$ & 1.19E-05 & $9.71 \mathrm{E}-07$ & $1.45 \mathrm{E}-05$ & $4.92 \mathrm{E}-04$ & $3.03 \mathrm{E}-02$ \\
\hline $216-S-16 P$ & $1.97 \mathrm{E}-03$ & $1.29 \mathrm{E}-04$ & $1.05 \mathrm{E}-05$ & $1.58 \mathrm{E}-04$ & $5.33 \mathrm{E}-03$ & $1.05 E+00$ \\
\hline $216-S-17$ & $8.32 \mathrm{E}-04$ & $5.47 \mathrm{E}-05$ & $4.45 \mathrm{E}-06$ & $6.67 \mathrm{E}-05$ & $2.26 \mathrm{E}-03$ & $4.53 \mathrm{E}-02$ \\
\hline $216-S-19$ & $8.45 \mathrm{E}-05$ & $5.56 \mathrm{E}-06$ & $4.52 \mathrm{E}-07$ & $6.77 \mathrm{E}-06$ & $2.29 \mathrm{E}-04$ & 5.19E-02 \\
\hline $216-S-20$ & $3.70 \mathrm{E}-03$ & $2.43 \mathrm{E}-04$ & $1.98 \mathrm{E}-05$ & $2.97 \mathrm{E}-04$ & $1.00 \mathrm{E}-02$ & $1.26 \mathrm{E}-02$ \\
\hline $216-S-21$ & $5.77 \mathrm{E}-03$ & $3.79 \mathrm{E}-04$ & $3.09 \mathrm{E}-05$ & $4.62 \mathrm{E}-04$ & $1.56 \mathrm{E}-02$ & $1.33 \mathrm{E}-03$ \\
\hline $216-S-22$ & $3.13 \mathrm{E}-05$ & $2.06 \mathrm{E}-06$ & $1.68 \mathrm{E}-07$ & $2.51 \mathrm{E}-06$ & $8.49 \mathrm{E}-05$ & $1.68 \mathrm{E}-05$ \\
\hline $216-S-23$ & $2.27 \mathrm{E}-04$ & $1.5 \mathrm{E}-05$ & $1.22 \mathrm{E}-06$ & $1.82 \mathrm{E}-05$ & $6.17 \mathrm{E}-04$ & 9.75E-05 \\
\hline $216-S-25$ & $4.24 \mathrm{E}-06$ & $2.79 \mathrm{E}-07$ & $2.27 \mathrm{E}-08$ & $3.4 \mathrm{E}-07$ & $1.15 \mathrm{E}-05$ & $5.56 \mathrm{E}-02$ \\
\hline $216-S-26$ & $2.02 \mathrm{E}-07$ & $1.33 \mathrm{E}-08$ & $1.08 \mathrm{E}-09$ & $1.62 \mathrm{E}-08$ & $5.49 \mathrm{E}-07$ & $6.89 \mathrm{E}-05$ \\
\hline $216-S-3$ & $1.43 \mathrm{E}-03$ & $9.44 \mathrm{E}-05$ & $7.68 \mathrm{E}-06$ & $1.15 \mathrm{E}-04$ & $3.89 \mathrm{E}-03$ & $9.75 \mathrm{E}-05$ \\
\hline
\end{tabular}


Table 3.8. (contd)

\begin{tabular}{|c|c|c|c|c|c|c|}
\hline & \multicolumn{6}{|c|}{ Radionuclide Inventories ${ }^{*}$ in Curies ${ }^{\star \star}$} \\
\hline Site Name & C-14 & Cl-36 & I-129 & Se-79 & Tc-99 & $\mathrm{U}-238$ \\
\hline $216-\mathrm{S}-5$ & $1.73 \mathrm{E}-03$ & $1.14 \mathrm{E}-04$ & $9.26 \mathrm{E}-06$ & $1.39 \mathrm{E}-04$ & $4.69 \mathrm{E}-03$ & $9.05 \mathrm{E}-02$ \\
\hline $216-\mathrm{S}-6$ & $7.53 \mathrm{E}-03$ & $4.96 \mathrm{E}-04$ & $4.03 \mathrm{E}-05$ & $6.04 \mathrm{E}-04$ & $2.04 \mathrm{E}-02$ & $9.05 \mathrm{E}-02$ \\
\hline $216-\mathrm{S}-7$ & $4.61 \mathrm{E}-02$ & $3.03 \mathrm{E}-03$ & $2.46 \mathrm{E}-04$ & $3.69 \mathrm{E}-03$ & $1.25 \mathrm{E}-01$ & $8.62 \mathrm{E}-01$ \\
\hline $216-S-8$ & $3.22 \mathrm{E}-04$ & $2.12 \mathrm{E}-05$ & $1.73 \mathrm{E}-06$ & $2.58 \mathrm{E}-05$ & $8.74 \mathrm{E}-04$ & $6.49 \mathrm{E}-02$ \\
\hline $216-S-9$ & $1.90 \mathrm{E}-02$ & $1.25 \mathrm{E}-03$ & $1.02 \mathrm{E}-04$ & $1.52 \mathrm{E}-03$ & $5.15 \mathrm{E}-02$ & $1.13 \mathrm{E}-02$ \\
\hline $216-\mathrm{T}-1$ & $2.54 \mathrm{E}-06$ & $1.67 \mathrm{E}-07$ & $1.36 \mathrm{E}-08$ & $2.03 \mathrm{E}-07$ & $6.88 \mathrm{E}-06$ & $1.66 \mathrm{E}-03$ \\
\hline $216-\mathrm{T}-12$ & $2.84 \mathrm{E}-04$ & $1.87 \mathrm{E}-05$ & $1.52 \mathrm{E}-06$ & $2.28 \mathrm{E}-05$ & $7.71 \mathrm{E}-04$ & $1.50 \mathrm{E}-02$ \\
\hline $216-T-14$ & $1.34 \mathrm{E}-02$ & $8.79 \mathrm{E}-04$ & $1.83 \mathrm{E}-02$ & $1.07 \mathrm{E}-03$ & $1.15 \mathrm{E}+01$ & $9.98 \mathrm{E}-03$ \\
\hline $216-T-15$ & $2.95 \mathrm{E}-02$ & $1.94 \mathrm{E}-03$ & $4.03 \mathrm{E}-02$ & $2.36 \mathrm{E}-03$ & $2.54 \mathrm{E}+01$ & $8.99 \mathrm{E}-03$ \\
\hline $216-T-16$ & $1.49 \mathrm{E}-02$ & $9.78 \mathrm{E}-04$ & $2.03 \mathrm{E}-02$ & $1.19 \mathrm{E}-03$ & $1.28 \mathrm{E}+01$ & $7.32 \mathrm{E}-03$ \\
\hline $216-\mathrm{T}-17$ & $1.06 \mathrm{E}-02$ & $6.98 \mathrm{E}-04$ & $1.45 \mathrm{E}-02$ & $8.51 \mathrm{E}-04$ & $9.15 \mathrm{E}+00$ & $6.66 \mathrm{E}-03$ \\
\hline $216-T-18$ & $1.59 \mathrm{E}-03$ & $1.04 \mathrm{E}-04$ & $4.73 \mathrm{E}-03$ & $1.27 \mathrm{E}-04$ & $1.36 \mathrm{E}+00$ & $8.99 \mathrm{E}-03$ \\
\hline 216-T-19 & $1.15 \mathrm{E}-03$ & $7.54 \mathrm{E}-05$ & $3.45 \mathrm{E}-02$ & $9.19 \mathrm{E}-05$ & $9.89 \mathrm{E}+00$ & $3.33 \mathrm{E}-03$ \\
\hline $216-T-20$ & $2.88 \mathrm{E}-05$ & 1.9E-06 & $1.54 \mathrm{E}-07$ & $2.31 \mathrm{E}-06$ & $7.82 \mathrm{E}-05$ & $1.66 \mathrm{E}-03$ \\
\hline $216-T-21$ & $1.14 \mathrm{E}-02$ & $7.50 \mathrm{E}-04$ & 0.01557 & $9.14 \mathrm{E}-04$ & $9.83 \mathrm{E}+00$ & $3.33 \mathrm{E}-04$ \\
\hline $216-\mathrm{T}-22$ & $5.26 \mathrm{E}-02$ & $3.46 \mathrm{E}-03$ & $7.19 \mathrm{E}-02$ & $4.22 \mathrm{E}-03$ & $4.54 \mathrm{E}+01$ & \begin{tabular}{|c|}
$66 \mathrm{E}-04$ \\
\end{tabular} \\
\hline $216-T-23$ & $3.78 \mathrm{E}-02$ & $2.49 \mathrm{E}-03$ & $5.16 \mathrm{E}-02$ & $3.03 \mathrm{E}-03$ & $3.26 \mathrm{E}+01$ & $3.33 \mathrm{E}-04$ \\
\hline 216-T-24 & $4.04 \mathrm{E}-02$ & $2.66 \mathrm{E}-03$ & $5.52 \mathrm{E}-02$ & $3.24 \mathrm{E}-03$ & $3.49 \mathrm{E}+01$ & $2.66 \mathrm{E}-03$ \\
\hline $216-\mathrm{T}-25$ & $2.53 \mathrm{E}-01$ & $1.66 \mathrm{E}-02$ & $3.45 \mathrm{E}-01$ & $2.03 \mathrm{E}-02$ & $2.18 \mathrm{E}+02$ & $3.33 \mathrm{E}-04$ \\
\hline $216-\mathrm{T}-26$ & $4.95 \mathrm{E}-03$ & $3.26 \mathrm{E}-04$ & $1.50 \mathrm{E}-02$ & $3.97 \mathrm{E}-04$ & $4.29 \mathrm{E}+00$ & \begin{tabular}{|l}
$4.99 \mathrm{E}-02$ \\
\end{tabular} \\
\hline $216-T-27$ & $3.66 \mathrm{E}-03$ & $2.41 \mathrm{E}-04$ & $1.96 \mathrm{E}-05$ & $2.94 \mathrm{E}-04$ & $9.93 \mathrm{E}-03$ & $2.33 \mathrm{E}-03$ \\
\hline $216-T-28$ & $1.26 \mathrm{E}-02$ & $8.32 \mathrm{E}-04$ & $3.80 \mathrm{E}-02$ & $1.01 \mathrm{E}-03$ & $1.09 \mathrm{E}+01$ & $1.30 \mathrm{E}-01$ \\
\hline $216-T-3$ & $1.40 \mathrm{E}-03$ & $9.18 \mathrm{E}-05$ & $7.47 \mathrm{E}-06$ & $1.12 \mathrm{E}-04$ & $3.79 \mathrm{E}-03$ & $6.95 \overline{\mathrm{E}-03}$ \\
\hline $216-T-32$ & $6.36 \mathrm{E}-04$ & $4.18 \mathrm{E}-05$ & $1.97 \mathrm{E}-03$ & 5.1E-05 & $5.65 \mathrm{E}-01$ & $7.56 \mathrm{E}-01$ \\
\hline $216-T-33$ & $1.75 \mathrm{E}-05$ & $1.15 \mathrm{E}-06$ & $9.36 \mathrm{E}-08$ & $1.4 \mathrm{E}-06$ & $4.74 \mathrm{E}-05$ & $1.66 \mathrm{E}-03$ \\
\hline $216-T-34$ & $1.03 \mathrm{E}-02$ & $6.77 \mathrm{E}-04$ & $5.5 \mathrm{E}-05$ & $8.24 \mathrm{E}-04$ & $2.79 \mathrm{E}-02$ & $1.33 \mathrm{E}-03$ \\
\hline $216-T-35$ & $7.66 \mathrm{E}-04$ & $5.04 \mathrm{E}-05$ & $4.1 \mathrm{E}-06$ & $6.14 \mathrm{E}-05$ & $2.08 \mathrm{E}-03$ & $1.63 \mathrm{E}-02$ \\
\hline $216-T-36$ & $2.48 \mathrm{E}-04$ & $1.63 \mathrm{E}-05$ & $1.33 \mathrm{E}-06$ & $1.99 \mathrm{E}-05$ & $6.74 \mathrm{E}-04$ & $3.33 \mathrm{E}-04$ \\
\hline 216-T-4B & $4.08 \mathrm{E}-04$ & $2.68 \mathrm{E}-05$ & $2.18 \mathrm{E}-06$ & $3.27 \mathrm{E}-05$ & $1.11 \mathrm{E}-03$ & $2.32 \mathrm{E}-01$ \\
\hline $216-T-5$ & $2.04 \mathrm{E}-03$ & $1.34 \mathrm{E}-04$ & $2.77 \mathrm{E}-03$ & $1.63 \mathrm{E}-04$ & $1.75 \mathrm{E}+00$ & $1.66 \mathrm{E}-03$ \\
\hline $216-\mathrm{T}-6$ & $7.21 \mathrm{E}-03$ & $4.74 \mathrm{E}-04$ & $3.86 \mathrm{E}-05$ & $5.78 \mathrm{E}-04$ & $1.95 \mathrm{E}-02$ & $7.65 \mathrm{E}-03$ \\
\hline $216-\mathrm{T}-7$ & $1.39 \mathrm{E}-03$ & $9.14 \mathrm{E}-05$ & $4.14 \mathrm{E}-03$ & $1.11 \mathrm{E}-04$ & $1.19 \mathrm{E}+00$ & $3.00 \mathrm{E}-03$ \\
\hline $216-T-8$ & $2.63 \mathrm{E}-06$ & $1.73 \mathrm{E}-07$ & $1.41 \mathrm{E}-08$ & $2.11 \mathrm{E}-07$ & $7.13 \mathrm{E}-06$ & $1.66 \mathrm{E}-03$ \\
\hline $216-U-1 \& 2$ & $2.86 \mathrm{E}-04$ & $1.88 \mathrm{E}-05$ & $1.53 \mathrm{E}-06$ & $2.29 \mathrm{E}-05$ & $7.75 \mathrm{E}-04$ & $7.02 \mathrm{E}-01$ \\
\hline $216-\mathrm{U}-10$ & $7.21 \mathrm{E}-04$ & $4.74 \mathrm{E}-05$ & $3.86 \mathrm{E}-06$ & $5.78 \mathrm{E}-05$ & $1.95 \mathrm{E}-03$ & $1.88 \mathrm{E}+00$ \\
\hline $216-U-12$ & $3.71 \mathrm{E}-06$ & 2.44E-07 & $1.98 \mathrm{E}-08$ & $2.97 \mathrm{E}-07$ & $1.01 \mathrm{E}-05$ & $6.77 \mathrm{E}-01$ \\
\hline $216-U-13$ & $2.91 \mathrm{E}-06$ & $1.91 \mathrm{E}-07$ & $1: 56 \mathrm{E}-08$ & $2.33 \mathrm{E}-07$ & $7.89 \mathrm{E}-06$ & $1.20 \mathrm{E}-04$ \\
\hline 216-U-15 & $3.05 \mathrm{E}-06$ & $2 \mathrm{E}-07$ & $1.63 \mathrm{E}-08$ & $2.44 \mathrm{E}-07$ & $8.26 \mathrm{E}-06$ & $6.66 \mathrm{E}-04$ \\
\hline
\end{tabular}


Table 3.8. (contd)

\begin{tabular}{|c|c|c|c|c|c|c|}
\hline & \multicolumn{6}{|c|}{ Radionuclide Inventories ${ }^{*}$ in Curies } \\
\hline Site Name & C-14 & Cl-36 & I-129 & Se-79 & Tc-99 & U-238 \\
\hline $216-U-16$ & $1.08 \mathrm{E}-06$ & $7.11 \mathrm{E}-08$ & $5.79 \mathrm{E}-09$ & $8.66 \mathrm{E}-08$ & $2.93 \mathrm{E}-06$ & $5.99 \mathrm{E}-03$ \\
\hline $216-U-17$ & $2.67 \mathrm{E}-04$ & $1.76 \mathrm{E}-05$ & $1.43 \mathrm{E}-06$ & $2.14 \mathrm{E}-05$ & $7.24 \mathrm{E}-04$ & $3.33 \mathrm{E}-04$ \\
\hline $216-U-3$ & $2.84 \mathrm{E}-05$ & $1.87 \mathrm{E}-06$ & $1.52 \mathrm{E}-07$ & $2.28 \mathrm{E}-06$ & $7.71 \mathrm{E}-05$ & $5.99 \mathrm{E}-03$ \\
\hline $216-U-4 A$ & $1.21 \mathrm{E}-05$ & $7.97 \mathrm{E}-07$ & $6.49 \mathrm{E}-08$ & $9.71 \mathrm{E}-07$ & $3.29 \mathrm{E}-05$ & $3.00 \mathrm{E}-03$ \\
\hline $216-\mathrm{U}-4 \mathrm{~B}$ & $1.29 \mathrm{E}-05$ & $8.49 \mathrm{E}-07$ & 6.91E-08 & $1.03 \mathrm{E}-06$ & $3.5 \mathrm{E}-05$ & $4.39 \mathrm{E}-03$ \\
\hline $216-U-5$ & $8.57 \mathrm{E}-03$ & $5.64 \mathrm{E}-04$ & $4.59 \mathrm{E}-05$ & $6.87 \mathrm{E}-04$ & $2.32 \mathrm{E}-02$ & $1.21 \mathrm{E}-01$ \\
\hline 216-U-6 & 8.57E-03 & 5.64E-04 & $4.59 \mathrm{E}-05$ & $6.87 \mathrm{E}-04$ & $2.32 \mathrm{E}-02$ & $1.21 \mathrm{E}-01$ \\
\hline 216-U-7 & $4.37 \mathrm{E}-04$ & $2.87 \mathrm{E}-05$ & $2.34 \mathrm{E}-06$ & $3.5 \mathrm{E}-05$ & $1.18 \mathrm{E}-03$ & $4.71 \mathrm{E}-02$ \\
\hline $216-U-8$ & $2.98 \mathrm{E}-06$ & $1.96 \mathrm{E}-07$ & $1.6 \mathrm{E}-08$ & $2.39 \mathrm{E}-07$ & $8.09 \mathrm{E}-06$ & $8.00 \overline{\mathrm{E}}+00$ \\
\hline $216-Z-1 \& 2$ & $2.62 \mathrm{E}-06$ & $1.72 \mathrm{E}-07$ & $1.4 \mathrm{E}-08$ & $2.1 \mathrm{E}-07$ & $7.11 \mathrm{E}-06$ & $2.70 \mathrm{E}-02$ \\
\hline $216-Z-10$ & $4.55 \mathrm{E}-04$ & $2.99 \mathrm{E}-05$ & $2.44 \mathrm{E}-06$ & $3.65 \mathrm{E}-05$ & $1.24 \mathrm{E}-03$ & $8.71 \mathrm{E}-05$ \\
\hline $216-Z-12$ & $3.47 \mathrm{E}-06$ & $2.28 \mathrm{E}-07$ & $1.86 \mathrm{E}-08$ & $2.78 \mathrm{E}-07$ & 9.42E-06 & $1.7 \mathrm{E}-05$ \\
\hline $216-Z-16$ & $3.42 \mathrm{E}-05$ & $2.25 \mathrm{E}-06$ & $1.83 \mathrm{E}-07$ & $2.74 \mathrm{E}-06$ & $9.27 \mathrm{E}-05$ & $1.16 \mathrm{E}-02$ \\
\hline $216-Z-17$ & $2.37 \mathrm{E}-05$ & $1.56 \mathrm{E}-06$ & $1.27 \mathrm{E}-07$ & $1.9 \mathrm{E}-06$ & $6.44 \mathrm{E}-05$ & $5 \mathrm{E}-05$ \\
\hline $216-Z-18$ & $2.09 \mathrm{E}-01$ & $1.38 \mathrm{E}-02$ & $1.12 \mathrm{E}-03$ & $1.68 \mathrm{E}-02$ & $5.68 \mathrm{E}-01$ & $4.01 \mathrm{E}-02$ \\
\hline $216-Z-1 A$ & $1.05 \mathrm{E}-05$ & $6.89 \mathrm{E}-07$ & $5.61 \mathrm{E}-08$ & 8.4E-07 & $2.84 \mathrm{E}-05$ & $0.00 \mathrm{E}+00$ \\
\hline $216-Z-20$ & $5.66 \mathrm{E}-06$ & $3.72 \mathrm{E}-07$ & $3.03 \mathrm{E}-08$ & $4.54 \mathrm{E}-07$ & $1.54 \mathrm{E}-05$ & $1.34 \mathrm{E}-03$ \\
\hline $216-Z-3$ & $3.14 \mathrm{E}-06$ & $2.07 \mathrm{E}-07$ & $1.68 \mathrm{E}-08$ & $2.52 \mathrm{E}-07$ & $8.53 \mathrm{E}-06$ & $1.7 \mathrm{E}-05$ \\
\hline $216-Z-4$ & $2.29 \mathrm{E}-06$ & $1.51 \mathrm{E}-07$ & $1.23 \mathrm{E}-08$ & $1.84 \mathrm{E}-07$ & $6.22 \mathrm{E}-06$ & $1.7 \mathrm{E}-05$ \\
\hline $216-Z-5$ & $2.36 \mathrm{E}-04$ & $1.55 \mathrm{E}-05$ & $1.26 \mathrm{E}-06$ & $1.89 \mathrm{E}-05$ & $6.40 \mathrm{E}-04$ & $1.7 \mathrm{E}-05$ \\
\hline $216-Z-6$ & $2.29 \mathrm{E}-06$ & $1.51 \mathrm{E}-07$ & $1.23 \mathrm{E}-08$ & $1.84 \mathrm{E}-07$ & $6.22 \mathrm{E}-06$ & 1.7E-05 \\
\hline $216-Z-7$ & $1.31 \mathrm{E}-02$ & $8.62 \mathrm{E}-04$ & $7.01 \mathrm{E}-05$ & $1.05 \mathrm{E}-03$ & $3.55 \mathrm{E}-02$ & $1.66 \mathrm{E}-03$ \\
\hline $216-Z-8$ & $1.82 \mathrm{E}-05$ & $1.2 \mathrm{E}-06$ & $9.75 \mathrm{E}-08$ & $1.46 \mathrm{E}-06$ & $4.94 \mathrm{E}-05$ & $3.48 \mathrm{E}-06$ \\
\hline $216-Z-9$ & $3.41 \mathrm{E}-06$ & $2.24 \mathrm{E}-07$ & $1.82 \mathrm{E}-08$ & $2.73 \mathrm{E}-07$ & $9.24 \mathrm{E}-06$ & $1.7 \mathrm{E}-05$ \\
\hline
\end{tabular}

* Refer to Sections 3.4.5 for a detailed discussion of the development of CERCLA radionuclide inventories.

** Inventories decayed to a common date of 2050 . 
Table 3.9. Inventory of Key Radionuclides for US Ecology

\begin{tabular}{|l|c|c|c|c|c|c|}
\hline & \multicolumn{6}{|c|}{ Radionuclide Inventories ${ }^{\star}$ in Curies } \\
\hline \multicolumn{1}{|c|}{ Site Name } & $\mathbf{C - 1 4}$ & $\mathbf{C l}-36$ & $\mathbf{I - 1 2 9}$ & Se-79 & Tc-99 & U-238 \\
\hline US Ecology current & $3.66 \mathrm{E}+03$ & $3.44 \mathrm{E}+01$ & $5.63 \mathrm{E}+00$ & & $6.17 \mathrm{E}+01$ & $1.08 \mathrm{E}+04$ \\
\hline US Ecology future & $1.91 \mathrm{E}+02$ & $6.00 \mathrm{E}-02$ & $1.40 \mathrm{E}-01$ & & $3.91 \mathrm{E}+00$ & $1.21 \mathrm{E}+02$ \\
\hline
\end{tabular}

* Total inventories were taken from the Site Stabilization and Closure Plan for Low-Level Radioactive Waste Management Facility, US Ecology, Inc., Richland, Washington.(Grant Environmental, Chase Environmental Group, and US Ecology 1996).

** Inventories decayed to a common date of 2050 .

+ The absence of selenium-79 from the commercial low-level waste disposal is a result of commercial waste not having a significant source of this radionuclide.

Table 3.10. Inventory of Key Radionuclides for the Decommissioned Reactor Cores

\begin{tabular}{|l|c|c|c|c|c|c|}
\hline & \multicolumn{5}{|c|}{ Radionuclide Inventories $^{*}$ in Curies } \\
\hline \multicolumn{1}{|c|}{ Site Name } & C-14 & Cl-36 & $\mathbf{I - 1 2 9}^{+}$ & Se-79 $^{+}$ & Tc-99 & U-238 \\
\hline C Reactor & $4.47 \mathrm{E}+03$ & $1.20 \mathrm{E}+01$ & & & $2.00 \mathrm{E}-03$ & $4.00 \mathrm{E}-03$ \\
\hline D Reactor & $4.27 \mathrm{E}+03$ & $3.40 \mathrm{E}+01$ & & & $2.00 \mathrm{E}-03$ & $0.00 \mathrm{E}+00$ \\
\hline DR Reactor & $3.18 \mathrm{E}+03$ & $2.60 \mathrm{E}+01$ & & & $2.00 \mathrm{E}-03$ & $0.00 \mathrm{E}+00$ \\
\hline F Reactor & $3.68 \mathrm{E}+03$ & $3.30 \mathrm{E}+01$ & & & $2.00 \mathrm{E}-03$ & $0.00 \mathrm{E}+00$ \\
\hline H Reactor & $3.48 \mathrm{E}+03$ & $1.70 \mathrm{E}+01$ & & & $2.00 \mathrm{E}-03$ & $0.00 \mathrm{E}+00$ \\
\hline KE Reactor & $6.95 \mathrm{E}+03$ & $5.40 \mathrm{E}+01$ & & & $3.30 \mathrm{E}-02$ & $0.00 \mathrm{E}+00$ \\
\hline KW Reactor & $6.66 \mathrm{E}+03$ & $5.20 \mathrm{E}+01$ & & & $3.30 \mathrm{E}-02$ & $0.00 \mathrm{E}+00$ \\
\hline N Reactor & $9.49 \mathrm{E}+03$ & $7.50 \mathrm{E}+01$ & & & $3.30 \mathrm{E}-02$ & $0.00 \mathrm{E}+00$ \\
\hline
\end{tabular}

* Inventories were from Appendix A of the draft EIS Decommissioning of Eight Surplus Production Reactors at the Hanford Site, Richland, Washington (DOE 1989) for all reactors except $\mathrm{N}$ Reactor. The $\mathrm{N}$ Reactor inventory was provided by $\mathrm{V}$. G. Edens (from Interoffice Memorandum \#042809; Subject, "105N and 107N Hazardous Assessment [Inventories]"; sent by R. S. Day to V. G. Edens of Hanford Environmental Restoration contractor; February 11, 1997).

** Inventories were decayed to a common date of 2050.

+ Neither iodine- 129 nor selenium-79 were reported in the inventories for the decommissioned reactor cores. 
Table 3.11. Summary Table of Inventories Considered in the Composite Analysis

\begin{tabular}{|c|c|c|c|c|c|c|}
\hline & & Radic & puciude inv & entories i & Curies & \\
\hline Site Name & C-14 & $\mathrm{Cl}-36$ & $\mathrm{I}-129$ & Se-79 & Tc-99 & U-238 \\
\hline Agnew" All Tanks & $4.78 \mathrm{E}+03$ & & $6.30 \mathrm{E}+01$ & $7.73 E+02$ & $3.26 \mathrm{E}+04$ & $9.06 E+02$ \\
\hline Agnew"* Cribs & $1.24 \mathrm{E}+02$ & & $1.64 \mathrm{E}+00$ & $2.63 E+01$ & $8.68 \mathrm{E}+02$ & $1.31 \mathrm{E}+03$ \\
\hline Agnew"* Leaks & $1.44 \mathrm{E}+01$ & & 2.04E-01 & $1.85 \mathrm{E}+00$ & $1.07 \mathrm{E}+02$ & 4.63E-01 \\
\hline Agnew"* Total Site & $4.91 \mathrm{E}+03$ & & $6.48 \mathrm{E}+01$ & $8.01 E+02$ & $3.35 E+04$ & $2.22 E+03$ \\
\hline Kupfer ** Global Tank Inventories & $4.78 \mathrm{E}+03$ & & $6.61 \mathrm{E}+01$ & $7.73 E+02$ & $3.26 \mathrm{E}+04$ & $3.22 \mathrm{E}+02$ \\
\hline Schmittroth ${ }^{* * * *}$ Total & $7.69 \mathrm{E}+02$ & & $6.61 \mathrm{E}+01$ & $1.03 E+03$ & $2.72 E+04$ & $2.96 \mathrm{E}+02$ \\
\hline Total $^{+}$ & $5.00 \mathrm{E}+04$ & $3.45 \mathrm{E}+02$ & $1.71 \mathrm{E}+01$ & $1.05 E+03$ & $2.49 \mathrm{E}+04$ & $6.60 \mathrm{E}+04$ \\
\hline Total minus US Ecology & $4.62 E+04$ & $3.11 E+02$ & $1.13 E+01$ & $1.05 \mathrm{E}+03$ & $2.48 \mathrm{E}+04$ & $5.50 \mathrm{E}+04$ \\
\hline Total minus (cores + US Ecology) & $3.95 E+03$ & $7.60 \mathrm{E}+00$ & $1.13 E+01$ & $1.05 E+03$ & $2.48 E+04$ & $5.50 \mathrm{E}+04$ \\
\hline $\begin{array}{l}\text { Total minus (cores + US Ecology } \\
+ \text { ERDF) }\end{array}$ & $1.50 \mathrm{E}+02$ & $7.60 \mathrm{E}+00$ & $1.13 E+01$ & $1.05 \mathrm{E}+03$ & $2.48 \mathrm{E}+04$ & $8.00 E+02$ \\
\hline TWRS ILAW & $7.69 \mathrm{E}+00$ & $0.00 \mathrm{E}+00$ & $6.62 \mathrm{E}+00$ & $1.03 \mathrm{E}+03$ & $2.23 \mathrm{E}+04$ & $1.78 \mathrm{E}+01$ \\
\hline TWRS SST Leaks - cmplx ${ }^{++}$ & 3.15E-01 & $0.00 \mathrm{E}+00$ & 5.99E-02 & $5.60 \mathrm{E}-02$ & $5.22 \mathrm{E}+01$ & $2.45 \mathrm{E}-03$ \\
\hline TWRS SST Leaks - ncmplx & $4.11 E+00$ & $0.00 \mathrm{E}+00$ & $6.78 \mathrm{E}-01$ & 7.32E-01 & $4.59 E+02$ & $3.21 E-02$ \\
\hline TWRS SST Losses - cmplx & $1.44 \mathrm{E}-01$ & $0.00 \mathrm{E}+00$ & $2.88 \mathrm{E}-03$ & 7.47E-03 & $5.76 \mathrm{E}+00$ & 3.14E-04 \\
\hline TWRS SST Losses - ncmplx & $3.52 \mathrm{E}+00$ & $0.00 \mathrm{E}+00$ & $6.23 \mathrm{E}-01$ & $6.27 \mathrm{E}-01$ & $4.67 \mathrm{E}+02$ & 2.75E-02 \\
\hline TWRS SST Residuals - cmplx & $1.17 \mathrm{E}+00$ & $0.00 \mathrm{E}+00$ & $5.74 \mathrm{E}-03$ & $2.79 E-01$ & $3.84 \mathrm{E}+00$ & 3.24E-01 \\
\hline TWRS SST Residuals - ncmplx & $2.86 \mathrm{E}+01$ & $0.00 \mathrm{E}+00$ & $1.54 \mathrm{E}-01$ & $7.70 \mathrm{E}+00$ & $1.06 \mathrm{E}+02$ & $4.42 \mathrm{E}+00$ \\
\hline TWRS DST Residuals - cmplx & $8.28 \mathrm{E}+00$ & $0.00 E+00$ & $0.00 \mathrm{E}+00$ & $0.00 \mathrm{E}+00$ & $8.62 E+01$ & $0.00 \mathrm{E}+00$ \\
\hline TWRS DST Residuals - ncmplx & $1.49 \mathrm{E}+01$ & $0.00 \mathrm{E}+00$ & $0.00 \mathrm{E}+00$ & $0.00 \mathrm{E}+00$ & $1.25 E+02$ & $0.00 \mathrm{E}+00$ \\
\hline $216^{\varepsilon}$ liquid discharges $+241^{\text {हf }}$ & $3.65 \mathrm{E}+00$ & $2.40 \mathrm{E}-01$ & $1.94 \mathrm{E}+00$ & $2.93 \mathrm{E}-01$ & $9.37 \mathrm{E}+02$ & $1.57 \mathrm{E}+02$ \\
\hline $218^{\checkmark} 200 \mathrm{~W}^{\prime s}$ pre-1988 & $2.89 \mathrm{E}+01$ & $1.45 \mathrm{E}+00$ & $6.18 E-01$ & $1.77 \mathrm{E}+00$ & $6.01 \mathrm{E}+01$ & $1.92 E+02$ \\
\hline 218200 E pre-1988 & $7.94 \mathrm{E}+01$ & $5.22 \mathrm{E}+00$ & 4.25E-01 & $6.36 \mathrm{E}+00$ & $2.15 \mathrm{E}+02$ & $9.85 \mathrm{E}-01$ \\
\hline $218200 \mathrm{~W}$ post-1988 & $1.74 \mathrm{E}+01$ & 8.33E-01 & $2.10 \mathrm{E}-01$ & $1.07 \mathrm{E}+00$ & $5.15 \mathrm{E}+01$ & $3.46 \mathrm{E}+02$ \\
\hline 218200 E post- 1988 & $1.35 \mathrm{E}-01$ & 8.87E-03 & 4.21E-02 & $1.08 \mathrm{E}-02$ & 3.66E-01 & $6.68 \mathrm{E}-02$ \\
\hline ERDF & $3.80 \mathrm{E}+03$ & $0.00 \mathrm{E}+00$ & $0.00 \mathrm{E}+00$ & $0.00 \mathrm{E}+00$ & $6.57 \mathrm{E}+00$ & $5.43 \mathrm{E}+04$ \\
\hline Production Reactor Cores & $4.22 \mathrm{E}+04$ & $3.03 \mathrm{E}+02$ & $0.00 \mathrm{E}+00$ & $0.00 \mathrm{E}+00$ & $1.09 \mathrm{E}-01$ & $4.00 \mathrm{E}-03$ \\
\hline US Ecology & $3.85 \mathrm{E}+03$ & $3.44 \mathrm{E}+01$ & $5.77 \mathrm{E}+00$ & $0.00 \mathrm{E}+00$ & $6.56 \mathrm{E}+01$ & $1.09 \mathrm{E}+04$ \\
\hline * Inventories have be & to a cor & Ion date of & 2050 & & & \\
\hline ** See Agnew et al. (1997). & & & & & & \\
\hline *** See Kupfer et al. (1997). & & & & & & \\
\hline **** See Schmittroth et al. (1995 & & & & & & \\
\hline$+\quad$ Sum of estimated inventori & of sites i & luded in $\mathrm{t}$ & first iter & on of the & mposite Ar & nalysis. \\
\hline $\begin{array}{l}+ \text { cmplx }=\text { complexed wastes. } \\
++ \text { ncmplx = noncomplexed wa }\end{array}$ & stes. & & & & & \\
\hline$£ \quad 216$ refers to past-practice 1 & uid dispo & & & & & \\
\hline 241 refers to tanks associate & with reve & se wells. & & & & \\
\hline 218 refers to solid waste bur & al grounds. & & & & & \\
\hline $\mathrm{W}$ and $\mathrm{E}$ refer to the $200 \mathrm{We}$ & & & & & & \\
\hline
\end{tabular}




\subsection{Performance Analysis}

The Composite Analysis included calculations for source release, vadose zone transport, groundwater transport, atmospheric transport, and dose for the radionuclides of concern identified in Chapter 3. The performance analysis was completed for each of the existing or planned waste sites with radionuclide inventories within the 200 Area Plateau. This chapter describes the assumptions, implementation, results, and sensitivity analyses associated with each component of the performance analysis. Results from the Composite Analysis are compared with earlier performance assessments conducted for sites within the 200 Area. The results are summarized and compared to the dose limits in Chapter 5.

\subsection{Methodology and Results}

The performance analysis involved estimating cumulative radionuclide doses from both subsurface and atmospheric pathways. The surface pathway was not considered because surface water transport within the 200 Area Plateau rarely occurs. The points of assessment for the Composite Analysis were located on the Hanford Site between the buffer zone and the Columbia River. The area inside the buffer zone (see Figure 1.4) was excluded from the bulk of this analysis because in current land use plans, this portion of the Hanford Site will be used exclusively for waste management to minimize human exposure (DOE 1996a). Dose impacts inside the buffer zone are shown only for the industrial exposure scenario. Although the atmospheric pathway was included in the analysis, the primary exposure route for contaminants from the Hanford Site was through the groundwater pathway, involving source term release, transport through the vadose zone and groundwater, and exposure from pumping and using the contaminated groundwater in a variety of exposure pathways. The transport and exposure pathways considered in the Composite Analysis are illustrated in Figure 4.1.

Radiological doses from the subsurface transport pathway were analyzed for each source site considered in the Composite Analysis. The radionuclide inventory for each waste site was released to the vadose zone according to its release model. Transport within the vadose zone was estimated with a transient one-dimensional variably saturated vadose zone transport model. Travel times for annual releases of unit mass were defined by arrival of $50 \%$ of each unit mass. These travel times were used to translate annual releases from the waste into releases to the water table of the aquifer. The resulting fluxes into the water table were transported in the unconfined aquifer with a transient three-dimensional saturated groundwater transport model. The concentrations in the groundwater plumes for each radionuclide were translated into doses associated with agricultural, residential, recreational, and industrial exposures using dose conversion factors. Doses from the various source locations and various radionuclides were combined to estimate the cumulative dose. Uranium toxicity was also considered in the Composite Analysis.

Radiological doses from the atmospheric pathway only considered releases from the graphite cores of surplus production reactors that are planned to be relocated to the 200 West Area solid waste burial 
grounds (ROD 1993) prior to Hanford Site closure. The radionuclide inventory contained in the reactor cores was released based on the atmospheric release model. The doses at different locations were estimated with spatial distribution functions for unit releases and the predicted atmospheric transport developed from historical wind profiles at the Hanford Site.

The sequence of calculations required to estimate the cumulative dose was performed with a suite of software elements that were integrated across two computational environments. These software elements included: 1) an Excel ${ }^{\mathrm{TM}}$ workbook; 2) a dynamically linked library version of the Subsurface Transport Over Multiple Phases (STOMP) code (White and Oostrom 1996; White and Oostrom 1997; Nichols et al. 1997); 3) the Coupled Fluid, Energy, and Solute Transport (CFEST-96) code (Gupta 1997); and 4) the ARC-INFO'M Geographic Information System. ${ }^{(a)}$ Elements 1 and 2 were implemented on personal computers running either Windows $95^{\mathrm{TM}}$ or Windows NT ${ }^{\mathrm{TM}}$. Elements 3 and 4 were implemented on UNIX workstations. Figure 4.2 illustrates the relationship among the software elements.

The methodologies for calculating source release, vadose zone transport, groundwater transport, atmospheric transport, and cumulative dose are described in the following sections. The key assumptions (e.g., geometry, initial conditions, boundary conditions, and parameters) for each calculation are identified and discussed. The implementation of each model for the base case and the sensitivity analyses are also described.

\subsubsection{Source Release Models}

Because of the variety of waste sources within the exclusive waste management area that have released to the atmosphere or subsurface environment (or are expected to release in the future), a variety of source release models were used. For the first iteration of the Composite Analysis, seven idealized source release models were applied. Of the seven release models, one was for liquid releases to vadose zone, five were for leaching from various solid waste forms to the vadose zone, and one was for atmospheric releases.

\subsubsection{Background}

Each of the release models in the Composite Analysis involved different assumptions. The assumptions for each of the release models are discussed below. Each source was characterized in terms of its generic waste form type, contaminant inventories, volume, duration of disposal, and geometry to facilitate calculation of release. The liquid source release model was the simplest and the most common. The five models for leaching from solid waste forms are more complex and are discussed in Appendix D which contains a detailed discussion of the conceptual model and mathematical approach for each type of source and the rationale for choosing parameter values in the release model equations. The atmospheric release model followed the approach defined in the draft environmental impact statement (EIS),

(a) ARC/INFO is a registered trademark of Environmental Systems Research Institute, Inc., Redlands, California. 
Decommissioning of Eight Surplus Production Reactors at the Hanford Site, Richland, Washington (DOE 1989) and in the final EIS (DOE 1992). Table 4.1 describes the critical assumptions of the source release models and the likely impact of each assumption on the overall performance analysis.

\subsection{Liquid Release Model}

In the liquid source release model, contaminants were assumed uniformly distributed in the liquid effluent. Therefore, the remaining fraction of the undecayed inventory was assumed to be equal to the fraction of liquid remaining at any time. Releases were assumed to have occurred uniformly over the period of the specific site's operation and discharge of waste to the vadose zone. Based on the type of disposal facility, different flux rates were used. Once the liquid source enters the soil, it was assumed to move vertically downward through the vadose zone to the water table. Liquid releases were the most common release mechanism in the Composite Analysis and included sources from tank leaks, tank sluicing losses, trenches, ditches, ponds, reverse wells, French drains, and cribs.

\subsection{Soil-Debris Release Model}

In the soil-debris waste model, wastes are assumed to be mixed with soils. Waste sources included in this model were assumed to be permeable to percolating water. Thus, all surfaces of the waste were assumed to come into contact with percolating water. If contaminant inventories in the source were high enough, leaching of contaminant through the bottom of the source was controlled by the solubility of the contaminant in soil water. Otherwise, leaching was controlled by partitioning the radionuclides between aqueous and sorbed phases. The inventory was assumed to be perfectly mixed throughout the source volume during the entire release period. Assuming perfectly mixed conditions reduced the likelihood that solubility would control the release. The soil-debris model was the second most frequently used release model. It was employed for all the solid waste burial grounds, including the Environmental Restoration Disposal Facility (ERDF) and the commercial low-level waste (LLW) burial ground operated by US Ecology, except those involving grouted waste or high-integrity containers for waste stabilization.

\subsection{Cake Release Model}

In the cake release model consolidated tank wastes were assumed to be permeable to water and dissolved over time because a major structural component of the waste (in this case nitrate salt) dissolved in the water percolating through the waste form. As the solid waste dissolved at a constant rate controlled by the aqueous solubility of nitrate, the contaminants associated with the dissolved portion of the waste form were assumed to be released into the percolating water congruently at constant rates related to their concentration in the waste form. The cake model was employed for residual wastes remaining in both single-shell and double-shell tanks after tank waste recovery operations have been completed. This release model was applied in Tank Waste Remediation System (TWRS) EIS (DOE and Ecology 1996). 


\subsection{Glass Release Model}

In the glass release model, vitrified wastes are assumed to release contaminants into pore water through corrosion of the glass. For glass, the aqueous permeability was assumed to be sufficiently low such that aqueous transport within the waste form itself was essentially zero. Because of the rectangular box called for in contract specifications and the likelihood of glass fracturing, this waste form was assumed to be roughly cubical in shape. Release was assumed to occur with time by slow dissolution from the exterior surfaces of the glass. All of the contaminants associated with the dissolved portion of the waste form undergo congruent release into the surrounding pore water at rates related to their concentration in the waste form and the overall waste form dissolution rate at the given time. The dissolution rate for vitrified waste was taken from the contract specification as it appears in the interim performance assessment for immobilized low-activity waste (Mann et al. 1997). The glass release model was applied for both of the proposed TWRS glass waste disposal sites.

\subsection{Cement Release Model}

In the cement release model, the waste form is assumed to have permeability much lower than that of the surrounding soil. The pore space connectivity in the cementitious waste form is sufficiently high to allow contaminant mobility within the waste form by diffusion. Percolating water was assumed to surround this waste form, and contaminants inside the waste form were assumed to diffuse to the outer . surface and enter the percolating water. Therefore, overall contaminant release from the source zone was assumed to be controlled by the contaminant's effective diffusion coefficient in the waste form. The cement release model was only used for two soil waste burial grounds that contained cementitious waste forms (e.g., caissons).

\subsection{Reactor Block Release Model}

In the reactor release model, irradiated solids were assumed to release contaminants into the water percolating past them by unspecified loss processes from the solid matrix and by corrosion of the solid components themselves over time. Because of the absence of information regarding the conceptual and mathematical description of the processes occurring, release of contaminants from the reactor blocks was assumed to be described by rates calculated from experimental leach test data. The reactor block release model was used to simulate release from each of the surplus reactors. This release model was first developed and applied to the reactor blocks in the draft EIS, Decommissioning of Eight Surplus Production Reactors at the Hanford Site, Richland, Washington (DOE 1989).

\subsection{Atmospheric Release Model}

Atmospheric releases were only estimated for tritium and carbon-14 inventories in the surplus production reactor cores that are scheduled to be relocated to the 200 Area West solid waste burial 
grounds. The method was based on the same experimental leach rate data used for the reactor block model. This approach was also described in the draft environmental impact statement for the surplus reactors (DOE 1989).

\subsubsection{Source Term Release Model Implementation}

Each waste source considered in the Composite Analysis was categorized as one of the generic waste form types described in the previous section. The inventories of radioactive contaminants for each waste source were compiled (as described in Chapter 3). Models for liquid, solid, and atmospheric releases were implemented separately to facilitate calculations. All three groups of release models were implemented within the Composite Analysis.xls Excel ${ }^{\mathrm{TM}}$ workbook. Figure 4.3 illustrates the implementation of the release models. Table 4.2 describes each of the primary worksheets in the Composite Analysis.xls workbook.

The approach used to estimate the temporal distribution of radionuclide fluxes to the water table does not require any specific implementation of a release model for liquid releases because these releases were assumed to occur uniformly over the release period specified in the Source Site worksheet of the Composite Analysis.xls Excel ${ }^{\mathrm{TM}}$ workbook. Atmospheric releases were estimated independently in the Composite Analysis.xls Excel ${ }^{\mathrm{TM}}$ workbook. The spatial distributions of unit atmospheric releases were calculated separately and were provided for processing by the geographic information system.

The release models for solid waste forms (soil-debris, cake, glass, cement, and reactor block) were implemented within several worksheets and Excel ${ }^{\mathrm{TM}}$ macros in the Composite Analysis.xls Excel ${ }^{\mathrm{TM}}$ workbook. The Nuclides \& Release Model Data worksheet provided release parameters (such as · fractional release from glass, cement diffusion coefficient, fractional release from reactor) and general nuclide data (such as decay half-life and specific activity) for each nuclide for each of the various solid waste sites. For the soil-debris waste form, the overall volume of the source zone was used to estimate contaminant concentrations from inventories. For cake, glass, and cement waste forms, their actual volumes were used. The release model associated with the reactor block type of waste form did not contain volume and concentration considerations. These and other waste site and waste form geometry data required for the release models were retrieved from the Source Site worksheet (see Table 4.3). The chemical classification of the waste stream for each waste site is listed in Table 4.4 .

The estimates of the volumetric water content of the source zone and the sorption coefficient required for the soil-debris waste form model were obtained from the $K_{d}$ and Release Model Classes worksheet. The recharge rates and release periods used in the source release and vadose zone transport models are summarized in Table 4.5. For many waste sites, the total inventory was assumed in place at the midpoint in the operational period. For those sites, the second and fourth columns in Table 4.5 represent the midpoint of the disposal or discharge operations and the end of operation. The MyRelease macro estimated the annual releases for 1500 years, beginning in 1944 when Hanford Site operations began, and stored these values in the Temp worksheet for later integration with results from the vadose zone simulation to achieve water table releases. 
The values of some waste site and release model parameters were specific to the conditions at a particular source site. In those cases, where it was believed that reasonable "Hanford Site-specific" values were known, they were used in the calculations. Most waste site and release/transport model parameter values were based on actual data. However, some were based on an assumed similarity in behavior with other radionuclides, and some values were set equal to "default" values when no other information was available.

The source term release models are closely linked to the vadose zone transport models. Results from the combined components of the model are summarized as cumulative release ( $\mathrm{Ci} / \mathrm{yr}$ ) to groundwater in the vadose zone transport (see Section 4.1.2.3). The sensitivity of results to the source term release models was investigated by varying the type of release model applied. As in the case of the results from the source-term release models, the results of sensitivity analyses will be summarized in the vadose zone transport (see Section 4.1.2.4).

\subsubsection{Vadose Zone Model}

Contaminants released from the various Hanford Site waste sources were transported downward through the vadose zone to the water table. The primary mechanism for transport in the vadose zone was water flow in response to gravitational and capillary forces. The radionuclide influx from each waste site release was accounted for in the Composite Analysis. Dry disposals such as the burial grounds, the immobilized low-activity waste (ILAW) disposal, the ERDF, and the reactor cores were placed at the assumed depths of disposal. After the waste disposal operations ceased, transient hydraulic conditions from different surface covers (including revegetation) that affect recharge were represented in the model. Recharge directly from precipitation or snowmelt infiltrates into the vadose zone. The recharge rate varies with operations and the placement of any covers for each of the waste sites. The geology and soils in the vadose zone are heterogeneous. Geochemical conditions in the vadose zone are similarly heterogeneous, with conditions near some waste sources more strongly influenced by the chemical nature of the waste itself. Because of the uncertainty in hydraulic and geochemical properties in the vadose zone, the uncertainties in the vadose zone model itself (DOE 1997a), and because the end states are not well defined for all waste sites at Hanford, vadose zone flow and transport predictions in the Composite Analysis are also uncertain. The data used in the vadose zone model are described in the remainder of this section.

\subsubsection{Background}

The vadose zone was modeled as a stratified one-dimensional column. In the first iteration of the Composite Analysis, it was not appropriate to represent the vadose zone with a multidimensional model because of the large number of waste sites modeled and the limited characterization of the vadose zone. Multidimensional modeling of the vadose zone has been determined to be important and has been performed for some waste forms (Mann et al. 1997; DOE 1997b), but is not practical for the first iteration of the Composite Analysis. The multidimensional effects will be accounted for in detailed modeling of 
individual waste sites and used to adjust the recharge rates and cross-sectional areas used for the onedimensional model in future iterations of the Composite Analysis. Multidimensional modeling will be considered in future iterations of the Composite Analysis as well.

In the remainder of this section, the stratigraphy, hydraulic properties, recharge, and geochemical conditions used in the first iteration of the Composite Analysis are described.

\subsection{Stratigraphy}

The stratigraphy used in the model was consistent with the major geologic formations found in the vadose zone beneath the 200 Area Plateau and was based on work documented in Thorne and Chamness (1992), Thorne et al. (1993), and Thorne et al. (1994). The geology at each site was defined as a set of strata consistent with nearest available well log. Each of the well logs included location, ground surface elevation, and the thickness of the various major sediment types. A summary of the geologic well logs used in the Composite Analysis appears in Table 4.6.

Seven sediment types and one rock type (basalt) were identified and used to define the stratigraphy at each profile location. The sediment types are: East Hanford Gravel, East Hanford Sand, East Ringold, West Hanford Sand, West Early Palouse, Plio Pleistocene, and West Ringold. The definitions of "east" or "west" were used to distinguish sediment types found only in the 200 East or 200 West Areas, respectively. The East Hanford Gravel also appears in the spreadsheet as Lower East Hanford Gravel, but the same soil moisture characteristics are applied to both. At most, four different sediment types occurred above the basalt at any location. In the vadose zone model, the basalt rock type was regarded as impermeable and was used to define the default bottom of the vadose zone profile. If the water table fell below the top of the basalt, the vadose zone was still assumed to be limited to the basalt surface.

\subsection{Hydraulic Characteristics}

Modeling water flow and radionuclide transport through the vadose zone required a description of the relationship between moisture content, pressure head, and unsaturated hydraulic conductivity. These relationships, called soil moisture characteristics, are highly nonlinear. In the Composite Analysis, nonhysteretic relationships were assumed for Hanford Site soils because few measurements have been made for Hanford Site soils to characterize hysteresis, and it is believed to be of secondary importance. The hydraulic properties of Hanford Site soils are highly variable, both between the Hanford and Ringold formations and within each of the formations (Khaleel and Freeman 1995).

In the Composite Analysis, different sediment types were used to define the one-dimensional columns beneath the waste sites. The hydraulic properties of the sediment types were assumed to be uniform with each sediment layer. Preferential flow paths in the form of wells and clastic dikes were not considered in the Composite Analysis because use of one-dimensional models can not represent their local influence in a three-dimensional environment. The potential influence of preferential flow paths, especially clastic dikes, have been addressed in the performance assessments for the solid waste burial 
grounds (Wood et al. 1995; Wood et al. 1996) and more recently by Ward, Gee, and White (1997). Wood et al. (1995) and Wood et al. (1996) concluded that clastic dikes were insufficiently large and insufficiently continuous to provide a true preferential pathway.

The model of soil hydraulic properties based on the van Genuchten (1980) and Mualem (1976) analytical expressions was used as the basis for the relationships between moisture content, pressure head, and unsaturated hydraulic conductivity. This model has been applied in previous vadose zone studies at the Hanford Site. Parameters for the van Genuchten and Mualem models have been determined by fitting experimental data for Hanford Site sediments to the classic analytic expressions of these models. These results are described in several Hanford Site documents, but the parameters used in the initial iteration of the Composite Analysis were compiled by Khaleel and Freeman (1995).

For the Composite Analysis, unsaturated flow parameters were established for each of the vadose zone sediment types defined above. The sediment types and associated sets of parameters used in the Composite Analysis unsaturated flow modeling are shown in Table 4.7. It should be noted that the laboratory-measured moisture retention and saturated conductivity data in Table 4.7 have been corrected for the gravel fraction ( $>2 \mathrm{~mm}$ ) present in the bulk sample.

\subsection{Recharge Rates}

Initial investigations in the Composite Analysis demonstrated that the significant changes in the recharge rates throughout the 1000-year study period require an analysis of transient vadose zone flow and transport. At the Hanford Site, data on the current distribution of soil moisture and contaminants in the vadose zone at the majority of waste sites are inadequate to define present initial conditions for modeling, so simulations were begun at the initiation of each waste source site's release to the vadose zone. Therefore, initial conditions in the Composite Analysis were based on expected conditions before operations started in the 200 Area; i.e., based on steady-state recharge under natural recharge conditions with no contaminants in the vadose zone. The recharge rate was allowed to vary, representing a range of surface cover conditions, from undisturbed surfaces with natural vegetation, to disturbed surfaces maintained free of vegetation, to engineered surface barriers designed for long-term service.

The current recharge rate into coarse surface sediments maintained free of vegetation was estimated as $75 \mathrm{~mm} / \mathrm{yr}$, based on data from a nonvegetated gravel-covered lysimeter on the Hanford Site. ${ }^{\text {(a) }}$ For a revegetated site, the recharge rate was estimated by Wood et al. (1996) to drop to $5 \mathrm{~mm} / \mathrm{yr}$. If a Hanford Protective Barrier was installed, the recharge was estimated to drop to $0.5 \mathrm{~mm} / \mathrm{yr}$ (Wing 1994). A variety of end states was proposed for the different waste sites by the different U.S. Department of Energy (DOE) programs queried for information supporting the first iteration of the Composite Analysis. For example, the solid waste burial grounds were assumed to have a long-term surface barrier limiting'

.(a) From an electronic mail message dated July 30, 1997 sent by M. J. Fayer, Pacific Northwest National Laboratory to C. T. Kincaid and L.W. Vail, Pacific Northwest National Laboratory; subject, "Recharge in Tank Farms." 
annual average recharge to no more than $5 \mathrm{~mm} / \mathrm{yr}$ while the ERDF trench, TWRS ILAW disposal facility, tank farms, and surplus reactor cores were assumed to employ a Hanford Protective Barrier, with a $0.5-\mathrm{mm} / \mathrm{yr}$ recharge rate. Based on guidance from the Hanford Strategic Plan (DOE 1996d), it was assumed that liquid disposal sites will be closed in place with surface barriers such as the Hanford Protective Barrier.

Infiltration rates for liquid discharge sites during their active disposal period were estimated based on the type of disposal facility. For ponds, the recharge rate was assumed to be the maximum infiltration rate that sediments beneath the pond would allow under unit gradient conditions, i.e., the saturated hydraulic conductivity of the least conductive geohydrologic unit in the vadose zone profile. For example, infiltration from such facilities in the 200 West Area were governed by the saturated hydraulic conductivity of the Early Palouse sediment, or $3040 \mathrm{~cm} / \mathrm{yr}$. For cribs, ditches, specific retention trenches, reverse wells, and French drains that received lesser quantities of liquid discharge, the flux rate was assumed to be one third of the saturated hydraulic conductivity. Thus, the wetted cross section of the one-dimensional column was assumed to be three times that defined by the saturated hydraulic conductivity and the assigned discharge rate of the facility. A reduced flux rate over a larger area was employed to represent the spreading or lateral dispersion that would occur during migration of contaminants in the vadose zone.

\subsection{Distribution Coefficients}

In the initial iteration of the Composite Analysis, the linear sorption isotherm model was used in transport calculations. This model was selected because it was the only approach for which model parameters (distribution coefficients) were available for a broad range of waste sites and radionuclides. At some waste sites the chemistry of the waste streams disposed to ground at the Hanford Site appreciably altered the geochemistry of the near-field sediments. Such changes in the geochemistry likely altered the sorption properties of the altered sediment. An approach was used in the Composite Analysis that allows the distribution coefficient to vary with depth. Both near-field and far-field ${ }^{(a)}$ distribution coefficients were defined for six waste types (Appendix E) representing the waste chemistries disposed to the subsurface. The waste type is listed for each source site in Table 4.4. The location of the transition from near- to far-field was estimated from information available in post-mortem studies of waste sites (Fecht, Last, and Price 1977).

The depths at which distribution coefficients change were estimated from the maximum penetration depth of beta- and gamma-emitting radionuclides in or adjacent to facilities. These measurements mainly reflect cesium-137 and strontium-90. If measurements were available for a facility, then the measured penetration depth was used. If no measurements were available, then the depth was estimated from measurements at facilities that received the same types of waste. The assumption was made that cesium is essentially mobile to the transition depth and immobile after the transition depth is reached.

(a) "Near-field" and "far-field" are referred to as "high impact" and "intermediate impact" zones in Appendix E. 
However, total volume discharged was also examined, and for sites with relatively large discharge volumes, the transition depth was taken to be something less than the maximum depth of measured gamma and beta. The selection of distribution coefficients is discussed in detail in Appendix $\mathrm{E}$.

Assumptions for the vadose zone model, the rationale for the assumptions, and the expected impacts are listed in Table 4.8 .

\subsubsection{Vadose Zone Model Implementation}

The vadose zone flow and transport model was implemented within the Composite Analysis.xls workbook. Figure 4.3 illustrates implementation of the vadose model in the Excel ${ }^{T M}$ spreadsheet. The STOMP code (White and Oostrom 1996; White and Oostrom 1997; Nichols et al. 1997) was accessed from the workbook to perform the fate and transport portion of the calculation. Implementation of the vadose zone model resulted in estimates of the annual contaminant flux to the water table.

The STOMP code was developed under the Volatile Organic Compounds (VOC) Arid Demonstration Project through the DOE Office of Technology Development (White and Oostrom 1997). STOMP is based on the numerical solution of the three-dimensional Richard's equation for fluid flow and the advection-dispersion equation for contaminant transport. While STOMP is capable of threedimensional simulations, it is also designed to be efficient in performing one- and two-dimensional simulations. By selecting STOMP for the Composite Analysis, the same code can be used in subsequent iterations, even if dimensionality of the simulations change. The code is based on an integral-volume, finite-difference method and is designed to simulate a wide variety of multidimensional, nonlinear, nonisothermal, and multiphase situations. STOMP was selected for the Composite Analysis because of computational efficiency and flexibility, its prior application to the Hanford Site vadose zone (Ward, Gee, and White 1997), and its thorough documentation (Nichols et al. 1997; White and Oostrom 1997; White and Oostrom 1996). STOMP is a candidate code for future performance assessment simulations in support of the TWRS ILAW.

Vadose zone stratigraphy for the Composite Analysis was defined at nine locations in and near the 200 East Area and at nine locations in and near the 200 West Area (Table 4.6). All but one of the stratigraphic profiles were defined at well locations from the geologic $\log$ and supporting information for the well. One of the stratigraphic profiles, labeled $218-\mathrm{W}-5$, was defined from a suite of wells located around low-level waste (LLW) burial ground 218-W-5. This was the same stratigraphic profile applied in the performance assessment for this burial ground.

Water table elevations for future conditions at each waste site location were calculated with the groundwater flow model. This information was used in the vadose zone transport calculations to define the bottom of the vadose zone. The elevation of the top of the vadose zone at each source was calculated from land surface elevations and depth to the bottom of the source, which was tabulated for each waste site. Because the elevation for the top of the vadose zone at a particular source generally did not match the elevation at the top of the stratigraphic profile applied to that source, an adjustment was made. If the 
elevation at the source was less than the top of the stratigraphic column, the portion of the column above that elevation was ignored. If the elevation at the source was greater than the top of the stratigraphic column, then the upper stratigraphic layer thickness was increased to make up the difference. A similar adjustment was made if the bottom of the defined stratigraphic column was below the water table elevation at the source. In this case the thickness of the lowest stratigraphic layer was increased.

Figure 4.4 illustrates the method used to estimate the flux to the water table. The source with an inventory of 6 units was assumed to completely release in three years. Three units leave the source and enter the upper vadose zone in the first year. Two units were assumed to leave the source in the second year, and one unit was assumed to leave the source in the third year. In the STOMP simulation, a single unit was assumed to enter the upper boundary of the simulated domain each year. After 3 years, half of the first unit released was predicted by STOMP to have passed through the lower boundary into the aquifer. The entire mass that was estimated by the release model to enter the vadose zone in the first year was assumed to have transported through the vadose zone and entered the water table at this time. The cumulative release to water table curve illustrates this for each of the three years' releases. Taking the derivative over time of the cumulative release curve provides an estimate of the instantaneous release. If the time between changes in the cumulative release is greater than the time periods used in the CFEST-96 simulations, the instantaneous inputs to CFEST-96 can become sharp peaks.

\subsubsection{Vadose Zone Model Results}

Existing plumes in the unconfined aquifer are the first measure of the expected response of the vadose zone transport model in the Composite Analysis. In an effort to match the response of the vadose zone model to field observation, the mass of the technetium-99 plume in 1996 was compared to the release forecast from liquid discharge sites and past leaks from single-shell tanks. These two types of sources represent the logical origin of existing plumes. For some liquid discharge sites, a considerable volume of waste was discharged over a relatively short period of time. The theory of vadose zone hydraulics implies that infiltration of these wastes into the vadose zone is limited by the saturated hydraulic conductivity of the vadose zone sediments. Because the model is one-dimensional, the least conductive of the sediment layers underlying the discharge site will define the infiltration rate.

Based on the discharge volume and duration for a given facility, and the governing saturated hydraulic conductivity, the cross section of the one-dimensional model was calculated. To account for lateral dispersion or spreading of the contaminant plume in the vadose zone, a sensitivity case examined the effect of increasing the cross section. It was determined that increasing the cross section by a factor of three produced a release by 1996 of $181 \mathrm{Ci}$ of technetium- 99 . Greater cross sections and larger factors have a diminishing affect on the estimated amount of nuclide breaking through to the water table. Estimates of the observed mass of technetium-99 in the aquifer vary from 15.8 to $37.6 \mathrm{Ci}$. Use of a

sufficiently high factor to cause the estimated release to drop to approximately $37.6 \mathrm{Ci}$ is not reasonable. Therefore, the factor of three was applied to all liquid discharge site releases. Additional adjustments of the technetium-99 release to the aquifer to result in an improved match with the existing plume are described in Section 4.3. 
Using the above model, the estimated releases of key mobile radionuclides into the water table are shown in Figures 4.5 through 4.13. The releases are shown for the periods 1940-2150 and 1940-3000. Releases for US Ecology, pre-1988 solid waste burial grounds, post-1988 solid waste burial grounds, tank leaks, tank sluicing losses, and other liquid release are shown. In general, liquid releases arrive first, followed by tank leaks and sluicing losses. Radionuclides leached from pre-1988 solid waste burial grounds and US Ecology arrive later. Finally, post-1988 solid waste burial grounds reach the water table. Primarily because of the surface cover or barrier applied to each, the ERDF waste and TWRS ILAW do not reach the water table within the 1500-year period simulated. All the releases are undecayed estimates for inventories estimated for 2050 .

Figures $4.5 \mathrm{a}$ and $4.5 \mathrm{~b}$ show the cumulative release of technetium-99 to the water table for the period 1940 through 2150 and 1940 through 3000 . Liquid discharge sites, tank leaks and pre-1988 solid waste burial grounds dominated releases prior to 2150 . Some tank sluicing losses also contributed in this period. Initial technetium- 99 release from post-1988 solid waste burial grounds began in approximately 2200. Shortly after that, first release occurred from the commercial low-level waste disposal facility.

The bulk of the technetium-99 inventory at the Hanford Site will be encapsulated in the TWRS ILAW. Of the inventory in single- and double-shell tanks, any not in the ILAW will be encapsulated in the immobilized high-activity waste from the tanks and will eventually be shipped to the national highlevel waste repository. Of the $1900 \mathrm{Ci}$ assigned to liquid discharges, tank leaks, and tank sluicing losses, and the $275 \mathrm{Ci}$ assigned to pre-1988 solid waste burial grounds, in excess of $1200 \mathrm{Ci}$ of technetium-99 is forecast by the model to be in the unconfined aquifer by 2150 . However, the rate at which it is predicted to enter the aquifer is lower than the rate that created the present technetium-99 plumes, and predicted concentrations in groundwater would be lower than in the current plumes.

Figures $4.6 \mathrm{a}$ and $4.6 \mathrm{~b}$ show the cumulative release of iodine- 129 to the water table for the period 1940 through 2150 and 1940 through 3000 . Liquid discharge sites and tank leaks dominated releases prior to 2150 . Tank sluicing losses are a relatively minor contributor to releases by 2150 , and remain a minor contributor through the year 3000 . Best-estimate distribution coefficients for iodine are small but nonzero, and prevent releases from other disposals of iodine-129 from reaching the water table in the 1000 years following Hanford Site closure.

Of the total inventory of $66 \mathrm{Ci}$ of iodine- 129 estimated to be at the Hanford Site, only $4.3 \mathrm{Ci}$ of iodine-129 are included in liquid discharges, tank leaks, tank sluicing losses, and pre-1988 solid waste disposals. Of that, the Composite Analysis projected approximately $0.5 \mathrm{Ci}$ were released to the aquifer by 1996 . This compares with an estimate of between 1.2 and $7 \mathrm{Ci}$ based on an integration of field observations. These estimates of iodine- 129 in the aquifer are highly dependent on the assumed distribution coefficient for iodine.in that they take into account both the aqueous and adsorbed masses of the isotope. Potentially, more significant than the apparent underestimate of existing contamination in the aquifer, is the fact that present and planned disposals account for less than $11 \mathrm{Ci}$ of the total $66 \mathrm{Ci}$ estimated as generated in the production reactors. 
Figures $4.7 \mathrm{a}$ and $4.7 \mathrm{~b}$ show the cumulative release of carbon-14 to the water table for the period 1940 through 2150 and 1940 through 3000 . Tank leaks and liquid discharge sites dominated releases prior to 2150 , and tank sluicing losses are a relatively minor contributor. The best-estimate distribution coefficients for carbon are small but nonzero, and as in the case of iodine, they prevent other disposals from releasing carbon-14 to the water table in the 1000 years following Hanford Site closure.

Nearly $5000 \mathrm{Ci}$ of carbon-14 were estimated to have been generated in the Hanford Site production reactors. However, estimates of the carbon-14 in liquid discharges $(3.7 \mathrm{Ci})$, tank leaks $(4.4 \mathrm{Ci})$, tank sluicing losses $(3.7 \mathrm{Ci})$, pre-1988 solid waste burials ( $<110 \mathrm{Ci}$ ), and post-1988 solid waste burials $(<20 \mathrm{Ci})$ total to a much lower inventory. The estimated solid waste inventories are based on cesium-137 inventory and isotopic ratios in 10-year old fuel, and therefore, are highly uncertain. Clearly, the inventory that was originally generated is not accounted for in estimated current and future disposals. It is important to note that the vast majority of carbon-14 to remain at Hanford Site resides in the graphite cores of the production reactors and the Composite Analysis indicates they do not release to groundwater in the 1000 years following Hanford Site closure.

Figures $4.8 \mathrm{a}$ and $4.8 \mathrm{~b}$ show the cumulative release of chlorine- 36 to the water table for the period 1940 through 2150 and 1940 through 3000 . Releases from the pre-1988 solid waste burial grounds dominate prior to 2150 . Small inventories for chlorine-36 estimated in liquid discharges produce releases that can barely be observed. Both of these sources are hypothetical. They are based on an assumed impurity level of $1 \mathrm{ppm}$ chlorine-35 in fuel irradiated in the production reactors, on cesium-137 levels in disposals, and on isotope ratios in 10-year old fuel. The release of chlorine-36 shown for the commercial LLW disposal site occurs later and is real in the sense that the inventory is based on shipment manifest records. The greatest inventory of chlorine- 36 resides in the graphite reactor cores and the Composite Analysis indicates it does not release to groundwater in the 1000 years following Hanford Site closure.

The pronounced steps in the cumulative release curve for chlorine-36 are an artifact of the methodology used to translate releases from waste sources to the water table. The commercial LLW disposal facility operated by US Ecology contains over $82 \%$ of the total inventory of sites expected to have any release to the water table within the first 1500 years. Because of the high solubility and low sorption $\left(\mathrm{K}_{\mathrm{d}}=0\right)$ of chlorine-36, nearly $20 \%$ of US Ecology's total chlorine-36 inventory is predicted to have entered the aquifer by 3000 .

Figures $4.9 \mathrm{a}$ and $4.9 \mathrm{~b}$ show the cumulative release of selenium-79 to the water table for the period 1940 through 2150 and 1940 through 3000 . Pre-1988 solid waste burial grounds dominated releases prior to 2150. Secondary contributions were from tank leaks and liquid discharge sites with a very minor contribution from tank sluicing losses. The high mobility of selenium-79 allows both solid waste and liquid disposals to contribute to the cumulative release. 
Selenium-79 generation in the production reactors was estimated at $800 \mathrm{Ci}$ by Agnew et al. (1997) and $1030 \mathrm{Ci}$ by Schmittroth et al. (1995). However, this isotope was only recently identified as potentially significant with respect to long-term dose, and previously was not included in inventory estimates for liquid discharges, leaks, or solid wastes. The isotopic ratio of selenium-79 to cesium-137 in 10-year old fuel was used to estimate the quantity of this isotope in these waste discharges. Accordingly, the significance of sources is directly related to the inventories assigned them. Pre-1988 solid waste burials were assigned $\sim 8.1 \mathrm{Ci}$, tank leaks were assigned $\sim 0.78 \mathrm{Ci}$, tank sluicing losses were assigned $\sim 0.63 \mathrm{Ci}$, liquid discharge sites were assigned $\sim 0.3 \mathrm{Ci}$, and post-1988 solid waste burial grounds were assigned $\sim 1.1 \mathrm{Ci}$. The total of these inventories is less than $11 \mathrm{Ci}$ and the Composite Analysis indicates slightly more than $6 \mathrm{Ci}$ release prior to 2150 . In an effort to be conservative or bounding with respect to future tank wastes, the TWRS program has assumed the entire inventory of selenium-79 is in the tanks and will be contained in the ILAW. However, if selenium-79 were assumed to be as abundant as other highly mobile radionuclides (e.g., technetium-99) in liquid discharges, then because of its mobility, a greater near-term release of this radionuclide would result.

Figures $4.10 \mathrm{a}$ and $4.10 \mathrm{~b}$ show the cumulative release of uranium-238 to the water table for the periods 1940 through 2150 and 1940 through 3000 . The small but nonzero distribution coefficient assigned to uranium for all waste forms was sufficient to retard its migration and result in no release to the water table from solid waste burial grounds or the commercial LLW disposal facility. Liquid discharge sites, especially ponds, are among the largest sources of uranium-238, and the Composite Analysis indicates fewer than $9 \mathrm{Ci}$ released from these liquid discharge sites. These releases are forecast to occur in the next decade. The model did not predict the significant release of uranium from the 216-U- 1 and 2 crib site. This is a result of the unique events (e.g., mobilization of deposits, flushing by new crib discharges, preferential flow down an unsealed reverse well) that created this particular release (Baker et al. 1988) compared to the generic approach taken in the Composite Analysis to analyze all key radionuclide disposals and discharges to the environment.

A significant inventory of approximately $54,300 \mathrm{Ci}$ of uranium (total) is assumed to reside in the ERDF, but none is forecast to reach the water table in the next 1000 years. The ERDF is assigned a substantial and perhaps unrealistic inventory of uranium. It is a conservative or bounding inventory estimate based on the maximum observed uranium concentration for contaminated soils or sediments at the Hanford Site, and on the total disposal volume forecast for the ERDF trench.

In addition to the key mobile radionuclides, releases of cobalt-60, americium-241, and neptunium-237 were evaluated for potential release and migration from the vadose zone. Cobalt- 60 is of interest because there is an existing plume; however, this radionuclide's short decay half-life greatly diminishes its mass and health impact by the time of Hanford Site closure. While there are no plumes of americium-241 in the aquifer today, this radionuclide's potential mobility in chelated wastes was of interest. Finally, neptunium-237 was included because it is a uranium daughter of some interest and generally appears as a contributor to dose in longer-term assessments (e.g., 10,000 years and beyond). 
Figures $4.11 \mathrm{a}$ and $4.11 \mathrm{~b}$ show the cumulative release of cobalt- 60 to the water table for the period 1940 through 2150 and 1940 through 3000 . The cumulative flux of cobalt-60 is less than $0.004 \mathrm{Ci}$ by 3000. However, inventories are for a decay date 2050. Cobalt has a half-life of 5.27 years, and it experiences significant decay prior to Hanford Site closure. All cobalt-60 released to groundwater is from cribs and specific retention trenches.

Figures $4.12 \mathrm{a}$ and $4.12 \mathrm{~b}$ show the forecast of cumulative release of americium-241 to the water table for the period 1940 through 2150 and 1940 through 3000 . Liquid discharge sites that received wastes containing organic complexants and radionuclides were shown to release approximately $130 \mathrm{Ci}$ of americium-241 to the aquifer. The model indicates releases dating back to the $1950 \mathrm{~s}$ and $1960 \mathrm{~s}$, and a cumulative release in 1996 of more than $100 \mathrm{Ci}$. Americium-241 has not been found in the aquifer. Obviously, this release is being overestimated. Dominant physicochemical processes governing the release, migration, and fate of americium-241 in the presence of organic complexants are not appropriately represented in the release and vadose zone models.

Figures $4.13 \mathrm{a}$ and $4.13 \mathrm{~b}$ show the cumulative release of neptunium-237 to the water table for the period 1940 through 2150 and 1940 through 3000. The Composite Analysis indicates liquid discharge sites release less than $0.012 \mathrm{Ci}$ of neptunium-237 to the groundwater by 3000 . Most of the neptunium237 , in excess of $0.01 \mathrm{Ci}$, was released to the water table by 2010. The model indicated cribs and ponds, notably $216-A-8,216-A-25,216-B-3$, and 216-B-7A \& B were the dominant sources of neptunium.

\subsubsection{Vadose Zone Model Sensitivity}

The sensitivity of the vadose zone model was investigated by varying the cross-sectional areas of the one-dimensional columns, the recharge rates, initial conditions, and distribution coefficients. For liquid discharges a relationship between the cross-sectional area of the column and volume and duration of the discharge was developed. Different area factors were applied to illustrate the sensitivity of the results to this cross-sectional area. Figures $4.14 \mathrm{a}$ and $\mathrm{b}$ show the sensitivity of the cumulative release to the area factor for all liquid discharge sites (see Tables 4.3 and 4.5 for further description of these sites) releasing technetium-99 for the years 1940 through 2040 and 1940 through 3000 . Increasing the area factor (i.e., reducing the recharge rate) delays and reduces the cumulative release to the water table.

The impact of different initial soil moisture conditions, consistent with three different steady recharge rates, on the cumulative flux from liquid and solid waste sites for a $K_{d}=0$ was also investigated. Figure 4.15 shows the results of the sensitivity analysis for initial soil moisture profiles consistent with steady recharge rates of 75,5 , and $0.5 \mathrm{~mm} / \mathrm{yr}$. A dry site, $218-\mathrm{E}-10$, and a wet site, 216-B-37, were analyzed based on the inventory estimates of technetium-99 for each site (see Tables 4.3 and 4.5 for further description of these sites). The recharge values used in the Composite Analysis for disturbed and coarse surface sediments maintained free of vegetation, a 2- or more-meter-thick surface barrier with natural vegetation, and a Hanford Protective Barrier were 75,5 , and $0.5 \mathrm{~mm} / \mathrm{yr}$, respectively. 
For both the dry and wet sites, increasing the initial soil moisture (i.e., higher recharge rate) results in earlier breakthroughs. However, by 2020 any difference in cumulative release as a result of the initial soil moisture condition is undetectable.

The effect of different assumed distribution coefficients was investigated for both liquid and solid waste sites. Figure 4.16 shows the response of the release and vadose zone model to varying distribution coefficients for technetium-99 release from the liquid discharge and solid waste burial sites. (See Tables 4.3 and 4.5 for further description of these sites.) Inventory estimates of technetium-99 for each site were used in this analysis. (Note, the analysis is generic and could use any nuclide. Technetium- 99 was not modeled as adsorbed in the environment in any other case in the Composite Analysis.) Hypothetical distribution coefficient values of $0.0,0: 1$, and $0.15 \mathrm{~mL} / \mathrm{g}$ were analyzed. It is important to remember that both sites are subjected to time-varying recharge rates. In both cases, as the distribution coefficient increases, less of the contaminant breaks through. The dry site shows the most profound decrease with no breakthrough estimated for the distribution coefficient of $0.15 \mathrm{~mL} / \mathrm{g}$ within the 1500 -year period simulated. The release from the liquid site is decreased by over three orders of magnitude. The results demonstrate that cumulative releases of adsorbed radionuclides are very sensitive to the selection of the distribution coefficient.

\subsubsection{Groundwater Flow Model}

The Composite Analysis used an existing three-dimensional numerical model for groundwater flow and solute transport in the Hanford Site unconfined aquifer (Wurstner et al. 1995; Barnett et al. 1997; Cole et al. 1997). This three-dimensional model was developed and enhanced as part of the Hanford Groundwater Project (HGWP) (Thorne and Chamness 1992; Thorne et al. 1993; Thorne et al. 1994; Wurstner et al. 1995; Cole et al. 1997). The three-dimensional model was developed to increase the understanding of future changes in water levels and to enhance predictions of contaminant plume movement being monitored by the HGWP (Cole et al. 1997). Applications and developments made on the HGWP's three-dimensional sitewide model of the Hanford Site unconfined aquifer are routinely reported in the Hanford Site's annual groundwater monitoring reports (e.g., Hartman and Dresel 1997).

The geologic and hydrologic data used in the sitewide model used in this Composite Analysis are discussed and summarized in the conceptual model report by Thorne et al. (1994) and the status report on the three-dimensional model implementation by Wurstner et al. (1995). As discussed in Thorne et al. (1994), the data needed to develop the three-dimensional conceptual model were derived from a variety of previous studies and ongoing Hanford Site investigations, as well as from work conducted specifically to support the sitewide model.

Hydraulic property data were obtained from the results of hydraulic tests documented in Bierschenk (1959); Kipp and Mud (1973); Deju (1974); Lindberg and Bond (1979); Graham et al. (1981); DOE (1988a); Liikala and Aaberg (1988); Thorne and Newcomer (1992); Peterson (1992); Connelly, Ford, and Lindberg (1992); Connelly, Ford, and Borghese (1992); Swanson (1992); Thorne et al. (1993); Connelly (1994); and Swanson (1994). Information was also obtained from new tests and tests that were 
previously undocumented. Information on the subsurface geologic framework came primarily from interpreting geologic descriptions of samples acquired during well drilling. These interpretations were based on work by Lindsey, Bjornstad, and Connelly (1991); Lindsey (1992); Lindsey et al. (1992); Lindsey and Jaeger (1993); Lindberg (1993a, 1993b); Hartman and Lindsey (1993); and Swanson (1992) in the 100,200, and 300 Areas of the Hanford Site, which use the lithofacies units outlined in Lindsey (1991).

Many of the wells used to define the geologic framework were drilled to basalt as part of a study for a proposed nuclear power plant (PSPL 1982). Other information used in defining the top of basalt came from wells drilled for the Basalt Waste Isolation Project (DOE 1988a), which studied the basalts underlying the Hanford Site for disposal of high-level nuclear waste. Approximately 550 wells were used to define the three-dimensional hydrogeologic structure of the unconfined aquifer system. Many of these wells were used to determine the elevation of the top of basalt, and not all have been interpreted over their entire depth. Information on the southern part of the Hanford Site and the Richland area came from studies conducted by the U.S. Geological Survey (Ebbert et al. 1993), from Liikala (1994), and from private well logs filed with the State of Washington Department of Ecology (Ecology). Information on the construction of Hanford Site wells was obtained from Chamness and Merz (1993) and from the Hanford Environmental Information System (HEIS) database.

\subsubsection{Background}

The Hanford Site lies within the Pasco Basin, a structural depression that has accumulated a relatively thick sequence of fluvial, lacustrine, and glacio-fluvial sediments. The geologic and hydrologic data used in the model were summarized in Wurstner et al. (1995) and are based on a number of reports published for the Hanford Site. The Pasco Basin and nearby anticlines and synclines initially developed in the underlying Columbia River Basalt Group, a sequence of continental flood basalts covering more than $160,000 \mathrm{~km}^{2}$. Overlying the basalt within the Pasco Basin are fluvial and lacustrine sediments of the Ringold Formation and the glacio-fluvial Hanford formation. Together, these sedimentary deposits comprise the Hanford Site unconfined aquifer system. The saturated thickness of this unconfined aquifer system is greater than $61 \mathrm{~m}$ in some areas but the thickness decreases and pinches out along the flanks of the basalt ridges. Depth to the groundwater ranges from less than $0.3 \mathrm{~m}$ near the Columbia River to more than $100 \mathrm{~m}$ near the 200 Areas. Groundwater in this unconfined aquifer system generally flows from recharge areas in the west to the Columbia River to the north and east.

Natural recharge to the unconfined aquifer system occurs from infiltration of 1) runoff from elevated regions along the western boundary of the Hanford Site, 2) spring discharges originating from the confined basalt aquifer system, and 3) precipitation falling across the Hanford Site. Some recharge to the unconfined aquifer also occurs along the Yakima River in the southern portion of the Hanford Site. Natural recharge from runoff and irrigation in the Cold Creek and Dry Creek valleys, upgradient of the Hanford Site, provides a source of groundwater inflow where these valleys enter the area of interest. Areal recharge from precipitation falling on the Hanford Site is highly variable, both spatially and 
temporally, and depends on local climate, soil type, and vegetation as discussed in Fayer and Walters (1995). The spatial variability in recharge resulting from the sitewide variation of these controlling parameters is illustrated in Figure 4.17. This figure shows ranges in recharge to make it easier to see the different recharge patterns and to relate them to specific features. For example, note the high recharge in the sand dunes area in the central part of the Hanford Site near the Columbia River. When overlaid on the computational grid, the actual distribution of recharge can vary on a grid-by-grid basis. This same recharge estimate (Figure 4.17) was used in the earlier three-dimensional model development efforts (Wurstner et al. 1995) as well as in the current Composite Analysis. Fayer developed this distribution for the 1979 time period using the same methods discussed in Fayer and Walters (Section 4.4.1, 1995). However, this recharge distribution was based on a different vegetation distribution. The recharge distribution developed in Fayer and Walters (1995) is not appropriate for this analysis because it reflects the effects of a large fire on the vegetation distribution. This altered vegetation distribution was not appropriate for the 1979 time period for which the model was calibrated, nor for long-term future conditions since the Hanford Site is expected to return to more natural vegetation patterns.

The other source of recharge to the unconfined aquifer is artificial recharge from wastewater disposal. Over the past 50 years the large volume of wastewater discharged to disposal facilities at the Hanford Site has significantly affected groundwater flow and contaminant transport in the unconfined aquifer. The volume of artificial recharge has decreased significantly during the past 10 years and is continuing to decrease. The major discharge facilities considered in this analysis are summarized in Wurstner et al. (1995). The major wastewater discharges from both past and future sources are summarized in Cole et al. (1997).

The boundaries for the Hanford Site unconfined aquifer system are the Columbia River to the north and east and basalt ridges on the south and west. The Columbia River represents the regional discharge for the unconfined aquifer. The amount of groundwater discharging to the river at any location and time is a function of the local hydraulic gradient and the local aquifer properties (specifically the hydraulic conductivity and saturated thickness). The hydraulic gradient is highly variable at any given time, since it is affected directly by the river stage which changes on a seasonal basis in response to precipitation and temperatures within the entire Columbia River basin upstream of the Hanford Site. The river stage, and thus hydraulic gradient, are also affected by weekly and daily changes in river flows at dams on the river, as determined by electric power generation needs, fisheries resources management, and other dam operations.

Hydraulic properties important to the conceptual model include both horizontal and vertical hydraulic conductivity, storativity, and specific yield. To apply a numerical model, the distribution of these parameters must be specified for each hydrogeologic unit. Hydraulic properties have been measured for the unconfined aquifer during pumping tests and from laboratory permeability tests. The results of these tests have been documented in published and unpublished reports over the past 50 years and in more recent summaries (DOE 1988a; Thorne and Newcomer 1992). As indicated in these documents, the quality of results from aquifer tests at the Hanford Site varies widely and has been affected by both aquifer conditions and analysis procedures. Thorne and Newcomer (1992) and Wurstner et al. 
(1995) analyzed the aquifer tests, many of which were single-well pumping tests, and selected the set of aquifer transmissivity calibration data used in the two-dimensional inverse model. The locations of wells that were tested to provide hydraulic properties used for model calibration are illustrated in Figure 4.18. The values illustrated in the figure are aquifer test interpretations of transmissivity in $\mathrm{m}^{2} / \mathrm{d}$.

The model of the unconfined aquifer system was calibrated to match 1979 water-table conditions. This time period was assumed by Jacobson and Freshley (1990) to approximate steady-state conditions during Hanford Site operations based on the fact the well hydrographs were steady and site discharges were relatively constant during this time period.

Key assumptions made for development of the groundwater flow model are listed in Table 4.9.

\subsubsection{Groundwater Flow Model Selection, Chronology, and Implementation}

The three-dimensional groundwater flow and transport model developed for the Hanford Groundwater Project and used in the Composite Analysis was implemented numerically using the CFEST code (Gupta et al. 1987; Cole, Yabusaki, and Kincaid 1988; Gupta 1997). The CFEST code was originally designed to support the radioactive waste repository investigations under DOE's Civilian Radioactive Waste Management Program (Gupta et al. 1987). It has also been effectively used by the chemical waste management community for conducting exposure assessments, evaluating remediation alternatives, and designing extraction and control systems for aquifers.

Selection. Descriptions of the capabilities and approach used in the CFEST code and its selection for the Hanford Groundwater Project are included in Evans et al. (1988) and Wurstner et al. (1995). The chronology in the continuing development of the Pacific Northwest National Laboratory (PNNL) sitewide model of the unconfined aquifer is outlined below. CFEST is an approved code for working on Hanford Federal Facility Agreement and Consent Order (also known as the Tri-Party Agreement; Ecology, EPA, and DOE 1989) milestones related to risk assessment (DOE 1991). The CFEST software library was extensively tested and brought under strict software quality assurance/quality control procedures by the Office of Nuclear Waste Isolation (ONWI) when it was developed by ONWI for DOE's Civilian Radioactive Waste Management Program. The supercomputer version (CFEST-SC), developed to run on all major UNIX work stations (Cole, Yabusaki, and Kincaid 1988), was used for all flow and transport modeling prior to FY 1997. In FY 1997, the refinement of sitewide three-dimensional model continued with its application to contaminant transport of selected contaminant plumes (Cole et al. 1997). An updated version of the CFEST code called CFEST96 (Gupta 1997) was used in this effort and in the Composite Analysis.

Composite Analysis results from CFEST are graphically displayed using the ARC/NNFO geographic information system (GIS). The ARC/NFO® GIS package is also used to store fundamental hydrogeologic data and information used to represent the three-dimensional conceptual model and to 
construct the three-dimensional numerical model. The three-dimensional visualization software package known as EarthVision $\otimes^{(a)}$ is used to manipulate hydrogeologic data for the conceptual model.

Chronology. Summarizing from the chronology discussed in Wurstner et al. (1995), a sitewide flow and transport model has been under continuous development by Pacific Northwest National Laboratory staff since the early 1960s as part of PNNL's continuing involvement the Hanford Site's groundwater monitoring efforts. The sitewide flow model and transport model capability has been and continues to be refined and updated as additional information is gathered and as conditions and application needs change at the Hanford Site. Pacific Northwest National Laboratory's Hanford Site unconfined aquifer model consists of a conceptual model and database that defines current system understanding.

Early flow models were two dimensional (i.e., the Variable Thickness Transient [VTT] code, Kipp et al. 1972) and transport modeling, depending on the application, was either of the advective type (i.e., the Hanford Pathline Calculation code [Friedrichs, Cole, and Arnett 1977]); quasi-three-dimensional particle tracking type (i.e., the Multicomponent Mass Transport [MMT] code [Alhstrom et al. 1977]); or multiple streamtube type (i.e., the TRANSS code [Simmons, Kincaid, and Resienauer 1986]). Early flow model calibration was carried out using a streamtube approach that used available field measurements of transmissivity, river stage, disposal rates to ground, and head in an iterative approach to determine the Hanford Site unconfined aquifer transmissivity distribution (Transmissivity Iterative Calculation Routine [Cearlock, Kipp, and Friedrichs 1975]). Applications of the VTT, MMT, and TRANSS codes at the Hanford Site are described by Freshley and Graham (1988).

In the mid 1980s, the CFEST code was selected for upgrading of Pacific Northwest National Laboratory's two-dimensional modeling capability. CFEST has been used to model the Hanford Site and a number of other sites in three dimensions (Dove et al. 1982; Cole et al. 1984; Gale et al. 1987; Foley et al. 1995). Evans et al. (1988), in a Hanford Site groundwater monitoring report for 1987, discuss selection of CFEST code for application to modeling flow and transport in the Hanford Site's unconfined aquifer.

Initial flow modeling with the CFEST code was two-dimensional as it had been with the previous VTT code. New data were used to recalibrate the CFEST two-dimensional groundwater flow model of the Hanford Site unconfined aquifer. A steady-state finite-element inverse calibration method developed by Neuman and Yakowitz (1979) and modified by Jacobson (1985) was used in this effort. All available information on aquifer hydraulic properties (e.g., transmissivities), hydraulic heads, boundary conditions, and discharges to and withdrawals from the aquifer were included in this inverse calibration. Initial inverse calibration efforts are described by Evans et al. (1988), final calibration results are described by Jacobson and Freshley (1990), and the calibrated two-dimensional model of the unconfined aquifer is described in.Wurstner and Devary (1993).

(a) EarthVision is a registered trademark of Dynamic Graphics, Inc., Alameda, California. 
Two-dimensional flow models used extensively at the Hanford Site prior to cessation of disposal operations were generally adequate for predicting aquifer head changes and directions of groundwater flow. This is because groundwater levels were somewhat stable through time across the Hanford Site. However, in the early 1990 s it was recognized that a three-dimensional model was needed for accurate calculation of future aquifer head changes, directions of groundwater flow, mass transport, and predictions of contaminant concentrations. The three-dimensional model was needed because there is significant vertical heterogeneity in the unconfined aquifer and the water table is dropping over most of the Hanford Site in response to cessation of large liquid disposals to ground. Development of a threedimensional model began in 1992 (Thorne and Chamness 1992) and was completed in 1995 (Wurstner et al. 1995). In the interpretation of the geohydrology of the Hanford Site unconfined aquifer, Thorne et al. (1994) indicate that it is composed of alternating series of transmissive units that are separated from each other in most places by less transmissive or mud units. Accounting for this vertical heterogeneity is particularly important for unconfined aquifer predictions at the Hanford Site as future water-table changes result in the dewatering of hydrogeologic layers. The water table is near the contact between the Hanford formation and the underlying, and much less permeable, Ringold Formation over a large part of the Hanford Site. Water level declines caused by decreased discharge at disposal facilities is causing and will continue to cause dewatering of the highly permeable Hanford formation sediments in some areas (Wurstner and Freshley 1994). This may result in aquifer transmissivity changes of an order of magnitude or more that would not be properly accounted for by two-dimensional flow and transport models that average vertical properties at each spatial location. As a result, changes in groundwater levels, groundwater flow direction, and contaminant transport can not be accurately simulated by a twodimensional model because the three-dimensional routing of groundwater flow and contaminant mass resulting from the vertical heterogeneity can not be properly accounted for. These three-dimensional effects are especially important to the Composite Analysis because the purpose of a composite analysis is to add together different plumes by accounting for the superposition of plumes from different sources through time. Changes along the migrating front of desaturating sediments can provide the means for plumes emanating from different places and at different times to composite. Such issues can not be properly addressed by a two-dimensional model or even a two- or three-layer, three-dimensional model because there is no ability (two-dimensional model) or limited ability (simple three-dimensional model) for one plume to migrate under another.

The initial three-dimensional model of the Hanford Site unconfined aquifer (Wurstner et al. 1995) was calibrated in a two-step process. In the first step the two-dimensional model was recalibrated with a steady-state, statistical inverse method implemented with the CFEST-INV computer code Devary (1987). The two-dimensional transmissivity distribution from this inverse modeling was preserved during the calibration of the three-dimensional model as is described in Wurstner et al. (1995).

The final improvements and calibration of the Pacific Northwest National Laboratory sitewide model used in this Composite Analysis were carried out during FY 1996 and FY 1997 as part of the HGWP. The purpose of this effort was to assist the HGWP in interpreting monitoring data; to investigate contaminant mass transport issues and evaluate the future movement of existing contaminant plumes; and to identify and quantify potential groundwater quality problems for onsite and offsite use. The report 
on this effort (Cole et al. 1997) describes the improvements to the three-dimensional model, the model recalibration, and the application of the model to predict the future transport of existing contaminant plumes in the unconfined aquifer. The Cole et al. (1997) report presents predicted changes in transientflow conditions in the unconfined aquifer to the year 4000 . This provided the hydrologic basis for simulating migration of existing contaminant plumes presented in the Cole et al. (1997) report as well as the future contaminant plume migration simulated as part of the Composite Analysis. The contaminant migration results used in the Composite Analysis that are described in the Cole et al. (1997) report include: the transport of the tritium plume resulting from future operations of the State-Approved Land Disposal Site (SALDS), and the transport of the existing tritium, iodine-129, technetium-99, uranium, and strontium-90 plumes originating from the 200 Areas. Tritium plume migration resulting from future operations of the SALDS is presented in more detail in Barnett et al. (1997).

Implementation. The lateral extent and relationships between the nine hydrogeologic units of the Ringold Formation and Hanford formation determined to be sufficient to adequately represent the unconfined aquifer were defined by determining geologic contacts between these layers at as many wells as possible. These interpreted distributions and thicknesses were integrated into EarthVision@, which was used to construct a database for formulation of the three-dimensional Hanford Site conceptual model. The resulting numerical model contains nine hydrogeologic units above the top of the underlying basalt. A brief summary of each of these units, based on descriptions in Wurstner et al. (1995), is provided in Table 4.10 .

A depiction of the surface finite-element grid and boundary conditions used in the three dimensional flow (and transport) model is illustrated in Figure 4.19. The finite-element grid was designed for the Composite Analysis to increase the overall effectiveness of the three-dimensional model in simulating transport problems. Most of the interior surface elements are regular elements that are $375 \mathrm{~m}$ on a side. Surface elements away from the 200 Area Plateau are larger. The total number of surface elements in the three-dimensional model is 2991 elements. The three-dimensional model, based on this surface grid, comprises a total of 23128 elements (2991 surface and 20137 subsurface elements) and 23668 nodes.

The Columbia River boundary in the updated three-dimensional model extended from the Hanford Site shore of the river to the middle of the river channel to reflect the hydraulic interaction of the unconfined aquifer and the river. The surface node at the river boundary was simulated as a prescribed head boundary condition reflective of the assumed river stage that was based on a long-term river stage average. The Columbia River was represented as a constant head boundary along half of its width by having the constant head nodes at both the edge and centerline of the river. Nodes below the surface, along the centerline of the river, were simulated as no-flow boundaries. This design provides a more accurate approximation of the upward movement of groundwater controlled by the hydraulic gradient between the aquifer and the river. The CHARIMA river simulation model (Walters, Richmond, and Gilmore 1994) was used to generate long-term average water surface elevations for the Columbia River based on 1979 conditions. 
At the Cold Creek and Dry Creek valleys (Figure 4.19), the unconfined aquifer extends westward beyond the boundary of the Hanford Site groundwater flow model and as a result the unconfined aquifer is recharged from these sources. Additionally the unconfined aquifer also is recharged from springs and runoff that infiltrate the aquifer along the northern side of the Rattlesnake Hills along the western edge of the model (Figure 4.19). To approximate the groundwater flux entering the modeled area from these valleys and the Rattlesnake Hills, both prescribed head and prescribed flux boundary conditions were defined. For the steady-state model calibration runs, a prescribed head boundary condition was specified for Cold Creek and Dry Creek Valleys as well as along the Rattlesnake Hills. Once calibrated, the steady-state model was used to calculate the flux condition that was then used in the transient simulations. The prescribed flux boundary was used because it better represents the response of the boundary to a declining water table than a prescribed head boundary.

Since the description of the sitewide model provided in Wurstner et al. (1995), a number of changes have been made to the extent of the model, model boundary conditions, and model grid. These changes were made to reflect the most recent understanding and interpretation of the unconfined aquifer system by the Hanford Groundwater Project. The most significant changes incorporated in the current version of the sitewide models were derived from reinterpretation of the 1979 water table surface of the unconfined aquifer and the top of the basalt. The reinterpretation led to changes in both internal and lateral boundary conditions, including:

- Moving the model boundary inward along Rattlesnake Ridge and the Yakima River to more closely approximate the location where basalt intersects the water table surface

- Increasing the extent of basalt subcrops above the water table surface in areas south and east of Gable Mountain and northwest of Gable Butte, to more closely approximate the location where basalt intersects the water table surface.

Simulations of Hanford Site water table conditions for the Composite Analysis focused on predicting the impact of ceasing the wastewater discharges that have been used extensively as a part of waste management practices. Previous analyses of post-Hanford Site unconfined aquifer conditions have considered land uses such as large-scale irrigation on the Hanford Site that could significantly alter the long-term behavior of the unconfined aquifer beneath the Hanford Site. The potential for large-scale agricultural irrigation at on the Hanford Site in the future was examined for the Composite Analysis. Consultations with staff from the Agricultural Research Service at the Agricultural Experiment Station in Prosser, Washington, resulted in the conclusion that the prospect of large-scale irrigation occurring on the Hanford Site is unlikely for the following reasons.

- Public acceptance of food products grown on the Hanford Site, regardless of the actual risk associated with agricultural development, is uncertain.

- Sufficient water rights within the Pasco Basin for development of crops requiring large-scale irrigation on the Hanford Site are unavailable. If agriculture should develop on the Hanford Site, it is 
likely that the crops to be planted will use the efficient and focused irrigation methods (e.g., drip irrigation) that are used in fruit orchards or vineyards.

- New technologies and advanced resource management practices will likely eliminate or significantly curtail over-irrigation of crops.

- The availability of sufficient water rights and land in the East High portion of the Columbia Basin Project suggests, in the event of a developing national or international need for increased agricultural production, that other areas of the State of Washington would be developed before the Hanford Site would be used.

Prior to conducting contaminant transport simulations with the three-dimensional model, the previous steady-state, two-dimensional model of the unconfined aquifer system was calibrated to 1979 water table conditions with a statistical inverse method implemented in the CFEST-INV computer code Devary (1987). The three-dimensional model was calibrated by preserving the spatial distribution of transmissivity from the two-dimensional inverse modeling. The vertical distribution of hydraulic conductivity at each spatial location was interpreted based on the inverse transmissivity value and the available three-dimensional hydraulic property data, that included: data on the geologic structure, facies data, generic property values based on facies descriptions. A complete description of the seven-step process used to distribute the transmissivity distribution derived from the inverse calibration among the major conductive hydrogeologic units is described in Cole et al. (1997).

The transient behavior of the three-dimensional flow model was calibrated by adjusting model storage properties (specific yield) until transient water-table predictions approximated observed watertable elevations between 1979 and 1996. Following the steady-state and transient calibrations, the threedimensional model was applied to predict the future response of the water table to postulated changes in Hanford Site operations.

\subsubsection{Groundwater Flow Model Results}

The three-dimensional model was used to simulate transient-flow conditions from 1996 through the year 4000 , based on the distribution of hydraulic conductivity from the steady-state calibration and the distribution of specific yields developed from the transient calibration ( 0.25 for Hanford formation layers and 0.1 for the Ringold Formation layers). The water table contours estimated for the years 2000 (Figure 4.20), 2100 (Figure 4.21), 2200 (Figure 4.22), and 2350 (Figure 4.23) with the three-dimensional model, predict an overall decline in the water table and hydraulic gradient across the entire site. The different areas approach steady state at varying rates, as illustrated in Cole et al. (1997). The areas north of the gap between Gable Butte and Gable Mountain along the Columbia River have the shortest time constants, and water levels in this region reach steady state by the year 2100 . The area between the Gable Butte and Gable Mountain reach steady-state conditions sometime between the years 2200 and 2300. The rest of the Hanford Site, including the area south of Gable Mountain and east of the 200 West Area, all are predicted to reach steady-state conditions by the year 2350 . 
Over about a 300-year period following elimination of wastewater discharges to the ground at the Hanford Site, the water table is predicted by the model to decline significantly and return to near preHanford Site conditions that were estimated to exist in 1944, Kipp and Mudd (1974). Over this period, the water table is predicted to drop as much as $11 \mathrm{~m}$ beneath the 200 West Area near U Pond and 7 to $8 \mathrm{~m}$ beneath the 200 East Area near B Pond. The areas of the model predicted to be different from the estimated 1944 conditions include: the area west of the 200 West Area, where higher predicted hydraulic heads reflect the effects of increased irrigation from upgradient regions; and the area of the North Richland well field, where annual injection and withdrawal sequences are assumed to continue.

Flow modeling results also suggested that as water levels drop in the vicinity of central areas in the model, the saturated thickness of the unconfined aquifer greatly decreases and may eventually dry out south of Gable Mountain along the south east extension of the Gable Butte anticline. This could cause the unconfined aquifer to the north and south of this line to become hydrologically separated. As a result, flow paths from the 200 West Area and the northern half of 200 East Area that currently extend through the gap between Gable-Butte and Gable Mountain, effectively may be cut off in the future. In time, the overall water table, including groundwater mounds near the 200 East Area will decline. As a result, the groundwater movement from the 200 Area Plateau would shift to a more west-to-east pattern of flow toward points of discharge along the Columbia River between the old Hanford town site and the Washington Public Power Supply System facility.

\subsubsection{Groundwater Transport Model}

A groundwater transport model based on the CFEST-96 code, discussed above, was developed and implemented for the Composite Analysis. This model was used to evaluate the future migration and fate of existing contaminant plumes (Cole et al. 1997) as well as the development and migration of plumes from future sources of unconfined aquifer contamination predicted by the source term release and vadose zone transport model discussed earlier.

\subsubsection{Background}

Transport simulations of both existing plumes and plumes from future sources were based on the previously described three-dimensional flow model. Transient flow conditions were used to provide the basis for all Composite Analysis modeling transport predictions.

Additional model parameters are required to model the contaminant transport processes of dispersion and adsorption. These additional model parameters include longitudinal and transverse dispersivities $\left(D_{1}\right.$ and $\left.D_{\mathfrak{k}}\right)$ and contaminant retardation factors $\left(R_{f}\right)$. The key assumptions made in the development of the contaminant transport model are listed in Table 4.11. 


\subsubsection{Groundwater Transport Model Implementation}

Dispersivity "the most elusive of the solute transport parameters" (Freeze and Cherry 1979) cannot be directly measured in the field or laboratory. It is determined by inverse modeling of tracer test breakthrough curves from tests performed at the transport scale of interest and in the geohydrologic system of interest (Farmer 1986). Freeze and Cherry (1979) indicate that values of longitudinal and transverse dispersivities are significantly larger than values obtained in laboratory experiments on homogeneous materials and materials with simple heterogeneity. No field tests have been conducted at the Hanford Site to develop an estimate for this parameter at the scale of transport appropriate for the Composite Analysis.

General studies indicate that dispersivity is a function of both time and transport distance because of unaccounted for temporal changes and unaccounted for heterogeneities. The U.S. Environmental Protection Agency (EPA), in their guidance for water quality assessment screening for toxic and conventional pollutants in surface and groundwater (Mills et al. 1985), indicates "A rough estimate of longitudinal dispersivity in saturated porous media may be made by setting $D_{1}(\mathrm{~cm})$ equal to $10 \%$ of the mean travel distance." This rule of thumb is based on analysis of tracer tests performed over a large range of laboratory and field scales and for a wide variety of aquifers.

The original work was performed by Lallemand-Barres and Peaudecerf (1978) and expanded by Gelhar and Axness (1981). Later in 1992, Gelhar, Welty, and Rehfeldt reexamined the data and indicated that because of the potential unreliability of the data that no definite conclusion regarding the rule could be reached beyond transport distances of $100 \mathrm{~m}$. However, this was later refuted by Neuman (1993).

Dispersivity is theoretically expected to have an asymptotic value that can be related to the scale of uncharacterized aquifer heterogeneity (Farmer 1986). In contaminant transport simulations, large values of dispersivity result in lower peak concentration estimates, but give rise to earlier first arrival times that can increase arrival concentrations of radionuclides with short half-lives. For the Composite Analysis, a longitudinal dispersivity, $\mathrm{D}_{\mathrm{l}}$, of $95 \mathrm{~m}$ was selected. This is not inconsistent with observations made in Freeze and Cherry (1979) that longitudinal dispersivities as large as $100 \mathrm{~m}$ and lateral dispersivities as large as $50 \mathrm{~m}$ have been used in migration studies of large contaminant plumes. As discussed in Wurstner et al. (1995), the 1/10 approach has generally been used in the past for determining dispersivity values for Hanford Site transport modeling. Law (1992) used values of $D_{1}=43 \mathrm{~m}$ and $D_{t}=12 \mathrm{~m}$ for a scale of $9500 \mathrm{~m}$ based on values compiled in Gelhar et al. (1985). An earlier model (Golder Associates 1990) used values of $15 \mathrm{~m}$ and $1.5 \mathrm{~m}$, respectively, for longitudinal and transverse dispersivity, which were also based on Gelhar et al. (1985).

It should be also recognized that the dispersivity values, determined from field tests at 59 different sites compiled by Gelhar, Welty, and Rehfeldt (1992), included results from two investigations at the Hanford Site. The first was a $1950 \mathrm{~s}$ tracer test that resulted in values of $D_{1}=6 \mathrm{~m}$ for the Hanford formation and $D_{1}=460 \mathrm{~m}$ for the Ringold Formation, as reported by Bierschenk (1959). Also included 
are values of $D_{1}=30.5 \mathrm{~m}$ and $D_{t}=18.3 \mathrm{~m}$ for a scale of $20,000 \mathrm{~m}$. These were calculated from twodimensional transport modeling of the 200 East Area tritium plume as reported in Ahlstrom et al. (1977).

Dispersivity is likely to vary across the Hanford Site depending on the degree of heterogeneity and the temporal variability of flow gradients. Ahlstrom et al. (1977) noted that the ratio of $D_{1}$ to $D_{t}$ calculated from their model of the Hanford Site was much higher than the ratio expected. They attributed the high ratio to heterogeneity. However, horizontal dispersion may have been enhanced by temporal variations in flow gradients caused by disposal practices. The flow paths for the tritium transport from the 200 East Area have gradually shifted from due east to a south-easterly direction, in response to wastewater discharges to B Pond and the 200 East Area. This shift in the flow path has enhanced the apparent dispersion of the tritium plume emanating from the 200 East Area. More recent sitewide modeling studies (Law et al. 1996) used values of $D_{1}$ and $D_{t}$ of $30.5 \mathrm{~m}$ and $3 \mathrm{~m}$ respectively, which appear to be related to the transport grid spacing of $100 \mathrm{~m}$. In the recent Hanford Low-level Tank Waste Interim Performance Assessment (Mann et al. 1997) the horizontal dispersivity for aquifer transport was set at $10 \%$ of the travel length in the direction of flow and in the vertical direction at $1 \%$ of the travel length. The Draft Hanford Remedial Action EIS (DOE 1996a) set transverse dispersivity at $1 / 5$ of the longitudinal value: Longitudinal dispersivities were based on the scale dependency relationships between longitudinal dispersivity and mean travel distance discussed in Walton (1985).

While the value of $D_{1}=95 \mathrm{~m}$ is not based on any Hanford Site data, it satisfies all three of the following constraints on its value:

1. The numerical constraint is related to the grid Peclet number, $P_{e}=\left(\right.$ grid spacing) $/ D_{1}$. For finite element transport simulations $\mathrm{P}_{e}<4$ are required for acceptable solutions (Campbell, Longsine, and Reeves 1981). The 95-m dispersivity estimate is approximately one quarter of the grid spacing in the finest part of the model grid in the 200 Area Plateau where the smallest grid spacing is on the order of about $375 \mathrm{~m}$ by $375 \mathrm{~m}$ (Figure 4.19).

2. At the grid scale of $375 \mathrm{~m}$ used in this modeling, the modeled system is homogeneous. Heterogeneities at scales less than 375 meters are uncharacterized. The $95-\mathrm{m}$ dispersivity value selected satisfies this constraint.

3. Finally, because it is more than $10 \mathrm{~km}$ from the closest source in the 200 East Area to the Columbia River, a nonasymptotic value of $1000 \mathrm{~m}$ for the longitudinal dispersivity could be appropriate. Because large values of dispersivity are not conservative in transport simulations, the 95-m dispersivity value selected for use in the Composite Analysis is the smallest value that could be used with the grid spacing selected. Applying the rule of thumb, discussed above, estimates of concentration $950 \mathrm{~m}$ from the source should be accurate and for greater distances they should be conservative. 
With regard to transverse dispersivity the following is noted:

- EPA guidance (Mills et al. 1985 ) is $1 / 3$ for the ratio of $D_{1} / D_{t}$.

- Freeze and Cherry (1979) indicate transverse dispersivity is lower by a range of 5 to 20 (i.e., 0.2 to 0.05 ).

- Walton (1985) states that reported ratios of $D_{l} / D_{\mathfrak{t}}$ vary from 1 to 24 but that common values are $1 / 5$ and $1 / 10$.

The transverse dispersivity, $\mathrm{D}_{t}$ used in these simulations was assumed to be approximately $20 \%$ of the longitudinal dispersivity. Thus, a transverse dispersivity of $20 \mathrm{~m}$ was used in all simulations.

With regard to sensitivity, a 45-m grid spacing was used in the recent 200-West Effluent Treatment Facility study (Barnett et al. 1997) with dispersivities of $20 \mathrm{~m}$ and $2 \mathrm{~m}$ (longitudinal and lateral respectively). Comparing these results with the Composite Analysis results the peak values and resolution were less because of the larger grids but the general character of the predicted plumes was much the same (see Section 4.3).

The vertical grid spacing for the transport (as well as the flow) model consisted of multiple transport layers that subdivided the nine hydrostratigraphic units. The basic thickness of these transport layers was $8 \mathrm{~m}$. The transport layers were defined from the water table surface to the basalt to account for the overall declining water table and to adequately represent contaminant concentrations in the threedimensional model. At every model node each of the nine hydrostratigraphic units below the water table was represented by at least one transport model layer. Nonconductive (e.g., mud units) below the water table were always represented by at least 2 transport model layers regardless of their saturated thickness in order to assure the vertical flow and transport through these units was appropriately represented. For units whose saturated thickness was $<12 \mathrm{~m}$ thick, the layer thickness was set to the actual saturated thickness of the unit. Nonconductive and conductive units whose saturated thickness was $>12 \mathrm{~m}$ were divided into multiple transport model layers in the same manner. For all units with thickness $>12 \mathrm{~m}$, the transport layering algorithm is as follows: create as many uniform 8-m transport layers as possible until the remaining unaccounted for saturated thickness is $>12 \mathrm{~m}$ but $<=16 \mathrm{~m}$, then create two additional transport layers set to half of the remaining saturated thickness of the hydrostratigraphic unit being layered.

Calculation of the effective contaminant retardation factors required estimates of contaminantspecific distribution coefficients as well as estimates of effective bulk density and porosity of the aquifer materials. Detail on contaminant-specific distribution coefficients measured or estimated for the unconfined aquifer is summarized in Appendix E. No adsorption was accounted for in simulation of the tritium and technetium-99 plumes in the Composite Analysis. However, for the iodine-129, uranium, and strontium-90 plumes, best-estimate distribution coefficients were developed and applied. 
In addition to the estimated distribution coefficient, calculation of contaminant-specific retardation factors used in the transport model required estimates of the effective bulk density and porosity. A bulk density of $1.9 \mathrm{~g} / \mathrm{cm}^{3}$ was used for the calculation of retardation factors in all groundwater transport simulations in the Composite Analysis. The effective porosity was estimated from specific yields obtained from multiple-well aquifer tests, which ranged from 0.01 to $0.37 \mathrm{~cm}^{3} / \mathrm{cm}^{3}$. Laboratory measurements of porosity available for samples from a few Hanford Site wells, which ranged from 0.19 to $0.41 \mathrm{~cm}^{3} / \mathrm{cm}^{3}$, were also considered. The few tracer tests conducted at the Hanford Site indicated a range in effective porosity from 0.1 to $0.25 \mathrm{~cm}^{3} / \mathrm{cm}^{3}$. Based on the ranges of values considered, a bestestimate value of the effective porosity of $0.25 \mathrm{~cm}^{3} / \mathrm{cm}^{3}$ was used for the calculation of retardation factors in all groundwater transport simulations in the Composite Analysis.

Transport simulations were developed to evaluate the future migration and fate of selected existing contaminant plumes, and to identify and quantify potential radiological impacts of offsite use of groundwater. Monitoring of groundwater in the unconfined aquifer has detected a number of radioactive contaminant plumes emanating from various operational areas (Hartman and Dresel 1997). The most widespread plumes are from tritium and iodine-129. Smaller plumes of strontium-90, technetium-99, and plutonium contain concentration levels of these constituents exceeding EPA and the State of Washington interim drinking water standards (DWS). Uranium concentrations are also found at levels greater than the proposed DWS. In recent years, areas of groundwater contaminated by cesium-137 and cobalt- 60 have also been found at or exceeding the DWS.

The existing contaminant plumes in the unconfined aquifer simulated for the Composite Analysis included the tritium, iodine-129, technetium-99, uranium, and strontium-90 plumes. Each of the transport simulations was based on the predicted future transient-flow conditions and the high-resolution finite-element grid designed to resolve areas of future plume transport. Interpreted plume maps for 1996 (Hartman and Dresel 1997) were used to represent initial conditions for the existing plume simulations. The initial conditions for the existing tritium, iodine-129, technetium-99, uranium, and strontium-90 plumes are illustrated in Cole et al. (1997).

Transport of future contaminant releases to the unconfined aquifer for source areas in the exclusive waste management area were evaluated to examine the future movement of contaminant plumes resulting from these releases to areas outside of the buffer zone. Radionuclides evaluated include future releases of technetium-99, iodine-129, carbon-14, chlorine-36, selenium-79, and uranium.

\subsubsection{Groundwater Transport Model Results}

Groundwater transport simulation results used in the Composite Analysis were performed in two steps. Transport of the tritium plume resulting from future operations of the SALDS, and the transport of the existing tritium, iodine-129, technetium- 99 , uranium, and strontium- 90 plumes originating from the 200 Areas were simulated as part of the Hanford Groundwater Project effort (Cole et al. 1997) discussed above. All other plumes related to future sources were simulated as part of the Composite Analysis 
using the same model presented in Cole et al. (1997). The existing contaminant plumes in the unconfined aquifer were transported from their current distributions with the hydraulic gradients that are projected for the future as the groundwater system responds to cessation of wastewater discharges. As discussed in Cole et al. (1997), simulations for all existing plumes except for tritium began in 1996. The initial conditions for these simulations were based on the plumes presented in the Hanford Site groundwater monitoring report for FY 1996 (Hartman and Dresel 1997). The tritium plume simulation was run from 1979 through 2100 and started with initial conditions interpreted from 1979 monitoring data and presented in Cole et al. (1997). Cole et al. (1997) compare simulation results for the 1996 tritium plume with interpretations from monitoring observations reported in Hartman and Dresel (1997).

Separating the analysis of plumes resulting from future leaching of contaminants from the vadose zone, from the analysis of the migration of existing plumes, facilitated interpretation of results. The existing contaminant plumes superimpose with the plumes generated by future releases of contaminants considered in the Composite Analysis. Radiological doses resulting from the separate simulations were simply added together in $\mathrm{ARC}_{\mathrm{ANFO}}{ }^{\circ}$ to produce the final results. To illustrate the fate and transport of contaminants considered in the Composite Analysis, the predicted distributions of the contaminant plumes are shown at their times of peak concentration in the unconfined aquifer (which is prior to the start of the compliance period).

The plan-view, maximum-concentration plots discussed in this subsection were prepared from the three-dimensional model results through a sampling process that determined the maximum at each location in space. This process involved sampling the vertical stack of nodes at each plan view location in the grid (Figure 4.19) in order to find the maximum concentration calculated at any depth in the profile. The contour plots of concentration shown represent the spatial distribution of maximum concentration values. The radiological doses resulting from the separate plume simulations were constructed from these maximum plan-view concentration distributions and added together in $\mathrm{ARC} / \mathrm{INFO}{ }^{\circledR}$ to produce the final results.

Figure 4.24a illustrates the predicted distribution of tritium in the unconfined aquifer in 1997, and Figure $4.24 \mathrm{~b}$ illustrates the predicted tritium distribution in 2050 , the start of the compliance period. All of the tritium considered in the Composite Analysis is from existing plumes or SALDS disposal. Figure 4.25a illustrates the distribution of technetium-99 from existing sources in 1996, the time of peak concentration, and Figure 4.25b illustrates the predicted technetium-99 distribution in 2049, approximately the start of the compliance period. Figure 4.26 illustrates the distribution of technetium- 99 from all sources in 2036 , at a time when the technetium- 99 produces a secondary peak in the groundwater. Figure 4.27a illustrates the distribution of iodine-129 in groundwater in 2036, and Figure 4.27b illustrates the predicted iodine-129 distribution in 2049, approximately the start of the compliance period. Strontium-90 peaks from existing plumes in 1996; carbon-14 from future sources peaks in 2027; chlorine-36 from future sources peaks in 2019; selenium-79 from future sources peaks in 2005, and uranium (total) from existing sources peaks in 1996. Concentration plots at time of peak concentration 
and at 2049, approximately the start of the compliance period (i.e., 2050) are shown for strontium-90; carbon-14; chlorine-36; selenium-79, and uranium (total) in Figures 28 (a-b), 29 (a-b), 30 (a-b), 31 (a-b), and $32(a-b)$, respectively.

\subsubsection{Groundwater Transport Model Sensitivity}

Wastes from some sites will be released to the groundwater pathway in the far future. To investigate this issue, a series of nine transport model sensitivity runs were made. These runs examined the expected variation in transport model response to source location in the far future to determine if plume formation at various waste sites was significantly different once the water table reached steady state. In each of these transport sensitivity runs $1 \mathrm{Ci}$ per year of a hypothetical long-lived radionuclide was released each year for a 20-year period starting in 3899. The total release over the 20-year period would thus be $20 \mathrm{Ci}$. The year 3899 has no particular significance. This time period was chosen for these sensitivity runs because transient flow simulation results for this far future time period were available, and it was believed that results for this time period would better represent future steady-state conditions when effects of previous Hanford Site discharge mounds would be minimal.

Four node locations were selected in the 200 East Area to represent hypothetical releases from the AX and AY Tank Farms, the BX and BY Tank Farms, the C Tank Farm, and the future TWRS ILAW. Similarly four node locations were selected in the 200 West Area to represent release from the T Tank Farm, the TX and TY Tank Farm, the U Tank Farm, and the S and SX Tank Farm. The ninth location selected was the node that would best represent release from the US Ecology site. Results of these runs in the form of maximum concentration versus time plots are shown in Figure 4.33. These plots show the predicted maximum concentration (at any depth) versus time at each of the nine source location nodes. Analysis of these results indicates that the time required to reach the maximum concentration at a source node is generally shorter in the 200 East Area (3 years at BX-BY Tank Farm source node, 5 years at C Tank Farm source node, and approximately 10 years at AX-AY Tank Farm and TWRS ILAW disposal site source nodes) compared to more than 20 years at all four nodes representing losses from tank farm sites in the 200 West Area. Additionally, source node peak concentrations in the 200 East Area are lower (i.e., $679 \mathrm{pCi} / \mathrm{L}$ at the BX-BY Tank Farm source node, $2051 \mathrm{pCi} / \mathrm{L}$ at TWRS $\mathrm{LAW}$ source node, $2713 \mathrm{pCi} / \mathrm{L}$ at $\mathrm{AX}-\mathrm{AY}$ Tank Farm source node, and $2980 \mathrm{pCi} / \mathrm{L}$ at $\mathrm{C}$ Tank Farm source node) than 200 West Area source node peaks (i.e., $12866 \mathrm{pCi} / \mathrm{L}$ at S-SX Tank Farm node, and between 15000 and $16000 \mathrm{pCi} / \mathrm{L}$ at T, TX-TY, and U Tank Farm source nodes). The response at US Ecology is somewhat in between the 200 East Area and 200 West Area responses, although it is closer to the 200 West Area results. These results can be scaled up or down to investigate the effect of different postulated future release rates at sites in these areas.

\subsubsection{Atmospheric Model}

The atmospheric pathway was evaluated for a single suite of sources in the Composite Analysis. Based on a review of previously completed analyses that showed minimal contribution to all-pathways 
dose from the atmospheric pathway, only the graphite cores from the production reactors were assumed to release contaminants that could be transported via the atmospheric pathway.

\subsubsection{Background}

The evaluation of the atmospheric pathway in the Composite Analysis only considered potential exposures to individuals living in the vicinity of the releases. Radionuclides released to the atmosphere were transported downwind from the solid waste burial ground that contained the graphite cores. The location employed in this analysis was assumed and simply placed the cores in the northwestern portion of the 200 West Area.

The key assumptions made for development of the atmospheric transport model are listed in Table 4.12.

\subsubsection{Atmospheric Model Implementation}

Unit transport factors (UTFs) were calculated for the postulated release originating within the exclusive waste management area. The atmospheric transport of gaseous radionuclides was evaluated with the Multimedia Environmental Pollutant Analysis System (MEPAS). Buck et al. (1995) and Droppo and Buck (1996) describe the MEPAS code. The MEPAS code is based on the sector-averaged Gaussian model, which is the method recommended for dose calculations performed for releases from Hanford Site facilities (Schreckhise et al. 1993).

The UTFs provide estimates or air concentration and deposition rate to soil as a function of distance and direction from each source area. The UTFs were normalized to an annual release of $1 \mathrm{pCi}$ of each radionuclide and provided air concentration estimates in units of $\mathrm{pCi} / \mathrm{m}^{3}$ and deposition rates in units of $\mathrm{pCi} / \mathrm{m}^{2} / \mathrm{yr}$. The emission was assumed to occur uniformly over an area source $100 \mathrm{~m}$ by $600 \mathrm{~m}$. Recommended atmospheric data from Schreckhise et al. (1993) were used to perform the atmospheric transport calculations. The environmental settings for the transport calculations used for the Composite Analysis are described by Holdren et al. (1995).

\subsubsection{Atmospheric Model Results}

For simplicity, atmospheric transport away from the eight surplus reactor cores, which for the purposes of this Composite Analysis were located at a hypothetical burial site in the northwestern part of the 200 West Area, was treated as a radial transport directed away from the center of the source area. Because the source is a distributed source based on either the actual size of the reactor cores or the size of the burial ground cover under which the cores would be placed, the peak values for dose rate and concentration estimated at the actual source location center are not very meaningful resulting from the radial nature to the fall off. The model predictions at the source should be ignored at points inside the $100-\mathrm{m}$ by $600-\mathrm{m}$ source areas. No method was developed to partition the gas versus liquid phase for carbon-14 and tritium as it is released from the reactor cores. As a result, the 2050 inventories of tritium 
$(7,300 \mathrm{Ci})$ and carbon-14 (42,000 $\mathrm{Ci})$ associated with these eight reactor cores were accounted for twice in the Composite Analysis because these inventories were released both to air and vadose zone pathways. The estimated release rate for carbon-14 was taken from DOE (1989), which indicates a maximum potential release rate for carbon-14 from water-saturated graphite cores of $<1.5 \mathrm{Ci}$ per year per reactor, or $12 \mathrm{Ci}$ per year. Tritium release was derived using the reactor core release model used for all the vadose zone transport calculations (Appendix D). The tritium release rate, using this model, was estimated at $0.0073 \mathrm{Ci}$ per year in 2050 , the time when it was assumed that the cores would be placed in their hypothetical disposal area.

\subsubsection{Exposure and Dose Model}

Four exposure scenarios were used in the Composite Analysis to evaluate the potential impact on individuals from radionuclide releases to water and air. The exposure scenarios used in the Composite Analysis are those defined for the Hanford Site Risk Assessment Methodology (HSRAM) (DOE 1995). The HSRAM exposure scenarios were developed for the Hanford Site to facilitate evaluations of dose and risk related to Comprehensive Environmental Response, Compensation, and Liability Act (CERCLA) remedial investigations and Resource Conservation and Recovery Act (RCRA) facility investigations. The four HSRAM exposure scenarios are referred to as recreational, industrial, residential, and agricultural. These scenarios are summarized in this section of the report as described by Strenge and Chamberlain (1994). Additional detail on the exposure scenarios and unit dose factors is provided in Appendix F.

\subsubsection{Background}

The radiological dose impacts ${ }^{(a)}$ considered in the Composite Analysis were predicted with unit dose factors (UDFs) that relate concentration of a radionuclide in an environmental medium to the resulting radiation dose. The UDFs were evaluated for the radionuclides of interest, and for chemical effects of uranium, as specified in the Composite Analysis guidance (DOE 1996b). The UDFs were evaluated for each exposure scenario and environmental medium appropriate to the exposure scenarios. The environmental media considered include groundwater, air, and soil contaminated by airborne deposition. The contributions to dose from all exposure pathways defined for each scenario were included in the UDFs. Key assumptions for the exposure and dose model are listed in Table 4.13.

The industrial scenario was intended to represent potential exposures to workers in a commercial industrial setting. The industrial scenario primarily involved indoor activities, but outdoor activities (e.g., soil contact) were also included. The workers were assumed to wear no protective clothing; the scenario was not intended to represent exposure of remediation workers. The specific exposure pathways included in the industrial scenario are listed in Table 4.14 for both radionuclides and chemicals, and for each transport medium.

(a) All doses in the Composite Analysis (except where noted) are in units of mrem effective dose equivalent (EDE) in a year. 
The recreational scenario was intended to represent exposure to individuals engaging in recreational activities on the Hanford Site. Exposure pathways include soil contact, ingestion of water, and inhalation. The individuals were also assumed to hunt and eat game (deer) meat. The specific exposure pathways included in the recreational scenario are listed in Table 4.15 for both radionuclides and chemicals, and for each transport medium.

The residential scenario was intended to represent potential exposures to future individuals who may take up residence on the Hanford Site. The exposures were assumed to be continuous throughout the year. The specific exposure pathways included in the residential scenario are listed in Table 4.16.

The agricultural scenario was very similar to the residential scenario, with the addition of meat, game, and milk ingestion. The individual was assumed to take up residence on the Hanford Site and grow vegetables, fruit, and raise meat and milk animals. These food products were assumed to be consumed on the family farm. The specific exposure pathways included in the agricultural scenario are listed in Table 4.17.

\subsubsection{Exposure and Dose Model Implementation}

Unit dose factors for radionuclides were used to calculate the annual radiation dose received by an individual exposed in each of the defined HSRAM scenarios (DOE 1995). The dose is expressed in units of rem per year and represents the committed effective dose equivalent for one year of intake or exposure. The UDFs were evaluated for a unit concentration in a specific exposure medium. For example, with groundwater as the transport medium, the UDF was expressed per $\mathrm{pCi} / \mathrm{L}$ in the groundwater. When air was the transport medium, the UDF was expressed per $\mathrm{pCi} / \mathrm{m}^{3}$ in air.

The evaluation of annual radiation dose as the endpoint in the analysis represents a deviation from the HSRAM (DOE 1995). The HSRAM report describes evaluation of the lifetime cancer incidence risk from radionuclides using slope factors. The slope factors relate intake $(\mathrm{pCi})$ to the lifetime cancer incidence risk. However, the guidance for the Composite Analysis specifies evaluation of annual radiation dose (DOE 1996b). Therefore, the use of slope factors in the HSRAM guidance was replaced with radiation-dose-conversion factors in the Composite Analysis.

The evaluation of annual radiation dose in the Composite Analysis was based on radiation-doseconversion factors published in Federal Guidance Reports No. 11 and 12 (Eckerman, Wolbarst, and Richardson 1988; Eckerman and Ryman 1993). These dose factors are based on recommendation of the International Commission on Radiological Protection as given in ICRP (1979a, b). The resulting doses represent the effective-dose-equivalent received over a commitment period of 50 years following intake in the first year.

Consistent with the HSRAM scenarios, the radionuclide concentrations in transport media were assumed to be constant over the exposure duration. The concentrations were also assumed to be constant for a period of time prior to an exposure period in which deposited radionucides (from irrigation or 
atmospheric deposition, if appropriate to the scenario) were allowed to reach equilibrium with the soil. Equilibrium was assumed reached when the deposition rate was equal to leaching and radioactive decay losses from the soil. An analysis was performed to determine the time necessary for each radionuclide to reach equilibrium in the surface soil layer (see Appendix D). Mobile and short-lived radionuclides would reach equilibrium within a year. However, for the Composite Analysis, the longer-lived radionuclides and radionuclides that generate progeny radionuclides did not come to equilibrium within the 1000 years considered. Therefore, all UDFs were evaluated for 50 years of prior deposition and accumulation in the soil from air or irrigation water deposition. This assumption will represent nearequilibrium conditions for most radionuclides.

For uranium, the UDF was represented by the hazard quotient. The hazard quotient is defined by EPA as the average daily intake of a chemical (in this case uranium) divided by the Reference Dose (RfD) for that chemical. The hazard quotient was evaluated for both inhalation exposures and ingestion exposures with RfDs determined for each route.

The UDFs used in the Composite Analysis are summarized in Table 4.18.

\subsubsection{Exposure and Dose Model Results}

The radiological dose results consist of doses from individual radionuclides and the composite doses from all sources for the four exposure scenarios considered in the analysis. The multiple-step compositing process developed both the spatial distribution of composite dose rate outside the buffer area and the maximum composite dose rate versus time. For each time step calculated and for each contaminant plume for which calculations were performed (e.g., tritium, technetium-99 from tanks, technetium-99 from liquid discharge sources, technetium-99 from existing plumes, chlorine-36 from all sources) a planview representation of maximum concentration was prepared as discussed in Section 4.1.4.3. Once each of these spatial distributions of maximum concentration were prepared for each and every plume and time step, the spatial distributions of dose rate for each of the four scenarios was prepared for each time step. The spatial distribution of composite dose rate for a given scenario and at a given time step was calculated from these maximum concentration distributions. The composite dose rate at each plan-view location was calculated as the sum (over all contributing contaminant plumes such as tritium, technetium-99 from tanks, technetium-99 from liquid discharge sources, technetium-99 from existing plumes, chlorine-36 from all sources) of the product of maximum concentration for the contributing nuclide times the appropriate dose conversion factor. The individual dose results are presented as the maximum dose rates versus time outside the buffer zone for the agricultural exposure scenario, which resulted in the highest dose rates.

A review of existing radionuclide plumes in the unconfined aquifer revealed the presence of a strontium- 90 plume beneath the decommissioned Gable Mountain Pond. The observed peak concentration of strontium-90 in the vicinity of the retired pond was $1500 \mathrm{pCi} / \mathrm{L}$ in 1996 (Hartman and Dresel,

1997; Figure 6.10-10). Using the unit dose factor for strontium-90 from the agricultural scenario, this concentration in groundwater converts to a dose rate of $\sim 470$ mrem in a year. If the site is not 
remediated to remove the strontium-90 in groundwater and in the overlying vadose zone, it is recommended the exclusive waste management area be expanded to include this decommissioned pond. Furthermore, it is also recommended a buffer zone of $\sim 1000 \mathrm{~m}$ be established as a region of relatively clean groundwater surrounding the existing strontium-90 plume such that monitoring can detect movement of the strontium. Strontium is highly sorbed on aquifer sediments $\left(\mathrm{K}_{\mathrm{d}}=20 \mathrm{~mL} / \mathrm{g}\right)$ and its decay half-life is relatively short, 28.78 years (Parrington et al. 1996). It is anticipated the declining water table will cause strontium in the upper sediments of the aquifer to be suspended in the vadose zone, and, thereby, act to further isolate the contamination. To simplify the discussion of results in the Composite Analysis, it is assumed the exclusive waste management area and buffer zone will be expanded as recommended. Hence, discussion of dose outside the buffer zone assumes the region surrounding Gable Mountain Pond is included inside the exclusive waste management area and buffer zone.

Figure 4.34 illustrates individual maximum dose rate results outside the buffer zone for the agricultural scenario for a) maximum dose, b) all key nuclide contributions, c) tritium, d) strontium-90 from existing plumes, e) carbon-14, f) chlorine-36, g) selenium-79, h) technetium-99 from existing plumes, i) technetium-99 from liquid discharges, j) technetium-99 from tank sources, $k$ ) technetium-99 from solid waste sources, 1 ) iodine- 129 from existing plumes, $\mathrm{m}$ ) iodine- 129 from future sources, $\mathrm{n}$ ) total uranium from existing plumes, and o) total uranium from future sources. These graphs illustrate the maximum dose rates for each radionuclide in the unconfined aquifer outside the buffer zone regardless of location.

Figure 4.35 depicts the composite dose rates from all radionuclides and all sources presented as maxima versus time outside the buffer zone for the a) agricultural, b) residential, c) recreational, and d) industrial exposure scenarios. These graphs illustrate the maximum dose rates wherever they occur in the unconfined aquifer outside the buffer zone. The area of the unconfined aquifer predicted to be above the dose rate of 4 mrem in a year for the agricultural scenario decreases from more than $100 \mathrm{~km}^{2}$ in 1996 to $40 \mathrm{~km}^{2}$ in 2050 and zero by 2085 .

Comparison of the maximum composite dose rate versus time and the maximum dose rates from individual radionuclides shows that the dose rates from 1996 to 2020 are dominated by the contributions of tritium and iodine-129. The peak composite dose rate occurs in 1996, primarily from the existing tritium and iodine-129 plumes. After the tritium concentrations in the unconfined aquifer are reduced by dispersion and decay, and the iodine- 129 concentrations are reduced by dispersion, the largest contribution to the composite dose rate is technetium-99. Figure 4.34 shows this will occur very near the end of the 1000-year period and result in a maximum dose rate of $\sim 1 \mathrm{mrem}$ in a year.

Secondary peaks (beyond 1996) occur in the maximum composite dose rate in 2020 (23 mrem in a year for the agricultural scenario) and 2031 (14 mrem in a year), primarily from technetium-99 and iodine-129. The sources of the technetium-99 in these future peaks are tank leaks and contributions from liquid discharge waste sites. The primary source of the iodine-129 in the future peaks is predicted to be liquid discharge waste sites. 
Before site closure, the maximum composite dose rates are predicted to be above $30 \mathrm{mrem}$ in a year. However, because the Composite Analysis (DOE 1996b) is a post-closure analysis, maximum dose rates after 2050 were compared to the dose limit of $100 \mathrm{mrem}$ in a year and the dose constraint of $30 \mathrm{mrem}$ in a year. By site closure in 2050, the maximum composite dose for the agricultural scenario is predicted to be less than $6 \mathrm{mrem}$ in a year and by 2150 (loss of institutional control), the maximum composite dose rate is predicted to be $\sim 4$ mrem in a year.

The predicted distributions of composite dose rate for each of the exposure scenarios are illustrated for the time of peak dose rate (1996), near site closure (2049), and near the time of loss of institutional control outside the buffer zone (2159). Model results for the exact times of site closure (2050) and loss of institutional control (2150) were not shown because dose rate results were not modeled at those specific time planes. Figures 4.36 through 4.39 illustrate the distribution of composite dose rate in 1996 . for the agricultural, residential, recreational, and industrial exposures, respectively. Figures 4.40 through 4.42 illustrate the predicted distribution of composite dose rate in 2049 for the agricultural, residential, and industrial exposures, respectively. Figures 4.43 and 4.44 illustrate the predicted distribution of composite dose rate in 2159 for the agricultural and residential exposure scenarios, respectively. The dose rate results for the recreational scenario at 2049 and 2159 are not illustrated because the predicted dose rates were less than $0.4 \mathrm{mrem}$ in a year. Similarly, the dose rate results for the industrial scenario are not included for 2159 because those predicted doses were below $0.4 \mathrm{mrem}$ in a year.

The radiological dose rate results are presented for lands outside the buffer zone because the exposure scenarios (agricultural, residential, recreational, and industrial) are assumed to not apply inside the buffer zone. These portions of the Hanford Site will remain in exclusive use for waste management with a surrounding buffer area for protection of the public. It is assumed these lands will remain under federal control until they are determined to be safe for release to the public. To provide an indication of the potential impacts if groundwater inside the buffer zone was used, radiological dose rates resulting from the industrial exposure scenario were calculated for the area inside the buffer and exclusion zones. If groundwater inside the zone were used in the industrial scenario, the peak dose rate inside the buffer zone in 1996 (time of peak dose) would be $124 \mathrm{mrem}$ in a year. The maximum dose rate at 2049 (i.e., the approximate time assumed for Hanford. Site closure in 2050) would be $32 \mathrm{mrem}$ in a year, and the maximum dose rate at 2139 (i.e., the approximate time assumed for the end of institutional control in 2150 ) would be $3.6 \mathrm{mrem}$ in a year. These dose rates are from strontium- 90 in the groundwater at the 216-B-5 reverse well site. Strontium-90 also appears in groundwater beneath Gable Mountain Pond. Strontium dominated all exposure and dose scenario calculations inside the buffer zone during this period. The DOE intends to maintain the exclusive waste management area and buffer zone until they can be released to the public. The DOE has acknowledged that many low-level radioactive waste facilities may never be suitable for unconditional release to the public, and that deed restrictions on the future use of groundwater resources may be necessary. Consequently, these future doses will not be realized. 
The results for uranium treated as a hazardous chemical do not show any impacts outside the exclusion zone and are therefore not illustrated in a figure. These results were produced by estimating uranium impacts with a hazard quotient calculation.

Results of this analysis indicate that for all times the peak air and soil dose rates for tritium are more than 4 orders of magnitude below lowest dose estimate that is contoured (i.e., $0.4 \mathrm{mrem}$ in a year). Results of the carbon- 14 modeling indicate that peak air transport medium dose rates of 4.6 mrem in a year at the source occur at the time of disposal and remain essentially constant through time, decreasing only as a result of carbon-14 decay. No separate plots of air transport medium dose rate are shown because the $0.4 \mathrm{mrem}$ in a year contour essentially occurs at the reactor-core-disposal-area boundary and lower doses occur outside the buffer zone. In the soil (air/deposition) transport medium, soil concentrations are created by the continuous air releases, their subsequent deposition, and leaching by infiltration. Dose from contact with contaminated soils is virtually constant over the 1000-year analysis period for the long-lived radionuclides like carbon-14. For short-lived radionuclides like tritium, the maximum soil dose occurs at the beginning of the release. Figure 4.45 illustrates the maximum dose rate for atmospheric release from both the air transport medium and the soil (air deposition) transport medium. The values shown are for the agricultural scenario because it was the scenario showing the greatest impact. The $4 \mathrm{mrem}$ in a year dose rate contour is immediately above the source, and a dose rate of $0.4 \mathrm{mrem}$ in a year barely extends into the buffer zone. The dose rate falls off spatially very quickly and is well below the $0.4 \mathrm{mrem}$ in a year level outside the buffer zone. The industrial scenario, the only viable scenario inside the exclusive waste management area and buffer zone, yielded an 0.2 mrem in a year closed contour immediately above the source and also decreased very quickly at points away from the source.

\subsubsection{Exposure and Dose Model Sensitivity}

The sensitivity of the exposure and dose model was evaluated by considering different unit dose factors for the key radionuclides contributing to dose. The TWRS ILAW interim performance assessment (Mann et al. 1997) used somewhat different dose conversion factors than those used in the Composite Analysis. Table 4.19 provides a comparison of the dose factors. In the table, the unit dose factors for the radionuclides contributing the greatest amount to dose, (e.g., tritium, iodine-129, and technetium-99); are not appreciably different for the two analyses. Therefore, variations of the unit dose factor within the range presented would not produce significantly different dose rate results.

\subsection{Comparison with Other 200 Area Modeling Analyses}

Several independent modeling analyses have been performed as part of other environmental assessments for specific existing or proposed facilities within the exclusive waste management area. This section briefly compares the salient features of these independent assessments with the analysis performed for these specific sites in the Composite Analysis. 
Only three of the independent assessments estimate breakthrough from the waste site to the water table within the 1500-year period modeled in the Composite Analysis. One of these three assessments was for past tank leaks from a specific tank farm. The other two assessments with breakthroughs within the 1500-year period involved shallow land burial of wastes. Three other dry disposal assessments that estimated travel times to the water table in excess of 1500 years are discussed briefly.

Work toward the decontamination and decommissioning of canyon buildings and associated facilities has begun at the Hanford Site. However, this assessment has not obtained key mobile radionuclide inventories in canyon buildings and related facilities, and therefore, has not analyzed their migration and fate. The work has examined the potential migration of large inventories of cesium-137 and strontium90 from the B Plant and its sand and high-efficiency particulate air (HEPA) filters.

Besides using different models, each of these assessments employed different inventories, model parameters, and assessment points and times of compliance. Generally, it was found that site-specific assessments were more likely to use more conservative parameters than the "best-estimate" values employed in the Composite Analysis. While the results are not necessarily identical, they do suggest fundamental consistency between the site-specific analyses and the Composite Analysis.

\subsubsection{Hanford Tanks Initiative}

Recent interest in subsurface environmental impacts arising from past leaks and future losses from tanks has resulted in an ongoing analysis of leaks and losses from the tanks in the AX tank farm as part of the Hanford Tanks Initiative (HTI). Liquid losses from single-shell tanks may occur during the recovery of tank waste. This section compares the preliminary unpublished results ${ }^{(\mathrm{a})}$ of the HTI analysis with the Composite Analysis.

To estimate the cumulative release of an 8,000 -gallon $\left(30-\mathrm{m}^{3}\right)$ liquid waste loss from a single-shell tank to the water table, the HTI analysis employed a two-dimensional model of a vertical plane running from the AX tank farm to the water table. The Composite Analysis employed a one-dimensional model. Considerably more detail has been included in the spatial discretization of the soil properties of the HTI model than could be incorporated in the one-dimensional soil column of the Composite Analysis. Whereas the Composite Analysis released the liquid source over the entire tank bottom, the HTI analysis released from a much smaller area representing a header leak and allowed the hydrostratigraphic layers and subsurface properties to spread the plume during its downward migration. The technetium-99 inventories in the two analysis were $4.52 \mathrm{Ci}$ for the $\mathrm{HTI}$ assessment and $3.43 \mathrm{Ci}$ for the Composite Analysis assessment. The background recharge rates used were $10 \mathrm{~cm} / \mathrm{yr}$ for the HTI assessment and $7.5 \mathrm{~cm} / \mathrm{yr}$ for the Composite Analysis assessment.

(a) From two electronic mail messages, both dated December 30, 1997, sent by P. Rogers, Jacobs Engineering, Richland, Washington to L. W. Vail, Pacific Northwest National Laboratory, Richland, Washington; subjects, "Past Leak Flux" and "Cumulative Mass Files". 
Figures 4.46 and 4.47 compare the predicted cumulative release of technetium-99 from the AX Tank Farm for time periods from 1940-2150 and 1940-3000. Figure 4.46 shows generally earlier breakthrough for the Composite Analysis assessment. Figure 4.47 shows that the higher inventory used in the HTI assessment eventually releases, and its cumulative release surpasses that of the Composite Analysis during the 1000 years of the analysis.

As observed, a two-dimensional model should result in later breakthroughs and a more gradual draining of the soil column. This is likely because of the more complex patterns of spreading resulting from the more complex and heterogeneous representation of soil properties. The differences in total curies released at year 3000 are consistent with the different inventory estimates. The multidimensional HTI model has overtaken the one-dimensional Composite Analysis model in cumulative curies released to the water table. However, over $90 \%$ of the Composite Analysis inventory has been purged from the one-dimensional column while less than $75 \%$ of the HTI inventory has released. This may be attributed to the greater lateral dispersal permitted by the multidimensional model. It may also be a function of the combined effects of lateral dispersal and structural features in the multidimensional analysis that act to shelter some fraction of the release from direct leaching by recharge.

\subsubsection{Area Solid Waste Burial Ground Performance Assessments}

Performance assessments have been performed for the solid waste burial grounds in both the 200 East Area (Wood et al. 1996) and 200 West Area (Wood et al. 1995). These assessments were required to demonstrate that the disposal practice is in compliance with performance objectives in DOE Order 5820.2a (DOE 1998b). As part of these performance assessments, it was required to estimate the temporal distribution of contaminant flux to the water table. An identical calculation was made in the Composite Analysis for nonsorbed radionuclides. Similar calculations for sorbed radionuclides appear in both analyses; however, different distribution coefficients were employed.

Results from the Composite Analysis and the performance assessments for low-level burial grounds in the 200 West and 200 East Areas are somewhat but not remarkably different. Because of the difference in the stratigraphic profiles, and, hence, the unsaturated hydraulic properties of the vadose zone sediments, the transport of contaminants is generally slower through the vadose zone beneath the 200 West Area than for 200 East Area. For 200 West Area, the mean travel time for an advective (unit pulse) release reported in the performance assessment is approximately 1070 years. The Composite Analysis methodology estimates a mean travel time of 1054 using the same recharge rate of $0.5 \mathrm{~cm} / \mathrm{yr}$. For 218-E-10 and 218-E-12 burial grounds in 200 East Area, the estimated mean travel times using the performance assessment methodology were approximately 1150 and 650 years, respectively. For a variety of reasons, the Solid Waste Program plans to place the majority of future solid waste in the 200 West Area burial grounds. Therefore, a mean travel time of approximately 900 to 1000 years is indicative of the environmental response for these wastes.

Releases to the aquifer from the post-1988 solid waste burial grounds occur well after the peak releases from other sources that occur in the next few decades, and after the resulting maximum 
individual dose outside the buffer zone at the time of Hanford Site closure. Contributions to dose from burial ground releases outside the buffer zone occur later in the 1000-year period and contribute to lower doses.

\subsubsection{Commercial/Low Level Waste Site Assessment}

Analyses have been performed to demonstrate that the commercial low-level waste (LLW) disposal facility on the Hanford Site will meet the license requirements established by the State of Washington and the U.S. Nuclear Regulatory Commission. These analyses are detailed in the site stabilization and closure plan for the commercial LLW disposal site (Grant Environmental, Chase Environmental Group, and US Ecology 1996). The commercial LLW disposal site assessment assumed a steady recharge rate of $0.5 \mathrm{~cm} / \mathrm{yr}$, whereas the transient simulation of the Composite Analysis assumed a change in recharge rates. In the Composite Analysis, a recharge rate of $7.5 \mathrm{~cm} / \mathrm{yr}$ was assumed until site closure. Until that time it was assumed the cover soils were coarse and maintained free of vegetation. The Composite Analysis did not take any credit for the integrity of the packaging of the disposed waste and allowed leaching to occur during the period prior to cover placement. The Composite Analysis assumed the recharge rate dropped to $0.127 \mathrm{~cm} / \mathrm{yr}$ after closure of the presently used trenches in 2000 .

The commercial LLW disposal site assessment reported travel times of 140, 1110, and 3575 years for steady recharge rates of $5,0.5$, and $0.127 \mathrm{~cm} / \mathrm{yr}$, respectively. These all assumed the current depth to the water table is $81 \mathrm{~m}$. However, they estimated the water table beneath the site to drop as much as $13 \mathrm{~m}$ as a result of the end of significant liquid disposals from Hanford Site production operations. The Composite Analysis assumed the water table had already dropped to pre-Hanford Site levels before the plume reached the water table resulting in an estimated depth to water table of $87 \mathrm{~m}$. The commercial LLW disposal site assessment reported an estimated travel time of $4288 \mathrm{yr}$ with a recharge of $0.127 \mathrm{~cm} / \mathrm{yr}$ and a depth to water table of $96 \mathrm{~m}$. The Composite Analysis estimated breakthrough of a nonsorbed radionuclide in the present inventory to occur after 246 years. This time estimate reflects the impact of transient hydrology. Specifically, it reflects the assumed relatively dry initial conditions based

on $0.5 \mathrm{~cm} / \mathrm{yr}, 21$ years of relatively high recharge of $7.5 \mathrm{~cm} / \mathrm{yr}$, followed by low recharge of $0.127 \mathrm{~cm} / \mathrm{yr}$. Despite a relatively early breakthrough of nonsorbed radionuclides (e.g., chlorine-36 and technetium-99) in the 1000-year period, these releases do not coincide with the releases of the immediate future. Those occurring now and during the next few decades are associated with liquid discharge sites, tank leaks, losses from tanks, and pre-1988 solid waste burial grounds. These are the sources responsible for the maximum dose outside the buffer zone during the 1000-year period following Hanford Site closure. Releases from the commercial LLW disposal site occur later in the 1000-year period and contribute to lower doses.

\subsubsection{Remedial Investigation and Feasibility Study for the Environmental Restoration Disposal Facility}

Analyses were performed to evaluate alternatives for the placement of wastes in an ERDF. All wastes disposed in such a facility are to be generated during the remediation of past-practice sites at the 
Hanford Site. The analyses and their assumptions are documented in the ERDF Remedial Investigation and Feasibility Study (RI/FS) (DOE 1994b). Travel times for wastes leached from the ERDF and arriving at the water table were estimated using a simple analytical approach in the RI/FS. Several facility designs (i.e., various surface barrier and liner options) and two climate conditions were examined. The Composite Analysis simulated a single case that represented the facility design described as the preferred alternative in the record of decision for the ERDF (Amended ROD September 1997).

In the ERDF RI/FS analyses. travel times from the waste form to the water table were estimated using user-prescribed recharge rates and moisture contents, whereas in the Composite Analysis the moisture contents throughout the soil column were estimated using a physically based model and specific recharge rates. For the preferred alternative the RI/FS employed recharge rates of $0.01 \mathrm{~cm} / \mathrm{yr}$ for the base climate and $0.4 \mathrm{~cm} / \mathrm{yr}$ for the wetter climate conditions, respectively. The Composite Analysis assumed a recharge rate of $0.05 \mathrm{~cm} / \mathrm{yr}$. Because of the presence of a double liner, leaching was assumed to begin after site closure.

The ERDF RI/FS estimated travel times of 13,000 and 500 years for the base and wetter climate, respectively. The Composite Analysis simulated a period of 1500 years without detecting any breakthrough to the water table.

\subsubsection{Environmental Assessment of Surplus Production Reactors}

The record of decision on decommissioning the surplus production reactors at the Hanford Site states the preferred alternative is for disposal on the central plateau in the 200 West Area after up to 75 years of continued storage in their respective 100 Areas (ROD 1993). Once disposed within the exclusive waste management area, a potential pathway for environmental impact is the transport of radionuclides through the vadose zone to the water table. Analyses of the vadose zone and groundwater pathway are discussed in Appendix $C$ of the environmental impact statement (DOE 1989). The EIS analysis assumed a recharge rate of $0.1 \mathrm{~cm} / \mathrm{yr}$ for the Hanford protective surface barrier. Since the late $1980 \mathrm{~s}$, the Hanford Site Permanent Isolation Barrier Development Program adopted a design standard of $0.05 \mathrm{~cm} / \mathrm{yr}$ for allowable recharge rate. Accordingly, the Composite Analysis assumed a recharge rate of $0.05 \mathrm{~cm} / \mathrm{yr}$.

The draft EIS (DOE 1989) reported a travel time of 4,200 years. The Composite Analysis simulated a period of 1500 years without detecting any breakthrough to the water table from the production reactors.

\subsubsection{TWRS ILAW Performance Assessment}

The Hanford Low-Level Tank Waste Interim Performance Assessment (Mann et. al., 1997) examined the long-term environmental effects associated with the disposal of the low-level fraction of the Hanford single- and double-shell tank waste in a disposal facility located within the 200 East Area. A threedimensional computer code was used to simulate the flow and transport of contaminants from the 
waste form through the vadose zone to the groundwater. Sensitivity analyses included in this interim performance assessment considered uncertainty in the depth to water table, hydraulic parameters, geochemical parameters, and recharge rates.

The base case of the performance assessment assumed an initial recharge rate of $0.05 \mathrm{~cm} / \mathrm{yr}$ followed by a recharge rate of $0.3 \mathrm{~cm} / \mathrm{yr}$ after 1000 years. The Composite Analysis assumed a recharge rate of $0.05 \mathrm{~cm} / \mathrm{yr}$ throughout the 1500 year period simulated. The interim performance assessment reported a mean travel time of approximately 3,000 years. The Composite Analysis simulation stopped after 1,500 years without detecting any breakthrough to the water table from the immobilized low-activity waste. These wastes are the subject of a formal performance assessment with a planned submittal date of March 1998 ${ }^{(\mathrm{a})}$.

\subsubsection{Canyon Buildings}

As a screening analysis of possible releases from canyon buildings on the Hanford Site, releases of cesium-137 and strontium-90 from the B-plant and its permanent filters were considered. These facilities have a combined inventory of approximately $2.1 \times 10^{6} \mathrm{Ci}$ of cesium-137 and $4.2 \times 10^{5} \mathrm{Ci}$ of strontium-90. The combined information for the B Plant and its sand and HEPA filters was used to estimate a conservative value for the depth of the source to the water table. Assuming a Hanford Protective Barrier with a recharge rate of $0.05 \mathrm{~cm} / \mathrm{yr}$, the Composite Analysis methodology estimated no breakthrough to the water table within 1500 years for fully mobile radionuclides (i.e., distribution coefficient $=0 \mathrm{~mL} / \mathrm{g}$ ). This is a conservative representation for these nuclides because cesium and strontium in the most mobile waste forms have a finite nonzero distribution coefficient.

\subsection{Model Calibration and Comparisons of Results with Observations}

The first iteration of the Composite Analysis required complex calculations of contaminant release and transport through the vadose zone, groundwater, and atmosphere. This section discusses available information on the relationships among liquid discharge sites, inventory estimates for these sites, and existing plumes were used to perform a limited calibration or history match of the vadose zone model. The section also discusses the calibration of the Composite Analysis aquifer model and compares predicted contaminant concentrations with observations.

\subsubsection{Background}

At the Hanford Site, there are few specific data sets suitable for aquifer or vadose-zone transport model calibration and comparison of results with observations. The data sets potentially the most useful

(a) Mann, F. M., R. P. Puigh II, C. R. Eiholzer, Y. Chen, N. W. Kline, A. H. Lu, B. P. McGrail, P. D. Rittmann, G. F. Williamson, J. A. Voogd, N. R. Brown, and P. E. LaMont. 1998. Hanford Immobilized Low-Activity Tank Waste Performance Assessment. DOE/RL-97-69, Rev 0, U.S. Department of Energy, Richland, Washington. 
for calibration or comparison with observations would be isolated liquid disposal sites receiving large amounts of liquids containing highly mobile nuclides (e.g., tritium and technetium-99). For these type of sites, movement through the vadose zone would be rapid and the plume created in the aquifer may be unique and identifiable, yet large enough to adequately characterize. Other sites, such as past-practice landfills, are unlikely candidates for calibration or comparison with observations because of the uncertainty associated with the waste inventory, waste containment, and waste leaching.

While there are more than 175 liquid discharge sites in the 200 Areas at Hanford, none are completely adequate for calibration or comparison with observations. This is because early records on liquid disposals are spotty and the information on radiological content of the highly mobile radionuclides was often limited to gross alpha and beta counts.

There are no specific, liquid-discharge site data sets available for use in vadose-zone model calibration and the subsequent comparison of model predictions with observations. As a result, the vadose-zone model calibrations and comparisons used in this Composite Analysis were done through a more global, mass-balance approach described in Section 4.3.2.

The best data for a limited calibration of transport in the aquifer is information on the tritium plume. Information on liquid disposals to ground and the tritium content of these liquids is available starting in the mid 1970s. There are also yearly estimates of the "near-water-table" concentrations of the tritium plume based on groundwater monitoring data. However, there is only very limited information on the vertical distribution of the tritium or any other contamination in the aquifer. This lack of information on the vertical distribution of the contamination poses an additional calibration difficulty, because tritium disposal prior to 1979 is the largest contributor to the total inventory estimated to be in the existing plume. Therefore, the lack of a good inventory for the tritium disposal that gave rise to the plume, and the lack of knowledge of the spatial variation of concentration with depth poses a problem in developing initial conditions for the existing plume simulations. This uncertainty in the initial conditions poses a problem when trying to compare model results with observations because the effects of the disposals after 1979 on future plumes cannot be separated from the problem with the initial conditions. As a result, it can not be determined whether the inability to match future plumes (post-1979) is related to a poor aquifer model or an inappropriate vertical distribution of initial conditions. In the process of simulating the existing plumes (Cole et al. 1997), a limited calibration effort was undertaken to address the issue related to the vertical depth of contamination assigned to existing plumes when imposing initial conditions.

Two depths for assigning initial conditions were examined. In the initial model, concentrations, as interpreted from monitoring reports, were assigned to all calculational nodes within $6 \mathrm{~m}$ of the water table. This depth corresponds to the screen height of most monitoring wells as a result of an assessment by Eddy, Myers, and Raymond (1978) that the bulk of the contamination was believed to located in the uppermost 5 to $10 \mathrm{~m}$ of the aquifer. In the final model of existing plumes, initial condition concentrations were applied to all nodes within $25 \mathrm{~m}$ of the water table. Comparison of these modeling results with observations indicated that the $25 \mathrm{~m}$ depth provided a better match. This is the depth that was used 
to model all existing plumes (Cole et al. 1997). Comparison of the sitewide aquifer model results with observations for the tritium plume is discussed in Section 4.3.3 to provide information on the quality and uncertainty in sitewide aquifer model predictions.

\subsubsection{Predicted Contaminant Releases to Groundwater from the Vadose Zone}

Contaminant releases to the groundwater in the Composite Analysis were evaluated as a combined waste form release and vadose zone transport calculation. The information on vadose zone transport presented in Section 4.1 consisted of cumulative releases of the various radionuclides from the vadose zone to the groundwater. The STOMP code was used to predict the one-dimensional transport of contaminants through the vadose zone and determine the time of release to the water table of the contaminant mass leaving the source during each time step.

The Composite Analysis results demonstrate that pre-1988 solid waste burial grounds can be expected to release to the water table in the coming decades. Significant portions of their inventories are predicted to release within the next 100 years. However, the active and planned disposal actions are dry disposals that include placement of surface covers to reduce recharge, and thus their releases occur over a much longer time frame. The uncertainty in container integrity, and thus in the actual contaminant quantity released, makes pre-1988 burial ground data useless for model calibration. As a result the data available for determining how realistic predicted vadose zone contaminant releases to the water table are, are restricted to data from past-practice or liquid discharge sites.

At the Hanford Site, there are only a limited number vadose-zone.data sets that could be used to compare vadose-zone models with observations. Sisson and Lu (1984) and Fayer et al. (1995) report on model comparisons with a field injection experiment conducted in the 200 East Area. In this experiment, a dilute, mixed-salt solution containing radionuclides was injected $4.5 \mathrm{~m}$ belowgrade and migration was monitored through a collection of 32 wells surrounding the injection point to a depth of $18 \mathrm{~m}$. This solution contained calcium, chloride, nitrate, and trace amounts of barium, cesium-134, rubidium, and strontium-85. Water contents and gamma scanning data were collected during the experiment and Fayer et al. (1995) reported on logging of the wells with a high-resolution spectral gamma logging system. Because of the scale of the experiment and the specific radionuclides examined, the experiment was not applicable to the Composite Analysis model calibration problem. Field studies in response to tank leaks (Freeman-Pollard, Caggiano, and Trent 1994) and liquid discharges (DOE 1993a, 1994a) are also incomplete with respect to data requirements for model calibration. As a result, data on the existing technetium-99 plumes, technetium-99 inventory associated with liquid discharges, data on liquid discharge breakthrough (including those from tank leaks), and the uncertainty in these estimates were used for adjusting vadose-zone model parameters and for comparison with model results.

The basic assumption used in the vadose zone model calibration was that contaminant mass estimates for existing plumes combined with spatial and temporal knowledge on the first appearance and suspected source of these various plumes could be used to adjust vadose-zone model assumptions and/or parameters. The existing radionuclide plumes in the unconfined aquifer characterized by groundwater 
monitoring (Hartman and Dresel 1997) include strontium-90, uranium, iodine-129, tritium, and technetium-99. Data on other important Composite Analysis radionuclides (e.g. carbon-14, chlorine-36, selenium-79) can not be compared with observations because monitoring data either do not exist or are limited. The release of significant amounts of uranium to groundwater resulted from unique events that caused the mobilization of uranium in the vadose zone beneath one crib, by flushing of water from another crib and preferential flow down the unsealed annulus of a reverse well (Baker et al. 1988). A significant release of strontium-90 to the aquifer has created a plume beneath 200 East Area, however, it resulted from discharge to a reverse well that was completed in the aquifer. The generic approach used in the Composite Analysis does not account for this level of detail in the conceptual and numerical models and as a result, the data on these existing plumes can not be used for model calibration. The iodine-129 inventory and iodine's retardation factor are both uncertain. No credible inventory of iodine129 discharge to ground during the last PUREX campaign (1984-1986) was found. However, the existing iodine-129 plume appears to be well correlated with this PUREX operation and the absence of release data makes this data set useless for calibration of the release and vadose zone contaminant migration model. The tritium data are not useful for vadose zone model calibration because the various plumes have commingled and there have been so many sources it is impossible to relate specific sources to specific plumes. As a result of the available existing plume data, only the technetium-99 data set was found to be appropriate for vadose-zone model calibration. In an effort to match the response of the release and vadose zone transport models to field observations, the predicted release of technetium-99 from all sources in the Composite Analysis was compared with the mass estimated to be in the aquifer. Mass in the aquifer was estimated from the 1996 groundwater concentration contours interpreted from groundwater monitoring data and presented in Hartman and Dresel (1997). The release and vadose zone transport models were then adjusted to match the observed mass of technetium-99 in the unconfined aquifer with the mass of technetium-99 predicted to be released to the water table before 1996 . However, as discussed below, both model parameters and the uncertain inventory estimates for technetium-99 disposed at liquid-discharge sites had to be reconciled during the history matching process.

Mean cross-sectional area associated with the liquid discharge or tank leak was varied in the model calibration, because this parameter directly affects the travel time of the contaminant through the vadose zone and it is a highly uncertain parameter. In the early modeling of tank leaks and liquid discharge sites, a conservative approximation was made to estimate this parameter. At each site where a onedimensional model was developed, the infiltration rate was assumed to be limited by the saturated hydraulic conductivity of the least conductive of the sediment layer in the hydrostratigraphic column assigned to that site in the Composite Analysis. With this approximation, the cross-sectional area for each discharge facility or leak is estimated based on the saturated hydraulic conductivity of the limiting layer, an assumption of a unit gradient, and a liquid discharge volume and discharge duration for each respective site. The cross-sectional area was very small, except for ponds, producing results that were not consistent with observations, both in terms the observed spatial distribution of contaminants and the total inventory estimated for plumes in 1996. 
Using the initial cross-sectional area approximation, all mobile constituents from tank leaks arrived at the water table within a few years and even iodine- 129 with a distribution coefficient $\left(\mathrm{K}_{\mathrm{d}}\right)$ of $0.5 \mathrm{~mL} / \mathrm{g}$ was predicted to arrive where no iodine-129 plumes have been observed. During the model calibration effort a cross-sectional area equal to the area of a tank bottom yielded results that were most consistent with field observations. This revised cross-sectional area approximation for tank leaks and sluicing losses (as discussed earlier in Section 4.1.2.3) predicted $0.5 \mathrm{Ci}$ of iodine-129 would be released to the aquifer prior to 1996 compared to estimates, based on monitoring data, of $7 \mathrm{Ci}$ of iodine-129 in the aquifer, most of which is believed to be from PUREX operations in the mid-1980s.

Based on the above model, the amount of technetium-99 predicted to release before 1996 was $5 \mathrm{Ci}$. The upper estimate of the observed mass of technetium- 99 in the aquifer is $37.6 \mathrm{Ci}$, based on integration of the existing plume distribution. This left 32.6 or $~ 33 \mathrm{Ci}$ of technetium-99 attributed to liquid discharge sites. Prior to the discovery that the technetium- 99 inventory data for the liquid-discharge sites from the Waste Site Groupings report (DOE 1997b) and the Environmental Restoration program (i.e., 5.1 Ci total) were significantly lower than the Waite (1991) inventory estimates for these sites (i.e., $930 \mathrm{Ci}$ ), there was no inventory estimate that could justify the existing technetium-99 plume which is estimated to contain between 15.8 and $37.6 \mathrm{Ci}$. All the technetium- 99 was predicted to release before 1996 , but the entire inventory of $5.1 \mathrm{Ci}$ was less than the $\sim 16$ to $38 \mathrm{Ci}$ of technetium-99 estimated to be in the existing plume. However, the Waite (1991) inventory estimates created the opposite dilemma; with the initial cross-sectional area approximation much more technetium-99 (i.e., $\sim 300 \mathrm{Ci}$ ) was predicted to be released than could be accounted by the existing plumes.

To delay the arrival of the technetium-99 at the water table and account for lateral dispersion, or spreading of the contaminant plume in the vadose zone for the liquid discharges, other than ponds, the effect of increasing the cross-sectional area was examined (see Section 4.1.2.4). These studies indicated increasing cross-sectional areas had a diminishing effect on the estimated amount of technetium-99 released to the water table. Increasing the cross-sectional area by a factor of three reduced the predicted release of technetium- 99 prior to 1996 from $300 \mathrm{Ci}$ to $\sim 181 \mathrm{Ci}$. The release of technetium- 99 from past tank leaks was calculated to be approximately $5 \mathrm{Ci}$, which left $\sim 33 \mathrm{Ci}$ of technetium- 99 to be associated with liquid-discharge sites prior to 1996 . The cross-sectional area required to match the $33 \mathrm{Ci}$ of technetium-99 was unreasonably high (e.g., greater than 10). Therefore, the three-fold increase in crosssectional area was adopted. This factor was applied to all liquid discharge sites, except ponds, for all radionuclides.

Based on these modeling results, the Waite (1991) estimated inventory of technetium-99 released to liquid discharge sites was believed to be too high, so the inventory for the base case was scaled from $930 \mathrm{Ci}$ to $\sim 167 \mathrm{Ci}$ in order for the predicted pre-1996 release to the water table of $\sim 181 \mathrm{Ci}$ to match the $\sim 33 \mathrm{Ci}$ estimated to be in the unconfined aquifer based on monitoring data. A sensitivity case was also used to demonstrate the effects of using the higher inventory estimate. In this case the pre-1996 release was the same as the base case. However, the post-1996 release rate was scaled up so that the full Waite (1991) estimate of $930 \mathrm{Ci}$ would be achieved. Figure 4.48 shows the cumulative release of technetium99 from all sources to the water table from 1940 to 3000 for the three inventory and release scenarios 
described for the liquid-discharge sites. The plot shows the results for the full Waite inventory for liquid discharge sites ( $930 \mathrm{Ci}$ ) (as was shown in Figure 4.5b), the scaled or base-case inventory estimate $(\sim 167 \mathrm{Ci})$, and the sensitivity case with the enhanced post-1996 release rate that achieves the full Waite (1991) inventory estimate for liquid discharge sites.

Using the adjusted parameters resulting from the qualitative calibration of the high-volume liquid discharges, the Composite Analysis model predict rapid release to the water table that has already occurred or will occur in the near future, consistent with observations. The model results for the past tank leaks show current impacts (releases) and future impacts to the aquifer, consistent with recent observations at several of the tank farms documented by Johnson and Chou (1998) and Hodges (1998).

In comparison with the liquid disposals, few if any observations are available for model comparison and parameter adjustment for the dry disposals. As previously described, the dry disposals include placement of surface covers to reduce recharge and their releases occur over a much longer time frame. A mean travel time of approximately 1000 years was associated with burial grounds that will receive the majority of future solid waste disposals. Forecasts of release from the pre-1988 burial indicate these sites have not released yet. Therefore, data are not available for determining how realistic the predicted vadose zone contaminant releases are for the dry disposals.

One method for establishing confidence in the models used to predict radionuclide releases from dry disposals was to compare Composite Analysis predictions with other performance assessments. These comparisons were made in Section 4.2 and demonstrated that dry disposal sites will release in the future. The time frames for release predicted with the Composite Analysis model for post-1988 disposals of low-level waste are consistent with those in other performance assessment calculations.

\subsubsection{Predicted Groundwater Contaminant Concentrations in the Aquifer}

Prior to conducting simulations of the contaminant transport summarized in this report, confidence in the three-dimensional model of the unconfined aquifer system was established by calibration of the model to 1979 water table conditions, which was a time of quasi-steady state, as described in Cole et al. (1997). The resulting distribution of hydraulic properties developed for the three-dimensional model were derived from the original transmissivity distributions developed for the two-dimensional version of the sitewide aquifer system and a statistical inverse method described in Jacobson and Freshley (1990). A seven-step process, described in Cole et al. (1997), was used to derive the three-dimensional distribution of hydraulic properties. This seven-step process used hydrostratigraphic and facies descriptions while preserving the calibrated spatial distribution of transmissivities determined from the twodimensional inverse modeling.

Confidence in the transient behavior of the three-dimensional flow model was established by evaluating its ability to approximate changes in the water table in response to transient liquid discharges to the unconfined aquifer between 1979 and 1996. The evaluation examined a range of model storage properties (specific yield) until transient water table predictions approximated observed water table 
changes during this period. Transient simulation results and comparisons of predicted and observed transient water table changes are presented in Cole et al. (1997). These results indicate that the best approximation was achieved when a specific yield of 0.1 was used for units in the Ringold Formation and a specific yield of 0.25 was used for the Hanford formation.

Model simulations of projected declines in artificial discharges at the site presented in Cole et al. (1997) showed that, over about a 300-year period, the water table would decline significantly and return to near pre-Hanford water table conditions that were estimated to exist in 1944. The predicted water table was estimated to be very close to steady state within 100 years. Over the 300 -year period, model results show that the water table will drop as much as $11 \mathrm{~m}$ in the 200 West Area near the retired U Pond and $10 \mathrm{~m}$ in the 200 East Area near B Pond. Modeled areas that differed from the estimated 1944 hindcast included:

- the area west of the 200 Area Plateau, where higher predicted hydraulic heads reflect boundary conditions that consider the effect of increased irrigation from areas upgradient of the modeled region

- the area north of Richland, where the model included the hydraulic effect of the North Richland well field.

Results generated by the Composite Analysis three-dimensional model (Cole et al. 1997) were consistent with the post-Hanford analysis of the water table changes reported by Chiaramonte et al. (1997).

Prior to simulating the future transport of existing plumes and future source of contaminants, confidence in the three-dimensional transport model was evaluated by examining the ability of the model to simulate the transient behavior of the existing plume of tritium from 1979 to 1996 . The tritium plume was selected for evaluation because estimates of tritium discharges were available and the plume was monitored during this period (1979 to 1996). A comparison of predicted and observed tritium plume transport, presented in Cole et al. 1997, suggests that the three-dimensional model provides a reasonable approximation of the overall transport of the tritium plume during the period of concern. Results of simulation were also in reasonable agreement with the transport behavior of the tritium plume over the same period performed by Chiaramonte et al. (1997).

Initial conditions used in the transport simulations of existing plumes (tritium, technetium-99, iodine-129, uranium, and strontium-90), were derived from interpreted areal distributions of existing plumes presented in Hartman and Dresel (1997). As discussed above, contamination was assumed to be uniformly spread from the water table to $25 \mathrm{~m}$ below the water table. The existing plumes model (Cole et al. 1997) and the groundwater model used in the Composite Analysis are exactly the same except for initial conditions and radionuclide source terms. The SALDS model (Barnett et al. 1997) not only has different initial conditions and radionuclide source terms, but a different grid resolution and assigned dispersivity. Since the SALDS tritium plume was modeled at lower resolution with the coarse grid 
model (Cole et al. 1997) and with the locally refined grid model and smaller dispersivity discussed in Barnett et al. (1997), a comparison of these results allows the effect of grid resolution and dispersivity on predicted results to be examined. The SALDS model used a local-scale horizontal grid spacing of $45 \mathrm{~m}$ by $45 \mathrm{~m}$ in the vicinity of the SALDS and a $\sim 6 \mathrm{~m}$ vertical grid all the way to the basalt. Lateral and transverse dispersivities were set to $20 \mathrm{~m}$ and $2 \mathrm{~m}$, respectively. The existing plumes analysis used a horizontal grid spacing of $375 \mathrm{~m}$ by $375 \mathrm{~m}$ and the vertical grid spacing was variable (minimum thickness of $8 \mathrm{~m}$ ). Lateral and transverse dispersivities were $95 \mathrm{~m}$ and $20 \mathrm{~m}$, respectively. The SALDS model contours for the tritium plume from Barnett et al. (1997) are shown in Figures 4.49a and $b$ for the years 2020 and 2045, while existing plumes modeling results for the SALDS from Cole et al. (1997) for these same times are shown in Figure 4.50 and Figure $4.24 \mathrm{~b}$ respectively. From a comparison of the general shape and movement of both predicted plumes one can conclude that the results are very similar. Plumes of the two models were compared by measuring the width of the plumes at their widest point for a given contour level (e.g. concentration). In 2020, the coarse-grid, large-dispersivity model predicted the plume diameter above $2,000 \mathrm{pCi} / \mathrm{L}$ to be $2.4 \mathrm{~km}$ and the high-resolution, small-dispersivity model prediction was $1.6 \mathrm{~km}$. Comparisons of high- and low-resolution results for the $20,000 \mathrm{pCi} / \mathrm{L}$ contour were $1.1 \mathrm{~km}$ and $1.2 \mathrm{~km}$ respectively. Similar comparisons for the $2,000 \mathrm{pCi} / \mathrm{L}$ contour in 2050 , after the centroid of the plume had moved $0.7 \mathrm{~km}$ from the disposal site, were $1.5 \mathrm{~km}$ for the high resolution model and $1.6 \mathrm{~km}$ for the low resolution model. A comparison of all the results of these two models would show that the low-resolution, large-dispersivity model missed the estimated peak values directly below the SALDS during the disposal phase. Small areas ( $100 \mathrm{~m}$ in diameter) were predicted to be above $2 \times 10^{6} \mathrm{pCi} / \mathrm{L}$ by the high-resolution model while no concentrations above that level were predicted by the low-resolution model. However, a comparison of results through time indicates that the overall areal extent and concentration levels predicted for the SALDS tritium plume using the low-resolution model from the start of operations through site closure and until 2100 , when all predicted levels by both models were below $500 \mathrm{pCi} / \mathrm{L}$, were very consistent with results produced by the high-resolution localscale model.

In Cole et al. (1997), model-predicted concentrations of selected contaminants were also evaluated with respect to observations. As illustrated in the above high- and low-resolution comparison, the 375-m grid resolution being used in the Composite Analysis model means that model-estimated concentration levels near small individual source locations are expected to be lower than observations made in wells near contaminant sources. However, the dispersion predicted by the model away from the sources and outside the buffer zone is likely to be consistent with the amount of dispersion that has been observed in monitoring data. Since, the Composite Analysis model predicts relatively fast reduction of plume concentrations as they migrate from the source, it is important to evaluate the reasonableness of the Composite Analysis model predicted fall off in concentration levels with migration distance. This can be accomplished by comparing simulated reduction of modeled concentrations to the observed reduction of groundwater concentrations at different migration distances from the source. Tritium groundwater concentrations measured in wells near the PUREX facility during its early operations and more recent measurements in observation wells located within the tritium plume outside of the buffer zone provide the data sets for evaluating the reasonableness of model predicted plume dispersion with distance. 
Process condensate liquid waste containing tritium from PUREX operations was discharged to ground at the $216-A-10$ crib south of PUREX in the 200 East area. The crib was initially operated for a 4-month period in 1956. In 1961, the crib received PUREX effluent continuously until 1973; it then received waste sporadically in 1977,1978 , and 1981. In 1982, effluent discharges resumed on a continuous basis until the crib was taken out of service and replaced by the $216-A-45 \mathrm{crib}$ in 1987 . The effect of the effluent discharges on groundwater near the 216-A-10 crib have been monitored in two wells, 299-E17-1 and 299-E24-2 since the 1961 start of operations. Long-term concentration histories at these two wells demonstrate that groundwater concentrations of tritium were at their highest within 1 to 2 years after the start of operations. A maximum tritium concentration of $4.6 \times 10^{7} \mathrm{pCi} / \mathrm{L}$ was measured in well 299-E24-2 in 1963 (Figure 4.51).

Approximately 10 to $12 \mathrm{~km}$ downgradient from the PUREX facility, maximum tritium levels observed in the plume, which has now moved toward the Columbia River, are just above $300,000 \mathrm{pCi} / \mathrm{L}$. One example of these observations is the tritium levels in well 699-42-12A (Figure 4.52) where concentrations between 300,000 and $360,000 \mathrm{pCi} / \mathrm{L}$ were observed between 1976 and 1988 . The peak values are approximately 150 times lower than levels that were originally observed near the PUREX facility in 1963. If decay of tritium is considered (i.e., a factor of 2), concentration levels of tritium following its migration to this area over a 12- to 13-year period would be about 75 times lower than maximum levels originally observed near PUREX.

The increases in tritium levels suspected to originate from near the PUREX facilities have also been observed in numerous wells within 5 to $6 \mathrm{~km}$ downgradient of the PUREX facilities just outside the buffer zone. Concentration histories for two wells, 699-31-31 and 699-34-39A (Figure 4.53) illustrate the rise and fall of elevated tritium concentrations with time in the area just outside of the 200 East Area southeast of PUREX. At these locations, tritium concentrations rose to levels of 4 to 5 million $\mathrm{pCi} / \mathrm{L}$ in the early 1960s. These levels are about a factor of about ten lower than levels observed near PUREX.

Composite Analysis results simulated with the current model (Cole et al. 1997) are consistent with the early observations of dispersion of the tritium plume resulting from early PUREX discharges. Composite Analysis existing plume results of tritium transport for the period from 1979 to 1996, which incorporated the restart of discharges to PUREX in the mid-1980s, were compared with the well observations made 5 to $6 \mathrm{~km}$ downgradient of the PUREX facilities discussed above. Model transport results from Cole et al. (1997) for 1985 (Figure 4.54), the period of maximum simulated tritium concentrations at PUREX, show approximately an order of magnitude decline of tritium concentrations as the resultant tritium plume migrates outside the buffer zone boundary southeast of PUREX. This result is generally consistent with order of magnitude decrease in tritium levels that were observed in wells 5 to $6 \mathrm{~km}$ downgradient of PUREX in the early 1960s (Figures 4.51 and 4.53). 
Table 4.1. Summary of Key Assumptions for the Source Release Models

\begin{tabular}{|l|l|l|}
\hline \multicolumn{1}{|c|}{ Assumption } & \multicolumn{1}{|c|}{ Rationale } & \multicolumn{1}{c|}{ Impact } \\
\hline $\begin{array}{l}\text { Instantaneous response to } \\
\text { changes in recharge rates. }\end{array}$ & $\begin{array}{l}\text { Sites are generally shallow and } \\
\text { should respond quickly to } \\
\text { changes in recharge relative to } \\
\text { the 1000-year study period. }\end{array}$ & $\begin{array}{l}\text { Changes in recharge at deeper } \\
\text { sites will occur gradually over } \\
\text { many years. Since decreased } \\
\text { recharge results in decreased } \\
\text { release from the waste form for } \\
\text { each of the release models, when } \\
\text { recharge rates decrease the model } \\
\text { will underestimate the release for } \\
\text { the next few years. }\end{array}$ \\
\hline $\begin{array}{l}\text { Uniform release of contaminants } \\
\text { in liquid releases. }\end{array}$ & $\begin{array}{l}\text { Insufficient data were available to } \\
\text { justify distributing the mass of } \\
\text { contaminants released in liquid } \\
\text { discharges in any specific } \\
\text { distribution. }\end{array}$ & $\begin{array}{l}\text { If the majority of mass releases } \\
\text { occurred early in the operation of } \\
\text { the liquid disposals, the approach } \\
\text { employed in the Composite } \\
\text { Analysis would underestimate the } \\
\text { cumulative mass release at the } \\
\text { water table. However, within a } \\
\text { few hundred years it can be } \\
\text { expected that the cumulative } \\
\text { releases would be approximately } \\
\text { equal. }\end{array}$ \\
\hline $\begin{array}{l}\text { Water content in soil-debris waste } \\
\text { form is constant and equal to } \\
\text { estimated pre-Hanford soil } \\
\text { moisture content of surrounding } \\
\text { soil. }\end{array}$ & $\begin{array}{l}\text { Soil hydraulic properties of soil- } \\
\text { debris waste forms are generally } \\
\text { unavailable. }\end{array}$ & $\begin{array}{l}\text { In the soil-debris release model, } \\
\text { given a specific recharge rate, } \\
\text { lowering the soil moisture would } \\
\text { result in earlier cumulative } \\
\text { releases. Using a low moisture } \\
\text { content (estimated from the } \\
\text { hydraulic properties of adjacent } \\
\text { soil and a steady infiltration rate } \\
\text { of 5 mm/yr) would result in } \\
\text { earlier cumulative releases except } \\
\text { in cases where a barrier reduces } \\
\text { the recharge to below 5 mm/yr. } \\
\text { However, none of the solid waste } \\
\text { disposals with barriers considered } \\
\text { in the Composite Analysis } \\
\text { provide breakthrough within the } \\
\text { 1000 years. }\end{array}$ \\
& &
\end{tabular}


Table 4.1. (contd)

\begin{tabular}{|c|c|c|}
\hline Assumption & Rationale & Impact \\
\hline $\begin{array}{l}\text { Only a single release model was } \\
\text { considered for each site. }\end{array}$ & $\begin{array}{l}\text { Inadequate data were available to } \\
\text { estimate inventories that may } \\
\text { have been disposed in different } \\
\text { waste types at the same site. } \\
\text { However, tanks were treated as } \\
\text { three separate sites: tank leaks; } \\
\text { tank losses; and tank residuals. }\end{array}$ & $\begin{array}{l}\text { Highly mobile wastes may be } \\
\text { handled separately from less- } \\
\text { mobile wastes. For instance, } \\
\text { highly mobile waste may be } \\
\text { packaged differently (e.g., } \\
\text { cement waste forms) and } \\
\text { disposed in a solid waste burial } \\
\text { ground with less-mobile wastes. } \\
\text { The Composite Analysis selected } \\
\text { the release model that would } \\
\text { result in the earliest cumulative } \\
\text { release. }\end{array}$ \\
\hline $\begin{array}{l}\text { Soil-debris release models } \\
\text { assumed the waste form was } \\
\text { continuously mixed. }\end{array}$ & $\begin{array}{l}\text { The parameters and distributions } \\
\text { of inventories within the waste } \\
\text { forms were highly uncertain. } \\
\text { Using a completely stirred tank } \\
\text { reactor model is a reasonable } \\
\text { approximation. }\end{array}$ & $\begin{array}{l}\text { Completely mixing the waste } \\
\text { form can result in earlier releases } \\
\text { by sufficiently diluting the } \\
\text { inventory to prevent any local } \\
\text { controls on the release (e.g., } \\
\text { solubility controls around a hot } \\
\text { spot in the waste form). }\end{array}$ \\
\hline $\begin{array}{l}\text { Soil-debris release models } \\
\text { assumed the waste form was } \\
\text { continuously mixed. }\end{array}$ & $\begin{array}{l}\text { The parameters and distributions } \\
\text { of inventories within the waste } \\
\text { forms were highly uncertain. } \\
\text { Using a completely stirred tank } \\
\text { reactor model is a reasonable } \\
\text { approximation. }\end{array}$ & $\begin{array}{l}\text { Completely mixing the waste } \\
\text { form would decrease the early } \\
\text { cumulative releases by } \\
\text { continuously redistributing the } \\
\text { mass into the upper portions of } \\
\text { the waste form. Therefore, this is } \\
\text { not a conservative assumption. } \\
\text { The magnitude of the impact } \\
\text { varies for each site. It is most } \\
\text { likely to affect releases of highly } \\
\text { mobile wastes by delaying their } \\
\text { release. }\end{array}$ \\
\hline
\end{tabular}


Table 4.2. Description of Worksheets in the Composite Analysis.xls Workbook

\begin{tabular}{|c|c|c|c|}
\hline Worksheet & Function & Primary Fixed Fields & Primary Derived Fields \\
\hline Source Site & $\begin{array}{l}\text { Contains most of the primary data regarding geometry, } \\
\text { geochemistry, and timing of releases and recharge for all } \\
\text { of the sources considered. }\end{array}$ & $\begin{array}{l}\text { Location } \\
\text { - Northing }(\mathrm{m}) \\
\text { - Easting }(\mathrm{m}) \\
\text { Depth of Source }(\mathrm{m}) \\
\text { Release Model Class } \\
\text { Waste Type } \\
\text { Area }\left(\mathrm{m}^{2}\right) \\
\mathrm{K}_{\mathrm{d}} \text { Switch Depth }(\mathrm{m}) \\
\text { Volume }\left(\mathrm{m}^{3}\right) \\
\text { Recharge Dates }(\mathrm{yr}) \\
\text { Recharge Rates }(\mathrm{cm} / \mathrm{yr}) \\
\text { Water Table Elevation } \\
\end{array}$ & $\begin{array}{l}\text { Column Name } \\
\text { Layer thicknesses }(\mathrm{m}) \\
\text { Corrected Area }\left(\mathrm{m}^{2}\right)\end{array}$ \\
\hline Soil & $\begin{array}{l}\text { Contains soil hydraulic parameters for each of the soils } \\
\text { considered. }\end{array}$ & $\begin{array}{l}\text { van Genuchten alpha }(-) \\
\text { van Genuchten } \mathrm{n}(\mathrm{L} / \mathrm{cm}) \\
\text { Residual water content }(-) \\
\text { Porosity }(-) \\
\text { Saturated hydraulic conductivity }(\mathrm{cm} / \mathrm{s}) \\
\text { Bulk Density }\left(\mathrm{g} / \mathrm{cm}^{3}\right) \\
\text { Gravel Fraction }(\%)\end{array}$ & Initial Saturation $^{(1)}$ \\
\hline Column & $\begin{array}{l}\text { Contains description of stratigraphy of Hanford from } \\
\text { available columns. }\end{array}$ & $\begin{array}{l}\text { Location } \\
\text { - Northing (m) } \\
\text { - Easting (m) } \\
\text { Stratigraphy } \\
\text { - Thickness (m) } \\
\text { - Soil Type }\end{array}$ & \\
\hline Recharge & Contains actual values for various recharge classes. & Recharge Rates (cm/yr) & \\
\hline $\begin{array}{l}\text { Ground Surface \& Water } \\
\text { Table }\end{array}$ & $\begin{array}{l}\text { Contains gridded ground surface and gridded water table } \\
\text { elevations based on CFEST simulation for } 1979 \text {. }\end{array}$ & $\begin{array}{l}\text { Location } \\
\text { - Northing (m) } \\
\text { - Easting (m) } \\
\text { Elevation } \\
\text { - Ground surface (m) } \\
\text { - Water Table (m) }\end{array}$ & \\
\hline $\begin{array}{l}\mathrm{K}_{\mathrm{d}} \text { and Release Model } \\
\text { Classes }\end{array}$ & $\begin{array}{l}\text { Contains best estimates of } K_{d} \text { for both near-field and far- } \\
\text { field for each waste class }\end{array}$ & $\begin{array}{l}\text { Waste Classes } \\
\mathrm{K}_{\mathrm{d}} \text { for both near-field and far-field }\end{array}$ & \\
\hline
\end{tabular}


Table 4.3. (contd)

\begin{tabular}{|c|c|c|c|}
\hline Worksheet & Function & Primary Fixed Fields & Primary Derived Fields \\
\hline Nuclides \& Release Data & $\begin{array}{l}\text { Contains the parameters for each radionuclide for each of } \\
\text { the release models. }\end{array}$ & $\begin{array}{l}\text { Atomic Number }(-) \\
\text { Aqueous Solubility (Ci/L) } \\
\text { Fractional release from glass (\%) } \\
\text { Cement diffusion coefficient }\left(\mathrm{cm}^{2} / \mathrm{yr}\right) \\
\text { Fractional release from reactor }(\%) \\
\text { Half-life (yr) }\end{array}$ & Specific activity \\
\hline Inventory & $\begin{array}{l}\text { Contains radionuclide inventories for each site assembled } \\
\text { from a variety of independent Excel }{ }^{T M}{ }_{\text {workbooks. }}\end{array}$ & & Inventory decayed to $2050(\mathrm{Ci})$ \\
\hline CFEST-time-step-ends & $\begin{array}{l}\text { Contains the time steps for which CFEST is set to accept } \\
\text { estimates of flux to the water table. }\end{array}$ & Times (yr) & \\
\hline Source CFEST-nodes map & $\begin{array}{l}\text { Contains the distribution of each site's instantaneous flux } \\
\text { to one or more CFEST nodes. }\end{array}$ & Fractional distribution of flux (\%) & \\
\hline CFEST input & $\begin{array}{l}\text { Contains the decayed instantaneous fluxes to the water } \\
\text { table at each of the respective CFEST nodes for each of } \\
\text { the CFEST time steps. }\end{array}$ & & Decayed instantaneous fluxes (Ci) \\
\hline Flux & $\begin{array}{l}\text { Contains the undecayed (2050) annual cumulative flux } \\
\text { for each site that breaks through to the water table within } \\
1500 \text { years. }\end{array}$ & & Annual cumulative flux (Ci) \\
\hline Temp & $\begin{array}{l}\text { Contains the unit release breakthrough times from } \\
\text { STOMP simulation and the annual releases from waste } \\
\text { form to upper vadose zone predicted with the appropriate } \\
\text { inventory and release model for the site. }\end{array}$ & $\begin{array}{l}\text { Cumulative unit flux predicted by } \\
\text { STOMP }(-)\end{array}$ & $\begin{array}{l}\text { Annual cumulative release to } \\
\text { upper vadose zone }(\mathrm{Ci}) \\
\text { Annual cumulative release to } \\
\text { water table }(\mathrm{Ci})\end{array}$ \\
\hline
\end{tabular}

(1) Estimate based on steady-state flux of $0.5 / \mathrm{cm} / \mathrm{yr}$ using algorithm developed by Rockhold, Simmons, and Fayer (1997). 
Table 4.3. Source Geometry Data Required for Release Models in the Source Site Worksheet

\begin{tabular}{|c|c|c|c|c|c|c|c|c|c|}
\hline Site Name & $\begin{array}{c}\text { Northing } \\
(\mathrm{m})^{n}\end{array}$ & $\begin{array}{l}\text { Easting } \\
(\mathrm{m})^{\mathrm{An}}\end{array}$ & $\begin{array}{l}\text { Depth } \\
(\mathrm{m})^{\mathrm{nan}}\end{array}$ & $\begin{array}{c}\text { Water Table } \\
\text { Elevation(m) }{ }^{+}\end{array}$ & \begin{tabular}{|c|} 
Ground \\
Surface \\
Elevation (m)
\end{tabular} & $\begin{array}{l}\text { Column } \\
\text { Name }^{+}\end{array}$ & $\begin{array}{c}\text { Release } \\
\text { Model } \\
\text { Class }\end{array}$ & $\begin{array}{c}\text { Source } \\
\text { Type Name }\end{array}$ & $\begin{array}{l}\text { Volume } \\
\left(\mathrm{m}^{3}\right)^{++1}\end{array}$ \\
\hline $207-U$ & 135,044 & 566,973 & 3.00 & 140 & 208 & 299-W14-7 & Liquid & Retention Basin & $5.0 \mathrm{E}+03$ \\
\hline $216-A-1$ & 136,082 & 575,522 & 4.57 & 122 & 214 & 299-E25-2 & Liquid & Crib & $9.8 \mathrm{E}+01$ \\
\hline $216-\mathrm{A}-10$ & 135,440 & 574,978 & 13.72 & 122 & 221 & 299-E24-7 & Liquid & Crib & $3.2 \mathrm{E}+06$ \\
\hline $216-\bar{A}-18$ & 136,236 & 575,580 & 4.57 & 122 & 209 & 299-E25-2 & Liquid & Trench & $4.9 \mathrm{E}+02$ \\
\hline $216-\bar{A}-19$ & 136,278 & 575,665 & 4.57 & 122 & 203 & 299-E25-2 & Liquid & Trench & $1.1 \mathrm{E}+03$ \\
\hline $216-A-2$ & 135,529 & 575,180 & 8.23 & 122 & 221 & 299-E25-2 & Liquid & Trench & $2.3 \mathrm{E}+02$ \\
\hline $216-A-20$ & 136,249 & 575,707 & 4.57 & 122 & 203 & 299-E25-2 & Liquid & Crib & $9.6 \mathrm{E}+02$ \\
\hline $216-A-21$ & 135,462 & 575,215 & 5.79 & 122 & 221 & 299-E25-2 & Liquid & Crib & $7.8 \mathrm{E}+04$ \\
\hline $216-A-24$ & 136,397 & 575,852 & 4.57 & 122 & 197 & 299-E26-8 & Liquid & Crib & $8.2 \mathrm{E}+05$ \\
\hline $216-A-25$ & 139,654 & 574,935 & 10.00 & 123 & 169 & 218-E-12B & Liquid & Pond & $3.1 \mathrm{E}+08$ \\
\hline $216-A-27$ & 135,401 & 575,197 & 4.27 & 122 & 221 & 299-E25-2 & Liquid & Crib & $2.3 \mathrm{E}+04$ \\
\hline $216-A-28$ & 135,779 & 575,083 & 3.35 & 122 & 216 & 299-E25-2 & Liquid & Crib & $3.0 \mathrm{E}+01$ \\
\hline $216-A-3$ & 135,820 & 575,100 & 4.88 & 122 & 216 & 299-E25-2 & Liquid & Crib & $3.1 \mathrm{E}+03$ \\
\hline $216-A-30$ & 135,508 & 575,981 & 3.66 & 122 & 210 & 299-E25-2 & Liquid & Crib & $7.1 \mathrm{E}+06$ \\
\hline $216-A-31$ & 135,484 & 575,166 & 7.32 & 122 & 221 & 299-E25-2 & Liquid & Crib & $1.0 \mathrm{E}+01$ \\
\hline $216-\mathrm{A}-36 \mathrm{~A} / \mathrm{B}$ & 135,345 & $\overline{575,106}$ & 6.71 & 122 & 221 & 299-E24-7 & Liquid & Crib & $3.2 \mathrm{E}+05$ \\
\hline $216-A-37-1$ & 135,679 & 575,842 & 3.35 & 122 & 211 & 299-E25-2 & Liquid & Crib & $3.8 \mathrm{E}+05$ \\
\hline $216-A-37-2$ & 135,526 & 576,170 & 4.57 & 122 & 210 & 299-E25-2 & Liquid & Crib & $1.1 \mathrm{E}+06$ \\
\hline $216-A-4$ & 135,529 & 575,217 & 7.92 & 122 & 221 & 299-E25-2 & Liquid & Crib & $6.2 \mathrm{E}+03$ \\
\hline $216-\mathrm{A}-45$ & 135,161 & 574,908 & 11.43 & 122 & 221 & 299-E24-7 & Liquid & Crib & $1.0 \mathrm{E}+05$ \\
\hline $216-A-5$ & 135,493 & 575,048 & 9.75 & 122 & 221 & 299-E24-7 & Liquid & Crib & $1.6 \mathrm{E}+06$ \\
\hline $216-A-6$ & 135,648 & 575,591 & 5.79 & 122 & 214 & 299-E25-2 & Liquid & Crib & $3.4 \mathrm{E}+06$ \\
\hline $216-A-7$ & 136,044 & 575,506 & 4.57 & 122 & 214 & 299-E25-2 & Liquid & Crib & $3.3 \mathrm{E}+02$ \\
\hline$\overline{216-A-8}$ & 136,194 & 575,780 & 4.27 & 122 & 203 & 299-E25-2 & Liquid & Crib & $1.2 \mathrm{E}+06$ \\
\hline $216-A-9$ & 136,036 & 575,099 & 3.96 & 122 & 216 & 299-E25-2 & Liquid & Crib & $9.8 \mathrm{E}+05$ \\
\hline $216-\mathrm{B}-10 \mathrm{~A}$ & 136,340 & 573,473 & 6.10 & 122 & 220 & 299-E28-16 & Liquid & Crib & $1.0 \mathrm{E}+04$ \\
\hline $216-\mathrm{B}-10 \mathrm{~B}$ & 136,340 & 573,451 & 6.10 & 122 & 220 & 299-E28-16 & Liquid & Crib & $2.8 \mathrm{E}+01$ \\
\hline 216-B-11A\&B & 137,419 & 573,851 & 12.19 & 122 & 197 & 218-E-12B & Liquid & Reverse Well & $3.0 \mathrm{E}+04$ \\
\hline
\end{tabular}


Table 4.3. (contd)

\begin{tabular}{|c|c|c|c|c|c|c|c|c|c|}
\hline Site Name & $\begin{array}{l}\text { Northing } \\
(\mathrm{m})^{\star}\end{array}$ & $\begin{array}{l}\text { Easting } \\
(\mathrm{m})^{\mathrm{nn}}\end{array}$ & $\begin{array}{l}\text { Depth } \\
(\mathrm{m})^{\mathrm{nnn}}\end{array}$ & $\begin{array}{c}\text { Water Table } \\
\text { Elevation }(\mathrm{m})^{+}\end{array}$ & \begin{tabular}{|c|} 
Ground \\
Surface \\
Elevation (m) \\
\end{tabular} & $\begin{array}{l}\text { Column } \\
\text { Name }^{++}\end{array}$ & $\begin{array}{c}\text { Release } \\
\text { Model } \\
\text { Class } \\
\end{array}$ & $\begin{array}{c}\text { Source } \\
\text { Type Name }\end{array}$ & $\begin{array}{l}\text { Volume } \\
\left(\mathrm{m}^{3}\right)^{+1+}\end{array}$ \\
\hline $216-B-12$ & 136,600 & 573,128 & 7.92 & 122 & 220 & 299-E28-16 & Liquid & Crib & $5.2 \mathrm{E}+05$ \\
\hline 216-B-14 & 134,405 & 573,649 & 3.66 & 122 & 229 & 299-E13-20 & Liquid & Crib & $8.7 \mathrm{E}+03$ \\
\hline $216-B-15$ & 134,432 & 573,607 & 4.57 & 122 & 229 & 299-E13-20 & Liquid & Crib & $6.3 \mathrm{E}+03$ \\
\hline $216-B-16$ & 134,366 & 573,625 & 3.66 & 122 & 229 & 299-E13-20 & Liquid & Crib & $5.6 \mathrm{E}+03$ \\
\hline 216-B-17 & 134,390 & 573,583 & 4.27 & 122 & 229 & 299-E13-20 & Liquid & Crib & $3.4 \mathrm{E}+03$ \\
\hline $216-B-18$ & 134,323 & 573,601 & $\overline{4.27}$ & 122 & 229 & 299-E13-20 & Liquid & Crib & $8.5 \mathrm{E}+03$ \\
\hline $216-\mathrm{B}-19$ & 134,347 & 573,559 & 4.27 & 122 & 229 & 299-E13-20 & Liquid & Crib & $6.4 \mathrm{E}+03$ \\
\hline 216-B-20 & 134,376 & 573,417 & 3.05 & 122 & 229 & 299-E13-20 & Liquid & Trench & $4.7 \mathrm{E}+03$ \\
\hline $216-\mathrm{B}-21$ & 134,376 & 573,383 & 3.05 & 122 & 229 & 299-E13-20 & Liquid & Trench & $4.7 \mathrm{E}+03$ \\
\hline $216-\bar{B}-2-1$ & 137,089 & 574,524 & 1.83 & 122 & 203 & $218-E-12 B$ & Liquid & Ditch & $1.5 \mathrm{E}+08$ \\
\hline $216-\mathrm{B}-22$ & 134,380 & 573,349 & 3.66 & 122 & 229 & 299-E13-20 & Liquid & Trench & $4.7 \mathrm{E}+03$ \\
\hline $216-\mathrm{B}-2-2$ & 137,068 & 574,517 & 2.44 & 122 & 203 & 218-E-12B & Liquid & Ditch & $5.0 \mathrm{E}+04$ \\
\hline $216-\mathrm{B}-23$ & 134,235 & 573,289 & 2.44 & 122 & 226 & 299-E13-20 & Liquid & Trench & $4.5 \mathrm{E}+03$ \\
\hline $216-\mathrm{B}-2-3$ & 137,036 & 574,468 & 2.44 & 122 & 203 & 218-E-12B & Liquid & Ditch & $1.9 \mathrm{E}+03$ \\
\hline $216-\mathrm{B}-24$ & 134,205 & 573,289 & 2.44 & 122 & 226 & 299-E13-20 & Liquid & Trench & $4.7 \mathrm{E}+03$ \\
\hline $216-\bar{B}-25$ & 134,174 & 573,289 & 3.05 & 122 & 226 & 299-E13-20 & Liquid & Trench & $3.8 \mathrm{E}+03$ \\
\hline $216-\mathrm{B}-26$ & 134,144 & 573,289 & 2.44 & 122 & 226 & 299-E13-20 & Liquid & Trench & $5.9 \mathrm{E}+03$ \\
\hline $216-\mathrm{B}-27$ & 134,113 & 573,289 & 2.44 & 122 & 225 & 299-E13-20 & Liquid & Trench & $4.4 \mathrm{E}+03$ \\
\hline $216-\mathrm{B}-28$ & 134,081 & 573,289 & 3.96 & 122 & 225 & 299-E13-20 & Liquid & Trench & $5.1 \mathrm{E}+03$ \\
\hline 216-B-29 & 134,439 & 573,089 & 3.05 & 122 & 231 & 299-E13-20 & Liquid & Trench & $4.8 \mathrm{E}+03$ \\
\hline $216-B-3$ & 136,687 & 576,899 & 10.00 & 127 & 178 & 299-E26-8 & Liquid & Pond & $2.4 \mathrm{E}+08$ \\
\hline $216-\mathrm{B}-30$ & 134,402 & 573,089 & 3.35 & 122 & 231 & 299-E13-20 & Liquid & Trench & $4.8 \mathrm{E}+03$ \\
\hline $216-\bar{B}-31$ & 134,361 & 573,089 & 3.05 & 122 & 229 & 299-E13-20 & Liquid & Reactor & $4.7 \mathrm{E}+03$ \\
\hline $216-\mathrm{B}-32$ & 134,325 & 573,089 & 3.05 & 122 & 229 & 299-E13-20 & Liquid & Trench & $4.8 \mathrm{E}+03$ \\
\hline $216-\mathrm{B}-33$ & 134,286 & 573,089 & 3.05 & 122 & 229 & 299-E13-20 & Liquid & Trench & $4.7 \mathrm{E}+03$ \\
\hline $216-\bar{B}-34$ & 134,250 & 573,089 & 3.05 & 122 & 229 & 299-E13-20 & Liquid & Trench & $4.9 \mathrm{E}+03$ \\
\hline $216-\mathrm{B}-35$ & 137,274 & 573,439 & 3.05 & 122 & 206 & 218-E-10 & Liquid & Trench & $1.1 \mathrm{E}+03$ \\
\hline $216-\mathrm{B}-36$ & 137,292 & 573,439 & 3.05 & 122 & 206 & 218-E-10 & Liquid & Trench & $1.9 \mathrm{E}+03$ \\
\hline 216-B-37 & 137,318 & 573,439 & 3.05 & 122 & 206 & $218-\mathrm{E}-10$ & Liquid & Trench & $4.3 \mathrm{E}+03$ \\
\hline
\end{tabular}


Table 4.3. (contd)

\begin{tabular}{|c|c|c|c|c|c|c|c|c|c|}
\hline Site Name & $\begin{array}{l}\text { Northing } \\
\text { (m) }\end{array}$ & $\begin{array}{l}\text { Easting } \\
(\mathrm{m})^{n \star n}\end{array}$ & $\begin{array}{l}\text { Depth } \\
(\mathrm{m})^{\text {ana }}\end{array}$ & $\begin{array}{c}\text { Water Table } \\
\text { Elevation }(\mathbf{m})^{+}\end{array}$ & $\begin{array}{c}\text { Ground } \\
\text { Surface } \\
\text { Elevation (m) }\end{array}$ & $\begin{array}{l}\text { Column } \\
\text { Name }^{++}\end{array}$ & $\begin{array}{c}\text { Release } \\
\text { Model } \\
\text { Class }\end{array}$ & $\begin{array}{c}\text { Source } \\
\text { Type Name }\end{array}$ & $\begin{array}{l}\text { Volume } \\
\left(\mathrm{m}^{3}\right)^{++1}\end{array}$ \\
\hline $216-B-38$ & 137,345 & 573,439 & 3.05 & 122 & 206 & 218-E-10 & Liquid & Trench & $1.4 \mathrm{E}+03$ \\
\hline $216-\mathrm{B}-39$ & 137,373 & 573,439 & 3.05 & 122 & 206 & 218-E-10 & Liquid & Trench & $1.5 \mathrm{E}+03$ \\
\hline $216-\mathrm{B}-40$ & 137,400 & 573,439 & 3.05 & 122 & 201 & $218-E-10$ & Liquid & Trench & $1.6 \mathrm{E}+03$ \\
\hline $216-B-41$ & 137,427 & 573,439 & 3.05 & 122 & 201 & 218-E-10 & Liquid & Trench & $1.4 \mathrm{E}+03$ \\
\hline 216-B-43 & 137,614 & 573,625 & 4.57 & 122 & 201 & 218-E-10 & Liquid & Crib & $2.1 \mathrm{E}+03$ \\
\hline $216-B-44$ & 137,640 & 573,625 & 4.57 & 122 & 193 & $218-E-10$ & Liquid & Crib & $5.6 \mathrm{E}+03$ \\
\hline $216-\mathrm{B}-45$ & 137,666 & 573,625 & 4.57 & 122 & 193 & $218-E-10$ & Liquid & Crib & $4.9 \mathrm{E}+03$ \\
\hline $216-\mathrm{B}-46$ & 137,692 & 573,625 & 4.57 & 122 & 193 & $218-E-10$ & Liquid & Crib & $6.7 \mathrm{E}+03$ \\
\hline $216-B-47$ & 137,614 & 573,582 & 4.57 & 122 & 201 & $218-\mathrm{E}-10$ & Liquid & Crib & $3.7 \mathrm{E}+03$ \\
\hline 216-B-48 & 137,640 & 573,582 & 4.57 & 122 & 193 & 218-E-10 & Liquid & Crib & $4.1 \mathrm{E}+03$ \\
\hline 216-B-49 & 137,666 & 573,582 & 4.57 & 122 & 193 & $218-\mathrm{E}-10$ & Liquid & Crib & $6.7 \mathrm{E}+03$ \\
\hline $216-\mathrm{B}-5$ & 136,732 & 573,781 & 92.05 & 122 & 215 & 299-E28-22 & Liquid & Reverse Well & $3.1 \mathrm{E}+04$ \\
\hline $216-\mathrm{B}-50$ & 137,692 & $57,3,582$ & 4.57 & 122 & 193 & $218-\mathrm{E}-10$ & Liquid & Crib & $5.5 \mathrm{E}+04$ \\
\hline $216-\mathrm{B}-52$ & 134,271 & 573,296 & 3.05 & 122 & 226 & 299-E13-20 & Liquid & Trench & $8.5 \mathrm{E}+03$ \\
\hline $216-\mathrm{B}-53 \mathrm{~A}$ & 134,441 & 573,235 & 3.05 & 122 & 229 & 299-E13-20 & Liquid & Trench & $5.5 \mathrm{E}+02$ \\
\hline $216-\mathrm{B}-53 \mathrm{~B}$ & 134,423 & 573,241 & 2.44 & 122 & 229 & 299-E13-20 & Liquid & Trench & $1.5 \mathrm{E}+01$ \\
\hline $216-B-54$ & 134,379 & 573,242 & 2.44 & 122 & 229 & 299-E13-20 & Liquid & Trench & $1.0 \mathrm{E}+03$ \\
\hline $216-\mathrm{B}-55$ & 136,495 & 573,092 & 3.66 & 122 & 221 & 299-E28-16 & Liquid & Crib & $1.2 \mathrm{E}+06$ \\
\hline $216-\mathrm{B}-57$ & 137,579 & 573,499 & 3.05 & 122 & 201 & $218-\mathrm{E}-10$ & Liquid & Crib & $8.4 \mathrm{E}+04$ \\
\hline$2 \longdiv { 2 1 6 - \mathrm { B } - 5 8 }$ & 134,349 & 573,242 & 2.44 & 122 & 226 & 299-E13-20 & Liquid & Trench & $4.1 \mathrm{E}+02$ \\
\hline $216-\mathrm{B}-59$ & 136,636 & 573,851 & 3.66 & 122 & 215 & 299-E28-22 & Liquid & Retention Basin & $2.5 \mathrm{E}+02$ \\
\hline $216-B-60$ & 136,470 & 573,365 & 12.19 & 122 & 220 & 299-E28-16 & Liquid & crib & $1.9 \mathrm{E}+01$ \\
\hline $216-\mathrm{B}-62$ & 136,815 & 573,075 & 5.49 & 122 & 215 & 299-E28-16 & Liquid & Crib & $2.8 \mathrm{E}+05$ \\
\hline $216-\overline{-B}-63$ & 137,199 & 574,189 & 3.05 & 122 & 196 & $218-\mathrm{E}-12 \mathrm{~B}$ & Liquid & Ditch & $7.2 \mathrm{E}+06$ \\
\hline 216-B-7A\&B & 137,393 & 573,799 & 4.27 & 122 & 197 & 218-E-12B & Liquid & Crib & $\overline{4.4 \mathrm{E}+04}$ \\
\hline $216-\mathrm{B}-8$ & 137,505 & 573,808 & 7.01 & 122 & 197 & 218-E-12B & Liquid & Crib & $2.7 \mathrm{E}+04$ \\
\hline $216-\mathrm{B}-9$ & 136,850 & 573,852 & 9.14 & 122 & 215 & 299-E28-22 & Liquid & Crib & $3.6 \mathrm{E}+04$ \\
\hline $216-\mathrm{C}-1$ & 136,304 & 574,580 & 3.96 & 122 & 215 & 299-E28-22 & Liquid & Crib & $2.3 \mathrm{E}+04$ \\
\hline $216-C-10$ & 136,314 & 574,697 & 2.13 & 122 & 214 & 299-E28-22 & Liquid & Crib & $9.0 \mathrm{E}+02$ \\
\hline
\end{tabular}


Table 4.3. (contd)

\begin{tabular}{|c|c|c|c|c|c|c|c|c|c|}
\hline Site Name & $\begin{array}{l}\text { Northing } \\
\text { (m) }\end{array}$ & $\begin{array}{l}\text { Easting } \\
(\mathrm{m})^{* *}\end{array}$ & $\begin{array}{l}\text { Depth } \\
\text { (m) }\end{array}$ & $\begin{array}{c}\text { Water Table } \\
\text { Elevation(m) }\end{array}$ & \begin{tabular}{|c|} 
Ground \\
Surface \\
Elevation (m)
\end{tabular} & $\begin{array}{l}\text { Column } \\
\text { Name }{ }^{++}\end{array}$ & $\begin{array}{c}\text { Release } \\
\text { Model } \\
\text { Class }\end{array}$ & $\begin{array}{c}\text { Source } \\
\text { Type Name }\end{array}$ & $\begin{array}{l}\text { Volume } \\
\left(\mathrm{m}^{3}\right)^{+1+}\end{array}$ \\
\hline $216-C-3$ & 136,300 & 574,534 & 3.05 & 122 & 215 & 299-E28-22 & Liquid & Crib & $5.0 \mathrm{E}+03$ \\
\hline $216-\mathrm{C}-4$ & 136,305 & 574,522 & 4.88 & 122 & 215 & 299-E28-22 & Liquid & Crib & $1.7 \mathrm{E}+02$ \\
\hline $216-C-5$ & 136,292 & 574,543 & 4.88 & 122 & 215 & 299-E28-22 & Liquid & Crib & $3.8 \mathrm{E}+01$ \\
\hline $216-C-6$ & 136,288 & 574,632 & 4.88 & 122 & 214 & 299-E28-22 & Liquid & Crib & $5.3 \mathrm{E}+02$ \\
\hline $216-C-7$ & 136,283 & 574,448 & 3.66 & 122 & 215 & 299-E28-22 & Liquid & Crib & $6.0 \mathrm{E}+01$ \\
\hline $216-C-9$ & 136,478 & 574,585 & 7.62 & 122 & 215 & 299-E28-22 & Liquid & Pond & $1.0 \mathrm{E}+06$ \\
\hline $216-\mathrm{N}-2$ & 140,380 & 569,829 & 2.13 & 127 & 175 & 299-W6-1 & Liquid & Trench & $7.6 \mathrm{E}+03$ \\
\hline $216-\mathrm{N}-3$ & 140,371 & 569,818 & 1.83 & 127 & 175 & 299-W6-1 & Liquid & Trench & $7.6 \mathrm{E}+03$ \\
\hline $216-\mathrm{N}-4$ & 139,933 & 570,754 & 0.91 & 123 & 177 & $218-E-10$ & Liquid & Pond & $9.5 \mathrm{E}+05$ \\
\hline $216-N-5$ & 140,374 & 570,635 & 1.83 & 123 & 177 & $218-\mathrm{E}-10$ & Liquid & Trench & $7.6 \mathrm{E}+03$ \\
\hline $216-N-6$ & 139,895 & 571,643 & 0.91 & 122 & 176 & $218-E-10$ & Liquid & Pond & $9.5 \mathrm{E}+05$ \\
\hline $216-\mathrm{N}-7$ & 140,384 & 571,434 & 1.83 & 122 & 173 & $218-\mathrm{E}-10$ & Liquid & Trench & $7.6 \mathrm{E}+03$ \\
\hline $216-S-1 \& 2$ & 134,260 & 566,980 & 10.67 & 139 & 206 & $299-W 22-24$ & Liquid & Crib & $1.6 \mathrm{E}+05$ \\
\hline $216-S-10 D$ & 133,440 & 566,650 & 1.83 & 139 & 203 & $299-W 22-24$ & Liquid & Ditch & $4.3 \mathrm{E}+06$ \\
\hline $216-S-11$ & 133,270 & 566,473 & 3.00 & 139 & 203 & $299-W 22-24$ & Liquid & Pond & $2.2 \mathrm{E}+06$ \\
\hline $216-S-12$ & 134,120 & 567,531 & 3.05 & 138 & 210 & $299-W 22-24$ & Liquid & Trench & $6.8 \mathrm{E}+01$ \\
\hline $216-\mathrm{S}-13$ & 134,011 & 567,155 & 10.36 & 138 & 209 & $299-W 22-24$ & Liquid & Crib & $5.0 \mathrm{E}+03$ \\
\hline $216-S-16 P$ & 133,254 & 565,033 & 0.91 & 141 & 191 & $299-W 18-21$ & Liquid & Pond & $4.1 \mathrm{E}+07$ \\
\hline $216-S-17$ & 133,248 & 565,991 & 3.05 & 140 & 199 & $299-W 18-21$ & Liquid & Pond & $6.4 \mathrm{E}+06$ \\
\hline $216-S-19$ & 133,435 & 567,678 & 10.00 & 137 & 206 & $299-W 22-24$ & Liquid & Pond & $1.3 \mathrm{E}+06$ \\
\hline $216-S-20$ & 133,917 & 567,554 & 9.14 & 138 & 210 & $299-W 22-24$ & Liquid & Crib & $1.4 \mathrm{E}+05$ \\
\hline $216-S-22$ & 133,989 & 567,608 & 3.05 & 138 & 210 & $299-W 22-24$ & Liquid & Crib & $9.8 \mathrm{E}+01$ \\
\hline $216-S-23$ & 134,692 & 567,114 & 8.23 & 140 & 208 & $299-W 22-24$ & Liquid & Crib & $3.4 \mathrm{E}+04$ \\
\hline $216-\mathrm{S}-25$ & 134,287 & 566,570 & 3.05 & 139 & 204 & $299-W 18-21$ & Liquid & Crib & $2.9 \mathrm{E}+05$ \\
\hline $216-S-26$ & 133,760 & 567,595 & 3.66 & 138 & 211 & $299-W 22-24$ & Liquid & Crib & $1.6 \mathrm{E}+05$ \\
\hline $216-S-3$ & 134,438 & 566,893 & 1.83 & 139 & 207 & $299-W 22-24$ & Liquid & French Drain & $4.2 \mathrm{E}+03$ \\
\hline $216-S-5$ & 133,440 & 566,430 & 4.57 & 139 & 199 & $299-W 22-24$ & Liquid & Crib & $4.1 \mathrm{E}+0.6$ \\
\hline 216-S-6 & 133,596 & 566,217 & 4.57 & 139 & 202 & $299-W 18-21$ & Liquid & Crib & $4.5 \mathrm{E}+06$ \\
\hline $216-\mathrm{S}-7$ & 134,176 & 567,168 & 6.71 & 139 & 208 & 299-W22-24 & Liquid & Crib & $3.9 \mathrm{E}+05$ \\
\hline
\end{tabular}


Table 4.3. (contd)

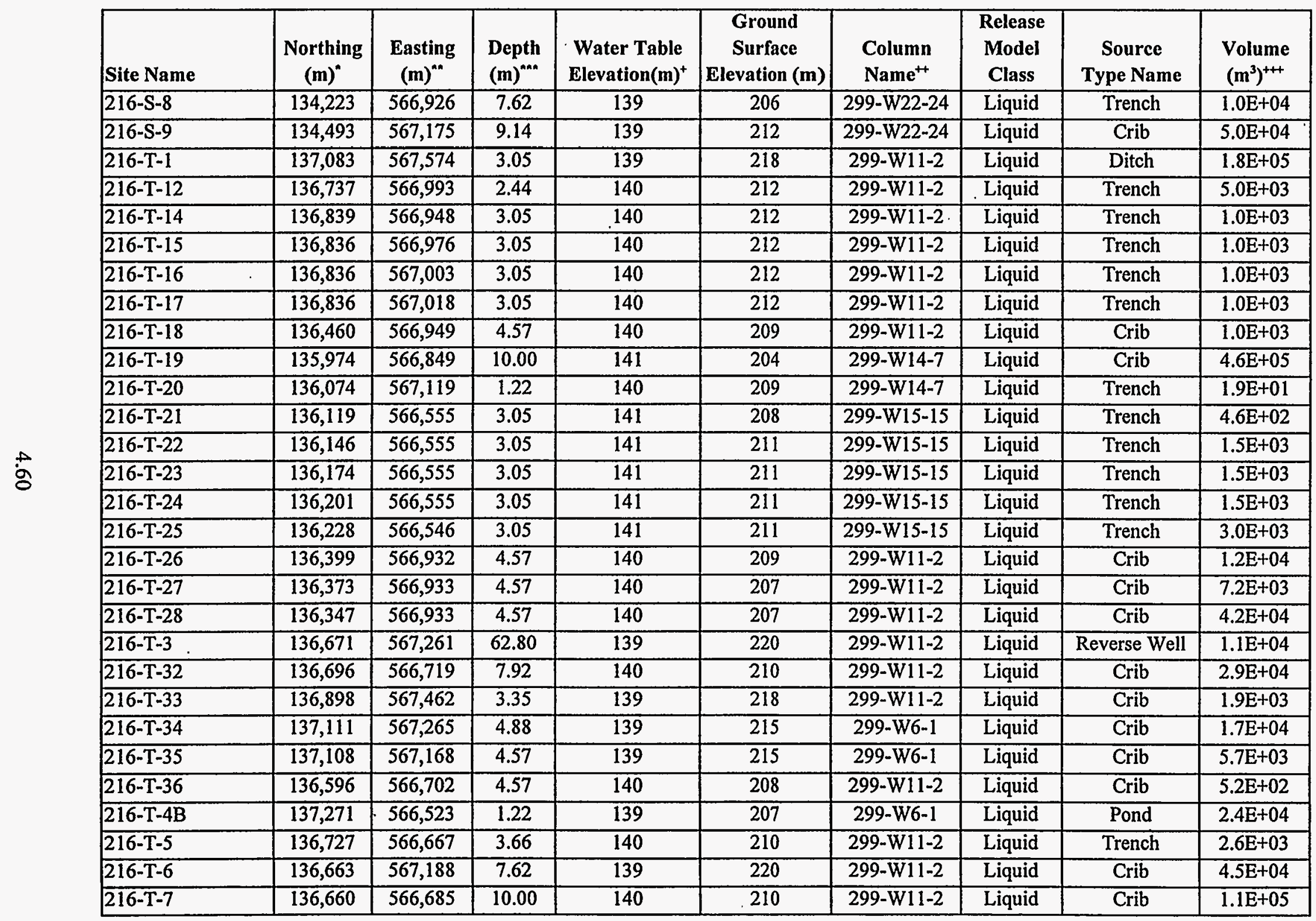


Table 4.3. (contd)

\begin{tabular}{|c|c|c|c|c|c|c|c|c|c|}
\hline Site Name & $\begin{array}{l}\text { Northing } \\
\text { (m) }\end{array}$ & $\begin{array}{l}\text { Easting } \\
(\mathrm{m})^{\mathrm{n}}\end{array}$ & $\begin{array}{l}\text { Depth } \\
\text { (m) }\end{array}$ & $\begin{array}{c}\text { Water Table } \\
\text { Elevation(m) }\end{array}$ & \begin{tabular}{|c|} 
Ground \\
Surface \\
Elevation $(m)$
\end{tabular} & $\begin{array}{l}\text { Column } \\
\text { Name }^{++}\end{array}$ & $\begin{array}{c}\text { Release } \\
\text { Model } \\
\text { Class }\end{array}$ & $\begin{array}{c}\text { Source } \\
\text { Type Name }\end{array}$ & $\begin{array}{l}\text { Volume } \\
\left(\mathrm{m}^{3}\right)^{+1+}\end{array}$ \\
\hline $216-T-8$ & 136,727 & 567,651 & 6.10 & 138 & 223 & 299-W11-2 & Liquid & Crib & $5.0 \mathrm{E}+02$ \\
\hline $216-U-1 \& 2$ & 135,002 & 567,243 & 7.32 & 140 & 212 & 299-W14-7 & Liquid & Crib & $4.6 \mathrm{E}+04$ \\
\hline $216-U-10$ & 134,624 & 566,372 & 10.00 & 140 & 201 & $299-W 18-21$ & Liquid & Pond & $1.7 \mathrm{E}+08$ \\
\hline $216-\mathrm{U}-12$ & 134,502 & 567,592 & 3.96 & 139 & 212 & 299-W22-24 & Liquid & Crib & $1.5 \mathrm{E}+05$ \\
\hline $216-U-15$ & 135,127 & 567,371 & 4.57 & 140 & 215 & 299-W14-7 & Liquid & Trench & $6.8 \mathrm{E}+01$ \\
\hline $216-U-16$ & 134,861 & 567,236 & 10.00 & 139 & 211 & 299-W22-24 & Liquid & Crib & $4.1 \mathrm{E}+05$ \\
\hline $216-U-17$ & 134,904 & 567,839 & 10.00 & 139 & 216 & $299-W 22-24$ & Liquid & Crib & $2.1 \mathrm{E}+03$ \\
\hline $216-U-3$ & 134,928 & 566,845 & 3.66 & 140 & 202 & 299-W18-21 & Liquid & French Drain & $7.9 \mathrm{E}+02$ \\
\hline $216-U-4 A$ & 135,111 & 567,580 & 22.86 & 139 & 213 & $299-W 22-24$ & Liquid & Reverse Well & $5.5 \mathrm{E}+02$ \\
\hline $216-U-4 B$ & 135,121 & 567,615 & 22.86 & 139 & 213 & 299-W14-8A & Liquid & Reverse Well & $3.3 \mathrm{E}+01$ \\
\hline $216-U-5$ & 135,359 & 567,673 & 3.05 & 139 & 220 & 299-W14-8A & Liquid & Trench & $4.5 \mathrm{E}+03$ \\
\hline $216-U-7$ & 135,204 & 567,611 & 5.18 & 139 & 221 & 299-W14-8A & Liquid & French Drain & $7.0 \mathrm{E}+00$ \\
\hline $216-U-8$ & 134,698 & 567,617 & 9.45 & 139 & 212 & $299-W 22-24$ & Liquid & Crib & $3.8 \mathrm{E}+05$ \\
\hline $216-Z-1 \& 2$ & $1.35,469$ & 566,547 & 6.40 & 141 & 211 & $299-W 14-7$ & Liquid & Crib & $3.4 \mathrm{E}+04$ \\
\hline $216-Z-10$ & 135,897 & 566,567 & 45.72 & 141 & 208 & 299-W15-15 & Liquid & Reverse Well & $1.0 \mathrm{E}+03$ \\
\hline $216-Z-12$ & 135,423 & 566,365 & 6.10 & 141 & 212 & 299-W15-15 & Liquid & Crib & $2.8 \mathrm{E}+05$ \\
\hline $216-Z-16$ & 135,991 & 566,430 & 4.57 & 141 & 208 & 299-W15-15 & Liquid & Crib & $1.0 \mathrm{E}+05$ \\
\hline $216-Z-17$ & 135,863 & 566,603 & 2.44 & 141 & 208 & 299-W14-7 & Liquid & Ditch & $3.7 \mathrm{E}+04$ \\
\hline $216-Z-18$ & 135,286 & 566,440 & 10.00 & 140 & 208 & 299-W18-21 & Liquid & Crib & $3.9 \mathrm{E}+03$ \\
\hline $216-Z-1 A$ & 135,419 & 566,549 & 10.00 & 141 & 211 & 299-W14-7 & Liquid & Crib & $5.3 \mathrm{E}+03$ \\
\hline $216-Z-20$ & 135,299 & 566,624 & 10.00 & 140 & 208 & 299-W14-7 & Liquid & Crib & $3.8 \mathrm{E}+06$ \\
\hline $216-Z-3$ & 135,459 & 566,577 & 7.62 & 141 & 211 & 299-W14-7 & Liquid & Crib & $1.8 \mathrm{E}+05$ \\
\hline $216-Z-4$ & 135,921 & 566,586 & 4.57 & 141 & 208 & 299-W14-7 & Liquid & Trench & $1.1 \mathrm{E}+01$ \\
\hline $216-Z-5$ & 135,949 & 566,555 & 10.00 & 141 & 208 & $299-W 15-15$ & Liquid & Crib & $3.1 \mathrm{E}+04$ \\
\hline $216-Z-6$ & 135,876 & 566,579 & 2.44 & 141 & 208 & 299-W15-15 & Liquid & Crib & $9.8 \mathrm{E}+01$ \\
\hline $216-Z-7$ & 135,927 & 566,701 & 1.52 & 141 & 204 & 299-W14-7 & Liquid & Crib & $7.9 \mathrm{E}+04$ \\
\hline $216-Z-8$ & 135,653 & 566,654 & 5.18 & 141 & 204 & 299-W14-7 & Liquid & Crib & $1.0 \mathrm{E}+01$ \\
\hline $216-Z-9$ & 135,611 & 566,758 & 6.40 & 141 & 205 & 299-W14-7 & Liquid & Crib & $4.1 \mathrm{E}+03$ \\
\hline 218-EC-9(a) & 136,465 & 574,658 & 6.71 & 122 & 212 & 299-E28-22 & Soil/debris & Burial Site & $1.9 \mathrm{E}+03$ \\
\hline
\end{tabular}


Table 4.3. (contd)

\begin{tabular}{|c|c|c|c|c|c|c|c|c|c|}
\hline Site Name & $\begin{array}{c}\text { Northing } \\
\text { (m)" }\end{array}$ & $\begin{array}{l}\text { Easting } \\
(\mathrm{m})^{\mathrm{nn}}\end{array}$ & $\begin{array}{l}\text { Depth } \\
(\mathrm{m})^{\mathrm{nnn}}\end{array}$ & $\begin{array}{c}\text { Water Table } \\
\text { Elevation(m) }\end{array}$ & $\begin{array}{c}\text { Ground } \\
\text { Surface } \\
\text { Elevation (m) } \\
\end{array}$ & $\begin{array}{l}\text { Column } \\
\text { Name }^{++} \\
\end{array}$ & $\begin{array}{c}\text { Release } \\
\text { Model } \\
\text { Class } \\
\end{array}$ & $\begin{array}{c}\text { Source } \\
\text { Type Name } \\
\end{array}$ & $\begin{array}{l}\begin{array}{l}\text { Volume } \\
\left(\mathrm{m}^{3}\right)^{++1+}\end{array} \\
\end{array}$ \\
\hline $218-\mathrm{EC}-9(\mathrm{~b})^{\mathrm{HAH}}$ & 136,465 & 574,658 & 6.50 & 122 & 212 & 299-E28-22 & Soil/debris & Burial Site & $5.7 \mathrm{E}+03$ \\
\hline 218-E-1(b) & 135,575 & 574,755 & 6.50 & 122 & 222 & 299-E24-7 & Soil/debris & Burial Site & $3.0 \mathrm{E}+03$ \\
\hline $218-\mathrm{E}-10(\mathrm{~b})$ & 137,268 & 572,945 & 6.50 & 122 & 210 & $218-\mathrm{E}-10$ & Soil/debris & Burial Site & $2.1 \mathrm{E}+04$ \\
\hline 218-E-10(a) & 137,268 & 572,945 & 6.50 & 122 & 210 & 218-E-10 & Soil/debris & Burial Site & $3.6 \mathrm{E}+03$ \\
\hline $218-\mathrm{E}-12 \mathrm{~A}(\mathrm{~b})$ & 136,803 & 574,938 & 6.50 & 122 & 202 & 218-E-12B & Soil/debris & Burial Site & $1.5 \mathrm{E}+04$ \\
\hline $218-\mathrm{E}-12 \mathrm{~B}(\mathrm{~b})$ & 137,447 & 574,796 & 6.50 & 122 & 188 & 218-E-12B & Soil/debris & Burial Site & $5.1 \mathrm{E}+04$ \\
\hline 218-E-12B(a) & 137,447 & 574,796 & 6.50 & 122 & 188 & 218-E-12B & Soil/debris & Burial Site & $3.7 \mathrm{E}+04$ \\
\hline 218-E-2(b) & 137,078 & 573,511 & 6.50 & 122 & 209 & 299-E28-16 & Soil/debris & Burial Site & $9.0 \mathrm{E}+03$ \\
\hline 218-E-4(b) & 136,891 & 573,497 & 6.50 & 122 & 209 & 299-E28-16 & Soil/debris & Burial Site & $1.6 \mathrm{E}+03$ \\
\hline 218-E-5(b) & 137,080 & 573,417 & 6.50 & 122 & 209 & 299-E28-16 & Soil/debris & Burial Site & $3.2 \mathrm{E}+03$ \\
\hline 218-E-5A(b) & 137,088 & 573,356 & 6.50 & 122 & 211 & 299-E28-16 & Soil/debris & Burial Site & $6.2 \mathrm{E}+03$ \\
\hline 218-E-8(b) & 137,225 & 575,116 & 6.50 & 122 & 189 & $218-E-12 B$ & Soil/debris & Burial Site & $2.3 \mathrm{E}+03$ \\
\hline 218-W-1(b) & 136,222 & 566,205 & 6.50 & 140 & 212 & 299-W15-15 & Soil/debris & Burial Site & $7.2 \mathrm{E}+03$ \\
\hline $218-W-11(b)$ & 136,319 & 566,205 & 6.50 & 140 & 212 & $299-W 15-15$ & Soil/debris & Burial Site & $1.2 \mathrm{E}+03$ \\
\hline $218-W-1 A(b)$ & 137,184 & 567,060 & 6.50 & 139 & 214 & 299-W6-1 & Soil/debris & Burial Site & $1.4 \mathrm{E}+04$ \\
\hline $218-\mathrm{W}-2(\mathrm{~b})$ & 136,062 & 566,205 & 6.50 & 141 & 208 & $299-W 15-15$ & Soil/debris & Burial Site & $8.2 \mathrm{E}+03$ \\
\hline $218-W-2 A(b)$ & 136,891 & 566,425 & 6.50 & 140 & 210 & $218-W-5$ & Soil/debris & Burial Site & $5.0 \mathrm{E}+04$ \\
\hline $218-W-3(b)$ & 136,745 & 566,166 & 6.50 & 140 & 213 & $218-W-5$ & Soil/debris & Burial Site & $2.2 \mathrm{E}+04$ \\
\hline $218-W-3 A(b)$ & 137,282 & 566,226 & 6.50 & 140 & 210 & $218-W-5$ & Soil/debris & Burial Site & $9.5 \mathrm{E}+04$ \\
\hline $218-W-3 A(a)$ & 137,282 & 566,226 & 6.50 & 140 & 210 & $218-W-5$ & Soil/debris & Burial Site & $2.4 \mathrm{E}+04$ \\
\hline $218-W-3 A E(b)$ & 137,391 & 566,616 & 6.50 & 139 & 210 & 299-W6-1 & Soil/debris & Burial Site & $1.1 \mathrm{E}+04$ \\
\hline 218-W-3AE(a) & 137,391 & 566,616 & 6.50 & 139 & 210 & 299-W6-1 & Soil/debris & Burial Site & $6.7 \mathrm{E}+04$ \\
\hline $218-W-4 A(b)$ & 136,491 & 566,228 & 6.50 & 140 & 210 & 299-W15-15 & Soil/debris & Burial Site & $1.8 \mathrm{E}+04$ \\
\hline $218-W-4 B-c(b)$ & 135,881 & 566,191 & 6.50 & 141 & 208 & $299-W 15-15$ & Cement & Burial Site & $1.0 \mathrm{E}+04$ \\
\hline $218-W-4 B-c(a)$ & 135,881 & 566,191 & 6.50 & 141 & 208 & 299-W15-15 & Cement & Burial Site & $2.7 \mathrm{E}+01$ \\
\hline $218-W-4 C(a)$ & 135,086 & 566,458 & 6.50 & 140 & 207 & $299-\mathrm{W} 18-21$ & Soil/debris & Burial Site & $2.7 \mathrm{E}+04$ \\
\hline $218-W-4 C(b)$ & 135,086 & 566,458 & 6.50 & 140 & 207 & 299-W18-21 & Soil/debris & Burial Site & $1.0 \mathrm{E}+04$ \\
\hline $218-W-5(b)$ & 137,165 & 565,870 & 6.50 & 140 & 219 & $218-W-5$ & Soil/debris & Burial Site & $6.3 \mathrm{E}+03$ \\
\hline $218-W-5(a)$ & 137,165 & 565,870 & 6.50 & 140 & 219 & $218-W-5$ & Soil/debris & Burial Site & $1.8 \mathrm{E}+05$ \\
\hline
\end{tabular}


Table 4.3. (contd)

\begin{tabular}{|c|c|c|c|c|c|c|c|c|c|}
\hline Site Name & $\begin{array}{l}\text { Northing } \\
\text { (m)* }\end{array}$ & $\begin{array}{l}\text { Easting } \\
(\mathrm{m})^{m-m}\end{array}$ & $\begin{array}{l}\text { Depth } \\
\text { (m) }\end{array}$ & $\begin{array}{l}\text { Water Table } \\
\text { Elevation(m) }\end{array}$ & \begin{tabular}{|c|} 
Ground \\
Surface \\
Elevation $(\mathrm{m})$
\end{tabular} & $\begin{array}{l}\text { Column } \\
\text { Name }^{++}\end{array}$ & $\begin{array}{c}\text { Release } \\
\text { Model } \\
\text { Class }\end{array}$ & $\begin{array}{c}\text { Source } \\
\text { Type Name }\end{array}$ & $\begin{array}{l}\text { Volume } \\
\left(\mathrm{m}^{3}\right)^{++1}\end{array}$ \\
\hline $218-W-7$ & 133,865 & 567,485 & 6.50 & 138 & 211 & $299-W 22-24$ & Soil/debris & Burial Site & $1.6 \mathrm{E}+02$ \\
\hline $218-W-8$ & 136,775 & 567,638 & 6.50 & 138 & 223 & 299-W11-2 & Soil/debris & Burial Site & $6.8 \mathrm{E}+01$ \\
\hline $218-W-9(b)$ & 134,307 & 567,189 & 6.50 & 139 & 208 & 299-W22-24 & Soil/debris & Burial Site & $4.9 \mathrm{E}+02$ \\
\hline $\begin{array}{l}\text { TWRS glass grout } \\
\text { vault }\end{array}$ & 135,787 & 576,019 & 6.5 & 122 & 222 & 299-E24-7 & Glass & Burial Site & $n / a$ \\
\hline $\begin{array}{l}\text { TWRS glass new } \\
\text { site }\end{array}$ & 135,298 & $574 ; 371$ & 6.5 & 122 & 222 & 299-E24-7 & Glass & Burial Site & $\mathrm{n} / \mathrm{a}$ \\
\hline US Ecology current & 134,188 & 572,175 & 13.7 & 123 & 224 & 299-E19-1 & Soil/debris & Burial Site & $4.2 \mathrm{E}+05$ \\
\hline US Ecology future & 134,188 & 572,175 & 13.7 & 123 & 224 & 299-E19-1 & Soil/debris & Burial Site & $1.0 \mathrm{E}+06$ \\
\hline ERDF & 134,422 & 568,900 & 14 & 135 & 222 & 299-W21-1 & Soil/debris & Burial Site & $3.0 \overline{\mathrm{E}}+06$ \\
\hline C Reactor & 136,852 & 567,570 & 22 & 139 & 222 & 299-W11-2 & Reactor & Reactor & \\
\hline D Reactor & 136,852 & 567,570 & 22 & 139 & 222 & 299-W11-2 & Reactor & Reactor & \\
\hline DR Reactor & 136,852 & 567,570 & 22 & 139 & 222 & 299-W11-2 & Reactor & Reactor & \\
\hline F Reactor & 136,852 & 567,570 & 22 & 139 & 222 & 299-W11-2 & Reactor & Reactor & \\
\hline H Reactor & 136,852 & 567,570 & 22 & 139 & 222 & $299-W 11-2$ & Reactor & Reactor & \\
\hline KE Reactor & 136,852 & 567,570 & 22 & 139 & 222 & 299-W11-2 & Reactor & Reactor & \\
\hline KW Reactor & 136,852 & 567,570 & 22 & 139 & 222 & $299-W 11-2$ & Reactor & Reactor & \\
\hline N Reactor & 136,852 & 567,570 & 22 & 139 & 222 & 299-W11-2 & Reactor & Reactor & \\
\hline TK-A-S & 136,060 & 575,353 & 16.6 & 122 & 217 & 299-E25-2 & Liquid & Tank & $1.8 \mathrm{E}+02$ \\
\hline TK-A-L & 136,060 & 575,353 & 16.6 & 122 & 217 & 299-E25-2 & Liquid & Tank & $1.1 \mathrm{E}+03$ \\
\hline TK-A-R & 136,060 & 575,353 & 16.6 & 122 & 217 & 299-E25-2 & Cake & Tank & \\
\hline TK-AN-R-1 & 136,423 & 575,381 & 17 & 122 & 198 & 299-E26-8 & Cake & Tank & \\
\hline TK-AN-R-2 & 136,423 & 575,381 & 17 & 122 & 198 & 299-E26-8 & Cake & Tank & \\
\hline TK-AP-R-1 & 135,822 & 575,556 & 17 & 122 & 214 & 299-E25-2 & Cake & Tank & \\
\hline TK-AP-R-2 & 135,822 & 575,556 & 17. & 122 & 214 & 299-E25-2 & Cake & Tank & \\
\hline TK-AW-R & 135,858 & 575,356 & 17 & 122 & 210 & 299-E25-2 & Cake & Tank & \\
\hline TK-AX-S-1 & 136,189 & 575,409 & 16.6 & 122 & 209 & 299-E25-2 & Liquid & Tank & $6.1 \mathrm{E}+01$ \\
\hline TK-AX-S-2 & 136,189 & 575,409 & 16.6 & 122 & 209 & 299-E25-2 & Liquid & Tank & $6.1 \mathrm{E}+01$ \\
\hline TK-AX-L-1 & 136,189 & 575,409 & 16.6 & 122 & 209 & 299-E25-2 & Liquid & Tank & $1.1 \mathrm{E}+01$ \\
\hline
\end{tabular}


Table 4.3. (contd)

\begin{tabular}{|c|c|c|c|c|c|c|c|c|c|}
\hline Site Name & $\begin{array}{l}\text { Northing } \\
\text { (m) }\end{array}$ & $\begin{array}{l}\text { Easting } \\
(\mathrm{m})^{\mathrm{nn}}\end{array}$ & $\begin{array}{l}\text { Depth } \\
(\mathrm{m})^{\text {nan }}\end{array}$ & $\begin{array}{c}\text { Water Table } \\
\text { Elevation }(\mathbf{m})^{+}\end{array}$ & \begin{tabular}{|c|} 
Ground \\
Surface \\
Elevation $(m)$
\end{tabular} & $\begin{array}{l}\text { Column } \\
\text { Name }^{++}\end{array}$ & $\begin{array}{l}\text { Release } \\
\text { Model } \\
\text { Class }\end{array}$ & $\begin{array}{c}\text { Source } \\
\text { Type Name }\end{array}$ & $\begin{array}{l}\text { Volume } \\
\left(\mathrm{m}^{3}\right)^{+1+}\end{array}$ \\
\hline TK-AX-L-2 & 136,189 & 575,409 & 16.6 & 122 & 209 & 299-E25-2 & Liquid & Tank & $3.0 \mathrm{E}+01$ \\
\hline TK-AX-R-1 & 136,189 & 575,409 & 16.6 & 122 & 209 & 299-E25-2 & Cake & Tank & \\
\hline TK-AX-R-2 & 136,189 & 575,409 & 16.6 & 122 & 209 & 299-E25-2 & Cake & Tank & \\
\hline TK-AY-R-1 & 136,188 & 575,312 & 17 & 122 & 210 & 299-E25-2 & Cake & Tank & \\
\hline TK-AY-R-2 & 136,188 & 575,312 & 17 & 122 & 210 & 299-E25-2 & Cake & Tank & \\
\hline TK-AZ-R & 136,311 & 575,397 & 17 & 122 & 209 & 299-E25-2 & Cake & Tank & \\
\hline TK-B-S & 137,299 & 573,826 & 10.3 & 122 & 204 & $218-E-12 B$ & Liquid & Tank & $4.8 \mathrm{E}+02$ \\
\hline TK-B-L & 137,299 & 573,826 & 10.3 & 122 & 204 & 218-E-12B & Liquid & Tank & $2.0 \mathrm{E}+02$ \\
\hline TK-B-R & 137,299 & 573,826 & 10.3 & 122 & 204 & $218-\mathrm{E}-12 \mathrm{~B}$ & Cake & Tank & \\
\hline TK-BX-S & 137,347 & 573,614 & 12.5 & 122 & 206 & $218-E-10$ & Liquid & Tank & $3.6 \mathrm{E}+02$ \\
\hline TK-BX-L & 137,347 & 573,614 & 12.5 & 122 & 206 & 218-E-10 & Liquid & Tank & $3.7 \mathrm{E}+02$ \\
\hline TK-BX-R & 137,347 & 573,614 & 12.5 & 122 & 206 & $218-E-10$ & Cake & Tank & \\
\hline TK-BY-S & 137,501 & 573,613 & 14.3 & 122 & 201 & 218-E-10 & Liquid & Tank & $3.6 \mathrm{E}+02$ \\
\hline TK-BY-L & 137,501 & 573,613 & 14.3 & 122 & 201 & 218-E-10 & Liquid & Tank & $1.6 \mathrm{E}+02$ \\
\hline TK-BY-R & 137,501 & 573,613 & 14.3 & 122 & 201 & $218-\mathrm{E}-10$ & Cake & Tank & \\
\hline TK-C-S-1 & 136,559 & 575,151 & 10.3 & 122 & 203 & 299-E26-8 & Liquid & Tank & $1.2 \mathrm{E}+02$ \\
\hline TK-C-S-2. & 136,559 & 575,151 & 10.3 & 122 & 203 & 299-E26-8 & Liquid & Tank & $3.3 \mathrm{E}+02$ \\
\hline TK-C-L-1 & 136,559 & 575,151 & 10.3 & 122 & 203 & 299-E26-8 & Liquid & Tank & $7.6 \mathrm{E}+00$ \\
\hline TK-C-L-2 & 136,559 & 575,151 & 10.3 & 122 & 203 & 299-E26-8 & Liquid & Tank & $1.0 \mathrm{E}+02$ \\
\hline TK-C-R-1 & 136,559 & 575,151 & 10.3 & 122 & 203 & 299-E26-8 & Cake & Tank & \\
\hline TK-C-R-2 & 136,559 & 575,151 & 10.3 & 122 & 203 & 299-E26-8 & Cake & Tank & \\
\hline TK-S-S & 134,236 & 566,804 & 14.3 & 139 & 202 & $299-W 22-24$ & Liquid & Tank & $3.6 \mathrm{E}+02$ \\
\hline TK-S-L & 134,236 & 566,804 & 14.3 & 139 & 202 & 299-W22-24 & Liquid & Tank & $9.1 \mathrm{E}+01$ \\
\hline TK-S-R & 134,236 & 566,804 & 14.3 & 139 & 202 & 299-W22-24 & Cake & Tank & \\
\hline TK-SX-S-1 & 134,456 & 566,804 & 16.6 & 140 & 203 & $299-W 22-24$ & Liquid & Tank & $3.0 \mathrm{E}+01$ \\
\hline TK-SX-S-2 & 134,456 & 566,804 & 16.6 & 140 & 203 & $299-W 22-24$ & Liquid & Tank & $4.2 \mathrm{E}+02$ \\
\hline TK-SX-L-2 & 134,456 & 566,804 & 16.6 & 140 & 203 & $299-W 22-24$ & Liquid & Tank & $6.3 \mathrm{E}+02$ \\
\hline TK-SX-R-1 & 134,456 & 566,804 & 16.6 & 140 & 203 & 299-W22-24 & Cake & Tank & \\
\hline TK-SX-R-2 & 134,456 & 566,804 & 16.6 & 140 & 203 & 299-W22-24 & Cake & Tank & \\
\hline
\end{tabular}


Table 4.3. (contd)

\begin{tabular}{|c|c|c|c|c|c|c|c|c|c|}
\hline Site Name & $\begin{array}{l}\text { Northing } \\
(\mathrm{m})^{\circ}\end{array}$ & $\begin{array}{l}\text { Easting } \\
(\mathrm{m})^{\mathrm{m}}\end{array}$ & $\begin{array}{l}\text { Depth } \\
\text { (m) }\end{array}$ & $\begin{array}{c}\text { Water Table } \\
\text { Elevation(m) }\end{array}$ & \begin{tabular}{|c|} 
Ground \\
Surface \\
Elevation (m)
\end{tabular} & $\begin{array}{l}\text { Column } \\
\text { Name }\end{array}$ & $\begin{array}{c}\text { Release } \\
\text { Model } \\
\text { Class }\end{array}$ & $\begin{array}{c}\text { Source } \\
\text { Type Name }\end{array}$ & $\begin{array}{l}\text { Volume } \\
\left(\mathrm{m}^{3}\right)^{+++}\end{array}$ \\
\hline TK-SY-R-1 & 134,541 & 566,883 & 17 & 139 & 207 & $299-W 22-24$ & Cake & Tank & \\
\hline TK-SY-R-2 & 134,541 & 566,883 & 17 & 139 & 207 & $299-W 22-24$ & Cake & Tank & \\
\hline TK-T-S & 136,719 & 566,806 & 10.3 & 140 & 210 & $299-W 11-2$ & Liquid & Tank & $4.8 \mathrm{E}+02$ \\
\hline TK-T-L & 136,719 & 566,806 & 10.3 & 140 & 210 & $299-W 11-2$ & Liquid & Tank & $5.1 \mathrm{E}+02$ \\
\hline TK-T-R & 136,719 & 566,806 & 10.3 & 140 & 210 & 299-W11-2 & Cake & Tank & \\
\hline TK-TX-S & 136,217 & 566,759 & 14.3 & 140 & 207 & $299-W 14-7$ & Liquid & Tank & $5.5 \mathrm{E}+02$ \\
\hline TK-TX-L & 136,217 & 566,759 & 14.3 & 140 & 207 & 299-W14-7 & Liquid & Tank & $2.2 \mathrm{E}+02$ \\
\hline TK-TX-R & 136,217 & 566,759 & 14.3 & 140 & 207 & 299-W14-7 & Cake & Tank & \\
\hline TK-TY-S & 136,416 & 566,758 & 14.3 & 140 & 208 & $299-W 11-2$ & Liquid & Tank & $1.8 \mathrm{E}+02$ \\
\hline TK-TY-L & 136,416 & 566,758 & 14.3 & 140 & 208 & $299-W 11-2$ & Liquid & Tank & $2.3 \mathrm{E}+02$ \\
\hline TK-TY-R & 136,416 & 566,758 & 14.3 & 140 & 208 & $299-W 11-2$ & Cake & Tank & \\
\hline TK-U-S & 135,058 & 566,812 & 10.3 & 140 & 202 & 299-W14-7 & Liquid & Tank & $4.8 \mathrm{E}+02$ \\
\hline TK-U-L & 135,058 & 566,812 & 10.3 & 140 & 202 & 299-W14-7 & Liquid & Tank & $3.8 \mathrm{E}+02$ \\
\hline TK-U-R & 135,058 & 566,812 & 10.3 & 140 & 202 & 299-W14-7 & Cake & Tank & \\
\hline
\end{tabular}

* Refers to north coordinate in Washington State Plane NAD83 coordinate system.

** Refers to east coordinate in Washington State Plane NAD83 coordinate system.

*** Refers to the depth of the source below the ground surface.

+ Water table elevation estimated for 1979 using CFEST groundwater model. Because of a reduction in liquid disposals, water table elevations are predicted to decline further.

+ See Table 4.6 for description of columns.

+++ For liquid disposals, "volume" refers to the volume of the liquid released. For a solid waste site, "volume" refers to the volumetric capacity of the site.

\# (a) refers to waste disposed after September 30, 1988.

\#\# (b) refers to waste disposed before September 30, 1988. 
Table 4.4. Chemical Classification of Waste Sites

\begin{tabular}{|c|c|c|}
\hline Site Name & Waste Type Name & $\begin{array}{l}\mathrm{K}_{\mathrm{d}} \text { Switch } \\
\text { Depth (m) }\end{array}$ \\
\hline $207-U$ & Low Organic - Low Salts - Near Neutral & 0.0 \\
\hline $216-A-1$ & Low Organic - Low Salts - Near Neutral & 0.0 \\
\hline $216-A-10$ & Low Organic - Low Salts - Near Neutral & 0.0 \\
\hline $216-A-18$ & Low Organic - Low Salts - Near Neutral & 0.0 \\
\hline $216-\mathrm{A}-19$ & Low Organic - Low Salts - Near Neutral & 0.0 \\
\hline $216-A-2$ & High Organic - Near Neutral & 0.0 \\
\hline $216-\mathrm{A}-20$ & Low Organic - Low Salts - Near Neutral & 0.0 \\
\hline $216-A-21$ & Low Organic - Low Salts - Near Neutral & 0.0 \\
\hline $216-A-24$ & High Organic - Near Neutral & 0.0 \\
\hline $216-\mathrm{A}-25$ & Low Organic - Low Salts - Near Neutral & 0.0 \\
\hline $216-\mathrm{A}-27$ & Low Organic - Low Salts - Near Neutral & 0.0 \\
\hline $216-\mathrm{A}-28$ & Low Organic - Low Salts - Near Neutral & 0.0 \\
\hline $216-\mathrm{A}-3$ & Low Organic - Low Salts - Near Neutral & 0.0 \\
\hline $216-A-30$ & Low Organic - Low Salts - Near Neutral & 0.0 \\
\hline $216-\mathrm{A}-31$ & High Organic - Near Neutral & 0.0 \\
\hline $216-\mathrm{A}-36 \mathrm{~A} / \mathrm{B}$ & Low Organic - Low Salts - Near Neutral & $0.0^{\circ}$ \\
\hline $216-A-37-1$ & Low Organic - Low Salts - Near Neutral & 0.0 \\
\hline $216-\mathrm{A}-37-2$ & Low Organic - Low Salts - Near Neutral & 0.0 \\
\hline $216-\mathrm{A}-4$ & Low Organic - Low Salts - Near Neutral & 0.0 \\
\hline $216-A-45$ & Low Organic - Low Salts - Near Neutral & 0.0 \\
\hline $216-\mathrm{A}-5$ & Low Organic - Low Salts - Near Neutral & 0.0 \\
\hline $216-A-6$ & Low Organic - Low Salts - Near Neutral & 0.0 \\
\hline $216-A-7$ & High Organic - Near Neutral & 0.0 \\
\hline $216-A-8$ & High Organic - Near Neutral & 0.0 \\
\hline $216-A-9$ & Low Organic - Low Salts - Near Neutral & 0.0 \\
\hline $216-B-10 A$ & Low Organic - Low Salts - Near Neutral & 0.0 \\
\hline 216-B-10B & Low Organic - Low Salts - Near Neutral & 0.0 \\
\hline 216-B-11A\&B & Low Organic - Low Salts - Near Neutral & 0.0 \\
\hline $216-B-12$ & Low Organic - Low Salts - Near Neutral & 0.0 \\
\hline $216-\mathrm{B}-14$ & Chelates - High Salts & 0.0 \\
\hline $216-\mathrm{B}-15$ & Chelates - High Salts & 0.0 \\
\hline $216-B-16$ & Chelates - High Salts & $\overline{0.0}$ \\
\hline 216-B-17 & Chelates - High Salts & 0.0 \\
\hline $216-\mathrm{B}-18$ & Chelates - High Salts & 0.0 \\
\hline $216-\bar{B}-19$ & Chelates - High Salts ${ }^{\circ}$. & 0.0 \\
\hline $216-B-20$ & Chelates - High Salts & 0.0 \\
\hline $216-\bar{B}-21$ & Chelates - High Salts & 0.0 \\
\hline 216-B-2-1 & Low Organic - Low Salts - Near Neutral & 0.0 \\
\hline
\end{tabular}


Table 4.4. (contd)

\begin{tabular}{|c|c|c|}
\hline Site Name & Waste Type Name & $\begin{array}{l}\mathrm{K}_{\mathrm{d}} \text { Switch } \\
\text { Depth (m) }\end{array}$ \\
\hline $216-B-22$ & Chelates - High Salts & 0.0 \\
\hline $216-\mathrm{B}-2-2$ & Low Organic - Low Salts - Near Neutral & 0.0 \\
\hline $216-\mathrm{B}-23$ & Chelates - High Salts & 0.0 \\
\hline $216-\mathrm{B}-2-3$ & Low Organic - Low Salts - Near Neutral & 0.0 \\
\hline $216-\mathrm{B}-24$ & Chelates - High Salts & 0.0 \\
\hline $216-\bar{B}-25$ & Chelates - High Salts & 0.0 \\
\hline $216-\mathrm{B}-26$ & Chelates - High Salts & 0.0 \\
\hline 216-B-27 & Chelates - High Salts & 0.0 \\
\hline $216-B-28$ & Chelates - High Salts & 0.0 \\
\hline $216-\mathrm{B}-29$ & Chelates - High Salts & 0.0 \\
\hline $216-\mathrm{B}-3$ & Low Organic - Low Salts - Near Neutral & 0.0 \\
\hline $216-B-30$ & Chelates - High Salts & 0.0 \\
\hline $216-\mathrm{B}-31$ & Chelates - High Salts & 0.0 \\
\hline $216-\mathrm{B}-32$ & Chelates - High Salts & 0.0 \\
\hline $216-\bar{B}-33$ & Chelates - High Salts & 0.0 \\
\hline 216-B-34 & Chelates - High Salts & 0.0 \\
\hline $216-\mathrm{B}-35$ & Very High Salts - Very Basic & 7.0 \\
\hline $216-\mathrm{B}-36$ & Very High Salts - Very Basic & 7.0 \\
\hline $216-\mathrm{B}-37$ & Very High Salts - Very Basic & 7.0 \\
\hline $216-\mathrm{B}-38$ & Very High Salts - Very Basic & 7.0 \\
\hline $216-B-39$ & Very High Salts - Very Basic & 7.0 \\
\hline $216-\mathrm{B}-40$ & Very High Salts - Very Basic & 7.0 \\
\hline $216-\bar{B}-41$ & Very High Salts - Very Basic & 7.0 \\
\hline $216-\bar{B}-43$ & Chelates - High Salts & 0.0 \\
\hline $216-\mathrm{B}-44$ & Chelates - High Salts & 0.0 \\
\hline $216-B-45$ & Chelates - High Salts & 0.0 \\
\hline $216-B-46$ & Chelates - High Salts & 0.0 \\
\hline $216-\mathrm{B}-47$ & Chelates - High Salts & 0.0 \\
\hline $216-\mathrm{B}-48$ & Chelates - High Salts & 0.0 \\
\hline 216-B-49 & Chelates - High Salts & 0.0 \\
\hline $216-B-5$ & Very High Salts - Very Basic & 0.0 \\
\hline $216-B-50$ & Low Organic - Low Salts - Near Neutral & 0.0 \\
\hline $216-\mathrm{B}-52$ & Chelates - High Salts & 0.0 \\
\hline $216-\bar{B}-53 \mathrm{~A}$ & Low Organic - Low Salts - Near Neutral & 0.0 \\
\hline 216-B-53B & Low Organic - Low Salts - Near Neutral & 0.0 \\
\hline $216-\bar{B}-54$ & Low Organic - Low Salts - Near Neutral & 0.0 \\
\hline $216-\mathrm{B}-55$ & Low Organic - Low Salts - Near Neutral & 0.0 \\
\hline 216-B-57 & Low Organic - Low Salts - Near Neutral & 0.0 \\
\hline
\end{tabular}


Table 4.4. (contd)

\begin{tabular}{|c|c|c|}
\hline Site Name & $\begin{array}{r}\text { Waste Type Name } \\
\end{array}$ & $\begin{array}{l}\mathbf{K}_{d} \text { Switch } \\
\text { Depth (m) }\end{array}$ \\
\hline $216-\mathrm{B}-58$ & Low Organic - Low Salts - Near Neutral & 0.0 \\
\hline $216-\bar{B}-59$ & Low Organic - Low Salts - Near Neutral & 0.0 \\
\hline $216-\mathrm{B}-60$ & Low Organic - Low Salts - Near Neutral & 0.0 \\
\hline $216-B-62$ & Low Organic - Low Salts - Near Neutral & 0.0 \\
\hline $216-\overline{B-63}$ & Low Organic - Low Salts - Near Neutral & 0.0 \\
\hline $216-B-7 A \& B$ & Very High Salts - Very Basic & 35.7 \\
\hline $216-\mathrm{B}-8$ & Very High Salts - Very Basic & 33.0 \\
\hline $216-B-9$ & Very High Salts - Very Basic & 0.9 \\
\hline $216-C-1$ & Very High Salts - Very Basic & 0.0 \\
\hline $216-C-10$ & Low Organic - Low Salts - Near Neutral & 0.0 \\
\hline $216-C-3$ & Low Organic - Low Salts - Near Neutral & 0.0 \\
\hline $216-\mathrm{C}-4$ & High Organic - Near Neutral & 0.0 \\
\hline $216-\mathrm{C}-5$ & Low Organic - Low Salts - Near Neutral & 0.0 \\
\hline $216-C-6$ & Low Organic - Low Salts - Near Neutral & 0.0 \\
\hline $216-\mathrm{C}-7$ & Low Organic - Low Salts - Near Neutral & 0.0 \\
\hline $216-C-9$ & Low Organic - Low Salts - Near Neutral & 0.0 \\
\hline $216-N-2$ & Low Organic - Low Salts - Near Neutral & 0.0 \\
\hline $216-N-3$ & Low Organic - Low Salts - Near Neutral & 0.0 \\
\hline $216-N-4$ & Low Organic - Low Salts - Near Neutral & $\overline{0.0}$ \\
\hline $216-N-5$ & Low Organic - Low Salts - Near Neutral & $\overline{0.0}$ \\
\hline $216-N-6$ & Low Organic - Low Salts - Near Neutral & 0.0 \\
\hline $216-\mathrm{N}-7$ & Low Organic - Low Salts - Near Neutral & 0.0 \\
\hline $216-S-1 \& 2$ & Low Organic - Low Salts - Acidic & 35.3 \\
\hline 216-S-10D & Low Organic - Low Salts - Near Neutral & 0.0 \\
\hline $216-S-11$ & Low Organic - Low Salts - Near Neutral & 0.0 \\
\hline $216-S-12$ & Low Organic - Low Salts - Near Neutral & 0.0 \\
\hline $216-S-13$ & High Organic - Near Neutral & 0.0 \\
\hline $216-S-16 \mathrm{P}$ & Low Organic - Low Salts - Near Neutral & 0.0 \\
\hline $216-S-17$ & Low Organic - Low Salts - Near Neutral & 0.0 \\
\hline $216-S-19$ & Low Organic - Low Salts - Near Neutral & 0.0 \\
\hline $216-\mathrm{S}-20$ & Low Organic - Low Salts - Near Neutral & 0.0 \\
\hline $216-S-22$ & Low Organic - Low Salts - Near Neutral & 0.0 \\
\hline $216-S-23$ & Low Organic - Low Salts - Near Neutral & 0.0 \\
\hline $216-S-25$ & Low Organic - Low Salts - Near Neutral & 0.0 \\
\hline $2.16-S-26$ & Low Organic - Low Salts - Near Neutral & 0.0 \\
\hline $216-\mathrm{S}-3$ & Low Organic - Low Salts - Near Neutral & 0.0 \\
\hline $216-S-5$ & Low Organic - Low Salts - Near Neutral & 0.0 \\
\hline $216-S-6$ & Low Organic - Low Salts - Near Neutral & 0.0 \\
\hline
\end{tabular}


Table 4.4. (contd)

\begin{tabular}{|c|c|c|}
\hline Site Name & Waste Type Name & $\begin{array}{l}\mathrm{K}_{\mathrm{d}} \text { Switch } \\
\text { Depth (m) }\end{array}$ \\
\hline $216-S-7$ & Low Organic - Low Salts - Near Neutral & 0.0 \\
\hline $216-5-8$ & Low Organic - Low Salts - Near Neutral & 0.0 \\
\hline $216-S-9$ & Low Organic - Low Salts - Acidic & 32.9 \\
\hline $216-\mathrm{T}-1$ & Low Organic - Low Salts - Near Neutral & 0.0 \\
\hline $216-\mathrm{T}-12$ & Low Organic - Low Salts - Near Neutral & 0.0 \\
\hline $216-T-14$ & Very High Salts - Very Basic & 7.0 \\
\hline $216-T-15$ & Very High Salts - Very Basic & 7.0 \\
\hline $216-T-16$ & Very High Salts - Very Basic & 7.0 \\
\hline $216-T-17$ & Very High Salts - Very Basic & 7.0 \\
\hline $216-T-18$ & Very High Salts - Very Basic & 5.4 \\
\hline $216-T-19$ & High Organic - Near Neutral & 0.0 \\
\hline $216-T-20$ & Low Organic - Low Salts - Near Neutral & 0.0 \\
\hline $216-T-21$ & Very High Salts - Very Basic & 7.0 \\
\hline $216-\mathrm{T}-22$ & Very High Salts - Very Basic & 7.0 \\
\hline $216-T-23$ & Very High Salts - Very Basic & 7.0 \\
\hline $216-T-24$ & Very High Salts - Very Basic- & 7.0 \\
\hline $216-T-25$ & Very High Salts - Very Basic & 17.0 \\
\hline $216-T-26$ & Chelates - High Salts & 0.0 \\
\hline $216-T-27$ & Low Organic - Low Salts - Near Neutral & 0.0 \\
\hline $216-T-28$ & Low Organic - Low Salts - Near Neutral & 0.0 \\
\hline $216-\mathrm{T}-3$ & Very High Salts - Very Basic & 0.0 \\
\hline $216-T-32$ & Very High Salts - Very Basic & 32.1 \\
\hline $216-T-33$ & Low Organic - Low Salts - Near Neutral & 0.0 \\
\hline $216-T-34$ & Low Organic - Low Salts - Near Neutral & 0.0 \\
\hline $216-T-35$ & Low Organic - Low Salts - Near Neutral & 0.0 \\
\hline $216-T-36$ & Low Organic - Low Salts - Near Neutral & 0.0 \\
\hline $216-\mathrm{T}-4 \mathrm{~B}$ & Low Organic -.Low Salts - Near Neutral & 0.0 \\
\hline $216-T-5$ & Very High Salts --Very Basic & 26.3 \\
\hline $216-\bar{T}-6$ & Very High Salts - Very Basic & 12.4 \\
\hline $216-\mathrm{T}-7$ & Very High Salts - Very Basic & 20.0 \\
\hline $216-\mathrm{T}-8$ & Low Organic - Low Salts - Near Neutral & 0.0 \\
\hline $216-U-1 \& 2$ & Low Organic - Low Salts - Acidic & 22.7 \\
\hline $216-U-10$ & Low Organic - Low Salts - Near Neutral & 0.0 \\
\hline $216-U-12$ & Low Organic - Low Salts - Near Neutral & 0.0 \\
\hline $216-U-15$ & High Organic - Near Neutral & 0.0 \\
\hline $216-U-16$ & Low Organic - Low Salts - Near Neutral & 0.0 \\
\hline 216-U-17 & Low Organic - Low Salts - Near Neutral & 0.0 \\
\hline $216-U-3$ & Low Organic - Low Salts - Near Neutral & 0.0 \\
\hline
\end{tabular}


Table 4.4. (contd)

\begin{tabular}{|c|c|c|}
\hline Site Name & Waste Type Name & $\begin{array}{l}\mathrm{K}_{\mathrm{d}} \text { Switch } \\
\text { Depth (m) }\end{array}$ \\
\hline $216-U-4 A$ & Low Organic - Low Salts - Near Neutral & 0.0 \\
\hline $216-U-4 B$ & Low Organic - Low Salts - Near Neutral & 0.0 \\
\hline $216-U-5$ & Low Organic - Low Salts - Near Neutral & 0.0 \\
\hline $216-\mathrm{U}-6$ & Low Organic - Low Salts - Near Neutral & 0.0 \\
\hline $216-U-7$ & Low Organic - Low Salts - Near Neutral & 0.0 \\
\hline $216-U-8$ & Low Organic - Low Salts - Acidic & 20.6 \\
\hline $216-Z-1 \& 2$ & High Organic - Near Neutral & 0.0 \\
\hline $216-Z-10$ & Low Organic - Low Salts - Near Neutral & 0.0 \\
\hline $216-Z-12$ & High Organic - Near Neutral & 0.0 \\
\hline $216-Z-16$ & Low Organic - Low Salts - Near Neutral & 0.0 \\
\hline $216-Z-17$ & Low Organic - Low Salts - Near Neutral & 0.0 \\
\hline $216-Z-18$ & High Organic - Very Acidic & 10.0 \\
\hline $216-Z-1 A$ & High Organic - Very Acidic & 1.0 \\
\hline $216-Z-20$ & Low Organic - Low Salts - Near Neutral & 0.0 \\
\hline $216-2-3$ & High Organic - Very Acidic & 92.4 \\
\hline $216-Z-4$ & Low Organic - Low Salts - Near Neutral & 0.0 \\
\hline $216-2-5$ & Low Organic - Low Salts - Near Neutral & 0.0 \\
\hline $216-Z=6$ & Low Organic - Low Salts - Near Neutral & 0.0 \\
\hline $216-Z-7$ & High Organic - Very Acidic & 98.5 \\
\hline $216-Z-8$ & Low Organic - Low Salts - Near Neutral & 0.0 \\
\hline $216-Z_{-9}^{-}$ & High Organic - Very Acidic & 7.6 \\
\hline 218-EC-9(a)** & Low Organic - Low Salts - Near Neutral & 0.0 \\
\hline $218-\overline{E C}-9(\mathrm{~b})^{* * *}$ & Low Organic - Low Salts - Near Neutral & 0.0 \\
\hline $218-\mathrm{E}-1(\mathrm{~b})$ & Low Organic - Low Salts - Near Neutral & 0.0 \\
\hline 218-E-10(b) & Low Organic - Low Salts - Near Neutral & 0.0 \\
\hline 218-E-10(a) & Low Organic - Low Salts - Near Neutral & 0.0 \\
\hline 218-E-12A(b) & Low Organic - Low Salts - Near Neutral & 0.0 \\
\hline $218-E-12 B(b)$ & Low Organic - Low Salts - Near Neutral & 0.0 \\
\hline 218-E-12B(a) & Low Organic - Low Salts - Near Neutral & 0.0 \\
\hline $218-\mathrm{E}-2(\mathrm{~b})$ & Low Organic - Low Salts - Near Neutral & 0.0 \\
\hline $218-E-4(b)$ & Low Organic - Low Salts - Near Neutral & 0.0 \\
\hline 218-E-5(b) & Low Organic - Low Salts - Near Neutral & 0.0 \\
\hline $218-\mathrm{E}-5 \mathrm{~A}(\mathrm{~b})$ & Low Organic - Low Salts - Near Neutral & 0.0 \\
\hline $218-E-8(b)$ & Low Organic - Low Salts - Near Neutral & 0.0 \\
\hline $218-W-1(b)$ & Low Organic - Low Salts - Near Neutral & 0.0 \\
\hline $218-W-11(b)$ & Low Organic - Low Salts - Near Neutral & 0.0 \\
\hline $218-W-1 A(b)$ & Low Organic - Low Salts - Near Neutral & 0.0 \\
\hline $218-W-2(b)$ & Low Organic - Low Salts - Near Neutral & 0.0 \\
\hline
\end{tabular}


Table 4.4. (contd)

\begin{tabular}{|c|c|c|}
\hline Site Name & Waste Type Name & $\begin{array}{l}\mathrm{K}_{\mathrm{d}} \text { Switch } \\
\text { Depth (m) }\end{array}$ \\
\hline $218-W-2 A(b)$ & Low Organic - Low Salts - Near Neutral & 0.0 \\
\hline $218-W-3(b)$ & Low Organic - Low Salts - Near Neutral & 0.0 \\
\hline $218-W-3 A(b)$ & Low Organic - Low Salts - Near Neutral & 0.0 \\
\hline $218-W-3 A(a)$ & Low Organic - Low Salts - Near Neutral & 0.0 \\
\hline $218-W-3 A E(b)$ & Low Organic - Low Salts - Near Neutral & 0.0 \\
\hline $218-W-3 \mathrm{AE}(\mathrm{a})$ & Low Organic - Low Salts - Near Neutral & 0.0 \\
\hline $218-W-4 A(b)$ & Low Organic - Low Salts - Near Neutral & 0.0 \\
\hline $218-W-4 B-c(b)$ & Low Organic - Low Salts - Near Neutral & 0.0 \\
\hline 218-W-4B-c(a) & Low Organic - Low Salts - Near Neutral & 0.0 \\
\hline $218-W-4 C(a)$ & Low Organic - Low Salts - Near Neutral & 0.0 \\
\hline $218-W-4 C(b)$ & Low Organic - Low Salts - Near Neutral & 0.0 \\
\hline $218-W-5(b)$ & Low Organic - Low Salts - Near Neutral & 0.0 \\
\hline 218-W-5(a) & Low Organic - Low Salts - Near Neutral & 0.0 \\
\hline $218-W-7$ & Very High Salts - Very Basic & 0.0 \\
\hline $218-W-8$ & Low Organic - Low Salts - Near Neutral & 0.0 \\
\hline $218-W-9(b)$ & Low Organic - Low Salts - Near Neutral & 0.0 \\
\hline TWRS glass grout vault & Low Organic - Low Salts - Near Neutral & 0.0 \\
\hline TWRS glass new site & Low Organic - Low Salts - Near Neutral & 0.0 \\
\hline US Ecology current & Low Organic - Low Salts - Near Neutral & 0.0 \\
\hline US Ecology future & Low Organic - Low Salts - Near Neutral & 0.0 \\
\hline ERDF & Low Organic - Low Salts - Near Neutral & 0.0 \\
\hline C Reactor & Low Organic - Low Salts - Near Neutral & 0.0 \\
\hline D Reactor & Low Organic - Low Salts - Near Neutral & 0.0 \\
\hline DR Reactor & Low Organic - Low Salts - Near Neutral & 0.0 \\
\hline F Reactor & Low Organic - Low Salts - Near Neutral & 0.0 \\
\hline H Reactor & Low Organic - Low Salts - Near Neutral & 0.0 \\
\hline KE Reactor & Low Organic - Low Salts - Near Neutral & 0.0 \\
\hline KW Reactor & Low Organic - Low Salts - Near Neutral & 0.0 \\
\hline N Reactor & Low Organic - Low Salts - Near Neutral & 0.0 \\
\hline TK-A-S & Very High Salts - Very Basic & 23.4 \\
\hline TK-A-L & Very High Salts - Very Basic & 23.4 \\
\hline TK-A-R & Very High Salts - Very Basic & 23.4 \\
\hline TK-AN-R-1 & Chelates - High Salts & 0.0 \\
\hline TK-AN-R-2 & Very High Salts - Very Basic & 23.0 \\
\hline TK-AP-R-1 & Chelates - High Salts & 0.0 \\
\hline TK-AP-R-2 & Very High Salts - Very Basic & 23.0 \\
\hline TK-AW-R & Very High Salts - Very Basic & 23.0 \\
\hline TK-AX-S-1 & Chelates - High Salts & 0.0 \\
\hline
\end{tabular}


Table 4.4. (contd)

\begin{tabular}{|c|c|c|}
\hline Site Name & Waste Type Name & $\begin{array}{c}\mathrm{K}_{\mathrm{d}} \text { Switch } \\
\text { Depth (m) }\end{array}$ \\
\hline TK-AX-S-2 & Very High Salts - Very Basic & 23.4 \\
\hline TK-AX-L-1 & Chelates - High Salts & 0.0 \\
\hline TK-AX-L-2 & Very High Salts - Very Basic & 23.4 \\
\hline TK-AX-R-1 & Chelates - High Salts & 0.0 \\
\hline TK-AX-R-2 & Very High Salts - Very Basic & 23.4 \\
\hline TK-AY-R-1 & Chelates - High Salts & 23.0 \\
\hline TK-AY-R-2 & Very High Salts - Very Basic & 23.0 \\
\hline TK-AZ-R & Very High Salts - Very Basic & 23.0 \\
\hline TK-B-S & Very High Salts - Very Basic & 29.7 \\
\hline TK-B-L & Very High Salts - Very Basic & 29.7 \\
\hline$\overline{T K}-\bar{B}-\mathrm{R}$ & Very High Salts - Very Basic & 29.7 \\
\hline TK-BX-S & Very High Salts - Very Basic & 27.5 \\
\hline TK-BX-L & Very High Salts - Very Basic & 27.5 \\
\hline$\overline{T K}-\mathrm{BX}-\bar{R}$ & Very High Salts - Very Basic. & 27.5 \\
\hline TK-BY-S & Very High Salts - Very Basic & 25.7 \\
\hline TK-BY-L & Very High Salts - Very Basic & 25.7 \\
\hline TK-BY-R & Very High Salts - Very Basic & 25.7 \\
\hline TK-C-S-1 & Chelates - High Salts & 29.7 \\
\hline TK-C-S-2 & Very High Salts - Very Basic & 29.7 \\
\hline TK-C-L-1 & Chelates - High Salts & 29.7 \\
\hline TK-C-L-2 & Very High Salts - Very Basic & 29.7 \\
\hline TK-C-R-1 & Chelates - High Salts & 0.0 \\
\hline TK-C-R-2 & Very High Salts - Very Basic & 29.7 \\
\hline TK-S-S & Very High Salts - Very Basic & 25.7 \\
\hline TK-S-L & Very High Salts - Very Basic & 25.7 \\
\hline TK-S-R & Very High Salts - Very Basic & 25.7 \\
\hline TK-SX-S-1 & Chelates - High Salts & 0.0 \\
\hline TK-SX-S-2 & Very High Salts - Very Basic & 23.4 \\
\hline TK-SX-L-2 & Very High Salts - Very Basic & 23.4 \\
\hline TK-SX-R-1 & Chelates - High Salts & 0.0 \\
\hline TK-SX-R-2 & Very High Salts - Very Basic & 23.4 \\
\hline TK-SY-R-1 & Chelates - High Salts & 0.0 \\
\hline TK-SY-R-2 & Very High Salts - Very Basic & 23.0 \\
\hline TK-T-S & Very High Salts - Very Basic & 29.7 \\
\hline TK-T-I & Very High Salts - Very Basic & 29.7 \\
\hline TK-T-R & Very High Salts - Very Basic & 29.7 \\
\hline TK-TX-S & Very High Salts - Very Basic & 25.7 \\
\hline TK-TX-L & Very High Salts - Very Basic & 25.7 \\
\hline
\end{tabular}


Table 4.4. (contd)

\begin{tabular}{|l|l|c|}
\hline \multicolumn{1}{|c|}{ Site Name } & \multicolumn{1}{|c|}{ Waste Type Name } & $\begin{array}{c}\text { Kow }_{\text {Switch }} \\
\text { Depth (m) }\end{array}$ \\
\hline TK-TX-R & Very High Salts - Very Basic & 25.7 \\
\hline TK-TY-S & Very High Salts - Very Basic & 25.7 \\
\hline TK-TY-L & Very High Salts - Very Basic & 25.7 \\
\hline TK-TY-R & Very High Salts - Very Basic & 25.7 \\
\hline TK-U-S & Very High Salts - Very Basic & 29.7 \\
\hline TK-U-L & Very High Salts - Very Basic & 29.7 \\
\hline TK-U-R & Very High Salts - Very Basic & 29.7 \\
\hline
\end{tabular}

* Refers to depth below ground surface at which the site's $\mathrm{K}_{d}$ is assumed to switch from near-field to far-field values.

** (a) refers to waste disposed after September 30, 1988.

*** (b) refers to waste disposed before September 30, 1988. 
Table 4.5. Recharge Rates Applied to Waste Sites

\begin{tabular}{|c|c|c|c|c|c|c|c|c|}
\hline Site Name & $\underset{1^{*}}{\text { Time }}$ & $\begin{array}{c}\text { Time } \\
2\end{array}$ & $\begin{array}{c}\text { Time } \\
3\end{array}$ & $\begin{array}{c}\text { Time } \\
4\end{array}$ & $\begin{array}{c}\text { Recharge } \\
1^{* *}\end{array}$ & \begin{tabular}{|c|} 
Recharge \\
2 \\
\end{tabular} & \begin{tabular}{|c|} 
Recharge \\
3 \\
\end{tabular} & $\begin{array}{c}\text { Recharge } \\
4 \\
\end{array}$ \\
\hline $207-U$ & 1952 & 1994 & 2017 & 2050 & 1 & 4 & 6 & 7 \\
\hline $216-A-1$ & 1955 & 1955.1 & 2018 & 2050 & 1 & 4 & $\overline{6}$ & 7 \\
\hline $216-A-10$ & 1956 & 1987.2 & 2015 & 2050 & 1 & 2 & 6 & 7 \\
\hline $216-A-18$ & 1955 & 1956.2 & 2018 & 2050 & 1 & 4 & 6 & 7 \\
\hline $216-A-19$ & 1955 & 1956.2 & 2018 & 2050 & 1 & $\overline{4}$ & 6 & 7 \\
\hline $216-A-2$ & 1956 & 1963 & 2015 & 2050 & 1 & 4 & 6 & 7 \\
\hline $216-A-20$ & 1955 & 1955.2 & 2018 & 2050 & I & 4 & 6 & 7 \\
\hline $216-A-21$ & 1957 & 1964.7 & 2015 & 2050 & 1 & 4 & 6 & 7 \\
\hline $216-A-24$ & 1958 & 1965.7 & 2018 & 2050 & 1 & 4 & 6 & 7 \\
\hline $216-\mathrm{A}-25$ & 1957 & 1986.1 & 2024 & 2050 & 1 & 4 & 6 & 7 \\
\hline $216-A-27$ & 1965 & 1970.1 & 2015 & 2050 & 1 & 4 & 6 & 7 \\
\hline $216-A-28$ & 1958 & 1966.9 & 2015 & 2050 & 2 & 4 & 6 & 7 \\
\hline $216-A-3$ & 1956 & 1981.3 & 2015 & 2050 & 1 & 4 & 6 & 7 \\
\hline $216-A-30$ & 1961 & 1995 & 2015 & 2050 & 1 & 4 & 6 & 7 \\
\hline $216-A-31$ & 1964 & 1966.3 & 2015 & 2050 & 3 & $\overline{4}$ & 6 & 7 \\
\hline $216-\mathrm{A}-36 \mathrm{~A} / \mathrm{B}$ & 1965 & 1987 & 2015 & 2050 & 1 & 4 & 6 & 7 \\
\hline $216-A-37-1$ & 1977 & 1994.8 & 2015 & 2050 & 1 & 4 & 6 & 7 \\
\hline $216-A-37-2$ & 1983 & 1995 & 2015 & 2050 & 1 & $\overline{4}$ & 6 & 7 \\
\hline $216-A-4$ & 1955 & 1958 & 2015 & 2050 & 1 & $\overline{4}$ & 6 & 7 \\
\hline $216-A-45$ & 1987 & 1990.8 & 2015 & 2050 & 1 & 4 & 6 & 7 \\
\hline $216-A-5$ & 1955 & 1965.8 & 2015 & 2050 & 1 & 4 & 6 & 7 \\
\hline $216-A-6$ & 1955 & 1969.2 & 2015 & 2050 & 1 & 4 & 6 & 7 \\
\hline $216-\mathrm{A}-7$ & 1955 & 1966 & 2018 & 2050 & 1 & 4 & 6 & 7 \\
\hline $216-A-8$ & 1955 & 1990.2 & 2018 & 2050 & 1 & 4 & 6 & 7 \\
\hline $216-A-9$ & 1956 & 1969.4 & 2015 & 2050 & 1 & 4 & 6 & 7 \\
\hline $216-B-10 A$ & 1949 & 1951.1 & 2025 & 2050 & 1 & 4 & 6 & 7 \\
\hline $216-\mathrm{B}-10 \mathrm{~B}$ & 1969 & 1973.3 & 2020 & 2050 & 2 & 4 & 6 & 7 \\
\hline $216-\bar{B}-11 \mathrm{~A} \& \mathrm{~B}$ & 1952 & 1955 & 2021 & 2050 & 1 & 4 & 6 & 7 \\
\hline $216-\bar{B}-12$ & 1952 & 1973 & 2019 & 2050 & 1 & 4 & 6 & 7 \\
\hline $216-B-14$ & 1956 & 1956.1 & 2019 & 2050 & 1 & 4 & 6 & 7 \\
\hline $216-B-15$ & 1956 & 1957.7 & 2019 & 2050 & 1 & 4 & 6 & 7 \\
\hline $216-\bar{B}-16$ & 1956 & 1956.3 & 2019 & 2050 & 1 & 4 & 6 & 7 \\
\hline $216-\bar{B}-17$ & 1956 & 1956.1 & 2019 & 2050 & 1 & $\overline{4}$ & 6 & 7 \\
\hline $216-\bar{B}-18$ & 1956 & 1956.1 & 2019 & 2050 & 1 & 4 & 6 & 7 \\
\hline $216-B-19$ & 1957 & 1957.7 & 2019 & 2050 & 1 & 4 & 6 & 7 \\
\hline $216-B-20$ & 1956 & 1956.1 & 2018 & 2050 & 1 & 4 & 6 & 7 \\
\hline $216-\bar{B}-21$ & 1956 & 1956.1 & 2019 & 2050 & 1 & 4 & 6 & 7 \\
\hline $216-\mathrm{B}-2-1$ & 1945 & 1963.6 & 2018 & 2050 & 1 & 4 & 6 & 7 \\
\hline
\end{tabular}


Table 4.5. (contd)

\begin{tabular}{|c|c|c|c|c|c|c|c|c|}
\hline Site Name & $\underset{1 *}{\text { Time }}$ & $\underset{2}{\text { Time }}$ & $\begin{array}{c}\text { Time } \\
3\end{array}$ & $\begin{array}{c}\text { Time } \\
4\end{array}$ & $\underset{1^{* *}}{\text { Recharge }}$ & \begin{tabular}{|} 
Recharge \\
2
\end{tabular} & \begin{tabular}{|c|} 
Recharge \\
3
\end{tabular} & $\begin{array}{c}\text { Recharge } \\
4\end{array}$ \\
\hline $216-\mathrm{B}-22$ & 1956 & 1997.1 & 2019 & 2050 & 2 & 4 & 6 & 7 \\
\hline $216-B-2-2$ & 1963 & 1969.5 & 2018 & 2050 & 1 & 4 & 6 & 7 \\
\hline $216-B-23$ & 1956 & 1997.1 & 2019 & 2050 & 2 & 4 & 6 & 7 \\
\hline $216-B-2-3$ & 1970 & 1987 & 2018 & 2050 & 1 & 4 & 6 & 7 \\
\hline $216-B-24$ & 1956 & 1956.1 & 2019 & 2050 & 1 & 4 & 6 & 7 \\
\hline $216-B-25$ & 1956 & 1956.1 & 2019 & 2050 & 1 & 4 & 6 & 7 \\
\hline $216-B-26$ & 1956 & 1956.2 & 2019 & 2050 & 1 & 4 & 6 & 7 \\
\hline $216-B-27$ & 1957 & 1957.2 & 2019 & 2050 & 1 & 4 & 6 & 7 \\
\hline $216-\mathrm{B}-28$ & 1957 & 1957.2 & 2019 & 2050 & 1 & 4 & 6 & 7 \\
\hline $216-B-29$ & 1957 & 1957.1 & 2019 & 2050 & 1 & 4 & 6 & 7 \\
\hline $216-B-3$ & 1945 & 1997.5 & 2018 & 2050 & 1 & 4 & 6 & $\overline{7}$ \\
\hline 216-B-30 & 1957 & 1957.1. & 2019 & 2050 & $\overline{1}$ & 4 & 6 & 7 \\
\hline $216-B-31$ & 1957 & 1957.1 & 20.19 & 2050 & 1 & 4 & 6 & 7 \\
\hline $216-B-32$ & 1957 & 1957.1 & 2019 & 2050 & 1 & 4 & 6 & 7 \\
\hline $216-B-33$ & 1957 & 1957.1 & 2019 & 2050 & 1 & 4 & 6 & 7 \\
\hline 216-B-34 & 1957 & 1957.1 & 2019 & 2050 & $I$ & 4 & 6 & 7 \\
\hline $216-B-35$ & 1954 & 1954.1 & 2020 & 2050 & 1 & 4 & 6 & 7 \\
\hline $216-B-36$ & 1954 & 1954.1 & 2020 & 2050 & 1 & 4 & 6 & 7 \\
\hline $216-B-37$ & 1954 & 1954.1 & 2020 & 2050 & 1 & 4 & 6 & 7 \\
\hline $216-\mathrm{B}-38$ & 1954 & 1954.2 & 2020 & 2050 & 1 & 4 & 6 & 7 \\
\hline $216-\mathrm{B}-39$ & 1953 & 1954.9 & 2020 & 2050 & 1 & 4 & 6 & 7 \\
\hline $216-\mathrm{B}-40$ & 1954 & 1954.2 & 2020 & 2050 & 1 & 4 & 6 & 7 \\
\hline $216-\bar{B}-41$ & 1954 & 1954.1 & 2020 & 2050 & 1 & 4 & 6 & 7 \\
\hline $216-B-43$ & 1954 & 1954.1 & 2013 & 2050 & 1 & 4 & 6 & 7 \\
\hline $216-B-44$ & 1954 & 1954.3 & 2013 & 2050 & 1 & 4 & 6 & 7 \\
\hline $216-B-45$ & 1955 & 1955.2 & 2013 & 2050 & 1 & 4 & 6 & 7 \\
\hline $216-B-46$ & 1955 & 1955.2 & 2013 & 2050 & 1 & 4 & 6 & 7 \\
\hline $216-B-47$ & 1955 & 1955.1 & 2013 & 2050 & 1 & 4 & 6 & 7 \\
\hline $216-B-48$ & 1955 & 1955.1 & 2013 & 2050 & 1 & 4 & 6 & $\overline{7}$ \\
\hline $216-\bar{B}-49$ & 1955 & 1955.1 & 2013 & 2050 & 1 & 4 & 6 & 7 \\
\hline $216-B-5$ & 1945 & 1947.5 & 2025 & 2050 & 1 & 4 & 6 & 7 \\
\hline $216-\mathrm{B}-50$ & 1965 & 1974 & 2013 & 2050 & 1 & 4 & 6 & 7 \\
\hline $216-\bar{B}-52$ & 1957 & 1957.1 & 2019 & 2050 & 1 & 4 & 6 & 7 \\
\hline 216-B-53A. & 1965 & 1965.1 & 2019 & 2050 & 1 & 4 & 6 & 7 \\
\hline $216-B-53 B$ & 1962 & 1962.3 & 2019 & 2050 & 1 & 4 & 6 & 7 \\
\hline $216-B-54$ & 1963 & 1965.6 & 2019 & 2050 & 2 & 4 & 6 & 7 \\
\hline 216-B-55 & 1967 & 1994.4 & 2021 & 2050 & 1 & 4 & 6 & 7 \\
\hline $216-B-57$ & 1968 & 1973.3 & 2013 & 2050 & 1 & 4 & 6 & 7 \\
\hline
\end{tabular}


Table 4.5. (contd)

\begin{tabular}{|c|c|c|c|c|c|c|c|c|}
\hline Site Name & $\underset{1^{*}}{\text { Time }}$ & $\begin{array}{c}\text { Time } \\
2\end{array}$ & $\begin{array}{c}\text { Time } \\
3\end{array}$ & $\underset{4}{\text { Time }}$ & $\begin{array}{c}\text { Recharge } \\
1^{* *}\end{array}$ & $\begin{array}{c}\text { Recharge } \\
2\end{array}$ & $\begin{array}{c}\text { Recharge } \\
3\end{array}$ & $\begin{array}{c}\text { Recharge } \\
4\end{array}$ \\
\hline $216-B-58$ & 1965 & 1966.6 & 2019 & 2050 & 1 & 4 & 6 & 7 \\
\hline $216-B-59$ & 1967 & 1967.1 & 2025 & 2050 & 1 & 4 & 6 & 7 \\
\hline $216-B-60$ & 1967 & 1967.1 & 2025 & 2050 & 1 & 4 & 6 & 7 \\
\hline $216-\bar{B}-62$ & 1973 & 1990.8 & 2021 & 2050 & 1 & 4 & 6 & 7 \\
\hline $216-\bar{B}-63$ & 1970.3 & 1993 & 2017 & 2050 & 1 & $\overline{4}$ & 6 & 7 \\
\hline $216-\mathrm{B}-7 \mathrm{~A} \& \mathrm{~B}$ & 1946 & 1976.6 & 2020 & 2050 & 1 & $\overline{4}$ & 6 & 7 \\
\hline $216-\bar{B}-8$ & 1948 & 1953.3 & 2020 & 2050 & 1 & 4 & 6 & 7 \\
\hline 216-B-9 & $\overline{1948}$ & 1950.9 & 2025 & 2050 & 1 & 4 & 6 & 7 \\
\hline $216-\mathrm{C}-1$ & 1953 & 1957.4 & 2017 & 2050 & 1 & 4 & 6 & 7 \\
\hline $216-C-10$ & 1964 & 1968.9 & 2017 & 2050 & 1 & 4 & 6 & 7 \\
\hline $216-C-3$ & 1953 & 1954.2 & 2017 & 2050 & 1 & 4 & 6 & 7 \\
\hline $216-C-4$ & 1955 & 1964.8 & 2017 . & 2050 & 2 & 4 & 6 & 7 \\
\hline $216-C-5$ & 1955 & 1955.3 & 2017 & 2050 & 1 & 4 & 6 & 7 \\
\hline $216-C-6$ & 1955 & 1964 & 2017 & 2050 & 1 & 4 & 6 & 7 \\
\hline $216-C-7$ & 1961 & 1982.7 & 2017 & 2050 & 3 & 4 & 6 & 7 \\
\hline $216-C-9$ & 1953 & 1983.5 & 2017 & 2050 & 1 & 4 & 6 & 7 \\
\hline $216-N-2$ & 1947 & 1947.1 & 2021 & 2050 & 1 & 4 & 6 & 7 \\
\hline $216-\mathrm{N}-3$ & 1952 & 1952.1 & 2021 & 2050 & 1 & 4 & 6 & 7 \\
\hline $216-N-4$ & 1944 & 1951.8 & 2021 & 2050 & 1 & 4 & 6 & $\overline{7}$ \\
\hline $216-N-5$ & 1952 & 1952.1 & 2021 & 2050 & 1 & 4 & 6 & 7 \\
\hline $216-N-6$ & 1944 & 1951.8 & 2021 & 2050 & 1 & 4 & 6 & 7 \\
\hline $216-N-7$ & 1952 & 1952.1 & 2021 & 2050 & 1 & 4 & 6 & 7 \\
\hline $216-S-1 \& 2$ & 1952 & 1956 & 2026 & 2050 & 1 & 4 & 6 & 7 \\
\hline $216-S-10 \mathrm{D}$ & 1951 & 1990.2 & 2019 & 2050 & 1 & $\overline{4}$ & 6 & 7 \\
\hline $216-S-11$ & 1954 & 1965.3 & 2019 & 2050 & 1 & 4 & 6 & 7 \\
\hline $216-S-12$ & 1954 & 1954.1 & 2025 & 2050 & 1 & 4 & 6 & 7 \\
\hline $216-S-13$ & 1952 & 1972.5 & 2026 & 2050 & 1 & 4 & 6 & 7 \\
\hline $216-S-16 P$ & 1957 & 1975.1 & 2019 & 2050 & I & $\overline{4}$ & 6 & 7 \\
\hline $216-5-17$ & 1951 & 1953.5 & 2019 & 2050 & 1 & 4 & 6 & 7. \\
\hline $216-S-19$ & 1952 & 1984.7 & 2019 & 2050 & 1 & 4 & 6 & 7 \\
\hline $216-S-20$ & 1952 & 1973.3 & 2025 & 2050 & 1 & 4 & 6 & 7 \\
\hline $216-S-22$ & 1957 & 1966.3 & 2024 & 2050 & 2 & 4 & 6 & 7 \\
\hline $216-\mathrm{S}-23$ & 1969 & 1972.5 & 2025 & 2050 & 1 & 4 & 6 & 7 \\
\hline $216-S-25$ & 1973 & 1994.2 & 2019 & 2050 & 1 & 4 & 6 & 7 \\
\hline $216-S-26$ & 1984 & 1994.3 & 2025 & 2050 & 1 & 4 & 6 & 7 \\
\hline $216-S-3$ & 1953 & 1955.9 & 2026 & 2050 & 1 & 4 & 6 & $\overline{7}$ \\
\hline $216-S-5$ & 1954 & 1957 & 2019 & 2050 & 1 & 4 & 6 & 7 \\
\hline $216-S-6$ & 1954 & 1971.7 & 2019 & 2050 & 1 & $\overline{4}$ & 6 & 7 \\
\hline
\end{tabular}


Table 4.5. (contd)

\begin{tabular}{|c|c|c|c|c|c|c|c|c|}
\hline Site Name & $\underset{1^{*}}{\text { Time }}$ & $\begin{array}{c}\text { Time } \\
2\end{array}$ & $\begin{array}{c}\text { Time } \\
3\end{array}$ & $\begin{array}{c}\text { Time } \\
4\end{array}$ & $\begin{array}{c}\text { Recharge } \\
1^{* *}\end{array}$ & \begin{tabular}{|c|} 
Recharge \\
2
\end{tabular} & $\begin{array}{c}\text { Recharge } \\
3\end{array}$ & $\begin{array}{c}\text { Recharge } \\
4\end{array}$ \\
\hline $216-S-7$ & 1956 & 1965.5 & 2026 & 2050 & 1 & 4 & 6 & 7 \\
\hline $216-5-8$ & 1951 & 1951.3 & 2026 & 2050 & 1 & 4 & 6 & 7 \\
\hline $216-S-9$ & 1965 & 1968.5 & 2026 & 2050 & 1 & 4 & 6 & 7 \\
\hline $216-\mathrm{T}-1$ & 1944 & 1995.1 & 2021 & 2050 & I & 4 & 6 & 7 \\
\hline $216-T-12$ & 1954 & 1954.1 & 2025 & 2050 & 1 & 4 & 6 & 7 \\
\hline $216-T-14$ & 1954 & 1954.1 & 2025 & 2050 & 1 & 4 & 6 & 7 \\
\hline $216-\mathrm{T}-15$ & 1954 & 1954.1 & 2025 & 2050 & 1 & 4 & 6 & 7 \\
\hline $216-T-16$ & 1954 & 1954.1 & 2025 & 2050 & 1 & 4 & 6 & 7 \\
\hline $216-\mathrm{T}-17$ & 1954 & 1954.3 & 2025 & 2050 & 1 & 4 & 6 & 7 \\
\hline $216-T-18$ & 1953 & 1953.1 & 2024 & 2050 & I & 4 & 6 & 7 \\
\hline $216-\mathrm{T}-19$ & 1951 & 1979.9 & 2024 & 2050 & 1 & $\overline{4}$ & 6 & 7 \\
\hline $216-T-20$ & 1952 & 1952.1 & 2024 & 2050 & 1 & 4 & 6 & 7 \\
\hline $216-T-21$ & 1954 & 1954.2 & 2024 & 2050 & 1 & 4 & 6 & 7 \\
\hline $216-T-22$ & 1954 & 1954.1 & 2024 & 2050 & 1 & 4 & 6 & 7 \\
\hline $216-T-23$ & 1954 & 1954.1 & 2024 & 2050 & I & 4 & 6 & 7 \\
\hline $216-T-24$ & 1954 & 1954.1 & 2024 & 2050 & 1 & 4 & 6 & 7 \\
\hline $216-T-25$ & 1954 & 1954.1 & 2024 & 2050 & 1 & 4 & 6 & 7 \\
\hline $216-T-26$ & 1955 & 1956.3 & 2024 & 2050 & 1 & 4 & 6 & 7 \\
\hline $216-T-27$ & 1965 & 1965.2 & 2024 & 2050 & 1 & 4 & 6 & 7 \\
\hline $216-T-28$ & 1960 & 1966 & 2024 & 2050 & 1 & $\overline{4}$ & 6 & 7 \\
\hline $216-T-3$ & 1945.5 & 1946.8 & 2021 & 2050 & 1 & 4 & 6 & 7 \\
\hline $216-T-32$ & 1946 & 1951.5 & 2024 & 2050 & 1 & 4 & 6 & 7 \\
\hline $216-T-33$ & 1963 & 1963.1 & 2021 & 2050 & 1 & 4 & 6 & 7 \\
\hline $216-T-34$ & 1966 & 1966.8 & 2021 & 2050 & 1 & 4 & 6 & 7 \\
\hline $216-\mathrm{T}-35$ & 1967 & 1967.8 & 2021 & 2050 & 1 & 4 & 6 & 7 \\
\hline $216-T-36$ & 1967 & 1968.8 & 2024 & 2050 & 1 & 4 & 6 & 7 \\
\hline $216-\mathrm{T}-4 \mathrm{~B}$ & 1972 & 1995.6 & 2025 & 2050 & 1 & 4 & 6 & 7 \\
\hline $216-T-5$ & 1955 & 1955.1 & 2024 & 2050 & 1 & 4 & 6 & 7 \\
\hline $216-T-6$ & 1946 & 1950.8 & 2025 & 2050 & 1 & 4 & 6 & 7 \\
\hline $216-\mathrm{T}-7$ & 1948 & 1955.6 & 2024 & 2050 & 1 & 4 & 6 & 7 \\
\hline $216-T-8$ & 1950 & 1951.3 & 2021 & 2050 & 1 & 4 & 6 & 7 \\
\hline $216-U-1 \& 2$ & 1951 & 1966.6 & 2017 & 2050 & 1 & 4 & 6 & 7 \\
\hline $216-U-10$ & 1944 & 1986.5 & 2017 & 2050 & 1 & 4 & 6 & 7 \\
\hline $216-U-12$ & 1960 & 1987.9 & 2017 & 2050 & 1 & 4 & 6 & 7 \\
\hline $216-U-15$ & 1957 & 1957.1 & 2017 & 2050 & 1 & 4 & 6 & 7 \\
\hline $216-U-16$ & 1984 & 1986.5 & 2017 & 2050 & 1 & 4 & 6 & 7 \\
\hline $216-U-17$ & 1988 & 1994.6 & 2017 & $2050^{\circ}$ & 2 & 4 & 6 & 7 \\
\hline $216-U-3$ & 1954 & 1955.3 & 2017 & 2050 & 1 & 4 & 6 & 7 \\
\hline
\end{tabular}


Table 4.5. (contd)

\begin{tabular}{|c|c|c|c|c|c|c|c|c|}
\hline Site Name & $\begin{array}{c}\text { Time } \\
1^{*}\end{array}$ & $\begin{array}{c}\text { Time } \\
2\end{array}$ & $\begin{array}{c}\text { Time } \\
3\end{array}$ & $\begin{array}{c}\text { Time } \\
4\end{array}$ & $\begin{array}{c}\text { Recharge } \\
1^{* *}\end{array}$ & \begin{tabular}{|} 
Recharge \\
2
\end{tabular} & $\begin{array}{c}\text { Recharge } \\
3\end{array}$ & $\begin{array}{c}\text { Recharge } \\
4\end{array}$ \\
\hline $216-U-4 A$ & 1955 & 1970 & 2017 & 2050 & 1 & 4 & 6 & 7 \\
\hline $216-\mathrm{U}-4 \mathrm{~B}$ & 1960 & 1968.7 & 2017 & 2050 & 1 & 4 & 6 & 7 \\
\hline $216-U-5$ & 1952 & 1952.1 & 2017 & 2050 & 1 & 4 & 6 & 7 \\
\hline $216-U-6$ & 1952 & 1952.1 & 2017 & 2050 & 1 & 4 & 6 & 7 \\
\hline $216-\mathrm{U}-7$ & 1952 & 1957.3 & 2017 & 2050 & 2 & 4 & 6 & 7 \\
\hline $216-\mathrm{U}-8$ & 1952 & 1959.8 & 2017 & 2050 & $T$ & 4 & 6 & 7 \\
\hline $216-Z-1 \& 2$ & 1949 & 1968.8 & 2019 & 2050 & 1 & 4 & 6 & 7 \\
\hline $216-Z-10$ & 1945 & 1945.3 & 2019 & 2050 & 1 & 4 & 6 & 7 \\
\hline $216-Z-12$ & 1959 & 1973.2 & 2019 & 2050 & 1 & 4 & 6 & 7 \\
\hline $216-Z-16$ & 1968 & 1976.8 & 2019 & 2050 & 1 & 4 & 6 & 7 \\
\hline $216-Z-17$ & 1967 & 1968 & 2019 & 2050 & 1 & 4 & 6 & 7 \\
\hline $216-Z-18$ & 1969 & 1973.1 & 2019 & 2050 & 2 & 4 & 6 & 7 \\
\hline $216-Z-1 A$ & 1949 & 1969 & 2019 & 2050 & 3 & 4 & 6 & 7 \\
\hline $216-Z-20$ & 1981 & 1994.3 & 2017 & 2050 & 1 & $\overline{4}$ & 6 & 7 \\
\hline $216-Z-3$ & 1952 & 1958.8 & 2019 & 2050 & 1 & 4 & 6 & 7 \\
\hline $216-Z-4$ & 1945 & 1945.1 & 2019 & 2050 & 1 & 4 & 6 & 7 \\
\hline $216-Z-5$ & 1945 & 1946.7 & 2019 & 2050 & 1 & $\overline{4}$ & 6 & 7 \\
\hline $216-Z-6$ & 1945 & 1945.1 & 2019 & 2050 & 1 & 4 & 6 & 7 \\
\hline $216-Z-7$ & 1947 & 1967 & 2019 & 2050 & 1 & 4 & 6 & 7 \\
\hline $216-Z-8$ & 1955 & 1961.8 & 2019 & 2050 & $\overline{2}$ & 4 & 6 & 7 \\
\hline $216-Z-9$ & 1955 & 1961.9 & 2019 & 2050 & 1 & 4 & 6 & 7 \\
\hline $218-\mathrm{EC}-9(\mathrm{a})^{+}$ & 1989 & 2018 & & & 4 & $\overline{6}$ & & \\
\hline $218-E C-9(b)^{+}$ & 1987 & 2018 & & & 4 & 6 & & \\
\hline $218-\mathrm{E}-1(\mathrm{~b})$ & 1949 & 2018 & & & 4 & $\overline{6}$ & & \\
\hline 218-E-10(b) & 1974 & 2018 & & & 4 & $\overline{6}$ & & \\
\hline 218-E-10(a) & 2002 & 2018 & & & 4 & 6 & & \\
\hline $218-E-12 A(b)$ & 1960 & 2018 & & & 4 & $\overline{6}$ & & \\
\hline $218-\mathrm{E}-12 \mathrm{~B}(\mathrm{~b})$ & 1978 & 2018 & & & $\overline{4}$ & 6 & & \\
\hline 218-E-12B(a) & 2002 & 2018 & & & 4 & 6 & & \\
\hline $218-E-2(b)$ & 1949 & 2018 & & & 4 & 6 & & \\
\hline 218-E-4(b) & 1956 & 2018 & & & 4 & $\overline{6}$ & & \\
\hline 218-E-5(b) & 1955 & 2018 & & & 4 & $\overline{6}$ & & \\
\hline $218-\mathrm{E}-5 \mathrm{~A}(\mathrm{~b})$ & 1958 & 2018 & & & 4 & $\overline{6}$ & & \\
\hline 218-E-8(b) & 1959 & 2018 & & & 4 & 6 & & \\
\hline 218-W-1(b) & 1948 & 2018 & & & 4 & 6 & & \\
\hline $218-W-11(b)$ & 1960 & 2018 & & & 4 & 6 & & \\
\hline $218-W-1 A(b)$ & 1949 & 2018 & & & 4 & 6 & & \\
\hline $218-W-2(b)$ & 1955 & 2018 & & & 4 & 6 & & \\
\hline
\end{tabular}


Table 4.5. (contd)

\begin{tabular}{|c|c|c|c|c|c|c|c|c|}
\hline Site Name & $\begin{array}{c}\text { Time } \\
1^{*}\end{array}$ & $\begin{array}{c}\text { Time } \\
2\end{array}$ & $\begin{array}{c}\text { Time } \\
3\end{array}$ & $\begin{array}{c}\text { Time } \\
4\end{array}$ & $\begin{array}{c}\text { Recharge } \\
1^{* *}\end{array}$ & $\begin{array}{c}\text { Recharge } \\
2\end{array}$ & \begin{tabular}{|c|} 
Recharge \\
$\mathbf{3}$
\end{tabular} & $\begin{array}{c}\text { Recharge } \\
4\end{array}$ \\
\hline 2 218-W-2A(b) & 1970 & 2018 & & & 4 & 6 & & \\
\hline $218-\mathrm{W}-3(\mathrm{~b})$ & 1959 & 2018 & & & 4 & 6 & & \\
\hline $218-W-3 A(b)$ & 1979 & 2018 & & & 4 & 6 & & \\
\hline $218-\mathrm{W}-3 \mathrm{~A}(\mathrm{a})$ & 2003 & 2018 & & & 4 & 6 & & \\
\hline 218-W-3AE(b) & 1986 & 2018 & & & 4 & $\overline{6}$ & & \\
\hline $218-\mathrm{W}-3 \mathrm{AE}(\mathrm{a})$ & 2000 & 2018 & & & 4 & 6 & & \\
\hline $218-W-4 A(b)$ & 1965 & 2018 & & & 4 & 6 & & \\
\hline $218-W-4 B-c(b)$ & 1978 & 2018 & & & 4 & 6 & & \\
\hline $218-W-4 B-c(a)$ & 1999 & 2018 & & & 4 & 6 & & \\
\hline $218-W=4 C(a)$ & 1998 & 2018 & & & 4 & 6 & & \\
\hline $218-W-4 C(b)$ & 1983 & 2018 & & & 4 & 6 & & \\
\hline $218-W-5(b)$ & 1987 & 2018 & & & 4 & 6 & & \\
\hline $218-\mathrm{W}-5(\mathrm{a})$ & 2002 & 2018 & & & 4 & 6 & & \\
\hline $218-\mathrm{W}-7$ & 1956 & & & & 6 & & & \\
\hline $218-W-8$ & 1948 & & & & $\overline{6}$ & & & \\
\hline $218-W-9(b)$ & 1954 & 2018 & & & 4 & 6 & & \\
\hline $\begin{array}{l}\text { TWRS glass } \\
\text { grout vault }\end{array}$ & 2007 & & & & 7 & & & \\
\hline $\begin{array}{l}\text { TWRS glass } \\
\text { new site }\end{array}$ & 2018 & & & & 7 & & & \\
\hline $\begin{array}{l}\text { US Ecology } \\
\text { current }\end{array}$ & 1979 & 2000 & & & 4 & 9 & & \\
\hline $\begin{array}{l}\text { US Ecology } \\
\text { future }\end{array}$ & 2025.5 & & & & 9 & & & \\
\hline ERDF & 2021 & & & & 7 & & & \\
\hline C Reactor & 2050 & & & & 7 & & & \\
\hline D Reactor & 2050 & & & & 7 & & & \\
\hline DR Reactor & 2050 & & & & 7 & & & \\
\hline F Reactor & 2050 & & & & 7 & & & \\
\hline H Reactor & 2050 & & & & 7 & & & \\
\hline KE Reactor & 2050 & & & & 7 & & & \\
\hline KW Reactor & 2050 & & & & 7 & & & \\
\hline N Reactor & 2050 & & & & 7 & & & \\
\hline TK-A-S & 2004 & 2016 & 2025 & & 1 & 4 & 7 & \\
\hline TK-A-L & 1963 & 1987 & 2025 & & 1 & 4 & 7 & \\
\hline TK-A-R & 2525 & & & & 7 & & & \\
\hline TK-AN-R-1 & 2525 & & & & 7 & & & \\
\hline TK-AN-R-2 & 2525 & & & & 7 & & & \\
\hline
\end{tabular}


Table 4.5. (contd)

\begin{tabular}{|c|c|c|c|c|c|c|c|c|}
\hline Site Name & $\begin{array}{c}\text { Time } \\
1 *\end{array}$ & $\begin{array}{c}\text { Time } \\
2\end{array}$ & $\begin{array}{c}\text { Time } \\
3\end{array}$ & $\begin{array}{c}\text { Time } \\
4\end{array}$ & $\begin{array}{c}\text { Recharge } \\
1^{* *}\end{array}$ & $\begin{array}{c}\text { Recharge } \\
2\end{array}$ & \begin{tabular}{|c|} 
Recharge \\
3
\end{tabular} & $\begin{array}{c}\text { Recharge } \\
4\end{array}$ \\
\hline TK-AP-R-1 & 2525 & & & & \begin{tabular}{|l|}
7 \\
\end{tabular} & & & \\
\hline TK-AP-R-2 & 2525 & & & & 7 & & & \\
\hline TK-AW-R & 2525 & & & & 7 & & & \\
\hline TK-AX-S-1 & 2003 & 2008 & 2025 & & 1 & 4 & 7 & \\
\hline TK-AX-S-2 & 2005 & 2008 & 2025 & & 1 & 4 & 7 & \\
\hline TK-AX-L-1 & 1988 & 1989 & 2025 & & 1 & 4 & 7 & \\
\hline TK-AX-L-2 & 1977 & 1978 & 2025 & & 1 & 4 & 7 & \\
\hline TK-AX-R-1 & 2525 & & & & 7 & & & \\
\hline TK-AX-R-2 & 2525 & & & & 7 & & & \\
\hline TK-AY-R-1 & 2525 & & & & 7 & & & \\
\hline TK-AY-R-2 & 2525 & & & & 7 & & & \\
\hline TK-AZ-R & 2525 & & & & 7 & & & \\
\hline TK-B-S & 2012 & 2018 & 2025 & & 1 & 4 & 7 & \\
\hline TK-B-L & 1974 & 1984 & 2025 & & 1 & 4 & 7 & \\
\hline TK-B-R & 2525 & & & & 7 & & & \\
\hline TK-BX-S & 2012 & 2018 & 2025 & & 1 & 4 & 7 & \\
\hline TK-BX-L & 1971 & 1984 & 2025 & & 1 & $\overline{4}$ & 7 & \\
\hline TK-BX-R & 2525 & & & & $\overline{7}$ & & & \\
\hline TK-BY-S & 2014 & 2019 & 2025 & & $\overline{1}$ & 4 & 7 & \\
\hline TK-BY-L & 1972 & 1984 & 2025 & & 1 & 4 & 7 & \\
\hline TK-BY-R & 2525 & & & & 7 & & & \\
\hline TK-C-S-1 & 2007 & 2016 & 2025 & & 1 & 4 & 7 & \\
\hline TK-C-S-2 & 1998 & 2009 & 2025 & & 1 & 4 & 7 & \\
\hline TK-C-L-1 & 1984 & 1985 & 2025 & & 1 & 4 & 7 & \\
\hline TK-C-L-2 & 1968 & 1988 & 2025 & & 1 & 4 & 7 & \\
\hline TK-C-R-1 & 2525 & & & & 7 & & & \\
\hline TK-C-R-2 & 2525 & & & & 7 & & & \\
\hline TK-S-S & 2012 & 2018 & 2025 & & I & 4 & 7 & \\
\hline TK-S-L & 1968 & 1969 & 2025 & & 1 & 4 & $\overline{7}$ & \\
\hline TK-S-R & 2525 & & & & 7 & & & \\
\hline TK-SX-S-1 & 2018 & 2019 & 2025 & & 1 & 4 & 7 & \\
\hline TK-SX-S-2 & 2004 & 2020 & 2025 & & 1 & 4 & 7 & \\
\hline TK-SX-L-2 & 1962 & 1988 & 2025 & & 1 & $\overline{4}$ & 7 & \\
\hline TK-SX-R-1 & 2525 & & & & 7 & & & \\
\hline TK-SX-R-2 & 2525 & & & & 7 & & & \\
\hline TK-SY-R-1 & 2525 & & & & 7 & & & \\
\hline TK-SY-R-2 & 2525 & & & & 7 & & & \\
\hline $\mathrm{TK}-\mathrm{T}-\mathrm{S}$ & 2017 & 2019 & 2025 & & 1 & 4 & 7 & \\
\hline
\end{tabular}


Table 4.5. (contd)

\begin{tabular}{|c|c|c|c|c|c|c|c|c|}
\hline Site Name & $\begin{array}{c}\text { Time } \\
1^{*}\end{array}$ & $\begin{array}{c}\text { Time } \\
2\end{array}$ & $\begin{array}{c}\text { Time } \\
3\end{array}$ & $\begin{array}{c}\text { Time } \\
4\end{array}$ & $\begin{array}{c}\text { Recharge } \\
1^{* *}\end{array}$ & \begin{tabular}{|c|} 
Recharge \\
2
\end{tabular} & $\begin{array}{c}\text { Recharge } \\
3\end{array}$ & $\begin{array}{c}\text { Recharge } \\
4\end{array}$ \\
\hline TK-T-L & 1973 & 1992 & 2025 & & 1 & 4 & 7 & \\
\hline TK-T-R & 2525 & & & & 7 & & & \\
\hline TK-TX-S & 2012 & 2019 & 2025 & & 1 & 4 & 7 & \\
\hline TK-TX-L & 1974 & 1984 & 2025 & & 1 & 4 & 7 & \\
\hline$\overline{\mathrm{TK}-\mathrm{TX}-\mathrm{R}}$ & 2525 & & & & 7 & & & \\
\hline TK-TY-S & 2016 & 2018 & 2025 & & 1 & 4 & $\overline{7}$ & \\
\hline TK-TY-L & 1959 & 1981 & 2025 & & 1 & 4 & 7 & \\
\hline TK-TY-R & 2525 & & & & 7 & & & \\
\hline TK-U-S & 2007 & 2019 & 2025 & & 1 & 4 & 7 & \\
\hline TK-U-L & 1959 & 1980 & 2025 & & 1 & 4 & 7 & \\
\hline TK-U-R & 2525 & & & & 7 & & & \\
\hline
\end{tabular}

* "Time" refers to the year that the application of the corresponding recharge begins. Therefore, recharge 1 is assume to begin at time 1 and end at time 2 .

** "Recharge" refers to the index of recharge rate ( $\mathrm{cm} / \mathrm{yr}$ ) applied over the time interval specified in the "time" field. An index volume of 1 indicates the recharge rate will be calculated as discussed in Section 4.1.2.1. Index values of $2,3,4,5,6,7,8$, and 9 specify recharge rates of $50,20,7.5,5$, $0.5,0.05,0.01$, and $0.172 \mathrm{~cm} / \mathrm{yr}$, respectively.

+ (a) refers to waste disposed after September 30, 1988.

+ (b) refers to waste disposed before September 30, 1988. 
Table 4.6. Geologic Well Logs for the Vadose Zone Model

\begin{tabular}{|l|c|c|c|c|c|c|c|c|c|c|}
\hline Column & $\begin{array}{c}\text { Elevation } \\
(\mathbf{m})\end{array}$ & $\begin{array}{c}\text { Northing } \\
(\mathbf{m})^{*}\end{array}$ & $\begin{array}{c}\text { Easting } \\
(\mathbf{m})^{* *}\end{array}$ & ${\text { Soil } \mathbf{1}^{+}}^{\text {Surface }}$ & $\begin{array}{c}\text { Thickness } \\
(\mathbf{m})\end{array}$ & Soil 2 & $\begin{array}{c}\text { Thickness } \\
(\mathrm{m})\end{array}$ & Soil 3 & $\begin{array}{c}\text { Thickness } \\
(\mathbf{m})\end{array}$ & $\begin{array}{c}\text { Thickness } \\
\text { Soil } \mathbf{4}^{++}\end{array}$ \\
\hline $\mathbf{m})$
\end{tabular}

* Refers to north coordinate in Washington State Plane NAD83 coordinate system.

** Refers to east coordinate in Washington State Plane NAD83 coordinate system.

+ "Soil 1" refers to the upper soil layer.

++ "Soil 4 " refers to the lowest soil layer simulated. 
Table 4.7. Sediment Types and Unsaturated Flow Model Parameters Used in the Composite Analysis

\begin{tabular}{|c|c|c|c|c|c|c|c|c|}
\hline Soil Name & Code & $\begin{array}{c}\text { van } \\
\text { Genuchten } \\
\text { alpha (-) }\end{array}$ & $\begin{array}{c}\text { van } \\
\text { Genuchten } \\
n(1 / \mathrm{cm})\end{array}$ & $\begin{array}{c}\text { Residual } \\
\text { Water } \\
\text { Content } \\
\left(\mathrm{cm}^{3} / \mathrm{cm}^{3}\right) \\
\end{array}$ & $\begin{array}{c}\text { Saturated } \\
\text { Water } \\
\text { Content } \\
\left(\mathrm{cm}^{3} / \mathrm{cm}^{3}\right) \\
\end{array}$ & $\begin{array}{c}\text { Saturated } \\
\text { Hydraulic } \\
\text { Conductivity } \\
(\mathrm{cm} / \mathrm{s}) \\
\end{array}$ & $\begin{array}{c}\text { Bulk } \\
\text { Density } \\
\left(\mathrm{g} / \mathrm{cm}^{3}\right) \\
\end{array}$ & Gravel \%* \\
\hline East Hanford Gravel & EHG & $8.11 \mathrm{E}-03$ & 1.58 & 0.0146 & 0.119 & $1.76 \mathrm{E}-03$ & 1.97 & $41.70 \%$ \\
\hline Lower East Hanford Gravel & LEHG & $8.11 \mathrm{E}-03$ & 1.58 & 0.0146 & 0.119 & $1.76 \mathrm{E}-03$ & 1.97 & $41.70 \%$ \\
\hline East Hanford Sand & EHS & $1.30 \mathrm{E}-01$ & 2.10 & 0.0257 & 0.337 & $.1 .19 \mathrm{E}-02$ & 1.78 & $17.30 \%$ \\
\hline East Ringold & ER & $8.19 \mathrm{E}-03$ & 1.53 & 0.0262 & 0.124 & $3.97 \mathrm{E}-04$ & 2.04 & $43.30 \%$ \\
\hline West Hanford Sand & WHS & $1.44 \mathrm{E}-02$ & 2.20 & 0.0519 & 0.382 & $3.98 \mathrm{E}-04$ & 1.64 & $3.60 \%$ \\
\hline Early Palouse & WEP & $6.27 \mathrm{E}-03$ & 2.53 & 0.0300 & 0.379 & $9.69 \mathrm{E}-05$ & 1.68 & $2.00 \%$ \\
\hline Plio-Pleistocene & WPP & $1.55 \mathrm{E}-02$ & 1.78 & 0.0616 & 0.337 & $5.79 \mathrm{E}-02$ & 1.65 & $8.40 \%$ \\
\hline West Ringold & WR & $3.14 \mathrm{E}-02$ & $1: 65$ & 0.0236 & 0.226 & $5.76 \mathrm{E}-02$ & 2.04 & $43.30 \%$ \\
\hline
\end{tabular}

* Only fine particles were assumed to contribute to sorption of radionuclides. The impact of larger particles was corrected using Gravel \%.

Data are from Khaleel and Freeman (1995). A normal distribution was assumed for the parameters "van Genuchten n," "Residual Water

Content," and "Saturated Water Content," and the mean was calculated accordingly. A log-normal distribution was assumed for the parameters "van Genuchten alpha" and "Saturated Hydraulic Conductivity," and the mean was calculated accordingly. If the sample size was less than 10, the parameters "van Genuchten alpha" and "Saturated Hydraulic Conductivity" were determined using the geometric mean. 
Table 4.8. Summary of Key Assumptions for the Vadose Zone Model

\begin{tabular}{|c|c|c|}
\hline Assumption & Rationale & Impact \\
\hline $\begin{array}{l}\text { Mass released from a waste site } \\
\text { was assumed to enter the aquifer } \\
\text { directly beneath the site. }\end{array}$ & $\begin{array}{l}\text { Data to characterize the } \\
\text { multidimensional flow patterns } \\
\text { beneath most sites are inadequate. }\end{array}$ & $\begin{array}{l}\text { Sites with significant horizontal } \\
\text { migration within the vadose zone } \\
\text { may enter the aquifer at some } \\
\text { other location than directly } \\
\text { beneath the site. }\end{array}$ \\
\hline $\begin{array}{l}\text { The vadose zone was represented } \\
\text { as a vertical soil column. }\end{array}$ & $\begin{array}{l}\text { Data to characterize the } \\
\text { multidimensional flow patterns } \\
\text { beneath most sites are inadequate. }\end{array}$ & $\begin{array}{l}\text { In order to ensure simulations } \\
\text { with the one-dimensional model } \\
\text { do not predict ponding, the } \\
\text { infiltration rate was not allowed } \\
\text { to exceed the infiltration capacity } \\
\text { of the strata with the lowest } \\
\text { infiltration rate. These specified } \\
\text { infiltration rates were generally } \\
\text { much less than for other layers. } \\
\text { Lowering the infiltration rates, } \\
\text { particularly in the upper layers, } \\
\text { delays the predicted cumulative } \\
\text { breakthrough to the water table. } \\
\text { Additionally, the increased } \\
\text { volume in the column simulated, } \\
\text { provides additional volume } \\
\text { subject to gradually draining } \\
\text { which also delays the cumulative } \\
\text { breakthrough. This is not a } \\
\text { conservative assumption. }\end{array}$ \\
\hline $\begin{array}{l}\text { For cribs, trenches, and ditches, } \\
\text { the simulated area of the } \\
\text { discharge was assumed to equal } \\
\text { three times the area required to } \\
\text { pass the recharge through the } \\
\text { strata with the lowest saturated } \\
\text { hydraulic conductivity without. }\end{array}$ & $\begin{array}{l}\text { Plumes spread significantly from } \\
\text { these sources as they move } \\
\text { downward through the vadose } \\
\text { zone. }\end{array}$ & $\begin{array}{l}\text { Increasing the simulated area } \\
\text { delays the calculated cumulative } \\
\text { breakthrough to the water table. } \\
\text { Sensitivity of cumulative release } \\
\text { to assumed area is discussed in } \\
\text { Section } 4.1 .2 .4 \text {. }\end{array}$ \\
\hline $\begin{array}{l}\text { For ponds, the simulated area of } \\
\text { the discharge was assumed to } \\
\text { equal the area required to } \\
\text { infiltrate the recharge through the } \\
\text { strata with the lowest saturated } \\
\text { hydraulic conductivity without } \\
\text { ponding. }\end{array}$ & $\begin{array}{l}\text { The area of ponds was large } \\
\text { enough to limit spreading to a } \\
\text { relatively small area around the } \\
\text { edges. }\end{array}$ & $\begin{array}{l}\text { Increasing the simulated area } \\
\text { delays the calculated cumulative } \\
\text { breakthrough to the water table. }\end{array}$ \\
\hline $\begin{array}{l}\text { For tank leaks and tank sluicing } \\
\text { losses, the area of the discharge } \\
\text { was assumed to equal the area of } \\
\text { the affected tank bottoms. }\end{array}$ & $\begin{array}{l}\text { The simulated area should be } \\
\text { related to the number of affected } \\
\text { tanks. }\end{array}$ & $\begin{array}{l}\text { Increasing the area delays the } \\
\text { calculated cumulative } \\
\text { breakthrough to the water table. }\end{array}$ \\
\hline
\end{tabular}


Table 4.8. (contd)

\begin{tabular}{|c|c|c|}
\hline Assumption & Rationale & Impact \\
\hline $\begin{array}{l}\text { The initial soil moisture was } \\
\text { estimated based on a steady } \\
\text { recharge of } 5 \mathrm{~mm} / \mathrm{yr} \text {. }\end{array}$ & $\begin{array}{l}5 \mathrm{~mm} / \mathrm{yr} \text { is estimated to be the } \\
\text { recharge before natural } \\
\text { vegetation was disturbed. }\end{array}$ & $\begin{array}{l}\text { The senstivity of calculated } \\
\text { cumulative breakthrough to the } \\
\text { water table is discussed in } \\
\text { Section } 4.1 .2 .4 \text {. Any impacts of } \\
\text { the initial water content are lost } \\
\text { within a relatively short period of } \\
\text { time. }\end{array}$ \\
\hline $\begin{array}{l}\text { The model was assumed to } \\
\text { instantaneously response to } \\
\text { changes in recharge rates. }\end{array}$ & $\begin{array}{l}\text { Waste sites are generally shallow } \\
\text { and should respond quickly to } \\
\text { changes recharge relative to the } \\
1000 \text {-year study period. }\end{array}$ & $\begin{array}{l}\text { Changes in recharge at deeper } \\
\text { sites will occur gradually over } \\
\text { many years. Since decreased } \\
\text { recharge results in decreased } \\
\text { release from the waste form for } \\
\text { each of the release models, when } \\
\text { recharge rates decrease the model } \\
\text { will underestimate the predicted } \\
\text { release to the water table for the } \\
\text { next few years. }\end{array}$ \\
\hline $\begin{array}{l}\text { Barriers were assumed to affect } \\
\text { the entire soil profile under } \\
\text { consideration. }\end{array}$ & $\begin{array}{l}\text { Barriers are expected to be } \\
\text { sufficiently extensive that the } \\
\text { flow from a waste form beneath a } \\
\text { barrier will not be influenced by } \\
\text { the recharge rates occurring } \\
\text { beyond the barrier. }\end{array}$ & $\begin{array}{l}\text { If the barrier is small relative to } \\
\text { the depth to the water table this } \\
\text { assumption will not be valid. } \\
\text { This assumption will delay the } \\
\text { predicted discharge to the water } \\
\text { table. }\end{array}$ \\
\hline $\begin{array}{l}\text { Adjacent sites were assumed to } \\
\text { not interfere with each other. }\end{array}$ & $\begin{array}{l}\text { Simulating the vadose zone } \\
\text { transport in multiple dimensions } \\
\text { for the entire } 200 \text { Plateau Area at } \\
\text { the Hanford Site was not practical } \\
\text { for the first iteration of the } \\
\text { Composite Analysis. }\end{array}$ & $\begin{array}{l}\text { Interference will generally } \\
\text { increase the flux to the water } \\
\text { table. This is not a conservative } \\
\text { assumption. }\end{array}$ \\
\hline $\begin{array}{l}\text { The soils were represented with a } \\
\text { total of seven main soil groups. }\end{array}$ & $\begin{array}{l}\text { Inadequate data exist to } \\
\text { characterize the soil properties } \\
\text { beneath most sites beyond the } \\
\text { seven main soil groups } \\
\text { considered. }\end{array}$ & $\begin{array}{l}\text { Several thin, very low } \\
\text { permeability strata have been } \\
\text { observed in the vadose zone } \\
\text { beneath the } 200 \text { Area Plateau at } \\
\text { the Hanford Site. These strata } \\
\text { would tend to reduce the flux to } \\
\text { the water table. Neglecting these } \\
\text { very low permeability strata } \\
\text { would tend to increase the } \\
\text { predicted cumulative flux to the } \\
\text { water table. }\end{array}$ \\
\hline
\end{tabular}


Table 4.8. (contd)

\begin{tabular}{|c|c|c|}
\hline Assumption & Rationale & Impact \\
\hline $\begin{array}{l}\text { Liquid releases were assumed to } \\
\text { occur uniformly over the period } \\
\text { of operation. }\end{array}$ & $\begin{array}{l}\text { Inadequate data exist to distribute } \\
\text { the volume of the liquid releases } \\
\text { and the associated inventories } \\
\text { over time. }\end{array}$ & $\begin{array}{l}\text { Many of the liquid releases had } \\
\text { very transient behaviors. } \\
\text { Assuming that the estimated } \\
\text { volume of the specific site is } \\
\text { released uniformly over the entire } \\
\text { period of operation will generally } \\
\text { increase the predicted cumulative } \\
\text { flux to the water table, since a } \\
\text { larger area would be required to } \\
\text { handle the transient release. }\end{array}$ \\
\hline $\begin{array}{l}\text { The depth that } \mathrm{K}_{\mathrm{d}} \mathrm{s} \text { change is } \\
\text { time-invariant. }\end{array}$ & $\begin{array}{l}\text { Inadequate data exist to describe } \\
\text { the temporal variation in the } \\
\text { depth that the } K_{d} \text { changes from } \\
\text { near-field to far-field. }\end{array}$ & $\begin{array}{l}\text { This assumption is conservative if } \\
\text { the near-field } \mathrm{K}_{d} \text { is less than the } \\
\text { far-field } \mathrm{K}_{d} \text { because it will } \\
\text { underestimate the depth of the } \\
\text { far-field early in the release. } \\
\text { However, if the near-field } \mathrm{K}_{d} \text { is } \\
\text { greater than the far-field } \mathrm{K}_{d} \text { this } \\
\text { assumption will underestimate } \\
\text { the influence to the far-field } \mathrm{K}_{d} \\
\text { on early releases. Generally, } \mathrm{K}_{d} s \\
\text { increase from near-field to far- } \\
\text { field. }\end{array}$ \\
\hline $\begin{array}{l}\text { Preferential flow paths were not } \\
\text { considered in the first iteration of } \\
\text { the Composite Analysis. }\end{array}$ & $\begin{array}{l}\text { Inadequate data exist to } \\
\text { characterize the soil properties } \\
\text { beneath most sites beyond the } \\
\text { seven main soil groups } \\
\text { considered. }\end{array}$ & $\begin{array}{l}\text { Preferential flow paths can } \\
\text { significantly increase the } \\
\text { predicted cumulative flux to the } \\
\text { water table. This is not a } \\
\text { conservative assumption. }\end{array}$ \\
\hline $\begin{array}{l}\text { A value of } 0.4 \mathrm{~m} \text { was used for } \\
\text { dispersivity in the STOMP } \\
\text { calculations. }\end{array}$ & $\begin{array}{l}\text { STOMP was only used to } \\
\text { estimate the travel times of unit } \\
\text { releases from the waste form to } \\
\text { the water table. The actual mass } \\
\text { flux is estimated using the } \\
\text { convolution approach discussed } \\
\text { in Section 4.1.2.2. }\end{array}$ & $\begin{array}{l}\text { Increasing the dispersivity value } \\
\text { will result in earlier break- } \\
\text { throughs to the water table. } \\
\text { However, a higher dispersivity } \\
\text { value will also result in the mass } \\
\text { flux to be spread out over a } \\
\text { longer time period. }\end{array}$ \\
\hline
\end{tabular}

The depths at which distribution coefficients change, were estimated from the maximum penetration depth of beta and gamma observed in or adjacent to facilities. These measurements mainly reflect cesium- 137 and strontium-90. If measurements were available for a facility, then the measured penetration depth was used. If no measurements were available, then the depth was estimated from measurements at facilities that received the same types of waste. The assumption was made that cesium is essentially mobile to the transition depth and immobile after the transition depth is reached. However, total volume discharged was also examined, and for sites with relatively large discharge volumes, the transition depth was taken to be something less than the maximum depth of measured gamma and beta. The selection of distribution coefficients is discussed in detail in Appendix E. 
Table 4.9. Summary of Key Assumptions for the Groundwater Flow Model

\begin{tabular}{|c|c|c|}
\hline Assumption & Rationale & Impact \\
\hline $\begin{array}{l}\text { The unconfined aquifer system, } \\
\text { overlying the basalts, can be } \\
\text { adequately represented by nine } \\
\text { hydrostratigraphic units. }\end{array}$ & $\begin{array}{l}\text { Flow of water (and transport of } \\
\text { radionuclides) is assumed to } \\
\text { occur in three dimensions. Nine } \\
\text { hydrostratigraphic units are } \\
\text { considered adequate to represent } \\
\text { flow in this unconfined aquifer } \\
\text { system over a wide range of } \\
\text { conditions. Nine units are } \\
\text { supported by available . } \\
\text { hydrogeologic data and represent } \\
\text { all major and areally extensive } \\
\text { conductive and nonconductive } \\
\text { geohydrologic units above the } \\
\text { basalt. }\end{array}$ & $\begin{array}{l}\text { Additional units would better } \\
\text { represent local flow conditions } \\
\text { and hydrogeology. However, } \\
\text { data are not currently available to } \\
\text { improve this interpretation on a } \\
\text { sitewide basis and other } \\
\text { uncertainties could nullify the } \\
\text { effect of this improvement. } \\
\text { Additionally, simulation times } \\
\text { would be adversely affected. }\end{array}$ \\
\hline $\begin{array}{l}\text { Natural recharge is variable } \\
\text { across the Hanford Site and is } \\
\text { included as a surface condition in } \\
\text { the flow (and transport) model. }\end{array}$ & $\begin{array}{l}\text { Variability of recharge across the } \\
\text { Hanford Site is based on the } \\
\text { distribution of surface cover, } \\
\text { ranging from natural shrub- } \\
\text { steppe vegetation to gravel } \\
\text { surfaces in some of the } 200 \\
\text { Areas. The differences in } \\
\text { recharge based on surface cover } \\
\text { have been well documented for } \\
\text { the Hanford Site (Fayer and } \\
\text { Walters 1995). }\end{array}$ & $\begin{array}{l}\text { The surface recharge affects the } \\
\text { flow model calibration by adding } \\
\text { water to the system. The result is } \\
\text { a distribution of higher hydraulic } \\
\text { conductivity than would occur } \\
\text { without recharge. Recharge } \\
\text { affects the transport model by } \\
\text { diluting the contaminant plumes } \\
\text { and driving the maximum plume } \\
\text { concentrations below the surface } \\
\text { nodes. }\end{array}$ \\
\hline $\begin{array}{l}\text { The Columbia River is treated as } \\
\text { a constant head boundary using } \\
\text { hydraulic heads for } 1979 \text { to } \\
\text { represent the long-term average } \\
\text { conditions. }\end{array}$ & $\begin{array}{l}\text { Performing simulations with } \\
\text { transient river stage boundary } \\
\text { conditions would not be } \\
\text { appropriate since the inland areas } \\
\text { that are the focus of this analysis } \\
\text { are not greatly affected by river } \\
\text { stage variations because they } \\
\text { damp out before they reach the } \\
200 \text { Areas. Additionally, how the } \\
\text { future river stage might vary is } \\
\text { not known, and it would be too } \\
\text { costly computationally at the } \\
\text { Hanford Site-wide scale of the } \\
\text { Composite Analysis. }\end{array}$ & $\begin{array}{l}\text { Including the highly variable } \\
\text { river stage conditions in the } \\
\text { Hanford Site-wide Composite } \\
\text { Analysis model would not affect } \\
\text { the long-term results. }\end{array}$ \\
\hline
\end{tabular}


Table 4.9. (contd)

\begin{tabular}{|c|c|c|}
\hline Assumption & Rationale & Impact \\
\hline $\begin{array}{l}\text { Post-Hanford conditions do not } \\
\text { include large-scale irrigation } \\
\text { impacts. }\end{array}$ & $\begin{array}{l}\text { The prospect of large-scale } \\
\text { irrigation occurring on the } \\
\text { Hanford Site is unlikely for the } \\
\text { following reasons. } \\
\text { - Public acceptance of food } \\
\text { products grown on the } \\
\text { Hanford Site, regardless of } \\
\text { the actual risk associated } \\
\text { with agricultural } \\
\text { development is uncertain. } \\
\text { Sufficient water rights within } \\
\text { the Columbia Basin for } \\
\text { development of crops } \\
\text { requiring large-scale } \\
\text { irrigation on the Site are } \\
\text { unavailable. If agriculture } \\
\text { should develop on the } \\
\text { Hanford Site, it is likely that } \\
\text { the crops to be planted will } \\
\text { use the efficient and focused } \\
\text { irrigation methods (e.g. drip } \\
\text { irrigation) that are used in } \\
\text { fruit orchards or vineyards. } \\
\text { New technologies and } \\
\text { advanced resource } \\
\text { management practices will } \\
\text { likely eliminate or } \\
\text { significantly curtail over- } \\
\text { irrigation of crops. }\end{array}$ & $\begin{array}{l}\text { The impact of this assumption } \\
\text { can be significant depending on } \\
\text { the scenario that is used. } \\
\text { Previous sitewide analyses such } \\
\text { as the Hanford Defense Waste } \\
\text { Environmental Impact Statement } \\
\text { (DOE 1987) included significant } \\
\text { agricultural irrigation scenarios, } \\
\text { which can alter the overall flow } \\
\text { system in the unconfined aquifer } \\
\text { and control the direction and rate } \\
\text { of groundwater flow and } \\
\text { contaminant transport. }\end{array}$ \\
\hline
\end{tabular}


Table 4.10. Major Hydrogeologic Units Used in the Site-Wide Three-Dimensional Model

\begin{tabular}{|c|l|l|}
\hline $\begin{array}{c}\text { Unit } \\
\text { Number }\end{array}$ & \multicolumn{1}{|c|}{ Hydrogeologic Unit } & \multicolumn{1}{c|}{ Lithologic Description } \\
\hline 1 & Hanford Formation & Fluvial gravels and coarse sands \\
\hline 2 & Palouse Soils & Fine-grained sediments and eolian silts \\
\hline 3 & Plio-Pleistocene Unit & $\begin{array}{l}\text { Buried soil horizon containing caliche and basaltic } \\
\text { gravels }\end{array}$ \\
\hline 4 & Upper Ringold Formation & Fine-grained fluvial/lacustrine sediments \\
\hline 5 & $\begin{array}{l}\text { Middle Ringold } \\
\text { (Unit E) }\end{array}$ & Semi-indurated coarse-grained fluvial sediments \\
\hline 6 & $\begin{array}{l}\text { Middle Ringold } \\
\text { (Unit C) }\end{array}$ & $\begin{array}{l}\text { Fine-grained sediments with some interbedded coarse- } \\
\text { grained sediments }\end{array}$ \\
\hline 7 & $\begin{array}{l}\text { Middle Ringold } \\
\text { (Unit B and D) }\end{array}$ & Coarse-grained sediments \\
\hline 8 & $\begin{array}{l}\text { Lower Mud Sequence } \\
\text { (Lower Ringold and part of } \\
\text { Basal Ringold) }\end{array}$ & Lower blue or green clay or mud sequence \\
\hline 9 & Basal Ringold (Unit A) & Fluvial sand and gravel \\
\hline 10 & Columbia River Basalt & Basalt \\
\hline
\end{tabular}


Table 4.11. Summary of Key Assumptions for the Groundwater Transport Model

\begin{tabular}{|c|c|c|}
\hline Assumption & Rationale & Impact \\
\hline $\begin{array}{l}\mathrm{K}_{\mathrm{d}} \mathrm{s} \text { were selected based on } \\
\text { information documented in } \\
\text { Appendix } \mathrm{E} \text {. }\end{array}$ & $\begin{array}{l}\mathrm{K}_{\mathrm{d}} \mathrm{s} \text { were based on available } \\
\text { geochemical data at the Hanford } \\
\text { Site and by analogy to other } \\
\text { waste forms. Best-estimate } \\
\text { values were used in the } \\
\text { Composite Analysis. }\end{array}$ & $\begin{array}{l}\text { Some of the } \mathrm{K}_{d} \mathrm{~s} \text { for specific } \\
\text { radionuclides may be uncertain } \\
\text { and result in different predictions } \\
\text { than actually have occurred and } \\
\text { will occur in the future. }\end{array}$ \\
\hline $\begin{array}{l}\text { A grid spacing of } 375 \mathrm{~m} \text { on a side } \\
\text { was used for the transport } \\
\text { simulations. }\end{array}$ & $\begin{array}{l}\text { This grid spacing was sufficient } \\
\text { to represent transport on the } \\
\text { sitewide scale used for the } \\
\text { Composite Analysis. This grid } \\
\text { spacing was a compromise } \\
\text { between resolution of predicted } \\
\text { contaminant plumes and } \\
\text { computational time. }\end{array}$ & $\begin{array}{l}\text { The grid spacing is too coarse to } \\
\text { adequately resolve predicted } \\
\text { concentrations at distances less } \\
\text { than } 1 \mathrm{~km} \text { from the contaminant } \\
\text { sources. Away from the sources } \\
\text { and beyond the exclusion and } \\
\text { buffer zones, the grid spacing is } \\
\text { adequate to represent the } \\
\text { contaminant plumes. }\end{array}$ \\
\hline $\begin{array}{l}\text { The basic vertical resolution of } \\
\text { the transport grid was } 8 \mathrm{~m} \text {. Each } \\
\text { of the nine units was represented } \\
\text { with as many } 8 \text {-m layers as } \\
\text { needed to represent its entire } \\
\text { thickness. Nonconductive (e.g., } \\
\text { mud units) were always repre- } \\
\text { sented by at least two transport } \\
\text { layers while conductive units } \\
\text { (e.g., sand-gravel units) were } \\
\text { only represented with one } \\
\text { transport layer if they were less } \\
\text { than } 8 \text {-m thick. Creation of } \\
\text { excessively thick and thin } \\
\text { transport layers to achieve total } \\
\text { unit thickness was prevented by } \\
\text { the layering algorithm. }\end{array}$ & $\begin{array}{l}\text { The 8-m transport layers were } \\
\text { selected based on simulations } \\
\text { previously performed for the } \\
\text { Effluent Treatment Facility (Cole } \\
\text { et al. 1997). }\end{array}$ & $\begin{array}{l}\text { Adding additional transport } \\
\text { layers would improve } \\
\text { representation of the vertical } \\
\text { distribution of contaminants, but } \\
\text { at the expense of computational } \\
\text { efficiency. }\end{array}$ \\
\hline $\begin{array}{l}\text { The longitudinal dispersivity } \\
\text { assumed for all contaminant } \\
\text { transport simulations was } 95 \mathrm{~m} \text {. } \\
\text { The transverse dispersivity was } \\
\text { assumed to be } 20 \mathrm{~m}(\sim 20 \% \text { of } \\
\text { the longitudinal dispersivity). }\end{array}$ & $\begin{array}{l}\text { Dispersivity is not a directly } \\
\text { measurable value and no sitewide } \\
\text { scale estimates are available. The } \\
\text { value selected was the smallest } \\
\text { value that satisfies all three } \\
\text { theoretical constraints on its } \\
\text { value, which include grid Peclet } \\
\text { numerical constraint, scale of } \\
\text { uncharacterized heterogeneities } \\
\text { constraint, and transport scale of } \\
\text { interest constraint. A transverse } \\
\text { dispersivity that is } 1 / 5 \text { of the } \\
\text { longitudinal dispersivity is typical } \\
\text { for transport simulations (Freeze } \\
\text { and Cherry 1979). }\end{array}$ & $\begin{array}{l}\text { Dispersivity parameters assumed } \\
\text { for contaminant transport directly } \\
\text { affect predicted concentrations. } \\
\text { Lower dispersivities result in } \\
\text { higher predicted concentrations } \\
\text { near the source but later first } \\
\text { arrival times; higher dispersivities } \\
\text { result in lower predicted } \\
\text { concentrations near the source } \\
\text { but earlier first arrival time which } \\
\text { can be important for radio- } \\
\text { nuclides with short half-lives. }\end{array}$ \\
\hline
\end{tabular}


Table 4.11. (contd)

\begin{tabular}{|c|c|c|}
\hline Assumption & Rationale & Impact \\
\hline $\begin{array}{l}\text { An effective porosity of } 0.25 \text { was } \\
\text { assumed for calculation of the } \\
\text { retardation factor in all } \\
\text { contaminant transport } \\
\text { simulations. }\end{array}$ & $\begin{array}{l}\text { This value of effective porosity } \\
\text { was based on measurements } \\
\text { available for Hanford Site } \\
\text { unconfined aquifer sediments. } \\
\text { Tracer tests conducted at the } \\
\text { Hanford Site have revealed a } \\
\text { range of effective porosity from } \\
0.1 \text { to } 0.25 \mathrm{~cm}^{3} / \mathrm{cm}^{3} \text {. }\end{array}$ & $\begin{array}{l}\text { Use of the highest value of } \\
\text { effective porosity to calculate } \\
\text { retardation factor yields a low } \\
\text { estimate of sorption in Hanford } \\
\text { sediments, and is therefore biased } \\
\text { toward a conservative (i.e., } \\
\text { maximum) estimate of contam- } \\
\text { inant migration in groundwater. }\end{array}$ \\
\hline
\end{tabular}


Table 4.12. Summary of Key Assumptions for the Atmospheric Model

\begin{tabular}{|l|l|l|}
\hline \multicolumn{1}{|c|}{ Assumption } & \multicolumn{1}{|c|}{ Rationale } & \multicolumn{1}{c|}{ Impact } \\
\hline $\begin{array}{l}\text { The graphite reactor cores source } \\
\text { was the only significant } \\
\text { contributor to dose via the } \\
\text { atmospheric pathway. }\end{array}$ & $\begin{array}{l}\text { Previous performance } \\
\text { assessments and environmental } \\
\text { impact statement analyses } \\
\text { demonstrated only negligible } \\
\text { impacts via the atmospheric } \\
\text { pathway. }\end{array}$ & $\begin{array}{l}\text { This assumption was not } \\
\text { conservative }\end{array}$ \\
\hline $\begin{array}{l}\text { The entire fraction of the } \\
\text { inventory predicted to have been } \\
\text { released from the reactor was } \\
\text { assumed to enter the atmosphere. }\end{array}$ & $\begin{array}{l}\text { Inadequate data exist to estimate } \\
\text { the fraction of the released } \\
\text { inventory that will move } \\
\text { downward through the vadose } \\
\text { zone and the fraction that will } \\
\text { enter the atmosphere. }\end{array}$ & $\begin{array}{l}\text { Because no credit is taken for the } \\
\text { fraction of the inventory } \\
\text { migrating through the vadose } \\
\text { zone, this is a conservative } \\
\text { assumption. }\end{array}$ \\
\hline $\begin{array}{l}\text { Atmospheric emissions were } \\
\text { assumed to occur uniformly over } \\
\text { an area source of } 100 \mathrm{~m} \text { by } \\
600 \mathrm{~m} .\end{array}$ & $\begin{array}{l}\text { The area assumed to release } \\
\text { reflects the dimension of the } \\
\text { likely source. }\end{array}$ & Negligible. \\
\hline
\end{tabular}


Table 4.13. Summary of Key Assumptions for the Exposure and Dose Model

\begin{tabular}{|c|c|c|}
\hline Assumption & Rationale & Impact \\
\hline $\begin{array}{l}\text { The exclusive waste management } \\
\text { area and buffer zone were } \\
\text { assumed to remain under federal } \\
\text { control until the lands are safe for } \\
\text { release to the public. }\end{array}$ & $\begin{array}{l}\text { Safe stewardship of land used by } \\
\text { the DOE requires that DOE retain } \\
\text { control of the land and ground- } \\
\text { water inside the buffer zone until } \\
\text { it is safe to release. }\end{array}$ & $\begin{array}{l}\text { Radiological doses were not } \\
\text { presented for the portion of the } \\
\text { Hanford Site inside the buffer } \\
\text { zone. }\end{array}$ \\
\hline $\begin{array}{l}\text { The Unit Dose Factor was used to } \\
\text { calculate doses in the Composite } \\
\text { Analysis. }\end{array}$ & $\begin{array}{l}\text { Guidance for the completion of } \\
\text { the Composite Analysis required } \\
\text { the simulation of annual radiation } \\
\text { dose. }\end{array}$ & $\begin{array}{l}\text { Calculation and presentation of } \\
\text { only the annual radiation dose is } \\
\text { a deviation from the guidance in } \\
\text { the HSRAM which calls for a } \\
\text { lifetime risk assessment from } \\
\text { both chemicals and radionuclides. }\end{array}$ \\
\hline $\begin{array}{l}\text { The exposure scenarios included } \\
\text { in the Composite Analysis were } \\
\text { recreational, industrial, } \\
\text { residential, and agricultural. }\end{array}$ & $\begin{array}{l}\text { These exposure scenarios cover } \\
\text { the range of possible post- } \\
\text { Hanford land uses, and formally } \\
\text { published in the HSRAM report. }\end{array}$ & $\begin{array}{l}\text { Some potential impacts may not } \\
\text { be covered by the conditions spe- } \\
\text { cified in these scenario descrip- } \\
\text { tions, e.g., recently defined } \\
\text { Native American scenarios. }\end{array}$ \\
\hline $\begin{array}{l}\text { Radionuclide concentrations in } \\
\text { transport media were assumed to } \\
\text { be constant over exposure } \\
\text { durations analyzed (e.g., annual } \\
\text { radiation dose). }\end{array}$ & $\begin{array}{l}\text { Impacts predicted for the } \\
\text { Composite Analysis are for } 1000 \\
\text { years. Groundwater transport is } \\
\text { simulated using relatively short } \\
\text { time steps, but not as short as } \\
1 \text { year. Therefore, the concen- } \\
\text { tration applied in the exposure } \\
\text { duration is constant for the 1-year } \\
\text { period. }\end{array}$ & $\begin{array}{l}\text { The impact is negligible. Release } \\
\text { calculations were made on a } \\
\text { l-year time interval. Greater } \\
\text { resolution of exposures would not } \\
\text { be consistent with the prior } \\
\text { simulation steps. }\end{array}$ \\
\hline $\begin{array}{l}\text { Radionuclides are assumed to } \\
\text { reach equilibrium with soils in a } \\
\text { time period not exceeding } \\
50 \text { years, and the maximum value } \\
\text { was not varied with time in the } \\
\text { Unit Dose Factor calculation. }\end{array}$ & $\begin{array}{l}\text { Unit Dose Factors are based on } \\
\text { constant deposition over the } \\
\text { duration period. This simplifi- } \\
\text { cation was also needed in order to } \\
\text { precalculate the UDF values. }\end{array}$ & $\begin{array}{l}\text { The agricultural scenario is well } \\
\text { represented with only iodine-129, } \\
\text { uranium-233, and uranium- } 235 \\
\text { assigned somewhat lower buildup } \\
\text { in soils over } 50 \text { years than are } \\
\text { predicted to occur over longer } \\
\text { time frame. Exposures to native } \\
\text { soils, e.g., in the recreational } \\
\text { scenario, are underestimated } \\
\text { when using the } 50 \text {-year soil } \\
\text { contamination buildup levels } \\
\text { because their low leach rates } \\
\text { cause a continuous buildup over } \\
1000 \text {-year period. }\end{array}$ \\
\hline
\end{tabular}


Table 4.14. Industrial Scenario Exposure Pathways

\begin{tabular}{|l|l|l|l|}
\hline \multicolumn{1}{|c|}{ Transport Medium } & \multicolumn{1}{|c|}{ Exposure Pathway } & \multicolumn{1}{c|}{ Chemical } & \multicolumn{1}{c|}{ Radioactive } \\
\hline \multirow{4}{*}{ Soil (air deposition) } & Ingestion & Yes & Yes \\
\cline { 2 - 5 } & External & No & Yes \\
\cline { 2 - 5 } & Dermal Contact & Yes & No \\
\cline { 2 - 5 } & Suspension - Inhalation & Yes & Yes \\
\hline Air & Inhalation & Yes & Yes \\
\hline Groundwater & Ingestion & Yes & Yes \\
\cline { 2 - 5 } & Dermal Contact & Yes & No \\
\hline
\end{tabular}


Table 4.15. Recreational Scenario Exposure Pathways

\begin{tabular}{|l|l|l|l|}
\hline \multicolumn{1}{|c|}{ Transport Medium } & \multicolumn{1}{|c|}{ Exposure Pathway } & \multicolumn{1}{c|}{ Chemical } & \multicolumn{1}{c|}{ Radioactive } \\
\hline \multirow{5}{*}{ Soil (air deposition) } & Ingestion & Yes & Yes \\
\cline { 2 - 4 } & External & No & Yes \\
\cline { 2 - 5 } & Dermal Contact & Yes & No \\
\cline { 2 - 5 } & Suspension - Inhalation & Yes & Yes \\
\cline { 2 - 5 } & Biota - game (deer) & Yes & Yes \\
\hline \multirow{5}{*}{ Air } & Inhalation & Yes & Yes \\
\cline { 2 - 5 } & Biota - game (deer) & Yes & Yes \\
\hline \multirow{3}{*}{ Groundwater } & Ingestion & Yes & Yes \\
\cline { 2 - 5 } & Biota - game (deer) & Yes & Yes \\
\cline { 2 - 5 } & Dermal Contact (bathing) & Yes & No \\
\hline
\end{tabular}


Table 4.16. Residential Scenario Exposure Pathways

\begin{tabular}{|l|l|l|l|}
\hline \multicolumn{1}{|c|}{ Transport Medium } & \multicolumn{1}{|c|}{ Exposure Pathway } & \multicolumn{1}{c|}{ Chemical } & \multicolumn{1}{c|}{ Radioactive } \\
\hline \multirow{5}{*}{ Soil (air deposition) } & Ingestion & Yes & Yes \\
\cline { 2 - 4 } & External & No & Yes \\
\cline { 2 - 5 } & Dermal Contact & Yes & No \\
\cline { 2 - 5 } & Biota - Fruit & Yes & Yes \\
\cline { 2 - 5 } & Biota - Vegetables & Yes & Yes \\
\cline { 2 - 5 } & Suspension - Inhalation & Yes & Yes \\
\hline \multirow{5}{*}{ Air } & Inhalation & Yes & Yes \\
\cline { 2 - 5 } & Biota - Fruit & Yes & Yes \\
\cline { 2 - 5 } & Biota - Vegetables & Yes & Yes \\
\hline \multirow{5}{*}{ Groundwater } & Ingestion & Yes & Yes \\
\cline { 2 - 5 } & Dermal Contact (bathing) & Yes & No \\
\cline { 2 - 5 } & Biota - Fruit & Yes & Yes \\
\cline { 2 - 5 } & Biota - Vegetables & Yes & Yes \\
\hline
\end{tabular}


Table 4.17. Agricultural Scenario Exposure Pathways

\begin{tabular}{|c|c|c|c|}
\hline Transport Medium & Exposure Pathway & Chemical & Radioactive \\
\hline \multirow[t]{9}{*}{ Soil (air deposition) } & Ingestion & Yes & Yes \\
\hline & External & No & Yes \\
\hline & Dermal Contact & Yes & No \\
\hline & Biota - Dairy & Yes & Yes \\
\hline & Biota - Meat & Yes & Yes \\
\hline & Biota - Game (deer) & Yes & Yes \\
\hline & Biota - Fruit & Yes & Yes \\
\hline & Biota - Vegetables & Yes & Yes \\
\hline & Suspension - Inhalation & Yes & Yes \\
\hline \multirow[t]{6}{*}{ Air } & Inhalation & Yes & Yes \\
\hline & Biota - Dairy & Yes & Yes \\
\hline & Biota - Meat & Yes & Yes \\
\hline & Biota - Game (deer) & Yes & Yes \\
\hline & Biota - Fruit & Yes & Yes \\
\hline & Biota - Vegetables & Yes & Yes \\
\hline \multirow[t]{8}{*}{ Groundwater } & Ingestion & Yes & Yes \\
\hline & Dermal Contact (bathing) & Yes & No \\
\hline & Biota - Dairy & Yes & Yes \\
\hline & Biota - Meat & Yes & Yes \\
\hline & Biota - Game (deer) & Yes & Yes \\
\hline & Biota - Fruit & Yes & Yes \\
\hline & Biota - Vegetables & Yes & Yes \\
\hline & Inhalation indoor & Yes & Yes (Radon) \\
\hline
\end{tabular}


Table 4.18. Unit Dose Factors (UDFs) Used in the Composite Analysis

\begin{tabular}{|l|l|l|l|l|}
\hline \multicolumn{1}{|c|}{ Radionuclide } & $\begin{array}{c}\text { Agricultural } \\
\text { Scenario } \\
\mathbf{m r e m} /(\mathbf{p C i} / \mathrm{L})\end{array}$ & $\begin{array}{c}\text { Residential } \\
\text { Scenario } \\
\mathbf{m r e m} /(\mathbf{p C i} / \mathrm{L})\end{array}$ & $\begin{array}{c}\text { Industrial } \\
\text { Scenario } \\
\mathbf{m r e m} /(\mathbf{p C i} / \mathrm{L})\end{array}$ & $\begin{array}{c}\text { Recreational } \\
\text { Scenario } \\
\mathbf{m r e m} /(\mathbf{p C i} / \mathrm{L})\end{array}$ \\
\hline $\mathrm{H}-3$ & $5.69 \mathrm{E}-05$ & $4.85 \mathrm{E}-05$ & $1.57 \mathrm{E}-05$ & $1.05 \mathrm{E}-06$ \\
\hline $\mathrm{C}-14$ & $4.09 \mathrm{E}-02$ & $1.52 \mathrm{E}-02$ & $5.22 \mathrm{E}-04$ & $2.99 \mathrm{E}-05$ \\
\hline $\mathrm{Cl}-36$ & $1.08 \mathrm{E}-01$ & $1.76 \mathrm{E}-02$ & $7.58 \mathrm{E}-04$ & $5.29 \mathrm{E}-05$ \\
\hline Se-79 & $1.21 \mathrm{E}-02$ & $6.77 \mathrm{E}-03$ & $2.17 \mathrm{E}-03$ & $1.28 \mathrm{E}-04$ \\
\hline Sr-90 & $3.12 \mathrm{E}-01$ & $2.53 \mathrm{E}-01$ & $3.58 \mathrm{E}-02$ & $2.01 \mathrm{E}-03$ \\
\hline Tc-99 & $3.66 \mathrm{E}-03$ & $1.36 \mathrm{E}-03$ & $3.65 \mathrm{E}-04$ & $2.10 \mathrm{E}-05$ \\
\hline I-129 & $6.19 \mathrm{E}-01$ & $2.27 \mathrm{E}-01$ & $6.90 \mathrm{E}-02$ & $3.95 \mathrm{E}-03$ \\
\hline & $\mathrm{mrem} /(\mu \mathrm{g} / \mathrm{L})$ & $\mathrm{mrem} /(\mu \mathrm{g} / \mathrm{L})$ & mrem/( $\mu \mathrm{g} / \mathrm{L})$ & $\mathrm{mrem} /(\mu \mathrm{g} / \mathrm{L})$ \\
\hline U-total & $1.86 \mathrm{E}-01$ & $1.69 \mathrm{E}-01$ & $5.27 \mathrm{E}-02$ & $2.96 \mathrm{E}-03$ \\
\hline Hazard Factor & Hazard Index/ & Hazard Index/ & $\begin{array}{l}\text { Hazard Index/ } \\
(\mu-\mathrm{g} / \mathrm{L})\end{array}$ & $\begin{array}{l}\text { Hazard Index/ } \\
(\mu-\mathrm{g} / \mathrm{L})\end{array}$ \\
\hline U-Total & $(\mu-\mathrm{g} / \mathrm{L})$ & $1.19 \mathrm{E}-02$ & $3.48 \mathrm{E}-03$ & $1.89 \mathrm{E}-04$ \\
\hline
\end{tabular}


Table 4.19. Comparison of Unit Dose Factors Between the TWRS Low-Level Tank Waste Performance Interim Assessment and the Composite Analysis

\begin{tabular}{|l|l|l|l|l|}
\hline & $\begin{array}{c}\text { Ingestion Factor } \\
\text { Composite } \\
\text { Analysis } \\
\text { Radionuclide }\end{array}$ & $\begin{array}{c}\text { Ingestion Factor } \\
\text { TWRS Low- } \\
\text { Level Tank } \\
\text { Waste IPA } \\
\text { (rem/pCi) }\end{array}$ & $\begin{array}{c}\text { Inhalation } \\
\text { Factor } \\
\text { Composite } \\
\text { Analysis } \\
\text { (rem/pCi) }\end{array}$ & $\begin{array}{c}\text { Inhalation Factor } \\
\text { TWRS Low- } \\
\text { Level Tank } \\
\text { Waste IPA } \\
\text { (rem/pCi) }\end{array}$ \\
\hline Carbon-14 & $2.09 \mathrm{E}-09$ & $2.1 \mathrm{E}-09$ & $2.09 \mathrm{E}-09$ & $2.1 \mathrm{E}-09$ \\
\hline Chlorine-36 & $3.03 \mathrm{E}-09$ & $3.0 \mathrm{E}-03$ & $2.19 \mathrm{E}-09$ & $2.1 \mathrm{E}-09$ \\
\hline Tritium & $6.3 \mathrm{E}-11$ & $6.3 \mathrm{E}-11$ & $6.3 \mathrm{E}-11$ & $6.3 \mathrm{E}-11$ \\
\hline Iodine-129 & $2.67 \mathrm{E}-07$ & $2.8 \mathrm{E}-07$ & $1.74 \mathrm{E}-07$ & $1.8 \mathrm{E}-07$ \\
\hline Selenium-79 & $8.7 \mathrm{E}-09$ & $8.3 \mathrm{E}-09$ & $9.84 \mathrm{E}-09$ & $8.9 \mathrm{E}-09$ \\
\hline Strontium-90 & $1.42 \mathrm{E}-07$ & $1.3 \mathrm{E}-07$ & $1.3 \mathrm{E}-06$ & $1.3 \mathrm{E}-06$ \\
\hline Technetium-99 & $1.46 \mathrm{E}-09$ & $1.3 \mathrm{E}-09$ & $5.33 \mathrm{E}-09$ & $7.5 \mathrm{E}-09$ \\
\hline Uranium-234 & $2.83 \mathrm{E}-07$ & $2.6 \mathrm{E}-07$ & $1.32 \mathrm{E}-04$ & $1.3 \mathrm{E}-04$ \\
\hline Uranium-235 & $2.66 \mathrm{E}-07$ & $2.5 \mathrm{E}-07$ & $1.23 \mathrm{E}-04$ & $1.2 \mathrm{E}-04$ \\
\hline Uranium-238 & $2.55 \mathrm{E}-07$ & $2.3 \mathrm{E}-07$ & $1.18 \mathrm{E}-04$ & $1.2 \mathrm{E}-04$ \\
\hline
\end{tabular}

IPA $=$ Interim Performance Assessment

TWRS $=$ Tank Waste Remediation System 


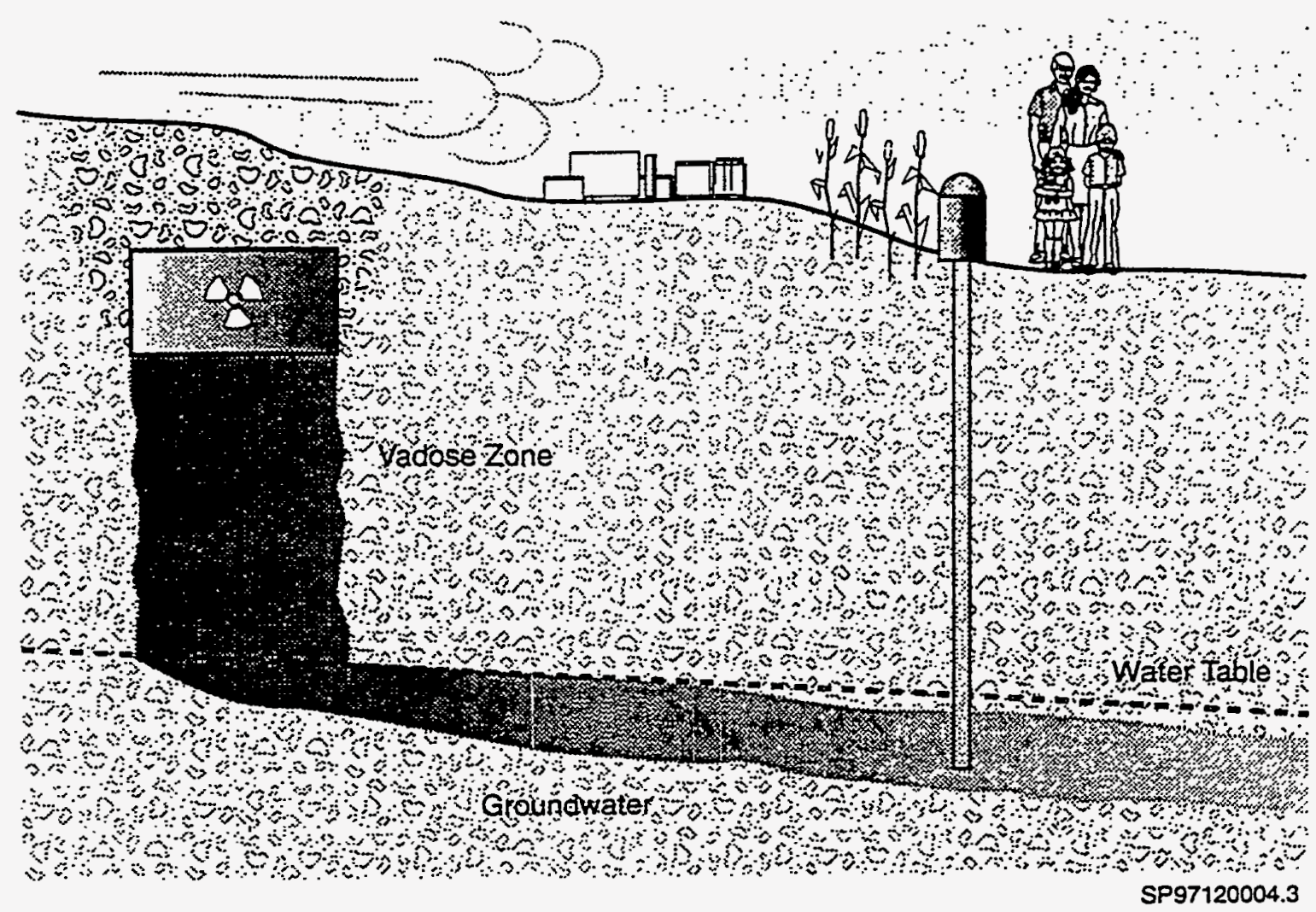

Figure 4.1. Transport and Exposure Pathways Considered in the Composite Analysis 


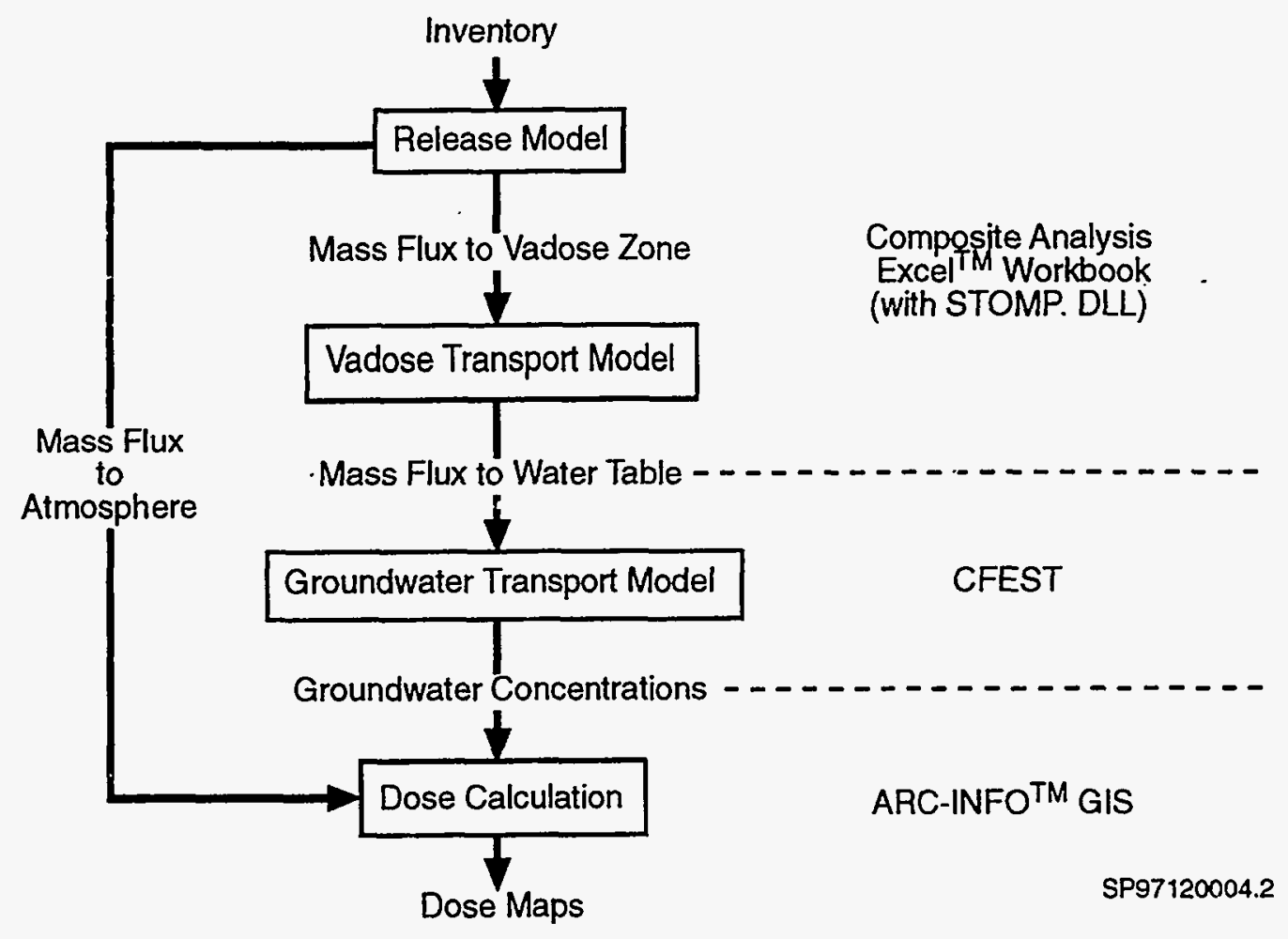

Figure 4.2. Relationship Among Software Elements in the Composite Analsysis 
7әәчุspeəدdS

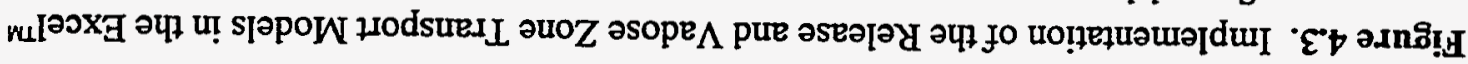
$\llcorner+\$ 000 Z 1 \angle 6 d S$
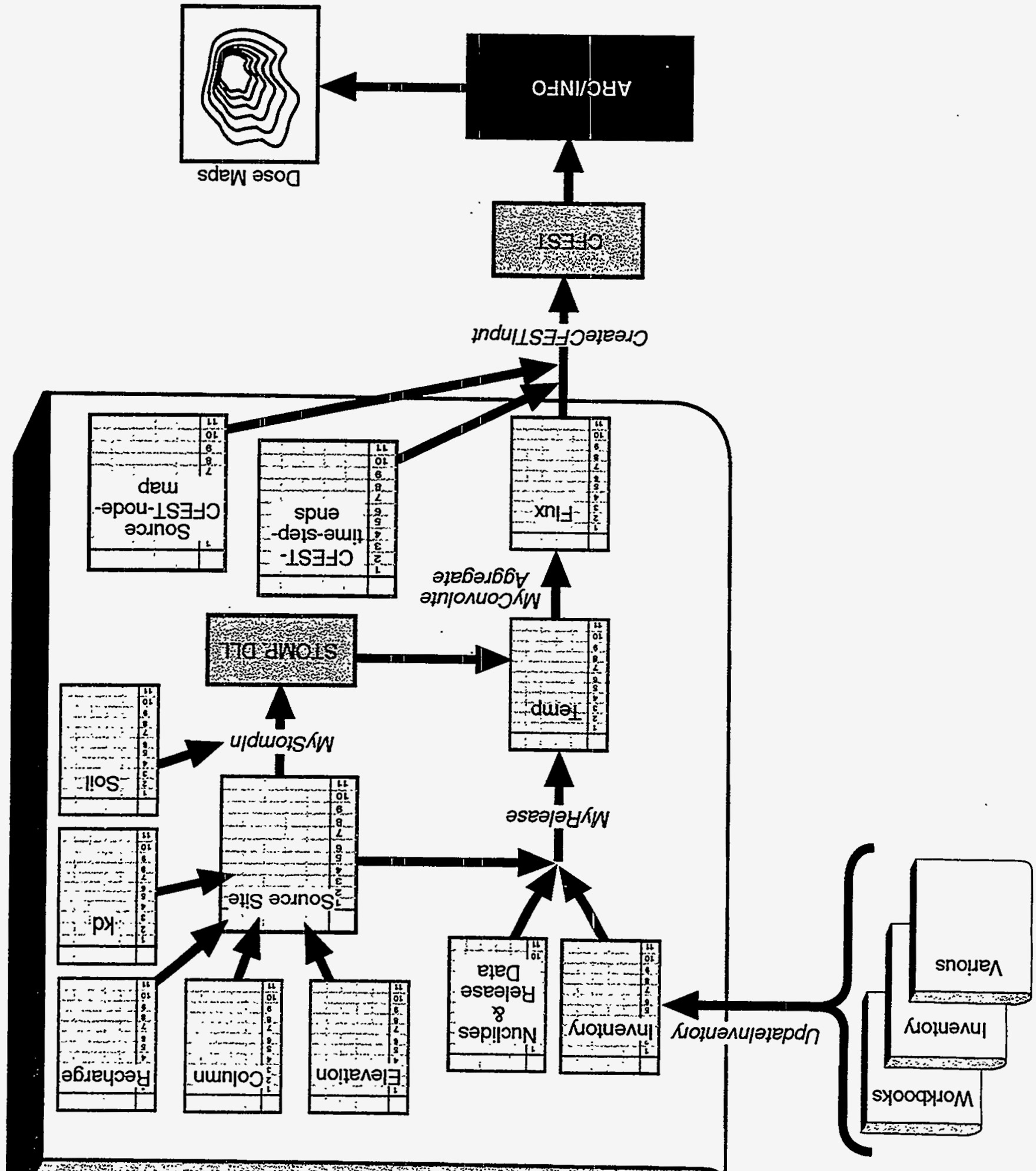


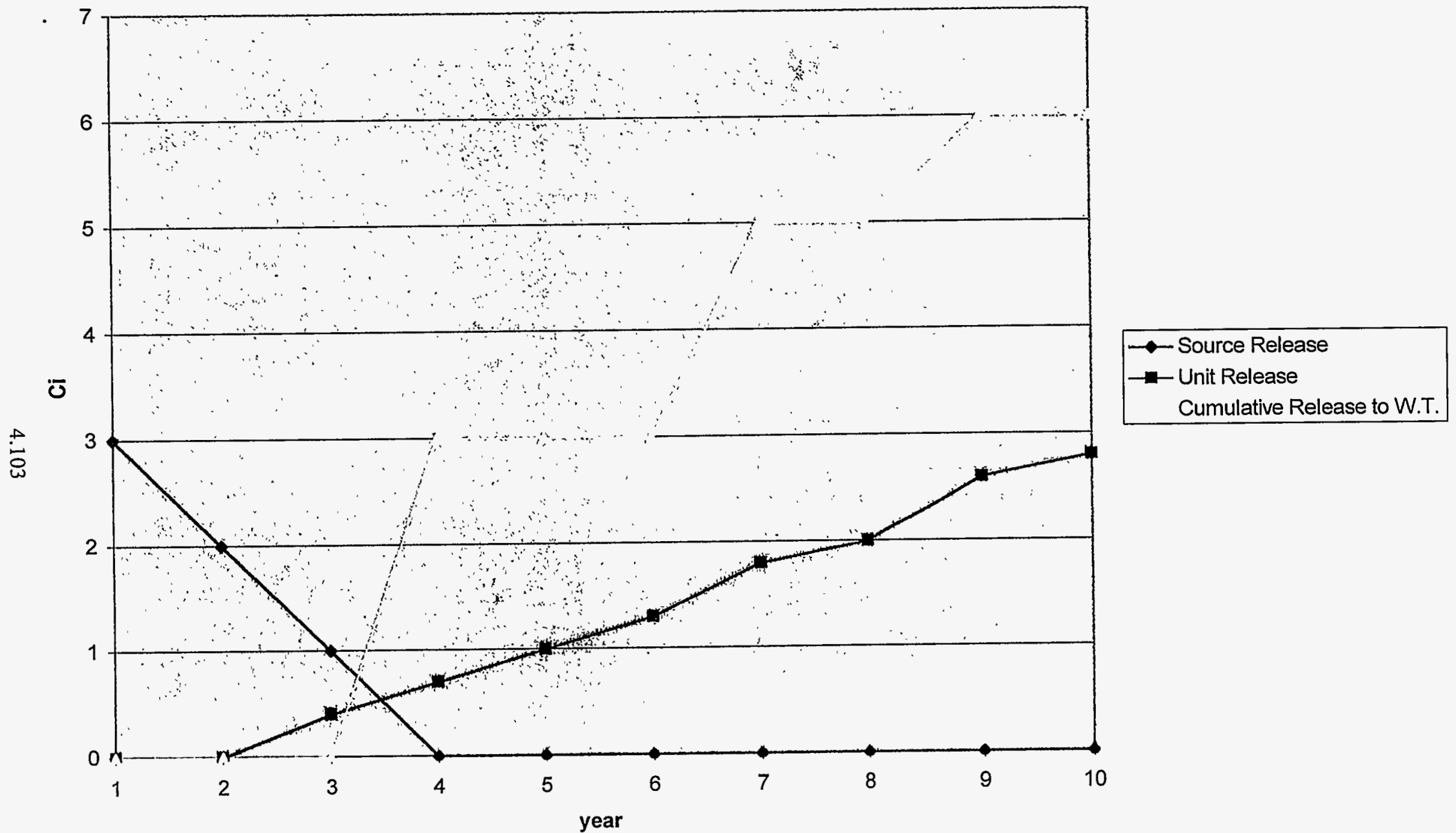

Figure 4.4. Convolution Method for Estimating Cumulative Flux to the Water Table 


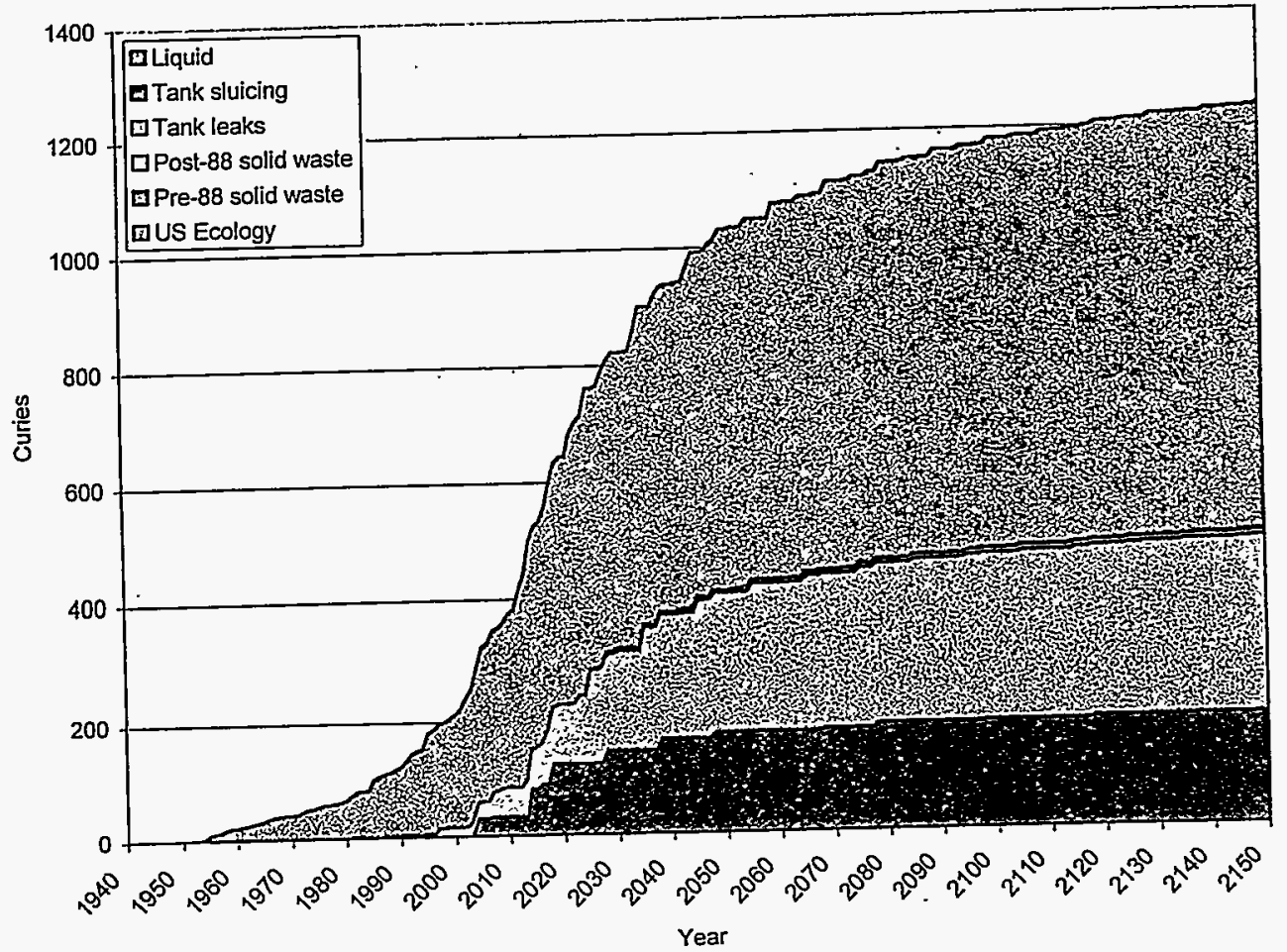

Figure 4.5a. Cumulative Release of Technetium-99 from All Sources to the Water Table from 1940 to 2150

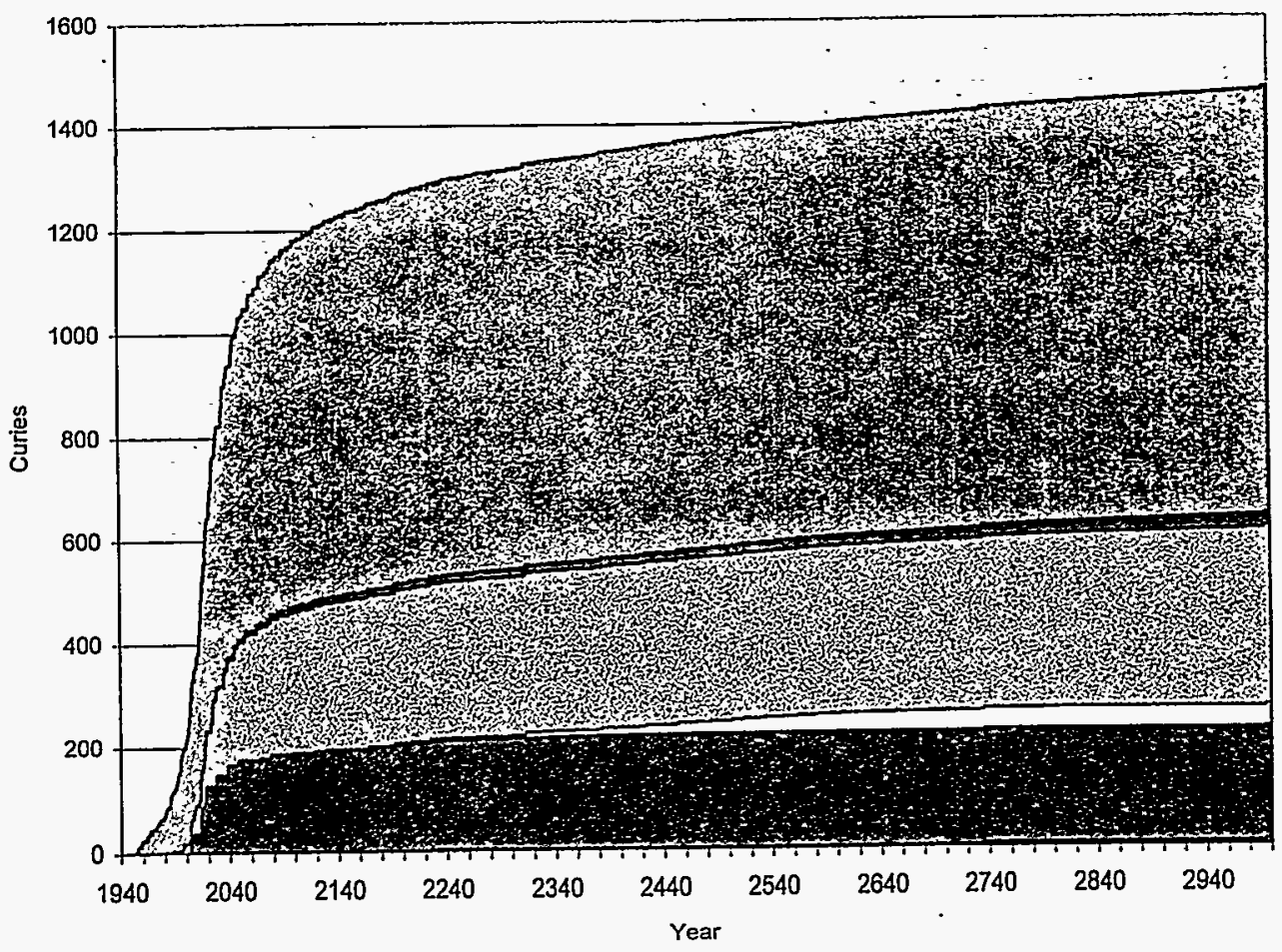

Figure 4.5b. Cumulative Release of Technetium-99 from All Sources to the Water Table from 1940 to 3000 


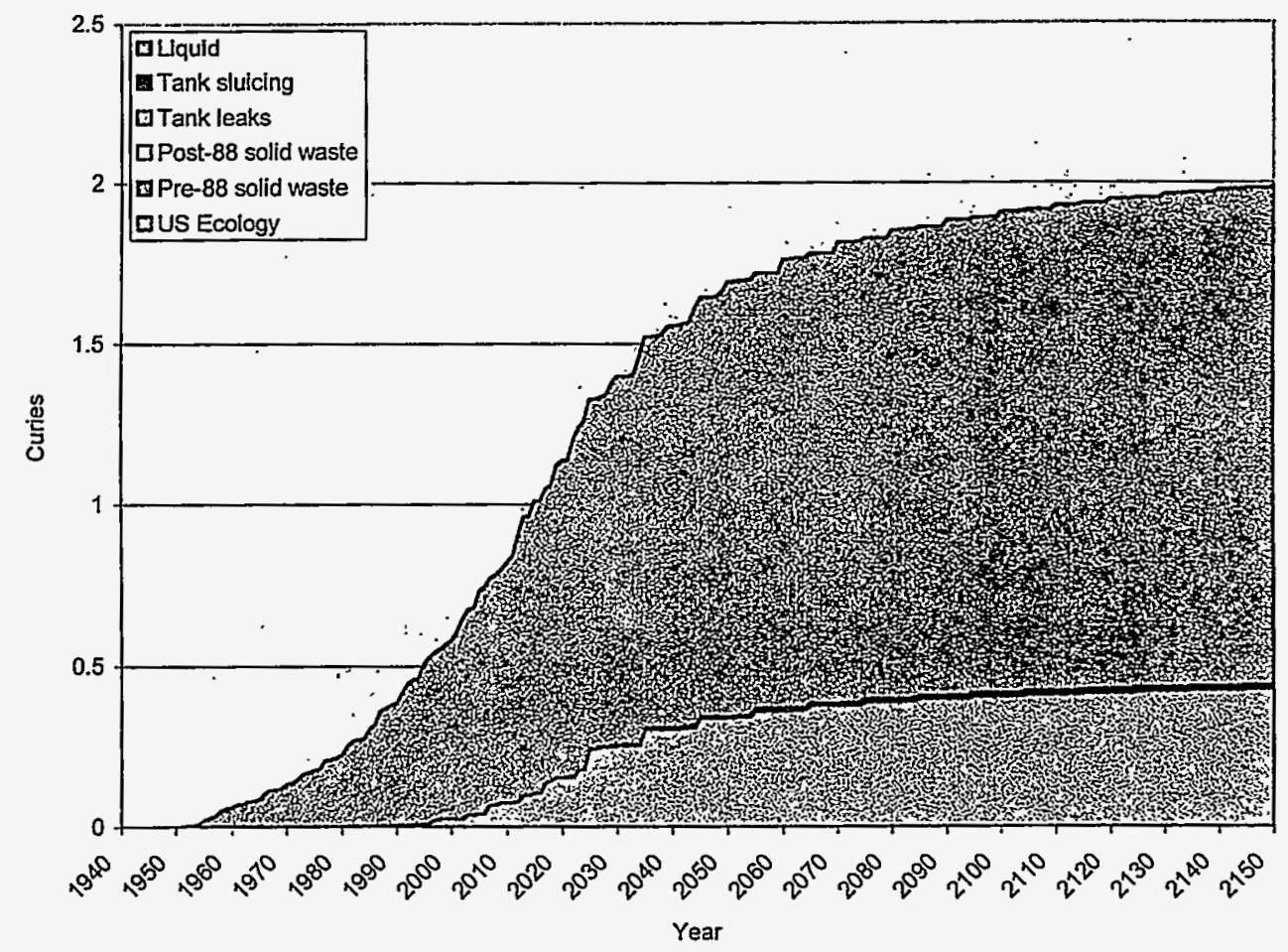

Figure 4.6a. Cumulative Release of Iodine-129 from All Sources to the Water Table from 1940 to 2150

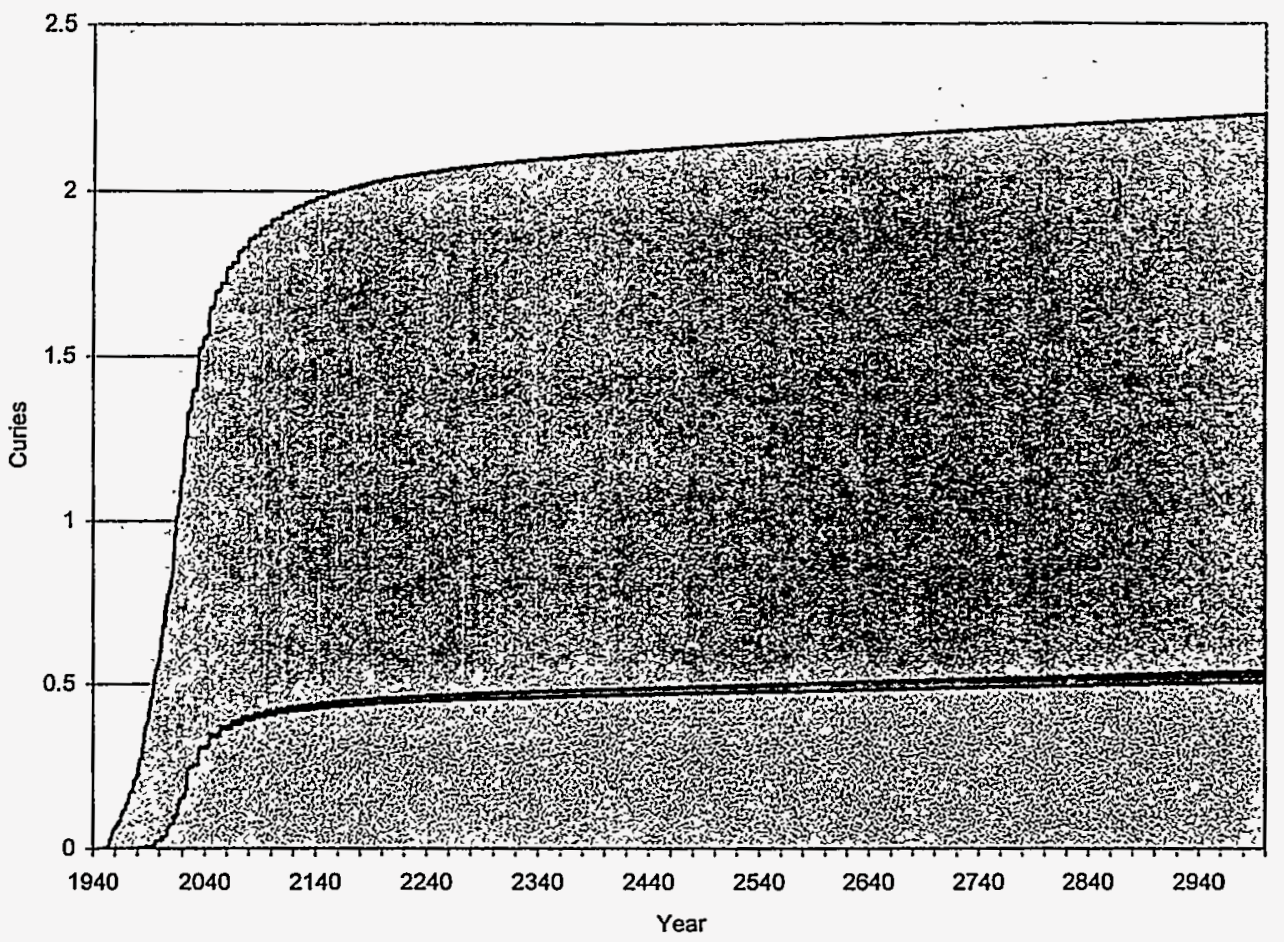

Figure 4.6b. Cumulative Release of Iodine-129 from All Sources to the Water Table from 1940 to 3000 


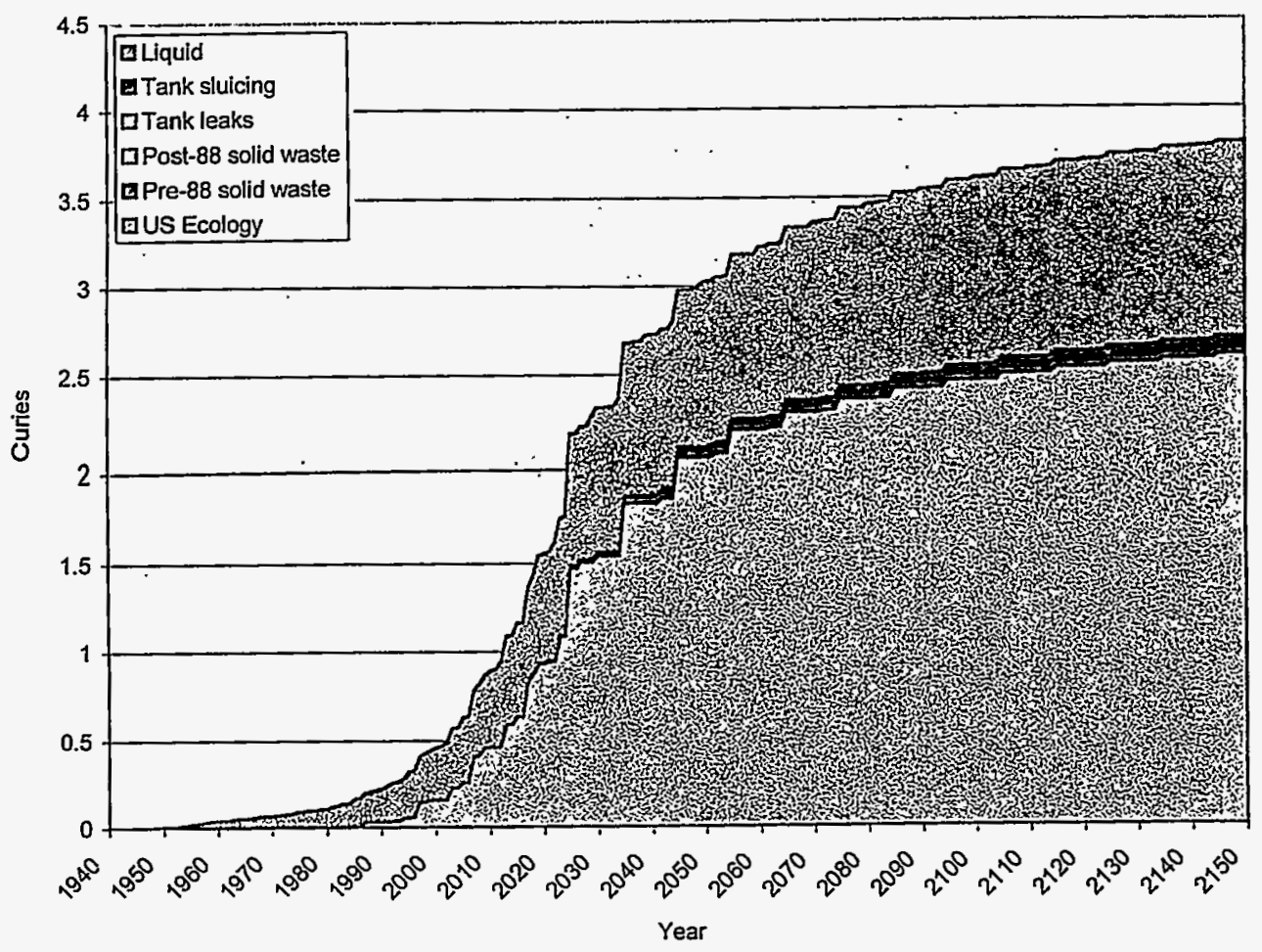

Figure 4.7a. Cumulative Release of Carbon-14 from All Sources to the Water Table from 1940 to 2150

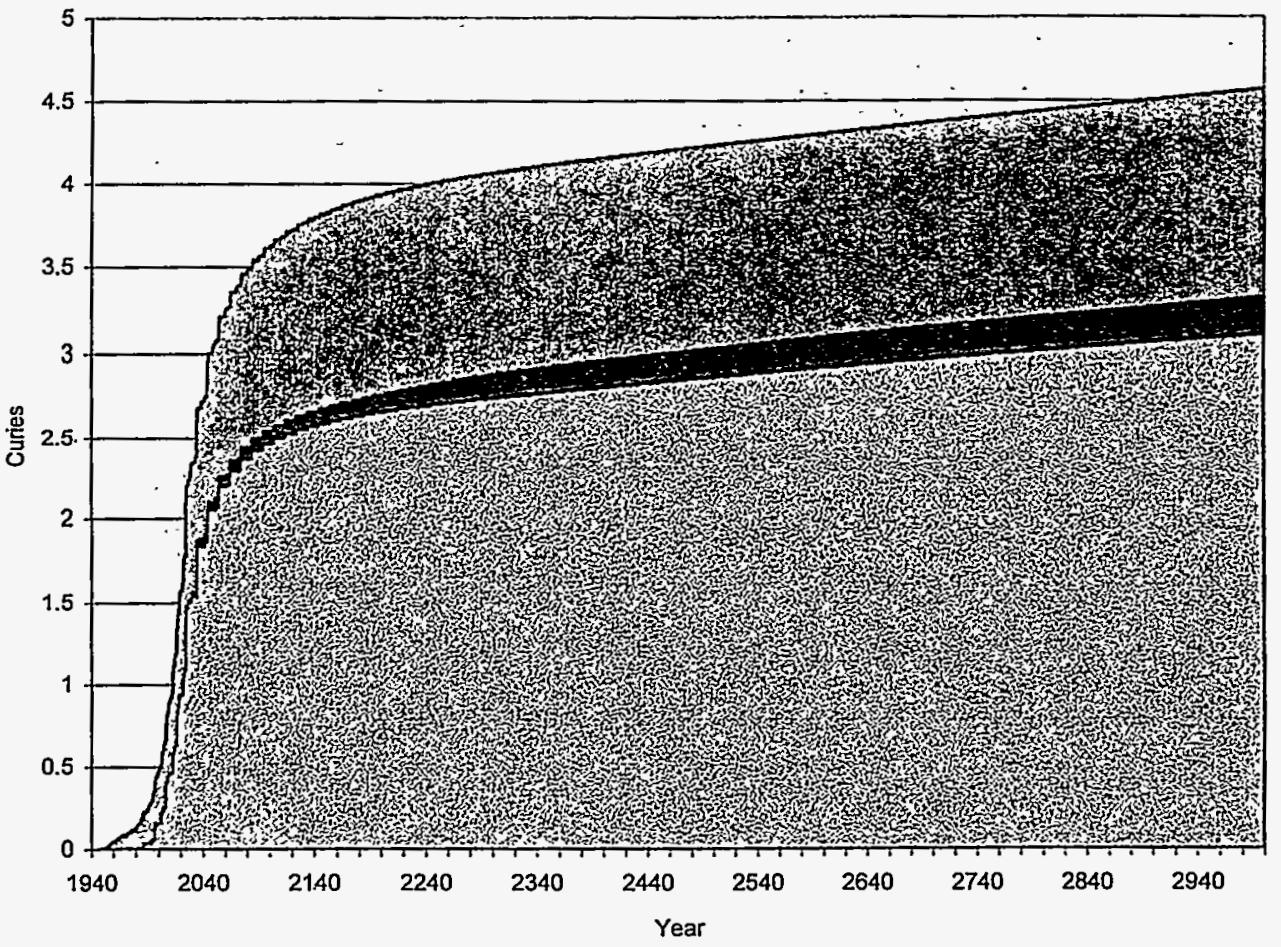

Figure 4.7b. Cumulative Release of Carbon-14 from All Sources to the Water Table from 1940 to 3000 


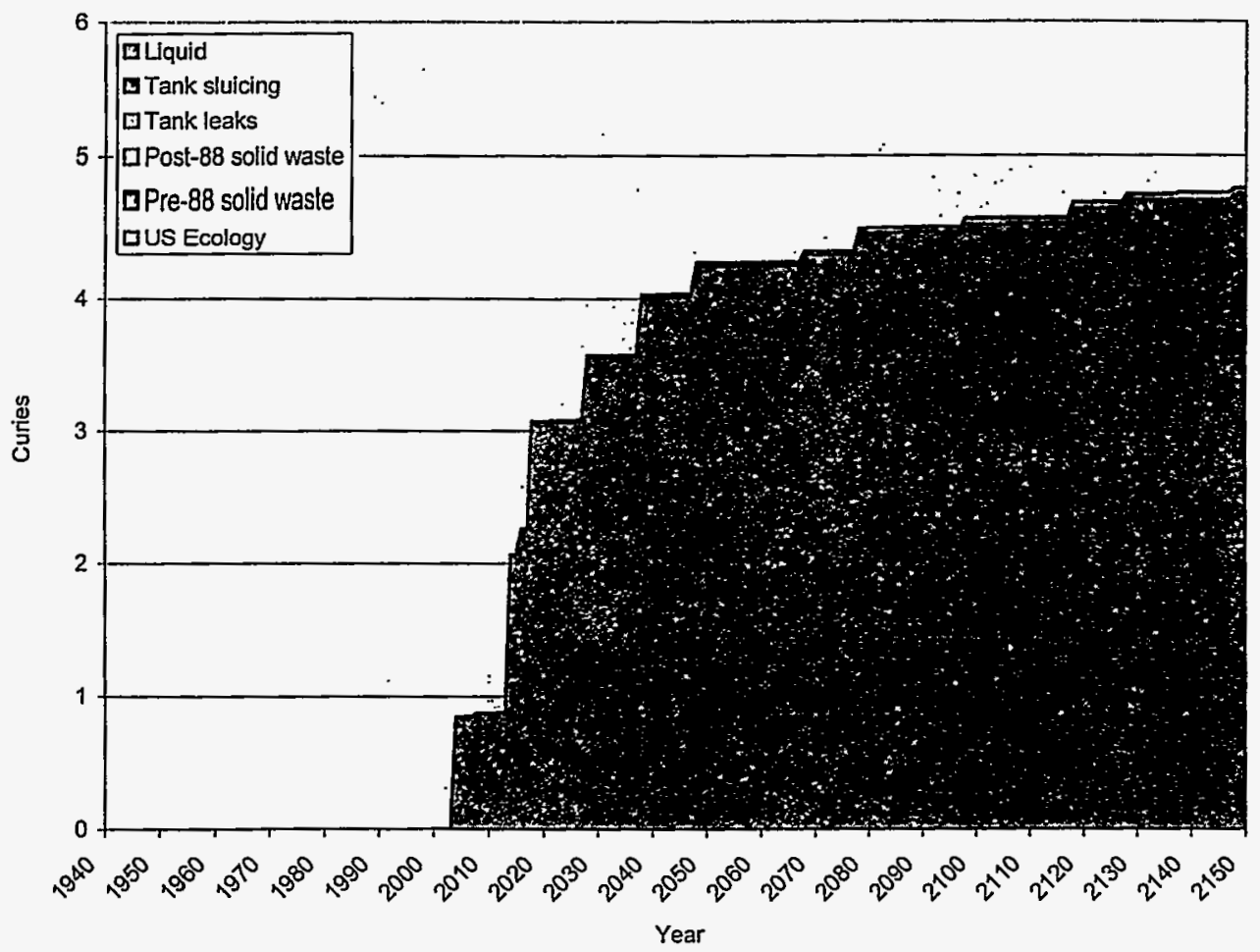

Figure 4.8a. Cumulative Release of Chlorine-36 from All Sources to the Water Table from 1940 to 2150

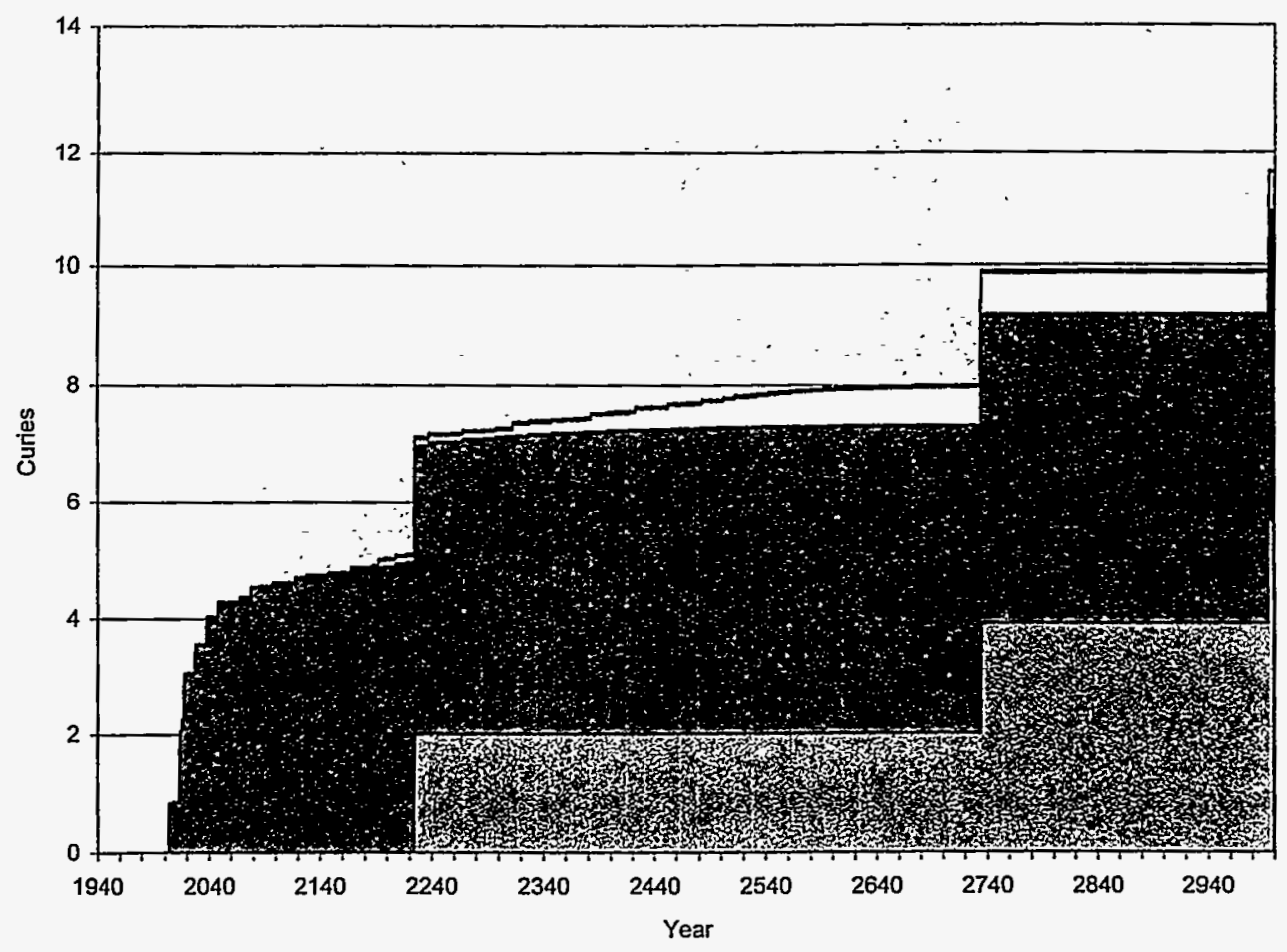

Figure 4.8b. Cumulative Release of Chlorine-36 from All Sources to the Water Table from 1940 to 3000 


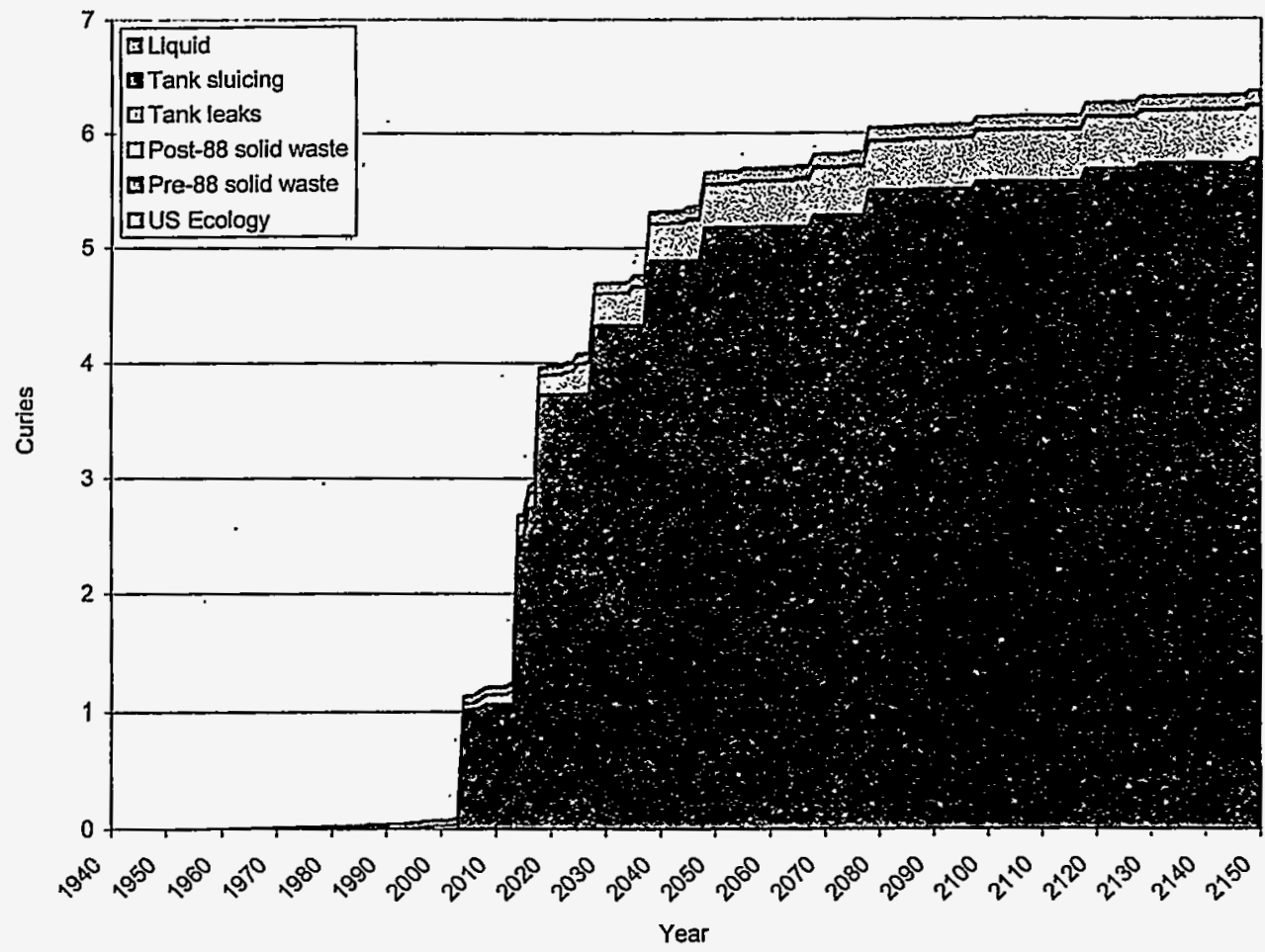

Figure 4.9a. Cumulative Release of Selenium-79 from All Sources to the Water Table from 1940 to 2150

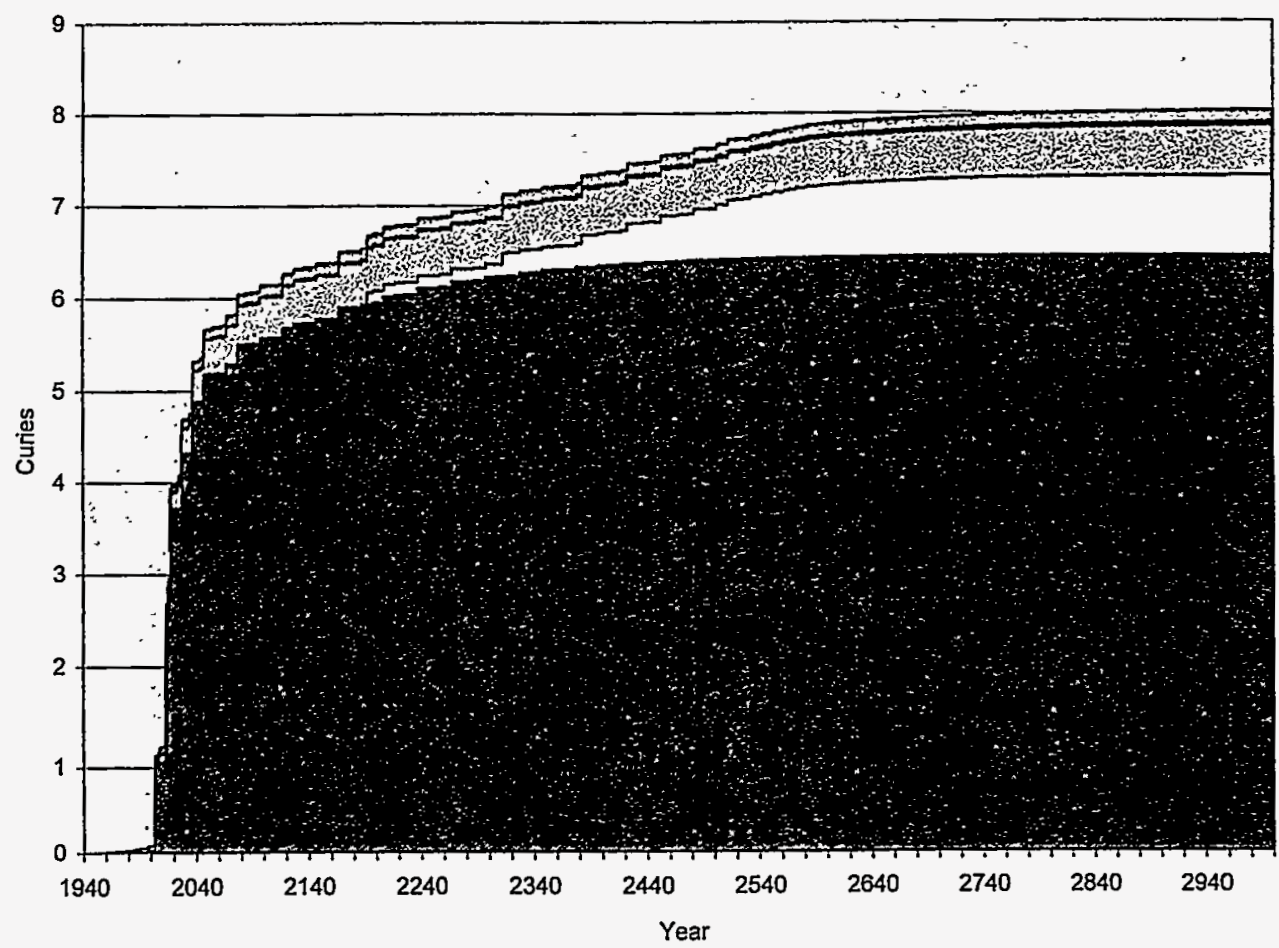

Figure 4.9b. Cumulative Release of Selenium-79 from All Sources to the Water Table from 1940 to 3000 


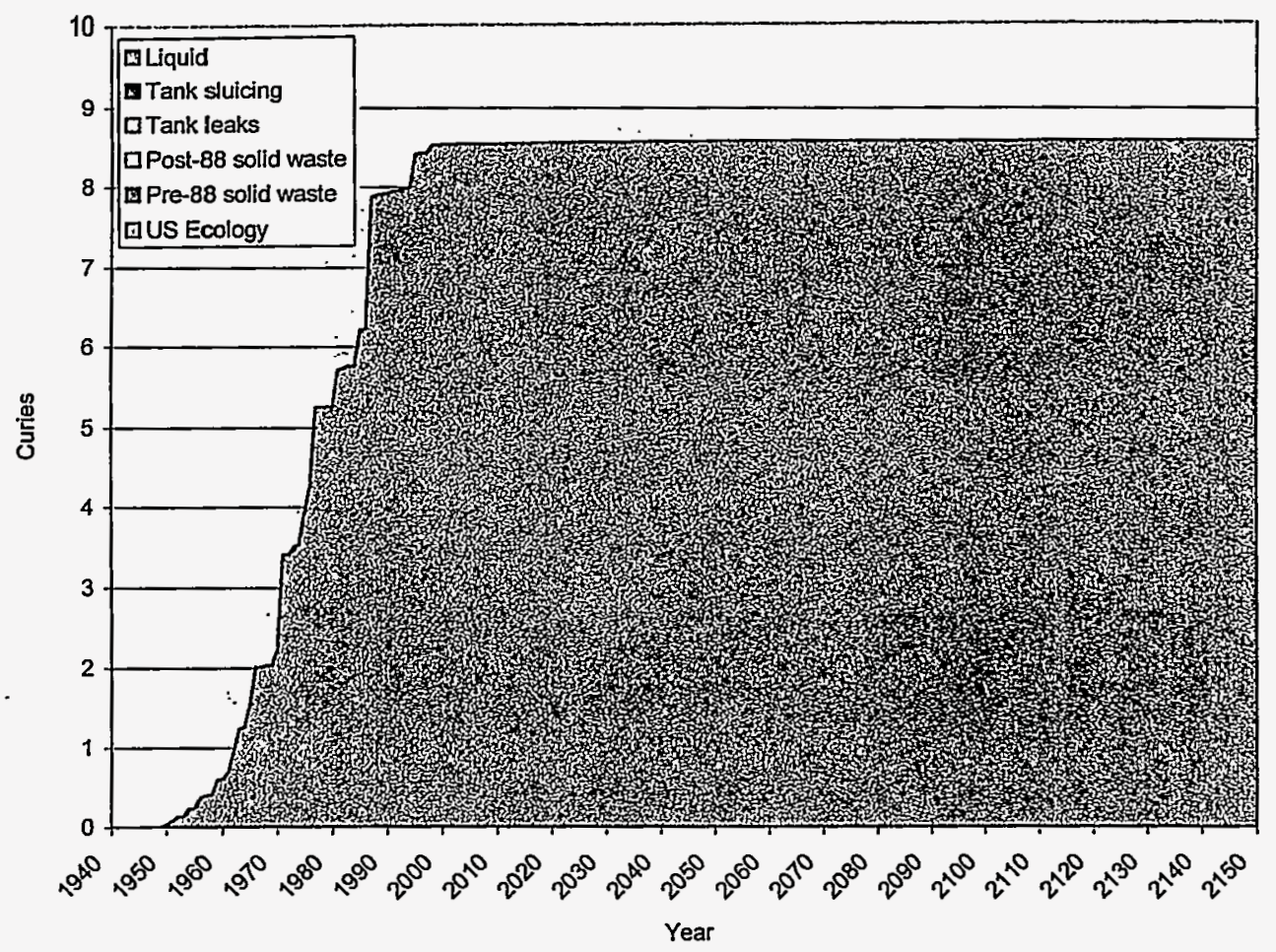

Figure 4.10a. Cumulative Release of Uranium-238 from All Sources to the Water Table from 1940 to 2150

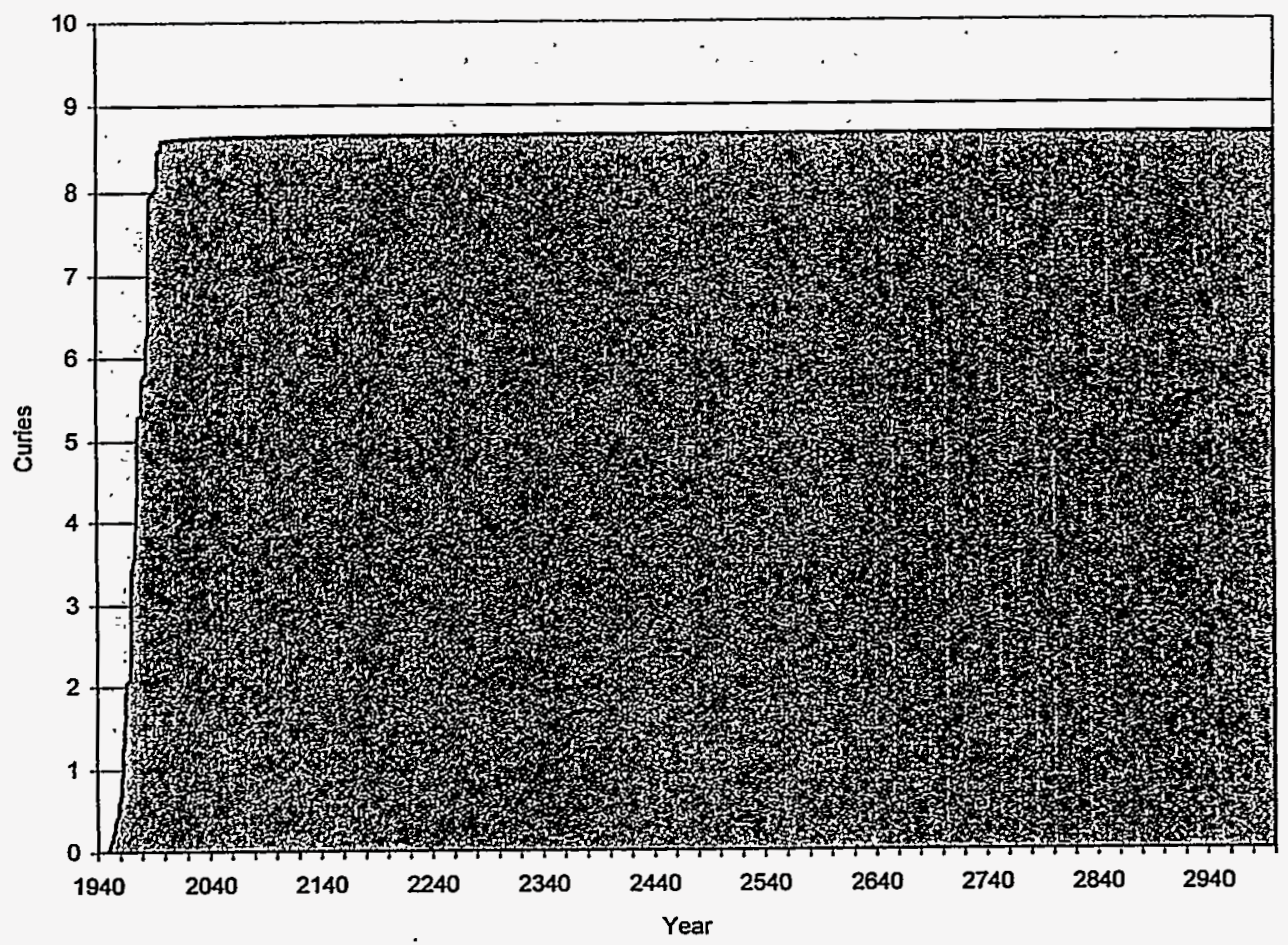

Figure 4.10b. Cumulative Release of Uranium-238 from All Sources to the Water Table from 1940 to 3000 


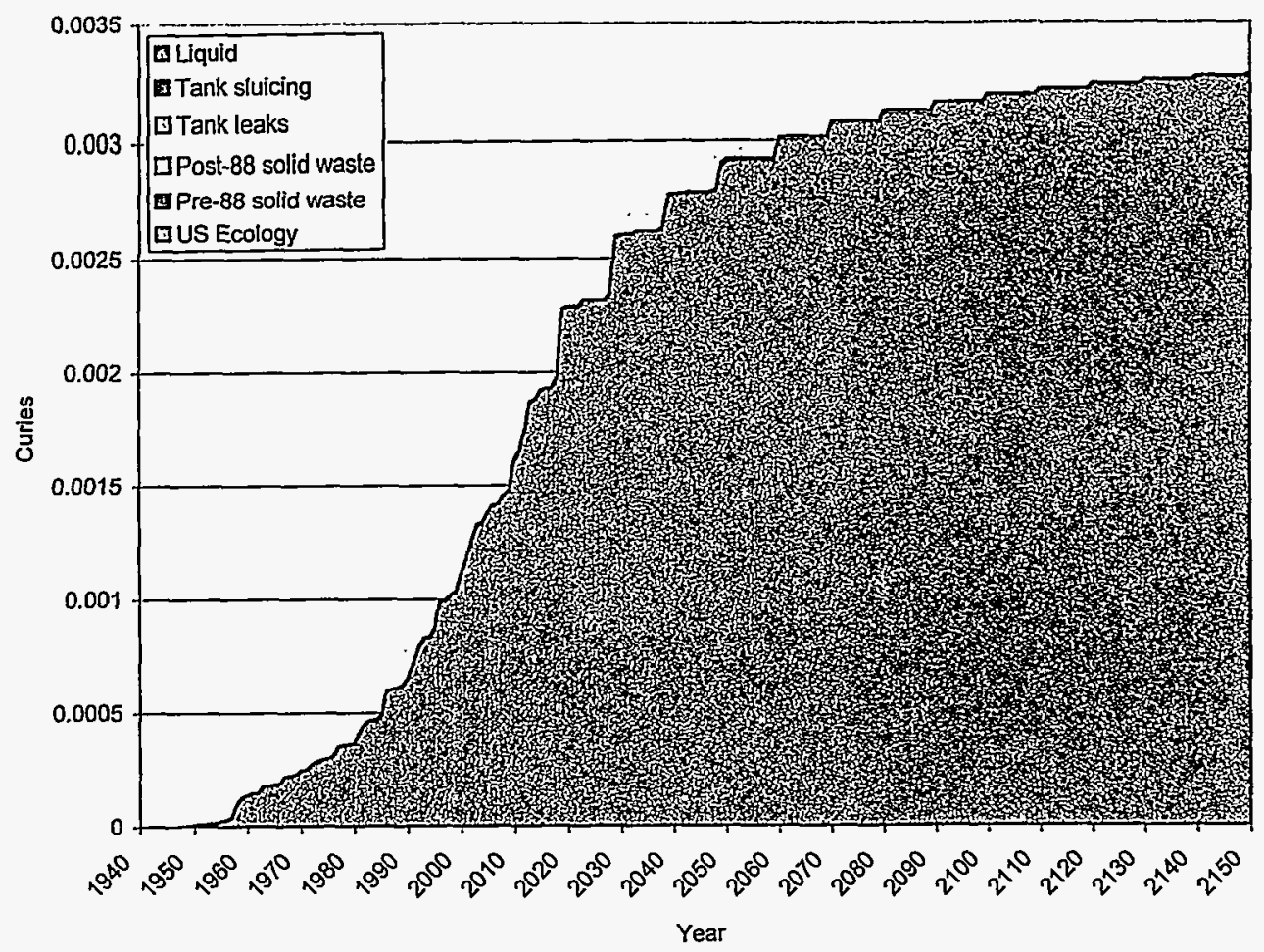

Figure 4.11a. Cumulative Release of Cobalt-60 from All Sources to the Water Table from 1940 to 2150

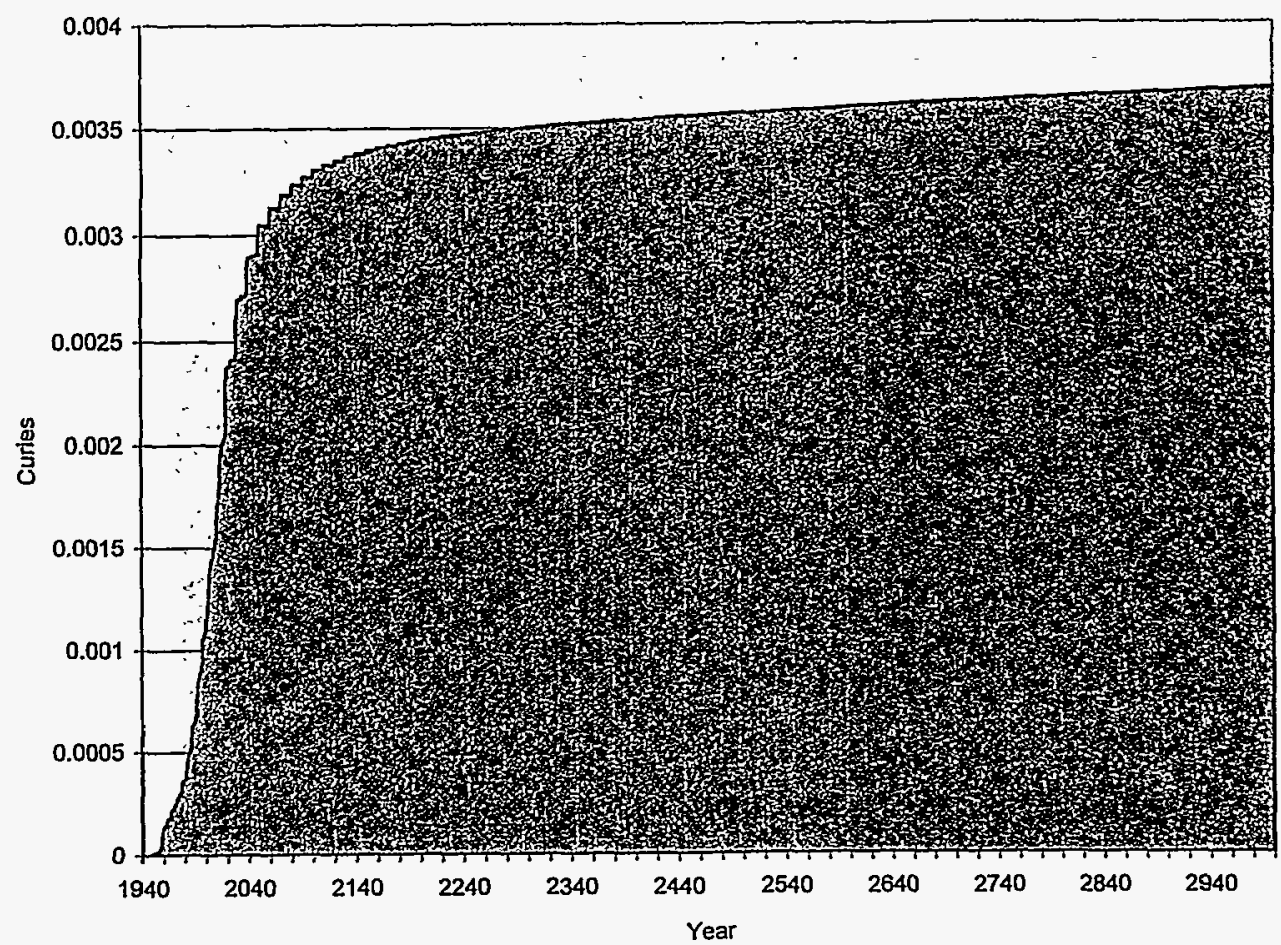

Figure 4.11b. Cumulative Release of Cobalt-60 from All Sources to the Water Table from 1940 to 3000 


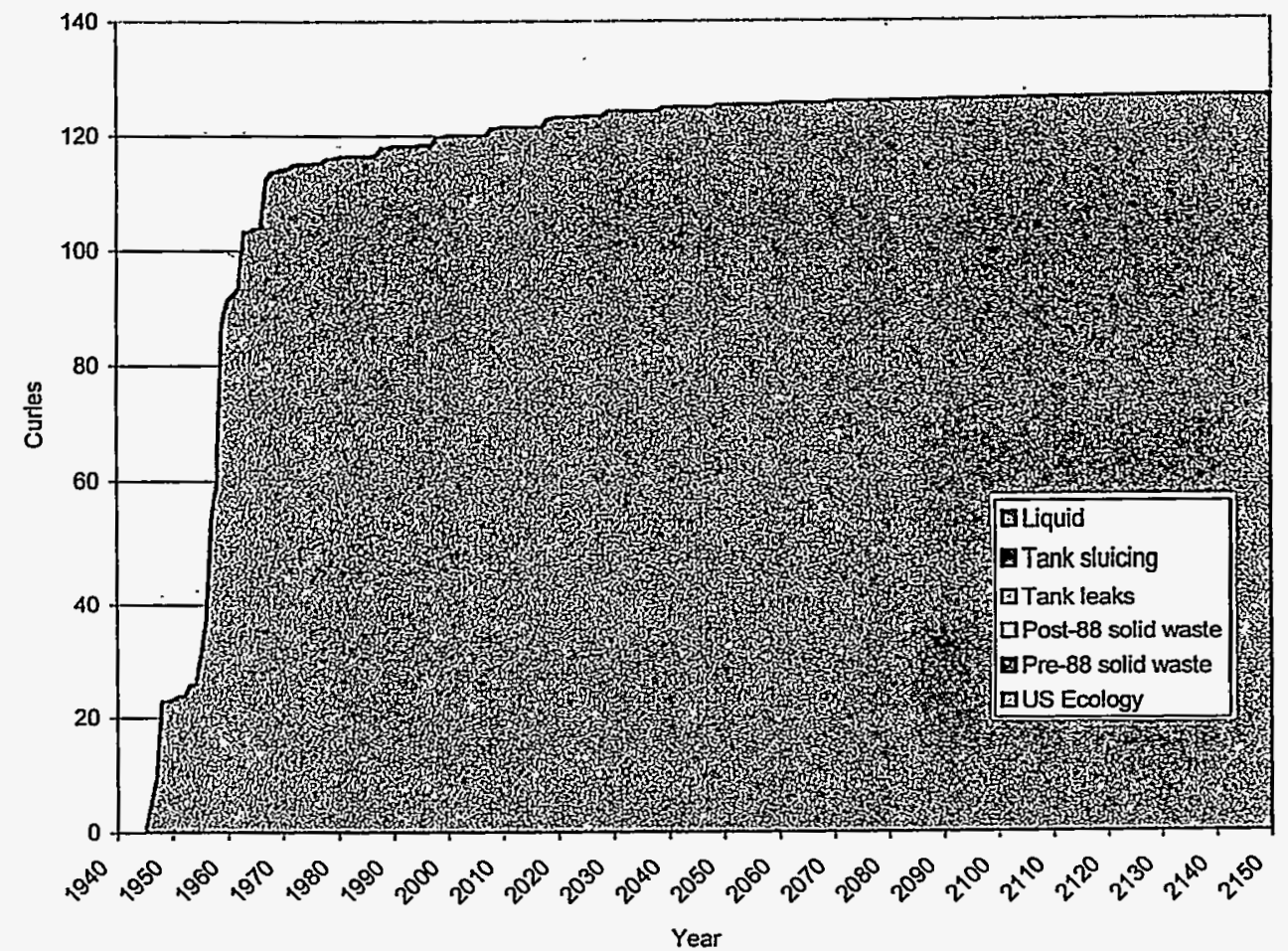

Figure 4.12a. Cumulative Release of Americium-241 from All Sources to the Water Table from 1940 to 2150

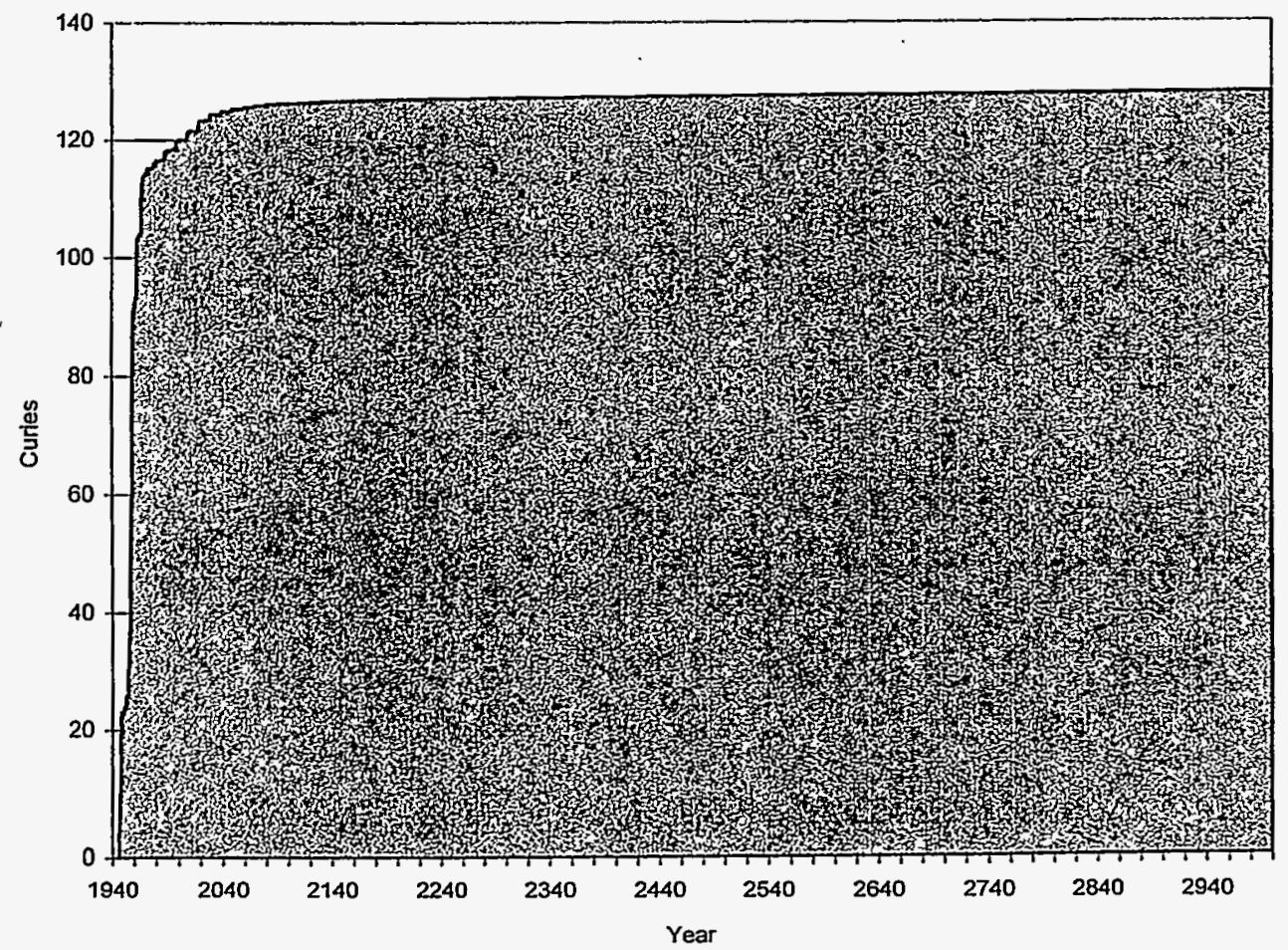

Figure 4.12b. Cumulative Release of Americium-241 from All Sources to the Water Table from 1940 to 3000 


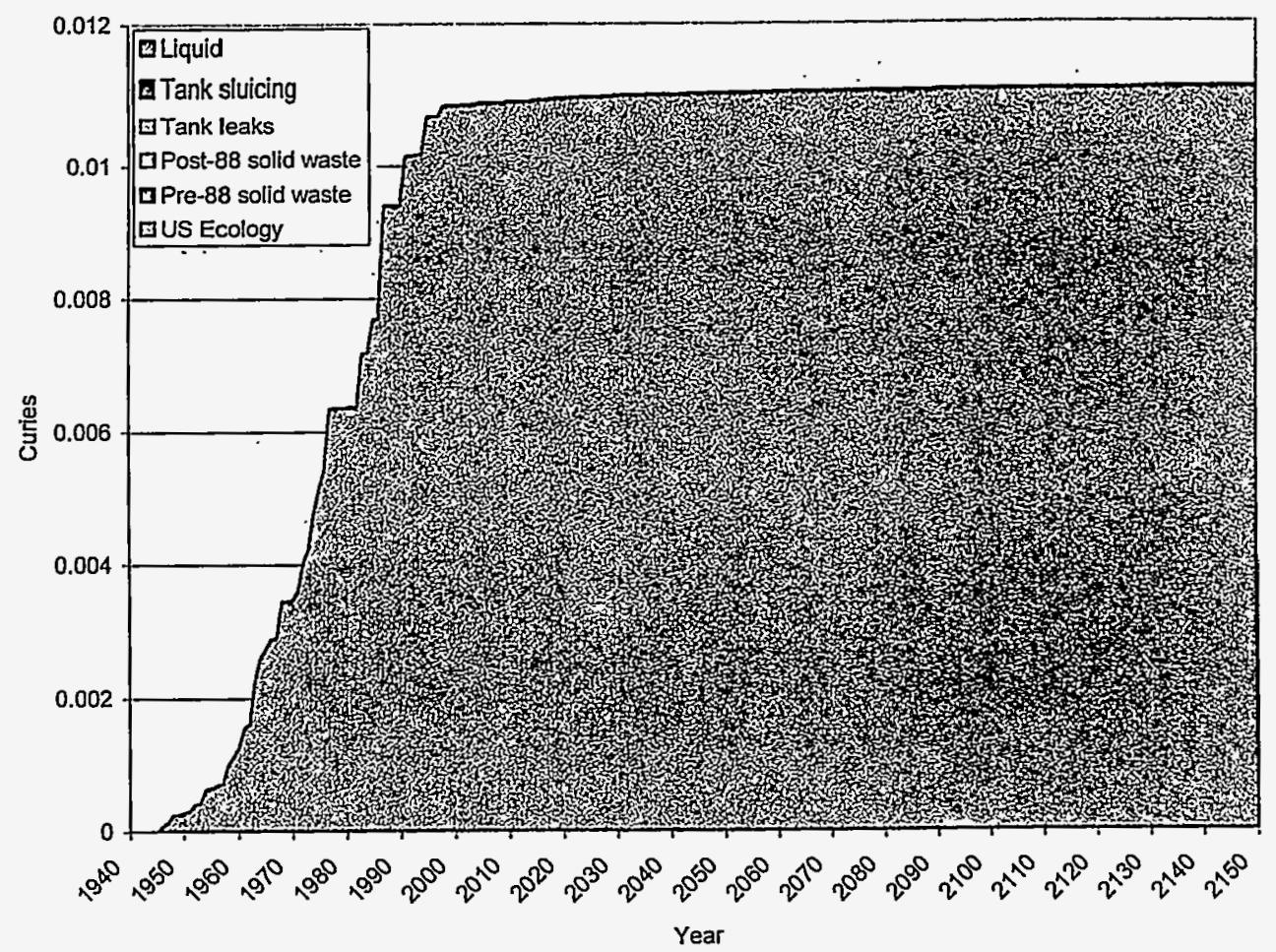

Figure 4.13a. Cumulative Release of Neptunium-237 from All Sources to the Water Table from 1940 to 2150

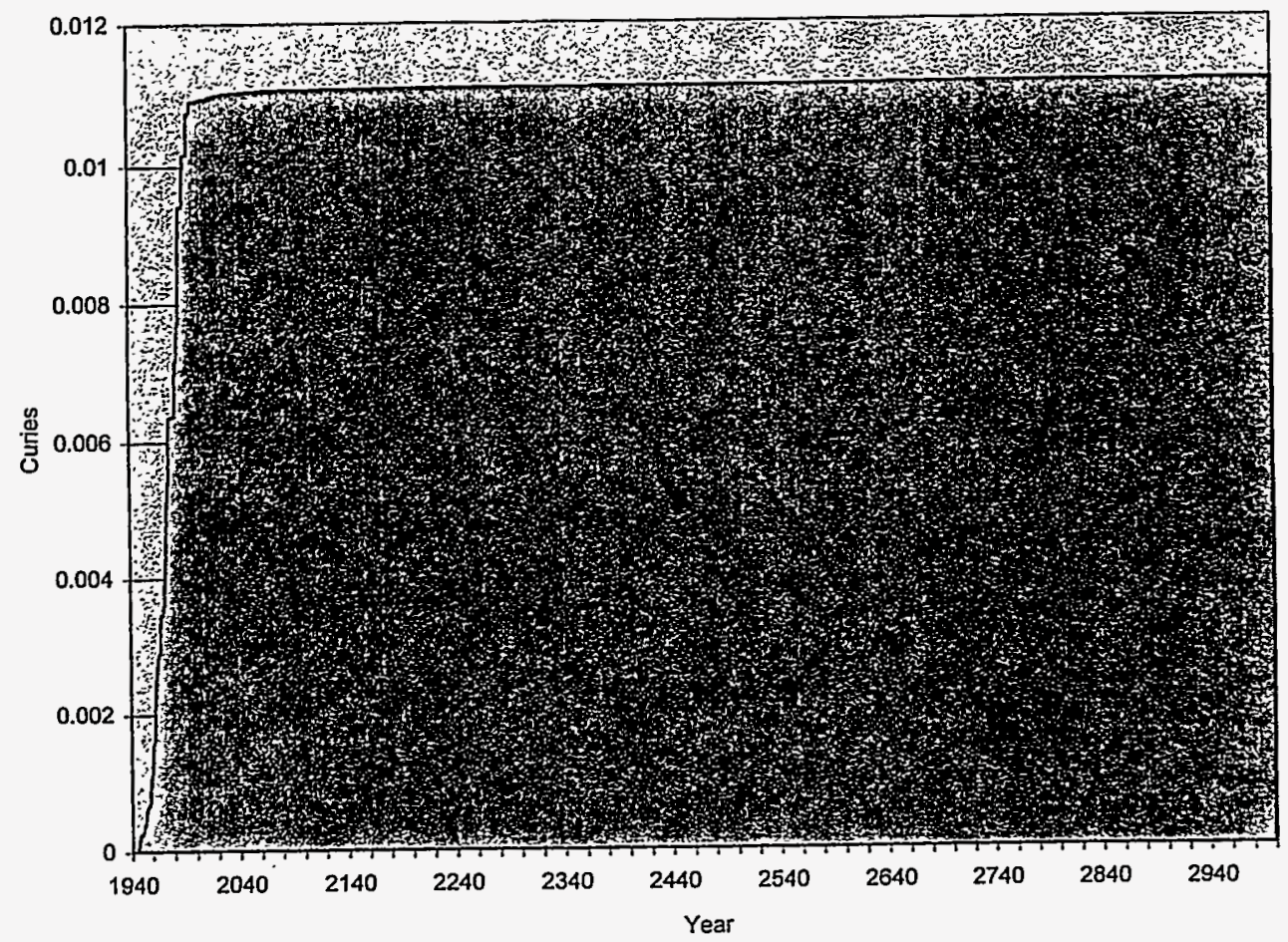

Figure 4.13b. Cumulative Release of Neptunium-237 from All Sources to the Water Table from 1940 to 3000 


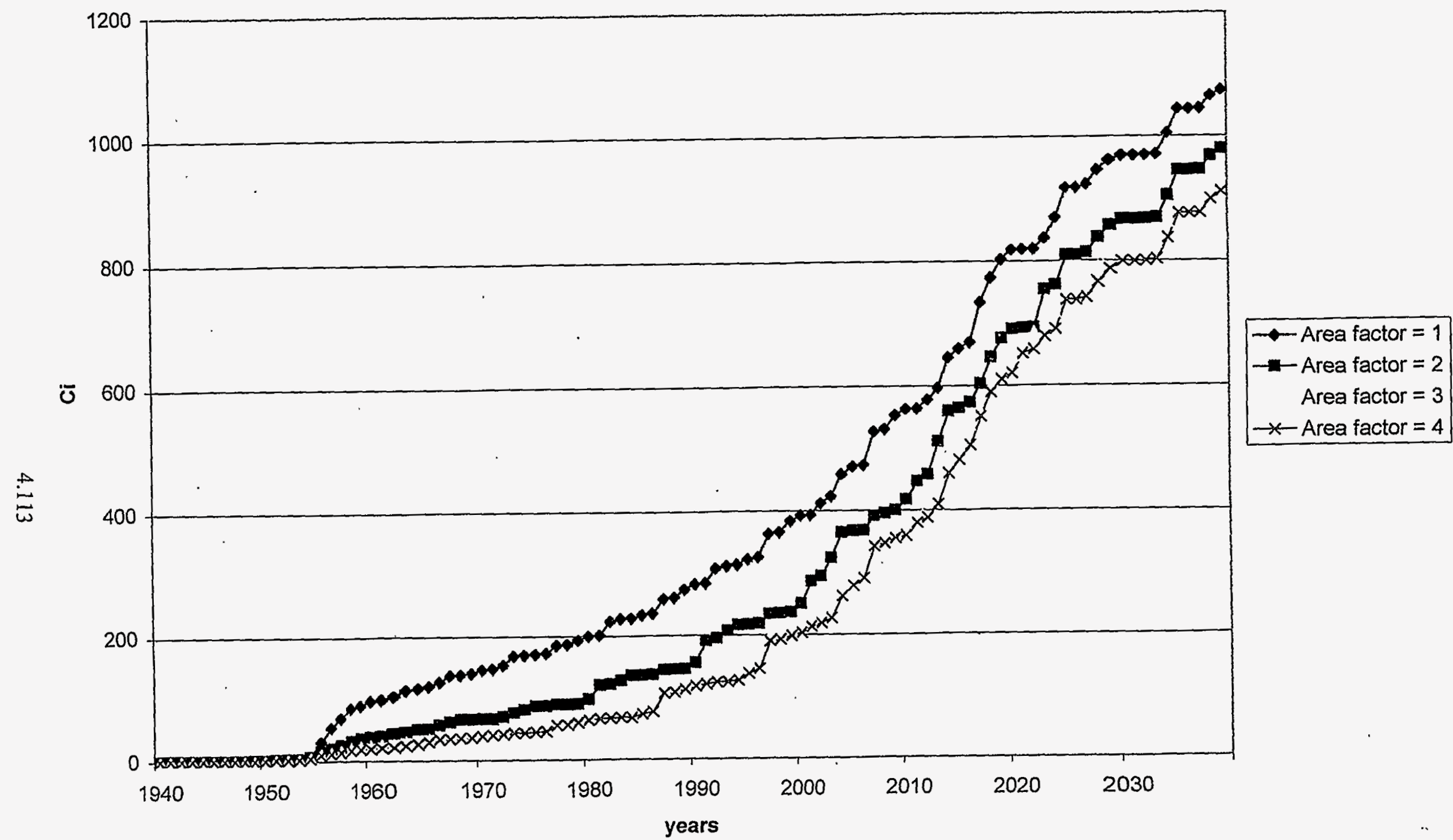

Figure 4.14a. Cumulative Release of Technetium-99 to Groundwater from Liquid Discharge Sources for Different Cross Section Area Factors from 1940 to 2040 


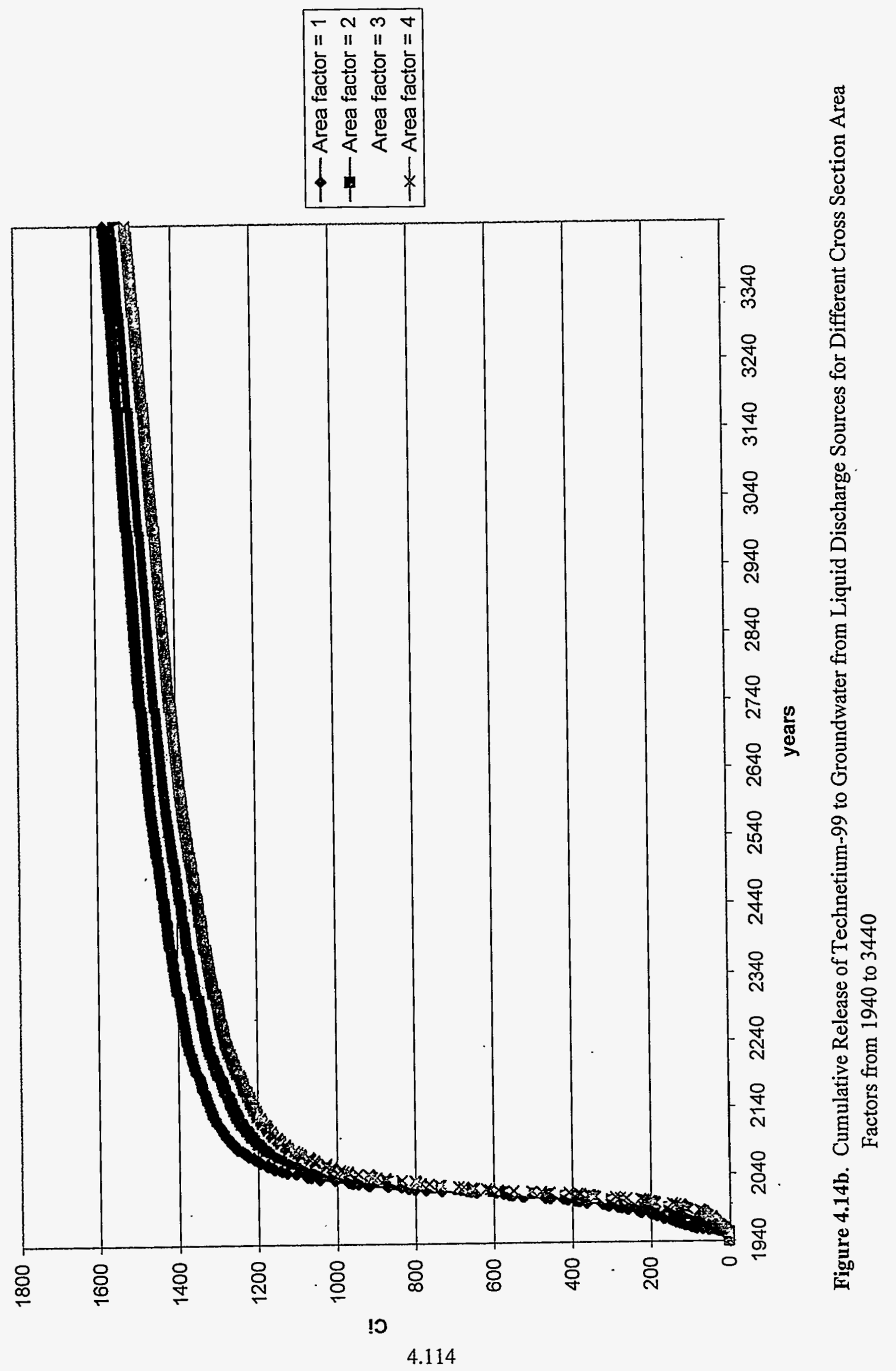




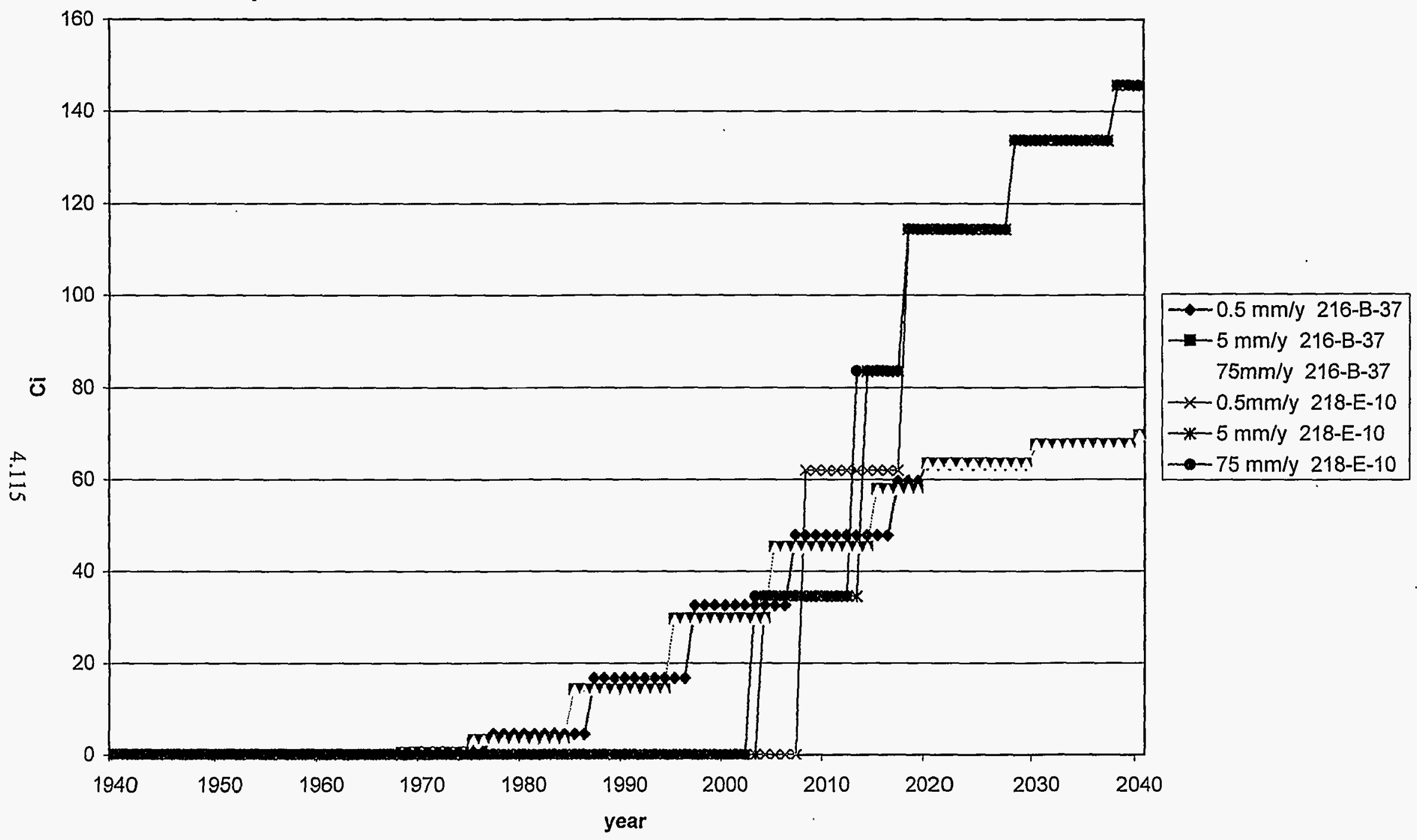

Figure 4.15. Cumulative Release of Technetium-99 from Liquid and Solid Waste Sites for Different Initial Soil Moisture Conditions 


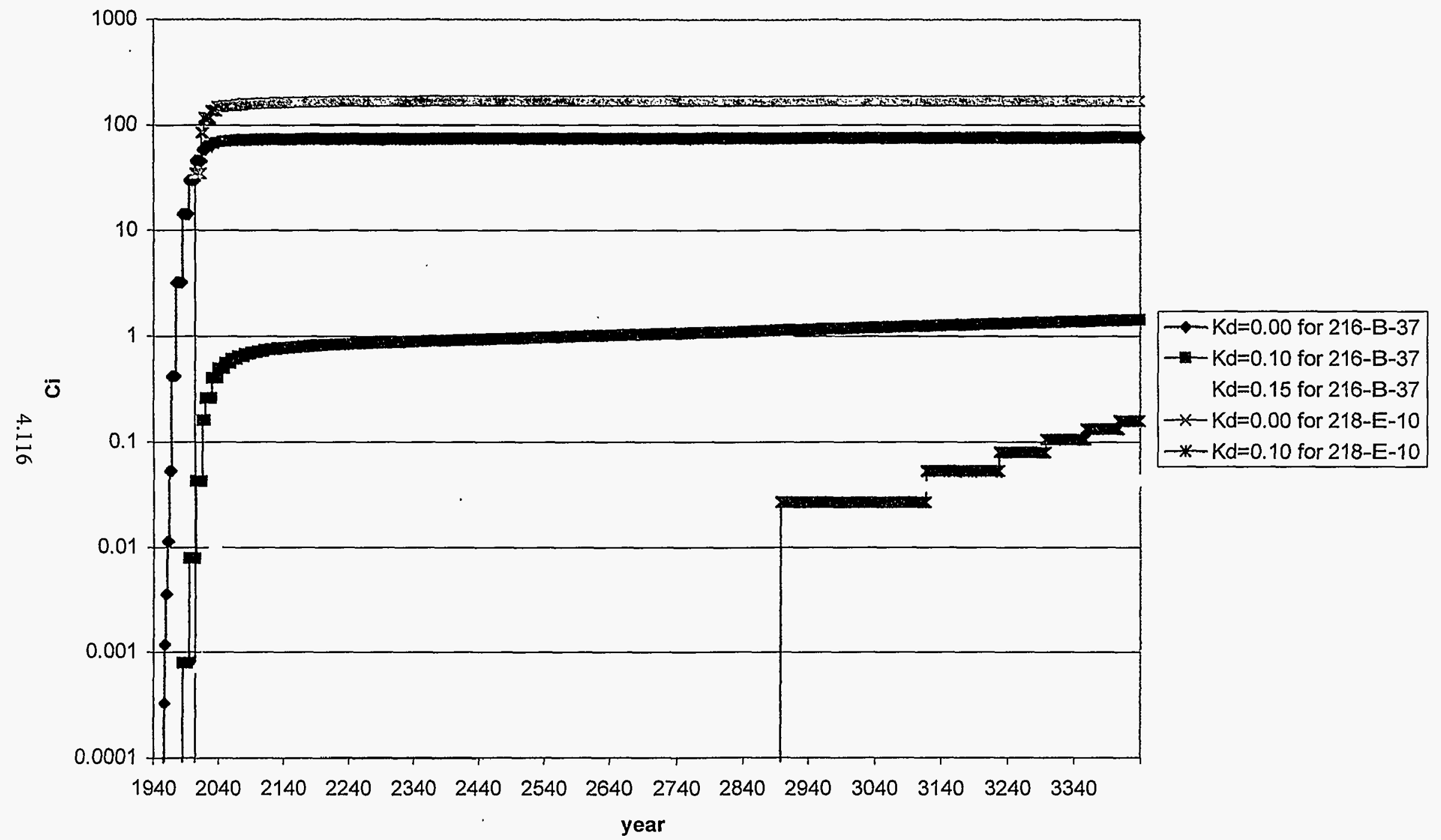

Figure 4.16. Cumulative Release of Technetium-99 from Liquid and Solid Waste Sites for Different Distribution Coefficients 


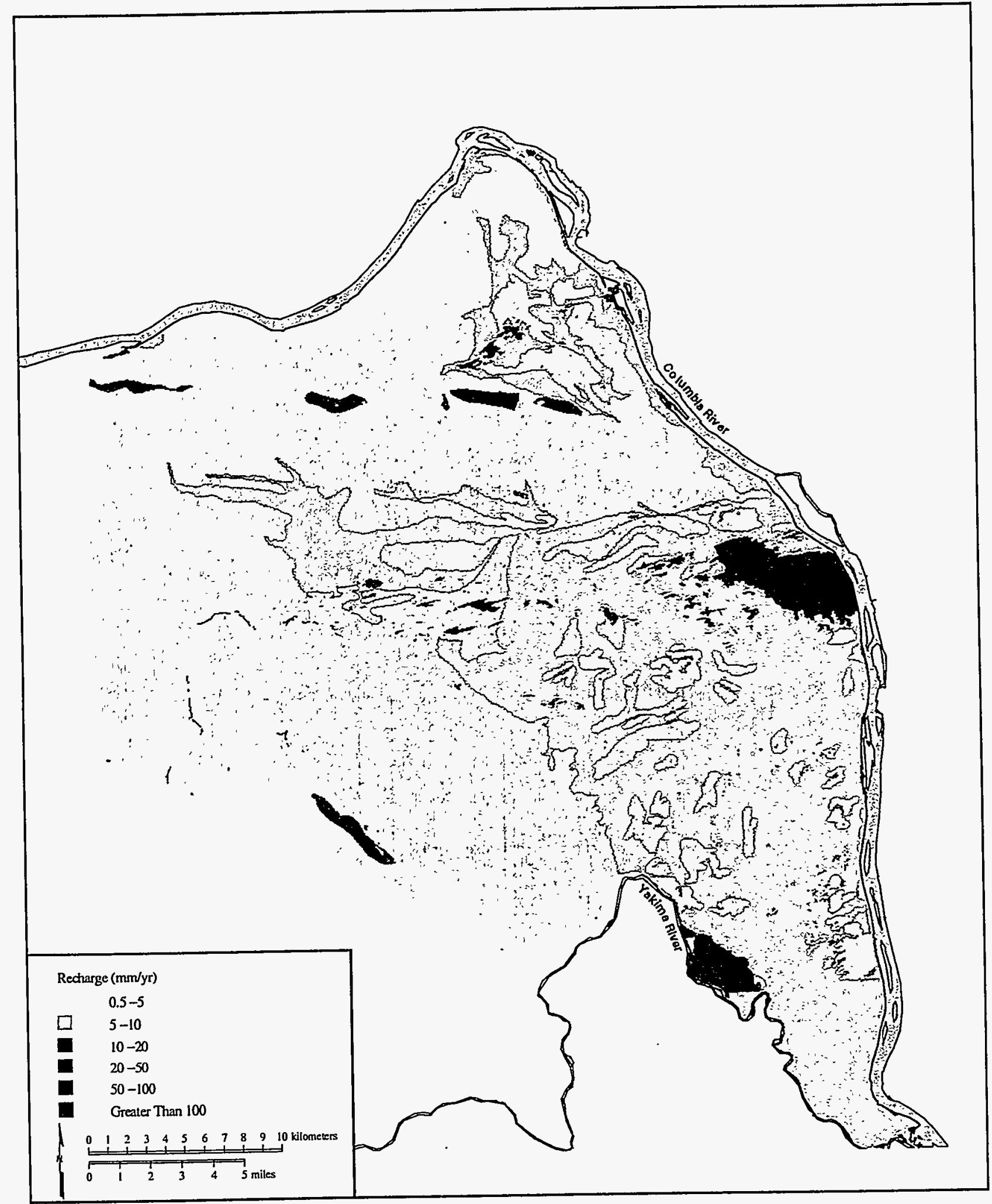

skw9800 l.eps January 02, 1998

Figure 4.17. Sitewide Distribution of Recharge at the Hanford Site Used in the Composite Analysis 


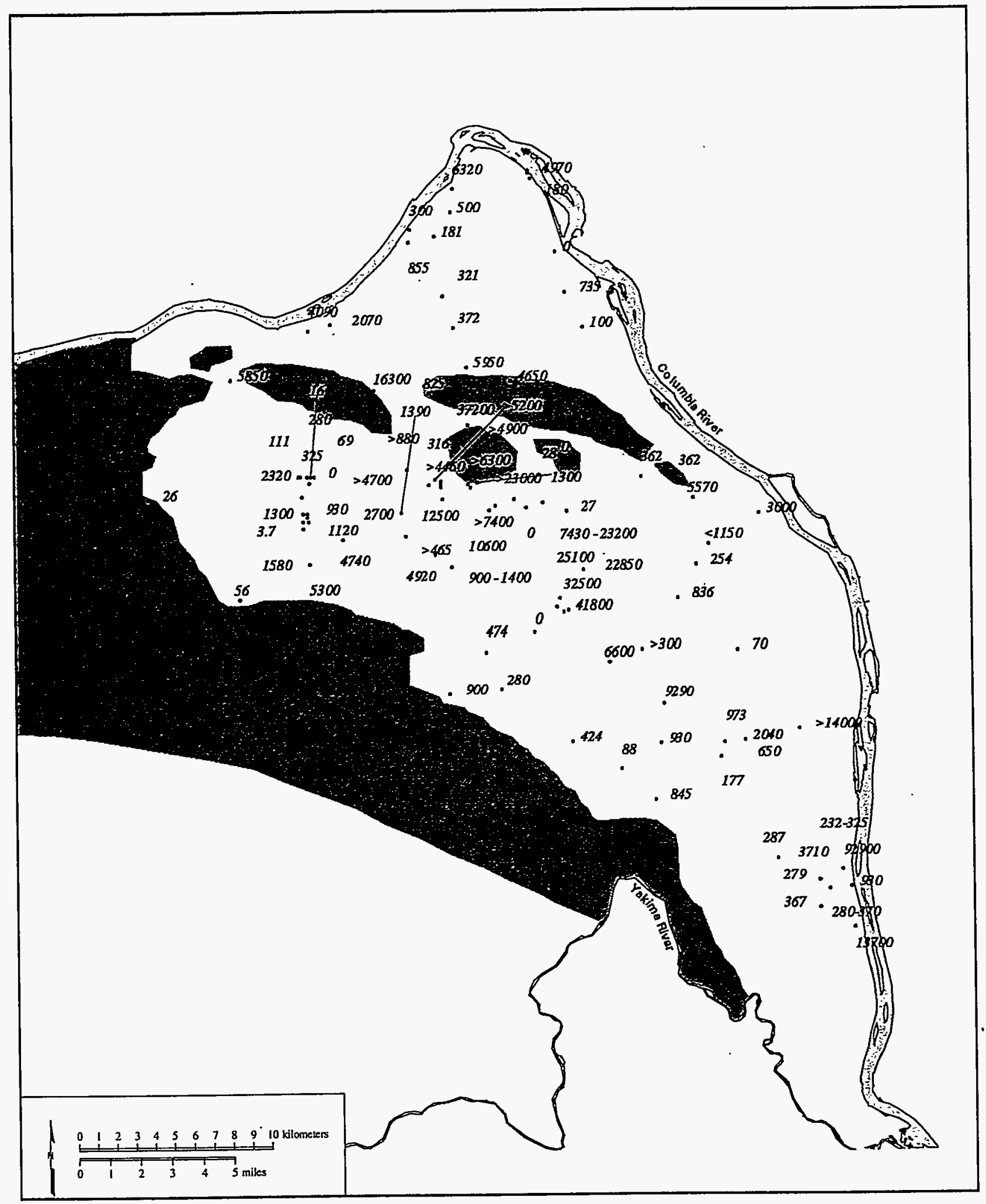

skw98002 eps January 02, 1998

Figure 4.18. Locations of Hanford Site Wells with Aquifer Test Results Used in the Groundwater Model Calibration (Values Shown are the Interpreted Transmissivity in $\mathrm{m}^{2} / \mathrm{d}$ ) 


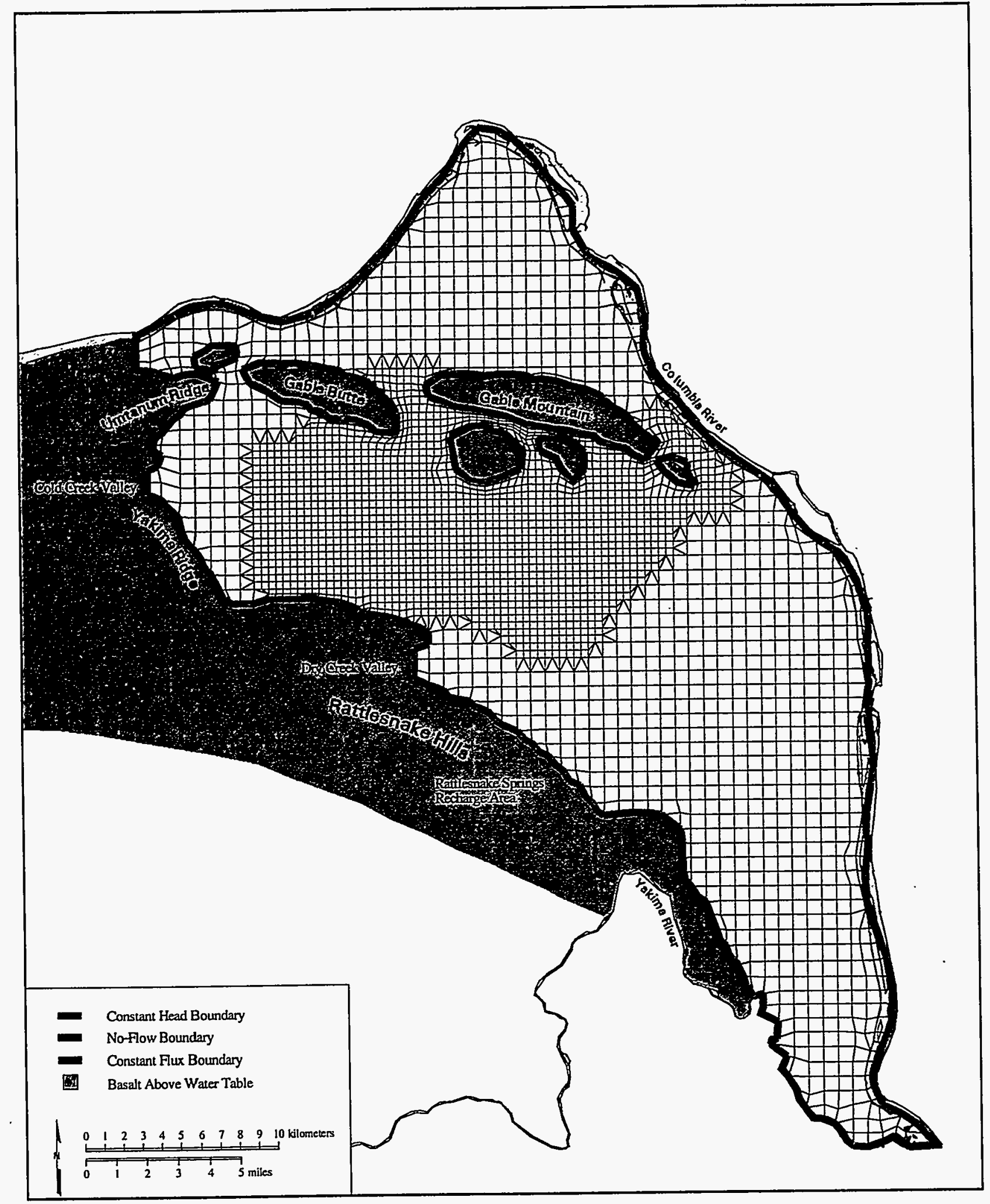

skw98003.eps January 02, 1998

Figure 4.19. Finite Element Grid and Boundary Conditions Used in the Groundwater Model of the Unconfined Aquifer for the Composite Analysis 


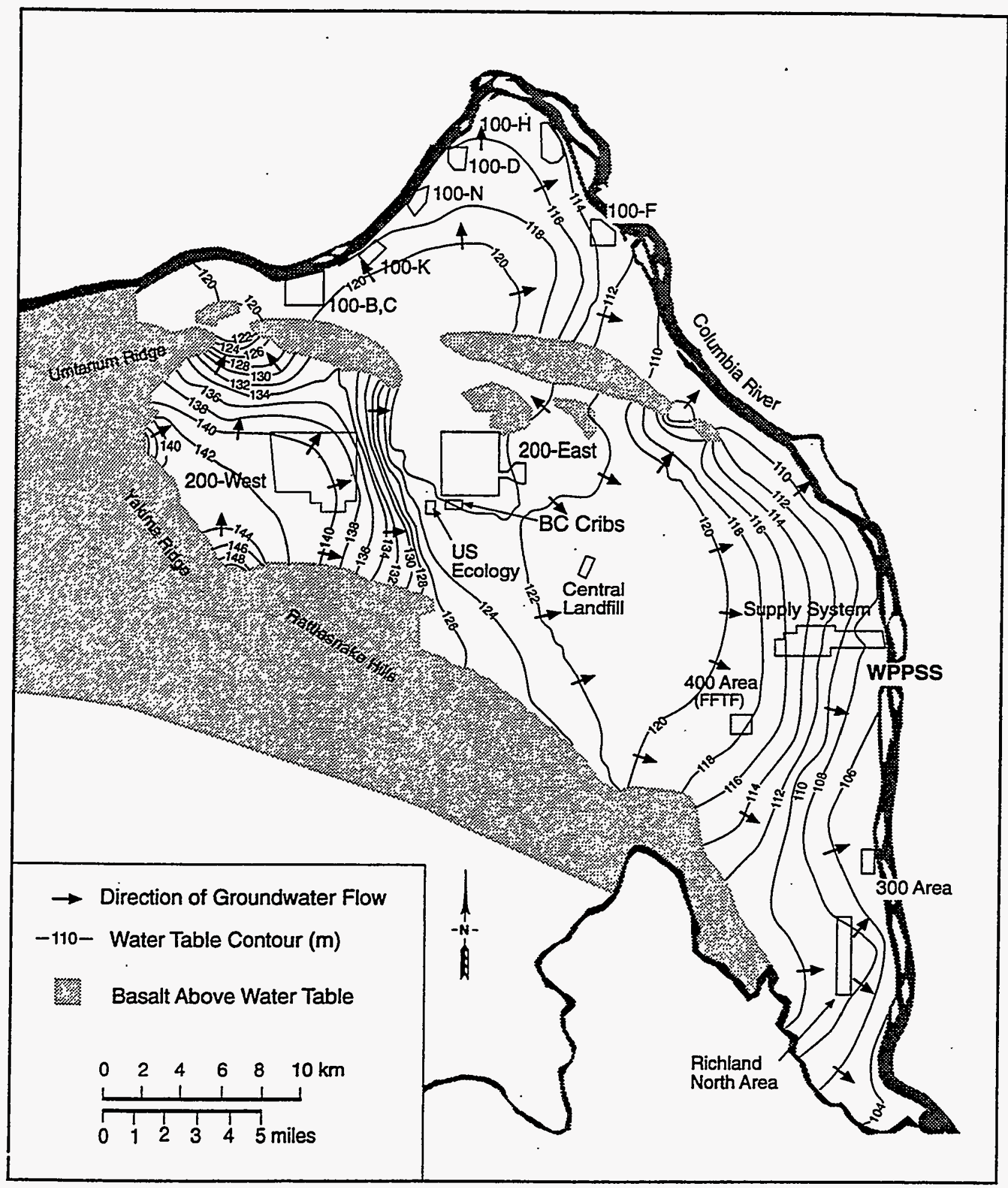

SG97090277.7

Figure 4.20. Water Table Predicted in 2000 with the Three-Dimensional Model 


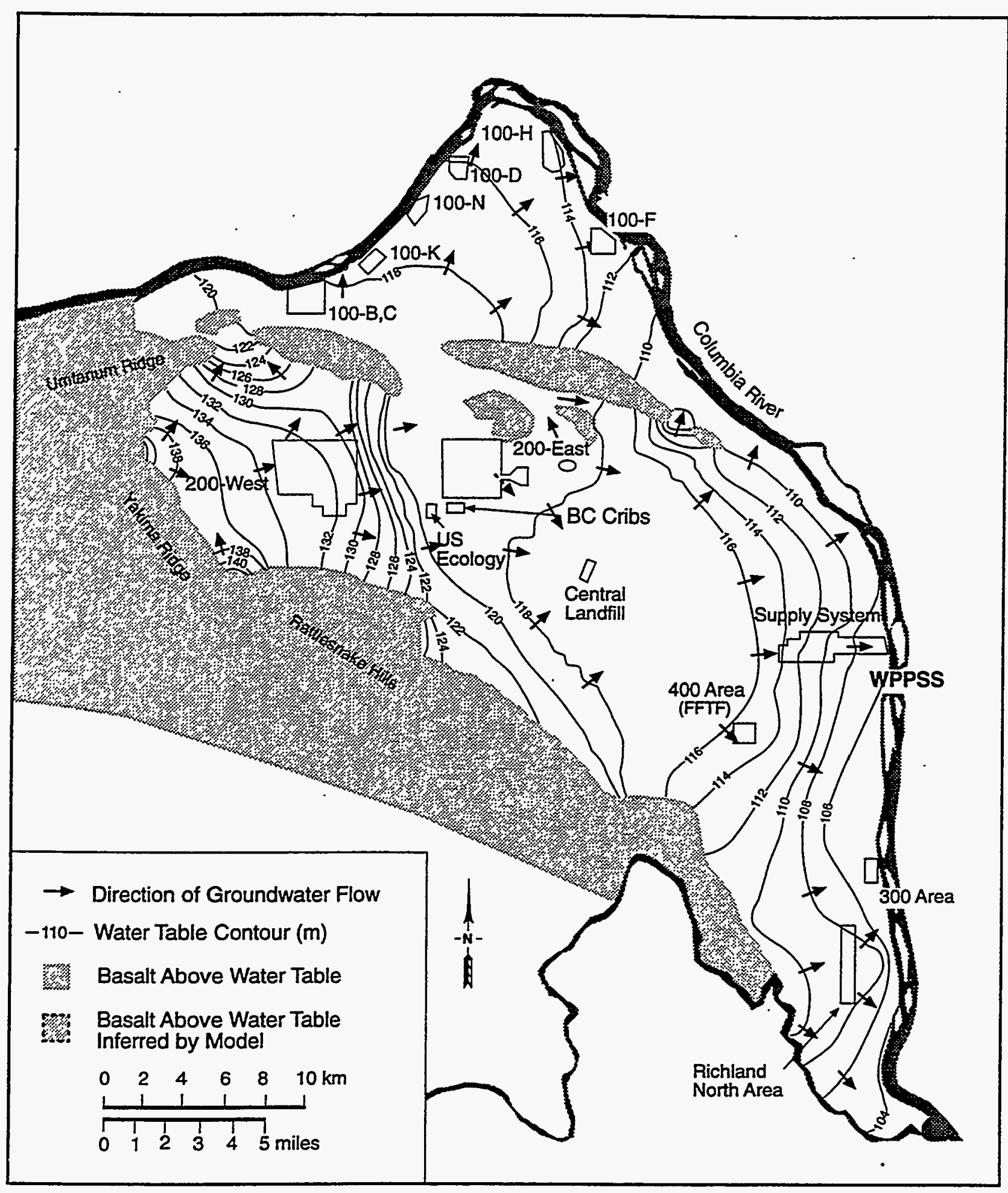

SG97090277.8

Figure 4.21. Water Table Predicted in 2100 with the Three-Dimensional Model 


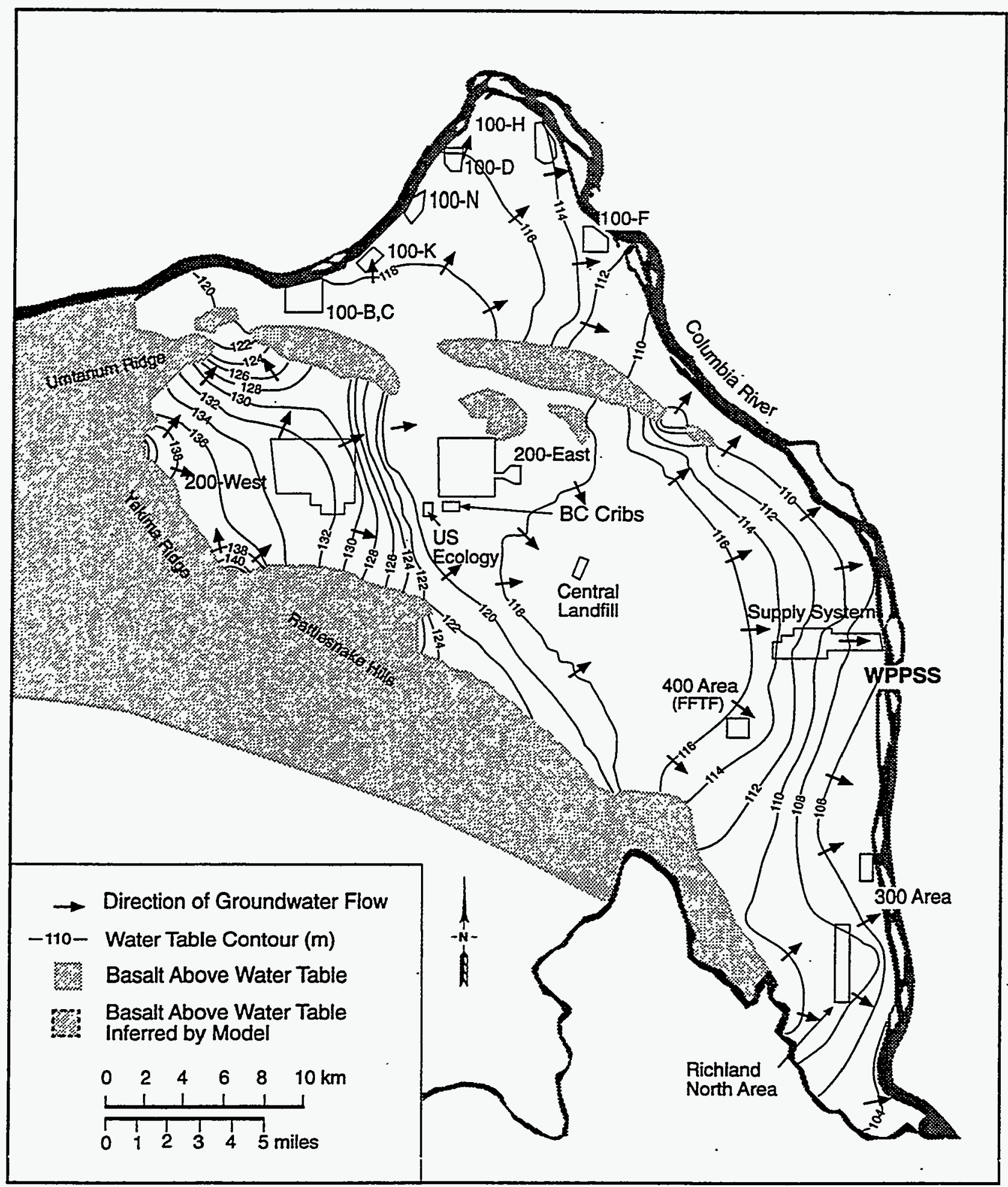

SG97090277.9

Figure 4.22. Water Table Predicted in 2200 with the Three-Dimensional Model 


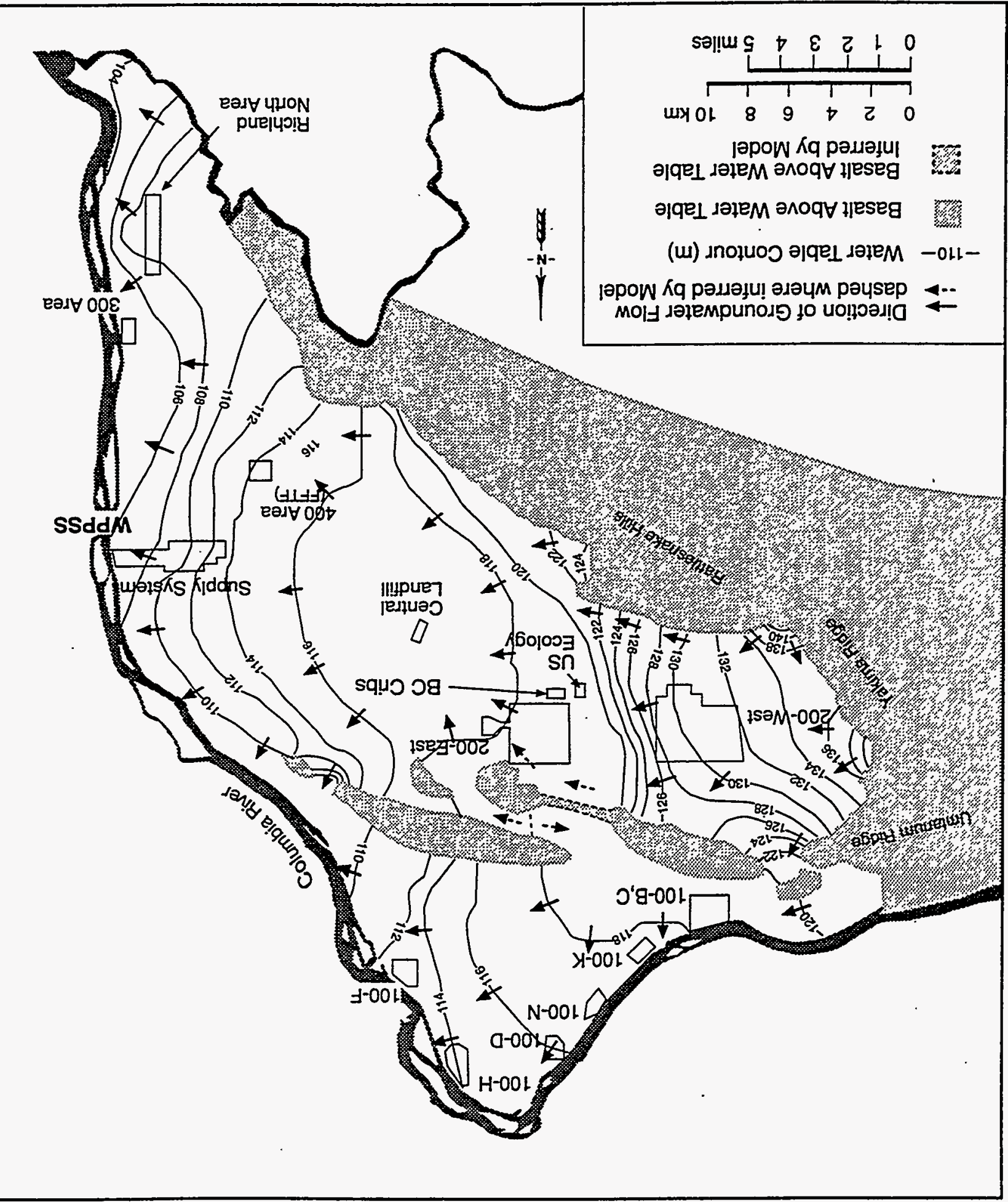




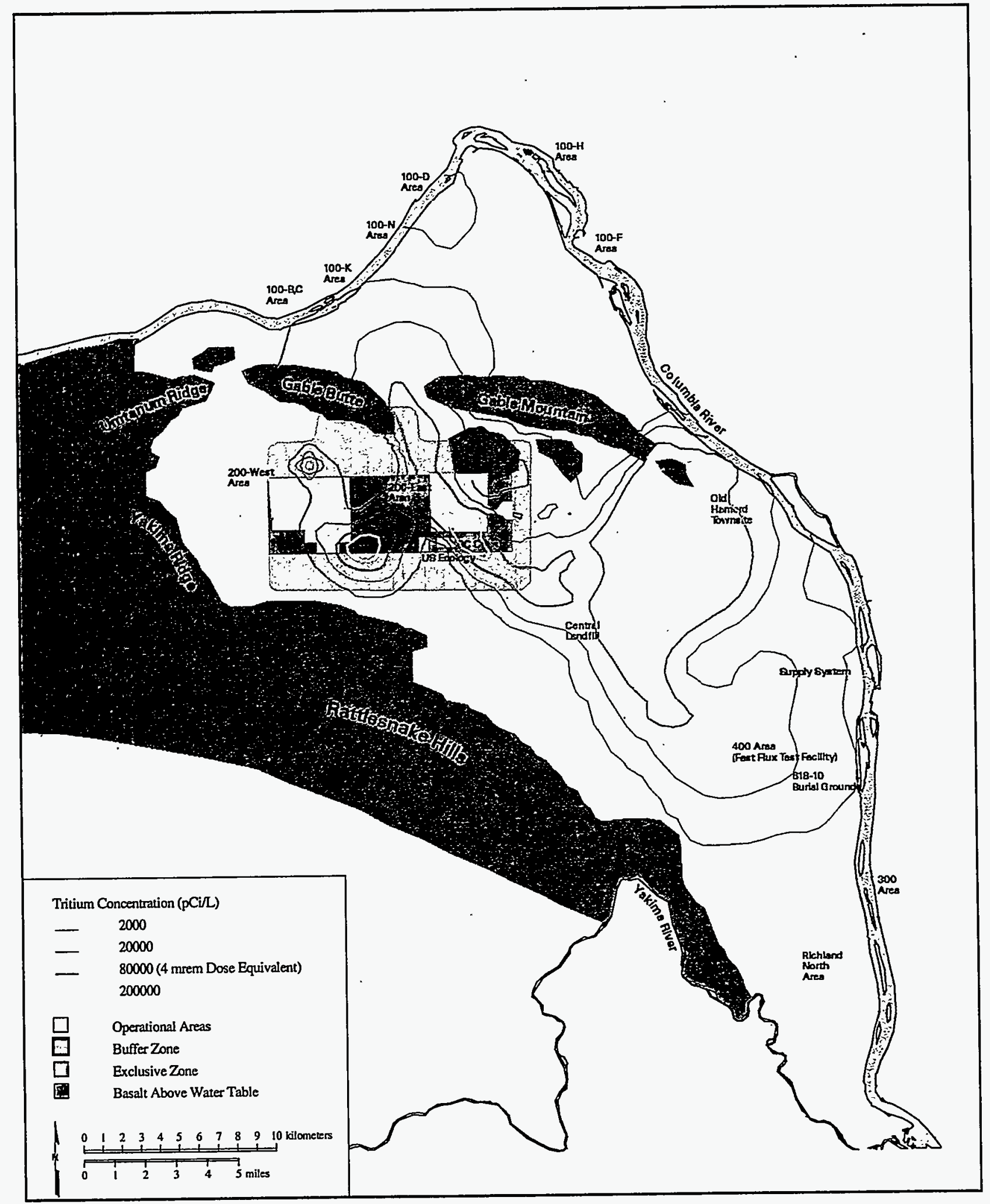

skw98037.eps December 23, 1997

Figure 4.24a. Predicted Distribution of Tritium in the Unconfined Aquifer from All Sources in 1997 (Time of Peak Concentration) 


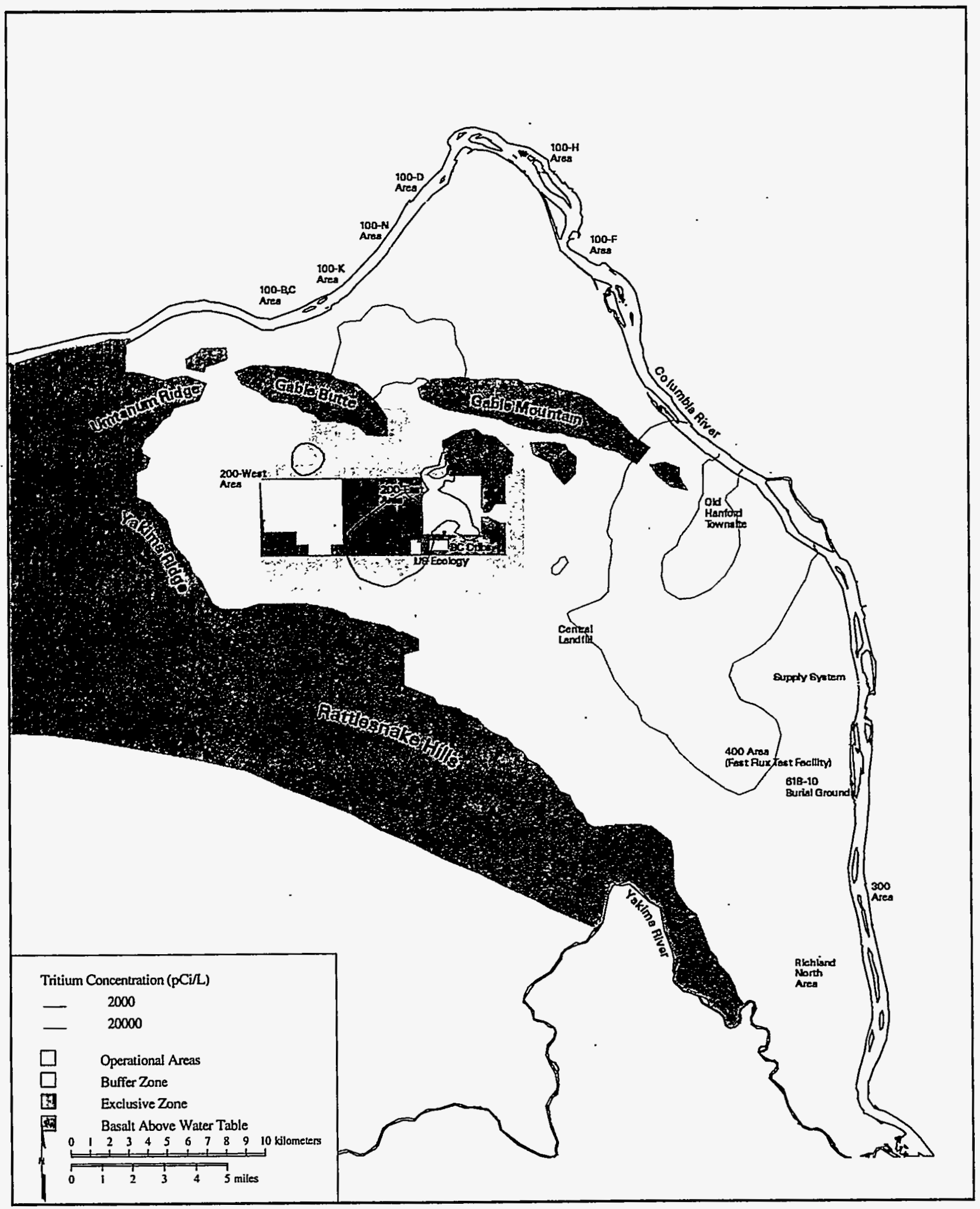

skw98050.eps December 23. 1997

Figure 4.24b. Predicted Distribution of Tritium in the Unconfined Aquifer from All Sources in 2050 


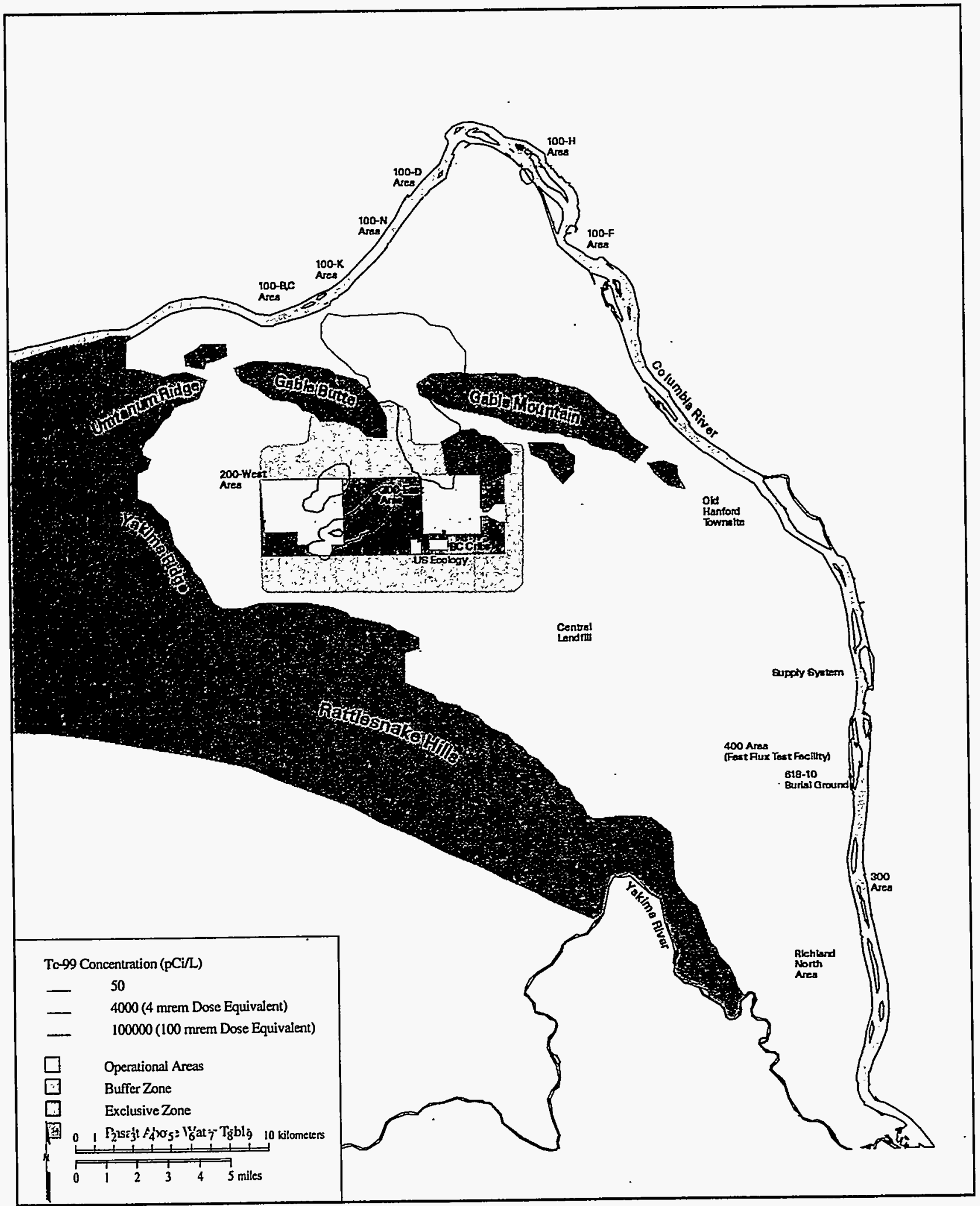

skw98042eps Docember 23. 1997

Figure 4.25a. Distribution of Technetium-99 in the Unconfined Aquifer from Existing Plumes in 1996 (Time of Peak Concentration) 


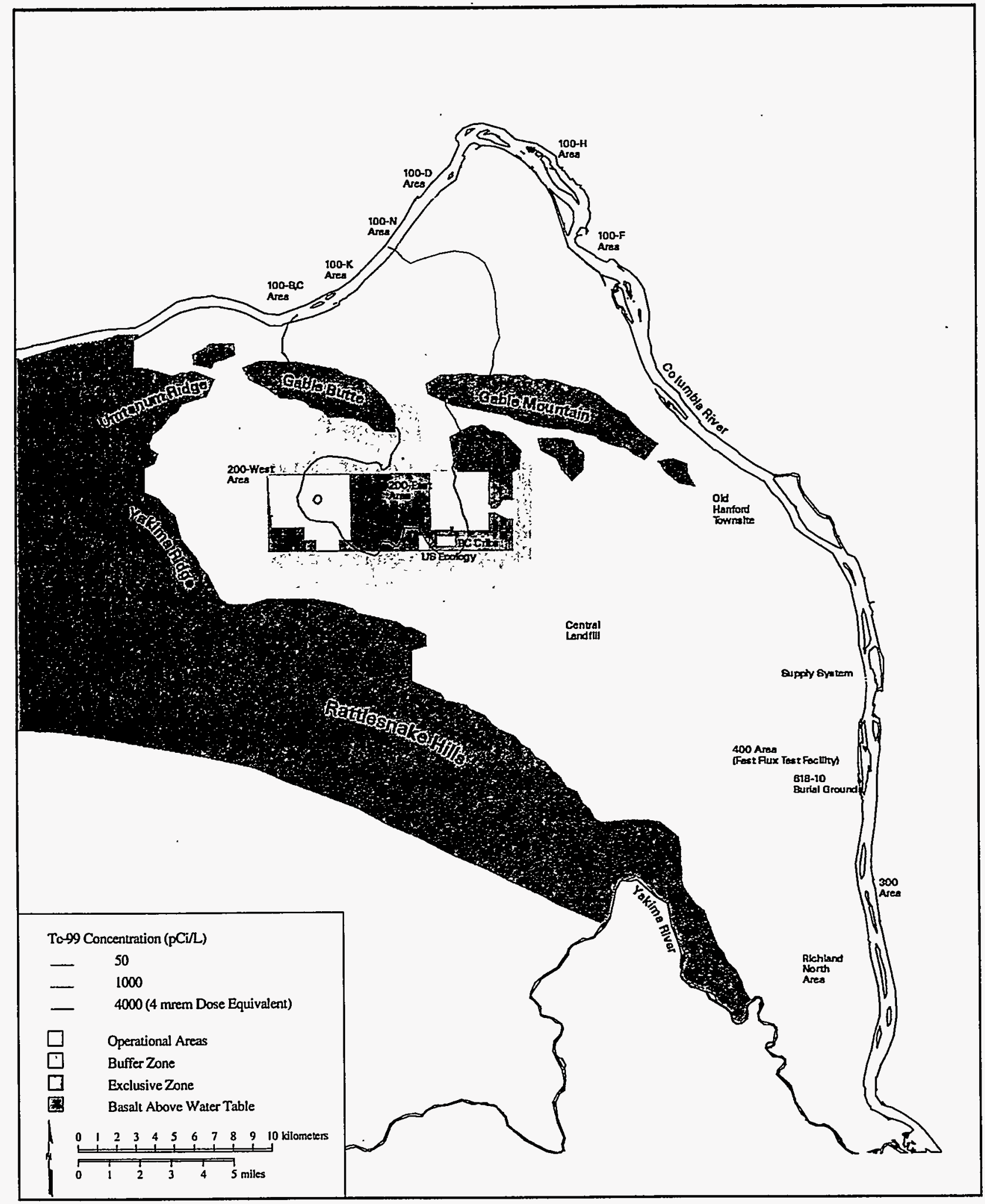

skw98055.eps December 23, 1997

Figure 4.25b. Predicted Distribution of Technetium-99 in the Unconfined Aquifer from All Sources in 2049 


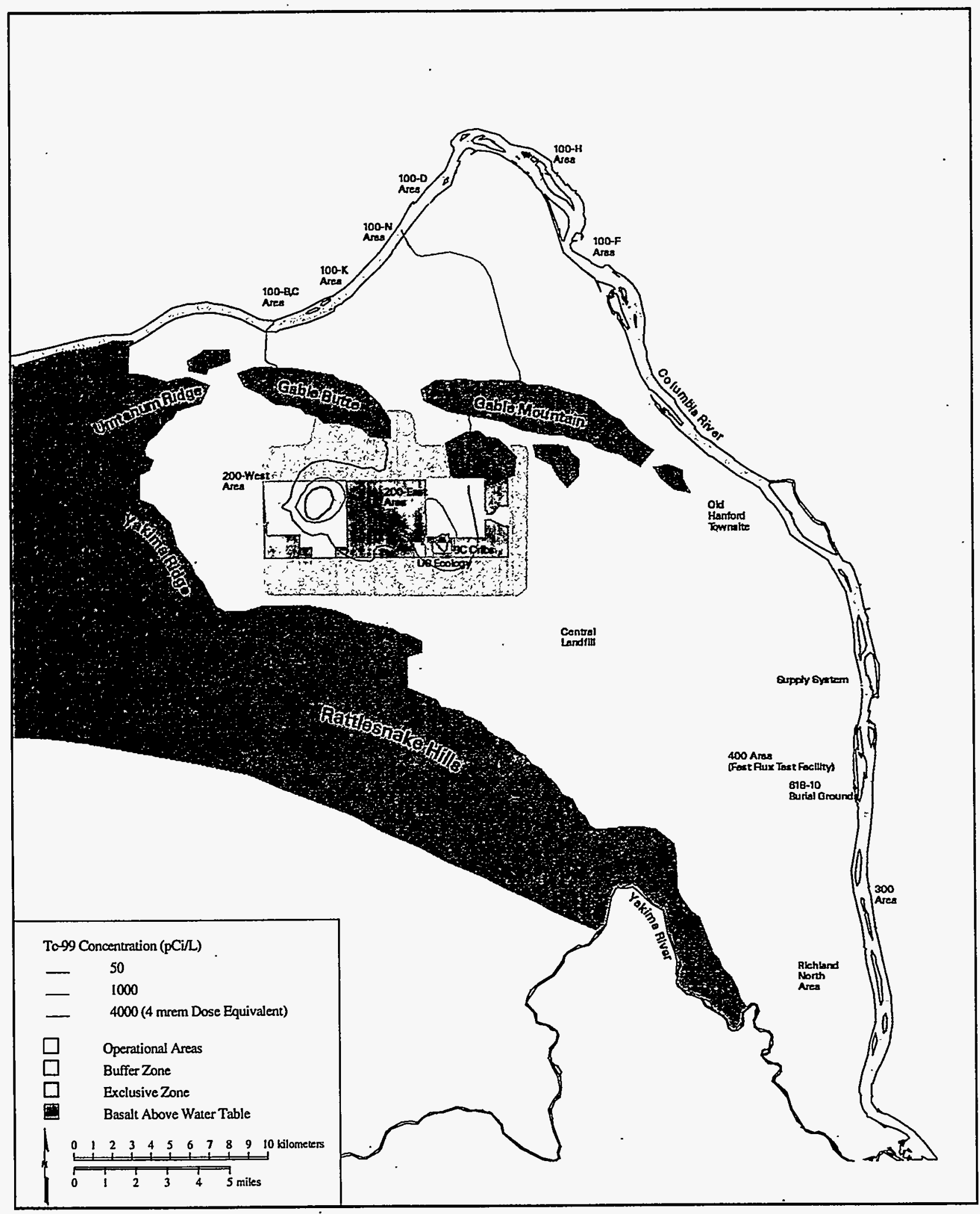

skw98058.eps December 23. 1997

Figure 4.26. Predicted Distribution of Technetium- 99 in the Unconfined Aquifer from All Sources in 2036 (Time of Secondary Peak Concentration) 


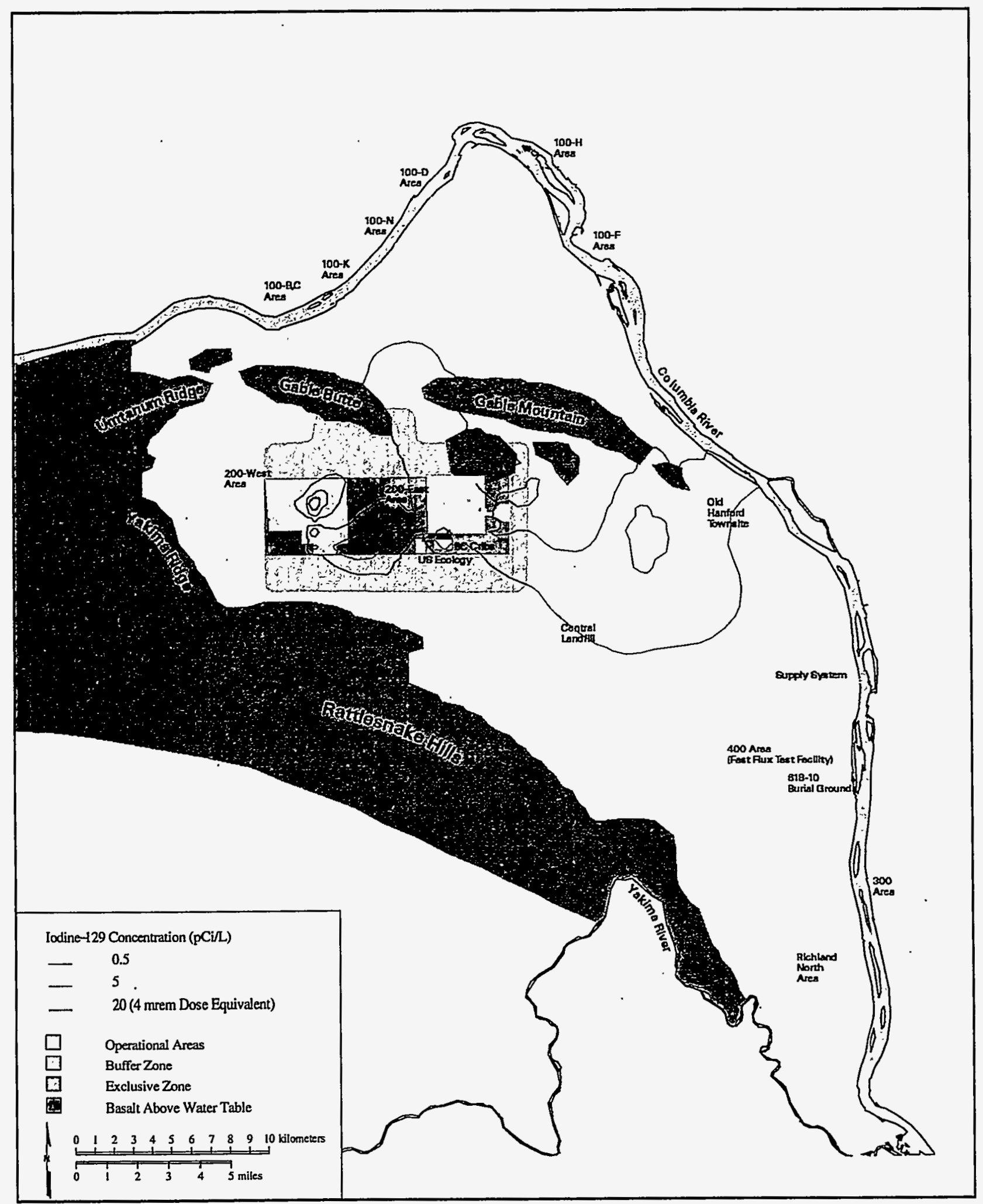

skw98059.eps December 23. 1997

Figure 4.27a. Predicted Distribution of Iodine-129 in the Unconfined Aquifer from All Sources in 2036 (Time of Peak Concentration) 


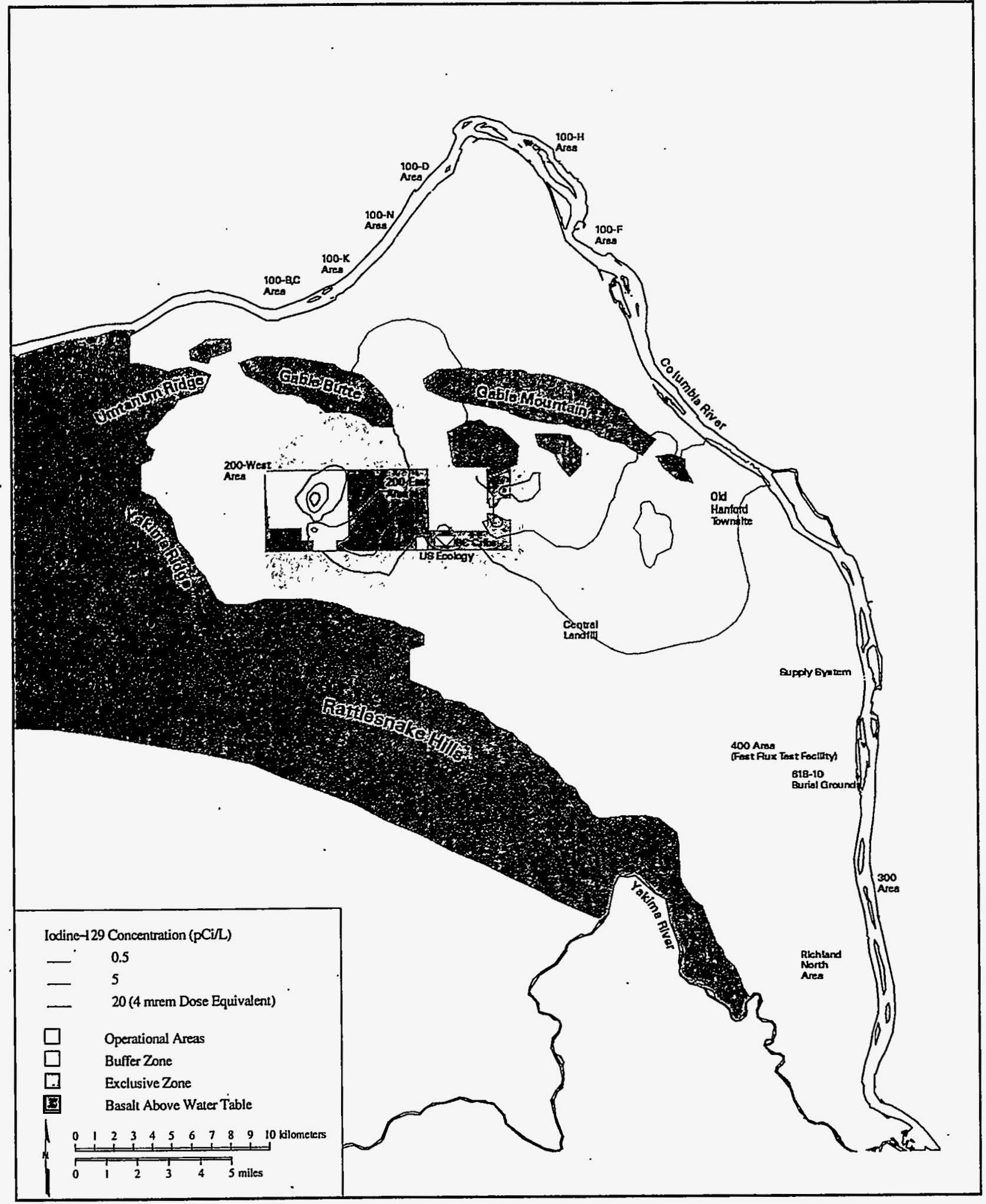

skw98056.eps March 03, 1998

Figure 4.27b. Predicted Distribution of Iodine-129 in the Unconfined Aquifer from All Sources in 2049 


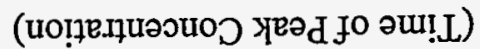

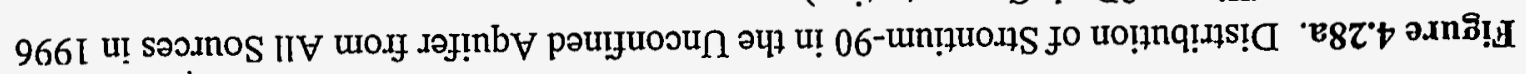
L66I ' $\varepsilon$ roquw2000 sdo. It086mys

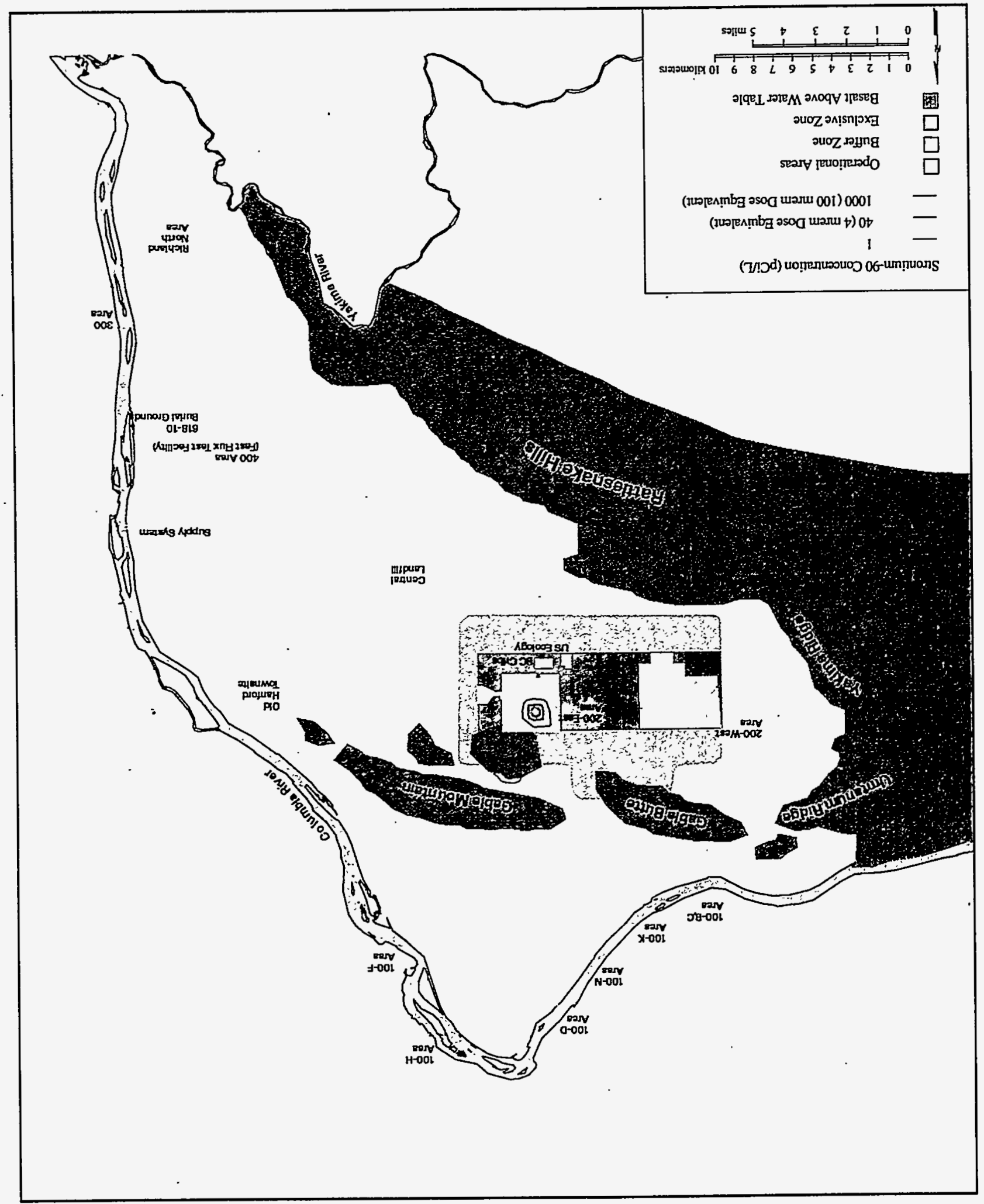




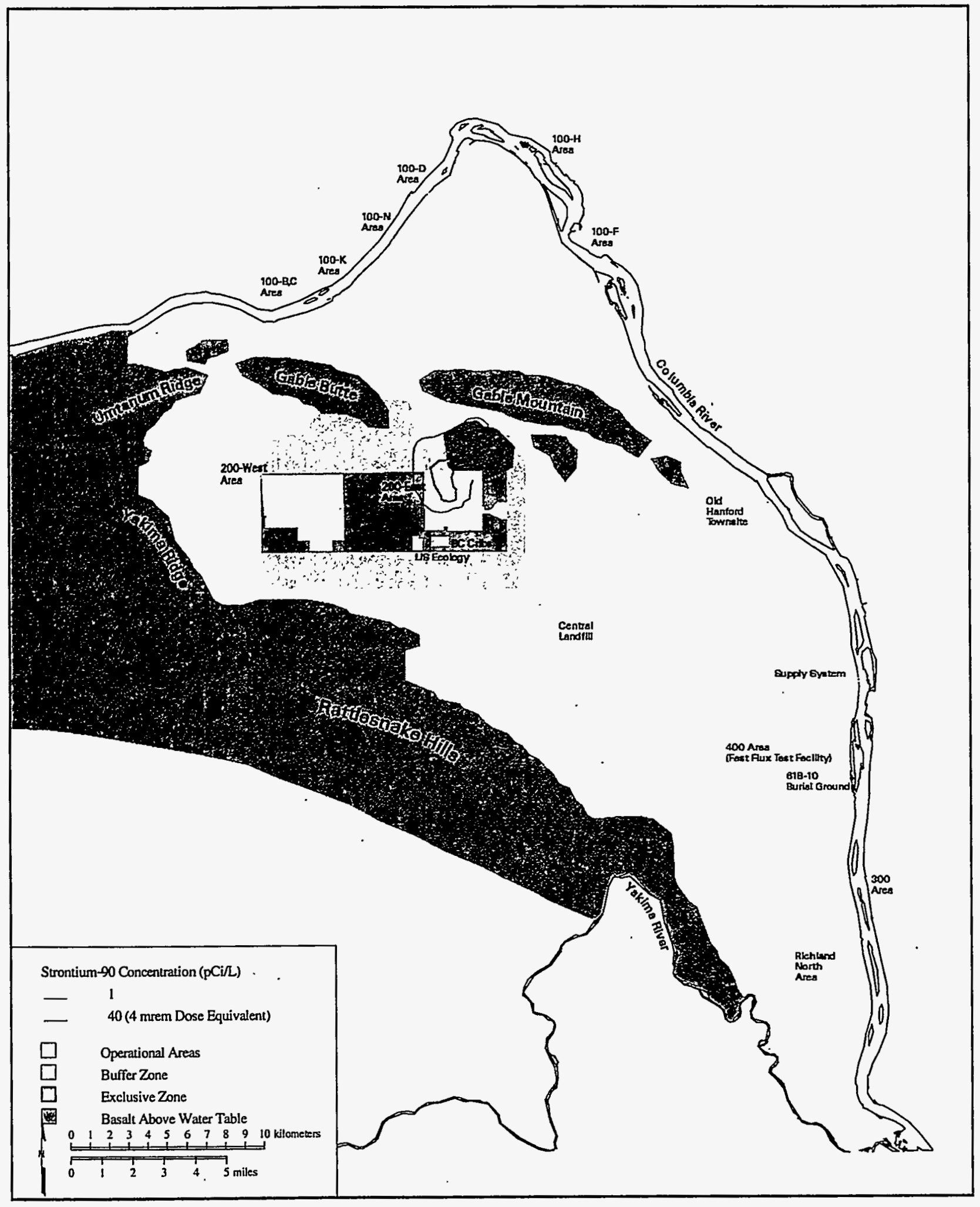

skw98054.eps December 23, 1997

Figure 4.28b. Predicted Distribution of Strontium-90 in the Unconfined Aquifer from All Sources in 2049 


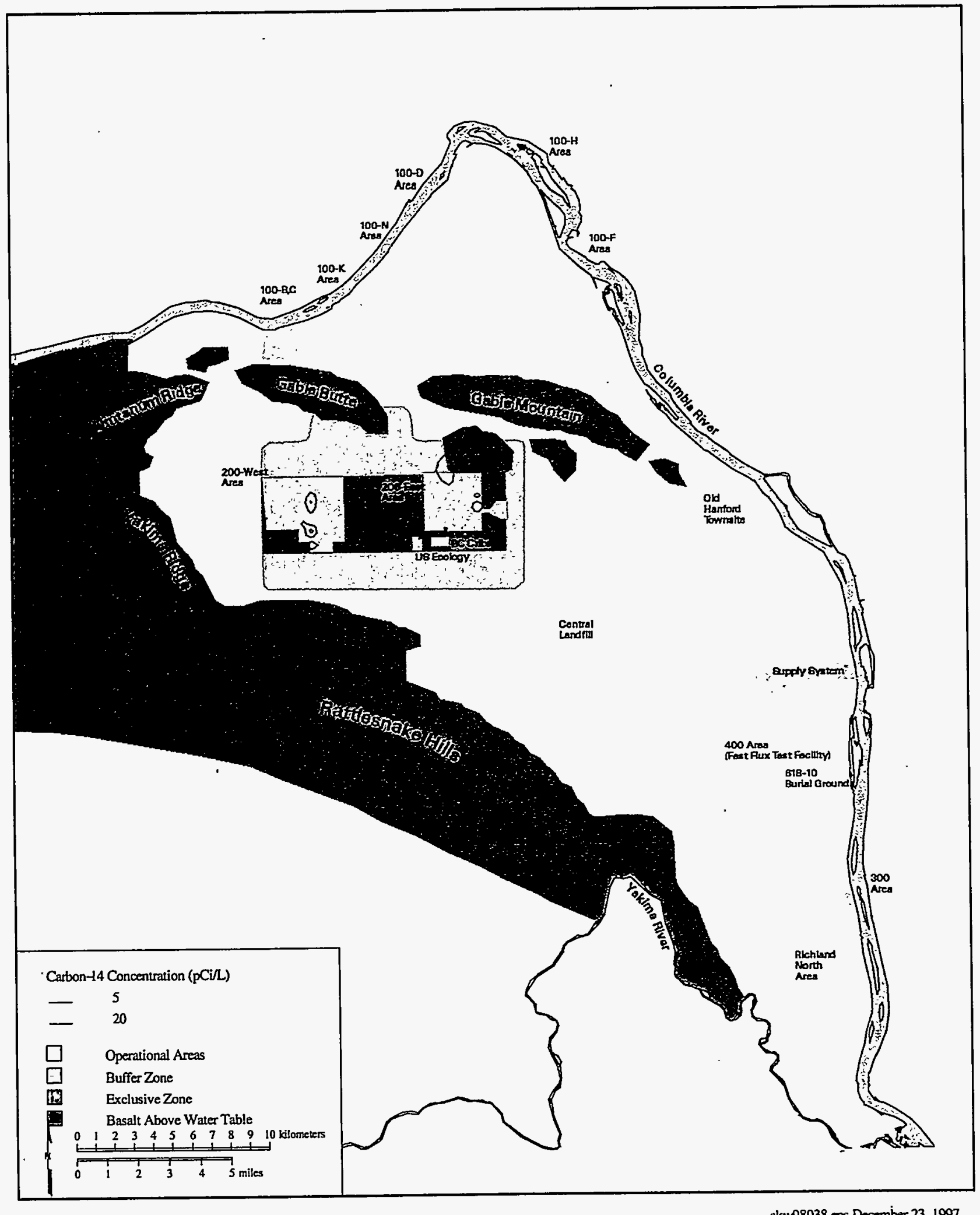

skw98038.qps December 23, 1997

Figure 4.29a. Predicted Distribution of Carbon-14 in the Unconfined Aquifer from All Sources in 2027 (Time of Peak Concentration) 


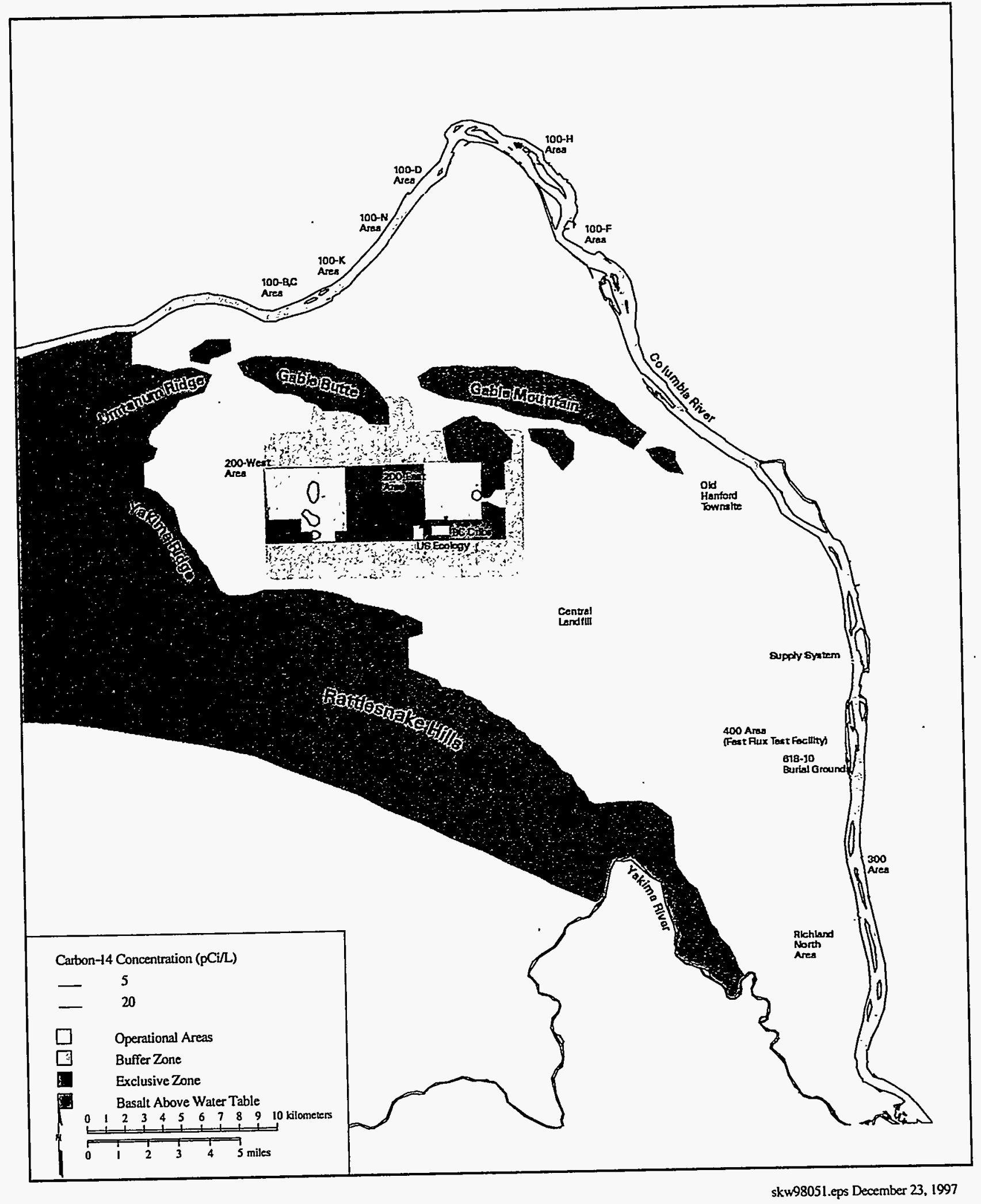

Figure 4.29b. Predicted Distribution of Carbon-14 in the Unconfined Aquifer from All Sources in 2049 


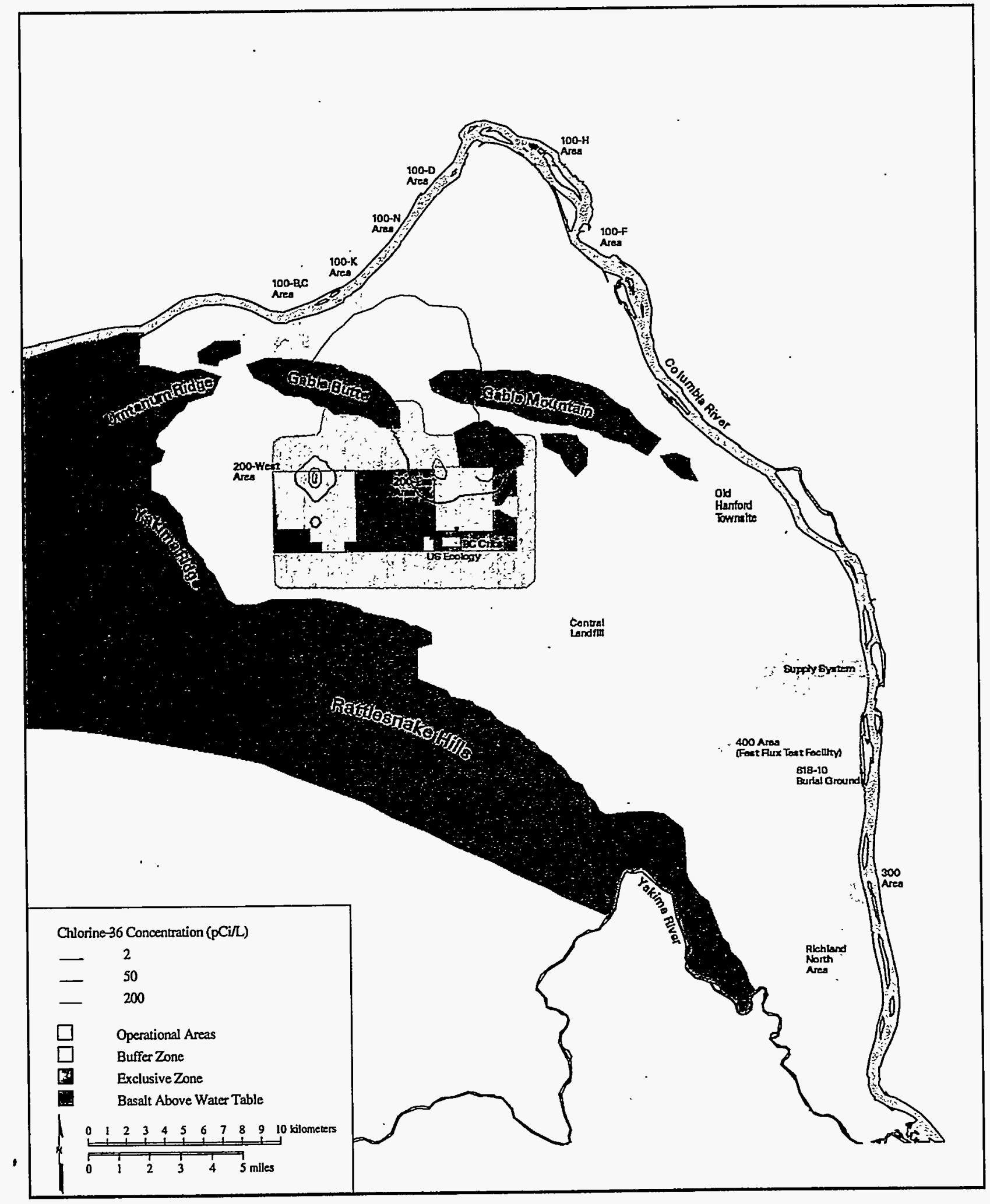

skw98039.eps December 23, 1997

Figure 4.30a. Predicted Distribution of Chlorine-36 in the Unconfined Aquifer from All Sources in 2019 (Time of Peak Concentration) 


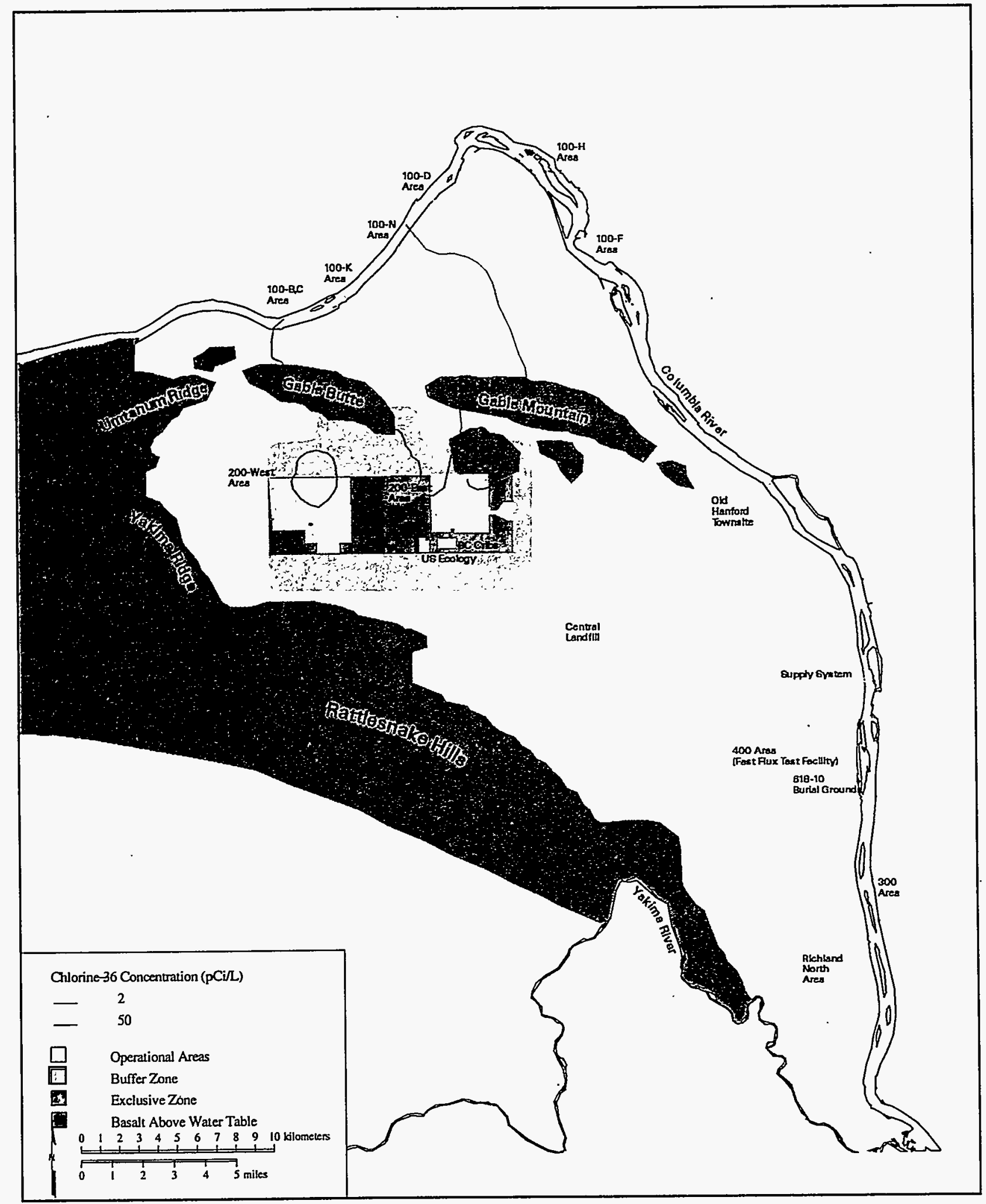

skw98052.eps December 23, 1997

Figure 4.30b. Predicted Distribution of Chlorine-36 in the Unconfined Aquifer from All Sources in 2049 


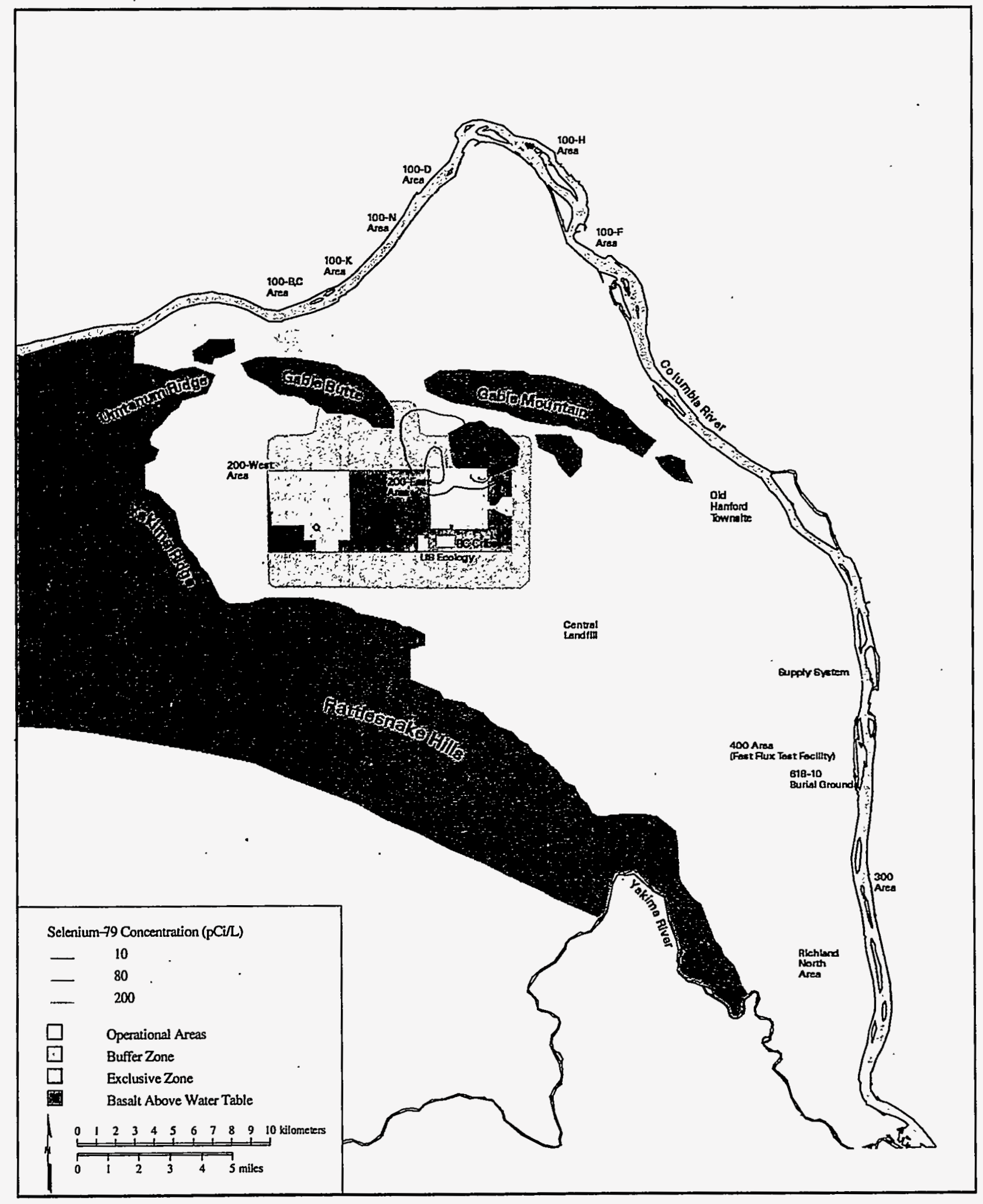

skw98040.eps December 23, 1997

Figure 4.31a. Predicted Distribution of Selenium-79 in the Unconfined Aquifer from All Sources in 2005 (Time of Peak Concentration) 


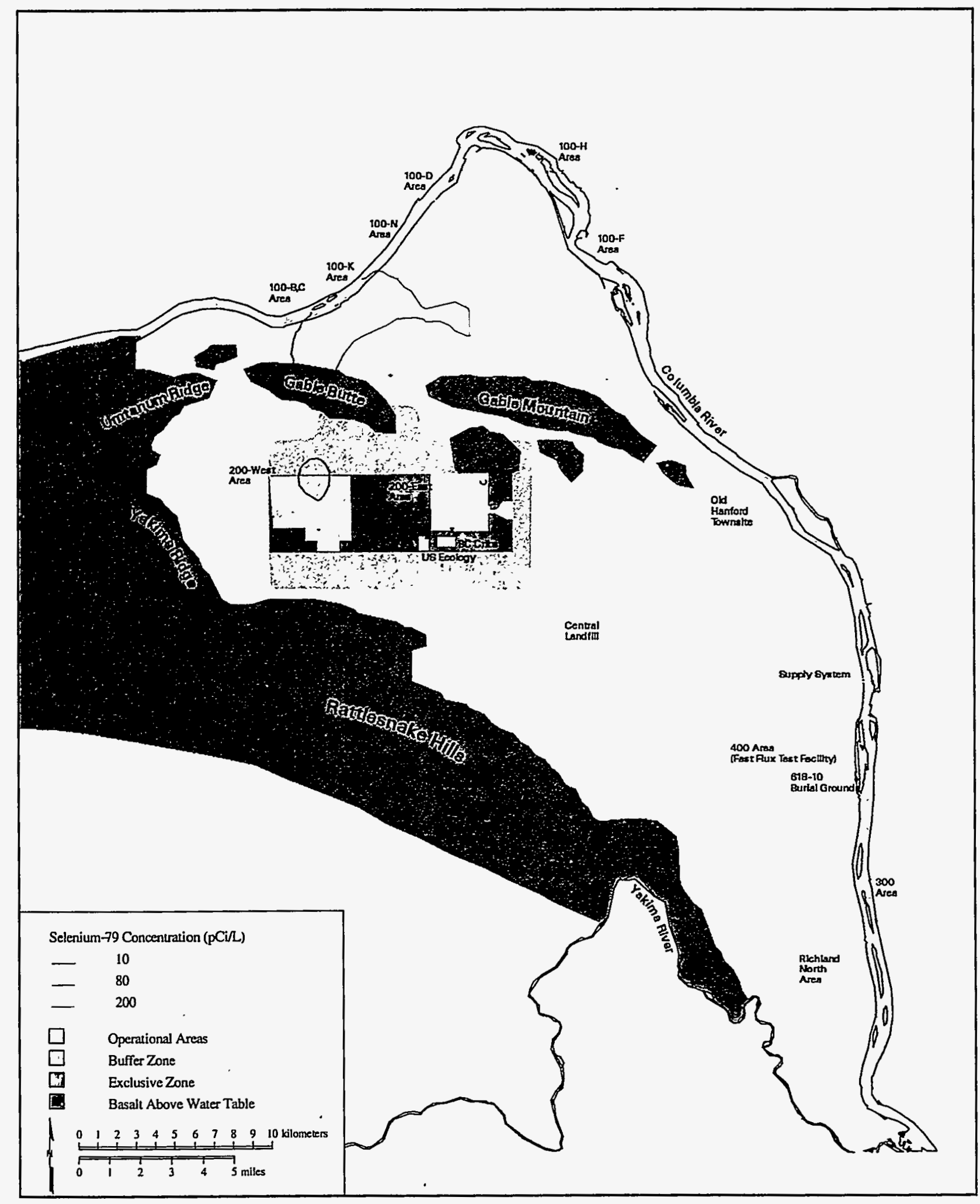

skw98053.eps December 23, 1997

Figure 4.31b. Predicted Distribution of Selenium-79 in the Unconfined Aquifer from All Sources in 2049 


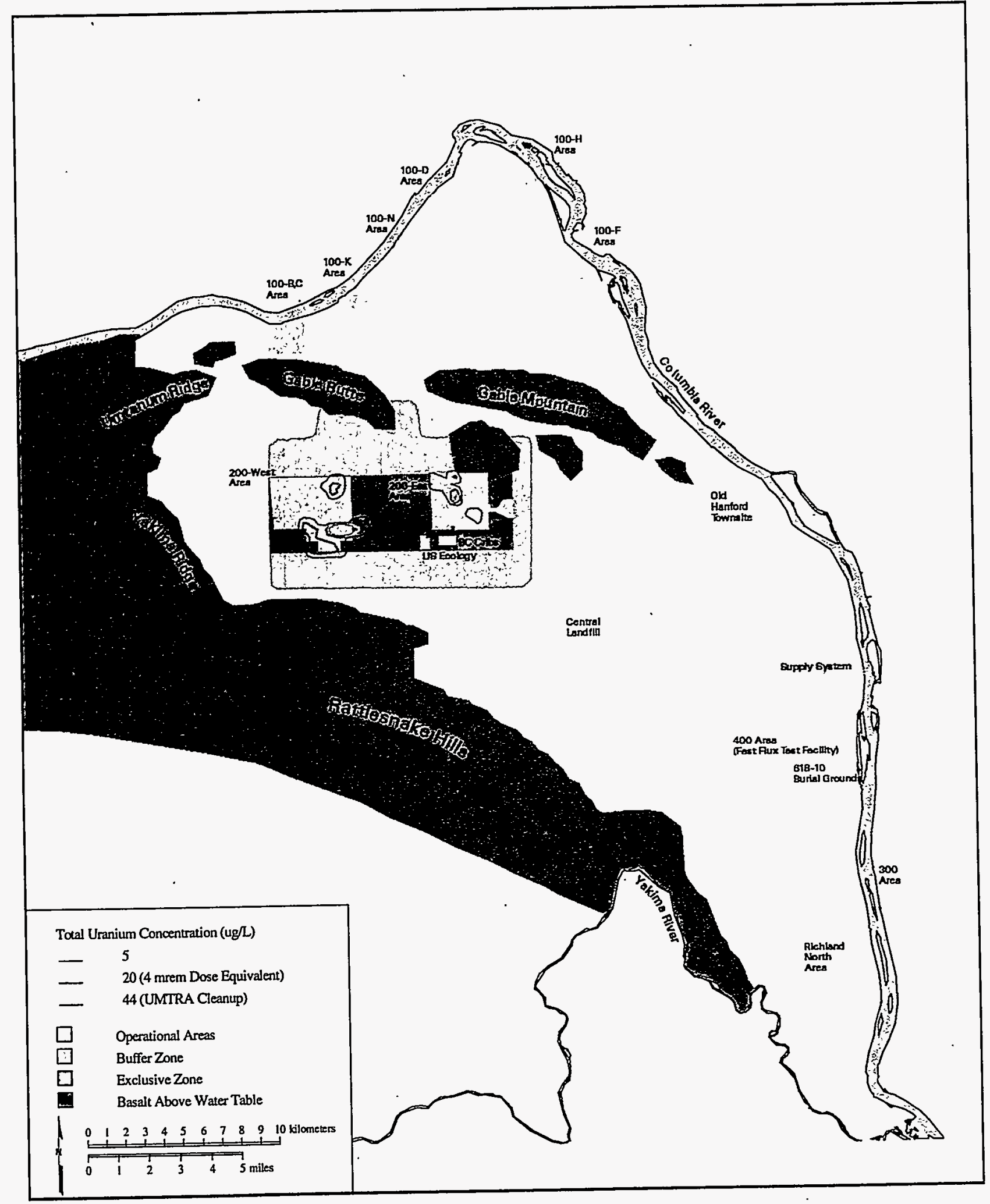

skw98048.eps December 23, 1997

Figure 4.32a. Distribution of Uranium (Total) in the Unconfined Aquifer from All Sources at 1996 (Time of Peak Concentration) 


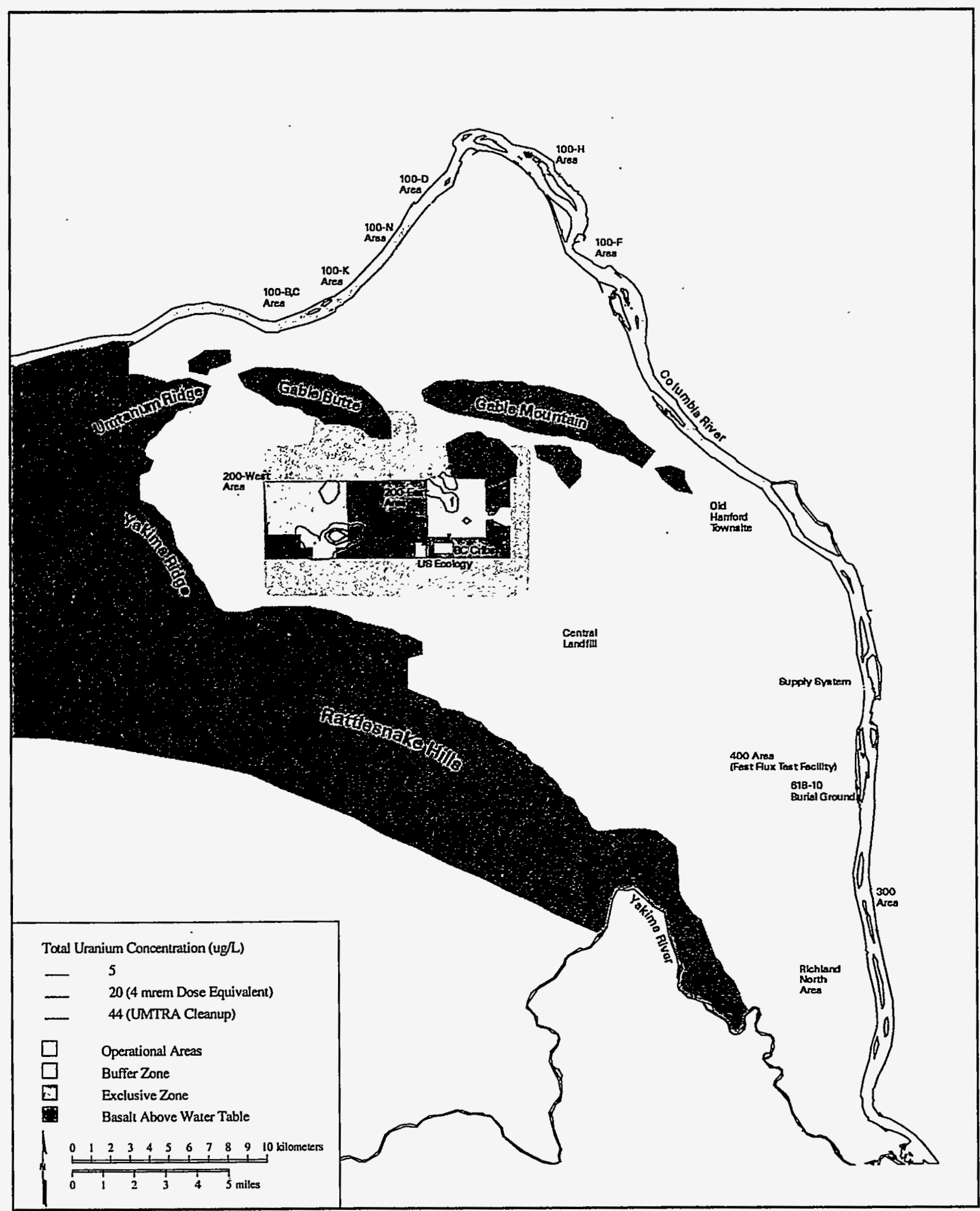

skw98057.eps December 23, 1997

Figure 4.32b. Predicted Distribution of Uranium (Total) in the Unconfined Aquifer from All Sources in 2049 
(a) Unit_source at TWRS

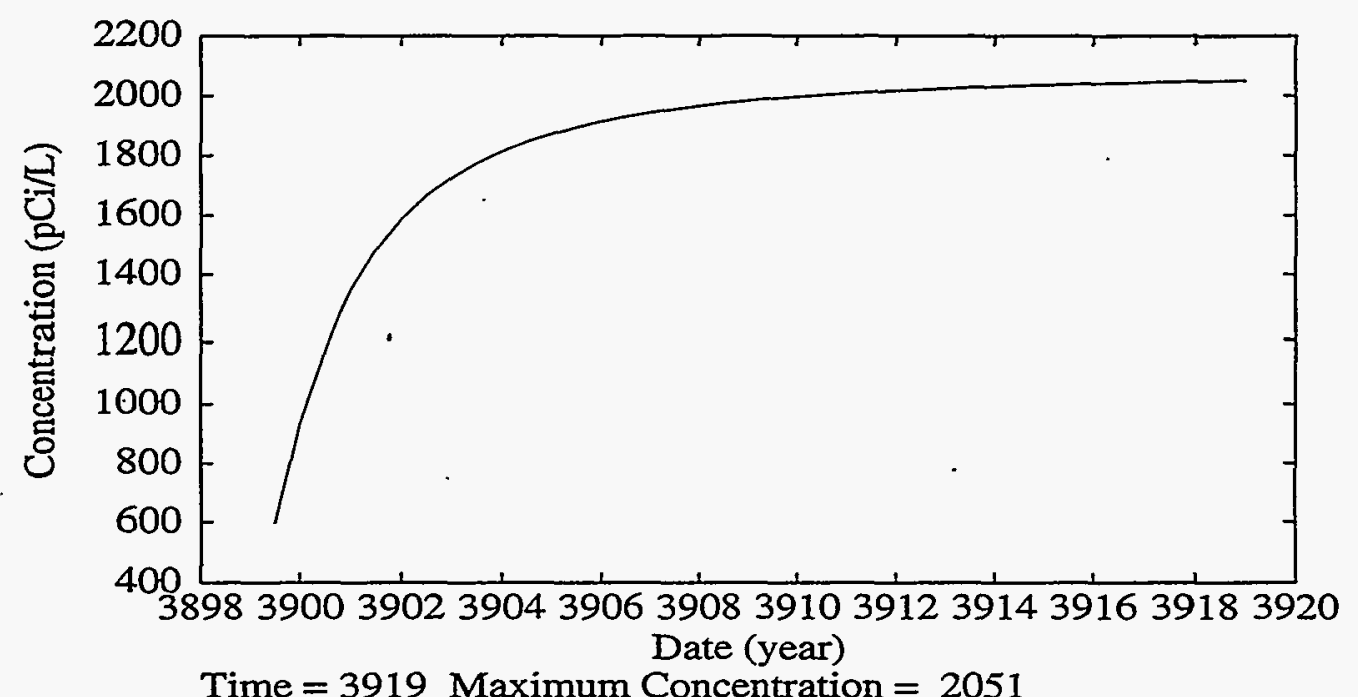

Time $=3919$ Maximum Concentration $=2051$

(b) Unit_source at AX and AY Tank Farms

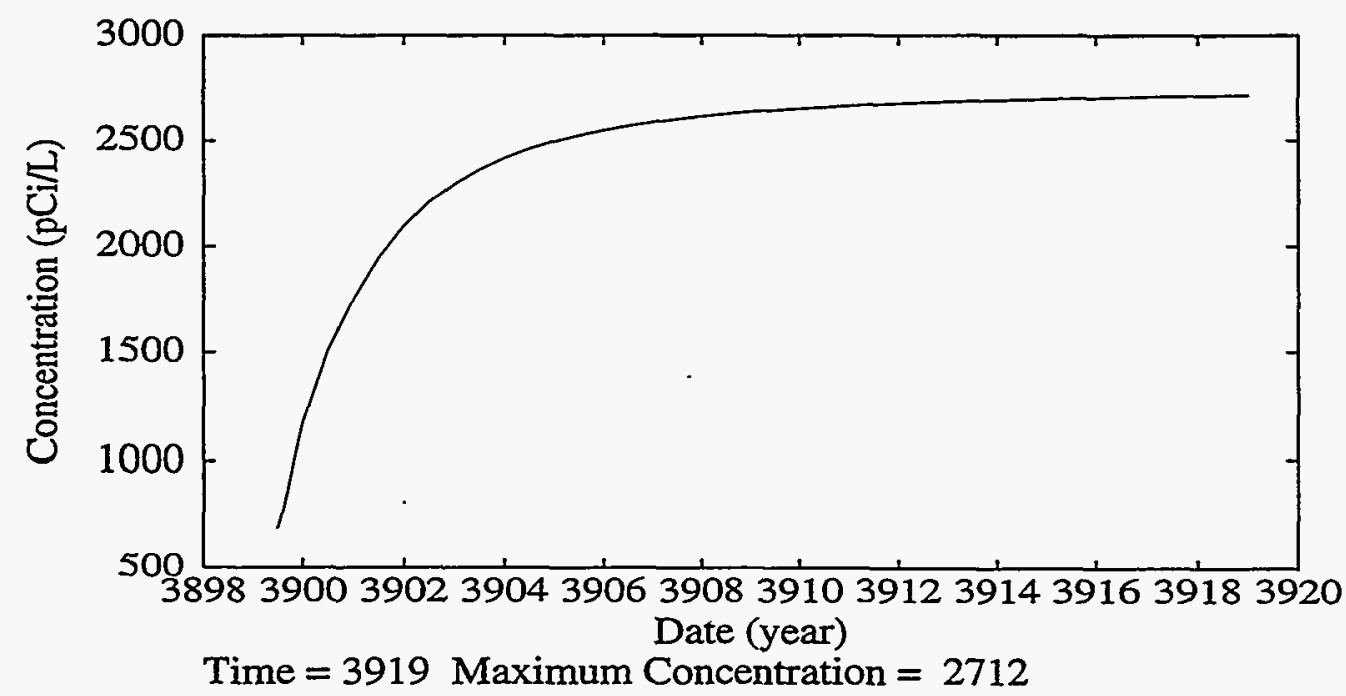

Figure 4.33. Results from a Series of Nine Transport Model Location Sensitivity Studies. Shown are Maximum Concentration Versus Time Plots for Unit Curie Sources at the Following Locations: a) TWRS Disposal Site, b) AX and AY Tank Farms. 
(c) Unit_source at BX and BY Tank Farms

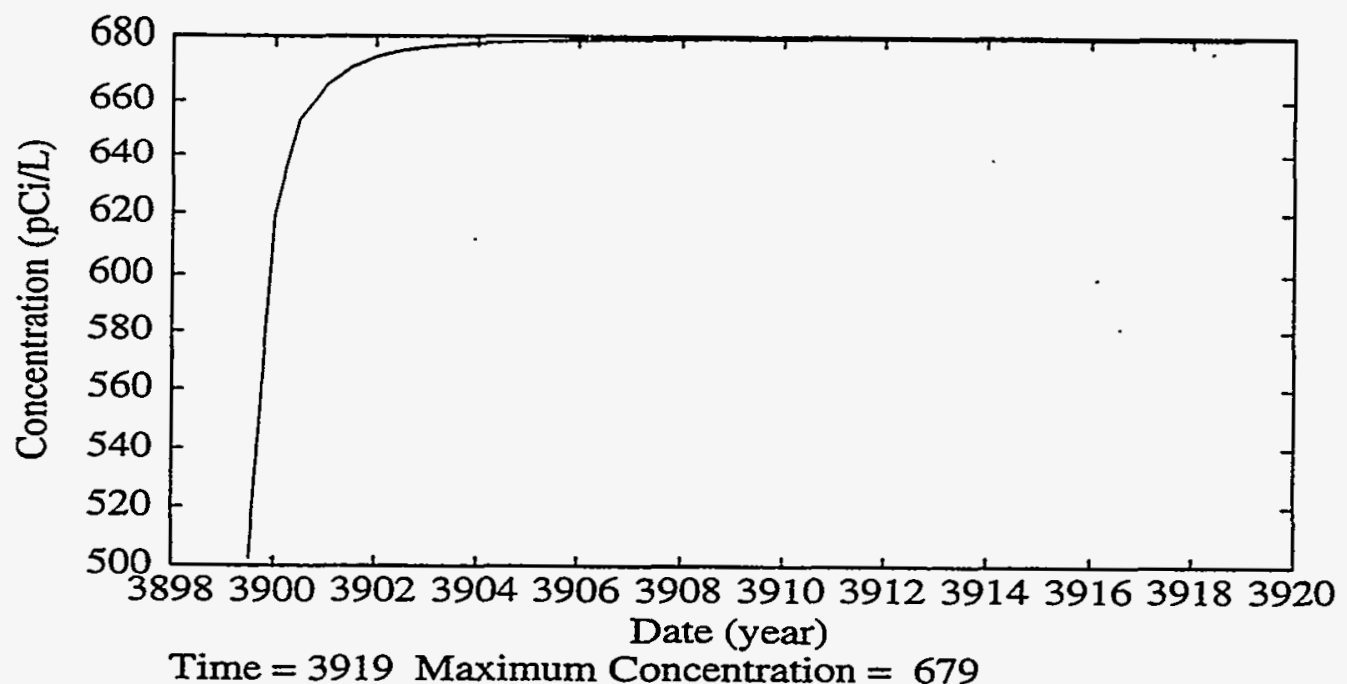

(d) Unit_source at C Tank Farm

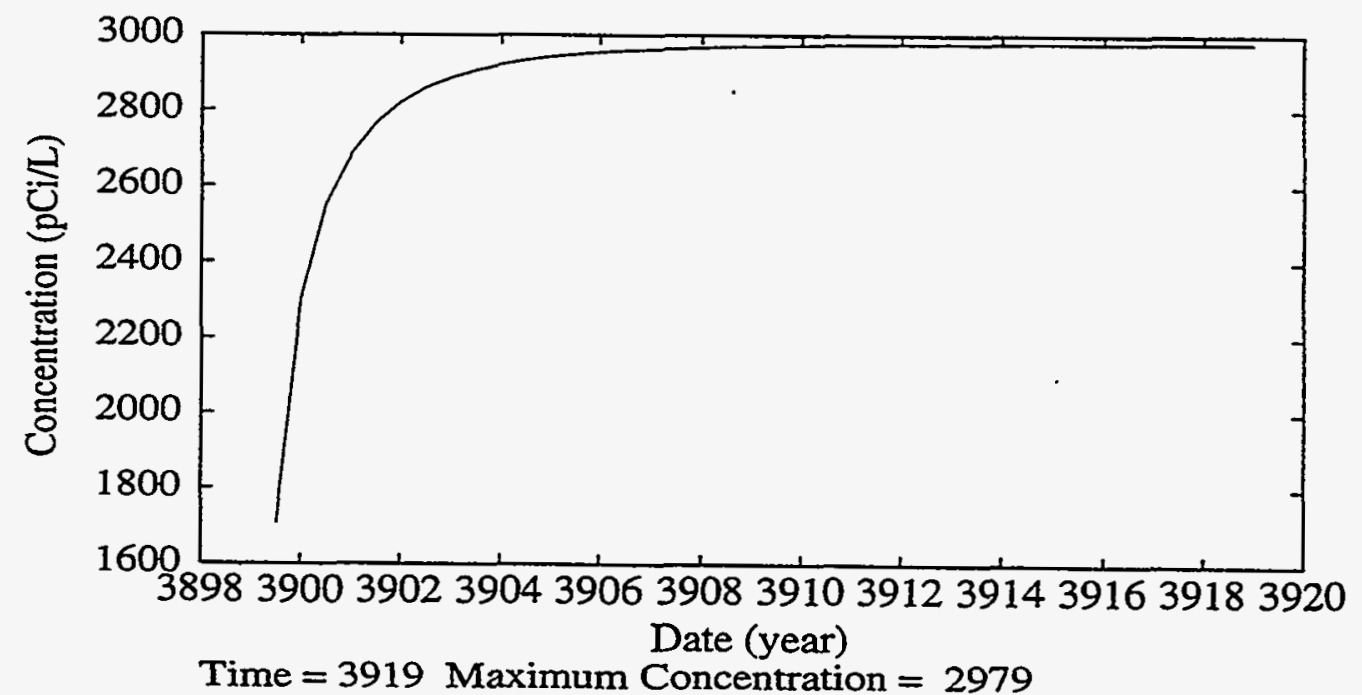

Figure 4.33. Results from a Series of Nine Transport Model Location Sensitivity Studies. Shown are Maximum Concentration Versus Time Plots for Unit Curie Sources at the Following Locations: c) BX and BY Tank Farms, d) C Tank Farm. 
(e) Unit_source at T Tank Farm

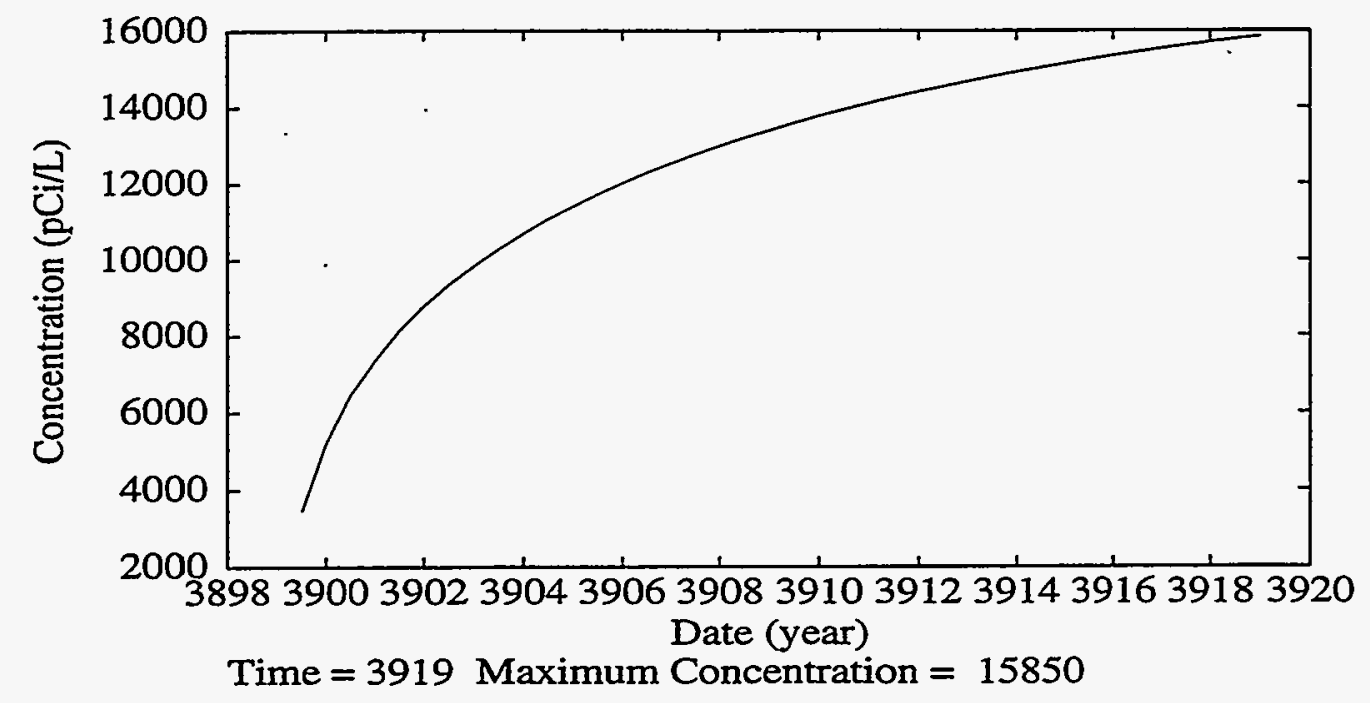

(f) Unit_source at TX and TY Tank Farms

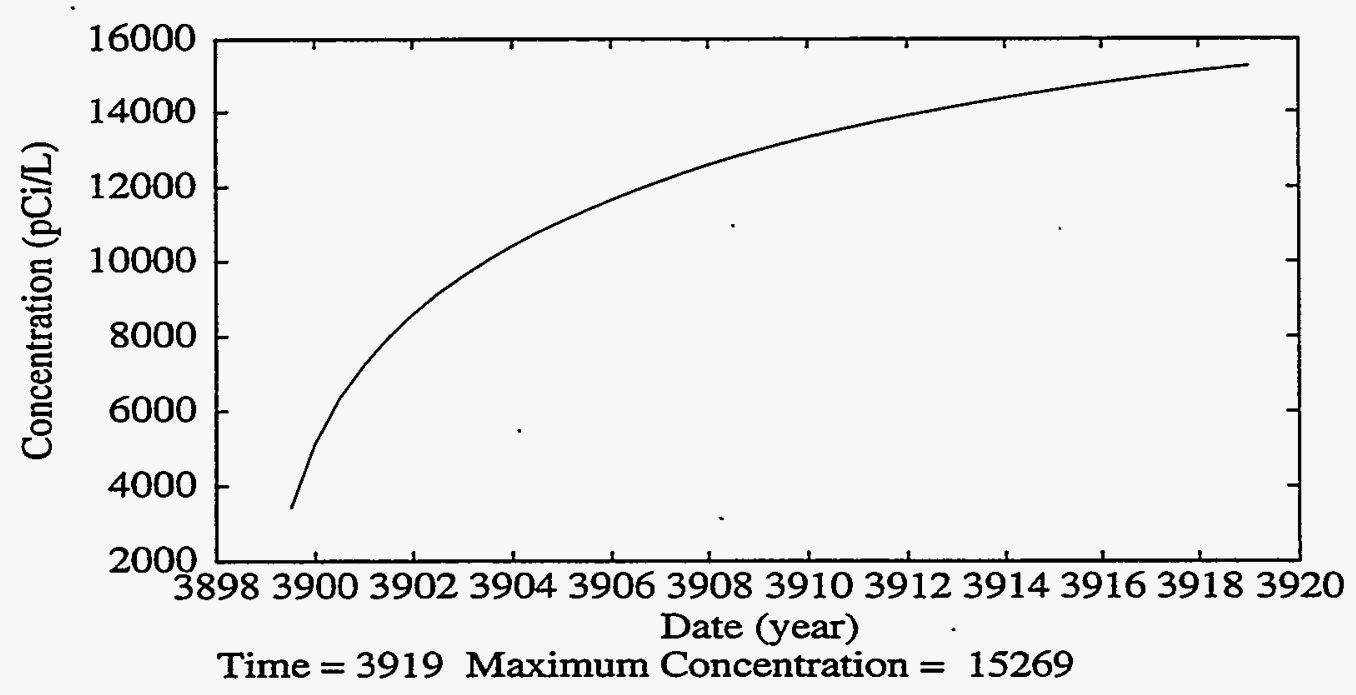

Figure 4.33. Resuits from a Series of Nine Transport Model Location Sensitivity Studies. Shown are Maximum Concentration Versus Time Plots for Unit Curie Sources at the Following Locations: e) T Tank Farm, f) TX and TY Tank Farms. 
(g) Unit_source at U Tank Farm

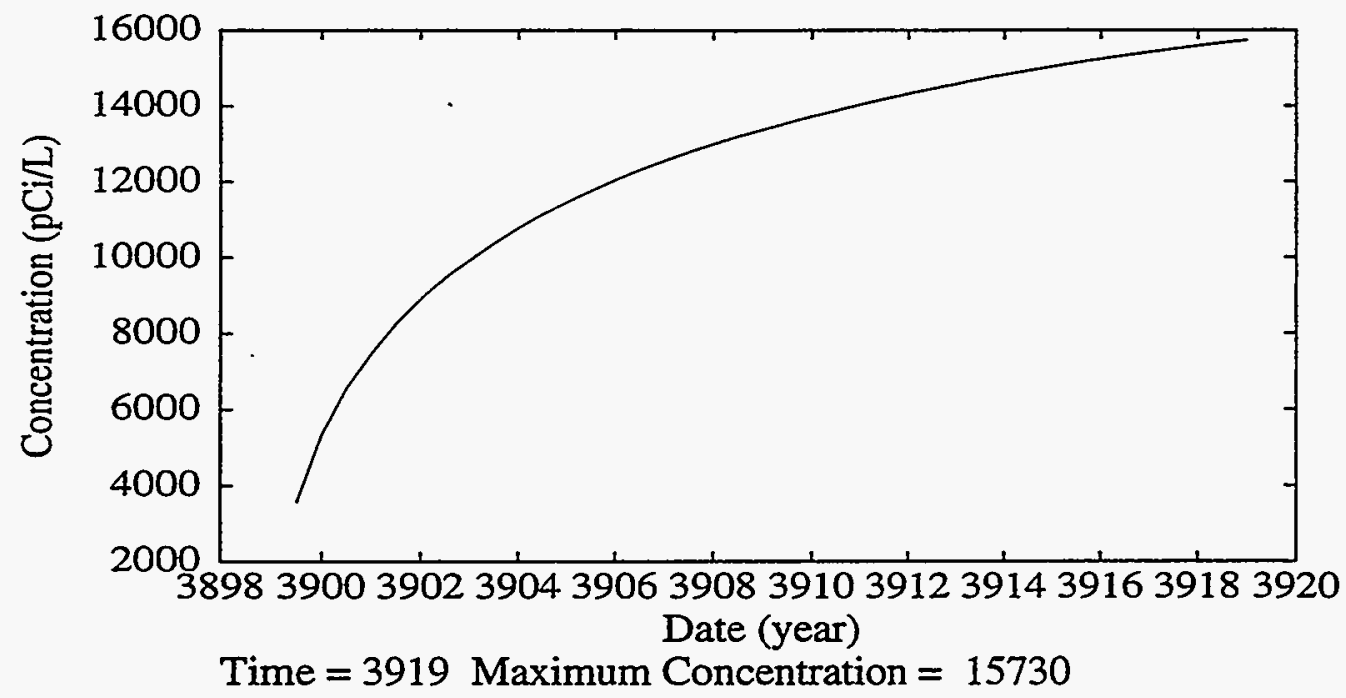

(h) Unit_source at S-SX Tank Farms

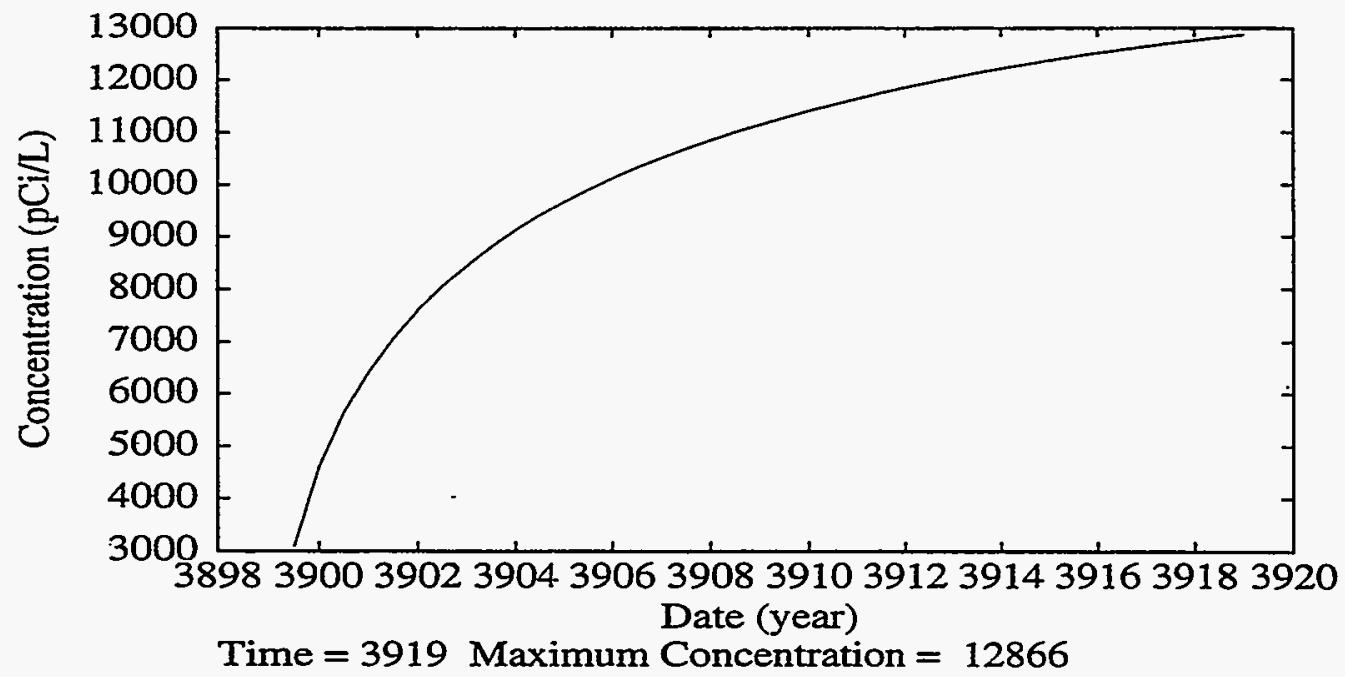

Figure 4.33. Results from a Series of Nine Transport Model Location Sensitivity Studies. Shown are Maximum Concentration Versus Time Plots for Unit Curie Sources at the Following Locations: g) U Tank Farm, h) S and SX Tank Farms. 
(i) Unit_source at US Ecology

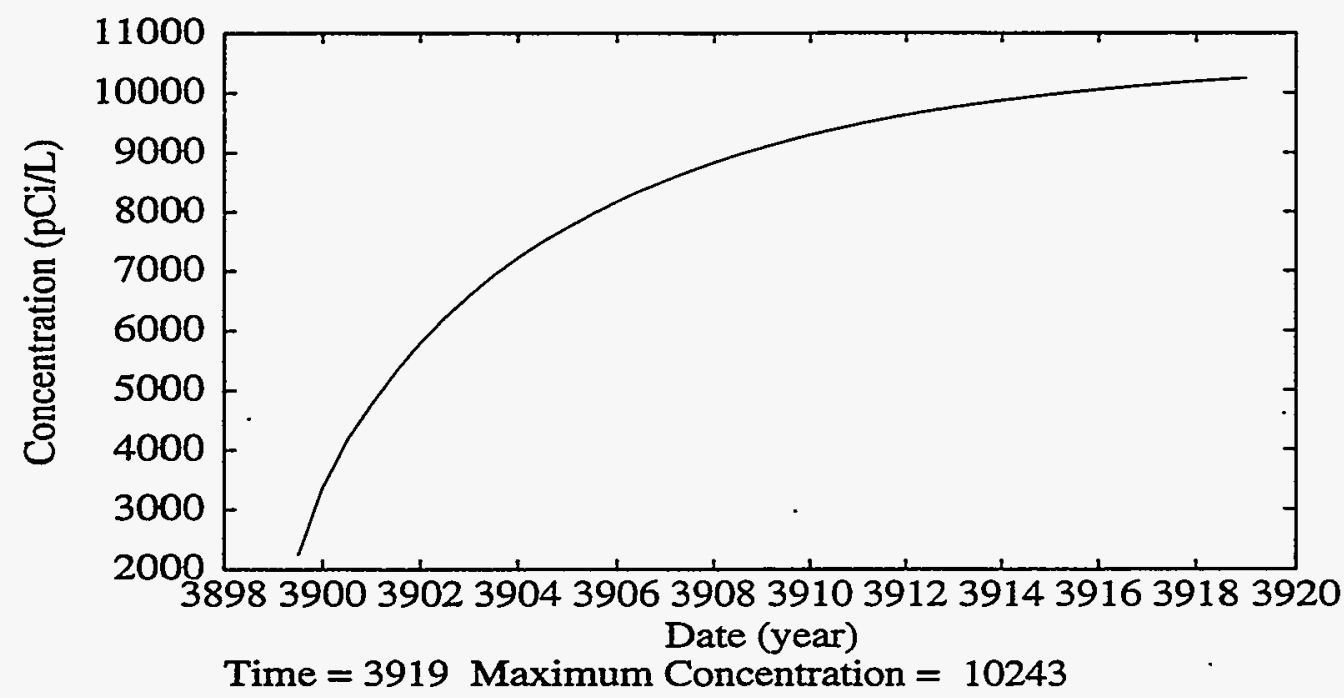

Figure 4.33. Results from a Series of Nine Transport Model Location Sensitivity Studies. Shown are Maximum Concentration Versus Time Plots for Unit Curie Sources at the Following Location: i) U.S. Ecology Site. 
(a) Maximum Dose for Agricultural Scenario

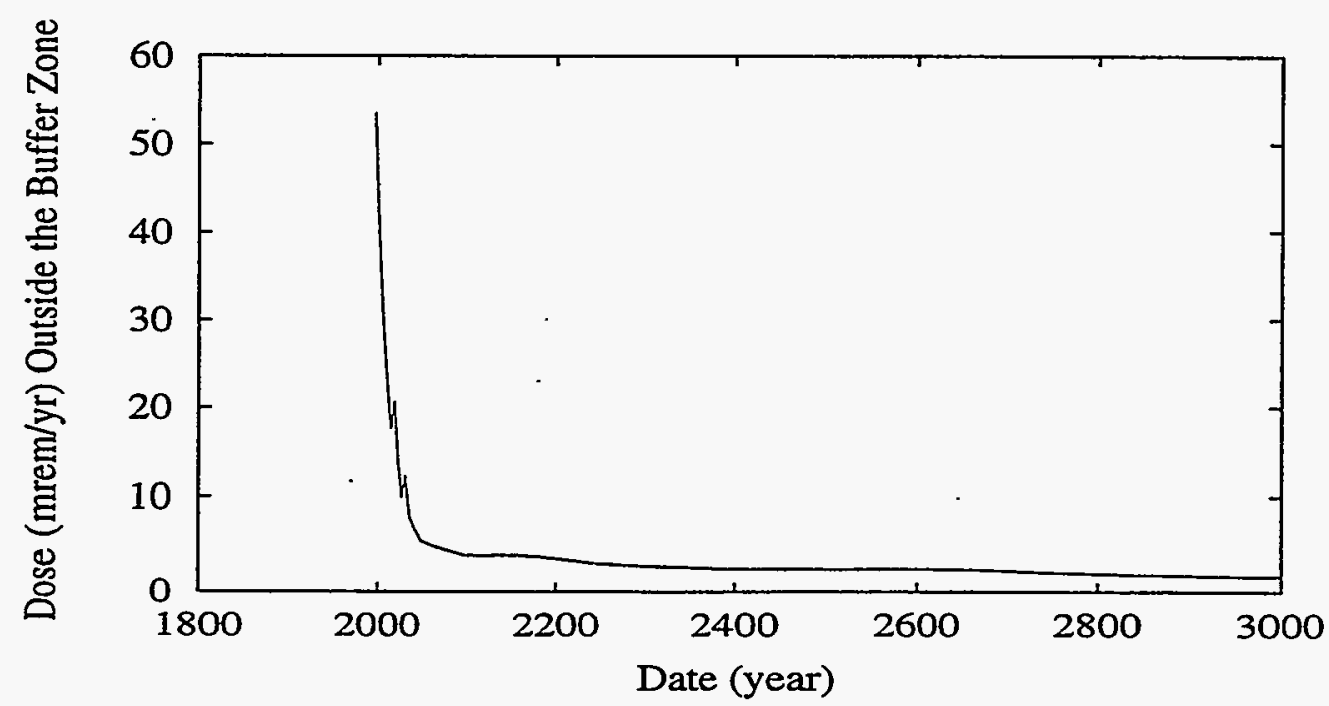

(b) All Dose Components for Agricultural Scenario

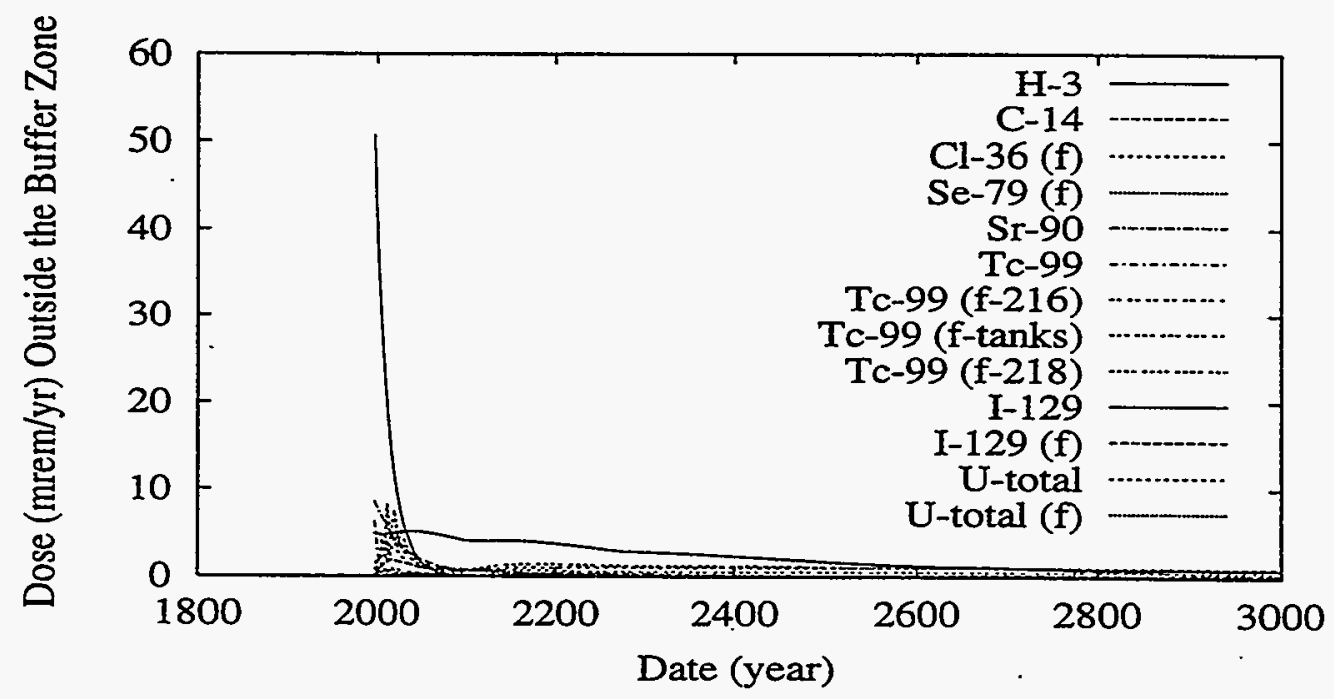

Figure 4.34. Maximum Dose Versus Time Outside the Buffer Zone for the Agricultural Scenario and the Dose the Various Radionuclides/Sources Contribute. Shown are:

a) Maximum Dose, b) All Contributions on Same Scale. 
(c) Dose from existing H-3 Plumes for Agricultural Scenario

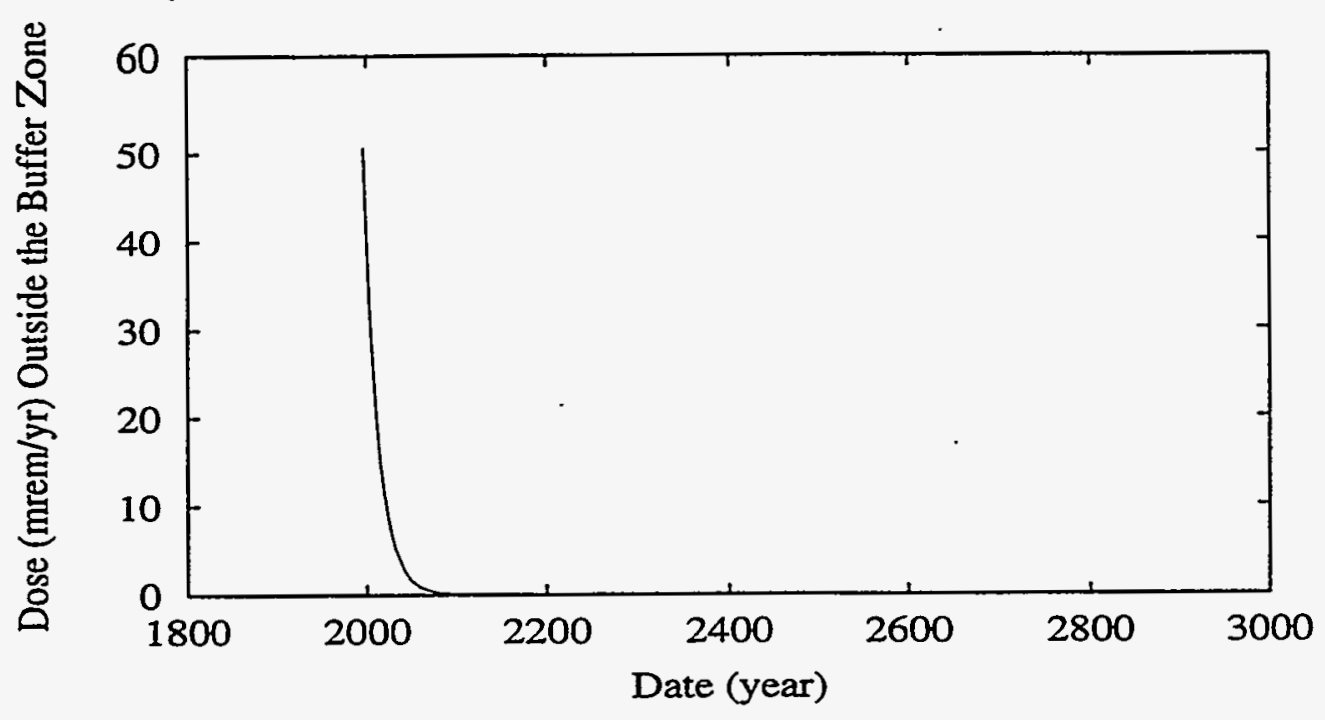

(d) Dose from existing Sr-90 Plumes for Agricultural Scenario

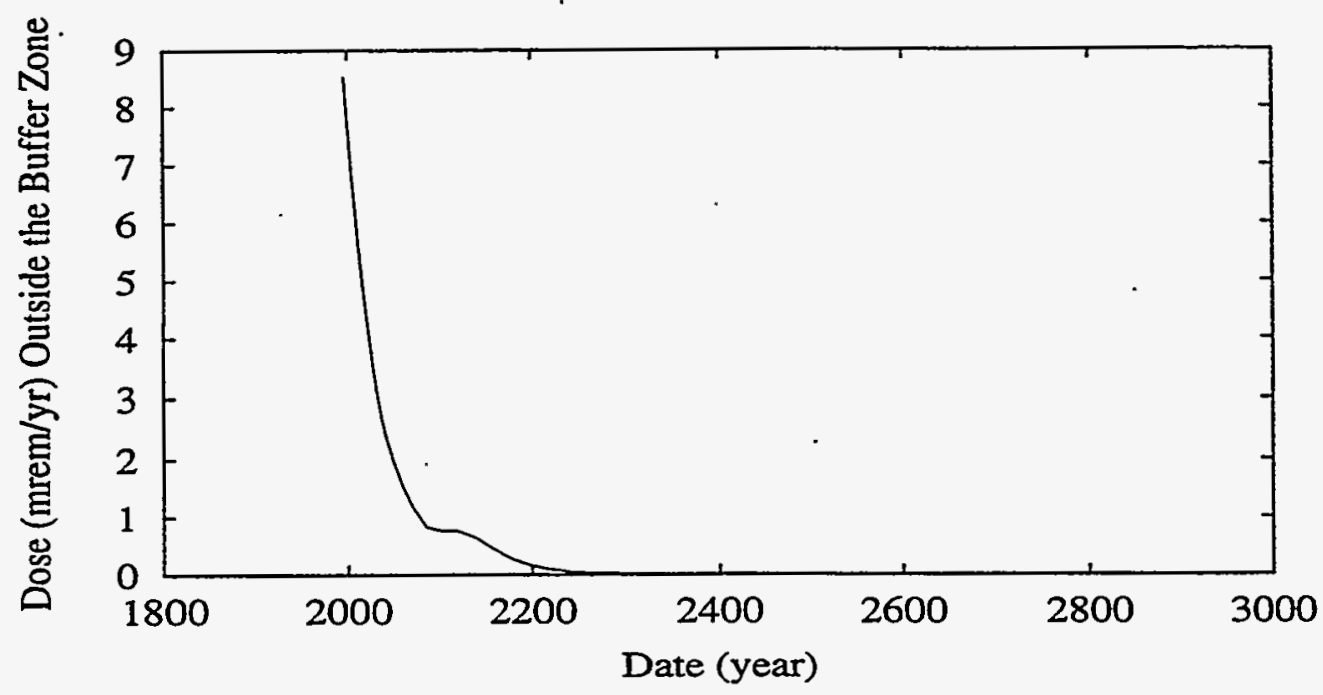

Figure 4.34. Maximum Dose Versus Time Outside the Buffer Zone for the Agricultural Scenario and the Dose the Various Radionuclides/Sources Contribute. Shown are: c) Tritium Contribution, d) Strontium-90. 
(e) Dose from future C-14 Sources for Agricultural Scenario

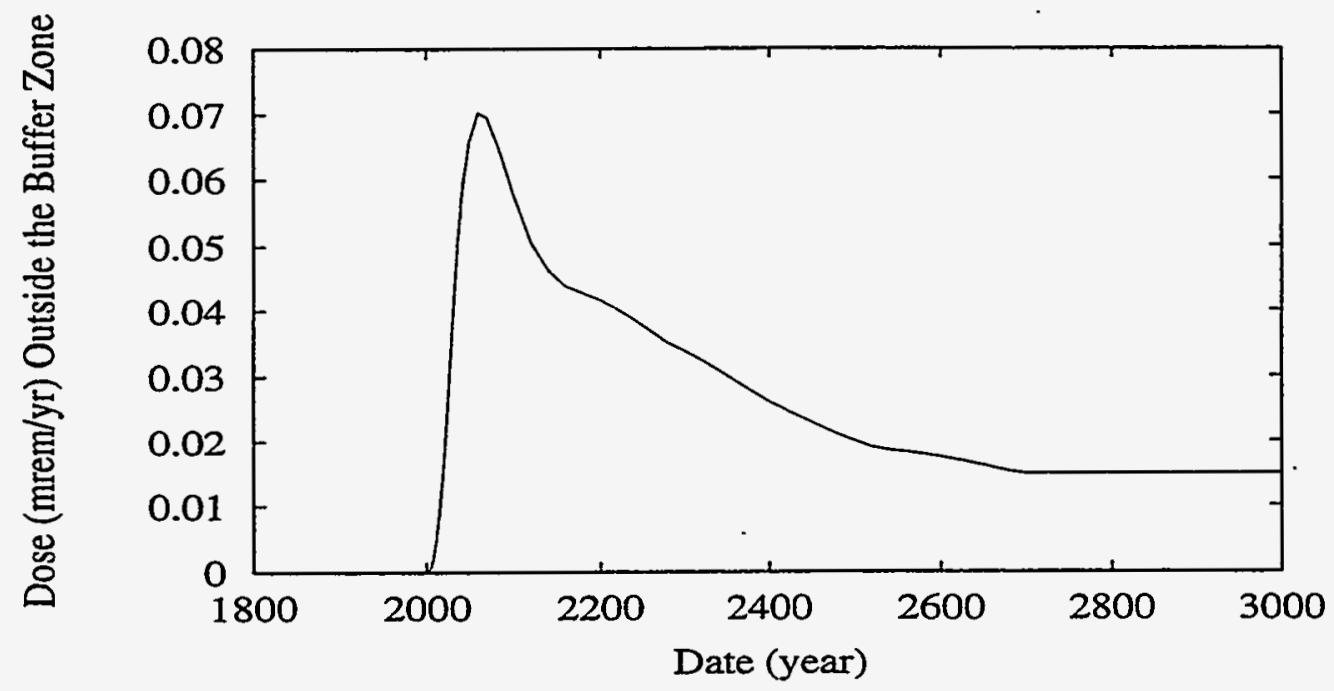

(f) Dose from future Cl-36 Sources for Agricultural Scenario

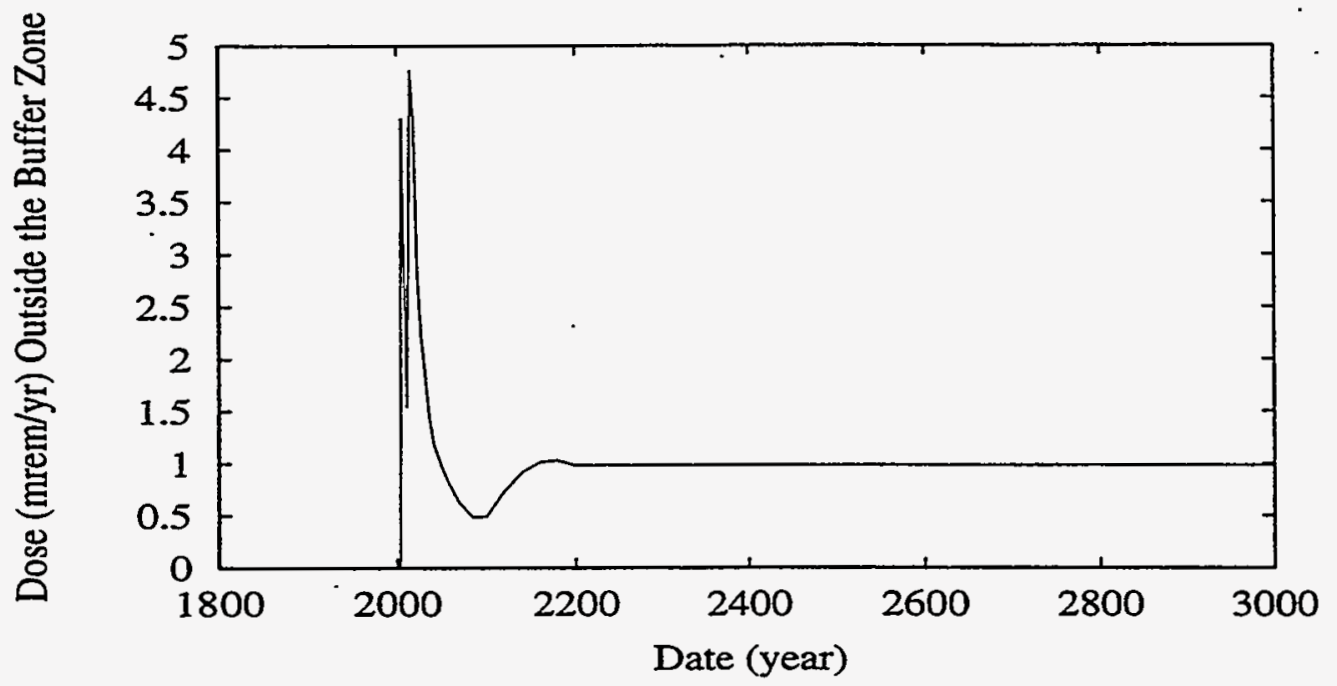

Figure 4.34. Maximum Dose Versus Time Outside the Buffer Zone for the Agricultural Scenario and the Dose the Various Radionuclides/Sources Contribute. Shown are:

e) Carbon-14, f) Chlorine-36. 
(g) Dose from future Se-79 Sources for Agricultural Scenario

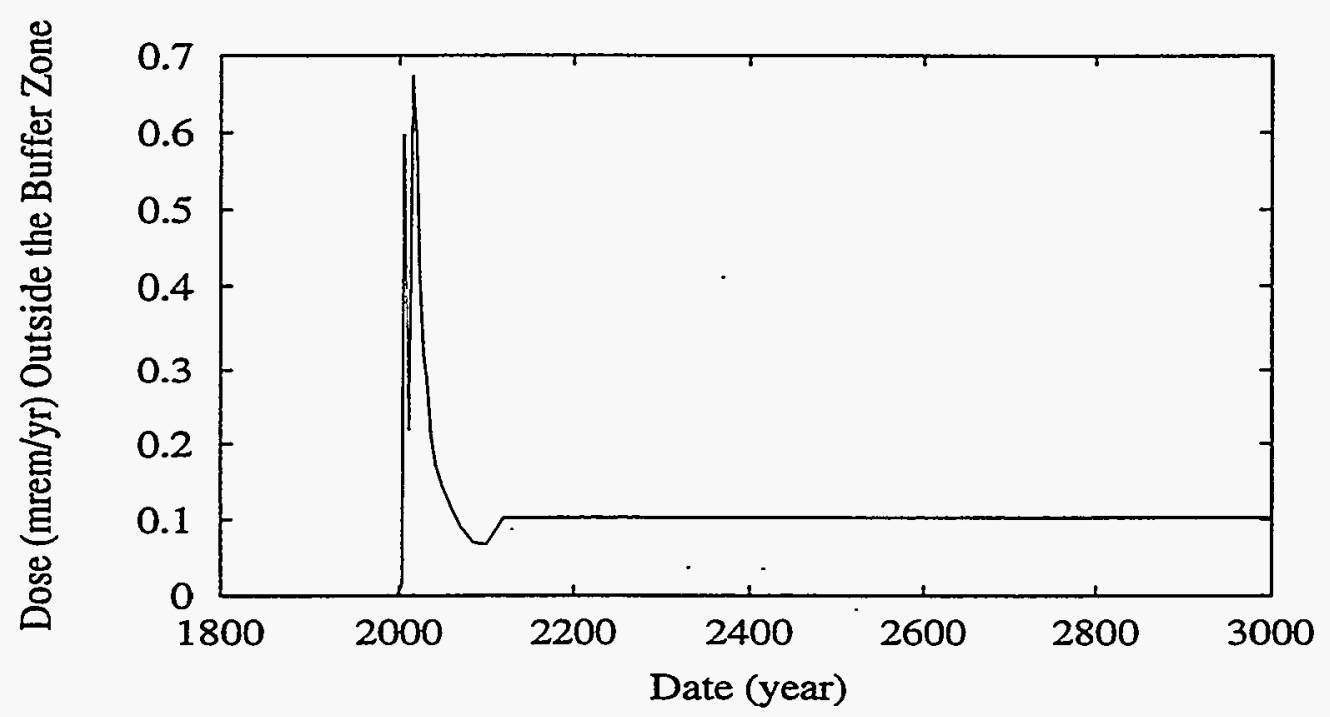

(h) Dose from existing Tc-99 Plumes for Agricultural Scenario

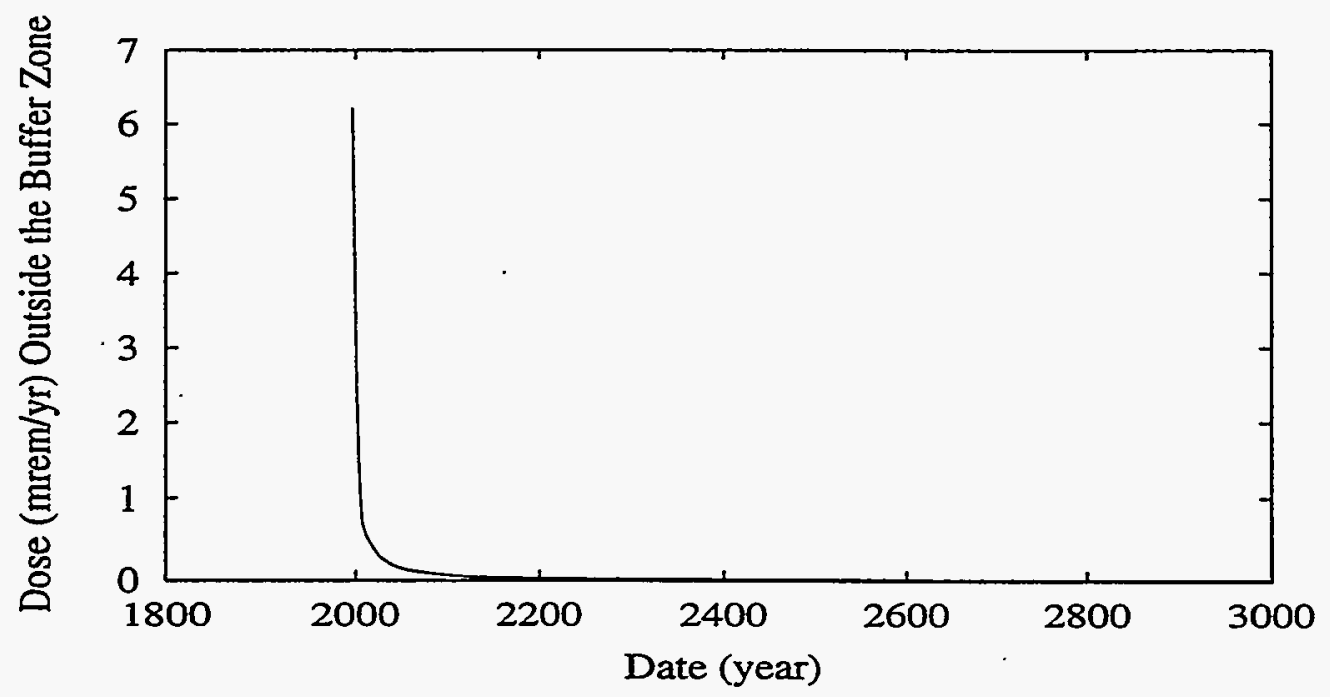

Figure 4.34. Maximum Dose Versus Time Outside the Buffer Zone for the Agricultural Scenario and the Dose the Various Radionuclides/Sources Contribute. Shown are:

g) Selenium-79, h) Technetium-99 Contribution from Existing Plumes. 
(i) Dose from future Tc-99 from 216 Sources for Agricultural Scenario

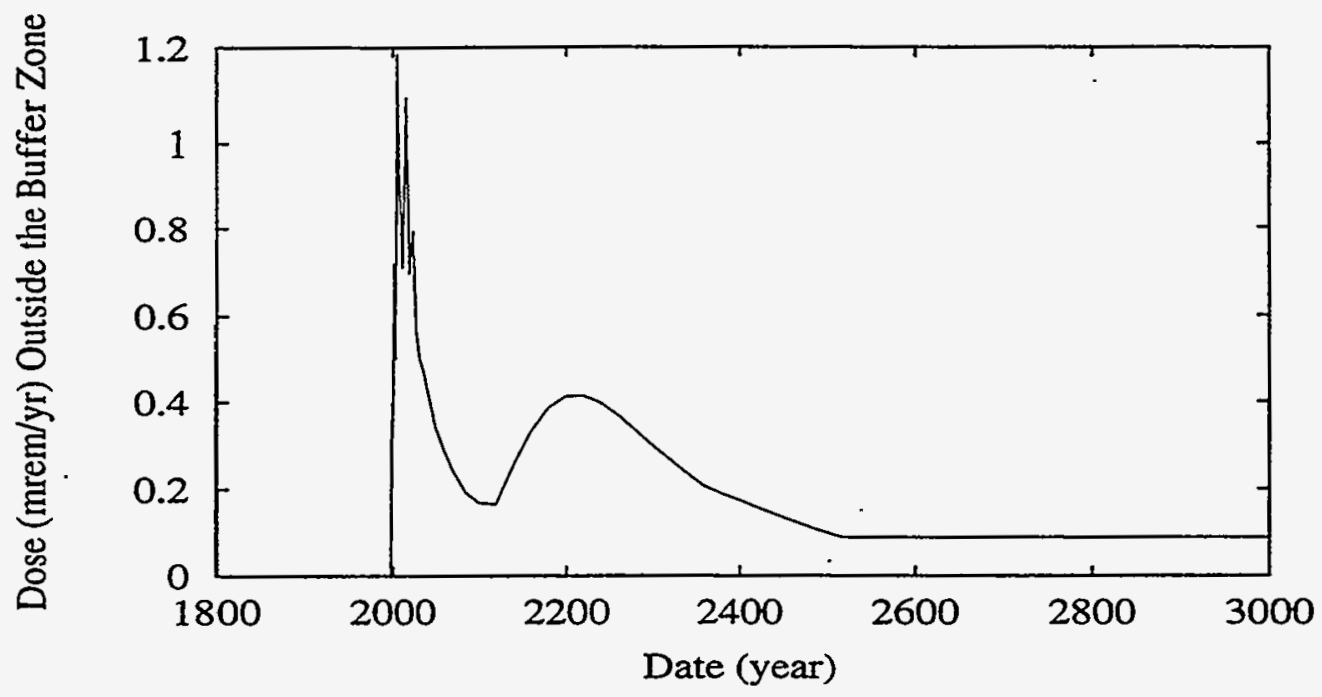

(j) Dose from future Tc-99 from Tank Sources for Agricultural Scenario

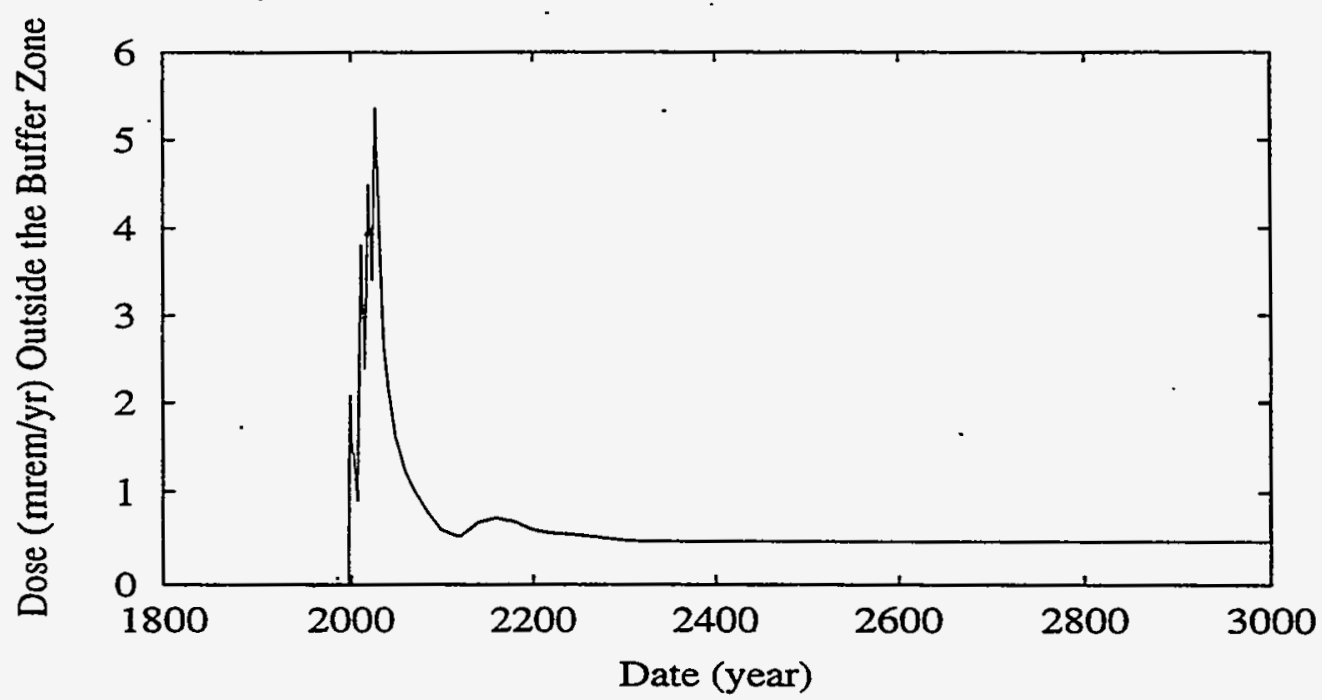

Figure 4.34. Maximum Dose Versus Time Outside the Buffer Zone for the Agricultural Scenario and the Dose the Various Radionuclides/Sources Contribute. Shown are:

i) Technetium-99 Contribution from Liquid Discharges, j) Technetium-99 Contribution from Tank Sources. 
(k) Dose from future Tc-99 from 218 Sources for Agricultural Scenario

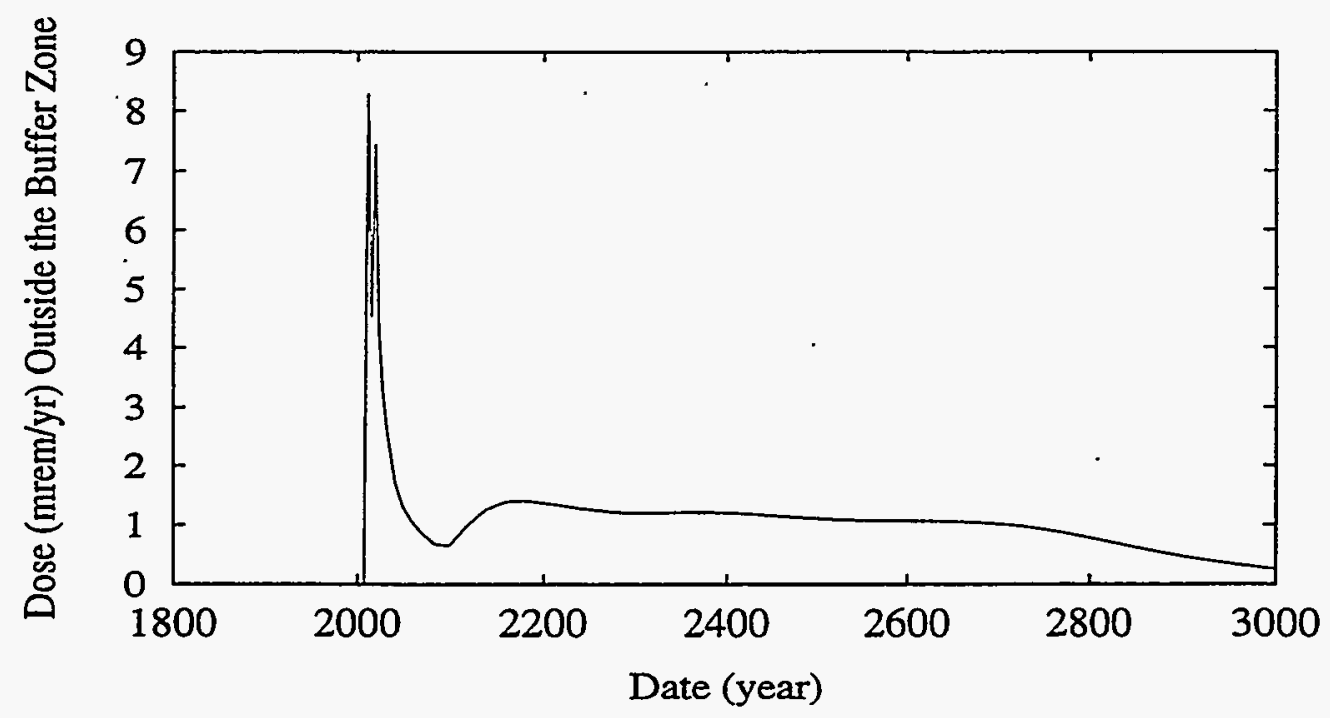

(1) Dose from existing I-129 Plumes for Agricultural Scenario

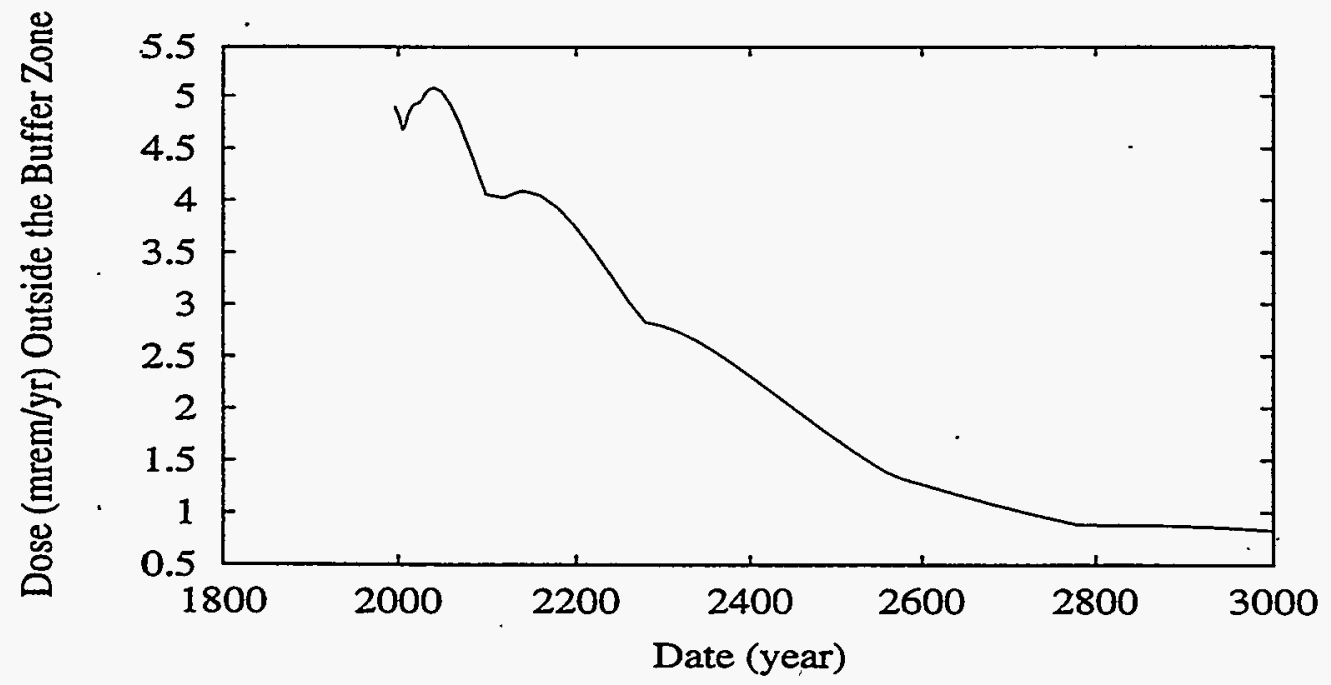

Figure 4.34. Maximum Dose Versus Time Outside the Buffer Zone for the Agricultural Scenario and the Dose the Various Radionuclides/Sources Contribute. Shown are: k) Technetium-99 Contribution from Solid Waste Sources, 1) Iodine-129 Contribution from Existing Plumes. 
(m) Dose from future I-129 Sources for Agricultural Scenario

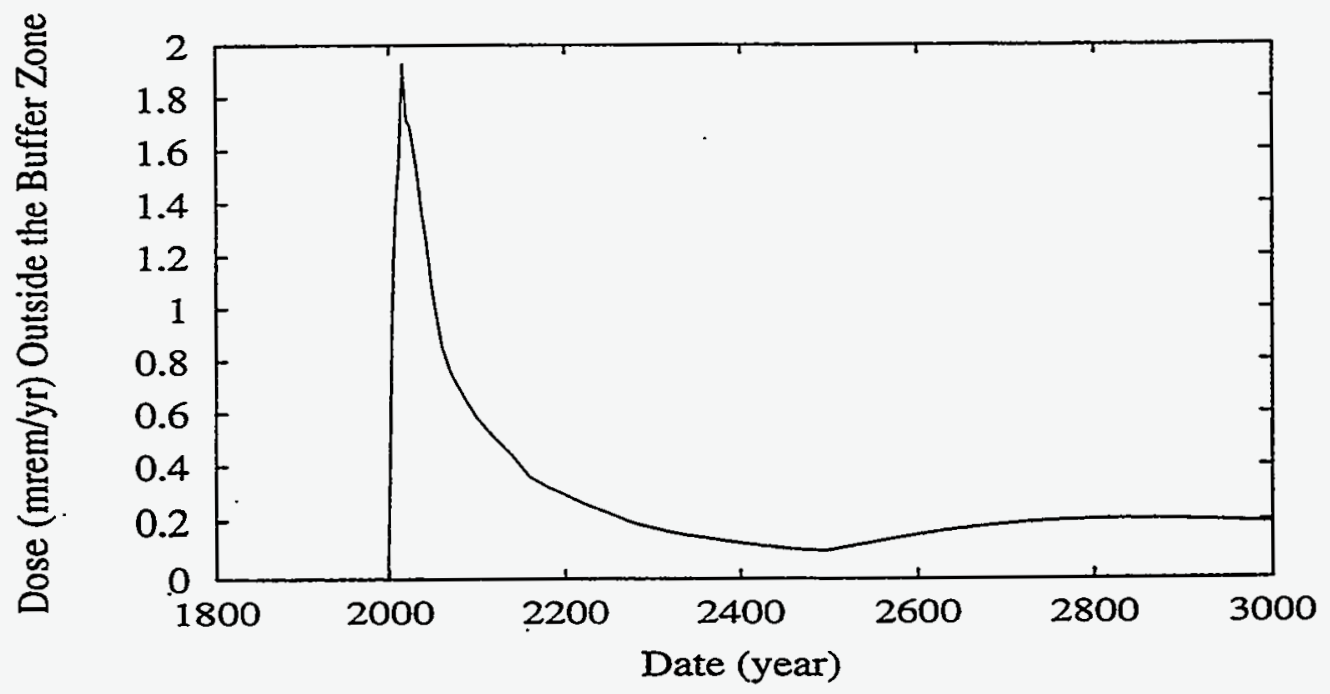

(n) Dose from existing U-total Plumes for Agricultural Scenario

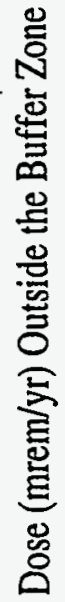

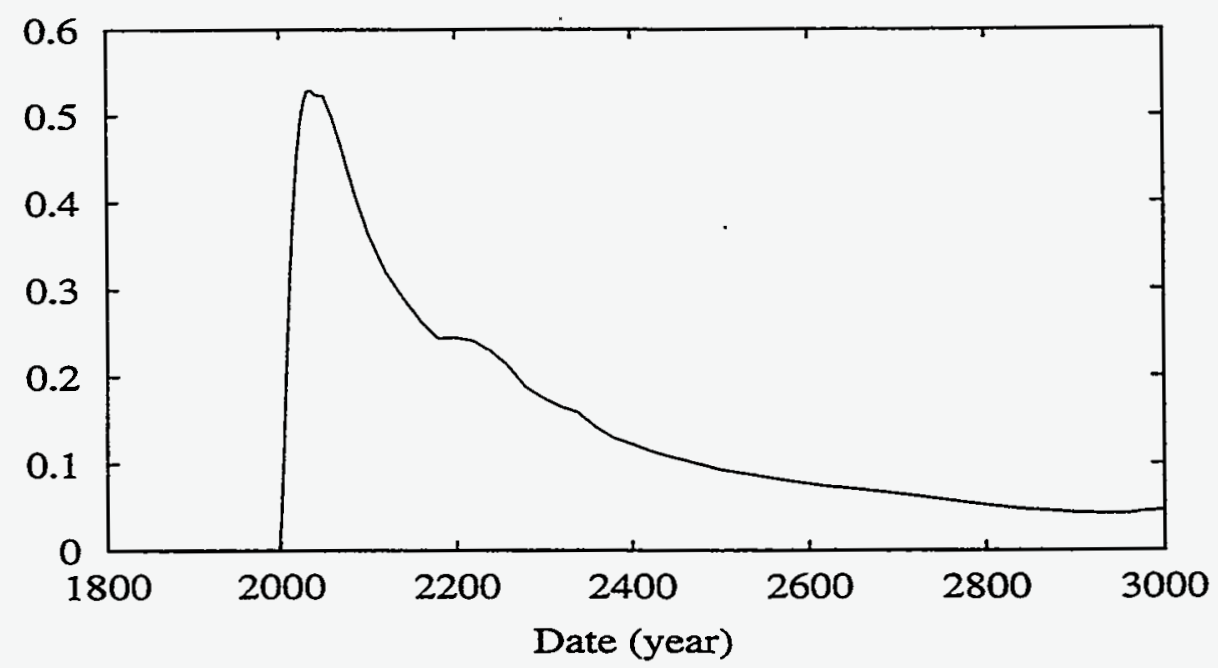

Figure 4.34. Maximum Dose Versus Time Outside the Buffer Zone for the Agricultural Scenario and the Dose the Various Radionuclides/Sources Contribute. Shown are:

m) Iodine-129 Contribution from Future Sources, n) Uranium (Total) from Existing Plumes. 
(o) Dose from future U-total Sources for Agricultural Scenario

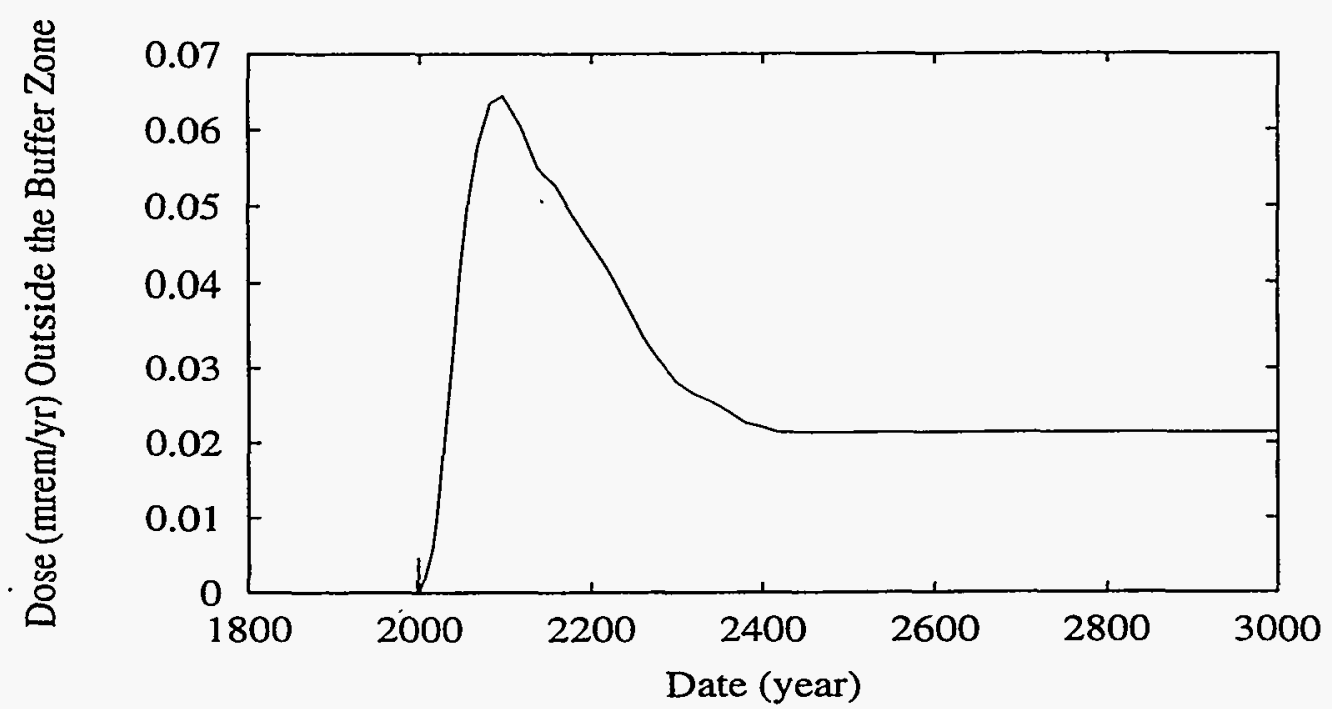

Figure 4.34. Maximum Dose Versus Time Outside the Buffer Zone for the Agricultural Scenario and the Dose the Various Radionuclides/Sources Contribute. Shown is:

o) Uranium (Total) from Future Sources. 
(a) Maximum Dose for Agricultural Scenario

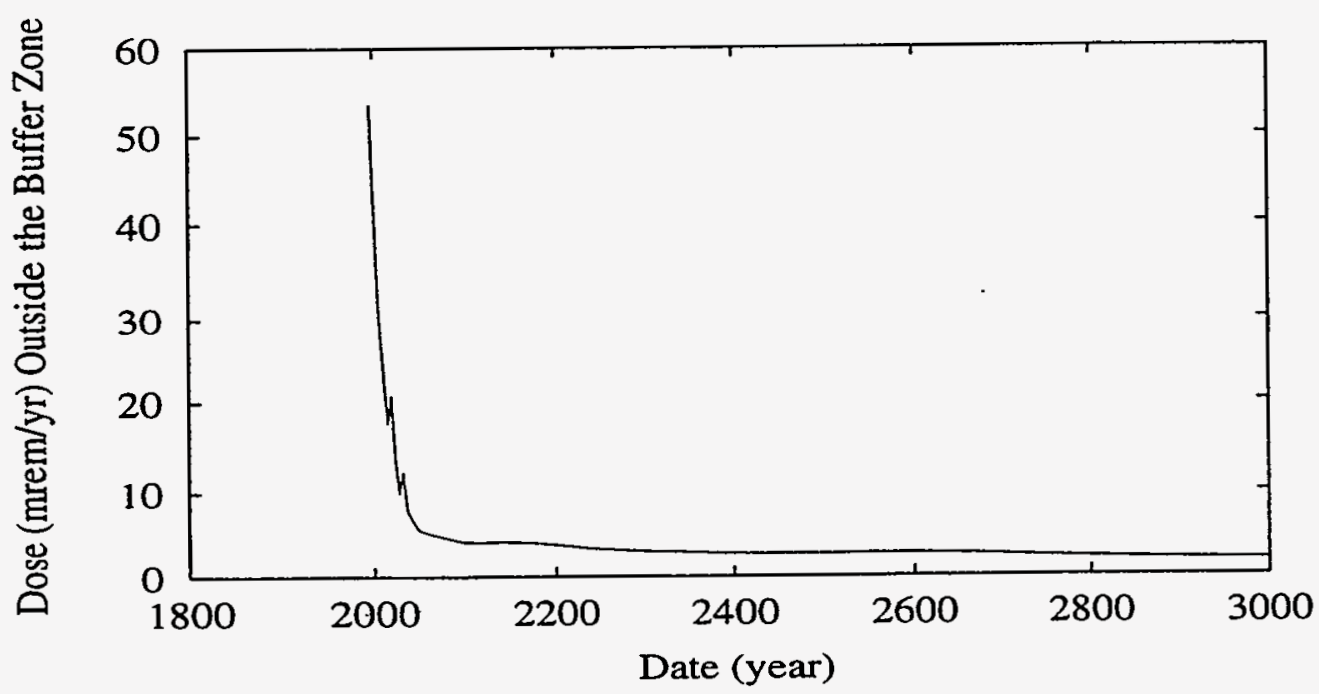

(b) Maximum Dose for Residential Scenario

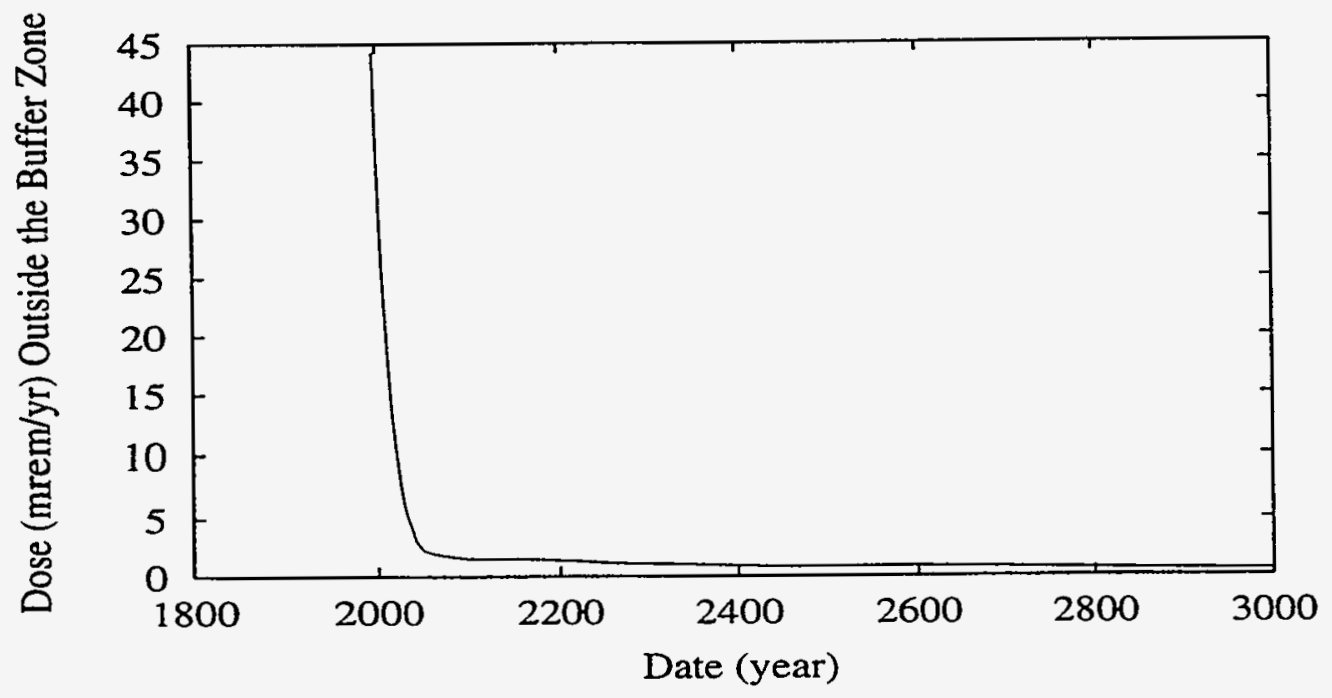

Figure 4.35. Composite Dose Outside the Buffer Zone from All Radionuclides and All Sources Modeled Versus Time for the a) Agricultural Scenario and b) Residential Scenario. 
(c) Maximum Dose for Recreational Scenario

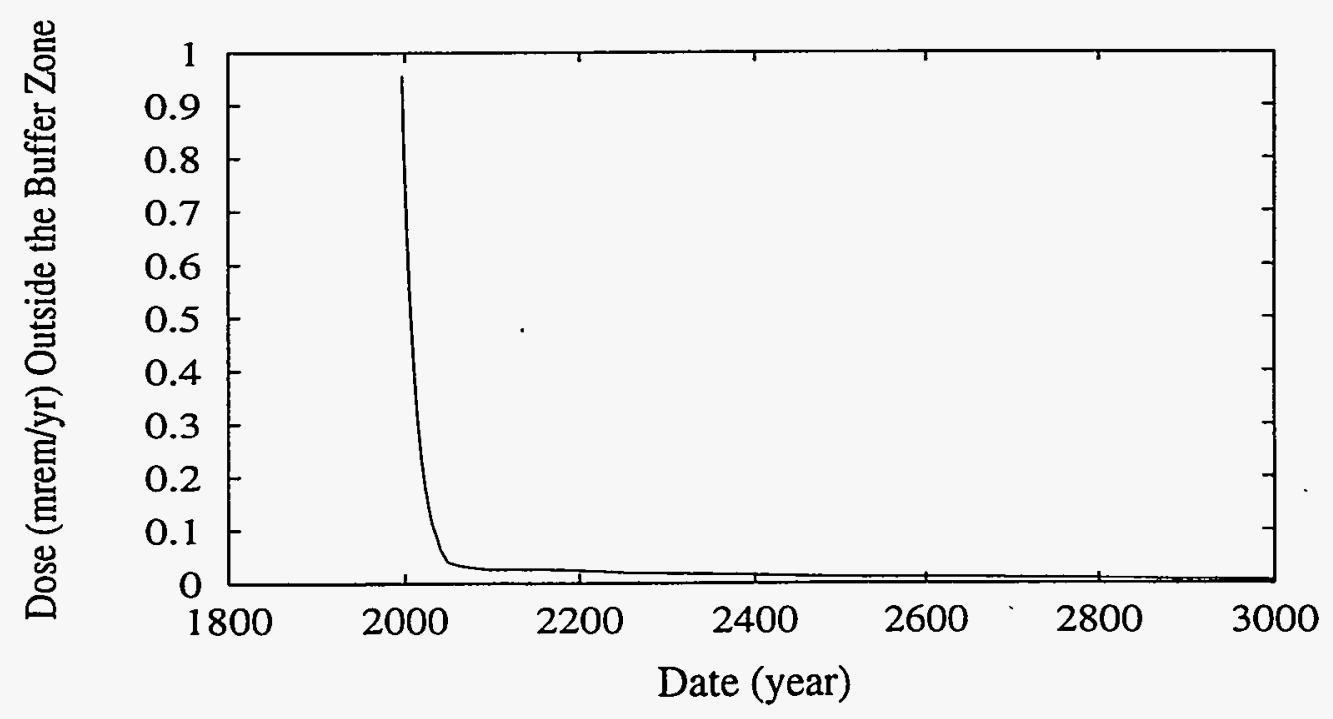

(d) Maximum Dose for Industrial Scenario

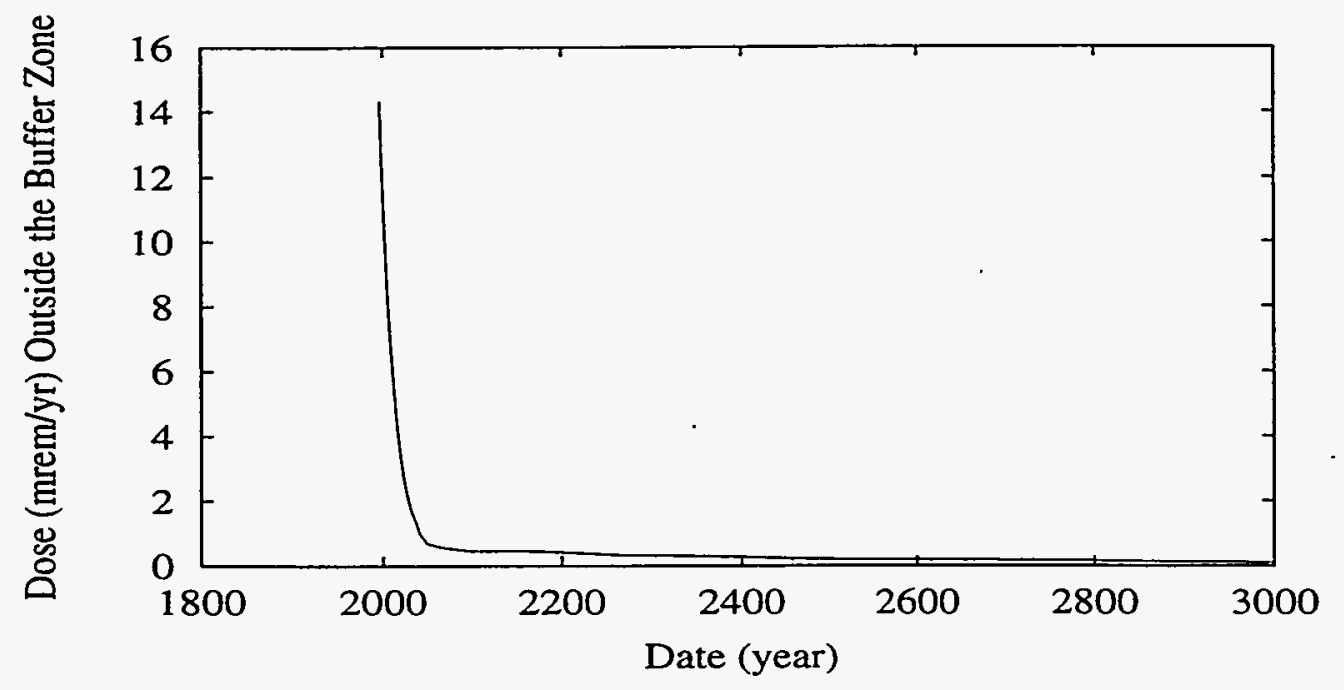

Figure 4.35. Composite Dose Outside the Buffer Zone from All Radionuclides and All Sources Modeled Versus Time for the c) Recreational Scenario and d) Industrial Exposure Scenario. 


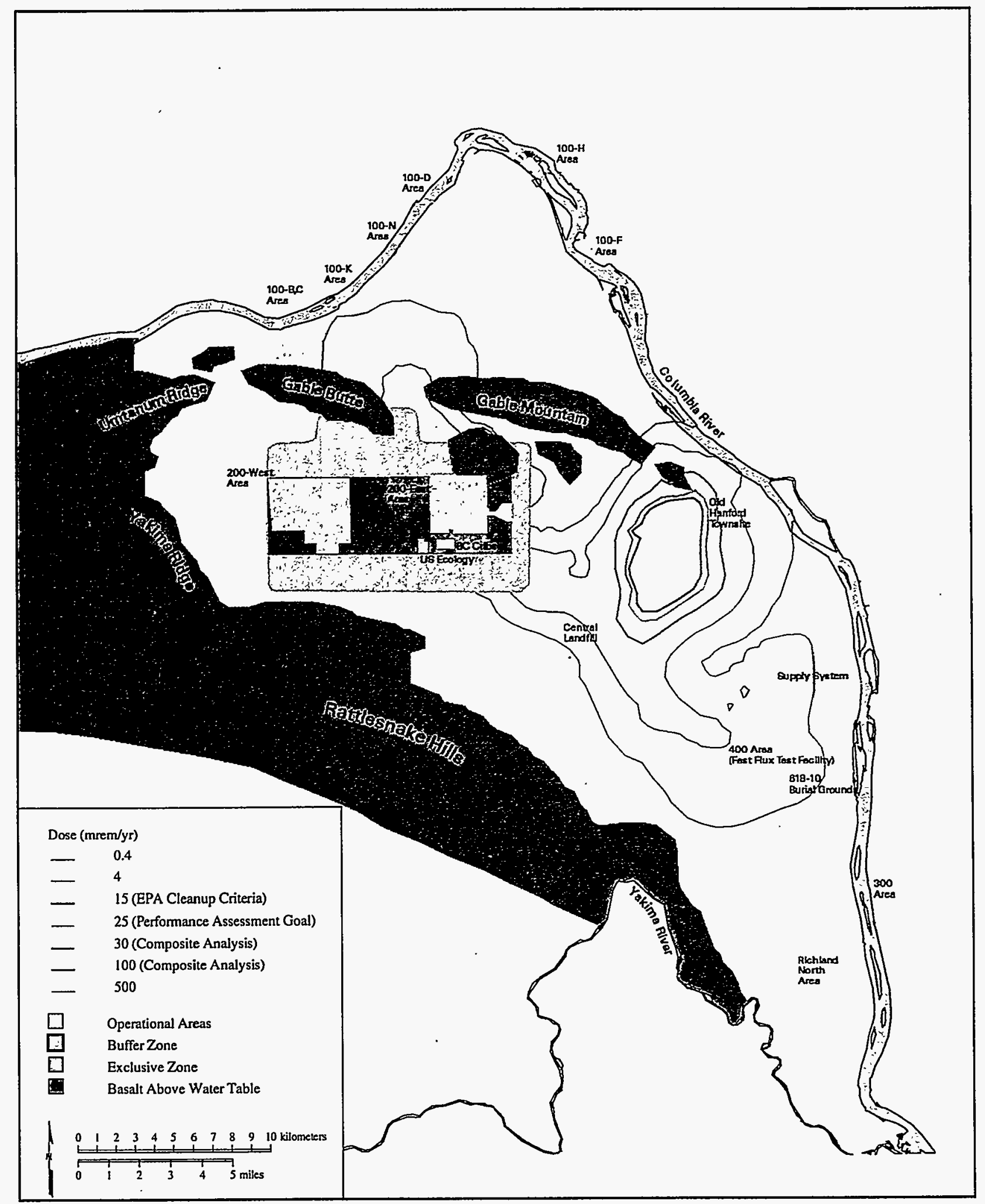

skw98022.eps December 19, 1997

Figure 4.36. Predicted Distribution of Composite Dose for the Agricultural Exposure Scenario in 1996 


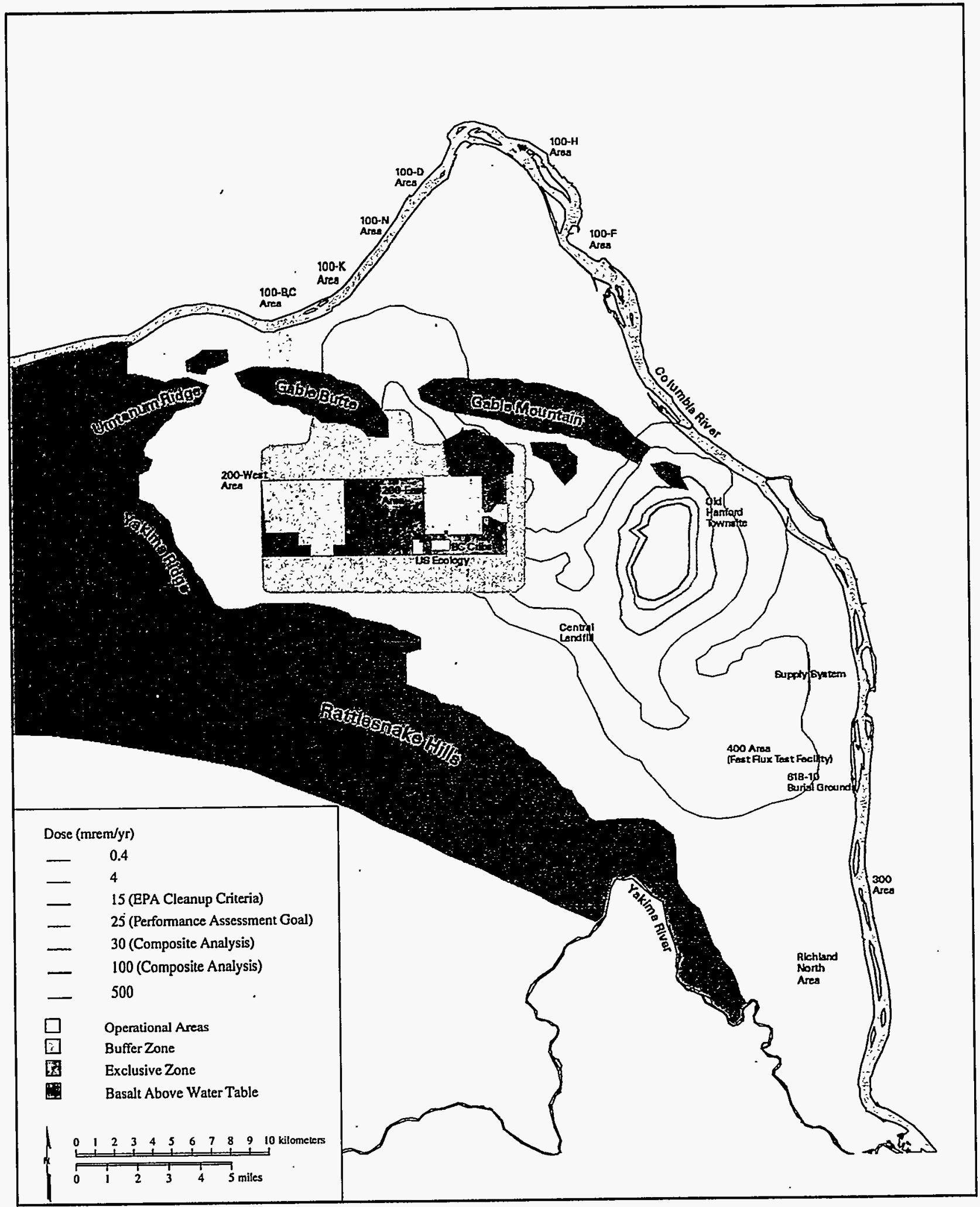

skw98023.eps December 19, 1997

Figure 4.37. Predicted Distribution of Composite Dose for the Residential Exposure Scenario in 1996 


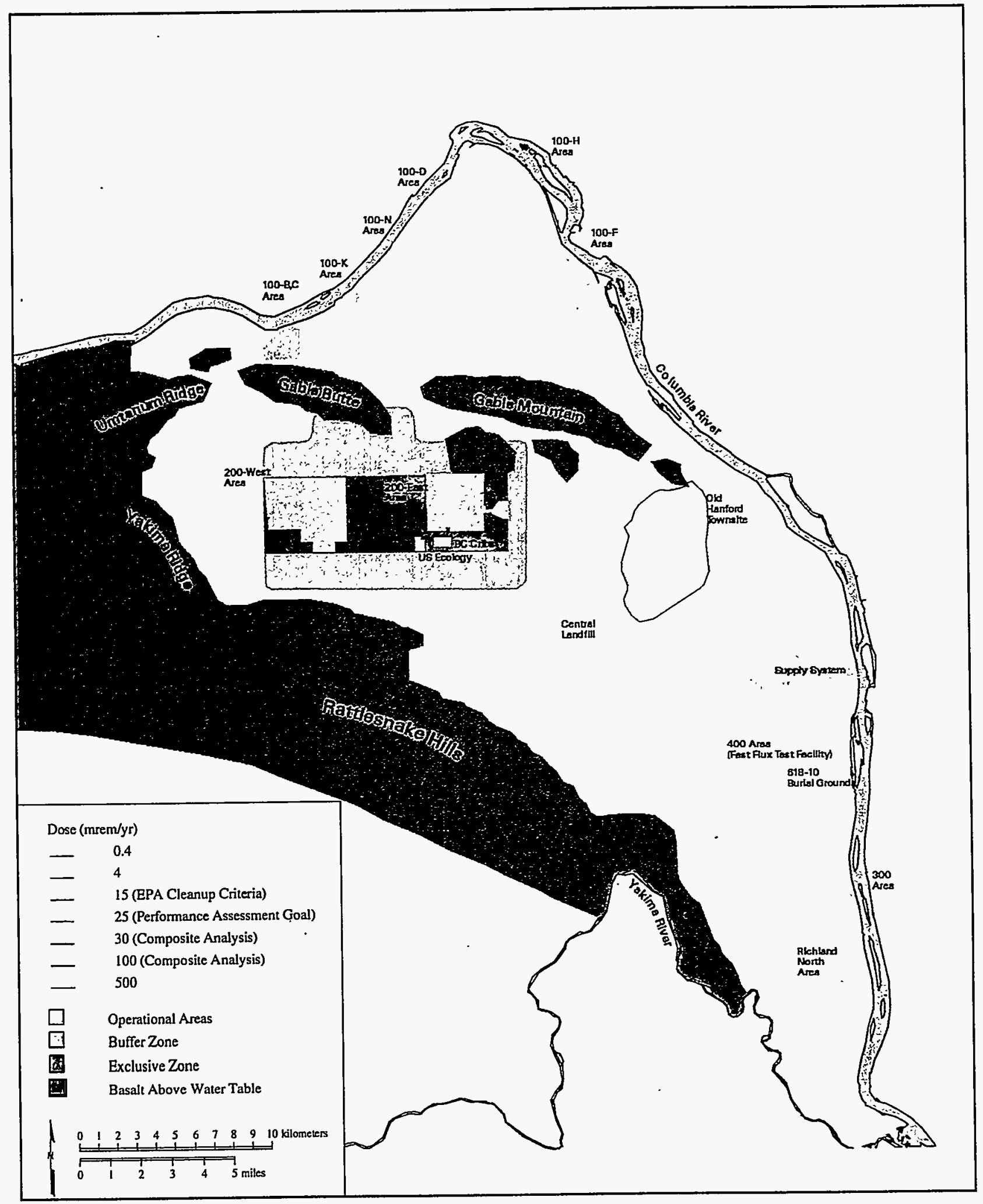

skw98024.eps December 19, 1997

Figure 4.38. Predicted Distribution of Composite Dose for the Recreational Exposure Scenario in 1996 


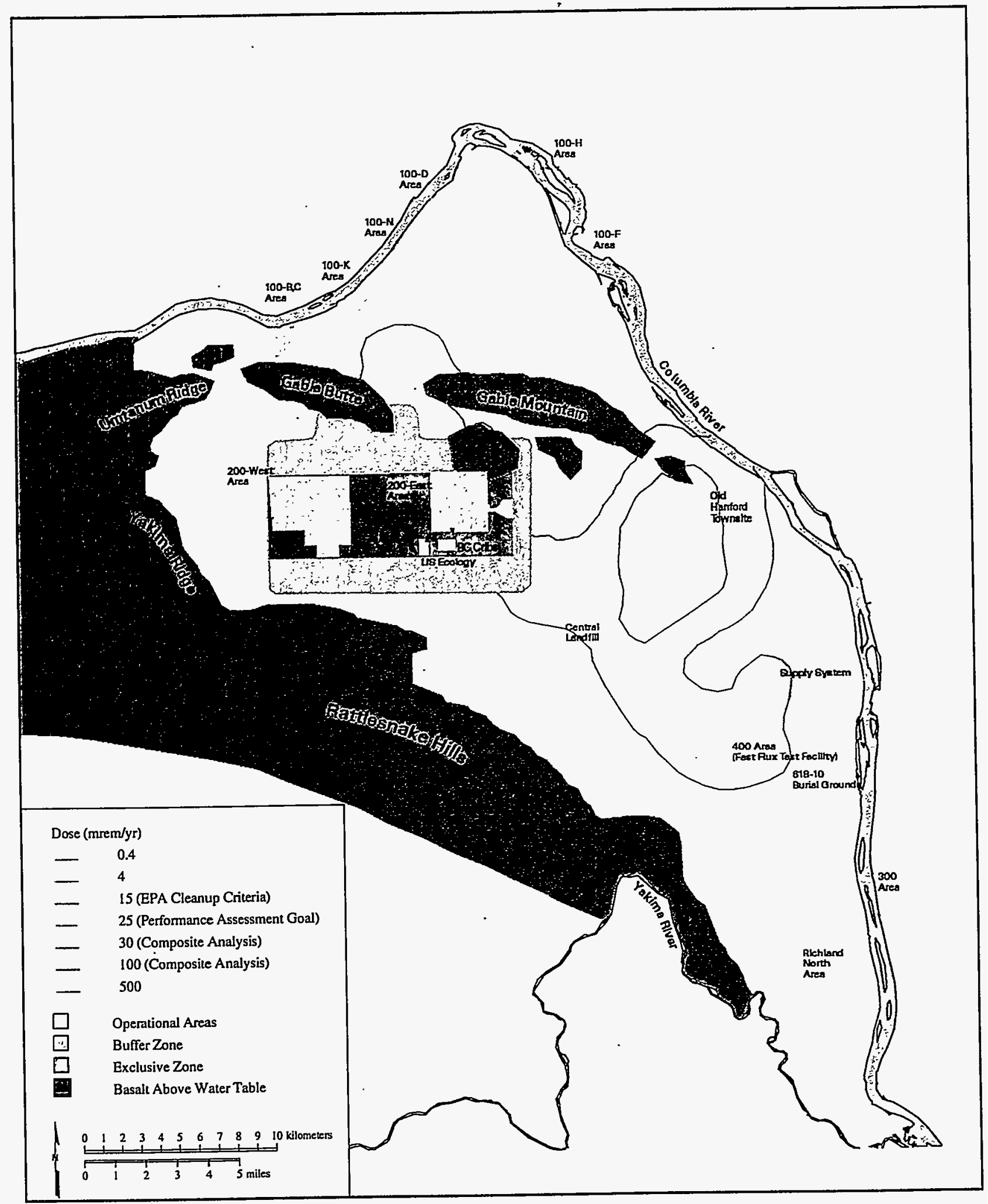

skw98025.eps December 19, 1997

Figure 4.39. Predicted Distribution of Composite Dose for the Industrial Exposure Scenario in 1996 


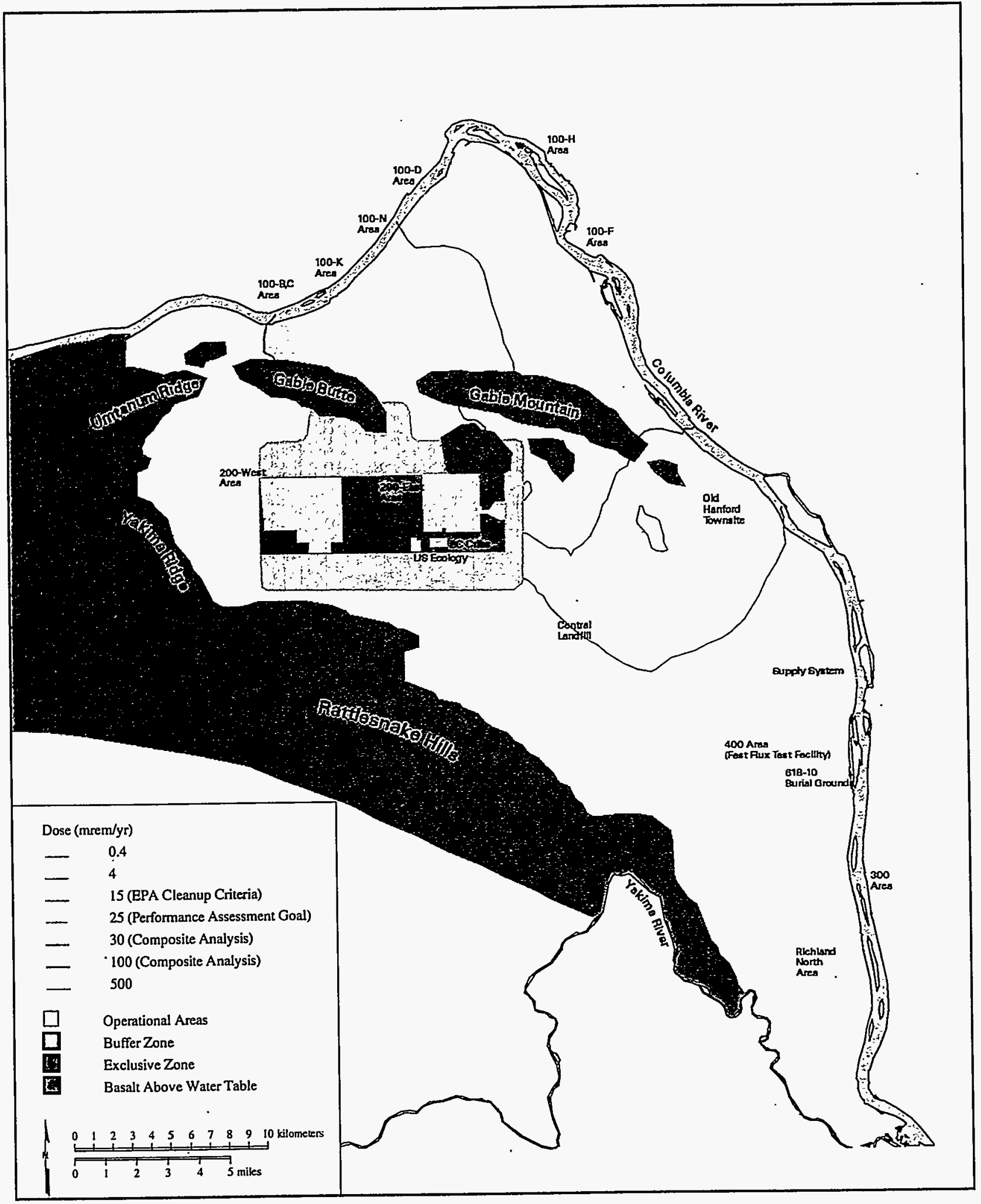

skw98027.eps December 19, 1997

Figure 4.40. Predicted Distribution of Composite Dose for the Agricultural Exposure Scenario in 2049 


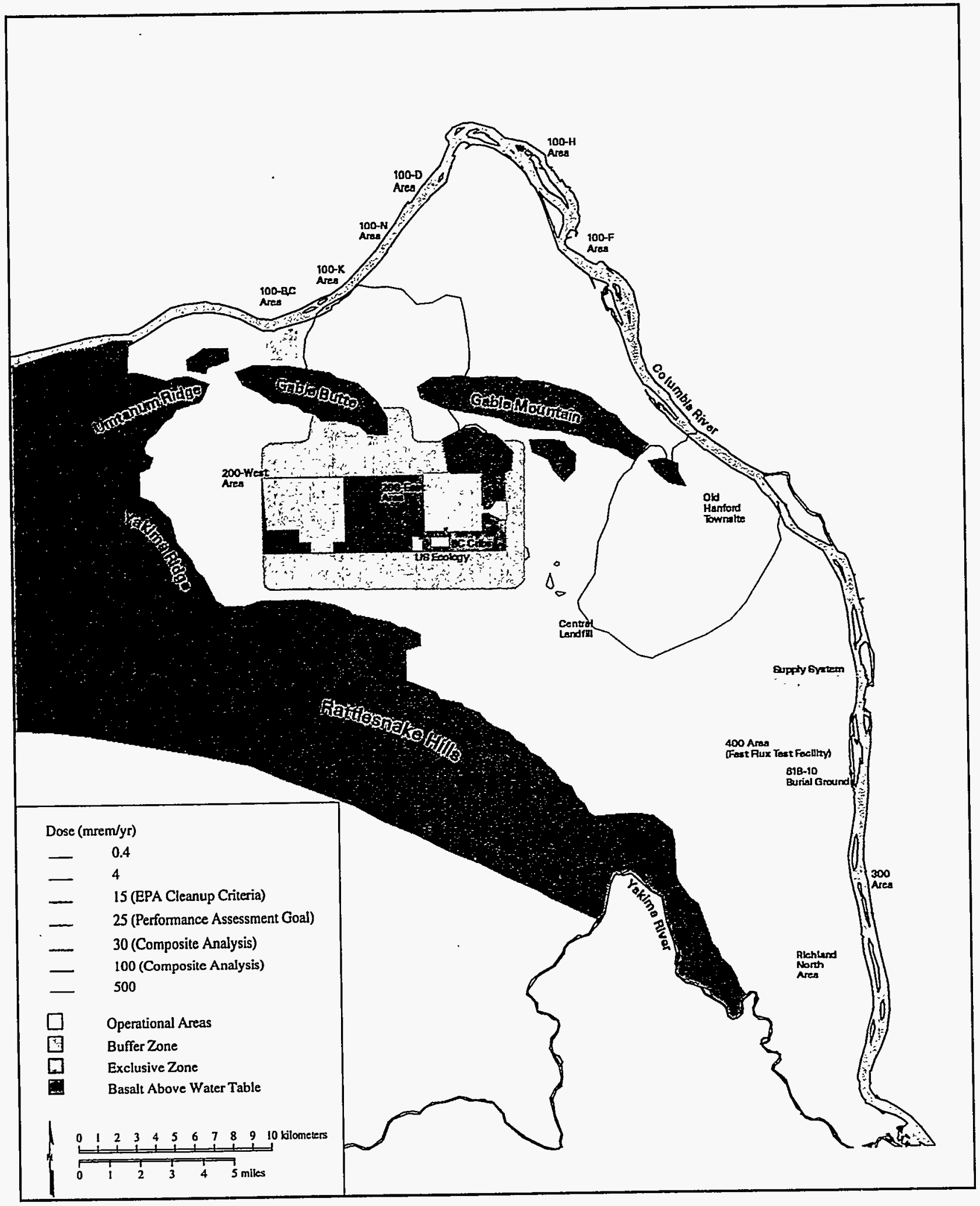

skw98028.eps December 19, 1997

Figure 4.41. Predicted Distribution of Composite Dose for the Residential Exposure Scenario in 2049 


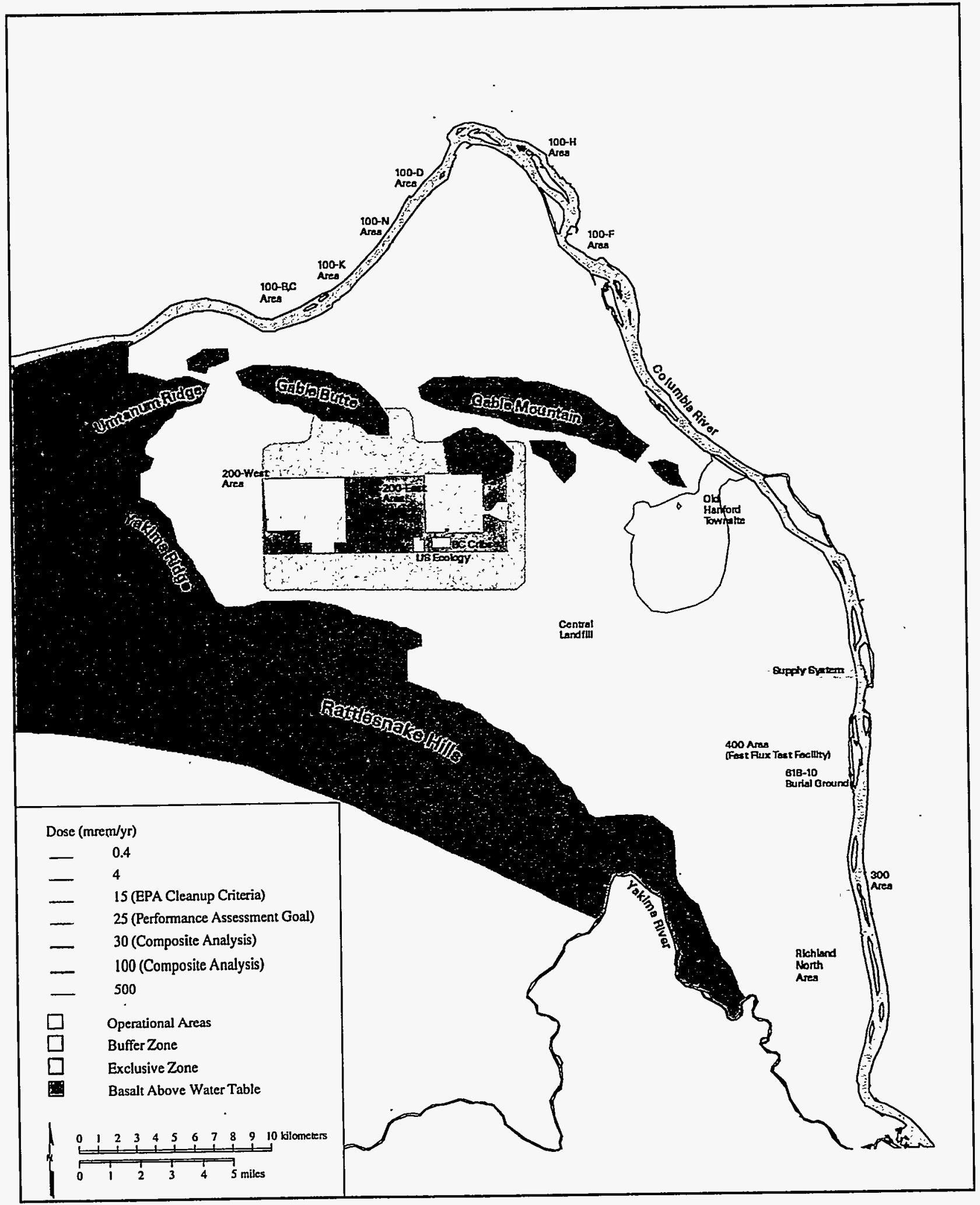

skw98030.eps December 19, 1997

Figure 4.42. Predicted Distribution of Composite Dose for the Industrial Exposure Scenario in 2049 


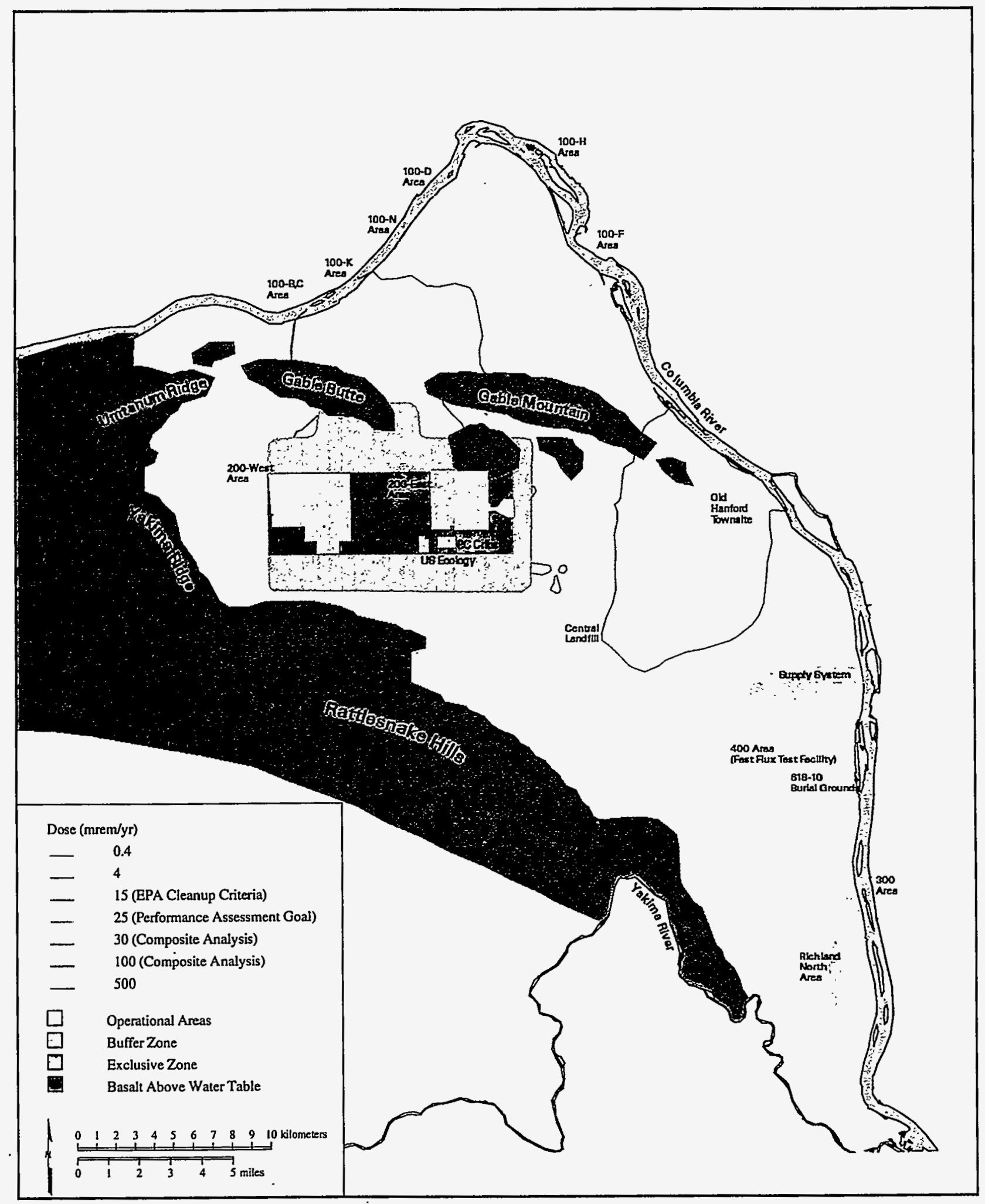

skw98032.eps December 19, 1997

Figure 4.43. Predicted Distribution of Composite Dose for the Agricultural Exposure Scenario in 2159 


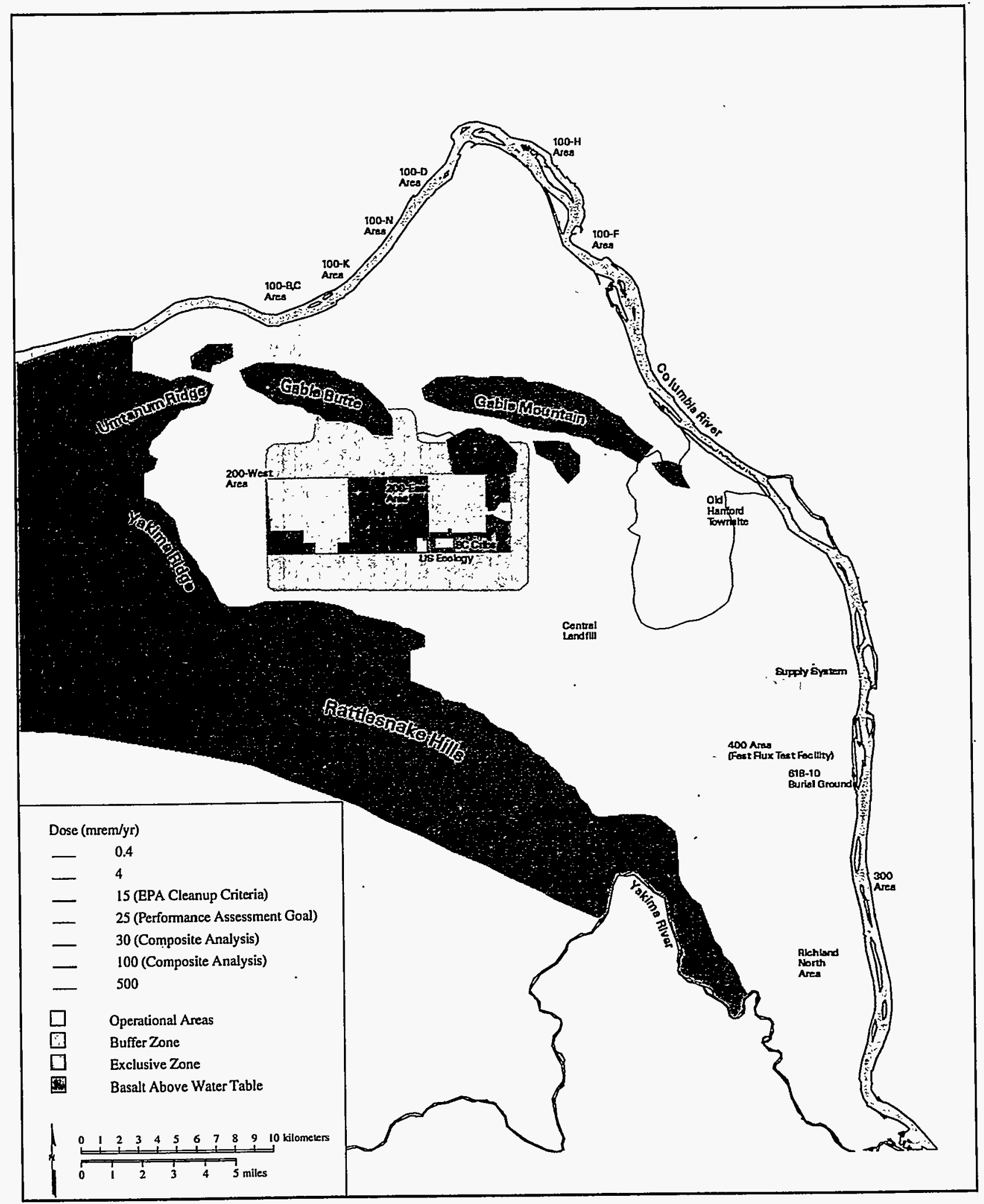

skw98033.eps December 19, 1997

Figure 4.44. Predicted Distribution of Composite Dose for the Residential Exposure Scenario in 2159 


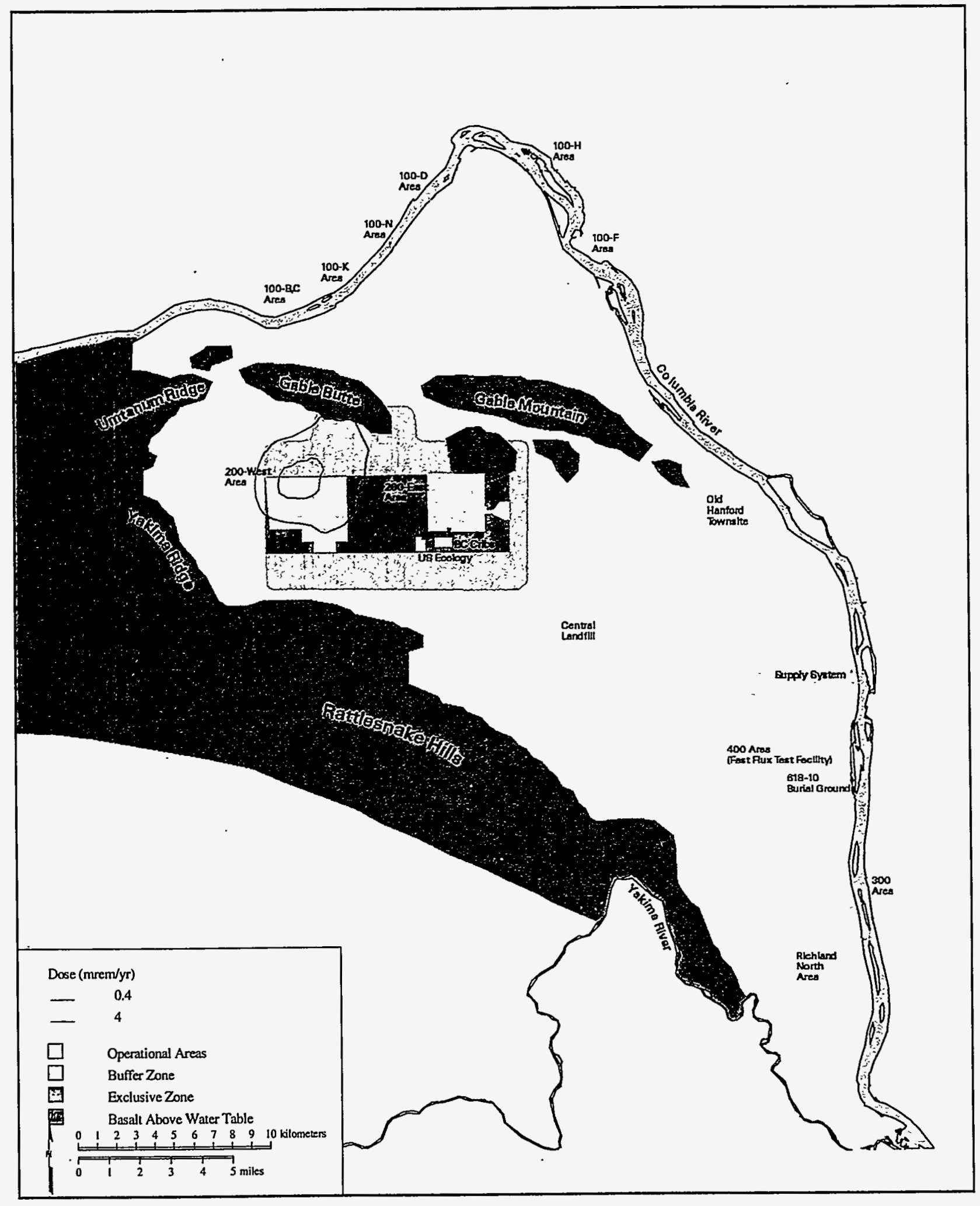

skw98062.eps January 06, 1998

Figure 4.45. Predicted Air Pathway Dose at 2999 (Time of Peak Dose Inside the Buffer Zone) 


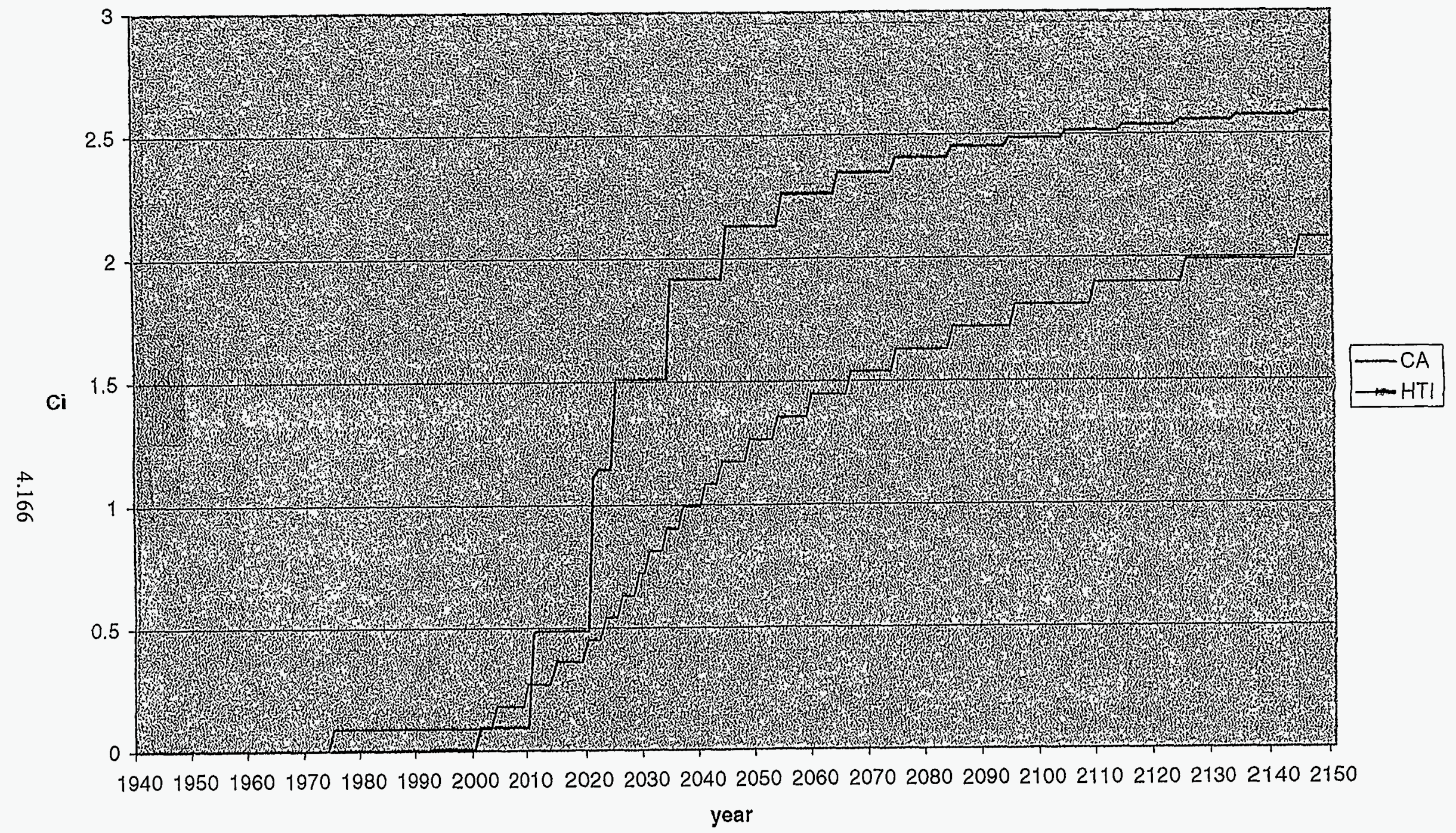

Figure 4.46. Comparison of Cumulative Release of Technetium-99 for AX Tank Farm Leaks (1940-2150) 

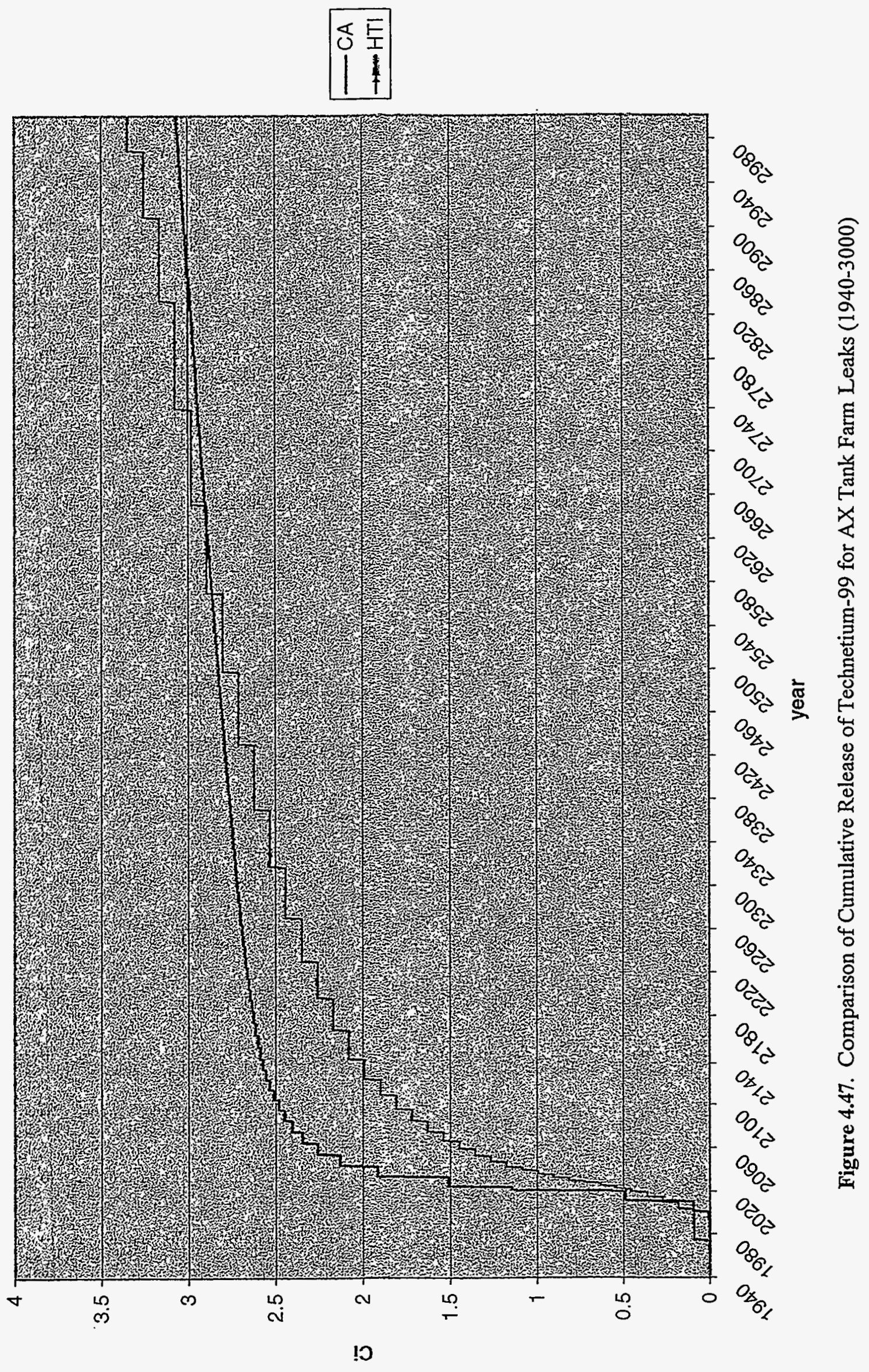


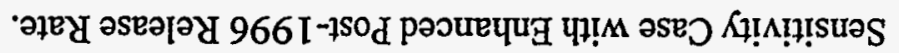

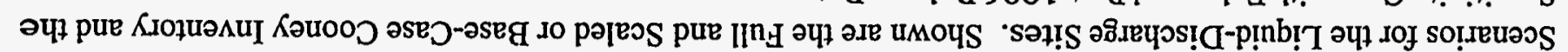

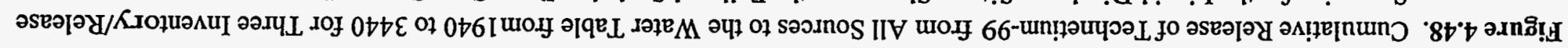

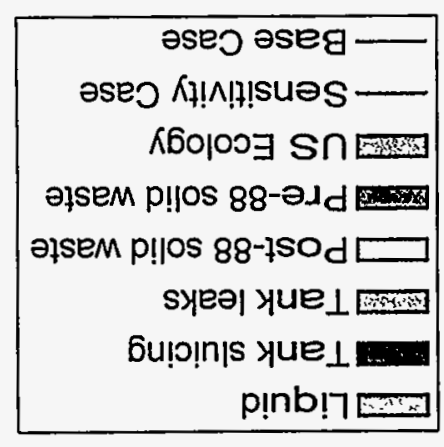

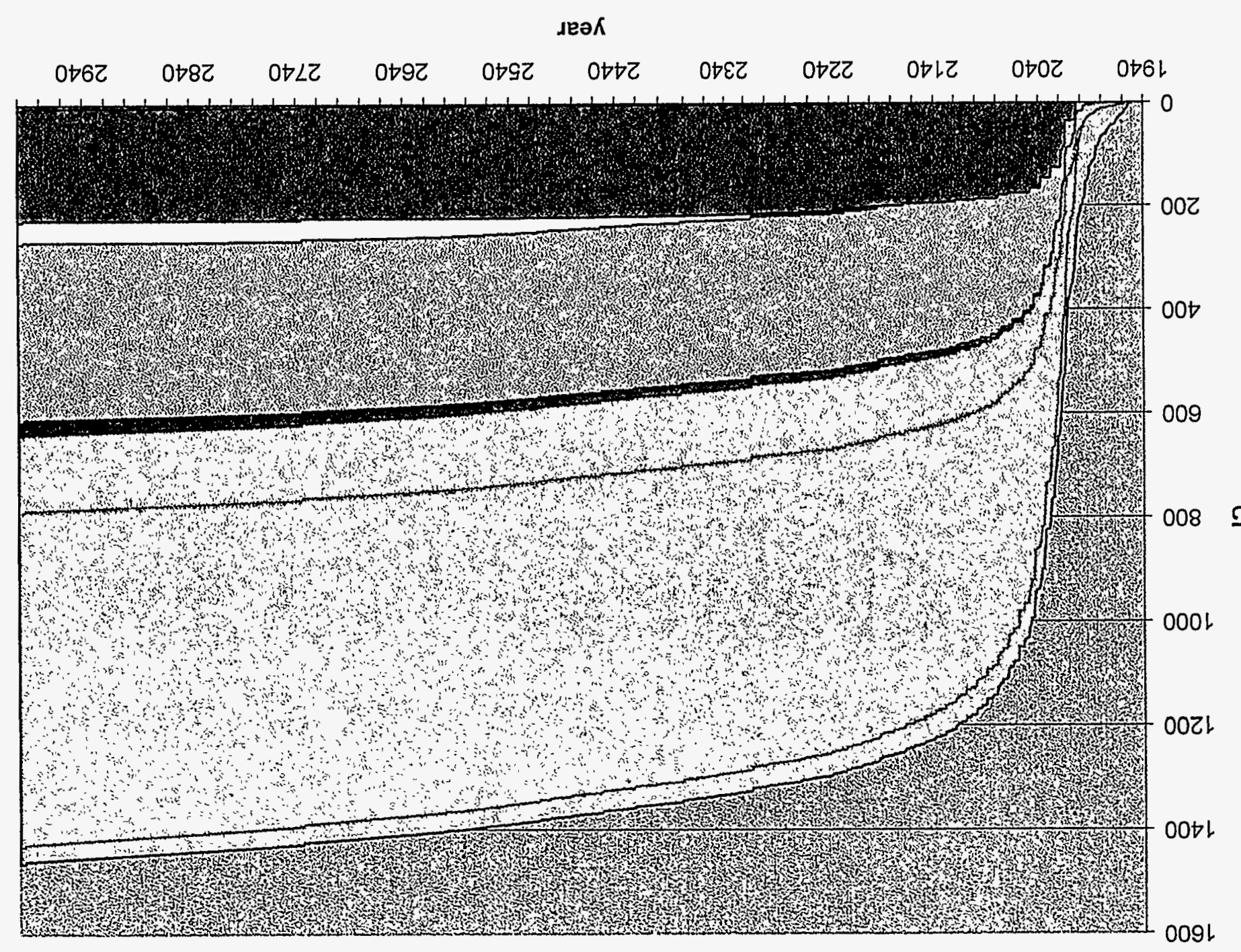




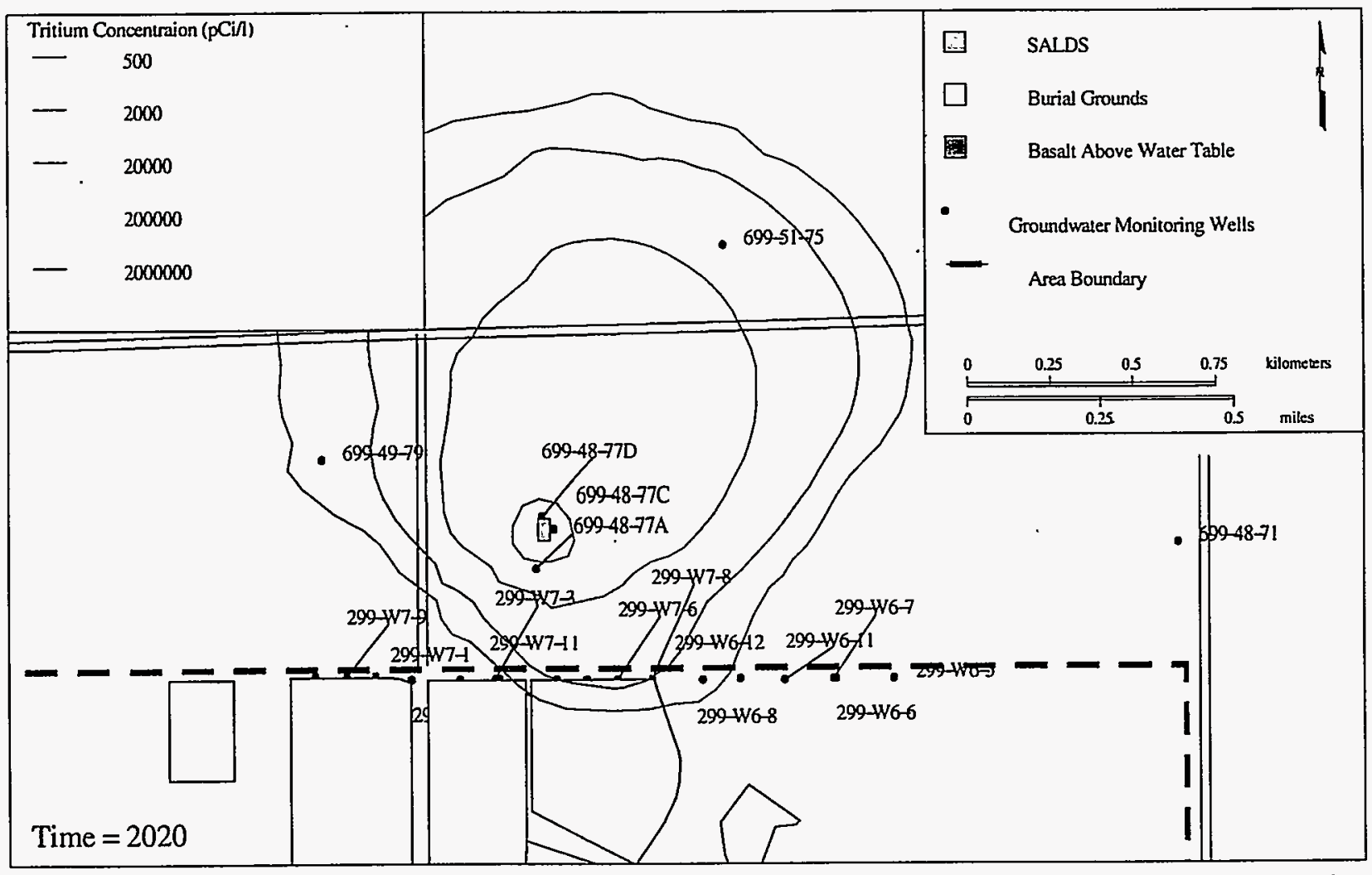

Figure 4.49a. Areal Distribution of Tritium Predicted in 2020 Near the SALDS with the High-Resolution, Three-Dimensional, Transport Model (from Barnett et al. 1997) 


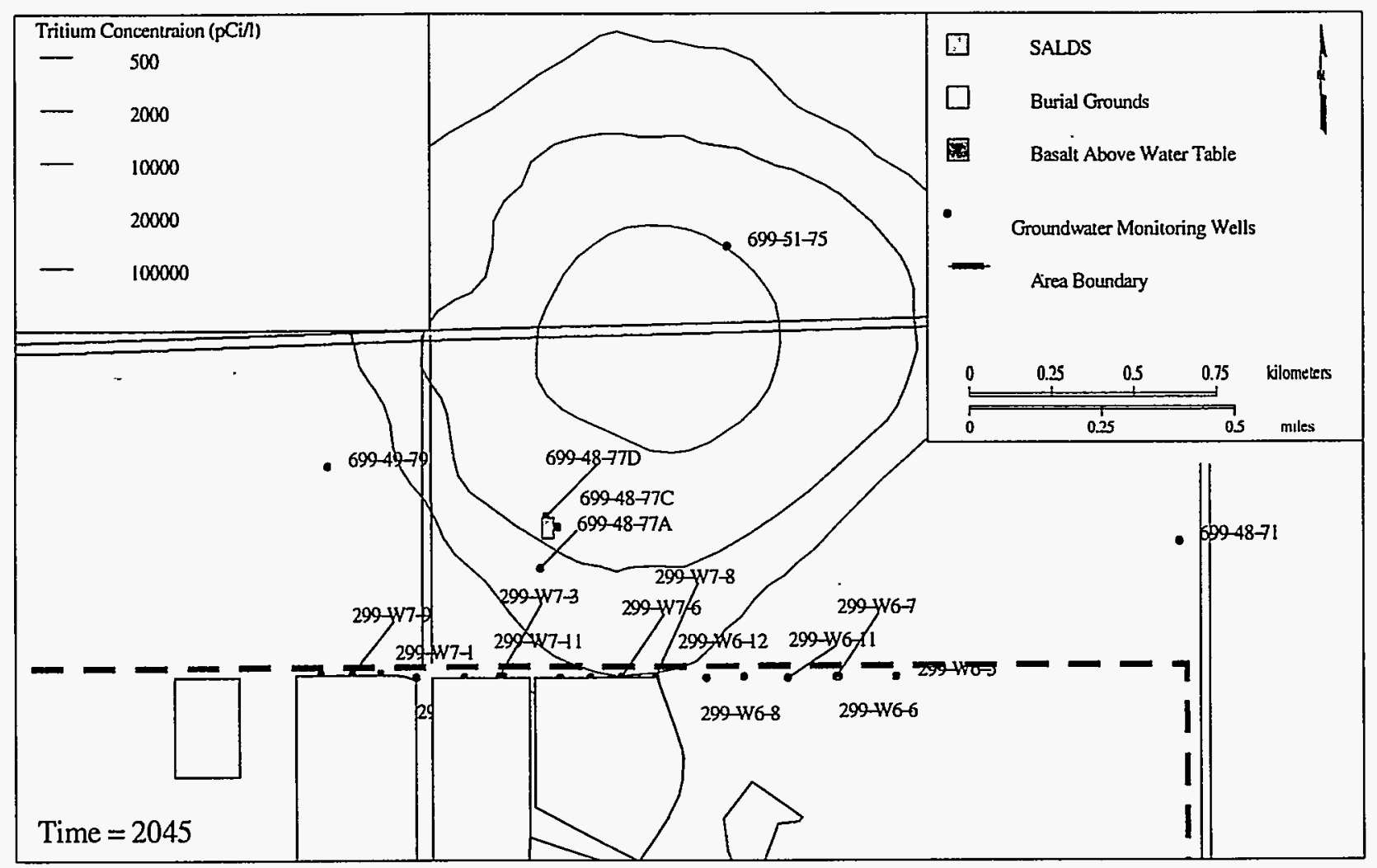

Figure 4.49b. Areal Distribution of Tritium Predicted in 2045 Near SALDS with the High-Resolution, Three-Dimensional, Transport Model (from Barnett et al. 1997) 


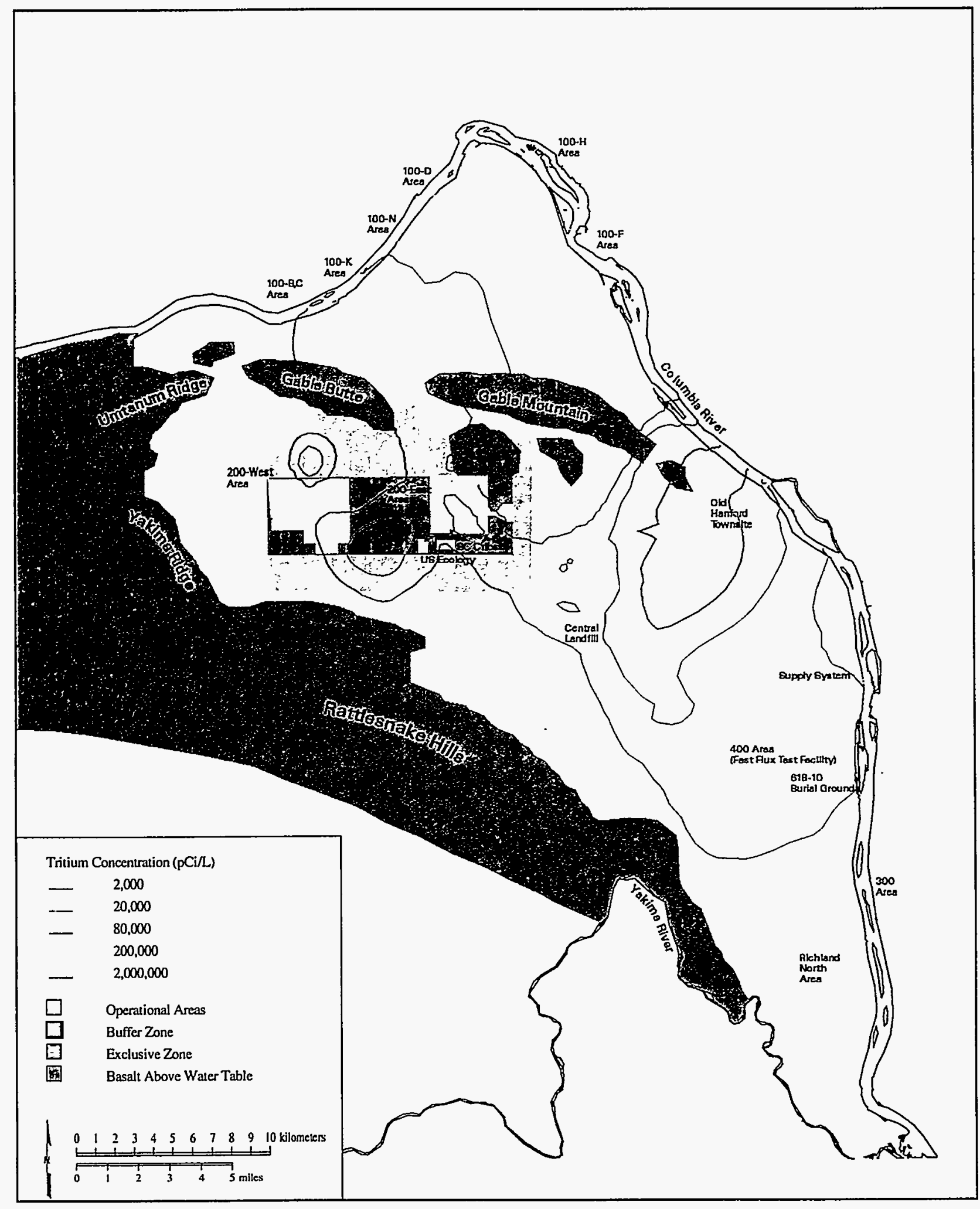

97skw034.eps December 30, 1997

Figure 4.50. Areal Distribution of Tritium in 2020 as Predicted with the Three-Dimensional Transport Model (from Cole et al. 1997) 


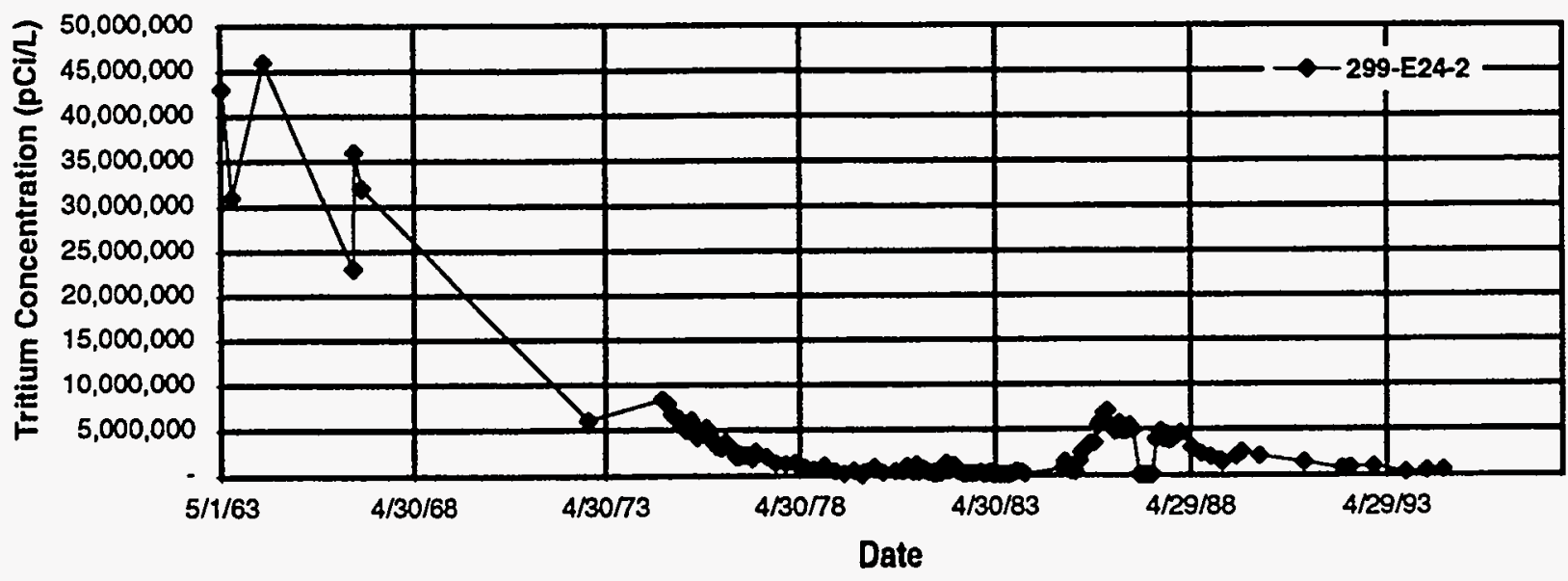

Figure 4.51. Tritium Concentration History for Observation Well 299-E24-2

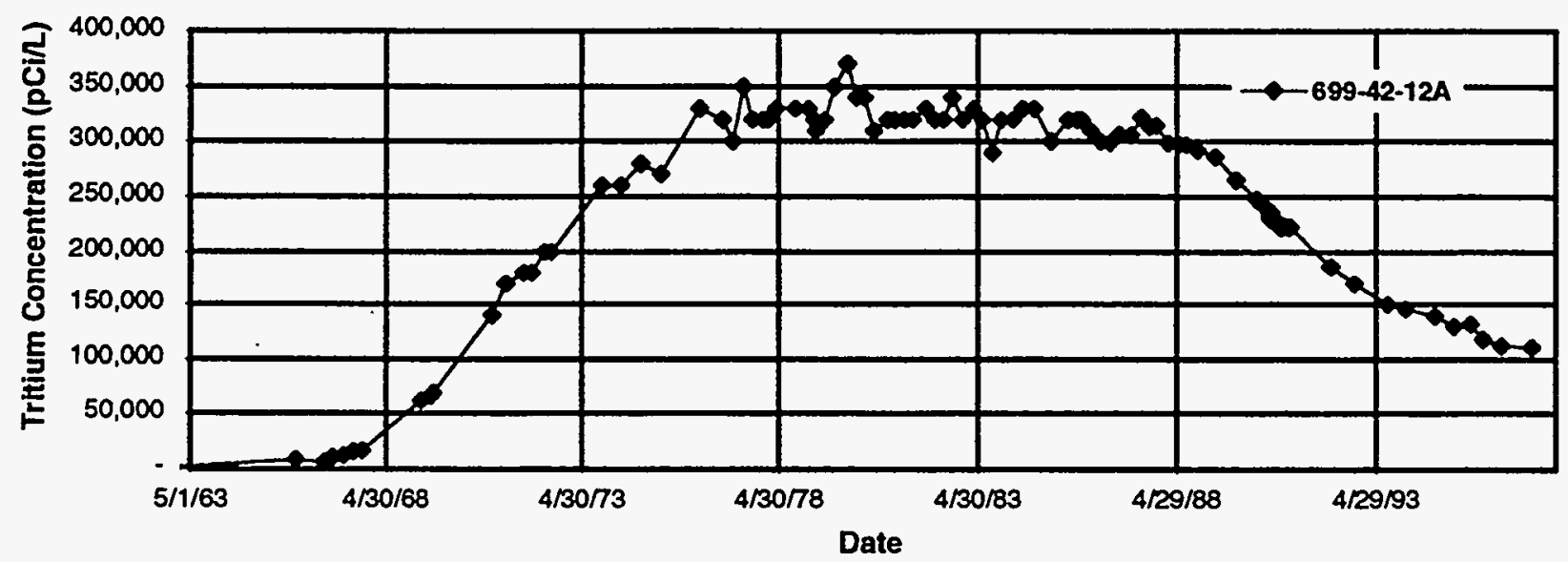

Figure 4.52. Tritium Concentration History for Observation Well 699-42-12A

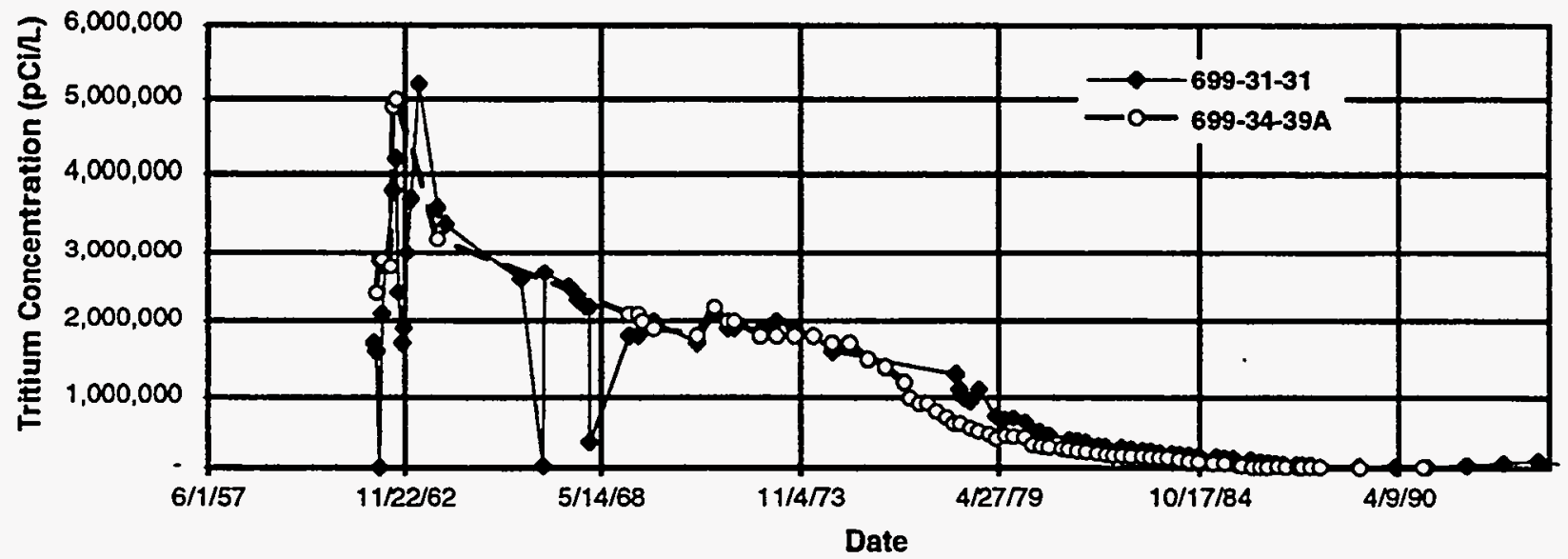

Figure 4.53. Tritium Concentration History for Observation Wells 699-31-31 and 699-34-39A 


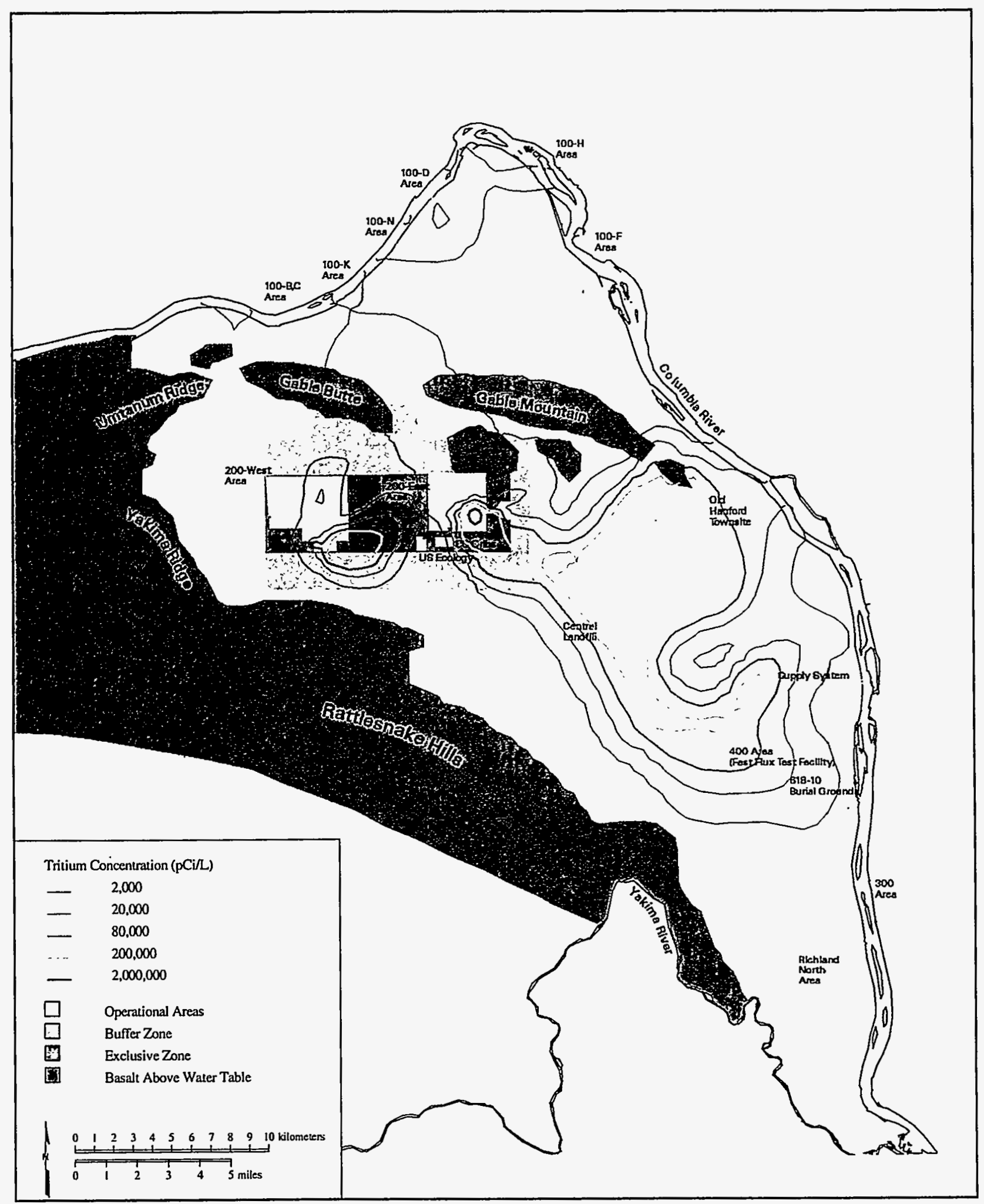

97skw031.eps December 30, 1997

Figure 4.54. Areal Distribution of Tritium in 1985 as Predicted with the Three-Dimensional Transport Model (from Cole et al. 1997) 


\subsection{Interpretation of Results}

This chapter discusses the results of the Composite Analysis in comparison with the primary dose limit and the dose constraint. It includes discussions of the principle sources of uncertainty, and the implications they have for results of the base case. The results of the Composite Analysis are interpreted and salient issues are discussed. A brief qualitative ALARA (as low as reasonably achievable) assessment is presented to quantify the value to society of a detailed options analysis and ALARA assessment for alternate remediations. Finally, suggestions are made for further study in preparation for the second and subsequent iterations of the Composite Analysis.

\subsection{Discussion of Results}

The U.S. Department of Energy (DOE) primary dose limit of 100 mrem effective dose equivalent (EDE) in a year applies to a hypothetical future member of the public. This all-pathways dose to the maximally exposed offsite individual is calculated for 1000 years at points on the Hanford Site that a future member of the public could access. The point of access nearest the waste disposals in the future is defined by the boundary of a buffer zone designed to separate the public from the exclusive waste management area on the 200 Area Plateau (Figure 1.4). The dose constraint is defined as 30 mrem EDE in a year to the maximally exposed offsite individual for 1000 years (DOE 1996b), and is used to ensure that no single source, practice, or pathway uses an extraordinary portion of the primary dose limit. If the dose to the maximally exposed individual is above either 100 or $30 \mathrm{mrem}$ in a year, an options analysis and an ALARA assessment must be performed to evaluate alternate actions the DOE could take to reduce the dose. If the dose is below $30 \mathrm{mrem}$ in a year, a qualitative ALARA assessment should be performed to determine whether a quantitative ALARA analysis would be cost-beneficial.

\subsubsection{Comparison with the Primary Dose Limit}

To quantify potential impacts from alternate future land uses, four scenarios were used in the Hanford Site Composite Analysis to quantify dose to the hypothetical future member of the public. In order of significance with respect to the dose they yield, they are based on agricultural, residential, industrial, and recreational land use assumptions. Each of these scenarios was applied to the region of the present Hanford Site outside the buffer zone surrounding the exclusive waste management area. Maximum dose within the exclusion area and buffer zone was not compared to the dose limit.

As described in Chapter 4, a review of existing radionuclide plumes in the unconfined aquifer revealed the presence of a strontium- 90 plume beneath the decommissioned Gable Mountain Pond. The observed peak concentration of strontium-90 in the vicinity of the retired pond was $1500 \mathrm{pCi} / \mathrm{L}$ in 1996 (Hartman and Dresel 1997; Figure 6.10-10). Using the unit dose factor for strontium-90 from the agricultural scenario, this concentration in groundwater converts to a dose of $\sim 470$ mrem in a year. If the site is not remediated to remove the strontium- 90 in groundwater and in the overlying vadose zone, it is 
recommended the exclusive waste management area be expanded to include this decommissioned pond. Furthermore, it is also recommended a buffer zone of $\sim 1000 \mathrm{~m}$ be established as a region of relatively clean groundwater surrounding the existing strontium- 90 plume such that monitoring can detect movement of the strontium. Strontium is highly sorbed on aquifer sediments $\left(\mathrm{K}_{d}=20 \mathrm{~mL} / \mathrm{g}\right)$ and its decay half-life is relatively short, 28.78 years. It is anticipated the declining water table will cause strontium in the upper sediments of the aquifer to be suspended in the vadose zone, and thereby act to further isolate the contamination. To simplify the discussion of results in the Composite Analysis, it is assumed the exclusive waste management area and buffer zone will be expanded as recommended. Hence, discussion of dose outside the buffer zone assumes the region surrounding Gable Mountain Pond. is included inside the exclusive waste management area and buffer zone.

For the agriculture scenario, which exhibits the greatest dose, the maximum dose simulated from the cumulative releases is less than $6 \mathrm{mrem}$ in a year during the regulatory period of 1000 years following Hanford Site closure for all lands outside the buffer zone. For this exposure scenario, the area extent of dose greater than 4 mrem in a year was projected to correspond with an area of $40 \mathrm{~km}^{2}$ in the unconfined aquifer outside the buffer zone at the year 2050, the time of Hanford Site closure. The aquifer area outside the buffer zone associated with this level of dose is projected to vanish by 2085 . Neither the primary limit, nor the dose constraint level is exceeded. During the regulatory period of 1000 years following Hanford Site closure the maximum doses simulated for the other scenarios are residential, 2.2 mirem in a year; industrial, $0.7 \mathrm{mrem}$ in a year; and recreational, $0.04 \mathrm{mrem}$ in a year.

This analysis has shown that in the first 1000 years after Hanford Site closure, maximum dose to an individual outside the buffer zone occurs at the time of closure and diminishes thereafter. Current groundwater contamination and its corresponding dose are a result of liquid discharges and tank leaks to the subsurface. For the post-1988 solid waste burial grounds, an initial period of relatively high recharge $(75 \mathrm{~mm} / \mathrm{yr}$ ) was assumed to apply until a surface barrier is constructed. Under this condition, releases to groundwater of the most mobile radionuclides (e.g., selenium-79, technetium-99) were simulated to occur from the active and planned burial grounds in the next 200 years. However, sorbed radionuclides including carbon-14, iodine-129 and uranium (total) do not release in the 1000-year period. Dose at the boundary of the accessible environment (the buffer zone) resulting from releases from the post-1988 solid waste burial grounds, the Environmental Restoration Disposal Facility (ERDF), and the immobilized low-activity waste (ILAW) from Hanford Site tanks cannot be distinguished from background levels resulting from the earlier releases during the 1000-year period following Hanford Site closure.

Present calculated doses to a hypothetical onsite individual are the result of groundwater plumes originating from operational discharges that have been discontinued and early releases from accidental tank leaks. Secondary dose peaks occur in the 2020 to 2030 time frame. These secondary peaks are a result of the calculated breakthrough of radioactive contamination from accidental tank leaks, projected losses from single-shell tanks during future tank waste recovery operations, and early releases from solid waste burial grounds closed prior to September 26, 1988. At present and for several years to come, doses 
calculated at points outside the exclusion area and buffer zone are dominated by tritium from past operations. By the assumed time of Hanford Site closure in 2050, doses are dominated by iodine-129 in the remnants of existing plumes.

The actual position and mobility of wastes in the vadose zone beneath liquid discharge facilities are not well known. Accordingly, analyses of liquid discharges to the aquifer are uncertain. However, existing groundwater contaminant plumes are a result of the past liquid discharges. Remnants of these wastes that remain in the vadose zone are deeper in the profile than dry wastes originally disposed in relatively shallow trenches. Many liquid wastes discharged to ground were very acidic or very basic waste streams; therefore, they may be under geochemical conditions more favorable for migration than neutralized solid waste in dry and relatively shallow disposals. Consequently, forecasts of relatively early releases from liquid discharge sites, past tank leaks, and losses during tank waste recovery operations are credible.

The analysis illustrates that, in comparison to the releases from liquid disposals or leaks, releases to the water table from the four active and planned low-level waste disposals will be delayed by hundreds or thousands of years. These disposals are essentially dry disposals. Releases from the ERDF and Tank Waste Remediation System (TWRS) ILAW disposal facilities do not release the most mobile radionuclides to the water table in the first 1000 years after Hanford Site closure. First releases of the most mobile radionuclides from the post-1988 solid waste burial grounds in 200 West and 200 East Areas appear approximately 200 years after Hanford Site closure. Minimally retarded radionuclides, including iodine- $129(0.5 \mathrm{~mL} / \mathrm{g})$ and uranium $(3 \mathrm{~mL} / \mathrm{g})$, do not release to the water table from the post1988 solid waste burial grounds in the 1000-year period following Hanford Site closure. The maximum dose from these dry disposals to the hypothetical future member of the public in the accessible environment outside the buffer zone is indistinguishable from background values within the regulatory period.

This analysis concludes that releases from the four dry disposals do not present a significant impact to the health and safety of an individual outside the buffer zone during the 1000-year regulatory period. Consequently, the impacts of these disposals do not require completion of a quantitative options analysis and an ALARA assessment.

\subsubsection{The Influence of Uncertain Inventories and Contaminant Mobility}

The original guidance (DOE 1996b) called for sensitivity analyses to be conducted on the issues of alternate future uses of DOE lands and alternate remediations of contaminated sites. Four land-use options were explored through the application of exposure and dose scenarios characteristic of long-term agricultural, residential, recreational, or industrial development. The analysis examined a single basic remediation alternative (i.e., the "leave undisturbed and cover with a surface barrier" alternative). Many groups view such an action as a virtual no-action alternative because wastes are not removed or further immobilized in their present setting. By this analysis, the DOE is not suggesting a preference for the alternative examined. Alternate remediations to be examined in the remedial investigations and 
feasibility studies (RI/FSs) for these sites will be decided jointly by the DOE, U.S. Environmental Protection Agency (EPA), and State of Washington Department of Ecology (Ecology) agencies as part of the Comprehensive Environmental Response, Compensation, and Liability Act (CERCLA) or Resource Conservation and Recovery Act (RCRA) process. Consequently, sensitivity analyses examining the impacts of alternate remediations for the variety of past-practice units including pre-1988 solid waste burial grounds, liquid discharge sites, canyon buildings, and tank farms were neither proposed nor analyzed in this first iteration of the Composite Analysis.

Aside from land use and remediation, potentially significant sources of uncertainty in this analysis lie in the assigned inventory of radionuclides and the combination of parameters assigned to influence the mobility of contaminants in the environment.

\subsubsection{The Influence of an Uncertain Inventory}

Inventory information gathered by several independent programs was used to assemble the inventory for the first iteration of the Composite Analysis. An examination of the total inventory assembled for the key radionuclides revealed a combination of issues that cannot be easily or quickly remedied, including: a) conservative estimates by individual programs, b) conservative estimates of individual radionuclides, c) no final identified disposal, d) absence of key mobile radionuclides, and e) failure to use all available Hanford Site surveillance data and process knowledge. Each of these issues is briefly discussed in this section.

The Composite Analysis is the only analysis conducted in recent years requiring an inventory compilation that applies to all the radioactive wastes that will reside at the Hanford Site after closure. The method of assembling inventory data from independent sources has proven difficult to implement. When basic records focus on major radionuclides such as cesium-137 and various isotopes of uranium and plutonium, methods of estimating the abundance of the key mobile radionuclides became central to the inventory uncertainty issue. The second iteration of the Composite Analysis would benefit greatly from the creation of an inventory that honors, or reconciles, radionuclide generation data, import data, export data, process flow sheets, and waste transaction records. The inventory should be in a form, perhaps as a model, that permits the generation of uncertainty estimates or equally likely realizations.

Conservative Estimates by Individual Programs. A conservative approach to environmental analyses is incorporated into performance assessment and risk assessment guidance and has gained acceptance. Whenever compliance to an environmental standard can be shown using a conservative analysis, there is little reason for a more accurate analysis. When a waste form can be shown to safely dispose of the entire Hanford Site-generated inventory of a radionuclide, there may be no reason for the program or project developing the waste form performance information to invest resources to better understand the true inventory. Using this logic, the TWRS program standard inventory has assigned all carbon-14, selenium-79, and iodine-129 generated in the reactors at the Hanford Site to reside in the single- and double-shell tanks. This effectively overestimates the amount of these radionuclides assigned to the tanks today, and, therefore, to the future ILAW. Similarly, the inventory estimate for the 
ERDF trench was based on maximum observed contamination levels in remediation site wastes applied to the total volume of wastes to be disposed. This must result in an overestimated inventory; however, the Composite Analysis indicated leachate from this facility will not reach groundwater in the 1000-year period following Hanford Site closure. Consequently, estimated environmental performance alone will not require the development of a realistic or best-basis inventory for the ERDF trench.

Conservative Estimates of Individual Radionuclides. The quantity of selenium-79 was based on the Oak Ridge Isotope Generation and Depletion Code (ORIGEN2) simulations of the fuel irradiated in the production reactors at the Hanford Site. Among other data, those simulations relied on the decay half-life of the isotope. The half-life of this isotope was recently revised from $<6.5 \times 10^{4}$ years to $<6.5 \mathrm{x}$ $10^{5}$ years. As a result, the amount of selenium-79 generated in the fuel and introduced to the chemical separation plants will decline by a factor of up to eight. Because selenium-79 is overestimated in the current inventory, results indicating safe disposal at current inventory levels are conservative. When the total inventory of selenium-79 is revised, waste forms that now account for selenium-79 will show a decline in inventory and associated dose impacts.

No Final Disposition Identified. In some cases, the final disposition of the radionuclide inventory in waste and contained in closed facilities is not well defined. In the Composite Analysis estimates of the inventory and its location were needed. Thus, when using the calculated total inventory generated in the reactors, where that inventory will reside at the time of Hanford Site closure must be determined. Because that time is sufficiently far away, several DOE programs have not developed an understanding of their inventories and where they will finally reside. Iodine-129 is a good example. The amount of iodine-129 lost to the atmosphere and trapped in scrubbers and disposed elsewhere (e.g., solid waste burial grounds or Plutonium Uranium Extraction [PUREX] Plant tunnels) is highly uncertain, and therefore, not quantified in the standard or best-basis inventory developed by the TWRS program. Consequently, in an effort to be conservative and bound the iodine-129 issue with regard to tank waste, all iodine- 129 was assigned to the tanks. Of that total, $10 \%$ is assigned to the ILAW to bound the potential dose impact of this radionuclide on ILAW performance. However, this is a conservatively high estimate of the amount of this highly volatile isotope that will be trapped in vitrified waste. The ultimate disposition of the iodine presumed in the tanks, (i.e., $66 \mathrm{Ci}$ ), is not well defined.

In nearly all analyses of closed facilities conducted to date (e.g., hazard assessments, waste volume estimates) the inventory data included only major radionuclides (e.g., cesium-137, strontium-90, uranium, and plutonium), or an inventory of the total fission products and total activation products. Neither of these types of inventory provides sufficient information to perform all-pathways exposure and dose analyses. This has made the simulation of some facilities and waste sites intractable for the first iteration of the Composite Analysis.

Absence of Key Mobile Radionuclides. In general, the radionuclides that have been identified as key to the estimation of maximum all-pathways dose have long decay half-lives and are relatively mobile in the subsurface environment. They are carbon- 14 , chlorine- 36 , selenium- 79 , technetium- 99 , iodine129 , and uranium (total). Chlorine- 36 is included because of its known presence in the graphite reactor 
cores. Its potential impact in other waste has also been studied in this analysis by incorporating a hypothetical amount in irradiated fuel and therefore, in the Hanford Site waste streams. Three of the radionuclides, carbon-14, iodine-129, and uranium (total), exhibit a small degree of sorption in the environment. With the exception of uranium, the mobile and long-lived radionuclides were not routinely measured and reported during the production period at the Hanford Site.

While it is common to find cesium-137, strontium-90, uranium (isotopic or total), and plutonium (isotopic or total) reported in inventory records for specific facilities, the others are not commonly found. The abundance of the mobile and long-lived radionuclides in irradiated fuel is estimated in the total standard inventory. However, records are incomplete with respect to their quantities discharged to the environment as gaseous atmosphere releases, to liquid discharge sites (e.g., cribs, specific retention trenches, reverse or discharge wells), or to solid waste burial grounds. Improved confidence in the quantities of the mobile radionuclides assigned to liquid discharges, tank leaks, and solid waste requires an accepted means of estimating with confidence the abundance of carbon-14, selenium-79, technetium-99, iodine-129, and perhaps chlorine-36 for gaseous, liquid, and solid waste disposals.

Failure to Use All Available Data and Process Knowledge. It is fundamentally important to use process knowledge and transfer records to estimate the timing, volume, and inventory of wastes discharged or lost to the environment. The combined 216-U-1\&2 crib site is an example where a combination of process knowledge and field observations could yield an improved estimate of the original release to a liquid discharge site. A series of events culminated in the detection of a release of uranium in solution to the water table in the vicinity of this crib site in February 1985. The observed uranium plume in the groundwater was and is significant. Periodically it has been the subject of pumpand-treat programs since its discovery. A significant technetium plume appeared at the same time and occupies the same groundwater. Its source is assumed to be the same crib site. However, technetium-99 disposed to the 216-U-1\&2 cribs has not been estimated, based on either the likelihood that technetium99 followed uranium in the U Plant process that generated the waste stream, or the knowledge that a substantial quantity of technetium-99 is now in the aquifer beneath these cribs. Because the Composite Analysis was based on the assembled inventory, the analysis of liquid discharges does not predict the present technetium-99 plume beneath the 216-U-1\&2 cribs. This plume was modeled as an existing plume, and results show that prior to its migration from the buffer zone, it will disperse and its dose consequences will greatly diminish. Existing databases that reveal the temporal and spatial extent of contamination in the environment are a valuable asset in the assessment of the original discharges. While these data may be incomplete, they do provide valuable clues to the presence of specific radionuclides and their relative abundance.

Case for a Single Inventory Estimate. The absence of an inventory generated with a view toward a best estimate of the final location and inventory of all wastes makes it virtually impossible to perform a meaningful study of sensitivity because too many possible realizations could be generated and improbable realizations would be admitted. In the study of an isolated facility or waste form, (i.e., as in a performance assessment), the influence of various levels of inventory can be examined. However, an assessment of uncertainty in inventory for the Composite Analysis requires alternate inventories in terms 
of both location and quantity. It is the potential cumulative impact of multiple disposals at a moment in time and point in space that creates the maximum composite dose. Thus, the uncertainty in composite dose is a function of uncertainties in inventory, release, migration, and exposure. The total or global estimate of inventory, based on reactor operation, is the best information on inventory at the Hanford Site. Upper bound estimates of inventory disposed at most facilities are unknown and unknowable. Thus, inventories assigned to each facility or type of facility must be associated with the known range of inventory for each. In other words, the inventory realizations generated must be equally probable for the assessment of uncertainty to be meaningful. For example, if less of a specific isotope is in the tanks today (and will eventually be in ILAW and immobilized high-level waste), more should be assigned to the inventory of one or more of the following:

- lost in gaseous form to the atmosphere during chemical separation processes

- disposed in liquid form to the subsurface

- disposed in solid form to burial grounds

- residing in canyon building vessels or structures or filters

- residing in PUREX tunnels in process vessels.

The perturbations possible in the assignment of inventories to specific waste disposal facilities must be constrained by our knowledge of processes and field observations. Before useful sensitivity cases capturing our uncertainty in the inventory can be formulated, the internal dependencies or correlation of the inventory problem must be incorporated into a collective best-estimate model. Only then can the true significance of real uncertainties be determined through simulation of environmental consequence.

Bounding estimates of inventory may be meaningful in the Composite Analysis when examining a single facility or class of facilities. However, with few exceptions, reaching consensus on a bounding inventory for a specific facility or waste form could be difficult. Such estimates may be useful when attempting to determine the maximum potential influence of a facility outside the buffer zone. Similarly, one may wish to determine the inventory required in a facility to cause a given impact outside the buffer zone. Such analyses would be useful in evaluating the need to retain a given facility or class of facilities in the Composite Analysis. Certainly, as in any environmental assessment, if the release processes or migration pathway act to constrain the dose impact to levels well below the performance standard, then bounding inventories could be assigned to all sources to simply illustrate the ultimate safety of the waste form or physical setting. However, simulations of this type do not quantify the influence of an uncertain inventory. They provide a reasonable assurance of no impact from any reasonable inventory.

Inventory Uncertainty with Respect to Dry Disposals. Given the assumptions regarding future land use, the results obtained in the first iteration of the Composite Analysis illustrate that inventories assigned to the active and planned disposals will not yield significant releases in the 1000-year period following Hanford Site closure. The analysis also indicated that increased inventories assigned to these disposals would not yield significant releases in the 1000 years. To ensure that no significant releases from the burial grounds are possible, waste acceptance criteria and procedures (WHC 1993) were applied to screen each waste package for mobile radionuclide content (e.g. carbon-14, selenium-79, 
technetium-99, iodine-129, and uranium) prior to disposal and to determine if mobile radionuclide inventories are sufficiently high to require additional isolation from the environment. Typical immobilization processes are encapsulation of waste packages in thick concrete boxes or direct grouting of the waste material. This protocol ensures that no one package can provide a substantial portion of allowable dose. Neither the ERDF nor the TWRS ILAW disposal facilities are predicted to release any radionuclides in the 1000 -year regulatory period.

Thus, the inventories analyzed yield maximum dose well below the DOE dose limit and constraint levels. Additional inventories, if identified for future disposals in the burial grounds, would be immobilized prior to disposal and be determined to be safe for long-term disposal prior to acceptance. Therefore, further analysis of the radioactive waste inventory with respect of incremental dose impacts from solid waste burial grounds, the ERDF, and the TWRS ILAW would not yield additional insight and are not included in the Composite Analysis.

\subsubsection{Uncertainty in Contaminant Mobility}

A recent peer review of vadose zone contamination beneath single-shell waste tank 241-SX-109 was critical of Hanford Site knowledge of contaminant migration resulting from a tank leak (DOE 1997a). The panel found insufficient information to defend a single conceptual model of the physical path and chemical mobility of cesium-137 and other radionuclides leaked to the subsurface. Alternate conceptual models stress the potential roles of natural heterogeneity, man-made preferential flow paths, fluid density, and geochemical mobility on the migration and fate of contaminants. The TWRS Vadose Zone Program is underway to gather data to better define the present distribution and future mobility of contaminants in the vadose zone beneath tank leaks. In addition, the DOE has created a program, the Hanford Groundwater and Vadose Zone Integration Project, to coordinate the study of the vadose zone across the various environmental management and environmental restoration programs at the Hanford Site. Developing confidence in models of contaminant migration and fate for the vadose zone beneath liquid discharge sites and leaking tanks must await completion of the early stages of these programs. The second and subsequent iterations of the Composite Analysis will benefit from these programs.

Geochemical Mobility. Unlike previous sitewide analyses, the Composite Analysis of wastes within the exclusive waste management area and buffer zone distinguishes among six waste types that were discharged or leaked to the subsurface environment. Based on the waste characteristics and an assumed amount of contact with subsurface sediments, the chemical elements were assigned distribution coefficients for each of the waste types and three geologic settings; an upper vadose zone, the lower vadose zone, and the unconfined aquifer (Appendix E). Thus, in this analysis process waste streams with a high organic content and very acidic $\mathrm{pH}$ have been assigned a different mobility than those wastes with a low organic content and a near-neutral $\mathrm{pH}$.

As described in Section 4.1.2.1.4, based on post-mortem studies of cribs, specific retention trenches, and tank leaks conducted during the late 1970 s and early 1980 s, the vadose zone was divided into two segments. The waste type governed the selection of the distribution coefficient in the upper segment. A 
combination of waste-type and sediment interactions governed the assigned value in the lower segment. In general, although not always, the wastes are more mobile in the upper segment and less mobile in the lower segment. This conceptual model applies to the liquid discharges to the sediment profile including tank leaks and losses from tanks during recovery operations.

Wastes in the dry disposal sites, including all solid waste burial grounds, the ERDF trench, and the TWRS ILAW disposal facility, were assumed to have a low-organic content, a low-salt content, and a near-neutral $\mathrm{pH}$. Accordingly, chemical elements in these wastes were assigned a single distribution coefficient that applies throughout the sediment column. Best-estimate values of distribution coefficients for carbon, iodine, and uranium were $5,0.5$, and $3 \mathrm{~mL} / \mathrm{g}$, respectively. Chlorine, selenium, and technetium were all assigned $0 \mathrm{~mL} / \mathrm{g}$ and assumed to move with the water. Conservative values for carbon, iodine, and uranium are $0.5,0.3$, and $0.6 \mathrm{~mL} / \mathrm{g}$, respectively. None of the sorbed contaminants from dry disposal sites are predicted to reach the water table in the period analyzed. The more mobile chlorine, selenium, and technetium radionuclides behave identically in both cases. It is interesting to note that if carbon and uranium were simulated using their conservative values of distribution coefficients ( 0.5 and $0.6 \mathrm{~mL} / \mathrm{g}$, respectively), their behavior would be similar to that of iodine with its best-estimate value of distribution coefficient $(0.5 \mathrm{~mL} / \mathrm{g})$. Neither would reach the reach the aquifer in 1000 years. Thus, a sensitivity analysis regarding the geochemical mobility of wastes disposed in the solid waste burial grounds, the ERDF trench and the TWRS ILAW disposal facility would not reveal significantly different results in the 1000 -year regulatory period.

Hydrogeologic Mobility. There is a fundamental difference between liquid discharges (including tank leaks), and dry disposals. Liquid discharges carry the contaminants into the vadose zone beneath the liquid discharge facility. This liquid, including radioactive contamination, seeks to redistribute in the vadose zone such that it comes into equilibrium with the surrounding soil water. Continuous liquid discharges move liquid waste and associated contamination deep into the vadose zone, eventually resulting in breakthrough to the water table. Short-term and lower-quantity discharges displace the resident soil water and then are driven more slowly by natural recharge as they also migrate downward to the water table.

The hydrologic driver for dry disposals is the recharge rate. Solid waste burial grounds at the Hanford Site are typical. Once in place, they are covered by $2 \mathrm{~m}$ of backfill pending placement of a final surface barrier system. In the base case, a sequence of recharge rates indicative of a site covered with coarse soil and maintained free of vegetation $(75 \mathrm{~mm} / \mathrm{yr})$ followed by the site covered with a surface barrier $(5 \mathrm{~mm} / \mathrm{yr}$ ) has been examined. One important nuance of this recharge and release scenario is that wastes were leached throughout both periods, i.e., it was assumed waste containers did not present a barrier to direct and immediate leaching by pore water during the period prior to surface barrier construction. In this scenario, pre-1988 solid waste burial grounds release waste to the water table in the first decades of the next century. Post-1988 disposals exhibited releases of the most mobile contaminants (chlorine-36, selenium-79, and technetium-99) in approximately 200 years but no release of less mobile contaminants (carbon-14, iodine-129, and uranium) in 1000 years. 
The comparison case for solid waste burial grounds examined the scenario where leaching of the waste did not begin until after the surface barrier with a recharge rate of $5 \mathrm{~mm} / \mathrm{yr}$ was constructed over the trenches. Essentially, this case is based on the assumption that waste containers minimize or preclude direct leaching of the solid waste until the barrier is in place. This case duplicates an essential feature of the analyses presented in the performance assessments for the post-1988 solid waste burial grounds (Wood et al. 1995; Wood et al. 1996). Because of the integrity and durability of containers employed since 1984, the comparison case is believed to be a better representation of future burial ground performance. In this comparison case, post-1988 disposals exhibited mean travel times of approximately 1070 years from burial grounds in the 200 West Area, 1150 years from the 218-E-10 burial ground, and 650 years from the $218-\mathrm{E}-12 \mathrm{~b}$ burial ground. The majority of future solid waste is destined for 200 West Area burial grounds, and, therefore, the mean travel times on the order of 1000 years will govern the majority of future releases.

The consequences of not constructing a surface barrier and applying a higher recharge rate (e.g., $50 \mathrm{~mm} / \mathrm{yr}$ ) over the long-term were studied in the published performance assessments (Wood et al. 1995; Wood et al. 1996). They concluded that a surface barrier tailored to the site and waste conditions should be designed and constructed over the burial grounds.

It is unlikely that more rapid leaching of the solid waste could occur than is characterized in the base case. The comparison case performed for the Composite Analysis captures a more likely scenario; however, low-level waste (LLW) containers are not specifically designed to defeat leaching by pore water for extended periods of time. When solid waste is dry and not corrosive, it is likely the container will survive and protect the waste from leaching phenomena. Further, moisture inside waste packages is largely eliminated by waste acceptance criteria requiring free liquid to be sorbed inside the package (WHC 1993). The comparison case may be extreme in the sense of preventing any release until the low recharge rate influences both the release and its subsequent migration.

The model employed in the Composite Analysis to represent contaminant transport in the vadose zone is one-dimensional. One shortcoming of such a model is its inability to quantify the multidimensional aspects of the analysis. Placement of a surface barrier implies an immediate and complete change in the recharge rate that is leaching the source and driving contaminants through the vadose zone. Edge effects of a barrier are neglected. In general, the distance from the land surface to the water table beneath the exclusive waste management area is less than $100 \mathrm{~m}$. The physical size of the four disposal facilities under consideration suggests their barriers will be in excess of $100 \mathrm{~m}$ across, and it is anticipated that the barrier will be extended well beyond the disposal facility, e.g., trench, vault. Consequently, the opportunity for edge effects, i.e., moisture moving under the barrier in the vicinity of its edge, to leach the disposed waste or accelerate its migration to the water table is less than might be envisioned. For example, only those wastes near the edge of the barrier could be exposed to greater leaching, and if the barrier is extended well beyond the edge of the disposal this is less likely to occur. Similarly, the ability of the edge-effect recharge to affect the transport pathway also will decrease with 
the length of extension. Thus, assuming that the future barrier design will include sufficient edge extension beyond buried waste deposits, the base case and sensitivity cases capture the range of likely environmental responses.

Uncertain Mobility with Respect to Dry Disposals. Although dry disposals in solid waste burial grounds, the ERDF trench, and the TWRS ILAW disposal facility are a primary focus of the Composite Analysis, this first iteration of the analysis has shown the importance of liquid releases (e.g., liquid discharges and tank leaks) and their migration and fate. Dry disposals are influenced by the recharge rates through disturbed surfaces and engineered surface barriers as compared to higher rates experienced at liquid release sites. Similarly, solid wastes are subject to more favorable geochemical mobility factors (i.e., distribution coefficients) than some liquid release sites. The cases reported in the Composite Analysis capture the range of conditions most likely to govern the mobility of these wastes, and illustrate the safety of these dry disposals.

\subsection{Interpretation of Composite Analysis Results}

While not as detailed as either a performance assessment or CERCLA analysis of LLW sites, this Composite Analysis is a reasonable first assessment of cumulative impacts at the Hanford Site. It includes impacts from active and planned LLW disposal facilities, and other sources of radioactive contamination that could interact with these LLW disposals and affect the dose to future members of the public. This Composite Analysis provides insight into what could occur at the Hanford Site in the next 1000 years and informs the DOE of the safety of active and planned LLW disposal.

By design (DOE 1996b) and out of necessity, the Composite Analysis is less rigorous than a sitespecific performance assessment or an RI/FS analysis. The 200 Area Plateau at the Hanford Site will be the final disposal location for a variety of waste forms and a considerable radionuclide inventory. A less sophisticated modeling approach and a sitewide scale were justified in this first iteration of the Composite Analysis because of the required scope of the analysis (e.g., the number and variety of sites) and the level of information readily available. Portions of the modeling effort have been less rigorous (i.e., simple zero-dimensional release models and a one-dimensional vadose zone model were employed). However, model results have been matched qualitatively with observed releases to the unconfined aquifer. A more sophisticated aquifer model than appears in previous performance assessment and RI/FS analyses has been applied.

\subsubsection{Consistency with Previous Performance Assessments and with the ERDF RI/FS}

The Composite Analysis is a companion document to four site- or waste-form specific studies. These studies are the performance assessments for the 200 West and 200 East Area solid waste burial grounds (Wood et al. 1995; Wood et al. 1996), the RI/FS completed for the ERDF trench (DOE 1994b), and the interim performance assessment for the ILAW now in the single- and double-shell tanks (Mann et al. 1997). The performance assessment for ILAW is scheduled for submittal in the spring of 1998. 
Performance assessments for the solid waste burial grounds (Wood et al. 1995; Wood et al. 1996) show first release of the most mobile radionuclides within the 1000-year period. However, these releases occur late in the 1000-year period and they result in a projected all-pathways dose that is orders of magnitude below the standard. These published performance assessments differ from the Composite Analysis in their approach to uranium release and migration. Wood et al. (1995) and Wood et al. (1996) make a conservative assumption regarding uranium mobility and assign it a distribution coefficient of $0(\mathrm{~mL} / \mathrm{g})$ in the subsurface sediments. They also modeled the release of most of the uranium inventory by applying a solubility controlled release model. Solubility values were assumed to be controlled by the local geochemical environment that was dominated by soil water reactions or cement-water reactions if the uranium was encapsulated in grout or disposed in concrete boxes. The net result is a much lower rate of uranium release to the subsurface followed by a more rapid migration through the vadose zone. In the first iteration of the Composite Analysis uranium was assigned a best-estimate value of $3 \mathrm{~mL} / \mathrm{g}$ for distribution coefficient and does not release from the vadose zone in the 1000-year period following Hanford Site closure. The simulation of another radionuclide, iodine, using a distribution coefficient of $0.5 \mathrm{~mL} / \mathrm{g}$ also revealed no release. The conservative estimate of uranium sorption is $0.6 \mathrm{~mL} / \mathrm{g}$ (Appendix E). Consequently, it is not necessary to apply the more realistic but complex release model because uranium is predicted to not release from the vadose zone in the 1000-year period of regulatory concern. Note, if the Composite Analysis had shown uranium release to the aquifer from dry disposals, it would be important to apply the combined solubility and sorption model as was done in the performance assessment. Only through the application of models for both processes can the concentration and timing of the release and transport be realistically modeled.

Both the ERDF RI/FS (DOE 1994b) and the interim performance assessment for TWRS ILAW (Mann et al. 1997) call for a high-integrity surface barrier. The Hanford Protective Barrier has a design standard recharge rate of $0.5 \mathrm{~mm} / \mathrm{yr}$, and it is assumed this barrier, or a similarly effective one, will be placed over the both the ERDF trench and the TWRS ILAW facilities soon after disposals are completed. The ERDF trench is double-lined to prevent releases during disposal operations, and the surface barrier is to be applied immediately after the trench is full. Thus, no releases from the trench are anticipated prior to barrier placement. Accordingly, releases will be a result of long-term leaching at a rate defined by the recharge rate through the surface barrier. Cases in the RI/FS (DOE 1994b) that examined surface barrier and liner conditions similar to those considered in the Composite Analysis showed no release to the water table in 10,000 years. The Composite Analysis results show no releases from the ERDF to the water table in the 1500 years analyzed.

While design features of disposal facilities for ILAW have not been finalized, it is apparent that barriers to recharge will be constructed to limit infiltration soon after waste placement. The TWRS ILAW performance assessment has shown the earliest releases to the water table of the most mobile radionuclides will occur in approximately 1000 years. However, the performance assessment has employed a somewhat higher dispersivity for the vadose zone than the Composite Analysis.

Consequently, the TWRS ILAW performance assessment would be expected to show an earlier release because of greater longitudinal dispersion. Because of the higher vertical resolution (i.e., finer grid) 
possible with the one-dimensional model, this iteration of the Composite Analysis used a dispersivity of $80 \mathrm{~cm}$ and predicted no release from the TWRS ILAW facilities in the base case for the 1500 -year period analyzed.

\subsubsection{Other Sites in the Exclusive Waste Management Area and Buffer Zone}

In addition to the active and planned disposals of LLW, this Composite Analysis examined existing plumes and future releases from pre-1988 solid waste burial grounds, liquid discharge sites, tank leaks, tank losses during recovery operations, tank residuals, and graphite cores from the production reactors. This analysis has shown a marked separation of environmental response to liquid discharges and leaks to the subsurface environment, and dry disposals of the recent past and the future. There are two episodes of groundwater contamination: the near-term contamination of the aquifer by liquid discharges, tank leaks, tank losses, and past-practice or pre-1988 burial grounds; and the long-term events associated with recent and future dry disposals.

\subsubsection{Existing Plumes}

Recent reports have examined existing plumes, their future migration, and their fate (Chiaramonte et al. 1997; Cole et al. 1997). Projections of groundwater contaminant plume migration in this analysis are based on the model described by Cole et al. (1997).

Plumes of tritium, cobalt-60, strontium-90, technetium-99, iodine-129, and uranium (total) are found in the unconfined aquifer. Because of its discharge from the aquifer and decay haif-life of 12.3 years, dose from tritium in the unconfined aquifer in 2050 is less than $2 \mathrm{mrem}$ in a year, and by 2100 its dose contribution has virtually vanished. Cobalt-60, with its decay half-life of 5.3 years and much lower inventory in the aquifer, will be of even less significance. Because of its strong sorption on aquifer sediments, strontium-90 is shown to remain within the exclusive waste management area and buffer zone, and does not significantly contribute to dose outside the buffer zone. As stated earlier, for the purpose of simplifying this discussion, it is assumed the exclusive waste management area has been expanded to include Gable Mountain Pond. At present, if groundwater were pumped and used as the scenarios assume, dose from tritium would dominate the maximum dose outside the buffer zone. Between now and the assumed time of Hanford Site closure, iodine-129 in the remnant of the existing plume becomes the dominant contributor to dose outside the buffer zone as the impact of tritium diminishes. Future releases from past liquid discharge sites, past tank leaks, future tank losses during tank waste recovery operations, and pre-1988 solid waste burial grounds also contribute to near-term doses. Existing plumes are evidence of environmental response to past liquid discharges and tank leaks. Only recently have tank wastes been identified as contributing to existing plumes (Johnson and Chou 1998; Hodges 1998). Thus, historical plume observations are a direct response from prior liquid discharges to the subsurface. In general, existing plumes are a result of large volume liquid discharges of process plant waste streams. They range from waste streams that were usually directed to tanks, to large volumes of cooling water carrying dilute contaminant concentrations. Sediment columns contaminated 
with these discharges will continue to drain and discharge to the water table aquifer. This Composite Analysis estimated dose from these releases to be maximum now, and to decline with time.

An inconsistency exists between the technetium-99 inventory assigned to liquid discharge sites and the estimated inventory of technetium- 99 in observed groundwater contaminant plumes. The release and vadose zone transport models for these discharges were driven by significant liquid discharge rates and they estimated significant inventories of technetium-99 were released to the aquifer. An estimated 930 $\mathrm{Ci}$ of technetium-99 were disposed to ground in these facilities (Waite 1991). The transport model routed these wastes to the groundwater rather rapidly. The model predicted a cumulative activity of 181.2 $\mathrm{Ci}$ of technetium-99 released to the water table from liquid discharge sites by 1996 . However, based on field observations, the existing plumes of technetium-99 were estimated to contain between 15.8 and $37.6 \mathrm{Ci}$, depending on the assumed thickness of contamination in the aquifer. While the integrated mass of contaminant in the aquifer is uncertain, it is believed the unconfined aquifer does not contain the amount of technetium-99 predicted by the source release model. The inconsistency was remedied by assuming the estimated mass in the aquifer was correct. As noted in Section 4.3, the model estimate of tank leak contribution to technetium-99 plumes was $\sim 5 \mathrm{Ci}$ in 1996 . Consequently, the inventory estimated as released to liquid discharge sites for the base case was scaled down to result in a release to groundwater of $32.6 \mathrm{Ci}$ by 1996.

If the full $930 \mathrm{Ci}$ inventory of technetium-99 were disposed, it could increase the contribution to dose from liquid discharge sites by a factor of $\sim 6.6$. However, the resulting increase in maximum dose outside the buffer zone would be less than 2 mrem in a year in 2050, and less than 3 mrem in a year during the 1000-year period following Hanford Site closure. Such increases to the overall maximum individual dose would only occur if points in space and moments in time for maximum dose from liquid discharge site releases coincided with other maximum or high contributions to dose. This sensitivity case was analyzed and yielded a maximum dose of less than $7.5 \mathrm{mrem}$ in a year for exposure outside the buffer zone in 2050 from the agricultural scenario. This was the maximum dose for the 1000 -year period following Hanford Site closure. The maximum dose obtained for the period after 2150 was less than $6 \mathrm{mrem}$ in a year.

\subsubsection{Liquid Discharge Sites}

This analysis includes contaminant releases from the ditches, ponds, reverse (or injection) wells, cribs, and specific retention trenches located on the 200 Area Plateau. There are no known prior analyses of the large number of liquid discharge sites examined. However, several post-mortem studies of specific facilities have been conducted, and results of those field studies have been used to qualitatively fit model results to field observation.

Large discharges of cooling water were made to a variety of ditches and ponds in the 200 Areas. These discharges had a significant influence on the water table, and, therefore, on the groundwater flow direction and rate. This is revealed in the history of groundwater mounds beneath the $U$ Pond in 200 West Area, the B Pond in 200 East Area, and the Gable Mountain Pond to the north of 200 East 
Area. These mounds are now declining, but they will continue to influence the groundwater flow pattern for several decades (Chiaramonte et al. 1997; Cole et al. 1997). Other than their influence on groundwater velocities and the direction groundwater plumes have moved, the large releases to ponds have not significantly affected water quality.

Several significant discharges during the early operation period were made to reverse wells. Some of these facilities discharged into the vadose zone above the water table; some discharged directly into the water table. Plumes associated with the reverse wells, notably that associated with the 216-B-5 reverse well, exhibit significant levels of radionuclides either in the water table or deep in the vadose zone. However, radionuclides in these discharges such as strontium- 90 are highly sorbed and have exhibited minimal contaminant migration in the past 50 years. Strontium- 90 and other highly sorbed radionuclides (cesium-137 and plutonium-239/240) presently at these retired reverse well sites are forecast to remain inside the exclusive waste management area and buffer zone. The use of reverse wells was virtually discontinued very early in the Hanford Site operations. Reverse wells completed into the vadose zone were used after the mid-1950s at only two locations, the U Plant and the hot semi-works (Law and Lu 1982). Both of these disposals involved relatively low volumes and low inventories compared to other similar facilities. Use of the reverse wells at the U Plant and hot semi-works was discontinued by 1970 and 1988, respectively (DOE 1996a).

In comparison to ponds, smaller but still relatively large discharges were made to cribs and specific retention trenches. These discharges contained significantly greater radionuclide inventories than the cooling water discharges. Some of these discharges were tank wastes. Waite (1991) estimated these wastes contained $930 \mathrm{Ci}$ of technetium-99 and $1.8 \mathrm{Ci}$ of iodine-129. In the base case, the technetium-99 inventory was scaled down because of inconsistencies between estimates of technetium- 99 released to the water table and existing in groundwater plumes, and only $167 \mathrm{Ci}$ of technetium- 99 were discharged to cribs and trenches. Some of the larger-volume discharges of these wastes were made to cribs that discharged to the aquifer. In an effort to contain discharged liquids permanently in the vadose zone, smaller-volume discharges of these wastes were made to specific retention trenches. This type of facility was designed to avoid discharges to the aquifer, however, the design was based on the assumption there was no recharge in the deep vadose zone deposits of the Hanford Site. Research and field observations have shown that under a variety of conditions this assumption is not true. Therefore, based on soil physics and contaminant transport theory, the present analysis forecasts releases from these facilities (i.e., specific retention trenches) to the water table.

The precise position and mobility today of wastes beneath liquid discharge facilities is not well known. Thus, analyses of liquid discharges to the aquifer are highly uncertain. However, existing plumes in the groundwater are a result of liquid discharges. Furthermore, remnants of these wastes that remain in the soil column are likely to be deeper in the vadose zone profile than dry wastes originally disposed in relatively shallow trenches. Many liquid waste discharges were very acidic or very basic waste streams, and therefore, they may be under geochemical conditions more favorable for migration than neutralized solid wastes in dry and relatively shallow disposals. Consequently, forecasts of 
relatively early releases from liquid discharge sites are credible. The characteristic of relatively early release separates releases to the aquifer by liquid discharge sites from those of recent and future dry disposals that will release much later.

In all cases, existing plumes are the result of relatively large liquid discharges to the subsurface. These releases to the water table have occurred from ponds, reverse wells, cribs, and specific retention trenches. The Composite Analysis illustrates that, depending on the mobility of the nuclides released and the quantity of liquid discharged, inventories retained in the soil column at these sites will continue to leach into the groundwater for decades. However, those contaminants in the aquifer today are a result of the early discharge of large quantities of liquid waste or direct injection at reverse well sites. Some discharges were virtually continuous, and others were periodic. Some of these discharges were tank wastes or first derivatives of tank waste that contained significant concentrations of key radionuclides. Consequently, the resulting plumes had relatively high concentrations, and they continue to exhibit relatively high peak values today despite years of groundwater transport, radioactive decay, and dispersal.

This is illustrated by the more recent modeling of the tritium plume (Cole et al. 1997). This modeling simulated the tritium plume buildup and migration from 1979 to 2100 . Effects of tritium discharges to ground prior to 1979 were accounted for through the simulation's initial conditions that were based on monitoring well measurements made in 1979. It also included both the projected future discharge of $1000 \mathrm{Ci}$ of tritium at the State-Approved Land Disposal Site (SALDS) starting in 1996, and all known past large liquid discharges to ground during the 1979 to 1996 time period. These past liquid discharges included $30,000 \mathrm{Ci}$ of tritium (1979 to 1996). The majority of the tritium disposal $(\sim 24,000 \mathrm{Ci})$ during the 1979 to 1996 time period occurred during 1984, 1985, and 1986 at 216-A-10 crib site as a result of PUREX operations. Tritium and liquid discharges to the $216-\mathrm{A}-10 \mathrm{crib}$ during this three-year period averaged $\sim 12000 \mathrm{Ci}$ per year and $\sim 400 \mathrm{~m}^{3} /$ day respectively. These rates are orders of magnitude higher than any predicted future release rates to groundwater.

Future releases to the aquifer from the liquid discharge sites, tank leaks, tank losses, and burial grounds will occur, but with a greatly diminished driving force as compared to the past releases, because the future leaching and movement is driven by natural recharge rates, not large liquid releases. Even though more curies of specific radionuclides like technetium-99 will leach into the aquifer in the future than are present today, they will be introduced at lower rates. Since the general magnitude of groundwater flow in the aquifer flowing under these various sites will remain relatively constant through time, these lower projected release rates from the sources will create plumes with lower peak concentrations. Consequently, this analysis has shown that future doses through the time of Hanford Site closure and beyond will be dominated by the existing plumes of tritium and iodine-129. Because the total curies of tritium presently disposed to ground are far greater than for any other nuclide, the tritium in existing plumes will dominate dose estimates until it either discharges to the river or decays away. Order of magnitude estimates for tritium in the 1973 plume (ERDA 1975) indicated that it might contain as many as 35 million $\mathrm{Ci}$. However, this estimate was based on the assumption that the tritium concentration was uniform over the entire thickness of the unconfined aquifer (i.e., from the water table surface to the base' 
of the unconfined aquifer). The recent plume modeling assessment (Cole et al. 1997) assumed the thickness of the initial 1979 tritium plume was $\sim 25 \mathrm{~m}$. As a result the total curies of tritium remaining in the plume in 1996 was estimated to be $\sim 160,000 \mathrm{Ci}$. While this is a large number of curies, it is significantly less than the U.S. Energy Research and Development Administration estimate (ERDA 1975). As the tritium concentrations are reduced by migration to the river, dispersion, and decay, the iodine-129, which is assumed to be less mobile, then begins to dominate dose because of its very high dose conversion factor.

\subsubsection{Past Tank Leaks, Future Tank Losses, and Tank Residuals}

Data on release volumes and leak dates from Hanlon (1997) were augmented with waste concentration data gathered from tank characterization reports to provide a basis for the simulation of past tank leaks in the Composite Analysis. Release and vadose zone model parameters have been adjusted to qualitatively match the recent releases (e.g., $~ 5 \mathrm{Ci}$ of technetium-99 from tank leaks is estimated to have reached the aquifer by 1996). The remainder of these releases is forecast to occur over the next century; however, its contribution to dose outside the buffer zone is relatively small.

Plans are now being made to recover tank wastes from the single- and double-shell tanks. Between 2003 and 2020 all of the single-shell tank wastes will be recovered. While the decision has not been made as to the methodology to be applied, the Composite Analysis assumed the sluicing method of tank waste retrieval was applied as described and analyzed in the TWRS environmental impact statement (EIS) (DOE and Ecology 1996). While the TWRS EIS analyzed consequences of an average 4000-gallon loss from each single-shell tank, the Composite Analysis uses the current estimate of 8000 gallons per tank. The model applied to tank losses is identical to that applied to tank leaks. These losses, like the tank leaks, were simulated as migrating through the vadose zone and releasing to the aquifer over the next century with most of the release coming before Hanford Site closure.

Finally, tank residuals, estimated as $1 \%$ by volume of current tank contents, are assumed to remain in the remediated and stabilized tanks. As in the TWRS EIS, the tank structure and remediation were assumed to protect the residual from leaching for 500 years. The Composite Analysis indicated this waste would not release into the unconfined aquifer during the 1500-year period analyzed.

\subsubsection{Pre-1988 Solid Waste Burial Grounds}

There are no published analyses of future waste migration from the pre- 1988 burial grounds. The response of wastes disposed in these burial grounds may be much different than that of the post-1988 wastes in similar facilities. In the Composite Analysis, burial grounds without permanent surface barriers were assumed to be leached by recharge rates indicative of covers of coarse soils maintained free of vegetation $(75 \mathrm{~mm} / \mathrm{yr}$ ). Under this assumption, some older burial grounds could experience more than 50 years of relatively high recharge and leaching. During the time of their operation, it is known that containers were less substantial and more susceptible to leaching than waste containers used today. 
Consequently, this analysis indicates releases from these facilities could begin in the near future, peak in the next few decades, and continue at low rates over the next century.

\subsubsection{Graphite Cores}

The graphite cores of the production reactors are to be transported to the 200 West Area for disposal in the burial grounds (ROD 1993). These cores have unique features: they contain the greatest estimated chlorine-36 inventory on the Hanford Site, and they were identified in the first iteration of the Composite Analysis as the only waste with the potential to make a significant atmospheric pathway contribution to the all-pathways dose.

Tritium and carbon-14 inventories were analyzed for vapor-phase migration upward and lateral transport in the atmosphere. The calculated atmospheric release yielded only a minor contribution of approximately $0.4 \mathrm{mrem}$ in a year to the all-pathways dose from this atmospheric release. This dose was the result of soil contamination that included the continuous buildup of contamination in surface soils over the full 1000-year period following Hanford Site closure. With regard to the timing of maximum dose, the maximum contribution from the atmospheric pathway would not superimpose on maximum doses from groundwater contamination since the latter occur at the time of Hanford Site closure. With regard to the spatial location of maximum dose, the atmospheric and groundwater pathways are also separated because the maximum contribution from the soil-atmospheric pathway occurred on the western edge of the 200 West Area. It will not superimpose on maximum groundwater contamination points to the east and south of the exclusive waste management area and buffer zone. A lower dose from the atmospheric pathway at the buffer zone boundary would result from placing the production reactor cores in the center of the western portion of the 200 West Area rather than near the boundary of the exclusive waste management area. Because of the assumed placement of a Hanford Protective Barrier or equivalent cover over the graphite cores, they are shown to not release to the groundwater aquifer during the time period analyzed in the Composite Analysis.

\subsubsection{Chemical Separation Plants and Associated Facilities}

For the first iteration of the Composite Analysis insufficient inventory and waste-form data prevented a truly credible analysis of the major facilities including the chemical separation plants or canyon buildings and their buried filters. No inventory exists for most key radionuclides, (e.g., carbon14, selenium-79, technetium-99, and iodine-129) in these facilities. Typically, inventories for only cesium-137, strontium-90, uranium (total), and plutonium (total), are provided. The physical and chemical forms of these wastes are also poorly defined. Programs responsible for the cleanup of the facilities often describe the waste as fixed in place and immobile.

With respect to these facilities, the Composite Analysis results are preliminary. Calculations were performed to demonstrate that the massive inventories of cesium-137 and strontium-90 in B Plant and in its sand and high-efficiency particulate air (HEPA) filters would not contribute to releases to the unconfined aquifer within the next 1500 years. In the calculation, wastes in the B Plant canyon building 
and HEPA filters were assumed to reside in a cementitious material, (i.e., the concrete floor and a grout matrix, respectively). The sand filter was assumed to be a simple sand deposit. Both waste deposits were assumed to be protected from infiltration by a Hanford Protective Barrier. Applying the most conservative distribution coefficients to describe adsorption, this analysis demonstrated no release of radionuclides to the unconfined aquifer over the next 1500 years.

\subsubsection{Commercial Low-Level Radioactive Waste Disposal Facility}

The commercial low-level radioactive waste disposal facility operated by US Ecology has unique features and inventory aspects. Located southwest of the 200 East Area inside the exclusive waste management area, this facility uses deep unlined trenches to dispose of commercial LLW. These trenches are excavated in a thick deposit of sand and silt. The inventory for this facility contains the second largest amount of chlorine- 36 onsite, $34.4 \mathrm{Ci}$, and a significant inventory of uranium, greater than $10,000 \mathrm{Ci}$. This facility also contains $5.77 \mathrm{Ci}$ of iodine-129 and $65.6 \mathrm{Ci}$ of technetium-99.

In the Composite Analysis, chorine-36 is predicted to release in the near-term period prior to Hanford Site closure. However, the maximum contribution of chlorine-36 to the all-pathways dose from all sources will be less than 1 mrem in a year at the boundary of the buffer zone. With the assigned distribution coefficients of 3 and $0.5 \mathrm{~mL} / \mathrm{g}$, uranium and iodine did not release to the water table during the 1500-year period of the Composite Analysis. The analysis indicated 1\% of the original technetium99 inventory will release to the water table in the 1000-year period following Hanford Site closure.

\subsection{ALARA Assessment}

The Composite Analysis indicates an all pathways dose well under the $30 \mathrm{mrem}$ EDE in a year level that would trigger the need for a full and detailed options analysis and ALARA assessment of alternate remedial actions. A brief qualitative ALARA assessment is provided to evaluate the potential value of a more detailed analysis of alternatives.

The first iteration of the Composite Analysis has demonstrated that groundwater contamination at the Hanford Site will undergo two distinct episodes in the future. The first is more severe than the second and involves releases from numerous liquid discharge sites, tank leaks, tank losses during waste recovery operations, and past-practice solid-waste burial grounds. This first episode began with Hanford Site operations and will continue through 2050 , the assumed date of Hanford Site closure. The dose prediction for the base case and the agricultural exposure scenario in 2050 is $\sim 5.5 \mathrm{mrem}$ in a year to the maximally exposed offsite individual (Figure 4.35). The dose predictions continue to decline through 2150, 100 years after Hanford Site.closure, and are in the neighborhood of $4 \mathrm{mrem}$ in a year to the maximally exposed offsite individual at that time. The second episode begins with releases from the post- 1988 solid waste burial grounds and extends well beyond the 1500 years analyzed in this.Composite Analysis. Earliest releases are predicted about 200 years from present and may not occur until very near the end of the 1000-year regulatory period. Dose predictions from the base case for the second episode, 
which yielded first release in approximately 200 years, are less than 4 mrem in a year to the maximally exposed individual outside the exclusive waste management area and its buffer zone. The dose projection establishes a plateau at $\sim 3$ mrem in a year during the middle of the 1000 -year period before falling to less than $2 \mathrm{mrem}$ in a year at the close of the period.

The unconfined aquifer underlying the Hanford Site has a low capacity and cannot support extensive irrigated agriculture. Its recharge is limited by being in the rain shadow of the Cascade Mountains, and it is not fed by recharge from upland areas that receive substantial precipitation. The Cold Creek and Dry Creek Valleys and Rattlesnake Mountains are the origins of this aquifer. The aquifer flows to the east and north from its sources and discharges into the Columbia River. The Yakima River borders the aquifer to the south, but otherwise plays a negligible role, especially with regard to that portion of the aquifer that underlies the 200 Area Plateau. Contamination from the exclusive waste management area will enter the aquifer from above over a very small portion of the land area of the aquifer. Thus, relatively few groundwater wells placed immediately downgradient of the buffer zone boundary would be able to withdraw contaminated groundwater from the aquifer, and correspondingly few individuals would be exposed to the contamination.

A small family farm would require on the order of $1.8 \times 10^{4} \mathrm{~m}^{3}$ of water each year. This is based on the rate of groundwater usage $\left(150 \mathrm{~L} / \mathrm{m}^{2} /\right.$ month for 6 months; Appendix F) from the agricultural scenario, and a family farm of 2 hectares (Kincaid et al. 1995). Thus, if a single-family farm supported an average family of 5 individuals, the aquifer would be required to supply $3.6 \times 10^{6} \mathrm{~m}^{3}$ of water each year to support a population of 1000 people.

Assuming that existing industrial discharges will be discontinued before Hanford Site closure, and that the groundwater system upgradient and beneath the 200 Area Plateau will come to an approximate steady state soon thereafter, one can approximate the groundwater discharge in the vicinity of the exclusive waste management area and buffer. Only a fraction of the flow of the unconfined aquifer passes beneath the 200 Area Plateau. A crude estimate of this quantity is given by the sum of groundwater entering the aquifer from the Cold Creek Valley, through the northern segment of Dry Creek Valley, and as natural recharge upgradient of the waste management area. Cole et al. (1997) estimated groundwater flux crossing the Cold Creek and northern Dry Creek boundaries as $1.05 \mathrm{x}$ $10^{6} \mathrm{~m}^{3} / \mathrm{yr}$ and $4.41 \times 10^{5} \mathrm{~m}^{3} / \mathrm{yr}$, respectively. The land area upgradient of the site represents less than 25 percent of the Hanford Site. Twenty-five percent of the natural recharge to the site is $-2.12 \times 10^{6} \mathrm{~m}^{3} / \mathrm{yr}$ (Fayer and Walters 1995). This represents an estimate of all contributions to the aquifer upgradient of the exclusive waste management area and buffer zone, and is $\sim 3.6 \times 10^{6} \mathrm{~m}^{3} / \mathrm{yr}$. Not all of this water resource would pass beneath the exclusive waste management area and buffer zone, and become contaminated. Thus, it is an overestimate. This analysis suggests that approximately 200 family farms and 1000 people could be supported by the unconfined aquifer immediately downgradient of the exclusive waste management area and buffer zone.

By making the following assumptions, the potential value of a full ALARA assessment can be appraised. 
- The period of interest is the 1000 years following loss of institutional control (DOE 1996b).

- The agricultural scenario yields the greatest dose and should be the basis for the long-term population impact assessment.

- A population of fewer than 1000 could consume Hanford Site groundwater and be exposed to its water quality.

- The exposure would continue for 1000 years without detection and remediation.

- A range of between $\$ 1000$ and $\$ 10,000$ per person-rem captures the cost to society from dose (DOE 1996b).

The dose estimate for the agricultural scenario shows a continual decline following present day maximums and is $\sim 5.5 \mathrm{mrem}$ in a year in 2050. It drops to less than 2 mrem in a year after 1000 years. During the 1000-year period, $4 \mathrm{mrem}$ in a year is a reasonable yet high average value for dose from the agricultural scenario. This representative individual dose applied to 1000 people for 1000 years results in a 4000-person-rem population dose. The resulting cost to society would range between $\$ 4$ million and $\$ 40$ million. This cost does not justify a more detailed ALARA assessment because the cost to society of further analysis and implementation of alternatives would likely be equal to or greater than this amount.

In addition to not being justified on a cost/benefit basis, it is important to note that a more detailed ALARA assessment involving a variety of remediation options could not be performed at this time. Aside from the analyses for the four low-level waste disposals to which this Composite Analysis is a companion, the other DOE sites included in this iteration of the Composite Analysis are subject to remediation under the CERCLA and RCRA programs at the Hanford Site. Analyses of sites and their alternate remedial actions completed under these programs are being and will be conducted jointly with representatives from Ecology and the EPA. Future iterations of the Composite Analysis will involve DOE, EPA, and Ecology, and if necessary, will include evaluations of alternate remedial actions.

\subsection{Suggestions for Further Study}

Improved confidence in the second and subsequent iterations of the Composite Analysis will come from improvements in a number of areas. Based on the experience gained in the first iteration, the most fruitful areas for improvement are the inventory, waste handling and engineered barriers, environmental mobility and models, and inclusion of additional sources.

\subsubsection{The Inventory}

Much has been accomplished in the past two decades to document the inventories of radionuclides and chemicals present at the Hanford Site. Process knowledge and waste transfers have been 
documented. The Track Radioactive Components (TRAC) model was developed (Jungfleisch 1980, 1983) and has been superseded by the Hanford Defined Waste (HDW) model (Agnew et al. 1997). The HDW model uses all available information; however, its development has been driven by the need to estimate the contents of single- and double-shell tanks. As mentioned above, some inventory entries are conservative estimates.

While appropriate for individual programs, conservative or bounding estimates are not as useful for the Composite Analysis. For the Composite Analysis, a conservative assessment implies a sequence of events that cause multiple plumes to arrive simultaneously at a point in space and moment in time. Performance assessments differ from the Composite Analysis because the former examine contaminants from a single source passing a single point in space. By using a conservative inventory estimate, one maximizes the dose consequence. This is not true of the Composite Analysis unless conservative estimates of inventory are used for all sources.

The inventory for the Hanford Site should be viewed in a holistic sense as a conserved quantity. Ideally, each nuclide has a known inventory for the Hanford Site based on the quantity imported or generated in the reactors. For those sites with potentially significant releases to the water table, it is important to examine the tradeoffs of inventory uncertainty. A greater inventory assigned to liquid discharge sites should correspond to a smaller inventory assigned to existing tank waste and future ILAW disposal. An estimate of the inventory emitted to the atmosphere should be accounted for in the overall inventory. That portion recovered by scrubbers and disposed at the Hanford Site should also be traced through the inventory to its final disposition. Ultimately, the most meaningful uncertainty analysis of inventory would be based on a best-estimate rather than bounding estimate.

Finally, if a full options analysis and ALARA assessment must be completed to evaluate alternate remediations, the Composite Analysis is used to identify those disposals most responsible for the dose. Alternate remediations must be proposed and studied for the wastes having the greatest impact rather than others of less significance. If bounding inventories have been used in the Composite Analysis, the analysis may need to be redone prior to proceeding with the options analysis and ALARA assessment.

Thus, the sitewide inventory assembled for the second iteration Composite Analysis should be a balanced and best estimate. The estimate should be balanced in the sense that gaseous, liquid, and solid waste inventories should be accounted for, should be consistent, and should be linked. This would enable the generation of sensitivity cases that examine the implications of a greater inventory lost to the atmosphere or sent in the liquid waste streams to cribs or tanks. The estimate needs to be centered about a best estimate that places waste where it is most likely to reside at the conclusion of Hanford Site operations. Sensitivity to inventory estimates could be analyzed as independent realizations that would be created by routing more or less waste to the atmosphere, to the liquid discharges sites, to the singleand double-shell tanks, and to the solid waste burial grounds. Reviewed and accepted methods of estimating the key mobile radionuclides of greatest importance to long-term health and safety studies should be incorporated into the inventory model. 
Such an inventory should be based on the HDW (Agnew et al. 1997) or a similar model of the Hanford Site inventory and the standard or best-basis inventory of Kupfer et al. (1997). It would then be possible to examine perturbations in the inventories assigned to specific waste disposal facilities. The assignments would be conditioned on the knowledge of processes and constrained by the knowledge of waste transfers. The influence of assumptions could be traced through the inventory estimates. For example, the assumed split of iodine-129 between gaseous and liquid phase, and the assumed effectiveness of silver-nitrate saddles in removing the iodine from the gaseous phase could be traced through to their resulting inventories assigned to the atmosphere, the solid waste landfills, and the liquids stored in tanks. Such a model would remedy the present issue of full accountability for the final disposal of key radionuclides including carbon-14 and iodine- 129 .

\subsubsection{Waste Handling and Engineered Barriers}

A major finding of the first iteration Composite Analysis is the separation in time of two release episodes. The first is the result of liquid discharges and tank leaks, and the second is the result of dry disposals. Confidence in this finding relies on the waste and its protective barriers, and estimates of contaminant migration and fate in the vadose zone. To a significant extent, confidence that dry disposals since 1988 will not release to the water table for hundreds of years relies on our confidence in engineered waste forms and barriers to infiltration and leaching. The following assumptions were made.

- Any large contributions to the key mobile nuclide inventories of the solid waste burial grounds will be detected prior to acceptance of the waste, and such a waste would be placed in a high-integrity waste form (e.g., mixed with a waste form material such as grout), or placed in a high-integrity container.

- Engineered systems such as the double liner and surface barrier of the ERDF will function to specifications.

- Engineered surface barriers placed over other wastes will perform to their design standards.

- The TWRS ILAW will meet performance specifications that have been the basis of its simulation in this analysis.

Confidence in the results of this and future Composite Analyses depend on efforts that justify the assumptions regarding the waste handling protocols, waste form performance, engineered barriers, and infiltration rates.

Increased confidence in long-term aspects of contaminant release and migration implies greater confidence in the performance of surface covers and protective barriers. Covers and barriers are included in disposal facility design to control or limit a number of impacts including intrusion by plants, small mammals, and humans, and especially the infiltration of water into the waste. Not all wastes will require the same cover or barrier. Consequently a graded approach to barrier design is needed. An 
understanding of the performance of the various design features or components of typical covers and protective barriers will enable $\mathrm{DOE}$ programs to incorporate into their designs only those cover features essential to the long-term performance of their waste. Studies should quantify the roles of surface soils, capillary interfaces between layers, climate variability, and plant dynamics in determining infiltration through the cover. Because barriers are assumed to function for decades and centuries, studies should seek to quantity the long-term durability of the components of typical covers and protective barriers. Studies should also quantify the potential for water to move laterally from the edge of the cover or barrier toward the waste form. This redistribution of water beneath the cover system may result in leaching of deep waste deposits including liquid discharge sites. Studies may show that covers have an influence over a finite depth, and that their ability to reduce infiltration rate or recharge in the deep vadose zone is mitigated by the layering of natural sediment deposits that act to spread surface infiltration laterally. Certainly, it will be important to fully understand and quantify infiltration rates applicable both before and after final covers are applied to waste sites.

\subsubsection{Environmental Mobility and Models}

The review of the 241-SX-109 tank leak experience (DOE 1997a) has placed previously accepted vadose zone conceptual models in question. Ongoing field studies with the purpose of developing better information on the physical extent and chemical mobility of tank wastes leaked to the subsurface are underway. This knowledge will enhance our ability to quantify the environmental response of liquids discharged to specific retention trenches and lost from tanks during waste recovery operations.

Through field study of leaks from tanks and discharges to cribs, the pathways and mobility of contaminants will become better understood. Based on the field evidence and our knowledge of the waste, alternate conceptual models of waste migration and fate in the subsurface can be posed. Conceptual models capture the physical features and physicochemical processes that produced the observed situation. They can be further studied through numerical simulation, and the alternate explanations of events can be narrowed to the few or one that best explain all of the field observations.

Issues that require resolution include explanations for the initially high mobility of some wastes and an evaluation of their ability to create or follow preferential flow paths. Another issue involves the ability of barriers to prevent leaching of the waste and ensure a slow flow and transport path to the water table (i.e., quantification of the edge effect of a surface barrier). Of particular importance to the second iteration Composite Analysis will be the development of confidence in estimates of long-term leaching and migration of wastes from dry disposals. The behavior of these dry disposal sites is difficult to study in the field because of the low release rates of dry waste. Under a dry regime, the migration of the release is less likely to find and then follow geologic formations (e.g., interfaces between coarse- and fine-grained sediments) that may represent preferential flow paths under wetter or saturated conditions. The release and migration will require special attention because they occur in a much drier regime than at the liquid disposal sites. Because of the time they have been exposed to potentially greater infiltration rates, the pre-1988 solid waste burial grounds may provide an opportunity to measure release and migration from burial grounds under less-than-optimal conditions. 
Our increasing knowledge of the physical position and chemical character of radionuclides in the vadose zone beneath tank leaks and liquid discharge facilities should be incorporated into the conceptual and mathematical models. By necessity, a one-dimensional model of the vadose zone was employed to simulate the numerous waste sites within the exclusive waste management area in the first iteration Composite Analysis. The greater understanding of contaminant migration in the vadose zone that will come from the ongoing and future vadose zone studies will either lead to the creation of more comprehensive, applicable, and accepted one-dimensional models or point to the need to perform multidimensional simulations of specific facilities or wastes. Certainly, the decision to proceed with the development and application of more sophisticated vadose zone transport models will be based on the perceived value of their predictive capability. An evaluation of their potential value may be approached through simulations with simpler models tailored to bound the potential impacts of the unresolved processes (e.g., multiphase physics, aqueous speciation, adsorption, precipitation) and geometries (e.g., two- or three-dimensional phenomena, preferential pathways) of a more sophisticated model. Studies may also conclude that probabilistic models are required. Regardless, completion of these studies and the implementation of the next generation models will lead to greater confidence in future iterations of the Composite Analysis.

\subsubsection{Inclusion of Additional Sources}

Numerous liquid discharge sites and canyon facilities were not modeled in this iteration of the Composite Analysis. In the case of liquid discharge sites, this is only justified by the belief that the most significant releases have been estimated and therefore included in current inventories. However, as inventory estimates are created for liquid-release and leak sites and canyon facilities not included in the first iteration, they will be included in future iterations of the Composite Analysis. The canyon buildings, their immovable underground filter assemblies, and the PUREX tunnels are another group of sources that need to be included in future analyses. The potential impacts of cesium-137 and strontium90 in the B Plant and in its sand and HEPA filters were included as a preliminary analysis of these major radionuclides. However, the inventory and location of the key mobile radionuclides in these structures need to be developed as the basis of a credible analysis of their potential impact.

\subsubsection{Use of Data Quality Objectives}

During this first iteration Composite Analysis, the concept of Data Quality Objectives (DQOs) as applied to a simulation-based analysis has been examined. Because the analysis was to use only information already at hand (DOE 1996b), the DQO process in this first iteration analysis became a dataacceptance or data-qualification process. While constrained in this iteration to not gather samples for analysis and use off-the-shelf information and capabilities, subsequent iterations will be expected to apply the DQO process. In light of the iterative character of Composite Analysis, the role of DQO in the next and subsequent iterations is important to understand.

The standard DQO process was developed in response to a need to define the quantity and quality of characterization data required for decisions at CERCLA and RCRA cleanup sites. As applied to a field 
characterization problem, the standard DQO process yields the sample size and equipment quality selection criteria that will meet the needs of the decision maker. This is achieved by balancing the risk and cost of making a decision error, against the cost of increasing the number of samples and achieving greater confidence in the field characterization.

This standard DQO approach can not be directly applied to the dose or risk forecast problem. The future state of the system is unknown and unknowable. Consequently, where in the field sampling problem one could completely sample the site and know the truth, there is no ability to know the true future. In the modified DQO process, the problem is one of balancing the cost of increasing model confidence or reducing uncertainty, against the cost or consequences of making an incorrect decision based on an incorrect model forecast. The poor decision may be to cleanup a site when it is not necessary, or to leave a site unremediated that deserves cleanup. A fully consistent DQO-based program would require a probabilistic analysis that considered sources of uncertainty in the inventory, release, vadose zone migration, aquifer migration, exposure mechanism, and dose or health consequence. Furthermore, the analysis would need to consider the propagation and compounding of uncertainty throughout the sequence of calculations. It is unlikely that sufficient data or resources exist to perform this analysis.

The second iteration Composite Analysis will gather information on the range and distribution of data. This information will enable an analysis of the sensitivity of the results to the range of estimated inventory, source term release, and environmental response. Subsequent Composite Analyses may use a probabilistic methodology and address the full uncertainty analysis. Prior to undertaking either sensitivity or uncertainty analyses during the second iteration Composite Analysis, it will be important to establish a baseline confidence in all elements of the analysis including the inventory, the release models and data, and the models and data for the vadose zone, groundwater, and exposure pathways.

\subsubsection{Linkage to the Hanford Groundwater Project, the 200 Area Characterization Program, and the TWRS Hanford Tanks Initiative}

Field observations must play a greater role in determining the base case conditions at the Hanford Site. Existing plumes in the vadose zone and groundwater are evidence of contaminant release and mobility. In response to CERCLA and RCRA guidance and DOE Orders 5400.1 and 5400.5 , a groundwater protection management plan is routinely issued (Barnett et al. 1995) for the groundwater resources at the Hanford Site. This plan describes the ongoing monitoring efforts. Before the next iteration of the Composite Analysis is completed, efforts should be made to include in this plan the work necessary to sample the aquifer for more of the key mobile radionuclides identified in the Composite Analysis including selenium-79 and chlorine-36. Similarly, efforts should be made to determine the distribution of key mobile radionuclides in the vadose zone beneath cribs, specific retention trenches, reverse wells, and tank farms.

Special efforts should be undertaken to sample groundwater and characterize the vadose zone in the vicinity of liquid discharge facilities (cribs, specific retention trenches, and reverse wells) and tank leaks 
that received the largest quantities of waste having large inventories of key mobile radionuclides. Recently identified radionuclides such as selenium- 79 and perhaps chlorine-36 should be added to laboratory analyses of water samples. In part, such efforts should attempt to substantiate the estimate of inventory (mass) and contaminant concentration discharged to the environment.

The sampling strategy should be designed to yield results suitable to provide an estimate of the mass of contaminant in the aquifer or vadose zone. Point samples taken at moments in time are prone to miss peak concentrations. Sampling a substantial interval of aquifer provides an integrated sample biased toward the water quality of the most conductive strata intercepted by the sample. Sampling short intervals over the depth of the vadose zone or aquifer borehole could provide valuable insight on contaminant distribution. Analysis of small intervals could identify sediment layers responsible for adsorption or precipitation phenomena in the vadose zone. Such an analysis of saturated sediments would begin to reveal the three-dimensional distribution of contaminants throughout the aquifer. Methods of estimating the contaminant mass, including a best estimate and range, are needed for comparison to model results of key mobile radionuclide discharges. Inventory estimates of key radionuclides discharged to cribs and leaked from tanks should be conditioned by our knowledge of the mass of those radionuclides found in the vadose zone and aquifer. 


\subsection{References}

Agnew S. F., J. Boyer, R. A. Corbin, T. B. Duran, J. R. Fitzpatrick, K. A. Jurgensen, T. P. Ortiz, B. L. Young. 1997. Hanford Tank Chemical and Radionuclide Inventories: HDW Model Rev. 4. LA-UR-96-3860, Los Alamos National Laboratory, Los Alamos, New Mexico.

Ahlstrom, S. W., H. P. Foote, R. C. Arnett, C. R. Cole, and R. J. Serne. 1977. Multicomponent Mass Transport Model: Theory and Numerical Implementation (Discrete-Parcel-Random-Walk Version). BNWL-2127, Battelle Northwest Laboratory, Richland, Washington.

Baker, S. M., R. F. Lorang, R. P. Elmore, A. J. Rossi, and M. D. Freshley. 1988. U1/U2 Uranium Plume Characterization, Remedial Action Review and Recommendation for Future Action. WHC-EP-0133, Westinghouse Hanford Company, Richland, Washington.

Barnett, D. B., J. S. Schmid, S. S. Lowe, W. L. Allen, N. A. Ballantyne, C. H. Dohrer, M. J. Hartman, F. N. Hodges, D. G. Horton, V. G. Johnson, K. J. Lueck, D. J. Ortiz, A. J. Knepp, B. H. Ford, S. J. Hope, D. K. Tyler, R. D. Hildebrand, D. E. Olson, R. E. Peterson, G. L. Kasza, D. A. Myers, S. P. Lattrell, P. D. Thorne, and R. K. Moser. 1995. Hanford Site Ground Water Protection Management Plan. DOE/RL-89-12, Rev. 2, U.S. Department of Energy, Richland, Washington.

Barnett, D. B., M. D. Freshley, M. P. Bergeron, S. K. Wurstner, and C. R. Cole. 1997. Tritium Monitoring in Groundwater and Evaluation of Model Predictions for the Honford Site 200 Area Effluent Treatment Facility. PNNL-11665, Pacific National Northwest Laboratory, Richland, Washington.

Bergsman, K. H. 1993. Hanford Irradiated Fuel Inventory Baseline. WHC-SD-CP-TI-175, Rev. 1, Westinghouse Hanford Company, Richland, Washington.

Bierschenk, W. H. 1959. Aquifer Characteristics and Ground-Water Movement at Hanford. HW-60601, General Electric Company, Hanford Atomic Products Operation, Richland, Washington.

Black, P. K., M. D. Neptune, R. T. Ryti, and D. D. Hickmott. 1994. "Professional Judgment in the Data Quality Objectives Process: A Bayesian Approach to Screening Assessment." In the Proceedings of the Federal Environmental Restoration III and Waste Minimization II Conference and Exhibition, New Orleans, Louisiana, April 25-29, 1994. LA-UR-94-1459, Los Alamos National Laboratory, Los Alamos, New Mexico.

Black, P., M. Hooten, K. Black, B. Moore, and B. Crowe. 1997. "Common-Sense Probabilistic Approach to Assessing the Inadvertent Human Intrusion into Low-Level Radioactive Waste at the Nevada Test Site." In Conference Proceedings of Waste Management 97, Tucson, Arizona, March 2-7, 1997. LA-UR-97-728, Los Alamos National Laboratory, Los Alamos, New Mexico. 
Buck, J. W., G. Whelan, J. G. Droppo, Jr., D. L. Strenge, K. J. Castleton, J. P. McDonald, C. Sato, and G. P. Streile. 1995. Multimedia Environmental Pollutant Assessment System (MEPAS), Application Guide. PNL-10395, Pacific Northwest Laboratory, Richland, Washington.

Campbell, J. E., D. E. Longsine, and M. Reeves. 1981. "Distributed velocity method of solving the convective-dispersion equation: 1 . Introduction, mathematical theory, and numerical implementation." Adv. Water Resources 4:102.

Cearlock, D. B., K. L. Kipp, and D. R. Friedrichs. 1975. The Transmissivity Iterative Calculation Routine - Theory and Numerical Implementation. BNWL-1706, Battelle Northwest Laboratory, Richland, Washington.

Chamness, M. A., and J. K. Merz. 1993. Hanford Wells. PNL-8800, Pacific Northwest Laboratory, Richland, Washington.

Chiaramonte, G. R., C. W. Denslow, A. J. Knepp, R. D. Landon, and S. Panday. 1997. Hanford Sitewide Groundwater Remediation Strategy - Groundwater Contaminant Predictions. BHI-00469, Rev. 1, Bechtel Hanford, Inc., Richland, Washington.

Clark, R. 1995. Solid Waste Information and Tracking System (SWITS) Software Design Descriptions (SDD). WHC-SD-WM-SWD-009, Rev. 8. Westinghouse Hanford Company, Richland, Washington.

Cole, C. R., F. W. Bond, S. M. Brown, and G. W. Dawson. 1984. Demonstration/Application of Ground-Water Modeling Technology for Evaluation of Remedial Action Alternatives.

Contract 68-03-3116, Municipal Environmental Research Laboratory, U. S. Environmental Protection Agency, Cincinnati, Ohio.

Cole, C. R., S. B. Yabusaki, and C. T. Kincaid. 1988. CFEST-SC, Coupled Fluid, Energy, and Solute Transport Code, SuperComputer Version, Documentation and User's Manual. Battelle, Pacific Northwest Laboratories, Richland, Washington.

Cole, C. R., S. K. Wurstner, M. P. Bergeron, M. D. Williams, and P. D. Thorne. 1997. ThreeDimensional Analysis of Future Groundwater Flow Conditions and Contaminant Plume Transport in the Hanford Site Unconfined Aquifer System: FY 1996 and 1997 Status Report. PNNL-11801, Pacific Northwest National Laboratory, Richland, Washington.

Comprehensive Environmental Response, Compensation, and Liability Act of 1980, as amended, Public Law 96-510, 94 Stat. 2767, 42 USC 9601 et seq.

Connelly, M. P., B. H. Ford, and J. W. Lindberg. 1992. Hydrogeologic Model for the 200 East Groundwater Aggregate Area. WHC-SD-EN-TI-019, Westinghouse Hanford Company, Richland, Washington. 
Connelly, M. P., B. H. Ford, and J. V. Borghese. 1992. Hydrogeologic Model for the 200 West Groundwater Aggregate Area. WHC-SD-EN-TI-014, Westinghouse Hanford Company, Richland, Washington

Connelly, M. P. 1994. Capture Zone Analyses for the 200-ZP-1 and 200-UP-1 Pilot Scale Pump-andTreat Tests. WHC-SD-EN-TI-252, Westinghouse Hanford Company, Richland, Washington.

Croff, A. G. 1980. ORIGEN2 - A Revised and Updated Version of the Oak Ridge Isotope Generation and Depletion Code. ORNL-5621, Oak Ridge National Laboratory, Oak Ridge, Tennessee.

Deju, R. A. 1974. The Hanford Field Testing Program. ARHC-00004-201, Atlantic Richfield Hanford Company, Richland, Washington.

Devary, J. L. 1987. The CFEST-INV Stochastic Hydrology Code: Mathematical Formulation, Application, and User's Manual. ICF Northwest, Richland, Washington.

DiCenso, A. T., and B. C. Simpson. 1994. Tank Characterization Report for Double-Shell Tank 241-AW-105. WHC-SD-WM-ER-364, Rev. 0, Westinghouse Hanford Company, Richland, Washington.

Dirkes, R. L., and R. W. Hanf (eds.) 1997. Hanford Site Environmental Report for Calendar Year 1996. PNNL-11472, Pacific Northwest National Laboratory, Richland, Washington.

DOE - See U.S. Department of Energy (DOE).

Dove, F. H., C. R. Cole, M. G. Foley, F. W. Bond, R. E. Brown, W. J. Deutsch, M. D. Freshley, S. K. Gupta, P. J. Gutknecht, W. L. Kuhn, J. W. Lindberg, W. A. Rice, R. Schalla, J. F. Washburn, and J. T. Zellmer. 1982. AEGIS Technology Demonstration for a Nuclear Waste Repository in Basalt. PNL-3632, Pacific Northwest Laboratory, Richland, Washington.

Droppo, J. G., Jr., and J. W. Buck. 1996. Multimedia Environmental Pollutant Assessment System (MEP AS): Atmospheric Pathway Formulation. PNNL-1 1080, Pacific Northwest National Laboratory, Richland, Washington.

Ebbert, J. C., S. E. Cox, B. W. Drost, and K. M. Shurr. 1993. Distribution and Sources of Nitrates, and Presence of Fluoride and Pesticides, in Parts of the Pasco Basin, Washington, 1986-88. Water Resources Investigations Report 93-4197, U.S. Geological Survey, Tacoma, Washington.

Eckerman, K. F., A. B. Wolbarst, and A. C. B. Richardson. 1988. Limiting Values of Radionuclide Intake and Air Concentration and Dose Conversion Factors for Inhalation, Submersion, and Ingestion, Federal Guidance Report No. 11. EPA-520/1-88-202. U.S. Environmental Protection Agency, Office of Radiation Programs, Washington, D.C. 
Eckerman, K. F., and Ryman, J. C. 1993. External Exposure to Radionuclides in Air, Water, and Soil, Federal Guidance Report No. 12. EPA 402-R-93-081, U.S. Environmental Protection Agency, Office of Radiation Programs, Washington, D.C.

Eddy, P. A., D. A. Myers, and J. R. Raymond. 1978. Vertical Contamination in the Unconfined Groundwater at the Hanford Site, Washington. PNL-2724, Pacific Northwest National Laboratory, Richland, Washington.

Evans, J. C., D. I. Dennison, R. W. Bryce, P. J. Mitchell, D. R. Sherwood, K. M. Krupka, N. W. Hinman, E. A. Jacobson, and M. D. Freshley. 1988. Honford Site Ground-Water Monitoring for July Through December 1987. PNL-6315-2, Pacific Northwest Laboratory, Richland, Washington.

Farmer, C. L. 1986. The Dispersal of Contaminants in Heterogeneous Aquifers: A Review of Methods of Estimating Scale Dependent Parameters. AEEW R-2058, Atomic Energy Establishment, Winfrith, Dorchester, Dorset, England.

Fayer, M. J., and T. B. Walters. 1995. Estimated Recharge Rates at the Hanford Site. PNL-10285, Pacific Northwest Laboratory, Richland, Washington.

Fayer, M. J., R. E. Lewis, R. E. Engelman, A. J. Pearson, C. J. Murray, J. L. Smooth, R. Randall, W. H. Wegener, and A. H. Lu. 1995. Re-Evaluation of a Subsurface Injection Experiment for Testing Flow and Transport Models. PNL-10860, Pacific Northwest National Laboratory, Richland, Washington.

Fecht, K. R., G. V. Last, K. R. Price. 1977. Evaluation of Scintillation Probe Profiles from 200 Area Cribs Monitoring Wells, Volumes II and III. ARH-ST-156, Atlantic Richfield Hanford Company, Richland, Washington.

Foley, M. G., D. J. Bradley, C. R. Cole, J. P. Hanson, K. A. Hoover, W. A. Perkins, and M. D. Williams. 1995. Hydrogeology of the West Siberian Basin and Tomsk Region. PNL-10585, Pacific Northwest Laboratory, Richland, Washington.

Freeman-Pollard, J. R., J. A. Caggiano, and S. J. Trent. 1994. Engineering Evaluation of the GAO-RCED-89-157, Tank 241-T-106 Vadose Zone Investigation. BHI-00061, Rev. 0, Bechtel Hanford, Inc., Richland, Washington.

Freeze, R. A., B. James, J. Massmann, T. Sperling, and L. Smith 1992. "Hydrogeological Decision Analysis: 4. The Concept of Data Worth and its Use in the Development of Site Investigation Strategies." Ground Water 30(4):574-588.

Freeze, R. A., and J. A. Cherry. 1979. Groundwater. Prentice-Hall, Inc., Englewood Cliffs, New Jersey. Freshley, M. D., and M. J. Graham. 1988. Estimation of Ground-Water Travel Time at the Hanford Site: Description, Past Work, and Future Needs. PNL-6328, Pacific Northwest Laboratory, Richland, Washington. 
Friedrichs, D. R., C. R. Cole, and R. C. Arnett. 1977. Hanford Pathline Calculational Program Theory, Error Analysis and Applications. ARH-ST-149, Atlantic Richfield Hanford Company, Richland, Washington.

Gale J., R. Macleod, J. Welhan, C. R. Cole, and L. W. Vail. 1987. Hydrogeological Characterization of the Stripa Site. Technical Report 87-15, SKB, Stockholm, Sweden.

Gelhar, L. W. and C. L. Axness. 1981. Stochastic Analysis of Macro-Dispersion in Three-Dimensionally Heterogeneous Aquifers. Report No. H-8, Hydraulic Research Program, New Mexico Institute of Mining and Technology, Soccorro, New Mexico.

Gelhar, L. W., A. Mantoglou, C. Welty, and K. R. Rehfeldt. 1985. A Review of Field-Scale Physical Solute Transport Processes in Saturated and Unsaturated Porous Media. EPRI EA-4190, Project 2485-5, Electric Power Research Institute, Palo Alto, California.

Gelhar, L. W., C. Welty, and K. R. Rehfeldt. 1992. "A critical review of data on field-scale dispersion in aquifers." Water Resour. Res. 28:1955-1974.

Gilbert, R.O., E. A. Bittner, and E. H. Essington. 1995. "On the Use of Uncertainty Analyses to Test Hypotheses Regarding Deterministic Model Predictions of Environmental Processes." J. Environ. Radioactivity, Vol. 27, No. 3, pp.231-260.

Golder Associates. 1990. Travel Time Estimates for Alternative Tritium Crib Locations, Hanford Site, Washington. WHC-SD-EN-EE-002, Rev. 0, Westinghouse Hanford Company, Richland, Washington.

Graham, M. J., M. D. Hall, S. R. Strait, and W. R. Brown. 1981. Hydrology of the Separations Area. RHO-ST-42, Rockwell Hanford Operations, Richland, Washington.

Grant Environmental, Chase Environmental Group, and US Ecology, Inc. 1996. Site Stabilization and Closure Plan for Low-Level Radioactive Waste Management Facility, US Ecology, Inc., Richland, Washington. US Ecology, Inc., Richland, Washington.

Gupta, S. K., C. R. Cole, C. T. Kincaid, and A. M. Monti. 1987. Coupled Fluid, Energy, and Solute Transport (CFEST) Model: Formulation and User's Manual. Report BMI/ONWI-660, Battelle Memorial Institute, Columbus, Ohio.

Gupta, S. K. 1997. Draft User's Manual, CFEST-96 Flow and Solute Transport, Constant/Variable Density, Computationally Efficient, and Low Disk PC/Unix Version. Consultant for Environmental System Technologies, Irvine, California.

Hanford Future Site Uses Work Group (HFSWG). 1992. The Future for Hanford: Uses and Cleanup, the Final Report of the Hanford Future Site Uses Working Group. Hanford Future Site Uses Working Group, Westinghouse Hanford Company, Richland, Washington. 
Hanlon, B. M. 1996. Waste Tank Summary Report for Month Ending August 31, 1996.

WHC-EP-0182-101, Westinghouse Hanford Company, Richland, Washington.

Hanlon, B. M. 1997. Waste Tank Summary Report for Month Ending February 28, 1997.

WHC-EP-0182-107, Westinghouse Hanford Company, Richland, Washington.

Hartman, M. J., and K. A. Lindsey. 1993. Hydrogeology of the 100-N Area, Hanford Site, Washington. WHC-SD-EN-EV-027, Westinghouse Hanford Company, Richland, Washington.

Hartman, M. J.; and P. E. Dresel (eds.) 1997. Hanford Site Groundwater Monitoring for Fiscal Year 1996. PNNL-11470, Pacific Northwest National Laboratory, Richland, Washington.

Hodges, F. N. 1998. Results of Phase I Groundwater Quality Assessment for Single-Shell Tank Waste Management Areas T and TX-TY at the Hanford Site. PNNL-11809, Pacific Northwest National Laboratory, Richland, Washington.

Holdren, G. R., C. S. Glanz, L. K. Berg, K. Delinger, C. J. Fosmire, S. M. Goodwin, J. R. Rustad, R. Schalla, and J. A. Schramke. 1995. Environmental Settings for Selected U.S. Department of Energy Installations - Support Information for the Programmatic Environmental Impact Statement and the Baseline Environmental Management Report. PNL-10550, Pacific Northwest Laboratory, Richland, Washington.

International Atomic Energy Agency (IAEA). 1989. Evaluating the Reliability of Predictions Made Using Environmental Transfer Models. Safety Series No. 100, Vienna, Austria.

International Commission on Radiological Protection (ICRP). 1979a. ICRP Publication 30, Part 1, Limits for Intakes of Radionuclides by Workers. Annals of the ICRP, Vol. 2, No. 3 and 4, Pergamon Press, Elmsford, New York.

International Commission on Radiological Protection (ICRP). 1979b. ICRP Publication 30, Supplement to Part 1, Limits for Intakes of Radionuclides by Workers. Annals of the ICRP, Vol. 3, No. 1-4, Pergamon Press, Elmsford, New York.

Jacobson, E. A. 1985. A Statistical Parameter Estimation Method Using Singular Value Decomposition With Application to Avra Valley Aquifer in Southern Arizona. Dissertation, Department of Hydrology and Water Resources, University of Arizona, Tucson, Arizona.

Jacobson, E. A., and M. D. Freshley. 1990. An Initial Inverse Calibration of the Groundwater Model for the Hanford Unconfined Aquifer. PNL-7144, Pacific Northwest Laboratory, Richland, Washington.

Johnson, V. G., and C. J. Chou. 1998. Results of Phase I Groundwater Quality Assessment for SingleShell Tank Waste Management Areas S-SX at the Hanford Site. PNNL-11810, Pacific Northwest National Laboratory, Richland, Washington. 
Jungfleisch, F. M. 1980. Hanford High-Level Defense Waste Characterization - A Status Report. RHO-CD-1019, Rockwell Hanford Operations, Richland, Washington.

Jungfleisch, F. M. 1983. Supplementary Information for the Preliminary Estimation of Waste Tank Inventories in Hanford Tanks through 1980. SD-WM-TI-058, Rockwell Hanford Operations, Richland, Washington.

Kaplan, D. I., and R. J. Serne. 1995. Distribution Coefficient Values Describing Iodine, Neptunium, Selenium, Technetium, and Uranium Sorption to Hanford Sediments. PNL-10379, Sup. 1, Pacific Northwest Laboratory, Richland, Washington.

Kaplan, D. I., R. J. Serne, A. T. Owen, J. Conca, T. W. Wietsma, and T. L. Gervais. 1996. Radionuclide Adsorption Coefficients Measured in Hanford Sediments for the Low Level Waste Performance Assessment Project. PNNL-11385, Pacific Northwest National Laboratory, Richland, Washington.

Khaleel, R., and E. J. Freeman. 1995. Variability and Scaling of Hydraulic Properties for 200 Area Soils, Hanford Site. WHC-EP-0883, Westinghouse Hanford Company, Richland, Washington.

Kincaid, C. T., J. W. Shade, G. A. Whyatt, M. G. Piepho, K. Rhoads, J. A. Voogd, J. H. Westsik, Jr., M. D. Freshley, K. A. Blanchard, and B. G. Lauzon. 1995. Volumes 1 and 2: Performance Assessment of Grouted Double-Shell Tank Waste Disposal at Hanford. WHC-SD-WM-EE-004, Rev. 1, Westinghouse Hanford Company, Richland, Washington.

Kipp, K. L., A. E. Reisenauer, C. R. Cole, and L. A. Bryan. 1972. Variable Thickness Transient Groundwater Flow Model: Theory and Numerical Implementation. BNWL-1703, Battelle Northwest Laboratory, Richland, Washington.

Kipp, K. L., and R. D. Mudd. 1973. Collection and Analysis of Pump Test Data for Transmissivity Values. BNWL-1709, Battelle Northwest Laboratory, Richland, Washington.

Kipp, K. L., and R. D. Mudd. 1974. Selected Water Table Contour Maps and Well Hydrographs for the Hanford Reservation, 1944-1973. BNWL-B-360, Battelle Northwest Laboratory, Richland, Washington.

Kirkbride, R. A., G. K. Allen, P. J. Certa, A. F. Manuel, R. M. Orme, L. W. Shelton, E. J. Slaathaung, R. S. Wittman, G. T. MacLean, and D. L. Penwell. 1997. Tank Waste Remediation System Operation and Utilization Plan Vol. I and II. HNF-SD-WM-SP-012, Rev. 0, Numatec Hanford Corporation, Richland, Washington.

Kupfer, M. J., A. L. Boldt, B. A. Higley, K, M. Hodgson, L. W. Sheiton, B. C. Simpson, R. A. Watrous, M. D. Le Clair, G. L. Borsheim, R. T. Winward, R. M. Orme, N. G. Colton, S. L. Lambert, D. E. Place, and W. W. Schulz. 1997. Standard Inventories of Chemicals and Radionuclides in Hanford Site Tank Wastes. HNF-SD-WM-TI-740, Rev. 0, Lockheed Martin Hanford Corporation, Richland, Washington. 
Lallemand-Barres, A. and P. Peaudecerf. 1978. "Recherche des Relations Entre La Valeur de la Dispersivite Macroscopique D'un Milieu Aquifere, Ses Autres Caracteristiques et les Conditions de Mesure.” Bulletin de Recherches Geologiques Minieres, 2e Serie, Section III, Number 4, Orleans, France.

Law, A.G., and A.H. Lu. 1982. Extrapolation of Results from the 216-B-5 Reverse Well Study. RHO-HS-EV-9, Rockwell Hanford Operations, Richland, Washington.

Law, A. G. 1992. Groundwater Modeling and Plume Migration Analyses for Candidate Soil Column Disposal Sites Hanford, Washington. WHC-SD-EN-ES-022, Westinghouse Hanford Company, Richland, Washington.

Law, A., S. Panday, C. Denslow, K. Fecht, and A. Knepp. 1996. Hanford Site-wide Groundwater Flow and Transport Model Calibration Report. BHI-00608, Bechtel Hanford, Inc., Richland, Washington.

Liikala, T. L., and R. L. Aaberg. 1988. Geohydrologic Characterization of the Area Surrounding the 183-H Solar Evaporation Basins. PNL-6728, Pacific Northwest Laboratory, Richland, Washington.

Liikala, T. L. 1994. Hydrogeology Along the Southern Boundary of the Hanford Site Between the Yakima and Columbia Rivers, Washington. PNL-10094, Pacific Northwest Laboratory, Richland, Washington.

Lindberg, J. W., and F. W. Bond. 1979. Geohydrology and Ground-Water Quality Beneath the 300 Area, Hanford Site, Washington. PNL-2949, Pacific Northwest Laboratory, Richland, Washington.

Lindberg, J. W. 1993a. Geology of the 100-B/C Area, Hanford Site, South-Central Washington. WHCSD-EN-TI-133, Westinghouse Hanford Company, Richland, Washington.

Lindberg, J. W. 1993b. Geology of the 100-K Area, Hanford Site, South-Central Washington. WHC-SDEN-TI-155, Westinghouse Hanford Company, Richland, Washington.

Lindsey, K. A., B. N. Bjornstad, and M. P. Connelly. 1991. Geologic Setting of the 200 West Area: An Update. WHC-SD-EN-TI- 008, Westinghouse Hanford Company, Richland, Washington.

Lindsey, K. A. 1991. Revised Stratigraphy for the Ringold Formation, Hanford Site, South-central Washington. WHC-SD-EN-EE-004, Rev. 0, Westinghouse Hanford Company, Richland, Washington.

Lindsey, K. A., B. N. Bjornstad, J. W. Lindberg, and K. M. Hoffman. 1992. Geologic Setting of the 200 East Area: An Update. WHC-SD-EN-TI-012, Westinghouse Hanford Company, Richland, Washington.

Lindsey, K. A. 1992. Geology of the Northern Part of the Hanford Site: An Outline of Data Sources and the Geologic Setting of the 100 Areas. WHC-SD-EN-TI-011, Rev. 0, Westinghouse Hanford Company, Richland, Washington. 
Lindsey, K. A. and G. K. Jaeger. 1993. Geologic Setting of the 100-HR-3 Operable Unit, Hanford Site, South-Central Washington. WHC-SD-EN-TI-132, Westinghouse Hanford Company, Richland, Washington.

Lowe, S. S. 1993. Engineering Study of Tank Leaks Related to Hydraulic Retrieval of Sludge from Tank 241-C-106. WHC-SD-WM-ES-218, Rev. 1, Westinghouse Hanford Company, Richland, Washington.

Mann, F. M. 1995. Data Packages for the Hanford Low-Level Tank Waste Interim Performance Assessment. WHC-SD-WM-RPT-166, Rev. 0, Westinghouse Hanford Company, Richland, Washington.

Mann, F. M., C. R. Eiholzer, Y. Chen, N. W. Kline, A. H. Lu, B. P. McGrail, P. D. Rittmann, G. F. Williamson, J. A. Voogd, N. R. Brown, and P. E. LaMont. 1997. Hanford Low-level Tank Waste Interim Performance Assessment. HNF-EP-0884, Rev. 1, Lockheed Martin Hanford Corporation, Richland, Washington.

Mills, D. B., D. B. Porcella, M. J. Ungs, S. A. Gherini, K. V. Summers, Lingfung Mok, G. L. Rupp, G. L. Bowie, and D. A. Haith. 1985. WATER QUALITY ASSESSMENT: A screening Procedure for Toxic and Conventional Pollutants (Revised 1985) Part II. EPA/600/6-85/002b, Environmental Research Laboratory Office of Research and Development, U. S. Environmental Protection Agency, Athens, Georgia.

Morgan, G. M. and M. Henrion. 1990. Uncertainty, A Guide to Dealing with Uncertainty in Quantitative Risk and Policy Analysis. Cambridge University Press, New York, New York.

Mualem, Y. 1976. "A New Model for Predicting the Hydraulic Conductivity of Unsaturated Porous Media." Water Resources Research 12:513.

Napier, B. N., R. A. Peloquin, D. L. Strenge, and J. V. Ramsdell. 1988. Conceptual Representation, Volume 1 of GENII - The Hanford Environmental Radiation Dosimetry Software System. PNL-6584, Vol. 1, Pacific Northwest Laboratory, Richland, Washington.

National Council on Radiation Protection (NCRP). 1996. "A Guide for Uncertainty Analysis in and Risk Assessments Related to Environmental Contamination." NCRP Commentary No. 14, National Council on Radiation Protection and Measurements, Bethesda, Maryland.

Neitzel, D. A. (ed.) 1997. Hanford Site National Environmental Policy Act (NEPA) Characterization. PNNL-6415, Rev. 9, Pacific Northwest National Laboratory, Richland, Washington.

Neuman, S. P. 1993. "Comment On 'A Critical Review of Data on Field-Scale Dispersion in Aquifers' by L. W. Gelhar, C. Welty, and K. R. Rehfeldt.” Water Resour. Res. 29(6):1863-1865.

Neuman, S. P. and S. Yakowitz. 1979. "A Statistical Approach to the Inverse Problem of Aquifer Hydrology, 1, Theory." Water Resour. Res. 15(4):845-860. 
Nichols, W. E., Aimo, N. J., M. Oostrom, and M. D. White. 1997. STOMP Subsurface Transport Over Multiple Phases: Application Guide. PNNL-11216, Pacific Northwest National Laboratory, Richland, Washington.

Oak Ridge National Laboratory (ORNL). 1997. Integrated Data Base for 1997: U.S. Spent Fuel and Radioactive Waste Inventories, Projections, and Characteristics. DOE/RW-0006, Rev. 13, U.S. Department of Energy, Washington, D.C.

Parrington, J. R., H. D. Knox, S. L. Breneman, E. M. Baum, and F. Feiner. 1996. Nuclides and Isotopes, Fifteenth Edition. General Electric Nuclear Energy, San Jose, California.

Penwell, D. L., C. E. Grenard, and R. S. Wittman. 1996. Initial Retrieval Sequence and Blending Strategy. WHC-SD-WM-RPT-229, Rev. 0, Westinghouse Hanford Company, Richland, Washington.

Peterson, C. A. 1996. Technical Basis for Classification of Low-Activity Waste Fraction from Hanford Site Tanks. WHC-SD-WM-TI-699, Rev. 1, Westinghouse Hanford Company, Richland, Washington.

Peterson, R. E. 1992. Hydrologic and Geologic Data Available for the Region North of Gable Mountain, Hanford Site, Washington. WHC-SD-EN-TI-006, Westinghouse Hanford Company, Richland, Washington.

Puget Sound Power and Light (PSPL). 1982. Skagit/Hanford Nuclear Project, Preliminary Safety Analysis Report. Appendix 20, Amendment 23, Puget Sound Power and Light Company, Bellevue, Washington.

Record of Decision (ROD). September 16, 1993. U.S. Department of Energy. "Record of Decision: Decommissioning of Eight Surplus Production Reactors at the Hanford Site, Richland, WA." 58 Federal Register 48509-48513. (Cited in text as ROD 1993.)

Record of Decision (ROD). 1995. Declaration of the Record of Decision, U.S. DOE Hanford Environmental Restoration Disposal Facility, Hanford Site, Benton County, Washington. U.S. Department of Energy, Richland, Washington. (Cited in text as ROD 1995.)

Record of Decision (ROD). February 26, 1997. U.S. Department of Energy. "Record of Decision for the Tank Waste Remediation System, Hanford Site, Richland, WA.” 62 Federal Register 8693-8704. (Cited in text as ROD 1997.)

Record of Decision (Amended ROD). September 25, 1997. U.S. Department of Energy Environmental Restoration Disposal Facility, Hanford Site-200 Area, Benton County, Washington, Amended Record of Decision, Decision Summary and Responsiveness Summary. U.S. Environmental Protection Agency, Seattle, Washington. (Cited in text as Amended ROD 1997.)

Resource Conservation and Recovery Act of 1976, as amended, Public Law 94-580, 90 Stat. 3221, 42 USC 6901 et seq. 
Rockhold, M. L, C. S. Simmons, and M. J. Fayer. 1997. "An analytical solution technique for onedimensional, steady vertical water flow in layered soils." Water Resources Research 33(4):897-902.

Schmittroth, F. A., T. H. DeLorenzo, D. W. Wootan, and D. Y. Garbrick. 1995. Inventories for LowLevel Tank Waste. WHC-SD-WM-RPT-164, Rev. 0, Westinghouse Hanford Company, Richland, Washington.

Schreckhise, R. G., K. Rhoads, J. S. Davis, B. A. Napier, and J. V. Ramsdell. 1993. Recommended Environmental Dose Calculation Methods and Hanford-Specific Parameters. PNL-3777, Rev. 2, Pacific Northwest Laboratory, Richland, Washington.

Simmons, C. S., C. T. Kincaid, and A. E. Reisenauer. 1986. A Simplified Model for Radioactive Contaminant Transport: The TRANSS Code. PNL-6029, Pacific Northwest Laboratory, Richland, Washington.

Sisson, J: B. and A. H. Lu. 1984. Field Calibration of Computer Models for Application to Buried Liquid Discharges: A Status Report. RHO-ST-46P, Rockwell Hanford Operations, Richland, Washington.

State of Washington Department of Ecology (Ecology), U.S. Environmental Protection Agency (EPA), and U.S. Department of Energy (DOE). 1989. Hanford Federal Facility Agreement and Consent Order Between the U.S. Environmental Protection Agency, the U.S. Department of Energy, and the State of Washington Department of Ecology, May 15, 1989, as amended. Olympia, Seattle, and Richland, Washington.

Strenge, D. L., and P. J. Chamberlain. 1994. Evaluation of Unit Risk Factors in Support of the Hanford Remedial Action Environmental Impact Statement. PNL-10190, Pacific Northwest Laboratory, Richland, Washington.

Superfund Amendments and Reauthorization Act of 1986, as amended, Public Law 99-499, 100 Stat. 1613,42 USC 11001 et seq.

Swanson, L. C. 1992. Phase 1 Hydrogeologic Summary of the 300-FF-5 Operable Unit, 300 Area. WHC-SD-EN-TI-052, Westinghouse Hanford Company, Richland, Washington.

Swanson, L. C. 1994. 1994 Characterization Report for the State Approved Land Disposal Site. WHCSD-CO18H-RPT-003, Westinghouse Hanford Company, Richland, Washington.

Thorne, P. D., and M. A. Chamness. 1992. Status Report on the Development of a Three-Dimensional Conceptual Model for the Hanford Site Unconfined Aquifer System. PNL-8332,.Pacific Northwest Laboratory, Richland, Washington. 
Thorne, P. D., and D. R. Newcomer. 1992. Summary and Evaluation of Available Hydraulic Property Data for the Hanford Site Unconfined Aquifer System. PNL-8337, Pacific Northwest Laboratory, Richland, Washington.

Thorne, P. D., and M. A. Chamness., F. A. Spane Jr., V. R. Vermeul, and W. D. Webber. 1993. ThreeDimensional Conceptual Model of the Hanford Site Unconfined Aquifer System, FY 93 Status Report. PNL-8971, Pacific Northwest Laboratory, Richland, Washington.

Thorne, P. D., and M. A. Chamness., V. R. Vermeul, Q. C. MacDonald, and S. E. Schubert. 1994. Three-Dimensional Conceptual Model for the Hanford Site Unconfined Aquifer System, FY 1994 Status Report. PNL-10195, Pacific Northwest Laboratory, Richland, Washington.

U.S. Department of Energy (DOE). 1987. Final Environmental Impact Statement, Disposal of Hanford Defense High-Level, Transuranic and Tank Waste, Hanford Site, Richland, Washington. DOE/EIS-0113, Vol. 1-5, U.S. Department of Energy, Richland, Washington.

U.S. Department of Energy (DOE). 1988a. Consultation Draft, Site Characterization Plan, Reference Repository Location, Hanford Site, Washington. DOE/RW-0164, Vol. 1 and 2, U.S. Department of Energy, Richland, Washington.

U. S. Department of Energy (DOE). 1988b. "Radioactive Waste Management." DOE Order 5820.2A.

U.S. Department of Energy (DOE). 1989. Decommissioning of Eight Surplus Production Reactors at the Hanford Site, Richland, Washington. DOE/EIS-0119D, Draft Environmental Impact Statement, U.S. Department of Energy, Washington, D.C.

U.S. Department of Energy (DOE). 1991. Description of Codes and Models to be Used in Risk Assessment. DOE/RW-9144, U.S. Department of Energy, Richland, Washington.

U.S. Department of Energy (DOE). 1992. Decommissioning of Eight Surplus Production Reactors at the Hanford Site, Richland, Washington, Addendum. DOE/EIS-0119F, U.S. Department of Energy, Washington, D.C.

U.S. Department of Energy (DOE). 1993a. Phase I Investigation Report for 200-BP-I Operable Unit. DOE/RL-92-70, Rev. 0, Volume 1, U.S. Department of Energy, Richland, Washington.

U.S. Department of Energy (DOE). 1993b. "Radiation Protection of the Public and the Environment." DOE Order 5400.5, Change 2.

U.S. Department of Energy (DOE). 1994a. Feasibility Study Report for the 200-BP-1 Operable Unit. DOE/RL-93-95, Rev. 1, U.S. Department of Energy, Richland, Washington. 
U.S. Department of Energy (DOE). 1994b. Remedial Investigation and Feasibility Study Report for the Environmental Restoration Disposal Facility. DOE/RL-93-99, Rev. 1, U.S. Department of Energy, Richland, Washington.

U.S. Department of Energy (DOE). 1995. Hanford Site Risk Assessment Methodology. DOE/RL-91-45, Rev. 3, U.S. Department of Energy, Richland, Washington.

U.S. Department of Energy (DOE). 1996a. Draft Hanford Remedial Action Environmental Impact Statement and Comprehensive Land Use Plan. DOE/EIS-0222D, U.S. Department of Energy, Washington, D.C.

U.S. Department of Energy (DOE). 1996b. Guidance for a Composite Analysis of the Impact of Interacting Source Terms. on the Radiological Protection of the Public from Department of Energy LowLevel Waste Disposal Facilities. U.S. Department of Energy, Washington, D.C.

U.S. Department of Energy (DOE). 1996c. Hanford Facility Dangerous Waste Permit Application, PUREX Storage Tunnels. DOE/RL 90-24, Rev. 3, U.S. Department of Energy, Richland, Washington.

U.S. Department of Energy (DOE). 1996d. Hanford Strategic Plan. DOE/RL-96-92, U.S. Department of Energy, Richland, Washington.

U.S. Department of Energy (DOE). 1996e. Implementation Plan, Defense Nuclear Facilities Safety Board Recommendation 94-2, Conformance with Safety Standards at Department of Energy Low-Level Nuclear Waste and Disposal Sites. Rev. 1, U.S. Department of Energy, Washington, D.C.

U.S. Department of Energy (DOE). 1996f. Interim Format and Content Guide and Standard Review Plan for U.S. Department of Energy Low-Level Waste Disposal Facility Performance Assessments. U.S. Department of Energy, Washington, D.C.

U.S. Department of Energy (DOE). 1996g. Remedial Design Report/Remedial Action Work Plan for the 100 Area. DOE/RL-96-17, Rev. 0, U.S. Department of Energy, Richland, Washington.

U.S. Department of Energy (DOE). 1996h. Revised Interim DOE Policy on Management, Disposal, Direction, and Oversight of Low-Level Radioactive Waste Management and Disposal. July 31, 1996, U.S. Department of Energy, Washington, D.C.

U.S. Department of Energy (DOE). 1996i. 200 Areas Soil Remediation Strategy-Environmental Restoration Program. DOE/RL-96-67, Rev. 0, U.S. Department of Energy, Richland, Washington.

U.S. Department of Energy (DOE). 1997a. TWRS Vadose Zone Contamination Issue, Expert Panel Status Report. DOE/RL-97-49, Rev. 0, U.S. Department of Energy, Richland, Washington.

U.S. Department of Energy (DOE). 1997b. Waste Site Groupings for 200 Areas Soil Investigations. DOE/RL-96-81, U.S. Department of Energy, Richland, Washington. 
U.S. Department of Energy (DOE) and State of Washington Department of Ecology (Ecology). 1996. Tank Waste Remediation System, Hanford Site, Richland, Washington, Final Environmental Impact Statement. DOE/EIS-0189, U.S. Department of Energy, Richland, Washington.

U.S. Environmental Protection Agency (EPA). 1994. Guidance for the Data Quality Objectives Process. EPA QA/G-4, U.S. Environmental Protection Agency, Washington, D.C.

U.S. Energy Research \& Development Administration (ERDA). 1975. Final Environmental Statement, Waste Management Operations, Hanford Reservation, Richland Washington. ERDA-1538, Volume $1 \mathrm{pp}$ II.1-68. National Technical Information Service, Springfield, Virginia.

van Genuchten, M. 1980. "A Closed-Form Equation for Predicting the Hydraulic Conductivity of Unsaturated Soils.” Soil Sci. Am. J. 44:892-898.

Waite, J. L. 1991. Tank Wastes Discharged Directly to the Soil at the Hanford Site. WHC-MR-0227, Westinghouse Hanford Company, Richland, Washington.

Walters, W. H., M. C. Richmond, and B. G. Gilmore. 1994. Reconstruction of Radionuclide Concentrations in the Columbia River from Hanford, Washington to Portland, Oregon, January 1950January 1971. BNWD-2225 HEDR, Battelle Pacific Northwest Division, Richland, Washington.

Walton, W. C. 1985. Practical Aspects of Groundwater Modeling. Second Edition, National Water Well Association, Worthington, Ohio.

Ward, A. L., G. W. Gee, and M. D. White. 1997. A Comprehensive Analysis of Contaminant Transport in the Vadose Zone Beneath Tank SX-109. PNNL-11463, Pacific Northwest National Laboratory, Richland, Washington.

Washington State Office of Financial Management. 1995. Population Trends for Washington State. Forecasting Division, Olympia, Washington.

Watrous, R. A., and D. W. Wootan. 1997. Activity of Fuel Batches Processed Through Hanford Separations Plants, 1944 Through 1989. HNF-SD-WM-TI-794, Rev. 0, Lockheed Martin Hanford Corporation, Richland, Washington.

Westinghouse Hanford Company (WHC). 1993. Hanford Site Solid Waste Acceptance Criteria. WHC-EP-0063-4, Westinghouse Hanford Company, Richland, Washington.

Westinghouse Hanford Company (WHC). 1996. Operational Tank Leak Detection and Minimization During Retrieval. WHC-SD-WM-ES-377, Rev. 0, Westinghouse Hanford Company, Richland, Washington.

White, M. D., and M. Oostrom. 1996. STOMP Subsurface Transport Over Multiple Phases: User's Guide. PNNL-11217, Pacific Northwest National Laboratory, Richland, Washington. 
White, M. D., and M. Oostrom. 1997. STOMP Subsurface Transport Over Multiple Phases: User's Guide. PNNL-11218, Pacific Northwest National Laboratory, Richland, Washington.

Wing, N. R. 1994. Permanent isolation Surface Barrier Development Plan. WHC-EP-0673, Westinghouse Hanford Company, Richland, Washington.

Winkelman, W. D. 1996. Tank Characterization Report for Single-Shell Tank 241-BX-112. WHC-SD-WM-ER-602, Rev. 0, Westinghouse Hanford Company, Richland, Washington.

Wodrich, D. D. 1991. "Historical Perspective of Radioactively Contaminated Liquid and Solid Wastes Discharged or Buried in the Ground at Hanford." TRAC-0151-VA, Presentation to the U.S. Nuclear Regulatory Commission, Westinghouse Hanford Company, Richland, Washington.

Wood, M. I., R. Khaleel, P. D. Rittmann, A. H. Lu, S. H. Finfrock, R. J. Serne, K. J. Cantrell, and T. H. DeLorenzo. 1995. Performance Assessment for the Disposal of Low-Level Waste in the 200 West Area Burial Grounds. WHC-ED-0645, Westinghouse Hanford Company, Richland, Washington.

Wood, M. I., R. Khaleel, P. D. Rittmann, S. H. Finfrock, T. H. DeLorenzo, and D. Y. Garbrick. 1996. Performance Assessment for the Disposal of Low-Level Waste in the 200-East Area Burial Grounds. WHC-SD-WM-TI-730, Rev. 0, Westinghouse Hanford Company, Richland, Washington.

Wurstner, S. K. and J. L. Devary. 1993. Hanford Site Ground-Water Model: Geographic Information System Linkages and Model Enhancements FY 1993. PNL-8991. Pacific Northwest National Laboratory, Richland, Washington.

Wurstner, S. K. and M. D. Freshley. 1994. Predicted Impacts of Future Water Level Decline on Monitoring Wells Using a Ground-Water Model of the Hanford Site. PNL-10196. Pacific Northwest Laboratory, Richland, Washington.

Wurstner, S. K., P. D. Thorne, M. A. Chamness, M. D. Freshley, and M. D. Williams. 1995. Development of a Three-Dimensional Groundwater Model of the Hanford Site Unconfined Aquifer System: FY 1995 Status Report. PNL-10886, Pacific Northwest Laboratory, Richland, Washington. 


\section{Appendix A}

\section{Solid Waste Inventories}




\title{
Appendix A
}

\section{Solid Waste Inventories}

\author{
G. A. Whyatt and C. T. Kincaid
}

\section{A.1 Introduction}

This appendix provides radionuclide inventory values for solid waste disposal. At the end of this appendix, Tables A.1 through A.6 present the inventories by burial ground. The data for actinides and their daughters are provided in a table separate from fission and activation products. The six waste categories are listed and described as follows:

$\operatorname{dec} 96$

sept88

New_96_88

Suspect_TRU
This inventory table includes total unsegregated and post-1970 segregated nontransuranic (non-TRU) solid waste disposal from the start of operations through December 1996. To ensure this original table is retained without revision, a new table "new_dec_96" (Table A.1) was created and used to develop the pre- and post1988 inventories.

This inventory table includes total unsegregated and post-1970 segregated non-TRU solid waste disposal from the start of operations through September 1988. To ensure this original table is retained without revision, a new table "new_sept_88" (Table A.2) was created and used to develop the pre- and post-1988 inventories.

This inventory table (Table A.3) is the difference between the dec 96 and sept88 tables and represents the inventory disposed after September 1988 to which U.S. Department of Energy (DOE) Order 5820.2A applies. This table replaced an earlier version, i.e., the "post_sept88" table, which was not based on the most current "dec96" and "sept88" tables.

This inventory table provides estimates of radionuclides that are contained in suspect transuranic (TRU) waste that are expected to be reclassified from TRU to low-level waste (LLW) and be disposed of onsite. This inventory represents waste disposed as TRU between 1970 and 1986 using a greater than or equal to $10 \mathrm{nCi} / \mathrm{g}$ definition of TRU. It is anticipated that some of this waste will be reclassified as LLW and disposed onsite because of the current definition of TRU (i.e., greater than or equal to $100 \mathrm{nCi} / \mathrm{g}$ ). A copy of this table named "new_suspect_TRU" (Table A.4) is used to develop the pre- and post-19.88 inventory estimates. 
New_future

This inventory table (Table A.5) provides a projection of potential future disposal based on a linear projection of disposal occurring after September 1988. The "new_96_88" table is multiplied by the factor "360 (months) / 99 (months)" to estimate the inventory disposed during the proposed 30-year operational period that began in 1988. This is the estimated post-1988 inventory that is regulated under DOE Order 5820.2a.

New_pre_1988 Table A.6 is simply the sum of the "new_sept_88" and "new_suspect_TRU" spreadsheets and provides an estimate of the total LLW inventory disposed in the pre-1988 burial grounds. These burial grounds will be closed under the Comprehensive Environmental Response, Compensation, and Liability Act (CERCLA) program.

\section{A.2 Explanation of Basis for Solid Waste Inventories}

Inventories of radionuclides disposed in each of the 200 East and 200 West solid waste burial grounds were estimated and are provided in Tables A.1 through A.6 at the end of this appendix. The inventory data are derived from the Solid Waste Information Tracking System (SWITS) database (Clark 1995). The Oak Ridge Isotope Generation and Depletion (ORIGEN2) code (Croff 1980; Wittekind 1989) was used to estimate the abundance of minor radionuclides that could potentially be present but are not be reported in the SWITS database.

Activities of cesium-137, and masses of uranium and plutonium disposed were obtained directly from the SWITS database. The following SWITS database reports were obtained on 1/4/96 to provide the inventory information:

- SWIR328D and SWIR328E: Unsegregated Waste Burial Ground Areas Waste Volumes Buried and Non-decayed Curie Content from Startup through September 30th, 1988. ("D" contains uranium and plutonium masses and " $E$ " contains cesium-137 Ci)

- SWIR328G and SWIR328H: Post-1970 Non-transuranic Waste Burial Ground Areas Waste Volumes Buried or Stored and Non-decayed Curie Content through September 30, 1988. ("G" contains uranium and plutonium masses and " $\mathrm{H}$ " contains cesium-137 $\mathrm{Ci}$ )

The reports above were also generated for dates of startup through December 31,1996 . The inventories of uranium, plutonium, and cesium-137 disposed were totaled between the unsegregated disposal inventory and the segregated non-TRU inventory (thus excluding the TRU waste that is not expected to remain onsite). The startup through 12/31/96 (99 months) represents all the waste of interest disposed as solid waste. DOE Order 5820.2A applies to waste disposed after $9 / 26 / 88$ so it was desired to 
provide a separate inventory estimate for disposal after this date. The inventory of waste disposed after $9 / 30 / 88^{(a)}$ was determined by subtracting the waste disposed through $9 / 30 / 88$ from the total disposal through 12/31/96.

\section{A.2.1 Suspect TRU Waste}

Prior to 1970, TRU waste was not segregated before disposal. After 1970, TRU waste, which was defined as greater than or equal to $10 \mathrm{nCi} / \mathrm{g}$, was segregated before disposal so that it could be retrieved and eventually be disposed offsite. In 1984, the definition of TRU waste was changed from $>10 \mathrm{nCi} / \mathrm{g}$ to $>100 \mathrm{nCi} / \mathrm{g}$. Therefore, it is possible that some quantity of segregated TRU waste disposed between 1970 and 1984 may be reclassified as LLW and be disposed of on the Hanford Site.

It is most likely that the waste would be assayed within the disposal trench and never leave the trench in order to avoid changing the Resource Conservation and Recovery Act (RCRA) "disposed waste" classification. Thus, the waste is assumed to remain in the burial grounds where it currently resides and it is assumed that DOE Order 5820.2A would not apply to such wastes. Thus, the estimated inventory of TRU waste reclassified as LLW is added to the inventory of LLW through September 30, 1988 ("new_sept_88") to create the pre-September 1988 inventory.

Inventory values for suspect TRU waste were based on an estimate that $50 \%$ of drums and $15 \%$ of burial boxes would be reclassified as LLW and disposed of onsite. This waste volume was assumed to have a density of $200 \mathrm{~kg} / \mathrm{m}^{3}$ and contain $100 \mathrm{nCi} / \mathrm{g}$ of TRU radionuclides (i.e., the maximum possible value for waste that could be reclassified as LLW). The alpha activity calculated to be in the reclassified waste was then used to calculate the fraction of alpha activity in suspect TRU waste, which was presumed to be reclassified as LLW. This calculation indicates that roughly $0.3 \%$ of alpha activity in drums and $1.1 \%$ of alpha activity in boxes may be present in the reclassified waste. This fraction of the total alpha activity was then used to calculate the activity of each radionuclide in the reclassified waste by multiplying by the total estimated inventory of the suspect TRU waste.

\section{A.2.2 Future Disposable Inventories}

There is substantial uncertainty in the future solid waste disposal inventories because of the change in the mission of the Hanford Site from the production of special nuclear materials to the safe cleanup and management of the site's legacy wastes. A simple approach was used in which the inventory disposed between September 30, 1988 and December 31, 1996 was used to extrapolate for an additional 30 years of disposal assuming a constant rate of disposal. The inventory values were compared to projections made in the East and West Area Solid Waste Performance Assessments (Wood et al. 1995, 1996). In all cases, the linear extrapolation of waste disposal over 30 years exceeded the inventories assumed in the

(a) The SWITS query was performed through September 30, 1988 before the precise effective date for DOE Order 5820.2A was determined. The 4-day discrepancy was not considered significant enough to warrant generating an additional set of reports. 
solid waste performance assessments. The projected inventory values were associated with the disposal areas where waste disposal has occurred over the last 8 years, but actual future disposal may or may not occur in the same disposal areas.

\section{A.2.3 Estimation of Non-Reported Radionuclides}

While uranium, plutonium, and cesium-137 are relatively well reported within the SWITS database, a number of minor nuclides may also be present but are not consistently reported. Some of these minor radionuclides are identified as key nuclides in performance assessment calculations. In an effort to estimate inventories of minor radionuclides, ORIGEN2 was used to estimate the relative abundance of minor radionuclides compared to the major radionuclides that were reported.

ORIGEN2 calculations were made for single-pass reactor irradiations and for $\mathrm{N}$ Reactor irradiations to determine radionuclide concentrations in spent fuel and cladding. Impurities in the fuel and the cladding were included in the model. The quantities are based on Bergsman (1993), and are given in Table A.7. The brazing was also included in the model. It was assumed that the single-pass reactor fuel was all natural uranium as opposed to the actual situation where $25 \%$ of it was slightly enriched uranium. The average burnup of the single-pass reactor fuel was $728 \mathrm{MWd} / \mathrm{MTU}$. It was also assumed that all of the $\mathrm{N}$ Reactor fuel was enriched to $0.947 \%$ uranium-235 when in fact some of it was of higher enrichment. The average burnup of the N Reactor fuel was $1045 \mathrm{MWd} / \mathrm{MTU}$. The power density was assumed to be $10 \mathrm{MW} / \mathrm{MTU}$ for all of the fuel. For long decay times the radionuclide concentrations are insensitive to the power density. About $90 \%$ of the fuel reprocessed at Hanford was irradiated in the single-pass reactors. A weighted average between the single pass and $\mathrm{N}$ Reactor nuclide concentrations was used to estimate the overall average nuclide composition.

Inventories of potentially unidentified fission products in solid waste burial grounds were estimated by multiplying the undecayed cesium-137 inventory from SWIS by the ratio of the curies per $\mathrm{kg}$ concentration of the radionuclide of interest to the concentration of cesium-137 from the ORIGEN2 calculation at 10 years after discharge from the reactor. Estimates based on 1-year decay would be more conservative for radionuclides with half-lives less than that of cesium-137 (30 years) while estimates based on 10 years of decay prior to disposal are more conservative for radionuclides with half-lives of more than 30 years. The inventory estimates have been based on the fuel aged for 10 years after discharge from the reactor. In any instance, where the activity of a fission product increased over time beyond 1 year, the maximum activity between 1 and 3000 years was used to calculate the ratio to cesium- 137 at 10 years.

The SWITS database reports provided information on uranium disposed, including both a mass of uranium that was not identified by isotope, and a quantity of uranium isotopes that were specifically identified. The ORIGEN2 results were used to divide the uranium that was not isotropically identified among the uranium isotopes and to estimate the quantity of other actinides (except plutonium) that might be present along with this uranium mass. The quantity of other actinides was estimated by multiplying the uranium mass reported in SWITS by the ratio of activity of actinide (or daughter) to uranium mass in discharged fuel at 1 year. Similar to the fission products, estimates are provided based on 10-year decay. 
As in the case of fission products, the maximum actinide or daughter activity between 1 and 3000 years in the ORIGEN2 calculation was used to calculate the ratio to uranium mass.

For plutonium, the approach was similar to that used for uranium. Plutonium reported without isotopic distribution was divided into isotopes based on the relative abundances indicated in the ORIGEN2 calculation at 10 years. Quantities of plutonium that were reported in SWITS as specific isotopes of plutonium were then added to arrive at total plutonium isotopic values.

Chlorine- 36 is a potentially important radionuclide that may be formed by the irradiation of chloride impurities in the fuel or cladding. No data on the chlorine-35 impurity levels within metallic uranium fuel were available and it is not known whether the impurity level was negligible. However, because of the uncertainty, a calculation was performed assuming a $1 \mathrm{ppm}$ by weight impurity in the fuel. The chlorine36 in waste was then ratioed to cesium-137 as for fission products. The purpose of including chlorine-36 in the inventory is to determine if the nuclide is potentially important to the results of the performance assessment. If the results indicate it is potentially important, then a more in-depth investigation into chlorine-36 may be justified. However, if results indicate it is many orders of magnitude below a level of concern, then additional effort may not be warranted.

The choice of using ORIGEN2-predicted ratios of nuclides in aged fuel to cesium-137 content at disposal was based on previous work by Wood et al. (1996). Their work provided a proposed breakdown of time after discharge at disposal by year in which the time after discharge at disposal varied between 1 and 10 years depending on the year of generation. For wastes disposed from 1945 through 1973, 1 year was determined to be appropriate. However, wastes disposed in the most recent years may originate from waste discharged from the reactor 10 years (or more) prior to disposal. Between 1 and 10 years after discharge, the cesium- 137 inventory declines by about $20 \%$. As a result, the inventories of long-lived fission products in some wastes estimates, based on cesium- 137 content, were about $20 \%$ higher when the 10-year assumption was made. Overall, the difference between the 1- and 10-year assumption is probably small relative to the uncertainty related to using a ratio of nuclides in aged fuel to cesium-137 content that implicitly assumes that the isotopic ratios in the waste are similar to those in the discharged, irradiated fuel.

\section{A.2.4 Specific Isotope Inventory Values Used from SWITS}

The approach of estimating fission products based on a ratio of cesium-137 and estimating actinides based on a ratio to uranium is potentially in error. There are three possibilities: a high estimate, a good estimate, and a low estimate. The fundamental assumption being made when the fuel-ratio method is applied is that the abundance of fission products, cesium-137, actinides, and uranium in aged fuel is a good approximation to that in waste streams.

A high estimate of fission products or actinides will result whenever the proportion of cesium or uranium in the waste is high in comparison to the fission products or actinides. This could occur if the process creating the waste is biased toward the production of high quantities of cesium or uranium in the waste stream. Process steps in the separation of cesium or uranium from Hanford wastes could have a feed stream rich in cesium or uranium, and therefore, could yield waste streams with this characteristic. 
Accordingly, the quantity of fission product or actinides in the waste stream would be overestimated by the aged-fuel-ratio method when the waste stream is rich in cesium or uranium. A low estimate of fission products and actinides will result when the opposite situation arises, i.e., the waste stream carries relatively low quantities of cesium and uranium. When a process feed stream is low in cesium or uranium because it was removed in a prior process step, waste streams could be higher in fission products and actinides than an aged-fuel ratio would indicate.

B Plant wastes reported to contain significant levels of cesium are likely to be associated with processes designed to separate cesium and produce feed streams and wastes disproportionately high in cesium. These feed streams and wastes would be disproportionately low in fission products. Accordingly, application of the aged-fuel-ratio methodology to estimate fission products would produce significant overestimates of the fission products in solid waste burial grounds. Similarly, wastes originating from the uranium extraction processes and reported to contain significant levels of uranium are likely to be disproportionately high in uranium. Feed streams and waste streams with high uranium contents may contain proportionately lower quantities of actinides. Application of the aged-fuel ratio would produce significant overestimates of the actinides in solid wastes. Certainly, examples resulting in low estimates of fission products or actinides are also possible. A detailed review of process waste steams produced during previous operations would be required to determine the true character of the error resulting from the use of the aged-fuel-ratio method to estimate fission products and actinides.

Such a detailed review was beyond the scope of the first iteration Composite Analysis. In the interim, the SWITS database was checked for curies of potentially key radionuclides (e.g., carbon-14, selenium-79, technetium-99, iodine-129, neptunium-237, and thorium-232) disposed in each solid waste burial ground. Where the SWITS database reported a larger inventory than projected, based on ratio to cesium-137 or uranium, the SWITS value was used for that burial ground. Furthermore, waste acceptance criteria are being revised to force waste generators to evaluate and report mobile radionuclides. This is being done to make the necessary inventory data available for future disposals, and eliminate the need to estimate key fission product and actinide inventories.

\section{A.2.5 Special Inventory Items}

Trench 94 of burial ground 218E-12B contains U.S. Navy ship reactor compartments. These wastes consist of activation products within corrosion-resistant metals, primarily Inconel Alloy 600. Because of the immobilized nature of the radionuclides within these activated metals, waste inventory identified in SWITS as being from offsite sources and disposed in Trench 94 of 218E-12B has been excluded from the inventory.

The SWITS inventory information for wastes from the 100 Area suggest that these wastes are activation products rather than fission products. While it is possible that the radionuclides exist in corrosion-resistant metals, this is not known for certain, as in the case of the reactor compartments. Therefore, the inventory of carbon-14 is reported separately for 100 Area wastes. Any non-100 Area wastes that were specifically identified as activated metal were included in the 100-Area carbon-14 inventory. 
Trench 218W4C contains 74.8 Ci of carbon-14 and 14.62 Ci of technetium-99 which were disposed of in grouted containers. Based on performance calculations made prior to waste acceptance, this portion of the inventory is expected to be significantly less mobile than the inventory in other wastes and therefore was not included within the inventory tables. If desired, these radionuclides could be included in a separate release model and the source term added to the source term from the remainder of the burial ground.

A separate inventory prepared by Wood ${ }^{(a)}$ was developed based on total beta-gamma measurements on waste packages. An assumed mix of fission products or activation products was then used to account for total beta-gamma not accounted for by cesium-137. In most cases this resulted in a smaller inventory than estimating using the ratio to cesium-137. However, in a few instances, a larger inventory was predicted. In these cases, the larger value predicted using the beta-gamma measurement was used in place of that predicted based on cesium-137.

Similarly, in comparing inventories to those produced by Wood, ${ }^{(a)}$ some discrepancies that were traced to differences in SWITS database reports were noted. The reasons for the discrepancies were not determined but in each case, the larger inventory value was adopted.

\section{A.3 Comparison to Previous Performance Assessment Inventories}

Performance assessments were previously performed for the disposal of solid waste in the 200 West and 200 East Areas (Wood et al. 1995, 1996). This section provides a comparison between the currently recommended inventory to the inventory recommended in the performance assessments. This is provided for information only, since Wood no longer recommends use of the inventory values in those performance assessments. He recommends the use of future inventory estimates based on the longer period of record of disposals now available. The comparison for a few of the mobile radionuclides is provided in Tables A.8 and A.9.

Projected uranium inventories in future 200 West Area burial ground disposals are much higher in this document. These higher projected inventories for uranium are probably a direct result of recent disposals of large quantities of uranium associated with the cleanup of facilities, and the use of a simple linear assumption for forecasting future disposals. Carbon-14 values for burial grounds in both 200 East and 200 West Areas are larger in the current inventory. One possible explanation for the larger carbon-14 values is that the published performance assessments may have eliminated more waste on the basis that it was in activated metal and not available to be released. In this document, the values of the technetium- 99 inventory for the 200 West Area burial grounds are also higher by an order of magnitude. Again, recent disposals and.linear projections may account for the increase in estimates. In comparing the currently recommended inventory to the previous performance assessment inventories, it should be noted that waste disposal has occurred between the time of the previous performance assessment and the present, and therefore the bases for the two estimates are quite different.

(a) Electronic mail message regarding West Area Burial Ground Inventories. Sent by M. I. Wood of Waste Management Federal Services of Hanford to G. A. Whyatt on December 19, 1996. 


\section{A.4 Information on Radionuclides of Interest}

This section provides information on the radionuclides within the inventory including half-life, sources, and inventories predicted by an ORIGEN2 calculation. All ORIGEN2 inventories are a weighted average of single pass and $N$ Reactor fuels, and they represent the activity in irradiated fuel and cladding in units of curies per $\mathrm{kg}$ of uranium. Compositions of single-pass and $\mathrm{N}$ Reactor fuels and cladding used in the ORIGEN2 simulation are provided in Table A.7. These simulations and the averaging of results were performed prior to development and publication of the standard inventory (Kupfer et al. 1997; Watrous and Wootan 1997). The ORIGEN2 inventory values were used to calculate inventories for nonreported radionuclides.

\section{A.4.1 Highest Mobility Nuclides}

Tritium

$T_{1 / 2}=12.3$ years. For this nuclide, activation dominates fission by a factor of $1 E+04$. It is a negligible source in waste. ORIGEN2 calculation indicates the following inventories: 1 year $=7.385 \mathrm{E}-02 \mathrm{Ci}$ and 10 years $=7.068 \mathrm{E}-03 \mathrm{Ci}$. Beta decays to helium-3 (stable).

Carbon-14 - $\quad T_{1 / 2}=5730$ years. This nuclide beta decays to nitrogen-14 (stable). It is generated as both a fission and an activation product, although activation is the more important source. Activation is from various neutron interactions with nitrogen-14, nitrogen-15, oxygen-16, oxygen-17, and carbon-13. ORIGEN2 calculation indicates the following inventories: 1 year $=1.247 \mathrm{E}-04 \mathrm{Ci}$ and 10 years $=1.246 \mathrm{E}-04 \mathrm{Ci}$. Sources other than the fuel and cladding may also be important and are not included in this value (e.g., production within graphite moderator).

Chlorine-36

$T_{1 / 2}=3.01 E+05$ years. This nuclide results from the activation of chlorine-35 impurity in fuel or cladding. Assuming a $1 \mathrm{ppm}$ by weight contaminant level of chlorine in the fuel, the ORIGEN2 calculation indicates a 1-year inventory of 1.228E-07 Ci. 200 East Area performance assessments (Wood et al. 1996) estimated inventory only in connection with reactor compartments. The use of inventory based on $1 \mathrm{ppm}$ contaminant level may allow determination if further investigation is warranted. Decays by beta to argon- 36 or by positron emission to sulfur-36, both stable. Another potential source is from chloride contaminant in materials other than fuel as well.

Selenium-79 . $\quad \mathrm{T}_{1 / 2}=6.5 \mathrm{E}+04$ years. This nuclide is a fission product only. ORIGEN2 calculation indicates the following inventories: 1 year $=1.016 \mathrm{E}-05 \mathrm{Ci}$ and 10 years $=1.015 \mathrm{E}-05$. Beta decays to bromine-79 (stable). 
Technetium-99

$\mathrm{T}_{1 / 2}=2.13 \mathrm{E}+05$ years. This nuclide is a fission product with minor activation source. ORIGEN2 calculation indicates the following inventories at 1 year: 3.434E-04 $\mathrm{Ci}$ fission product and $6.484 \mathrm{E}-11 \mathrm{Ci}$ activation. Beta decays to ruthenium-99 (stable).

\section{A.4.2 Moderately Mobile Nuclides}

Iodine-129

$\mathrm{T}_{1 / 2}=1.57 \mathrm{E}+07$ years. This nuclide is a fission product with a minor activation source. ORIGEN2 calculation indicates the following inventories for 1 year: 6.774E-07 Ci fission product, and 1.149E-25 Ci activation.

Uranium-232 $\quad \mathrm{T}_{1 / 2}=68.9$ years. This nuclide is a daughter of relatively short-lived parent plutonium-236 ( $\mathrm{T}_{1 / 2}=2.85$ years). ORIGEN2 calculation indicates an inventory . at 1 year $=3.93 \mathrm{E}-10 \mathrm{Ci}$ with maximum inventory of about $4.3 \mathrm{E}-08 \mathrm{Ci}$ at 10 years.

Uranium-233

$\mathrm{T}_{1 / 2}=1.592 \mathrm{E}+05$ years. This nuclide is an ingrowth daughter product, with longlived neptunium-237 parent. ORIGEN2 calculation indicates an inventory of 3.93E- $10 \mathrm{Ci}$ at 1 year, still growing in at $1.03 \mathrm{E}-07 \mathrm{Ci}$ at 3000 years. It is a potentially significant source if thorium-232 is used as fertile material [reactions are $(n, \gamma), \beta, \beta]$ to produce fissile uranium-233. Thorium was used not only for targets for uranium-233 production but also for neutron shielding which could add inventories of uranium-233 depending on where the material is eventually disposed.

Uranium-234 $\quad T_{1 / 2}=2.454 \mathrm{E}+05$ years. This nuclide is a daughter within uranium-238 decay chain. Inventory in ORIGEN2 calculation is essentially constant at 3.49E-04 Ci from 1 through 3000 years.

Uranium-235 $T_{1 / 2}=7.037 \mathrm{E}+08$ years. This nuclide is the primary fissile isotope in fuel, and its concentration depends on enrichment and burnup. ORIGEN2 calculation indicates a 1-year discharge at $1.425 \mathrm{E}-05 \mathrm{Ci}$. Minor source from plutonium-239 alpha decay causes an increase to $1.439 \mathrm{E}-05 \mathrm{Ci}$ over 3000 years after discharge.

Uranium-236

$T_{1 / 2}=2.342 \mathrm{E}+07$ years. This nuclide has slow ingrowth from long-lived plutonium-240 alpha decay. ORIGEN2 calculation indicates an inventory of 8.616E-06 $\mathrm{Ci}$ at 1 year, increasing to $9.41 \mathrm{E}-06 \mathrm{Ci}$ at 3000 years.

Uranium-237

$\mathrm{T}_{1 / 2}=6.75$ days. This nuclide may be generated in a reactor through $(\mathrm{n}, \gamma),(\mathrm{n}, \gamma)$ reactions from uranium-235 although initial inventory quickly decays because of short half-life. A more important source at 1 year is through a minor alpha decay route $(0.0024 \%)$ of plutonium- 241 . This holds concentration up and for 
Uranium-238

Uranium-239

Uranium-240

Neptunium-237 performance assessment purposes, uranium-237 should be considered as a shortlived daughter of plutonium-241. The uranium-237 beta decays to neptunium237 and over time, the contribution to neptunium-237 is non-negligible. After 1 year ORIGEN2 calculation indicates that an inventory of $1.177 \mathrm{E}-05 \mathrm{Ci}$ of uranium-237 remains.

$\mathrm{T}_{\mathrm{I} / 2}=4.468 \mathrm{E}+09$ years. Primary uranium isotope, which alpha decays to thorium-234 with a long decay chain. ORIGEN2 calculation indicates 1-year inventory of $3.335 \mathrm{E}-04 \mathrm{Ci}$, and $991.6 \mathrm{~g}$.

$\mathrm{T}_{1 / 2}=23.5$ minutes. This nuclide beta decays to neptunium-239. ORIGEN2 calculation indicates zero inventory after 1 year. This nuclide was not included in the inventory.

$\mathrm{T}_{1 / 2}=14.1$ hours. This nuclide is formed in a reactor by $(\mathrm{n}, \gamma),(\mathrm{n}, \gamma)$ with uranium-238 but initial inventory quickly decays. It is produced as daughter from plutonium-244 alpha decay and should be considered as short-lived daughter of plutonium-244. Beta decays to neptunium-240, and then beta decays again $\left(T_{1 / 2}=67\right.$ minutes) to plutonium-240. Uranium-240 activity at 1 year $=$ 2.45E-15 Ci.

$\mathrm{T}_{1 / 2}=2.14 \mathrm{E}+06$ years. This nuclide is generated by uranium-235 $(\mathrm{n}, \gamma) \rightarrow$ uranium-236(n,y) $\rightarrow$ Uranium-237( $\beta) \rightarrow$ neptunium-237 reactions. It is also formed by alpha decay of americium- 241 and by a plutonium- 241 alpha decay $(0.0024 \%)$ followed by uranium -237 beta decay. Decay chain includes alpha to protactinium-233, beta to uranium-233 and then follows the uranium-233 decay chain. ORIGEN2 calculation indicates the following inventories: 1 year $=$ 5.181E-06 $\mathrm{Ci}$ and 3000 year $=8.542 \mathrm{E}-06 \mathrm{Ci}$. Much of this increase after 1 year is the result of the plutonium-241 to uranium-237 to neptunium-237 decay route.

\section{A.4.3 Moderately Immobile Nuclides}

Protactinium-231 . $T_{1 / 2}=3.28 \mathrm{E}+04$ years. This nuclide is a daughter of thorium-231 beta decay within the uranium-235 decay chain. Inventory slowly grows in over time. ORIGEN2 calculations indicate the following inventories: after 1 year $=3.9 \mathrm{E}-10$ $\mathrm{Ci}$ and after 3000 years $=8.8 \mathrm{E}-07 \mathrm{Ci}$.

Radium-222

$\mathrm{T}_{1 / 2}=38$ seconds. This nuclide is a daughter product resulting from uranium-230 alpha decay $\left(\mathrm{T}_{1 / 2}=20.8\right.$ days $)$ to thorium-226 $\left(\mathrm{T}_{1 / 2}=30.9\right.$ minutes $)$ which alpha decays to radium-222. Because of the short half-life of parent nuclides, this nuclide decays to negligible levels within 3 years and need not be considered in the Composite Analysis. This nuclide was not included in inventory. 
Radium-223

$T_{1 / 2}=11.435$ days. This nuclide is a daughter in the uranium- 235 decay chain and grows in over time. ORIGEN2 calculation indicates the following inventories: 1 year $=7.8 \mathrm{E}-12 \mathrm{Ci}$ and 3000 year $=8.8 \mathrm{E}-07 \mathrm{Ci}$.

Radium-224

$\mathrm{T}_{1 / 2}=3.66$ days. This nuclide is a daughter in the thorium-232 decay chain. ORIGEN2 calculation indicates minor initial ingrowth $(8.1 \mathrm{E}-09 \mathrm{Ci}$ at 1 year) with a peak at $4.0 \mathrm{E}-08 \mathrm{Ci}$ at 10 years and a decline to $2.4 \mathrm{E}-12 \mathrm{Ci}$ at 3000 years.

Radium-225

$\mathrm{T}_{1 / 2}=14.8$ days. This nuclide is a daughter in the neptunium-237/uranium-233 decay chain. It grows in over time. ORIGEN2 calculation indicates the following inventories: $6.4 \mathrm{E}-14 \mathrm{Ci}$ at 1 year and $1.3 \mathrm{E}-08 \mathrm{Ci}$ at 3000 years.

Radium-226

$\mathrm{T}_{1 / 2}=1.6 \mathrm{E}+03$ years. This nuclide is a daughter in the uranium-238/uranium-234 decay chain. It grows in over time. ORIGEN2 calculation indicates the following inventories: $1.0 \mathrm{E}-12 \mathrm{Ci}$ at 1 year and $4.12 \mathrm{E}-6 \mathrm{Ci}$ at 3000 years.

Radium-228

$\mathrm{T}_{1 / 2}=5.76$ years. This nuclide is a thorium-232 daughter. ORIGEN2 calculation indicates ingrowth to $2.6 \mathrm{E}-17 \mathrm{Ci}$ at 1 year, and $1.3 \mathrm{E}-12 \mathrm{Ci}$ over 3000 years.

Ruthenium-106

$\mathrm{T}_{1 / 2}=367$ days. This nuclide is a fission product with very minor activation source. ORIGEN2 calculation indicates the following inventories: 1 year $=$ $5.914 \mathrm{Ci}$, and 10 year $=1.214 \mathrm{E}-02 \mathrm{Ci}$. Beta decays to short-lived daughter rhodium-106, which beta decays to palladium-106 (stable).

\section{A.4.4 Highly Immobile Nuclides}

Nickel-59

$\mathrm{T}_{1 / 2}=7.5 \mathrm{E}+04$ years. This nuclide is an activation product. It decays by electron capture (99+\%) to cobalt-59 (stable). ORIGEN2 calculation indicates inventory at 1 year $=1.841 \mathrm{E}-05 \mathrm{Ci}$.

Cobalt- 60

$\mathrm{T}_{1 / 2}=5.271$ years. This nuclide is an activation product which $\beta^{-}$decays to nickel-60 (stable). ORIGEN2 calculation indicates the following inventories: 1 year $=2.65 \mathrm{E}-03 \mathrm{Ci}$ and 10 year $=8.111 \mathrm{E}-04 \mathrm{Ci}$.

Nickel-63

$T_{1 / 2}=100$ years. This nuclide is an activation product. It decays by beta to copper-63 (stable). ORIGEN2 calculation indicates the following inventories: 1 year $=2.272 \mathrm{E}-03 \mathrm{Ci}$ and 10 years $=2.123 \mathrm{E}-03 \mathrm{Ci}$.

Strontium-90

$T_{1 / 2}=28.8$ years. This nuclide is a fission product. It beta decays to yttrium-90, then beta decays again ( $T_{1 / 2}=64.1$ hours) to zirconium-90 (stable). ORIGEN2 calculation indicates the following inventories: 1 year $=2.039 \mathrm{Ci}$ and 10 year $=$ $1.646 \mathrm{Ci}$. 
Zirconium-93

Niobium-93m

Niobium-94

Palladium-107

(Tin) Sn-126

Cesium-135

Cesium-137

Cerium-141

Cerium-144
$T_{1 / 2}=1.5 \mathrm{E}+06$ years. This nuclide is primarily a fission product with minor activation source. It decays by $\beta^{-}$to niobium-93m then niobium-93 (stable). ORIGEN2 calculation indicates the following inventories: 1 year $=4.72 \mathrm{E}-05 \mathrm{Ci}$ from fission, 1 year $=2.19 \mathrm{E}-07 \mathrm{Ci}$ from activation; 10 year $=4.720 \mathrm{E}-05 \mathrm{Ci}$ from fission, 10 year $=2.188 \mathrm{E}-07$ Ci from activation.

$\mathrm{T}_{1 / 2}=13.6$ years. This nuclide is a daughter of fission product with a much smaller activation source. It decays by isomeric transition to niobium-93 (stable). The source from zirconium-93 decay causes inventory to grow in over about 300 years to a level of $4.483 \mathrm{E}-05 \mathrm{Ci}$ (from ORIGEN2 calculation).

$\mathrm{T}_{1 / 2}=2.0 \mathrm{E}+4$ years. This nuclide is a fission product with very minor activation source. It beta decays to molybdenum-94 (stable). ORIGEN2 calculation indicates that the inventory at 1 year $=1.55 \mathrm{E}-09 \mathrm{Ci}$.

$\mathrm{T}_{1 / 2}=6.5 \mathrm{E}+06$ years. This nuclide is a fission product with negligible activation source. It decays by $\beta^{-}$to (silver) Ag-107 (stable). ORIGEN2 calculation indicates that the inventory at 1 year $=1.21 \mathrm{E}-06 \mathrm{Ci}$.

$\mathrm{T}_{1 / 2}=1 \mathrm{E}+05$ years. This nuclide is a fission product with a very small activation source. It beta decays to (antimony) Sb-126, then beta decays again ( $\mathrm{T}_{1 / 2}=12.4$ days) to tellurium-126 (stable). ORIGEN2 calculation indicates that the inventory at 1 year $=1.65 \mathrm{E}-05 \mathrm{Ci}$.

$\mathrm{T}_{1 / 2}=3.0 \mathrm{E}+06$ years. This nuclide is a fission product that beta decays to barium-135 (stable). ORIGEN2 calculation indicates that the inventory at 1 year $=8.325 \mathrm{E}-06 \mathrm{Ci}$.

$T_{1 / 2}=30.17$ years. This nuclide is a fission product. Beta decays through shortlived barium-137m to barium-137 (stable). ORIGEN2 calculation indicates the following inventories: 1 year $=2.379 \mathrm{Ci}$ and 10 year $=1.932 \mathrm{Ci}$.

$T_{1 / 2}=32.5$ days. This nuclide is a fission product, which beta decays to praseodymium-141 (stable). ORIGEN2 calculation indicates that the inventories are $0.158 \mathrm{Ci}$ at 1 year and approximately zero at 10 years. Because of a short half-life and lack of an ingrowth source to maintain activity, this nuclide is unlikely to be important in groundwater analysis. However, this radionuclide has been included in the inventory.

$\mathrm{T}_{1 / 2}=284$ days. This nuclide is a fission product that beta decays to praseodymium-144 ( $T_{1 / 2}=17.3$ minutes $)$ and beta decays again to neodymium$144\left(\mathrm{~T}_{1 / 2}=2.1 \mathrm{E}+15\right.$ years $)$. Activity decays normally after 1 year, with no source supporting activity. ORIGEN2 calculation indicates the following inventories: 1 year $=29.44 \mathrm{Ci}$ and 10 years $=9.735 \mathrm{E}-03 \mathrm{Ci}$. 
Samarium-147

Samarium-151

Europium-152

Europium-154

Europium-155

(Lead) $\mathrm{Pb}-205$

Actinium-225

Actinium-227

Thorium-227

Thorium-228

Thorium-229
$\mathrm{T}_{1 / 2}=1.06 \mathrm{E}+11$ years. This nuclide grows in as a daughter of fission product promethium-147. ORIGEN2 calculation predicts a maximum inventory of $2.378 \mathrm{E}-10 \mathrm{Ci}$ at about 40 years. Alpha decays to neodymium-143 (stable).

$T_{1 / 2}=90$ years. This nuclide is a fission product, that decays by $\beta^{-}$to europium151 (stable). ORIGEN2 calculation indicates the following inventories: 1 year $=$ $4.435 \mathrm{E}-02 \mathrm{Ci}$ and 10 years $=4.138 \mathrm{E}-02 \mathrm{Ci}$.

$T_{1 / 2}=13$ years. This nuclide is a fission product with no apparent decay-chain source. ORIGEN2 calculation indicates the following inventories: 1 year $=$ 6.133E-05 $\mathrm{Ci}$ and 10 year $=3.877 \mathrm{E}-05 \mathrm{Ci}$.

$\mathrm{T}_{1 / 2}=8.5$ years. This nuclide is a fission product with no apparent decay-chain source. ORIGEN2 calculation indicates the following inventories: 1 year = $1.392 \mathrm{E}-02 \mathrm{Ci}$ and 10 year $=6.739 \mathrm{E}-03 \mathrm{Ci}$.

$T_{1 / 2}=4.9$ years. This nuclide is a fission product with no apparent decay-chain source. ORIGEN2 calculation indicates the following inventories: 1 year $=$ 5.613E-02 $\mathrm{Ci}$ and 10 year $=1.596 \mathrm{E}-02 \mathrm{Ci}$.

$\mathrm{T}_{1 / 2}=1.4 \mathrm{E}+07$ years. This nuclide is an activation product with no apparent decay-chain source. ORIGEN2 calculation indicates that the inventory at 1 year $=8.041 \mathrm{E}-14 \mathrm{Ci}$.

$T_{1 / 2}=10$ days. This nuclide is a daughter product within the neptunium237/uranium-233 decay chain. ORIGEN2 calculation indicates that the inventory at 1 year $=6.387 \mathrm{E}-14 \mathrm{Ci}$, and grows in to $1.27 \mathrm{E}-08 \mathrm{Ci}$ at 3000 years.

$\mathrm{T}_{1 / 2}=21.773$ years. This is a daughter in the uranium-235 decay chain. ORIGEN2 calculation indicates that the inventory at 1 year $=7.77 \mathrm{E}-12 \mathrm{Ci}$, and grows in to a level of $8.81 \mathrm{E}-07 \mathrm{Ci}$ at 3000 years.

$T_{1 / 2}=18.718$ days. This nuclide is a daughter within the uranium-235 decay chain. ORIGEN2 calculation indicates that the inventory at 1 year $=7.67 \mathrm{E}-12 \mathrm{Ci}$ and grows in over time to $8.69 \mathrm{E}-07 \mathrm{Ci}$ at 3000 years.

$T_{1 / 2}=1.931$ years. This nuclide is a daughter within the thorium-232 decay chain. ORIGEN2 calculation indicates that the inventory grows in from 1 year $=$ 8.1E-09 $\mathrm{Ci}$ to 10 year $=4.0 \mathrm{E}-08 \mathrm{Ci}$, to 3000 year $=2.4 \mathrm{E}-12 \mathrm{Ci}$.

$\mathrm{T}_{1 / 2}=7.3 \mathrm{E}+03$ years. This nuclide is a daughter within the neptunium-237 decay chain. ORIGEN2 calculation indicates that the inventory grows in from 1 year $=$ $6.4 \mathrm{E}-14 \mathrm{Ci}$ to 3000 years $=1.27 \mathrm{E}-08 \mathrm{Ci}$. 
Thorium-230

Thorium-231

Thorium-232

Thorium-234

Plutonium-236

Plutonium-237

Plutonium-238

Plutonium-239

Plutonium-240
$\mathrm{T}_{1 / 2}=8.0 \mathrm{E}+04$ years. This nuclide is a daughter within the uranium-238/ uranium-234 decay chain. ORIGEN2 calculation indicates that the inventory grows in from 1 year $=3.8 \mathrm{E}-09 \mathrm{Ci}$ to 3000 years $=9.32 \mathrm{E}-06 \mathrm{Ci}$.

$T_{1 / 2}=25.52$ hours. This nuclide is a daughter within the uranium-235 decay chain. ORIGEN2 calculation indicates a fairly constant inventory level at $1.43 \mathrm{E}-5 \mathrm{Ci}$.

$\mathrm{T}_{1 / 2}=1.41 \mathrm{E}+10$ years. This is a natural thorium isotope. ORIGEN2 calculation indicates that it grows in from uranium-236 alpha decay from $4.72 \mathrm{E}-16 \mathrm{Ci}$ at 1 year to $1.337 \mathrm{E}-12 \mathrm{Ci}$ at 3000 years.

$\mathrm{T}_{1 / 2}=24.1$ days. This nuclide is a uranium-238 daughter. ORIGEN2 calculation indicates fairly steady concentration at 1 year at $3.34 \mathrm{E}-04 \mathrm{Ci}$.

$\mathrm{T}_{1 / 2}=2.85$ years. Generation at low levels from a secondary beta decay route $(9 \%)$ of neptunium-236 prevents this isotope from decaying to zero. ORIGEN2 calculation indicates inventories of $5.36 \mathrm{E}-07 \mathrm{Ci}$ at 1 year, $6.00 \mathrm{E}-08 \mathrm{Ci}$ at 10 years, with decay leveling off at a level of about $1.0 \mathrm{E}-12 \mathrm{Ci}$.

$T_{1 / 2}=45.4$ days. This nuclide decays through electron capture to form neptunium-237, but this is not an important source of neptunium-237. Plutonium-237 has a source from curium-241 alpha decay, which causes the isotope to be present longer than expected from its half-life, although it is still gone within 30 years. ORIGEN2 calculation indicates the following inventories: 1 year $=1.19 \mathrm{E}-08 \mathrm{Ci}$ and 10 years $=2.38 \mathrm{E}-30 \mathrm{Ci}$.

$T_{1 / 2}=87.74$ years. Sources from alpha decay of curium-242 and from beta decay of neptunium-238 are insignificant after 1 year and the activity decays very nearly as if there were no source. ORIGEN2 calculation indicates the following inventories: 1 year $=2.70 \mathrm{E}-03 \mathrm{Ci}$ and 10 years $=2.514 \mathrm{E}-03 \mathrm{Ci}$.

$T_{1 / 2}=2.41 E+04$ years. Uranium-238, which constitutes the majority of the metallic uranium fuel, undergoes an $(n, \gamma)$ reaction during reactor operations to form uranium-239. The uranium-239 then undergoes two beta decays to form first neptunium-239 and then plutonium-239. Curium-243 decay is an insignificant source of plutonium-239. ORIGEN2 calculation indicates that the inventory at 1 year $=4.89 \mathrm{E}-02 \mathrm{Ci}$.

$\mathrm{T}_{1 / 2}=6.57 \mathrm{E}+03$ years. In the reactor, plutonium- 240 may be produced either directly through an $(n, \gamma)$ reaction with plutonium-239 or through an $(n, \gamma)$ reaction with uranium-239 followed by two beta decays. Curium-244 decay is an insignificant source of plutonium-240. ORIGEN2 calculation indicates that the inventory at 1 year $=1.05 \mathrm{E}-02 \mathrm{Ci}$. 
Plutonium-241

Plutonium-242

Plutonium-243

Plutonium-244

Americium-241

Americium-242m

Americium-243

Curium-242
$\mathrm{T}_{1 / 2}=14.4$ years. During reactor operations, plutonium-241 is produced through an $(\mathrm{n}, \gamma)$ reaction with plutonium-240. Curium-245 decay is an insignificant source of plutonium-241. ORIGEN2 calculation indicates the following inventories: 1 year $=4.80 \mathrm{E}-01 \mathrm{Ci}$ and 10 years $=0.3111 \mathrm{Ci}$.

$\mathrm{T}_{1 / 2}=3.76 \mathrm{E}+05$ years. During reactor operations, plutonium-242 is produced through an $(n, \gamma)$ reaction with plutonium-241. Curium-246 decay is an insignificant source of plutonium-242. ORIGEN2 calculation indicates that the inventory at 1 year $=4.64 \mathrm{E}-07 \mathrm{Ci}$.

$\mathrm{T}_{1 / 2}=4.956$ hours. During reactor operations, plutonium -243 is produced through an $(n, \gamma)$ reaction with plutonium-242. Curium-247 alpha decay also contributes to the plutonium-243 inventory. ORIGEN2 calculation indicates a fairly steady concentration at $8.6 \mathrm{E}-20 \mathrm{Ci}$.

$\mathrm{T}_{1 / 2}=8.1 \mathrm{E}+07$ years. During reactor operations, plutonium-244 is produced through an $(n, \gamma)$ reaction with plutonium-243. Curium-248 alpha decay is an insignificant source of plutonium-244. Its long half-life maintains relatively constant concentration in ORIGEN2 calculation at 2.46E-15 Ci.

$T_{1 / 2}=433$ years. This nuclide's source is plutonium-241 beta decay. ORIGEN2 calculation indicates that the inventory at 1 year $=8.30 \mathrm{E}-04 \mathrm{Ci}$, and grows to maximum of $1.47 \mathrm{E}-02 \mathrm{Ci}$ at 100 years.

$\mathrm{T}_{1 / 2}=16.01$ hours. This nuclide is the short-lived daughter of the isomeric transition of americium-242m. It beta decays to plutonium-242. ORIGEN2 calculation indicates the following inventories: 1 year $=6.91 \mathrm{E}-07 \mathrm{Ci}$ and 10 year $=6.63 \mathrm{E}-07 \mathrm{Ci}$.

$\mathrm{T}_{1 / 2}=152$ years. This nuclide decays to americium-242. ORIGEN2 calculation indicates the following inventories: 1 year $=6.94 \mathrm{E}-07 \mathrm{Ci}$ and 10 year $=$ 6.67E-07 Ci.

$\mathrm{T}_{1 / 2}=7.37 \mathrm{E}+03$. This nuclide has no source after 1 year. It decays by alpha to neptunium-239, which then beta decays to plutonium-239. This source of plutonium-239 is not significant. ORIGEN2 calculation indicates the following inventories: 1 year $=1.58 \mathrm{E}-07 \mathrm{Ci}$ and 10 year $=1.576 \mathrm{E}-07 \mathrm{Ci}$.

$T_{1 / 2}=162.8$ days. This nuclide alpha decays to plutonium-238, although this source of plutonium-238 is not important after 1 year. ORIGEN2 calculation indicates the following inventories: 1 year $=3.634 \mathrm{E}-04 \mathrm{Ci}$ and 10 years $=$ 5.488E-07 Ci. 
Curium-244

Curium-245

Curium-246
$\mathrm{T}_{1 / 2}=18.11$ years. This nuclide alpha decays to plutonium-240, although this source of plutonium-240 is not important after 1 year. ORIGEN2 calculation indicates the following inventories: 1 year $=1.54 \mathrm{E}-05 \mathrm{Ci}$ and 10 year $=$ 1.092E-05 Ci.

$T_{1 / 2}=8.5 \mathrm{E}+03$ years. This nuclide alpha decays to plutonium-241, although this source of plutonium-241 is not important after 1 year. ORIGEN2 calculation indicates the following inventories: 1 year $=8.323 \mathrm{E}-11 \mathrm{Ci}$ and 10 years $=$ 8.317E-11 Ci.

$\mathrm{T}_{1 / 2}=4.7 \mathrm{E}+03$ years. This nuclide alpha decays to plutonium-242, although it is an insignificant source of plutonium-242. ORIGEN2 calculation indicates the following inventories: 1 year $=6.925 \mathrm{E}-13 \mathrm{Ci}$ and 10 year $=6.916 \mathrm{E}-13 \mathrm{Ci}$.

\section{A.5 References}

Bergsman, K. H. January 1993. Hanford Irradiated Fuel Inventory Baseline. WHC-SD-CP-TI-175, Rev. 1, Westinghouse Hanford Company, Richland, Washington.

Clark, R. 1995. Solid Waste Information and Tracking System (SWITS) Software Design Descriptions (SDD). WHC-SD-WM-SWD-009, Rev. 8, Westinghouse Hanford Company, Richland, Washington.

Comprehensive Environmental Response, Compensation and Liability Act of 1980, as amended, Public Law 96-510, 94 Stat. 2767, 42 USC 9601 et seq.

Croff, A. G. 1980. ORIGEN2 - A Revised and Updated Version of the Oak Ridge Isotope Generation and Depletion Code. ORNL-5621, Oak Ridge National Laboratory, Oak Ridge, Tennessee.

Kupfer, M. J., A. L. Boldt, B. A. Higley, K. M. Hodgson, L. W. Shelton, B. C. Simpson, R. A. Watrous, M. D. LeClair, G. L. Borsheim, R. T. Winward, R. M. Orme, N. G. Colton, S. L. Lambert, D. E. Place, and W. W. Schulz. 1997. Standard Inventories of Chemicals and Radionuclides in Hanford Site Tank Wastes. HNF-SD-WM-TI-740, Rev. 0, Lockheed Martin Hanford Corporation, Richland, Washington.

Resource Conservation and Recovery Act of 1976, as amended, Public Law 94-580, 90 Stat. 3221, 42 USC 6901 et seq.

U.S. Department of Energy (DOE). 1988. "Radioactive Waste Management.” DOE Order 5820.2A.

Watrous, R. A. and D. W. Wootan. 1997. Activity of Fuel Batches Processed Through Hanford Separations Plants, 1944 Through 1989. HNF-SD-WM-TI-794, Rev. 0, Lockheed Martin Hanford Corporation, Richland, Washington.

Wittekind, W. D. 1989. Software Certification Package for the ORIGEN2 Computer Code. WHC-SDNR-SWD-006, Rev. 0, Westinghouse Hanford Company, Richland, Washington. 
Wood, M. I., R. Khaleel, P. D. Rittmann, A. H. Lu, S. H. Finfrock, R. J. Serne, K. J. Contrell, T. H.

DeLorenzo. 1995. Performance Assessment for the Disposal of Low-Level Waste in the 200 West Area Burial Grounds. 'WHC-EP-0645, Westinghouse Hanford Company, Richland, Washington.

Wood, M. I., R. Khaleel, P. D. Rittmann, S. H. Finfrock, T. H. DeLorenzo, and D. Y. Garbrick. 1996. Performance Assessment for the Disposal of Low-Level Waste in the 200 East Area Burial Grounds. WHC-SD-WM-TI-730, Rev. 0, Westinghouse Hanford Company, Richland, Washington. 
Table A.1. Solid Waste Inventory for "new_dec_96" Category (page 1 of 5)

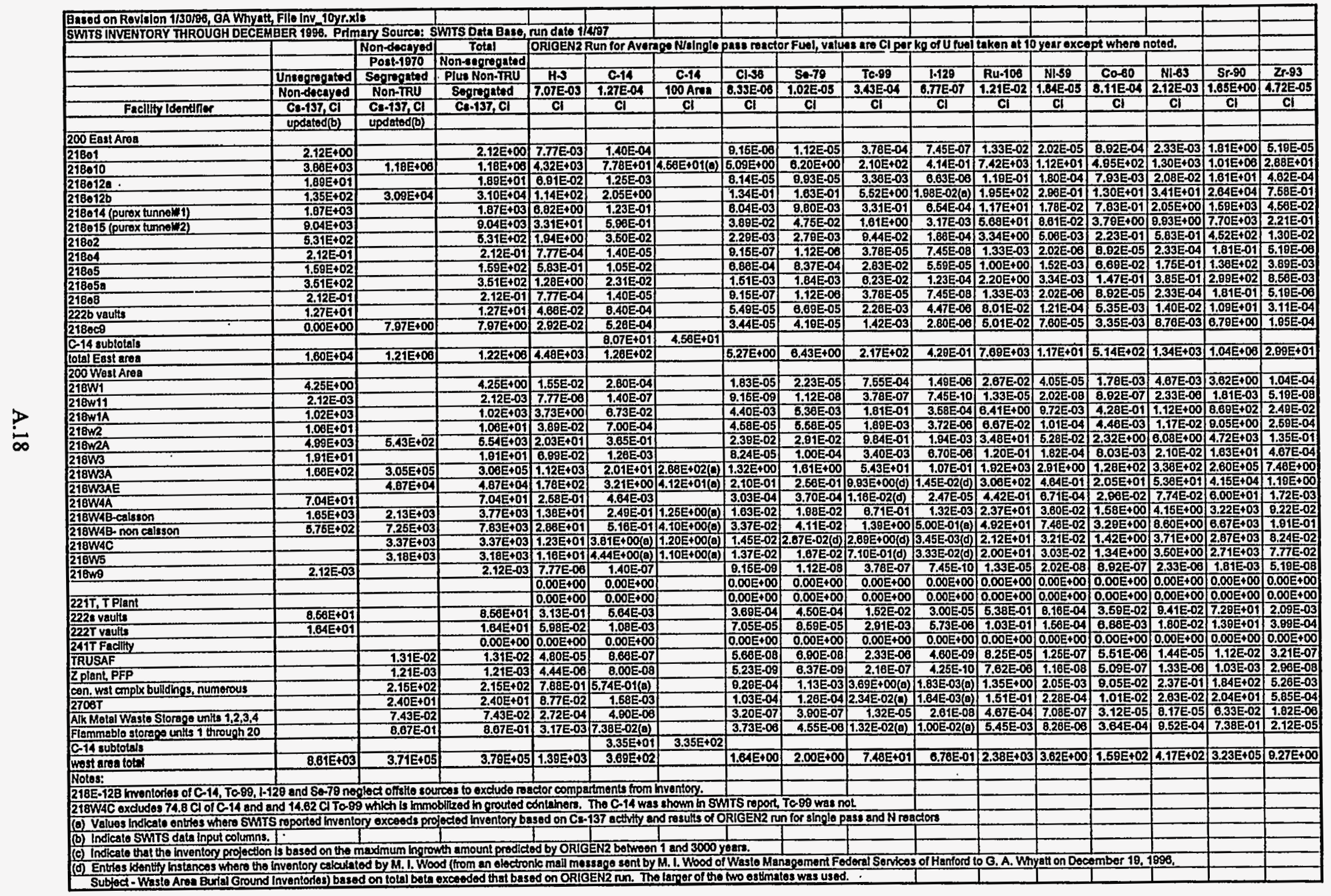


Table A.1. (page 2 of 5)

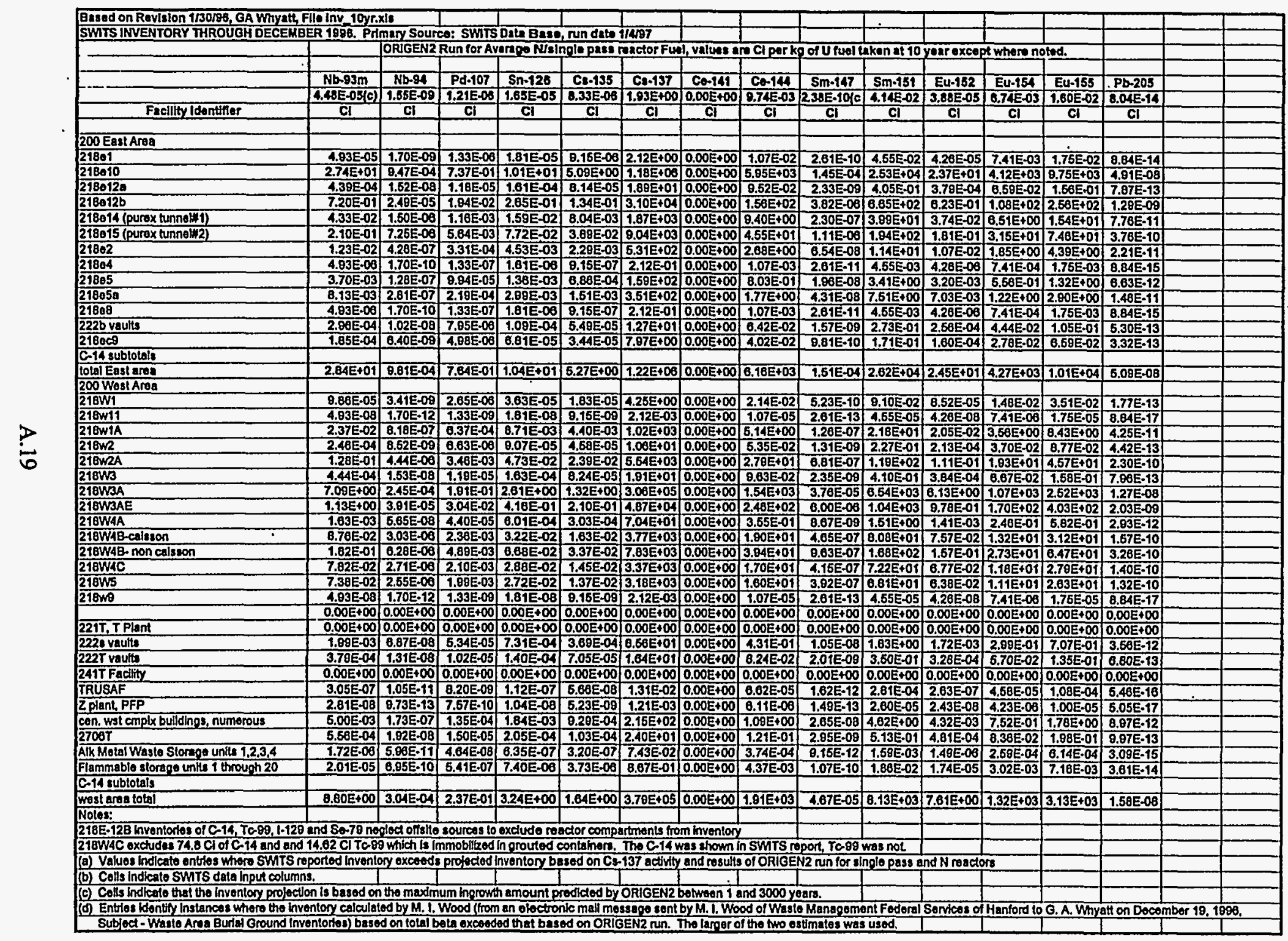


Table A.1. (page 3 of 5)

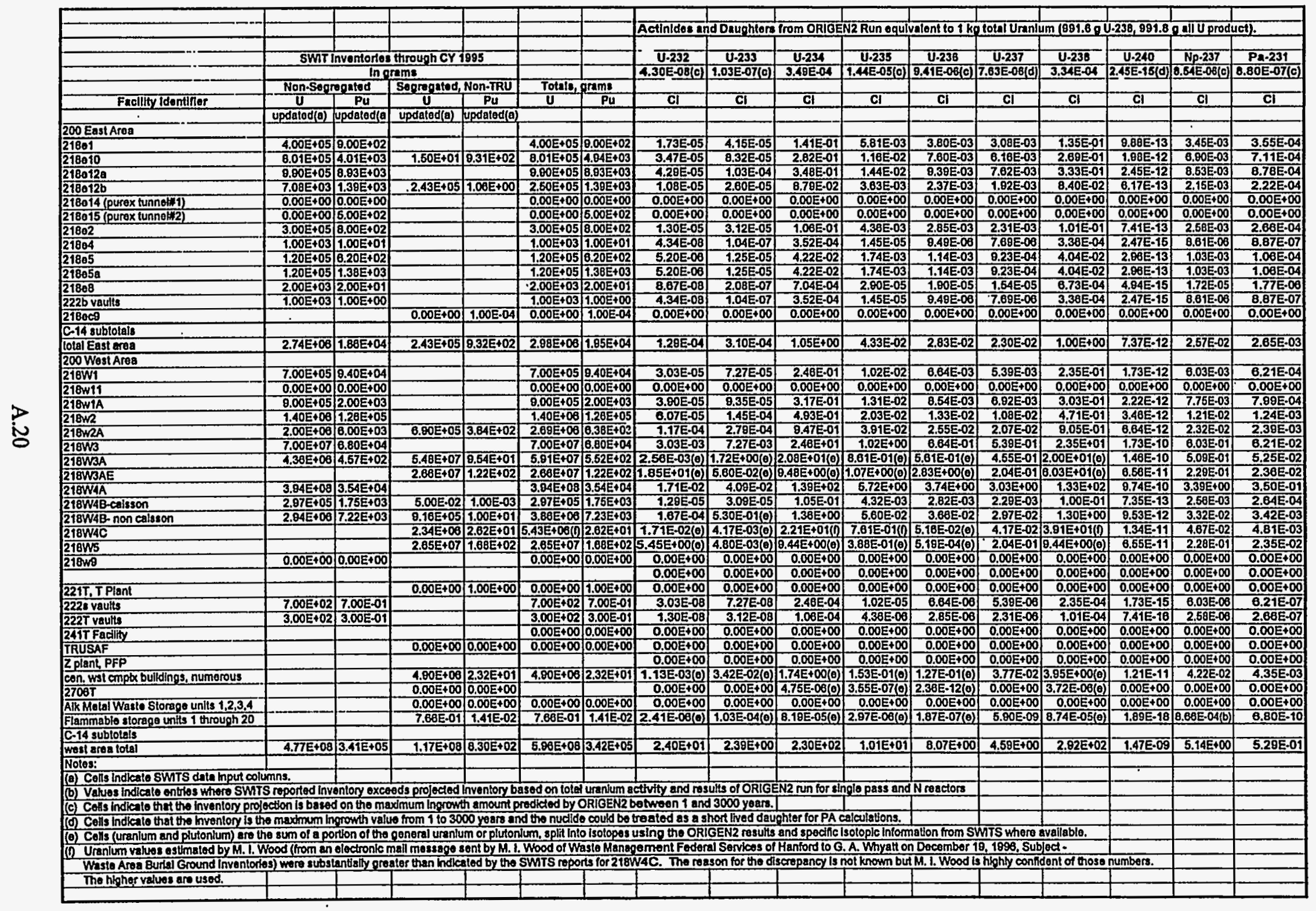


Table A.1. (page 4 of 5)

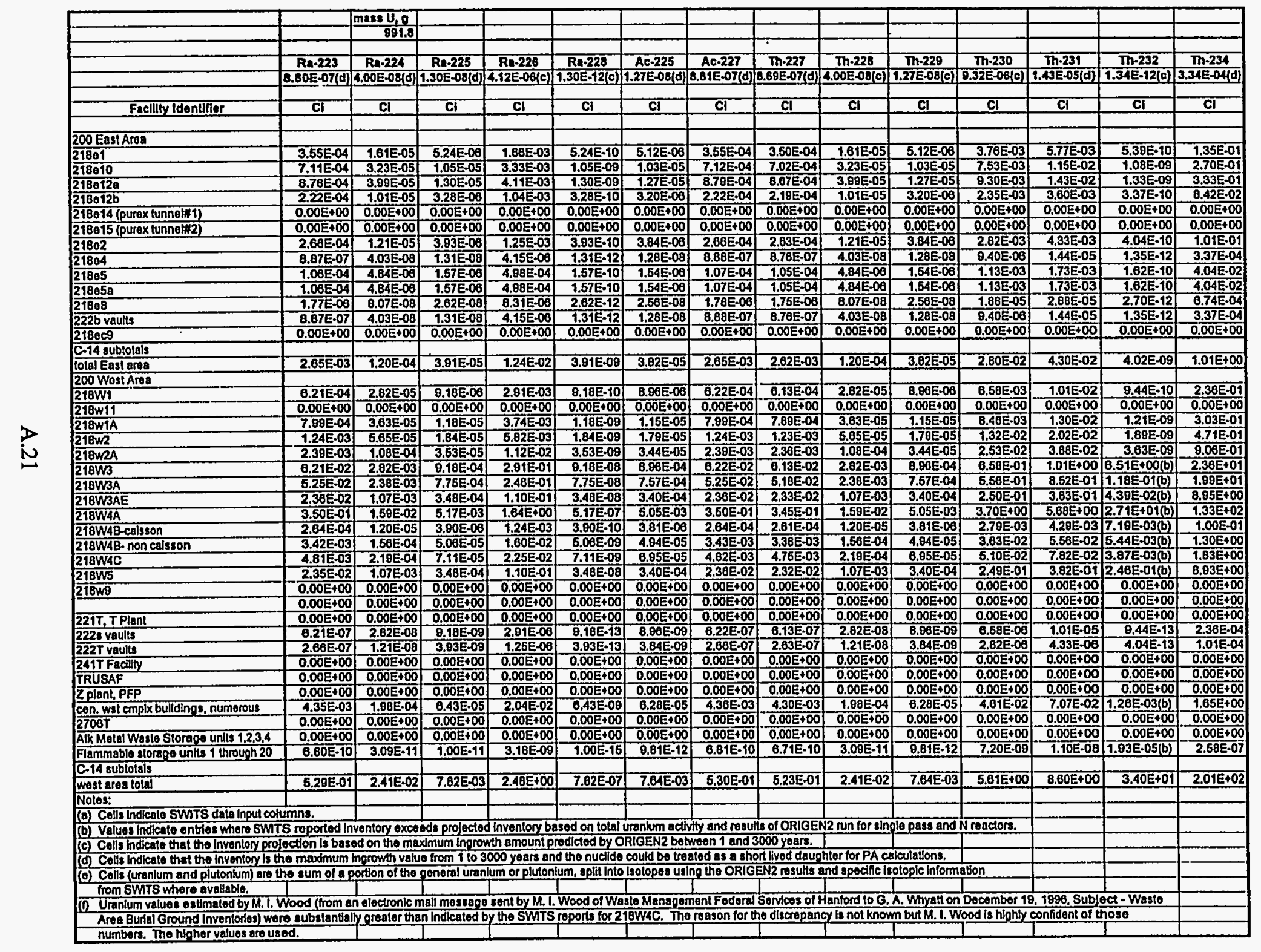


Table A.1. (page 5 of 5)

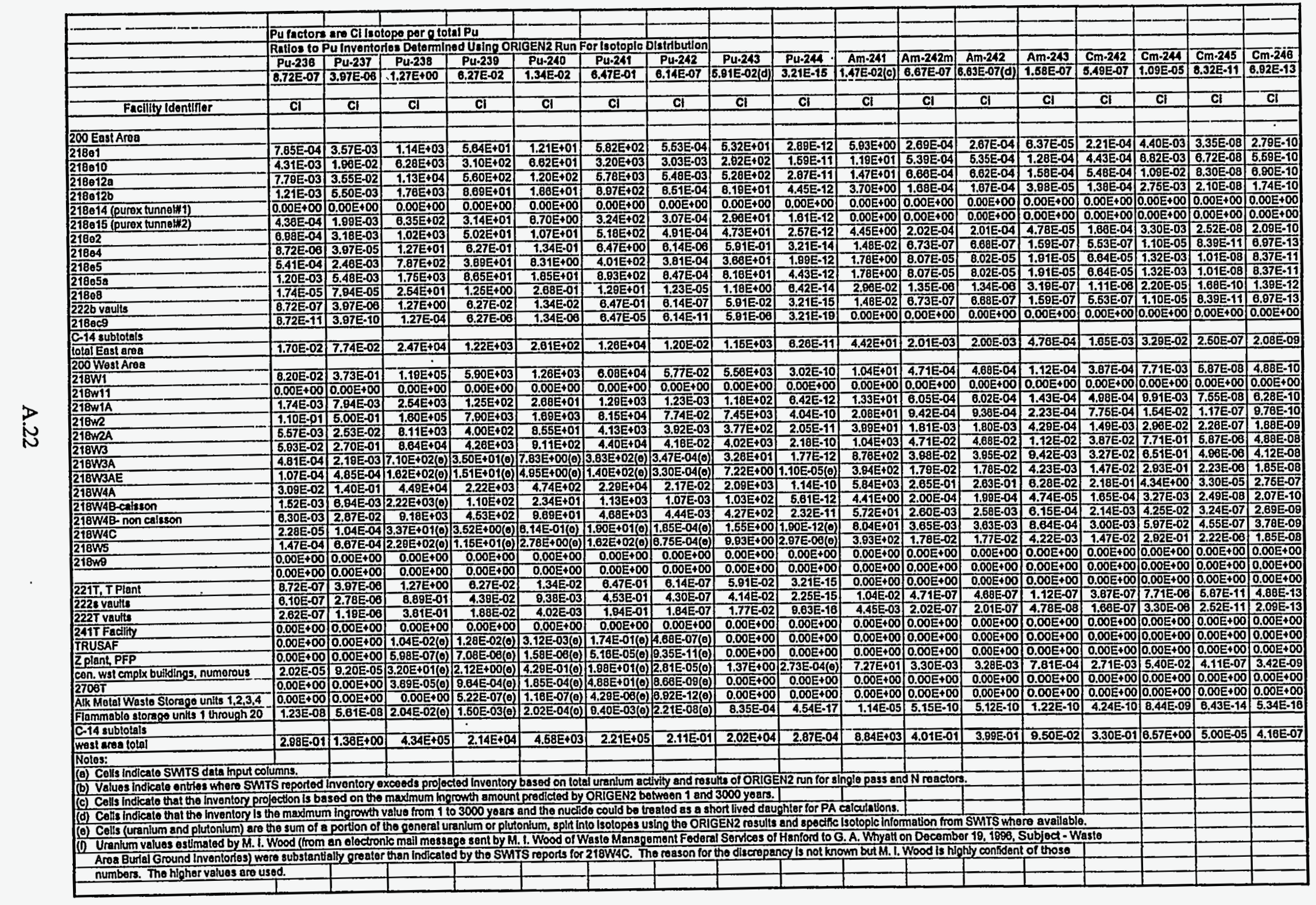


Table A.2. Solid Waste Inventory for "new_sept_88" Category (page 1 of 5)

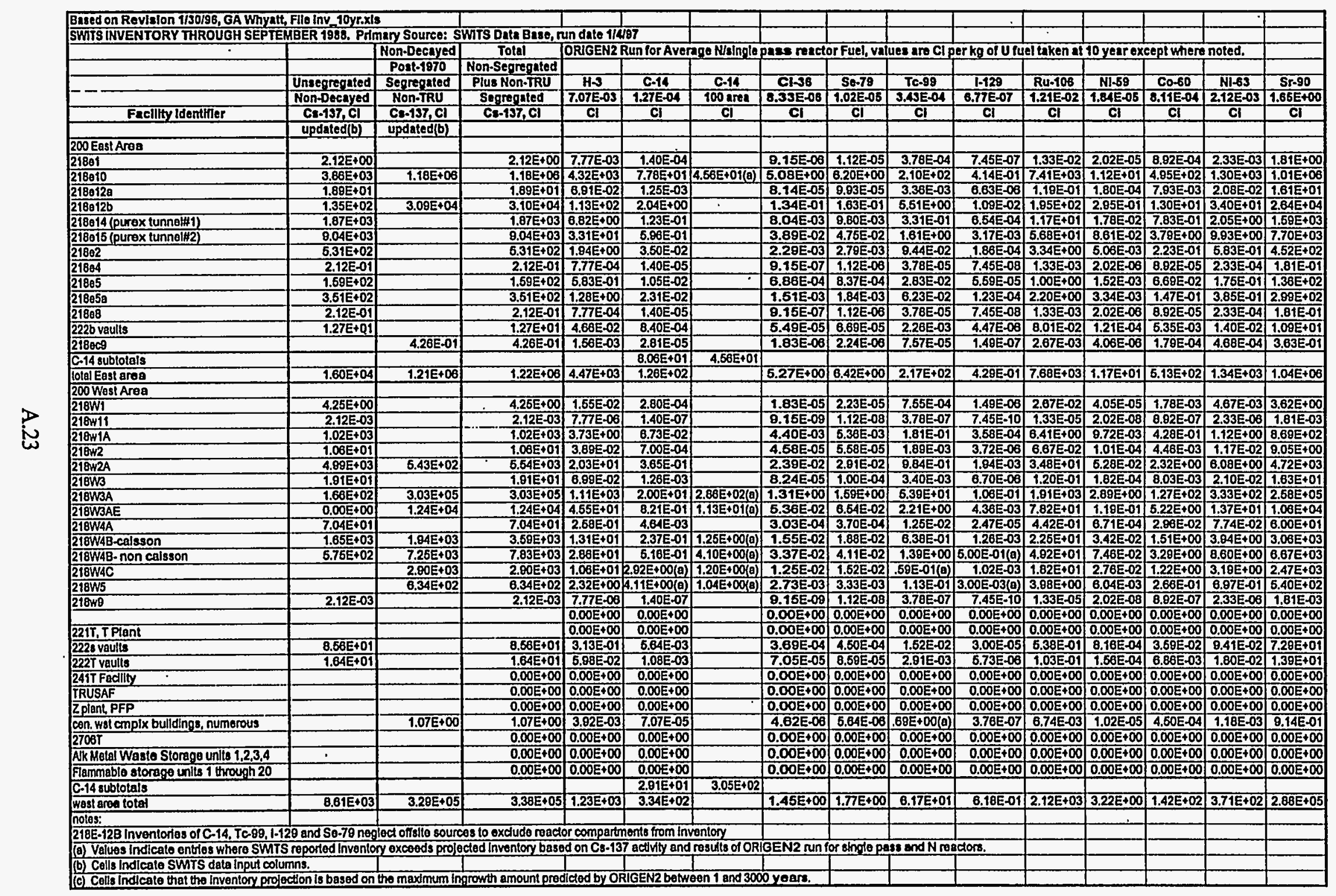


Table A.2. (page 2 of 5)

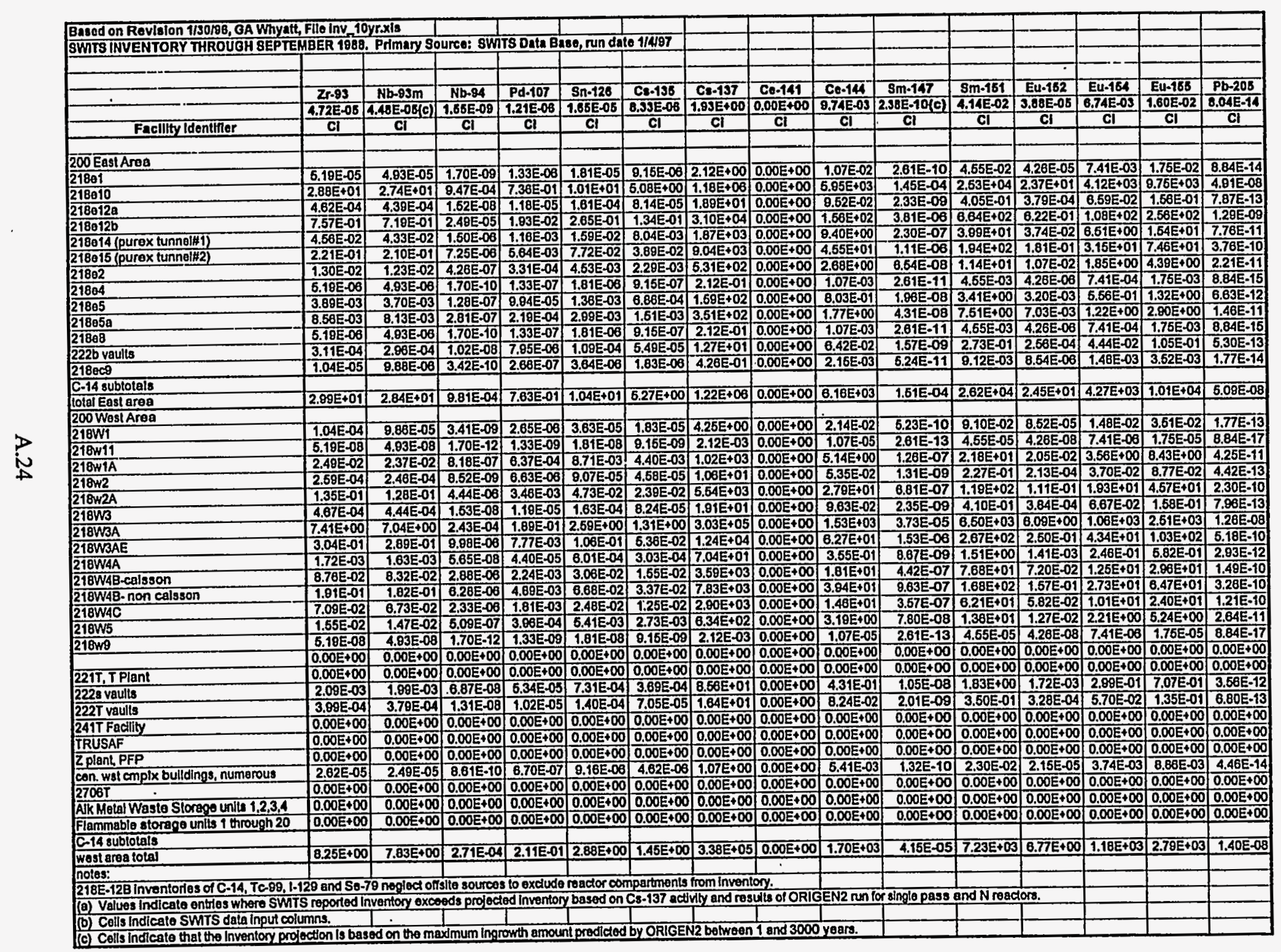


Table A.2. (page 3 of 5)

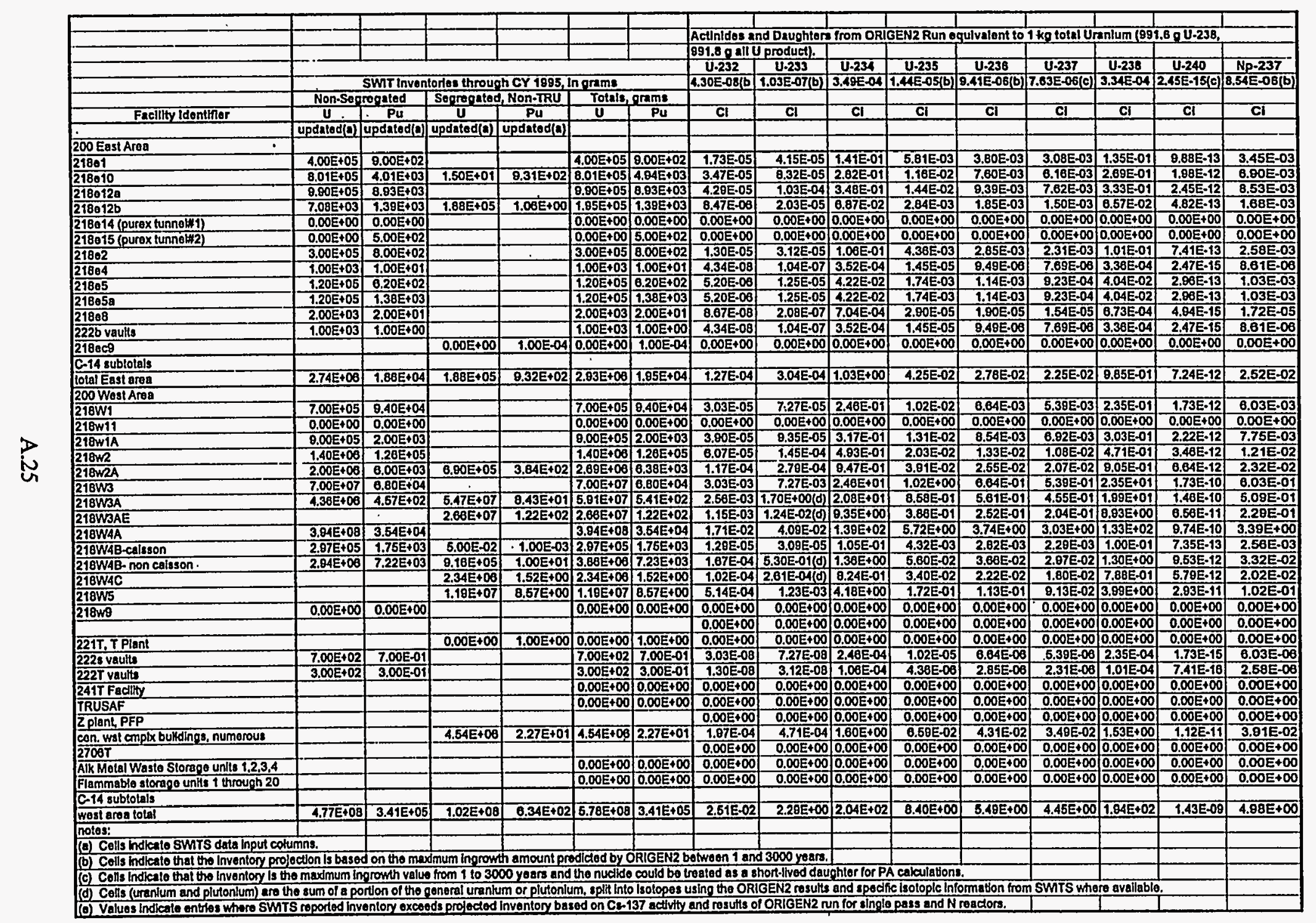


Table A.2. (page 4 of 5)

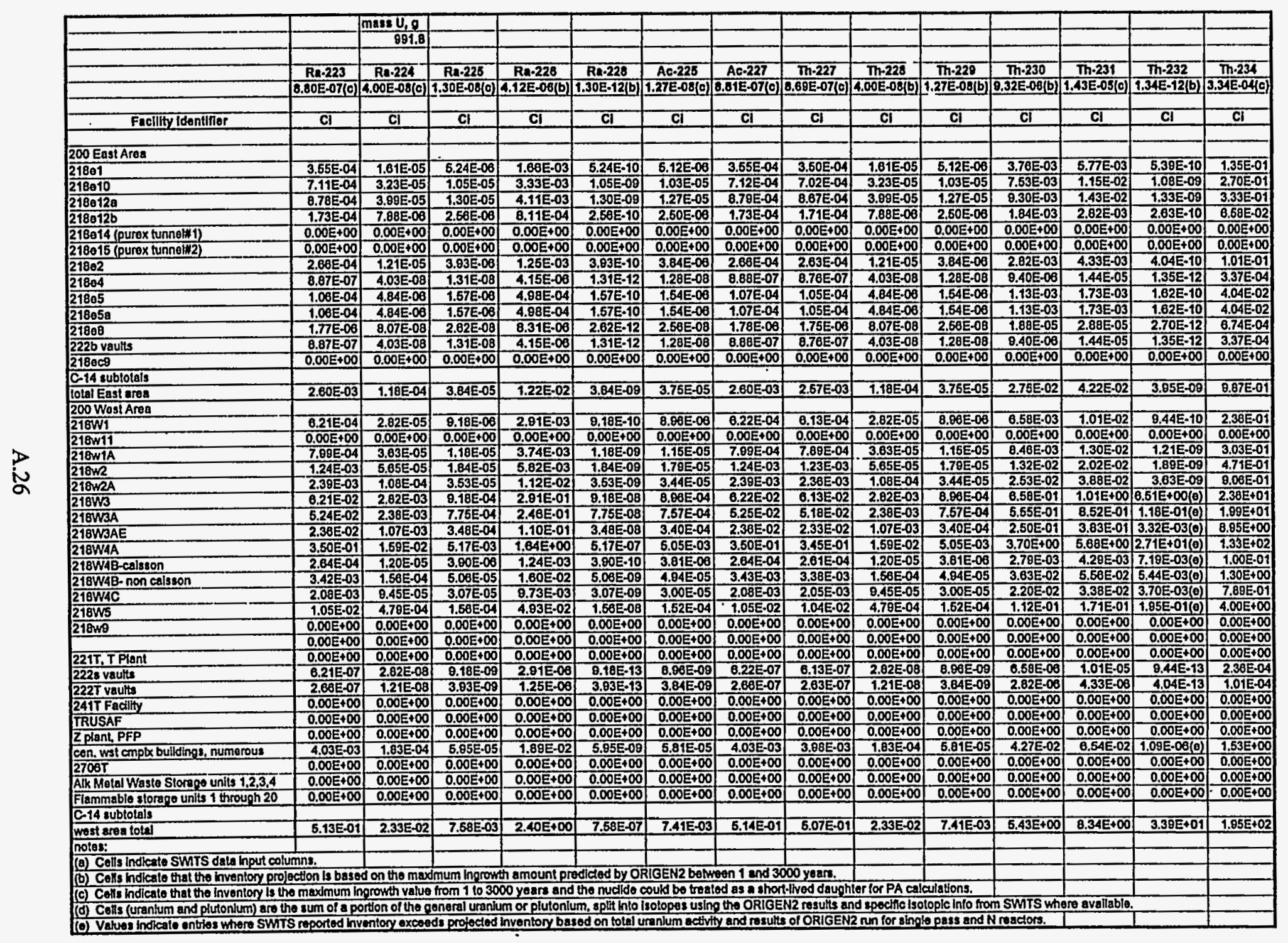


Table A.2. (page 5 of 5)

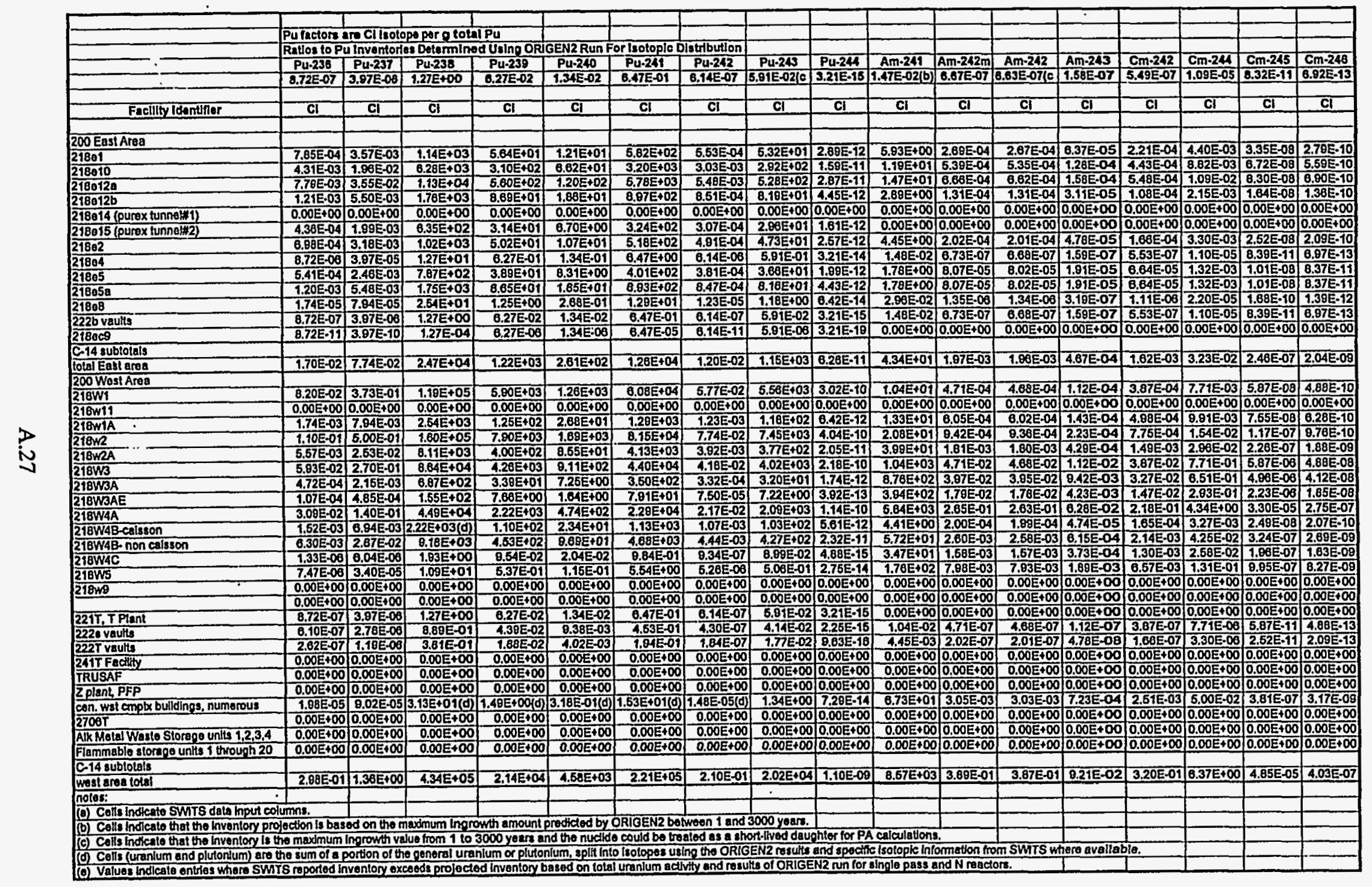


Table A.3. Solid Waste Inventory for "new_96_88" Category (page 1 of 6)

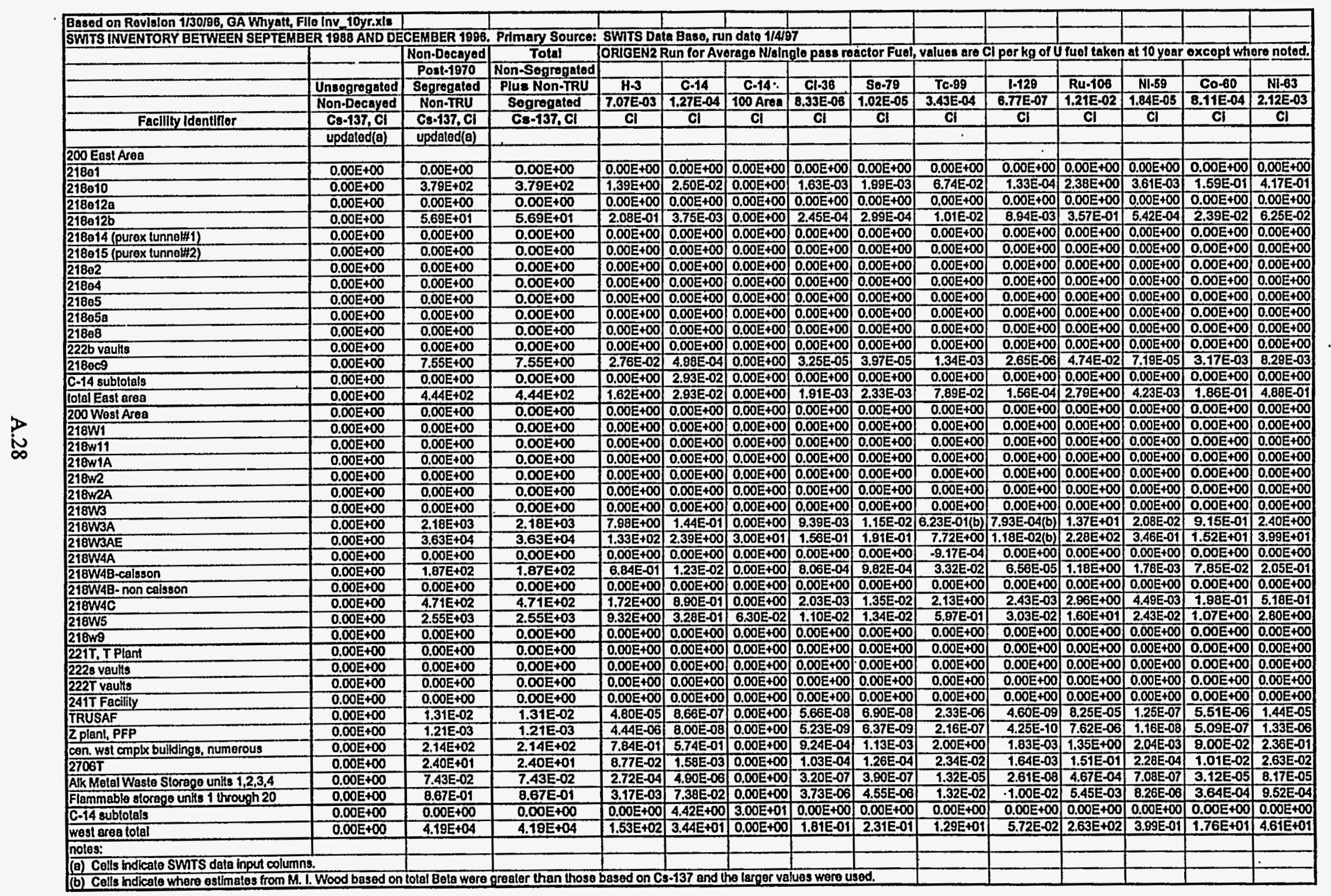


Table A.3. (page 2 of 6)

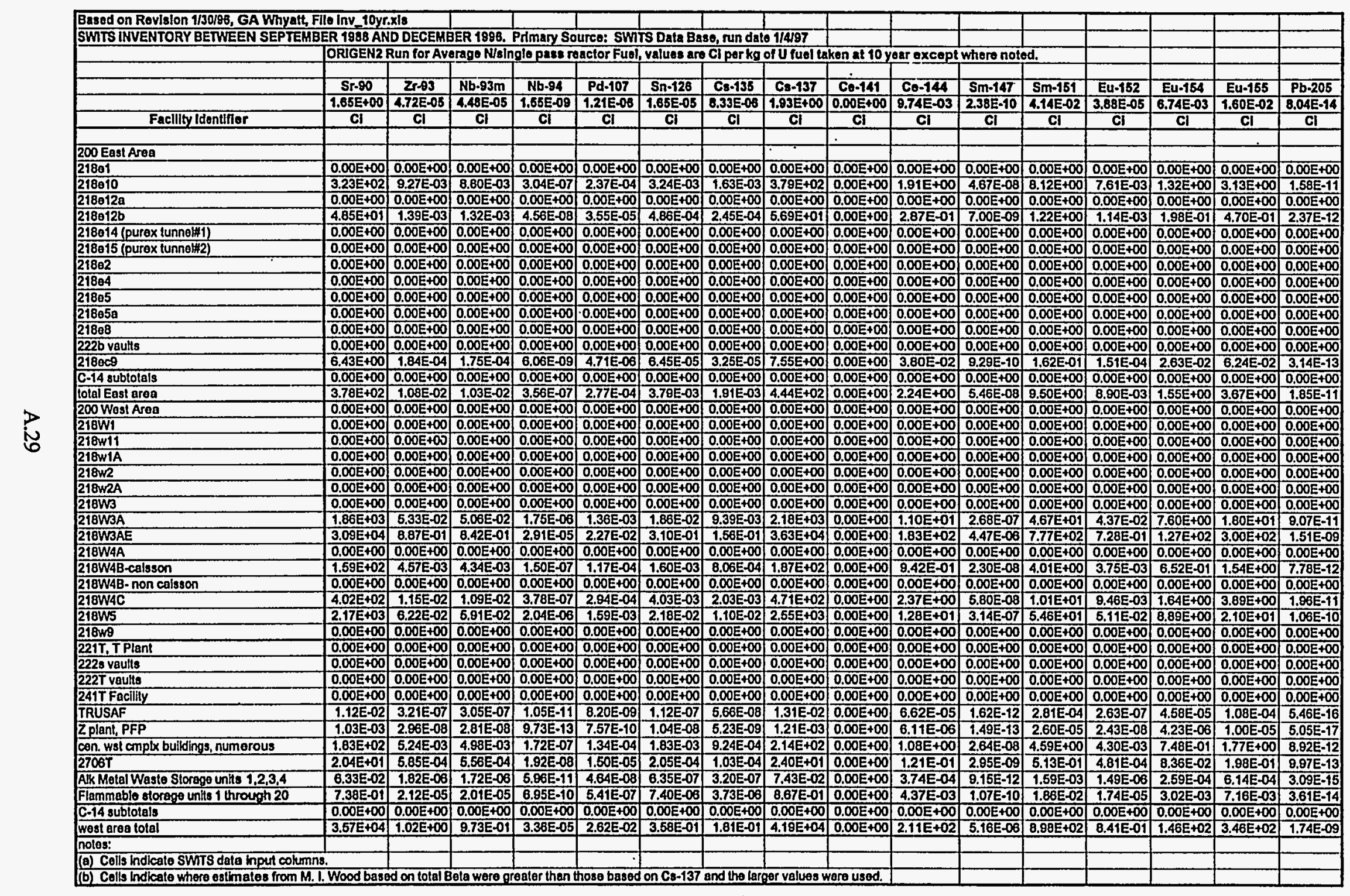


Table A.3. (page 3 of 6 )

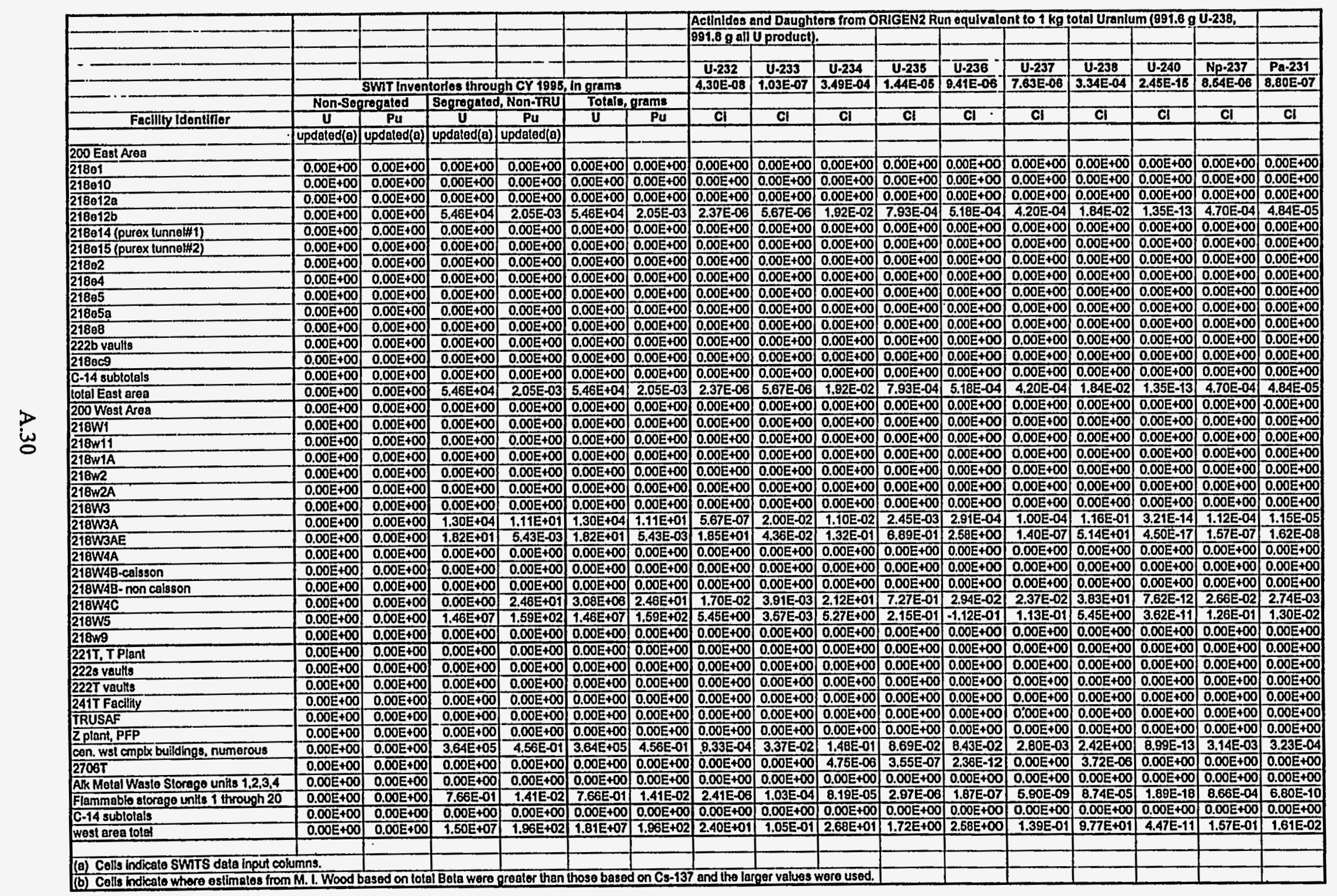


Table A.3. (page 4 of 6 )

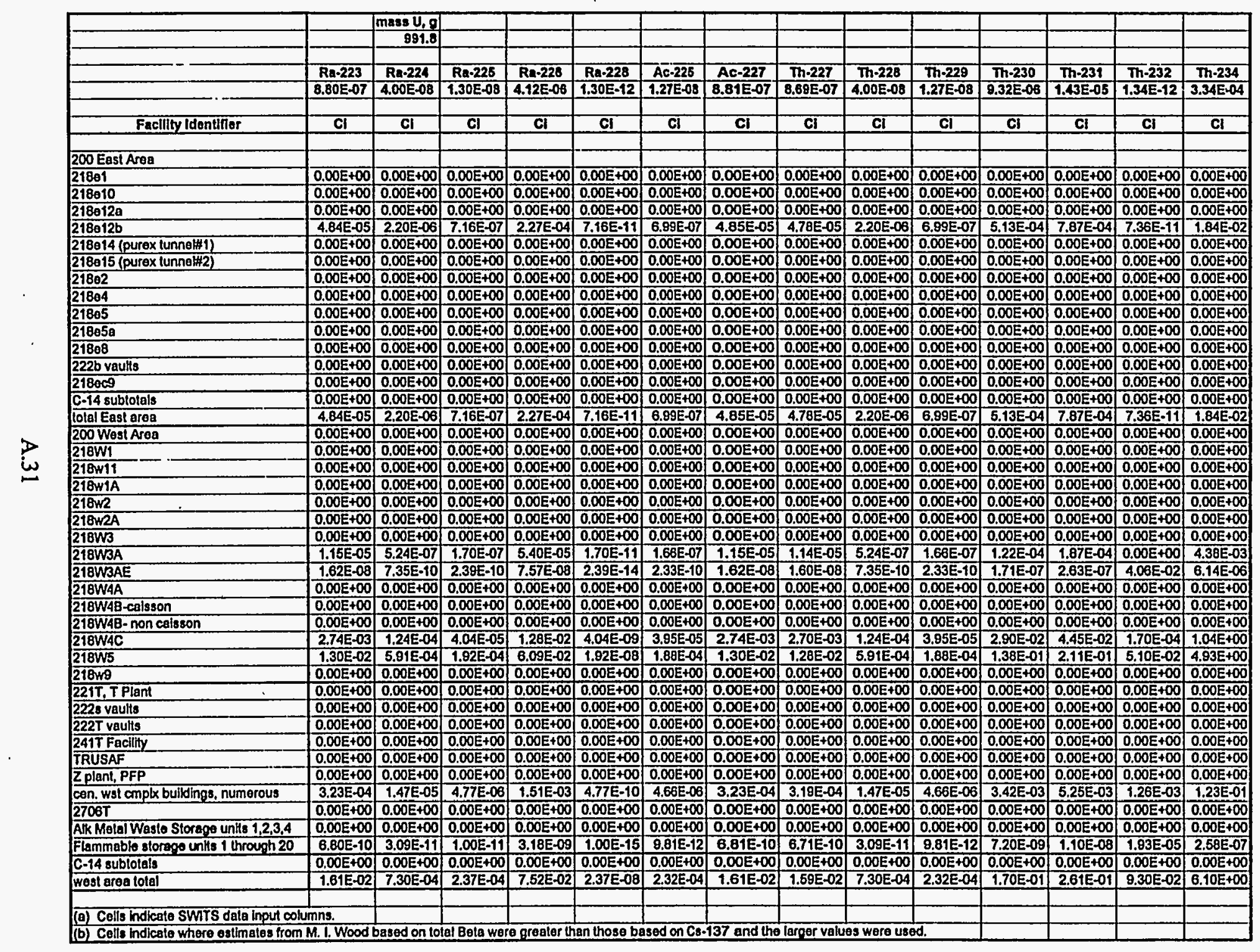


Table A.3. (page 5 of 6)

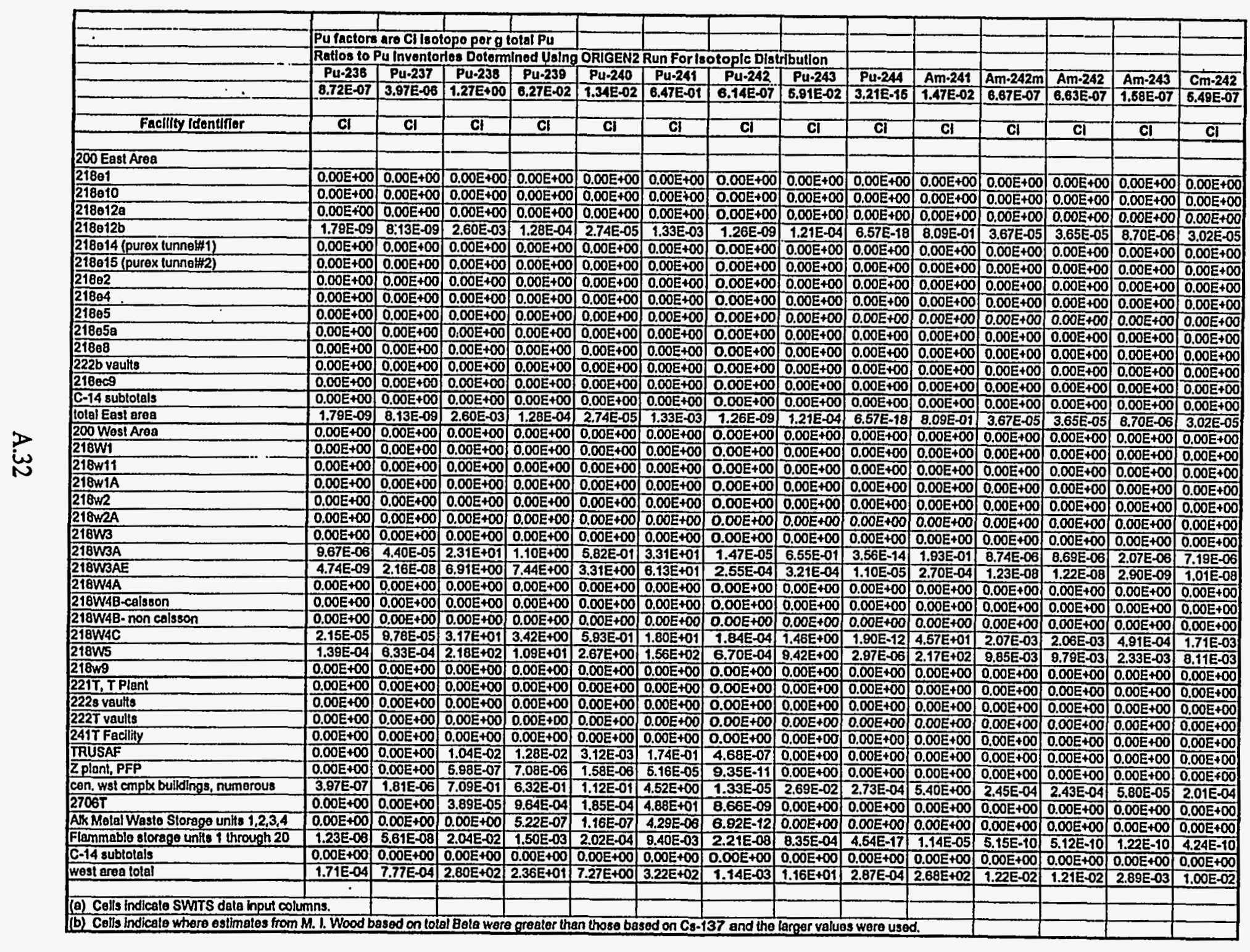


Table A.3. (page 6 of 6 )

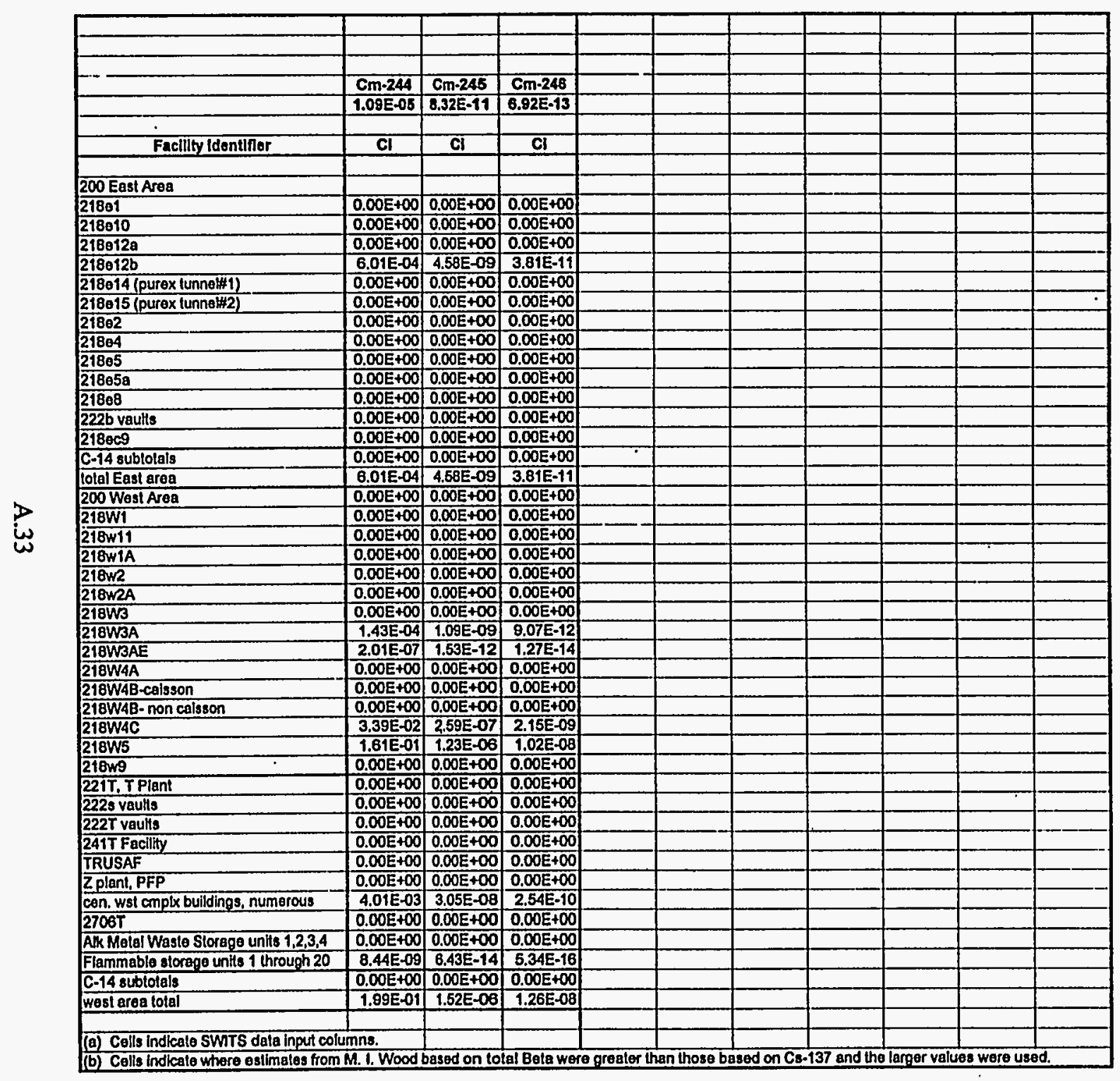


Table A.4. Solid Waste Inventory for "new_suspect_TRU" Category (page 1 of 7)

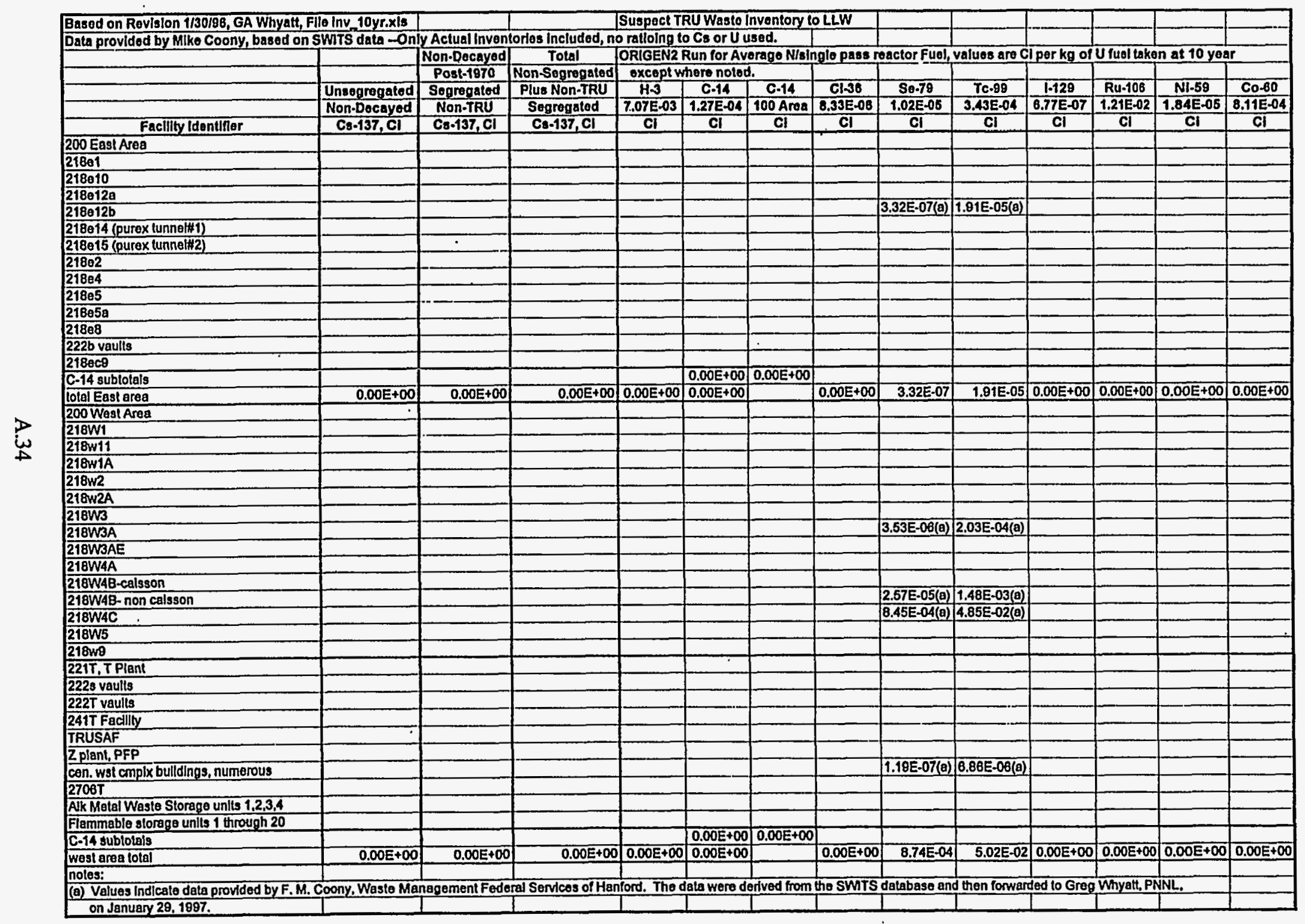


Table A.4. (page 2 of 7)

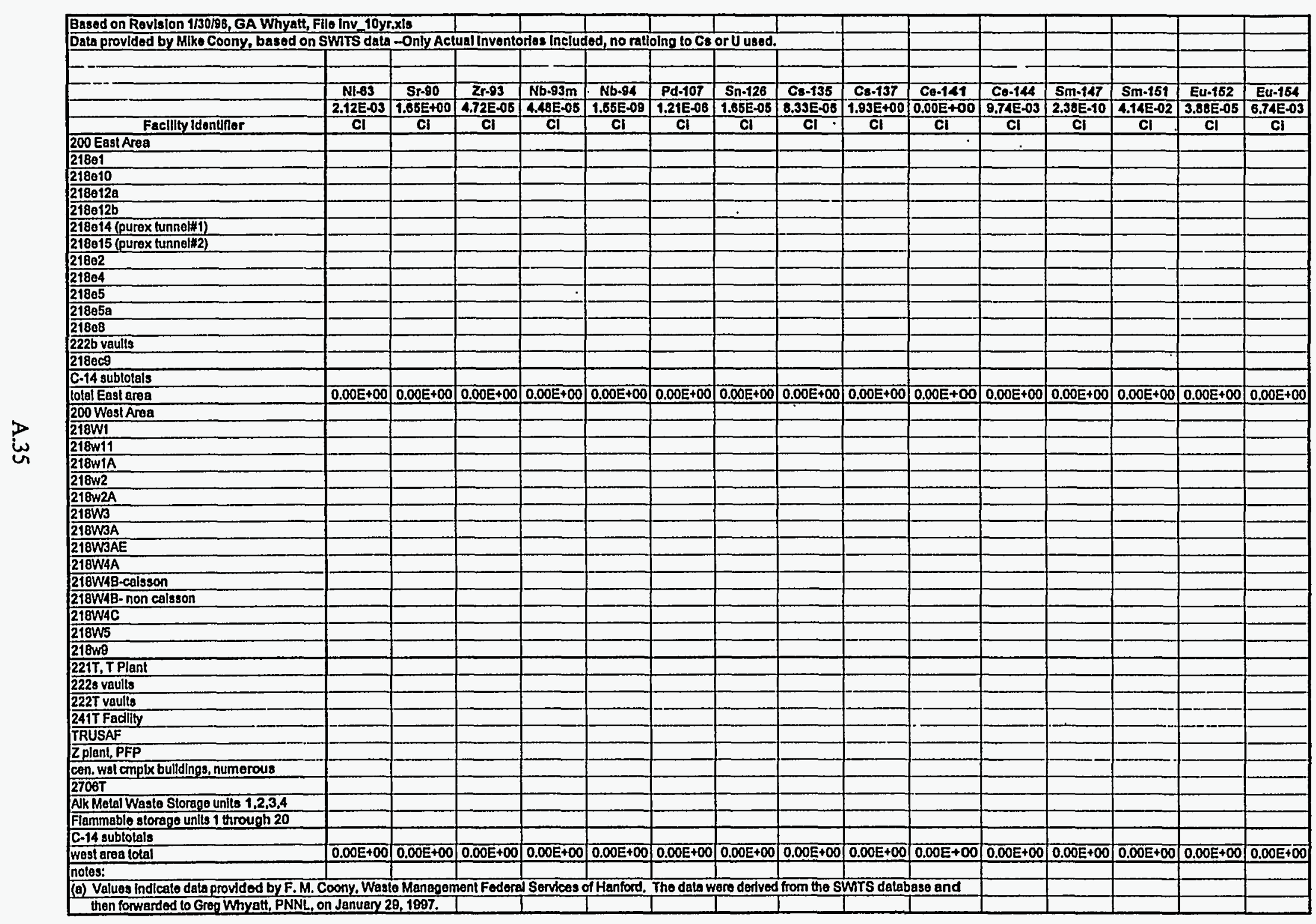


Table A.4. (page 3 of 7)

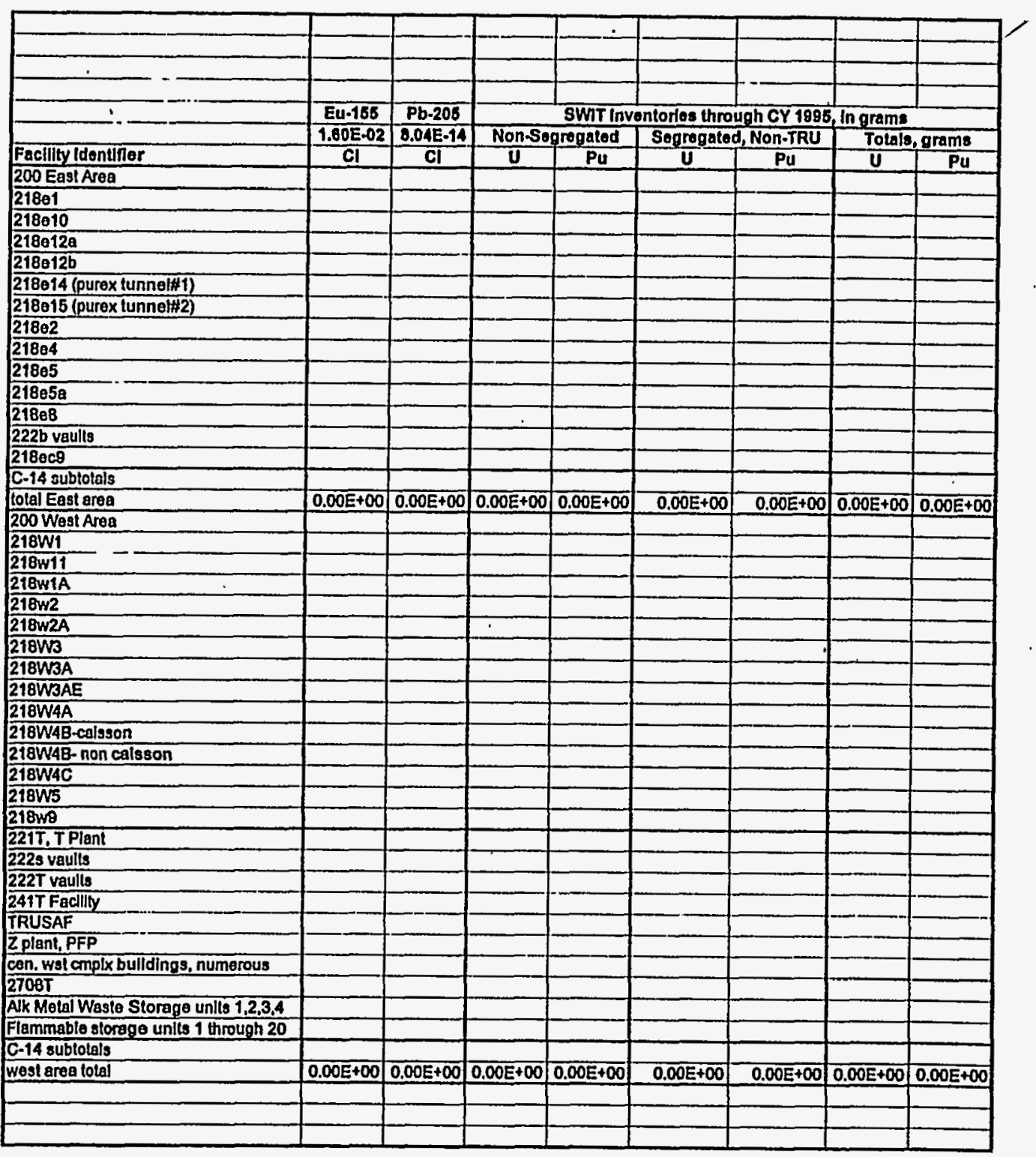




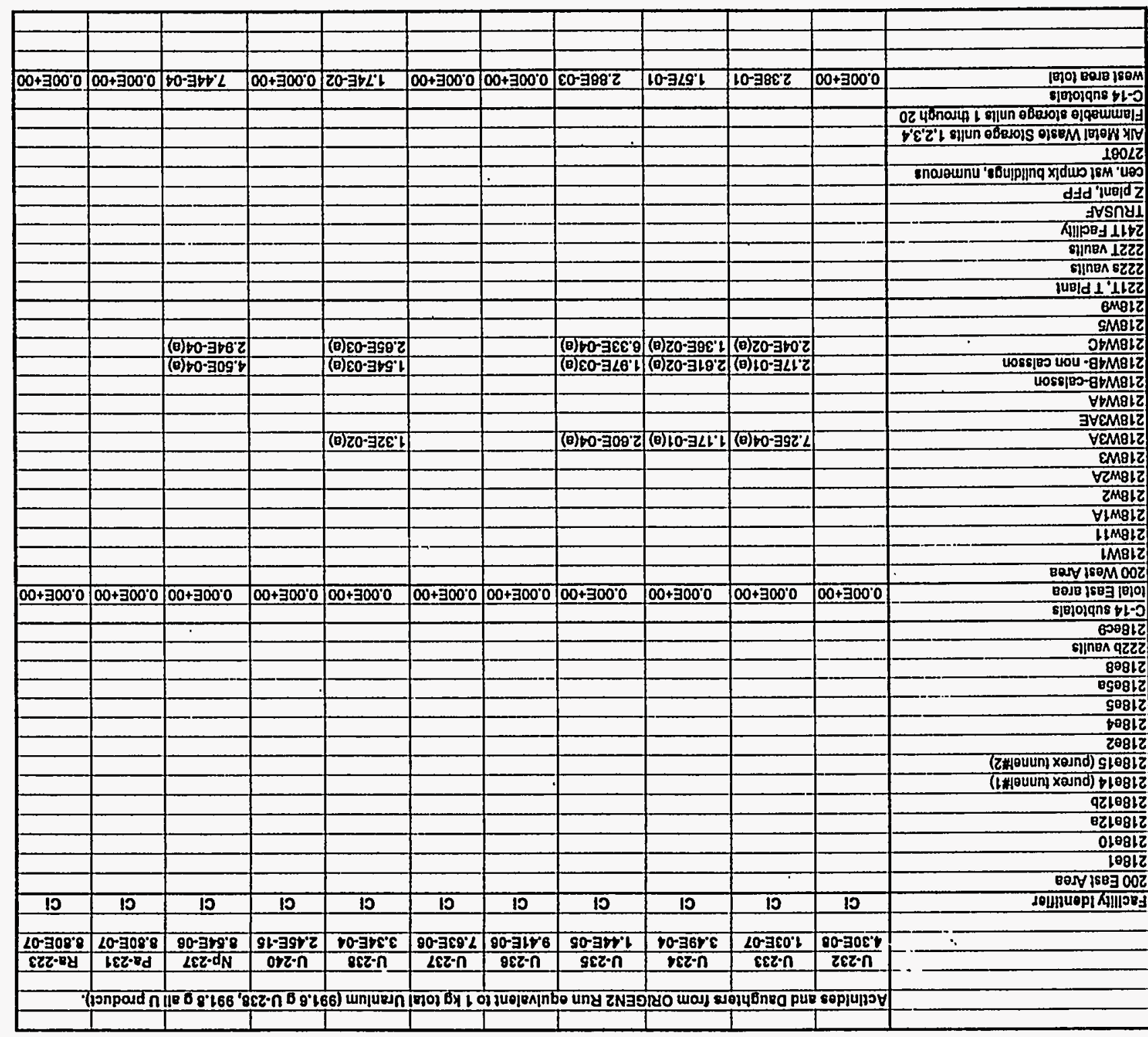

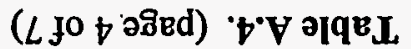




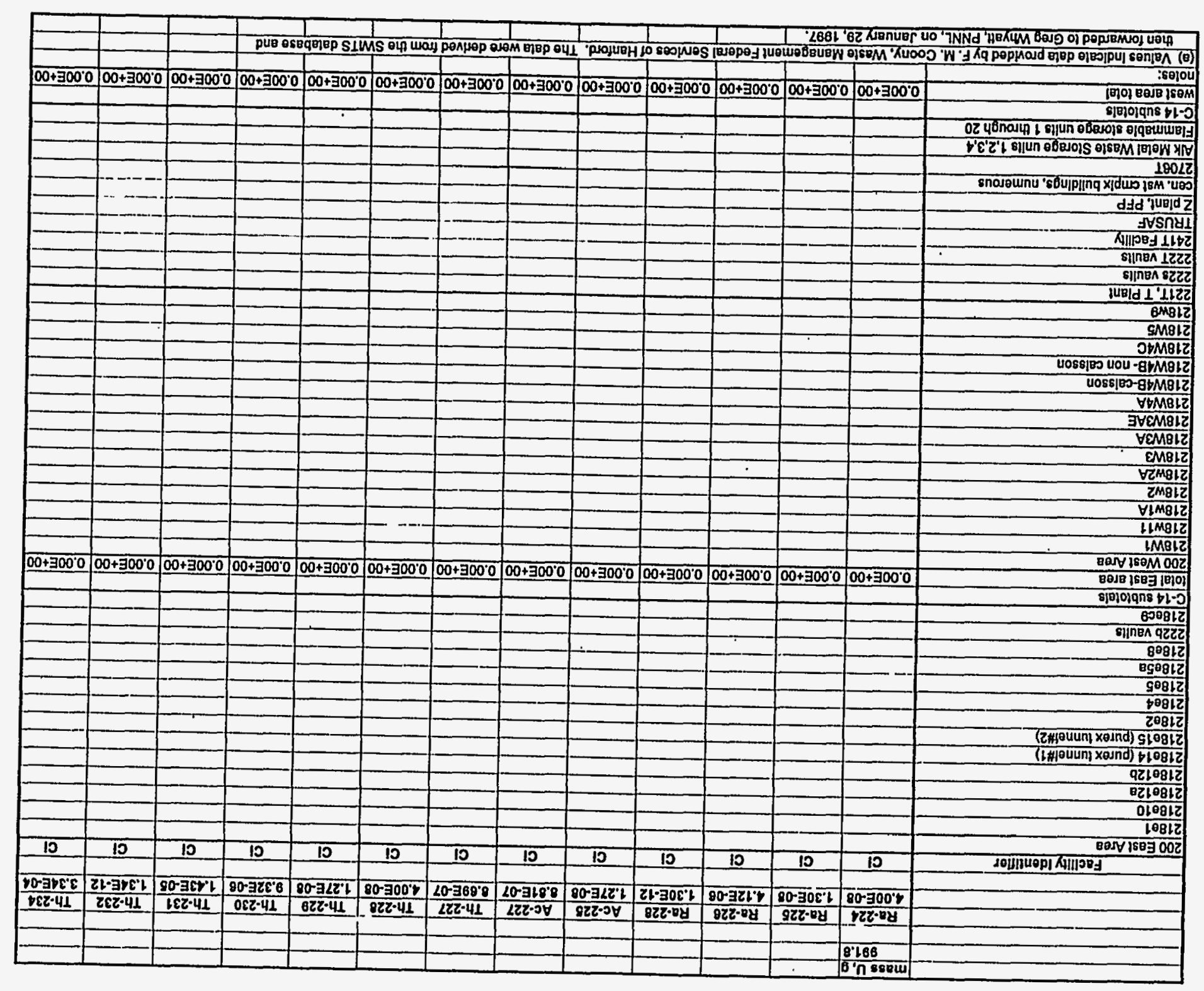

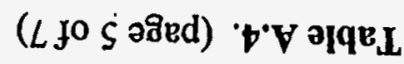


Table A.4. (page 6 of 7)

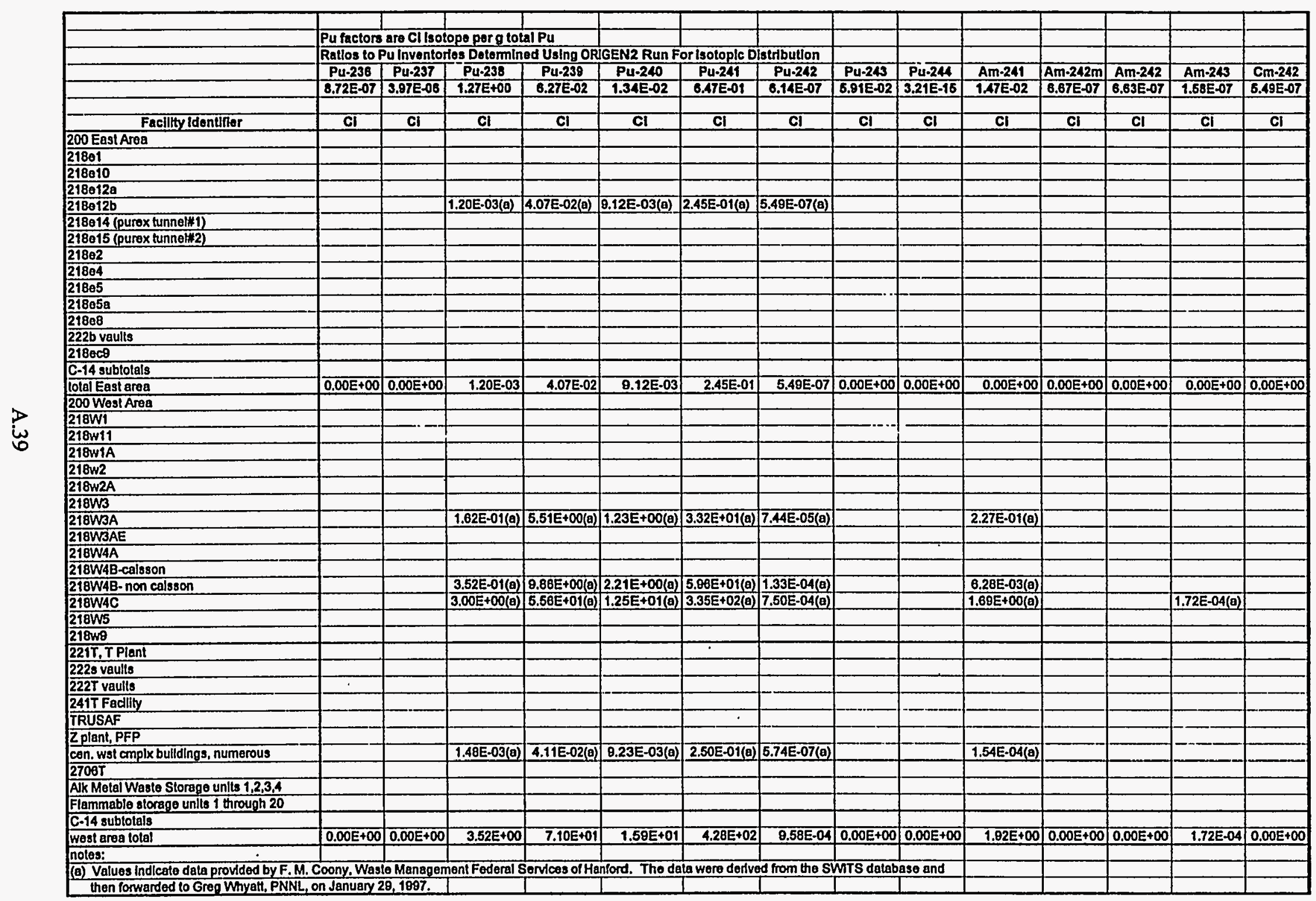




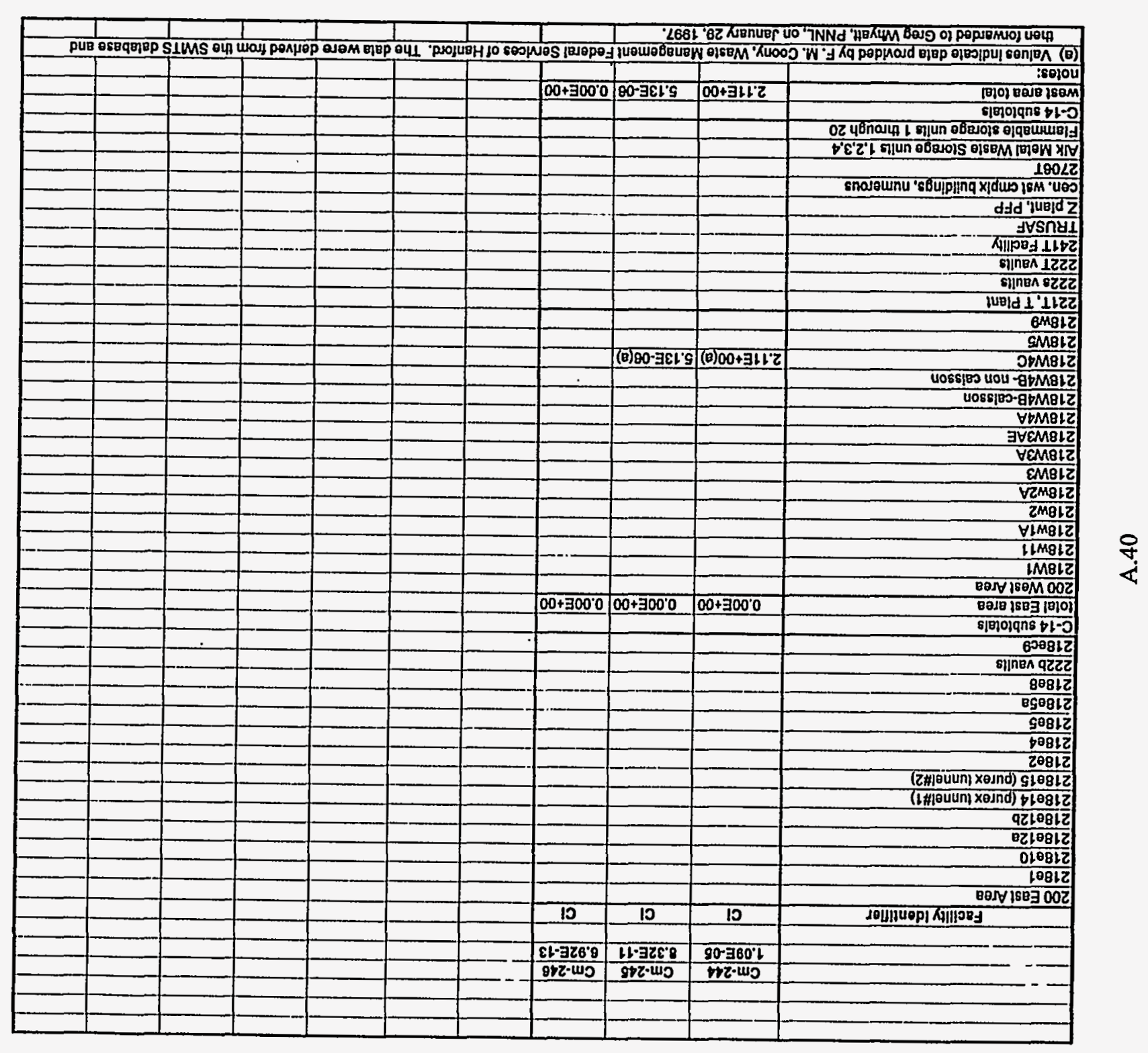

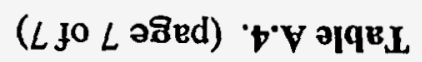


Table A.5. Solid Waste Inventory for "new_future" Category (page 1 of 6)

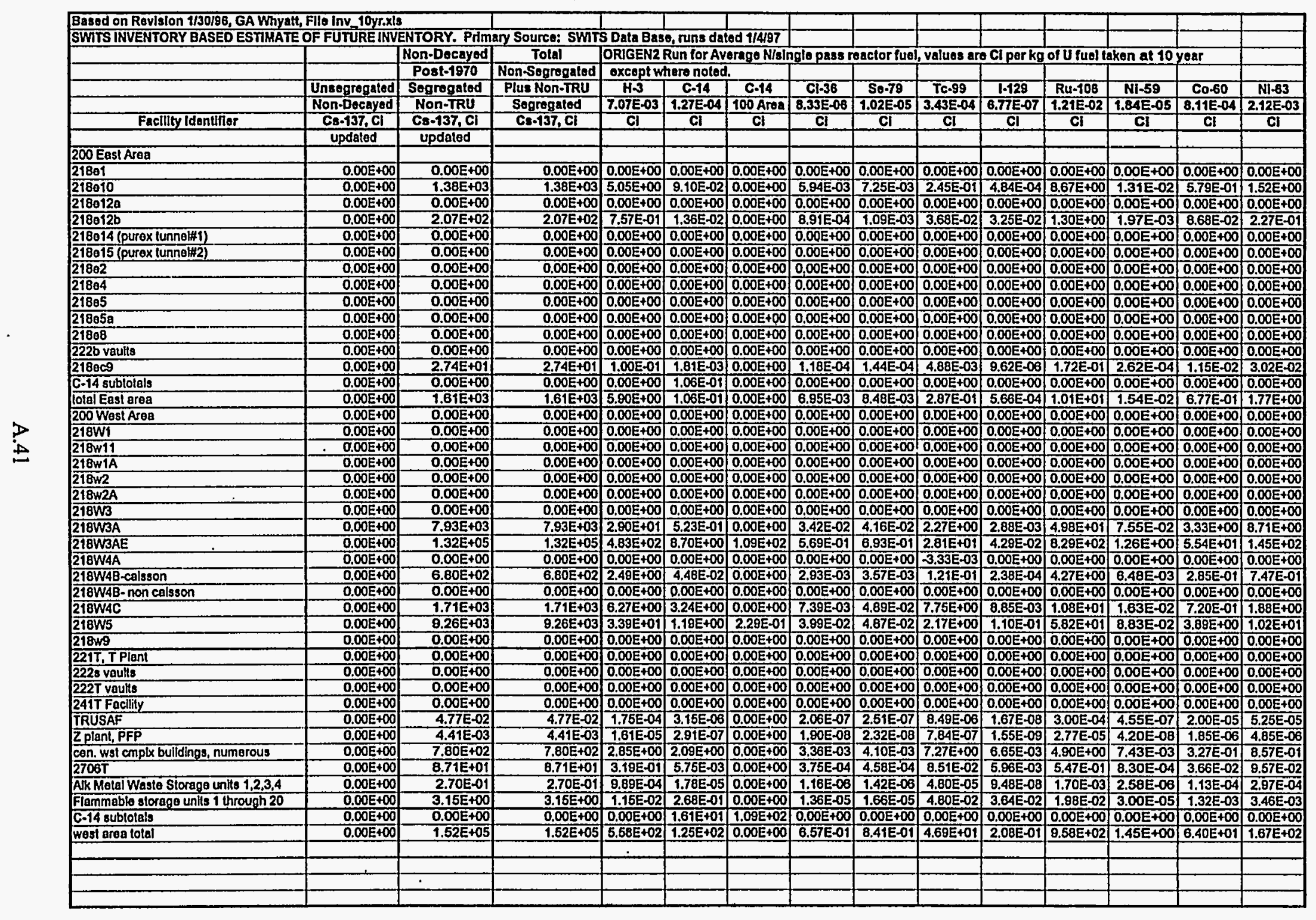


Table A.5. (page 2 of 6)

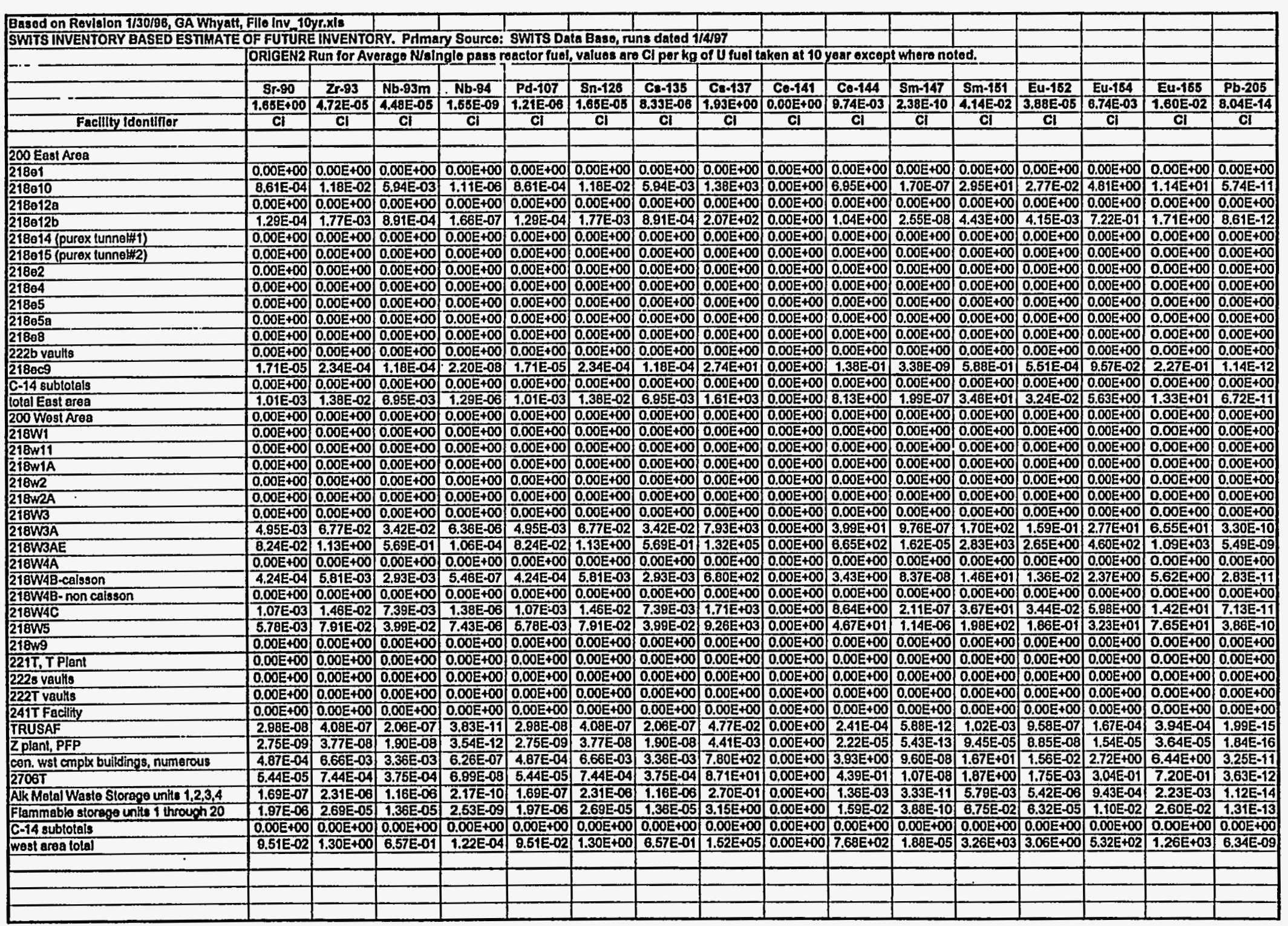


Table A.5. (page 3 of 6)

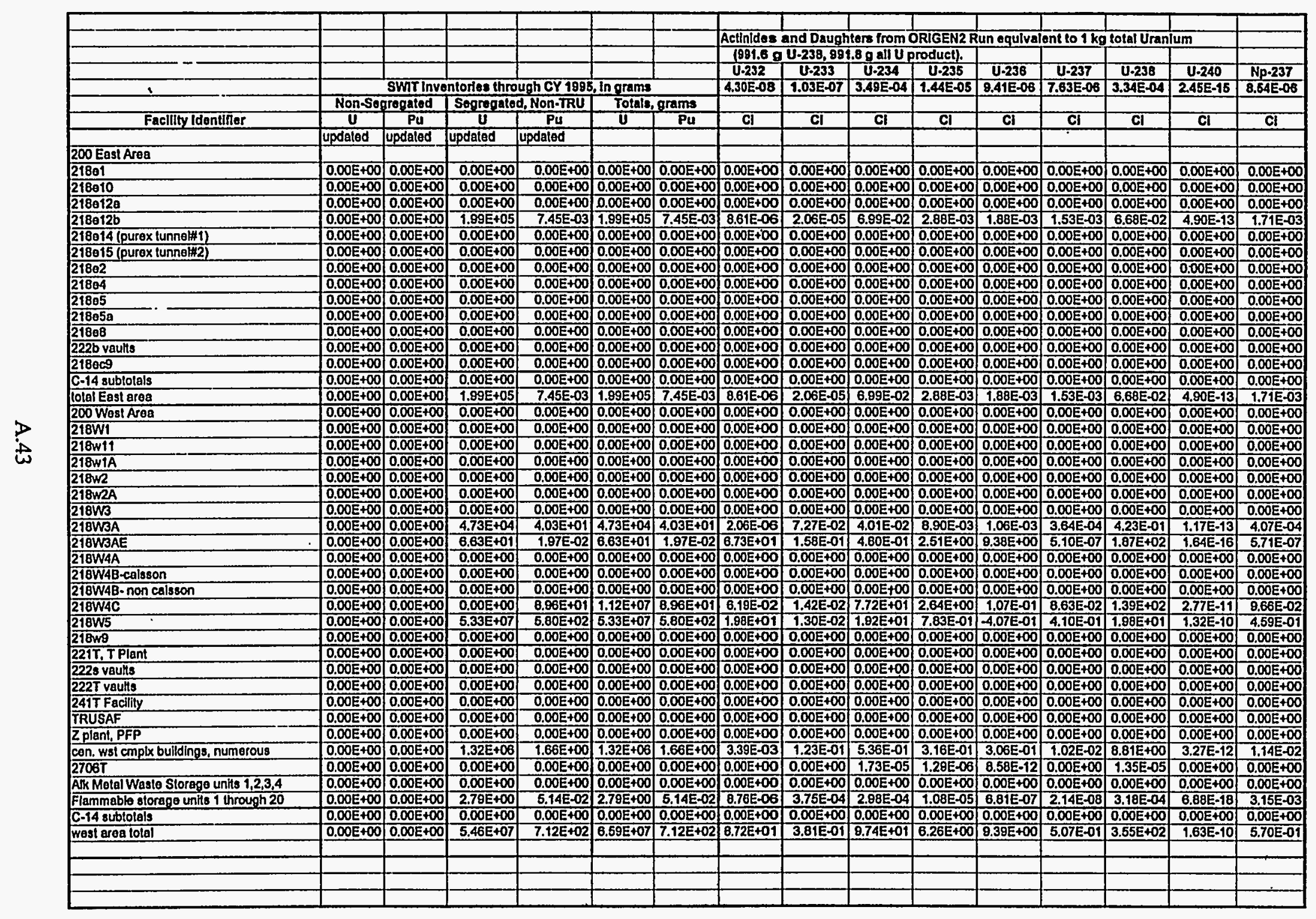


Table A.5. (page 4 of 6 )

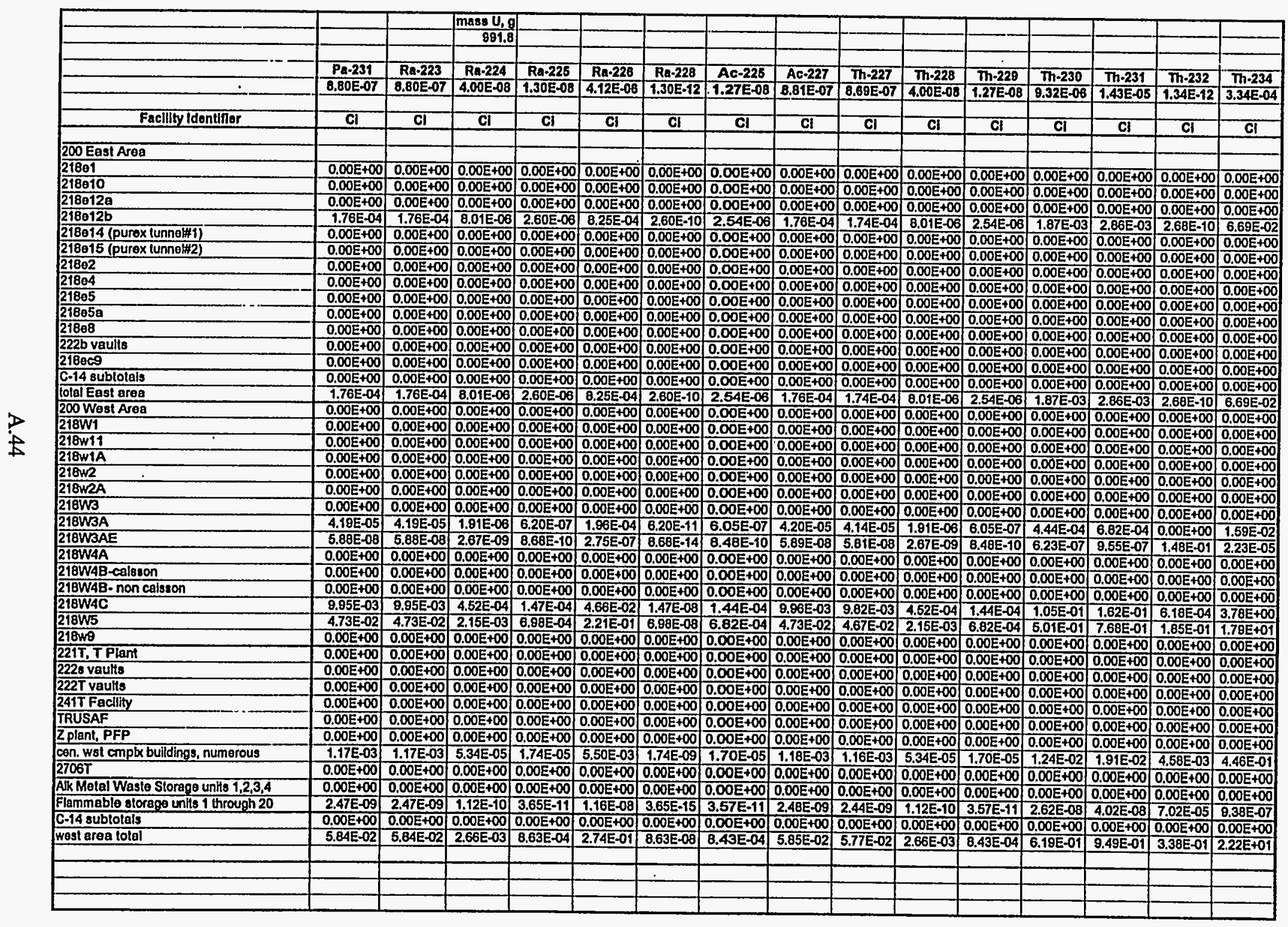


Table A.5. (page 5 of 6 )

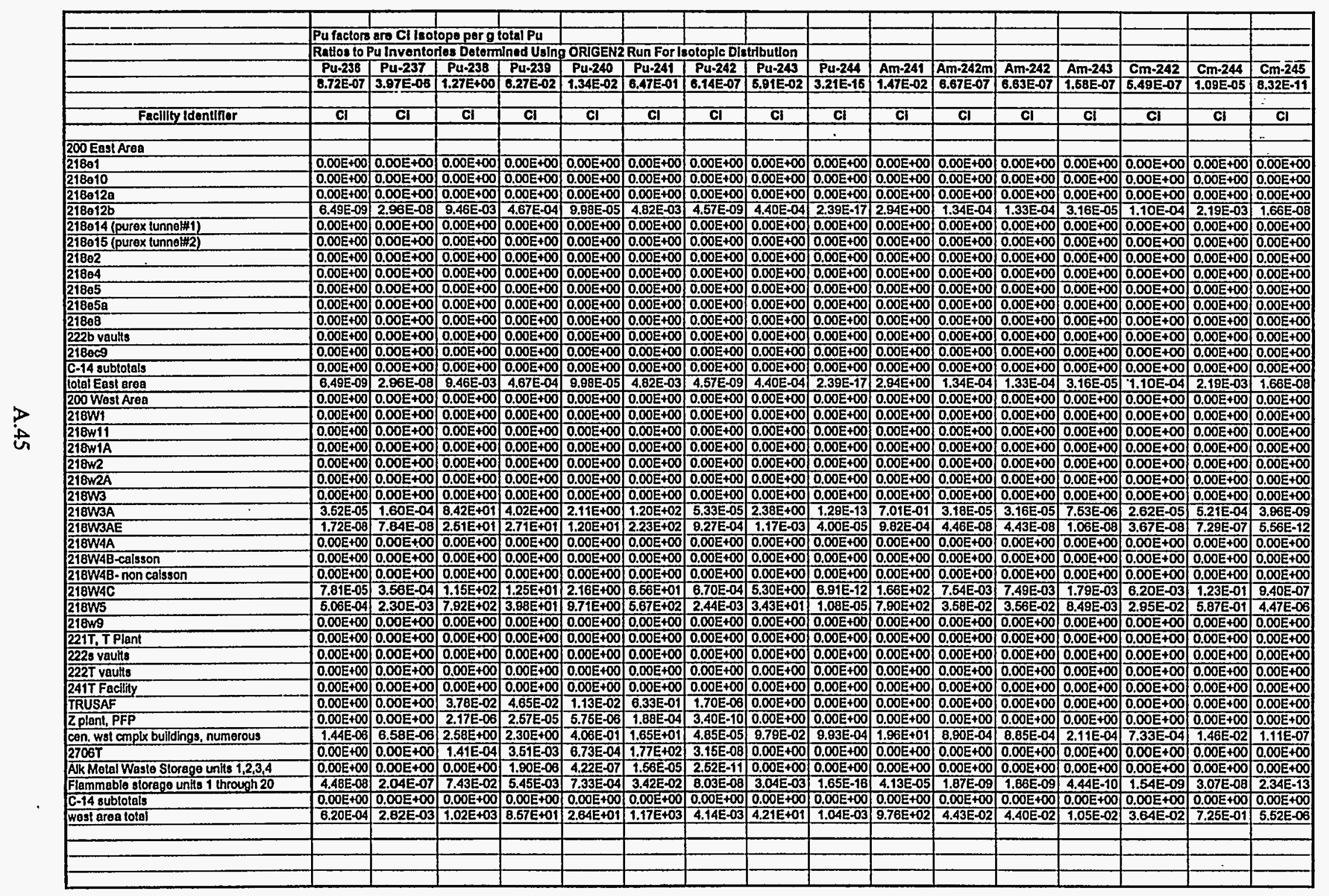


Table A.5. (page 6 of 6 )

\begin{tabular}{|c|c|c|c|c|c|c|c|c|}
\hline \multicolumn{9}{|c|}{ 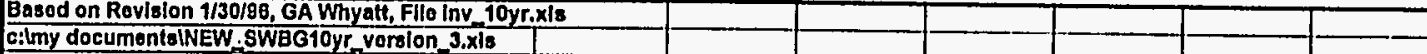 } \\
\hline \multicolumn{9}{|c|}{ Now_Futuro Sproadshoot summary for Key Nucildos } \\
\hline & $\mathrm{Cm} \cdot 246$ & & & & & & & \\
\hline & 6.92E-13 & C.14 & C.14 & C1.3B & S0.79 & TC-99 & 1.128 & U.238 \\
\hline & & & 100 Aroas & & & & & \\
\hline Facllity Identifler & Cl & Cl & CI. & CI & CI & $\mathrm{Cl}$ & CI & CI \\
\hline \multicolumn{9}{|l|}{200 East Area } \\
\hline 21801 & $0.00 E+\infty 0$ & $0.00 E+\infty$ & $0.00 E+00$ & $0.00 E+00$ & $0,00 E+\infty 0$ & $0.00 E+00$ & $0.00 E+00$ & \\
\hline 218010 & $0.00 E+00$ & $9.10 \mathrm{E}-02$ & $0.00 E+\infty$ & $5.84 \mathrm{E}-03$ & $7.25 E-03$ & $2.45 \mathrm{E}-01$ & $\frac{.06 C T}{4.84 E-04}$ & $\frac{0.00 E+\infty 0}{0.00 E+00}$ \\
\hline $218012 \theta$ & $0.00 E+00$ & $0.00 E+00$ & $0.00 E+00$ & $0.00 E+00$ & $0.00 E+00$ & $0.00 E+00$ & $0.00 E+00$ & $0.00 \mathrm{E}+00$ \\
\hline $218012 \mathrm{~b}$ & $1.38 \mathrm{E}-10$ & $1.36 E-02$ & $0.00 \mathrm{E}+00$ & $8.91 E-04$ & 1.09E-03 & $3.6 \mathrm{BE}-02$ & $3.25 \mathrm{E}-02$ & $6.68 E-02$ \\
\hline 218014 (purex (unnellin)(a) & $0.00 E+00$ & $0.00 E+00(\theta)$ & $0.00 E+00(a)$ & $0.00 \mathrm{E}+00(\mathrm{a})$ & $0.00 E+00(a)$ & $0.00 E+00(8)$ & $0.00 E+00(\mathrm{a})$ & $0.00 \mathrm{E}+00(\mathrm{a})$ \\
\hline 218015 (purex funnel\#2)(a) & $0.00 E+00$ & $0.00 \mathrm{E}+00(\mathrm{a})$ & $0.00 E+00(a)$ & $0.00 E+00(a)$ & $0.00 \mathrm{E}+00(\mathrm{a})$ & $0.00 E+00(a)$ & $0.00 E+00(a)$ & $0.00 \mathrm{E}+00(\mathrm{a})$ \\
\hline 21802 & $0.00 E+00$ & $0.00 \mathrm{E}+00$ & $0.00 E+00$ & $0.00 E+00$ & $0.00 E+00$ & $0.00 E+00$ & $0.00 \mathrm{E}+00$ & $0.00 \mathrm{E}+00$ \\
\hline 21804 & $0.00 E+00$ & $0.00 E+00$ & $0.00 E+\infty$ & $0.00 E+00$ & $0.00 \mathrm{E}+00$ & $0.00 \mathrm{E}+00$ & $0.00 E+\infty$ & $0.00 \mathrm{E}+00$ \\
\hline 21805 & $0.00 \mathrm{E}+00$ & $0.00 E+00$ & $0.00 E+00$ & $0.00 E+00$ & $0.00 \mathrm{E}+00$ & $0.00 E+00$ & $0.00 E+00$ & $0.00 \mathrm{E}+00$ \\
\hline $21805 \mathrm{a}$ & $0.00 E+\infty 0$ & $0.00 E+00$ & $0.00 \mathrm{E}+00$ & $0.00 E+00$ & $0.00 E+\infty 0$ & $0.00 E+00$ & $0.00 E+00$ & $0.00 E+00$ \\
\hline 21808 & $0.00 \mathrm{E}+0.0$ & $0.00 E+00$ & $0.00 E+00$ & $0.00 E+00$ & $0.00 \mathrm{E}+\infty$ & $0.00 E+00$ & $0.00 E+00$ & $0.00 E+00$ \\
\hline 222 b vaults & $0.00 E+00$ & $0.00 E+00$ & $0.00 E+00$ & $0.00 E+00$ & $0.00 \mathrm{E}+00$ & $0.00 E+00$ & $0.00 E+00$ & $0.00 \mathrm{E}+00$ \\
\hline 218000 & $0.00 E+\infty 0$ & 1.81E-03 & $0.00 E+00$ & $1.18 E-04$ & $1.44 E-04$ & $4.88 E-03$ & $8.62 E-06$ & $0.00 \mathrm{E}+\infty$ \\
\hline C-14 subtotals & $0.00 \mathrm{E}+00$ & $1.06 \mathrm{E}-01$ & $0.00 \mathrm{E}+00$ & $0.00 E+00$ & $0.00 E+00$ & $0.00 E+00$ & $0.00 E+00$ & $0.00 \mathrm{E}+00$ \\
\hline tolal East area(b) & $1.38 E-10$ & $1.08 \mathrm{E}-01(\mathrm{~b})$ & $0.00 E+00(b)$ & $8.95 E-03(\mathrm{~b})$ & $8.48 \mathrm{E}-03(\mathrm{~b})$ & $2.87 \mathrm{E}-01(\mathrm{~b})$ & $3.30 E-02(b)$ & $60-02(b)$ \\
\hline 200 Wost Area & $0.00 E+00$ & $0.00 \mathrm{E}+00$ & $0.00 E+00$ & $0.00 E+00$ & $0.00 \mathrm{E}+00$ & $0.00 E+00$ & $0.00 E+00$ & $0.00 E+00$ \\
\hline $218 W_{1}$ & $0.00 E+00$ & $0.00 E+00$ & $0.00 \mathrm{E}+00$ & $0.00 E+00$ & $0.00 E+00$ & $0.00 \mathrm{E}+00$ & $0.00 E+\infty 0$ & $0.00 \mathrm{E}+00$ \\
\hline $216 w 11$ & $0.00 E+00$ & $0.00 E+00$ & $0.00 \mathrm{E}+00$ & $0.00 E+00$ & $0.00 E+00$ & $0.00 E+00$ & $0.00 \mathrm{E}+\infty 0$ & $0.00 \mathrm{E}+00$ \\
\hline $218 \mathrm{W1A}$ & $0.00 E+00$ & $0.00 E+00$ & $0.00 \mathrm{E}+00$ & $0.00 E+\infty 0$ & $0.00 E+00$ & $0.00 \mathrm{E}+00$ & $0.00 \mathrm{E}+00$ & $0.00 E+00$ \\
\hline $218 w 2$ & $0.00 E+00$ & $0.00 E+00$ & $0.00 E+00$ & $0.00 E+00$ & $0.00 E+00$ & $0.00 E+00$ & $0.00 E+00$ & $0.00 \mathrm{E}+00$ \\
\hline $218+2 A$ & $0.00 E+00$ & $0.00 E+00$ & $0.00 E+\infty$ & $0.00 E+\infty$ & $0.00 E+\infty 0$ & $0.00 E+00$ & $0.00 E+00$ & $0.00 \mathrm{E}+00$ \\
\hline $218 \mathrm{W3}$ & $0.00 E+00$ & $0.00 \mathrm{E}+00$ & $0.00 E+00$ & $0.00 \mathrm{E}+00$ & $0.00 E+00$ & $0.00 \mathrm{E}+00$ & $0.00 \mathrm{E}+00$ & $0.00 E+00$ \\
\hline 218W3A & $3.30 E-11$ & $5.23 \mathrm{E}-01$ & $0.00 E+\infty$ & $3.42 E-02$ & $4.16 E-02$ & $2.27 E+00$ & $2.86 E-03$ & $4.23 E-01$ \\
\hline 218WBAE & $4.62 E-14$ & $8.70 E+00$ & $1.09 \mathrm{E}+02$ & $5.69 \mathrm{E}-01$ & $6.93 E-01$ & $2.81 E+01$ & $4.29 E-02$ & $1.87 E+02$ \\
\hline 210 WAA & $0.00 E+00$ & $0.00 E+\infty$ & $0.00 E+00$ & $0.00 \mathrm{E}+\infty 0$ & $0.00 E+00$ & $0.00 E+00$ & $0.00 \mathrm{E}+00$ & $0.00 \mathrm{E}+00$ \\
\hline 218 W4B-calisson & $0.00 \mathrm{E}+00$ & $4.48 \mathrm{E}-02$ & $0.00 E+00$ & $2,93 E-03$ & $3.57 E-03$ & $1.21 E-01$ & $2.38 E-04$ & $0.00 \mathrm{E}+00$ \\
\hline 218W4B-non calsson & $0.00 E+00$ & $0.00 E+00$ & $0.00 E+00$ & $0.00 E+00$ & $0.00 E+00$ & $0.00 E+00$ & $0.00 \mathrm{E}+00$ & $0.00 E+\infty 0$ \\
\hline $218 W 4 C$ & $7.82 E-09$ & $3.24 \mathrm{E}+00$ & $0.00 E+00$ & $7.39 E-03$ & $4.89 \mathrm{E}-02$ & $7.75 E+00$ & $8.85 E-03$ & $1.39 E+02$ \\
\hline 218 W5 & $3.71 E-08$ & $1.19 \mathrm{E}+00$ & $2.29 E-01$ & $3.99 \mathrm{E}-02$ & $4.87 \mathrm{E}-02$ & $2.17 E+00$ & $1.10 E-01$ & $1.98 \mathrm{EE}+01$ \\
\hline $218 \mathrm{wg}$ & $0.00 E+\infty$ & $0.00 E+00$ & $0.00 E+00$ & $0.00 E+\infty 0$ & $0.00 E+00$ & $0.00 \mathrm{E}+00$ & $0.00 E+00$ & $0.00 E+00$ \\
\hline 221 T. TPlant(a) & $0.00 E+00$ & $0.00 E+00(\mathrm{a})$ & $0.00 E+00(a)$ & $0.00 E+00(a)$ & $0.00 E+00(a)$ & $0.00 \mathrm{E}+00(\mathrm{a})$ & $0.00 E+00$ (a) & \\
\hline 2228 vaults & $0.00 E+00$ & $0.00 \mathrm{E}+00$ & $0.00 E+\infty$ & $0.00 E+00$ & $0.00 E+00$ & $0.00 E+00$ & $0.00 \mathrm{E}+00$ & $\frac{0.00 \mathrm{E}+00}{0}$ \\
\hline 222 T vaults & $0.00 E+00$ & $0.00 E+00$ & $0.00 \mathrm{E}+00$ & $0.00 E+00$ & $0.00 \mathrm{E}+00$ & $0.00 \mathrm{E}+00$ & $0.00 \mathrm{E}+00$ & $0.00 \mathrm{E}+\infty$ \\
\hline 241 T Facility(a) & $0.00 E+00$ & $0.00 \mathrm{E}+00(\mathrm{a})$ & $0.00 \mathrm{E}+00(0)$ & $0.00 E+00(a)$ & $0.00 \mathrm{E}+00(\mathrm{a})$ & $0.00 E+00(a)$ & $0.00 E+00(0)$ & $0.00 E+00(a)$ \\
\hline TRUSAF(a) & $0.00 E+00$ & $3.15 \mathrm{E}-06(0)$ & $0.00 \mathrm{E}+00(\mathrm{a})$ & $2.06 \mathrm{E}-07$ (a) & $2.51 E-07(0)$ & $8.49 \mathrm{E}-06(0)$ & $1.67 \mathrm{E}-08(\mathrm{a})$ & $0.00 \mathrm{E}+00(\mathrm{a})$ \\
\hline Zplant,PFP(a) & $0.00 E+\infty$ & $2.91 E-07(a)$ & $0.00 E+00(0)$ & $1.90 E-08(\theta)$ & $2.32 E-08(\mathrm{a})$ & $7.84 E-07(a)$ & $1.55 \mathrm{E}-09(\mathrm{a})$ & $0,00 E+00(a)$ \\
\hline con. wst cmplx buildings, numerous(a) & $9.23 E-10$ & $2.09 E+00(a)$ & $0.00 E+00(\theta)$ & $3.36 \mathrm{E}-03(\mathrm{a})$ & $4.10 \mathrm{E}-03(\mathrm{a})$ & $7.27 \mathrm{E}+00(\mathrm{a})$ & $6.65 \mathrm{E}-03(\mathrm{a})$ & $8.81 \mathrm{E}+00(\mathrm{a})$ \\
\hline $2706 \mathrm{~T}(\mathrm{a})$ & $0.00 E+00$ & $5.75 E-03(a)$ & $0.00 \mathrm{E}+00(\mathrm{a})$ & $3.75 E-04(8)$ & $4,58 E-04(a)$ & $8.51 E-02(a)$ & $5.06 \mathrm{E}-03(\mathrm{a})$ & $1.35 \mathrm{E}-05(\mathrm{a})$ \\
\hline Ak Molal Waste Slorago units 1,2,3,4(e) & $0.00 E+00$ & \begin{tabular}{|l|}
$.78 \mathrm{E}-05(\mathrm{a})$ \\
\end{tabular} & $0.00 E+00(a)$ & $1.16 \mathrm{E}-06(\mathrm{\theta})$ & $1.42 E .06(\mathrm{a})$ & $4.80 E-05(a)$ & $9.48 \mathrm{E}-08(\mathrm{a})$ & $0.00 \mathrm{E}+00(\mathrm{a})$ \\
\hline Flammable sloraga units 1 through 20(a) & 1.94E-15 & $2.68 \mathrm{E}-01(0)$ & $0.00 E+00(a)$ & $1.36 E-05(0)$ & $1.66 \mathrm{E}-05(0)$ & $4.80 E-02(a)$ & \begin{tabular}{|l|l|}
$3.64 E-02(a)$ \\
\end{tabular} & $3.18 E-04(\mathrm{a})$ \\
\hline G.14 sublotols & $0.00 E+00$ & $1.37 E+01$ & \begin{tabular}{|l|}
$1.09 E+02$ \\
\end{tabular} & & & & & \\
\hline West area lotal(b) & 4.59E-08 & $1.23 \mathrm{E}+02(\mathrm{~b})$ & & $8.53 \mathrm{E}-01(\mathrm{~b})$ & $8.36 \mathrm{E}-01(\mathrm{~b})$ & $4.04 \mathrm{E}+01(\mathrm{~b})$ & $1.65 \mathrm{E}-01(\mathrm{~b})$ & $3.46 E+02(b)$ \\
\hline lotal of East end Wost Aroas in SWOG & & $1.23 E+02$ & & $6.60 \mathrm{E}-01$ & $8.45 E-01$ & $4.07 E+01$ & 1.98E-01 & $3.46 \mathrm{E}+02$ \\
\hline \multirow{2}{*}{\multicolumn{9}{|c|}{$\begin{array}{l}\text { nolos: } \\
\text { (a) Indicales faclitles nol modeled boc }\end{array}$}} \\
\hline & & & & & & & & \\
\hline & & & & & & & & \\
\hline
\end{tabular}


Table A.6. Solid Waste Inventory for "new _pre_88" Category (page 1 of 6)

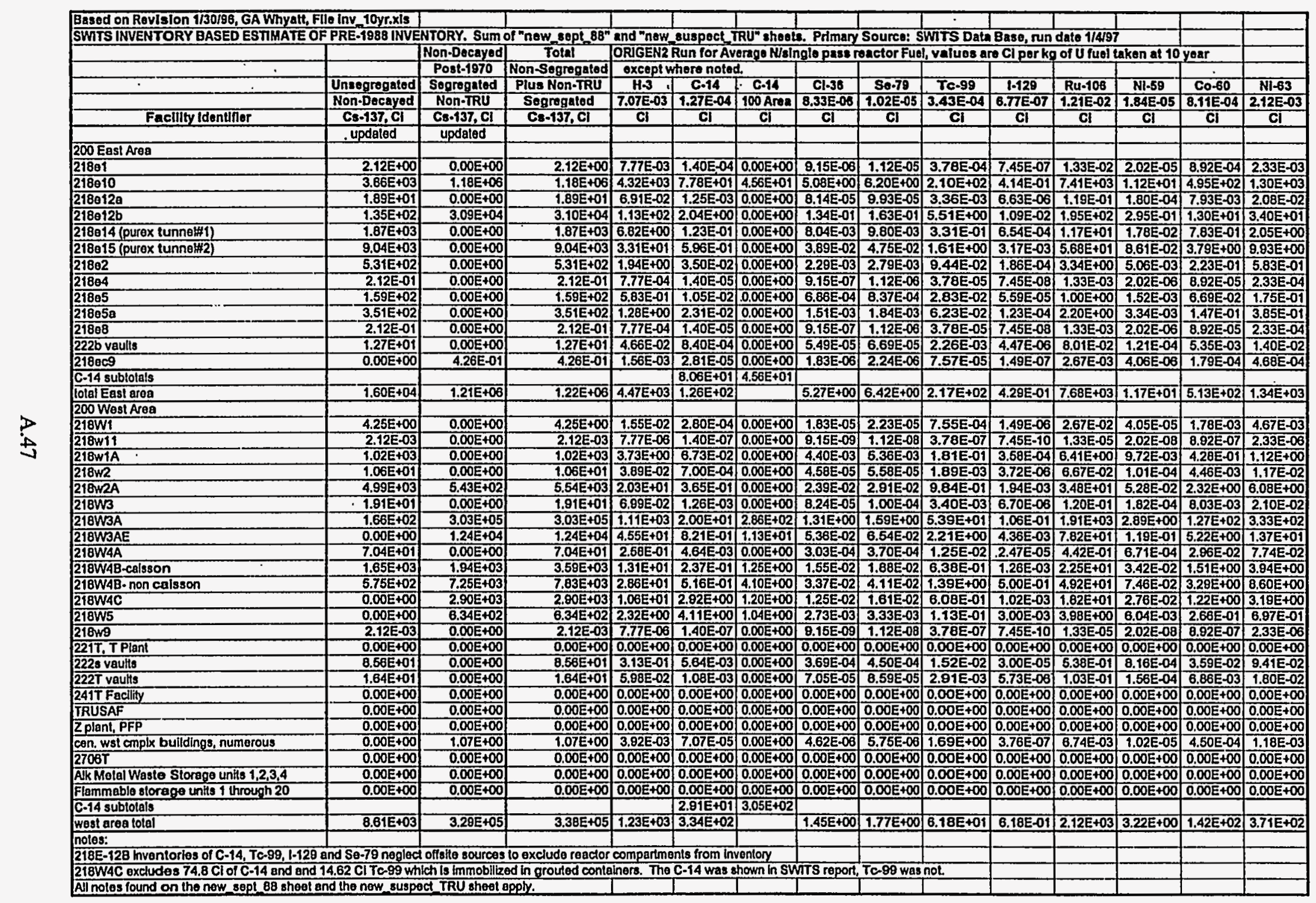


Table A.6. (page 2 of 6)

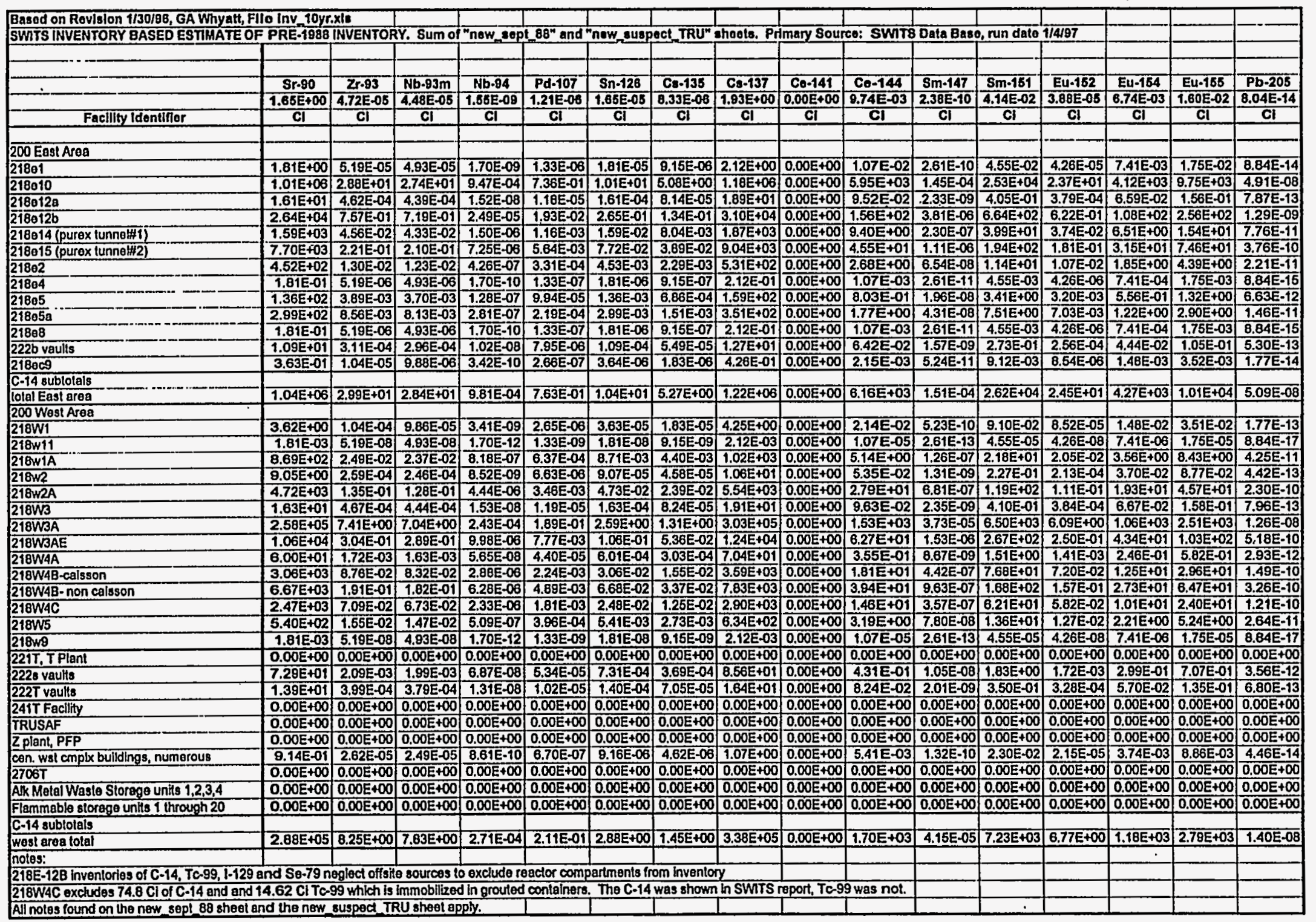




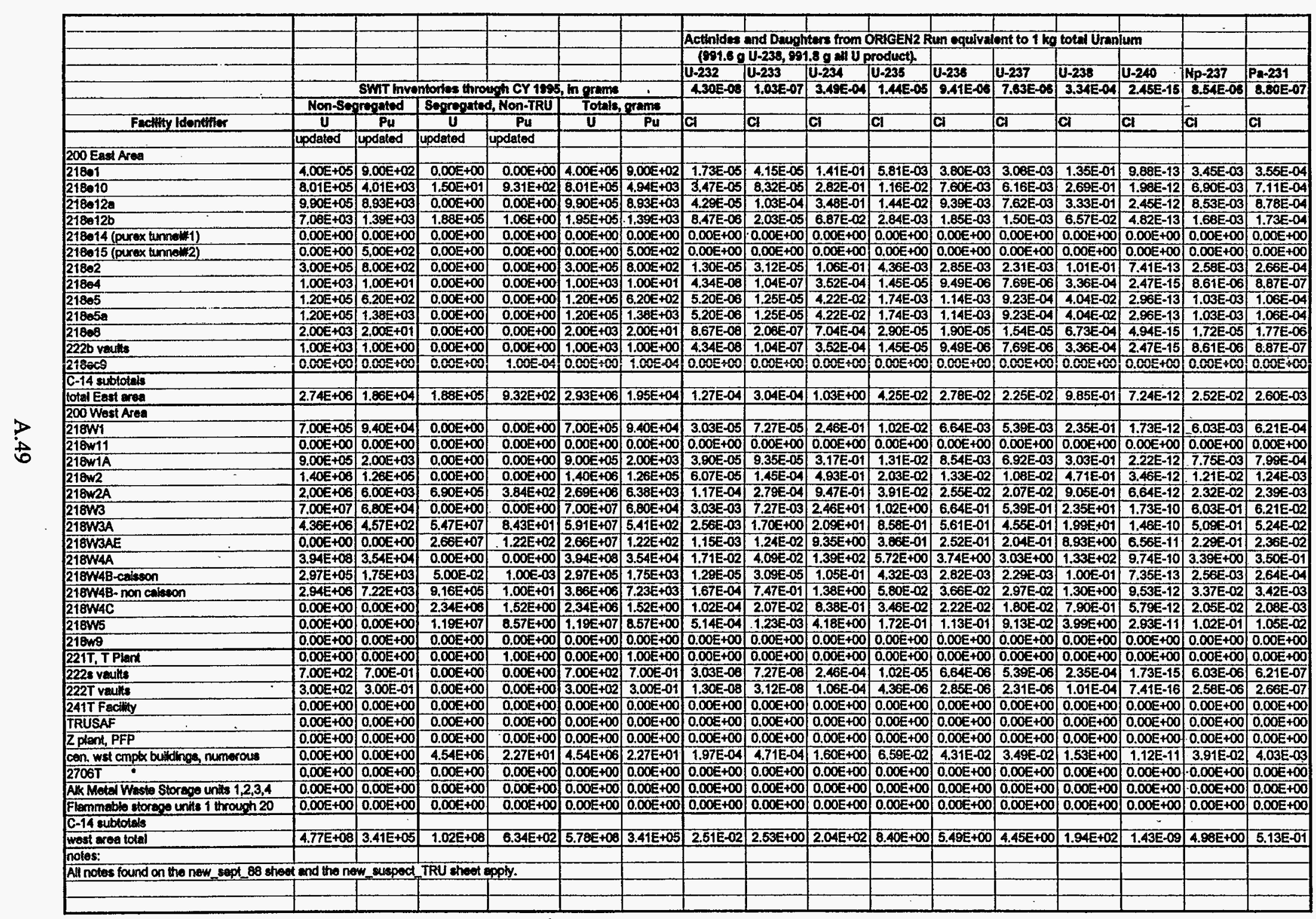




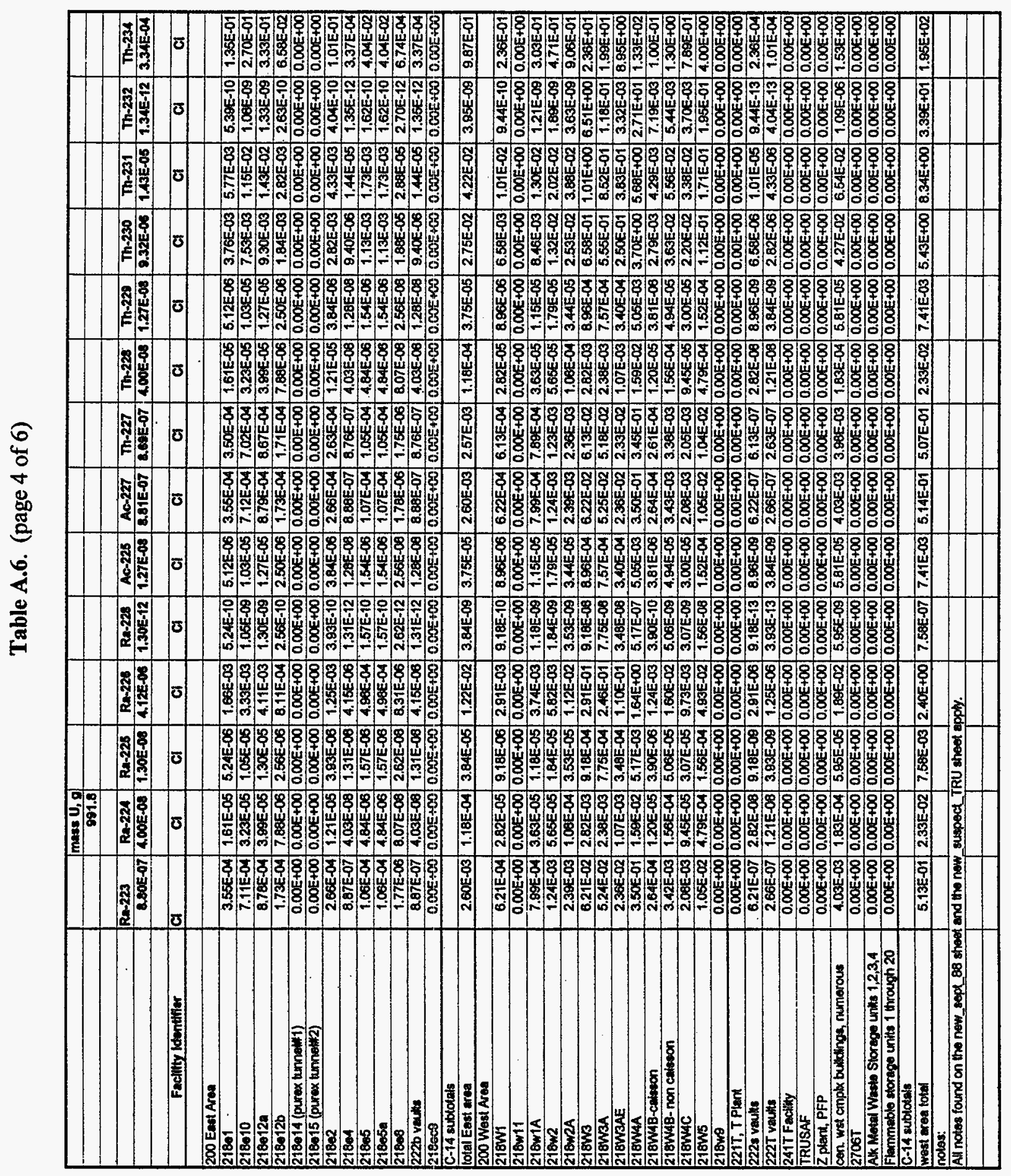

A. 50 
Table A.6. (page 5 of 6 )

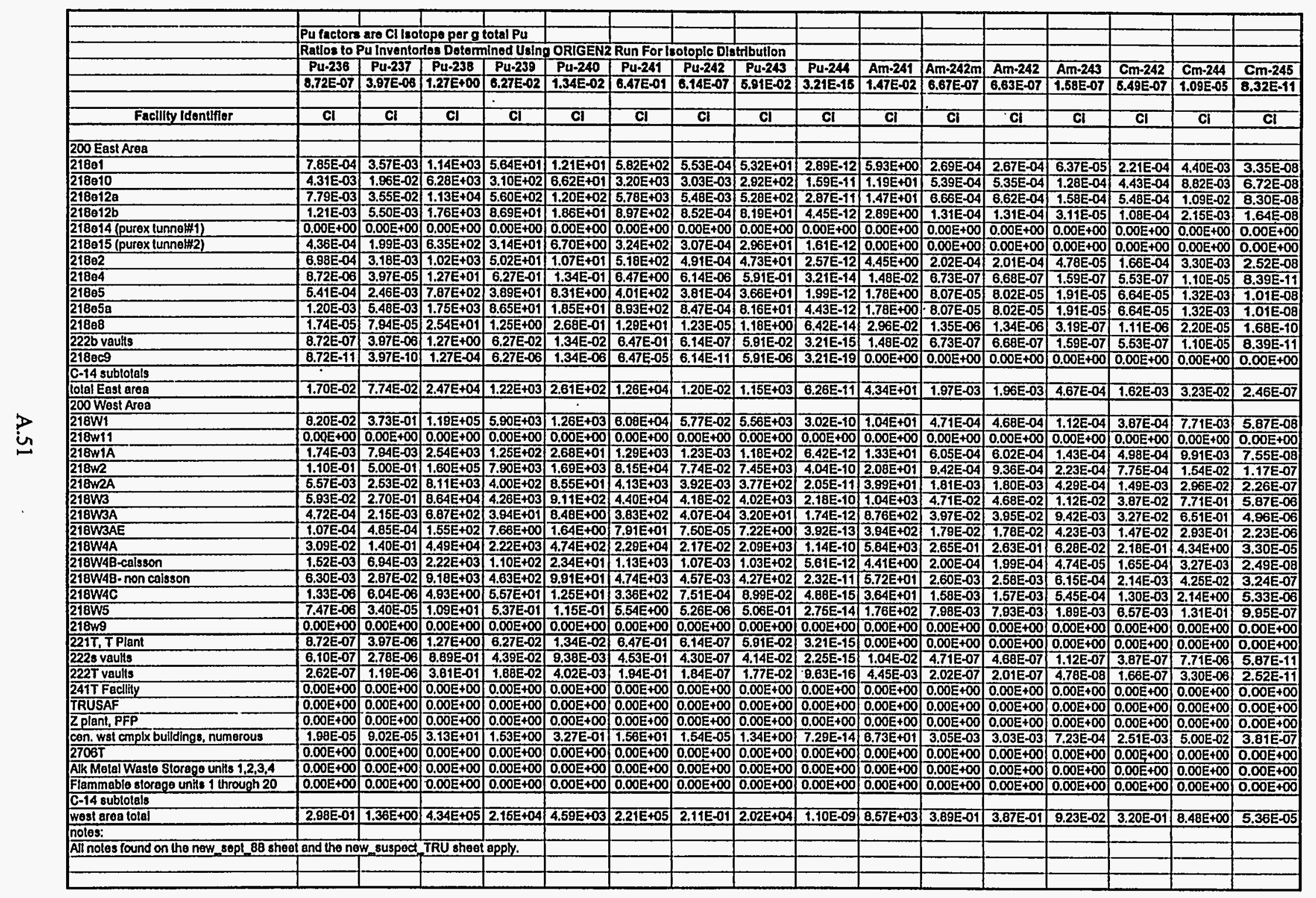


Table A.6. (page 6 of 6)

\begin{tabular}{|c|c|c|c|c|c|c|c|c|}
\hline \multicolumn{9}{|c|}{ Based on Rovision 1/30/86, GA Whyaft, Fllo inv 10yr.x/s } \\
\hline \multicolumn{9}{|c|}{ Colmy documontalNEW SWBG10yt verolon $3 . \times 18$} \\
\hline \multicolumn{9}{|c|}{ Now Pre 1980 Sproadoheot summary for Koy Nuclldos } \\
\hline & $\mathrm{Cm}-246$ & & & & & & & \\
\hline & $6.92 \mathrm{E}-13$ & C.14 & 0.14 & C1.3B & 80.79 & Tc-99 & 1.129 & $\mathbf{U} \cdot 238$ \\
\hline & & & 100Aroa & & & & & \\
\hline Faclity loentlifor & CI & CI & Ci & CI & Ci & Ci & C) & C) \\
\hline \multirow{2}{*}{\multicolumn{9}{|c|}{200 East Area }} \\
\hline & $2.79 E-10$ & $1.40 E-04$ & $0.00 \mathrm{E}+00$ & $9.15 E-06$ & 1.12E-05 & $3.78 E-04$ & $7.45 E-07$ & \\
\hline$\frac{27801}{218010}$ & $5.59 E-10$ & $7.7 \overline{8 E+01}$ & $4.56 \mathrm{E}+01$ & $5.08 E+00$ & $8.20 E+00$ & $2.10 E+02$ & $4.14 E-01$ & $2.69 E-01$ \\
\hline 2180120 & $6.90 \mathrm{E}-10$ & $1.25 E-03$ & $0.00 E+00$ & $8.14 E-05$ & $9.03 \mathrm{E}-05$ & $3.36 E-03$ & $6.63 E-06$ & $3.33 \mathrm{E}-01$ \\
\hline$\frac{1106 \mathrm{ac}}{218 \mathrm{e} 12 \mathrm{~b}}$ & $1.36 \mathrm{E}-10$ & $2.04 E+00$ & $0.00 E+00$ & 1.34E-01 & $1.63 \mathrm{E}-01$ & $5.51 E+00$ & $1.09 E-02$ & $6.57 \mathrm{E}-02$ \\
\hline 218014 (purex turnelli)(a) & $0.00 \mathrm{E}+00$ & $1.23 \mathrm{E}-01(\mathrm{a})$ & $0.00 \mathrm{E}+00(\mathrm{a})$ & $8.04 \mathrm{E}-03(\mathrm{a})$ & $8.80 E-03(a)$ & $3.31 \mathrm{E} .01(\mathrm{a})$ & $6.54 \mathrm{E}-04(\mathrm{\theta})$ & $0.00 \mathrm{E}+00(\mathrm{a})$ \\
\hline 218015 (purex (unne'ti2)(a) & $0.00 E+00$ & $5.96 \mathrm{E}-01(\mathrm{\theta})$ & $0.00 E+00(a)$ & $3.89 \mathrm{E}-02(\mathrm{a})$ & 4.75E-02(a) & $1.16 E-00$ (1) & $3.17 \mathrm{E}-03(a)$ & $0,00 E+00(a)$ \\
\hline$\frac{11802}{218}$ & $2.09 \mathrm{E}-10$ & $3.50 \mathrm{E}-02$ & $0.00 \mathrm{E}+00$ & $2.29 E-03$ & 2.79E-03 & $9.44 E-02$ & $1.86 \mathrm{E}-04$ & 1.01E-01 \\
\hline$\frac{10064}{21804}$ & $6.97 E-13$ & $1.40 \mathrm{E}-05$ & $0.00 E+00$ & $9.15 E-07$ & $1.12 E-06$ & $3.78 E-05$ & $7.45 \mathrm{E}-08$ & $3.36 E-04$ \\
\hline 21805 & $8.37 \mathrm{E}-11$ & $1.05 E-02$ & $0.00 E+00$ & $6.86 \mathrm{E}-04$ & $8.37 E-04$ & $2.83 E-02$ & $5.59 \mathrm{E} .05$ & $4.04 E-02$ \\
\hline $21805 \mathrm{a}$ & $8.37 E-11$ & $2.31 E .02$ & $0.00 E+00$ & $1.51 \mathrm{E}-03$ & $9.84 E-03$ & $6.23 \mathrm{E}-02$ & $1.23 \mathrm{E}-04$ & $4.04 \mathrm{E}-02$ \\
\hline$\frac{10800}{21808}$ & $1.39 E-12$ & $1.40 E-05$ & $0.00 E+00$ & $9,15 E-07$ & $1.12 E-06$ & $3.78 E-05$ & $7.45 E-08$ & $\frac{4.04 E-02}{6.73 E-04}$ \\
\hline 27808 & & & & $\frac{9100}{6-105015}$ & 669505 & 236503 & DA7F-n & \\
\hline 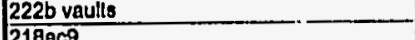 & 6.97E-13 & $8.40 E-04$ & $0.00 E+00$ & $5.49 \mathrm{E}-05$ & $6.69=0.05$ & 2.2050 .03 & 4.47E-06 & $3.36 \mathrm{E}-04$ \\
\hline 218009 & $0.00 E+00$ & 2.81E-05 & $0.00 E+00$ & 1.83E-06 & $2.24 E-06$ & $7.57 E-05$ & $1.49 \mathrm{E}-07$ & $0.00 \mathrm{E}+00$ \\
\hline C-14 sublotals & & $7.99 \mathrm{E}+01$ & $-4.56 E+01$ & & & & & \\
\hline total East area(b) & $2.04 E-09$ & $1.26 E+02(b)$ & & $5.22 \mathrm{E}+00(\mathrm{~b})$ & $6.37 \mathrm{E}+00(\mathrm{~b})$ & $2.15 E+02(b)$ & $4.25 \mathrm{E}-01(\mathrm{~b})$ & $9.85 E-01(b)$ \\
\hline \multicolumn{9}{|l|}{200 West Area } \\
\hline 218W1 & $4.88 E-10$ & $2.80 E-04$ & $0.00 E+00$ & 1.83E-05 & 2.23E-05 & $7.55 E-04$ & $1.49 \mathrm{E}-06$ & $2.35 E-01$ \\
\hline $218 w 11$ & $0.00 E+00$ & $1.40 E-07$ & $0.00 E+00$ & $9.15 E-09$ & 1.12E-08 & $3.78 E-07$ & $7.45 E-10$ & $0.00 E+00$ \\
\hline $218 w 1 A$ & $6.28 \mathrm{E}-10$ & $6.73 E-02$ & $0.00 \mathrm{E}+\infty 0$ & $.4 .40 \mathrm{E}-03$ & $5.36 \mathrm{E}-03$ & 1.81E-01 & $3.58 \mathrm{E}-04$ & $3,03 \mathrm{E}-01$ \\
\hline \multicolumn{9}{|c|}{$9.76 \mathrm{E}-10$} \\
\hline \multirow{2}{*}{\multicolumn{9}{|c|}{$\frac{1.88 E-09}{4.88 E-0.8}$}} \\
\hline & & & & & & & & \\
\hline \multirow{2}{*}{\multicolumn{9}{|c|}{$4.12 E-08$}} \\
\hline \multirow{2}{*}{\multicolumn{9}{|c|}{$218 \mathrm{WBAE}$}} \\
\hline \multirow{2}{*}{\multicolumn{7}{|c|}{$218 W 4 A$}} & & \\
\hline \multirow{2}{*}{\multicolumn{9}{|c|}{ 218W4B-calsson }} \\
\hline & & & & & & & & \\
\hline \multicolumn{4}{|l|}{ 218W4B-non caisson } & & & & & \\
\hline 21865 & $8.27 E-09$ & $4.11 E+\infty$ & $1.04 \mathrm{E}+\infty 0$ & $2.73 \mathrm{E}-03$ & $3.33 \mathrm{E}-03$ & $1.13 E-01$ & $3,00 E-03$ & \\
\hline \multicolumn{9}{|c|}{\begin{tabular}{|l|l|}
$1.40 \mathrm{E}-07$ & $0.00 \mathrm{E}+00$ \\
\end{tabular}} \\
\hline \multicolumn{9}{|c|}{$0.00 E+00 \mid 0.00 E+00(a)[0.00 E+00(a)] 0.00 E+00(a)[0.00 E+00(a)][0.00 E+00(a)] 0.00 E+00(a)] 0.00 E+00(a)$} \\
\hline 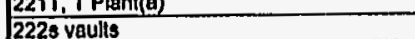 & $4.08 \mathrm{E}-13$ & $\frac{0.00 \mathrm{LT}, \mathrm{a}}{5.64 \mathrm{E}-03}$ & $0.00 \mathrm{E}+00$ & 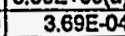 & $4.50 \mathrm{E}-04$ & $1.52 \mathrm{E} \cdot 02$ & $3.00 \mathrm{E}-05$ & $2.35 \mathrm{E}-04$ \\
\hline $222 T$ vauts & $2.09 E-13$ & $1.00 E .03$ & $0.00 E+00$ & $7.05 \mathrm{E}-05$ & $8.59 E-05$ & 2.91E-03 & $5.73 E-06$ & $1.01 \mathrm{E} .04$ \\
\hline 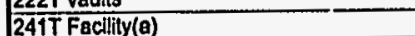 & $0.00 E+00$ & $0.00 \mathrm{E}+00(\mathrm{\theta})$ & $0.00 E+00(a)$ & $0.00 E+00(\mathrm{a}$ & $0.00 \mathrm{E}+00(\mathrm{a})$ & $0.00 E+00(a)$ & $0.00 E+00(a)$ & $0.00 E+00(0)$ \\
\hline TRUSAF(a) & $0.00 E+00$ & $0.00 \mathrm{E}+00(\mathrm{\theta})$ & $0.00 E+00(a)$ & $0.00 E+00(a)$ & $0.00 \mathrm{E}+00(\mathrm{a})$ & $0.00 E+001 a$ & $0.00 E+00(a)$ & $0.00 \mathrm{E}+00(\mathrm{\theta})$ \\
\hline Zplant, PFP(B) & $0.00 E+00$ & $0.00 E+00(0)$ & $0.00 E+00(\theta)$ & $0.00 \mathrm{E}+00(\mathrm{a}$ & $0.00 \mathrm{E}+00(\mathrm{a})$ & $0.00 E+00 / 0$ & $0.00 E+00(a)$ & $0.00 E+00(a)$ \\
\hline cen. wst cmplx butldings, numerous(a) & 3.17E-09 & $7.07 E-05(a)$ & $0.00 E+00(8)$ & 4.62E-06) & $5.75 E-06(0)$ & $1.69 E+\infty 00$ & & $1.53 E+00(\mathrm{a})$ \\
\hline $2706 \mathrm{~T}(\theta)$ & $0.00 \mathrm{E}+\infty$ & $0.00 \mathrm{E}+00(\mathrm{a})$ & $0.00 \mathrm{E}+00(8)$ & $0.00 \mathrm{E}+00$ & $0.00 E+00(\theta)$ & $0.00 \mathrm{E}+00 \mathrm{a}$ & $0.00 \mathrm{E}+00(\mathrm{a})$ & $0.00 E+00(\theta)$ \\
\hline Alk Melal Wasto Storago unlis 1,2,3,4(0) & $0.00 E+00$ & $0.00 \mathrm{E}+00(\mathrm{a})$ & $0.00 E+C O(a)$ & $0.00 E+00$ & $0.00 E+00(0)$ & $0.00 E+00(a)$ & $0.00 E+00(a)$ & $0.00 E+00(a)$ \\
\hline 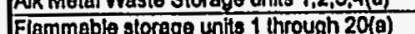 & $0.00 E+\infty$ & $0.00 \mathrm{E}+00(0)$ & $0.00 E+00(a)$ & $0.00 \mathrm{E}+00 \mathrm{Oa}$ & $0,00 E+00(\theta)$ & $0.00 E+00(a)$ & $0.00 E+000$ & $0.00 \mathrm{E}+00(\mathrm{a})$ \\
\hline C-14 sublotals & & $2.91 E+01$ & $3.05 E+02$ & & & & & \\
\hline wast area total(b) & 4.03E-07 & $3.34 E+02(b)$ & & $1.45 \mathrm{E}+00(\mathrm{D}$ & $1.77 E+00(b)$ & $6.01 E+01(\mathrm{~b})$ & $6.18 \mathrm{E}-01(\mathrm{~b})$ & $1.03 E+02(b)$ \\
\hline Total East and Wost & & $4.60 \mathrm{E}+02$ & & $6.68 \mathrm{E}+\infty$ & $\begin{array}{r}1.77 E+00 \\
\end{array}$ & 1. $2.75 E+02$ & $1.04 E+\infty$ & $1.94 E+02$ \\
\hline notos: & & & & & & & & \\
\hline I(a) Faciltities nol & & thaniordS & oin & entories & & & & \\
\hline (b) Asea totals. & & & & & & & & \\
\hline
\end{tabular}


Table A.7. Composition of Hanford Reactor Fuel and Cladding Used in ORIGEN2 Calculation

\begin{tabular}{|c|c|c|}
\hline Isotope & N-Reactor Fuel, g/kg U & Single-Pass Reactor Fuel, g/kg U \\
\hline${ }^{234} U$ & 0.077 & 0.055 \\
\hline${ }^{235} \mathrm{U}$ & 9.47 & 7.11 \\
\hline${ }^{238} \mathrm{U}$ & 990.45 & 992.84 \\
\hline Element & N-Reactor Fuel Impurities, g/kg U & Single-Pass Reactor Fuel Impurities, g/kg U \\
\hline $\mathrm{Al}$ & 0.900 & \\
\hline $\mathrm{Be}$ & 0.010 & \\
\hline B & 0.00025 & \\
\hline $\mathrm{Cd}$ & 0.00025 & \\
\hline $\mathrm{C}$ & 0.735 & 0.750 \\
\hline $\mathrm{Cr}$ & 0.065 & 0.065 \\
\hline $\mathrm{Cu}$ & 0.075 & \\
\hline $\mathrm{H}$ & 0.002 & \\
\hline $\mathrm{Fe}$ & 0.400 & 0.150 \\
\hline $\mathrm{Mg}$ & 0.025 & 0.025 \\
\hline $\mathrm{Mn}$ & 0.025 & 0.025 \\
\hline $\mathrm{Ni}$ & 0.100 & 0.100 \\
\hline $\mathbf{N}$ & 0.075 & 0.100 \\
\hline $\mathrm{Si}$ & 0.124 & 0.075 \\
\hline $\mathrm{Zr}$ & 0.065 & \\
\hline & N-Reactor Cladding, g/kg Zr & Single-Pass Reactor Cladding, g/kg Al \\
\hline $\mathrm{Al}$ & $0: 075$ & 960.3 \\
\hline $\mathrm{Be}$ & 0.390 & \\
\hline B & 0.0005 & 0.010 \\
\hline $\mathrm{Cd}$ & 0.0005 & 0.030 \\
\hline $\mathrm{C}$ & 0.275 & \\
\hline $\mathrm{Cr}$ & 1.5 & \\
\hline Co & 0.010 & 0.010 \\
\hline
\end{tabular}


Table A.7. (contd)

\begin{tabular}{|l|l|l|}
\hline $\mathrm{Cu}$ & 0.050 & 1.50 \\
\hline $\mathrm{Hf}$ & 0.200 & \\
\hline $\mathrm{H}$ & 0.025 & \\
\hline $\mathrm{Fe}$ & 2.0 & 7.0 \\
\hline $\mathrm{Pb}$ & 0.100 & \\
\hline $\mathrm{Li}$ & & 0.080 \\
\hline $\mathrm{Mg}$ & 0.020 & \\
\hline $\mathrm{Mn}$ & 0.050 & \\
\hline $\mathrm{Mo}$ & 0.050 & \\
\hline $\mathrm{Ni}$ & 0.8 & 13.0 \\
\hline $\mathrm{N}$ & 0.080 & \\
\hline $\mathrm{O}$ & 0.018 & \\
\hline $\mathrm{Si}$ & 0.100 & 18.1 \\
\hline $\mathrm{Na}$ & 0.020 & \\
\hline $\mathrm{Sn}$ & 17.0 & \\
\hline $\mathrm{Ti}$ & 0.050 & \\
\hline $\mathrm{W}$ & 0.050 & \\
\hline $\mathrm{V}$ & 0.050 & \\
\hline $\mathrm{Zr}$ & 977.1 & \\
\hline
\end{tabular}


Table A.8. Comparison of Post-88 Inventory to 200 East Area Performance Assessment

\begin{tabular}{|l|l|l|l|}
\hline & \multicolumn{3}{|c|}{ Post-September 1988 Inventory, Ci ${ }^{\text {(a) }}$} \\
\cline { 2 - 4 } & $\begin{array}{c}\text { East Area PA, } \\
\text { Wood et al. } \\
(1996)\end{array}$ & $\begin{array}{c}1^{\text {st }} \text { Projection Based } \\
\text { on Fuel Aged 10 } \\
\text { Years and Erroneous } \\
\text { "post_sept88" Table }\end{array}$ & $\begin{array}{c}2^{\text {nd }} \text { Projection Based } \\
\text { on Fuel Aged 10 } \\
\text { Years and Corrected } \\
\text { "new_96_88” Table }\end{array}$ \\
\hline Technetium-99 & 0.13 & 0.366 & 0.0287 \\
\hline Iodine-129 & 0.0002 & 0.0421 & 0.0330 \\
\hline Carbon-14 & $1.3 \mathrm{E}-06$ & $0.136^{(\mathrm{b})}$ & $0.106^{(\mathrm{b})}$ \\
\hline Selenium-79 & 0.002 & 0.0108 & 0.00848 \\
\hline Uranium-238 & $0.0183^{(\mathrm{c})}$ & 0.0851 & 0.0668 \\
\hline
\end{tabular}

(a) Excludes reactor compartment waste.

(b) Excludes activated metal and wastes from 100 Areas that show characteristics of activated metal.

(c) Wood et al. (1996) reported 54,500 grams uranium. If all were uranium-238 at a specific activity of $3.36 \mathrm{E}-07 \mathrm{Ci} / \mathrm{g}$, then this represents $1.83 \mathrm{E}-02 \mathrm{Ci}$.

$\mathrm{PA}=$ Performance assessment

Table A.9. Comparison of Post-88 Inventory to 200 West Area Performance Assessment

\begin{tabular}{|l|l|l|l|}
\hline & \multicolumn{3}{|c|}{ Post-September 1988 Inventory, Ci } \\
\cline { 2 - 4 } & $\begin{array}{c}\text { West Area PA, } \\
\text { Wood et al. } \\
(1995)\end{array}$ & $\begin{array}{c}\mathbf{1}^{\text {st }} \text { projection based on } \\
\text { fuel aged 10 years and } \\
\text { erroneous } \\
\text { "post_sept88" table }\end{array}$ & $\begin{array}{c}\mathbf{2}^{\text {nd }} \text { projection based } \\
\text { on fuel aged 10 years } \\
\text { and corrected } \\
\text { "new_96_88" table }\end{array}$ \\
\hline Technetium-99 & 1.6 & 51.5 & 40.4 \\
\hline Iodine-129 & 0.18 & 0.210 & 0.165 \\
\hline Carbon-14 & 5.2 & $17.5^{(0)}$ & $13.7^{\text {(v) }}$ \\
\hline Selenium-79 & (c) & 1.07 & 0.836 \\
\hline Uranium-238 & 20 & 22.86 & 346 \\
\hline
\end{tabular}

(a) Excludes graphite cores of the production reactors.

(b) Excludes activated metal and wastes from 100 Areas which show characteristics of activated metal.

(c) Not incluced.

PA $=$ Performance assessment 


\section{Appendix B}

\section{Environmental Restoration Waste Site Inventories}




\section{Appendix B}

\section{Environmental Restoration Waste Site Inventories}

Appendix B is a spreadsheet obtained from the Environmental Restoration Contractor (ERC),

Bechtel Hanford, Inc. This appendix comprises six tables that present current inventory data on 444 sites at Hanford.

The inventory data available are not complete for all sites. A summary of available inventory information for radionuclides is provided in each of the tables as follows:

- Table B.1 - cesium-137, strontium-90, ruthenium-106, and total plutonium

- Table B.2 - plutonium-238, $-239,-240$, and -241

- Table B.3 - plutonium-242, total uranium, gross uranium, and uranium-235

- Table B.4 - uranium-238, alpha emitters, beta emitters, and americium-241

- Table B.5 - tritium, cobalt-60, carbon-14, and europium-154

- Table B.6 - promethium-147, tin (Sn-113), and iodine-129. 
Table B.1. Environmental Restoration Waste Site Inventories for Cesium-137, Strontium-90, Ruthenium-106, and Total Plutonium (page 1 of 14)

\begin{tabular}{|c|c|c|c|c|c|c|c|c|}
\hline Sitto Codo & $\begin{array}{c}\text { Wasto Managoment } \\
\text { Unit Type }\end{array}$ & Wasto Managomen£ Unit & Waate Typo & $\left|\begin{array}{c}\text { Operable } \\
\text { Unit }\end{array}\right|$ & \begin{tabular}{|c|} 
Ceslum-137, \\
Units (CI)
\end{tabular} & $\begin{array}{c}\text { Strontlum-90, } \\
\text { Unite (CI) }\end{array}$ & $\left|\begin{array}{c}\text { Ruthonlum-108, } \\
\text { Units (CI) }\end{array}\right|$ & $\begin{array}{l}\text { Total Plutonlum, Units (g, } \\
\text { unloss otherwise stated) }\end{array}$ \\
\hline & & $212-\mathrm{N}$ to $216-\mathrm{N}-1$ Pipeline & & & & & & \\
\hline & & 212-P Hazandous Wasto Staging Area & & & & & & \\
\hline & & 212-P lo 216-N-4 Plpelino & & & & & & \\
\hline & & 212-P Transformer Oil Tank & & & & & & \\
\hline & & 212-R to 216-N-6 Plpeline & & & & & & \\
\hline & & 241-C Waste Line Unplanned Release No. 1 & & & & & & \\
\hline & & 241-C Waste Line Unplanned Release No. 2 & & & & & & \\
\hline & & $241-2$ Diversion Box No. 1 & & & & & & \\
\hline & & 241-Z Diversion Box No. 2 & & & & & & \\
\hline & & Sanitary Crib & & & & & & \\
\hline 200-E BP & Burtal Stte & 200-E Bumling PHt & Debris & $P O-6$ & & & & \\
\hline 200.EPAP & Burial Sto & 200-E Ash Pit & & SS-1 & & & & \\
\hline 200-EPD & Ditches & 200 East Powerhouse Ditch & Cooling Water & so-1 & & & & \\
\hline $200-E-4$ & French Drain & Crttlcal Mass Laboratory Dry Woll North & Mlscellaneous Drainago & $50-1$ & & & & \\
\hline $200-N-3$ & Burial Stte & Ballast Pits & Dobris & NO-1 & & & & \\
\hline $200-W A D B$ & Burtal Site & 200-W Ash Disposal Basin & Ash & SS-2 & & & & \\
\hline 200-WADS & Burial Sito & 200-W Ash Pit Demolittion SHo & N/A & SS-2 & & & & \\
\hline $200-W B P$ & Burial Stto & 200-W Buming PHt & Debris & SS-2 & & & & \\
\hline 200-W PAP & Burial Stte & 200-W Powerhouse Ash PH & Ash & SS-2 & & & & \\
\hline 200-WPP & Ponds & 200-W Powerhouse Pond & Coolling Water & $\mathrm{TP}-2$ & & & & \\
\hline $201-C$ & Building & 201-C Process Building & Process Condensato & so-1 & & 8000 & & 68.3 \\
\hline $207-A$ & & $207-A$ & & & & & & \\
\hline $207 \cdot 8$ & Retention Basin & 207-Bb/Retention Basin & Cooling Water & $\mathrm{BP}-8$ & & & & \\
\hline $207-5$ & Rotention Basin & $207-5$ & Cooling Water & R0.2 & & & & \\
\hline 207. SL & Retention Basin & $207-S L$ & Lab Waste & RO-3 & & & & \\
\hline $207-T$ & Retention Basin & 207-T Retention Basin & Cooling Water & TP-3 & & & & \\
\hline $207-2$ & Retention Basin & 207-Z Rolention Basin & Steam Condensate & $2 \mathrm{PP}-2$ & & & & \\
\hline 209-E-WS-1 & French Drain & Crittcal Mass Laboratory Dry Woll East & Miscellaneous Drainago & so-1 & & & & \\
\hline 209-E-WS-2 & French Drain & Critical Mass Laboratory Dry Woll South & Miscellaneous Drainago & so-1 & & & & \\
\hline 200-E-WS-3 & Diveraton Box & Critlcal Mass Laboratory Vaivo Plt & Process Wasto & so-1 & & & & \\
\hline 2101-M POND & Ponds & 2101-M Pond & Lab Wasto & ss-1 & & & & \\
\hline $216-A-1$ & Cribs & 216-A-1 & Process Wasio & PO-5 & 0.0444 & 0.0422 & $2.75 \mathrm{E}-12$ & 0.1 \\
\hline $216-A-10$ & Cribs & $216-A-10$ & Process Condensate & $\overline{P O-2}$ & 80.5 & 82.5 & . 0.308 & 350 \\
\hline
\end{tabular}


Table B.1. (page 2 of 14)

\begin{tabular}{|c|c|c|c|c|c|c|c|c|}
\hline Site Couls & $\begin{array}{c}\text { Waste Mariggement } \\
\text { Unlt Type }\end{array}$ & Waste Management Unit. & Waste Type & $\underset{\substack{\text { Operable } \\
\text { Unit }}}{2}$ & $\begin{array}{l}\text { Coslum-137, } \\
\text { Units (Ci) }\end{array}$ & $\begin{array}{l}\text { Strontlum.90, } \\
\text { Units (Ci) }\end{array}$ & $\begin{array}{c}\text { Ruthenlum-106, } \\
\text { Units (C)) }\end{array}$ & $\begin{array}{l}\text { Total Plutonlum, Units (g, } \\
\text { unless otherwise stated) }\end{array}$ \\
\hline $216-A-11$ & French Drain & $216-A-11$ & Miscellaneous Drainage & PO-2 & & & & \\
\hline $216-A-12$ & French Drain & $216-\bar{A}-12$ & Miscellaneous Drainage & PO-2 & & & & \\
\hline 216-A-13 & French Drain & $216-A-13$ & Mlsceilaneous Drainago & $\mathrm{PO}-2$ & & & & \\
\hline $216-A-14$ & French Drain & $\longdiv { 2 1 6 - A - 1 4 }$ & Miscellaneous Drainage & $\overline{P 0-2}$ & & & & \\
\hline $216-A-15$ & French Drain & $216-A-15$ & Process Condensale & $\overline{\mathrm{PO}}-2$ & & & & \\
\hline $216-A-16$ & French Drain & $\overline{216-A-16}$ & Chemical Sewer & PO.5 & & & & \\
\hline 216-A-17 & French Drain & 216-A-17 & Chemical Sewer & PO-5 & & & & \\
\hline 216-A-18 & Trench & $2 \overline{216-A-18}$ & Process Waste & $\overline{P O-5}$ & 0.0444 & 0.042 & 2.75E-12 & $\overline{0.1}$ \\
\hline 216-A-19 & Trench & 216-A-19 & Process Wasto & $\overline{P 0.5}$ & 0.0444 & 0.042 & $2.75 \mathrm{E}-12$ & 0.1 \\
\hline 216-A-2 & Cribs & $216-A-2$ & Process Wasto & $\overline{\mathrm{PO}-2}$ & 1.45 & 0.921 & $7.83 E-08$ & 130 \\
\hline$\overline{216-A-20}$ & Trench & $\overline{216-A-20}$ & Process Waste & $\overline{P O-5}$ & 0.0444 & 0.042 & 2.75E-12 & 0.1 \\
\hline 216-A-21 & Cribs & $2 \overline{216-A-21}$ & Lab Waste & PO-2 & 78.5 & 7.51 & 0.00000145 & 150 \\
\hline $216-A-22$ & French Drain & $216-A-22$ & Mlscellaneous Dralnage & $\mathrm{PO}-2$ & & & & . \\
\hline $216-A-23 A$ & French Drain & $216-A-23 A$ & Process Condensale & PO-5 & & & & \\
\hline $216 \cdot \bar{A}-23 B$ & French Drain & $216-A-23 B$ & Process Condensale & PO-5 & & & & \\
\hline $218-A-24$ & Cribs . & $216-A-24$ & Process Condensalo & $\overline{P O-5}$ & 268 & 18.3 & 0.00000132 & 5.06 \\
\hline 216-A-25 & Ponds & 216-A-25 Pond & Cooling Water & IU-6 & 204 & 257 & 0.000162 & 428 \\
\hline $216-A-26$ & French Drain & $216-A-28$ & Miscellaneous Dralnage & PO-2 & & & & \\
\hline$\overline{216-\bar{A}-26 \mathrm{~A}}$ & French Draln & $216 \cdot A-28 A$ & Miscellanéous Drainago & PO-2 & & & & \\
\hline $216-A-27$ & Cribs & $216 \cdot A-27$ & Mlscellaneous Drainage & P0.2 & 32.4 & 24.5 & 0.0000138 & 98.5 \\
\hline $216-A-28$ & Cribs & $216-A-28$ & Process Condensate & $\overline{P O-2}$ & & & & \\
\hline $216-A-29$ & \begin{tabular}{|l|l|} 
Ditches \\
\end{tabular} & 216-A-29 & Chemical Sewer & $\overline{B P-11}$ & & & & \\
\hline $216-\bar{A}-3$ & Cribs & $216-A-3$ & Procoss Wasto & $\mathrm{PO} 0.2$ & 0.0455 & 0.0431 & 0.000000152 & 0.2 \\
\hline $216-A-30$ & Cribs & $216 \cdot A-30$ & Steam Condensato & PO-4 & 117 & 102 & 0.0814 & $\overline{73.1}$ \\
\hline$\overline{216-A-31}$ & Cribs & $216 \cdot A-31$ & Procoss Waste & PO-2 & 82 & 1.05 & 0.0013 & 8 \\
\hline $216-A-32$ & Cribs & $216-A-32$ & Miscellaneous Drainage & $\mathrm{PO}-2$ & & & & \\
\hline $216-A-33$ & French Drain & $216-A-33$ & Miscellaneous Drainage & $\mathrm{PO}-2$ & & & & . \\
\hline $216-A-34$ & Cribs & $216 \cdot A-34$ & Process Condensale & PO-5 & & & & \\
\hline $216-A-35$ & Fronch Draln & $216-A-35$ & Miscellaneous Drainago & PO.2 & & & & \\
\hline $216-A-36 A$ & Cribs & $216-A-3 B A$ & Process Wasto & $\mathrm{PO} 2$ & 847 & 978 & 0.000116 & 80 \\
\hline $216-A-36 B$ & Cribs & $216-A-36 B$ & Process Wasto & $\mathrm{PO-2}$ & 350 & 331 & 3.17 & 178 \\
\hline $216-A-37-1$ & Cribs & $216-A-37-1$ & Process Condensale & PO-4 & 0.0947 & 0.0542 & 0.0415 & 0.0283 \\
\hline$\longdiv { 2 1 0 - A - 3 7 - 2 }$ & Cribs & $216-A-37-2$ & Steam Condensate & PO-4 & 0.204 & 0.307 & 0.0407 & \\
\hline
\end{tabular}


Table B.1. (page 3 of 14)

\begin{tabular}{|c|c|c|c|c|c|c|c|c|}
\hline Site Code & $\begin{array}{c}\text { Wasto Management } \\
\text { Unl: Typo }\end{array}$ & Wasto Managomont Unit & Waste Typo & $\begin{array}{c}\text { Operable } \\
\text { Unit }\end{array}$ & $\begin{array}{c}\text { Ceslum-137, } \\
\text { Untis (CI) }\end{array}$ & $\begin{array}{c}\text { Strontlum-90, } \\
\text { Units (Ci) }\end{array}$ & $\begin{array}{c}\text { Ruthenium-106, } \\
\text { Units (CI) }\end{array}$ & $\begin{array}{l}\text { Total Plutonlum, Units (g, } \\
\text { unless othorwise stated) }\end{array}$ \\
\hline $216-A-38-1$ & Cribs & $216-A-38-1$ & N/A & $\mathrm{PO}-2$ & & & & \\
\hline $216-A-39$ & Ditches & $216-A-38$ & Miscellaneous Drainago & $\mathrm{PO}-3$ & 14.3 & & & \\
\hline $216-A-4$ & Cribs & $216-A-4$ & Lab Wasto & PO-2 & 6.93 & 4.39 & 4.38E-08 & 140 \\
\hline $216-A-40$ & Retention Basin & $216-A-40$ & Steam Condensate & $\mathrm{PO}-2$ & & & & \\
\hline $216-A-41$ & Cribs & $216-A-41$ & Mlscellaneous Drainage & $P O-2$ & & & & \\
\hline $216-A-42$ & Retentlon Basin & $216-A-42$ & Cooling Water & $\longdiv { \mathrm { PO } - 4 }$ & & & & \\
\hline $216-A-45$ & Cribs & $216-A-45$ & Process Condensate & po-2 & 0.0097 & 0.00834 & 0.0133 & \\
\hline $216-A-5$ & Cribs & $216-A-5$ & Process Condensate & PO-2 & 12.1 & 41.6 & 0.000000108 & 65 \\
\hline $216-A-524$ & Diversion Box & $216-A-524$ & & PO-5 & & & & \\
\hline $216-A-6$ & Cribs & $216-A-6$ & Steam Condensate & $\mathrm{PO}-4$ & 105 & 44.1 & 0.0000055 & 35.6 \\
\hline 216-A-7 & Cribs & $216-A-7$ & Process Waste & P0-5 & 2.31 & 0.431 & 0.00000011 & \\
\hline 216-A-8 & Cribs & $216 \cdot \bar{A}-8$ & Process Condensate & PO-5 & 522 & 51.5 & 0.0000469 & 50 \\
\hline $216 \cdot A-9$ & Cribs & $216-A-9$ & Cooling Water & PO-2 & 4.65 & 11 & $3.63 \mathrm{E}-08$ & 0.5 \\
\hline 216-B-10A & Cribs & 216-B.10A Crib & Lab Waste & BP-6 & 0.401 & 1.80 & 0 & 9.8 \\
\hline 216-B-10B & Criks. & 216-B-10B Crib & Lab Waste & BP-6 & 0.0001 & 0.0002 & 0 & 0 \\
\hline 216-B-11A\&B & Reverse Well & 216-B-11A\&B Reverse Wells & Process Condensate & $\mathrm{BP}-4$ & 21.3 & 2.01 & 0.425 & 4 \\
\hline $216-B-12$ & Cribs & $218-\mathrm{B}-12 \mathrm{Crb}$ & Process Condensale & $\overline{B P-9}$ & 716 & 79.3 & 0.0001 & 374 \\
\hline $216-8-13$ & French Drain & 216-B-13 Fronch Draln & Miscellaneous Drainage & BP.6 & & & & \\
\hline $218-8-14$ & Cribs & $216-\mathrm{B}-14 \mathrm{Crib}$ & Scavenged Waste & $B P-2$ & 114 & 172 & 0 & 25 \\
\hline $216 \cdot \mathrm{B}-15$ & Cribs & 216-8-15 Crib & Scavenged Wasto & BP-2 & 92.4 & 87.3 & 0 & 5 \\
\hline 216-8-16 & Cribs & 216-8-16 Crib & Scavenged Waste & $B P-2$ & 296 & 302 & 0 & 10 \\
\hline 216-B-17 & Cribs & 216-B-17 Crib & Scavenged Wasle & BP-2 & 100 & 68.8 & 0 & 10 \\
\hline 216-B-18 & Cribs & 216-8-18 Crib & Scavenged Wasto & $\overline{\mathrm{BP}-2}$ & 114 & 81.8 & 0 & 10 \\
\hline $216-8-19$ & Cribs & 216-B-19 Cतb & Scavenged Waste & $B P-2$ & 126 & 88.3 & 0 & 10 \\
\hline $216-B-2-1$ & Ditches & 216-8-2-1 Dilchb/ & Coofling Water & $B P-11$ & 93.5 & 101 & 1.42 & 250 \\
\hline 216-B-2-2 & Ditches & 216-8-2-2 DHchal & Coolling Wator & BP-11 & 0.314 & 147 & & $\overline{0.042}$ \\
\hline $216-8-2-3$ & Ditches & 216-8-2-3 Dlich & Coolling Water & BP-11 & 0.314 & 432 & & \\
\hline $216-8-20$ & Trench & 216-8-20 Trench & Scavenged Waste & $\overline{B P-2}$ & 684 & 340 & 0 & 1.3 \\
\hline $216-8-21$ & Trench & 216-B-21 Trench & Scavenged Waste & $B P-2$ & 169 & 318 & 0 & 10.3 \\
\hline $216-8-22$ & Trench & 216-8-22 Trench & Scavenged Waste & $\overline{B P-2}$ & 20.5 & 176 & of & 2.6 \\
\hline $216-B-23$ & Trench & 216-B-23 Trench & Scavenged Waste & $8 \mathrm{P}-2$ & 50.8 & 62.5 & 0 & 1.8 \\
\hline $216-8-24$ & Trench & 216-B-24 Trench & Scavenged Wasto & $\mathrm{BP}-2$ & 58.6 & 78 & 0 & 77 \\
\hline $216-B-25$ & Trench & 216-B-25 Trench & Scavenged Waste & $8 P-2$ & 25.5 & 88.3 & 0 & \\
\hline
\end{tabular}


Table B.1. (page 4 of 14)

\begin{tabular}{|c|c|c|c|c|c|c|c|c|}
\hline Site Codo & $\begin{array}{c}\text { Waste Management } \\
\text { Unit Type }\end{array}$ & Wasto Management Unit & Wasto Typo & $\begin{array}{c}\text { Operable } \\
\text { Unit }\end{array}$ & $\begin{array}{c}\text { Ceslum-137, } \\
\text { Units (CI) }\end{array}$ & $\begin{array}{c}\text { Strontlum.90, } \\
\text { Units (CI) }\end{array}$ & $\begin{array}{c}\text { Ruthenlum-106, } \\
\text { Units (Ci) }\end{array}$ & $\begin{array}{l}\text { Total Plutonlum, Units (g, } \\
\text { unless otherwise stated) }\end{array}$ \\
\hline 216-8-26 & Trench & 216-B-26 Trench & Scavenged Wasto & $\overline{B P-2}$ & 438 & 475 & 0 & $\begin{array}{rr}2.5 \\
\end{array}$ \\
\hline 216-B-27 & Trench & 216-B-27 Trench & Scavenged Waste & $\overline{\mathrm{BP}-2}$ & 15.8 & 263 & 0 & 70 \\
\hline 216-B-28 & Trench & 216-B-28 Trench & Scavenged Waste & BP-2 & 10.7 & 49.5 & 0 & 5.6 \\
\hline 216-8-29 & Trench & 216-B-29 Trench & Scavenged Waste & BP-2 & 27.4 & 84.8 & 0 & 1.1 \\
\hline 216-B-3 & Ponds & 216-B-3 Ponde/ & Cooling Water & 8P-11 & 93.5 & 101 & 1.42 & 250 \\
\hline 216-B-3-1 & Ditches & 216-B-3-1 Ditchb/ & Cooling Water & $8 \bar{P}-11$ & & & & \\
\hline 216-B-3-2 & Ditches & 216-B-3-2 Ditchal & Cooling Water & $\mathrm{BP}-11$ & & & & \\
\hline $216-B-3-3$ & Ditchos & 218-B-3-3 Ditch & Cooling Water & $\overline{B P-11}$ & & & & \\
\hline $216-B-30$ & Trench & 216-B-30 Trench & Scavenged Waste & $B P-2$ & 1570 & 265 & o & 2.1 \\
\hline 216-8-32 & Trench & 216-B-32 Trench & Scavenged Waste & BP-2 & 58.6 & 113 & 0 & 2.6 \\
\hline 216-8-33 & Trench & 216-B-33 Trench & Scavenged Waste & $\overline{\mathrm{BP}-2}$ & 127 & 18.1 & 0 & 11.8 \\
\hline 216-B-34 & Trench & 216-B-34 Trench & Scavenged Waste & $\mathrm{BP}-2$ & 7.91 & 18.1 & 0 & 5.7 \\
\hline 216-B-35 & Trench & 216-B.35 Trench & Tank Farm Waste & $B P-3$ & 185 & 96.4 & 0 & 1.2 \\
\hline $216-\bar{B}-36$ & Trench & 216-8-36 Trench & Tank Farm Waste & $\mathrm{BP}-3$ & 336 & 199. & 0 & 0.8 \\
\hline 216-B-37 & Trench & 216-B-37 Trench & Process Waste & $\mathrm{BP}-3$ & 1350 & 6.56 & 0 & 2 \\
\hline 216-B-38 & Trench & 216-B-38 Trench & Tank Farm Wasto & $\overline{B P}-3$ & 221 & 750 & 0 & 1.2 \\
\hline $216-B-39$ & Trench & 216-B-39 Trench & Tank Farm Waste & $\mathrm{BP}-3$ & 192 & 9.27 & o & 1.51 \\
\hline $216-8-3 A$ & Ponds & 216-B-3A Pond & Cooling Water & BP-11 & & & & \\
\hline 216-B-3B & Ponds & 216-B-3B Pond & Cooling Water & BP-11 & & & & \\
\hline $216-B-3 C$ & Ponds & 216-B-3C Pond & Coollng Water & $8 P-11$ & & & & \\
\hline $216-8-4$ & Reverse Weill & 216-B-4 Reverse Well & Miscellaneous Dralnage & $B P-6$ & 可 & 0 & 0 & \\
\hline $216-8-40$ & Trench & 216-B-40 Trench & Tank Farm Wasto & BP-3 & 153 & 115 & 可 & \\
\hline $216-B-41$ & Trench & 216-B-41 Trench & Tank Farm Wasto & $\mathrm{BP}-3$ & 386 & 19.3 & 0 & 0.3 \\
\hline $216-8-42$ & Trench & 216-B-42 Trench & Scavenged Waste & BP-3 & 42.7 & 463 & 0 & 10 \\
\hline $216-B-43$ & Cribs & 216-B-43 Crib & Scavenged Waste & $\mathrm{BP}-1$ & 130 & 574 & 0 & 0.5 \\
\hline $216-B-44$ & Cribs & 216-B-44 Crib & Scavenged Wasto & $\mathrm{BP}-1$ & 309 & 1200 & 0 & 15 \\
\hline $216 \cdot B-45$ & Cribs & 216-B-45 Crib & Scavenged Waste & $B P-1$ & 666 & 1180 & 0 & 10 \\
\hline $216-8-46$ & Cribs & 216-B-46 Crib & Scavenged Waste & BP-1 & 88.9 & 631 & 0 & 20 \\
\hline $216-B-47$ & Cribs & 216-B-47 Crịb & Scavenged Waste & $\mathrm{BP}-1$ & 66.6 & 261 & 0 & 5 \\
\hline $216 \cdot B-48$ & Cribs & 216-B-4B Crib & Scavenged Wasto & $B P-1$ & 200 & 547 & 0 & 5 \\
\hline $216 \cdot 8-49$ & Cribs & 216-B-49 Crib & Scavenged Wasto & BP-1 & 182 & 1140 & 0 & 15 \\
\hline $216-8-5$ & Reverso Well & 216-B-5 Reverse Well & Process Waste & BP.6 & 29.2 & 25.5 & $1.03 \mathrm{E}-11$ & 4270 \\
\hline $216-8-50$ & Cribs & 216-B-50 Crib & Process Condensato & $\mathrm{BP}-1$ & 51.2 & 3.39 & 0 & $\overline{0.239}$ \\
\hline
\end{tabular}


Table B.1. (page 5 of 14)

\begin{tabular}{|c|c|c|c|c|c|c|c|c|}
\hline Stto Codo & \begin{tabular}{|c|} 
Wasto Management \\
Unit Typo
\end{tabular} & Waste Stanagement Unit & Waste Typo & $\begin{array}{c}\text { Oporablo } \\
\text { Unit }\end{array}$ & $\begin{array}{c}\text { Coslum-137, } \\
\text { Units (C)) }\end{array}$ & $\begin{array}{c}\text { Strontlum-90, } \\
\text { Units (Ci) }\end{array}$ & $\begin{array}{l}\text { Ruthenlum-106, } \\
\text { Unito (CI) }\end{array}$ & $\begin{array}{l}\text { Total Plutonlum, Unlts ( }(g, \\
\text { unless otherwise stated) }\end{array}$ \\
\hline 216-B-51 & French Draln & 216-B-51 French Drain & MIscellaneous Drainago & BP-4 & & & & \\
\hline $216-B-52$ & Trench . & 218-B-52 Trench & Scavenged Waste & $\overline{B P-2}$ & 160 & 4.92 & 0 & 19 \\
\hline 216-B-53A & Trench & 218-B-53A Trench & Lab Wasle & $\overline{\mathrm{BP}-2}$ & 0.0559 & 0.0538 & 0 & 100 \\
\hline $216-B-53 B$ & Trench & 216-B-53B Trench & LabWaste & $\overline{\mathrm{BP}-2}$ & 3.7 & 5.06 & 0 & 5 \\
\hline $216 \cdot 8-54$ & Trench & 216-8-54 Tranch & Lab Waste & $\overline{\mathrm{BP}-2}$ & 0.0547 & 0.0525 & 0 & 5 \\
\hline 216-B-55 & Cribs & 216-B.55 Crib & Steam Condensate & $B P-9$ & 13.7 & 7.23 & 0.0000501 & $\overline{0.653}$ \\
\hline $216-8.56$ & Cribs & 216-8-56 Crib & N/A & BP-6 & & & & \\
\hline $218-8-57$ & Cribs & 216-B-57 Crib & Process Condensate & BP-1 & 226 & 1.83 & 0 & 0.187 \\
\hline 216-B-58 & Trench & 216-B-58 Trench & Lab Wasto & $\mathrm{BP}-2$ & 4.4 & 5.55 & 0) & 6.7 \\
\hline 216-B-59 & Retention Basin & 216-8-59 Basin & Coollng Water & BP.6 & 0.012 & 0.0289 & & \\
\hline $216-8-6$ & Reverse Well & 216-B-6 Reverse Well & Lab Waste & BP.6 & 0 & 0 & 0 & 0 \\
\hline $216-8-60$ & Cribs & 216-B-60 Crib & Decon Wasto & $\mathrm{BP}-8$ & & & & \\
\hline 216-8-61 & Cribs & 216-B-61 Crib & N/A & $B P-1$ & & & & \\
\hline$\overline{216-8-62}$ & Cribs & $216-\mathrm{B}-62 \mathrm{Crb}$ & Process Condensate & BP-9 & 135 & 74.6 & 0.0049 & 0.755 \\
\hline $216 \cdot 8 \cdot-83$ & Ditches & 216-B-63 T:ench & Chemical Sewer & BP-11 & 0.625 & 2.41 & 0.000000239 & 0.573 \\
\hline $216-8-64$ & Retentlon Basin & 216-B-64 Basin & N/A & $B P-9$ & & & & \\
\hline 216-B-7A\&B & Cribs & 216-B-7A\&B Crib & Process Waste & $\mathrm{BP}-4$ & 43.2 & 2200 & 0 & 4300 \\
\hline 216-B-8 & Cribs & 216-B-8TF Crib & Process Wasto & $B P-4$ & 19.8 & 5.58 & 0 & 30 \\
\hline $216-B-9$ & Cribs & 218-B-9TF Crib & Process Waste & BP-6 & 3.92 & 5.52 & 0 & 174 \\
\hline $216-\mathrm{C}-1$ & Cribs & 216-C-1 Crib & Process Condensale & SO-1 & 0.0455 & 85.5 & $1.89 \mathrm{E}-08$ & 8 \\
\hline $216-C-10$ & Cribs & $216-C-10$ Crib & Process Condensate & 50-1 & 0.0855 & 3.45 & $8.95 E-08$ & 0.15 \\
\hline$\overline{216-C+2}$ & Reverse Well & 216-C-2 Roverse Well & Miscellaneous Drainage & SO-1 & & & & \\
\hline 216-C-3 & Cribs & 218-C-3 Crib & Process Wasto & So-1 & 0.0424 & 8.04 & 8.3E-11 & $\overline{1}$ \\
\hline $216-C-4$ & Cribs & $216-\mathrm{C}-4 \mathrm{Crib}$ & Process Waste & $50-1$ & 0.0433 & 11.8 & 5.35E-10 & \\
\hline $216-C-5$ & Cribs & 216-C-5 Crib & Process Wasto & 50.1 & 0.0444 & 4.2 & $1.38 \mathrm{E}-10$ & 1 \\
\hline $216-C-6$ & Cribs & 216-C-6 Crib & Process Condensate & so-1 & 0.0465 & 28.8 & $2.73 E-08$ & 0.1 \\
\hline $216-C-7$ & Cribs & 216-C.7 Crib & Process Waste & So-1 & 0.0534 & 0.0512 & $1.06 E-08$ & 9.1 \\
\hline $216-C-8$ & French Drain & $216-C-8$ & Process Wasto & PO-3 & & & & \\
\hline $216-C-9$ & Ponds & 216-C-9 Pond & Cooling Water & so-1 & 0.703 & 2.43 & $8.66 \mathrm{E}-08$ & $\overline{0.338}$ \\
\hline $218-E-28$ & Ponds & 216-E-28 Pond & N/A & Bp-11 & & & & \\
\hline $216-N-1$ & Ponds & 216-N-1 Pond & Cooling Waler & NO-1 & & & & \\
\hline $216 \cdot N-2$ & Trench & 216-N-2 Trench & Coollng Water & No-1 & 0.0785 & 0.0687 & $4.73 \mathrm{E}-14$ & \\
\hline $216-\mathrm{N}-3$ & Trench & 216-N-3 Trench & Cooling Water & NO.1 & 0.0881 & 0.0777 & $1.49 E-12$ & \\
\hline
\end{tabular}


Table B.1. (page 6 of 14)

\begin{tabular}{|c|c|c|c|c|c|c|c|c|}
\hline Site Code & $\begin{array}{c}\text { Wasto Management } \\
\text { Unlt Typo }\end{array}$ & Waste Management Li:H & Wasto Type & $\begin{array}{c}\text { Operablo } \\
\text { Unit }\end{array}$ & $\begin{array}{c}\text { Cesilum-137, } \\
\text { Units (Ci) }\end{array}$ & $\begin{array}{l}\text { Strontium-90, } \\
\text { Units (CI) }\end{array}$ & $\begin{array}{c}\text { Ruthenlum-106, } \\
\text { Units (CI) }\end{array}$ & $\begin{array}{l}\text { Total Plutonlum, Units (g, } \\
\text { unless otherwlso stated) }\end{array}$ \\
\hline $216-N-4$ & Ponds & 216-N-4 Pond & Coolling Water & NO-1 & $\overline{0.0813}$ & 0.0713 & 3.32E-13 & 1 \\
\hline $216-N-5$ & Trench & 216-N-5 Trench & Coolling Water & NO-1 & 0.0881 & 0.0777 & 1.49E-12 & \\
\hline $216-N-6$ & Ponds & 216-N-G Pond & Cooling Water & NO-1 & 0.0813 & 0.0713 & $3.32 E-13$ & 1 \\
\hline $216-N-7$ & Trench & 216-N-7 Trench & Cooling Water & NO-1 & 0.0881 & 0.0777 & 1.49E-12 & \\
\hline $216-N-8$ & Ponds & 216-N-8 Pond & & IUU-6 & & & & \\
\hline $216-S-182$ & Cribs & $216 \cdot S \cdot 1 \& 2$ & Process Condensato & RO-2 & 1100 & 1250 & $6.19 \mathrm{E}-08$ & 1200 \\
\hline 216-S-10D & Ditches & $216-\mathrm{S} \cdot 10 \mathrm{D}$ & Chemical Sewer & RO-1 & 1.24 & 1.07 & 0.346 & 0.1 \\
\hline $216-S-10 P$ & Ponds & 216-S-10P & Chemical Sewer & RO-1 & & & & \\
\hline $216-5-11$ & Ponds & $\overline{216-S-11}$ & Chemical Sewer & RO-1 & 0.82 & 0.814 & 0.292 & $\overline{0.31}$ \\
\hline 2 & Trench & $216-\mathrm{S}-12$ & Miscellaneous Drainage & RO-3 & 0.434 & 0.41 & $1.38 \mathrm{BE}-11$ & 1 \\
\hline $216-5-13$ & Cribs & $216-5-13$ & Process Wasto & $\overline{R 0-2}$ & .2 .77 & 0.0204 & 0.00000236 & 8 \\
\hline 216-S-14 & Trench & $216-5-14$ & Process Wasto. & $\longdiv { R O - 3 }$ & & & & \\
\hline $216-\mathrm{S}-15$ & Ponds & $216-S-15$ & Cooling Water & RO-2 & & & & \\
\hline $216-S-16 D$ & Diltchos & $216-5-160$ & Coolling Water & RO-1 & & & & \\
\hline $216-S-16 \mathrm{P}$ & Ponds & 216-S-16P & Coolling Water & RO-1 & 30 & 45.1 & 0.00000447 & \\
\hline$\overline{216-S-17}$ & Ponds & 216-S-17 & Cooling Water & RO-1 & 12.7 & 15.9 & $3.12 E-10$ & 3 \\
\hline $216-\mathrm{S}-172$ & Diverston Box & $216-5-172$ & Coolling Water & RO-1 & & & & \\
\hline $216-S-18$ & Trench & $216-\mathrm{S}-18$ & Debris & R0-2 & & & & \\
\hline 216-S-19 & Ponds & 216-5-10 & Lab Wasle & RO-1 & 1.29 & 1.3 & 0.000000389 & 20.6 \\
\hline 216-S-20 & Cribs & $216-5-20$ & Lab.Wasto & RO-3 & 56.5 & 22.7 & 0.000000249 & $\overline{171}$ \\
\hline $216-S-22$ & Cribs & 216-S-22 & Procoss Wasto & RO-3 & 0.478 & 0.455 & $1.41 E-09$ & 0.101 \\
\hline $216-S-23$ & Cribs & $216-5-23$ & Process Condensate & RO-2 & 3.47 & 1.14 & 0.0000349 & 0.994 \\
\hline $216-S-25$ & Cribs & $216-5-25$ & Steam Condensale & RO-1 & 0.0647 & $\overline{0.041}$ & 0.000016 & 0.0466 \\
\hline $216-S-28$ & Cribs & $216-5-28$ & Lab Waste & RO-3 & 0.00309 & 0.00183 & & \\
\hline $216-5-3$ & French Drain & $216-5-3$ & Process Condensalo & RO-2 & 21.0 & 0.414 & $1.09 \mathrm{E}-09$ & 0.5 \\
\hline$\overline{216-5-4}$ & French Drain & $216-5-4$ & Process Condensale & UP-2 & & & & \\
\hline $216-S-5$ & Cribs & $216-5 \cdot 5$ & Coolling Water & $\longdiv { R O - 1 }$ & 26.4 & 54.1 & $7.14 E-10$ & 580 \\
\hline $216-\mathrm{S}-6$ & Cribs & $216-5-6$ & Cooling Water & RO-1 & 115 & 204 & 0.00000589 & $\overline{473}$ \\
\hline 216-S-7 & Cribs & $216-5-7$ & Process Condensato & RO-2 & 703 & 1390 & 0.0000013 & 440 \\
\hline $216-5-8$ & Trench & $216-\mathrm{S}-8$ & Process Wasto & RO-2 & 4.92 & 0.386 & $1.3 \mathrm{E}-10$ & $\overline{2}$ \\
\hline $216-5-9$ & Cribs & $216-5-9$ & Process Condensato & RO-2 & 290 & 86.3 & 0.000287 & 65 \\
\hline 216-T-1 & Ditches & 216-T-1 Ditch & Cooling Water & TP-4 & 0.0387 & 0.0363 & 4.39E-13 & 0.1 \\
\hline 216-T-10 & Trench & 216-T-10 Trench & Decon Waste & TPA & & & & \\
\hline
\end{tabular}


Table B.1. (page 7 of 14)

\begin{tabular}{|c|c|c|c|c|c|c|c|c|}
\hline Site Code & $\begin{array}{c}\text { Waste Management } \\
\text { Untt Type }\end{array}$ & Waste Management Unit & Wasto Typo & $\begin{array}{c}\text { Operablo } \\
\text { Unit }\end{array}$ & $\begin{array}{c}\text { Cosium-137, } \\
\text { Units (CI) }\end{array}$ & $\begin{array}{c}\text { Strontlum-90, } \\
\text { Units (Cl) }\end{array}$ & $\begin{array}{c}\text { Ruthenlum-106, } \\
\text { Units (CI) }\end{array}$ & $\begin{array}{l}\text { Total Plutonlum, Unlts (g, } \\
\text { unless othorwise stated) }\end{array}$ \\
\hline $216-T-11$ & Trench & 216-T-11 Trench & Decon Wasto & $T P-4$ & & & & \\
\hline $216-T-12$ & Trench & 216-T-12 Trench & Cooling Water & TP=3 & 4.34 & 2.05 & $1.38 E-10$ & 1 \\
\hline$\overline{216-T-13}$ & Trench & 216-T-13 Trench & Decon Waste & TP-2 & & & & \\
\hline $216-T-14$ & Trench & 216-T-14 Trench & Tank Fam Wasto & $T P-3$ & 204 & 2.46 & $2.07 E-10$ & 0.88 \\
\hline $216-T-15$ & Trench & 216-T-15 Trench & Tank Farm Waste & TP.3 & 450 & 8.62 & $1.66 E-10$ & 0.94 \\
\hline $216-T-16$ & Trench & 216-T-18 Trench & Tank Farm Waste & TP-3 & 227 & 3.28 & 1.79E-10 & 0.65 \\
\hline $216-T-17$ & Trench & 216-T-17 Trench & Tank Farm Waste & TP-3 & 162 & 1.23 & $1.38 \mathrm{E}-10$ & 0.53 \\
\hline $216-T-18$ & Cribs & 218-T-18 Crib & Tank Farm Waste & TP-2 & 24.2 & 2.8 & $1.38 \mathrm{E}-09$ & 1800 \\
\hline 216-T-19 & Cribs & 216-T-19TF Crib and Tile Field & Process Wasto & $\overline{\mathrm{TP}-2}$ & 17.5 & 27.8 & 0.00000603 & 14.4 \\
\hline 216-T-2 & Reverse Well & 216-T-2 Reverse Well & Lab Waste & TP.4 & & & & \\
\hline 216-T-20 & Trench & 216-T-20 Trench & Process Waste & TP-2 & 0.44 & 0.388 & $7.44 E-12$ & \\
\hline $216-T-21$ & Trench & 216-T-21 Tranch & Tank Farm Wasto & TP-1 & 174 & 3.28 & $8.56 \mathrm{E}-10$ & 1 \\
\hline 216-T-22 & Trench & 216-T-22 Trench & Tank Farm Waste & TP-1 & 803 & 20.9 & 4.14E-10 & 2 \\
\hline $216-T-23$ & Trench & 216-T-23 Trench & Tank Farm Wasto & $\mathrm{TP}-1$ & 577 & 16.82 & $3.59 \mathrm{E}-10$ & 1 \\
\hline 216-T-2! & Trench & 216-T-24 Trench & Tank Farm Wasto & TP-1 & 617 & 16.4 & $4.42 \mathrm{E}-10$ & 2 \\
\hline $210 \cdot T-25$ & Trench & 216-T-25 Trench & Process Waste & TP-1 & 3860 & 1.64 & 1.38E-09 & 1 \\
\hline 216-T.26 & Cribs & 216-T-28 Crib & Tank Farm Wasto & $\mathrm{TP}-2$ & 75.6 & 282 & $8.02 \mathrm{E}-08$ & 59 \\
\hline 216-T-27 & Cribs & 216-T-27 Crib & Lab Wasto & TP-2 & 55.9 & 75.3 & 0.0000409 & 13 \\
\hline $218-T-28$ & Cribs & 216-T-28 Crib & Decon Wasto & TP-2 & 193 & 108 & 0.0000196 & 70 \\
\hline $218-T-29$ & Cribs & 216-T-29.Crib & Miscellaneous Drainago & TP-4 & & & & \\
\hline 216-T-3 & Reverse Well & 216-T-3 Reverse Woll & Process Wasto & TP-4 & 21.3 & 18.6 & $5.22 \mathrm{E}-12$ & 3350 \\
\hline $218-T-31$ & French Draln & 216-T-31 French Drain & Mlscolleneous Drainago & $\mathrm{TP}-2$ & & & & \\
\hline $216-T-32$ & Cribs & $216-T-32$ Crib & Process Wasto & TP.1 & 0.71 & 10.9 & $4.44 E-11$ & 3200 \\
\hline 216-T-33 & Cribs & 216-T-33 Crib & Docon Wasto & TP-4 & 0.267 & 0.256 & 6.86E-08 & 5 \\
\hline 216-T-34 & Cribs & 216-T-34 Crib & Lab Waste & TP-4 & 157 & 178 & 0.00000598 & 107 \\
\hline 216-T-35 & Cribs & 216-T-35 Grib & LabWasto & TP-4 & 11.7 & 11.4 & 0.0000144 & 66.2 \\
\hline 216-T-36 & Cribs & 216-T-36 Crib & Steam Condensate & $\mathrm{TP}-1$ & 3.79 & 4.36 & 0.00000524 & 2.48 \\
\hline $216-T-4-10$ & Ditches & 216-T-A-10 Dilch & Cooling Water & TP-3 & & & & \\
\hline $216-T-4-2$ & Ditchos & 216-T-4-2 Ditch & Steam Condensate & TP.3 & & & & \\
\hline 216-T-4A & Ponds & 216-T-AA Pond & Cooting Water & $\mathrm{TP}-3$ & & & & \\
\hline $216-T-4 B$ & Ponds & 216-T-4B Pond & Cooling Water & $T P-3$ & 6.23 & 3.37 & 0.000000867 & 3.71 \\
\hline $216-T-5$ & Tronch & 216-T-5 Trench & Tank Farm Wasto & $T P-1$ & 31.1 & 0.42 & $8.25 E-10$ & 180 \\
\hline 216-T-6 & Cribs & 216-T-6 Crib & Procoss Wasto & TP-3 & 110 & 124 & 6.07E-11 & 390 \\
\hline
\end{tabular}


Table B.1. (page 8 of 14)

\begin{tabular}{|c|c|c|c|c|c|c|c|c|}
\hline Slte Codo & $\begin{array}{c}\text { Waste nlanagement } \\
\text { Untt Typo }\end{array}$ & Waste Managoment Unit & Waste Type & $\begin{array}{c}\text { Operable } \\
\text { Unit }\end{array}$ & $\begin{array}{c}\text { Cosium-137, } \\
\text { Units (Ci) }\end{array}$ & $\begin{array}{c}\text { Strontium-90, } \\
\text { Units (CI) }\end{array}$ & $\begin{array}{c}\text { Ruthonlum-106, } \\
\text { Units (Ci) }\end{array}$ & $\begin{array}{l}\text { Total Plutonlum, Unlts (g, } \\
\text { unless otherwise stated) }\end{array}$ \\
\hline $216-T-7$ & Cribs & 216-T-7TF Crib and Tile Field & Tank Farm Wasto & TP-1 & 21.2 & \begin{tabular}{|l|}
24 \\
\end{tabular} & 2.02E-09 & 130 \\
\hline $216-T-8$ & Cribs & 216-T-8 Crib & Lab Waste & TP-4 & 0.0401 & 0.376 & 6.63E-12 & \\
\hline 216-T-9 & Trench & 216-T-9 Trench & Decon Waste & TP-4 & & & & \\
\hline $216-U-182$ & Cribs & $218-U-1 \& 216-U-2$ & Process Condensate & UP-2 & 4.36 & 2.11 & 0.0000006 & 42.6 \\
\hline $216-U-10$ & Ponds & $216-U-10$ & Coolling Water & UP-2 & 11 & 11 & 0.0000278 & 8000 \\
\hline $216-U-11$ & Ditches & $216-U-11$ & Coolling Water & UP-2 & & & & \\
\hline $216-U-12$ & Cribs & 2 & Process Condensate & UP-2 & 0.0566 & 55.8 & 0.00000218 & \\
\hline $\begin{array}{l}216-U-13 \\
-13\end{array}$ & Trench & 216-U-13 (same as UN-200-W. 125) & Decon Waste & UP-2 & 0.0444 & 0.042 & & 0.1 \\
\hline$\overline{216-U-14}$ & Ditches & $216-U-14$ & Coolling Water & $\mathrm{UP}-2$ & & & & \\
\hline $216-U-15$ & Trench & $216 \cdot U-15$ & Process Waste & UP-2 & 0.0465 & 0.0442 & & 0.1 \\
\hline $216-U-16$ & Cribs & $216-U \cdot 16$ & Process Condensale & UP-2 & 0.0165 & 0.0092 & & \\
\hline $216-U-17$ & Cribs & $216-U-17$ & Process Condensale & $\mathrm{UP}-2$ & & & & \\
\hline $216-U-21$ & & 216-U-21 & & & 85.5 & 21.8 & 0.00000139 & 2.08 \\
\hline $218-\mathrm{U}-3$ & French Draln & $216-U-3$ & Miscellaneous Drainago & $\mathrm{UP}-2$ & 0.434 & 0.041 & & 0.1 \\
\hline$\overline{218-U-4}$ & Reverse Well & $216--4-4$ & Lab Wasle & UP-2 & & & & \\
\hline $216-U-4 A$ & French Drain & $216-U-4 A$ & Miscellaneous Drainago & UP-2 & 0.185 & 0.0159 & 0.00000012 & 0.009 \\
\hline $216 . U-4 B$ & French Draln & $216-4 \cdot 4 B$ & Miscellaneous Drainago & UP-2 & 0.187 & 0.00165 & & 0.054 \\
\hline $216-U-5$ & Trench & $218-U-5 \& 216-U-6$ & Process Waste & UP-2 & 0.0207 & 0.0195 & & 0.05 \\
\hline 216-U.7 & French Draln & $218 \cdot 0 \cdot 7$ & Miscellaneous Dralnage & UP-2 & & & & \\
\hline $216-U-8$ & Cribs & $218 \cdot U \cdot 8$ & Process Condensato & UP-2 & 0.0455 & 0.0431 & 0.00000001 & 370 \\
\hline 216-U-9 & Diliches & 216-U-9 & Cooling Water & RO-1 & & & & \\
\hline $216-W-L W C$ & Cribs & 218-W-LWC Crib & Chomlcal Sewer & SS-2 & & & & \\
\hline$\overline{216-2-1822}$ & Cribs & $216-z-1 \& 216-Z-2$ Cribs & Process Wasto & $\mathrm{ZP}-2$ & 0.04 & 0.037 & $1.6 \mathrm{E}-11$ & 7000 \\
\hline $216-Z-10$ & Reverse Well & 216-z-10 Reverse Well & Procoss Wasto & $\mathrm{ZP}-2$ & & & & 50 \\
\hline $216-2-11$ & Dinches & $216-z-11$ & Cooling Water & UP-2 & & & & \\
\hline $216-2-12$ & Cribs & 216-Z-12 Crib & Process Wasto & $2 \mathrm{PP}-2$ & 0.053 & 0.051 & 0.00000003 & 25000 \\
\hline $216-2-13$ & French Drain & 216-Z-13 French Draln & Miscelleneous Drainage & $\mathrm{ZP}-2$ & & & & \\
\hline $216-2-14$ & French Drain & 216-z-14 French Draln & Miscellaneous Drainage & $2 \mathrm{P}-2$ & & & & \\
\hline $216-z-15$ & French Draln & 216-Z-15 French Draln & Miscellaneous Drainage & $2 \mathrm{P}-2$ & & & & \\
\hline $216-2-16$ & Cribs & 216-z-16 Crib & Lab Waste & $2 \mathrm{P}-2$ & & & & 72 \\
\hline$\overline{218-2-17}$ & Ditches & 216-Z-17 Trench & Lab Wasto & $2 \mathrm{P}-2$ & & & & 50 \\
\hline $218-z-18$ & Cribs & $216-Z-18$ Crib & Process Wasto & ZP.2 & & & & 23000 \\
\hline 216-Z-19 & Dilches & $218-2-19$ & Coolling Water & UP-2 & & & & \\
\hline
\end{tabular}


Table B.1. (page 9 of 14)

\begin{tabular}{|c|c|c|c|c|c|c|c|c|}
\hline Slle Codo & $\begin{array}{c}\text { Wasto Management } \\
\text { Unit Type }\end{array}$ & Wasto Managomont Unit & Wasto Typo & $\begin{array}{c}\text { Operable } \\
\text { Unit }\end{array}$ & $\begin{array}{c}\text { Ceslum-137, } \\
\text { Units (Ci) }\end{array}$ & $\begin{array}{c}\text { Strontlum-90, } \\
\text { Units (Ci) }\end{array}$ & $\begin{array}{l}\text { Ruthonlum-106, } \\
\text { Units (CI) }\end{array}$ & $\begin{array}{l}\text { Total Plutonilum, Units (g, } \\
\text { unloss othorwise statod) }\end{array}$ \\
\hline $216-Z-1 A$ & Cribs & 216-Z-1A Tile Field & Process Waste & ZP-2 & 0.18 & 0.15 & 0.0000052 & 57000 \\
\hline 216-Z-1D & Ditches & 216-Z-10 & Coolling Water & UP-2 & & & & \\
\hline$\overline{216-2-20}$ & Cribs & $216-2-20$ & Cooling Water & $\overline{U P-2}$ & 0.0864 & 0.083 & 0.000107 & 0.148 \\
\hline$\overline{216-2-21}$ & Retenllon Basin & 216-z-21 Seepage Basin & Coolling Water & $\mathrm{ZP}=2$ & & & & \\
\hline $216-z-3$ & Cribs & 216-Z-3 Crib & Process Waste & $\overline{\mathrm{ZP}-2}$ & 0.048 & 0.045 & 0.000000006 & 5700 \\
\hline$\overline{216-Z-4}$ & Trench & 216-Z-4 Trench & Procoss Wasto & $2 \mathrm{ZP}-2$ & 0.035 & 0.033 & $2.7 E-14$ & \\
\hline 216-Z-5 & Cribs & $216-z-5$ Crib & Process Waste & $2 \mathrm{ZP}-2$ & 3.6 & 1.7 & 5.2E-12 & 340 \\
\hline 216-z-6 & Cribs & $216-z-6$ Crib & Process Waste & 2 & 0.035 & 0.033 & $2.7 E-14$ & \\
\hline $216-2-7$ & Cribs & 216-z-7 Crib & LabWaste & $\mathrm{ZP}-2$ & 200 & 200 & $0.000005 t$ & 2000 \\
\hline $216-Z-8$ & Cribs & 216-2-8 French Drain & Process Waste & $2 \mathrm{P}-2$ & & & & \\
\hline $216-z-9$ & Cribs & 216-2-9 Trench & Process Waste & $2 \mathrm{PP}-2$ & 0.052 & 0.048 & 0.000000018 & 48000 \\
\hline 218-C-9 & Burial Site & 218.C-9 Burial Ground & LLW-SOLID & so-1 & 8.1 & & 0.0000054 & 0.0001 \\
\hline 218-E-1 & Burial Site & 218-E-1 & LLW-SOLID & $\overline{\mathrm{PO}-2}$ & 0.8166 & 0.7165 & $7.69 \mathrm{E}-12$ & 900 \\
\hline $218-E-10$ & Burial Site & 218-E-10 Burial Ground & LLW-SOLID & BP-10 & 931000 & 768000 & 0.771 & 4900 \\
\hline $218-E-12 A$ & Burial Site & 218-E-12A & LLW-SOLID & PO.6 & 10.99 & 9.056 & 0.000001222 & 8930 \\
\hline $\begin{array}{l}\text { 218-E-12B } \\
\text { (1) }\end{array}$ & Burfal Sitte & $218-E-12 B$ & LLW-SOLID & PO.6 & & & & \\
\hline $218-E-13$ & Burial.Sitte & $218 \cdot E-13$ & & $\mathrm{PO}-2$ & & & & \\
\hline $218-\mathrm{E}-2$ & Burial Site & 218-E-2 Burral Grọund & LLW-SOLIO & $B P-10$ & 213 & 187 & 0 & 800 \\
\hline 218-E-2A & Burial Site & 218-E-2A Burial Ground & LLW-SOLID & $8 \mathrm{P}-10$ & & & & \\
\hline $218-E-4$ & Eurial sito & 218-E-4 Burial Ground & LLW-SOLID & BP-10 & 940 & 0.0833 & 0 & 10 \\
\hline 218-E-5 & Burial Site & $218-E-5$ Burial Ground & LLW-SOLLID & EP-10 & 70.7 & 62.7 & of & 620 \\
\hline 218-E-5A & Burial She & 218-E-5A Burál Ground & LLW-SOLID & $B P-10$ & 165 & 147 & of & 1380 \\
\hline$\longdiv { 2 1 8 - E - 6 }$ & Burial Site & 218-E-6 Burial Ground & Debris . & $B P-6$ & & & & \\
\hline 218-E-7 & Burtal SHe & 218-E-7 Burral Ground & Lab Wasto & $B P-6$ & 4.96 & 4.36 & & \\
\hline $218-E-8$ & Burla Site & $218-E-8$ & TRU Solld Wasto & $\mathrm{PO}-6$ & 0.1017 & 0.09058 & $1.177 E-10$ & 20 \\
\hline 218-E-9 & Burlal Site & 218-E-9 Burial Ground & LLW-SOLIO & BP-10 & & & & \\
\hline $218-W-1$ & Burlal Sthe & 218-W-1 Burfal Ground & TRU Solld Waste & $\mathrm{ZP}=3$ & 1.63 & 1.44 & $8.83 E-12$ & 94000 \\
\hline $218-W-11$ & Burial Site & 218-W-11 Burlal Ground & LLW-SOLID & $\mathrm{ZP}-3$ & 0.002 & 0.0009 & $1,6 E-09$ & \\
\hline $218-W-1 A$ & Burial Sthe & 218-W-1A Burial Ground & LLW-SOLID & $2 \mathrm{ZP}-3$ & 359 & 359 & $5.23 E-09$ & 2000 \\
\hline $218-W-2$ & Burlal Site & 218-W-2 Burial Ground & TRU Solid Wasto & $\mathrm{ZP}-3$ & 4.86 & 4.1 & $5.72 E-10$ & 126000 \\
\hline $218-W-2 A$ & Burial SHo & $218-\mathrm{W}-2 \mathrm{~A}$ Burial Ground & LLW-SOLID & $\mathrm{ZP}-3$ & 2766 & 2467 & 0.0025 & \\
\hline $218-W-3$ & Burial Site & 218-W-3 Burial Ground & TRU Solld Wasto & $2 \mathrm{p}-3$ & 9.15 & 8.15 & $1.31 \mathrm{E}-08$ & 68000 \\
\hline $218-W-3 A$ & Eurial Stito & $218-W-3 A$ Burial Ground & TRU Solld Waslo & $2 \mathrm{PP}-3$ & 302000 & 101000 & 12.7 & 29300 \\
\hline
\end{tabular}


Table B.1. (page 10 of 14)

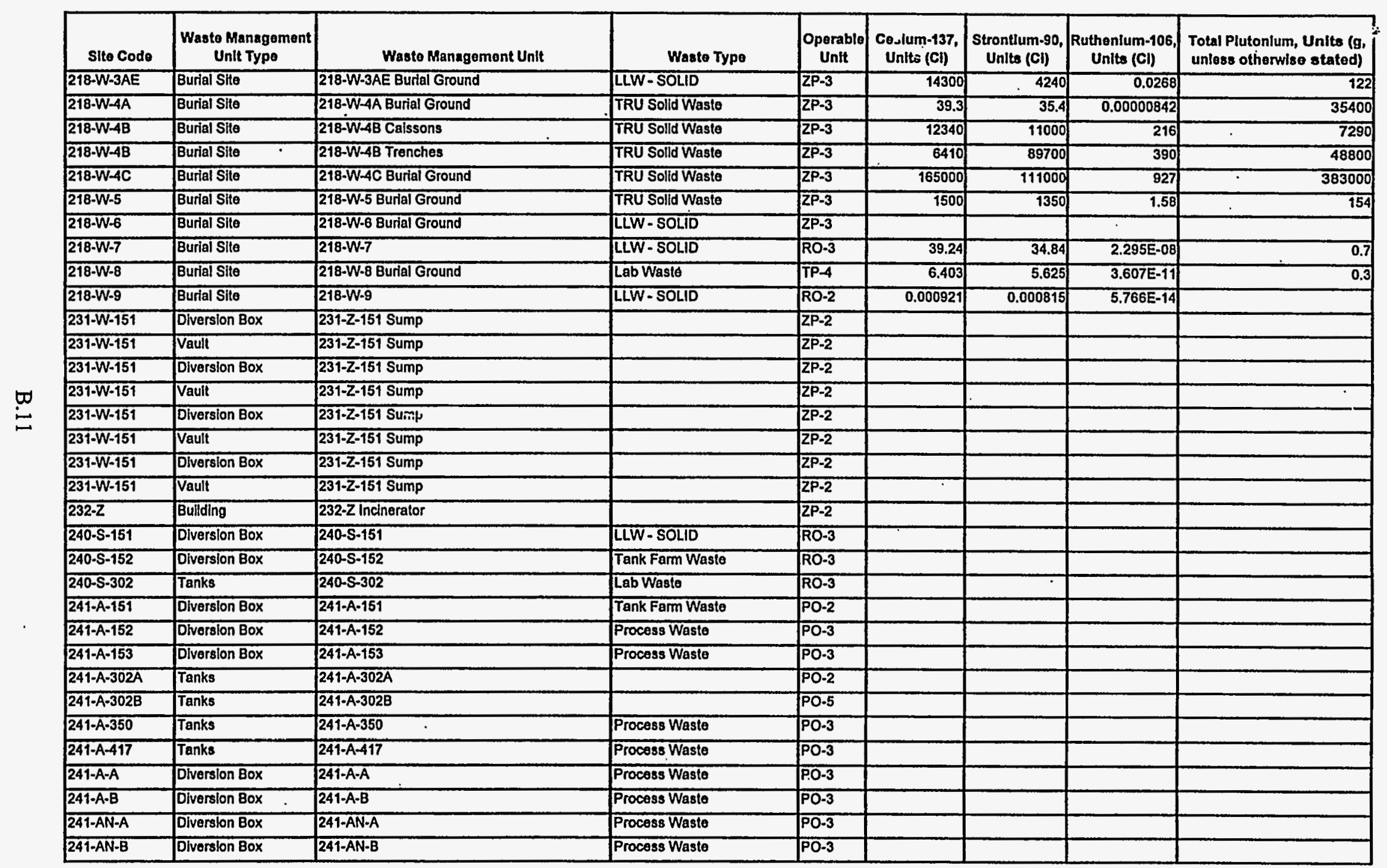


Table B.1. (page 11 of 14)

\begin{tabular}{|c|c|c|c|c|c|c|c|c|}
\hline Sito Codo & $\begin{array}{c}\text { Wasto Management } \\
\text { Unlt Typo }\end{array}$ & Wasto Management Unit & \begin{tabular}{|c} 
Wasto Typo \\
\end{tabular} & $\begin{array}{c}\text { Operable } \\
\text { Unit }\end{array}$ & $\begin{array}{c}\text { Cesislum-137, } \\
\text { Units (CI) }\end{array}$ & $\begin{array}{c}\text { Strontlum-90, } \\
\text { Units (CI) }\end{array}$ & $\begin{array}{c}\text { Ruthonlum-106, } \\
\text { Units (C) }\end{array}$ & $\begin{array}{l}\text { Total Plutonlum, Units (g, } \\
\text { unloss otherwlse stated) }\end{array}$ \\
\hline 241-APVP & Valve Pit & $\sqrt{241-A P}$ & Process Waste & PO-3 & & & & \\
\hline$\overline{241-A R-151}$ & Diversion Box & 241-AR-151 & Process Waste & PO-3 & & & & \\
\hline $241-A W-A$ & Diversion Box & $241-A W \cdot A$ & Process Waste & $\overline{P O-3}$ & & & & \\
\hline 241-AW-B & Diveralon Box & 241-AW'B & Process Waste & PO-3 & & & & \\
\hline $241-A X-151$ & Diversion Box & $241-A X-151$ & Process Waste & PO-3 & & & & \\
\hline 241-AX-152DS & Tanks & 241-AX-152DS & Procoss Waste & PO-3 & & & & \\
\hline $241-A X-155$ & Diversion Box & $241-A X-155$ & Tank Fam Waste & $\overline{P O .3}$ & & & & \\
\hline$\overline{241-A X-501}$ & Valve PII & $241-A X-501$ & & $\overline{\mathrm{PO}-3}$ & & & & \\
\hline $241-A X-A$ & Diverslon Box & $241-A X-A$ & & $\overline{\mathrm{PO}-3}$ & & & & \\
\hline $241-A X-B$ & Dlversion Box & $241-A X-B$ & & PO-3 & & & & \\
\hline $241-A Y-151$ & Diversion Box & $241-A Y-151$ & Process Waste & $\overline{\mathrm{PO}-3}$ & & & & \\
\hline $241-A Y-152$ & Diversion Box & $241-A Y-152$ & Process Waste & $\mathrm{PO}-3$ & & & & \\
\hline $241-A Z-1510 S$ & Diversion Box & 241-AZ-1510S & & $\overline{\mathrm{PO}-3}$ & & & & \\
\hline $241 \cdot A Z-152$ & Diversion Box & $241-A Z-152$ & & $\mathrm{PO}-3$ & & & & \\
\hline $241 \cdot C-151$ & Diversion Box & $241-\mathrm{C}-151$ & & PO-3 & & & & \\
\hline $241-C-152$ & Diversion Box & $\begin{array}{l}241-C-152 \\
\end{array}$ & & $\overline{\mathrm{PO}-3}$ & & & & \\
\hline $241-C-153$ & Diverston Box & $241-\mathrm{C}-153$ & & $\overline{\mathrm{PO}-3}$ & & & & \\
\hline $241-C-154$ & Diversion Box & 241-C-154 Diversion Box & Process Waste & SO-1 & & & & \\
\hline $241-C-252$ & Diversion Box & $241-C-252$ & & PO-3 & & & & \\
\hline $241-C-301 C$ & Tanks & $241-\mathrm{C}-301 \mathrm{C}$ & & $\overline{\mathrm{PO}-3}$ & & & & \\
\hline $241-C R-151$ & Diverston Box & $241-C R-151$ & & $\overline{\mathrm{PO}-3}$ & & & & . \\
\hline $241-C R-152$ & Diversion Box & $241-C R-152$ & & PO-3 & & & & \\
\hline $241-C R-153$ & Diverston Box & $241-\mathrm{CR}-153$ & & PO-3 & & & & \\
\hline $241 \cdot C X-T K-70$ & Tanks & 241-CX-70 Storage Tank & Tank Farm Waste & SO-1 & & & & \\
\hline $241 \cdot C X \cdot T K-71$ & Tanks & 241-CX-71 Storage Tank & Process Condensate & SO-1 & 0.0496 & 83 & & \\
\hline 241-CX-TK-72 & Tanks & 241-CX-72 Storage Tank & Process Waste & SO-1 & 15000 & 0.0000028 & & 200 \\
\hline 241-ER-153 & Diversion Box & 241-ER-153 & & PO-3 & & & & \\
\hline$\overline{241-S-151}$ & Diversion Box & $241-S-151$ & LLW-SOLID & $\overline{\mathrm{RO}-2}$ & & & & \\
\hline $241-S-152$ & Diversion Box & $241-5-152$ & Tank Farm Wasto & RO-4 & & & & \\
\hline $241-\mathrm{S}-302 \mathrm{~A}$ & Tanks & 241-S-302A & Lab Waste & RO-2 & & & & \\
\hline $241-S-302 B$ & Tanks & $241-S-3028$ & LLW-SOLID & $\overline{R O-4}$ & & & & \\
\hline $241-S-A$ & Diversion 8ox & $241-S-A$ & Tank Farm Waste & ROA & & & & \\
\hline $241-S-B$ & Diversion Box & 241-S-B & Tank Farm Waste & RO-4 & & & & \\
\hline
\end{tabular}


Table B.1. (page 12 of 14)

\begin{tabular}{|c|c|c|c|c|c|c|c|c|}
\hline Site Code & $\begin{array}{l}\text { Wasto Management } \\
\text { Unit Typo }\end{array}$ & Wasto Managoment Unit & Wasto Type & $\begin{array}{c}\text { Operable } \\
\text { Unit }\end{array}$ & $\begin{array}{l}\text { Cosium-137, } \\
\text { Units (Ci) }\end{array}$ & $\begin{array}{c}\text { Strontium-90, } \\
\text { Units (CI) }\end{array}$ & $\begin{array}{c}\text { Ruthenlum-106, } \\
\text { Uisilts (CI) }\end{array}$ & $\begin{array}{l}\text { Total Plutonlum, Units ( } g \text {, } \\
\text { unless otherwise stated) }\end{array}$ \\
\hline $241-S-C$ & Diversion Box & 241-S-C & Tank Farm Wasto & RO-4 & & & & \\
\hline 241-S-D & Diversion Box & 241-S-D & Tank Farm Waste & RO-4 & & & & \\
\hline $241-S X-151$ & Dlversion Box & $241-S X-151$ & Tank Farm Waste & ROA & & & & \\
\hline $241-S X-152$ & Diversion Box & $241-5 X-152$ & Tank Farm Waste & RO.4 & & & & \\
\hline $241-S X-302$ & Tanks & $241-5 X-302$ & & RO-2 & & & & \\
\hline $241-S X-A$ & Diversion Box & $241-S X-A$ & & RO-4 & & & & \\
\hline 241-SX-B & Diversion Box & $241-S X-B$ & & RO-4 & & & & \\
\hline 241-SY-A & Diversion Box & $241-S Y-A$ & & ROA-4 & & & & \\
\hline 241-SY-A & Diversion Box & $241-S Y-A$ & & ROA-4 & & & & \\
\hline 241-SY-B & Diversion Box & 241-SY-B & & RO-4 & & & & \\
\hline 241-SY-B & Diversion Box & 241-SY-B & $\cdot$ & RO-4 & & & & \\
\hline $241-T-151$ & Divérsion Box & 241-T-151 Diversion Box & Tank Farm Waste & TP-6 & & & & \\
\hline $241-T-152$ & Diversion BOX & 241-T-152 Diversion Box & Tank Farm Waste & TP.6 & & & & \\
\hline $241-T-153$ & Diversion Box & 241-T-153 Diversion Box & Tank Farm Waste & TP-B & & & & \\
\hline $241-T-252$ & Diverston Box & 241-T-252 Diversion Box & Tank Farm Waste & TP-6 & & & & \\
\hline $241-T-301$ & Tanks & 241-T-301 Catch Tank & Tank Fam Wasto & TP-6 & & & & \\
\hline $241-T-302$ & Tanks & 241-T-302 Catch Tank & Tank Fam Wasto & TP-6 & & & & \\
\hline $241-T-381$ & Tanks & 241-T-361 Settling Tank & Process Waste & TP-4 & & & & $15500 \mathrm{C}$ \\
\hline $24 t-T R-152$ & Diversion Box & 241-TR-152 Dlversion Box & Tank Farm Wasto & $T P-6$ & & & & \\
\hline 241-TR-153 & Diversion Box & 241-TR-153 Diversion Box & Tank Farm Waste & TP-6 & & & & \\
\hline $241-T X-152$ & Diversion Box & 241-TX-152 Dlvarslon BoX & Tank Farm Wasto & TP-2 & & & & \\
\hline $241-T X-153$ & Diversion Box & 241-TX-153 Dlversion BoX & Tank Farm Waste & TP-S & & & . & \\
\hline $241-T X-154$ & Diversion Box & 241-TX-154 Dlversion BoX & Tank Farm Wasto & TP-4 & & & & \\
\hline $241-T X-155$ & Diversion Box & 241-TX-155 Dlversion Box & Tank Farm Wasto & TP-2 & & & & \\
\hline $241-T X-302 A$ & Tanks & 241-TX-302A Catch Tank & Tank Farm Wasto & TP.5 & & & & \\
\hline $241-T X-302 B$ & Tanks & 241-TX-302B Calch Tank & Tank Farm Waste & $T P-2$ & & & & \\
\hline $241-\mathrm{TX}-302 \mathrm{C}$ & Tanks & 241-TX-302C Catch Tank & Tank Farm Waste & TPA & & & & \\
\hline 241-TXR-151 & Diversion Box & 241-TXR-151 Diversion Box & Tank Farm Wasto & TP-5 & & & & \\
\hline 241-TXR-152 & Diverston Box & 241-TXR-152 Dlveralon Box & Tank Farm Wasto & TP-5 & & & & \\
\hline $241-T X R-153$ & Dhersion Box & 241-TXR-153 Diversion Box & Tank Farm Waste & TP.5 & & & & \\
\hline 241-TY-163 & Diversion Box & 241-TY-153 Diversion Box & Tank Farm Wasto & TP-5 & & & & \\
\hline 241-TY-302A & Tanks & 241-TY-302A Catch Tank & Tank Farm Waste & TP.5 & & & & \\
\hline 241-TY-3028 & Tanks & 241-TY-302B Catch Tank & Tank Farm Waste & TP-5 & & & & \\
\hline
\end{tabular}


Table B.1. (page 13 of 14)

\begin{tabular}{|c|c|c|c|c|c|c|c|c|}
\hline Site Codo & \begin{tabular}{|c|}
$\begin{array}{c}\text { Wasto Management } \\
\text { Untt Type }\end{array}$ \\
\end{tabular} & Wasto Managementi Unit & \begin{tabular}{|l} 
Waste Typs \\
\end{tabular} & $\begin{array}{c}\text { Oparable } \\
\text { Unit }\end{array}$ & $\begin{array}{c}\text { Cesium-137, } \\
\text { Units (Ci) }\end{array}$ & $\begin{array}{c}\text { Strontlum }-90, \\
\text { Units (CI) }\end{array}$ & $\begin{array}{l}\text { Ruthonlum-106, } \\
\text { Units (CI) }\end{array}$ & $\begin{array}{l}\text { Total Plutonlum, Units (g, } \\
\text { ulitess otherwlse statod) }\end{array}$ \\
\hline $241-z-361$ & \begin{tabular}{|l|l} 
Tanks \\
\end{tabular} & 241-Z-361 Settlling Tank & Procoss Waste & \begin{tabular}{|l|l|}
$\mathrm{PP}-2$ \\
\end{tabular} & & & & 75000 \\
\hline 241-Z-TK-8 & Tanks & 216-Z-8 Settling Tank & Process Waste & $2 \mathrm{PP}-2$ & & & & 1600 \\
\hline 241-Z-TK-D5 & Tanks & 241-Z Treatment Tank & Process Wasto & $\mathrm{ZP}-2$ & & & & \\
\hline $242-T-151$ & \begin{tabular}{|l|} 
Diverston Box \\
\end{tabular} & 242-T-151 Diversion Box & Process Condensate & TP-5 & & & & \\
\hline 244-A RT & Tanks & $244-A$ & Procoss Wasto & $\mathrm{PO}-3$ & & & & \\
\hline 244-AR VAULT & Vauit & 244-AR & Process Wasto & PO-3 & & & & \\
\hline 244-CR VAULT & Vault & $244-C R$ & Process Waste & PO.3 & & & & \\
\hline 244-S RT & Tranks & 244-S Receiver Tank & & RO-2 & & & & \\
\hline 244-TXRT & Tanks & 244-TX Receiving Tank & & TP-5 & & & & \\
\hline 244-TXR & Vault & 244-TXR Vault & Tank Farm Waste & TP-5 & & & & \\
\hline $2607-E 5$ & Septlic System & 2607-E-5 Septic Tank and Drain Fleld & Sanitary Wasto & 50-1 & & & & \\
\hline 2607-EB & Septic System & 2607-E6 & Sanitary Wasto & $\mathrm{PO}-2$ & & & & \\
\hline 2607-E7A & Septlc Systom & 2607-E-7A Septlic Tank and Drain Field & Sanitary Waste & 50.1 & & & & \\
\hline $2607-\mathrm{EA}$ & Septlic System & 2607-EA & Santtary Waste & PO-2 & & & & \\
\hline 2607-EC & Septic System & 2607-EC & Sanitary Was!3 & PO-5 & & & & \\
\hline 2607 -ED & Septlc System & 2607-ED & Sanitary Waste & $\overline{P O-3}$ & & & & \\
\hline 2607-EE & Septlic System & $2607-E L$ & Santtany Waste & $\mathrm{PO}-2$ & & & & \\
\hline 2607 -EG & Septic System & 2607-EG & Sanitary Wasto & PO-3 & & & & \\
\hline $2607-\mathrm{EJ}$ & Seplic System & 2607 -EJ & Santtary Waste & $\overline{P 0-3}$ & & & & \\
\hline $2607-\mathrm{N}$ & Seplic System & 2807-N Septlc Tank/Drain Fiald & Sanitary Waste & $\mathrm{NO}$ & & & & \\
\hline$\frac{2607-P}{2607-R}$ & Seplic System & 2807-P Septic Tank/Drain Fleld & Sanltary Wasto & NO-1 & & & & \\
\hline $\begin{array}{l}2607-R \\
2607-W 1\end{array}$ & \begin{tabular}{|l} 
Septlc System \\
Septic System
\end{tabular} & $\frac{2607-R \text { Septic Tank/Drain Field }}{2607-\text { W1 Septle Tank }}$ & $\begin{array}{l}\text { Sanitary Waste } \\
\text { Sanitary Wasto }\end{array}$ & $\frac{\mathrm{Na}-1}{\mathrm{sS-2}}$ & & & & \\
\hline 2607-W/2 & Septic System & 2607-W2 Soptle Tank & Sanilary Waste & SS-2 & & & & \\
\hline$\overline{2607-W_{3}^{3}}$ & Soptic System & 2607-W3 Septlc Tank & Saniltary Wasto & TP-4 & & & & \\
\hline $2607-\mathrm{W}^{4}$ & Septlc System & 2607-W4 Septic Tank & Sanitary Waste & $T P-4$ & & & & \\
\hline 2607-WB & Sepllic System & 2607-WB & Sanitary Waste & RO-3 & & & & \\
\hline 2607-W8 & Septic System & 2807-W-8 Seplle Tank and Drain Field & Santlary Wasto & $\mathrm{ZP}-2$ & & & & \\
\hline 2807-WA & Septic System & 2007-WA Septlc Tank and Drain Field & Sanittary Waste & $\mathrm{ZP}-2$ & & & & \\
\hline 2607-WB & & 2607-WB Septic Tank and Drain Fleld & & & & & & \\
\hline 2607-WT & Soptle System & 2607-WT Septle Tank & Sanitary Waste & TP-5 & & & & \\
\hline$\frac{2607-W I X}{2607-W z}$ & $\begin{array}{l}\text { Septle System } \\
\text { Septic System }\end{array}$ & $\frac{2607-W T X \text { Septic Tank }}{2607-W Z}$ & \begin{tabular}{|l} 
Sanitary Wasto \\
Sanitary Wasto
\end{tabular} & $\frac{T P-5}{R O-1}$ & & & & \\
\hline 2607.2 & Seplic System & 2607-2 Septic Tank and Draln Field & Sanitary Wasto & $2 P-2$ & & & & \\
\hline
\end{tabular}


Table B.1. (page 14 of 14)

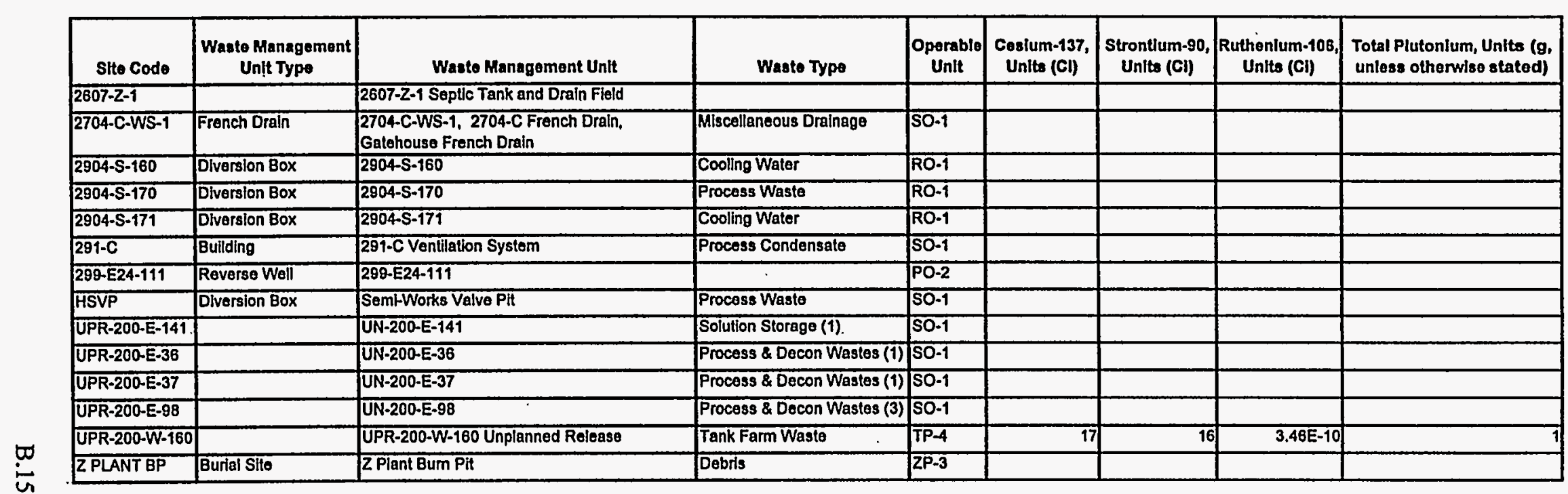


Table B.2. Environmental Restoration Waste Site Inventories for Plutonium-238, -239, -240, and -241 (page 1 of 14)

\begin{tabular}{|c|c|c|c|c|c|c|c|c|}
\hline Stte Code & $\begin{array}{c}\text { Waate Management } \\
\text { Unit Type. }\end{array}$ & Waste Management Unit & Wasto Typo & $\left|\begin{array}{c}\text { Operablo } \\
\text { Unit }\end{array}\right|$ & $\begin{array}{l}\text { Plutonlum-238, } \\
\text { Units (CI) }\end{array}$ & $\begin{array}{l}\text { Plutonlum-239, } \\
\text { Units (CI) }\end{array}$ & $\begin{array}{l}\text { Plutonlum-240, } \\
\text { Units (C) }\end{array}$ & $\begin{array}{l}\text { Plutonlum-241, } \\
\text { Units (CI) }\end{array}$ \\
\hline & & $212-N$ to 216-N-1 Pipeline & & & & & & \\
\hline & & 212-P Hazardous Waste Slaglng Area & & & & & & \\
\hline & & 212-P to 216-N-4 Plpeline & & & & & & \\
\hline & & 212-P Transformer Oil Tank & & & & & & \\
\hline & & 212-R to 216-N-6 Plpeline & & & & & & \\
\hline & & 241-C Waste Line Unplanned Reloase No. 1 & & & & & & \\
\hline & & 241.C Waste Line Unplanned Release No. 2 & & & & & & \\
\hline & & 241-Z Diversion Box No. 1 & & & & & & \\
\hline & & 241-Z Dlversion Box No. 2 & & & & & & \\
\hline & & Sanitary Crib & & & & & & \\
\hline 200-E BP & Burial Site & 200-E Buming PHt & Debris & $\overline{P 0.6}$ & & & & \\
\hline 200-E PAP & Burial Sito & 200-EAsh PIt & & SS-1 & & & & \\
\hline 200-EPD & Ditches & 200 East Powerhouse Ditch & Coolling Water & 50.1 & & & & \\
\hline $200-E-4$ & Fronch Drailn & Critical Mass Laboratory Dry Well North & Miscellaneous Drainage & so-1 & & & & \\
\hline $200-\mathrm{N}-3$ & Burial Site & Ballast Pits & Debris & NO-1 & & & & \\
\hline $200-W$ ADB & Burdal Sitio & 200-WAsh Disposal Basin & $\overline{\text { Ash }}$ & sS-2 & & & & \\
\hline 200-WADS & Burial Sito & 200-WAsh Pit Demolition Sito & N/A & ss-2 & & & & \\
\hline $200-W B P$ & Burial Site & 200-W Bumlng Plf & Debris & Ss-2 & & & & \\
\hline 200-WPAP & Eurlal Site & 200-W Powerhouso Ash Pit & $\overline{\text { Ash }}$ & SS-2 & & & & \\
\hline 200-WPP & Ponds & 200-WPowerhouse Pond & Cooling Water & $T \mathrm{TP}=2$ & & & & \\
\hline $201-C$ & Bullding & 201-C Process Bullding & Process Condensate & SO-1 & 3.7 & 4.9 & & \\
\hline$\overline{207-A}$ & & $207-\mathrm{A}$ & & & & & & \\
\hline $207-B$ & Retention Basin & 207-Bb/Retention Basin & Coollng Water & BP-8 & & & & \\
\hline $207-5$ & Retention Basin & $207-5$ & Cooling Water & $\overline{R O .2}$ & & & & \\
\hline$\overline{\text { 207-SL }}$ & Retention Basin & 207-SL & Lab Wasto & RO-3 & & & & \\
\hline$\overline{207-T}$ & Retention Basin & 207-T Retention Basin & Cooling Water & TP-3 & & & & \\
\hline $207-2$ & Retentlon Basin & 207-Z Retention Basin & Steam Condensate & $2 \mathrm{P}-2$ & & & & \\
\hline$\overline{\text { 200-E-WS-1 }}$ & French Drain & Critical Mass Laboratory Dry Well East & Miscellaneous Drainage & so-1 & & & & \\
\hline$\overline{\text { 209-E.WS-2 }}$ & Fronch Drain & Crtical Mass Laboratory Dry Well South & Miscellaneous Dralnage & $50-1$ & & & & \\
\hline 209-E-WS-3 & Diversion Box & Critheal Mass Laboratory Valve Plt & Procoss Wasto & SO-1 & & & & \\
\hline 2101-MPOND & Ponds & 2101-MPond & LabWaste & SS-1 & & & & \\
\hline $216-A-1$ & Cribs & $216-A-1$ & Process Wasto & PO-5 & & 0.00571 & 0.00154 & \\
\hline $216-A-10$ & Cribs & $216-A-10$ & Process Condensato & PO-2 & 0.329 & 3.49 & & 42.3 \\
\hline
\end{tabular}


Table B.2. (page 2 of 14)

\begin{tabular}{|c|c|c|c|c|c|c|c|c|}
\hline Site Code & $\begin{array}{c}\text { Waste Management } \\
\text { Unlt Type }\end{array}$ & - Waste Management Unit & Wasto Typo & $\begin{array}{c}\text { Operablo } \\
\text { Unit }\end{array}$ & $\begin{array}{c}\text { Plutonium-238, } \\
\text { Units (CI) }\end{array}$ & $\begin{array}{l}\text { Plutonlum-239, } \\
\text { Unitts (CI) }\end{array}$ & $\begin{array}{l}\text { Plutonium-240, } \\
\text { Units (CI) }\end{array}$ & \begin{tabular}{|c} 
Pilutonium-241, \\
Units (CI)
\end{tabular} \\
\hline $216-A-11$ & French Drain & $216-A-11$ & Miscellaneous Drainage & PO-2 & & & & \\
\hline$\overline{216-A-12}$ & French Drain & $216-A-12$ & Miscellaneous Drainage & PO-2 & & & & \\
\hline $216-A-13$ & French Draln & $216-A-13$ & Miscellaneous Drainage & PO-2 & & & & \\
\hline $216-A-14$ & French Draln & $216-A-14$ & Miscellaneous Drainago & PO-2 & & & & \\
\hline $216-A \cdot 15$ & French Draln & $216-A-15$ & Process Condensate & PO-2 & & & & \\
\hline $216-A-16$ & French Drain & $216-A-16$ & Chemlcal Sewer & PO-5 & & & & \\
\hline $216-A-17$ & French Drain & $216-A-17$ & Chemical Sewer & PO-5 & & & & \\
\hline $216-A-18$ & Trench & $216-A-18$ & Process Waste & $\mathrm{PO}-5$ & & 0.00571 & 0.00154 & \\
\hline$\overline{216 \cdot A-19}$ & Trench & $216-A-19$ & Process Waste & PO-5 & & 0.00571 & 0.00154 & \\
\hline $216 \cdot A \cdot 2$ & Cribs & $216-A-2$ & Process Waste & PO-2 & & 7.42 & 2 & \\
\hline $216-A-20$ & Trench & $\overline{216-A-20}$ & Process Waste & PO-5 & & $\overline{0.00571}$ & 0.00154 & \\
\hline $216-A-21$ & Cribs & 216-A-21 & Lab Waste & PO-2 & & 8.56 & 2.31 & \\
\hline $216-A-22$ & French Drain & $216-A-22$ & Miscellaneous Drainage & $\mathrm{PO}-2$ & & & & \\
\hline 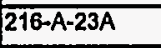 & French Drain & $216-A-23 A$ & Process Condensate & PO-5 & & & & \\
\hline 216-A-23B & French Drain & $216-A-23 B$ & Process Condensate & PO-5 & & & & \\
\hline $\begin{array}{l}216-A \cdot 24 \\
\end{array}$ & Cribs & $216-A-24$ & Process Condensato & PO-5 & & 0.289 & 0.0779 & \\
\hline $216 \cdot A-25$ & Ponds & 216-A-25 Pond & Cooling Waler & IU.6 & & . & & \\
\hline $216-A-26$ & French Drain & $216-A-2 B$ & Miscellaneous Dralnage & PO.2 & & & & \\
\hline $216-A-26 A$ & French Drain & 2 & Miscellaneous Dralnage & PO-2 & & & & \\
\hline $216 \cdot A-27$ & Cribs & $218-A-27$ & Miscellaneous Dralnage & PO-2 & & 5.51 & 1.49 & \\
\hline $216-A-28$ & Cribs & $218-A-28$ & Process Condensate & PO-2 & & & & \\
\hline $216-A-28$ & Ditches & 216-A-29 & Chemical Sewer & BP-11 & & & & \\
\hline $216-A-3$ & Cribs & $216-A-3$ & Process Waste & $\sqrt{\mathrm{PO}-2}$ & & & & \\
\hline $216 \cdot A \cdot 30$ & Cribs & $216-A-30$ & Steam Condensate & PO-4 & & 0.0751 & & \\
\hline $216-A-31$ & Cribs & 216-A-31 & Procoss Waste & $\overline{\mathrm{PO}-2}$ & & 0.514 & 0.139 & \\
\hline $216-A-32$ & Cribs & $216-A-32$ & Miscellaneous Drainago & PO-2 & & & & \\
\hline$\sqrt{216 \cdot A-33}$ & French Drain & $216-A-33$ & Miscellaneous Dralnage & PO-2 & & & & \\
\hline $218-A-34$ & Cribs & $216-A-34$ & Process Condensate & PO-5 & & & & \\
\hline $216-A-35$ & French Drain & $216-A-35$ & Miscellaneous Drainage & PO-2 & & & & \\
\hline $216-A-36 \bar{A}$ & Cribs & $216-A-36 A$ & Process Waslo & PO-2 & & 4.57 & 1.23 & \\
\hline $216-A-36 B$ & Cribs & $216-A-36 B$ & Process Wasto & PO-2 & & 0.0569 & & 0.558 \\
\hline 216-A-37-1 & Cribs & $216-A-37-1$ & Procoss Condensalo & PO-A & & 0.000201 & & \\
\hline $\mid$ & Cribs & $216-A-37-2$ & Steam Condensate & PO-4 & & 373 & & \\
\hline
\end{tabular}


Table B.2. (page 3 of 14)

\begin{tabular}{|c|c|c|c|c|c|c|c|c|}
\hline Site Code & $\begin{array}{c}\text { Wasto Maragement } \\
\text { Unit Typo }\end{array}$ & Wasto Management Unit & Waste Typo & $\begin{array}{c}\text { Operablo } \\
\text { Unit }\end{array}$ & $\begin{array}{l}\text { Plutonlum-238, } \\
\text { Units (C1) }\end{array}$ & $\begin{array}{l}\text { Plutonlum-239, } \\
\text { Units (Cl) }\end{array}$ & $\begin{array}{l}\text { Piutonlum-240, } \\
\text { Units (Ci) }\end{array}$ & $\begin{array}{l}\text { Plutonium-241, } \\
\text { Units (CI) }\end{array}$ \\
\hline $216-A-38-1$ & Cribs & $216-A-38-1$ & N/A & PO-2 & & & & \\
\hline 216-A-39 & Ditches & $216-A-3 \theta$ & Miscellaneous Drainago & $\mathrm{PO}-3$ & & & & \\
\hline $216-A-4$ & Cribs & $216-A-4$ & Lab Wasto & $\overline{\mathrm{PO}-2}$ & & 7.99 & 2.16 & \\
\hline $216-A-40$ & Relentlon Basin & $216 \cdot A-40$ & Steam Condensate & $\overline{\mathrm{PO}-2}$ & & & & \\
\hline $216-A-41$ & Cribs & $216-A-41$ & Miscellaneous Drainage & $\longdiv { \mathrm { PO } - 2 }$ & & & & \\
\hline $216-A-42$ & Relention Basin & $216-A-42$ & Coolhg Water & $\overline{P O A}$ & & & & \\
\hline $216-A-45$ & Cribs & $216-A-45$ & Process Condensate & $\overline{P O-2}$ & 0.00613 & 0.0556 & & 0.658 \\
\hline $216-A-5$ & Cribs & 216-A-5 & Process Condensate & PO-2 & & 3.71 & 1 & \\
\hline $216-A-524$ & Diverslon Box & $216-A-524$ & & PO-5 & & & & \\
\hline $216-A-6$ & Cribs & $216-A-6$ & Steam Condensato & $\overline{\mathrm{PO}-4}$ & & 2.09 & 0.548 & \\
\hline $216-A-7$ & Cribs & 216-A-7 & Process Wasto & PO-5 & & 0.0571 & 0.0154 & \\
\hline $216-A-B$ & Cribs & $216-A-6$ & Process Condensato & PO-5 & & & & \\
\hline $216-A-9$ & cribs & $216-A-9$ & Cooling Water & PO-2 & & 0.02851 & 0.0077 & \\
\hline 216-B-10A & Cribs & 216-B-10A Crib & Lab Waste & BP.6 & 0 & $\overline{0.56}$ & & 0 \\
\hline 216-8-10B & Cribs & 216-8-10B Crib & Lab Wasto & BP.6 & 0 & 0 & & 0) \\
\hline 216-B-11A\&B & Reverse Well & 216-B-11A\&B Reverse Welis & Process Condensate & BP-4 & 0 & 0.228 & & 0 \\
\hline $216-\mathrm{B}-12$ & Cribs & 216-B-12 Crib & Process Condensate & BP-9 & 0 & 21.4 & & 0 \\
\hline 216-B-13 & French Drain & 216-8-13 French Drain & Miscellaneous Dralnage & BP.6 & & 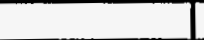 & & \\
\hline 216-B-14 & Cribs & $216 \cdot 8-14 \mathrm{Crb}$ & Scavenged Waste & BP-2 & 0 & 1.43 & & 0 \\
\hline 216-B-15 & Cribs & $216-8-15$ Crib & Scavenged Waste & BP-2 & 0 & 0.285 & & 0 \\
\hline $216-B-16$ & Cribs & 216-B-16 Crib & Scavenged Waste & BP-2 & 0 & 0.571 & & 0 \\
\hline $218-B-17$ & Cribs & 216-8-17 Crib & Scavenged Waste & BP-2 & 0 & 0.571 & & 0 \\
\hline 216-8-18 & Cribs & 216-B-18 Crib & Scavenged Wasto & BP-2 & 0 & 0.571 & & 0 \\
\hline 216-8-19 & Cribs & 216-B-19 Crib & Scavenged Wasto & $\overline{B P-2}$ & 0 & 0.571 & & 0 \\
\hline$\overline{216-8-2-1}$ & Ditches & 216-B-2-1 DHchb/ & Cooling Waler & $B P-11$ & 0.0026 & 0.799 & & \\
\hline $216-8-2-2$ & Ditches & 216-8-2-2 Ditchel & Cooling Waler & BP-11 & & 0.0024 & & $\overline{0}$ \\
\hline $216-B-2-3$ & Ditches & 216-B-2-3 Ditch & Cooling Water & $\mathrm{BP}-11$ & & & & \\
\hline $216-8-20$ & Trench & 216-8-20 Trench & Scavenged Waste & $B P-2$ & 0 & 0.0742 & & 0 \\
\hline $210-B-21$ & Trench & 216-8-21 Trench & Scavenged Waste & BP-2 & 0 & 0.58 & & 0 \\
\hline $216-8-22$ & Trench & 216-8-22 Trench & Scavenged Wasto & BP-2 & 0 & 0.148 & & 0 \\
\hline $216-B-23$ & Trench & 216-B-23 Trench & Scavenged Waste & BP-2 & - & 0.102 & & 0 \\
\hline $216-B-24$ & Trench & 216-9-24 Trench & Scavonged Wasto & BP-2 & 0 & 0.44 & & 0 \\
\hline $216-B-25$ & Trench & 216-B-25 Trench & Scavenged Waste & BP-2 & 0 & 0.114 & & 0 \\
\hline
\end{tabular}


Table B.2. (page 4 of 14)

\begin{tabular}{|c|c|c|c|c|c|c|c|c|}
\hline Stte Code & $\begin{array}{c}\text { Weste Management } \\
\text { Unit Type }\end{array}$ & Waste Management Unit & Waste Type & $\begin{array}{c}\text { Operablo } \\
\text { Unit }\end{array}$ & $\begin{array}{c}\text { Plutonium-238, } \\
\text { Units (Ci) }\end{array}$ & $\begin{array}{c}\text { Plutonlum-239, } \\
\text { Units (CI) }\end{array}$ & $\begin{array}{l}\text { Plutonlum-240, } \\
\text { Units (CI) }\end{array}$ & $\begin{array}{l}\text { Plutonium-241, } \\
\text { Units (Ci) }\end{array}$ \\
\hline 216-8-26 & \begin{tabular}{|l|} 
Trench \\
\end{tabular} & 216-8-26 Trench & Scavenged Wasto & BP-2 & 0 & 0.143 & & \\
\hline$\overline{216-8-27}$ & Trench & 216-8-27 Trench & Scavenged Waste & BP-2 & 0 & 0.04 & & \\
\hline $216-B-28$ & Trench & 216-B-28 Trench & Scavenged Waste & BP-2 & o & 0.32 & & \\
\hline 216-8-29 & Trench & 216-B-29 Trench & Scavenged Wasto & $\mathrm{BP}-2$ & 0 & 0.0628 & & \\
\hline$\overline{216-\mathrm{B}-3}$ & Ponds & 216-B-3 Pondel & Cooling Water & $\mathrm{BP}-11$ & 0.0026 & 0.799 & & \\
\hline$\overline{216 \cdot B-3-1}$ & Ditches & 216-B-3-1 Ditchb/ & Cooling Water & BP-11 & & & & \\
\hline 216-B-3-2 & Ditches & 216-B-3-2 Ditchal & Cooling Water & $B P-11$ & & & & \\
\hline $\begin{array}{l}216-8-3-3 \\
\end{array}$ & Ditches & 216-B-3-3 Ditch & Cooling Water & BP-11 & & & & \\
\hline $216 \cdot B \cdot 30$ & Trench & 216-B-30 Trench & Scavenged Waste & $\overline{B P-2}$ & 0 & 0.12 & & \\
\hline $218-B-32$ & Trench & 216-B-32 Trench & Scavenged Wasto & $B P-2$ & $\overline{0}$ & 0.148 & & \\
\hline$\overline{216-B-33}$ & Trench & 216-B-33 Trench & Scavenged Waste & $\longdiv { B P - 2 }$ & 0 & 0.674 & & \\
\hline 216-B-34 & Trench & 216-B-34 Tronch & Scavenged Waste & $B \mathrm{PP}-2$ & 0 & 0.325 & & \\
\hline $216-\mathrm{B}-35$ & Trench & 216-B-35 Trench & Tank Farm Wasto & BP-3 & 0 & 0.0685 & & \\
\hline 216-B-36 & Trench & 216-B-36 Trench & Tank Farm Wasto & BP-3 & 0 & 0.0457 & & \\
\hline 216-8-37 & Trench & 216-B-37 Trench & Process Waste & $B P-3$ & 0 & 0.114 & & \\
\hline 218-B-38 & Trench & 216-B-38 Trench & Tank Farm Waste & $B P-3$ & 0 & 0.0685 & & \\
\hline 216-8-39. & Trench & 216-8-39 Trench & Tank Farm Wasto & $B P-3$ & 0 & 0.0826 & & \\
\hline $216-8-3 A$ & Ponds & 218-8-3A Pond & Cooling Water & BP-11 & & & & \\
\hline 216-B-3B & Ponds & 216-8-3B Pond & Coolling Water & $\overline{B P}-11$ & & & & \\
\hline $216-8-3 C$ & Ponds & 216-B-3C Pond & Cooling Water & BP-11 & & & & \\
\hline $216 \cdot 8-4$ & Reverso Well & 210-B-4 Reverse Well & Miscellaneous Drainage & BP-6 & 0 & 0 & & \\
\hline $216-B-40$ & Trench & 216-8-40 Trench & Tank Farm Wasto & BP-3 & $\overline{0}$ & 0.0571 & & \\
\hline $216 \cdot 8-41$ & Trench & 216-8-41 Trench & Tank Farm Waste & $B P-3$ & & 0.0171 & & \\
\hline $216-B-42$ & Trench & 216-B-42 Trench & Scavenged Waste & $\mathrm{BP}-3$ & 0 & 0.0571 & & \\
\hline $216-8-43$ & Cribs & 216-B-43 Crib & Scavenged Waste & BP-1 & 0 & 0.0285 & & \\
\hline $218-B-44$ & Cribs & $216-B-44$ Crib & Scavenged Waste & BP-1 & $\overline{0}$ & 0.856 & & \\
\hline $216-8-45$ & Cribs & $\longdiv { 2 1 6 - B - 4 5 \text { Crib } }$ & Scavenged Waste & BP-1 & $\overline{0}$ & 0.571 & & \\
\hline $216-B-48$ & Cribs & $216-B-A B$ Crib & Scavenged Waste & $\mathrm{BP}-1$ & & 1010 & & \\
\hline $216-8-47$ & Cribs & 216-B-47 Crib & Scavenged Waste & BP-1 & 0 & 0.285 & & \\
\hline $218-B-48$ & Cribs & 216-B-A8 Crib & Scavenged Wasto & BP-1 & $\overline{0}$ & 0.285 & & \\
\hline $218-B-49$ & Cribs & 216-B-49 Crib & Scavenged Waste & BP-1 & $\overline{0}$ & 0.856 & & \\
\hline $218-B \cdot 5$ & Reverse Well & 216-B-5 Reverse Well & Procoss Waste & $8 \mathrm{PP}-6$ & 0 & 244 & & \\
\hline 218-B-50 & Cribs & 210-B-50 Críb & Process Condensato & BP-1 & 0 & 0.0136 & & \\
\hline
\end{tabular}


Table B.2. (page 5 of 14)

\begin{tabular}{|c|c|c|c|c|c|c|c|c|}
\hline Slte Codo & $\begin{array}{c}\text { Wasto Managemont } \\
\text { Unit Typo }\end{array}$ & Waste Managoment Unit & - Waste Type & $\begin{array}{c}\text { Operablo } \\
\text { Unit }\end{array}$ & $\begin{array}{l}\text { Plutonium-238, } \\
\text { Units (CI) }\end{array}$ & $\begin{array}{l}\text { Plutonium-239, } \\
\text { Unlts (Ci) }\end{array}$ & $\begin{array}{l}\text { Plutonium-240, } \\
\text { - Unlts (Ci) }\end{array}$ & $\begin{array}{l}\text { Plutonlum-241, } \\
\text { Units (Cl) }\end{array}$ \\
\hline $216-8-51$ & French Drain & 216-8-51 French Draln & Miscellaneous Drainage & $8 P-4$ & & & & \\
\hline $216-\mathrm{B}-52$ & Trench & $218-8.52$ Trench & Scavenged Waste & BP-2 & $\overline{0}$ & 1.08 & & $\overline{0}$ \\
\hline$\overline{216-B-53 A}$ & Trench & 216-B-53A Trench & Lab Wasto & $\overline{B P-2}$ & $\overline{0}$ & 5.71 & & $\overline{0}$ \\
\hline $216 \cdot B-53 B$ & Trench & 216-8-53B Trench & Lab Wasto & BP-2 & $\overline{0}$ & 0.285 & & $\overline{0}$ \\
\hline $216-8-54$ & Trench & 216-B-54 Trench & Lab Waste & BP-2 & $\overline{0}$ & 0.285 & & $\overline{0}$ \\
\hline $216-8-55$ & Cribs & 216-B-55 Crib & Sleam Condensate & $B P-9$ & 0 & 0.0000038 & & $\overline{0}$ \\
\hline $216-8-58$ & Cribs & 216-8-56 Crib & N/A & $\overline{B P-6}$ & & & & \\
\hline $216-8 \cdot 57$ & Cribs & 216-B-57 Crib & Process Condensate & $\overline{B P-1}$ & $\overline{0}$ & 0.0106 & & 0 \\
\hline $216-8-58$ & Trench & 216-9-58 Trench & Lab Waste & BP-2 & 0 & 0.393 & & $\overline{0}$ \\
\hline 216-8-59 & Rolention Basin & 216-B-59 Basin & Coolling Water & $\overline{B P-6}$ & & & & \\
\hline $216-8-6$ & Reverse Well & 216-B-6 Reverse Woil & LabWaste & $\overline{B P} \cdot 6$ & $\overline{0}$ & 0 & & 0 \\
\hline $216-\mathrm{B}-60$ & Cribs & 216-8-60 Crib & Decon Wasto & BP-6 & & & & \\
\hline $216-B-61$ & Cribs & 216-8-61 Crib & N/A & $\overline{\mathrm{BP}-1}$ & & & & \\
\hline $216-B-62$ & Cribs & 216-B-62 Crib & Process Condensato & BP-9 & & 0.0023 & & \\
\hline $216 \cdot 8 \cdot 63$ & Ditchos & 216-B-63 Trench & Chemical Sewer & $\overline{B P-11}$ & & 0.0108 & & \\
\hline $216-B-64$ & Retenlion Basin & 216-B-64 Basin & N/A & $B P-9$ & & & & \\
\hline 216-B-7A\&B & Cribs & 216-B-7A\&B Crib & Process Wáste & $\overline{B P-4}$ & $\overline{0}$ & 246 & & $\overline{0}$ \\
\hline $216 \cdot 8 \cdot 8$ & Cribs & 216-B-8TF Crib & Process Waste & $\longdiv { B P - 4 }$ & 0 & 1.7 & & $\overline{0}$ \\
\hline 216-B-9 & Cribs & 216-B-9TF Crib & Process Wasto & BP-6 & 0 & 8.94 & & $\overline{0}$ \\
\hline 216-C-1 & Cribs & $216-C-1$ Crib & Process Condensate & SO-1 & & 0.4579 & 0.123 & \\
\hline$\overline{216-C-10}$ & Cribs & $216-C-10 \mathrm{Crib}$ & Process Condensato & $50-1$ & & & & \\
\hline $210-C-2$ & Roverse Well & 216-C-2 Reverse Well & Mlscellaneous Drainago & SO.1 & & & & \\
\hline 218-C-3 & Cribs & 216-C-3 Crib & Process Wasto & SO-1 & & & & \\
\hline $216-C-4$ & Cribs & 218-C-4 Crib & Procoss Waste & $50-1$ & & & & \\
\hline$\overline{216-C-5}$ & Cribs & 216-C-5 Crib & Process Wasto & SO-1 & & & & \\
\hline $218-C-6$ & Cribs & 216-C.6 Crb & Process Condensate & $50-1$ & & & & \\
\hline 216-C-7 & Cribs & 216-C-7 Crib & Process Waste & SO-1 & & & & \\
\hline $216-C-8$ & French Draln & $216-C-8$ & Process Wasto & PO-3 & & & & \\
\hline 216-c-9 & Ponds & 216-C-8Pond & Cooling Water & $50-1$ & & & & \\
\hline 216-E-28 & Ponds & 216-E-28 Pond & N/A & BP-11 & & & & \\
\hline $216-N-1$ & Ponds & 216-N-1 Pond & Coolling Water & NO-1 & & & & \\
\hline $216-N-2$ & Trench & 216-N-2 Trench & Cooling Water & NO-1 & & & & \\
\hline $216-N-3$ & Trench & 216-N-3 Trench & Cooling Water & NO-1 & & & & \\
\hline
\end{tabular}


Table B.2. (page 6 of 14)

\begin{tabular}{|c|c|c|c|c|c|c|c|c|}
\hline Site Code & $\begin{array}{c}\text { Wasto Management } \\
\text { Unit Typo. }\end{array}$ & Wasto Management Unit & Wasto Typo & $\left.\begin{array}{c}\text { Oporable } \\
\text { Unit }\end{array}\right]$ & $\begin{array}{c}\text { Plutonlum-238, } \\
\text { Units (Ci) }\end{array}$ & $\begin{array}{l}\text { Plutonlum-239, } \\
\text { Unlts (C) }\end{array}$ & $\begin{array}{l}\text { Plutonlum-240, } \\
\text { Units (C) }\end{array}$ & $\begin{array}{l}\text { Plutonlum-241, } \\
\text { Unito (CI) }\end{array}$ \\
\hline $216-\mathrm{N}-4$ & Ponds & $216-\mathrm{N}-4$ Pond & Cooling Water & \begin{tabular}{|l|}
$N O-1$ \\
\end{tabular} & & 0.0571 & 0.0154 & \\
\hline $216-\mathrm{N}-5$ & Trench & 218-N-5 Trench & Coolling Water & NO-1 & & & & \\
\hline $216-N-6$ & Ponds & 216-N-6 Pond & Cooling Water & NO-1 & & 0.0571 & 0.0132 & \\
\hline $216-\mathrm{N}-7$ & Trench & 216-N-7 Trench & Cooling Water & NO-1 & & & & \\
\hline $216-\mathrm{N}-8$ & Ponds & 218-N-8 Pond & & IU-6 & & & & \\
\hline $216-\mathrm{S}-182$ & Cribs & $216-S-1 \& 2$ & Process Condensale & RO-2 & & & & \\
\hline$\overline{216-S-100}$ & Ditches & $\begin{array}{l}218-S-10 D \\
\end{array}$ & Chemical Sewer & RO-1 & & 0.00468 & & \\
\hline 216-S-10P & Ponds & 216-S-10P & Chemical Sewer & RO-1 & & & & \\
\hline$\overline{216-S-11}$ & Ponds & 216-S-11 & Chemical Sewer & RO-1 & & . & & \\
\hline$\overline{216-S-12}$ & Trench & $\overline{216-S-12}$ & Miscellaneous Dralnage & RO-3 & & & & \\
\hline$\overline{216-S-13}$ & Cribs & 216-S-13 & Process Waste & $\sqrt{\mathrm{RO}-2}$ & & & & \\
\hline $216-5-14$ & Trench & $216-S-14$ & Process Wasto & $\longdiv { R O - 3 }$ & & & & \\
\hline 216-S-15 & Ponds & $\begin{array}{l}216-S-15 \\
\end{array}$ & Coolling Water & RO-2 & & & & \\
\hline$\overline{216-S-160}$ & Ditches & 216-S-16D & Cooling Water & RO-1 & & & & \\
\hline 216-S-16P & Ponds & 216-S-16P & Cooling Water & RO-1 & & & & \\
\hline $\begin{array}{l}16-\mathrm{S}-17 \\
217\end{array}$ & Ponds & $\begin{array}{l}216-S-17 \\
\end{array}$ & Coollng Water & RO-1 & & & & \\
\hline$\overline{216-S-172}$ & Diversion Box & $\sqrt{216-S-172}$ & Coolling Water & RO-1 & & & & \\
\hline 216-S-18 & Tranch. & \begin{tabular}{|l|}
$216-S-18$ \\
\end{tabular} & Debris & $\longdiv { R 0 - 2 }$ & & & & \\
\hline$\overline{216-5-19}$ & Ponds & $218 \cdot S-19$ & Lab Waste & RO-1 & & & & \\
\hline 216-S-20 & Cribs & $218-5-20$ & Lab Wasto & RO-3 & & & & \\
\hline $216-5-22$ & Cribs & $216-5-22$ & Process Wasto & शि-3 & & & & \\
\hline 216-S-23 & Cribs & $216-5-23$ & Process Condensalo & Rि-2 & & & & \\
\hline $216-\mathrm{S}-25$ & Cribs & $216-\mathrm{S}-25$ & Steam Condensate & RO-1 & & & & \\
\hline 216-S-26 & çribs & $216-S-26$ & Lab Wasto & RO-3 & & 0.000172 & & \\
\hline $218-S-3$ & French Draln & 216-S-3 & Process Condensale & RO-2 & & & & \\
\hline$\overline{216-S-4}$ & French Draln & $216-5-4$ & Process Condensale & UP-2 & & & & \\
\hline 216-S-5 & Cribs & $\sqrt{216-S \cdot 5}$ & Cooling Water & Rू-1 & & & & \\
\hline$\overline{216-S-6}$ & Cribs & $216-5-6$ & Coolling Water & RO-1 & & & & \\
\hline $216-\mathrm{S}-7$ & Cribs & 216-S-7 & Process Condensato & $\overline{R O-2}$ & & & & \\
\hline 216-S-8 & Trench & $216-5-8$ & Process Wasto & RO-2 & & & & \\
\hline 216-S-9 & Cribs & $216-S-9$ & Process Condensale & $\overline{R O-2}$ & & & & \\
\hline 216-T-1 & Ditches & 216-T-1. Ditch & Cooling Water & $T P=-4$ & & & & \\
\hline 216-T-10 & Trench & 216-T-10 Trench & Decon Wasto & $T \mathrm{TP}-4$ & & & & \\
\hline
\end{tabular}


Table B.2. (page.7 of 14)

\begin{tabular}{|c|c|c|c|c|c|c|c|c|}
\hline Site Code & $\begin{array}{c}\text { Waate Management } \\
\text { Unlt Typo }\end{array}$ & Wasto Masiagoment Unit & Wasto Typo & $\begin{array}{c}\text { Operablo } \\
\text { Unit }\end{array} \mid$ & $\begin{array}{c}\text { Plutonium-238, } \\
\text { Units (C) }\end{array}$ & $\begin{array}{l}\text { Plutonlum-239, } \\
\text { Units (C) }\end{array}$ & $\begin{array}{c}\text { Plutonlum-240, } \\
\text { Unlts (C)) }\end{array}$ & $\begin{array}{l}\text { Plutonlum-241, } \\
\text { Units (CI) }\end{array}$ \\
\hline 216-T-11 & Trench & 216-T-11 Trench & Decon Waste & \begin{tabular}{|l|l|}
$T P-4$ \\
\end{tabular} & & & & \\
\hline$\overline{216-T-12}$ & Trench & 216-T-12 Trench & Coolling Water & TP-3 & & 0.0571 & 0.0154 & \\
\hline $216-T-13$ & Trench & 216-T-13 Trench & Decon Waste & TP-2 & . & & & \\
\hline$\overline{216-T-14}$ & Trench & 216-T-14 Trench & Tank Farm Waste & $\overline{\mathrm{TP}-3}$ & & 0.0502 & 0.135 & \\
\hline 216-T-15 & Trench & 216-T-15 Trench & Tank Farm Waste & TP-3 & & 0.0537 & 0.0145 & \\
\hline 216-T-16 & Trench & 216-T-18 Trench & Tank Farm Waste & TP-3 & & 0.0372 & 0.101 & \\
\hline $216-T-17$ & Trench & 216-T-17 Trench & Tank Farm Wasto & TP-3 & & 0.303 & 0.00816 & \\
\hline$\overline{216-T-18}$ & Cribs & 216-T-18 Crib & Tank Farm Wasto & $\sqrt{\mathrm{TP}-2}$ & & .903 & 27.7 & \\
\hline 216-T-19 & Cribs & 216-T-19TF Crib and Tile Fleld & Process Waste & $T P-2$ & & & & \\
\hline $216-T-2$ & Reverso Well & \begin{tabular}{|l|} 
216-T-2 Reverse Well \\
\end{tabular} & Lab Wasto & TP-4 & & & & \\
\hline$\longdiv { 2 1 8 - T - 2 0 }$ & Trench & 216-T-20 Trench & Process Waste & $\overline{T P}-2$ & & & & \\
\hline 216-T-21 & Trench & 216-T-21 Trench & Tank Farm Waste & TP-1 & & 0.571 & 0.154 & \\
\hline $216-T-22$ & Trench & 216-T-22 Trench & Tank Farm Waste & TP-1 & & 0.114 & 0.308 & \\
\hline $216-\mathrm{T}-23$ & Trench & 216-T-23 Trench & Tank Farm Wasto & $\overline{T P}-1$ & & 0.0571 & 0.0154 & \\
\hline $\begin{array}{l}216-T-24 \\
\end{array}$ & Trench & 216-T-24 Trenct: & Tank Farm Waste & TP-1 & & 0.114 & 0.0306 & \\
\hline 216-T-25 & Trench & 216-T-25 Trench & Process Waste & $T P-1$ & & 0.571 & 0.154 & \\
\hline $216-T-26$ & Cribs & $216-T-26$ Crib & Tank Farm Waste & TP-2 & & 3.37 & 0.908 & \\
\hline $216-T-27$ & Cribs & 216-T-27 Crib & Lab Wasto & $\mathrm{TP}-2$ & & 0.742 & 0.2 & \\
\hline $218-T-28$ & Cribs & $216-\mathrm{T}-28 \mathrm{Crib}$ & Decon Waste & $T \mathrm{TP}-2$ & & 4 & 1.08 & \\
\hline $216-T-28$ & Cribs & $216-\mathrm{T}-29 \mathrm{Crb}$ & Milscellaneous Drainage & $T \mathrm{~T}-4$ & & & & \\
\hline $216-T-3$ & Reverse Well & 216-T-3 Reverso Well & Process Wasto & TP-4 & & 191 & 51.5 & \\
\hline 216-T-31 & French Drain & 216-T-31 French Drain & Miscellaneous Dralnage & $\overline{T P}-2$ & & & & \\
\hline $216-T-32$ & Cribs & $216-\mathrm{T}-32 \mathrm{Crib}$ & Process Wasto & TP-1 & & 1.83 & 49.3 & \\
\hline$\overline{216-T \cdot 33}$ & Cribs & $216-T-33 \mathrm{Crib}$ & Decon Wasto & TP-4 & & 0.285 & 0.077 & \\
\hline $216-T-34$ & Cribs & 216-T-34 Crib & Lab Wasle & $T P-4$ & & 6.11 & 1.65 & \\
\hline 216-T-35 & Cribs & $216-T-35$ Crib & Lab Wasle & $\mathrm{TP}-4$ & & 3.78 & 1.02 & \\
\hline 216-T-36 & Cribs & $216-T-36$ Crib & Steam Condensate & $T \mathrm{TP}-1$ & & 0.142 & 0.0381 & \\
\hline $216-T-4-10$ & Ditches & 216-T-4-10 DHch & Coolling Water & TP -3 & & & & \\
\hline $216-T-4-2$ & Ditches & 216-T-4-2 Dltch & Steam Condensale & $T P-3$ & & & . & \\
\hline 216-T-AA & Ponds & 218-T-4A Pond & Cooling Water & $T \mathrm{P}-3$ & & & & \\
\hline 21B-T-AB & Ponds & 21B-T-4B Pond & Cooling Water & $\widehat{T P}-3$ & & & & \\
\hline 218-T-5 & Trench & 216-T-5 Trench & Tank Farm Waste & TP-1 & & 10.3 & 2.77 & \\
\hline 216-T-6 & Cribs & 216-T-6 Crib & Process Waste & TP-3 & & 22.3 & 6.01 & \\
\hline
\end{tabular}


Table B.2. (page 8 of 14)

\begin{tabular}{|c|c|c|c|c|c|c|c|c|}
\hline Sito Codo & $\begin{array}{c}\text { Waste Management } \\
\text { Unit Typo }\end{array}$ & Wasto Managemont Unit & Wasto Typo & $\begin{array}{c}\text { Operable } \\
\text { Unit }\end{array}$ & $\begin{array}{l}\text { Plutonlum-238, } \\
\text { Units (CI) }\end{array}$ & $\begin{array}{l}\text { Plutonlum-239, } \\
\text { Units (C) }\end{array}$ & $\begin{array}{l}\text { Plutonlum-240, } \\
\text { Units (Ci) }\end{array}$ & $\begin{array}{c}\text { Plutonium-241, } \\
\text { Units (Ci) }\end{array}$ \\
\hline 216-T-7 & Cribs & 216-T-7TF Crib and Tilo Field & Tank Farm Waste & TP-1 & & 7.42 & 2 & \\
\hline $216-T-8$ & Cribs & 216-T-B Crib & Lab Wasto & TP-4 & & 0.285 & 0.077 & \\
\hline $216-T-9$ & Trench & 216-T-9 Trench & Decon Waste & TP-4 & & & & \\
\hline $216-U-1 \& 2$ & Cribs & $216-U-1 \& 216-U-2$ & Procoss Condensate & $\overline{\mathrm{UP}-2}$ & & 2.43 & 0.656 & \\
\hline 216-U-10 & Ponds & $216-U-10$ & Coolling Waler & UP-2 & & 0.768 & & \\
\hline $216-U-11$ & Ditches & 216-U.11 & Coolling Water & UP-2 & & & & \\
\hline $216-U-12$ & Cribs & $216-U-12$ & Process Condensate & UP-2 & & 0.0123 & & \\
\hline 21G-U-13 & Trench & $216-U-13$ (same as UN-200-W-125) & Decon Wasto & $\mathrm{UP}-2$ & & 0.00571 & 0.00154 & \\
\hline$\sqrt{216-U-14}$ & Ditches & $216-U \cdot 14$ & Cooling Water & UP-2 & & & & \\
\hline $216-U-15$ & Trench & $216 \cdot U \cdot 15$ & Process Waste & UP-2 & & 0.00571 & 0.00154 & \\
\hline $216-U-16$ & Cribs & $216 \cdot U \cdot 16$ & Process Condensate & UP-2 & & 0.0902 & & \\
\hline $216-U-17$ & Cribs & $216-U-17$ & Process Condensato & UP-2 & & 0.0000296 & & \\
\hline $216-U-21$ & & $216-U-21$ & & & & 0.119 & 0.032 & \\
\hline 216-U-3 & French Draln & $218.4 \cdot 3$ & Miscelleneous Drainage & UP-2 & & 0.00571 & 0.00154 & \\
\hline $216-U-4$ & Revorso Well & $216-U-4$ & Lab Waste & UP-2 & & & & \\
\hline $216-U-4 A$ & French Drain & $216-U-4 A$ & Miscellaneous Drainage & UP-2 & & 0.00051 & 0.00013 & \\
\hline $216-U-4 B$ & French Draln & $216-U-4 B$ & Mlscellaneous Drainage & UP-2 & & 0.00308 & 0.00083 & \\
\hline 216-U-5 & Trench & $216-U-5 \& 216-U-6$ & Process Waste & UP-2 & & 0.00285 & 0.00077 & \\
\hline 216-U-7 & French Draln & $218-U-7$ & Miscollaneous Drainage & UP-2 & & & & \\
\hline 218-U-8 & Cribs & $216-U-8$ & Process Condensate & UP-2 & & 21.8 & 5.7 & \\
\hline $216 \cdot$ U.9 & Ditches & 216-U-9 & Cooling Water & RO-1 & & & & \\
\hline 216-W-LWC & Cribs & 216-W-LWC Crib & Chemical Sewer & SS-2 & & & & \\
\hline $216-Z-182$ & Cribs & $216-z-1 \&$ 216-Z-2 Cribs & Process Waste & $Z \mathrm{PP}-2$ & & 2680 & 992 & \\
\hline $216-Z-10$ & Reverso Well & 216-z-10 Reverso Well & Process Wasto & $\mathrm{ZP}-2$ & 0.14 & 2.85 & 0.77 & $\overline{2}$ \\
\hline $216-2-11$ & Ditches & $216-2-11$ & Coolling Water & UP-2 & & 137 & 37 & \\
\hline $216-2-12$ & Cribs & 216-z-12 Crib & Procoss Wasto & $2 \mathrm{P}-2$ & & 1430 & 386 & \\
\hline $216-Z-13$ & French Draln & 216-z-13 French Drain & Mlscellaneous Drainage & $\mathrm{ZP}-2$ & . & & & \\
\hline$\overline{216 \cdot 2-14}$ & French Drain & 216-2-14 French Drain & Miscollaneous Drainage & $\mathrm{ZP}-2$ & & & & \\
\hline 216-Z-15 & French Drain & 216-z-15 French Drain & Miscollaneous Drainage & ZP-2 & & & & \\
\hline $216-z-16$ & Cribs & $210-2-16$ Crib & Lab Wasto & $\mathrm{ZP}-2$ & & 4.09 & 1.1 & \\
\hline $216-2-17$ & Ditches & 216-Z-17 Trench & Lab Waste & $\overline{Z P-2}$ & & 2.87 & 0.225 & \\
\hline 216-Z-18 & Cribs & 216-Z-18 Crib & Process Waste & $\overline{\mathrm{ZP}-2}$ & & 1310 & 353 & \\
\hline $216-2-19$ & Ditches & 216-2-19 & Cooling Water & UP-2 & & & & \\
\hline
\end{tabular}


Table B.2. (page 9 of 14)

\begin{tabular}{|c|c|c|c|c|c|c|c|c|}
\hline Sito Code & $\begin{array}{c}\text { Waste Management } \\
\text { Unit Type }\end{array}$ & Waste Management Unit & Waste Typo & $\begin{array}{c}\text { Operablo } \\
\text { Unit }\end{array} \mid$ & $\begin{array}{c}\text { Piutonlum-238, } \\
\text { Units (CI) }\end{array}$ & $\begin{array}{c}\text { Plutonlum-239, } \\
\text { Unilts (Ci) }\end{array}$ & \begin{tabular}{|} 
Plutonium-240, \\
Units (Ci)
\end{tabular} & $\begin{array}{l}\text { Plutonlum-241, } \\
\text { Units (CI) }\end{array}$ \\
\hline $216-Z-1 A$ & Cribs & 216-Z-1A Tile Field & Process Waste & $2 p-2$ & & 137 & $\begin{array}{r}37 \\
\end{array}$ & \\
\hline $216-Z-1 D$ & Ditches & $216-Z-10$ & Coolling Water & $U P=-2$ & & 137 & 37 & \\
\hline $216-2-20$ & Cribs & $218-z-20$ & Cooling Water & UP-2 & 0.0153 & 2.03 & & 2.5 \\
\hline $216-2-21$ & Retention Basin & 218-z-21 Seopege Basin & Cooling Water & $2 \mathrm{PP}-2$ & & & & \\
\hline $216-Z-3$ & Cribs & 2 & Process Waste & $\mathrm{ZP}-2$ & & 325 & 87.8 & \\
\hline $216 \cdot z-4$ & Trench & 216-Z-4 Trench & Process Wasto & $2 \mathrm{P}-2$ & & & & \\
\hline 216-Z-5 & Cribs & 216-Z-5 Crib. & Process Wasto & $\mathrm{ZP}-2$ & & 19.4 & 5.24 & \\
\hline $216-2-6$ & Cribs & $216-2 \cdot 6$ Crib & Process Waste & $2 \mathrm{PP}-2$ & & 0.28 & 0.077 & \\
\hline $216-2-7$ & Cribs & 216-Z-7 Crib & Lab Waste. & $2 \mathrm{P}-2$ & & 114 & 30.8 & \\
\hline 216-Z-8 & Cribs & 216-z-8 French Drain & Process Waste & $\mathrm{ZP}-2$ & 0.13 & 2.76 & 0.745 & \\
\hline $216 \cdot Z-9$ & Cribs & 216-Z-9 Trench & Process Waste & $2 p-2$ & & 2190 & 580 & \\
\hline $218-C-9$ & Burial Site & 218-C-9 Burial Ground & LLW-SOLID & SO-1 & & & & \\
\hline $218-E-1$ & Burlal Site & $218-E-1$ & LLW-SOLID & $\overline{\mathrm{PO}-2}$ & & 51.4 & 13.9 & \\
\hline $218-E-10$ & Burial Silo & 218-E-10 Burlal Ground & LLW-SOLID & 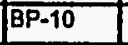 & & & & \\
\hline 218-E-12A & Burial Site & $218-E-12 A$ & LLW-SOLID & PO-6 & & 510 & 138 & \\
\hline $218-E-12 B$ & Burial Silo & $218-E-128$ & LLW-SOLID & PO-6 & & & & \\
\hline $218-E-13$ & Burial Silto & $218-E-13$ & & PO-2 & & & & \\
\hline $218-E-2$ & Burial Sile & 218-E-2 Burial Ground & LLW-SOLID & $\overline{B P-10}$ & & & & \\
\hline $218-E-2 A$ & Burlal site & 218-E-2A Burial Ground & LLW-SOLID & $8 P-10$ & & & & \\
\hline $218-E-4$ & Burial Stie & 218-E-4 Burial Ground & LLW-SOLIO & $B P-10$ & & & & \\
\hline $218-E-5$ & Burial Sthe & 218-E-5 Burlal Ground & LLW-SOLID & $B P-10$ & & & & \\
\hline 218-E-5A & Burtal Stito & 218-E-5A Burlal Ground & LLW-SOLID & BP-10 & & & & \\
\hline $218-E-6$ & Burial Sitte & 218-E-6 Buntal Ground & Dabris & $B P-6$ & & & & \\
\hline 218-E-7 & Burial Sito & 218-E-7 Burlal Ground & Lab Wasto & BP-6 & & & & \\
\hline $218-E \cdot 8$ & Burlal Sto & 218-E-8 & TRU Sold Waste & PO-6 & & 1.14| & 0.308 & \\
\hline 218-E-9 & Burial SHto & 218-E-9 Burial Ground & LLW-SOLID & BP-10 & & & & \\
\hline 218-W-1 & Burial Site & 218-W-1 Burial Ground & TRU Solid Wasto & $2 \mathrm{P}-3$ & & 5370 & 1450 & \\
\hline $218-W-11$ & Burlal Site & 218-W-11 Burial Ground & LLW-SOLID & $2 \mathrm{PP}-3$ & & & & \\
\hline $218-W-1 A$ & Burlal Sito & 218-W-1A Burlal Ground & LLW-SOLID & $2 \mathrm{P}-3$ & & 1114 & 30.8 & \\
\hline 218-W-2 & Burial Sitto & 218-W-2 Bural Ground & TRU Solld Wasto & ZP-3 & & 7190 & 1940 & \\
\hline $218-W-2 A$ & Burial Sitto & 218-W-2A Burial Ground & LLW-SOLID & $\mathrm{ZP}-3$ & & & & \\
\hline 218-W-3 & Burial Stte & 218-W-3 Bural Ground & TRU Solld Waste & $2 \mathrm{P}-3$ & & 3880 & 1050 & \\
\hline $218-W-3 A$ & Burial Sito & 218-W-3A Burial Ground & TRU Solld Waste & $2 \mathrm{P} \cdot 3$ & & & & \\
\hline
\end{tabular}


Table B.2. (page 10 of 14)

\begin{tabular}{|c|c|c|c|c|c|c|c|c|}
\hline Sito Code & $\begin{array}{c}\text { Waste Mal.agement } \\
\text { Unit Typz }\end{array}$ & Waste Managoment Unit & Waste Typo & $\begin{array}{c}\text { Operablo } \\
\text { Unit }\end{array}$ & $\begin{array}{l}\text { Plutonlum-238, } \\
\text { UnIts (G) }\end{array}$ & $\begin{array}{c}\text { Plutonlum-239, } \\
\text { Units (C) }\end{array}$ & $\begin{array}{c}\text { Plutonlum-240, } \\
\text { Units (Ci) }\end{array}$ & $\begin{array}{l}\text { Piutonlum-241, } \\
\text { Unlts (CI) }\end{array}$ \\
\hline $218-W-3 A E$ & \begin{tabular}{|l|l} 
Burial Site \\
\end{tabular} & 218-W-3AE Burial Ground & LLW-SOLID & $2 \mathrm{P}-3$ & & & & \\
\hline $218-W-4 A$ & Burial Sile & 218-W-AA Burial Ground & TRU Solld Wasto & $2 \mathrm{P}-3$ & & & & \\
\hline 218-W-4B & Burfal Silte & 218-W-AB Calssons & TRU Solld Waste & $\overline{\mathrm{ZP}-3}$ & & & & \\
\hline $218-W-4 B$ & Burial Sile & 218-W-4B Trenches & TRU Solid Waste & $2 P-3$ & & & & \\
\hline $218-W-4 C$ & Burial Sllo & 218-W-4C Burial Ground & TRU Solld Wasle & $2 \mathbf{P - 3}$ & & & & \\
\hline $218-W-5$ & Burial Sile & 218-W-5 Burial Ground & TRU Solld Wasie & $2 \mathrm{PP}-3$ & & & & \\
\hline $218-W-6$ & Burial Sile & 218-W.6 Burial Ground & LLW-SOLID & $2 \mathrm{PP}-3$ & & & & \\
\hline $218-W-7$ & Burial Silo & $\begin{array}{l}218-W-7 \\
\end{array}$ & LLW-SOLID & RO-3 & & & & \\
\hline $218-W-8$ & Burial SHo & 218-W-8 Burial Ground & Lab Waste & TP-4 & & 0.171 & 0.00462 & \\
\hline $218-W-9$ & Burial Sito & 218-W-9 & LLW-SOLID & $\overline{R O}-2$ & & & & \\
\hline 231-W-151 & Diversion Box & 231-Z-151 Sump & & PP-2 & & & & \\
\hline $231-W-151$ & Vault & 231-Z-151 Sump & & $\mathrm{PP}=2$ & & & & \\
\hline $231-W-151$ & Diversion Box & 231-Z-151 Sump & & $2 \mathrm{ZP}-2$ & & & & \\
\hline 231-W-151 & Vault & 231-Z-151 Sump & & $2 \mathrm{ZP}-2$ & & & & \\
\hline 231-W-151 & Diveralon Box & 231-Z-151 Sump & & $\mathrm{ZP-2}$ & & & & \\
\hline 231-W-151 & Vault & 231-Z-151 Sump & & $2 \mathrm{PP}-2$ & & & & \\
\hline 231-W-151 & Diverston Box & 231-z-151 Sump & & $2 \mathrm{P}-2$ & & & & \\
\hline 231-W-151 & Vault & 231-2-151 Sump & & $2 \mathrm{ZP}-2$ & & & & \\
\hline $232-2$ & gutlding & 232.2 Incinerator & & $\mathrm{ZP}-2$ & & & & \\
\hline $240-S-151$ & Diversion Box & $240-5-151$ & LLW-SOLID & $\overline{R O-3}$ & & & & \\
\hline $240-S-152$ & Diversion Box & $240-5-152$ & Tank Farm Wasto & RO-3 & & & & \\
\hline $240-5-302$ & Tanks & $\begin{array}{l}240-5-302 \\
\end{array}$ & Lab Waste. & $\longdiv { 8 0 - 3 }$ & & & & \\
\hline $241-A-151$ & Diversion Box & $241-A-151$ & Tank Farm Waste & PO-2 & & & & \\
\hline $241-A-152$ & Diversion Box & $241-A-152$ & Process Waste & P0.3 & & & & \\
\hline 241-A-153 & Diversion Box & $241-A-153$ & Process Waste & $\mathrm{PO}-3$ & & & & \\
\hline $241-A-302 A$ & Tanks & $241-A-302 A$ & & PO-2 & & & & \\
\hline $241-A-302 B$ & Tanks & $241-A-302 B$ & & PO-5 & & & & \\
\hline $241-A-350$ & Tanks & $241-A-350$ & Process Waste & PO-3 & & & & \\
\hline $241-A-417$ & Tanks & $241-A-417$ & Procoss Waste & $\overline{P O}-3$ & & & & \\
\hline $241-A-A$ & Diversion Box & $241-A-A$ & Process Wasto & PO-3 & & & & \\
\hline $241-\mathrm{A}-\mathrm{B}$ & Diversion Box & $241-A-B$ & Process Wasto & PO-3 & & & & \\
\hline 241-AN-A & Diversion Box & 241-AN-A & Process Waste & $\mathrm{PO}-3$ & & & & \\
\hline $241-\mathrm{AN}-\mathrm{B}$ & Divorsion Box & $241-A N-B$ & Process Wasto & PO-3 & & & & \\
\hline
\end{tabular}


Table B.2. (page 11 of 14)

\begin{tabular}{|c|c|c|c|c|c|c|c|c|}
\hline Site Code & \begin{tabular}{|c|}
$\begin{array}{c}\text { Waste Management } \\
\text { Untt Typo }\end{array}$ \\
\end{tabular} & Waste Managoment Unit & \begin{tabular}{|l} 
Waste Typo \\
\end{tabular} & $\begin{array}{c}\text { Operable } \\
\text { Unit }\end{array}$ & $\begin{array}{c}\text { Plutonlum-238, } \\
\text { Units (C) }\end{array}$ & $\begin{array}{c}\text { Plutonium-239, } \\
\text { Units (C) }\end{array}$ & $\begin{array}{c}\begin{array}{c}\text { Plutonlum-240, } \\
\text { Units (Ci) }\end{array} \\
\end{array}$ & $\begin{array}{l}\text { Plutonlum-241, } \\
\text { Units (C) }\end{array}$ \\
\hline 241-AP VP & \begin{tabular}{|l|l} 
Valva Pit \\
\end{tabular} & $241-A P$ & Process Wasto & \begin{tabular}{|l|l|}
$P 0-3$ & \\
\end{tabular} & & & & \\
\hline 241-AR-151 & Diversion Box & 241-AR-151 & Process Waste & $\overline{P O-3}$ & & & & \\
\hline 241-AW-A & Diversion Box & 241-AW-A & Process Waste & $\overline{\mathbf{P O}-3}$ & & & & \\
\hline 241-AW-B & Diversion Box & 241-AW-B & Process Waste & PO.3 & & & & \\
\hline $241-A X-151$ & Diversion Box & $241-A X-151$ & Process Wasto & $\overline{P 0.3}$ & & & & \\
\hline 241-AX-152DS & Tanks & 241-AX-152DS & Process Waste & PO-3 & & & & \\
\hline $241-A X-155$ & Diversion Box & $241-A X-165$ & Tank Farm Waste & $\overline{\mathrm{PO}-3}$ & & & & \\
\hline $241-A X-501$ & Valve Pit & 241-AX-501 & & $\mathrm{PO}-3$ & & & & \\
\hline $241-A X \cdot A$ & Diversion Box & $241 \cdot A X-A$ & & PO-3. & & & & \\
\hline $241-A X-B$ & Dlversion Box & $241-\overline{A X} \cdot-\mathrm{B}$ & & PO-3 & & & & \\
\hline $241-A Y \cdot 151$ & Diversion Box & $241-A Y-151$ & Process Waste & $\overline{\mathrm{PO}-3}$ & & & & \\
\hline $241-A Y-152$ & Diversion Box & $241-A Y-152$ & Procoss Wasțo & $\overline{\mathrm{PO}-3}$ & & & & \\
\hline 241-AZ-1510S & Diversion Box & 241-AZ-1510S & & $\overline{\mathrm{PO}-3}$ & & & & \\
\hline $241-A Z-152$ & Diversion Box & $241-A Z-152$ & & PO-3 & & & & \\
\hline $241-C-151$ & Diversion Box & $241-\mathrm{C}-151$ & & PO-3 & & & & \\
\hline $241-C-152$ & Dlverston Box & $241-C-152$ & & $\overline{\mathrm{PO}-3}$ & & & & \\
\hline $241-\mathrm{C}-153$ & \begin{tabular}{|l|l|} 
Diversion 80x \\
\end{tabular} & $241-\mathrm{C}-153$ & & $\overline{P O-3}$ & & & & \\
\hline $241-C-154$ & Diversion Box & $241-\mathrm{C}-154$ Diversion Box & Process Waste & SO-1 & & & & \\
\hline $241-\mathrm{C}-252$ & Dlverston Box & $241-\mathrm{C}-252$ & & $\overline{\mathrm{PO}-3}$ & & & & \\
\hline $241 \cdot \mathrm{C}-301 \mathrm{C}$ & Tanks. & $241-\mathrm{C}-301 \mathrm{C}$ & & PO-3. & & & & \\
\hline 241-CR-151 & \begin{tabular}{|l} 
Diversion Box \\
\end{tabular} & 241-CR-151 & & PO-3 & & & & \\
\hline 241-CR-152 & Dlversion Box & 241-CR-152 & & $\mathrm{PO} \cdot 3$ & & & & \\
\hline 241-CR-153 & Diversion Box & 241-CR-153 & & $\mathrm{PO}-3$ & & & & \\
\hline 241-CX-TK-70 & Tanks & 241-CX-70 Storage Tank & Tank Farm Waste & SO-1 & & & & \\
\hline 241-CX-TK-71 & Tanks & 241-CX-71 Storage Tank & Procoss Condensate & SO-1 & & 0.4579 & 0.123 & \\
\hline 241-CX-TK-72 & Tanks & 241-CX-72 Storage Tank & Process Waste & so-1 & & & & \\
\hline 241-ER-153 & Diversion Box & $241-E R-153$ & & PO-3 & & & & \\
\hline $241-S-151$ & Diversion Box & 241-S-151 & LLW-SOLID & RO-2 & & & & \\
\hline$\frac{241-S-152}{241-S-302 A}$ & \begin{tabular}{|l} 
Dlverslon Box \\
Tanks
\end{tabular} & $\frac{241-S-152}{241-S-302 A}$ & $\frac{\text { Tank Farm Waste }}{\text { Lab Wasto }}$ & $\frac{R O-4}{\mathrm{RO}-2}$ & & & & \\
\hline 241-S-302B & Tanks & $241-S-3028$ & LLW-SOLID & $R 0-4$ & & & & \\
\hline $241-S \cdot A$ & Dlverston Box & 241-S-A & Tank Farm Waste & $\mathrm{RO}-4$ & & & & \\
\hline 241-S-B & Diversion Box & 241-S-B & Tank Farm Wasto & RO-4 & & & & \\
\hline
\end{tabular}


Table B.2. (page 12 of 14)

\begin{tabular}{|c|c|c|c|c|c|c|c|c|}
\hline Sitto Code & $\begin{array}{c}\text { Waste Management } \\
\text { Unitt Type }\end{array}$ & Waste Management Unit & Waste Type & $\left|\begin{array}{c}\text { Operabto } \\
\text { Unit }\end{array}\right|$ & $\begin{array}{l}\text { Plutonlum-238, } \\
\text { Units (CI) }\end{array}$ & $\begin{array}{l}\text { Plutonlum-239, } \\
\text { Untts (CI) }\end{array}$ & $\begin{array}{l}\text { Plutonlum-240, } \\
\text { Units (Ci) }\end{array}$ & $\begin{array}{l}\text { Plutonlum-241, } \\
\text { Units (CI) }\end{array}$ \\
\hline $241-\mathrm{S}-\mathrm{C}$ & Diversion 80x & $241-S-C$ & Tank Farm Wasle & $R 0-4$ & & & & \\
\hline 241-S-D & Diversion Box & 241-S.D & Tank Farm Wasto & RO-4 & & & & \\
\hline $241-S X-151$ & Diversion Box & $241-S X-151$ & Tank Farm Wasto & RO-4 & & & & \\
\hline $241-S X-152$ & Diversion Box & $241-S X-152$ & Tank Farm Wasto & RO-4 & & & & \\
\hline $241-5 X-302$ & Tanks & $241-S X-302$ & & $\sqrt{\mathrm{RO}-2}$ & & & & \\
\hline $241-S X-A$ & Diversion Box & $24.1-S X-A$ & & RO-4 & & & & \\
\hline $241-S X-B$ & Diversion Box & $241-S X-B$ & & RO-4 & & & & \\
\hline$\overline{241-S Y-A}$ & Diversion Box & $241-S Y-A$ & & RO-4 & & & & \\
\hline 241-SY-A & Diversion Box & $241-S Y-A$ & & $R 0-4$ & & & & \\
\hline $241-$ SY-B & Diversion Box & $241-S Y \cdot B$ & & $\overline{R O-4}$ & & & & \\
\hline $241-S Y-B$ & Diversion Box & $241 \cdot S Y \cdot B$ & & $\overline{R O-4}$ & & & & \\
\hline 241-T-151 & Diversion Box & $\mid$ & Tank Farm Waste & TP.6 & & & & \\
\hline $241-T-152$ & Diversion $80 x$ & 241-T-152 Diversion Box & Tank Farm Waste & TP-6 & & & & \\
\hline $241-T-153$ & Diverslon Box & 241-T-153 Diversion Box & Tank Farm Waste & $T \mathrm{P}-6$ & & & & \\
\hline $241-T-252$ & Diversion Box & 241-T-252 Diversion Box & Tank Farm Waste & $T P-6$ & & & & \\
\hline $241-T-301$ & Tanks & 241-T-301 Catch Tank & Tank Farm Wasto & $\mid \overline{P P}-6$ & & & & \\
\hline $241-T-302$ & Tanks & 241-T-302 Catch Tank & Tank Farm Waste & $T \mathrm{PP} \cdot 6$ & & & & \\
\hline 241-T-361 & Tanks & 241-T-361 Sottling Tank & Process Waste & TP-4 & & & & \\
\hline $241-T R-152$ & Diversion Box & 241-TR-152 Dlverslon Box & Tank Farm. Wasto & TP-8 & & & & \\
\hline $241-T R-153$ & Diversion Box & 241-TR-153 Diversion Box & Tank Farm Wasto & TP-8 & & & & \\
\hline $241-T X-152$ & Diversion Box & 241-TX-152 Diverston BoX & Tank Farm Wasto & $T P-2$ & & & & \\
\hline $241-T X-153$ & Diversion BOX & 241-TX-153 Divorslon BoX & Tank Farm Wasle & TP-5 & & & & \\
\hline $244-T X-154$ & Diversion Box & 241-TX-154 Diversion Box & Tank Farm Wasto & TP-4 & & & & \\
\hline $241-T X-155$ & Diversion Box & 241-TX-155 Dlvarsion Box & Tank Farm Wasto & TP-2 & & & & \\
\hline $241-\mathrm{TX}-302 \mathrm{~A}$ & Tanks & 241-TX-302A Catch Tank & Tank Farm Wasto & TP-5 & & & & \\
\hline $241-\mathrm{TX}$-302B & Tanks & 241-TX-302B Catch Tank & Tank Farm Wasto & TP-2 & & & & \\
\hline $241-\mathrm{TX}-302 \mathrm{C}$ & Tanks & 241-TX-302C Catch Tank & Tank Farm Waste & TP-4 & & & & \\
\hline $241-\mathrm{TXR}-151$ & Diversion Box & 241-TXR-151 Diversion Box & Tank Farm Waste & TP-5 & & & & \\
\hline$\overline{241-T X R-152}$ & Diverslon Box & 241-TXR-152 Diverslon Box & Tank Farm Wasto & TP-5 & & & & \\
\hline $241-\mathrm{TXR}-153$ & Dlversion Box & 241-TXR-153 Diverslon Box & Tank Farm Waste & TP-5 & & & & \\
\hline $241-T Y-153$ & Diverslon Box & $219-T Y$-153 Dlversion Box & Tank Farm Wasto & TP-5 & & & & \\
\hline $241-T Y-302 A$ & Tanks & 241-TY-302A Catch Tank & Tank Farm Wasto & TP-5 & & & & \\
\hline $241-T Y-3028$ & Tanks & 241-TY-302B Catch Tank & Tank Farm Wasto & TP-5 & & & & \\
\hline
\end{tabular}


Table B.2. (page 13 of 14)

\begin{tabular}{|c|c|c|c|c|c|c|c|c|}
\hline sto Codo & $\begin{array}{c}\text { Wasto Management } \\
\text { Unit Type }\end{array}$ & Waste Managoment Unit & Waste Typo & $\begin{array}{c}\text { Operablo } \\
\text { Unit }\end{array}$ & $\begin{array}{l}\text { Plutonlum-238, } \\
\text { Unlts (C) }\end{array}$ & $\begin{array}{l}\text { Piutonlum-239, } \\
\text { Inits (CI) }\end{array}$ & $\begin{array}{l}\text { Plutonlum-240, } \\
\text { Unlts (CI) }\end{array}$ & $\begin{array}{l}\text { Plutonlum-241, } \\
\text { Units (CI) }\end{array}$ \\
\hline $241-Z-361$ & Tanks & 241-Z-361 Settling Tank & Process Wasto & $2 p-2$ & & & & \\
\hline 241-Z-TK-8 & Tanks & 216-Z-8 Settling Tank & Process Waste & $\mathrm{ZP}-2$ & & & & \\
\hline 241-Z-TK-D5 & Tanks & 241-Z Treatment Tank & Process Waste & $\overline{\mathrm{ZP}-2}$ & & & & \\
\hline $242-T-151$ & Diversion Box & 242-T-151 Diversion Box & Process Condensalo & TP-5 & & & & \\
\hline 244-ART & Tanks & $244-A$ & Process Wasle & $\mathrm{FO}-3$ & & & & \\
\hline 244-AR VAULTT & Vault & 244-AR & Process Waste & $\overline{\mathrm{PO}-3}$ & & & & \\
\hline 244-CR VAULT & Vault & 244-CR & Process Waste & PO-3 & & & & \\
\hline 244-SRT & Tanks & 244-S Recelver Tank & & $\sqrt{R 0-2}$ & & & & \\
\hline 244-TXRT & Tanks & 244-TXRecoiving Tank & & TP-5 & & & & \\
\hline 244-TX̣R & Vault & 244-TXR Vault & Tank Farm Wasto & TP.5 & & & & \\
\hline 2807-E5 & Sepllc System & 2 B07-E-5 Septic Tank and Draln Field & Sanitary Wasto & SO-1 & & & & \\
\hline $2607-E 6$ & Septic System & 2607-E6 & Sanitary Waste & PO.2 & & & & \\
\hline 2607-E7A & Sepllc System & 2607-E-7A Septlic Tank and Drain Field & Sanitary Waste & SO-1 & & & & \\
\hline 2607-EA & Septic System & $2607-E A$ & Sanitary Wasto & PO-2 & & & & \\
\hline F07-EC & Seplic Systom & 2607-EC & Sanitary Wasto & PO-5 & & & & \\
\hline$\overline{2607 \cdot E D}$ & Seplic System & 2807-ED & Sanitary Waste & $\mathrm{PO}-3$ & & & & \\
\hline 2607-EE & Septlc System & $2607-\mathrm{EL}$ & Sanitary Waste & PO-2 & & & & \\
\hline 2807-EG & Seplle Systom & 2607-EG & Sanitary Wasto & PO-3 & & & & \\
\hline 2607-EJ & Septlc System & $2607 \cdot \mathrm{EJ}$ & Sanltary Wasto & $\overline{\mathrm{PO}-3}$ & & & & \\
\hline $2607-N$ & Septlc System & 2607-N Septic Tank/Drain Fleld & Sanitary Waste & NO-1 & & & & \\
\hline $2607-P$ & Septle System & 2607.P Septlc TankJDraln Field & Sanitary Waste & NO-1 & & & & \\
\hline $2 \overline{2607-R}$ & Septlc System & 2607-R Sepllc Tank/Drain Field & Sanitary Waste & NO-1 & & & & \\
\hline$\overline{2607-W 1}$ & Septlc System & 2607-W1 Septlc Tank & Sanitary Waste & $\overline{S S-2}$ & & & & \\
\hline$\overline{2607-W 2}$ & Septlc System & 2607-W2 Septle Tank & Sanitary Wasto & SS-2 & & & & \\
\hline $2607-W_{3}$ & Septic System & 2607-W3 Septlc Tank & Sanitary Wasto & $\overline{T P-4}$ & & & & \\
\hline $2607-W 4$ & Septc System & 2607-W4 Septlc Tank & Sanitary Wasto & TP-4 & & & & \\
\hline 2607-W6 & Septic System & $2607-W 6$ & Sanitary Wasto & RO-3 & & & . & \\
\hline $2607-W B$ & Seplic System & 2607-W-8 Septic Tank and Drain Field & Sanitary Waste & $\mathrm{ZP}-2$ & & & & \\
\hline 2607-WA & Septic System & 2607-WA Septic Tank and Drain Fiold & Sanilary Wasto & $2 \mathrm{P}-2$ & & & & \\
\hline 2607-WB & & 2607-WB Septlc Tank and Draln Fleld & & & & & & \\
\hline$\overline{2607-W T}$ & Septle System & 2607-WT Septic Tank & Sanitary Waste & TP-5 & & & & \\
\hline $2607-W T X$ & Seplic System & 2607-WTX Septle Tank & Sanitary Wasto & TP-5 & & & & \\
\hline $2607-W z$ & Seplic System & $2607-W z$ & Sanitary Waste & RO-1 & & & & \\
\hline
\end{tabular}


Table B.2. (page 14 of 14)

\begin{tabular}{|c|c|c|c|c|c|c|c|c|}
\hline SItto Codo & $\begin{array}{c}\text { Wasto Management } \\
\text { Unit Type }\end{array}$ & Wasto Managomont Unit & Wasto Typo & $\begin{array}{c}\text { Operablo } \\
\text { Unit }\end{array}$ & $\begin{array}{l}\text { Plutonlum-238, } \\
\text { Unlts (Ci) }\end{array}$ & $\begin{array}{l}\text { Plutonlum-239, } \\
\text { Units (C)) }\end{array}$ & $\begin{array}{l}\text { Plutonlum-240, } \\
\text { Units (CI) }\end{array}$ & $\begin{array}{l}\text { Plutonium-241, } \\
\text { Units (G) }\end{array}$ \\
\hline $2607-2$ & \begin{tabular}{|l|l|} 
Soptlic System & \\
\end{tabular} & $2607-2$ Septle Tank and Drain Fleld & Sanitary Wasto & \begin{tabular}{|l|l|}
$\mathrm{ZP}-2$ & \\
\end{tabular} & & & & \\
\hline $2607-2-1$ & & 2607-Z-1 Septlic Tank and Drain Field & & & & & & \\
\hline 2704-C-WS-1 & Fronch Drain & $\begin{array}{l}\text { 2704-C-WS-1, 2704-C French Draln, } \\
\text { Gatohouss French Drain }\end{array}$ & Mlscenlaneous Dralnage & SO.1 & & & & \\
\hline 2904-S-160 & Diversion Box & $2904-5 \cdot 160$ & Cooling Water & RO-1 & & & & \\
\hline 2904-S-170 & Diversion Box & 2904-S-170 & Process Waste & $\mathrm{RO} 0.1$ & & & & \\
\hline 2904-S-171 & Diversion Box & 2904-S-171 & Coolling Water & RO-1 & & & & \\
\hline $291-\mathrm{C}$ & Building & 291-C Ventilation System & Process Condensate & SO-1 & & & & \\
\hline 299-E24-111 & Reverse Well & \begin{tabular}{|l|l|l|} 
299-E24-111 \\
\end{tabular} & & $\overline{\mathrm{PO}-2}$ & & & & \\
\hline HSVP & Diversion Box & Seml-Works Valve Pit & Process Waste & SO-1 & & & & \\
\hline UPR-200-E-141 & & UN-200-E-141 & Solution Storage (1) & so-1 & & & & \\
\hline UPR-200-E-36 & & UN-200-E-36 & Process \& Decon Wastes (1) & SO-1 & & & & \\
\hline UPR-200-E-37 & & UN-200-E-37 & Process \& Decon Wastes (1) & SO-1 & & & & \\
\hline UPR-200-E-98 & & UN-200-E-98 & Process \& Decon Wastes (3) & SO-1 & & & & \\
\hline UPR-200-W-160 & & UPR-200-W-160 Unplanned Releaso & Tank Farm Wasie & TP-4 & & & & \\
\hline ZPLANT BP & Burial Sito & Z Plant Bum Pit & Debris & $\mathrm{ZP}-3$ & & & & \\
\hline
\end{tabular}


Table B.3. Environmental Waste Site Inventories for Plutonium-242, Total Uranium, Gross Uranium, and Uranium-235 (page 1 of 14)

\begin{tabular}{|c|c|c|c|c|c|c|c|c|}
\hline Slte Code & \begin{tabular}{|c|} 
Waste Management \\
Unlt Type
\end{tabular} & Wasto Management Unit & Wasto Typo & $\begin{array}{c}\text { Oporablo } \\
\text { Unit }\end{array}$ & $\begin{array}{c}\text { Plutonlum-242, } \\
\text { Unlts (C) }\end{array}$ & $\begin{array}{l}\text { Total Uranlum, Units (Cl, } \\
\text { unloss otherwise stated) }\end{array}$ & $\begin{array}{c}\text { Gross Uranlum, } \\
\text { Units (CI) }\end{array}$ & $\begin{array}{l}\text { Uranlum-236, } \\
\text { Units (CI) }\end{array}$ \\
\hline & & 212-N to 216-N-1 Plpeline & & & & & & \\
\hline & & 212.P Hazardous Wasto Slaging Area & & & & & & \\
\hline & & 212.P to 216-N-4 Plpellne & & & & & & \\
\hline & & 212.P Transformer Oli Tank & & & & & & \\
\hline & & 212-R to 216-N-6 Pipeline & & & & & & \\
\hline & & 241.C Wasto Line Unplanned Releaso No. 1 & & & & & & \\
\hline & & 241.C Waste Lire Unplanned Releaso No. 2 & & & & & & \\
\hline & & 241-Z Diversion Box No. 1 & & & & & & \\
\hline & & 241-Z Dlversion Box No. 2 & & & & & & \\
\hline & & Sanflary Crib & & & & & & \\
\hline 200-E BP & Burlal SHe & 200-E Bumlng PH & Debris & PO-6 & & & & \\
\hline 200-E PAP & Burial SHe & 200-E Ash PH & & SS-1 & & & . & \\
\hline 200-EPD & Ditches & 200 East Powerhouso DHch & Coollng Water & So-1 & & & & \\
\hline $200-E-4$ & French Drain & Critlcal Mass Laboralory Dry Well North & Miscellanoous Drainage & SO-1 & & & & \\
\hline $20 \Omega N-3$ & Burlal Site & Eallast PHs & Debris & NO-1 & & & & \\
\hline 200-WADB & Burial Stle & 200-WAsh Dlsposal Bash & Ash & SS-2 & & & & \\
\hline 200-WADS & Burfal SHo & 200-WAsh PH Demolition Site & N/A & SS-2 & & & & \\
\hline 200 WBP & Burlai Sito & 200-W Buming PH & Debris & SS-2 & & & & . \\
\hline 200-WPAP & Burial SHe & 200-W Powertiouse Ash Plt & Ash & SS-2 & & & & \\
\hline 200-WPP & Ponds & 200-W Powertiouse Pond & Coollng Water & TP-2 & & & & \\
\hline $201-\mathrm{C}$ & Bullding & 201-C Procoss Bullding & Process Condensale & so-1 & & & & \\
\hline $207-A$ & & 207-A & & & & & & \\
\hline $207 \cdot 8$ & Retention Basin & 207.Bb/ Retentlon Basin & Coollng Waler & BP-8 & & & & \\
\hline $207-5$ & Retentlon Basin & 207.5 & Coolling Water & RO-2 & & & & \\
\hline 207-SL & Retenilon Basin & $207-\mathrm{SL}$ & Lab Waste & RO.3 & & & & \\
\hline $207-T$ & Retentlon Basin & 207-T Retentlon Basin & Cooling Waler. & PP-3 & & & & \\
\hline $207-Z$ & Retention Basin & 207-2 Rotention Basin & Steam Condensate & $2 \mathrm{P}-2$ & & . & & \\
\hline 209-E-WS-1 & French Draln & Critlcal Mass Laboratory Dry Well East & Miscellaneous Drainage & SO-1 & & & & \\
\hline 209-E-WS-2 & French Drain & Crilical Mass Laboratory Dry Well South & Miscallaneous Drainage & So-1 & & & & \\
\hline 209-E-WS-3 & Diversion Box & Critleal Mass Laboratory Valve PH & Process Waste & SO-1 & & & & \\
\hline 2101-MPOND & Ponds & 2101-M Pond & Lab Waste & SS-1 & & & & \\
\hline $216-A-1$ & Cribs & $216-A-1$ & Process Waste & PO-5 & & 0.0514 & & \\
\hline 216-A-10 & Cribs & $216-A-10$ & Process Condensate & PO-2 & & 0.081 & & \\
\hline
\end{tabular}


Table B.3. (page 2 of 14)

\begin{tabular}{|c|c|c|c|c|c|c|c|c|}
\hline Sito Codio & $\begin{array}{c}\text { Wasto Management } \\
\text { Unlt Type }\end{array}$ & Waste Management Untt & Wasto Typo & $\begin{array}{c}\text { Operablo } \\
\text { Unit }\end{array}$ & $\begin{array}{c}\text { Plutonlum-242, } \\
\text { Units (C) }\end{array}$ & $\begin{array}{l}\text { Total Uranlum, Units (Cl, } \\
\text { unless otherwise stated) }\end{array}$ & $\begin{array}{l}\text { Gross Uranium, } \\
\text { Units (CI) }\end{array}$ & $\begin{array}{l}\text { Uranlum-235, } \\
\text { Unlts (CI) }\end{array}$ \\
\hline $216-A-11$ & French Draln & $216-A-11$ & Miscellaneous Dralnage & PO-2 & & & & \\
\hline 216-A-12 & French Drain & $\overline{216-A-12}$ & Miscellaneous Drainago & $\overline{P O .2}$ & & & & . \\
\hline $216-A-13$ & French Drain & $216-A-13$ & Miscellaneous Drainago & $\overline{P 0-2}$ & & & & \\
\hline $216-A-14$ & French Drain & $216-A-14$ & Mlscellaneous Drainago & PO-2 & & & & \\
\hline 216-A-15 & French Draln & 216-A-15 & Process Condensato & PO-2 & & & & \\
\hline $216-A-16$ & French Drain & $216-A-16$ & Chemical Sewer & PO-5 & & & & \\
\hline $216-A-17$ & French Draln & $216-A-17$ & Chemlcal Sewer & PO-5 & & & & \\
\hline $216-A-18$ & Trench & $216-A-18$ & Process Wasto & P0.5 & & 0.469 & & \\
\hline $216-A-19$ & Trench & $216-A-19$ & Process Waste & P0-5 & & 13 & & \\
\hline $216-A-2$ & Cribs & $216-A-2$ & Process Waste & $\overline{\mathrm{PO}-2}$ & & 0.026 & & \\
\hline $216-A-20$ & Tronch & $216-A-20$ & Process Waste & PO-5 & & 0.135 & & \\
\hline $216-A-21$ & Cribs & $\overline{216-A-21}$ & Lab Waste & $\overline{P O-2}$ & & 0.065 & & \\
\hline $216-A-22$ & French Drain & $216-A-22$ & Miscellaneous Dralnage & PO-2 & & & & \\
\hline $216-\bar{A}-23 A$ & French Drain & $216-A-23 A$ & Process Condensate & P0.5 & & & & \\
\hline $216-A-23 B$ & French Draln & 216-A-23B & Process Condensate & $\widehat{P O-5}$ & & & & \\
\hline $216-A-24$ & Cribs & $216-A-24$ & Process Condensale & PO-5 & & 0.0167 & & \\
\hline 216-A-25 & Ponds & 216-A-25 Pond & Cooling Water & IU-6 & & 4.24 & & \\
\hline $216-A-28$ & French Draln & $216-A-26$ & MIscellaneous Drainage & $\overline{\mathrm{PO}-2}$ & & & & \\
\hline 216-A-26A & French Draln & 216-A-26A & Miscellaneous Dralnage & $\overline{\mathrm{PO}-2}$ & & & & \\
\hline 216-A-27 & Cribs & $216-A-27$ & Miscellaneous Dralnage & PO-2 & & 0.0227 & & \\
\hline $216-A-28$ & Cribs & $216-A-28$ & Process Condensato & $\mathrm{PO}-2$ & & 0.211 & & \\
\hline $216-A-29$ & DHches & $216-A-29$ & Chemical Sewar & 丽-11 & & & & \\
\hline $216-A-3$ & Cribs. & $216-A-3$ & Procoss Wasto & $\overline{\mathrm{PO} .2}$ & & 0.559 & & \\
\hline $216-A-30$ & Cribs & $216-A-30$ & Steam Condensato & P0.4 & & 0.1 & & \\
\hline 216-A-31 & Cribs & $216-A-31$ & Procoss Waste & $\overline{P O-2}$ & & 0.00683 & & \\
\hline $216-A-32$ & Cribs & $21,6-A-32$ & Miscellaneous Dralnago & $\overline{\mathrm{PO}-2}$ & & & & \\
\hline $218-A-33$ & French Draln & $216-A-33$ & Miscellaneous Dralnago & $\overline{\mathrm{PO}-2}$ & & & & \\
\hline $216-A \cdot 34$ & Cribs & $216-A-34$ & Process Condensalo & P0.5 & & & & \\
\hline $216-A-35$ & French Drain & $216-A-35$ & Miscellaneous Drainage & PO-2 & & & & \\
\hline $216-A-36 A$ & Cribs & 216-A-36A & Process Wasto & PO-2 & & 0.0484 & & \\
\hline $216-A-36 B$ & Cribs & $216-A-36 B$ & Process Waste & $\mathrm{PO}-2$ & & 0.0398 & & \\
\hline $216-A-37.1$ & Cribs & $216-A-37-1$ & Process Condensate & $\mathrm{PO}-4$ & & 0.0109 & & \\
\hline 216-A-37-2 & Cribs & $216-A-37-2$ & Steam Condensate & PO-4 & & 0.0172 & & \\
\hline
\end{tabular}


Table B.3. (page 3 of 14)

\begin{tabular}{|c|c|c|c|c|c|c|c|c|}
\hline Sito Codo & $\begin{array}{c}\text { Waate Managemont } \\
\text { Unlt Type }\end{array}$ & Waste Managoment Unlt & Waste Type & $\begin{array}{c}\text { Oporable } \\
\text { Unit }\end{array}$ & $\begin{array}{c}\text { Plutonlum-242, } \\
\text { Units (CI) }\end{array}$ & $\mid \begin{array}{l}\text { Total Uranium, Units (CI, } \\
\text { uniess otherwise stated) }\end{array}$ & $\begin{array}{c}\text { Gross Uranlum, } \\
\text { Unlts (CI) }\end{array}$ & $\begin{array}{l}\text { Uranlum-235, } \\
\text { Units (CI) }\end{array}$ \\
\hline $216-A-3 B-1$ & Cribs & $216-A-38-1$ & NIA & $\mathrm{PO}-2$ & & & & \\
\hline $216-A-39$ & DHthes & $216-A-39$ & Miscellaneous Dralnago & $\mathrm{PO} 0.3$ & & & & \\
\hline $216-A-4$ & Cribs & $216-A-4$ & Lab Wasto & PO-2 & & 0.133 & & \\
\hline $216-A-40$ & Retenllon Bas/n & $216-A-40$ & Steam Condensato & PO-2 & & & & \\
\hline $216-A-41$ & Cribs & $216-A-41$ & Miscellaneous Dralnage & $\overline{P 0-2}$ & & & & \\
\hline $216-A-42$ & $\longdiv { \text { Retention Basin } }$ & $216-A-42$ & Coollng Water & $\overline{\mathrm{PO}-4}$ & & & & \\
\hline $216-A-45$ & Cribs & $216-A-45$ & Process Condensate & $\overline{\mathrm{PO}-2}$ & & 0.00225 & & \\
\hline $216-A-5$ & Cribs & $216-A \cdot 5$ & Process Condensate & $\overline{\mathrm{PO}-2}$ & & 0.0877 & & \\
\hline $216-A-524$ & Dlversion Box & $216-A-524$ & & PO-5 & & & & \\
\hline $216-A \cdot 6$ & Cribs & $216-A-6$ & Steam Condensale & $P O-4$ & & 0.055 & & \\
\hline $216-A-7$ & Cribs & $216-A-7$ & Process Waste & PO.5 & & 0.00227 & & \\
\hline $216-A-8$ & Cribs & $216-A-8$ & Process Condensate & P0.5 & & 0.123 & & \\
\hline 216-A-9 & Crlbs & 216-A-9 & Cooling Waler & PO-2 & & 0.0000757 & & \\
\hline $216-B-10 A$ & Cribs & 216-B-10A Crib & Lab Wasto & $\mathrm{BP}-6$ & & 0.00302 & & $\overline{0}$ \\
\hline 216-B-10B & Cribs & 216-B-10B Crib & Lab Waste & BP-6 & & 0.000000249 & & $\overline{0}$ \\
\hline $216-B-11 A \& B$ & Reverse Well & 216-B-11A\&B Reverse Wells & Process Condensale & $8 P-4$ & & 0.00454 & & $\overline{0}$ \\
\hline $216-B-12$ & Cribs & $216-\mathrm{B} \cdot 12 \mathrm{Crib}$ & Process Condensate & BP-9 & & 6.96 & & $\overline{0}$ \\
\hline $216-B-13$ & French Draln & 216-B-13 French Drain & Mlscellaneous Drainage & BP-6 & & & & \\
\hline 216-B-14 & Cribs & 216-B-14 Crib & Scavenged Waste & BP-2 & & 0.0726 & & $\overline{0}$ \\
\hline $216-B-15$ & Cribs & 218-B-15 Crib & Scavenged Waste & $\overline{8 P-2}$ & & 0.0348 & & $\overline{0}$ \\
\hline $216-B-16$ & Cribs & 216-8.18 Crib & Scavenged Waste & BP-2 & & 0.107 & & $\overline{0}$ \\
\hline 216-B-17 & Cribs & 210-B-17 Crb. & Scavenged Waste & BP-2 & & 0.118 & & $\overline{0}$ \\
\hline $216-B-18$ & Cribs & 210-B-18 Chb & Scavenged Wasto & BP-2 & & 0.0786 & & 0 \\
\hline $21 \mathrm{~B}-\mathrm{B}-19$ & Cribs & 216-B-19 Crb & Scavenged Wasto & $\mathrm{BP}-2$ & & 0.0605 & & $\overline{0}$ \\
\hline $216-B-2-1$ & Ditches & 218-B-2-1 Ditchb/ & Cooling Water & BP-11 & & 2.1 & & \\
\hline $216-8-2-2$ & Ditches & 216-8-2-2 DHchal & Coolling Water & $8 P-11$ & & 0.0000157 & & $\overline{0}$ \\
\hline $216-B-2-3$ & Dilches & 216-B-2-3 Dich & Cooling Water & BP-11 & & & & \\
\hline $216-B-20$ & Trench & 216-B-20 Trench & Scavenged Waste & $8 p-2$ & & 0.117 & & $\overline{0}$ \\
\hline $216-\mathrm{B}-21$ & Trench & 216-B-21 Trench & Scavenged Wasto & BP-2 & & 0.225 & & $\overline{0}$ \\
\hline $216-B-22$ & Trench & 210-B-22 Trench & Scavenged Waste & BP-2 & & 0.139 & & 0 \\
\hline $216-8-23$ & Trench & 218-B-23 Trench & Scavenged Waste & BP.2 & & 0.052 & & 0 \\
\hline $216-8-24$ & Trench & 216-B-24 Trench & Scavenged Waste & BP-2 & & 0.082 & & 0 \\
\hline $216-8-25$ & Trench & 216-8-25 Trench & Scavenged Wasle & BP-2 & & 0.0051 & & $\overline{0}$ \\
\hline
\end{tabular}


Table B.3. (page 4 of 14)

\begin{tabular}{|c|c|c|c|c|c|c|c|c|}
\hline Sito Codo & $\begin{array}{c}\text { Waste Managemont } \\
\text { Unlt Type }\end{array}$ & Waste Managemont Unit & Waste Typo & $\begin{array}{c}\text { Operablo } \\
\text { Unit }\end{array}$ & $\begin{array}{l}\text { Plutonium-242, } \\
\text { Units (Cl) }\end{array}$ & $\begin{array}{l}\text { Total Uranlum, Units (Cl, } \\
\text { unless otherwise stated) }\end{array}$ & $\begin{array}{c}\text { Gross Uranlum, } \\
\text { Units (C) }\end{array}$ & $\begin{array}{l}\text { Uranlum-235, } \\
\text { Urilts (CI) }\end{array}$ \\
\hline $216-B-2 B$ & \begin{tabular}{|l|} 
Trench \\
\end{tabular} & 216-B-26 Trench & Scavenged Waste & $\mathrm{BP}-2$ & & 0.196 & & 0 \\
\hline 216-8-27 & Trench & 216-8-27 Trench & Scavenged Waste & BP-2 & & 0.114 & & $\overline{0}$ \\
\hline $216-8-28$ & Trench & 216-B-2B Trench & Scavenged Wasto & $\overline{B P-2}$ & & 0.1 & & $\overline{0}$ \\
\hline$\overline{216-8 \cdot 29}$ & Trench & 216-8-29 Trench & Scavenged Waste & BP-2 & & 0.115 & & 0 \\
\hline $216-B-3$ & Ponds & 216-8-3 Ponde/ & Cooling Water & BP-11 & & 2.1 & & \\
\hline $216-8-3-1$ & Dilches & 216-8-3-1 DHchbl & Cooling Water & BP-11 & & & & \\
\hline $216-8-3-2$ & Ditches & 216-8-3-2 DHchel. & Coolling Water & BP-11 & & & & \\
\hline$\longdiv { 2 1 6 - 8 - 3 - 3 }$ & Ditches & 216-B-3-3Ditch & Coollng Water & BP-11 & & & & \\
\hline $216 \cdot B \cdot 30$ & Trench & 216-8-30 Trench & Scavenged Waste & BP-2 & & 0.0293 & & 0 \\
\hline $216-8 \cdot 32$ & Trench & 216-B-32 Trench & Scavenged Wasto & BP-2 & & 0.00367 & & 0 \\
\hline $216-8 \cdot 33$ & Trench & 216-8-33 Trench & Scavenged Waste & BP-2 & & 0.00687 & & 0 \\
\hline 216-B-34 & Trench & 216-8-34 Tronch & Scavenged Waste & BP.2 & & 0.0283 & & o \\
\hline $216-B-35$ & Trench & 216-8-35 Trench & Tank Farm Wasto & $\overline{B P-3}$ & & 0.00557 & & $\overline{0}$ \\
\hline $216-B-36$ & Trench & 216-B-36 Trench & Tank Farm Wasto & BP-3 & & 0.00532 & & 0 \\
\hline $216-B-37$ & Trench & 216-8-37 Trench & Process Waste & BP-3 & & 0.00121 & & 0 \\
\hline 216-B-38 & Trench & 216-8-38 Trench & Tank Farm Wasto & BP-3 & & 0.0141 & & 0 \\
\hline$\overline{216-8-39}$ & Trench & 216-8-39 Trench & Tank Farm Waste & BP-3 & & 0.00193 & & 0 \\
\hline 218-B-3A & Ponds & 216-B-3A Pond & Cooling Water & $\overline{B P-11}$ & & & & \\
\hline $216-8-3 B$ & Ponds & 216-8-3B Pond & Coolling Water & $8 \mathrm{P}-11$ & & & & \\
\hline 216-B-3C & Ponds & 216-B-3C Pond & Coolling Water & BP-11 & & & & \\
\hline $216-8-4$ & Reverso Woll & 216-B-4 Reverso Woil & Miscollaneous Drainage & $\overline{B P-6}$ & & $\overline{0}$ & & 0 \\
\hline$\overline{216-8-40}$ & Trench & 216-B-40 Trench & Tank Farm Wasto & BP-3 & & 0.017 & & $\overline{0}$ \\
\hline $216-B-41$ & Trench & 216-B-41 Trench & Tank Farm Wasto & $\overline{B P-3}$ & & 0.0025 & & 0 \\
\hline $218-8-42$ & Trench & 216-B-42 Trench & Scavengod Wasto & BP-3 & & 0.227 & & 0 \\
\hline $216-B-43$ & Cribs & 216-B-43 Crib & Scavonged Wasto & BP-1 & & 0.00454 & & 0 \\
\hline$\overline{216-8-44}$ & Cribs & 216-B-44 Crib & Scavenged Wasto & $\overline{\mathrm{BP}-1}$ & & 0.000756 & & 0 \\
\hline $216-8-45$ & Cribs & 216-B-45 Crib & Scavenged Wasto & BP-1 & & 0.00227 & & 0 \\
\hline $216-B-46$ & Crlbs & 216-8-46 Crib & Scavenged Wasto & BP-1 & & 0.0635 & & 0 \\
\hline $216-B-47$ & Cribs & $216-8-47 \mathrm{Crb}$ & Scavenged Wasto & 8P-1 & & 0.00227 & & 0 \\
\hline $218-B-48$ & Cribs & $216-8-48 \mathrm{Cr} i \mathrm{~b}$ & Scavenged Wasto & $\overline{B P-1}$ & & 0.000757 & & 0 \\
\hline$\overline{216-B-49}$ & Cribs & 216-B-49 Cib & Scavenged Wasto & $\overline{B P-1}$ & & 0.106 & & $\overline{0}$ \\
\hline 216-B-5 & Reverse Well & 216-B-5 Reverso Well & Process Waste & $\mathrm{BP}-6$ & & 0 & & $\overline{0}$ \\
\hline$\overline{216 \cdot 8 \cdot 50}$ & Cribs & 216-B-50 Cतrb & Process Condensato & बि-1 & & 0.000095 & & 0 \\
\hline
\end{tabular}


Table B.3. (page 5 of 14)

\begin{tabular}{|c|c|c|c|c|c|c|c|c|}
\hline SIto Codo & $\begin{array}{c}\text { Wasto Management } \\
\text { Unlt Typo }\end{array}$ & Wasto Menagement Unit & Wasto Typo & $\begin{array}{c}\text { Operablo } \\
\text { Unlt }\end{array}$ & $\begin{array}{l}\text { Plutonlum-242, } \\
\text { Units (C) }\end{array}$ & $\begin{array}{l}\text { Total Uranlum, Units (C), } \\
\text { unless otherwise stated }\end{array}$ & $\begin{array}{l}\text { Gross Urantum, } \\
\text { Untts (C)) }\end{array}$ & $\begin{array}{l}\text { Uranilum-235, } \\
\text { Unles (C)) }\end{array}$ \\
\hline $216-\mathrm{B}-51$ & French Drain & 218-8-5i French Draln & MIscellaneous Drainage & BP-4 & & & & \\
\hline $216-8.52$ & Tranch & 216-8-52 Trench & Scavenged Waste & 的-2 & & 0.00998 & & \\
\hline $216-B-53 A$ & Trench & 216-B.53A Trench & Lab Wasle & $\sqrt{B P-2}$ & & 0.00756 & & \\
\hline 216-8-53B & Trench & 216-8.53B Trench & Lab Wasto & $\overline{B P-2}$ & & 0.00302 & & \\
\hline $216-8-54$ & Trench & 216-8-54 Tronch & Lab Waste & $\overline{8 P-2}$ & & 0.00302 & & \\
\hline $216-\bar{B}-55$ & Cribs & 216-B-55 Crib & Steam Condensate & 丽-9 & & 0.0268 & & \\
\hline $216-8-56$ & Cribs & $216-8-56$ Crib & N/A & $\sqrt{B P-6}$ & & & & \\
\hline $216-8-57$ & Cribs & 216-8-57 Crib & Process Condensate & BP-1 & & 0.000297 & & \\
\hline 216-B-5B & Trench & 216-B-58 Tronch & Lab Wasto & $\longdiv { B P - 2 }$ & & 0.00304 & & \\
\hline $216-8-59$ & Retentlon Basin & 216-8-59 Basin & Cooling Water & $\sqrt{\mathrm{BP} .6}$ & & & & \\
\hline $216-B \cdot 6$ & Reverse Well & 216-B-6 Reverse Well & Lab Waste & BP.6 & & . & & \\
\hline 218-B-60 & Cribs & 216-B-60 Crib & Decon Waste & $\longdiv { B P - 6 }$ & & & & \\
\hline $216-B \cdot 61$ & Cribs & 216-B-61 Crib & N/A & EP-1 & & & & \\
\hline $216-8-62$ & Cribs & 216-B-62 Crib & Process Condensate & BP-9 & & 0.01 & & \\
\hline $210-8-63$ & Ditches & 216-B-63 Tronch & Chemlcal Sewer & BP-11 & & 0.15 & & \\
\hline$\longdiv { 2 1 6 - 8 - 6 4 }$ & Retention Basin & 216-8-64 Basin & NIA & $B P-8$ & & & & \\
\hline 216-B-7A\&B & Cribs & 216-B-7A\&B CCHb & Process Waste & $\sqrt{8 P-4}$ & & 0.0606 & & \\
\hline 216-8-8 & cribs & 216-B-8TF Crib & Procoss Waste & 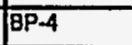 & & 0.0151 & & \\
\hline $216-8-9$ & cribs & 216-B-9TF Crib & Process Waste & 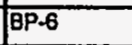 & & 0.0151 & & \\
\hline $216-C-1$ & Cribs & $218-C-1 \mathrm{Crb}$ & Procoss Condensale & SO-1 & & & & \\
\hline $216-C-10$ & Cribs & 216-C-10 Crib & Procoss Condensate & SO.1 & & & & \\
\hline$\overline{216-\mathrm{C}-2}$ & Reverso Well & 218-C-2 Reverse Woll & Miscollaneous Drahage & so-1 & & & & \\
\hline $216-C-3$ & Cribs & $\sqrt{216-\mathrm{C}-3 \mathrm{CHCHb}}$ & Process Waste & $50-1$ & & & & \\
\hline $216-C-4$ & Cribs & $\sqrt{216-C-4 \mathrm{Cnib}}$ & Procoss Wasto & 50.1 & & & & \\
\hline $216-C-5$ & Cribs & 216-C-5 Crib & Process Wasto & S0.1 & & & & \\
\hline $216-C-6$ & Cribs & 216-C.6 Crib & Procass Condensato & SO-1 & & & & \\
\hline $216-C-7$ & Cribs & 216-C-7 Crib & Process Waste & 50.1 & & & & \\
\hline $216-C-8$ & French Draln & $216-C-8$ & Process Wasto & $\mathrm{PO} \cdot 3$ & & & & \\
\hline $216-C-9$ & Ponds & 216-C-9Pond & Coolling Water & 50-1 & & & & \\
\hline $216-E-28$ & Ponds & 216-E-28 Pond & N/A & BP-11 & & & & \\
\hline $216-\mathrm{N}-1$ & Ponds & 216-N-1 Pond & Cooling Water & No.1 & & & & \\
\hline $216-\mathrm{N}-2$ & Trench & 216-N-2 Trench & Cooling Waler & NO-1 & & & & \\
\hline $216-\mathrm{N}-3$ & Tिrench & 216-N-3 Trench & Cooling Water & $\mathrm{NO}$ & & & & \\
\hline
\end{tabular}


Table B.3. (page 6 of 14)

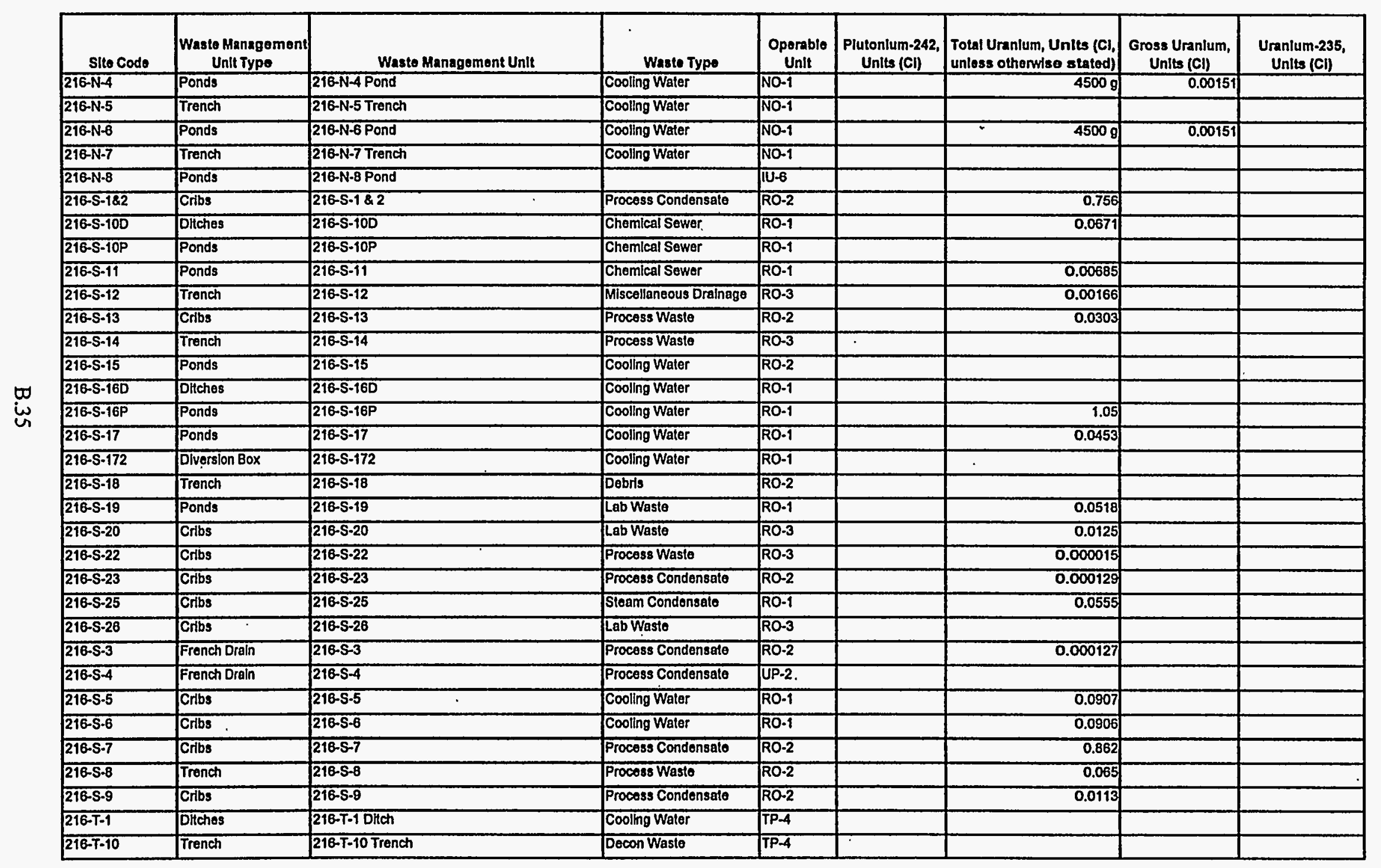


Table B.3. (page 7 of 14)

\begin{tabular}{|c|c|c|c|c|c|c|c|c|}
\hline Site Codo & $\begin{array}{c}\text { Wasto Management } \\
\text { Unlt Typo }\end{array}$ & Waste Management Unlt & Wasto Typo & $\begin{array}{c}\text { Operablo } \\
\text { Unit }\end{array}$ & $\begin{array}{l}\text { Plutonlum-242, } \\
\text { Units (Ci) }\end{array}$ & $\begin{array}{l}\text { Total Uranlum, Units (CI, } \\
\text { unless otherwise stafed) }\end{array}$ & $\begin{array}{c}\text { Gross Uranlum } \\
\text { Unlts (CI) }\end{array}$ & $\begin{array}{l}\text { Uranlum-236, } \\
\text { Units (C) }\end{array}$ \\
\hline 216-T-11 & Trench & 216-T-11 Trench & Decon Wasto & TP-4 & & & & \\
\hline $216-T-12$ & Trench & 216-T-12 Trench & Cooling Water & $\overline{T P}-3$ & & & & \\
\hline $216-T-13$ & Trench & 218-T-13 Trench & Decon Wasto & TP-2 & & & & \\
\hline $216-T-14$ & Trench & 216-T-14 Trench & Tank Farm Wasto & TP-3 & & & & \\
\hline $216-T-15$ & Trench & 216-T-15 Trench & Tank Farm Waste & TP-3 & & & & \\
\hline 216-T-16 & Trench & 216-T-16 Trench & Tank Farm Waslo & TP-3 & & & & \\
\hline 216-T-17 & Trench & 216-T-17 Trench & Tank Farm Wasto & TP-3 & & & & \\
\hline $216-\mathrm{T}-18$ & Cribs & 216-T-18 Crib & Tank Farm Wasle & TP-2 & & & & \\
\hline 216-T-19 & Cribs & 216-T-19Tf Crib and Tile Fleld & Process Waste & TP-2 & & & & \\
\hline 216-T-2 & Reverse Well & 216-T-2 Reverso Well & Lab Waste & TP-4 & & & & \\
\hline $216-T-20$ & Trench & 216-T-20 Trench & Process Waste & TP-2 & & & & \\
\hline $216-T-21$ & Tronch & 218-T-21 Trench & Tank Farm Waste & TP-1 & & & & \\
\hline 216-T-22 & Trench & 216-T-22 Trench & Tank Farm Waste & TP-1 & & & & \\
\hline 216-T-23 & Trench & 216-T-23 Trench & Tank Farm Waste & TP.1. & & & & \\
\hline 216-T-24 & Trench & 216-T-24 Trench & Tank Farm Wasto & TP-1 & & & & \\
\hline $216-T-25$ & Trench & 216-T-25 Trench & Process Wasle & TP-1 & & & & \\
\hline 216-T-26 & Cribs & 216-T-26 Crib & Tank Farm Waste & TP-2 & & & & \\
\hline 216-T-27 & Cribs & 216-T-27 Crlb & Lab Wasto & TP-2 & & & & \\
\hline $216-T-28$ & Cribs & 216-T-28 Crib & Decon Waste & TP-2 & & & & \\
\hline $216-T-29$ & Cribs & 216-T-29 Crib & Miscollaneous Dralnage & TP-4 & & & & \\
\hline 216-T-3 & Roverso Well & 216-T-3 Reverse Weil & Process Waste & TP-4 & & & & \\
\hline 216-T-31 & French Draln & 216-T-31 French Drain & Mlscollaneous Drainage & TP-2 & & & & \\
\hline $216-T-32$ & Cribs & 216-T-32 Crib & Process Waste & TP-1 & & & & . \\
\hline $216-T-33$ & Cribs & 216-T-33 Crib & Decon Waste & $\mathrm{TP}-4$ & & & & \\
\hline $216-T-34$ & Cribs & $216-T-34 \mathrm{Crib}$ & Lab Waste & TP-4 & & & . & \\
\hline 216-T-35 & Cribs & 216-T-35 Crib & Lab Wasle & TP-4 & & & & \\
\hline $216-T-36$ & Cribs & 216-T-36 Crib & Steam Condensale & TP.1 & & & & \\
\hline $210-T-4-10$ & Ditchios & 216-T-4-1D Ditch & Coolling Waler & TP-3 & & & & \\
\hline $216-T-4-2$ & Ditches & 216-T-4-2DHch & Steam Condensate & TP-3 & & & & \\
\hline 21Q-T-AA & Ponds & 216-T-4APond & Cooling Waler & TP.3 & & & & \\
\hline $216-T-4 B$ & Ponds & 216-T-4B Pond & Cooling Water & $\mathrm{TP}-3$ & & & & \\
\hline 216-T-5 & Trench & 216-T-5 Trench & Tank Farm Wasto & TP-1 & & & & \\
\hline $216-T-6$ & Cribs & 216-T-6 Crib & Process Wasto & TP-3 & & & & \\
\hline
\end{tabular}


Table B.3. (page 8 of 14)

\begin{tabular}{|c|c|c|c|c|c|c|c|c|}
\hline Sito Codo & $\begin{array}{c}\text { Wasto Managoment } \\
\text { Unit Typo }\end{array}$ & Wasto Management Unit & Wasto Typo & $\begin{array}{c}\text { Operable } \\
\text { Unit }\end{array}$ & $\begin{array}{l}\text { Plutonlum-242, } \\
\text { Units (C) }\end{array}$ & $\begin{array}{l}\text { Total Uranlum, Unlts (Cl, } \\
\text { unloss otherwise stated) }\end{array}$ & $\begin{array}{l}\text { Gross Uranlum, } \\
\text { Units (Cl) }\end{array}$ & $\begin{array}{l}\text { Urantum-235, } \\
\text { Units (C) }\end{array}$ \\
\hline $216-T-7$ & \begin{tabular}{|l|l|} 
Cribs \\
\end{tabular} & 216-T-7TF Crib and Tile Fleld & Tank Farm Waste & TP-1 & & & & \\
\hline $216-T-8$ & Cribs & 216-T-8 Crib & Lab Wasto & TP-4 & & & & \\
\hline 21G-T-9 & Trench & 216-T-9 Trench & Decon Waste & $T \frac{T P-4}{4}$ & & & & \\
\hline $216-U-182$ & Cribs & $216-U-1 \& 216-U-2$ & Process Condensate & UP-2 & & 0.702 & & \\
\hline $216-U \cdot 10$ & Ponds & $216-U-10$ & Coolling Water & UP-2 & & 1.88 & & \\
\hline $218-U \cdot 11$ & Ditches & $216-U-11$ & Coolling Water & $\overline{\mathrm{UP}-2}$ & & & & \\
\hline 218-U-12 & Cribs & $\longdiv { 2 1 6 - U - 1 2 }$ & Process Condensate & $\overline{U P-2}$ & & 0.677 & & \\
\hline $216-U-13$ & Trench & $216-U-13$ (same as UN-200-W-125) & Decon Waste & UP-2 & & & & \\
\hline $216-U-14$ & Ditches & $216-U-14$ & Coolling Water & UP-2 & & & & \\
\hline $216-U-15$ & Trench & 216-U-15 & Process Waste & UP-2 & & & & \\
\hline $216-U-16$ & Cribs & $216-U-16$ & Process Condensate & UP-2 & & 0.00592 & & \\
\hline $216-U-17$ & Cribs & $216-U-17$ & Process Condensate & UP-2 & & 0.000478 & & \\
\hline $216-U-21$ & & $216-U-21$ & & & & & & \\
\hline $216-U-3$ & French Drain & $216-U-3$ & Miscellaneous Drainage & UP-2 & & 0.00606 & & \\
\hline $216-U-4$ & Reverse Well & $216-U-4$ & LabWasto & $\overline{U P-2}$ & & & & \\
\hline $216-U-4 A$ & French Draln & 216-U-4A & Milscellaneous Dralnago & UP-2 & & 0.00297 & & \\
\hline $216-U-4 B$ & French Draln & $216-U-4 B$ & Mlscellaneous Dralnage & UP-2 & & & & \\
\hline $216-U-5$ & Trench & $216-U .5 \& 216-U .8$ & Process Waste & UP-2 & & & & \\
\hline $216-4-7$ & French Draln & $216-4.7$ & Miscellaneous Drainage & UP-2 & & & & \\
\hline $216-U \cdot 8$ & Cribs & $216-U-8$ & Process Condensate & UP-2 & & 8.04 & & \\
\hline $216-U-9$ & Ditches & $216-U-8$ & Coollng Water & RO-1 & & & & \\
\hline 216-W-LWC & Cribs & 216-W-LWC Crib & Chomlcal Sewer & SS-2 & & & & \\
\hline $216-2-1 \& 2$ & Cribs & 216-Z-1 \& 216-Z-2 Cribs & Process Wasle & $2 \mathrm{P}-2$ & & & & \\
\hline $216-z-10$ & Reverse Well & 218-Z-10 Reverse Well & Process Waste & $2 \mathrm{P}-2$ & 0.00004 & & & \\
\hline $216-2-11$ & Ditches & $216-z-11$ & Coollng Waler & UP-2 & & & & \\
\hline $216-z-12$ & Cribs & $216-Z-12 \mathrm{Crlb}$ & Process Waste & $2 \mathrm{P}-2$ & & & & \\
\hline $216-2-13$ & French Drain & 216-2-13 French Draln & Miscellaneous Drainage & $2 \mathrm{PP}-2$ & & & & \\
\hline $216-2-14$ & French Draln & 216-z-14 French Drain & Miscellaneous Drainage & $2 \mathrm{P}-2$ & & & & \\
\hline$\overline{216-2-15}$ & French Drain & 216-z-15 French Draln & Mlscellaneous Drainage & $2 \mathrm{P}-2$ & & & & \\
\hline $216-z-16$ & Cribs & 216-2-16 Crib & Lab Waste & $2 \mathrm{P}-2$ & & & & \\
\hline $216-z-17$ & Ditches & 216-2-17 Trench & Lab Waste & $\mathrm{ZP}-2$ & & & & \\
\hline $216-z-18$ & Cribs & $216-z-18$ Crib & Procoss Wasto & $2 \mathrm{P}-2$ & & & & \\
\hline $216-2-19$ & Ditches & $216-z-19$ & Cooling Water & UP-2 & & & & \\
\hline
\end{tabular}


Table B.3. (page 9 of 14)

\begin{tabular}{|c|c|c|c|c|c|c|c|c|}
\hline Slto Code & $\begin{array}{c}\text { Wasto Management } \\
\text { Unitt Type }\end{array}$ & Wasto Management Unlt & Waste Type & $\begin{array}{c}\text { Operable } \\
\text { Unit }\end{array}$ & $\begin{array}{l}\text { Plutonlum-242, } \\
\text { Unlts (C)) }\end{array}$ & $\begin{array}{l}\text { Total Urantum, Units (Cl, } \\
\text { unloss othorwiso stated) }\end{array}$ & $\begin{array}{l}\text { Gross Uranlum, } \\
\text { Units (CI) }\end{array}$ & $\begin{array}{l}\text { Uranlum-235, } \\
\text { Unlts (CI) }\end{array}$ \\
\hline $216-Z \cdot 1 A$ & Cribs & 216-Z-1A Tile Fild & Procoss Wasto & $\mathrm{ZP-2}$ & & & & \\
\hline $216-Z-10$ & Ditches & $216-Z-1 D$ & Coolling Water & UP-2 & & & & \\
\hline $216-2-20$ & Corlbs & $216-Z \cdot 20$ & Cooling Water & UP-2 & & & & \\
\hline 216-Z-21 & Relentlon Basin & 216-z-21 Seepage Basin & Cooling Water & $2 \mathrm{P}-2$ & & & & \\
\hline$\overline{216-z-3}$ & Cribs & $216-z-3 C_{\text {Crib }}$ & Process Wasto & $\overline{\mathrm{ZP}-2}$ & & & & \\
\hline $216-2-4$ & Trench & 216-Z-4 Trench & Process Waste & $\mathrm{ZP}-2$ & & & & \\
\hline $216-Z-5$ & Cribs & $216-Z-5$ Crib & Process Wasto & $\overline{\mathrm{ZP}-2}$ & & & & \\
\hline $216-Z-6$ & Cribs & $216-Z-6$ Crib & Process Waste & $\mathrm{ZP}-2$ & & & & \\
\hline 216-Z-7 & Cribs & 216-z-7 Crib & Lab Waste & $\mathrm{ZP}-2$ & & & & \\
\hline 216-Z-8 & Cribs & 216-2-8 French Draln & Process Waste & $\mathrm{ZP}-2$ & & & & \\
\hline$\overline{216-2-9}$ & Cribs & 218-Z-9 Trench & Process Wasto & $\overline{\mathrm{ZP}-2}$ & & & & \\
\hline 218-C.9 & Burlal SHe & 218-C-9 Burlal Ground & LLW-SOLID & SO-1 & & & & \\
\hline 218-E-1 & Burfal SHe & $218-E-1$ & LLW-SOLID & $\overline{\mathrm{PO}-2}$ & & 400000 & & \\
\hline 218-E-10 & Burlal Sitte & 218-E-10 Burlal Ground & LLW-SOLID & $\mathrm{BP}-10$ & & 800000 & & \\
\hline $218-E-12 A$ & Burial Ste & $218-E-12 A$ & LLW-SOLID & PO-6 & & 990000 & & \\
\hline 218-E-12B & Burfal SHE & 218-E-12B & LLW.SOLID & $\overline{P O-B}$ & & & & \\
\hline $218-E-13$ & Burfial Stro & $218-E-13$ & & $\mathrm{PO}-2$ & & & & \\
\hline$\overline{218-E-2}$ & Burfal SHO & 218-E-2 Burtal Ground & LLW-SOLID & BP.10 & & & & \\
\hline $218-E-2 A$ & Burlal SHto & 218-E-2A Burial Ground & LLW-SOLID & $\overline{B P-10}$ & & & & \\
\hline $218-E-4$ & Burial SHo & 218.E-4 Burlal Ground & LLW-SOLID & BP-10 & & & & \\
\hline $218-E \cdot 5$ & Burlal SHe & 218-E-5 Burfal Ground & LLW-SOLID & $B P-10$ & & & & \\
\hline 218-E-5A & Burlal Sito & 218-E-5A Burlal Ground & LLW-SOLID & BP-10 & & & & \\
\hline 218-E-6 & Burlal Sito & 218.E.6 Burlal Ground & Debris & BP-6 & & & & \\
\hline $218-E-7$ & Burlal Stho & 218-E-7 Burlal Ground & Lab Waste & $B P-6$ & & & & \\
\hline $218-E-8$ & Burlar SHo & 218-E-8 & TRU Solld Wasto & PO-6 & & 2000 & & \\
\hline $218-E-9$ & Burial SHe & 218-E-9 Burlal Ground & LLW-SOLID & BP-10 & & & & \\
\hline $218-W-1$ & Burial Site & 218-W-1 Burlal Ground & TRU Solld Wasto & $\mathrm{ZP}-3$ & & & & \\
\hline $218 \cdot W-11$ & Burlal SHe & 218.W-11 Burlal Ground & LLW.SOLID & $\mathrm{ZP}-3$ & & & & \\
\hline $218-W-1 A$ & Burlal Silie & 21B-W-1A Burlal Ground & LLW-SOLID & $2 \mathrm{P}-3$ & & & & \\
\hline $218-W-2$ & Burlal Sile & 218-W-2 Burlal Ground & TRU Solld Waste & $2 \mathrm{P}-3$ & & & & \\
\hline $218 \cdot W \cdot 2 A$ & Burial She & 218-W-2A Burial Ground & LLW-SOLID & $2 \mathrm{ZP}-3$ & & & & \\
\hline $218 \cdot W-3$ & Burlal Sto & 218 -W-3 Burlal Ground & TRU Solld Wasto & $2 \mathrm{P}-3$ & & & & \\
\hline $218-W-3 A$ & Burial Stio & 218-W-3A Eurlal Ground & TRU Solld Waste & $2 \mathrm{ZP}-3$ & & & & \\
\hline
\end{tabular}


Table B.3. (page 10 of 14)

\begin{tabular}{|c|c|c|c|c|c|c|c|c|}
\hline Site Code & $\begin{array}{c}\text { Waste Managoment } \\
\text { Unit Typo }\end{array}$ & Waste Management Uni: & Wasto Type & $\begin{array}{c}\text { Operablo } \\
\text { Unit }\end{array}$ & $\begin{array}{c}\text { Plutonlum-242, } \\
\text { Unlts (Cl) }\end{array}$ & \begin{tabular}{|l} 
Total Uranlum, Units (CI, \\
unless otherwise stated)
\end{tabular} & $\begin{array}{c}\text { Gross Uranlum, } \\
\text { Units (CI) }\end{array}$ & $\begin{array}{l}\text { Uranium-235, } \\
\text { Unlts (Ci) }\end{array}$ \\
\hline $218 \cdot W-3 A E$ & Burdal SHe & 218-W-3AE Burlal Ground & LLW-SOLID & $\overline{\mathrm{ZP} \cdot 3}$ & & & & \\
\hline $218 \cdot W-4 A$ & Burial Stio & 218-W-4A Burlal Ground & TRU Solld Wasto & $2 \mathrm{P}-3$ & & & & \\
\hline 218-W-4B & Burial SHe & 218-W-4B Calssons & TRU Solled Waste & $2 \mathrm{P}-3$ & & & & \\
\hline $218-W-4 B$ & Buriai Site & 218-W-4B Trenches & TRU Solld Waste & $2 \mathrm{ZP-3}$ & & & & \\
\hline $218-W-4 C$ & Burlal SHe & 218-W-4C Burlal Ground & TRU Solld Waste & $2 \mathrm{ZP}-3$ & & & & \\
\hline $218-W-5$ & Burlal SHo & 218-W-5 Burial Ground & TRU Solld Wasto & $2 \mathrm{PP}-3$ & & & & \\
\hline 218-W-6 & Burial SHe & 218-W-6 Burial Ground & LLW-SOLID & $2 \mathrm{PP}-3$ & & & & \\
\hline 218-W-7 & Burial SHito & 218-W-7 & LLW-SOLID & $\overline{R O-3}$ & & 7009 & & \\
\hline $218 \cdot W-8$ & Burlal SHo & 218-W-8 Burial Ground & Lab Waste & TP-4 & & & & \\
\hline $218-W \cdot 9$ & Guriai Stto & $218-W-9$ & LLW-SOLID & RO-2 & & & & \\
\hline $231-W \cdot 151$ & Dlverslon Box & 231-z-151 Sump & & $2 \mathrm{ZP}-2$ & & & & \\
\hline $231-W-151$ & Vault & $231-Z-151$ Sump & & $2 \mathrm{P} \cdot 2$ & & & & \\
\hline $231-W-151$ & Diversion Box & 231-z-151 Sump & & $\mathrm{ZP-2}$ & & & & \\
\hline $231-W-151$ & Vault & $231-z-151$ Sump & & $\mathrm{ZP}-2$ & & & & \\
\hline $231-W-151$ & Dlversion Box & 231-Z-151 Sump & & $\mathrm{ZP}-2$ & & & & \\
\hline $231-W-151$ & Vault & 231-Z-151 Sump & & ZP-2 & & & & \\
\hline $231-W-151$ & Diversion Box & 231-Z-151 Sump & & ZP-2 & & & & \\
\hline $231-W-151$ & Vault & 231-2-151 Sump & & $\mathrm{ZP}-2$ & & & & \\
\hline $232-Z$ & Bullding & 232-Z Inclnerator & & $2 \mathrm{P}-2$ & & & & \\
\hline $240-S-151$ & Dlverslon Box & $240-5-151$ & ILLW-SOLID & RO-3 & & & & \\
\hline $240-5-152$ & Dlversion Box & $240-5-152$ & Tank Farm Waste & $\overline{R O .3}$ & & & & \\
\hline $240-5 \cdot 302$ & Tanks & $240-5-302$ & Lab Wasto & RO.3 & & & & \\
\hline $241-A-151$ & Dlversion Box & 241-A-151 & Tank Farm Waste & $\overline{P O-2}$ & & & & \\
\hline $241-A-152$ & Dlverston Box & $241 \cdot A \cdot 152$ & Process Waste & PO-3 & & & & \\
\hline $241-A-153$ & Diversion Box & $241-A-153$ & Process Wasto & PO-3 & & & & \\
\hline $241-A-302 A$ & Tanks & $241-A-302 A$ & & $\overline{P O-2}$ & & & & \\
\hline $241-A \cdot 302 B$ & Tanks & $241-A-302 B$ & & PO.5 & & & & \\
\hline $241-A-350$ & Tanks & $241-A-350$ & Process Waste & PO-3 & & & & \\
\hline $241-A-417$ & Tanks & $241-A-417$ & Procoss Wasto & po-3 & & & & \\
\hline $241 \cdot A \cdot A$ & Diversion Box & $241-A \cdot A$ & Process Waste & PO-3 & & & & \\
\hline $241-A-B$ & Dlversion Box & 241-A-B & Process Waste & PO.3 & & & & \\
\hline $241-A N-A$ & Dlversion Box & 241-AN-A & Process Wasto & PO-3 & & & & \\
\hline 241-AN-B & Diversion Box & $241-A N-B$ & Process Waste & PO-3 & & & & \\
\hline
\end{tabular}


Table B.3. (page 11 of 14)

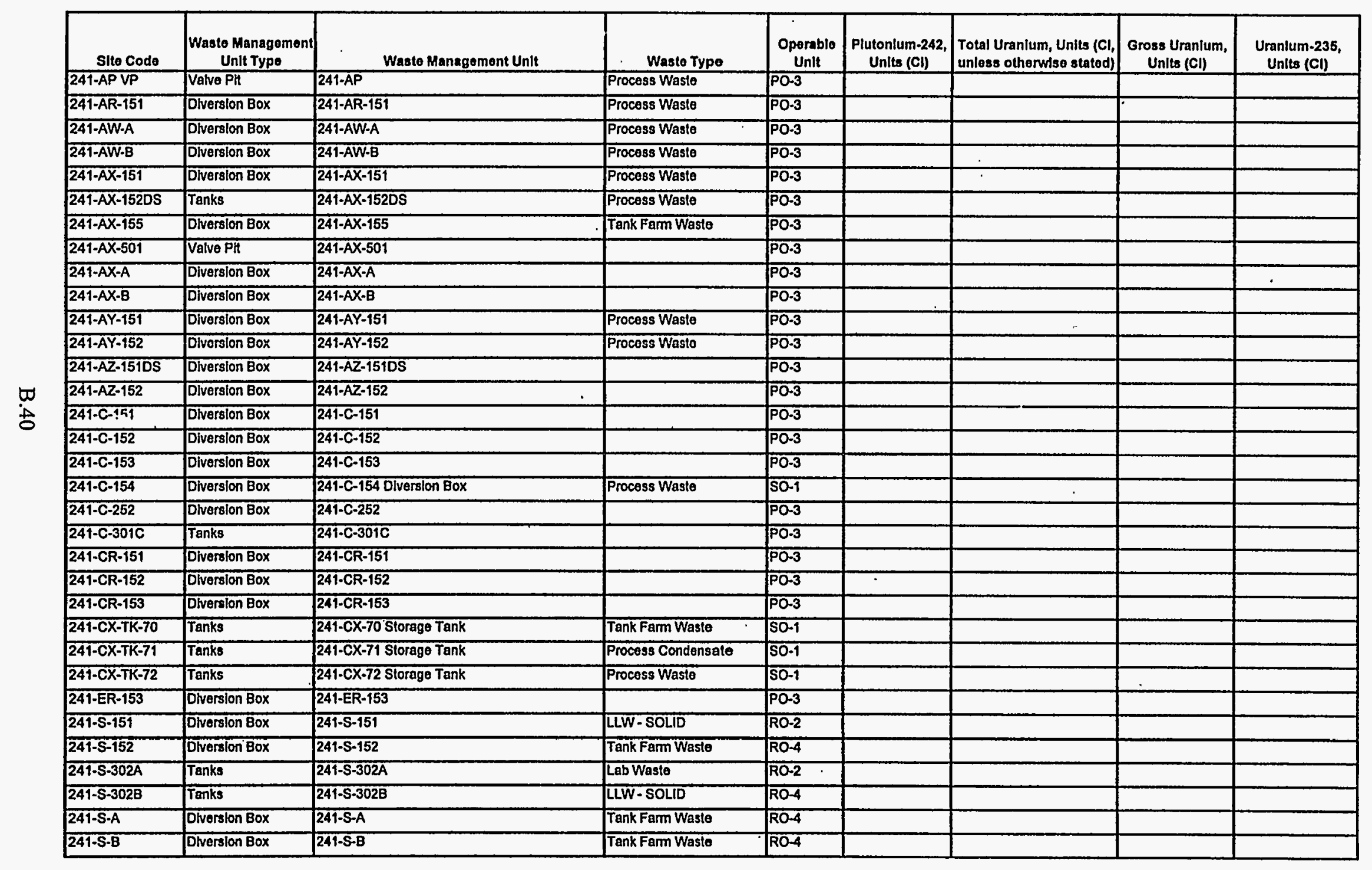


Table B.3. (page 12 of 14)

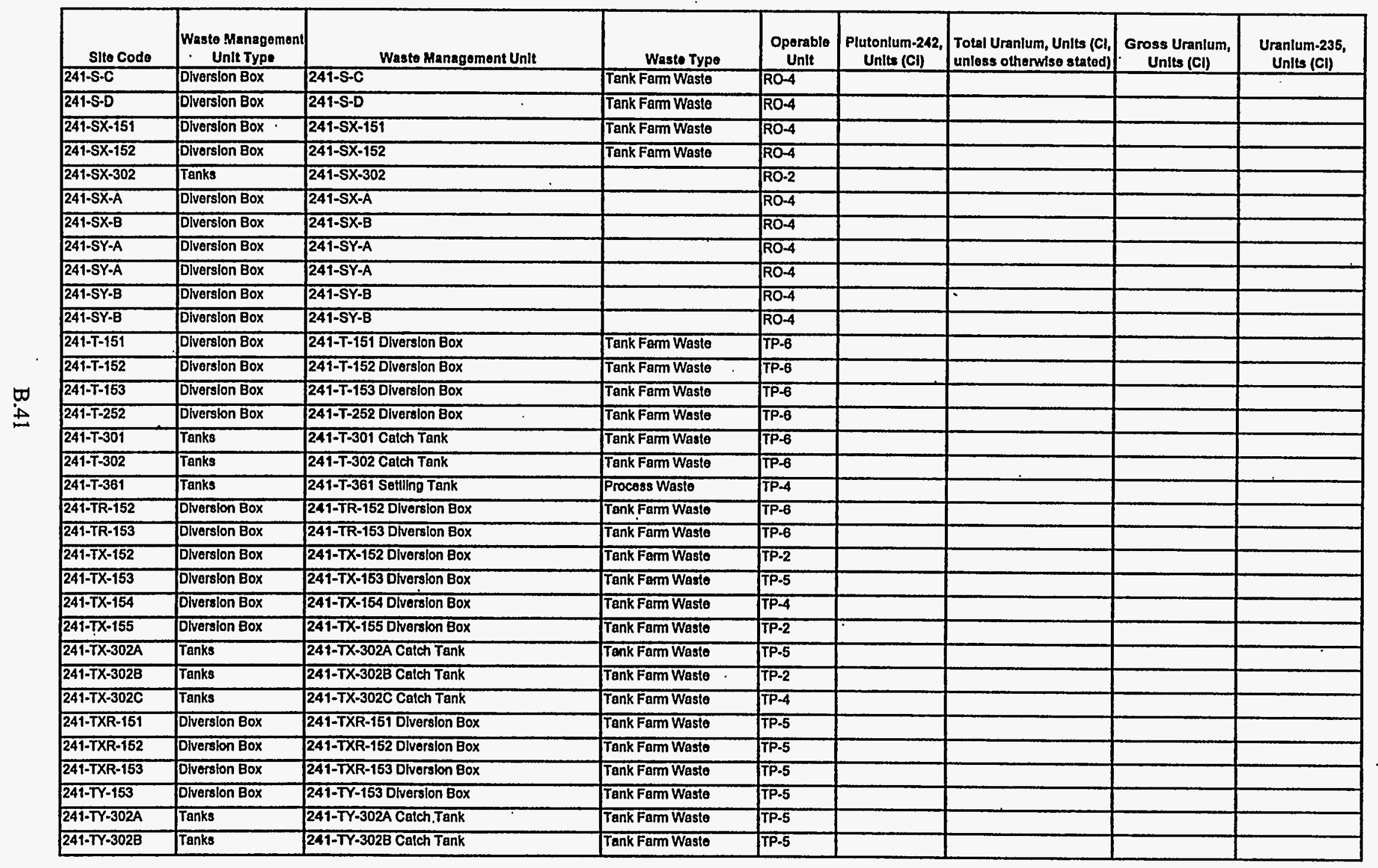


Table B.3. (page 13 of 14)

\begin{tabular}{|c|c|c|c|c|c|c|c|c|}
\hline Silo Codo & $\begin{array}{c}\text { Waste Management } \\
\text { Unit Type }\end{array}$ & Waste Management Unit & Wasto Typo & $\begin{array}{c}\text { Operable } \\
\text { Unit }\end{array}$ & $\begin{array}{c}\begin{array}{c}\text { Plutonlum-242, } \\
\text { Unlts (C) }\end{array} \\
\end{array}$ & $\begin{array}{l}\text { Total Uranlum, Units (Cl, } \\
\text { unless otherwlse stated) }\end{array}$ & $\begin{array}{c}\text { Gross Uranlum, } \\
\text { Units (C) }\end{array}$ & $\begin{array}{l}\text { Uranlum-235, } \\
\text { Units (C)) }\end{array}$ \\
\hline $241-2-381$ & Tranks & 241-Z-361 Settling Tank & Procoss Wasto & $\mathrm{ZP}-2$ & & & & \\
\hline 241-Z-TK-8 & Tanks & 218-2-8 Settling Tank & Procoss Wasto & $\overline{\mathrm{ZP}-2}$ & & & & \\
\hline 241-Z-TK.D5 & Tanks & 241-Z Treatment Tank & Process Waste & $Z \mathrm{PP}-2$ & & & & \\
\hline 242-T-151 & Diverslon Box & 242-T-151 Diversion Box & Process Condensate & TP-5 & & & & \\
\hline 244ART & Tanks & $244-A$ & Process Waste & $\overline{\mathrm{PO}-3}$ & & & & \\
\hline 244-AR VAULT & Vault & 244-AR & Process Waslo & PO-3 & & & & \\
\hline 244-CR VAULT & Vault & 244-CR & Process Wasto & PO-3 & & & & \\
\hline 244-S RT & Tanks & 244-S Recelver Tank & & RO-2 & & & & \\
\hline 244-TX RT & Tanks & 244-TX Receiving Tank & & TP-5 & & & & \\
\hline 244-TXR & Vault & 244-TXR Vault & Tank Farm Wasto & TP.5 & & & & \\
\hline 2607-E5 & Septlc System & $2607 \cdot$-E-5 Soptlc Tank and Drain Flofd & Santtary Wasle & 50-1 & & & & \\
\hline $2607-E 6$ & Soptlc System & $2807 \cdot E 6$ & Sanllary Waslo & $\overline{P 0-2}$ & & & & \\
\hline $2607-E 7 A$ & Septlc System & 2607-E-7A Septlc Tank and Draln Floid & Sanitary Waste & so-1 & & & & \\
\hline 2607-EA & Sepllc System & $2607 . E A$ & Santhary Waste & PO-2 & & & & \\
\hline 2607-EC & Soptle System & 2607-EC & Sanitary Waste & PO-5 & & & & \\
\hline 2607-ED & Septlc System & 2607-ED & Sanitery Waste & PO-3 & & & & \\
\hline 2807-EE & Soptlc System & 2607-EL & Sanitlany Wasto & PO-2 & & & & \\
\hline 2607-EG & Septlc System & $2607 \cdot E G$ & Santlary Waste & PO-3 & & & & \\
\hline 2607-EJ & Septic System & 2607-EJ & Saniltery Wasto & P0.3 & & & & \\
\hline $2607-N$ & Septlc System & 2607-N Septlc Tenk/Drain Fleld & Sanitary Waste & NO-1 & & & & \\
\hline $2607-\mathrm{P}$ & Soptle System & 2607-P Soplic Tank/Draln Fleld & Sanilary Waste & NO-1 & & & & \\
\hline $2607 \cdot R$ & Seplic System & 2607-R Septic TankJDrain Fleld & Santlary Waste & NO.1 & & & & \\
\hline 2607-W1 & Seplic System & 2607-W1 Sopllc Tank & Sanillary Wasto & SS-2 & & & & \\
\hline $2607 \cdot W 2$ & Sepllc System & 2607-W2 Septlc Tank & Santlary Wasto & sS-2 & & & & \\
\hline $2607-W 3$ & Septlc System & 2607-W3 Seplic Tank & Sanflary Wasto & TP-4 & & & & \\
\hline $2607-W 4$ & Septlc Syslem & 2607-W4 Septlc Tank & Sanflary Waste & TP-4 & & & & \\
\hline $2607-W 8$ & Seplic System & $2607-$ W6 & Santtary Wasto & RO-3 & & & & \\
\hline 2607-W8 & Septlc System & 2607-W-8 Septlc Tank and Drain Fleld & Saniltary Waste & $z P-2$ & & & & \\
\hline 2607-WA & Sepille System & 2607-WA Seplic Tank and Draln Fleld & Sentlary Wasto & $2 \mathrm{P} \cdot 2$ & & & & \\
\hline 2607-WB & & 2607-WB Seplic Tank and Drain Fleld & . & & & & & \\
\hline $2607-W T$ & Septlc System & 2607-Wr Soptle Tank & Sanitary Wasto & TP-5 & & & & \\
\hline 2607-WTX & Septle System & 2607-WTX Seplic Tank & Santtary Wasto & $T P-5$ & & & & \\
\hline $2607 \cdot W z$ & Septlc Systom & $2607 \cdot W 2$ & Sanitary Wasto & RO-1 & & & & \\
\hline
\end{tabular}


Table B.3. (page 14 of 14)

\begin{tabular}{|c|c|c|c|c|c|c|c|c|}
\hline Slto Codo & $\begin{array}{c}\text { Waste Management } \\
\text { Unil Type }\end{array}$ & Wasto Management Unit & Wasto Typo & $\begin{array}{c}\text { Oporablo } \\
\text { Unit }\end{array}$ & $\begin{array}{l}\text { Plutonlum-242, } \\
\text { Units (C)) }\end{array}$ & $\begin{array}{l}\text { Total Uranlum, Units (Cl, } \\
\text { unless otherwiso stated) }\end{array}$ & $\begin{array}{l}\text { Gross Uranlum, } \\
\text { Units (CI) }\end{array}$ & $\begin{array}{c}\text { Uranium-235, } \\
\text { Units (CI) }\end{array}$ \\
\hline $2607 . \bar{z}$ & Septle System & 2607-Z Seplic Tank and Draln Fleld & Santtary Wasto & $\mathrm{ZP}-2$ & & & & \\
\hline $2607 \cdot z-1$ & & 2607-2-1 Seplle Tank and Draln Fleld & & & & & & \\
\hline 2704 C-WS-1 & French Draln & $\begin{array}{l}\text { 2704-C-WS-1, 2704-C Fronch Drain, } \\
\text { Gatehouse French Drain }\end{array}$ & Miscollaneous Dralnago & SO-1 & & & & \\
\hline $2904-\mathrm{S}-160$ & Diversion Box & $2904-\mathrm{S}-160$ & Cooling Water & RO-1 & & & & \\
\hline 2904-S-170 & Diversion Box & 2004 S-170 & Process Wasto & RO-1 & & & & \\
\hline 2904-S-171 & Diversion Box & $2904-\mathrm{S}-171$ & Cooling Waler & RO-1 & & & & \\
\hline $291-\mathrm{C}$ & Bullding & 201-C Ventllation Systom & Process Condensalo & So-1 & & & & \\
\hline 299-E24-111 & Reverse Well & 299-E24-111 & & PO-2 & & & & \\
\hline HSVP & Diverslon Box & Somt-Works Valvo Pft & Process Waste & $50-1$ & & & & \\
\hline UPR-200-E-141 & & UN-200-E-141 & Solution Storage (1) & 50.1 & & & & \\
\hline UPR-200-E-38 & & UN-200-E-36 & Process \& Decon Wastes & 50.1 & & & & \\
\hline UPR-200-E-37 & & UN-200-E-37 & Frocess \& Decon Wastes & $50-1$ & & & & \\
\hline UPR-200-E-98 & & UN-200-E-98 & Process \& Decon Wastes & 50.1 & & & & \\
\hline UPR-200-W-160 & & UPR-200-W-160 Unplanned Release & Fitnk Farm Waste & $\sqrt{T P-4}$ & & & & \\
\hline Z PLANT BP & Eurial SHo & ZPlant Bum PHt & Debris & $2 \mathrm{PP}-3$ & & & & \\
\hline
\end{tabular}


Table B.4. Environmental Restoration Waste Site Inventories for Uraniunm-238, Alpha Emitters, Beta Emitters, and Americium-241 (page 1 of 14)

\begin{tabular}{|c|c|c|c|c|c|c|c|c|}
\hline Silte Code & $\begin{array}{c}\text { Wasto Managoment } \\
\text { Unlt Typo }\end{array}$ & Wasto Management Unlt & Waste Typo & $\begin{array}{c}\text { Oporable } \\
\text { Unit }\end{array}$ & $\begin{array}{l}\text { Urantum-238, } \\
\text { Units (Ci) }\end{array}$ & $\begin{array}{l}\text { Alpha Emittors, } \\
\text { Units (C)) }\end{array}$ & $\begin{array}{l}\text { Bola Emittors, } \\
\text { Units (CI) }\end{array}$ & $\begin{array}{l}\text { Americlum-241, } \\
\text { Units (CI) }\end{array}$ \\
\hline & & 212-N to 216-N-1 Pipeline & & & & & & \\
\hline & & 212-P Hazardous Wasle Slaging Area & & & & & & \\
\hline & & 212-P to 216-N-4 Plpeline & & & & & & \\
\hline & & 212.P Transformar Oll Tank & & & & & & \\
\hline & & 212-R to 218-N-6 Plpeiline & & & & & & \\
\hline & & 241-C Wasto Line Unplanned Release No. 1 & & & & & & \\
\hline & & 241.C Wasto Line Unplanned Release No. 2 & . & & & & & \\
\hline & & 241-2 Dlverslon Box No. 1 & & & & & & \\
\hline & & 241-Z Dlversion Box No. 2 & & & & & & \\
\hline & & Santiary Crib & & & & . & & \\
\hline 200-EBP & $\longdiv { \text { Burlal Stro } }$ & 200-E Buming PH & Debris & PO.6 & & & & \\
\hline 200-E PAP & Burial SHe & 200-EAsh PH & & SS-1 & & & & \\
\hline 200-E PD & Ditches & 200 East Powerhouse DHch & Coolling Water & So-1 & & & & \\
\hline $200-E-4$ & French Drain & Crttlcal Mass Laboratory Dry Well North & Miscellaneous Drainage & SO-1 & & & & \\
\hline $200-N-3$ & Burial SHo & Ballast PHs & Debrls & NO-1 & & & & \\
\hline 200-W ADB & Burlai Site & 200-WAsh Dlsposal Basin & Ash & $\widehat{S S-2}$ & & & & \\
\hline 200-WADS & Burlal Site & 200-WAsh PH Demolitlon SHo & NIA & SS-2 & & & & \\
\hline 200-WBP & Burial Stto & 200-W Buming PH & Debris & SS-2 & & & & \\
\hline 200-WPAP & Burial SHte & 200-W Powerhouse Ash PH & Ash & SS-2 & & & & \\
\hline 200-WPP & Ponds & 200-W Powerhouse Pond & Cooling Water & $\overline{\mathrm{TP}-2}$ & & & & \\
\hline $201 . \mathrm{C}$ & Bullding & 201-C Process Bullding & Process Condensate & 50.1 & & & & 0.2 \\
\hline $207-A$ & & $207-A$ & & & & & & \\
\hline $207 \cdot 8$ & Reiention Basin & 207-Bb/Retentlon Basin & Coolling Water & $\overline{B P .8}$ & & & & \\
\hline 207-S & Retenilon Basin & $207-5$ & Coolling Water & $\overline{R O-2}$ & & & & \\
\hline $207-S L$ & Retention Basin & $207-S L$ & Lab Wasto & RO-3 & & & & \\
\hline $207-T$ & Reiention Basin & 207-T Retentlon Basin & Cooling Water & TP.3 & & & & \\
\hline $207-2$ & Releniton Basin & 207-2 Retention Basin & Steam Condensale & $\mathrm{ZP}-2$ & & & & \\
\hline 209-E-WS-1 & French Drain & Crttlcal Mass Laboratory Dry Well East & Miscollaneous Dralnage & $50-1$ & & & & \\
\hline 209-E-WS-2 & French Drain & Critleal Mass Laboralory Dry Well South & Mlscellaneous Drainage & $50-1$ & & & & \\
\hline 209-E-WS-3 & Diversion Box & Critlical Mass Laboratory Valve PH & Process Waste & SO-1 & & & & \\
\hline 2101-M POND & Ponds & 2101-MPond & Lab Wasle & SS-1 & & & & \\
\hline $216-A-1$ & Cribs & $218-A-1$ & Process Wasto & P0.5 & 0.0516 & 0.00614 & 0.17 & \\
\hline $216-\bar{A}-10$ & Cribs & $216-A-10$ & Process Condensate & PO-2 & & 28.1 & 360 & 0.773 \\
\hline
\end{tabular}


Table B.4. (page 2 of 14)

\begin{tabular}{|c|c|c|c|c|c|c|c|c|}
\hline Site Code & $\begin{array}{l}\text { Waste Management } \\
\text {. Unit Type }\end{array}$ & Waste Management Unlt & Wasto Typo & $\begin{array}{c}\text { Operable } \\
\text { Unit }\end{array}$ & $\begin{array}{l}\text { Uranlum-238, } \\
\text { Unlts (CI) }\end{array}$ & $\begin{array}{c}\text { Alpha Emitters, } \\
\text { Units (Ci) }\end{array}$ & $\begin{array}{l}\text { Bota Emitters, } \\
\text { Unlts (C!) }\end{array}$ & $\begin{array}{l}\text { Americlum-241, } \\
\text { Units (Cl) }\end{array}$ \\
\hline $216-A-11$ & French Drain & $216-A-11$ & Miscollaneous Drainage & P0.2 & & & & \\
\hline $216-A-12$ & French Draln & $216-A-12$ & Mlscellaneous Drainage & PO-2 & & & & \\
\hline $216-A \cdot 13$ & French Drain & $216-A-13$ & Miscollaneous Drainago & $\overline{\mathrm{PO}-2}$ & & & & \\
\hline $216-A-14$ & French Drain & $216-A-14$ & Miscollaneous Drahago & PO-2 & . & & & \\
\hline $216-A-15$ & French Draln & $216-A-15$ & Process Condensale & PO-2 & & & & \\
\hline $216-A \cdot 16$ & French Drain & $216-A-16$ & Chemkal Sewer & PO-5 & & & & \\
\hline $216-A \cdot 17$ & French Draln & $216-A-17$ & Chemical Sewer & PO-5 & & & & \\
\hline $216-A-18$ & Trench & 2 & Process Waste & PO-5 & 0.472 & 0.00614 & 0.172 & \\
\hline $216-A-19$ & Trench & 216-A-19 & Process Waste & PO-5 & 13 & 0.00614 & 0.17 & \\
\hline $216-A-2$ & Cribs & 216-A-2 & Process Waste & $\mathrm{PO}-2$ & 0.0262 & 7.98 & 4.71 & \\
\hline $216-A-20$ & Trench & $\sqrt{216-A-20}$ & Process Waste & $\overline{\mathrm{PO}-5}$ & 0.135 & 0.00614 & 0.17 & \\
\hline $216-A-21$ & Cribs & $216-A-21$ & Lab Waste & $\overline{\mathrm{PO}-2}$ & 0.0653 & 9.21 & 166 & \\
\hline $216-A-22$ & French Drain & $216-A-22$ & Miscellaneous Dralnage & PO.2 & & & & \\
\hline $216-A-23 A$ & French Draln & $216-A-23 A$ & Process Condensale & PO-5 & & & & \\
\hline $216-A-23 B$ & French Drain & $216-A-23 B$ & Process Condensale & PO-5 & & & & \\
\hline $216-A-24$ & Cribs & $216-A-24$ & Process Condensate & PO-5 & 0.0168 & 0.311 & 552 & \\
\hline $216-A-25$ & Ponds & 216-A-25 Pond & Cooling Water & IU.6 & & 27.5 & 939 & 0.000528 \\
\hline $216-A-26$ & French Drain & $216-A-26$ & Mlscellaneous Drainage & $\overline{P O-2}$ & & & & \\
\hline $216-A-26 A$ & Franch Drain & $216-A-26 A$ & Mlscellaneous Dralnage & PO-2 & & & & \\
\hline $216-A-27$ & Cribs & 216-A-27 & Mlscellaneous Dralnage & PO-2 & 0.0228 & 5.92 & 112 & \\
\hline $216-A-28$ & Cribs & $216-A \cdot 2 B$ & Process Condensale & $\overline{P 0.2}$ & 0.212 & & 0.00747 & \\
\hline $216 \cdot A \cdot 29$ & Ditches & $216-A-29$ & Chemlcal Sower & BP-11 & & & & \\
\hline $216-A-3$ & Cribs & $216-A-3$ & Process Waste & PO-2 & & 0.0123 & 0.182 & \\
\hline 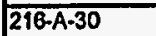 & Cribs & $216-A-30$ & Steam Condensalo & PO-4 & & 4.64 & 432 & 0.198 \\
\hline $216-A-31$ & Cribs & $216-A-31$ & Process Waste & $\overline{P 0.2}$ & 0.00886 & 0.553 & 162 & \\
\hline$\sqrt{216 \cdot A \cdot 32}$ & Cribs & $216-A-32$ & Miscollaneous Drainago & PO-2 & & & & \\
\hline 2 & French Draln & $216-A-33$ & Miscollaneous Drainago & $\overline{\mathrm{PO}-2}$ & & & & \\
\hline $216-A-34$ & Cribs & $\overline{216-A \cdot 34}$ & Procoss Condensato & PO-5 & & & & \\
\hline $218-A-35$ & Frentch Draln & 218-A-35 & Mlscellaneous Drainage & PO-2 & & & & \\
\hline $216-A-36 A$ & Cribs & $216-A-36 A$ & Process Wasto & $\longdiv { \mathrm { PO } - 2 }$ & 0.0486 & 4.91 & .3630 & \\
\hline $216-A-36 B$ & Cribs & $216-A-36 B$ & Process Waste & $\overline{P O-2}$ & & 11 & 1360 & 0.217 \\
\hline $216-A-37-1$ & Cribs & $216-A-37-1$ & Process Condensato & PO-4 & & 0.00845 & 0.508 & 0.000369 \\
\hline $216-A-37-2$ & Cribs & $216-A-37-2$ & Steam Condensale & PO-4 & & 0.105 & 1.85 & 0.0982 \\
\hline
\end{tabular}


Table B.4. (page 3 of 14)

\begin{tabular}{|c|c|c|c|c|c|c|c|c|}
\hline Silto Code & $\begin{array}{c}\text { Wasto Management } \\
\text { Unlt Typo }\end{array}$ & Wasto Management Unit & Waste Typo & $\begin{array}{c}\text { Operable } \\
\text { Unlt }\end{array}$ & $\begin{array}{l}\text { Uranlum-238, } \\
\text { Unlts (C)) }\end{array}$ & $\begin{array}{l}\text { Alpha Emiltors, } \\
\text { Units (Ci) }\end{array}$ & $\begin{array}{l}\text { Bota Emitters, } \\
\text { Unlts (Cl) }\end{array}$ & $\begin{array}{c}\text { Americlum-241, } \\
\text { Unlts (CI) }\end{array}$ \\
\hline $216-A-3 B-1$ & \begin{tabular}{|l|} 
Cribs \\
\end{tabular} & $216-A-38-1$ & N/A & $P O-2$ & & & & \\
\hline $216-A-39$ & Ditches & $216-A-39$ & Mlscellaneous Drainage & $\overline{P 0-3}$ & & & 27.5 & \\
\hline $216-\bar{A}-4$ & Cribs & 216-A-4 & Lab Wasto & PO-2 & 0.134 & 8.6 & 2.21 & \\
\hline $216-A-40$ & Relention Basin & $216-A-40$ & Steam Condensate & PO-2 & & & & \\
\hline $216-A-41$ & Cribs & $\overline{216-A-41}$ & Miscallaneous Drainage & PO-2 & & & & \\
\hline $216-A-42$ & Retention Basin & $216-A-42$ & Coolling Waler & PO-4 & & & & \\
\hline $216-A-45$ & Cribs & $216-A-45$ & Procoss Condensate & $\overline{\mathrm{PO}-2}$ & & 0.0551 & 0.112 & 0.11 \\
\hline $216-A-5$ & Cribs & $216-A-5$ & Process Condensate & PO-2 & 0.0881 & 3.99 & 109 & \\
\hline $216-A-524$ & Dlversion $80 x$ & $216-A-524$ & & p0.5 & & & & \\
\hline 216-A-6 & Cribs & $216-A-6$ & Steam Condensale & $\overline{P 0.4}$ & 0.0553 & 2.10 & 291 & \\
\hline $216-A-7$ & Cribs & $216-A-7$ & Process Waste & PO-5 & 0.00228 & 0.0614 & 5.29 & \\
\hline $216-A-8$ & $\overline{\text { Cribs }}$ & $216-A \cdot B$ & Process Condensato & PO-5 & & 3.07 & 1110 & \\
\hline $216-A-9$ & Cribs & $216-A \cdot 9$ & Cooling Water & PO-2 & 0.00008 & 0.0307 & 31 & \\
\hline $216-8-10 A$ & Cribs & $216-8-10 \mathrm{~A} \mathrm{Crib}$ & Lab Wasto & BP-6 & 0.00304 & 0.602 & 4.55 & $\overline{0}$ \\
\hline $216-B-10 B$ & Cribs & 216-B-10B Crib & Lab Wasto & BP-B & & 0.00000291 & 0.000000531 & 0 \\
\hline 216-B-11A\&B & Reverso Well & 216-B-11A\&B Reverse Wolls & Process Condensale & $B P-4$ & 0.00456 & 0.246 & 44.9 & 0 \\
\hline $216-8-12$ & Cribs & $216-\mathrm{B}-12 \mathrm{Crib}$ & Process Condensalo & BP-9 & 7 & 23 & 1540 & 0 \\
\hline 216-B-13 & French Drain & 216-B-13 French Draln & Mlscellaneous Dralnage & BP.6 & & & & \\
\hline $216-8-14$ & Cribs & 216-B-14 Crib & Scavenged Wasto & BP.2 & 0.073 & 1.53 & 567 & 0 \\
\hline 216-B-15 & Cribs & 216-B.15 CAb & Scavenged Wasto & $B P-2$ & 0.0348 & 0.307 & 357 & 0 \\
\hline $216-B-16$ & Cribs & 216-8-16 Crib & Scavenged Wasto & BP-2 & 0.108 & 0.614 & 1180 & 0 \\
\hline 218-B-17 & Cribs & 216-8-17 Crib & Scavenged Waste & BP-2 & 0.110 & 0.614 & 330 & 0 \\
\hline 216-B-18 & Cribs & 216-B-18 Crib & Scavenged Wasto & $B P-2$ & 0.0791 & 0.614 & 385 & 0 \\
\hline $216-B-19$ & Cribs & 216-B-19 Crib & Scavenged Wasto & BP-2 & 0.0606 & 0.614 & 418 & 0 \\
\hline $216-8-2-1$ & Ditches & 216-B-2-1 DHchb & Coolling Waler & BP-11 & & 16.2 & 390 & 3.96 \\
\hline $216-8 \cdot 2 \cdot 2$ & Diches & 216-B-2-2 Dlichad & Cooling Water & BP.11 & 0 & 0.00258 & 295 & \\
\hline $216-B-2-3$ & Ditches & 216-8-2-3DHch & Cooling Water & BP-11 & & & 864 & \\
\hline $216 \cdot 8 \cdot 20$ & Trench & 216-8-20 Trench & Scavenged Waste & BP-2 & 0.118 & 0.0798 & 2000 & व \\
\hline $216-B-21$ & Trench & 216-B-21 Trench & Scavenged Waste & Bp-2 & 0.226 & 0.632 & 965 & - \\
\hline $216-8-22$ & Trench & 216-B-22 Trench & Scavengod Wasle & QP-2 & 0.14 & 0.16 & 398 & \\
\hline 216-B-23 & Trench & 216-B-23 Trench & Scavenged Wasto & $8 \mathrm{P}-2$ & 0.0523 & 0.111 & 226 & 0 \\
\hline $216-8-24$ & Trench & 216-8-24 Trench & Scavenged Waste & BP-2 & 0.0825 & 0.473 & 0.274 & 0 \\
\hline $216-B-25$ & Trench & 216-B-25 Trench & Scavenged Waste & $B P-2$ & 0.513 & 0.123 & 229 & 0 \\
\hline
\end{tabular}


Table B.4. (page 4 of 14)

\begin{tabular}{|c|c|c|c|c|c|c|c|c|}
\hline Sito Codo & $\begin{array}{c}\text { Wasto Management } \\
\text { Unit Type }\end{array}$ & Wasto Manzigomont Unlt & Wasto Typo & $\begin{array}{c}\text { Operable } \\
\text { Unit }\end{array}$ & $\begin{array}{l}\text { Uranlam-238, } \\
\text { Unlts (CI) }\end{array}$ & $\begin{array}{l}\text { Alpha Emitters, } \\
\text { Units (CI) }\end{array}$ & $\begin{array}{l}\text { Beta Emlters, } \\
\text { Units (CI) }\end{array}$ & $\begin{array}{l}\text { Americlum-241, } \\
\text { Units (Cl) }\end{array}$ \\
\hline $216-B-26$ & \begin{tabular}{|l|} 
Trench \\
\end{tabular} & 216-8-26 Trench & Scavenged Waste & $B P-2$ & 0.197 & 0.0153 & 1800 & 0 \\
\hline $216-8-27$ & Tronch & 216-8-27 Trench & Scavenged Waste & $\overline{B P-2}$ & 0.115 & 0.043 & 560 & 0 \\
\hline 216-B-28 & Trench & 216-B-28 Trench & Scavenged Waste & $\overline{\mathrm{BP}-2}$ & 0.101 & 0.34 & 121 & 0 \\
\hline 21Q-B-29 & Trench & 216-B-29 Trench & Scavenged Wasie & BP-2 & 0.115 & 0.0675 & 226 & 0 \\
\hline 2 & Ponds & 218-B-3 Pondel & Coolling Water & $\overline{B P-11}$ & & 16.2 & 390 & 3.96 \\
\hline $216-B-3-1$ & Ditches & 216-8-3-1 Ditchb/ & Coolling Water & $\overline{B P-11}$ & & & & \\
\hline $216-\overline{B-3-2}$ & Ditches & 216-B-3-2D Dtthal & Cooling Water & बि-11 & & & & \\
\hline $216-B-3-3$ & Ditchos & 216-B-3-3D DHक & Cooling Water & $8 \mathrm{PP}-11$ & & & & \\
\hline $216-\mathrm{B}-30$ & Trench & 216-B-30 Trench & Scavenged Waste & BP-2 & 0.0295 & 0.129 & 3540 & 0 \\
\hline $216-8-32$ & Trench & 216-B-32 Trench & Scavenged Waste & $8 \mathrm{PP}-2$ & 0.00368 & 0.16 & 330 & 0 \\
\hline $216-8-33$ & Trench & 216-B-33 Trench & Scavenged Waste & BP-2 & 0.0067 & 0.724 & 281 & 0 \\
\hline $216-8-34$ & Trench & 216-B-34 Trench & Scavenged Waste & $\overline{\mathrm{BP}-2}$ & 0.0285 & 0.35 & 51.7 & 0 \\
\hline $216-8-35$ & Trench & 216-B-35 Trench & Tank Farm Waste & $\mathrm{BP}-3$ & 0.00559 & 0.0737 & 549 & .0 \\
\hline$\overline{216-8-36}$ & Trench & 216-B-36 Trench & Tank Farm Waste & BP-3 & 0.00532 & 0.0491 & 1040 & 0 \\
\hline $216-8-37$ & Trench & 216-8-37 Trench & Process Waste & $\overline{\mathrm{BP}-3}$ & 0.00121 & 0.123 & 2600 & $\overline{0}$ \\
\hline $216-8-38$ & Trench & 216-B-38 Trench & Tank Farm Waste & $\overline{\mathrm{BP}-3}$ & 0.0142 & 0.0737 & 1940 & 0 \\
\hline$\overline{216-8-39}$ & Trench & 216-B-39 Trench & Tank Farm Waste & $\overline{B P-3}$ & 0.00194 & 0.0927 & 387 & $\overline{0}$ \\
\hline$\overline{216-8-3 A}$ & Ponds & 216-B-3A Pond & Coolling Waler & $\overline{B P}-11$ & & & & \\
\hline $216-8 \cdot 3 B$ & Ponds. & 216-B-38 Pond & Coolling Water & $\overline{B P-11}$ & & & & \\
\hline $216-B-3 C$ & Ponds & 216-B-3C Pond & Cooling Water & BP-11 & & & & \\
\hline $216-8-4$ & Roverse Well & 216-B-4 Reverse Well & Miscellaneous Drainago & $\overline{B P-6}$ & of & 0 & 1 & $\overline{0}$ \\
\hline $216-8-40$ & Trench & 216-8-40 Tronch & Tank Farm Waste & $\overline{B P-3}$ & 0.00117 & 0.0614 & 523 & $\overline{0}$ \\
\hline 216-8-41 & Trench & 216-8-41 Trench & Tank Farm Wasio & BP-3 & 0.00251 & 0.0184 & 780 & 0 \\
\hline$\frac{3}{216-B-42}$ & Trench & 216-B-42 Trench & Scavenged Waste & BP-3 & 0.228 & 0.614 & 1010 & $\overline{0}$ \\
\hline$\longdiv { 2 1 6 - 8 - 4 3 }$ & Cribs & $216-8-43 \mathrm{Crlb}$ & Scavenged Wasto & $\overline{B P-1}$ & 0.00456 & 0.0307 & 1400 & $\overline{0}$ \\
\hline$\overline{216-8-44}$ & Cribs & 216-8-44 Crlb & Scavenged Waste & BP-1 & 0.00076 & 0.921 & 2990 & $\overline{0}$ \\
\hline $216-8-45$ & Cribs & $216-\mathrm{B}-45$ Crlb & Scavenged Wasle & $\overline{B P}-1$ & 0.00228 & 0.614 & 3640 & 0 \\
\hline$\overline{216-B-46}$ & Cribs & 216-B-46 CNIb & Scavenged Waste & $\overline{B P} \cdot 1$ & 0.0636 & 1.23 & 1440 & 0 \\
\hline 216-B-47 & Cribs & 216-B-47 Crib & Scavenged Waste & BP-1 & 0.00228 & 0.307 & 650 & 0 \\
\hline$\overline{216-8-48}$ & Cribs & 216-B-48 Crib & Scavenged Wasto & $\overline{B P-1}$ & 0.00076 & 0.307 & 1490 & 0 \\
\hline $216-8-49$ & Cribs & 218-B-49 Crib & Scavenged Waste & $B P-1$ & 0.106 & 262 & 2360 & 0 \\
\hline 216-B-5 & Reverso Woll & 216-B-5 Raverso Woll & Process Waste & BP.6 & 0 & 262 & 108 & 0 \\
\hline $216-\mathrm{B}-50$ & Cribs & 216-B-50 Crib & Process Condensale & $\overline{B P}-1$ & 0.0001 & 0.0147 & 105 & 0 \\
\hline
\end{tabular}


Table B.4. (page 5 of 14)

\begin{tabular}{|c|c|c|c|c|c|c|c|c|}
\hline Site Codo & $\begin{array}{c}\text { Waste Management } \\
\text { Unlt Typo }\end{array}$ & Waste Managoment Unit & Wasto Typo & $\begin{array}{c}\text { Oporable } \\
\text { Unit }\end{array}$ & $\begin{array}{l}\text { Uranlum-238, } \\
\text { Units (CI) } \\
\end{array}$ & $\begin{array}{l}\text { Alpna Emitters, } \\
\text { Unilts (CI) }\end{array}$ & $\begin{array}{l}\text { Beta Emitters, } \\
\text { Units (CI) }\end{array}$ & $\begin{array}{c}\text { Amortclum-241, } \\
\text { Unlts (CI) }\end{array}$ \\
\hline $216-B-51$ & French Drah & 216-B-51 French Draln & Miscellaneous Dralnage & BP-4 & & & & \\
\hline 216-B-52 & Trench & 216-B-52 Trench & Scavenged Wasto & EP-2 & 0.01 & 1.17 & 317 & \\
\hline 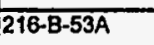 & Trench & 218-B-53A Tronch & Lab Waslo & BP.2 & 0.076 & 6.14 & 0.246 & \\
\hline $216-8-538$ & Trench & 216-8-53B Trench & LabWasto & BP.2 & 0.00303 & 307 & 17.2 & \\
\hline $216-\mathrm{B}-54$ & Trench & 216-B-54 Trench & Lab Waste & $\sqrt{B P-2}$ & 0.00303 & 0.307 & 0.945 & \\
\hline $216-8-55$ & Cribs & $216-8-55$ Crib & Steam Condensato & BP.9 & & 0.0423 & 40.8 & 0.0000038 \\
\hline 216-B-56 & Cribs & 216-B-56 Crib & N/A & BP-6 & & & & \\
\hline 216-B-57 & Cribs & 216-B-57 Crib & Process Condensate & BP-1 & 0.00029 & 0.0115 & 437 & \\
\hline $216-B-58$ & Trench & 216-8-58 Trench & Lab Waste & EP-2 & 0.00305 & 0.411 & 19.7 & \\
\hline $216-8-59$ & Retention Basin & 216-8-59 Easin. & Coolling Waler & BP.6 & & & 0.0832 & \\
\hline $216-8-6$ & Reverso Well & 216-B.6 Reverse Well & Lab Wasto & BP.B & 0 & 0 & 10 & \\
\hline $216-8-60$ & Cribs & 216-8-60 Crib & Decon Wasle & $\overline{8 P-6}$ & & & & \\
\hline $216-8-61$ & Cribs & 216-B-61 Crib & $\mathrm{N} / \mathrm{A}$ & $8 \mathrm{P}-1$ & & & & \\
\hline $216-8-62$ & Cribs & 216-B-62 Crlb & Process Condensate & EP.9 & & 0.105 & 418 & 0.103 \\
\hline $216-8-63$ & Ditches & 216-8-63 Trench & Chemical sewer & EP-11 & & 0.0742 & 6.32 & 0.0348 \\
\hline $216-8-64$ & Retention Basin & 216-8-64 Basin & N/A & $\overline{B P}-9$ & & & & \\
\hline $216-B-7 A \& B$ & Cribs & 216-B-7ABB CतID & Process Wasto & $\sqrt{B P-4}$ & 0.061 & 264 & 4490 & \\
\hline $216-B-8$ & Cribs & 216-B-8TF Crib & Process Wasto & $\sqrt{\mathrm{EP}-4}$ & 0 & 1.84 & 49.3 & \\
\hline $216-8-9$ & Cribs & 216-8.9TF Crib & Process Waste & BP. -8 & 0.0152 & 10.7 & 2 & \\
\hline $216-\mathrm{C}-1$ & Cribs & $216-\mathrm{C}-1 \mathrm{Crlb}$ & Process Condensale & SO-1 & 0.0988 & & & \\
\hline $216-C-10$ & Cribs & $218-C-10$ Crib & Process Condensale & $50-1$ & 0.00001 & & & \\
\hline $216-C-2$ & Roverso Well & 216-C-2 Reverse Well & Miscollaneous Dralnage & 50.1 & & & & \\
\hline $216-C-3$ & Cribs & $216-C-3$ Crib & Process Waste & SO-1 & 0.0153 & & & \\
\hline $218-C-4$ & Cribs & $216-\mathrm{C}-4 \mathrm{Crb}$ & Process Wasto & 50.1 & 0.0011 & & & \\
\hline $216-C-5$ & Cribs & $216-G-5$ Crib & Procoss Wasto & $50-1$ & 0.0182 & & & \\
\hline $216-c-6$ & Cribs & 216-C-6 Crib & Process Condensate & 50.1 & 0.0001 & & & \\
\hline $216-0-7$ & Cribs & 216-C-7 Crib & Process Wasto & so-1 & & & & \\
\hline $216-C-8$ & French Drain & $216-C-8$ & Process Wasto & $\mathrm{PO}-3$ & & & & \\
\hline $216 \cdot C \cdot 9$ & Ponds & 216-C-9 Pond & Cooling Water & 50.1 & & & & \\
\hline 216-E-28 & Ponds & 216-E-28 Pond & N/A & QP-11 & & & & \\
\hline $216-N-1$ & Ponds & 216-N-1 Pond & Coolling Water & NO-1 & & & & \\
\hline $216-\mathrm{N}-2$ & Trench & 218-N-2 Trench & Cooling Water & $\mathrm{NO}-1$ & & & 0.29 & \\
\hline $216-\mathrm{N}-3$ & Trench & 218-N-3 Trench & Cooling Water & NO-1 & & & 0.326 & \\
\hline
\end{tabular}


Table B.4. (page 6 of 14)

\begin{tabular}{|c|c|c|c|c|c|c|c|c|}
\hline Sito Codo & $\begin{array}{l}\text { Wasto Management } \\
\text { Unlt Typo }\end{array}$ & Wasto Managoment Unlt & Wasto Typo & $\begin{array}{c}\text { Operablo } \\
\text { Unll }\end{array} \mid$ & $\begin{array}{l}\text { Uranlum-238, } \\
\text { Unlts (CI) }\end{array}$ & $\begin{array}{l}\text { Alpha Emitters, } \\
\text { Units (Cl) }\end{array}$ & $\begin{array}{l}\text { Bota Emilters, } \\
\text { Unlts (C)) }\end{array}$ & $\begin{array}{l}\text { Amertclum-241, } \\
\text { Unlts (C)) }\end{array}$ \\
\hline $216-N-4$ & Ponds & $216-N-4$ Pond & Cooling Water & NO-1 & 0.00152 & 0.0614 & 0.3 & \\
\hline $216-N-5$ & Trench & 216-N-5 Trench & Coollng Water & NO-1 & & & 0.326 & \\
\hline 216-N-6 & Ponds & 216-N-6Pond & Cooling Water & NO.-1 & 0.00152 & 0.0614 & 0.3 & \\
\hline $216-N \cdot 7$ & Trench & 216-N-7 Trench & Cooling Water & Na-1 & & & 0.326 & \\
\hline $216-N \cdot 8$ & Ponds & 216-N-8 Pond & & IU-6 & & & & \\
\hline $\begin{array}{l}216-S-182 \\
\end{array}$ & Cribs & $218-S-1 \& 2$ & Process Condensate & RO-2 & & 73.7 & 4750 & \\
\hline 216-S-10D & Dithes & $\begin{array}{l}216-S-10 D \\
2100\end{array}$ & Chemlcal Sewer & RO-1 & . & 0.0244 & 3.51 & 0.0152 \\
\hline 216-S-10P & Ponds & 216-S-10P & Chemical Sewer & RO-1 & & & & \\
\hline 216-S-11 & Ponds & $216-S-11$ & Chemical Sewor & RO-1 & & 0.00553 & 1.94 & \\
\hline 216-S-12 & Trench & 216-S-12 & Miscellaneous Drainage & RO-3 & & 0.0614 & 1.66 & \\
\hline$\sqrt{216-S-13}$ & Cribs & 216-S-13 & Process Waste & RO-2 & & 0.491 & 5.5 & \\
\hline$\overline{216-S-14}$ & Trench & $216-S-14$ & Process Waste & RO-3 & & & $\cdot$ & \\
\hline $216-5-15$ & Ponds & 216-S-15 & Coollng Waler & RO-2 & & & & \\
\hline 216-S-160 & Ditches & 216-S-16D & Coolling Water & RO-1 & & & & \\
\hline 216-S-16P & Ponds & 216-S-16P & Coolling Water & RO.1 & & 22.6 & 148 & \\
\hline 216-S-17 & Ponds & 216-S-17 & Coollng Water & RO-1 & & 0.184 & 56.3 & \\
\hline$\overline{216-S-172}$ & Diversion Box & $\mid \overline{216-S-172}$ & Coolling Water & RO-1 & & & & \\
\hline $216-S-18$ & Trench & $216-5-18$ & Debris & RO-2 & & & & \\
\hline $216-\mathrm{S}-19$ & Ponds & 216-S-19 & Lab Wasto & RO-1 & & 1.26 & 5.12 & \\
\hline $216-S-20$ & Cribs & $218-S-20$ & Lab Wasto & RO-3 & & 10.5 & 156 & \\
\hline$\overline{216-S-22}$ & Cribs & $216-S-22$ & Process Waste & RO-3 & & 0.0062 & 1.83 & \\
\hline$\overline{216-S-23}$ & Cribs & $216-S-23$ & Process Condensate & $\longdiv { \text { RO-2 } }$ & & 0.0611 & 9.07 & \\
\hline$\overline{216-S-25}$ & Cribs & 216-S-25 & Stoam Condensato & RO-1 & & 0.012 & 0.247 & \\
\hline $216-S-26$ & Cribs & 216-S-28 & Lab Waste & RO-3 & & 0.000763 & 0.01 & 0.00058 \\
\hline $216-S-3$ & French Draln & $216-\mathrm{S}-3$ & Process Condensate & RO-2 & & 0.0307 & 43 & \\
\hline $216-S-4$ & French Draln & $218-5-4$ & Procoss Condensale & UP-2 & & & & \\
\hline 216-S-5 & Cribs & 216-S-5 & Coolling Water & RO-1 & & 35.6 & 159 & \\
\hline $216-\mathrm{S} \cdot 6$ & Cribs & $216-5-6$ & Cooling Water & RO-1 & & 28 & 630 & \\
\hline $216 \mathrm{~S} \cdot 7$ & Cribs & 216-S.7 & Procoss Condensale & RO-2 & & 27) & 4180 & \\
\hline 216-S-8 & Trench & 21Q-S-8 & Process Wasto & RO-2 & & 0.123 & 10.5 & \\
\hline 216-S-9 & Cribs & 216-S-9 & Procoss Condensalo & RO-2 & & 3.99 & 753 & \\
\hline$\overline{216-T-1}$ & Ditichos & 216-T-1 Dilch & Cooling Water & TP-4 & 0.0015 & & & \\
\hline 216-T-10 & Trench & 216-T-10 Trench & Decon Waste & TP-4 & & & & \\
\hline
\end{tabular}


Table B.4. (page 7 of 14)

\begin{tabular}{|c|c|c|c|c|c|c|c|c|}
\hline Site Codo & \begin{tabular}{|c|}
$\begin{array}{c}\text { Wasto Management } \\
\text { Unlt Typo }\end{array}$ \\
\end{tabular} & Wasto Management Unit & Wasto Type & $\begin{array}{c}\text { Operable } \\
\text { Unlt }\end{array}$ & $\begin{array}{l}\text { Urantum-238, } \\
\text { Units (C)) }\end{array}$ & $\begin{array}{c}\text { Alpha Emltters, } \\
\text { Units (CI) } \\
\end{array}$ & $\begin{array}{l}\text { Bota Emittors. } \\
\text { Units (C) }\end{array}$ & $\begin{array}{c}\text { Amariclum-241, } \\
\text { Units (Ci) }\end{array}$ \\
\hline 216-T-11 & Trench & 216-T-11 Trench & Decon Waste & \begin{tabular}{|l|}
$T P-4$ \\
\end{tabular} & & & & \\
\hline $216-T-12$ & Trench & 216-T-12 Trench & Coolling Water & TP-3 & 0.0152 & & & \\
\hline $216-T-13$ & Trench & 216-T-13 Trench & Decon Waste & $\overline{T P-2}$ & & & & \\
\hline $216-T-14$ & Trench & 216-T-14 Trench & Tank Farm Wasto & $\overline{T P-3}$ & 0.0102 & & & \\
\hline 216-T-15 & Trench & 216-T-15 Trench & Tank Farm Wasie & TP-3 & 0.00911 & & & \\
\hline 216-T-16 & Trench & 216-T-16 Trench & Tank Farm Waste & TP.3 & 0.00743 & & & \\
\hline $216-\mathrm{T}-17$ & Trench & 218-T-17 Trench & Tank Farm Waste & $\mathrm{TP}-3$ & 0.0068 & & & \\
\hline $216-T-18$ & Cribs & 216-T-18 Crib & Tank Farm Wasle & TP-2 & 0.00911 & & & \\
\hline 216-T-19 & Cribs & 216-T-19TF Crib and Tilo Fleld & Process Waste & Tि-2 & & & & 0.00982 \\
\hline$\overline{216-T \cdot 2}$ & Reverso Well & 216-T-2 Reverse Well & Lab Waste & TP-4 & & & & \\
\hline $216-T-20$ & Trench & $216-$ T-20 Trench & Process Wasto & TP-2 & 0.0167 & & & \\
\hline 216-T-21 & Trench & 216-T-21 Trench & Tank Farm Waste & TP-1 & 0.00033 & & & \\
\hline 216-T-22 & Trench & 216-T-22 Trench & Tank Farm Waste & $\overline{T P-1}$ & 0.00087 & & & \\
\hline $218-T-23$ & Trench & 216-T-23 Tronch & Tank Farm Wasto & TP-1 & 0.00034 & & & 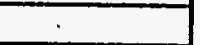 \\
\hline $216-T-24$ & Trench & 216-T-24 Trench & Tank Farm Wasto & TP-1 & 0.00278 & & & \\
\hline $216-\mathrm{T}-25$ & Trench & 210-T-25 Tronch & Procoss Wasle & TP-1 & 0.0003 & & & \\
\hline $216-T-26$ & Cribs & $210-T-26 \mathrm{Crlb}$ & Tank Farm Waste & TP-2 & 0.503 & & & \\
\hline $216-T-27$ & Cribs & $216-T-27$ Crib & Lab Wasto & TP-2 & 0.00243 & & & \\
\hline $216-T-28$ & Cribs & 216-T-28 C Crb & Decon Waste & TP?-2 & 0.131 & & & \\
\hline $216-\mathrm{T}-29$ & Cribs & 216-T-29 Crib & Miscollaneous Drainage & $T P-4$ & & & & \\
\hline 216-T-3 & Reverse Well & 216-T-3 Reverso Woll & Process Wasto & $\mathrm{TP}^{\mathrm{P}-4}$ & & & & \\
\hline 216-T-31 & Fronch Draln & 216-T-31 French Draln & Miscellaneous Drainage & $\mathrm{TP}-2$ & & & & \\
\hline 216-T-32 & Cribs & $216-\mathrm{T}-32 \mathrm{Crb}$ & \begin{tabular}{|l|} 
Process Wasto \\
Decon Wasto
\end{tabular} & $\frac{T P-1}{T P-4}$ & $\begin{array}{r}0.0076 \\
0.00152 \\
\end{array}$ & & & \\
\hline$\frac{216-T-33}{216-T-34}$ & \begin{tabular}{|l} 
Critbs \\
Crlbs
\end{tabular} & $\frac{\mid 216-T-33 \text { Crib }}{\text { 216-T-34 Crib }}$ & $\begin{array}{l}\text { Decon Wasto } \\
\text { Lab Waste }\end{array}$ & $\frac{P=4}{T P-4}$ & 0.00138 & & & \\
\hline$\frac{1010-1.04}{216-T \cdot 35}$ & Cribs & 218-T-35 Crib & Lab Wasto & TP-4 & 0.0184 & & & \\
\hline 216-T-36 & Cribs & 210-T-36 Crib & Steam Condensale & $T P-1$ & 0.00039 & & & \\
\hline $216-T-4-10$ & Ditches & 218-T-4-10 Ditch & Coolling Water & TP-3 & & & & \\
\hline $216-T-4 \cdot 2$ & Ditchies & 210-T-4-2 Ditch & Steam Condensato & TP-3 & & & & \\
\hline 216-T-4A & Ponds & 218-T-AA Pond & Coolling Water & TP-3 & & & & \\
\hline $218-T-4 B$ & Ponds & 216-T-AB Pond & Cooling Water & TP-3 & 0.232 & & & \\
\hline 216-T-5 & Trench & 218-T-5 Trench & Tank Farm Waste & TP-1 & 0.00152 & & & \\
\hline $216-T-6$ & Cribs & 216-T-6 Crib & Process Wasto & TP-3 & 0.0076 & & & \\
\hline
\end{tabular}


Table B.4. (page 8 of 14)

\begin{tabular}{|c|c|c|c|c|c|c|c|c|}
\hline Slto Codo & $\begin{array}{c}\text { Wasto Management } \\
\text { Unlt Type }\end{array}$ & Wasto Managoment Unlt & Wasto Typo & $\begin{array}{c}\text { Operablo } \\
\text { Unit }\end{array}$ & $\begin{array}{c}\text { Uranlum-238, } \\
\text { Units (Cl) }\end{array}$ & $\begin{array}{l}\text { Alpha Emitters, } \\
\text { Units (Ci) }\end{array}$ & $\begin{array}{l}\text { Bota Emittors, } \\
\text { Units (Cl) }\end{array}$ & $\begin{array}{c}\text { Amoriclum-241, } \\
\text { Units (Cl) }\end{array}$ \\
\hline $216-T-7$ & Cribs & 216-T-TTF Crib and Tlle Field & Tank Farm Wasto & TP-1 & 0.00304 & & & \\
\hline $216-T-8$ & Cribs & 216-T-8 Crib & Lab Waste & TP-4 & 0.0015 & & & \\
\hline $216-T-9$ & Trench & 216-T-9 Trench & Decon Waste & TP-4 & & & & \\
\hline $216-U-182$ & Cribs & $216-U-1 \& 216-U-2$ & Process Condensato & UP-2 & & 2.62 & 12.6 & \\
\hline 216-U-10 & Ponds & $216-U-10$ & Coolling Water & UP.-2 & & 505 & $\overline{44.2}$ & 0.492 \\
\hline $216-U \cdot 11$ & DHchos & $216-U \cdot 11$ & Cooling Water & UP-2 & & & & \\
\hline $216-U \cdot 12$ & Cribs & $216-U-12$ & Process Condensale & UP-2 & & 0.105 & 112 & 0.00645 \\
\hline $216-U-13$ & Trench & $216-U-13$ (same as UN-200-W-125) & Decon Waste & UP-2 & 0.00012 & 0.00614 & 0.176 & \\
\hline $216-U-14$ & Ditches & $216-U-14$ & Cooling Water & UP-2 & & & & \\
\hline $216-U-15$ & Trench & $216-4-15$ & Procoss Waste & UP-2 & 0.00076 & 0.00614 & 0.18 & \\
\hline $216-U-16$ & Cribs & $216-U-16$ & Process Condensate & UP-2 & & 0.00739 & 0.0515 & \\
\hline 216-U-17 & Cribs & $216-U-17$ & Process Condensate & UP-2 & & 0.000195 & & 0.000053 \\
\hline $216-U-21$ & & $216-U-21$ & & & 0.0014 & 0.128 & 208 & \\
\hline $216-U \cdot 3$ & French Drain & $216-U-3$ & Miscellaneous Dralnago & UP-2 & & 0.00814 & 0.1917 & \\
\hline $216-U-4$ & Reverse Well & $21 \frac{1-U-4}{4}$ & Lab Waste & Uि-2 & & & & \\
\hline $216-U 4 A$ & French Drah & $216-U-A A$ & Miscellaneous Dralnage & UP-2 & & 0.000553 & 0.387 & \\
\hline $216-U-4 B$ & French Drain & $216-U-4 B$ & Miscellaneous Drainage & UP-2 & & 0.00332 & 0.381 & \\
\hline $216-U-5$ & Trench & $216-U .5 \& 216-U-6$ & Procoss Waste & UP-2 & 0.122 & 0.00307 & 0.0792 & \\
\hline 216-U-7 & French Draln & 218.0 .7 & Miscellaneous Drainage & UP-2 & & & & \\
\hline 216-U-8 & Cribs & $218-1-8$ & Process Condensale & UP-2 & 8.04 & 22.7 & 0.65 & \\
\hline $216-U-9$ & Ditches & $216-U-9$ & Cooling Water & $\longdiv { R 0 - 1 }$ & & & & \\
\hline 216-W.LWC & Cribs & 216-W-LWC Crb & Chemical Sewer & SS-2 & & & & \\
\hline $216-z-182$ & Cribs & $216-2-1 \& 216-z-2$ Cribs & Process Waste & $2 \mathrm{PP}-2$ & 0.027 & & & \\
\hline $216-2-10$ & Reverse Well & 216-2-10 Reverso WeIl & Procoss Wasto & $\mathrm{ZP}-2$ & & & & 7 \\
\hline 216-Z-11 & Ditches & $216-z-11$ & Cooling Water & UP-2 & & & & \\
\hline $216-Z-12$ & Cribs & 216-z-12 Crib & Procoss Wasto & $\mathrm{ZP}-2$ & 0.000017 & & & \\
\hline $216-z-13$ & Frẹnch Drain & 216-2-13 French Drain & Mlscellaneous Drainage & $\overline{2 P-2}$ & & & & \\
\hline $216-Z-14$ & Fronch Draln & 216-2-14 French Drain & Mlscellaneous Dralnage & $\mathrm{ZP}-2$ & & & & \\
\hline $216-2-15$ & French Drain & 216-2-15 French Drah & Miscellaneous Drainage & $2 \mathrm{P}-2$ & & & & \\
\hline $216-2-16$ & Cribs & 216-z-16 Crib & Lab Wasto & $2 \mathrm{P}-2$ & & & & \\
\hline $216-z-17$ & Ditches & 216-2-17 Trench & Lab Wasto & $2 \mathrm{PP}-2$ & 0.00005 & & & \\
\hline$\overline{216-2-18}$ & Cribs & 216-2-18 Crib & Process Wasto & $\overline{Z P-2}$ & & & & \\
\hline$\widehat{216-Z-19}$ & Ditches & $216-2 \cdot 19$ & Cooling Water & UP-2 & & & & \\
\hline
\end{tabular}


Table B.4. (page 9 of 14)

\begin{tabular}{|c|c|c|c|c|c|c|c|c|}
\hline Site Codo & $\begin{array}{c}\text { Wasto Managoment } \\
\text { Unlt Typo }\end{array}$ & Wasto Manayoment Unit & Wasto Typo & $\begin{array}{c}\text { Operablo } \\
\text { Unit }\end{array}$ & $\begin{array}{l}\text { Uranlum-238, } \\
\text { Units (Ci) }\end{array}$ & $\begin{array}{l}\text { Aipha Emitters, } \\
\text { Unlts (CI) }\end{array}$ & $\begin{array}{l}\text { Bola Emitters, } \\
\text { Units (CI) }\end{array}$ & $\begin{array}{c}\text { Americlum-241, } \\
\text { Unlts (C)) }\end{array}$ \\
\hline $216-Z-1 A$ & Cribs & 216-2-1A Tllo Field & Procass Waste & $2 \mathrm{P}-2$ & & & & 3432 \\
\hline $216-2-10$ & Ditches & $216-2-10$ & Cooling Water & UP-2 & & & & \\
\hline $216-2-20$ & Cribs & 216-z-20 & Cooling Water & UP-2 & & 2.22 & 0.400 & 1.01 \\
\hline $218-2-21$ & Retention Basin & 218-z-21 Seopage Gasin & Cooling Water & $2 \mathrm{P}-2$ & & & & \\
\hline $216-2-3$ & Cribs & $216-2-3$ Crib & Process Waste & $\mathrm{ZP}-2$ & 0.000017 & & & \\
\hline $216-z-4$ & Trench & 216-Z-4 Trench & Process Wasto & $2 \mathrm{P}-2$ & 0.000017 & . & & \\
\hline $216-z-5$ & Cribs & $216-2 \cdot 5$ Crib & Process Waste & $2 \mathrm{P}-2$ & 0.000017 & & & \\
\hline $216-2-8$ & Cribs & 216-Z.6Crib & Process Wasto & $2 \mathrm{P}-2$ & 0.000017 & & & \\
\hline 216-z-7 & Cribs & $216-2-7 \mathrm{Crb}$ & Lab Wasto & $2 \mathrm{P}-2$ & 0.0015 & & & \\
\hline $216-2-8$ & Cribs & 216-Z-8 French Draln & Procoss Wasto & $\mathrm{ZP}-2$ & & & & 1373 \\
\hline 216-Z-9 & Cribs & 216-2-9 Trench & Process Waste & $\overline{\mathrm{zP}-2}$ & 0.000017 & & & 8580 \\
\hline $218-C \cdot 9$ & Burlal Stto & 218-C-9 Burial Ground & LLW-SOLID & so-1 & $\therefore$ & & & \\
\hline $218 \cdot E-1$ & Burtal Stio & $218 \cdot \mathrm{E}-1$ & LLW-SOLID & $\mathrm{PO}-2$ & 0.134 & & & \\
\hline $218-E-10$ & Burlal Stle & 218-E-10 Burtal Ground & LLW-SOLID & $\mathrm{BP}-10$ & & & 430000 & \\
\hline 218-E-12A & Burial Sife & 218-E-12A & LLW-SỌLID & $\overline{P O-6}$ & 0.332 & & & \\
\hline 218-E-12B & Burial Stte & 218-E-128 & LLW-SOLID & PO.6 & & & & \\
\hline 218-E-13 & Burial Site & $218-E-13$ & & $\mathrm{PO}-2$ & & & & \\
\hline 218-E-2 & Burfal Stte & 218-E-2 Burfal Ground & LLW-SOLID & Bp-10 & & & & \\
\hline 218-E-2A & Burial Silte & 218-E-2A Burial Ground & LLW-SOLID & $B P-10$ & & & & \\
\hline$\frac{218-E-4}{218-E-5}$ & $\begin{array}{l}\text { Bural She } \\
\text { Burial Site }\end{array}$ & 218-E-4 Burial Ground & LLW-SOLID & $\mathrm{EP}-10$ & & & & \\
\hline$\frac{218-E-5}{218-E-5 A}$ & $\begin{array}{l}\text { Burial site } \\
\text { Burial she }\end{array}$ & 218-E-5 Burfal Ground & LLW-SOLID & BP-10 & & & & - \\
\hline$\frac{218-E-E-6}{218-6}$ & $\begin{array}{l}\text { Burlal SHe } \\
\text { Burfal SHo }\end{array}$ & 218-E-5A Burial Ground & LLW-SOLID & $\mathrm{BP}-10$ & & & & \\
\hline 218-E-7 & $\frac{\text { Burtal Stto }}{\text { Burial Stto }}$ & 218-E-6 Burial Ground & Debrls & BPन-6 & & & & \\
\hline 218-E-8 & Burial Stto & 218-E-7 Burial Ground & LabWaste & BP.6 & & & & \\
\hline $218 \cdot E \cdot 9$ & $\frac{\text { Burlal Site }}{\text { Burfal Site }}$ & 218-E-8 & TRU Solid Wasto & $\mathrm{PO}-6$ & 0.00067 & & & \\
\hline $218-W-1$ & $\begin{array}{l}\text { Burfal Sire } \\
\text { Burial Stle }\end{array}$ & 218-E-9 Burfal Ground & LLW-SOLID & BP:10 & & & & \\
\hline $218-W-11$ & Burlal SHo & 218-W-1 Burial Ground & TRU Solld Wasto & $\frac{Z P-3}{70-3}$ & 0.0235 & & & \\
\hline $218-W \cdot 1 A$ & Bur|al SHO & $\frac{218-W-11 \text { Burlal Ground }}{218-W-1 A \text { Burial Ground }}$ & LLW-SOLID & $\frac{\mathrm{ZP}-3}{\mathrm{ZP}-3}$ & 0.302 & & & \\
\hline $218-W-2$ & Buria! Sto & 218-W-2 Burial Ground & LLW.SOLID & $\mathrm{ZP}-3$ & $\begin{array}{r}0.302 \\
46.9\end{array}$ & & & \\
\hline 218-W-2A & Burial SHe & $\begin{array}{l}\text { 218-W-2 Burial Ground } \\
\text { 218-W-2A Burtal Ground }\end{array}$ & TRU Solid Wasto & $\frac{Z \mathrm{PP}-3}{\mathrm{ZP}-3}$ & & & & \\
\hline $218-W-3$ & Burial SHe & $\begin{array}{l}\text { 218-W-2A Burlal Ground } \\
\text { 218-W-3 Burlal Ground }\end{array}$ & LLW-SOLID & $\frac{\mathrm{ZP}-3}{\mathrm{ZP}-3}$ & 23.5 & & & \\
\hline $218 \cdot W-3 A$ & Burial Slio & 218-W-3A Burlal Ground & TRU Solid Wasto & $\frac{\mathrm{ZP}-3}{\mathrm{ZP}-3}$ & & & & \\
\hline
\end{tabular}


Table B.4. (page 10 of 14)

\begin{tabular}{|c|c|c|c|c|c|c|c|c|}
\hline Slte Code & $\begin{array}{c}\text { Wasto Management } \\
\text { Unit Typo }\end{array}$ & Wasto Managomont Unit & Waste Type & $\mid \begin{array}{c}\text { Operable } \\
\text { Unit }\end{array}$ & $\begin{array}{l}\text { Uranlum-238, } \\
\text { Units (CI) }\end{array}$ & $\begin{array}{l}\text { Alpha Emitters, } \\
\text { Units (Ci) }\end{array}$ & $\begin{array}{l}\text { Beta Emitters, } \\
\text { Units (CI) }\end{array}$ & $\begin{array}{c}\text { Americlum-241, } \\
\text { Units (Ci) }\end{array}$ \\
\hline 218-W-3AE & \begin{tabular}{|l|} 
Burial SHo \\
\end{tabular} & 218-W-3AE Burlal Ground & LLW-SOLID & $\mathrm{ZP}-3$ & & & & \\
\hline $218-W-4 A$ & Burial Site & 218-W-4A Buthal Ground & TRU Solld Waste & $\mathrm{ZP}-3$ & & & & \\
\hline $218-W-4 B$ & Burlal Stre & 218-W-AB Calssons & TRU Solld Waste & $2 \mathrm{PP}-3$ & & & & \\
\hline 218.W-4B & Burtal SHo & 218-W-4B Trenches & TRU Solld Wasto & $\overline{\mathrm{ZP}-3}$ & & & & \\
\hline 218-W-4C & Eurtal Stte & 218-W-4C Burial Ground & TRU Solld Wasto & $2 \mathrm{P}-3$ & & & & \\
\hline 218-W-5 & Burfal SHe & 218-W-5 Burtal Ground & TRU Solld Wasto & $2 \mathrm{PP}-3$ & & & & \\
\hline $218-W \cdot 6$ & Burtal SHo & 218-W-6 Burial Ground & LLW-SOLID & $2 \mathrm{PP}-3$ & & & & \\
\hline 218-W-7 & Burlal SHo & 218-W-7 & LLW-SOLID & RO-3 & & & & \\
\hline 218-W-8 & Burlal Stte & 218-W-8 Burial Ground & Lab Wasto & TP-4 & 0.0001 & & & \\
\hline 218-W.9 & Burlal Stte & $218-W-9$ & LLW-SOLID & RO-2 & & & & \\
\hline $231-W-151$ & Diversion $B 0 x$ & 231-z-151 Sump & & $2 \mathrm{P}-2$ & & & & \\
\hline $231-W-151$ & Vauit & 231-Z-151 Sump & & $\mathrm{ZP}-2$ & & & & \\
\hline $231-W-151$ & Dlverslon Box & 231-z-151 Sump & & $\mathrm{ZP}-2$ & . & & & \\
\hline $231-W-151$ & Vault & 231-Z-151 Sump & & $\overline{2 P-2}$ & & & & \\
\hline $231-W-151$ & Diversion Box & $231-z-151$ Sump & & $\mathrm{ZP}-2$ & & & & \\
\hline $231-W-151$ & Vauffi & 231-z-151 Sump & & $2 \mathrm{PP}-2$ & & & & \\
\hline $231 \cdot W-151$ & Diversion Box & 231-z-151 Sump & & $\overline{\mathrm{ZP}-2}$ & & & & \\
\hline $231-W-151$ & Vault & 231-z-151 Sump & & $\mathrm{ZP}-2$ & & & & \\
\hline $232-2$ & Building & 232-ZInclnerator & & $2 \mathrm{PP}-2$ & & & & \\
\hline $240-5-151$ & Diverslon Box & $240-5-151$ & LLW-SOLID & RO-3 & & & & \\
\hline $240-s-152$ & Dlversion Box & $240-5-152$ & Tank Farm Wasto & RO-3 & & & & \\
\hline $240-5-302$ & Tanks & $240-5-302$ & Lab Waste & RO-3 & & & & \\
\hline $241-A-151$ & Diverston Box & 241-A-161 & Tank Farm Wasto & PO-2 & & & & \\
\hline $241 \cdot A-152$ & Diveraton Box & 2 211-A-152 & Process Waste & PO-3 & & & & - \\
\hline $241-A-153$ & Diversion Box & $241-A-153$ & Process Waste & $\overline{P O-3}$ & & & & \\
\hline $241-A-302 A$ & Tanks & $211-A-302 A$ & & $\overline{P O .2}$ & & & & \\
\hline $241-A-3028$ & Tenks & $241-A-302 B$ & & PO-5 & & & & \\
\hline $241-A-350$ & Tanks & $241-A-350$ & Procoss Waste & PO-3 & & & & \\
\hline $241-A-417$ & Tanks & $241 \cdot A-417$ & Process Waste & PO-3 & & & & \\
\hline $241-A-A$ & Dlversion Box & $241-A-A$ & Procoss Waste & $\mathrm{PO}-3$ & & & & \\
\hline $241-A-B$ & Diversion Box & $211 \cdot A \cdot B$ & Process Waste & $\mathrm{PO}-3$ & & & & \\
\hline 241-AN-A & Diversion Box & 241.AN-A & Process Waste & PO-3 & & & & \\
\hline 241-AN-B & Diversion Box & 241-AN-B & Process Waste & PO-3 & & & & \\
\hline
\end{tabular}


Table B.4. (page 11 of 14)

\begin{tabular}{|c|c|c|c|c|c|c|c|c|}
\hline Site Codo & $\begin{array}{l}\text { Waste Management } \\
\text { Unil Typo }\end{array}$ & Wasto Managoment Unlt & Wasto Type & $\begin{array}{c}\text { Operable } \\
\text { Unlt }\end{array}$ & $\begin{array}{l}\text { Uranlum-238, } \\
\text { Units (CI) }\end{array}$ & $\begin{array}{l}\text { Alpha Emitters, } \\
\text { UnIts (CI) }\end{array}$ & $\begin{array}{l}\text { Bota Emitters, } \\
\text { Units (G)). }\end{array}$ & $\begin{array}{l}\text { Amortcium-241, } \\
\text { Urilts (CI) }\end{array}$ \\
\hline 241-APVP & Valvo Plt & 241-AP & Procass Wasto & $\mathrm{PO}-3$ & & & & \\
\hline 241-AR-151 & Diversion Box & $241 \cdot A R-151$ & Process Wasto & PO-3 & & & & \\
\hline 241-AW-A & Diversion Box & $241-A W-A$ & Process Wasto & PO-3 & & & & \\
\hline $241 \cdot A W \cdot B$ & Diversion Box & 241-AW.8 & Procoss Waste & $\mathrm{PO}-3$ & & & & \\
\hline $241-A X-151$ & Diversion Box & $241-A X-151$ & Process Wasto. & $\mathrm{PO}-3$ & & & & \\
\hline $241-A X-152 D S$ & Tanks . & 241-AX-1520S & Process Waste & $\mathrm{PO}-3$ & & & & \\
\hline $241-A X-155$ & Diversion Box & $241-A X-155$ & Tank Farm Waste & PO-3 & & & & \\
\hline $241-A X-501$ & Valive Plt & 241-AX-501 & & $\mathrm{PO}-3$ & & & & \\
\hline$\frac{241 \cdot A X-A}{241-A X-B}$ & \begin{tabular}{|l} 
Diversion $B 0 x$ \\
\end{tabular} & $241-A X-A$ & & PO.3 & & & & \\
\hline$\frac{241-A X \cdot B}{241-A Y-151}$ & \begin{tabular}{|l|} 
Diversion Box \\
Diversion Box
\end{tabular} & 241-AX-B & & $\mathrm{PO}-3$ & & & & \\
\hline$\frac{241-A Y-151}{241-A Y-152}$ & \begin{tabular}{|l|} 
Diversion Box \\
Diversion Box
\end{tabular} & $241-A Y-151$ & Process Waste & PO-3 & & & & \\
\hline 241-AZ-151DS & \begin{tabular}{|l} 
Diversion Box \\
Dlversion 80x
\end{tabular} & $241-A Y-152$ & Procoss Waste & PO-3 & & & & \\
\hline $241-A Z-152$ & \begin{tabular}{|l|} 
Diversion $80 x$ \\
Diversion $B 0 x$ \\
\end{tabular} & 241-AZ-151DS & & $\mathrm{PO}-3$ & & & & \\
\hline$\sqrt{241-\mathrm{C}-151}$ & \begin{tabular}{|l} 
Diversion Box \\
Diversion Box.
\end{tabular} & $\frac{241-A Z-152}{241-C-151}$ & & $\mathrm{PO}-3$ & & & & \\
\hline $241-C-152$ & Dlverston Box & $\frac{244-C-151}{241-C-152}$ & & $\frac{\mathrm{PO}-3}{\mathrm{PO}-3}$ & & & & \\
\hline 241-C-153 & Diversion Box & $241 \cdot C-153$ & & PO-3 & & & & \\
\hline $241-C-154$ & Diversion Box & 241-C-154 Diversion Box & Procoss Wasto & $50-1$ & & & & \\
\hline $241-\bar{C}-252$ & Dalversion Box & $241-C-252$ & & PO-3 & & & & \\
\hline $241-C-301 C$ & Tanks & $241-C-301 C$ & & $\mathrm{PO} \cdot 3$ & & & & \\
\hline $241-C R-151$ & Diversion Box & $241-C R-151$ & . & $\mathrm{PO}-3$ & & & & \\
\hline $241-C R-152$ & Diversion Box & 241-CR-152 & & PO-3 & & & & \\
\hline 241-CR-153 & Diversion Box & $\frac{241-C R-153}{241-C X-70 \text { S10rage Tank }}$ & Tank Farm Wasto & $\mathrm{PO}-3$ & & & & \\
\hline$\frac{241-C X-T K-70}{241-C X-T K-71}$ & \begin{tabular}{|l} 
Tanks \\
Tanks
\end{tabular} & $\frac{241-C X-70 \text { Silorage lank }}{241-C X-71 \text { Storage Tank }}$ & Process Condensalo & $\frac{50.1}{50-1}$ & 0.0986 & & & \\
\hline $241 \cdot \mathrm{CX}-\mathrm{TK} \cdot-72$ & Tranks & 241-CX-72 Storage Tenk & Process Waste & so-1 & 0.000000533 & & & \\
\hline 241-ER-153 & Diversion Box & $241-E R-153$ & & PO-3 & & & & \\
\hline $241-\mathrm{S}-151$ & Dlversion Bax & $241-S-151$ & LLW-SOLID & R0-2 & & & & \\
\hline $241-S-152$ & Diversion Box & $241-S-152$ & Tank Farm Waste & RO-4 & & & & \\
\hline $241-\mathrm{S}-302 A$ & Tranks & $241-S-302 A$ & Lab Waste & $\longdiv { 2 0 - 2 }$ & & & & \\
\hline 241-5-3028 & Tanks & $\begin{array}{l}241-S-302 B \\
241-S-A\end{array}$ & LLW-SOLID & RO-4 & & & & \\
\hline$\frac{241-S-A}{241-S-B}$ & $\begin{array}{l}\text { Diversion Box } \\
\text { Diversion Box }\end{array}$ & $\frac{241-S-A}{241-S-B}$ & Tank Farm Wasto & $\frac{\mathrm{RO}-4}{\mathrm{R} 0-4}$ & & & & \\
\hline & & & 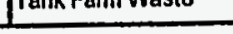 & (1) & & & & \\
\hline
\end{tabular}


Table B.4. (page 12 of 14)

\begin{tabular}{|c|c|c|c|c|c|c|c|c|}
\hline Slto Code & $\begin{array}{c}\text { Wasto Mana } \\
\text { Unlt Type }\end{array}$ & Wasto Management Unit & Waste Typo & $\begin{array}{c}\text { Operable } \\
\text { Unlt }\end{array}$ & $\begin{array}{c}\text { Uranlum-238, } \\
\text { Unlts (CI) }\end{array}$ & $\begin{array}{l}\text { Alpha Emilters, } \\
\text { Units (CI) }\end{array}$ & $\begin{array}{l}\text { Bota Emilters, } \\
\text { Unlis (CI) }\end{array}$ & $\begin{array}{c}\text { Amertclum-241, } \\
\text { Unlts (CI) }\end{array}$ \\
\hline $241-S \cdot C$ & Diverston Box & 241-S-C & Tank Farm Waste & RO-4 & & & & \\
\hline $241-S \cdot D$ & Dlversion Box & 241-S-D & Tank Farm Waslo & RO-4 & & & & \\
\hline $241-S X-151$ & Diverston Box & $241-S X-151$ & Tank Farm Wasle & $\overline{R O-4}$ & & & & \\
\hline $\begin{array}{l}241-S X-152 \\
\end{array}$ & Diversion Box & $241-S X-152$ & Tank Farm Wasle & $\overline{R O-4}$ & & & & \\
\hline $241-S X-302$ & Tanks & $241-S X-302$ & & RO-2 & & & . & \\
\hline $241-S X-A$ & Dlversion Box & $241-S X-A$ & & $\overline{R O-4}$ & & & & \\
\hline $241-S X-B$ & Diversion Box & $241-S X-B$ & & $\overline{R O-4}$ & & & & \\
\hline $241-S Y-A$ & Diversion Box & $\overline{241-S Y-A}$ & & RO-4 & & & & \\
\hline $241-\mathrm{SY}-\mathrm{A}$ & Diversion Box & 241 -SY-A & & $\overline{R 0-4}$ & & & & \\
\hline $241-S Y \cdot B$ & Diversion Box & $241-S Y-B$ & & $\overline{\mathrm{RO}-4}$ & & & & \\
\hline $241-S Y-B$ & Diverslon Box & $241-S Y \cdot B$ & & RO-4 & & & & \\
\hline 241-T-151 & Diversion Box & 241-T-151 Dlversion Box & Tank Fam Waste & TP-6 & & & & \\
\hline $241-T-152$ & Diversion Box & 241-T-152 Diversion 80x & Tank Fam Wasto & TP-6 & & & & \\
\hline 241-T-153 & Diverston Box & $241-\mathrm{T}-153$ Diversion Box & Tank Farm Wasio & TP-6 & & & & \\
\hline $241 \cdot T-252$ & Diversion $40 x$ & 241-T-252 DVersion Box & Tank Farm Wasto & TP-6 & & & & \\
\hline $241 \cdot T \cdot 301$ & Tanks & $\mid 241-T-301$ Calch Tank & Tank Farm Wasto & TP-8 & & & & \\
\hline $241-T \cdot 302$ & Tanks & 241-T-302 Calch Tank & Tank Fam Wasto & TP-8 & & & & \\
\hline $241-T \cdot 361$ & Tanks & 241-T-361 Seftlling Tank & Procoss Wasle & $\overline{T P A-4}$ & & & & \\
\hline $241-T R-152$ & Diversion Box & 241-TR-152 Dlversion Box & Tank Farm Waste & $T \mathrm{PP}-6$ & & & & \\
\hline $241-T R \cdot 153$ & Diversion Box & 241-TR-153 Dlversion Box & Trank Farm Waste & $\overline{T P-6}$ & & & & \\
\hline $241-T X-152$ & Dlversion Box & 241-TX-152 Dlverslon Box & Tank Farm Wasto & $\overline{T P-2}$ & & & & \\
\hline $241-T X-153$ & Diversion Box & 241-TX-153 Diveralon Box & Tank Fam Waste & TP-5 & & & & \\
\hline $241-\lceil X-154$ & Dlversion Box & 241-TX-154 Diversion Box & Tank Farm Wasto & $T P-4$ & & & & \\
\hline $241-T X-155$ & Diversion Box & $241-T X-155$ Dlyersion Box & Tank Ferm Waste & TP-2 & & & & \\
\hline $241-\mathrm{TX}-302 \mathrm{~A}$ & Tanks & 241-TX-302A Catch Tank & Tank Farm Wasto & $\overline{T P-5}$ & & & & \\
\hline $241-T X-302 B$ & Tanks & 241-TX-302B Catch Tank & Tank Farm Waste & $\mathrm{TP}-2$ & & & & \\
\hline $241 \cdot T \mathrm{TX}-302 \mathrm{C}$ & Tanks & 241-TX-302C Calch Tank & Tank Farm Waslo & TP-4 & & & & \\
\hline $241-T X R-151$ & Diverston Box & $241-T X R-151$ Dlversion Box & Tank Farm Waste & TP-5 & & & & \\
\hline $241-\mathrm{T} \times R-152$ & Diversion Box & 241-TXR-152 Diversion Box & Tank Fam Waste & TP-5 & & & & \\
\hline$\overline{241-T X R-153}$ & Diverston Box & 241-TXR-153 Dlverslon BoX & Trank Fam Wasto & TP.5 & & & & \\
\hline $241-T Y-153$ & Diversion Box & 241-TY-153 Dlverslon Box & Tank Farm Waste & TP-5 & & & & \\
\hline $241-T Y-302 A$ & Tanks & 241-TY-302A Catch Tank & Trank Farm Wasle & TP.5 & & & & \\
\hline $241-\overline{T Y}-302 B$ & Tanks & 241-TY-302B Catch Tank & Trank Fam Wasto & TP-5 & & & & \\
\hline
\end{tabular}


Table B.4. (page 13 of 14)

\begin{tabular}{|c|c|c|c|c|c|c|c|c|}
\hline SIto Codo & $\begin{array}{c}\text { Wasto Managoment } \\
\text { Unit Typo }\end{array}$ & Wasto Management Unit & Waste Type & $\begin{array}{c}\text { Oporablo } \\
\text { Untt }\end{array}$ & $\begin{array}{l}\text { Uranlum-238, } \\
\text { Un/ts (C), }\end{array}$ & $\begin{array}{l}\text { Alpha Emitters, } \\
\text { Unilts (Cl) }\end{array}$ & $\begin{array}{l}\text { Beta Emltters, } \\
\text { Units (CI) }\end{array}$ & $\begin{array}{l}\text { Amerlcium-241, } \\
\text { Unlts (CI) }\end{array}$ \\
\hline $241-2-361$ & \begin{tabular}{|l|} 
Tanks \\
\end{tabular} & 241-Z-361 Settllng Tank & Process Wasto & \begin{tabular}{|l|}
$\mathrm{ZP}-2$ \\
\end{tabular} & & & & \\
\hline 241-Z-TK-8 & Tank 3 & 216.Z.8 Settling Tank & Process Waste & $2 \mathrm{PP}-2$ & & & & \\
\hline 241-Z-TK-D5 & Tanks & 241-Z Treatment Tank & Process Waste & $2 \mathrm{PP}-2$ & & & & \\
\hline 242-T-151 & Dlversion Box & 242-T-151 Diversion Box & Process Condensale & TP-5 & & & & \\
\hline 244-ART & Tanks & $244-A$ & Process Wasio & PO-3 & & & & \\
\hline 244-AR VAULT & Vault & $244-A R$ & Process Waste & $\overline{\mathbf{P O}-3}$ & & & & \\
\hline $244-$ CR VAULT & Vault & $244-\mathrm{CR}$ & Procoss Waste & PO.3 & & & & \\
\hline 244-SRT & Tanks & 244-S Recolver Tank & & RO-2 & & & & \\
\hline 244-TXRT & Tanks & 244-TX Recelving Tank & & TP.5 & & & & \\
\hline 244-TXR & Vault & 244 -TXR Vault & Tank Farm Waste & TP.5 & & & & \\
\hline 2607-E5 & Seplle System & 2607-E-5 Seplic Tank and Draln Fleld & Saniflary Waste & SO-1 & & & & \\
\hline $2607-\mathrm{E} 6$ & Soptlic Syatem & $2607-E 6$ & Santlany Waste & $\overline{\mathrm{PO}-2}$ & & & & \\
\hline $2607-E 7 A$ & Septlc System & 2607-E-7A Soptlc Tank and Draln Field & Santlary Waste & so-1 & & & & \\
\hline 2607-EA & Septlc System & 2607.EA & Sanittary Waste & PO-2 & & & & \\
\hline $2607-\mathrm{EC}$ & Septic System & $2607 \cdot \mathrm{EC}$ & Sentlary Wasto & PO-5 & & & & \\
\hline 2607-ED & Septlc System & 2607-ED & Santlary Wasto & $\overline{\mathrm{PO}-3}$ & & & & \\
\hline 2607 -EE & Septlc System & 2607.EL & Santlary Waste & PO-2 & & & & \\
\hline 2607-EG & Sepplic System & $\overline{\text { 2807-EG }}$ & Sanithary Waste & $\overline{\mathrm{PO}-3}$ & & & & \\
\hline $2607-\mathrm{EJ}$ & Sepilc System & $2607 \cdot$ EJ & Sanitary Wasto & PO-3 & & & & \\
\hline $2607-\mathrm{N}$ & Septlc System & 2607-N Septlc Tank/Krobln Fleld & Șanittary Waste & NO-1 & & & & \\
\hline $2607-\mathrm{P}$ & Septlc Systom & 2607-P Septlc TankiDraln Fleld & Santlery Wasto & NO-1 & & & & \\
\hline $2607-R$ & Septle System & 2607-R Septle Tank/Drain Field & Sanittary Waste & NO-1 & & & & \\
\hline$\frac{2607-W 1}{2607-W 2}$ & \begin{tabular}{|l} 
Septic System \\
Septlc System
\end{tabular} & $\begin{array}{l}\text { 2607-W1 Septic Tank } \\
\text { 2607-W/2 Soptlc Tank }\end{array}$ & $\begin{array}{l}\text { Sanltary Wasto } \\
\text { Santtary Waste }\end{array}$ & $\frac{\mid S S-2}{S S-2}$ & & & & \\
\hline $2607-W 3$ & Seplle Systom & 2607-W3 Sepllc Tank & $\begin{array}{l}\text { Saninary Waste } \\
\text { Santlary Waste }\end{array}$ & $\frac{5 S-2}{T P-4}$ & & & & \\
\hline $2607-W 4$ & Seplic System & 2607-W4 Sepllc Tank & Santlary Wasto & $T$ TP-4 & & & & \\
\hline $2607 \cdot W_{6}$ & Septlc Systom & 2607-WB & Sanitary Waste & $\mathbf{R O - 3}$ & & & & \\
\hline 2607-WB & Sepilic Systom & $2607-$ W.8 Seplic Tank and Draln Fleld & Sanftary Wasto & $\mathrm{ZP}-2$ & & & & \\
\hline 2607-WA & Septle System & 2607-WA Septic Tank and Drain Fleld & Sanittary Waste & $2 \mathrm{PP}-2$ & & & & \\
\hline 2607-WB & & 2607-WB Septic Tank and Drain Fleld & & & & & & \\
\hline 2607-WT & Soptle Systom & 2607-WT Septlc Tank & Sanltary Waste & TP-5 & & & & \\
\hline$\frac{2607-W T x}{2607-W z}$ & \begin{tabular}{|l} 
Septce System \\
Sopltc Systom
\end{tabular} & $\frac{2607-W T X \text { Seplic Tank }}{2607-W Z}$ & $\frac{\text { Santtary Wasto }}{\text { Santitary Wasto }}$ & $\frac{\text { TP-5 }}{\text { RO-1 }}$ & & & & \\
\hline
\end{tabular}


Table B.4. (page 14 of 14)

\begin{tabular}{|c|c|c|c|c|c|c|c|c|}
\hline Sllo Codo & $\begin{array}{c}\text { Wasto Managoment } \\
\text { Unlt Type }\end{array}$ & Wasto Managomont Unit & Wasto Type & $\begin{array}{c}\text { Operable } \\
\text { Unit }\end{array}$ & $\begin{array}{l}\text { Uranlum-238, } \\
\text { Units (CI) }\end{array}$ & $\begin{array}{l}\text { Alpha Emitters, } \\
\text { Units (Cl) }\end{array}$ & $\begin{array}{l}\text { Bota Emlttors, } \\
\text { Unlis (CI) }\end{array}$ & $\begin{array}{l}\text { Amortclum-241, } \\
\text { Unlus (Ci) }\end{array}$ \\
\hline $2607-2$ & Septlc System & 2607-2 Sepllc Tank and Draln Fleld & Santlany Waste & \begin{tabular}{|l|}
$\mathrm{ZP}-2$ \\
\end{tabular} & & & & \\
\hline$\overline{2607-2-1}$ & & 2607-z-1 Sepllc Tank and Drain Fleld & & & & & & \\
\hline $2704-C-W S-1$ & French Draln & $\begin{array}{l}2704 \text {-C-WS-1, 2704C French Draln, Gatehouss } \\
\text { French Draln }\end{array}$ & 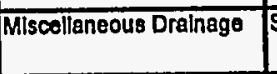 & SO-1 & & & & \\
\hline 2904-S-160 & Diversion Box & $2904-\mathrm{S}-160$ & Cooling Water & RO-1 & & & & \\
\hline 2904-S-170 & Diversion Box & $2904-\mathrm{S}-170$ & Process Waste & RO-1 & & & & \\
\hline $2904-\mathrm{S}-171$ & Diversion Box & $2904-\mathrm{S} \cdot 171$ & Cooling Waler & RO-1 & & & & \\
\hline $291-\mathrm{G}$ & Bullding & 291-C Ventiliallon System & Process Condensale & so-1 & & & & \\
\hline 299-E24-111 & Reverse Well & 299-E24-111 & & PO-2 & & & & \\
\hline HSVP & Diversion Box & Semi-Works Valve PH & Procoss Waste & SO-1 & & & & \\
\hline \begin{tabular}{|l|} 
UPR-200-E-141 \\
UPR-200E-36
\end{tabular} & & UN-200-E-141 & Solution Storage (1) & $50-1$ & & & & \\
\hline$\frac{U P R-200-E-36}{U P R-200-E-37}$ & & $\begin{array}{l}\text { UN-200-E-36 } \\
U N-200-E-37\end{array}$ & Procoss \& Decon Wastes & SO-1 & & & & \\
\hline UPR-200-E-98 & & \begin{tabular}{|l|}
$U N-200-E-37$ \\
$U N-200-E-98$
\end{tabular} & $\begin{array}{l}\text { Process \& Decon Wastes } \\
\text { Process \& Decon Wastes }\end{array}$ & $\frac{50-1}{50-1}$ & & & & \\
\hline UPR-200-W-160 & & UPR-200-W-160 Unplanned Reloaso & Fitnk Farm Waste & TP-4 & & & & \\
\hline ZPLANT BP & Bural Sitte & Z Plant Burn Plt & Debris & $2 \mathrm{PP}-3$ & & & & \\
\hline
\end{tabular}


Table B.5. Environmental Restoration Waste Site Inventories for Tritium, Cobalt-60, Carbon-14, and Europium-154 (page 1 of 14)

\begin{tabular}{|c|c|c|c|c|c|c|c|c|}
\hline SIto Code & $\begin{array}{c}\text { Waste Management } \\
\text { Unit Type }\end{array}$ & Waste Managoment Unit & Wasto Typo & $\begin{array}{c}\text { Operablo } \\
\text { Unit }\end{array}$ & $\begin{array}{l}\text { (Trittum) } \\
\text { Hydrogon-3, } \\
\text { Unlts (Ci) }\end{array}$ & $\begin{array}{l}\text { Cobalt-60, } \\
\text { Units (CI) }\end{array}$ & $\begin{array}{l}\text { Carbon-14, } \\
\text { Units (CI) }\end{array}$ & $\begin{array}{l}\text { Europlum-164, } \\
\text { Unlte (Cl) }\end{array}$ \\
\hline & & 212-N to 216-N-1 Plpeline & & & & & & \\
\hline & & 212-P Hazardous Waste Staging Area & & & & & & \\
\hline & & 212-P to 216-N-4 Pipellno & & & & & & \\
\hline & & 212-P Transformer Oll Tank & & & & & & \\
\hline & & $212-\mathrm{R}$ to $216-\mathrm{N}-\mathrm{B}$ Pipeline & & & & & & \\
\hline & & 241-C Waste Line Unplanned Release No. 1 & & & & & & \\
\hline & & 241-C Wasto Line Unplanned Release No. 2 & & & & & & \\
\hline & & $241-Z$ Dlversion Box No. 1 & & & & & & \\
\hline & & 241-Z Diversion Box No. 2 & & & & & & \\
\hline & & Sanitary Crib & & & & & & \\
\hline 200-E BP & Burial Site & 200-E Buming Pif & Debris & PO-6 & & & & \\
\hline 200-E PAP & Burial Sito & 200-E Ash Pit & & SS-1 & & & & \\
\hline 200-EPD & Dittches & 200 East Powerhouse Ditch & Cooling Water & so-1 & & & & \\
\hline 200-E-4 & French Drain & Critical Mass Laboratory Dry Well North & Miscenleneous Drainage & SO-1 & & & & \\
\hline $200-N-3$ & Burial Sffe & Ballas! PHts & Debris & NO-1 & & & & \\
\hline 200-WADB & Burial Site & 200-WAsh Dlsposa! Basin & Ash & SS-2 & & & & . \\
\hline 200-WADS & Burial Sito & 200-WAsh Plt Demolition SHe & NIA & SS-2 & & & & \\
\hline 200-WBP & Burtal Site & 200-W Buming Pit & Debris & SS-2 & & & & \\
\hline 200-WPAP & Burtal Site & 200.W Powerhouse Ash Pft & Ash & SS-2 & & & & \\
\hline 200-WPP & Ponds & 200-WPowerhouse Pond & Coollng Water & TP-2 & & & & \\
\hline 201-C & Building & 201-C Procoss Bulding & Process Condensato & SO-1 & & & & \\
\hline $207-A$ & & $207-A$ & & & & & & \\
\hline $207-\mathrm{B}$ & Retention Basin & 207-8b/Retentlon Basin & Cooling Water & BP-8 & & & & \\
\hline $207-\mathrm{S}$ & Retention Basin & $207-5$ & Cooling Water & RO-2 & & & & \\
\hline$\overline{207-S L}$ & Retention Basin & $207-$ SL & LabWaste & RO-3 & & & & \\
\hline $207-T$ & Retention Basln & 207-T Retentlon Basin & Coolling Water & TP-3 & & & & \\
\hline $207-Z$ & Retention Basin & 207-2 Retention Basin & Steam Condensato & $\mathrm{ZP}-2$ & & & & \\
\hline 209-E-WS-1 & Fronch Drain & Critcal Mass Laboratory Dry Woll East & Miscellanoous Drainage & SO-1 & & & & \\
\hline 209-E-WS-2 & Fronch Drain & Critteal Mass Laboratory Dry Woll South & Miscellaneous Drainago & SO-1 & & & & \\
\hline 209-E-WS-3 & Diversion Box & Crittcal Mass Laboratory Valvo PH & Process Wasto & SO-1 & & & & \\
\hline 2101-M POND & Ponds & 2101-M Pond & Lab Wasto & SS-1 & & & & \\
\hline $218-A-1$ & Cribs & $210-A-1$ & Procoss Wasto & PO-5 & & 0.00179 & & \\
\hline 218-A-10 & Cribs & $218-A-10$ & Process Condensate & PO-2 & 1850 & & & \\
\hline
\end{tabular}


Table B.5. (page 2 of 14)

\begin{tabular}{|c|c|c|c|c|c|c|c|c|}
\hline Site Codo & $\begin{array}{c}\text { Waste Management } \\
\text { Unit Type }\end{array}$ & Wasto Management Unit & Wasto Typo & $\begin{array}{c}\text { Operablo } \\
\text { Unit }\end{array}$ & $\begin{array}{l}\text { (Tritlum) } \\
\text { Hydrogen-3, } \\
\text { Units (Ci) }\end{array}$ & $\begin{array}{l}\text { Cobalt-60, } \\
\text { Units (CI) }\end{array}$ & $\begin{array}{l}\text { Carbon-14, } \\
\text { Units (CI) }\end{array}$ & $\begin{array}{l}\text { Europlum-154, } \\
\text { Units (CI) }\end{array}$ \\
\hline $216 \cdot A \cdot 11$ & French Drain & $216-A-11$ & Mlscellaneous Drainage & PO-2 & & & & \\
\hline $216-A-12$ & French Drain & $\mid 216-A-12$ & Misceillaneous Drainago & PO-2 & & & & \\
\hline $216-A-13$ & French Drain & $218-A-13$ & Mlscellaneous Drainago & $\mathrm{PO}-2$ & & & & \\
\hline $216-A-14$ & French Drain & $\longdiv { 2 1 6 - A - 1 4 }$ & Miscellaneous Drainage & $\mathrm{PO}-2$ & & & & \\
\hline $216-A-15$ & French Draln & $216-A-15$ & Process Condensate & PO-2 & & & & \\
\hline $216-A-16$ & French Drain & $216 \cdot A-16$ & Chemical Sewer & PO-5 & & & & \\
\hline $216-A-17$ & French Drain & $\overline{218-A-17}$ & Chemical Sewer & PO-5 & & & & \\
\hline $216-A-18$ & Trench & $216-A-18$ & Process Wasto & PO-5 & & 0.00179 & & \\
\hline 216-A-19 & Trench & $216-\bar{A}-19$ & Process Waste & PO-5 & & 0.00179 & & \\
\hline $216-A-2$ & Cribs & $216-A-2$ & Process Wasto & $\mathrm{PO}-2$ & & 0.0297 & & \\
\hline $216-A-20$ & Trench & $216-A-20$ & Process Waste & PO-5 & & 0.00179 & & \\
\hline $216-A-21$ & Cribs & $216 \cdot A-21$ & Lab Waste & PO-2 & & 0.471 & & \\
\hline $216-A-22$ & French Drain & $216-A-22$ & Miscellaneous Drainage & PO-2 & & & & \\
\hline $216-A-23 A$ & French Drain & $216-A-23 A$ & Process Condensate & PO-5 & & & & \\
\hline $216-A-23 B$ & French Draln & 216-A-23B & Process Condensate & PO-5 & & & & \\
\hline $216-A-24$ & Cribs & $216-A-24$ & Process Condensato & PO-5 & 1400 & 0.0219 & & \\
\hline $216-A-25$ & Ponds & $216 \cdot A-25$ Pond & Coofing Walor & $1 \mathrm{U}-6$ & 213 & & & \\
\hline$\overline{216-A-26}$ & French Drain & $216-A-26$ & Mlscellaneous Drainage & $\overline{P O-2}$ & & & & \\
\hline $216-A-26 A$ & French Drain & $216-A-2 B A$ & Miscellaneous Dralnage & PO.2 & & & & \\
\hline $216-A-27$ & Cribs & $216-A-27$ & Mlscellaneous Dralnage & $P 0-2$ & & 0.3 & & \\
\hline $216-A-28$ & Cribs & $216-A-28$ & Procoss Condensato & $\mathrm{PO}-2$ & & & & \\
\hline $216-A-29$ & Ditches & $216-A-28$ & Chemkal Sower & $B P-11$ & & & & \\
\hline $216-A-3$ & Cribs & $216-A-3$ & Procoss Waste & $\overline{P O-2}$ & & & & \\
\hline $216-A \cdot 30$ & Cribs & $216-A \cdot 30$ & Steam Condensate & $\mathrm{PO}-4$ & 16 & & & \\
\hline $216-A-31$ & Cribs & 216-A-31 & Process Wasto & $\overline{P O-2}$ & & 0.00588 & & \\
\hline $216 \cdot A \cdot 32$ & Cribs & $210-A-32$ & Miscollanoous Dralnage & PO-2 & & & & \\
\hline $216-A-33$ & French Drain & $216-A-33$ & Miscellaneous Drainago & PO-2 & & & & \\
\hline $216-A-34$ & Cribs & $216-\bar{A}-34$ & Process Condensate & PO-5 & & & & \\
\hline $216-A-35$ & French Drain & $216-A-35$ & Miscellaneous Drainago & PO-2 & & & & \\
\hline $216-A-36 A$ & Cribs & $216-A \cdot 36 A$ & Process Waste & $\mathrm{PO}-2$ & & 0.71 & & \\
\hline $216-A-36 B$ & Cribs & $216-A-36 B$ & Process Wasto & $\overline{P O-2}$ & 507 & & & \\
\hline $216-A-37-1$ & Cribs & $216-A-37-1$ & Process Condensate & PO-4 & 1600 & & & \\
\hline $216-A-37-2$ & Cribs & $216-A-37-2$ & Steam Condensate & POA & 6 & & & \\
\hline
\end{tabular}


Table B.5. (page 3 of 14)

\begin{tabular}{|c|c|c|c|c|c|c|c|c|}
\hline Site Code & \begin{tabular}{|c|}
$\begin{array}{c}\text { Waste Management } \\
\text { Untt Type }\end{array}$ \\
\end{tabular} & Waste Managoment Unit & Waste Type & $\begin{array}{c}\text { Oporable } \\
\text { Unft }\end{array}$ & $\begin{array}{c}\text { (Tritlum) } \\
\text { Hydrogen-3, } \\
\text { Units (C) }\end{array}$ & $\begin{array}{l}\text { Cobatt-60, } \\
\text { Units (Ci) }\end{array}$ & $\begin{array}{l}\text { Carbon-14, } \\
\text { Units (CI) }\end{array}$ & $\begin{array}{l}\text { Europlum-154, } \\
\text { Units (CI) }\end{array}$ \\
\hline $216-A-3 B-1$ & Cribs & $218 \cdot A-38-1$ & N/A & PO-2 & & & & \\
\hline $216-A-39$ & Ditches & $216 \cdot A \cdot 39$ & Mlscellaneous Drainage & $\overline{\mathrm{PO}-3}$ & & & & \\
\hline $216-A-4$ & Cribs & $216-A-4$ & Lab Waste & PO-2 & & 0.0226 & & \\
\hline $216-A-40$ & Retention Basin & $216-A-40$ & Steam Condensale & $\overline{\mathrm{PO}-2}$ & & & & \\
\hline $216-A-41$ & Cribs & $216-A-41$ & Miscellaneous Dralnage & $\overline{P O-2}$ & & & & \\
\hline $216 \cdot A-42$ & Retenllon Basin & $216-A-42$ & Cooling Water & $\overline{P O-4}$ & & & & \\
\hline $216-A-45$ & Cribs & $216-A-45$ & Procass Condensale & $\overline{\mathrm{PO}-2}$ & 3850 & & & \\
\hline $216-A-5$ & Cribs & $216-A-5$ & Process Condensalo & PO-2 & & 3.32 & & \\
\hline $216-\bar{A}-524$ & Diversion Box & $218-A-524$ & & PO-5 & & & & \\
\hline $216 \cdot A-6$ & Cribs & $216-A-6$ & Sleam Condensate & $\overline{\mathrm{PO}-4}$ & & 0.18 & & \\
\hline 216-A=7 & Cribs & $216-A-7$ & Process Waste & PO-5 & & 0.00204 & & \\
\hline $216-A \cdot B$ & Cribs & $216 \cdot A-8$ & Process Condensate & PO-5 & of & & & \\
\hline $216-A-9$ & Cribs & $216-A-9$ & Cooling Water & $\overline{\mathrm{PO}}-2$ & 4000 & 0.00583 & & \\
\hline $216-B-10 A$ & Cribs & 216-B-10A Crib & Lab Wasle & $\begin{array}{l}\mathrm{BP}-6 \\
\end{array}$ & 0 & 0.00098 & & \\
\hline 218-B-10B & Cribs & $216-B-10 B$ Crlb & Lab Waste & $\overline{B P .6}$ & of & 0 & & \\
\hline 216.B-11A\&B & Reverse Well & 216-8-11A\&B Reverse Wells & Process Condensato & $\overline{B P-4}$ & 0 & 0.00143 & & \\
\hline $218-B-12$ & Cribs & 216-B-12 Crib & Process Condensate & BP-9 & 0 & 0.232 & & \\
\hline $216-8-13$ & French Drain & 216-8-13 French Draln & Miscellaneous Drainage & BP-6 & $\cdot$ & & & \\
\hline $216-8-14$ & Cribs & $216-8-14$ Crib & Scavenged Waste & $8 \mathrm{P}-2$ & 0 & 0.103 & & \\
\hline $216-B-15$ & Cribs & 216-B-15 Crib & Scavenged Wasto & BP-2 & 0 & 0.109 & & \\
\hline $216-B-16$ & Cribs & $216-8-16 \mathrm{Crib}$ & Scavenged Waste & $B P-2$ & 450 & 0.103 & & \\
\hline $216-8-17$ & Cribs & 216-B-17 Crib & Scavenged Wasto & BP-2 & 0 & 0.0204 & & \\
\hline $216-8-18$ & Cribs & $218-\mathrm{B}-18 \mathrm{C}$ तb & Scavenged Wasto & BP-2 & of & 0.103 & & \\
\hline 216-8-19 & Cribs & 216-B-19 Crib & Scavenged Waste & BP-2 & 0 & 0.117 & & \\
\hline $216-8-2-1$ & Ditches & 216-8-2-1 Ditchb/ & Coolling Water & BP-11 & 790 & & & \\
\hline 216-B-2-2 & Ditches & 216-B-2-2 DItchd & Coolling Water & $8 \mathrm{P}-11$ & 0 & & & \\
\hline $216-8-2-3$ & Ditches & 216-8-2-3 Ditch & Cooling Water & $B P-11$ & & & & \\
\hline 216-B-20 & Trench & 216-B-20 Trench & Scavenged Waste & $B P-2$ & of & 0.0899 & & \\
\hline 216-B-21 & Trench & 216-B-21 Trench & Scavenged Waslo & $8 P-2$ & 0 & 0.133 & & \\
\hline $216-\mathrm{B}-22$ & Trench & 216-8-22 Trench & Scavenged Waslo & $\mathrm{BP}-2$ & 0 & 0.274 & & \\
\hline 216-B-23 & Trench & 216-B-23 Trench & Scavenged Wasto & $\mathrm{BP}-2$ & 0 & 0.137 & & \\
\hline 216-B-24 & Trench & 216-B-24 Trench & Scavenged Wasto & $\mathrm{BP}-2$ & 0 & 0.21 & & \\
\hline 216-B-25 & Trench & 216-B-25 Trench & Scavenged Wasto & BP-2 & 0 & 0.141 & & \\
\hline
\end{tabular}


Table B.5. (page 4 of 14)

\begin{tabular}{|c|c|c|c|c|c|c|c|c|}
\hline Sito Code & $\begin{array}{c}\text { Waste Management } \\
\text { Unit Type }\end{array}$ & Wasto Management Unit & Waste Typo & $\begin{array}{c}\text { Operable } \\
\text { Unit }\end{array}$ & $\begin{array}{l}\text { (Tritlum) } \\
\text { Hydrogen-3, } \\
\text { Units (Ci) }\end{array}$ & $\begin{array}{l}\text { Cobalt-60, } \\
\text { Units (CI) }\end{array}$ & $\begin{array}{l}\text { Carbon-14, } \\
\text { Units (CI) }\end{array}$ & $\begin{array}{l}\text { Europium-164, } \\
\text { Units (CI) }\end{array}$ \\
\hline$\overline{216-B-28}$ & Trench & 216-B-26 Trench & Scavenged Waste & BP-2 & 0 & 0.223 & & \\
\hline $216-8-27$ & Trench & 216-B-27 Trench & Scavenged Waste & $\overline{B P-2}$ & 0 & 0.177 & & \\
\hline$\overline{216-B-28}$ & Trench & 216-8-28 Trench & Scavenged Wasto & BP-2: & $\overline{0}$ & 0.0537 & & \\
\hline$\overline{216-B-29}$ & Trench & 216-B-29 Trench & Scavenged Wasto & BP-2 & 0 & 0.165 & & \\
\hline 216-B-3 & Ponds & 216-8-3 Pondel & Cooling Water & BP-11 & 790 & & & \\
\hline $216-B-3-1$ & Ditches & 216-8-3-1 Ditchb/ & Cooling Water & BP-11 & & & & \\
\hline $216-B-3-2$ & Ditches & 216-B-3-2 Ditchal & Cooling Water & BP-11 & & & & \\
\hline $216-B-3-3$ & Ditches & 216-B.3.3 Dltch & Cooling Water & BP-11 & & & & \\
\hline $216-B-30$ & Trench & 216-8-30 Trench & Scavenged Waste & $8 \mathrm{PP}-2$ & $\overline{0}$ & 0.0397 & & \\
\hline$\overline{218-B-32}$ & Trench & 216-8-32 Trench & Scavenged Waste & $\overline{\mathrm{BP}-2}$ & 0 & 0.0397 & & \\
\hline $216-\mathrm{B}-33$ & Trench & 216-8-33 Trench & Scavenged Wasto & $\overline{\mathrm{BP}-2}$ & $\overline{0}$ & 0.0327 & & \\
\hline 216-B-34 & Trench & 216-B-34 Trench & Scavenged Waste & BP-2 & 0 & 0.014 & & \\
\hline $216-B-35$ & Trench & 216-B-35 Trench & Tank Farm Wasto & BP-3 & 0 & 0.00047 & & \\
\hline 218-B-36 & Trench & 216-B-38 Trench & Tank Farm Wasto & $\overline{\mathrm{BP}-3}$ & 0 & 0.0011 & & \\
\hline $216-8-37$ & Tronch & 216-B-37 Trench & Procoss Wasto & $\overline{B P-3}$ & 0 & 0.0157 & & \\
\hline 216-8-38 & Trench & 216-B-38 Trench & Tank Farm Wasto & $\mathrm{BP}-3$ & 0 & 0.00094 & & \\
\hline $216-8-38$ & Trench & 216-B-39 Trench & Tank Farm Waste & $8 P-3$ & $\overline{0}$ & 0.0148 & & \\
\hline 216-B-3A & Ponds & 216-B-3A Pond & Cooling Water & BP-11 & & & & \\
\hline 21B-B-3B & Ponds & 216-B-3B Pond & Cooling Water & BP-11 & & & & \\
\hline $21 B \cdot B-3 C$ & Ponds & 216-B-3C Pond & Cooling Waler & $\overline{B P}-11$ & & & & \\
\hline $216-8-4$ & Reverse Well & 216-B-A Reverso Well & Mlscollaneous Drainago & $\overline{B P-6}$ & 0 & 0 & & \\
\hline $216-B-40$ & Trench & 216-B-40 Trench & Tank Farm Waste & $\overline{B P-3}$ & 0 & 0.00031 & & \\
\hline 216-8-41 & Trench & 218-B-A1 Trench & Tank Farm Waste & BP-3 & 0 & 0.00016 & & \\
\hline $216-8-42$ & Trench & 218-B-42 Trench & Scavenged Wasto & $\overline{B P-3}$ & 0 & 0.179 & & \\
\hline$\longdiv { 2 1 6 \cdot 8 - 4 3 }$ & Cribs & 216-B-43 Crib & Scavenged Waste & $\overline{B P-1}$ & $\overline{170}$ & 0.0157 & & \\
\hline$\overline{216-8-44}$ & Cribs & 216-B-44 CAib & Scavenged Wasto & BP-1 & 450 & 0.0848 & & \\
\hline $216-B-45$ & Cribs & 216-B-45 Crib & Scavenged Waste & $\overline{B P-1}$ & 390 & 0.0899 & & \\
\hline $216-8-46$ & Cribs & 216-B-46 Crib & Scavenged Waste & $\overline{\mathrm{BP}-1}$ & 536 & 0.0889 & & \\
\hline 216-B-A7 & Cribs & 216-B-47 Crib & Scavenged Waste & BP-1 & 0 & 0.0179 & & \\
\hline$\longdiv { 2 1 6 - 8 - 4 8 }$ & Cribs & 216-B-48 Crib & Scavenged Waste & $\longdiv { B P - 1 }$ & 327 & 0.0179 & & \\
\hline $216-8-49$ & Cribs & 216-B-49 Crib & Scavenged Waste & $\overline{B P-1}$ & $\overline{536}$ & 0.0899 & & \\
\hline $216 \cdot B \cdot 5$ & Reverse Well & 216-B-5 Reverso Well & Process Waste & BP.6 & 0 & 0 & & \\
\hline$\overline{216-8-50}$ & Cribs & 218-B-50 Crib & Process Condensate & BP-1 & 90 & 0.0283 & & \\
\hline
\end{tabular}


Table B.5. (page 5 of 14)

\begin{tabular}{|c|c|c|c|c|c|c|c|c|}
\hline Slte Codo & $\begin{array}{c}\text { Wasto Management } \\
\text { Unit Typo }\end{array}$ & Wasto Managemont Unit & Wasto Typo & $\begin{array}{c}\text { Operable } \\
\text { Unit }\end{array}$ & $\begin{array}{c}\text { (Trittum) } \\
\text { Hydrogen-3, } \\
\text { Units (CI) }\end{array}$ & $\begin{array}{l}\text { Cobalt-60, } \\
\text { Units (Ci) }\end{array}$ & $\begin{array}{l}\text { Carbon-14, } \\
\text { Unite (CI) }\end{array}$ & $\begin{array}{c}\text { Europlum-164, } \\
\text { Units (Ci) }\end{array}$ \\
\hline $216-8-51$ & French Drain & 218-B-51 French Drain & Mlscellaneous Drainage & $B P-4$ & & & & \\
\hline $216-8-52$ & Trench & 216-B-52 Trench & Scavenged Waste & BP-2 & 0 & 0.113 & & \\
\hline $216 \cdot 8-53 A$ & Trench & 216-B-53A Trench & LabWaste & BP-2 & 0 & 0.0335 & & \\
\hline $216-8-53 B$ & Trench & 216-B-53B Trench & Lab Waste & $\longdiv { B P - 2 }$ & 0 & 0.0483 & & \\
\hline $216-8-54$ & Trench & 216-8-54 Trench & Lab Waste & $\overline{B P-2}$ & 0 & 0.0059 & & \\
\hline 216-B-55 & Cribs & 216-B-55 Crib & Steam Condensate & $\overline{B P-9}$ & 2.68 & & & \\
\hline $216-\mathrm{B}-56$ & Cribs & $216-\mathrm{B}-56 \mathrm{Crib}$ & N/A & $B P-6$ & & & & \\
\hline 216-B-57 & Cribs & 216-B-57 Crib & Process Condensate & $\overline{B P-1}$ & 0 & 0.0147 & & \\
\hline 216-B-58 & Trench & 216-B-58 Trench & Lab Waste & $\overline{8 P-2}$ & 0 & 0.198 & & \\
\hline $216-8-59$ & Retention Basin & 216-B-59 Basin & Coolling Water & $B P-6$ & & & & \\
\hline$\overline{216-B-6}$ & Reverse Well & 216-B-6 Reverse Well & Lab Waste & $\longdiv { B P - 6 }$ & 0 & 0 & & \\
\hline 218-B-60 & Cribs & 216-B.60 Crib & Decon Waste & $\overline{B P-6}$ & & & & \\
\hline $216-8-61$ & Cribs & 216-B-61 Crib. & N/A & $B P-1$ & & & & \\
\hline 216-B-62 & Cribs & 216-B-62 Crib & Process Condensate & BP-9 & 14.7 & & & \\
\hline $216-8-63$ & Ditches & 216-B-63 Trench & Chemical Sewer & BP-11 & 2.12 & & & \\
\hline 216-B-64 & Rotention Basin & 216-B-64 Basin & N/A & BP-9 & & & & \\
\hline 216-B-7A\&B & Cribs : & 216-B-7A\&B Crib & Process Waste & BP-4 & 0 & 0.012 & & \\
\hline $216-B-B$ & Cribs & 216-B-8TF Crib & Process Waste & $\mathrm{BP}-4$ & 0 & 0.009 & & \\
\hline 216-B-8 & Cribs & 216-B-9TF Crib & Process Wasto & BP.6 & of & 0.0008 & & \\
\hline$\overline{216-c-1}$ & Cribs & 216-C-1Crib & Process Condensato & SO-1 & 70 & 0.002 & & \\
\hline $216-C-10$ & Cribs & 216-C-10 Crib & Process Condensate & SO-1 & & 0.0113 & . & \\
\hline $216-C-2$ & Reverse Well & 216-C-2 Reverse Well & Mlscellaneous Drainage & SO-1 & & & & \\
\hline $216-C-3$ & Cribs & 216-C-3 Crib & Process Waste & $50-1$ & & 0.0014 & & \\
\hline $216-C-4$ & Cribs & 216-C-4 Crib & Process Wasto & so-1 & & 0.0018 & & \\
\hline$\overline{216-C-5}$ & Cribs & $216-C-5 \mathrm{Crib}$ & Process Wasto & SO-1 & & 0.0018 & & \\
\hline $216-C-6$ & Cribs & 216-C-6 Crib & Process Condensate & SO-1 & & 0.0025 & & \\
\hline $216-C-7$ & Cribs & 216-C-7 Crib & Process Waste & so-t & & & & \\
\hline $216 \cdot \mathrm{C}-8$ & French Drain & $216 \cdot C-8$ & Process Wasto & PO-3 & & & & \\
\hline $216-\mathrm{C}-9$ & Ponds & 216-C-9 Pond & Coollng Waler & $50-1$ & & & & \\
\hline 216-E-28 & Ponds & 216-E-28 Pond & N/A & $\mathrm{BP}-11$ & & & & \\
\hline $216-N-1$ & Ponds & 216-N-1 Pond & Cooling Water & NO-1 & & & & \\
\hline $216-N-2$ & Tronch & 216-N-2 Trench & Cooling Water & NO-1 & & & & \\
\hline $216-\mathrm{N}-3$ & Trench & 216-N-3 Trench & Cooling Water & NO-1 & & & & \\
\hline
\end{tabular}


Table B.5. (page 6 of 14)

\begin{tabular}{|c|c|c|c|c|c|c|c|c|}
\hline Site Codo & $\begin{array}{c}\text { Waste Managemont } \\
\text { Unlt Type }\end{array}$ & Wasto Managomont Unit & Waste Typo & $\begin{array}{c}\text { Operable } \\
\text { Unit }\end{array}$ & $\begin{array}{c}\text { (Trittum) } \\
\text { Hydrogen-3, } \\
\text { Units (CI) }\end{array}$ & $\begin{array}{l}\text { Cobalt-60, } \\
\text { Unite (CI) }\end{array}$ & $\begin{array}{l}\text { Carbon-14, } \\
\text { Units (CI) }\end{array}$ & $\begin{array}{l}\text { Europlum-154, } \\
\text { Units (CI) }\end{array}$ \\
\hline $216-N-4$ & \begin{tabular}{|l|l|} 
Ponds \\
\end{tabular} & 216-N-4 Pond & Cooling Water & NO-1 & & & & \\
\hline $216-\mathrm{N}-5$ & Trench & 216-N-5 Trench & Cooling Waiter & NO-1 & & & & \\
\hline $216-N-6$ & Ponds & 216-N-6 Pond & Coolling Waler & NO-1 & & & & \\
\hline 216-N-7 & Trench & 216-N-7 Trench & Cooling Water & $\overline{N O-1}$ & & & & \\
\hline $216-\mathrm{N}-\mathrm{B}$ & Ponds & 216-N-8 Pond & & $\mid \overline{I U-6}$ & & & & \\
\hline$\overline{216-S-182}$ & Cribs & 216-S-1\&2 & Procoss Condensato & $\overline{\mathrm{RO}-2}$ & & & & \\
\hline$\overline{216-S-10 D}$ & Ditches & $216-\mathrm{S}-10 \mathrm{D}$ & Chemical Sewer & RO-1 & & & & \\
\hline 216-S-10P & Ponds & 218-S-10P & Chemical Sower & RO-1 & & & & \\
\hline $216-5-11$ & Ponds & $216-S-11$ & Chemical Sewer & RO-1 & & & & \\
\hline$\overline{216-S-12}$ & Trench & $216-S-12$ & Miscellaneous Dralnage & $\overline{R O-3}$ & & & & \\
\hline $216-5-13$ & Cribs & $\overline{216-S \cdot 13}$ & Process Waste & $\overline{R O-2}$ & & & & \\
\hline$\overline{216-S-14}$ & Trenchi & 216-S-14 & Process Waste & $\overline{\mathrm{RO}-3}$ & & & & \\
\hline $216-S-15$ & Ponds & $216-S-15$ & Cooling Water & $\overline{\text { RO-2 }}$ & & & & \\
\hline 216-S-16D & Ditchos & 216-S-160 & Coolling Water & RO-1 & & & & \\
\hline 216-S-16P & Ponds & 216-S-16P & Cooling Water & RO-1 & & & & \\
\hline $216-5-17$ & Ponds & 216-S-17 & Cooling Water & RO-1 & & & & \\
\hline 216-S-172 & Diversion Box & $\overline{216-5 \cdot 172}$ & Cooling Water & $\overline{R O-1}$ & & & & \\
\hline $216-S-18$ & Tranch & 218-S-18 & Debris & $\overline{\text { RO.2 }}$ & & & & \\
\hline $216-S-19$ & Ponds & 216-S-19 & Lab Wasto & RO-1 & 0.187 & & & \\
\hline$\overline{216-S-20}$ & Cribs & $210-5-20$ & Lab Wasto & RO-3 & & & & \\
\hline$\overline{216-S-22}$ & Cribs & $210-S-22$ & Process Wasto & $\overline{R O} \cdot 3$ & & & & \\
\hline 216-S-23 & Cribs & $210-5-23$ & Process Condensale & $\sqrt{\text { RO-2 }}$ & & & & \\
\hline 216-S-25 & Cribs & $216-S-25$ & Steam Condensato & $\overline{R O-1}$ & 148 & & & \\
\hline $216-S-2 B$ & Cribs & $216-S-26$ & Lab Wasto & $\overline{\mathrm{RO}-3}$ & & & & \\
\hline $216-S-3$ & Fronch Drain & $216-S-3$ & Procoss Condensato & $\overline{\mathrm{RO}-2}$ & & & & \\
\hline $216-5-4$ & French Drain & $216-5-4$ & Procoss Condensalo & UP-2 & 0.02 & & & \\
\hline $216-S-5$ & Cribs & $216 \cdot S-5$ & Coolling Water & RO-1 & & & & \\
\hline $216-S-6$ & Cribs & $216-5-8$ & Coollng Water & RO-1 & & & & \\
\hline 216-S-7 & Cribs & $218-5-7$ & Process Condensato & $\overline{R O-2}$ & & & & \\
\hline $216-\mathrm{S}-\mathrm{B}$ & Trench & $216-\mathrm{S}-8$ & Process Waste & RO-2 & & & & \\
\hline 216-S-9 & Cribs & $216-S-8$ & Process Condensale & RO-2 & & & & \\
\hline 216-T-1 & Ditches & 218-T-1 Ditch & Cooling Water & TP-4 & & & & \\
\hline 216-T-10 & Trench & 216-T-10 Trench & Decon Waslo & TP-4 & & & & \\
\hline
\end{tabular}


Table B.5. (page 7 of 14)

\begin{tabular}{|c|c|c|c|c|c|c|c|c|}
\hline Slte Codte & $\begin{array}{c}\text { Wasto Marsagement } \\
\text { Unit Typo }\end{array}$ & Waste Managoment Unit & Waste Typo & $\mid \begin{array}{c}\text { Operable } \\
\text { Unit }\end{array}$ & $\begin{array}{c}\text { (Trillum) } \\
\text { Hydrogen-3, } \\
\text { Units (CI) }\end{array}$ & $\begin{array}{l}\text { Cobalt-60, } \\
\text { Units (Ci) }\end{array}$ & $\begin{array}{l}\text { Carbon-14, } \\
\text { Units (Cl) }\end{array}$ & $\begin{array}{l}\text { Europlum-164, } \\
\text { Units (CI) }\end{array}$ \\
\hline 216-T-11 & Trench & 216-T-11 Trench & Decon Waste & TP-4 & & & & \\
\hline 216-T=12 & Trench & 216-T-12 Trench & Cooling Water & TP-3 & & 0.0341 & & \\
\hline 216-T-13 & Trench & 216-T-13 Trench & Decon Waste & $\sqrt{\mathrm{TP}-2}$ & & & & \\
\hline 216-T-14 & Tronch & 216-T-14 Trench & Tank Farm Wasto & TP-3 & 0.8 & 0.236 & & \\
\hline 216-T-15 & Trench & 216-T-15 Trench & Tank Farm Wasto & TP-3 & 0.8 & 0.188 & & . \\
\hline 216-T-16 & Trench & 216-T-16 Trench & Tank Farm Wasto & $\mathrm{TP}-3$ & 0.8 & 0.204 & & \\
\hline 216-T-17 & Trench & 216-T-17 Trench & Tank Farm Waste & TP-3 & 0.6 & 0.0157 & & \\
\hline $216-T-18$ & Cribs & 216-T-18 Crib & Tank Farm Wasto & TP-2 & 0.8 & 0.137 & & \\
\hline 216-T-19 & Cribs & 216-T-19TF Crib and Tile Fleld & Process Wasto & TP-2 & 4.25 & & & \\
\hline $216-T-2$ & Revorse Weil & 216-T-2 Reverse Woll & Lab Waste & TPA & & & & \\
\hline $216-T-20$ & Trench & 216-T-20 Trench & Process Wasto & $T P-2$ & & & & \\
\hline 216-T-21 & Trench & 216-T-21 Trench & Tank Farm Wasto & TP-1 & 0.4 & 0.314 & & \\
\hline 216-T-22 & Trench & 216-T-22 Trench & Tank Farm Waste & TP-1 & 1.2 & 0.0157 & & \\
\hline $216-T-23$ & Trench & 216-T-23 Trench & Tank Farm Waste & $T P-1$ & 1.2 & 0.0157 & & \\
\hline $216-T-24$ & Trench & 218-T-24 Tronch & Tank Farm Waste & TP-1 & 1.2 & 0.0157 & & \\
\hline 216-T-25 & Trench & 216-T-25 Trench & Process Wasto & TP-1 & 2.4 & 0.00157 & & \\
\hline 216-T-26 & Cribs & 216-T-26 Crib & Tank Farm Waste & TP-2 & & 0.0489 & & \\
\hline 216-T-27 & Cribs & 216-T-27 Crib & Lab Waste & TP-2 & & 0.087 & & \\
\hline 216-T-28 & Cribs & 216-T-28 Crib & Decon Wasto & TP-2 & & 0.319 & & \\
\hline $216-T-29$ & Cribs & 216-T-29 Crib & Miscellaneous Drainage & TP-4 & & & & \\
\hline $216-T-3$ & Roverso Well & 216-T-3 Roverso Well & Process Waute & $T P=4$ & & & & \\
\hline 216-T-31 & French Drain & 216-T-31 French Drain & Miscellaneous Drainage & TP-2 & & & & \\
\hline $210-T-32$ & Cribs & 216-T-32 Crib & Process Waste & $T P-1$ & & 0.00827 & & \\
\hline $216-T-33$ & Cribs & 216-T-33 Crib & Decon Waste & TP-A & & 0.0515 & & \\
\hline $216-T-34$ & Cribs & 216-T-34 Crib & Lob Wasto & TP-4 & & 0.585 & & \\
\hline 216-T.35 & Cribs & 216-T-35 Crib & Lab Waste & TPA & & 0.298 & & \\
\hline 216-T-36 & Cribs & 216-T-36 Crib & Steam Condensate & TP-1 & & 0.0487 & & \\
\hline 216-T-4-1D & Ditches & 216-T-4-10 DHch & Cooling Water & TP-3 & & & & \\
\hline 216-T-4-2 & Ditches & 216-T-4-2 Ottch & Steam Condensato & $\overline{T P-3}$ & & & & \\
\hline 216-T-4A & Ponds & 216-T-4A Pond & Cooling Water & TP-3 & & & & \\
\hline $218-T-A B$ & Ponds & 216-T-AB Pond & Coolng Waler & TP-3 & & & & \\
\hline $216-T-5$ & Trench & 216-T-5 Tranch & Tank Farm Waste & TP-1 & & 0.0899 & & \\
\hline $216-T-6$ & Cribs & 216-T-6 Crib & Procoss Wasto & TP-3 & & 0.0305 & & \\
\hline
\end{tabular}


Table B.5. (page 8 of 14)

\begin{tabular}{|c|c|c|c|c|c|c|c|c|}
\hline Slte Code & $\begin{array}{c}\text { Waste Management } \\
\text { Untt Typo }\end{array}$ & Waste Managemont Unit & Waste Typo & $\begin{array}{c}\text { Operablo } \\
\text { Untt }\end{array}$ & $\begin{array}{c}\text { (Trittum) } \\
\text { Hydrogen-3, } \\
\text { Units (CI) }\end{array}$ & $\begin{array}{l}\text { Cobalt-60, } \\
\text { Units (C)) }\end{array}$ & $\begin{array}{l}\text { Carbon-14, } \\
\text { Units (CI) }\end{array}$ & $\begin{array}{l}\text { Europlum-154, } \\
\text { Units (Cl) }\end{array}$ \\
\hline $216-T-7$ & Cribs & 216-T-7TF Crib and Tile Fleld & Tank Farm Wasto & TP-1 & & 0.0142 & & \\
\hline $216-T-8$ & Cribs & 216-T-8 Crib & Lab Waste & TP-4 & & 0.00099 & & \\
\hline$\overline{216-T-9}$ & Trench & 216-T-9 Trench & Decon Waste & TP-4 & & & & \\
\hline 216-U-1\&2 & Cribs & $216-U-1 \& 216-U-2$ & Process Condensate & UP-2 & & 0.00157 & & \\
\hline $216-U-10$ & Ponds & $216-U \cdot 10$ & Coolling Water & UP-2 & 196 & & & \\
\hline $216-U-11$ & Diltches & $216 \cdot U-11$ & Coollng Water & UP-2 & & & & \\
\hline $216-U-12$ & Cribs & $216-U-12$ & Process Condensate & UP-2 & 0.00188 & & & \\
\hline $216-U-13$ & Trench & 216-U-13 (same as UN-200-W- 125) & Decon Waste & |UP-2 & & 0.00179 & & \\
\hline 216-U-14 & Ditches & $216-U-14$ & Cooling Water & UP-2 & & & & \\
\hline $216-U-15$ & Trench & $216-U-15$ & Process Waste & UP-2 & & 0.00233 & & \\
\hline $\begin{array}{l}216-U-16 \\
\end{array}$ & Cribs & $216-U-16$ & Process Condensate & UP-2 & 0.233 & & & \\
\hline $216-U-17$ & Cribs & $216 \cdot \mathrm{U}-17$ & Process Condensate & UP-2 & 69.7 & & & \\
\hline$\overline{216-U-21}$ & & $216 \cdot \mathrm{U}-21$ & & & & 0.3333 & & \\
\hline $216-U \cdot 3$ & French Drain & $216-U-3$ & Mlscellaneous Dralnage & UP-2 & & 0.00157 & & \\
\hline$\sqrt{216-U-4}$ & Reverso Weil & $216-U-4$ & Lab Waste & UP-2 & & . & & \\
\hline $216-U-4 A$ & French Draln & $216-U-4 A$ & Miscellaneous Drainage & UP-2 & & & & \\
\hline $216 \cdot U-4 B$ & French Draln & $216-U-4 B$ & Miscellaneous, Dralnage & UP-2 & & & & \\
\hline $296 \cdot$ U.5 & Trench & $216-U-5 \& 216-U-6$ & Process Waslo & UP-2 & & 0.0006 & & \\
\hline $216-U-7$ & French Drain & $216-U-7$ & Mlscellaneous Drainage & |UP-2 & & & & \\
\hline $216-U-8$ & Cribs & $216-U-8$ & Process Condensale & UP-2 & & 0.00204 & & \\
\hline $216-U \cdot \theta$ & Ditches & $218-U-9$ & Cooling Water & RO-1 & & & & \\
\hline 216-W-LWC & Cribs & 216-W-LWC Crib & Chomical Sewer & SS-2 & & & & \\
\hline $216-z-182$ & Cribs & 216-Z-1 \& 216-z-2 Cribs & Process Waslo & $\mathrm{ZP}-2$ & & 0.0171 & & \\
\hline $216-z-10$ & Reverse Well & 216-Z-10 Reverse Well & Process Waslo & $\overline{Z P-2}$ & & & & \\
\hline 216-Z-11 & Ditches & $216-z-11$ & Cooling Water & UP-2 & & & & \\
\hline $216-2-12$ & Cribs & 216-Z-12 Crib & Process Wasto & $2 \mathrm{PP}-2$ & & 0.00515 & & \\
\hline 216-Z-13 & Franch Drain & 216-2-13 French Drain & Miscellaneous Dralnage & $\mathrm{ZP}-2$ & & & & \\
\hline $216-Z-14$ & French Drain & 216-Z-14 French Drain & Miscellaneous Drainage & $2 \mathrm{P}-2$ & & & & \\
\hline $216-2-15$ & French Drain & 218-2-15 Fronch Draln & Miscellaneous Drainago & $2 \mathrm{P}-2$ & & & & \\
\hline $216-2-16$ & Cribs & 216-z-16 Crlb & Lab Waste & $\mathrm{ZP}-2$ & & & & \\
\hline $216-2-17$ & Ditches & 216-2-17 Trench & Lab Waste & $2 \mathrm{P}-2$ & & & & \\
\hline $216-Z-18$ & Cribs & 216-z-18 Crib & Process Waste & $\mathrm{ZP}-2$ & & & & \\
\hline 216-z-19 & Ditches & $218-Z-19$ & Cooling Water & UP-2 & & & & \\
\hline
\end{tabular}


Table B.5. (page 9 of 14)

\begin{tabular}{|c|c|c|c|c|c|c|c|c|}
\hline Site Code & $\begin{array}{c}\text { Wasto Managoment } \\
\text { Unit Typo }\end{array}$ & Wasto Management Unit & Waste Typo & $\begin{array}{c}\text { Operablo } \\
\text { Unit }\end{array}$ & $\begin{array}{c}\text { (Trittum) } \\
\text { Mydrogen-3, } \\
\text { Units (CI) }\end{array}$ & $\begin{array}{l}\text { Cobalt-60, } \\
\text { Unite (Ci) }\end{array}$ & $\begin{array}{l}\text { Carbon-14, } \\
\text { Units (CI) }\end{array}$ & $\begin{array}{l}\text { Europlum-164, } \\
\text { Units (CI) }\end{array}$ \\
\hline $216-Z-1 A$ & Cribs & 216-Z-1A Tile Fiold & Process Waste & $2 \mathrm{P}-2$ & & & & \\
\hline 216-2-10 & Ditches & 216-Z-1D & Coollng Water & UP-2 & & & & \\
\hline $216-2-20$ & Cribs & $216-2-20$ & Cooling Water & UP-2 & & & & \\
\hline 216-Z-21 & Retention Basin & 216-Z-21 Seepage Basin & Coolling Waler & $\overline{\mathrm{ZP}-2}$ & & & & \\
\hline $216-2-3$ & Cribs & 216-z-3 Crib & Process Waste & $\mathrm{ZP}-2$ & & & & \\
\hline $216-Z-4$ & Trench & 216-Z-4 Trench & Process Waste & $\mathrm{ZP}-2$ & & & & \\
\hline $216-z-5$ & Cribs & $216-2-5$ Crib & Process Waste & $Z P-2$ & & 0.0026 & & \\
\hline $216-2-6$ & Cribs & 216-2-6 Crib & Process Wasto & $\mathrm{ZP}-2$ & & 0.00048 & & \\
\hline $216 \cdot Z \cdot 7$ & Cribs & 216-Z-7 Crib & Lab Waste & $Z \mathrm{P}-2$ & & 0.0765 & & \\
\hline $216-Z-8$ & Cribs & 216-Z-8 French Drain & Procoss Waste & $2 p-2$ & & & & \\
\hline 216-Z-9 & Cribs & 216-Z-9 Trench & Process Waste & $\overline{Z P-2}$ & & 0.00395 & & \\
\hline $218-C-9$ & Burial SHe & 218-C-9 Burfal Ground & LLW-SOLID & SO-1 & & & 0.000001 & \\
\hline 218-E-1 & Burial Site & $218-E-1$ & LLW-SOLID & PO-2 & & & & \\
\hline $218-E-10$ & Burlal Site & 218-E-10 Burial Ground & LLW-SOLID & $\overline{B P-10}$ & & 2470 & & \\
\hline 218-E-12A & Burial Sito & 218-E-12A & LLW-SOLID & PO-6 & & & & \\
\hline 218-E-12B & Burial Sito & 218-E-12B & LLW-SOLID & PO-6 & & & & \\
\hline $218-E-13$ & Burial Stte & 218-E-13 & & $\mathrm{PO}-2$ & & & & \\
\hline 218-E-2 & Burtal Site & 218-E-2 Burial Ground & LLW-SOLID & $B P-10$ & & & & \\
\hline $218-E-2 A$ & Burial Site & 218-E-2A Burial Ground & LLW-SOLID & $\overline{B P-10}$ & & & $\cdot$ & \\
\hline $218-E-4$ & Burial Sitto & 218-E-4 Burial Ground & LLW-SOLID & $B P-10$ & & & & \\
\hline 218-E-5 & Burial SHe & 218-E-5 Burial Ground & LLW-SOLID & BP-10 & & & & \\
\hline 218-E-5A & Burial Sito & 218-E-5A Burial Ground & LLW-SOLID & $\mathrm{BP}-10$ & & & & \\
\hline $218-E-6$ & Burial Stte & 218-E-6 Burial Ground & Debris & $B P-6$ & & . & & \\
\hline 218-E-7 & Eurial Site & 218-E-7 Burlal Ground & Lab Waste & BP-6 & & & & \\
\hline 218-E-8 & Burial Silte & $218-E-8$ & TRU Solid Wasto & PO-6 & & & & \\
\hline 218-E-9 & Burial Sito & 218-E-9 Burlal Ground & LLW-SOLID & BP-10 & & & & \\
\hline 218-W-1 & Burlal Sile & 218-W-1 Burial Ground & TRU Solid Wasle & $2 \mathrm{P}-3$ & & & & \\
\hline $218-W-11$ & Burial Site & 218-W-11 Burial Ground & LLW-SOLID & $\mathrm{ZP}-3$ & & & & \\
\hline 218-W-1A & Burial Site & 218-W-1A Burial Ground & LLW-SOLID & $2 \mathrm{P}-3$ & & & & \\
\hline $218-W-2$ & Burial Sito & 218-W-2 Burfal Ground & TRU Solid Waste & $2 \mathrm{P}-3$ & & & & \\
\hline $218-W-2 A$ & Burial Stte & 218-W-2A Burial Ground & LLW-SOLIO & $2 \mathrm{PP}-3$ & & 0.33 & & \\
\hline $218-W-3$ & Burial Site & 218-W-3 Burial Ground & TRU Solld Waste & $\mathrm{ZP}-3$ & & & & \\
\hline $218-W-3 A$ & Burial Site & 218-W-3A Burial Ground & TRU Solid Wasto & $2 P-3$ & 178000 & 9840 & 1.74 & 0.145 \\
\hline
\end{tabular}


Table B.5. (page 10 of 14)

\begin{tabular}{|c|c|c|c|c|c|c|c|c|}
\hline Slte Code & $\begin{array}{c}\text { Waste Management } \\
\text { Unit Typo }\end{array}$ & Wasto Management Unlt & Wasto Type & $\begin{array}{c}\text { Operablo } \\
\text { Unit }\end{array}$ & $\begin{array}{l}\text { (Trittlum) } \\
\text { Mydrogen-3, } \\
\text { Units (C) }\end{array}$ & $\begin{array}{l}\text { Cobalt-60, } \\
\text { Units (CI) }\end{array}$ & $\begin{array}{l}\text { Carbon-14, } \\
\text { Units (CI) }\end{array}$ & $\begin{array}{l}\text { Europlum-154, } \\
\text { Units (Cl) }\end{array}$ \\
\hline 218-W-3AE & Buriạl Site & 218-W-3AE Burial Ground & LLW-SOLID & $\mathrm{ZP}-3$ & 19500 & 299 & 0.321 & 0.141 \\
\hline 218-W-4A & Burial Sito & 218-W-4A Burial Ground & TRU Solid Waste & $2 \mathrm{P}-3$ & & & & \\
\hline $218-W-4 B$ & Burial Sito & 218-W-4B Caissons & TRU Solid Wasto & $\mathrm{ZP}-3$ & 786 & 76000 & & 0.211 \\
\hline $218-W-4 B$ & Burial Site & 218-W-4B Tronchos & TRU Solid Wasto & $\mathrm{ZP}-3$ & 68500 & & & \\
\hline $218-W-4 C$ & Burial Sito & 218-W-4C Burial Ground & TRU Solid Waste & $2 \mathrm{P}-3$ & 25.1 & 221000 & 7.85 & 288 \\
\hline $218-W-5$ & Burial Sito & 218-W-5 Burial Ground & TRU Solld Wasto & $\overline{\mathrm{ZP}-3}$ & 15200 & 3410 & 4.29 & 108 \\
\hline 218-W-6 & Burial Sito & 218-W-6 Burlal Ground & LLW-SOLID & $\mathrm{ZP}-3$ & . & & & \\
\hline $218-W-7$ & Burial Stte & $218-W-7$ & LLW-SOLID & RO-3 & & & & \\
\hline 218-W-8 & Burial Site & 218-W-8 Burial Ground & Lab Waste & $T P-4$ & & & & \\
\hline 218-W-9 & Burial Site & $218-W-9$ & LLW-SOLID & $R 0.2$ & & & & \\
\hline $231-W-15 i$ & Diversion Box & 231-Z-151 Sump & & $2 \mathrm{P}-2$ & & & & \\
\hline $231-W-151$ & Vault & 231-z-151 Sump & & $\mathrm{ZP}-2$ & & & & \\
\hline 231-W-151 & Diversion Box & 231-z-151 Sump & & $\mathrm{ZP}-2$ & & & & \\
\hline $231-W-151$ & Vaull & $231-z-151$ Sump & & $2 \mathrm{P}-2$ & & & & \\
\hline $231-W-151$ & Diversion Box & 231-Z-151 Sump & & $\mathrm{ZP}-2$ & & & & \\
\hline $231-W-151$ & Vauli & 231-z-151 Sump & & $\overline{Z P-2}$ & & & & \\
\hline $231-W-151$ & Diversion Box & 231-Z-151 Sump & & $\mathrm{ZP}-2$ & & & & \\
\hline $231-W-151$ & Vault & $231-z-151$ Sump & & $\mathrm{ZP}-2$ & & & & \\
\hline $232-2$ & Building & 232-z Incineralor & . & $2 \mathrm{P}-2$ & & & & \\
\hline $240-S-151$ & Diversion Box & $240-5-151$ & LLW-SOLID & RO-3 & & & & \\
\hline $240-S-152$ & Diverston Box & $240-5-152$ & Tank Farm Waste & RO-3 & & & & \\
\hline $240-5-302$ & Tanks & $240-5-302$ & Lab Wasto & RO-3 & & & & \\
\hline $241-A-151$ & Dlversion Box & $241-A-151$ & Tank Farm Wasto & PO-2 & & & & \\
\hline $241-A-152$ & Diversion Box & $241-A-152$ & Process Wasto & $\mathrm{PO}-3$ & & & & \\
\hline $241-A-153$ & Diversion Box & $241-A-153$ & Process Waste & PO-3 & & & & \\
\hline 241-A-302A & Tanks & $241-A-302 A$ & & PO-2 & & & & \\
\hline $241-A-302 B$ & Tanks & $241-A-302 B$ & & PO.5 & & & & \\
\hline $241-A-350$ & Tanks & $241-\bar{A}-350$ & Process Waste & PO-3 & & & & \\
\hline $241-A-417$ & Tanks & $241-A-417$ & Process Wasto & $\mathrm{PO}-3$ & & & & \\
\hline $241-A-A$ & Diversion Box & 241-A-A & Process Waste & $\mathrm{PO} .3$ & & & & \\
\hline 241-A-B & Diversion Box & $241 \cdot A-B$ & Process Wasto & $\mathrm{PO}-3$ & & & & \\
\hline $241-A N-A$ & Diverston Box & 241-AN-A & Process Wasto & $\overline{P O-3}$ & & & & \\
\hline 241-AN-B & Diversion Box & 241-AN-B & Process Wasto & PO-3 & & & & \\
\hline
\end{tabular}


Table B.5. (page 11 of 14)

\begin{tabular}{|c|c|c|c|c|c|c|c|c|}
\hline Slto Code & $\begin{array}{c}\text { Waste Management } \\
\text { Untt Type }\end{array}$ & Wasto Managomont Unit & Waste Typo & $\begin{array}{c}\text { Oporablo } \\
\text { Unit }\end{array}$ & $\begin{array}{l}\text { (Tritlum) } \\
\text { Hydragen-3, } \\
\text { Units (C1) }\end{array}$ & $\begin{array}{l}\text { Cobalt-60, } \\
\text { Units (CI) }\end{array}$ & $\begin{array}{l}\text { Carbon-14, } \\
\text { Units (CI) }\end{array}$ & $\begin{array}{l}\text { Europlum-154, } \\
\text { Units (CI) }\end{array}$ \\
\hline 241-APVP & \begin{tabular}{|l|} 
Valvo PH \\
\end{tabular} & 241-AP & Procoss Wasto & $\mathrm{PO}-3$ & & & & \\
\hline 241-AR-151 & Dlvereion Box & 241-AR-151 & Process Waste & $\mathrm{PO}-3$ & & . & & \\
\hline 241-AW-A. & Diversion Box & 241-AW-A & Process Waste & PO-3 & & & & \\
\hline 241-AW-B & Diversion Box & 241-AW-B & Process Wasto & PO-3 & & & & \\
\hline $241-A X-151$ & Diversion Box & $241-A X-151$ & Process Waste & PO-3 & & & & \\
\hline $241-A X-1520 S$ & Tanks & $241-A X-1520 S$ & Process Wasle & PO-3 & & & & \\
\hline $241-A X-155$ & Diversion Box & $241-A X-155$ & Tank Farm Waste & PO-3 & & & & \\
\hline $241-A X-501$ & Valve PIt & $241-A X-501$ & & PO.3 & & & & \\
\hline $241-A X-A$ & Diversion Box & $241-A X-A$ & & PO-3 & & & & \\
\hline 241-AX-B & Diversion Box & $241-A X-B$ & & PO-3 & & & & \\
\hline $241-A Y-154$ & Diversion Box & 241-AY-151 & Process Waste & PO-3 & & & & \\
\hline $241-A Y-152$ & Diversion Box & $241-A Y-152$ & Process Waste & PO-3 & & & & \\
\hline 241-AZ-151DS & Diversion Box & 241-AZ-151DS & & $\overline{P O}-3$ & & & & \\
\hline $241-A 2-152$ & Diverston Box & $241-A Z-152$ & & PO.3 & & & & \\
\hline $241-C-151$ & Diversion Box & 241-C-151 & & PO-3 & & & & \\
\hline $241 \cdot C-152$ & Diverston Box & $241 \cdot \mathrm{C}-152$ & & po.3 & & & & \\
\hline $241-C-153$ & Diversion Box & $241-C-153$ & & $\overline{P O-3}$ & & & & \\
\hline $241-\mathrm{C}-154$ & Diversion Box & 241-C-154 Diversion Box & Process Wasto & SO-1 & & & & \\
\hline $241-C-252$ & Diversion Box & $241-C-252$ & & PO-3 & & & & \\
\hline $241-C-301 C$ & Tanks & $241-C-301 C$ & & $\mathrm{PO}-3$ & & & & \\
\hline 241-CR-151 & Diversion Box & 241-CR-151 & & PO-3 & & & & \\
\hline 241-CR-152 & Diverston Box & $241-C R-152$ & & $\overline{\mathrm{PO}-3}$ & & & & \\
\hline 241-CR-153 & Diverslon 80x & $241-C R-153$ & & PO-3 & & & & \\
\hline 241-CX-TK-70 & Tanks & 241-CX-70 Storago Tank & Tank Farm Waste & $50-1$ & & & & \\
\hline 241-CX-TK-71 & Tanks & 241-CX-71 Storage Tank & Process Condensato & $50-1$ & 70 & 0.002 & & \\
\hline $241-C X-T K-72$ & Tanks & 241.CX-72 Storage Tank & Process Waste & So-1 & & & & \\
\hline 241-ER-153 & Diversion Box & 241-ER-153 & & $\overline{\mathrm{PO}-3}$ & & & & \\
\hline \begin{tabular}{|l}
$241-\mathrm{S}-151$ \\
$241-\mathrm{S}-152$
\end{tabular} & Diversion Box & 241-S-151 & LLW-SOLID & RO-2 & & & & \\
\hline $\mid \frac{241-S-152}{241-S-302 A}$ & \begin{tabular}{|l} 
Diversion Box \\
Tanks
\end{tabular} & $\frac{241-S-152}{241 \cdot S-302 A}$ & Tank Farm Wasto & RO-4 & & & & \\
\hline $241-S-302 B$ & \begin{tabular}{|l} 
Tanks \\
Tanks
\end{tabular} & $\frac{241 \cdot S-302 A}{241 \cdot S-302 B}$ & Lab Wasto & $R O-2$ & & & & \\
\hline $241-S-A$ & Diversion Box & $\frac{241 \cdot S-302 B}{241-S-A}$ & \begin{tabular}{|l|} 
LLW-SOLID \\
TankFarm Waste
\end{tabular} & RO-4 & & & & \\
\hline $241-\mathrm{S}-\mathrm{B}$ & Diverslon Box & $\frac{241-S-A}{241-S-8}$ & \begin{tabular}{|l} 
Tank Farm Waste \\
TankFarm Waste
\end{tabular} & RO-A & & & & \\
\hline & & & & RO-4 & & & & \\
\hline
\end{tabular}


Table B.5. (page 12 of 14)

\begin{tabular}{|c|c|c|c|c|c|c|c|c|}
\hline Stte Code & $\begin{array}{c}\text { Waste Managament } \\
\text { Unlt Typo }\end{array}$ & Waste Management Unit & Waste Type & $\begin{array}{c}\text { Oporablo } \\
\text { Unit }\end{array}$ & $\begin{array}{c}\text { (Trittum) } \\
\text { Hydrogen-3, } \\
\text { Units (Ci) }\end{array}$ & $\begin{array}{l}\text { Cobalt-60, } \\
\text { Units (C)) }\end{array}$ & $\begin{array}{l}\text { Carbon-14, } \\
\text { Units (Ci) }\end{array}$ & $\begin{array}{l}\text { Europlum-164, } \\
\text { Units (CI) }\end{array}$ \\
\hline $241-S-C$ & Diversion $80 x$ & 241-S-C & Tank Farm Wasto & RO-4 & & & & \\
\hline 241-S-D & Dlversion Box & 241-S-D & Tank Farm Wasto & $\longdiv { R O - 4 }$ & & & & \\
\hline $241-S X-151$ & Diversion Box & $241-5 X-151$ & Tank Farm Wasto & RO-4 & & & & \\
\hline $241-S X-152$ & Diversion Box & $241-S X-152$ & Tank Farm Waste & RO-4 & & & & \\
\hline $241-S X-302$ & Tanks & $241-5 X-302$ & & RO-2 & & & & \\
\hline $241-S X-A$ & Diversion 80x & $241-S X-A$ & & RO-4 & & & & \\
\hline $241-S X-B$ & Diversion Box & 241-SX-B & & RO-4 & & & & \\
\hline 241-SY-A & Diversion Box & 241-SY-A & & ROA & & & & \\
\hline 241-SY-A & Diverston Box & 241-SY-A & & RO-4 & & & & \\
\hline $241-S Y-B$ & Diversion Box & 241-SY-B & & ROA & & & & \\
\hline 241-SY-B & Diversion Box & $241-S Y-8$ & & RO-4 & & & & \\
\hline $241-T-151$ & Diversion Box & 241-T-151 Diversion Box & Tank Farm Waste & TP.6 & & & & \\
\hline $241-T-152$ & Diversion Box & 241-T-152 Diversion Box & Tank Farm Wasto & TP-6 & & & & \\
\hline $241-T-153$ & Diversion Box & 241-T-153 Diversion Box & Tank Farm Wasto & TP-6 & & & & \\
\hline $241-T-252$ & Dilyersion Box & 241-T-252 Dlversion Box & Tank Farm Wasto & TP-6 & & & & \\
\hline 241-T-301 & Tanks & 241-T-301 Catch Tank & Tank Farm Waste & TP.6 & & & & \\
\hline $241-T-302$ & Tanks & 241-T-302 Catch Tank & Tank Farm Waste & TP-6 & & & & \\
\hline $241-T-361$ & Tanks & 241-T-361 Sottling Tank & Process Wasto & TP-4 & & & & \\
\hline 241-TR-152 & Diversion Box & 241-TR-152 Diversion Box & Tank Farm Waste & TP-6 & & & & \\
\hline $241-T R-153$ & Diversion Box & 241-TR-153 Diversion Box & Tank Farm Wasto & TP-6 & & & & \\
\hline $241-T X-152$ & Diversion Box & $241-T X-152$ Diversion Box & Tank Farm Wasto & TP-2 & & & & \\
\hline $241-T X-153$ & Diversion Box & 241-TX-153 Divorsion Box & Tank Farm Wasto & TP-5 & & & & \\
\hline $241-T X-154$ & Diversion Box & 241-TX-154 Diversion BoX & Tank Farm Waste & TP-4 & & & & \\
\hline $241-T X-155$ & Diversion Box & 241-TX-155 Diverston BoX & Tank Farm Waste & TP-2 & & & & \\
\hline $241-T X-302 A$ & Tanks & 241-TX-302A Catch Tank & Tank Farm Wasto & TP-5 & & & & \\
\hline $241-\mathrm{TX}-302 \mathrm{~B}$ & Tanks & 241-TX-3028 Calch Tank & Tank Farm Wasto & TP-2 & & & & \\
\hline $241-T X-302 C$ & Tanks & 241-TX-302C Catch Tank & Tank Farm Waste & TP-4 & & & & \\
\hline$\overline{241-T X R-151}$ & Diversion 80x & 241-TXR-151 Diversion BoX & Tank Farm Waste & TP-5 & & & & \\
\hline $241-T \times R-152$ & Diversion Box & 241-TXR-152 Dlversion Box & Tank Farm Wasto & TP-5 & & & & \\
\hline $241-T X R-153$ & Diversion Box & 241-TXR-153 Diverslon BoX & Tank Farm Waste & TP-5 & & & & \\
\hline $241-T Y-153$ & Diversion Box & 241-TY-153 Diversion Box & Tank Farm Wasto & Tि-5 & & & & \\
\hline $241-T Y-302 A$ & Tanks & 241-TY-302A Catch Tank & Tank Farm Wasto & TP-5 & & & & \\
\hline $241-T Y-302 B$ & Tanks & 241-TY-302B Catch Tank & Tank Farm Waste & TP-5 & & & & \\
\hline
\end{tabular}


Table B.5. (page 13 of 14)

\begin{tabular}{|c|c|c|c|c|c|c|c|c|}
\hline Site Code & \begin{tabular}{|} 
Waste Management \\
Unit Type
\end{tabular} & Wasto Managoment Unit & Wasto Typo & $\begin{array}{c}\text { Operablo } \\
\text { Unit }\end{array}$ & $\begin{array}{l}\text { (Tritium) } \\
\text { Hydrogen-3, } \\
\text { Units (CI) }\end{array}$ & $\begin{array}{l}\text { Cobalt-60, } \\
\text { Units (CI) }\end{array}$ & $\begin{array}{l}\text { Carbon-14, } \\
\text { Unito (CI) }\end{array}$ & $\begin{array}{l}\text { Europlum-154, } \\
\text { Untts (CI) }\end{array}$ \\
\hline $241-2-361$ & Tank8 & 241-Z-361 Settling Tank & Procoss Waste & $2 \mathrm{p}-2$ & $\cdot$ & & & \\
\hline 241-Z-TK-8 & Tanks & 216-Z-8 Setting Tank & Process Waste & $2 \mathrm{P}-2$ & & & & \\
\hline 241-Z-TK-D5 & Tanks & 241-Z Trealment Tank & Process Waste & $\mathrm{ZP}-2$ & & & & \\
\hline 242-T-151 & Diversion 80x & 242-T-151 Diversion Box & Process Condensate & TP-5 & & & & \\
\hline 244-ART & Tanks & $244-A$ & Process Waste & PO.3 & & & & \\
\hline 244-AR VAULT & Vault & 244-ÁR & Process Waste & PO-3 & & & & \\
\hline 244-CR VAULT & Vault & 244-CR & Process Wasto & PO-3 & & & & \\
\hline 244-S RT & Tanks & 244-S Recolver Tank & & RO-2 & & & & \\
\hline 244-TXRT & Tanks & 244-TX Recelving Tank & & TP-5 & & & & \\
\hline 244-TXR & Vault & 244-TXR Vault & Tank Farm Wasto & TP-5 & & & & \\
\hline 2607-E5 & Septic System & 2607-E-5 Septle Tank and Drain Fleld & Sanitary Waste & SO-1 & & & & \\
\hline $2607-E_{6}$ & Soptlic System & $2607-E 6$ & Sanitary Waste & $\overline{P O-2}$ & & & & \\
\hline 2607-E7A & Septlc System & 2607-E-7A Septlc Tank and Drain Fiold & Sanitary Waste & $\overline{50.1}$ & & & & \\
\hline 2607-EA & Septic System & 2607-EA & Sanitary Waste & PO-2 & & & & \\
\hline 2607-EC & Septlc System & 2807-EC & Senitary Wasto & PO-5 & & & & \\
\hline 2607-ED & Septlc System & 2807-ED & Sanitary Waste & PO-3 & & & & \\
\hline 2607-EE & Septle System & 2607-EL & Sanitary Wasto & PO-2 & . & & & \\
\hline 2607-EG & Septle System & 2807-EG & Sanitary Waste & Po-3 & & & & \\
\hline 2607-EJ & Septlc System & $2807-E J$ & Sanitary Wasto & $\overline{P O-3}$ & & & & . \\
\hline $2607-N$ & Soptle System & 2607-N Septlc Tank/Draln Floidd & Sanilary Wasto & NO-1 & & & & \\
\hline $2607-P$ & Septic System & 2607-P Septlc Tank/Draln Fleld & Saniltary Wasto & NO-1 & & & & \\
\hline 2607-R & Septlc Systom & 2607-R Septic Tank/Drain Field & Sanitary Wasto & NO-1 & & & & \\
\hline $2607-W_{1}$ & Septlc System & 2607-WI Septic Tank & Sanitary Wasto & SS-2 & & & & . \\
\hline $2607-W / 2$ & Septic System & 2807-W/2 Septic Tank & Sanitary Wasto & SS-2 & & & & \\
\hline $2607-1 / 3$ & Septic Systom & 2607-WB Septic Tank & Saniltary Wasto & TP-4 & & & & \\
\hline $2607-W_{4}$ & Septle System & 2607-W4 Septic Tank & Sanitary Wasto & TP-4 & & & & \\
\hline 2607-W6 & Seplle System & $2607-1{ }^{8}$ & Sanitary Waste & RO-3 & & & & \\
\hline 2607-W8 & Seplle System & 2607-W-8 Seplic Tank and Drain Fleld & Sanitary Wasle & $2 \mathrm{P}-2$ & & & & \\
\hline 2607-WA & Septic System & 2607-WA Seplic Trank and Drain Fleld & Sanitary Waste & $\overline{2 P-2}$ & & & & \\
\hline $2607-W B$ & & 2607-WB Septic Tank and Drain Field & & & & & & \\
\hline $2607-W T$ & Seplle System & 2607-WT Septle Tank & Senttany Wasto & TP-5 & & & & \\
\hline 2607-WTX & Septic System & 2607-WTX Soptic Tank & Sanitary Waste & TP-5 & & & & \\
\hline $2607-W 2$ & Septic System & $2607 \cdot W \bar{z}$ & Sanitary Wasto & RO-1 & & & & \\
\hline
\end{tabular}


Table B.5. (page 14 of 14)

\begin{tabular}{|c|c|c|c|c|c|c|c|c|}
\hline Site Code & $\begin{array}{c}\text { Wasto Management } \\
\text { Unit Type }\end{array}$ & Wasto Managoment Unit & Waste Typo & $\begin{array}{c}\text { Operablo } \\
\text { Unit }\end{array}$ & $\begin{array}{c}\text { (Trittlum) } \\
\text { Hydrogen-3, } \\
\text { Units (CI) }\end{array}$ & $\begin{array}{l}\text { Cobalt-50, } \\
\text { Units (C) }\end{array}$ & $\begin{array}{l}\text { Carbon-14, } \\
\text { Units (CI) }\end{array}$ & $\begin{array}{l}\text { Europlum-154, } \\
\text { Units (CI) }\end{array}$ \\
\hline $2607-2$ & Septic System & 2607-Z Seplic Tank and Drain Field & Sanitary Wasle & \begin{tabular}{|l|l|}
$\mathrm{ZP}-2$ \\
\end{tabular} & & & & \\
\hline $2607-z-1$ & & 2607-Z-1 Soplic Tank and Drain Field & & & & & & \\
\hline $2704-C-W S-1$ & French Drain & $\begin{array}{l}\text { 2704-C-WS-1, 2704-C French Drain, } \\
\text { Gatehouse French Drain }\end{array}$ & Miscollaneous Drainage & SO-1 & & & & \\
\hline $2904-S-160$ & Diversion Box & $2904-S-160$ & Cooling Water & RO-1 & & & & \\
\hline $2904-S-170$ & Diversion Box & $2904-5-170$ & Process Waste & RO-1 & & & & \\
\hline $2904-S-171$ & Diversion Box & 2904-S-171 & Cooling Water & RO-1 & & & & \\
\hline $291-\mathrm{C}$ & Building & 291-C Ventilation System & Process Condensale & So-1 & & & & \\
\hline 299-E24-111 & Reverse Well & 299-E24-111 & & $\mathrm{PO}-2$ & & & & \\
\hline HSVP & Diversion Box & Seml-Works Valve Pit & Process Waste & SO-1 & & & & \\
\hline UPR-200-E-141 & & UN-200-E-141 & Solution Storage $(1)$ & SO-1 & & & & \\
\hline UPR-200-E-36 & & UN-200-E-36 & Process \& Decon Wastes & SO-1 & & & & \\
\hline UPR-200-E-37 & & UN-200-E-37 & Procoss \& Decon Wastes & SO-1 & & & & \\
\hline UPR-200-E-98 & & UN-200-E-98 & Process \& Decon Wastes & SO-1 & & & & \\
\hline UPR-200-W-160 & & UPR-200-W-160 Unplanned Releaso & Fink Farm Waste & TP-4 & & & & \\
\hline ZPLANT BP & Burial SHte & 2 Plant Burn Pit & $\overline{\text { Debris }}$ & $2 \mathrm{ZP}-3$ & & & & \\
\hline
\end{tabular}


Table B.6. Environmental Restoration Waste Site Inventories for Promethium-147, Tin (Sn-113), and lodine-129 (page 1 of 14)

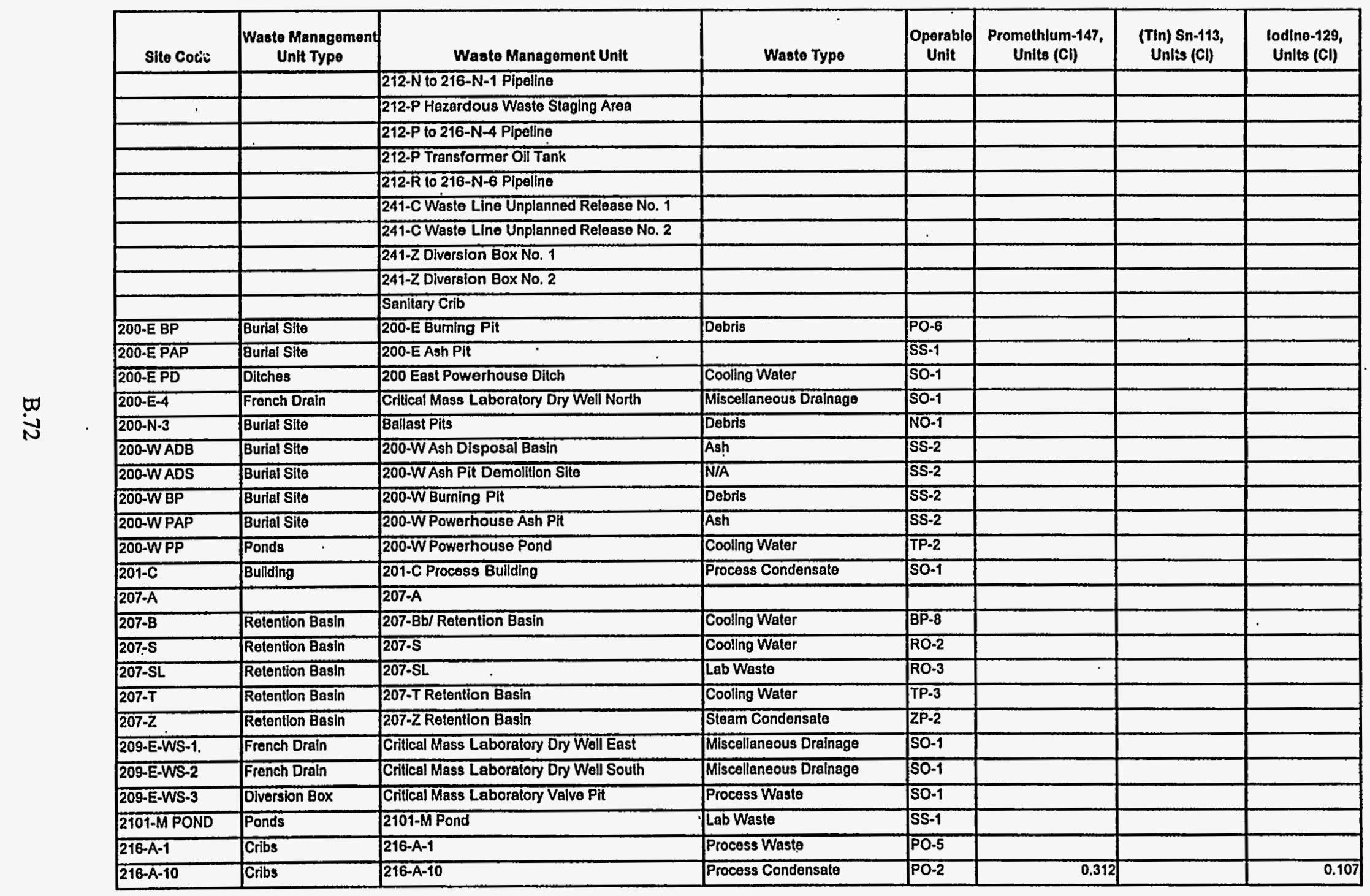


Table B.6. (page 2 of 14)

\begin{tabular}{|c|c|c|c|c|c|c|c|}
\hline Site Code & $\begin{array}{c}\text { Waste Managemen } \\
\text { Unit Type }\end{array}$ & Wasto Management Unit & Waste Type & $\begin{array}{c}\text { Operable } \\
\text { Unit }\end{array}$ & $\begin{array}{l}\text { Promethlum-147, } \\
\text { Units (CI) }\end{array}$ & $\begin{array}{c}\text { (Tnn) Sn-113, } \\
\text { Units (CI) }\end{array}$ & $\begin{array}{l}\text { Iodine-129, } \\
\text { Units (CI) }\end{array}$ \\
\hline $216-A-11$ & French Draln & $216-A-11$ & Miscellaneous Drainage & PO-2 & . & & \\
\hline $216-A-12$ & French Drainh & $216-A-12$ & Miscellaneous Drainage & $\overline{P O-2}$ & & & \\
\hline $216-A-13$ & French Drain & $216-A-13$ & Miscellaneous Drainage & PO-2 & & & \\
\hline $216-A-14$ & French Drain & $\longdiv { 2 1 6 - A - 1 4 }$ & Mlscellaneous Drainage & $\overline{P O}-2$ & & & \\
\hline $216-A-15$ & French Draln & $\sqrt{216-A-15}$ & Process Condensate & $\overline{P O-2}$ & & & \\
\hline $216-A-16$ & French Draln & $\begin{array}{l}216-A-16 \\
\end{array}$ & Chemical Sewer & PO-5 & . & & \\
\hline $216-A-17$ & French Drain & $\overline{216-A-17}$ & Chemlcal Sewer & PO-5 & & & \\
\hline$\overline{216-\bar{A}-18}$ & Trench & $\overline{216-A-18}$ & Process Wasto & PO-5 & & & \\
\hline $216-A-19$ & Trench & $216-A-19$ & Process Wasto & PO-5 & & & \\
\hline $216-A-2$ & Cribs & 216-A-2 & Process Waste & $\overline{\mathrm{PO}-2}$ & & & \\
\hline $216-A-20$ & Trench & $216-A-20$ & Process Waste & PO-5 & & & \\
\hline $216-A-21$ & Cribs & $216-A-21$ & Lab Waste & $\overline{P O-2}$ & & & \\
\hline $216-A-22$ & French Draln & $\sqrt{216-A-22}$ & Mlscellaneous Drainage & $\overline{P O-2}$ & & & \\
\hline $216-A-23 A$ & French Drain & $216-A-23 A$ & Process Condensate & PO-5 & & & \\
\hline $216-A-23 B$ & Frérseh Drain & $216-A-23 B$ & Process Condensate & PO-5 & & & \\
\hline $216-A-24$ & Cribs & 218-A-24 & Process Condensate & PO-5 & & & \\
\hline $216-A-25$ & Ponds & 216-A-25 Pond & Cooling Water & IU-6 & & & \\
\hline $216-A-2 B$ & French Drain & $216-A-26$ & Mlscellaneous Drainage & $\longdiv { \mathrm { PO } - 2 }$ & & & \\
\hline $216-A-26 A$ & French Drain & 216-A-26A & Mlscellaneous Drainage & $\mathrm{PO}-2$ & & & \\
\hline $216-A-27$ & Cribs & 218-A-27 & Miscellaneous Drainage & PO-2 & & & \\
\hline $216 \cdot A-28$ & Cribs & 216-A-2B & Process Condensate & PO-2 & & & \\
\hline $216-A-28$ & Ditchos & $218-A-29$ & Chemical Sewer & BP-11 & & & \\
\hline $216-A-3$ & Cribs & $216-A-3$ & Process Waste & $\mathrm{PO}-2$ & & & \\
\hline $216-A-30$ & Cribs & $216-A-30$ & Steam Condensate & $\mathrm{POA}$ & 0.429 & 0.00315 & \\
\hline $216-A-31$ & Cribs & $218 \cdot A \cdot 31$ & Process Waste & PO-2 & & & \\
\hline $216-A-32$ & Cribs & $216-A-32$ & Miscellaneous Drainage & PO-2 & & & \\
\hline $216 \cdot \bar{A}-33$ & French Drain & $216-A-33$ & Miscellaneous Dralnage & PO-2 & & & \\
\hline $216-A-34$ & Cribs & 216-A-34 & Process Condensate & PO-5 & & & \\
\hline 216-A-35 & French Drain & $216-A-35$ & Miscellaneous Dralnage & PO-2 & & & \\
\hline $216 \cdot A-36 A$ & Cribs & $216-A-36 A$ & Process Wasto & PO-2 & & & \\
\hline $216-A-36 B$ & Cribs & $210-A-36 B$ & Procoss Waste & PO-2 & 1.99 & 0.000579 & 0.00842 \\
\hline $216-A-37-1$ & Cribs & $216-A-37-1$ & Process Condensate & $\mathrm{PO}-4$ & 0.0919 & 0.00252 & 0.00426 \\
\hline $216-A-37-2$ & Cribs & $216-A-37-2$ & Steam Condensato & PO-4 & 0.196 & 0.00157 & \\
\hline
\end{tabular}


Table B.6. (page 3 of 14)

\begin{tabular}{|c|c|c|c|c|c|c|c|}
\hline Site Codo & $\begin{array}{c}\text { Waste Management } \\
\text { Unit Type }\end{array}$ & Wasto Management Unit & Waste Typo & $\begin{array}{c}\text { Operable } \\
\text { Unit }\end{array}$ & $\begin{array}{l}\text { Promethlum-147, } \\
\text { Unito (CI) }\end{array}$ & $\begin{array}{l}\text { (TIn) Sn-113, } \\
\text { Units (CI) }\end{array}$ & $\begin{array}{l}\text { lodine-129, } \\
\text { Units (CI) }\end{array}$ \\
\hline $216-A-38-1$ & Cribs & $216-A-38-1$ & N/A & $P 0.2$ & & & \\
\hline $216-A-39$ & Dilches & $216-A-39$ & Miscellaneous Drainage & $\overline{P O-3}$ & & & \\
\hline $216-A-4$ & Cribs & $216-A-4$ & Lab Waste & PO-2 & & & \\
\hline $216-A-40$ & Retention Basin & $216-A-40$ & Steam Condensale & $\mathrm{PO}-2$ & & & \\
\hline $216-A-41$ & Cribs & $2 \overline{216-A-41}$ & Mlscellaneous Drainage & PO-2 & & & \\
\hline $216-A-42$ & Retention Basin & $218-A-42$ & Cooling Waler & $\mathrm{PO}-4$ & & & \\
\hline $216-A-45$ & Cribs & $216-A-45$ & Process Condensate & PO-2 & 0.0421 & 0.0000656 & 0.011 \\
\hline$\overline{216-A-5}$ & Cribs & $216-A-5$ & Process Condensale & $\mathrm{PO}-2$ & & & \\
\hline $216-A-524$ & Diversion Box & $216 \cdot A-524$ & & PO-5 & & & \\
\hline $216-A-6$ & Cribs & $216-A-6$ & Steam Condensate & PO-4 & & & \\
\hline $216-A-7$ & Cribs & 216-A-7 & Process Wasle & PO-5 & & & \\
\hline $216-A-8$ & Cribs & $216-\bar{A}-8$ & Process Condensate & PO-5 & & & \\
\hline $216-A-9$ & Cribs & $216-A-9$ & Cooling Water & $\mathrm{PO}-2$ & & & \\
\hline $216-8 \cdot 10 A$ & Cribs & 216-B-10A Crib & Lab Wasto & BP-6 & & & \\
\hline 216-B-10B & Cribs & 216-8-10B Crib & Lab Waste & $\mathrm{BP}-6$ & & & \\
\hline 216-B-11A\&B & Reverse Weil & 216-B-11A\&B Reverse Wells & Process Condensate & $\mathrm{BP}-4$ & & & \\
\hline $216-B-12$ & Cribs & 216-B-12 Crib & Process Condensate & $\overline{B P}-9$ & & & \\
\hline $216-8-13$ & French Drain & 216-B-13 French Drain & Miscellaneous Drainago & $\mathrm{BP}-6$ & & & \\
\hline $216-8-14$ & Cribs & $216-\mathrm{B}-14 \mathrm{Crib}$ & Scavenged Waste & $\mathrm{BP}-2$ & & & \\
\hline $216-\overline{B-15}$ & Cribs & 216-8-15 Crib & Scavenged Waste & BP-2 & & & \\
\hline $216-\mathrm{B}-16$ & Cribs & 216-B-16 Crib & Scavenged Waste & $\mathrm{BP}-2$ & & & \\
\hline $216-\mathrm{B}-17$ & Cribs & 216-B-17 Crib & Scavenged Wasto & $\mathrm{BP}-2$ & & & \\
\hline $216-B-18$ & Cribs & 216-B-18 Crib & Scavenged Wasto & $\mathrm{BP}-2$ & & & \\
\hline 216-B-19 & Cribs & 216-B-19 Crib & Scavenged Waste & $8 \mathrm{P}-2$ & & & \\
\hline $216-B-2-1$ & Ditches & 216-B-2-1 Ditchb/ & Cooling Water & $8 \mathrm{P}-11$ & & & \\
\hline $216-8-2-2$ & Ditches & 216-B-2-2 Ditcha' & Cooling Water & BP-11 & & & \\
\hline $216-B-2-3$ & Ditches & 216-8-2-3 Difch & Cooling Water & $8 \mathrm{P}-11$ & & & \\
\hline $216-B-20$ & Trench & 216-B-20 Trench & Scavenged Wasto & $\mathrm{BP}-2$ & & & \\
\hline $216-B-21$ & Trench & 216-8-21 Trench & Scavenged Wasto & BP-2 & & & \\
\hline 216-B-22 & Trench & 216-8-22 Trench & Scavenged Wasto & $8 \mathrm{P}-2$ & & & \\
\hline $216-\mathrm{B}-23$ & Tronch & 216-8-23 Trench & Scavenged Waste & $\mathrm{BP}-2$ & & & \\
\hline 216-8-24 & Trench & 216-B-24 Trench & Scavenged Waste & $\overline{B P-2}$ & & & \\
\hline $216-8 \cdot 25$ & Trench & 216-B-25 Trench & Scavenged Waste & BP-2 & & & \\
\hline
\end{tabular}


Table B.6. (page 4 of 14)

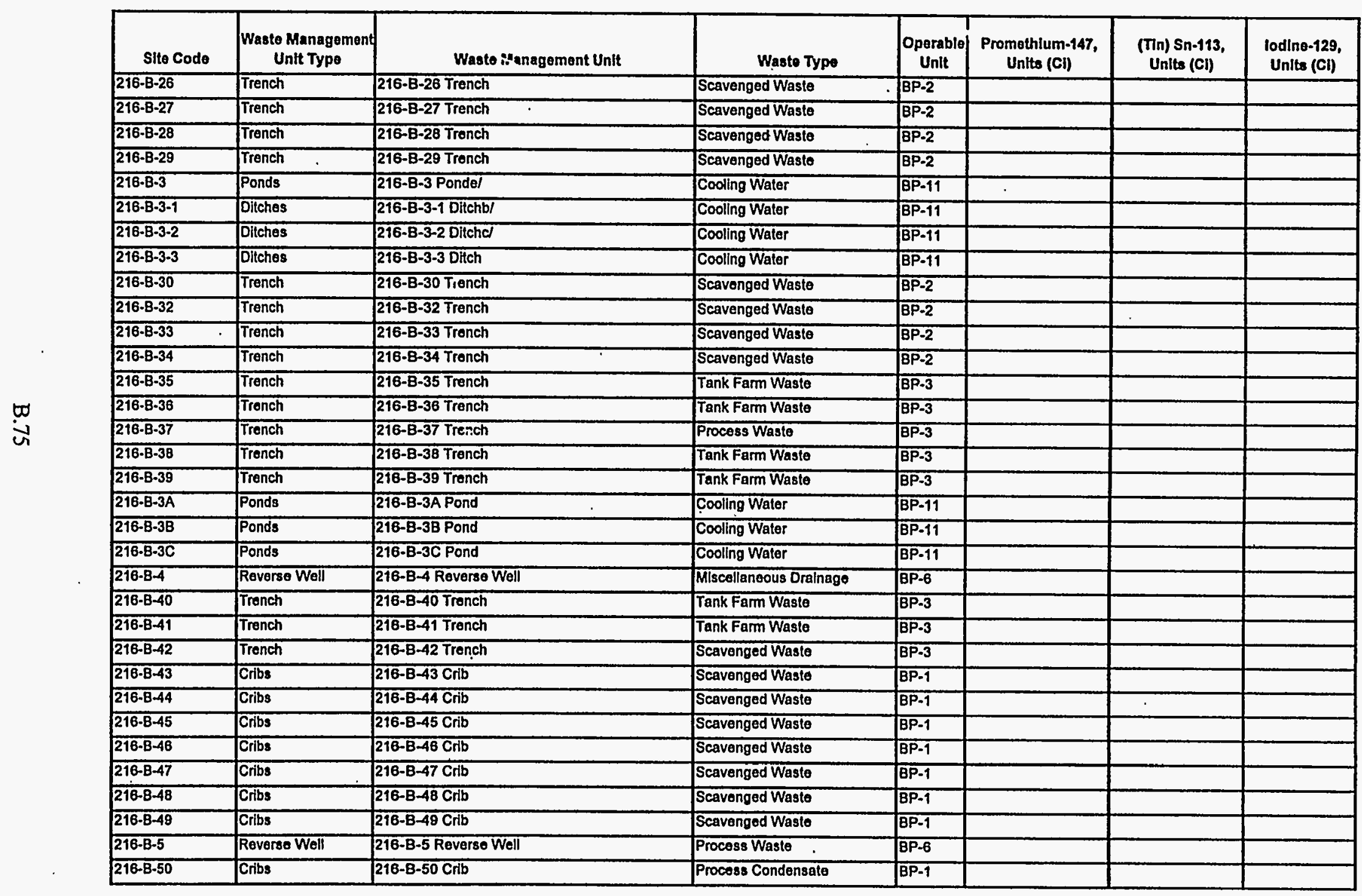


Table B.6. (page 5 of 14)

\begin{tabular}{|c|c|c|c|c|c|c|c|}
\hline Sito Codo & $\begin{array}{c}\text { Waste Managoment } \\
\text { Unit Typo }\end{array}$ & Waste Managoment inint & Wasto Typo & $\begin{array}{c}\text { Oporablo } \\
\text { Unit }\end{array}$ & $\begin{array}{l}\text { Promothium-147, } \\
\text { Units (CI) }\end{array}$ & $\begin{array}{l}\text { (TIn) Sn-113, } \\
\text { Units (CI) }\end{array}$ & $\begin{array}{l}\text { Iodine-129, } \\
\text { Unlte (Ci) }\end{array}$ \\
\hline $216-8-51$ & French Drain & 216-B-51 French Draln & Miscellaneous Drainage & BP-4 & & & \\
\hline $216 \cdot 8 \cdot 52$ & Trench & 216-B-52 Trench & Scavenged Waste & $\overline{\mathrm{BP}-2}$ & & & \\
\hline $216.9-53 \mathrm{~A}$ & Trench & 216-B-53A Trench & Lab Waste & $\overline{B P-2}$ & & & \\
\hline $216.8-53 B$ & Trench & 216-B-53B Trench & Lab Waste & $\overline{B P-2}$ & & & \\
\hline $216-8-54$ & Trench & 216-B-54 Trench & Lab Waste & $\overline{B P}-2$ & & & \\
\hline 216-8-55 & Cribs & 216-B-55 Crib & Steam Condensate & BP.9 & & & \\
\hline $216 \cdot 9 \cdot 56$ & Cribs & 216-B-56 Crib & N/A & $\overline{B P} \cdot 6$ & & & \\
\hline $216 \cdot 8-57$ & Cribs & 216-8-57 Crib & Process Condensate & $\overline{B P-1}$ & & & \\
\hline $216-\bar{B}-58$ & Trench & 216-B-58 Trench & Lab Waste & $\overline{B P-2}$ & & & \\
\hline $216 \cdot B-59$ & Retention Basin & 216-8-59 Basin & Coolling Water & $\overline{B P-6}$ & & & \\
\hline $216-8-6$ & Reverse Woll & 216-8-6 Reverso Well & Lab Waste & $\overline{B P-B}$ & & & \\
\hline $216-8-60$ & Cribs & 216-8-60 Crib & Decon Waste & BP-B & & & \\
\hline$\overline{216 \cdot \bar{B} \cdot 61}$ & Cribs & $216-8-61$ Crib & N/A & (6P-1 & & & \\
\hline $216 \cdot 8 \cdot 62$ & Cribs & 216-8-82 Crib & Process Condensate & BP-9 & & & \\
\hline$\overline{218-\bar{B}-63}$ & Ditches & 216-B-63 Trench & Chemical Sewer & $\overline{B P-11}$ & & & \\
\hline $216 \cdot B \cdot 64$ & Retention Basin & 216-B-64 Basin & NIA & BP-9 & & & \\
\hline 216-8-7A\&B & Cribs & 216-B-7A\&B Crib & Process Waste & $\longdiv { B P - 4 }$ & & & \\
\hline $216-B-8$ & Crtbs & 216-B-8TF Crib & Process Waste & $8 P-4$ & & & \\
\hline 216-8-9 & Cribs & 216-B-9TF Crib & Process Waste & $\overline{B P-6}$ & & & \\
\hline $216-C-1$ & Cribs & $216-C-1$ Crib & Process Condensate & SO-1 & . & & \\
\hline $216-C-10$ & Cribs & 216-C-10 Crib & Process Condensate & SO-1 & & & \\
\hline$\overline{216-C-2}$ & Reverse Well & 216-C-2 Reverse Well & Miscellaneous Drainago & So-1 & & & \\
\hline $216-C-3$ & Cribs & 216-C-3 Crib & Process Wasto & So.1 & & & \\
\hline $216-C-4$ & Cribs & 216-C-4 Crib & Process Waste & SO-1 & & & \\
\hline $216-C-5$ & Cribs & $216-C .5$ Crib & Process Waste & So-1 & & & \\
\hline $216 \cdot C-6$ & Cribs & 216-C-6 Crib & Process Condensale & So-1 & & & \\
\hline $216-C-7$ & Cribs & 216-C-7 Crib & Process Wasto & $\widehat{S O-1}$ & & & \\
\hline $216-\mathrm{C}-8$ & French Drain & $216-C-8$ & Process Waste & PO-3 & & & \\
\hline $216-C-9$ & Ponds & 216-C-9 Pond & Cooling Waler & SO-1 & & & \\
\hline $216-E-28$ & Ponds & 216-E-28 Pond & NIA & $B P-11$ & & & \\
\hline $216-\mathrm{N}-1$ & Ponds & 216-N-1 Pond & Cooling Water & NO-1 & & & \\
\hline $216-\mathrm{N}-2$ & Trench & 216-N-2 Trench & Coolling Water & NO-1 & & & \\
\hline $216-N-3$ & Trench & 216-N-3 Trench & Cooling Water & NO-1 & & & \\
\hline
\end{tabular}


Table B.6. (page 6 of 14)

\begin{tabular}{|c|c|c|c|c|c|c|c|}
\hline Site Codo & $\begin{array}{c}\text { Waato Management } \\
\text { Unlt Type }\end{array}$ & Wasto Managoment Unit & Wasto Typo & $\begin{array}{c}\text { Operable } \\
\text { Unit }\end{array}$ & $\begin{array}{c}\text { Promethlum-147, } \\
\text { Units (Ci) }\end{array}$ & $\begin{array}{c}\text { (Tin) Sn-113, } \\
\text { Unlts (CI) }\end{array}$ & $\begin{array}{l}\text { lodlne-129, } \\
\text { Units (Ci) }\end{array}$ \\
\hline $216 \cdot \mathrm{N}-4$ & Ponds & 216-N-4 Pond & Cooling Water & NO-1 & & & \\
\hline $216 \cdot N-5$ & Trench & 216-N-5 Trench & Cooling Water & NO-1 & & & \\
\hline $216-\mathrm{N}-6$ & Ponds & 21G-N-6 Pond & Coollng Water & NO-1 & & & \\
\hline $216-N-7$ & Trench & 218-N-7 Trench & Cooling Water & NO-1 & & & \\
\hline $216-N-8$ & Ponds & 216-N-8 Pond & & IUS-6 & & & \\
\hline $216 \cdot S-182$ & Cribs & $216-S-1 \& 2$ & Process Condensate & $\overline{R O-2}$ & & . & \\
\hline 216-S-10D & Ditches & $216-5-100$ & Chemlcal Sewer & RO-1 & & & \\
\hline 216-S-10P & Ponds & 216-S-10P & Chemical Sewer & $\overline{R O-1}$ & & & \\
\hline $218-5-11$ & Ponds & 216-S-11 & Chemical Sewer & RO-1 & & & \\
\hline$\longdiv { 2 1 6 - S - 1 2 }$ & Trench & $\mid 216-S-12$ & Miscellaneoús Drainage & RO-3 & & & \\
\hline $216-S-13$ & Cribs & $\sqrt{216-S-13}$ & Process Waste & $\overline{R O-2}$ & & & \\
\hline $216-5-14$ & Trench & 216-S-14 & Process Wasto & RO-3 & & & \\
\hline $216-S-15$ & Ponds & 216-S-15 & Coolling Water & RO-2 & & & \\
\hline 216-S-16D & Ditches & 216-S-160 & Coolling Water & RO-1 & & & \\
\hline 216-S-16? & Ponds & $216-5-16 P$ & Cooling Water & RO-1 & & & \\
\hline $216-S-17$ & Ponds & 216-S-17 & Cooling Water & RO-1 & & & \\
\hline $216-S-172$ & Diverslon Box & $216-5-172$ & Coollng Water & RO-1 & & & \\
\hline 216-S-18 & Trench & $216-\mathrm{S}-18$ & Debris & $\overline{R O-2}$ & & & \\
\hline $216-\mathrm{S}-19$ & Ponds & 216-S-19 & Lab Wasto & RO-1 & & & \\
\hline $216-S-20$ & Cribs & $216-\mathrm{S}-20$ & Lab Wasto & RO-3 & & & \\
\hline 216-S-22 & Cribs & 216-S-22 & Process Wasto & RO-3 & & & \\
\hline $216-5-23$ & Cribs & $216-5-23$ & Procoss Condensale & RO-2 & & & \\
\hline 216-S-25 & Cribs & $216-5-25$ & Steam Condensate & RO-1 & & & \\
\hline $216-\mathrm{S}-26$ & Cribs & 216-S-26 & LabWasto & $\overline{R O-3}$ & & & \\
\hline$\overline{216-S-3}$ & Fronch Drain & $216.5-3$ & Procoss Condensale & RO-2 & & & \\
\hline $216-5-4$ & French Drain & $216 \cdot 5-4$ & Process Condensalo & UP-2 & & & \\
\hline $216-S-5$ & Cribs & 216-5-5 & Cooling Water & RO-1 & & ? & \\
\hline $216-S \cdot 6$ & Cribs & $216 \cdot 5-6$ & Cooling Water & RO-1 & & . & \\
\hline $216-5-7$ & Cribs & 216-S-7 & Process Condensate & RO-2 & & & \\
\hline $216-5-8$ & Trench & 216-5-8 & Procoss Waste & RO-2 & & & \\
\hline 216-5-9 & Cribs & 216-S-9 & Process Condensale & $\overline{R O-2}$ & & & \\
\hline $216-T-1$ & Ditches & 216-T-1 Ditch & Cooling Water & TP-4 & & & \\
\hline $216-T-10$ & Trench & 216-T-10 Trench & Docon Waste & $T P-4$ & & & \\
\hline
\end{tabular}


Table B.6. (page 7 of 14)

\begin{tabular}{|c|c|c|c|c|c|c|c|}
\hline Site Codo & $\mid \begin{array}{c}\text { Waste Management } \\
\text { Unit Typo }\end{array}$ & Wasto Management Untt & Wasto Typo & $\begin{array}{c}\text { Operable } \\
\text { Unit }\end{array}$ & $\begin{array}{l}\text { Promethlum-147, } \\
\text { Units (CI) }\end{array}$ & $\begin{array}{l}\text { (Tin) Sn-113, } \\
\text { Units (CI) }\end{array}$ & $\begin{array}{l}\text { lodine-129, } \\
\text { Units (Cl) }\end{array}$ \\
\hline $216-T-11$ & Trench & 218-T-11 Trench & Decon Waste & $T P-4$ & & & \\
\hline 216-T-12 & Trench & 216-T-12 Trench & Cooling Water & $\mathrm{TP}-3$ & & & \\
\hline$\longdiv { 2 1 6 - T - 1 3 }$ & Trench & 216-T-13 Trench & Decon Wasto & TP-2 & & & \\
\hline 216-T-14 & Trench & 216-T-14 Trench & Tank Farm Wasto & TP-3 & & & \\
\hline$\overline{216-T-15}$ & Trench & 216-T-15 Trench & Tank Farm Wasto & $T \mathrm{P}-3$ & & & \\
\hline 216-T-16 & Trench & 216-T-16 Trench & Tank Farm Waste & $T P-3$ & & & \\
\hline 216-T-17 & Trench & 216-T-17 Trench & Tank Farm Wasto & TP-3 & & & \\
\hline 216-T-18 & Cribs & 216-T-18 Crib & Tank Farm Waste & TP-2 & & & \\
\hline 216-T-19 & Cribs & 216-T-19TF Crib and Tile Flold & Process Waste & $\overline{T P-2}$ & & $\cdot$ & \\
\hline $216-T-2$ & Reverse Well & 216-T-2 Reverso Well & Lab Waste & $T P=4$ & & & \\
\hline $216-T-20$ & Trench & 216-T-20 Trench & Process Waste & $\mathrm{TP}-2$ & & & \\
\hline 216-T-21 & Trench & 216-T-21 Trench & Tank Farm Wasto & TP-1. & & & \\
\hline 216-T-22 & Trench & 216-T-22 Trench & Tank Farm Waste & TP-1 & & & \\
\hline $216-T-23$ & Trench & 216-T-23 Trench & Tank Farm Waste & $\overline{T P-1}$ & & & \\
\hline$\overline{216-T-24}$ & Trench & 216-T-24 Trench & Tank Faim Waste & $\mathrm{TP}-1$ & & & \\
\hline 216-T-25 & Trench & 216-T-25 Trench & Process Wasto & $\mathrm{TP}=1$ & & & \\
\hline $216-T-26$ & Cribs & 216-T-26 Crib & Tank Farm Waste & TP-2 & & & \\
\hline $216-T-27$ & Cribs & 216-T-27 Crib & Lab Waste & $T P-2$ & & & \\
\hline $216-T-28$ & Cribs & $216-T-28$ Crib & Decon Waste & $\overline{T P-2}$ & & & \\
\hline 216-T-29 & Cribs & 216-T-29 Crib & Mlscellaneous Drainage & TP-4 & & & \\
\hline $216-T-3$ & Reverse Well & 216-T.3 Reverso Well & Process Waste & TP-A & & & \\
\hline $216-T-31$ & French Drain & 216-T-31 French Drain & Miscellaneous Drainage & TP-2 & & & \\
\hline $216-T-32$ & Cribs & $216-T-32$ Crib & Process Waste & $\mathrm{TP}-1$ & & & \\
\hline$\overline{216-T-33}$ & Cribs & 216-T-33 Crib & Decon Waste & $T P-4$ & & & \\
\hline $218-T-34$ & Cribs & 216-T-34 Crib & Lab Waste & $\overline{T P-4}$ & & & \\
\hline $216-T-35$ & Cribs & 216-T-35 Crib & Lab Waste & TP-4 & & & \\
\hline $216-T-36$ & Cribs & 216-T-36 Crib & Steam Condensale & $\mathrm{TP}-1$ & & & \\
\hline 216-T-4-1D & Ditches & 216-T-4-10 Ditch & Cooling Water & $T P_{-3}$ & & & \\
\hline $216-T-4-2$ & Ditches & 218-T-4-2 Ditch & Steam Condensate & TP-3 & & & \\
\hline $216-T-4 A$ & Ponds & 216-T-4A Pond & Cooling Waler & TP-3 & & & \\
\hline $216-T-4 B$ & Ponds & 210-T-AB Pond & Cooling Waler & TP.3 & & & \\
\hline $216-T-5$ & Tranch & 216-T-5 Trench & Tank Farm Wasle & TP-1 & & & \\
\hline 216-T-6 & Cribs & 216-T-6 Crib & Process Waste & TP.3 & & & \\
\hline
\end{tabular}




\begin{tabular}{|c|c|c|c|c|c|c|c|}
\hline & & & $z-d n$ & LOIEM Buj|000 & $61-2-912$ & so40Hล & $61-z-912$ \\
\hline & & & $z-d z$ & Oise M ssososd & $q u 081-z=912$ & squj & $81-z-912$ \\
\hline & & & $z-d z$ & 0158M987 & 40LP $\perp \angle L-Z-012$ & 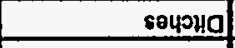 & $\angle 1-z-9 \mid 2$ \\
\hline & & & $z-d z$ & 0188M q87 & quo $91-z-\theta เ z$ & squo & $9 t-z-9+2$ \\
\hline & & & $z-d z$ & 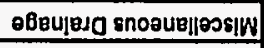 & 니미 40u러 S1-Z-912 & 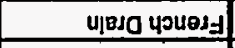 & st-z-9tz) \\
\hline & & & $z-d z$ & obeujeso snozur||вos|w & 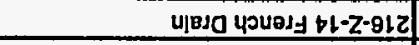 & 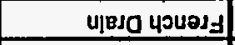 & $+1-z-9+2$ \\
\hline & & & $z-d z$ & 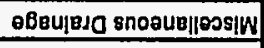 & 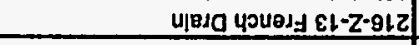 & 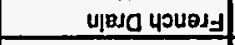 & $\varepsilon L-Z-9 L Z$ \\
\hline & & & $z-d z$ & . 0150M sseossd & qu$ว z t-z-9 t z$ & squo & $z L-z-9 l z$ \\
\hline & & & $z-d n$ & 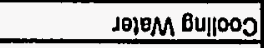 & $11-z-912$ & so4рuव & $1-z-9+2$ \\
\hline & $\therefore$ & & $z-d z$ & Oisem ssososd & 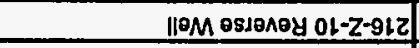 & ॥PM OSJOAOY & 0l-Z-9l2 \\
\hline & & & $z-d z$ & Q188M $85050 \mathrm{Jd}_{\mathrm{d}}$ & squo z-z-9lz \& $1-z-9 t z$ & squo & $281-2-912$ \\
\hline & & & z-ss & 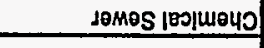 & qHO OMT-M-9LZ & squo & $O M 7-M-912$ \\
\hline & & & $1-0 y$ & 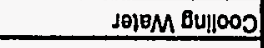 & $6-n-912$ & 804340 & 6-n-912 \\
\hline & & & $z-d n$ & өзвsuapuoj s8050Jd & 8-n-912 & squo & $8-n-912$ \\
\hline & & & $z-\mathrm{dn}$ & 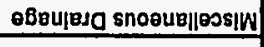 & $2-n-912$ & 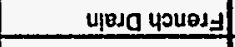 & $2-n-912$ \\
\hline & & & $z-d n$ & Qissom ssosojd & $9-n-9+2<8 \mathrm{~s}-n-9+2$ & 40ues & $s=n-912$ \\
\hline & & & $z-d n$ & ob8ufesa snooue||cos!w & gt-n-9lz & 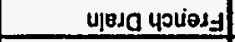 & $8 b-n-912$ \\
\hline & & & $z-d n$ & 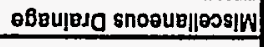 & $\forall t-n-912$ & 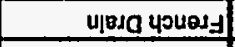 & $\forall 6-n-912$ \\
\hline & & & $z-d n$ & 보이 q日7 & $\forall n-912$ & llom Ossonoy & $t n-912$ \\
\hline & & & $z-d n$ & obeujejo snogue\|nesswW & $\varepsilon-\cap-912$ & Uipesa youes] & $\varepsilon-n-912$ \\
\hline & & & & & $12-0-912$ & & $1 z-n-91 z$ \\
\hline & & & $z-d n$ & gjesuapuoo ssosodd & $\angle 1-n-\theta 12$ & squo & $\angle L-n-9 L 2$ \\
\hline & & & $z-d n$ & Ojesuepuoj sseoodd & $9 t-n-912$ & squo & $9 l-n-9 b \dot{z}$ \\
\hline & & & $z-d n$ & 일M SsEjosd & st-n-9) & पशVOJ1 & st-n-9tZ \\
\hline & & & $2-d n$ & 18: M Bul1000 & $+2-n-912$ & 8өपगนด & th-n-9t2 \\
\hline & & & $z-d n$ & O),8EM 40000 & (sZL-M-00Z-Nn sB owes) $\varepsilon L-n-\theta l Z$ & पoued1 & $\varepsilon t-n-9 t z$ \\
\hline & & & $z-d n$ & Oigsuepuop ssososd & $z_{f-n-9 l 2}$ & squo & $21-n-912$ \\
\hline & & & $2-d n$ & LOjEM 6 U. & $1+-n-812$ & se404a & $\mid 1-n-9) 2$ \\
\hline & & & $z-d n$ & JOi,BM BuylooO & ol-n-9l2 & spuod & $01-n-912$ \\
\hline & & & $z-d n$ & Ojesuopuoj ssesold & $2-n-9128<-n-912$ & squo & $281-n-912$ \\
\hline & & & b-d1 & O1SBM U0000 & 40481 6-1-912 & 4PUอ]1 & $6-1-912$ \\
\hline & & & $t-d 1$ & O)ร8M 987 & qบ5 8-1-9lz & squs & $8-1-912$ \\
\hline & & & $t-d !$ & 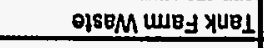 & 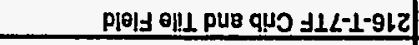 & squo & $\angle-1-912$ \\
\hline $\begin{array}{l}\text { (10) sभun } \\
\text { '6ZL-0uIpol }\end{array}$ & $\begin{array}{l}\text { (10) कqun } \\
\text { ELL-us (uLL) }\end{array}$ & 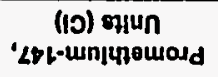 & $\begin{array}{c}\text { Hun } \\
\text { olqesodo }\end{array}$ & od $K_{L}$ OJ8EM & 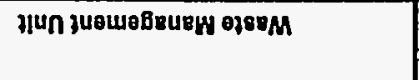 & 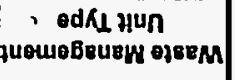 & opos ous \\
\hline
\end{tabular}

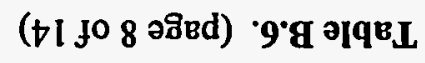


Table B.6. (page 9 of 14)

\begin{tabular}{|c|c|c|c|c|c|c|c|}
\hline Site Codo & $\begin{array}{c}\text { Wasto Managoment } \\
\text { Unit Typo }\end{array}$ & Waste Management Unit & Wasto Typo & $\mid \begin{array}{c}\text { Oparablo } \\
\text { Unit }\end{array}$ & $\begin{array}{c}\text { Promethium-147, } \\
\text { Unlts (CI) }\end{array}$ & $\begin{array}{c}\text { (T/n) Sn-113, } \\
\text { Units (CI) }\end{array}$ & $\begin{array}{l}\text { lodine-129, } \\
\text { Units (CI) }\end{array}$ \\
\hline $216-Z-1 A$ & Cribs & 216-Z-1A Tile Field & Process Waste & $2 \mathrm{P}-2$ & & & \\
\hline $216-2-10$ & Ditches & $216-2-10$ & Coolling Waler & UP-2 & & & \\
\hline $216-2-20$ & Cribs & $218-Z-20$ & Cooling Water & UP-2 & & & \\
\hline $216 \cdot Z-21$ & Retention Basin & 216-Z-21 Seepage 8asin & Coolling Water & $2 \mathrm{PP}-2$ & & & \\
\hline $216-Z-3$ & Cribs & 216-Z-3 Crib & Procass Wasto & $2 p-2$ & & & \\
\hline $216-2-4$ & Trench & 216-2-4 Trench & Process Waste & $2 \mathrm{PP}-2$ & & & \\
\hline $216-z-5$ & Cribs & 216-Z-5 Crib & Process Waste & $2 P-2$ & & & \\
\hline 216-Z-6 & Cribs & 216-2-6 Crib & Process Waste & $\mathrm{ZP}-2$ & & . & \\
\hline $216-z-7$ & Cribs & 216-Z-7 Crib & Lab Waste & $\mathrm{ZP}-2$ & & & \\
\hline $216 \cdot 2-8$ & Gribs & 216-Z-8 French Drain & Process Waste & $\overline{\mathrm{ZP}-2}$ & & & \\
\hline 216-2-9 & Cribs & 216-Z-9 Trench & Process Wasto & $2 \mathrm{ZP}-2$ & & & \\
\hline $218-C-9$ & Burial Site & 218.C-9 Burial Ground & LLW-SOLID & SO-1 & & & \\
\hline 218-E-1 & Burial Sito & 218-E-1 & LLW-SOLID & $\mathrm{PO}-2$ & & & \\
\hline 218-E-10 & Burial Sito & 218-E-10 Burial Ground & LLW-SOLID & BP-10 & & & \\
\hline 218-E-12A & Burial Site & 218-E-12A & LLW-SOLID & $\overline{P O-6}$ & . & & \\
\hline 218-E-12B & Burlal Sito & $218-E-128$ & LLW-SOLID & $\overline{P O-6}$ & & & \\
\hline $218-E-13$ & Burial Site & 218-E-13 & & PO-2 & & & \\
\hline$\overline{218-E-2}$ & Burial Sito & 218-E-2 Burial Ground & LLW-SOLID & $\overline{8 P-10}$ & & & \\
\hline $218-E-2 A$ & Burial Site & 218-E-2A Burial Ground & LLW-SOLID & BP-10 & & & \\
\hline $218-E-4$ & Burial Sito & 218-E-4 Burial Ground & LLW-SOLID & $B P-10$ & & & \\
\hline $218-E-5$ & Burial Sito & 218-E-5 Burial Ground & LLW-SOLID & BP-10 & & & \\
\hline 218-E-5A & Burlal Sitto & 218-E-5A Burial Ground & LLW-SOLID & $B P-10$ & & & \\
\hline $218-E-6$ & 8urlal Stte & 218-E-6 Burial Ground & Debris & $B P \cdot 6$ & & & \\
\hline 218-E-7 & Burial Sito & 218-E-7 Burtal Ground & Lab Wasto & $\overline{B P-6}$ & & & \\
\hline $218-E-8$ & Eurial Stlo & $218-E-8$ & TRU Solld Wasto & PO-6 & & & \\
\hline $218-E-9$ & Burial Sito & 218-E-9 Burial Ground & LLW-SOLID & BP-10 & & & \\
\hline $218-W-1$ & Burial Sile & 218-W-1 Burtal Ground & TRU Solid Waste & $\overline{\mathrm{ZP}-3}$ & & & \\
\hline $218-W-11$ & Burial Site & 218-W-11 Burial Ground & LLW-SOLID & $\overline{Z P-3}$ & & & \\
\hline $218-W-1 A$ & Burial Silo & 218-W-1A Burfal Ground & LLW-SOLID & $2 \mathrm{PP}-3$ & & & \\
\hline $218-W-2$ & Burial Sile & 218-W-2 Burial Ground & TRU Solld Wasto & $2 \mathrm{P}-3$ & & & \\
\hline 218-W-2A & Burial Site & 218-W-2A Burial Ground & LLW-SOLID & $2 \mathrm{P}-3$ & & & \\
\hline $218-W-3$ & Burtal Site & 218-W-3 Burial Ground & TRU Solld Wasle & ZP-3 & & & \\
\hline $218-W-3 A$ & Burial Site & 218-W-3A Burial Ground & TRU Solld Wasto & ZP-3 & & & \\
\hline
\end{tabular}


Table B.6. (page 10 of 14)

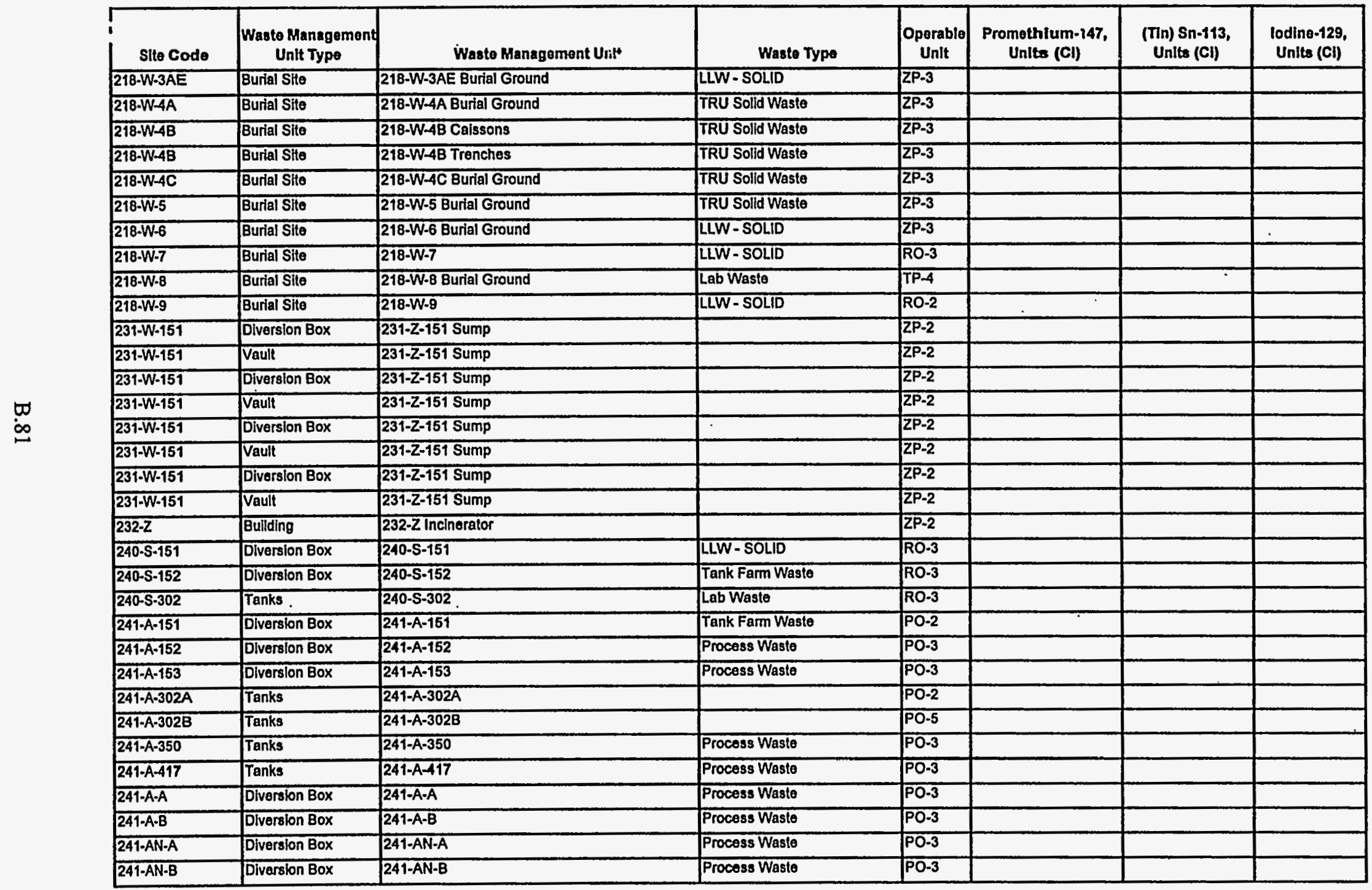


Table B.6. (page 11 of 14)

\begin{tabular}{|c|c|c|c|c|c|c|c|}
\hline Silto Codo & $\begin{array}{c}\text { Waste Management } \\
\text { Unit Type }\end{array}$ & Wasto Management Unit & Waste Type & $\begin{array}{c}\text { Operable } \\
\text { Unit: }\end{array}$ & $\begin{array}{c}\text { Promethlum-147, } \\
\text { Unlto (CI) }\end{array}$ & $\begin{array}{c}(\pi n) S n-113, \\
\text { Unito (C)! }\end{array}$ & $\begin{array}{l}\text { lodino-129, } \\
\text { Units (Cl) }\end{array}$ \\
\hline 241-AP VP & Valve Pit & 241-AP & Process Waste & PO.3 & & & \\
\hline 241-AR-151 & Diversion Box & 241-AR-161 & Process Waste & $\overline{P O-3}$ & & & \\
\hline 241-AW-A & Dlverslon Box & 241-AW-A & Process Waste & $\overline{P O-3}$ & & & \\
\hline 241-AW-B & Diversion Box & 241-AW-B & Process Wasto & $\overline{P O}-3$ & & & \\
\hline $241-A X-151$ & Diversion Box & $241-A X-151$ & Process Waste & $\overline{P O-3}$ & & & \\
\hline $241-A X-1520 S$ & Tanks & $241-A X-152 D S$ & Process Waste & $\mathrm{PO}-3$ & & & \\
\hline $241-A X-155$ & Diversion Box & $241-A X-155$ & Tank Farm Waste & PO-3 & & & \\
\hline $241-A X-501$ & Valve Pit & $241-A X-501$ & & $\overline{P O-3}$ & & & \\
\hline $241-A X-A$ & Diversion Box & 241-AX-A & & $\overline{\mathrm{PO}-3}$ & & & \\
\hline $241-A X-B$ & Diversion 80x & $241-A \bar{X}-B$ & & PO-3 & & & \\
\hline $241-A Y-151$ & Dlversion Box & $241-A Y-151$ & Process Waste & PO-3 & & & \\
\hline $241-A Y-152$ & Dlversion Box & $241-A Y-152$ & Process Wasto & $\overline{P O-3}$ & & & \\
\hline 241-AZ-151DS & Diversion Box & 241-AZ-151DS & & PO.3 & & & \\
\hline $241-A Z-152$ & Diverston Box & 241-A2-152 & & $\overline{P 0.3}$ & & & \\
\hline $241-C-151$ & Diversion Box & $241-\mathrm{C}-151$ & & PO-3 & & & \\
\hline $241-C-152$ & Dlversion Box & $241-C-152$ & & $\overline{\mathrm{PO}-3}$ & & & \\
\hline $241-C-153$ & Diversion Box & $241-C-153$ & & PO.3 & & & \\
\hline $241-C-154$ & Diverston Box & 241-C-154 Dlversion Box & Procoss Wasto & SO-1 & & & \\
\hline $241-C-252$ & Diversion Box & $241 \cdot C-252$ & & PO-3 & & & \\
\hline $241-C-301 C$ & Tanks & $241 . \mathrm{C}-301 \mathrm{C}$ & & $\mathrm{PO}-3$ & & & \\
\hline 241-CR-151 & Diversion Box & $241-C R-151$ & & $\mathrm{PO}-3$ & & & \\
\hline $241-C R-152$ & Diversion Box & 241-CR-152 & & PO-3 & & & \\
\hline $241-C R-153$ & Diversion Box & 241-CR-153 & & $\overline{P O-3}$ & & & \\
\hline 241-CX-TK-70 & Tanks & 241-CX-70 Slorage Tank & Tank Farm Waste & $\widehat{\text { SO-1 }}$ & & & \\
\hline 241-CX-TK-71 & Tanks & 241-CX-71 Siorage Tank & Process Condensate & SO-1 & & & \\
\hline 241-CX-TK-72 & Tanks & 241-CX-72 Storage Tank & Process Waste & So-1 & & & \\
\hline 241-ER-153 & Diversion Box & $241-E R-153$ & & PO-3 & & & \\
\hline $241-S-151$ & Diversion Box & $241-\mathrm{S}-151$ & LLW-SOLID & $\overline{R O .2}$ & & & \\
\hline $241-S-152$ & Dhersion Box & $241-S-152$ & Tank Farm Waste & RO-4 & & & \\
\hline $241-S-302 A$ & Tanks & $241-S-302 A$ & Lab Waste & $R 0.2$ & & & \\
\hline $241-5-302 B$ & Tanks & $241-\mathrm{S}-302 \mathrm{~B}$ & LLW-SOLID & $\longdiv { \mathrm { RO } - 4 }$ & & & \\
\hline 241-S-A & Diversion Box & $241-S-A$ & Tank Farm Waste & RO-4 & & & \\
\hline 241-S-B & Dlversion Box & 241-S-B & Tank Farm Waste & $\mathrm{RO}-4$ & & & \\
\hline
\end{tabular}


Table B.6. (page 12 of 14)

\begin{tabular}{|c|c|c|c|c|c|c|c|}
\hline Site Code & $\begin{array}{c}\text { Wasto Managemont } \\
\text { Unlt Typs }\end{array}$ & Waste Management Unlt & Wasto Type & $\begin{array}{c}\text { Operable } \\
\text { Unit }\end{array}$ & $\begin{array}{c}\text { Promethlum-147, } \\
\text { Unlts (Ci) }\end{array}$ & $\begin{array}{c}\text { (Tin) Sn-113, } \\
\text { Units (Ci) }\end{array}$ & $\begin{array}{l}\text { lodine-129, } \\
\text { Units (Cl) }\end{array}$ \\
\hline 241-S-C & Diversion Box & 241-S-C & Tank Farm Waste & RO-4 & & & \\
\hline 241-S-D & Diversion Box & 241-S-D & Tank Farm Waste & RO-4 & & & \\
\hline $241-S X-151$ & Divarsion Box & 241-SX-151 & Tank Farm Wasto & RO-4 & & & \\
\hline $241-5 X-152$ & Diversion Box & 241-SX-152 & Tank Farm Wasto & $\overline{R O-4}$ & & & \\
\hline $241-5 X-302$ & Tanks & $241-5 X-302$ & & RO-2 & & & \\
\hline $241-S X-A$ & Diversion Box & $241-S X-A$ & & ROA & & & \\
\hline $241-S X-B$ & Diverslon Box & $241-S X+B$ & & RO-4 & & & \\
\hline $241-S Y-A$ & Diversion Box & $241-S Y-A$ & & $\overline{R O-4}$ & & & \\
\hline$\overline{241-S Y-A}$ & Dlversion Box & $241-S Y-A$ & & RO-4 & & & \\
\hline 241-SY-B & Dlverslon Box & 241-SY-B & & RO-4 & & & \\
\hline 241-SY-B & Diversion Box & 241-SY-B & & RO-4 & & & \\
\hline $241-T-151$ & Divergion Box & $241-T-151$ Diversion Box & Tank Farm Wasto & $T+6-6$ & & & \\
\hline $241-T-152$ & Diversion Box & 241-T-152 Diversion Box & Tank Farm Waste & TP-6 & & & \\
\hline $241-T-153$ & Diversion Box & 241-T-153 Diversion Box & Tank Farm Waste & TP-6 & & & \\
\hline $241-T-252$ & Divers!on Box & 241-T-252 Diversion Box & Tank Fam, Waste & TP-6 & & & \\
\hline $241-T-301$ & Tanks & 241-T-301 Catch Tank & Tank Farm Waste & TP-6 & & & \\
\hline $241-T-302$ & Tanks & 249-T-302 Catch Tank & Tank Farm Waste & TPP-6 & & & \\
\hline 241-T-361 & Tanks & 244-T-361 Sotting Tank & Process Waste & TP-4 & & & \\
\hline 241-TR-152 & Diversion Box & 241-TR-162 Diversion Box & Tank Farm Wasto & TPP-6 & & & \\
\hline 241-TR-153 & Dlversion Box & 241-TR-153 Diversion Box & Tank Farm Wasto & TP-6 & & & \\
\hline $241-T X-152$ & Diversion Box & 241-TX-152 Diversion BoX & Tank Farm Wasto & TP-2 & & & \\
\hline $241-T X-153$ & Diversion Box & 241-TX-153 Diversion BoX & Tank Farm Waste & TP-5 & & & \\
\hline $241-T X-154$ & Diversion Box & 241-TX-154 Diveralon Box & Tank Farm Waste & TPP-4 & & & \\
\hline 241-TX-165 & Diversion Box & 241-TX-155 Diveralon Box & Tank Farm Wasto & TPP-2 & & & \\
\hline 241-TX-302A & Tanks & 241-TX-302A Catch Tank & Tank Farm Waste & TP-5 & & & \\
\hline $241-\mathrm{TX}-302 \mathrm{~B}$ & Tanks & 241-TX-302B Catch Tank & Tank Farm Wasto & TP-2 & & & \\
\hline 241-TX-302C & Tanks & 241-TX-302C Calch Tank & Tank Farm Wasto & $T P-4$ & & & \\
\hline 241-TXR-151 & Diversion Box & 241-TXR-151 Dlversion BoX & Tank Farm Waste & TP.5 & & & \\
\hline 241-TXR-152 & Diversion Box & 241-TXR-152 Dlversion Box & Tank Farm Wasto & TP-5 & & & \\
\hline 241-TXR-153 & Dlversion Box & 241-TXR-153 Diverslon Box & Tank Farm Wasto & TP-5 & & & \\
\hline $241-T Y-153$ & Diverston Box & 241-TY-153 Dlvorslon Box & Tank Farm Wasto & TP-5 & & & \\
\hline $241-T Y-302 A$ & Tanks & 241-TY-302A Catch Tank & Tank Farm Wasto & TP-5 & & & \\
\hline $241-T Y-3028$ & Tanks & $241-T Y-302 B$ Catch Tank & Tank Farm Waste & TP.5 & & & \\
\hline
\end{tabular}


Table B.6. (page 13 of 14)

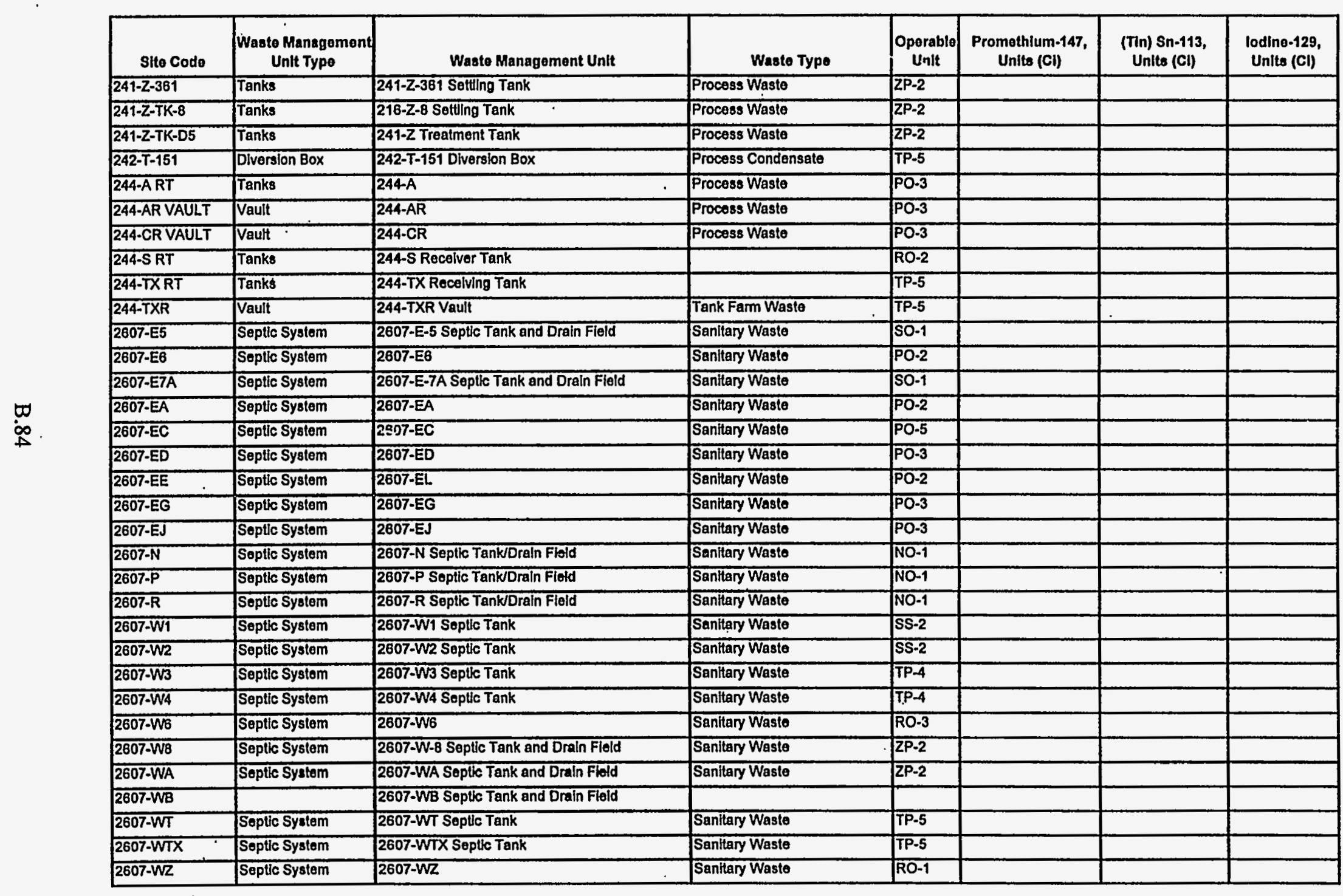


Table B.6. (page 14 of 14)

\begin{tabular}{|c|c|c|c|c|c|c|c|}
\hline Sito Codo & $\mid \begin{array}{c}\text { Wasto Managemont } \\
\text { Unit Typo }\end{array}$ & Waste Marizgement Unit & Wasto Typo & $\begin{array}{c}\text { Operable } \\
\text { Unit }\end{array}$ & $\begin{array}{l}\text { Promethlum-147, } \\
\text { Inlts (CI) }\end{array}$ & $\begin{array}{l}\text { (Tin) Sn-113, } \\
\text { Units (CI) }\end{array}$ & $\begin{array}{l}\text { loding-129, } \\
\text { Units (CI) }\end{array}$ \\
\hline$\overline{2607-2}$ & Septic System & 2607-Z Soptic Tank and Drain Fleld & Saniltary Waste & $2 \mathrm{PP}-2$ & & & \\
\hline $2607 \cdot 2-1$ & & 2607-Z-1 Septic Tank and Drain Fleld & & & & & \\
\hline 2704-C-WS-1 & French Drain & $\begin{array}{l}\text { 2704-C-WS-1, 2704-C French Draln, } \\
\text { Gatehouse Franch Drain }\end{array}$ & Miscellaneous Drainage & so-1 & & & \\
\hline $2904-\mathrm{S}-160$ & Diversion Box & $2904-S-160$ & Cooling Waiter & RO-1 & & & \\
\hline $2904-5-170$ & Diversion Box & $2904-S-170$ & Process Waste & RO-1 & & & \\
\hline 2904-S-171 & Diversion Box & $2904-S-171$ & Coolling Water & RO.1 & & & \\
\hline $291-\mathrm{C}$ & Building & 291-C Ventilation System & Process Condensale & $50-1$ & & & \\
\hline 299-E24-111 & Reverso Well & 299-E24-111 & & PO-2 & & & \\
\hline HSVP & Diversion Box & Semt-Works Valve Pft & Procoss Wasto & SO-1 & & & \\
\hline UPR-200-E-141 & & UN-200-E-141 & Solution Storage (1) & So-1 & & & \\
\hline UPR-200-E-36 & & UN-200-E-36 & Process \& Decon Wastes (1) & SO-1 & & & \\
\hline UPR-200-E-37 & & ONN-200-E-37 & Process \& Decon Wastes (1) & SO-1 & & & \\
\hline UPR-200-E-98 & & UN-200-E-98 & Procass \& Decon Wastes (3) & SO-1 & & & \\
\hline UPR-200-W-160 & & UPR-200-W-180 Unplanned Release & Tank Farm Waste & $T P-4$ & & & \\
\hline ZPLANT BP & Burial Site & 2 Plant Bum Plt & Debris & $2 \mathrm{PP}-3$ & & & \\
\hline
\end{tabular}




\section{Appendix C}

Environmental Restoration Sites Without Inventories 


\section{Appendix C}

\section{Environmental Restoration Sites Without Inventories}

Appendix $C$ is a spreadsheet obtained from the Environmental Restoration Contractor (ERC), Bechtel Hanford, Inc. This appendix consists of one table that lists 363 environmental restoration sites on the 200 Area Plateau at Hanford for which inventories have not been assigned. The table presents the following available information for each site listed: waste volume, waste description, type of waste (radioactive, chemical, or mixed), and an evaluation of whether the release constitutes a potentially significant source. 


\begin{tabular}{|c|c|c|c|c|c|}
\hline & & & ZLEOObOL & & $6 z-\forall-912$ \\
\hline & & & 1 & & $\forall \theta z-\forall-\theta l z$ \\
\hline & & & & & $9 z-\forall-91 z$ \\
\hline & & & Or & & $2 z-\forall-912$ \\
\hline & pox|w & Juanแม $358030 \mathrm{~J}_{\mathrm{d}}$ & 00001 & & $S L-\forall-9 L Z$ \\
\hline & paxiw & ө|esuepuos uieals & 1 & & $\forall L-\forall-9 L 2$ \\
\hline & paxiw & गәाBM & 001 & & $\varepsilon L-\bar{V} \cdot 9 L Z$ \\
\hline & pax!w & өjesuapuos ureats & 001 & & $21-\forall-912$ \\
\hline & pəx|w & elв8uepuos meers & 001 & & $11-\forall-\theta 12$ \\
\hline & & & & & 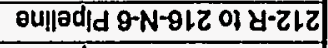 \\
\hline & & & & & oullod/d $-N-912$ ol $d-Z 1 Z$ \\
\hline & & $\cdot$ & & & 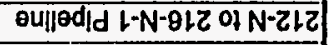 \\
\hline & ONIDEOIPEIUON & & & & aNOdW-LOLZ \\
\hline & өslloeolpey & Juenแร s9000dd & & & $\varepsilon-S M-\exists-602$ \\
\hline & өлl|projpey & 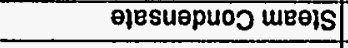 & & & $2-5 M-3 \cdot 602$ \\
\hline & en!lpeoppey & elesuepuos weeis & & & 1-SM-3-60Z \\
\hline & $\cdot$ & өөesuepuoj ureis & & & $2-20 z$ \\
\hline & en!lpeoppey & өןesuepuoj weels & & & $1-102$ \\
\hline & poxiW & fuenHy ssesold & & & $75-\angle 02$ \\
\hline & poxiw & fuentug ssasodd & & & $\mathrm{s}-\angle 02$ \\
\hline & & & & & g- L0Z \\
\hline & & & & & HLNOS- $\forall-\angle 0 Z$ \\
\hline & & & & & HLEON-V-LOZ \\
\hline & & & & & $6-M-002$ \\
\hline & Onlpeolpey & Iuouddinb & & & $L-M-002$ \\
\hline & pexin & yue $\perp$ abejols & & & $91-M-002$ \\
\hline & & & & & ddM-00Z \\
\hline & & & & & VISOM-002 \\
\hline & 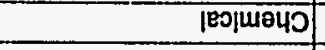 & 110 & & & $8 \cdot 3 \cdot 002$ \\
\hline & Enlpeolpey & ө)esuepuoj ureis & & & $6-9-002$ \\
\hline & |воршәчО & \|OS pejeu|uejuo & & & $92-3-002$ \\
\hline & & & & & $81-3-002$ \\
\hline & & JuenHy ssejosd & & & $+1-\exists-002$ \\
\hline & & & & & Od $3-002$ \\
\hline 80jON & $\theta d K_{\perp} 018 \mathrm{~B} M$ & 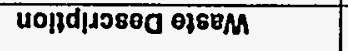 & $f_{f}(\mathrm{II})$ ounjon & 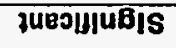 & opos ouls \\
\hline
\end{tabular}

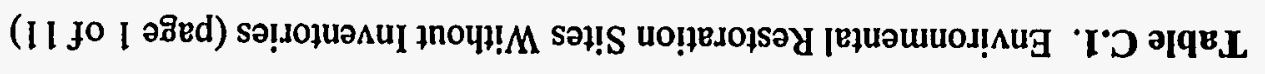




\begin{tabular}{|c|c|c|c|c|c|}
\hline & $p \theta \times|w|$ & Iuenuy ssesold & 0001 & & r-s.912 \\
\hline & pex!W & 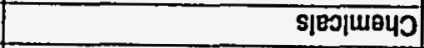 & & & $81-S \cdot 912$ \\
\hline & pexin & Luenug ssososd & & & $2 \angle 1-5-912$ \\
\hline & $\mathrm{p \partial x} \mid \mathrm{W}$ & IUenify s50501d & 00000 & & $0 \theta l-s-\theta l z$ \\
\hline & $-\ldots$ & juonug ssosodd & & & $61-s \cdot 912$ \\
\hline & 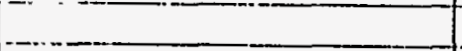 & & & & dol-s-9l2 \\
\hline & & & & & $8-N-912$ \\
\hline & & & 000966 & & L-N-912 \\
\hline & & & & & $82-3-912$ \\
\hline & & & & & xog uojsionia puod 6-5-9lz \\
\hline & & & & & $2-0.912$ \\
\hline & & & & 1 & $102-18-912$ \\
\hline & & & & $T$ & $+9-8-912$ \\
\hline & & ө)вsuepuos ureis & & & $19-8-912$ \\
\hline & & & 0009 & . & 8-8-912 \\
\hline & $\therefore$ & & & & $99-8-912$ \\
\hline & pex!W & juanLy] ssosodd & $L$ & & $19 \cdot 8 \cdot 912$ \\
\hline & & & 01 & & $6-9-912$ \\
\hline & & & & & $5 \varepsilon-8-912$ \\
\hline & & & & & $8 \varepsilon-8-912$ \\
\hline & & & & & $\forall \varepsilon-8-912$ \\
\hline & & & & & $\varepsilon-\varepsilon-\varepsilon-9 / z$ \\
\hline & & & 0000006 b & & $z-\varepsilon-\varepsilon-912$ \\
\hline & & & $0000006 t b$ & & $1-\varepsilon-8-9 l z$ \\
\hline & PEX!W & fuonlug ssesodd & 12 & & $\varepsilon+-8 \cdot 9 l 2$ \\
\hline & On!logolp8y & fuenแHa ssesodd & & & $6 Z S-\forall-912$ \\
\hline & & (1) & & & $z b-\gamma-91 z$ \\
\hline & & & 01 & & $1+\forall-912$ \\
\hline & enjpeolpey & ө̨esuepuoj weəIS & 966 & & Ob- $\forall-912$ \\
\hline & & & & & $1-8 \varepsilon-\forall-91 z$ \\
\hline & & & 101 & & SE- $\forall-912$ \\
\hline & & & & & $t \varepsilon \cdot \forall \cdot 91 z$ \\
\hline & & JOJEM 6Uf1005 & & & $\varepsilon \varepsilon-\forall-9 L Z$ \\
\hline & & & 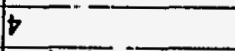 & & $2 \varepsilon-\forall-9 \mid z)$ \\
\hline SOION & $\theta d K_{\perp}$ oj8EM & 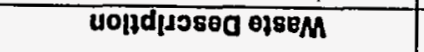 & 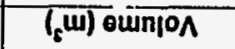 & fueगf|uBis & opoj olIs \\
\hline
\end{tabular}

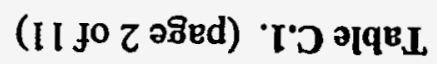




\begin{tabular}{|c|c|c|c|c|c|}
\hline & OAllFEO|pBY & juenLug ssejodd & & & $\forall z 0 \varepsilon-\forall-i b z$ \\
\hline & poxw & 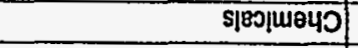 & & & 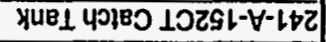 \\
\hline & әnpрeopey & juenLfHA Ssejodd & & & $191-\forall-1+2$ \\
\hline & paxiw & YuB 10 obejols & & & $20 \varepsilon-5 \cdot 0+2$ \\
\hline & paxiW & S|вग|waนว & & & $291-S-0 t z$ \\
\hline & poxiw & S|Eग|W & & & $19 t-5=0+2$ \\
\hline$\ldots . .$. & - & & & $\ldots$ & duns $เ S L-Z-I \varepsilon z$ \\
\hline & & & & & IGL-M-IEZ \\
\hline & pexiw & 8|в०јய840 & & & 8-bZz \\
\hline & & & & & $\forall Z-3-812$ \\
\hline & & elesuepuoj ureis & & & $12-2-912$ \\
\hline & әNlpeolpey & Iuenulu sseoold & 0001 & & $a b-2-912$ \\
\hline & өN|lJeO|pey & juen!Ha ssasodd & & & $6 l-z-912$ \\
\hline & pax|w & fuenน4 $350201 d$ & & . & Gl-Z-912 \\
\hline & әліproolpey & өjesuapuoo weels & & & $81-z-912$ \\
\hline & BAlpeopeduou/snodebuepuoN & olesuepuoj ubeis & & & $\varepsilon l-Z-9 l z$ \\
\hline & อмlpeoppey & juanug ssaoosd & & & IL-Z-912 \\
\hline & әn!peojpey & גө|вM & 0000021 & & DM7-M-912 \\
\hline & & & & & 6-n-912 \\
\hline & pax!W & IU日nlyg ssovold & & & $t-n-912$ \\
\hline & & & & & จl-n-9l2 \\
\hline & paxiw & fuen LHa sse20Jd & & & เ1-n-9!z \\
\hline & & & & & $102-\mu 1-912$ \\
\hline & OAlpeopejuou/snoJabuepuoN & Juanuy sseoold & & & $6-1-912$ \\
\hline & poxiw & өjesuepuoj weeis & $0000052 t$ & & $\forall b-\perp-9 l 2$ \\
\hline & OกIIOEOIPEY & өlesuopuoJ ureis & & & $2-t-1-912$ \\
\hline & pax|w & өlesuapuoj ureels & & & $a l-b-1-912$ \\
\hline & Oniloeoipey & өjesuepuoj ureeis & & & $|\varepsilon-1-9| 2$ \\
\hline & & & & & $0 \varepsilon-1-912$ \\
\hline & PEXIN & 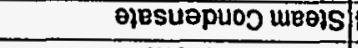 & $L$ & & $62-1-912$ \\
\hline & pexin & Twenug ssejodd & 0009 & & $2-1-912$ \\
\hline & pox!W & Juenufy ssosold & & & $\varepsilon l-1-9+2$ \\
\hline & On!lOEO!pedUOU/SnOJeGuepuON & fuenLy ssesodd & & & $11-1-912$ \\
\hline & On!peoppesuou/snosebuepuON & JuenแHa s9800dd & & & $01-1-912$ \\
\hline $8010 \mathrm{~N}$ & odKI $0188 M$ & Lopzdj20800 0180M & $f_{c}(w)$ ounjon & 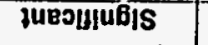 & opos oxा5 \\
\hline
\end{tabular}

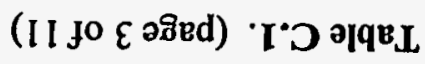




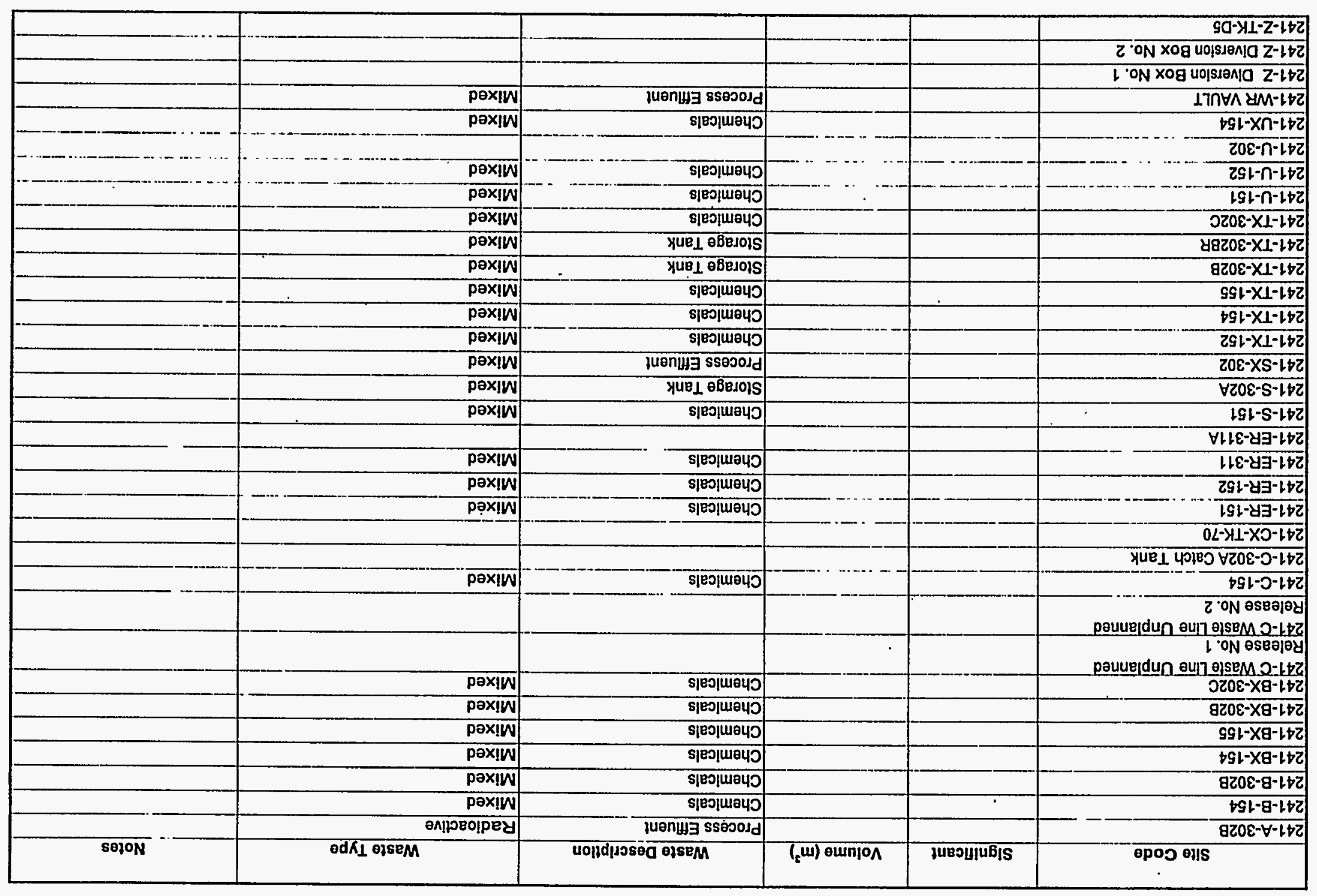

\section{(I รั}




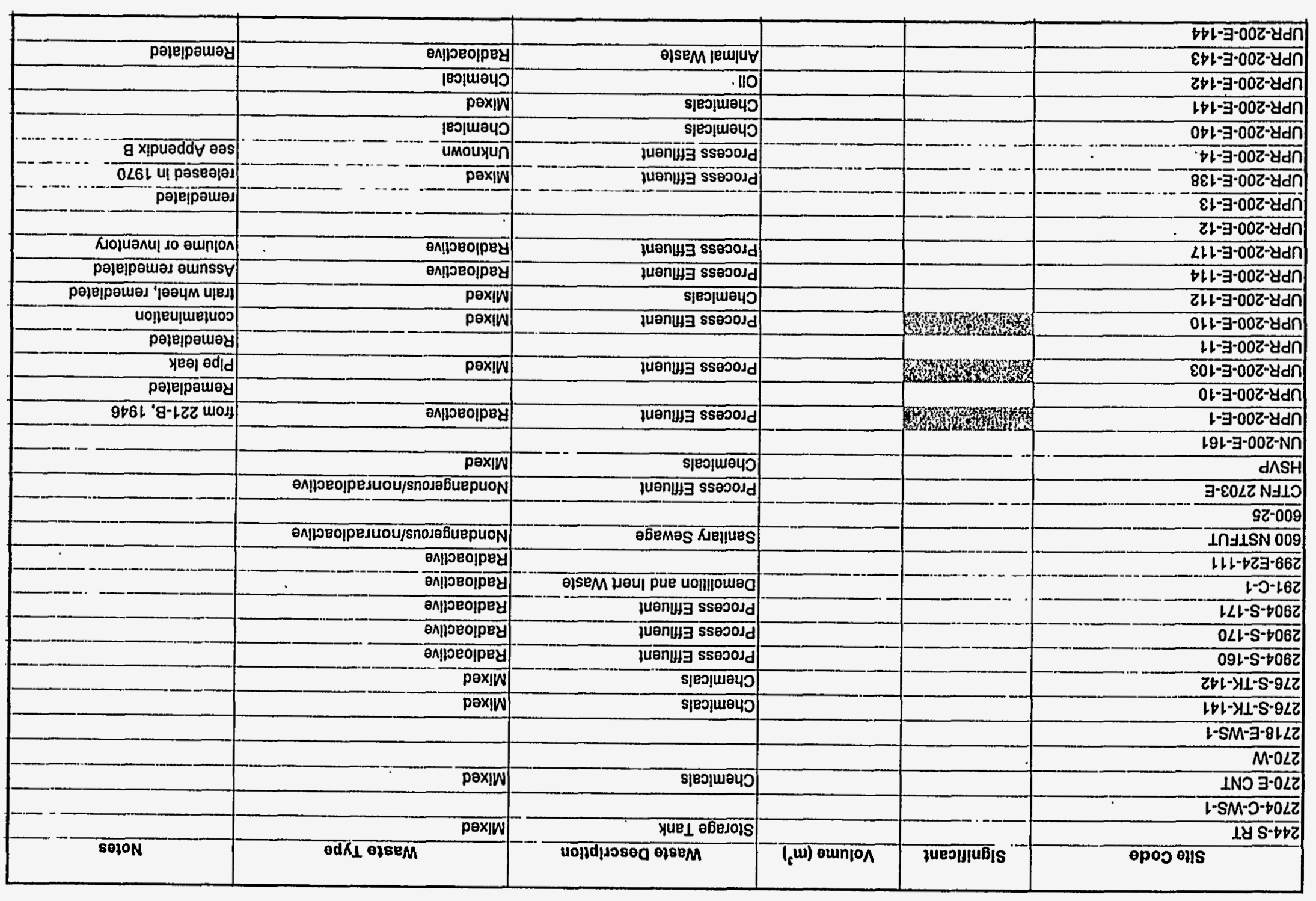

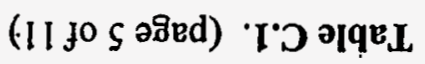


Table C.1. (page 6 of 11)

\begin{tabular}{|c|c|c|c|c|c|}
\hline Site Code & Significant & Volume $\left(\mathrm{m}^{3}\right)$ & Waste Description & Wasto Typo & Notes \\
\hline UPR-200-E-145 & & & Soil & Radioaclive & \\
\hline UPR-200-E-15 & & & Process Effluent & Radioaclive & crib \\
\hline UPR-200-E-18 & & & Process Effluent & Mixed & \\
\hline UPR-200-E-19 & & & Process. Effluent & Radioactive & Minor ground contamination \\
\hline UPR-200-E-2 & & & Process Effiuent & Mlxed & particle releases \\
\hline UPR-200-E-20 & & & Process Effluent & Radioactive & Minor spot contamination \\
\hline UPR-200-E-21 & & & & & assume minor \\
\hline UPR-200-E-22 & & & Chemlcal Release & & \\
\hline \multicolumn{6}{|l|}{ UPR-200-E-24 } \\
\hline UPR-200-E-25 & & & & & from steam release \\
\hline$\overline{\text { UPR-200-E-26 }}$ & & & & & Remedlated \\
\hline UPR-200-E-28 & & & & & steam \\
\hline UPR-200-E-29 & & & Process Effluent & Radioactive & \\
\hline UPR-200-E-3 & 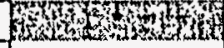 & & Process Effluent & Radioactive & 154 tank, no volume \\
\hline UPR-200-E-30 & & & Barrels/Drums/Buckets/Cans & Radioactive & \\
\hline UPR-200-E-32 & & & Process Effluent & Radloactlve & see Appendix B \\
\hline UPR-200-E-33 & & & Barrels/Drums/Buckets/Cans & Radioactive & Minor spot contamination \\
\hline UPR-200-E-34 & & & Process Effluent & Radioactive & see Appendix B \\
\hline UPR-200-E-35 & & & & & Appendix B \\
\hline UPR-200-E-36 & & & Process Effluent & Radioactive & \\
\hline UPR-200-E-37 & & & Process Effluent & Radioactive & \\
\hline UPR-200-E-39 & . & & Process Effluent & Mixed & Minor vapor release \\
\hline UPR-200-E-40 & & & Process Effluent & Radioactive & Minor spot contamination \\
\hline UPR-200-E-41 & & & Process Effluent & Radloactive & Remediated \\
\hline UPR-200-E-42 & & & Process Effluent & Radioactive & \\
\hline UPR-200-E-44 & & & Process Effluent & Radioactive & ine leak \\
\hline UPR-200-E-45 & & & Process Effluent & Radloactive & Remedlated \\
\hline UPR-200-E-49 & & & Process Effluent & Radioactive & \\
\hline UPR-200-E-50 & & & Equipment & Radloactive & \\
\hline UPR-200-E-51 & & & & & 216-B-3 Pond, see Appendix \\
\hline UPR-200-E-52 & & & & & probably $<100 \mathrm{~L}$ \\
\hline
\end{tabular}


Table C.1. (page 7 of 11)

\begin{tabular}{|c|c|c|c|c|c|}
\hline Site Code & Signifflcant & Volume $\left(\mathrm{m}^{3}\right)$ & Waste Description & Wasto Type & Notes \\
\hline UPRR-200-E-54 & & & & & Remediated \\
\hline UPR-200-E-55 & & & & & Remediated \\
\hline \multicolumn{6}{|l|}{ UPR-200-E-56 } \\
\hline \multicolumn{6}{|l|}{ UPR-200-E-58 } \\
\hline \multicolumn{6}{|l|}{ UPR-200-E-59 } \\
\hline \multicolumn{6}{|l|}{ UPR-200-E-60 } \\
\hline UPR-200-E-61 & & & Process Effluent & Radioactive & Remediated \\
\hline UPR-200-E-62 & & & Process Effluent & Radioactive & \\
\hline UPR-200-E-63. & & & Vegetation & Radioactive & removed \\
\hline UPR-200-E-64 & & & & & Mlnor specks \\
\hline UPR-200-E-65 & & & Process Effluent & Radioaclive & \\
\hline UPR-200-E-66 & & & Process Effluent & Radioactive & \\
\hline UPR-200-E-67 & & & Misc. Trash and Debris & Radioactive & \\
\hline UPR-200-E-69 & & & Barrels/Drums/Buckets/Cans & Radioactive & from equipment on flat car \\
\hline UPR-200-E-7 & & & Process Effluent & Radioactive & waste, no volume information \\
\hline UPR-200-E-77 & \multirow{8}{*}{$\begin{array}{l}-x^{2} \\
-3 \\
-3\end{array}$} & & Process Effluent & Radioactive & $1 \mathrm{Cl}$ process effluent leak \\
\hline UPR-200-E-78 & & & Process Effluent & Radioactive & volume information \\
\hline UPR-200-E-80 & & & Process Effluent & Mixed & volume information \\
\hline UPR-200-E-83 & & & Animal Waste & Radloactlve & colaminated rabbit and coyote \\
\hline UPR-200-E-84 & & & Process Effluent & Mixed & leak \\
\hline UPR-200-E-85. & & & Process Effuent & Mixed & effluent \\
\hline UPR-200-E-87 & & & Process Effuent & Mixed & contaminated 4-m2 area \\
\hline UPR-200-E-88 & & & Process Effluent & Radioactive & \\
\hline UPR-200-E-89 & & & Process Effiuent & Mixed & from BY tank farm, minor \\
\hline UPR-200-E-9 & & $\cdot$ & Process Effluent & Mixed & Remedlated \\
\hline UPR-200-E-90 & & & Process Effluent & Radioactive & No release \\
\hline UPR-200-E-92 & 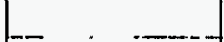 & & Chemicals & Mlxed & tumbleweeds \\
\hline UPR-200-E-93 & & & Chemicals & Mixed & from tumbleweeds. \\
\hline UPR-200-E-95 & & & Chemicals ... & Mixed & contamination - \\
\hline UPR-200-E-96 & & & Process Efluent & Radioactive & \\
\hline UPR-200-E-97 & & & Soll & Unknown & \\
\hline UPR-200-E-98 & & & Chemicals & Mixed & \\
\hline UPR-200-N-1 & & & & & contamination \\
\hline UPR-200-N-2 & & & & & remedialed \\
\hline
\end{tabular}




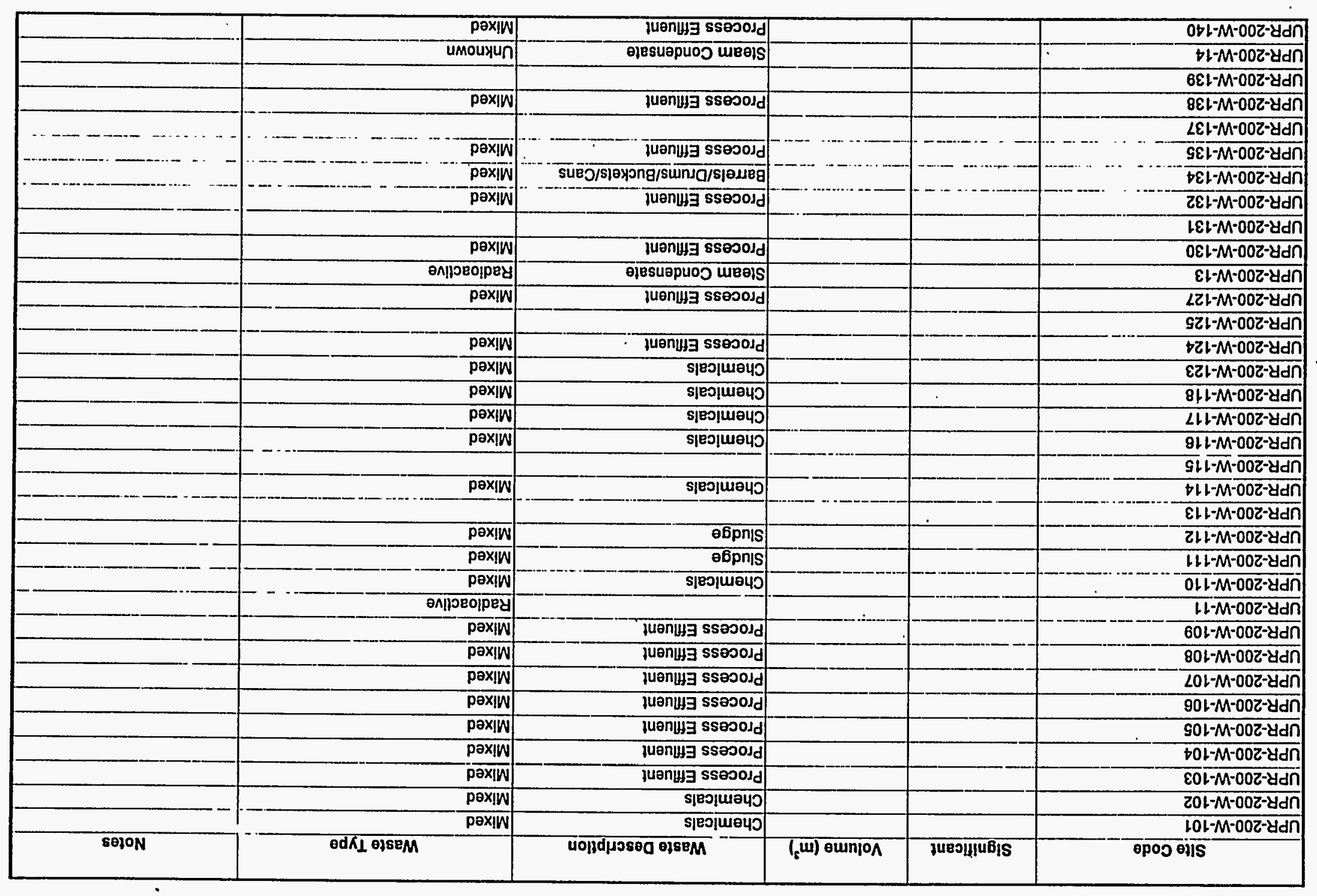

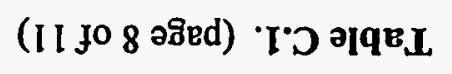




\begin{tabular}{|c|c|c|c|c|c|}
\hline . & & & & & $\nabla-M-00 z-4 d \cap$ \\
\hline & umouyun & & & & $6 \varepsilon-M-00 z-y d n$ \\
\hline & өл|prop|pey & fuanuy ssejodd & & & $8 \varepsilon-M-00 z-y d n$ \\
\hline & enjprojpey & 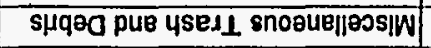 & & & $\angle \varepsilon-M-00 z-y d n$ \\
\hline 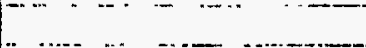 & 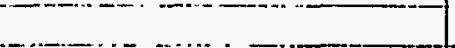 & 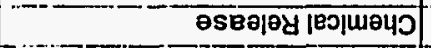 & & & $9 \varepsilon-M-00 z-y d n$ \\
\hline ... & umouyun & juanw3 ssesosd & & & $6 \varepsilon-M-00 z-\bar{d} d \Pi$ \\
\hline$\cdots-\cdots+\cdots-$ & $-5+\cdots-\cdots+$ & & & & $\varepsilon \varepsilon-M-00 z-2 d \cap$ \\
\hline & 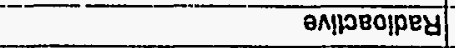 & 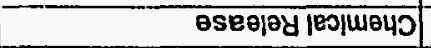 & & & $z \varepsilon-M-00 z-z d n$ \\
\hline & umouxun & IuenแH] ssejodd & & & $0 \varepsilon-M-00 z-y d n$ \\
\hline & enlpeolpey & & & & $\varepsilon-M-00 z-y d n$ \\
\hline & ONIIDEOJPEY & fuen|fy sseoold & & & $6 z-M-00 z-y d n$ \\
\hline & & & & & $8 z-M-00 z-y d n$ \\
\hline & & & & & $\angle Z-M-00 Z-8 d n$ \\
\hline & & & & & $9 z-M-00 z-8 d n$ \\
\hline & & & & & $\varepsilon z-M-00 z-y d \cap$ \\
\hline & & & & & $L Z-M-00 z-y d n$ \\
\hline & & & & & $0 z-M-00 z-z d n$ \\
\hline & $\therefore$ & & & & $61-M-00 z-y d n$ \\
\hline & & & & & $81-M-00 z-y d n$ \\
\hline & pox!w & 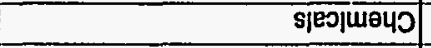 & & & $\angle \theta L-M-00 Z-Z d \Pi$ \\
\hline & өлlpeo!pey & 1105 & & & $99 l-M-00 z-z d d n$ \\
\hline & pexiW & s|eว|щ日) & & & S9l-M-00z-ydn \\
\hline & EAlfoBojpey & juenufy ssejodd & & & 601-M-00z-ydn \\
\hline & pex!W & puenug ssejodd & & & $\varepsilon \theta L-M-00 z-8 d n$ \\
\hline & pox!W & s;ejumeñ & & & $z 9 l-M-00 z-y d n$ \\
\hline & pex!W & 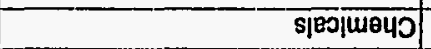 & & & $1 \theta L-M-00 z-y d n$ \\
\hline & pex!w & juenuy ssesosd & & & $091-M-00 z-4 d n$ \\
\hline & & & & & Ol-M-00z-zdn \\
\hline & pex!w & s|во|யа4ว & & & 6SL-M-00z-Zdd \\
\hline & & & & & $8 s L-M-00 z-8 d n$ \\
\hline & 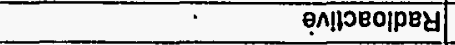 & өjesuөpuoj meajs & & & SL-M-00z-Zdn \\
\hline & pexin & Juenug ssejodd & & & $16 l-M-00 z-y d n$ \\
\hline $8010 \mathrm{~N}^{-}$ & od $K_{\perp}$ ojseM & uolfdjJosed ojseM & $T_{\text {f }}(11)$ ownjon & 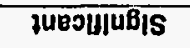 & ор00 өया \\
\hline
\end{tabular}

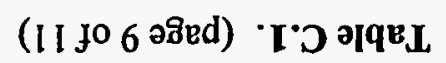


Table C.1. (page 10 of 11)

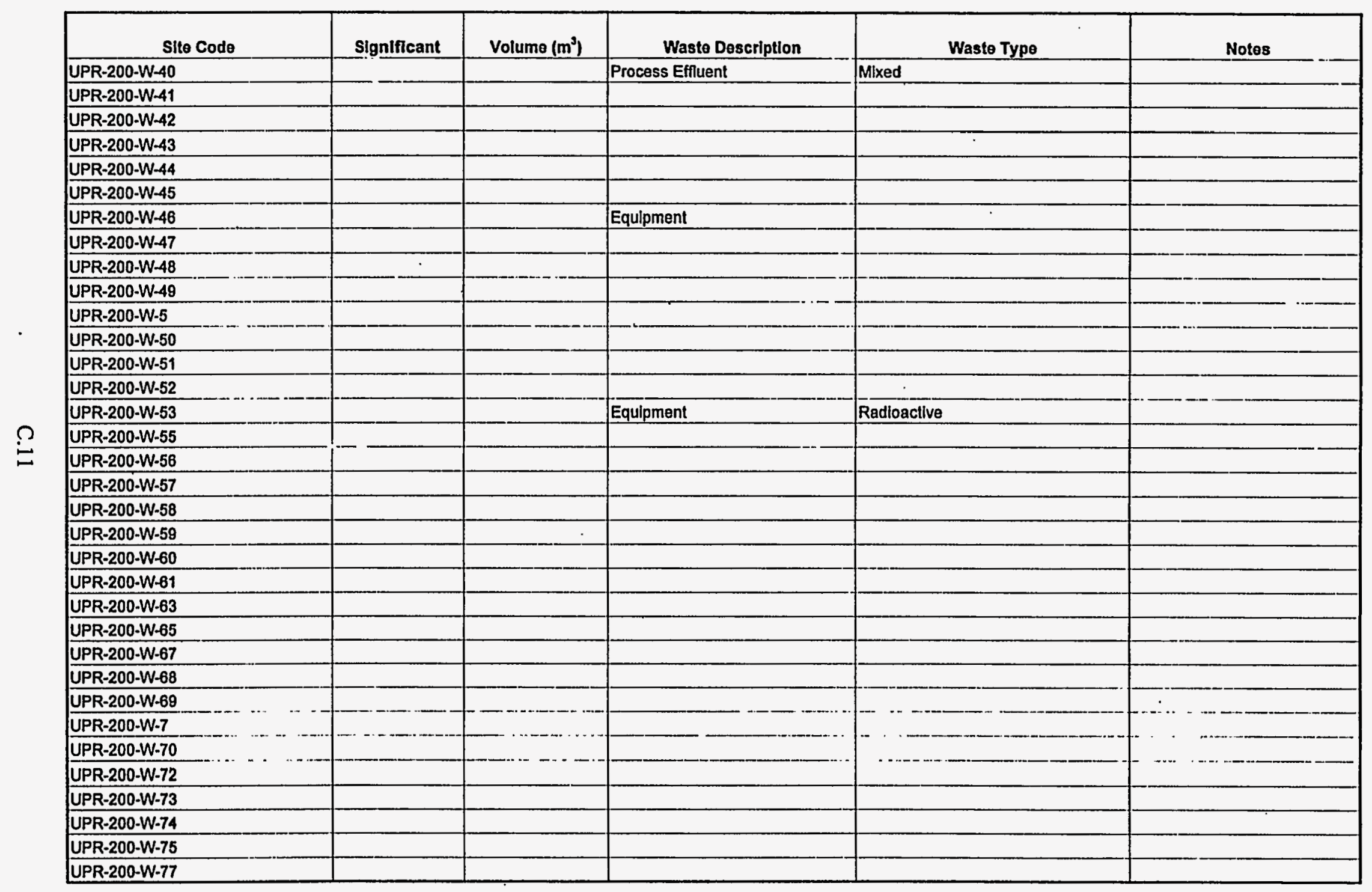


Table C.1. (page 11 of 11)

\begin{tabular}{|c|c|c|c|c|c|}
\hline Site Code & Significant & Volume $\left(m^{3}\right)$ & Waste Description & Waste Type & Notes \\
\hline \multicolumn{6}{|l|}{ UPR-200-W-78 } \\
\hline \multicolumn{6}{|l|}{ UPR-200-W-79 } \\
\hline UPR-200-W-8 & & & Miscellaneous Trash and Debris & Radioactlve & \\
\hline UPR-200-W-82 & & & Chemicals & Mixed & \\
\hline UPR-200-W-83 & & & Chemicals & Mixed & \\
\hline UPR-200-W-84 & & & Chemicals & Mixed & \\
\hline UPR-200-W-85 & & & Process Effluent & Mixed & \\
\hline \multicolumn{6}{|l|}{ UPR-200-W-86 } \\
\hline UPR-200-W-87 & & & Chemicals & Mixed & \\
\hline UPR-200-W-88 & & & Chemicals & Mixéd & \\
\hline UPR-200-W-89 & & & Chemicals & Mixed & \\
\hline UPR-200-W-90 & & & Chemicals & Mlxed & \\
\hline UPR-200-W-91 & & & Chemicals & Mixed & \\
\hline UPR-200-W-95 & & & Process Effluent & Mlxed & \\
\hline UPR-200-W-96 & & & Process Effluent & Mixed & \\
\hline UPR-200-W-98 & & & Process Efluent & Mlxed . . & \\
\hline UPR-200-W-99 & & & Chemicals & Radloactlve & \\
\hline \multicolumn{6}{|l|}{ UPR-216-W-25 } \\
\hline UPR-600-12 & & & Chemicals & Mixed & Remediated \\
\hline UPR-600-16 & & & & & Remedlated \\
\hline UPR-600-18 & & & & & Will be removed \\
\hline UPR-600-19 & & & & 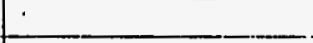 & Nonradioactlve \\
\hline UPR-600-20 & & & Process Effluent & Mixed & Minor specks \\
\hline UPR-600-21 & & & & & from tumbleweeds \\
\hline WBL & & & Miscellaneous Trash and Debris & Chemical & \\
\hline
\end{tabular}




\section{Appendix D}

Hanford Composite Analysis Source Term Release Models 


\title{
Appendix D
}

\section{Hanford Composite Analysis Source-Term Release Models}

\author{
G. P. Streile
}

\section{D.1 Types of Contaminant Sources, and Source Zone Attributes}

There are many different types of contaminant sources at Hanford that release, or could release, contamination to the vadose zone. Consequently, many different types of quantitative release models could be required to perform a detailed release analysis for every type of source zone. However, for the scope of this effort, only five idealized generic types of contaminant source zones (i.e., generic waste form types) were considered for the conceptual model of release: soil-debris, cake waste, glass waste, cement waste, and reactor block waste. Each source zone at the Hanford Site was characterized in terms of its generic waste form type, contaminant inventories, volume, and horizontal crosssectional area. Only radionuclide contaminants are considered in the present analyses.

\section{D.1.1 Soil-Debris Waste Form Type}

The first generic waste form type consists of unconsolidated wastes mixed with soil material, and is referred to as the "soil-debris" type of waste form. Source zones composed of this waste form type are permeable to percolating water; and thus all surfaces of the waste come into contact with the percolating water as it passes through the zone in a manner similar to how infiltrating water passes through natural vadose zone material. If contaminant inventories in the source zone are high enough, leaching of contaminant out of the bottom of the source zone is controlled by the solubility of the contaminant in the percolating water. Otherwise, the leaching is controlled by partitioning of the contaminant between aqueous and sorbed phases. Unconsolidated wastes in this waste form type could be further subdivided into those having either high or low surface-area-to-volume (S/V) ratios. Contaminants from 'wastes in the low S/V category (e.g., waste containers, personal protection equipment, and metal process equipment) readily leach into the surrounding soil; and therefore their release from the source zone is controlled by the properties of the surrounding soil in the source zone. Contaminants from wastes in the high S/V category (e.g., sludge, soil, and spent filters/ adsorbents) can have high surface adsorption coefficients. Therefore, their release from the source zone is controlled by the properties of the waste material itself. However, because the availability of physical and chemical data regarding these wastes is limited, and because of the scope of this effort, it has been assumed that the properties of the surrounding soil can also be used to calculate release in this case. (This assumption is understood to be conservative because the sorptive properties of the surrounding soil would be lower than that of the waste material.)

\section{D.1.2 Cake Waste Form Type}

The second generic waste form type consists of consolidated waste that is permeable to water, and that dissolves over time because some major structural component of the solid waste dissolves in the water percolating through the waste form. Tank waste consisting of salt cake and sludge is a waste form of this type. This is referred to as the "cake" type of waste form. As the solid waste form dissolves (at a constant rate controlled by the aqueous solubility of the major structural component) 
all of the contaminants associated with the portion of the waste form that dissolved are released into the percolating water congruently at constant rates related to their concentration in the waste form.

\section{D.1.3 Glass Waste Form Type}

The third generic waste form type consists of solidified wastes whose permeability is much lower than that of the surrounding soil, and is also so low that contaminant mobility within the waste form is essentially zero. This is referred to as the "glass" type of waste form. It is assumed that this waste form is composed of pieces of "glass" that are roughly cubical in shape, and that only the "glass" surfaces are exposed to water percolating through the source zone. Furthermore, the waste form is assumed to be slowly dissolving (from the exterior surfaces of the "glass") with time; i.e., over time the pieces of "glass" are slowly shrinking in size. The overall rate of dissolution of the waste form changes over time because the surface area of the waste form (exposed to the percolating water) changes as the pieces of "glass" shrink. All of the contaminants associated with the portion of the waste form that dissolved are released into the percolating water congruently at rates related to their concentration in the waste form and the overall waste form dissolution rate at the given time.

\section{D.1.4 Cement Waste Form Type}

The fourth generic waste form type consists of solidified wastes whose permeability is much lower than that of the surrounding soil (i.e., low enough that advective water flow within the waste form is essentially zero), but is sufficiently high to allow some contaminant mobility within the waste form. This is referred to as the "cement" type of waste form. Percolating water tends to move around this type of waste form, and contaminants are only leached from the waste form's outer surface. As this occurs, contaminants inside the waste form are assumed to diffuse toward the outer surface. Therefore, overall contaminant release from the source zone is assumed to be controlled by the contaminant's effective diffusion coefficient in the waste form.

\section{D.1.5 Reactor Block Waste Form Type}

The fifth generic waste form type consists of irradiated solids that release contaminants into the water percolating past them via unspecified loss processes from the solid matrix as well as via corrosion of the solid components themselves over time. This is referred to as the "reactor block" type of waste form. Because of the lack of information regarding the conceptual and mathematical description of the actual processes occurring, release of contaminants is assumed to be described by rates calculated from experimental leach test data.

\section{D.1.6 Assumptions Made About Waste Form Types}

Contaminants released from cake, glass, cement, and reactor block waste form types may initially enter some kind of soil material surrounding them, if these waste forms are present in a larger, overall source zone that also contains soil. It is possible that the ultimate release from the overall source zone could be limited by the release from this surrounding soil. However, the analyses now assume that the release from the waste form itself is the limiting step in the total release process. This could be modified in the future to compare the release rate from the specific waste form type to that from the surrounding soil, and then use the lower of these two values.

Analyses now assume that cement waste forms stay intact for all time during the simulation. This could be modified to allow the waste form to catastrophically fail at some specified time, after which the source zone acts like a soil-debris waste form type. 
In addition to the primary waste forms and surrounding soil, the source zone may initially contain other material, such as facilities/buildings, waste containers, waste-zone structural components (e.g., asphalt pads, and plywood sheets separating layers of waste containers). In these analyses, no credit is taken for the ability of these other materials to inhibit contaminant release (i.e., the analyses now assume that these components degrade rapidly and offer no protection for the five generic waste form types for essentially the entire simulation time).

Each source zone on the Hanford Site considered in this Composite Analysis was categorized into one of the above five generic waste form types. The inventories of all relevant radionuclide contaminants for that source zone were compiled. If a source zone contained more than one of the waste form types, the contaminant inventories were appropriately apportioned among the different waste form types; separate release calculations were performed for each waste form type; and the resulting losses into the vadose zone for any specific contaminant were summed.

For the soil-debris type of waste form, the overall volume of the source zone was used to obtain contaminant concentrations (needed for the mathematical model of release) from inventories. For cake, glass, and cement waste forms, it is possible that some of the source zone also contains soil material (which is not considered in the mathematical model of contaminant release). So, for these waste form types, the actual volume of the cake, glass, or cement waste form in the source zone was used to obtain concentrations from inventories. The release model associated with the reactor block type of waste form does not contain these volume and concentration considerations.

For the soil-debris type of waste form, the horizontal cross-sectional area of the overall source zone (the perpendicular area seen by water percolating through the source zone) was used to calculate the water and contaminant fluxes for leaching losses. (This area is also needed by the vadose zone transport component of the Composite Analysis.) For the other four generic waste form types, the effective horizontal cross-sectional area used to calculate the water and contaminant fluxes for leaching losses may be less than that of the overall source zone if it were determined that part of the water percolating through the overall source zone did not really come into contact with the waste form.

\section{D.2 Contaminant Release Models}

In all cases, the Composite Analysis assumes that the (radionuclide) contaminants are lost from the source zone only via radioactive decay within the source zone and leaching from the bottom of the source zone along with water percolating through or around the waste form types. Additional potential contaminant loss processes (e.g., volatilization, wind suspension of contaminated particles, and water erosion of contaminated particles) are not considered in the primary analyses.

The release model appropriate to a specific source zone depends on the overall waste form type and the potential for geochemical controls to limit the release. The soil-debris, cake, glass, and reactor block source zones are assumed to be so-called "well-mixed reactors"; i.e., the properties (contaminant concentrations) are assumed to be spatially uniform throughout. The cement source zone is assumed to contain concentration gradients within the waste form.

The mathematical approach to the entire release and transport problem is as follows. It is assumed that the impact of progeny products is negligible, and that the ingrowth and transport of progeny products need not be accounted for. Because of this assumption, each contaminant can be analyzed individually. Furthermore, with this conceptualization, the mathematical problem of leaching release coupled with radioactive decay in the source zone (as well as transport coupled with decay in the vadose zone and aquifer) can be reduced to an associated mathematical problem that 
considers only leaching release and vadose zone/aquifer transport of a nondecaying species. After this associated scenario is analyzed to produce concentration breakthrough curves at receptor points for the nondecaying species, the actual concentration breakthrough curves for the original problem scenario can be obtained by decaying the contaminant concentrations at the receptor point based on contaminant arrival time and decay half-life. This simplifies the source zone release and transport models, and reduces the number of transport simulations that must be done.

The primary required output from the source-term release component of the Hanford Composite Analysis is the fraction of initial inventory remaining in the source zone for each contaminant as a function of time. This function is used as an input boundary condition to the vadose zone transport component of the Composite Analysis. The different source zone release models described below calculate the fraction remaining for the nondecaying species of the associated mathematical problem. Table D.1 defines the source-term release model notation used in the following sections.

\section{D.2.1 Variable Transform Method of Formulating the Mathematical Problem}

Mathematical expressions for contaminant release from the source zone are based on the total activity of the radionuclide in the source zone:

$$
M_{i}^{*}(t)=\int_{V} C_{T i}^{*}(t) d V
$$

where $\quad M_{i}^{*}=$ the total activity of contaminant $i$ in the source zone for the original mathematical problem (that includes decay) (Ci)

$t=$ the time since initial condition of the source zone $(y r)$

$\mathrm{C}_{\mathrm{Ti}}=$ the total concentration of contaminant $i$ (in all forms) in the source zone for the original mathematical problem (that includes decay) $\left(\mathrm{Ci} \mathrm{cm}^{-3}\right)$

$V=$ the volume of the source zone $\left(\mathrm{cm}^{3}\right)$.

(In Equation D.1 and in all of the following equations, the symbol "•" in the superscript of a variable related to radionuclide quantity means that the variable is associated with the original mathematical problem [i.e., the real-world situation where decay as well as leaching is occurring].) At this point in the mathematical development, it is not necessary to assume that the total concentration of the contaminant is spatially uniform throughout the source zone.

With leaching and decay being the only loss processes assumed, the rate of change of contaminant activity in the source zone can be expressed as

$$
\frac{\mathrm{dM}_{\mathrm{i}}^{*}}{\mathrm{dt}}=\left[\frac{\mathrm{d \textrm {M } _ { \mathrm { i } } ^ { * }}}{\mathrm{dt}}\right]_{\text {leach }}+\left[\frac{\mathrm{dM}_{\mathrm{i}}^{*}}{\mathrm{dt}}\right]_{\text {decay }}=\left[\frac{\mathrm{dM}_{\mathrm{i}}^{*}}{\mathrm{dt}}\right]_{\text {leach }}-\lambda_{\mathrm{i}} \mathrm{M}_{\mathrm{i}}^{*}
$$

where $\quad \lambda_{i}=$ the first-order decay coefficient for contaminant $i\left(\mathrm{yr}^{-1}\right)$. 
Equation D.2 implicitly assumes that the first-order decay coefficient of the contaminant is independent of the phase (aqueous, sorbed, or precipitated) in which the contaminant resides (this is strictly true for radionuclides).

The flux density of a contaminant entering the vadose zone below the source zone (because of leaching from the source zone) can be expressed as

$$
\mathrm{q}_{\mathrm{ci}}^{\cdot}(\mathrm{t})=-\frac{1}{\mathrm{~A}}\left[\frac{\mathrm{d} \mathrm{M}_{\mathrm{i}}^{\bullet}}{\mathrm{dt}}\right]_{\text {leach }}
$$

where $\quad q_{c i}^{*}=$ the flux density of contaminant $i$ entering the vadose zone below the source zone for the original mathematical problem (that includes decay) $\left(\mathrm{Ci} \mathrm{cm}^{-2} \mathrm{yr}^{-1}\right)$

$A=$ the effective horizontal cross-sectional area of the contaminant source zone $\left(\mathrm{cm}^{2}\right)$.

Note that Equation D.3 can be interpreted as a definition of an average leaching flux density over the effective horizontal cross-sectional area, A, of the bottom of the source zone. The source-term calculations need a single value of flux density at this vertical location (at any particular time) because a one-dimensional, vertical transport scenario is assumed for the vadose zone below the source zone. Therefore, at this point in the mathematical development, it is still not necessary to assume that the total concentration of the contaminant in the source zone is spatially uniform. However, if the flux density variable is assumed to be an actual, horizontally uniform value (rather than a horizontally spatially averaged value), then the source zone should now be assumed to be horizontally spatially uniform.

This flux density, which is a function of time, is a necessary input to (i.e., a boundary condition for) the mathematical transport problem in the vadose zone that must be solved subsequently. However, in order to avoid complications that arise from using discrete time intervals in the solution to the coupled source-zone/vadose-zone problem, the two zones are linked via a function that represents the fraction of contaminant remaining in the source zone over time (rather than the flux density of contaminant out of the source zone over time):

$$
f_{r i}^{*}(t)=\frac{M_{i}^{*}(t)}{M_{o i}^{*}}
$$

where $\quad f_{\mathrm{ri}}=$ the fraction of the initial inventory of contaminant $i$ remaining in the source zone for the original mathematical problem that includes decay (unitless)

$\mathrm{M}_{\mathrm{oi}}^{*}=$ the initial total activity of contaminant $i$ in the source zone for the original mathematical problem that includes decay $(\mathrm{Ci})$.

If Equation D.2 is rearranged and substituted into Equation D.3, and then Equation D.4 is rearranged and substituted into that equation, it can be shown that the relationship between the flux density and the fraction remaining is

$$
\mathrm{q}_{\mathrm{ci}}^{\cdot}=-\frac{\mathrm{M}_{\mathrm{oi}}}{\mathrm{A}}\left(\frac{\mathrm{df_{ \textrm {ri } }}}{\mathrm{dt}}+\lambda_{\mathrm{i}} \mathrm{f}_{\mathrm{ri}}^{\cdot}\right)
$$


Now, let us define the following variable transformation:

$$
M_{i}^{0}(t) \equiv M_{i}(t) e^{-\lambda_{i} t}
$$

where $\quad \mathrm{M}_{\mathrm{i}}=$ the total activity of contaminant $i$ in the source zone for the transformed mathematical problem (Ci).

If Equation D.6 is differentiated with respect to time, and if that expression is then substituted into Equation D.2, and if the resulting expression is then simplified, we obtain the expression

$$
\frac{\mathrm{dM}_{\mathrm{i}}}{\mathrm{dt}}=\left[\frac{\mathrm{dM}}{\mathrm{dt}}\right]_{\text {leach }}
$$

In deriving Equation D.7, the leaching loss term (on the right hand side of the equation) was explicitly defined to be

$$
\left[\frac{\mathrm{dM}_{\mathrm{i}}}{\mathrm{dt}}\right]_{\text {leach }} \equiv \mathrm{e}^{\lambda_{\mathrm{i}} \mathrm{t}}\left[\frac{\mathrm{d \textrm {M } _ { \mathrm { i } } ^ { * }}}{\mathrm{dt}}\right]_{\text {leach }}
$$

Furthermore, if Equation D.8 is rearranged and substituted into Equation D.3, and if the resulting expression is simplified, we obtain the expression

$$
\mathrm{q}_{\mathrm{ci}}(\mathrm{t})=-\frac{1}{\mathrm{~A}}\left[\frac{\mathrm{dM}_{\mathrm{i}}}{\mathrm{dt}}\right]_{\text {leach }}
$$

where $\mathrm{q}_{\mathrm{ci}}=$ the flux density of contaminant $i$ entering the vadose zone below the source zone for the transformed mathematical problem $\left(\mathrm{Ci} \mathrm{cm}^{-2} \mathrm{yr}^{-1}\right)$.

In deriving Equation D.9, we made use of the definition:

$$
q_{c i}^{\cdot}(t) \equiv q_{c i}(t) e^{-\lambda_{i} t}
$$

Furthermore, if Equation D.6 is substituted into Equation D.4, and if we make use of the fact that the initial total activities of the radionuclide must be the same for the two mathematical problems, i.e.,

$$
\mathrm{M}_{\mathrm{oi}} \equiv \mathrm{M}_{\mathrm{oi}}
$$

where $\mathrm{M}_{\mathrm{oi}}=$ the initial total activity of contaminant $i$ in the source zone for the transformed mathematical problem that includes decay $(\mathrm{Ci})$

we obtain the expression

$$
f_{r i}(t)=\frac{M_{i}(t)}{M_{o i}}
$$


where $\mathrm{f}_{\mathrm{ri}}=$ the fraction of the initial mass of contaminant $i$ remaining in the source zone for the transformed mathematical problem (unitless).

In deriving Equation D.12, we made use of the definition:

$$
f_{r i}^{0}(t) \equiv f_{r i}(t) e^{-\lambda_{i} t}
$$

Furthermore, if Equations D.10, D.11, and D.13 are substituted into Equation D.5, and if the resulting equation is then simplified, we obtain the expression

$$
q_{c i}=-\frac{M_{o i}}{A} \frac{d f_{r i}}{d t}
$$

The original mathematical problem (describing the actual real-world situation of loss by leaching and decay) given by Equations D.2, D.3, D.4, and D.5 has been transformed into an analogous problem given by Equations D.7, D.9, D.12, and D.14 (by using the variable transform definitions in Equations D.6, D.8, D.10, and D.13). Note further that Equations D.7, D.9, D.12, and D.14 have the proper mathematical form to describe a situation where the contaminants do not decay, and loss occurs only by leaching. To illustrate this, just let $\lambda_{\mathrm{i}}$ go to zero in Equations D.2, D.3, D.4, and D.5, and they reduce to the mathematical forms of Equations D.7, D.9, D.12 and D.14. In other words, the variable transform mathematics show that we really only need to solve the source zone release problem assuming that the contaminants do not decay. As long as we develop the leaching loss expression as the product of the actual real-world expression and the exponential factor (according to Equation D.8), we can use the variable transform definitions in Equations D.6, D.10, and D.13 to obtain the actual values of source zone activity, flux density, and fraction remaining if we so desire.

Next, a similar procedure can be applied to the mathematical problems of reactive transport in the vadose zone and aquifer. Beginning with the vadose zone, the differential equation of transport can be written as

$$
\frac{\partial}{\partial t}\left(\theta_{w} C_{w i}^{\cdot}+\beta C_{s i}^{\cdot}\right)=-\frac{\partial}{\partial z}\left(-\theta_{w} D_{s i} \frac{\partial C_{w i}^{\cdot}}{\partial z}+q_{w} C_{w i}^{\cdot}\right)-\lambda_{i}\left(\theta_{w} C_{w i}^{\cdot}+\beta C_{s i}^{*}\right)
$$

where $\theta_{w}=$ the volumetric water content of the source zone soil or vadose zone soil (unitless; $\mathrm{cm}^{3} \mathrm{~cm}^{-3}$ )

$\mathrm{C}_{\mathrm{wi}}^{\cdot}=$ the concentration of contaminant $i$ in the aqueous phase for the original mathematical problem (that includes decay) $(\mathrm{Ci} \mathrm{cm}-3)$

$B=$ the bulk density of the source zone soil or vadose zone soil $\left(\mathrm{g} \mathrm{cm}^{-3}\right)$

$\mathrm{C}_{\mathrm{si}}=$ the concentration of contaminant $i$ in the sorbed phase for the original mathematical problem (that includes decay) $\left(\mathrm{Ci} \mathrm{cm}^{-3}\right)$

$\mathrm{z}=$ the vertical spatial coordinate $(\mathrm{cm})$

$\mathrm{D}_{\mathrm{si}}=$ the effective diffusion coefficient of contaminant $i$ in the soil $\left(\mathrm{cm}^{2} \mathrm{yr}^{-1}\right)$

$\mathrm{q}_{\mathrm{w}}=$ the Darcy flux density of water flowing through the source zone or vadose zone $\left(\mathrm{cm} \mathrm{yr}^{-1}\right)$.

It is assumed that the contaminant sorption process is linear, reversible, and at equilibrium, i.e., that it can be described by a single sorption coefficient, $\mathrm{K}_{\mathrm{di}}$, for each contaminant for each porous medium. 
Therefore:

$$
\mathrm{C}_{\mathrm{si}}^{\cdot}=\mathrm{K}_{\mathrm{di}} \mathrm{C}_{\mathrm{wi}}^{\cdot}
$$

where $\mathrm{K}_{\mathrm{di}}=$ the linear equilibrium sorption coefficient for contaminant $i$ to the source zone soil or vadose zone soil $\left(\mathrm{cm}^{3} \mathrm{~g}^{-1}\right)$.

Substituting Equation D.16 in Equation D.15, and assuming that

$$
\mathrm{v}_{\mathrm{p}}=\frac{\mathrm{q}_{\mathrm{w}}}{\theta_{\mathrm{w}}}
$$

where $\quad v_{p}=$ the pore water velocity $\left(\mathrm{cm} \mathrm{yr}^{-1}\right)$.

Equation D.15 can be simplified to

$$
\frac{\partial C_{w i}^{\bullet}}{\partial t}=\frac{D_{s i}}{R_{i}} \frac{\partial^{2} C_{w i}^{\bullet}}{\partial z^{2}}-\frac{v_{p}}{R_{i}} \frac{\partial C_{w i}^{\bullet}}{\partial z}-\lambda_{i} C_{w i}^{\bullet}
$$

where $\quad \mathrm{R}_{\mathrm{i}}=$ the retardation factor, or phase apportionment factor, for contaminant $i$ (unitless) and is given by

$$
R_{i}=1+\left(\frac{\beta K_{d i}}{\theta_{w}}\right)
$$

Equation D.18 is the transport differential equation solved for the vadose zone. To complete the specification of the mathematical problem, the initial condition is given by

$$
\mathrm{C}_{\mathrm{wi}}^{\cdot}(\mathrm{z}, 0)=0
$$

the upper boundary condition (at the bottom of the source zone) is given by

$$
\left[-\theta_{w} D_{s i} \frac{\partial C_{w i}^{\cdot}}{\partial z}+q_{w} C_{w i}^{\cdot}\right]_{z=z_{s z}}=q_{c i}^{\cdot}
$$

where $\quad z_{s z}=$ the location of the bottom of the source zone $(\mathrm{cm})$

and the lower boundary condition (at the water table) is given by

$$
\left[\frac{\partial C_{w i}^{\cdot}}{\partial z}\right]_{z=z_{w z}}=0
$$


where $z_{\mathrm{wt}}=$ the location of the water table $(\mathrm{cm})$.

Now, let us define the following variable transformation:

$$
\mathrm{C}_{w i}^{\cdot}(\mathrm{t}) \equiv \mathrm{C}_{w i}(\mathrm{t}) \mathrm{e}^{-\lambda_{\mathrm{i}} \mathrm{t}}
$$

where $\mathrm{C}_{\mathrm{wi}}=$ the concentration of contaminant $i$ in the aqueous phase for the transformed mathematical problem $\left(\mathrm{Ci} \mathrm{cm}^{-3}\right)$.

This definition is consistent with the variable transform definition for total activity in the source zone given by Equation D.6. In fact, if there were no precipitated-phase contaminant in the source zone, we could have used Equation D.23 as the variable transform definition for the source zone; and then derived Equation D.6 from Equation D.23 using Equations D.1 and D.16, along with the standard relationship for the total concentration in terms of the concentrations in aqueous and sorbed phases. By substituting Equation D.23 into Equations D.18, D.20, D.21, and D.22 (and then differentiating, rearranging, and simplifying as was done for the source zone equations), we obtain the following set of equations for the transformed mathematical problem:

$$
\begin{gathered}
\frac{\partial C_{w i}}{\partial t}=\frac{D_{s i}}{R_{i}} \frac{\partial^{2} C_{w i}}{\partial z^{2}}-\frac{v_{p}}{R_{i}} \frac{\partial C_{w i}}{\partial z} \\
C_{w i}(z, 0)=0 \\
{\left[-\theta_{w} D_{s i} \frac{\partial C_{w i}}{\partial z}+q_{w} C_{w i}\right]_{z=z_{s z}}=q_{c i}} \\
{\left[\frac{\partial C_{w i}}{\partial z}\right]_{z=z_{w t}}=0}
\end{gathered}
$$

Equations D.24 through D.27 have the proper mathematical form to describe a transport situation where the contaminants do not decay. (To illustrate this, just let $\lambda_{i}$ go to zero in Equations D.18, D.20, D.21, and D.22, and they reduce to the mathematical forms of Equations D.24 through D.27.) Once this set of equations is solved (using the transformed leaching flux density from the source zone calculations in Equation D.26), we can use the variable transform definition in Equation D.23 to obtain the actual value of aqueous concentration if we so desire. We can also then calculate the contaminant flux density versus time function at the water table, which represents contaminant input to the groundwater aquifer. Furthermore, by using Equation D.14 (i.e., the relationship between the flux density and the fraction remaining), the vadose zone problem could also be formulated to use the fraction remaining as part of the upper boundary condition.

Next, a similar procedure can be applied to the mathematical problem of reactive transport in the groundwater aquifer. The approach and the equation development are very similar to that described above for the vadose zone problem. The differential equation includes a three-dimensional representation of dispersion, and the boundary conditions are more numerous and slightly different; but the logic is the same. Therefore, the full derivation is not presented. Suffice it to say that the 
transformed groundwater transport problem can be solved to obtain contaminant concentrations as functions of time at desired receptor points. The actual (real-world) concentrations at the receptors can be obtained from these values by applying the transform definition in Equation D.23.

With the above understanding of the mathematical approach to the problem, all that is left to do to calculate the release from the source zone is to derive the specific transformed fraction remaining functions (Equation D.12) based on the leaching loss terms (Equations D.7 and D.8) appropriate to each specific type of waste form category.

\section{D.2.2. Equations Used for the Soil-Debris Waste Form Type}

The source zone is conceptualized as unconsolidated porous material, and the contaminants are assumed to be uniformly distributed throughout for all times. Because only radionuclide contaminants are considered, the conceptual model assumes that no organic liquid phase (immiscible with the aqueous phase) is present. It is also assumed that there is no competition between contaminants for sorption sites, and no other significant chemical interaction between contaminants. Because of these assumptions, partitioning of the mass/activity of a specific contaminant between phases depends only on the amount of that contaminant itself, rather than on the amounts of all contaminants jointly. Furthermore, it is also assumed that partitioning of the radionuclides into the vapor phase is negligible. Therefore, only aqueous and sorbed phases, and possibly a precipitated phase, are assumed to exist.

The maximum amount of contaminant that can be accommodated in the aqueous and sorbed phases of a source zone (without a precipitated phase) can be expressed as

$$
M_{\operatorname{maxi}}^{*}=\left(\theta_{w} C_{w i}^{s o l}+\beta K_{d i} C_{w i}^{s o l}\right) \cdot V
$$

where $\mathrm{M}_{\operatorname{maxi}}^{*}=$ the maximum amount of contaminant $i$ possible in the source zone without a precipitated phase $(\mathrm{Ci})$

$$
\mathrm{C}_{\mathrm{wi}}^{\mathrm{sol}}=\text { the aqueous solubility of contaminant } i\left(\mathrm{Ci} \mathrm{cm}^{-3}\right) \text {. }
$$

If the volume of the source zone is given by

$$
\mathrm{V}=\mathrm{Ah}
$$

where $h=$ the average vertical thickness of the contaminant source zone $(\mathrm{cm})$

Equation D.28 can be rewritten as

$$
\mathrm{M}_{\operatorname{maxi}}=\theta_{\mathrm{w}} \mathrm{R}_{\mathrm{i}} \mathrm{C}_{\mathrm{wi}}^{\mathrm{sol}} \mathrm{Ah}
$$

If more than this amount $\left(\mathrm{M}_{\operatorname{maxi}}^{*}\right)$ of contaminant mass/activity exists in the source zone, a precipitated phase is assumed to be present and the aqueous concentration of the contaminant in the source zone is assumed to be solubility-controlled. In other words, if the following condition is true

$$
M_{i}^{*}-\theta_{w} R_{i} C_{w i}^{s o l} A h>0
$$


the actual aqueous concentration in the source zone is given by

$$
\mathrm{C}_{\mathrm{wi}}^{\cdot}=\mathrm{C}_{\mathrm{wi}}^{\mathrm{sol}}
$$

Using the variable transform definition in Equation D.23, this means that the transformed aqueous concentration in the source zone is given by

$$
C_{w i}=C_{w i}^{s o l} e^{\lambda_{i} t}
$$

(This is an unusual and important point to remember, and arises from the fact that the solubility limit must be applied to the actual scenario and not the transformed one.) If the condition in Equation D. 31 is false, the transformed aqueous concentration of the contaminant in the source zone is assumed to be desorption-controlled, and given by

$$
C_{w i}=\frac{M_{i}}{\theta_{w} R_{i} A h}
$$

Because of the similarity/interdependence of variable transform definitions (Equations D.6 and D.23) for total activity and aqueous concentration when no precipitated phase is present, the mathematical form of Equation D.34 is identical to the analogous expression for the original (realworld) variables.

The leaching process is assumed to occur by advective transport of the aqueous-phase contaminant out of the bottom face of the source zone along.with the percolating vadose zone water. Hence, the leaching flux is given by the product of the volumetric flux of water out of the source zone face and the aqueous concentration in the water at that time. The volumetric water flux is assumed to be in steady state, and is equal to the product of the Darcy water flux density and the horizontal cross-sectional area of the source zone. Therefore, the rate of loss of mass/activity from the source zone by leaching at any time is given by

$$
\frac{d M_{i}}{d t}=-q_{w} A C_{w i}
$$

In Equation D.35, the right-hand side of the equation is the leaching loss term given by the righthand side of Equation D.7; and it is equal to the right hand side of Equation D.8, as it is supposed to be. Furthermore, in Equation D.35, $\mathrm{C}_{\mathrm{wi}}$ is given by the expression in either Equation D.33 or D.34, depending on whether the system is solubility- or desorption-controlled.

If the condition given by Equation D. 31 is false at time $t=0$, the leaching process is desorptioncontrolled for all times. In this instance, Equation D.35 (along with Equation D.34) can be rearranged and integrated to obtain the following expression for the contaminant activity remaining in the source zone as a function of time:

$$
M_{i}(t)=M_{o i} \exp \left[-\frac{q_{w} t}{\theta_{w} R_{i} h}\right]
$$


To obtain Equation D.36, the lower limits of the integrals involved are $\mathrm{M}_{\mathrm{oi}}$ for contaminant activity and 0 for time. To obtain an expression for the fraction of contaminant activity remaining in the source zone as a function of time, Equation D. 36 is divided by the initial inventory of the contaminant. Therefore, when the initial contaminant inventory is low enough that the leaching is always desorption-controlled, the fraction remaining is given by

$$
f_{r i}(t)=\exp \left[-\frac{q_{w} t}{\theta_{w} R_{i} h}\right]
$$

If the condition given by Equation D.5 is true at time $t=0$, there will be a period of time when the contaminant is leaching from the source zone via solubility control. For this time period, Equation D.35 (along with Equation D.33) can be rearranged and integrated to obtain the following expression for the contaminant activity remaining in the source zone as a function of time:

$$
M_{i}(t)=M_{o i}-q_{w} A C_{w i}^{s o l}\left(\frac{e^{\lambda_{i} t}-1}{\lambda_{i}}\right)
$$

For radionuclides with sufficiently long half-lives (i.e., $\lambda_{i}$ approaching zero), the expression in Equation D.38 can be taken to the limit to obtain

$$
M_{i}(t) \approx M_{o i}-q_{w} A C_{w i}^{s o l} t
$$

which is the expression we would expect for a nondecaying contaminant.

The function given by Equation D.38 is only valid up until the time, $t_{c 0}$, when the source zone changes over to a desorption-controlled leaching regime. In other words, the type of leaching described by Equation D.38 will last until the activity of the contaminant in the source zone is reduced to the amount specified by Equation D.30. Thus, this change-over time can be calculated by first substituting Equation D.6 into Equation D.38 (to obtain a expression for the actual activity), then rearranging the resulting expression, then setting $M_{i}^{*}$ and $t$ in that expression to $M_{\text {maxi }}^{*}$ and $t_{c o}$, respectively, to obtain

$$
M_{\operatorname{maxi}}^{*}=M_{o i} e^{-\lambda_{i} t_{c o}}-q_{w} A C_{w i}^{s o l}\left(\frac{1-e^{-\lambda_{i} t_{c o}}}{\lambda_{i}}\right)
$$

where $\quad t_{c o}=$ the time at which leaching changes from solubility- to desorption-controlled (yr).

Equation D.40 must then be solved for t. This must be done by some type of root-finding algorithm. Again, for radionuclides with sufficiently long half-lives (i.e., $\lambda_{i}$ approaching zero), the expression in Equation D.40 can be taken to the limit, and then the resulting expression can be explicitly solved for $t_{c o}$ to obtain

$$
t_{c o}=\frac{\left(M_{o i}-M_{\operatorname{maxi}}\right)}{q_{w} A C_{w i}^{s o l}}
$$

D.12 
For times greater than $t_{c o}$, the leaching is desorption-controlled. In this instance, Equation D.35 (along with Equation D.34) can be rearranged and integrated to obtain the following expression for the contaminant activity remaining in the source zone as a function of time:

$$
M_{i}(t)=M_{\text {maxi }}^{*} \exp \left[-\frac{q_{w}\left(t-t_{c o}\right)}{\theta_{w} R_{i} h}\right]
$$

To obtain Equation D.42, the lower limits of the integrals involved are $M_{\text {maxi }}$ for contaminant activity and $t_{c o}$ for time (rather than $\mathrm{M}_{\mathrm{oi}}$ and 0 , which were used to obtain Equation D.36).

To obtain expressions for the fraction of contaminant activity remaining in the source zone as a function of time, Equations D.38 and D.42 are divided by the initial inventory of the contaminant. Therefore, when the initial contaminant inventory is high enough that a period of solubilitycontrolled release exists, the fraction remaining is given by

$$
f_{r i}(t)=\left\{\begin{array}{ll}
1-\left(\frac{q_{w} A C_{w i}^{s o l}}{M_{o i}}\right)\left(\frac{e^{\lambda_{i} t}-1}{\lambda_{i}}\right) & \text { for } 0 \leq t<t_{c o} \\
\frac{M_{\text {maxi }}^{0}}{M_{o i}} \exp \left[-\frac{q_{w}\left(t-t_{c o}\right)}{\theta_{w} R_{i} h}\right] & \text { for } t \geq t_{c o}
\end{array}\right\}
$$

where $t_{c o}$ is given by the solution of Equation D.40. Again, for radionuclides with sufficiently long half-lives (i.e., $\lambda_{i}$ approaching zero), the fraction remaining could be approximated by

$$
f_{r i}(t)=\left\{\begin{array}{ll}
1-\left(\frac{q_{w} A C_{w i}^{s o l}}{M_{o i}}\right) t & \text { for } 0 \leq t<t_{c o} \\
\frac{M_{m a x i}^{0}}{M_{o i}} \exp \left[-\frac{q_{w}\left(t-t_{c o}\right)}{\theta_{w} R_{i} h}\right] & \text { for } t \geq t_{c o}
\end{array}\right\}
$$

where $t_{c_{0}}$ is given by Equation D.41.

\section{D.2.3 Equations Used for the Cake Waste Form Type}

The source zone is conceptualized as consolidated porous material; and the contaminants are assumed to be uniformly distributed throughout for all times. The cake type of waste form also contains a given initial inventory, $\mathrm{M}_{\mathrm{msco}}$, of some major structural component that is also assumed to be uniformly distributed throughout for all times. The dissolution of this major structural component (via solubility control) into the water that percolates through the cake controls the dissolution of the overall cake. Therefore, the rate of loss of mass of the major structural component from the source zone by leaching at any time is given by

$$
\frac{\mathrm{dM}_{\mathrm{msc}}}{\mathrm{dt}}=-\mathrm{q}_{\mathrm{w}} A C_{\mathrm{wmsc}}^{\mathrm{sol}}
$$


where $M_{\mathrm{msc}}=$ the mass of the major structural component in the source zone (g)

$C_{\text {wmsc }}^{\text {sol }}=$ the aqueous solubility of the major structural component $\left(\mathrm{g} \mathrm{cm}^{-3}\right)$.

Equation D.45 can be rearranged and integrated to obtain the following expression for the mass of the major structural component remaining in the source zone as a function of time:

$$
M_{m s c}(t)=M_{m s c o}-q_{w} A C_{w m s c}^{s o l} t
$$

where $M_{\text {msco }}=$ the initial mass of the major structural component in the source zone $(\mathrm{g})$.

The time that it takes the percolating water to completely dissolve the cake can be calculated by letting $M_{m s c}(t)$ be equal to 0 in Equation D.46, and solving the resulting equation for $t$. Doing so produces the expression

$$
t_{\mathrm{dd}}=\frac{M_{\mathrm{msco}}}{\mathrm{q}_{\mathrm{w}} A \mathrm{C}_{\mathrm{wmsc}}^{\mathrm{sol}}}
$$

where $\quad t_{c d}=$ the cake dissolution time $(y r)$.

Because all of the contaminants in the waste form are leached congruently with the dissolving cake, the initial inventory of each nondecaying contaminant is lost at a constant rate over the time period $t_{c d}$. In other words,

$$
\left[\frac{\mathrm{dM}_{\mathrm{i}}}{\mathrm{dt}}\right]_{\text {leach }}=\frac{\mathrm{M}_{\mathrm{oi}}}{\mathrm{t}_{\mathrm{cd}}}=\mathrm{C}_{\mathrm{Ti}}\left(\frac{\mathrm{V}_{\mathrm{cao}}}{\mathrm{t}_{\mathrm{cd}}}\right)
$$

where $V_{\text {cao }}=$ the initial volume of the cake source zone $\left(\mathrm{cm}^{3}\right)$.

In Equation D.48, the term $\mathrm{V}_{\mathrm{cao}} / \mathrm{t}_{\mathrm{cd}}$ can be considered to be the volumetric rate of dissolution of the cake. For the real-world scenario, where the contaminant is decaying, the volumetric cake dissolution rate would be the same, but the total contaminant concentration in the remaining cake would be decreasing over time. This would be accounted for by including an exponential decay factor to the leaching loss term for the actual scenario; which means that the theory presented here is indeed consistent with the condition in Equation D.8. Therefore, the resulting expression for the activity of a nondecaying contaminant remaining in the source zone as a function of time is given by

$$
M_{i}(t)=M_{o i}-\left(\frac{M_{o i}}{t_{o d}}\right) t
$$

Substituting Equation D.47 into Equation D.49 results in

$$
M_{i}(t)=M_{o i}\left(1-\frac{q_{w} A C_{w m s c}^{s o l}}{M_{m s c o}}\right)
$$


To obtain an expression for the fraction of contaminant mass/activity remaining in the source zone as a function of time, Equation D.50 is divided by the initial inventory of the contaminant. Therefore, the fraction remaining is given by

$$
f_{r i}(t)=1-\left(\frac{q_{w} A C_{w m s c}^{s o l}}{M_{m s c o}}\right) t
$$

\section{D.2.4 Equations Used for the Glass Waste Form Type}

For the glass waste form types, the contaminant release mechanism is dissolution of the glass at the outer surface of the waste form as water percolates past it. Contaminants are assumed to be uniformly distributed throughout the glass for all times. The conceptual and mathematical models for release used for the Hanford Composite Analysis are the same as those used for the interim performance assessment of Hanford low-level tank waste (Mann et al. 1997). Therefore, the rate of loss of activity from the source zone by leaching at any time is given by

$$
\frac{d M_{i}}{d t}=-\frac{r_{g} A_{s g} M_{o i}}{V_{g o}}
$$

where $\quad r_{g}=$ the volumetric dissolution rate of glass per area of surface $\left(\mathrm{cm} \mathrm{yr}^{-1}\right)$

$A_{\mathrm{sg}}=$ the total external surface area of the glass waste form in the source zone $\left(\mathrm{cm}^{2}\right)$

$V_{\mathrm{go}}=$ the initial volume of the glass waste form in the source zone $\left(\mathrm{cm}^{3}\right)$.

In Equation D.52, $\mathrm{M}_{\mathrm{oi}} / \mathrm{V}_{\mathrm{go}}$ represents the volumetric total concentration of the nondecaying. contaminant in the glass. Hence, Equation D.52 describes the contaminant mass loss rate as the product of the volumetric glass dissolution rate per area of surface, the surface area, and the contaminant concentration in the glass. For the real-world scenario, where the contaminant is decaying, the total concentration in the remaining glass would be decreasing over time. This would be accounted for by including an exponential decay factor to the leaching loss term for the actual scenario; which means that the theory presented here is indeed consistent with the condition in Equation D.8.

$\mathrm{A}_{\mathrm{sg}}$ is not constant, but instead is changing over time as the glass dissolves. Therefore, Equation D.52 must be further developed by substituting into it an appropriate expression for how the surface area changes as a function of time. To develop this expression, the initial shape of the waste form is assumed to be roughly cubical. For this shape, the time-dependent surface area of a single waste form (six square sides) is given by

$$
A_{s g}=6\left(L_{o}-2 r_{g} t\right)^{2}
$$

where $\quad L_{0}=$ the initial linear dimension of the cubical glass waste form $(\mathrm{cm})$.

The time that it takes the percolating water to completely dissolve the glass can be calculated by letting $A_{s g}$ be equal to 0 in Equation D.53, and solving the resulting equation for $t$. Doing so produces the expression 


$$
t_{g d}=\frac{L_{0}}{2 r_{g}}
$$

where $\quad t_{g d}=$ the glass dissolution time $(y r)$.

By solving Equation D.54 for $\mathrm{L}_{\mathrm{o}}$ and substituting the resulting expression into Equation D.53, the surface area of the waste form can be expressed as

$$
A_{s g}=24 r_{g}^{2}\left(t_{g d}-t\right)^{2}
$$

By substituting Equation D.55 into Equation D.52, and then expressing the initial volume of the cube in terms of the initial linear dimension $\left(\mathrm{L}_{0}\right)$, and then expressing $\mathrm{L}_{\mathrm{o}}$ in terms of $t_{\mathrm{gd}}$ (via Equation D.54), the rate of loss of mass/activity from the source zone by leaching at any time can be expressed as

$$
\frac{d M_{i}}{d t}=-\frac{3\left(t_{g d}-t\right)^{2} M_{o i}}{t_{g d}^{3}}
$$

Now, an expression for the initial fractional contaminant release rate can be derived from Equation D.56, and is given by

$$
F_{\text {rrgoi }}=-\frac{1}{M_{o i}}\left[\frac{d M_{i}}{d t}\right]_{t=0}=\frac{3}{t_{g d}}
$$

where $\mathrm{F}_{\mathrm{rrgoi}}=$ the initial fractional release rate from a glass waste form for contaminant $i\left(\mathrm{yr}^{-1}\right)$.

The Request for Proposal (RFP) for the glass waste form to be produced for use at Hanford specifies the maximum average initial fractional release rate that will be allowed for different radionuclides (i.e, one value is specified for the release of technetium-99, and one value is specified for the release of all non-technetium- 99 radionuclides). The Composite Analysis calculations assume that the initial fractional contaminant release rate will be equal to the value specified in the RFP. Therefore, by solving Equation D.57 for $t_{\mathrm{gd}}$, and then substituting the resulting expression into Equation D.56, the rate of loss of activity from the source zone by leaching at any time can be expressed as

$$
\frac{d M_{i}}{d t}=-\frac{F_{\text {rrgoi }}^{3}\left(\frac{3}{F_{\text {rrgoi }}}-t\right)^{2} M_{o i}}{9} .
$$

Equation D.58 can now be rearranged and integrated to obtain the following expression for the contaminant mass/activity remaining in the source zone as a function of time:

$$
M_{i}(t)=M_{o i}\left(1-\frac{F_{\text {rgoi }} t}{3}\right)^{3}
$$


To obtain an expression for the fraction of contaminant mass/activity remaining in the source zone as a function of time, Equation D.59 is divided by the initial inventory of the contaminant. Therefore, the fraction remaining is given by

$$
f_{r i}(t)=\left(1-\frac{F_{\text {rrgoi }} t}{3}\right)^{3}
$$

\section{D.2.5 Equations Used for the Cement Waste Form Type}

For cement waste form types, the contaminant release mechanism to the leaching pathway is diffusion through the solidified waste material to the outer surface of the waste form where it is carried away by the water percolating past the surface. For this conceptualization, the rate of loss of activity from the source zone by leaching at any time is assumed to be given by

$$
\frac{d M_{i}}{d t}=-M_{o i}\left(\frac{A_{s c}}{V_{c e}}\right) \sqrt{\frac{D_{c i}}{\pi t}}
$$

where $\quad A_{s c}=$ the total external surface area of the cement waste form in the source zone (cm2)

$$
\begin{aligned}
V_{c e}= & \text { the volume of the cement waste form in the source zone }\left(\mathrm{cm}^{3}\right) \\
D_{\mathrm{ci}}= & \text { the effective diffusion coefficient of contaminant } i \text { within a cement waste form } \\
& \left(\mathrm{cm}^{2} \mathrm{yr}-1\right) .
\end{aligned}
$$

Equation D.61 is actually derived from the solution to the diffusion equation for mass/activity lost through an infinite plane that bounds a semi-infinite solid source when no decay occurs (Godbee et al. 1980). In Equation D.61, $\mathrm{M}_{\mathrm{oi}} / \mathrm{V}_{\mathrm{ce}}$ represents the total concentration of the nondecaying contaminant in the cement waste form. For the real-world scenario, where the contaminant is decaying, the total concentration in the cement would be decreasing over time. This would be accounted for by including an exponential decay factor to the leaching loss term for the actual scenario; which means that the theory presented here is indeed consistent with the condition in Equation D.8.

To go from an expression for flux density of contaminant lost from an. infinite plane to the expression (Equation D.61) for total flux lost from the finite cement waste form, the assumption is made that the flux density expression can merely be multiplied by the total external surface area of the cement in the source zone. The flux calculated by Equation D.61 is approximately equal to that coming from a finite solid source for early times. However, at later times, Equation D.61 will overpredict the flux by an increasing amount as time goes on. Furthermore, the larger the cement waste form, and the smaller the effective diffusion coefficient, the longer the flux given by Equation D.61 will be approximately equal to that diffusing from a finite waste form. In spite of the approximate nature of the above expression, this idealized approach was taken because more accurate flux expressions for finite solids would strongly depend on the specific shape of the cement waste forms. There could likely be a variety of waste form shapes encountered in the Composite Analysis (meaning that a number of different, more complicated, expressions would need to be derived for the diffusive release), and these were not known a priori.

It is also worth noting that Equation D.61 depends on the activity in the source zone initially, rather than on the activity in the source zone at any given time (as in the flux expressions used for the 
other generic types of source zone). This arises from the fact that the conceptual model used here requires that a spatial gradient in concentration be present within the waste form. This is in direct opposition to the "well-mixed reactor" assumption used to derive loss flux expressions in all of the other scenarios dealt with. In addition, because Equation D.61 depends on $M_{o i}$ rather than $M_{i}$, partial failure of a cement waste form cannot be simulated. However, total failure of a cement waste form at some designated time can, in principle, be simulated. In this case the leaching flux expression would revert back to that for a soil-debris waste form (with contaminant activity at the initial time of that phase equal to the mass/activity remaining in the cement waste form when it failed). However, the analyses now assume that the cement waste form never fails; and so Equation D.61 is used for all times.

Equation D.61 can now be rearranged and integrated to obtain the following expression for the contaminant mass/activity remaining in the source zone as a function of time:

$$
M_{i}(t)=M_{o i}\left[1-2\left(\frac{A_{s c}}{V_{c e}}\right) \sqrt{\frac{D_{c i} t}{\pi}}\right]
$$

To obtain an expression for the fraction of contaminant mass/activity remaining in the source zone as a function of time, Equation D.62 is divided by the initial inventory of the contaminant. Therefore, the fraction remaining is given by

$$
f_{r i}(t)=1-2\left(\frac{A_{s c}}{V_{c e}}\right) \sqrt{\frac{D_{c i} t}{\pi}}
$$

\section{D.2.6 Equations Used for the Reactor Block Waste Form Type}

This generic waste form type was developed to apply to the loss of radionuclides from irradiated graphite reactor blocks disposed of in the vadose zone. The conceptual and mathematical models of release are based on those reported in the draft environmental impact statement (EIS) for the decommissioning of the eight surplus production reactors at Hanford (DOE 1989). For the dosimetric analysis in that EIS, it was assumed that half of the released carbon-14 was leached to the vadose zone and half was volatilized to the atmosphere. However, for the Composite Analysis, it is assumed that all released contaminants are leached to the vadose zone.

The blocks release contaminants into the water percolating past them via unspecified loss processes from the solid graphite matrix as well as via corrosion of the solid graphite matrix and irradiated metal components over time. The surplus reactor EIS (DOE 1989) reports that no specific data are available regarding radionuclide release rates from the irradiated metal components; and so the EIS assumed that release from the metal was the same as from the graphite material of the reactor block. Several experimental studies of the loss of carbon-14 from graphite reactor block material indicate that there is an initial period of high release followed by a longer period of approximately steady-state release that is approximately two orders of magnitude lower. The surplus reactor EIS uses laboratory data to derive a correlation equation between carbon-14 release rate and time, and then uses the volume-to-surface area ratio of the Hanford reactor blocks to obtain a correlation equation that is equivalent to the following equation for predicting the loss of carbon-14 from the reactor block as a function of time and temperature: 


$$
\frac{\mathrm{dM}_{\mathrm{i}}}{\mathrm{dt}}=-\mathrm{M}_{\mathrm{oi}}(365)\left[565\left(1+100 \mathrm{e}^{-(0.08)(365) \mathrm{t}}\right) \mathrm{e}^{-6440 / \mathrm{T}}\right]
$$

where $\quad T=$ the absolute temperature of the reactor block $(K)$.

In Equation D.64, the factors of 365 have been added to convert time from the units used in the surplus reactor EIS (days) to those used here (years).

To put the time dependence of Equation D.64 in perspective, note that the release flux will fall to within $1 \%$ of its ultimate steady-state value at approximately $0.3 \mathrm{yr}$. Compared to the length of the Composite Analysis simulation time (which is on the order of 1000 to $2000 \mathrm{yr}$ ), the initial period of transient release is assumed to be insignificant. In addition, carbon-14 is the only radionuclide for which temperature-dependent release information is available. Because of this fact, the surplus reactor EIS assumed that the temperature of the reactor blocks was constant at $22^{\circ} \mathrm{C}$. By assuming constant temperature and steady-state release flux conditions, Equation D.64 reduces to a form that is identical to the form of the release model used in the surplus reactor EIS for other radionuclides. Specifically, for other radionuclides, the only information available was limited laboratory data on the steady-state fractional release rates at ambient temperature. These single values were corrected for the volume-to-surface area ratio of the Hanford reactor blocks to produce a table (which was reported in the surplus reactor EIS) of Hanford-specific fractional release rates for certain specific radionuclides. Furthermore, for certain additional radionuclides for which there were no release data, the surplus reactor EIS recommends using the fractional release rate values of specific tabulated radionuclides that are assumed to behave similarly.

Based on these considerations, the rate of loss of activity from the source zone by leaching at any time is assumed to be given by

$$
\frac{d M_{i}}{d t}=-M_{o i} F_{\text {rrri }}
$$

where $\quad \mathrm{F}_{\mathrm{rrri}}=$ the fractional release rate from a reactor block waste form for contaminant $i\left(\mathrm{yr}^{-1}\right)$.

Equation D.65 can now be rearranged and integrated to obtain the following expression for the contaminant activity remaining in the source zone as a function of time:

$$
M_{i}(t)=M_{o i}\left(1-F_{m i r i} t\right)
$$

To obtain an expression for the fraction of contaminant activity remaining in the source zone as a function of time, Equation D.66 is divided by the initial inventory of the contaminant. Therefore, the fraction remaining is given by

$$
f_{r i}(t)=1-F_{n r i} t
$$

\section{D.3 Rationale for Choosing Values for Radionuclide-Related Parameters in the Release Model Equations}

The radionuclide-related parameters required by the source zone release model are decay coefficient, aqueous solubility, distribution or sorption coefficient, initial fractional release rate from glass, effective diffusion coefficient in cement, and fractional release rate from reactor blocks. Some 
of these parameters were input directly while others were calculated from other parameters. Table D.2 presents a list of the radionuclides considered in the source zone calculations, along with the input values used for each parameter for each nuclide.

The values of some of these parameters would, in general, be specific to the conditions at a particular source site. In some cases, where it was believed that reasonable "Hanford Site-specific" values were known, these values were used in the calculations. However, note that the value used for a specific parameter for a specific nuclide was the same for all Hanford source sites (i.e., because of the scope of this effort, no attempt was made to examine physico-chemical conditions at each source site and determine a different individual value of a parameter for each site). Most parameter values are based on actual data. However, some values are based on assumed similarity in behavior with other radionuclides, and some values are set equal to "default" values when no other information is available.

\section{D.3.1 Decay Coefficient}

Radioactive decay coefficients are actually calculated from decay half-lives by the source zone release model, according to the equation

$$
\lambda_{\mathrm{i}}=\frac{\ln 2}{\mathrm{t}_{1 / 2 \mathrm{i}}}
$$

where $\quad t_{1 / 2 \mathrm{i}}=$ the decay half-life of contaminant $i(\mathrm{yr})$.

Values of radioactive decay half-lives for different radionuclides are unambiguous and well known. The specific values of half-life used for the source-term calculations were the values originally reported in a U.S. Environmental Protection Agency (EPA) Guidance report for radionuclides (Eckerman, Wolbarst, and Richardson 1988). These values had previously been incorporated into a computer database known as the Multimedia-Modeling Environmental Database and Editor (MMEDE) (Warren and Strenge 1994). For the source-term calculation effort of the Hanford Composite Analysis project, the MMEDE database was queried to produce an electronic file of tabulated half-lives for relevant radionuclides (that was subsequently incorporated into the sourceterm calculation spreadsheet).

\section{D.3.2 Aqueous Solubility}

First, the MMEDE database (Warren and Strenge 1994) was queried for values of aqueous solubility, for each radionuclide. (The database contains a reference for each solubility value it contains.) Unfortunately, other than for tritium, the database does not contain a value for aqueous solubility for the radionuclides considered here.

However, as part of recent prior efforts on preparation of the Waste Isolation Pilot Plant Supplementary Environmental Impact Statement (WIPP SEIS) (DOE 1997), the solubility values for some of these radionuclides were estimated based on geochemical calculations (using the MINTEQA2 computer code [Allison, Brown, and Novo-Gradoc 1991]) for Hanford Site-specific conditions. The specific radionuclides chosen for estimation were based on a screening of the WIPP SEIS contaminants to determine which were most likely to be solubility-controlled and have a major influence on ultimate risk. The screening process and geochemical calculations are described, and the resulting solubility values are reported, in Buck et al. (1996). These values were adopted for use in the Composite Analysis calculations. For all remaining radionuclides (for which there were no 
specific values available), the aqueous solubility was fixed at an arbitrarily high default value ( $1 \mathrm{x}$ $1010 \mathrm{mg} \mathrm{L}-1)$ so that the source zone release model would automatically select algorithms for desorption control rather than solubility control in these cases.

The source zone release model actually needs aqueous solubility values measured in units of $\mathrm{Ci} \mathrm{cm}{ }^{-3}$. Values measured in units of $\mathrm{mg} \mathrm{L}^{-1}$ were converted to units of $\mathrm{Ci} \mathrm{cm}^{-3}$ by multiplying by the specific activity of each radionuclide (along with appropriate units conversion factors). The specific activity, in turn, was calculated from the decay half-life and the atomic mass according to the formula (DOHEW 1970):

$$
\mathrm{a}_{\mathrm{spi}}=\frac{3.578 \times 10^{5}}{\mathrm{t}_{1 / 2 \mathrm{i}} \mathrm{m}_{\mathrm{ai}}}
$$

where $\quad a_{\text {spi }}=$ the specific activity of contaminant $i\left(\mathrm{Ci} \mathrm{g}^{-1}\right)$

$\mathrm{m}_{\mathrm{ai}}=$ the atomic mass of contaminant $i\left(\mathrm{~g} \mathrm{~mol}^{-1}\right)$.

Therefore, Table D.2 also includes values of specific activity and atomic mass.

\section{D.3.3 Sorption Coefficient}

A set of Hanford Site-specific $K_{d}$ values were developed specifically for the Composite Analysis project in a separate effort. The Hanford data used and the approach taken for developing the $\mathrm{K}_{\mathrm{d}}$ values are discussed in detail in Appendix E of the Composite Analysis.

\section{D.3.4 Initial Fractional Release Rate from Glass}

As stated previously, the conceptual and mathematical models for release from glass waste form types used for Hanford Composite Analysis are the same as those used for the interim performance assessment of Hanford low-level waste (Mann et al. 1997). Mann et al. (1997) also specify the initial fractional release rate to be used in the calculations. This value is part of the specifications for the waste form reported in the waste-form privatization RPF, and is the same for all radionuclides for these calculations.

\section{D.3.5 Effective Diffusion Coefficient in Cement}

First, specific values of effective diffusion coefficient in cement type waste forms for each radionuclide were chosen to be the values originally reported by Serne et al. (1989). These values had previously been incorporated into a computer database known as the MMEDE (Warren and Strenge 1994). For the source-term calculation effort of the Hanford Composite Analysis project, the MMEDE database was queried to produce an electronic file of tabulated diffusion coefficients for relevant radionuclides (which was subsequently incorporated into the source-term calculation spreadsheet).

However, as part of recent prior efforts on preparation of the WIPP SEIS, the diffusion coefficient values for some of these radionuclides were improved. The rationale for modifying the diffusion coefficients is described, and the resulting diffusion coefficient values are reported in Buck et al. 
(1996). These values were adopted for use in the Composite Analysis calculations. For some radionuclides (for which there were no specific values available), the diffusion coefficient was fixed at a reasonable conservatively high default value $\left(5 \times 10^{-8} \mathrm{~cm}^{2} \mathrm{~s}^{-1}\right)$.

\section{D.3.6 Fractional Release Rate from Reactor Blocks}

As stated previously, the conceptual and mathematical models of release from reactor blocks are based on those reported in the EIS for the decommissioning of the eight surplus production reactors at Hanford (DOE 1989). The surplus reactor EIS also reported values for fractional release rate (based on data) of some specific radionuclides from Hanford reactor blocks. It also made recommendations for what values to use for certain other radionuclides based on assumed similarity in behavior to radionuclides with measured data. These values were adopted for use in the Composite Analysis calculations.

\section{D.4.0 References}

Allison, J. D., D. S. Brown, and K. J. Novo-Gradac. 1991. MINTEQA2/PRODEFA2, A Geochemical Assessment Model for Environmental Systems: Version 3.0. User's Manual. EPA/600/3-91/021, U.S. Environmental Protection Agency, Athens, Georgia.

Buck, J. W., L. M. Bagaasen, M. P. Bergeron, G. P. Streile, L. H. Staven, K. J. Castleton, G. M. Gelston, D. L. Strenge, K. M. Krupka, and R. J. Serne. 1996. Draft Long-Term-Consequence Analysis of No Action Alternative 2: Support Information for the Waste Isolation Pilot Plant Disposal-Phase Supplemental Environmental Impact Statement. PNNL-11252, Pacific Northwest National Laboratory, Richland, Washington.

Eckerman, K. F., A. B. Wolbarst, and A. C. B. Richardson. 1988. Limiting Values of Radionuclide Intake and Air Concentration and Dose Conversion Factors for Inhalation, Submersion, and Ingestion, Federal Guidance Report No. 11. EPA-520/1-88-202. U.S. Environmental Protection Agency, Office of Radiation Programs, Washington, D.C.

Godbee, H., E. L. Compere, D. S. Joy, A. H. Kibbey, J. G. Moore, C. W. Nestor, Jr., O. U. Anders, and R. M. Neilson, Jr. 1980. "Applications of mass transport theory to the leaching of radionuclides from waste solids." Nuc. Chem. Waste Manag. 1:29-35.

Mann, F. M., C. R. Eiholzer, Y. Chen, N. W. Kline, A. H. Lu, B. P. McGrail, P. D. Rittmann, G. F. Williamson, J. A. Voogd, N. R. Brown, and P. E. LaMont. 1997. Hanford Low-Level Tank Waste Interim Performance Assessment. HNF-EP-0884, Rev. 1, Lockheed Martin Hanford Corporation, Richland, Washington.

Serne, R. J., W. J. Martin, R. O. Lokken, V. L. LeGore, C. W. Lindenmeier, and P. F. C. Martin. 1989. Leach and EP Toxicity Test on Grouted 106-AN Tank Waste. PNL-6960, Pacific Northwest Laboratory, Richland, Washington.

U.S. Department of Energy (DOE). 1989. Draft Environmental Impact Statement: Decommissioning of Eight Surplus Production Reactors at the Hanford Site, Richland, Washington. DOE/EIS-0119D, U.S. Department of Energy, Washington, D.C. 
U.S. Department of Energy (DOE). 1997. Waste Isolation Pilot Plant Disposal Phase Final Supplemental Environmental Impact Statement. DOE/EIS-0026-S-2, Carlsbad Area Office, Carlsbad, New Mexico.

U.S. Department of Health, Education, and Welfare (DOHEW). 1970. Radiological Training Handbook. Public Health Service, Rockville, Maryland.

Warren, B. R., and D. L. Strenge. 1994. Multimedia-Modeling Environmental Database Editor (MMEDE). PNNL-11562, Pacific Northwest National Laboratory, Richland, Washington. 
Table D.1. Definition of Source-Term Release Model Notation

\begin{tabular}{|c|c|c|}
\hline Notation & Definition & Units \\
\hline$a_{\text {spi }}$ & specific activity of contaminant $i$ & $\mathrm{Ci} \mathrm{g}^{-1}$ \\
\hline A & effective horizontal cross-sectional area of the contaminant source zone & $\mathrm{cm}^{2}$ \\
\hline$A_{s c}$ & total external surface area of the cement waste form in the source zone & $\mathrm{cm}^{2}$ \\
\hline$A_{s g}$ & total external surface area of the glass waste form in the source zone & $\mathrm{cm}^{2}$ \\
\hline $\mathrm{C}_{\text {si }}^{\bullet}$ & $\begin{array}{l}\text { concentration of contaminant } i \text { in the sorbed phase for the original } \\
\text { mathematical problem (that includes decay) }\end{array}$ & $\mathrm{Ci} \mathrm{cm}{ }^{-3}$ \\
\hline $\mathrm{C}_{\mathrm{T}}^{\bullet}$ & $\begin{array}{l}\text { total concentration of contaminant } i \text { (in all forms) in the source zone for the } \\
\text { original mathematical problem (that includes decay) }\end{array}$ & $\mathrm{Ci} \mathrm{cm}^{-3}$ \\
\hline $\mathrm{C}_{w i}$ & $\begin{array}{l}\text { concentration of contaminant } i \text { in the aqueous phase for the transformed } \\
\text { mathematical problem }\end{array}$ & $\mathrm{Ci} \mathrm{cm}^{-3}$ \\
\hline $\mathrm{C}_{\mathrm{w}}^{\text {sol }}$ & aqueous solubility of contaminant $i$ & $\mathrm{Ci} \mathrm{cm}^{-3}$ \\
\hline$C_{w}^{\bullet}$ & $\begin{array}{l}\text { concentration of contaminant } i \text { in the aqueous phase for the original } \\
\text { mathematical problem (that includes decay) }\end{array}$ & $\mathrm{Ci} \mathrm{cm} \mathrm{cm}^{-3}$ \\
\hline $\mathrm{C}_{\text {wms }}^{\text {sol }}$ & aqueous solubility of the major structural component & $\mathrm{g} \mathrm{cm}^{-3}$ \\
\hline$D_{c i}$ & effective diffusion coefficient of contaminant $i$ within a cement waste form & $\mathrm{cm}^{2} \mathrm{yr}^{-1}$ \\
\hline $\mathrm{D}_{\mathrm{si}}$ & effective diffusion coefficient of contaminant $i$ in the soil & $\mathrm{cm}^{2} \cdot \mathrm{yr}^{-1}$ \\
\hline$f_{i}$ & $\begin{array}{l}\text { fraction of the initial mass of contaminant } i \text { remaining in the source zone for } \\
\text { the transformed mathematical problem }\end{array}$ & unitless \\
\hline$\stackrel{\mathrm{f}_{\mathrm{r}}^{\bullet}}{ }$ & $\begin{array}{l}\text { fraction of the initial inventory of contaminant } i \text { remaining in the source zone } \\
\text { for the original mathematical problem (that includes decay) }\end{array}$ & unitless \\
\hline$F_{m i}$ & fractional release rate from a reactor block waste form for contaminant $i$ & $y r^{-1}$ \\
\hline$F_{\text {rigoi }}$ & initial fractional release rate from a glass waste form for contaminant $i$ & $\mathrm{yr}^{-1}$. \\
\hline h & average vertical thickness of the contaminant source zone & $\mathrm{cm}$ \\
\hline $\mathrm{K}_{\mathrm{di}}$ & $\begin{array}{l}\text { linear equilibrium sorption coefficient for contaminant } i \text { to the source zone soil } \\
\text { or vadose zone soil }\end{array}$ & $\mathrm{cm}^{3} \mathrm{~g}^{-1}$ \\
\hline $\mathrm{L}_{\mathrm{o}}$ & initial linear dimension of the cubical glass waste form & $\mathrm{cm}$ \\
\hline $\mathrm{m}_{\mathrm{ai}}$ & atomic mass of contaminant $i$ & $\mathrm{~g} \mathrm{~mol}^{-1}$ \\
\hline $\mathrm{M}_{\mathrm{i}}$ & $\begin{array}{l}\text { total activity of contaminant } i \text { in the source zone for the transformed } \\
\text { mathematical problem }\end{array}$ & $\mathbf{C i}$ \\
\hline
\end{tabular}


Table D.1. (contd)

\begin{tabular}{|c|c|c|}
\hline Notation & Definition & Units \\
\hline $\mathrm{M}_{\mathrm{i}}^{0}$ & $\begin{array}{l}\text { total activity of contaminant } i \text { in the source zone for the original mathematical } \\
\text { problem (that includes decay) }\end{array}$ & $\mathrm{Ci}$ \\
\hline$M_{\max }^{\bullet}$ & $\begin{array}{l}\text { maximum amount of contaminant } i \text { possible in the source zone without a } \\
\text { precipitated phase }\end{array}$ & $\mathrm{Ci}$ \\
\hline $\mathrm{M}_{\mathrm{msc}}$ & mass of the major structural component in the source zone & g \\
\hline $\mathrm{M}_{\mathrm{msco}}$ & initial mass of the major structural component in the source zone & g \\
\hline $\mathrm{M}_{\mathrm{oi}}$ & $\begin{array}{l}\text { initial total activity of contaminant } i \text { in the source zone for the transformed } \\
\text { mathematical problem (that includes decay) }\end{array}$ & $\mathrm{Ci}$ \\
\hline $\mathrm{M}_{\mathrm{oi}}^{\bullet}$ & $\begin{array}{l}\text { initial total activity of contaminant } i \text { in the source zone for the original } \\
\text { mathematical problem (that includes decay) }\end{array}$ & $\mathrm{Ci}$ \\
\hline $\mathfrak{q}_{\mathbf{i}}$ & $\begin{array}{l}\text { flux density of contaminant } i \text { entering the vadose zone below the source zone } \\
\text { for the transformed mathematical problem }\end{array}$ & $\mathrm{Ci} \mathrm{cm} \mathrm{cm}^{-2}$ \\
\hline$q_{\dot{c i}}^{\bullet}$ & $\begin{array}{l}\text { flux density of contaminant } i \text { entering the vadose zone below the source zone } \\
\text { for the original mathematical problem (that includes decay) }\end{array}$ & $\mathrm{Ci} \mathrm{cm} \mathrm{cm}^{-2} \mathrm{yr}^{-1}$ \\
\hline $\mathbf{q}_{\mathrm{w}}$ & Darcy flux density of water flowing through the source zone or vadose zone & $\mathrm{cm} \mathrm{yr^{-1 }}$ \\
\hline$r_{g}$ & volumetric dissolution rate of glass per area of surface & $\mathrm{cm} \mathrm{yr^{-1 }}$ \\
\hline $\mathrm{R}_{\mathrm{i}}$ & retardation factor, or phase apportionment factor, for contaminant $i$ & unitless \\
\hline $\mathbf{t}$ & time since initial condition of the source zone & $\mathrm{yr}$ \\
\hline$t_{1 / 2 i}$ & decay half-life of contaminant $i$ & yr \\
\hline$t_{c d}$ & cake dissolution time & yr \\
\hline$t_{c o}$ & time at which leaching changes from solubility- to desorption-controlled & yr \\
\hline$t_{g d}$ & glass dissolution time & yr \\
\hline $\mathrm{T}$ & absolute temperature of the reactor block & $\mathrm{K}$ \\
\hline $\mathrm{V}$ & volume of the source zone & $\mathrm{cm}^{3}$ \\
\hline $\mathrm{v}_{\text {cao }}$ & initial volume of the cake source zone & $\mathrm{cm}^{3}$ \\
\hline$v_{\infty}$ & volume of the cement waste form in the source zone & $\mathrm{cm}^{3}$ \\
\hline $\mathrm{v}_{\mathrm{go}}$ & initial volume of the glass waste form in the source zone & $\mathrm{cm}^{3}$ \\
\hline$v_{\mathrm{p}}$ & Pore water velocity & $\mathrm{Cm} \mathrm{yr^{-1 }}$ \\
\hline$z$ & vertical spatial coordinate & $\mathrm{cm}$ \\
\hline $\mathbf{z}_{\mathrm{sz}}$ & location of the bottom of the source zone & $\mathrm{cm}$ \\
\hline
\end{tabular}


Table D.1. (contd) .

\begin{tabular}{|l|l|c|}
\hline \multicolumn{1}{|c|}{ Notation } & \multicolumn{1}{|c|}{ Definition } & Units \\
\hline$z_{\mathrm{wt}}$ & location of the water table & $\mathrm{cm}$ \\
\hline$B$ & bulk density of the source zone soil or vadose zone soil & $\mathrm{g} \mathrm{cm}^{-3}$ \\
\hline$\lambda_{\mathrm{i}}$ & first-order decay coefficient for contaminant $i$ & $\mathrm{yr}^{-1}$ \\
\hline$\theta_{\mathrm{w}}$ & volumetric water content of the source zone soil or vadose zone soil & unitless $\left(\mathrm{cm}^{3} \mathrm{~cm}^{-3}\right)$ \\
\hline
\end{tabular}


Table D.2. Radionuclide-Specific Properties Needed as Inputs for the Composite Analysis

\begin{tabular}{|c|c|c|c|c|c|c|c|c|c|c|c|c|c|c|}
\hline \multirow[b]{2}{*}{ Radlónuclldo } & \multirow[b]{2}{*}{ ID Code } & \multicolumn{2}{|c|}{$\begin{array}{l}\text { Aquoous } \\
\text { Solublitity }\end{array}$} & \multirow{2}{*}{\begin{tabular}{|c|}
$\begin{array}{c}\text { Sorptlon } \\
\mathbf{K}_{\mathrm{d}}\end{array}$ \\
$(\mathrm{cm} 3 / \mathrm{g})$
\end{tabular}} & \multicolumn{2}{|c|}{$\begin{array}{c}\text { Fractional Roleaso } \\
\text { Rate }\end{array}$} & \multicolumn{2}{|c|}{$\begin{array}{c}\text { Cement } \\
\text { Diffusion Coefficient }\end{array}$} & \multicolumn{2}{|c|}{$\begin{array}{l}\text { Fractlonal Releaso } \\
\text { Rate }\end{array}$} & \multicolumn{2}{|c|}{$\begin{array}{c}\text { Decay } \\
\text { Half-Life }\end{array}$} & \multirow{2}{*}{$\begin{array}{c}\text { Speclfic } \\
\text { Actlvity } \\
\text { (Cllg) }\end{array}$} & \multirow{2}{*}{$\begin{array}{l}\begin{array}{c}\text { Atomic } \\
\text { Mass }\end{array} \\
\text { (g/mol) }\end{array}$} \\
\hline & & (mgh) & $\left.(\mathrm{C} / \mathrm{cm})^{3}\right)$ & & $(1 / s)$ & $(1 / y r)$ & $\left(\mathrm{cm}^{2} / \mathrm{s}\right)$ & $\left(\mathrm{cm}^{2} \mathrm{byr}\right)$ & $(1 / d)$ & $(1 / y r)$ & (d) & (yr) & & \\
\hline Actinium-224 & AC224 & $1.00 E+10$ & $4.82 E+10$ & 40 & $1.4 E-13$ & $4.31 E-08$ & $5.00 \mathrm{E}-08$ & $1.58 \mathrm{E}+00$ & & & 1.21E-01 & $3.31 E-04$ & $4.82 E+06$ & 224 \\
\hline Actinium-225 & AC225 & $1.00 E+10$ & $5.81 E+08$ & 40 & $1.4 E-13$ & $4.31 \mathrm{E}-06$ & $5.00 E-08$ & $1.58 E+00$ & & & $1.00 E+01$ & $2.74 \mathrm{E}-02$ & $5.81 \mathrm{E}+04$ & 225 \\
\hline Actinium-226 & AC226 & $1.00 E+10$ & $4.35 E+09$ & 40 & $1.4 \mathrm{E}-13$ & $4.31 E-06$ & $5.00 \mathrm{E}-08$ & $1.58 E+00$ & & & $1.33 E+00$ & $3.64 \mathrm{E}-03$ & $4.35 E+05$ & 226 \\
\hline Actinlum-227 & $A \overline{C 227}$ & $1.00 \mathrm{E}+10$ & $7.24 E+05$ & 40 & $1.4 \mathrm{E}-13$ & $4.31 \mathrm{E}-06$ & $5.00 \mathrm{E}-08$ & $1.58 \mathrm{E}+00$ & & & $7.95 E+03$ & $2.18 E+01$ & $7.24 E+01$ & 227 \\
\hline Actinium-228 & AC228 & $1.00 E+10$ & $2.25 \mathrm{E}+10$ & 40 & $1.4 \mathrm{E}-13$ & $4.31 \mathrm{E}-06$ & 5.00 E-0B & $1.58 E+00$ & & & $2.55 \mathrm{E}-01$ & $6.98 E-04$ & $2.25 E+06$ & 228 \\
\hline Americlum-237 & AM237 & $1.00 \mathrm{E}+10$ & $1.09 E+11$ & 40 & $1.4 \mathrm{E}-13$ & $4.31 \mathrm{E}-06$ & $5.00 E-08$ & $1.58 E+00$ & $8.00 \mathrm{E}-05$ & $2.92 \mathrm{E}-02$ & 5.07E-02 & $1.39 \mathrm{E}-04$ & $1.09 E+07$ & 237 \\
\hline Americium-238 & AM238 & $1.00 \mathrm{E}+10$ & $8.06 \mathrm{E}+10$ & 40 & $1.4 \mathrm{E}-13$ & $4.31 \mathrm{E}-06$ & $5.00 \mathrm{E}-08$ & $1.58 E+00$ & $8.00 E-05$ & $2.82 \mathrm{E}-02$ & $6.81 \mathrm{E}-02$ & $1.86 E-04$ & $8.06 E+06$ & 238 \\
\hline Americlum-239 & AM239 & $1.00 E+10$ & $1.10 E+10$ & 40 & $1.4 E-13$ & $4.31 \mathrm{E}-08$ & $5.00 E-08$ & $9.58 E+00$ & $8.00 \mathrm{E}-05$ & $2.02 \mathrm{E}-02$ & 4.96E-01. & $1.36 \mathrm{E}-03$ & $1.10 E+06$ & 239 \\
\hline Americlum-240 & AM240 & $1.00 E+10$ & $2.57 E+09$ & 40 & $1.4 \mathrm{E}-13$ & 4.31E-06 & $5.00 \mathrm{E}-08$ & $1.58 E+00$ & $8.00 E-05$ & $2.92 \mathrm{E}-02$ & $2.12 E+00$ & $5.80 \mathrm{E}-03$ & $2.57 E+05$ & 240 \\
\hline Americium-241 & AM241 & $1.00 \mathrm{E}+10$ & $3.43 E+04$ & 40 & $1.4 \mathrm{E}-13$ & $4.31 \mathrm{E}-06$ & $5.00 \mathrm{E}-13$ & $1.58 \mathrm{E}-05$ & 8.00E-05 & $2.92 \mathrm{E}-02$ & $1.58 E+05$ & $4.33 E+02$ & $3.43 \mathrm{E}+00$ & 241 \\
\hline Americium-242 & AM242 & $1.00 E+10$ & $8.06 \mathrm{E}+09$ & 40 & $1.4 \mathrm{E}-13$ & $4.31 E-0 B$ & $5.00 \mathrm{E}-13$ & $1.5 \mathrm{BE}-05$ & $8.00 E-05$ & $2.92 \mathrm{E}-02$ & $6.70 \mathrm{E}-01$ & $1.83 \mathrm{E}-03$ & $8.08 E+05$ & 242 \\
\hline Americium-242M & AM242M & $1.00 E+10$ & $9.73 E+04$ & 40 & $1.4 \mathrm{E}-13$ & $4.31 \mathrm{E}-06$ & $5.00 \mathrm{E}-13$ & $1.58 \mathrm{E}=05$ & $8.00 E-05$ & $2.92 \mathrm{E}-02$ & $5.55 E+04$ & $1.52 \mathrm{E}+02$ & $9.73 E+00$ & 242 \\
\hline Americium-243 & AM243 & $1.00 E+10$ & $1.99 E+03$ & 40 & $1.4 \mathrm{E}-13$ & $4.31 \mathrm{E}-06$ & $5,00 \mathrm{E}-13$ & $1.58 \mathrm{E}-05$ & $8.00 E-05$ & $2.92 \mathrm{E}-02$ & $2.70 E+06$ & $7.39 E+03$ & $1.99 \mathrm{E}-01$ & 243 \\
\hline Canton-14 & C-14 & $1.00 \mathrm{E}+10$ & $4.47 E+04$ & & $1.4 \mathrm{E}-13$ & 4.31E-0B & 1.00E-12 & $3.16 \mathrm{E}-05$ & & & $2.09 E+06$ & $5.72 E+03$ & $4.47 E+00$ & 14 \\
\hline Cerium-134 & CE134 & $1.00 \mathrm{E}+10$ & $3.25 \mathrm{E}+09$ & 40 & $1.4 \mathrm{E}-13$ & 4.31E-06 & $5.00 \mathrm{E}-11$ & $1.58 \mathrm{E}-03$ & & & $3.00 \mathrm{E}+00$ & $8.21 E-03$ & $3.25 \mathrm{E}+05$ & 134 \\
\hline Cerium-135 & CE135 & $1.00 \mathrm{E}+10$ & $1.32 E+10$ & 40 & $1.4 E-13$ & 4.31E-06 & $5.00 \mathrm{E}-11$ & $1.58 \mathrm{E}-03$ & & & $7.33 \mathrm{E}-01$ & 2.01E-03 & $1.32 E+06$ & 135 \\
\hline Cerium-137 & CE137 & $1.00 E+10$ & $2.54 E+10$ & 40 & $1.4 \mathrm{E}-13$ & $4.31 \mathrm{E}-06$ & $5.00 \mathrm{E}-11$ & $1.58 \mathrm{E}-03$ & & & $3.75 E-01$ & $1.03 \mathrm{E}-03$ & $2.54 E+06$ & 137 \\
\hline Cerium-137M & CE137M & $1.00 E+10$ & $6.67 \mathrm{E}+09$ & 40 & $1.4 \mathrm{E}-13$ & $4.31 E-06$ & $5.00 \mathrm{E}-11$ & $1.58 \mathrm{E}-03$ & & & $1.43 E+00$ & $3.92 \mathrm{E}-03$ & $6.67 E+05$ & 137 \\
\hline Cerium-139 & CE139 & $1.00 E+10$ & $6.81 E+07$ & 40 & $1.4 E-13$ & $4.31 \mathrm{E}-06$ & $5.00 \mathrm{E}-11$ & $1.58 \mathrm{E}-03$ & & & $1.38 E+02$ & 3.78E-01 & $6.81 E+03$ & 139 \\
\hline Cerium-144 & CE144 & $1.00 E+10$ & $3.20 \mathrm{E}+07$ & 40 & $1.4 \mathrm{E}-13$ & 4.31E-08 & $5.00 \mathrm{E}-11$ & $1.58 \mathrm{E}-03$ & & & $2.84 E+02$ & $7.78 \mathrm{E}-01$ & $3.20 E+03$ & 144 \\
\hline Cesium-130 & CS130 & $1.00 E+10$ & $4.83 E+11$ & 40 & $1.4 \mathrm{E}-13$ & $4.31 \mathrm{E}-06$ & $5.00 E-08$ & $1.58 E+00$ & 3.00E-05 & $1.10 E-02$ & $2.08 \mathrm{E}-02$ & $5.69 \mathrm{E}-05$ & $4.83 E+07$ & 130 \\
\hline Coslum-132 & CS132 & $1.00 E+10$ & $1.53 E+09$ & 40 & $1.4 \mathrm{E}-13$ & $4.31 \mathrm{E}-06$ & $5.00 \mathrm{E}-08$ & $1.58 E+00$ & $3.00 \mathrm{E}-05$ & $1.10 \mathrm{E}-02$ & $8.48 \mathrm{E}+00$ & $1.77 \mathrm{E}-02$ & $1.53 E+05$ & 132 \\
\hline Coslum-134 & CS134 & $1.00 \mathrm{E}+10$ & $1.30 E+07$ & 40 & $1.4 \mathrm{E}-13$ & $4.31 E-08$ & $5.00 \mathrm{E}-10$ & $1.58 \mathrm{E}-02$ & 3.00E-05 & $1.10 \mathrm{E}-02$ & $7.53 \mathrm{E}+02$ & $2.06 \mathrm{E}+00$ & $1.30 E+03$ & 134 \\
\hline Ceslum-135 & CS135 & $1.00 E+10$ & $1.15 E+01$ & 40 & $1.4 \mathrm{E}-13$ & $4.31 E-06$ & $5.00 \mathrm{E}-10$ & $1.58 E-02$ & $3.00 \mathrm{E}-05$ & $1.10 \mathrm{E}-02$ & $8.40 E+08$ & $2.30 \mathrm{E}+06$ & $1.15 \mathrm{E}-03$ & 135 \\
\hline Cosium-136 & CS138 & $1.00 \mathrm{E}+10$ & $7.34 E+08$ & 40 & $1.4 \mathrm{E}-13$ & $4.31 \mathrm{E}-06$ & $5.00 \mathrm{E}-08$ & $1.5 B E+00$ & $3.00 \mathrm{E}-05$ & $1.10 \mathrm{E}-02$ & $1.31 E+01$ & $3.59 \mathrm{E}-02$ & $7.34 E+04$ & 136 \\
\hline Cesium-137+D & CS137 & $1.00 \mathrm{E}+10$ & $8.67 E+05$ & 40 & $1.4 E-13$ & $4.31 \mathrm{E}-06$ & $5.00 E-10$ & $1.58 \mathrm{E}-02$ & $3.00 E-05$ & $1,10 \mathrm{E}-02$ & $1.10 E+04$ & $3.01 E+01$ & $8.67 E+01$ & 137 \\
\hline Cesium-138 & CS138 & $1.00 E+10$ & $4.23 E+11$ & 40 & $1.4 \mathrm{E}-13$ & $4.31 \mathrm{E}-06$ & 5.00 E.08 & $1.58 \mathrm{E}+00$ & $3.00 \mathrm{E}-05$ & $1.10 E \cdot 02$ & $2.24 \mathrm{E}-02$ & $6.13 \mathrm{E}-05$ & $4.23 E+07$ & 138 \\
\hline Chloring-36 & CL.36 & $1.00 \mathrm{E}+10$ & $3.30 E+02$ & 0 & $1.4 E-13$ & $4.31 E-06$ & $5.00 \mathrm{E}-08$ & $1.58 E+00$ & 1.00E-06 & $3.65 \mathrm{E}-04$ & $1.10 E+08$ & $3.01 E+05$ & $3.30 \mathrm{E}-02$ & 36 \\
\hline Cobalt-55 & Co55 & $1.00 E+10$ & $3.25 E+10$ & 40 & $1.4 \mathrm{E}-13$ & $4.31 \mathrm{E}-08$ & $5.00 \mathrm{E}-08$ & $1.58 E+00$ & $3.00 \mathrm{E}-05$ & $1.10 E-02$ & 7.31E-01 & $2.00 \mathrm{E}-03$ & $3.25 \mathrm{E}+08$ & 55 \\
\hline Cobalt-56 & $\cos 6$ & $1.00 E+10$ & $2.86 E+08$ & 40 & $1.4 \mathrm{E}-13$ & 4.31E-06 & $5.00 \mathrm{E}-08$ & $1.58 E+00$ & $3.00 \mathrm{E}-05$ & $1.10 \mathrm{E}-02$ & $7.88 \mathrm{E}+01$ & $2.16 E-01$ & $2.96 E+04$ & 58 \\
\hline Cobalt-57 & $\cos 7$ & $1.00 E+10$ & $8.46 \mathrm{E}+07$ & 40 & $1.4 \mathrm{E}-13$ & $4.37 E-08$ & $5.00 \mathrm{E}-11$ & $1.58 \mathrm{E} \cdot 03$ & $3.00 E-05$ & $1.10 \mathrm{E}-02$ & $2.71 E+02$ & 7.42E-01 & $8.46 E+03$ & 57 \\
\hline Cobalt-58 & $\cos 8$ & $1.00 \mathrm{E}+10$ & $3.18 \mathrm{E}+08$ & 40 & $1.4 \mathrm{E}-13$ & 4.31E-06 & $5.00 \mathrm{E}-08$ & $1.58 E+00$ & 3.00 E-05 & $1.10 \mathrm{E}-02$ & $7.08 \mathrm{E}+01$ & 1.84E-01 & $3.18 E+04$ & 58 \\
\hline Cobalt-58M & $\cos 8 \mathrm{M}$ & $1.00 E+10$ & $6.01 \mathrm{E}+10$ & 40 & $1.4 \mathrm{E}-13$ & $4.31 \mathrm{E}-06$ & $5.00 E-08$ & $1.58 \mathrm{E}+00$ & 3.00E-05 & $1.10 \mathrm{E}-02$ & $3.81 \mathrm{E}-01$ & $1.04 \mathrm{E} .03$ & $5.91 E+08$ & 58 \\
\hline Cobait-60 & 0060 & $1.00 \mathrm{E}+10$ & $1.13 E+07$ & 40 & $1.4 E-13$ & $4.31 \mathrm{E}-08$ & $5.00 \mathrm{E}-11$ & $1.58 \mathrm{E}-03$ & $3.00 \mathrm{E}-05$ & $1.10 E-02$ & $1.83 E+03$ & $5.28 \mathrm{E}+00$ & $1.13 E+03$ & 60 \\
\hline Curium-240 & CM240 & $1.00 E+10$ & $2.02 E+00$ & 40 & $1.4 \mathrm{E}-13$ & $4.31 \mathrm{E}-06$ & $5.00 E-08$ & $1.58 E+00$ & & & $2.70 E+01$ & $7.39 \mathrm{E}-02$ & $2.02 \mathrm{E}+04$ & 240 \\
\hline Curum-242 & CM242 & $1.00 E+10$ & $3.31 \mathrm{E}+07$ & 40 & $1.4 \mathrm{E}-13$ & 4.31 E-06 & $5.00 E-13$ & $1.5 \mathrm{BE}-05$ & & & $1.69 \mathrm{E}+02$ & $4.46 \mathrm{E}-01$ & $3.31 E+03$ & 242 \\
\hline Curium-243 & CM243 & $1.00 \mathrm{E}-03$ & $5.17 E-0 B$ & 40 & $1.4 \mathrm{E}-13$ & $4.31 E-08$ & $5.00 \mathrm{E}-11$ & $1,68 \mathrm{E} \cdot 03$ & & & $1.04 E+04$ & $2.85 E+01$ & $5.17 E+01$ & 243 \\
\hline Curium-244 & CM244 & $1.00 \mathrm{E}-03$ & $8.10 E-08$ & 40 & $1.4 \mathrm{E}-13$ & $4.31 \mathrm{E}-08$ & $5.00 \mathrm{E}-11$ & $1.58 \mathrm{E} .03$ & & & $8.61 \mathrm{E}+03$ & $1.81 \mathrm{E}+01$ & $8.10 E+01$ & 244 \\
\hline Curium-245 & CM245 & $1.00 E+10$ & $1.72 \mathrm{E}+03$ & 40 & $1,4 E-13$ & 4.31E-06 & $5.00 \mathrm{E}-13$ & $1.58 E-05$ & & & $3.10 E+06$ & $8.49 E+03$ & $1.72 E-01$ & 245 \\
\hline Curium-248 & CM248 & $1.00 E+10$ & $3.07 E+03$ & 40 & $1.4 \mathrm{E}-13$ & 4.31E.06 & $5.00 \mathrm{E}-08$ & $1.58 E+00$ & & & $1.73 E+06$ & $4.74 E+03$ & $3.07 E-01$ & 246 \\
\hline Curium-248 & CM248 & $1.00 E+10$ & $4.25 E+01$ & 40 & $1.4 E-13$ & $4.31 E .06$ & $5.00 E-08$ & $1.58 E+00$ & & & $1.24 \mathrm{E}+08$ & $3.39 \mathrm{E}+05$ & $4.25 \mathrm{E}-03$ & 248 \\
\hline Europlium-145 & EU145 & $1.00 \mathrm{E}+10$ & $1.52 E+09$ & 40 & $1.4 E-13$ & 4.31E-OB & $5.00 E-08$ & $1.68 E+00$ & $8.00 \mathrm{E}-05$ & $2.92 \mathrm{E}-02$ & $5.94 E+00$ & $1.63 \mathrm{E}-02$ & $1.52 E+05$ & 145 \\
\hline Europlum-146 & EU148 & $1.00 \mathrm{E}+10$ & $1.94 E+09$ & 40 & $1.4 E-13$ & $4: 31 \mathrm{E}-06$ & $6.00 \mathrm{E}-08$ & $1.58 E+00$ & $8.00 \mathrm{E}-05$ & $2.92 \mathrm{E}-02$ & $4.61 E+00$ & $1.26 \mathrm{E}-02$ & $1.94 \mathrm{E}+05$ & 146 \\
\hline Europlum-147 & EU147 & $1.00 E+10$ & $3.70 E+08$ & 40 & $1.4 \mathrm{E}-13$ & 4.31E-06 & $5.00 \mathrm{E}-08$ & $1.58 E+00$ & $8.00 E-05$ & $2.92 \mathrm{E}-02$ & $2.40 E+01$ & 6.57 E-02 & $3.70 \mathrm{E}+0.4$ & 147 \\
\hline Europium-14B & EU148 & $1.00 E+10$ & $1.62 \mathrm{E}+0 \mathrm{~B}$ & 40 & $1.4 E-13$ & $4.31 E^{206}$ & $5.00 \mathrm{E}-08$ & $1.58 E+00$ & $8.00 E-05$ & $2.92 \mathrm{E}-02$ & $5.45 E+01$ & $1.49 \mathrm{E} \cdot 01$ & $1.62 \mathrm{E}+04$ & 148 \\
\hline Europium-149 & EU149 & $1.00 E+10$ & \begin{tabular}{|l|}
$0.42 E+07$ \\
\end{tabular} & 40 & $1.4 \mathrm{E}-13$ & 4.37E-0B & $5.00 E-08$ & $1.58 \mathrm{E}+00$ & $8.00 E-05$ & $2.92 E-02$ & $9.31 E+0.1$ & $2.55 \mathrm{E}-01$ & $9.42 E+03$ & 148 \\
\hline
\end{tabular}


Table D.2. (contd)

\begin{tabular}{|c|c|c|c|c|c|c|c|c|c|c|c|c|c|c|}
\hline \multirow[b]{2}{*}{ Radionuclido } & \multirow[b]{2}{*}{ ID Codo } & \multicolumn{2}{|c|}{$\begin{array}{l}\text { Aqueous } \\
\text { Solubility }\end{array}$} & \multirow{2}{*}{$\begin{array}{c}\begin{array}{c}\text { Sorption } \\
K_{d}\end{array} \\
(\mathrm{~cm} 3 / \mathrm{g}) \\
\end{array}$} & \multicolumn{2}{|c|}{$\begin{array}{c}\begin{array}{c}\text { Fractlonal Release } \\
\text { Rate }\end{array} \\
\end{array}$} & \multicolumn{2}{|c|}{$\begin{array}{c}\text { Cement } \\
\text { Diffusion Coefficient }\end{array}$} & \multicolumn{2}{|c|}{$\begin{array}{c}\text { Fractional Release } \\
\text { Rate } \\
\end{array}$} & \multicolumn{2}{|c|}{$\begin{array}{c}\text { Decay } \\
\text { Half-Lifo }\end{array}$} & \multirow{2}{*}{$\begin{array}{l}\begin{array}{l}\text { Specific } \\
\text { Aclivity }\end{array} \\
\text { (C)/g) }\end{array}$} & \multirow{2}{*}{\begin{tabular}{|c}
$\begin{array}{c}\text { Atomlc } \\
\text { Mass }\end{array}$ \\
(gimol) \\
\end{tabular}} \\
\hline & & (mg/L) & $\left(\mathrm{C} / 1 \mathrm{~cm}^{3}\right)$ & & $(1 / 8)$ & $(1 / y n)$ & $\left(\mathrm{cm}^{2} / \mathrm{s}\right)$ & $\left(\mathrm{cm}^{2} / \mathrm{yr}\right)$ & (1/d) & (1/yr) & (d) & (vo) & & \\
\hline Europlum-150A & EU150A & $1.00 E+10$ & $1.66 \mathrm{E}+10$ & 40 & $9.4 E-13$ & 4.31E-06 & $5.00 \mathrm{E}-08$ & $1.58 E+00$ & $8.00 \mathrm{E}-05$ & $2.92 E-02$ & $5.25 \mathrm{E}-01$ & $1.44 \mathrm{E}-03$ & $1.66 \mathrm{E}+06$ & \\
\hline Europlum-1508 & EU150B & $9.00 E+10$ & $6.87 E+05$ & 40 & $1.4 E-13$ & $4.31 E-06$ & $5.00 \mathrm{E}-08$ & $1.58 E+00$ & $8.00 E-05$ & $2.92 \mathrm{E}-02$ & $1.25 E+04$ & $3.42 E+01$ & $6.97 E+01$ & 150 \\
\hline Europium-152 & EU152 & $1.00 E+10$ & $1.77 \mathrm{E}+06$ & 40 & $1.4 \mathrm{E}-13$ & $4.31 \mathrm{E}-06$ & $5.00 E-11$ & $1.58 \mathrm{E}-03$ & $8.00 E-05$ & $2.92 E-02$ & $4.87 E+03$ & $1.33 E+01$ & $1.77 E+02$ & 152 \\
\hline Europlum-154 & EU154 & $1.00 \mathrm{E}+10$ & $2.64 E+08$ & 40 & $1.4 \mathrm{E}-13$ & 4.31E-06 & $5.00 \mathrm{E}-11$ & 1.58 E-03 & $8.00 \mathrm{E} \cdot 05$ & $2.92 E-02$ & $3.21 E+03$ & $8.79 E+00$ & $2.64 E+02$ & 154 \\
\hline Europlum-155 & EU155 & $1.00 \mathrm{E}+10$ & $4.66 \mathrm{E}+0 \mathrm{~B}$ & 40 & $1.4 \mathrm{E}-13$ & 4.31E-06 & $5.00 \mathrm{E}-11$ & $1.58 \mathrm{E}-03$ & $8.00 \mathrm{E}-05$ & $2.02 \mathrm{E}-02$ & $1.81 \mathrm{E}+03$ & $4.86 E+00$ & $4.66 E+02$ & 155 \\
\hline Iodine-129 & 1129 & $1.00 E+10$ & $1.77 E+00$ & 0.6 & $1.4 \mathrm{E}-13$ & $4.31 \mathrm{E}-08$ & $5.00 \mathrm{E}-08$ & $1.58 E+00$ & & & $5.73 E+09$ & $1.57 E+07$ & $1.77 E-04$ & 129 \\
\hline loding-131 & 1131. & $1.00 E+10$ & $1.24 \mathrm{E}+09$ & 0.8 & $1.4 \mathrm{E}-13$ & 4.31E-08 & 5.00 E-08 & $1.58 E+00$ & & & $8.04 E+00$ & $2.20 \mathrm{E} \cdot 02$ & $1.24 E+05$ & 131 \\
\hline Ioding-135 & 1135 & $1.00 E+10$ & $3,52 E+10$ & 0.6 & $1.4 \mathrm{E}-13$ & $4.31 \mathrm{E}-06$ & $5.00 \mathrm{E}-08$ & $1.58 E+00$ & & & $2.75 E-01$ & $7.53 \mathrm{E}-04$ & $3.52 E+06$ & 135 \\
\hline Lead-203 & PB203 & $2.00 \mathrm{E}-01$ & $5.93 E-02$ & 40 & $1.4 \mathrm{E}-13$ & $4.31 E-08$ & $5.00 \mathrm{E}-08$ & $1.58 E+00$ & & & $2.17 E+00$ & $5.94 \mathrm{E} \cdot 03$ & $2.97 \mathrm{E}+05$ & 203 \\
\hline Lead-200 & PB209 & $2.00 \mathrm{E}-01$ & $9.20 \mathrm{E}-01$ & 40 & $1.4 \mathrm{E}-13$ & 4.31 E.06 & $5.00 E-08$ & $1.58 E+00$ & & & $1.36 E-01$ & $3.72 E-04$ & $4.60 E+08$ & 209 \\
\hline Lead-210 & PB210 & $2.00 \mathrm{E}-01$ & $1.53 \mathrm{E}-05$ & 40 & $1.4 \mathrm{E}-13$ & $4.31 E-06$ & $1.00 \mathrm{E}-11$ & $3.16 \mathrm{E}-04$ & & & $8.15 E+03$ & $2.23 E+01$ & $7.64 E+01$ & 210 \\
\hline Lead-211 & $P 8211$ & $2.00 \mathrm{E}-01$ & $4.84 E+00$ & 40 & $1.4 \mathrm{E}-13$ & $4.31 \mathrm{E}-06$ & $5.00 E-08$ & $1.58 E+00$ & & & $2.51 \mathrm{E}-02$ & $6.87 \mathrm{E}-05$ & $2.47 E+07$ & 211 \\
\hline Legd-212 & PB212 & $2.00 E-01$ & $2.78 E-01$ & 40 & $1.4 \mathrm{E}-13$ & 4.31E-06 & $1.00 \mathrm{E}-11$ & $3.16 \mathrm{E}-04$ & & & $4.43 \mathrm{E}-01$ & $1.21 \mathrm{E}-03$ & $1.39 E+06$ & 212 \\
\hline Neplunlum-237 & NP237 & $9.00 \mathrm{E}+02$ & $6.35 \mathrm{E}-07$ & 10 & $1.4 \mathrm{E}-13$ & $4.31 E-06$ & $5.00 \mathrm{E}-10$ & $1.58 \mathrm{E}-02$ & & & $7.82 E+08$ & $2.14 E+06$ & $7.05 \mathrm{E}-04$ & 237 \\
\hline Noplunium-238 & NP238 & $1.00 E+10$ & $2.59 E+09$ & 10 & $1.4 \mathrm{E}-13$ & $4.31 \mathrm{E}-06$ & $5.00 \mathrm{E}-13$ & $1.58 \mathrm{E}-05$ & & & $2.12 E+00$ & $5.80 \mathrm{E}-03$ & $2.59 E+05$ & 238 \\
\hline Neplunlum-239 & NP239 & $1.00 E+10$ & $2.32 E+00$ & 10 & $1.4 \mathrm{E}-13$ & $4.31 \mathrm{E}-06$ & $5.00 \mathrm{E}-13$ & $1.5 B E-05$ & & & $2.36 E+00$ & $6.46 \mathrm{E}-03$ & $2.32 E+05$ & 239 \\
\hline Nickel.56 & NI56 & $1.00 E+10$ & $3.83 E+09$ & 40 & $1.4 E-13$ & $4.31 \mathrm{E}-08$ & $5.00 \mathrm{E}-10$ & $1.58 \mathrm{E}-02$ & $1.00 \mathrm{E}-05$ & $3.65 \mathrm{E}-03$ & $6.10 E+00$ & $1.67 E-02$ & $3.83 E+05$ & 56 \\
\hline Nickel-57 & N157 & $1.00 \mathrm{E}+10$ & $1.53 \mathrm{E}+10$ & 40 & $1.4 \mathrm{E}-13$ & $4.31 \mathrm{E}-06$ & $5.00 \mathrm{E}-10$ & $1.58 \mathrm{E}-02$ & $1.00 \mathrm{E}-05$ & $3.65 \mathrm{E}-03$ & $1.50 \mathrm{E}+00$ & $4.11 \mathrm{E}-03$ & $1.53 \mathrm{E}+06$ & 57 \\
\hline$\frac{\text { Nickel.59 }}{\text { Nicke.63 }}$ & NI59 & $1.00 \mathrm{E}+10$ & $8.08 E+02$ & 40 & $1.4 \mathrm{E}-13$ & $4.39 \mathrm{E}-08$ & $5.00 \mathrm{E}-10$ & $1.58 \mathrm{~B} \cdot 02$ & $1.00 \mathrm{E}-05$ & $3.65 \mathrm{E}-03$ & $2.74 E+07$ & $7.50 E+04$ & $8.08 \mathrm{E} \cdot 02$ & 59 \\
\hline$\frac{\text { Nickel-63 }}{\text { Niobium-88 }}$ & NI63 & $1.00 \mathrm{E}+10$ & $5.91 \mathrm{E}+05$ & 40 & $1.4 E-13$ & $4.31 E-06$ & $5.00 E-10$ & $1.58 E-02$ & $1.00 \mathrm{E}-05$ & \begin{tabular}{|c|}
$3.65 E-03$ \\
\end{tabular} & $3.51 E+04$ & $0.61 E+01$ & $5.91 E+01$ & 63 \\
\hline Niobium-88 & NB68 & $1.00 \mathrm{E}+10$ & $1.50 \mathrm{E}+12$ & 40 & $1.4 \mathrm{E}-13$ & $4.31 \mathrm{E}-0 \mathrm{~B}$ & $5.00 \mathrm{E}-08$ & $1.58 E+00$ & & & $9.93 \mathrm{E}-03$ & $2.72 E-05$ & $1.50 E+08$ & 88 \\
\hline Nioblum-89A & NB88A & $1.00 E+10$ & $3.21 \mathrm{E}+11$ & 40 & $1.4 \mathrm{E}-13$ & 4.31E.06 & $5.00 \mathrm{E}-08$ & $1.58 \bar{E}+00$ & & & $4.58 \mathrm{E}-02$ & $1.25 \mathrm{E}-04$ & $3.21 E+07$ & 89 \\
\hline$\frac{\text { Nioblum-89B }}{\text { Ninblum-90 }}$ & NAB8B & $1.00 E+10$ & $1.74 E+11$ & 40 & $1.4 E-13$ & $4.31 E-06$ & $5.00 \mathrm{E}-08$ & $1.58 \mathrm{E}+00$ & & & $8.46 \mathrm{E}-02$ & $2.32 E-04$ & 1.74E+07 & 89 \\
\hline Nloblum-90 & NE90 & $1.00 E+10$ & $2.39 E+10$ & 40 & $1.4 \mathrm{E}-13$ & $4.31 \mathrm{E}-08$ & $5.00 \mathrm{E}-08$ & $1.58 E+00$ & & & $6.08 E-01$ & $1,66 \mathrm{E}-03$ & $2.39 E+08$ & 90 \\
\hline Nloblum-93M & NB93M & $1.00 \mathrm{E}+10$ & $2.83 E+06$ & 40 & $1.4 \mathrm{E}-13$ & $4.31 \mathrm{E}-06$ & $5.00 E-08$ & $1.58 E+00$ & & & $4.97 \mathrm{E}+03$ & $1.36 E+01$ & $2.83 E+02$ & 93 \\
\hline \begin{tabular}{|l} 
Nioblum-94 \\
Niobium-95
\end{tabular} & NB94 & $1.00 E+10$ & $1.88 \mathrm{~B}+03$ & 40 & $1.4 E-13$ & $4.31 \mathrm{E}-06$ & $5.00 E-08$ & $1.58 E+00$ & & & $7.41 E+06$ & $2.03 E+04$ & $1.88 \mathrm{E}-01$ & 94 \\
\hline \begin{tabular}{|l} 
Niobium-95 \\
Piulun-234
\end{tabular} & NB85 & $1.00 \mathrm{E}+10$ & $3.91 E+08$ & 40 & $1.4 E-13$ & $4.31 E-08$ & $5.00 \mathrm{E}-08$ & $1.58 E+00$ & & & $3.52 E+01$ & $9.64 E-02$ & $3.91 \mathrm{E}+04$ & 95 \\
\hline \begin{tabular}{|l} 
Plutonium-234 \\
Plutonlum-235
\end{tabular} & PU234 & $9.00 E+10$ & $1.52 E+10$ & 40 & $1.4 E-13$ & 4.31E-06 & $5.00 \mathrm{E}-08$ & 1.58E+00 & $8.00 \mathrm{E}-05$ & $2.92 \mathrm{E}-02$ & $3.67 \mathrm{E}-01$ & $1.00 \mathrm{E}-03$ & $1.52 E+06$ & 234 \\
\hline $\begin{array}{l}\text { Plutonium-235 } \\
\text { Plutonium-236 }\end{array}$ & PU235 & $1.00 E+10$ & $3.16 E+19$ & 40 & $1.4 \mathrm{E}-13$ & 4.31E.06 & $5.00 \mathrm{E} \cdot 08$ & $1.58 E+00$ & $8.00 \mathrm{E}-05$ & $2.92 E-02$ & $1.76 \mathrm{E}-02$ & 4.82E-05 & 3.16E+07 & 235 \\
\hline $\begin{array}{l}\text { Plutonium-236 } \\
\text { Plutonlum-237 }\end{array}$ & PU236 & $1.00 E+10$ & $5.32 E+06$ & 40 & $1.4 E-13$ & $4.31 \mathrm{E}-06$ & $5.00 \mathrm{E}-08$ & $1.58 \mathrm{E}+0 \mathrm{D}$ & $8.00 \mathrm{E}-05$ & 2.92E-02 & $1.04 E+03$ & $2.85 E+00$ & $5.32 E+02$ & 236 \\
\hline $\begin{array}{l}\text { Plutonlum-237 } \\
\text { Plutonium-238 }\end{array}$ & PU237 & $1.00 E+10$ & $1.22 \mathrm{E}+08$ & 40 & $1.4 \mathrm{E}-13$ & $4.31 E-08$ & $5.00 \mathrm{E}-08$ & $1.58 E+00$ & $8.00 \mathrm{E}-05$ & $2.92 E-02$ & $4.53 \mathrm{E}+01$ & 1.24E-01 & $1.22 \mathrm{E}+04$ & 237 \\
\hline \begin{tabular}{|l} 
Plutonium-238 \\
Plutonlum-239
\end{tabular} & PिU23B & $7.00 \mathrm{E}+01$ & $1.20 \mathrm{E}-03$ & 40 & $1.4 E-13$ & $4.31 E-06$ & $5.00 \mathrm{E}-11$ & $1.58 \mathrm{E}-03$ & $8.00 \mathrm{E}-05$ & $2.82 \mathrm{E}-02$ & $3.20 E+04$ & $8.76 E+01$ & $1.72 \mathrm{E}+01$ & 238 \\
\hline $\begin{array}{l}\text { Plutonlum-239 } \\
\text { Plutonlum-240 }\end{array}$ & PU239 & $7.00 \mathrm{E}+01$ & $4.35 \mathrm{E}-08$ & 40 & $1.4 E-13$ & $4.31 E-08$ & $5.00 \mathrm{E}-11$ & $1.58 \mathrm{E}-03$ & $8.00 \mathrm{E}-05$ & $2.92 \mathrm{E}-02$ & $8.79 E+0 B$ & $2.41 E+04$ & $6.22 \mathrm{E}-02$ & 239 \\
\hline \begin{tabular}{|l|} 
Plutonlum-240 \\
Plutonium-241
\end{tabular} & PU240 & $7.00 E+01$ & $1.59 \mathrm{E}-05$ & 40 & $1.4 E-13$ & $4.31 E-06$ & $5.00 \mathrm{E}-11$ & $1.58 \mathrm{E}-03$ & $8.00 \mathrm{E}-05$ & $2.92 \mathrm{E}-02$ & $2.39 E+06$ & $6.54 E+03$ & $2.28 \mathrm{E}-01$ & 240 \\
\hline Plutonium-241 & PU241 & $1.00 E+10$ & $1.03 E+08$ & 40 & $1.4 E-13$ & $4.31 E-06$ & $5.00 \mathrm{E}-11$ & $1.58 E-03$ & $8.00 \mathrm{E}-05$ & $2.82 \mathrm{E}-02$ & $5.26 \mathrm{E}+03$ & $1.44 E+01$ & $1.03 \mathrm{E}+02$ & 241 \\
\hline Plutonium-242 & PU242 & $1.00 E+10$ & $3.94 E+01$ & 40 & $1.4 \mathrm{E}-13$ & 4.31E-06 & $5.00 \mathrm{E}-08$ & $1.58 E+00$ & $8.00 \mathrm{E}-05$ & $2.82 \mathrm{E}-02$ & $1.37 \mathrm{E}+08$ & $3.75 E+05$ & $3.94 \mathrm{E}-03$ & 242 \\
\hline Protactinium-231 & PA231 & $1.00 \mathrm{E}+10$ & $4.71 E+02$ & 10 & $1.4 \mathrm{E}-13$ & $4.31 \mathrm{E}-06$ & $5.00 \mathrm{E} \cdot 08$ & $1.58 E+00$ & & & $1.20 E+07$ & $3.29 \mathrm{E}+04$ & $4.71 \mathrm{E}-02$ & 231 \\
\hline Protaclinium-233 & PA233 & $1.00 E+10$ & $2.08 E+08$ & 10 & $1.4 E-13$ & $4.31 E-06$ & $5.00 \mathrm{E}-08$ & $1.58 E+00$ & & & $2.70 E+01$ & $7.39 \mathrm{E}-02$ & $2.08 E+04$ & 233 \\
\hline $\begin{array}{l}\text { Radium-223 } \\
\text { Radium-224 }\end{array}$ & RA223 & $1.00 \mathrm{E}+10$ & $5.14 E+08$ & 10 & $1.4 E-13$ & $4.39 E-08$ & $5.00 \mathrm{E}-11$ & $1.58 \mathrm{E}-03$ & & & $1.14 E+01$ & $3.12 E-02$ & $5.14 E+04$ & 223 \\
\hline Radium-224 & RA224 & $1.00 E+10$ & $1.59 E+09$ & 10 & $1.4 E-13$ & $4.31 E-06$ & $5.00 \mathrm{E}-08$ & $1.58 E+00$ & & & $3.66 E+00$ & $1.00 \mathrm{E}-02$ & $1.59 E+05$ & 224 \\
\hline Radium-225 & RA225 & $1.00 E+10$ & $3.92 E+08$ & 10 & $1.4 \mathrm{E}-13$ & $4.31 E-08$ & $5.00 \mathrm{E}-11$ & $1.58 E-03$ & & & $1.48 E+01$ & $4.05 E-02$ & $3.92 E+04$ & 225 \\
\hline Radium-226 & RA226 & $1.00 E+10$ & $9.90 \mathrm{E}+03$ & 10 & $1.4 \mathrm{E}-13$ & 4.31E-06 & $5.00 \mathrm{E}-11$ & $9.58 \mathrm{E}-03$ & & & $5.84 E+05$ & $1.60 \mathrm{E}+03$ & $9.90 \mathrm{E}-01$ & 226 \\
\hline Radlum-228 & RA228 & $1.00 E+10$ & $2,73 E+06$ & 10 & $1.4 \mathrm{E}-13$ & $4.31 \mathrm{E}-06$ & $5.00 E-11$ & $9.58 \mathrm{E}-03$ & & & $2.10 E+03$ & $5.75 E+00$ & $2.73 E+02$ & 228 \\
\hline Ruthenium-103 & RU103 & $1.00 E+10$ & $3.23 E+08$ & 10 & $1.4 \mathrm{E}-13$ & 4.31E.06 & $5.00 E \cdot 08$ & $1.58 \mathrm{E}+00$ & & & $3.93 \mathrm{E}+01$ & $1.08 \mathrm{E}-01$ & $3.23 E+04$ & 103 \\
\hline Ruthenium-105 & RU105 & $1.00 E+10$ & $6.73 E+10$ & 10 & $1.4 E-13$ & $4.31 E-06$ & $5.00 \mathrm{E}-08$ & $1.58 E+00$ & & & 1.85E-01 & 5.07E-04 & $6.73 \mathrm{E}+06$ & 105 \\
\hline Ruthonium-106 & RUTO6 & $1.00 \mathrm{E}+10$ & $3.35 E+07$ & 10 & $1.4 E-13$ & $4.31 E-06$ & $5.00 \mathrm{E}-08$ & $1.58 E+00$ & & & $3.68 \mathrm{E}+02$ & $1.01 E+00$ & $3.35 E+03$ & 106 \\
\hline
\end{tabular}


Table D.2. (contd)

\begin{tabular}{|c|c|c|c|c|c|c|c|c|c|c|c|c|c|c|}
\hline & & \multicolumn{2}{|c|}{$\begin{array}{l}\text { Aqueous } \\
\text { Solubility }\end{array}$} & \multirow{2}{*}{\begin{tabular}{|c|}
$\begin{array}{c}\text { Sorption } \\
K_{d}\end{array}$ \\
$(\mathrm{~cm} 3 / \mathrm{g})$
\end{tabular}} & \multicolumn{2}{|c|}{$\begin{array}{c}\text { Fractional Release } \\
\text { Rate }\end{array}$} & \multicolumn{2}{|c|}{$\begin{array}{c}\text { Cement } \\
\text { Diffusion Coefficlent }\end{array}$} & \multicolumn{2}{|c|}{$\begin{array}{c}\text { Fractional Release } \\
\text { Rate }\end{array}$} & \multicolumn{2}{|c|}{$\begin{array}{c}\text { Decay } \\
\text { Half-Life }\end{array}$} & \multirow{2}{*}{$\begin{array}{c}\text { Specific } \\
\text { Activity } \\
\text { (CU/a) }\end{array}$} & \multirow{2}{*}{$\begin{array}{c}\begin{array}{c}\text { Atomlc } \\
\text { Mass }\end{array} \\
\text { (g/mol) }\end{array}$} \\
\hline Radionuclide & ID Codo & (mg/L) & $\left(\mathrm{C} / / \mathrm{cm}^{3}\right)$ & & $(1 / 8)$ & $(1 / y r)$ & $\left(\mathrm{cm}^{2} / \mathrm{s}\right)$ & $\left(\mathrm{cm}^{2} / \mathrm{yr}\right)$ & (1/d) & $(1 / y r)$ & (d) & $(y r)$ & & \\
\hline Solenium-70 & SE70 & $1.00 \mathrm{E}+10$ & $6.55 E+11$ & 0 & $1.4 \mathrm{E}-13$ & 4.31E-06 & $5.00 \mathrm{E}-08$ & $1.58 E+00$ & & & $2.85 \mathrm{E}-02$ & $7.80 \mathrm{E}-05$ & $6.55 E+07$ & $\frac{10}{70}$ \\
\hline Selenium-73 & SE73 & $1.00 E+10$ & $6.01 E+10$ & 0 & $1.4 \mathrm{E}-13$ & 4.31E-06 & $5.00 E-08$ & $1.58 \mathrm{E}+00$ & & & $2.98 \mathrm{E}-01$ & $8.16 E-04$ & $6.01 E+06$ & 73 \\
\hline Selenium-73M & SE73M & $1.00 E+10$ & $6.61 \mathrm{E}+11$ & 0 & $9.4 \mathrm{E}-13$ & 4.31E-06 & $5.00 E-08$ & $1.58 E+00$ & & & $2.71 \mathrm{E}-02$ & $7.42 \mathrm{E}-05$ & $6.61 E+07$ & 73 \\
\hline Selenium-75 & SE75 & $1.00 E+10$ & $1.45 E+08$ & 0 & $9.4 \mathrm{E}-13$ & 4.31E-06 & $5.00 \mathrm{E}-08$ & $1.58 \mathrm{E}+00$ & & & $1.20 E+02$ & $3.29 \mathrm{E}-01$ & $1.45 E+04$ & 75 \\
\hline Selenlum-79 & SE79 & $1.00 \mathrm{E}+10$ & $6.98 \mathrm{E}+02$ & 0 & $1.4 \mathrm{E}-13$ & 4.31E-06 & $2.00 E-10$ & $6.31 E-03$ & & & $2.37 E+07$ & $6.49 E+04$ & $6.98 \mathrm{E} \cdot 02$ & 79 \\
\hline Selenlum-81 & SE81 & $1.00 E+10$ & $1.26 E+12$ & $\frac{\pi}{0}$ & $1.4 \mathrm{E}-13$ & $4.31 E-06$ & $5.00 \mathrm{E}-08$ & $1.58 \mathrm{E}+00$ & & & $1.28 \mathrm{E}-02$ & $3.50 \mathrm{E}-05$ & $1.26 \mathrm{E}+08$ & 81 \\
\hline Strontium-85 & SR85 & $1.00 E+10$ & $2.37 E+08$ & 10 & $1.4 \mathrm{E}-13$ & $4.31 E-06$ & $5.00 \mathrm{E}-08$ & $1.58 \mathrm{E}+00$ & $3.00 E-05$ & $1.10 E-02$ & $6.48 E+01$ & $1.77 \mathrm{E}-01$ & $2.37 E+04$ & 85 \\
\hline Strontium-89 & SR89 & $1.00 E+10$ & $2.91 E+08$ & 10 & $1.4 E-13$ & $4.31 E-06$ & $5.00 \mathrm{E}-11$ & $1.58 \mathrm{E}-03$ & $3.00 \mathrm{E}-05$ & $1.10 \mathrm{E}-02$ & $5.05 E+01$ & $1.38 \mathrm{E}-01$ & $2.91 E+04$ & 89 \\
\hline Strontium-90 & SR90 & $1.00 \mathrm{E}+10$ & $1.37 E+06$ & 10 & $1.4 \mathrm{E}-13$ & $4.31 \mathrm{E} .06$ & $5.00 \mathrm{E}-11$ & $1.58 \mathrm{E}-03$ & 3.00 E-05 & $1.10 \mathrm{E}-02$ & $1.06 \mathrm{E}+04$ & $2.90 E+01$ & $1.37 E+02$ & 90 \\
\hline Strontium-91 & SR91 & $1.00 E+10$ & $3.63 E+10$ & 10 & $1.4 \mathrm{E}-13$ & $4.31 \mathrm{E}-06$ & $5.00 \mathrm{E}-08$ & $1.58 \mathrm{E}+00$ & $3.00 \mathrm{E}-05$ & $1.10 \mathrm{E}-02$ & $3.96 \mathrm{E}-01$ & $1.08 \mathrm{E}-03$ & $3.63 \mathrm{E}+08$ & 91 \\
\hline Technetium-101 & TC101 & $1.00 E+10$ & $1.31 E+12$ & 0 & $1.4 \mathrm{E}-13$ & $4.31 \mathrm{E}-06$ & 1.00 E-08 & $3.16 \mathrm{E}-01$ & & & $9.86 \mathrm{E}-03$ & $2.70 \mathrm{E}-05$ & $1.31 \mathrm{E}+08$ & 101 \\
\hline Technotium-97 & TC97 & $1.00 \mathrm{E}+10$ & $1.42 \mathrm{E}+01$ & 0 & $1.4 \mathrm{E}-13$ & $4.31 \mathrm{E}-06$ & $5.00 \mathrm{E}-08$ & $1.58 E+00$ & & & $9.49 \mathrm{E}+08$ & $2.60 E+06$ & $1.42 \mathrm{E} \cdot 03$ & 97 \\
\hline Technetium-98 & $\frac{1038}{1 \mathrm{C} 98}$ & $1.00 E+10$ & $8.72 E+00$ & 0 & $1.4 \mathrm{E}-13$ & $4.31 E-06$ & $5.00 \mathrm{E}-08$ & $1.58 E+00$ & & & $1.53 E+09$ & $4.19 E+06$ & $8.72 \mathrm{E} \cdot 04$ & $\frac{98}{98}$ \\
\hline Technotium-99 & TC99 & $1.00 E+10$ & $1.70 E+02$ & 0 & $1.4 \mathrm{E}-13$ & $4.31 \mathrm{E}-06$ & $1.00 \mathrm{E}-09$ & $3.16 \mathrm{E}-02$ & & & $7.78 E+07$ & $2.13 E+05$ & $1.70 \mathrm{E} \cdot 02$ & 99 \\
\hline Thorium-227 & TH227 & $1.00 \mathrm{E}+10$ & $3.08 E+08$ & 40 & $1.4 \mathrm{E}-13$ & $4.31 \mathrm{E}-06$ & $1.00 \mathrm{E}-12$ & $3.16 \mathrm{E} \cdot 05$ & & & $1.87 E+01$ & $5.12 \mathrm{E}-02$ & $3.08 E+04$ & 227 \\
\hline Thorlum-228 & TH228 & $1.00 E+10$ & $8.20 \mathrm{E}+06$ & 40 & $1.4 \mathrm{E}-13$ & 4.31E-06 & $1.00 \mathrm{E}-12$ & $3.16 \mathrm{E}-05$ & & & $6.99 \mathrm{E}+02$ & $1.91 E+00$ & $8.20 E+02$ & 228 \\
\hline Thorium-229 & TH229 & $1.00 E+10$ & $2.13 E+03$ & 40 & $1.4 \mathrm{E}-13$ & $4.31 E-06$ & $1.00 E-12$ & $3.16 \mathrm{E} \cdot 05$ & & & $2.68 E+06$ & $7.34 E+03$ & $2.13 \mathrm{E}-01$ & 229 \\
\hline Thorium-230 & TH230 & $1.00 E+10$ & $2.02 E+02$ & 40 & $1.4 \mathrm{E}-13$ & $4.31 E-06$ & $1.00 E-12$ & $3.16 \mathrm{E}-05$ & & & $2.81 E+07$ & $7.69 E+04$ & $2.02 \mathrm{E}-02$ & 230 \\
\hline Thorium-231 & TH231 & $1.00 \mathrm{E}+10$ & $5.34 E+09$ & 40 & $1.4 \mathrm{E}-13$ & $4.31 \mathrm{E}-06$ & $5.00 \mathrm{E}-08$ & $1.58 \mathrm{E}+00$ & & & $1.06 \mathrm{E}+00$ & $2.90 \mathrm{E}-03$ & $5.34 E+05$ & 231 \\
\hline Thorium-232 & TH232 & $1.00 E+10$ & $4.00 \mathrm{E}-01$ & 40 & $1.4 \mathrm{E}-13$ & $4.31 E-06$ & $1.00 \mathrm{E}-12$ & $3.16 \mathrm{E}-05$ & & & $1.41 E+10$ & $3.86 \mathrm{E}+07$ & $4.00 \mathrm{E}-05$ & 232 \\
\hline Thorium-234 & TH234 & $1.00 E+10$ & $2.32 E+08$ & 40 & $1.4 \mathrm{E}-13$ & $4.31 \mathrm{E}-06$ & $1.00 \mathrm{E}-12$ & $3.16 \mathrm{E}-05$ & & & $2.41 E+01$ & $6.60 \mathrm{E}-02$ & $2.32 E+04$ & 234 \\
\hline Tin-113 & SN113 & $1.00 \mathrm{E}+10$ & $1.01 \mathrm{E}+08$ & 40 & $1.4 \mathrm{E}-13$ & $4.31 \mathrm{E}-06$ & 5.00E-08 & $1.58 \mathrm{E}+00$ & & & $1.15 E+02$ & $3.15 \mathrm{E}-01$ & $1.01 \mathrm{E}+04$ & 113 \\
\hline$\frac{110}{\operatorname{Tin}-121}$ & SN121 & $1.00 E+10$ & $9.56 E+09$ & 40 & $1.4 \mathrm{E}-13$ & $4.31 \mathrm{E}-06$ & $5.00 \mathrm{E} \cdot 08$ & $1.58 \mathrm{E}+00$ & & & $1.13 \mathrm{E}+00$ & $3.09 \mathrm{E}-03$ & $9.56 \mathrm{E}+05$ & 121 \\
\hline Tin-123 & $\frac{1}{S N 123}$ & $1.00 E+10$ & $8.24 E+07$ & 40 & $1.4 \mathrm{E}-13$ & $4.31 E-06$ & $5.00 \mathrm{E}-08$ & $1.58 \mathrm{E}+00$ & & & $1.29 E+02$ & $3.53 \mathrm{E}-01$ & $8.24 \mathrm{E}+03$ & 123 \\
\hline Tin-125 & SN125 & $1.00 E+10$ & $1.08 \mathrm{E}+09$ & 40 & $1.4 \mathrm{E}-13$ & $4.31 \mathrm{E}-06$ & $5.00 \mathrm{E} \cdot 08$ & $1.58 \mathrm{E}+00$ & & & $9.64 E+00$ & $2.64 \mathrm{E}-02$ & $1.08 E+05$ & 125 \\
\hline Tin-126 & SN126 & $1.00 \mathrm{E}+10$ & $2.84 E+02$ & 40 & $1.4 E-13$ & 4.31E-08 & $5.00 \mathrm{E}-08$ & $1.58 \mathrm{E}+00$ & & & $3.65 E+07$ & $9.99 E+04$ & $2.84 \mathrm{E}-02$ & 126 \\
\hline Trillum (Elemental) & H3.EL & $3.80 \mathrm{E}-03$ & $3.69 \mathrm{E}-05$ & 0 & $1.4 \mathrm{E}-13$ & $4.31 E-06$ & $5.00 \mathrm{E}-08$ & $1.58 \mathrm{E}+00$ & 1.00 E-06 & $3.65 \mathrm{E}-04$ & $4.49 E+03$ & $1.23 \mathrm{E}+01$ & $9.70 E+03$ & 3 \\
\hline$\frac{1}{\text { Tritium }(\mathrm{H} 3)}$ & $\mathrm{H3}$ & $1.00 E+10$ & $9.70 E+07$ & 0 & $1.4 \mathrm{E}-13$ & $4.31 \mathrm{E}-06$ & $5.00 \mathrm{E}-08$ & $1.58 E+00$ & $1.00 \mathrm{E}-06$ & $3.65 \mathrm{E}-04$ & $4.49 E+03$ & $1.23 E+01$ & $9.70 \mathrm{E}+03$ & 3 \\
\hline Uranium-232 & U232 & $1.00 E+10$ & $2.14 E+05$ & 0.6 & $1.4 \mathrm{E}-13$ & $4.31 \mathrm{E}-06$ & $5.00 \mathrm{E}-08$ & $1.58 \mathrm{E}+00$ & & & $2.63 E+04$ & $7.20 \mathrm{E}+01$ & $2.14 E+01$ & 232 \\
\hline Uranium-233 & U233 & $1.00 E+10$ & $9.69 E+01$ & 0.6 & $1.4 \mathrm{E}-13$ & 4.31E-06 & $1.00 \mathrm{E}-12$ & $3.16 \mathrm{E}-05$ & & & $5.79 E+07$ & $1.59 E+05$ & $9.69 \mathrm{E}-03$ & 233 \\
\hline Uranlum-234 & U234 & $1.00 E+10$ & $6.25 \mathrm{E}+01$ & 0.8 & $1.4 E-13$ & $4.31 \mathrm{E} \cdot 08$ & $1.00 \mathrm{E}-12$ & $3.16 \mathrm{E}-05$ & & & $8.03 E+07$ & $2.44 E+05$ & $6.25 \mathrm{E} \cdot 03$ & 234 \\
\hline Uranium-235 & U235 & $1.00 \mathrm{E}+10$ & $2.16 E-02$ & 0.6 & $1.4 E-13$ & $4.31 \mathrm{E}-0 \mathrm{~B}$ & $1.00 \mathrm{E}-12$ & $3.16 \mathrm{E}-05$ & & & $2.57 E+11$ & $7.04 E+08$ & $2.16 \mathrm{E}-06$ & 235 \\
\hline Uranium-236 & U236 & $1.00 E+10$ & $6.48 \mathrm{E}-01$ & 0.6 & $1.4 E-13$ & $4.31 \mathrm{E}-08$ & $1.00 \mathrm{E}-11$ & $3.16 \mathrm{E}-04$ & & & $8.55 \mathrm{E}+09$ & $2.34 E+07$ & $6.48 \mathrm{E}-05$ & 236 \\
\hline Uranium-238 & U. & $1.00 E+10$ & \begin{tabular}{|l|}
$3.37 \mathrm{E}-03$ \\
\end{tabular} & 0.6 & $1.4 \mathrm{E}-13$ & $4.31 \mathrm{E}-06$ & $1.00 \mathrm{E}-11$ & $3.16 \mathrm{E}-04$ & & & $1.63 E+12$ & $4.46 E+09$ & $3.37 \mathrm{E}-07$ & 238 \\
\hline Uranium-239 & U239 & $1.00 E+10$ & $3.35 \mathrm{E}+11$ & 0.6 & $1.4 \mathrm{E}-13$ & $4.31 \mathrm{E} \cdot 06$ & $1.00 \mathrm{E}-11$ & $3.16 \mathrm{E}-04$ & & & $1.63 \mathrm{E}-02$ & $4.46 \mathrm{E}-05$ & $3.36 E+07$ & 239 \\
\hline \begin{tabular}{|l} 
Zirconium-89 \\
\end{tabular} & ZRB9 & $1.00 E+10$ & $4.49 E+09$ & 40 & $1.4 \mathrm{E}-13$ & $4.31 E-06$ & $1.00 \mathrm{E}-12$ & $3.16 E \cdot 05$ & & & $3.27 E+00$ & $8.95 \mathrm{E} \cdot 03$ & $4.49 E+05$ & 89 \\
\hline Zirconium-83 & $\frac{1}{\text { ZR93 }}$ & $1.00 \mathrm{E}+10$ & $2.51 E+01$ & 40 & $1.4 \mathrm{E}-13$ & $4.31 \mathrm{E} \cdot 06$ & $1.00 \mathrm{E}-12$ & $3.16 \mathrm{E}-05$ & & & $5.59 E+08$ & $1.53 E+08$ & $2.51 \mathrm{E}-03$ & 93 \\
\hline
\end{tabular}




\section{Appendix E}

\section{Distribution Coefficient $\left(K_{d}\right)$ Selection for the}

Composite Analysis 


\title{
Appendix E
}

\section{Distribution Coefficient $\left(K_{d}\right)$ Selection for the Composite Analysis}

\author{
D. I. Kaplan, R. J. Serne, V. G. Johnson, and C. T. Kincaid
}

\section{E.1 Background}

The purpose of the Composite Analysis is to estimate the projected cumulative impacts of all radioactive material in the ground that may interact with projected releases from existing or planned LLW disposal facilities. Guidance was issued for the Composite Analysis to examine how the variety of wastes to be permanently disposed at a DOE site might commingle and might exceed health protective limits.

The requirement to analyze, in a single analysis all wastes that will remain at the Hanford Site forced the examination of the numerous previous analyses of individual facilities and reconciliation of the conceptual models selected and model parameters applied to those analyses. The purpose of this appendix is to document the selections made for the geochemical adsorption/desorption distribution coefficients for the Composite Analysis.

\section{E.2 Approach}

For the Composite Analysis, several assumptions were made regarding the characteristics of sorption and the model that was employed. Adsorption was assumed to be fully reversible. Thus, a single distribution coefficient was used to represent both sorption and desorption. Because of its use in previous analyses at the Hanford Site (environmental impact statements, performance assessments, and CERCLA risk assessments), the linear sorption isotherm model was selected to represent the adsorption process. Other adsorption models exist, but their large data requirements cannot be met for the suite of radionuclides examined in the Composite Analysis. A distribution coefficient $\left(\mathrm{K}_{\mathrm{d}}\right)$ defined by the following equation:

$$
\mathrm{K}_{\mathrm{d}}=\text { mass of solute on solid phase per unit mass of solid phase/concentration of solute in solution }
$$

was selected for elements and applied to all isotopes of that element. Thus, the same $\mathrm{K}_{\mathrm{d}}$ value was applied to all isotopes of uranium considered in the analysis. The $\mathrm{K}_{\mathrm{d}}$ values assembled here are based on experiments on saturated sediments. While research is underway to study the dependence of adsorption on moisture content, results are not available for a general model and the suite of radionuclides of interest. 
In deriving the $\mathrm{K}_{\mathrm{d}}$ values for elements considered in the Composite Analysis, previous analyses were examined. Specifically, $\mathrm{K}_{\mathrm{d}}$ values from the following analyses were reviewed and evaluated:

- Performance assessments for the 200 West Area solid waste burial ground (Wood et al. 1995b); the 200 East Area solid waste burial ground (Wood et al. 1996); and the interim performance assessment for low-activity waste from Hanford tanks (Mann et al. 1996) and the remedial investigation/ feasibility study report for the Environmental Restoration Disposal Facility (ERDF) (DOE 1994; Wood et al. 1995a).

- Environmental impact statements completed for the surplus production reactors (DOE 1989, 1992), environmental restoration (DOE 1996), and the Tank Waste Remediation System (TWRS) program (DOE and State of Washington Department of Ecology [Ecology] 1996).

- The closure plan for the commercial LLW disposal site operated at Hanford by US Ecology (Grant, Environmental, Chase Environmental Group, and US Ecology, Inc. 1996).

$\mathrm{K}_{\mathrm{d}}$ values used in these previous analyses are summarized in Table E.1. This table illustrates that a consistent suite of $K_{d}$ values was not selected and used by the different programs evaluating LLW disposal at Hanford.

Because of the inconsistent definition of $\mathrm{K}_{\mathrm{d}}$ values from the previous analyses at Hanford, it was necessary to evaluate all of the available data and derive a consistent set of values to use in the Composite Analysis. The first attempt at deriving a consistent set of $K_{d}$ values involved use of a single $K_{d}$ for each element. In an effort to minimize the number of simulations that must be conducted, radionuclides were assigned a $\mathrm{K}_{d}$ value that is less than or equal to its actual $\mathrm{K}_{d}$ value. However, the results of the source term release model demonstrated that a single $\mathrm{K}_{d}$ approach did not adequately represent the complexity of the disposal environment and natural subsurface system.

In the Composite Analysis, $\mathrm{K}_{\mathrm{d}}$ values were assigned in a manner designed to recognize the impacts of waste chemistry and background chemistry. The concentrations of chelating agents, salts, and organic phases as well as $\mathrm{pH}$ have been demonstrated to greatly affect the magnitude of $\mathrm{K}_{\mathrm{d}}$ values measured in the laboratory or derived from field observations. To account for the impacts of waste chemistry manifested through these factors, the sources were first categorized according to their waste compositions. The six source term categories used in the Composite Analysis are described in Table E.2.

The $\mathrm{K}_{\mathrm{d}}$ values used in the Composite Analysis were further categorized based on the estimated impacts of background chemistry (Table E.3). Three distribution coefficient zones were established to represent changing geochemical conditions away from the source: 1) the high-impact zone near the source in the vadose zone, 2) an intermediate-impact zone away from the source, but still in the vadose zone, and 3) the groundwater zone. The high-impact zone is defined as the zone where the geochemistry of the vadose zone is greatly affected by the chemical composition of the waste source. The intermediateimpact zone differs from the high-impact zone in that the effect, if any, of the source-term $\mathrm{pH}$ on $\mathrm{K}_{\mathrm{d}}$ values has disappeared; the effects of salts and organics, if present, continue to affect $K_{d}$ values. The 
intermediate-zone was defined in the vadose zone, before contaminants reach the groundwater. The groundwater zone is defined as the zone where $\mathrm{K}_{d}$ values are not affected by the chemical composition of the waste source. The background chemical composition of the groundwater zone is assumed to be greatly diluted and does not affect $\mathrm{K}_{d}$ values. The presence of chelates in the waste source represents the only aqueous constituent that could influence $K_{d}$ values in the groundwater zone.

To accommodate the different waste source-term categories and $K_{d}$ zones, $K_{d}$ values had to be assigned to fill in a matrix of the six source types and three zones (Table E.4). Unique $K_{d}$ values were not needed for all eighteen categories generated from the matrix of the three zones and six source types. Many of the categories were effectively the same and only eight different categories of $K_{d}$ values were needed. The category identified in Table E. 4 as "F" represents the far-field $\mathrm{K}_{d}$ values; category " $\mathrm{C}$ " represents the far-field $K_{d}$ values affected by chelates.

Once the $\mathrm{K}_{\mathrm{d}}$ categories were established, the geochemistry literature was reviewed to identify measured values to assign to the matrix. A range of $\mathrm{K}_{d}$ values was selected for each cell in the matrix. Generally, the lowest value of the range was used to represent the conservative estimate of $K_{d}$ for each element. "Best"-estimate values for $\mathrm{K}_{d}$ were also identified. In some cases, values other than the lowest value in the range were assigned to the conservative value. In these cases expert judgement was applied to make the assignment. Where "best" estimates were based only on expert judgement, they are identified.

\section{E.3 $\mathbf{K}_{\mathbf{d}}$ Values for the Eight Source-Zone Categories}

$K_{d}$ values for each of the eight source-zone categories identified in Table E.4 are presented in Tables E.5 through E.12. Conservative (low), "best," and likely range of $K_{d}$ values are included in the table. Additionally, a brief outline of the justification and references used to make these estimates are also provided. The "best" estimates are presented to provide guidance on what the most likely $\mathrm{K}_{d}$ value is for a given condition. Table E.10 provides $K_{d}$ values for typical groundwater conditions at Hanford.

\section{E.4 Summary Tables}

Tables E.13 through E.17 provide summaries of the $\mathrm{K}_{d}$ values presented in Tables E.5 through E.12, but without the justifications and references. Table E.13 is a summary of the best estimate values used in the Composite Analysis. Table E.14 presents the summary of best estimate $\mathrm{K}_{d}$ values, adjusted for the maximum value of $40 \mathrm{~mL} / \mathrm{g}$ replacement of all values greater than $40 \mathrm{~mL} / \mathrm{g}$. This adjustment was made to reduce the number of $K_{d}$ values that had to be modeled. Constituents with $K_{d}$ values of $40 \mathrm{~mL} / \mathrm{g}$ and greater are considered immobile in the vadose zone and groundwater. Table E.15 is a summary of the conservative values. Table E. $1 \dot{6}$ presents the summary of conservative $\mathrm{K}_{d}$ values, adjusted for the maximum value of $40 \mathrm{~mL} / \mathrm{g}$ replacement of all values greater than $40 \mathrm{~mL} / \mathrm{g}$. Table E.17 provides a summary of the ranges of $K_{d}$ values. 


\section{E.5 References}

Ames, L. L., and D. Rai. 1978. Radionuclide Interactions with Soil and Rock Media. EPA 520/6-78-007, Vol. 1, U.S. Environmental Protection Agency, Office of Radiation Programs, Las Vegas, Nevada.

Ames, L. L., and R.'J. Serne. 1991. Compilation of Data to Estimate Groundwater Migration Potential for Constituents in Active Liquid Discharges at the Hanford Site. PNL-7660, Pacific Northwest Laboratory, Richland, Washington.

Barney, G. S. 1978. Variables Affecting Sorption and Transport of Radionuclides in Hanford Subsoils. RHO-SA-87, Rockwell Hanford Operations, Richland, Washington.

Benson, D. W. 1960. Review of Soil Chemistry Research at Hanford. HW-67201, General Electric Company, Richland, Washington.

Brown, D. J. 1967. Migration Characteristics of Radionuclides Through Sediments Underlying the Hanford Reservation. ISO-SA-32, Isochem, Richland, Washington.

Delegard, C. H., and G. S. Barney. 1983. Effects of Hanford High-Level Waste Components on Sorption of Cobalt, Strontium, Neptumium, Plutonium, and Americium on Hanford Sediments. RHO-RE-ST-1 P, Rockwell Hanford Operations, Richland, Washington.

DOE - See U.S. Department of Energy.

Garnier, J. M. 1985. "Retardation of Dissolved Radiocarbon Through a Carbonated Matrix." Geochimica et Cosmochimica Acta 49:683-693.

Grant Environmental, Chase Environmental Group, and US Ecology, Inc. 1996. Site Stabilization and Closure Plan for Low-Level Radioactive Waste Management Facility, US Ecology Inc., Richlond, Washington. US Ecology, Richland, Washington.

Hajek, B. F, and K. C. Knoll. 1966. Disposal Characteristics of Plutonium $2^{\text {nd }}$ Americium in a High Salt Acid Waste. BNWL-CG. 649, General Electric Company, Richland, Washington.

Haney, W. A. 1957. Disposal of High Cobalt-60 Scavenger Wastes. HW-48862, Westinghouse Hanford Company, Richland, Washington.

Hartman, M. J., and P. E. Dresel (eds.). 1997. Hanford Site Groundwater Monitoring for Fiscal Year 1996. PNNL-11470, Pacific Northwest National Laboratory, Richland, Washington.

Johnson, V. G. 1993. Groundwater Impact Assessment Report for the 216-Z-20 Crib, 200 West Area. WHC-EP-0674, Westinghouse Hanford Company, Richland, Washington. 
Kaplan, D. I., and R. J. Serne. 1995. Distribution Coefficient Values Describing Iodine, Neptunium, Selenium, Technetium, and Uranium Sorption to Hanford Sediments. PNL-10379, Sup. 1, Pacific Northwest Laboratory, Richland, Washington.

Kaplan, D. I., R. J. Serne, and M. G. Piepho. 1995. Geochemical Factors Affecting Radionuclide Transport Through Near and Far Field at a Low-Level Waste Disposal Site. PNL-10379, Pacific Northwest Laboratory, Richland, Washington.

Kaplan, D. I., R. J. Serne, A. T. Owen, J. Conca, T. W. Wietsma, and T. L. Gervais. 1996: Radionuclide Adsorption Distribution Coefficients Measured in Hanford Sediments for the Low Level Waste Performance Assessment Project. PNNL-11385, Pacific Northwest National Laboratory, Richland, Washington.

Knoll, K. C. 1969. Reactions of Organic Wastes in Soils. HW-67201, General Electric Company, Richland, Washington.

Mann, F. M., C. R. Eiholzer, Y. Chen, N. W. Kline, A. H. Lu, B. P. McGrail, P. D. Rittmann, G. F. Williamson, J. A. Voogd, N. R. Brown, and P. E. LaMont. 1997. Hanford Low-level Tank Waste Interim Performance Assessment. HNF-EP-0884, Rev. 1, Lockheed Martin Hanford Corporation, Richland, Washington.

Martin, W. J. 1996. Integration of Risk Analysis and Sorption Studies in the Subsurface Transport of Aqueous Carbon-14 at the Hanford Site. Ph. D. Dissertation, Washington State University, Pullman, Washington.

McHenry, J. R. 1954. Adsorption and Retention of Cesium by Soils of the Hanford Project. HW-31011, General Electric Company, Richland, Washington.

Mozeto, A. A., P. Fritz, and E. J. Reardon. 1983. "Experimental Observation of Carbon Isotope Exchange in Carbonate-Water System." Geochimica et Cosmochimica Acta 48:495-504.

Nelson, J. L. 1959. Soil Column Studies with Radiostrontium: Effects of Temperature and of Species of Accompany Ion. HW-62035, General Electric Company, Richland, Washington.

Pourbaix, M. 1966. Atlas of Electrochemical Equilibria. Pergamon Press, Oxford, England.

Price, S. M., R. B. Kasper, M. K. Additon, R. M. Smith, and G. V. Last. 1979. Distribution of Plutonium and Americium Beneath the 216-Z-1A Crib: A Status Report. RHO-ST-17, Rockwell Hanford Operations, Richland, Washington.

Prout, W. E. 1959. Adsorption of Fission Products by Savannah River Plant Soil. DP-394, Savannah River National Laboratory, Aiken, South Carolina. 
Raymond, J. R. 1964. Investigation of the Disposition and Migration of Gross Gamma Emitters Beneath Liquid Waste Disposal Sites. HW-81746, General Electric Company, Richland, Washington.

Raymond, J. R. 1965. Cesium and Strontium Distribution Beneath Liquid Waste Disposal Sites. BNWL-235, Battelle Northwest Laboratory, Richland, Washington.

Rhodes, D. W. 1956. Adsorption by Soil of Strontium from 216-S Crib Waste. HW-42699, General Electric Company, Richland, Washington.

Rhodes, D. W. 1957a. "The Effect of $\mathrm{pH}$ on the Uptake of Radioactive Isotopes from Solution by a Soil.” Soil Sci. Soc. Am. Proc. 21:389-392.

Rhodes, D. W. 1957b. "The Adsorption of Pu by Soil." Soil Sci. 84:465-471.

Rhodes, D. W., and J. L. Nelson. 1957. Disposal of Radioactive Liquid Wastes from the Uranium Recovery Plont. HW-54721, General Electric Company, Richland, Washington.

Rhodes K., B. N. Bjornstad, R. E. Lewis, S. S. Teel, K. J. Cantrell, R. J. Serne, J. L. Smooth, C. T. Kincaid, and S. K. Wurstner. 1992. Estimation of the Release and Migration of Lead Through Soils and Groundwater at the Hanford Site 218-E-12B Burial Ground. PNL-8356; Pacific Northwest Laboratory, Richland, Washington.

Routson, R. C., G. Jansen, and A. V. Robinson. 1976. "241-Am, 237-Np, and 99-Tc Sorption on Two United States Subsoils from Differing Weathering Intensity Areas.” Health Phys. 33:311-317.

Routson, R. C., G. S. Barney, and R. O. Seil. 1978. Measurement of Fission Product Sorption Parameters for Hanford 200 Area Sediment Types. Progress Report. RHO-LD-73, Rockwell Hanford Operations, Richland, Washington.

Routson, R. C., G. S. Barney, R. M. Smith, C. H. Delegard, and L. Jensen. 1981. Fission Product Sorption Parameters for Hanford 200 Area Sediment Types. RHO-ST-35, Rockwell Hanford Operations, Richland, Washington.

Serne, R. J., and J. F. Relyea. 1983. "The Status of Radionuclide Sorption-Desorption Studies Performed by the WRIT Program." In The Technology of High-Level Nuclear Waste Disposal, Vol. 1, pp. 203-254. DOE/TIC-621, Technical Information Center, U.S. Department of Energy, Oak Ridge, Tennessee.

Serne, R. J., J. L. Conca, V. L. LeGore, K. J. Cantrell; C. W. Lindenmeier, J. A. Campbell, J. E. Amonette, and M. I. Wood. 1993. Solid-Waste Leach Characteristics and Contaminant-Sediment Interactions. Volume 1: Batch Leach and Adsorption Tests and Sediment Characterization. PNL-8889, Vol. 1, Pacific Northwest Laboratory, Richland, Washington. 
Serne, R. J., A. R. Felmy, K. J. Cantrell, K. M. Krupka, J. A. Campbell, H. Bolton, Jr., and J. K. Fredrickson. 1995. Characterization of Radionuclide-Chelating Agent Complexes Found in LowLevel Radioactive Decontamination Waste: Literature Review. NUREG/CR-6124, U.S. Nuclear Regulatory Commission, Washington, D.C.

Serne, R. J., and D. S. Burke. 1997. Chemical Information on Tank Supernatants, Cs Adsorption From Tank Liquids Onto Hanford Sediments, and Field Observations of Cs Migration From Past Tank Leaks. PNNL-11495, Pacific Northwest National Laboratory, Richland, Washington.

Sheppard, J. C., J. A. Kittrick, and T. L. Hardt. 1976. Determination of Distribution Ratios and Diffusion Coefficients of Neptunium, Americium, and Curium in Soil-Aquatic Environments. RLO-222:1-T-12-2, Washington State University, Pullman, Washington.

Smith, R. M., and A. E. Martell. 1976. Critical Stability Constants Vol. 4: Inorganic Complexes. Plenum Press, New York.

Striegl, R. G., and D. E. Armstrong. 1990. "Carbon Dioxide Retention and Carbon Exchange on Unsaturated Quaternary Sediments." Geochimica et Cosmochimica Acta 54:2277-2283.

Thibault, D. H., M. I Sheppard, and P. A. Smith. 1990. A Critical Compilation $2^{\text {nd }}$ Review of Default Soil Solid/Liquid Partition Coefficients, $K_{d}$ For Use in Environmental Assessment. AECL-10125, White Shell Nuclear Research Establishment, Pinawa, Manitoba, Canada.

U.S. Department of Energy (DOE). 1988. "Radioactive Waste Management.” DOE Order 5820.2A.

U.S. Department of Energy (DOE). 1989. Draft Environmental Impact Statement: Decommissioning of Eight Surplus Production Reactors at the Hanford Site, Richland, Washington. DOE/EIS-0119D, U.S. Department of Energy, Washington, D.C.

U.S. Department of Energy (DOE). 1992. Addendum (Final Environmental Impact Statement); Decommissioning of Eight Surplus Production Reactors at the Hanford Site, Richland, Washington. DOE/EIS-0119F, U.S. Department of Energy, Washington, D.C.

U.S. Department of Energy (DOE). 1994. Remedial Investigation and Feasibility Study Report for the Environmental Restoration Disposal Facility. DOE/RL-93-99, Rev 1, U.S. Department of Energy, Richland, Washington.

U.S. Department of Energy (DOE). 1996. Draft Hanford Remedial Action Environmental Impact Statement and Comprehensive Land Use Plan. DOE/EIS-0222D, U.S. Department of Energy, Washington, D.C. 
U.S. Department of Energy (DOE) and State of Washington Department of Ecology (Ecology). 1996. Tank Waste Remediation System, Hanford Site, Richland, Washington, Final Environmental Impact Statement. DOE/EIS-0189, U.S. Department of Energy, Washington, D.C.

Wood, M. I., R. Khaleel, P. D. Rittmann, A. H. Lu, S. H. Finfrock, and T. H. DeLorenzo. 1995a. Environmental Restoration Disposal Facility Performance Assessment. BHI-00169, Bechtel Hanford Inc., Richland, Washington.

Wood, M. I., R. Khaleel, P. D. Rittmann, A. H. Lu, S. H. Finfrock, R. J. Serne, K. J. Cantrell, and T. H. DeLorenzo. 1995b. Performance Assessment for the Disposal of Low-Level Waste in the 200 West Area Burial Grounds. WHC-ED-0645, Westinghouse Hanford Company, Richland, Washington.

Wood, M. I., R. Khaleel, P. D. Rittmann, S. H. Finfrock, T. H. DeLorenzo, and D. Y. Garbrick. 1996. Performance Assessment for the Disposal of Low-Level Waste in the 200-East Area Burial Grounds. WHC-SD-WM-TI-730, Westinghouse Hanford Company, Richland, Washington.

Zhang, J., P. O. Quay, and D. O. Wilbur. 1995. "Carbon Isotope Fractionation During Gas-Water Exchange and Dissolution of $\mathrm{CO}_{2} . "$ Geochimica et Cosmochimica Acta 59:107-114. 
Table E.1. Summary of Distribution Coefficients (mL/g) Previously Assigned to Radionuclides

\begin{tabular}{|c|c|c|c|c|c|c|c|c|c|}
\hline \multirow[b]{2}{*}{ Element } & \multicolumn{9}{|c|}{ Distribution Coefficients Assigned in Previous Studies } \\
\hline & $\begin{array}{c}\text { Surplus } \\
\text { Reactors }^{(*)}\end{array}$ & ERDF $^{(b)}$ & $\begin{array}{l}200 \text { East } \\
\text { SWBG }^{(\mathrm{c})}\end{array}$ & $\begin{array}{l}\text { TWRS } \\
\text { EIS }^{(৯)}\end{array}$ & $\begin{array}{l}\text { HRA } \\
\text { EIS }^{(\boldsymbol{e})}\end{array}$ & $\begin{array}{c}\text { US } \\
\text { Ecology }\end{array}$ & $\begin{array}{c}\text { TWRS } \\
\text { ILAW(s) }\end{array}$ & $\begin{array}{l}\text { Low } \\
\mathbf{K}_{d}^{\left({ }^{(a)}\right.} \\
\end{array}$ & $\begin{array}{l}\text { High } \\
\mathbf{K}_{\mathbf{d}}^{\left({ }^{2}\right)} \\
\end{array}$ \\
\hline \multicolumn{10}{|c|}{ Group of Highly Mobile Elements Assigned a $\mathrm{K}_{d}$ of $0 \mathrm{~mL} / \mathrm{g}$} \\
\hline $\mathrm{H}$ & 0 & 0 & 0 & & 0 & & & & \\
\hline $\mathrm{Cl}$ & 0 & & 0 & & 0 & 0 & & & \\
\hline Se & & & 0 & 0 & 0 & & 0 & 0 & 0.78 \\
\hline Tc & 0 & 0 & 0 & 0 & 0 & 0 & 0 & 0 & 1.3 \\
\hline \multicolumn{10}{|c|}{ Group of Somewhat Mobile Elements Assigned a $\mathrm{K}_{\mathrm{d}}$ of $0.6 \mathrm{~mL} \mathrm{~g}$} \\
\hline I & & & - & - & - & - & 3 & 0.04 & 18 \\
\hline$\overline{\mathrm{U}}$ & 0 & 0 & 0 & 0 & 0 to 250 & 0 & 0.6 & 0.08 & 79.3 \\
\hline \multicolumn{10}{|c|}{ Group of Moderately Immobile Elements Assigned a $\mathrm{K}_{\mathrm{d}}$ of $10 \mathrm{~mL} / \mathrm{g}$} \\
\hline $\mathrm{Np}$ & & 2 & 10 & 0 & 0 to 500 & & 15 & 2.4 & 29.1 \\
\hline $\mathrm{Pa}$ & & & & 1 & 50 & & 6 & 10 & 1000 \\
\hline$\overline{\mathrm{Ra}}$ & & 10 & 10 & 10 & 20 & 200 & 15 & 24 & 100 \\
\hline $\mathbf{R u}$ & & & & 0 & 0 & & & 27 & 274 \\
\hline Sr & 0.64 & 10 & 10 & 10 & 10 & 0.64 & 3 & 5 & 173 \\
\hline \multicolumn{10}{|c|}{ Group of Highly Immobile Elements Assigned a $\mathrm{K}_{\mathrm{d}}$ of $40 \mathrm{~mL} / \mathrm{g}$} \\
\hline Ac & & & & 50 & & & 40 & 7 & 1330 \\
\hline $\mathrm{Am}$ & 76 & 100 & 100 & 50 & 50 & 810 & 40 & 67 & $>1200$ \\
\hline $\mathrm{Bi}$ & & & & 1 & 100 & & & & \\
\hline $\mathrm{Ce}$ & & & & & & & 100 & 100 & $>2000$ \\
\hline $\mathrm{Cm}$ & & & & 50 & 50 & & 100 & 106 & 1330 \\
\hline $\mathrm{Co}$ & 100 & 1 & 1 & & 12 & & 100 & 1200 & 12,500 \\
\hline Cs & 26 & 100 & 100 & 50 & 30 & & 100 & 540 & 3180 \\
\hline Eu & & 10 & 10 & 50 & & & 100 & 100 & 228 \\
\hline $\bar{K}$ & & 10 & 10 & & 0.2 & 0 & & & \\
\hline$\overline{\mathrm{Nb}}$ & & & & & 100 & 350 & 40 & 50 & 100 \\
\hline $\mathrm{Ni}$ & 100 & 100 & 100 & 1 & 12 & 100 & 40 & 50 & 2350 \\
\hline $\mathrm{Pb}$ & & & & 10 & 100 & & 100 & 13,000 & 79,000 \\
\hline Po & & & & & 100 & & & & \\
\hline $\mathrm{Pu}$ & 71 & 100 & 100 & 10 & I to 200 & 73 & 40 & 80 & $>1980$ \\
\hline$\overline{\operatorname{Re}}$ & & & 0 & & & & & & \\
\hline$\overline{S n}$ & & & & 10 & & & 100 & 100 & 230 \\
\hline $\mathrm{Th}$ & & 100 & 100 & 10 & 50 & 40 & 40 & 40 & 100 \\
\hline $\mathrm{Y}$ & & 50 & & 50 & 100 & & & & \\
\hline $\mathrm{Zr}$ & 2000 & & & 50 & 50 & & 40 & 90 & $>2000$ \\
\hline \multicolumn{10}{|c|}{ Special Case Elements } \\
\hline$C^{(1)}$ & 0 & 0 & 0 & 0 & 0 & 0 & 6 & 0 & 4 \\
\hline
\end{tabular}
(a) From DOE (1989).
(b) From DOE (1994).
(c) From Wood et al. (1996).
(d) From DOE and Ecology (1996).
(e) From DOE (1996).
(f) From Grant Environmental, Chase Environmental Group, and US Ecology, Inc. (1996).
(g) From Mann et al. (1997).
(h) From Kaplan and Serne (1995) and Kaplan, Serne, and Piepho (1995).
(i) Recent work by Martin (1996) suggests carbon-14 undergoes attenuation in the environment because of isotopic exchange or dilution through recrystallization of minerals. 
Table E.2. Source Term Categories

\begin{tabular}{|c|c|c|}
\hline Category & Description & Examples/Comments \\
\hline High Organic/Very Acidic & $\begin{array}{l}\text { Selected plutonium and organic- } \\
\text { rich condensate and process } \\
\text { wastes }\end{array}$ & $\begin{array}{l}\text { Z Plant, Carbon tetrachloride (with TBP, DBBP } \\
\text { or lard oil) and aqueous waste streams from } \\
\text { same facility }\end{array}$ \\
\hline $\begin{array}{l}\text { High Organic/Near } \\
\text { Neutral pH }\end{array}$ & $\begin{array}{l}\text { Organic rich process condensate } \\
\text { and process wastes }\end{array}$ & $\begin{array}{l}\text { REDOX, PUREX, Z Plant. Organics include } \\
\text { hexone, carbon tetrachloride, TBP, and DBBP }\end{array}$ \\
\hline $\begin{array}{l}\text { Very High Salt/Very } \\
\text { Basic }\end{array}$ & $\begin{array}{l}\text { Tank wastes and wastes } \\
\text { associated with small tanks, lines, } \\
\text { pits, and boxes }\end{array}$ & $\begin{array}{l}\text { Tank waste can contain chelators but the high } \\
\mathrm{pH} \text { tends to diminish impacts of organic } \\
\text { chelators on } \mathrm{K}_{\mathrm{d}} \text { values }\end{array}$ \\
\hline Chelates/High salts & $\begin{array}{l}\text { Tank wastes with organic } \\
\text { chelating or complexing agents }\end{array}$ & $\begin{array}{l}\text { By cribs, waste with ferrocyanide (used to } \\
\text { remove cesium) or EDTA additives (used to } \\
\text { remove strontium) }\end{array}$ \\
\hline $\begin{array}{l}\text { Low Organic/Low } \\
\text { Salts/Very Acidic }\end{array}$ & Uranium-rich process condensate & $\begin{array}{l}\text { Uranium recovery from bismuth-phosphate } \\
\text { wastes; PUREX; REDOX; and S-1/2, S-9, U- } \\
1 / 2, U-8 \text {, and U-12 cribs received acid waste }\end{array}$ \\
\hline $\begin{array}{l}\text { Low Organic/Low } \\
\text { Salts/Near Neutral pH }\end{array}$ & \multicolumn{2}{|c|}{$\begin{array}{l}\text { - Plutonium-rich process condensate and process wastes. Characterized as neutral- } \\
\text { basic wastes without organics, from Z Plant } \\
\text { - General process condensate and process wastes. Characterized as small } \\
\text { inventories of low-salt, neutral-basic wastes } \\
\text { - Steam condensate. Characterized as small inventories of low-salt, neutral-basic } \\
\text { wastes with high volumes } \\
\text { - Chemical sewers. Characterized as small inventories of low-salt, neutral-basic } \\
\text { wastes with high volumes } \\
\text { - Cooling water. Characterized as small inventories of low-salt, neutral-basic } \\
\text { wastes with high volumes } \\
\text { - Chemical laboratory wastes. Characterized as low-salt, neutral-basic wastes } \\
\text { - Miscellaneous wastes. Characterized as low-salt, neutral-basic wastes }\end{array}$} \\
\hline
\end{tabular}

TBP $=$ Tributyl phosphate

DBBP $=$ Dibutyl butyl phosphate

REDOX $=$ Reduction-Oxidation (S Plant)

PUREX = Plutonium-uranium extraction

EDTA $=$ Ethylenediaminetetraacetic acid 
Table E.3. Distribution Coefficient $\left(\mathrm{K}_{\mathrm{d}}\right)$ Zone Categories

\begin{tabular}{|l|l|l|}
\hline Zone Category & \multicolumn{1}{|c|}{ Description } & Generalized Effect on $\mathbf{K}_{d}$ \\
\hline High Impact & $\begin{array}{l}\text { This zone is located in the vadose zone near the } \\
\text { disposal facility inlet or ground surface. The } \\
\text { liquid phase is greatly affected by the chemical } \\
\text { composition of the contaminated liquid source. } \\
\text { Organic compounds, } \mathrm{pH} \text {, and salt, when present in } \\
\text { the source term may affect } \mathrm{K}_{d} \text { values. }\end{array}$ & Lowest $\mathrm{K}_{\mathrm{d}}$ values \\
\hline Intermediate Impact & $\begin{array}{l}\text { This zone is located in the vadose zone } \\
\text { immediately below the high-impact zone. The } \\
\text { upper boundary is defined as the depth where the } \\
\text { excessive acidic or basic nature of the waste has } \\
\text { been neutralized by the buffering capacity of the } \\
\text { natural soil. No pH effects of the plume remain. }\end{array}$ & Intermediate $\mathrm{K}_{\mathrm{d}}$ values \\
\hline Groundwater & $\begin{array}{l}\text { This zone is in the unconfined aquifer where } \mathrm{K}_{d} \\
\text { values are not affected by the chemical } \\
\text { composition of the plume. The waste source } \\
\text { chemical compositions in this zone are assumed to } \\
\text { be so greatly diluted they do not affect } \mathrm{K}_{d} \text { values. }\end{array}$ & Largest $\mathrm{K}_{d}$ values \\
\hline
\end{tabular}

Table E.4. Source and Distribution Coefficient $\left(\mathrm{K}_{\mathrm{d}}\right)$ Zone Categories ${ }^{(\mathrm{a})}$

\begin{tabular}{|l|c|c|c|}
\hline \multicolumn{1}{|c|}{ Source Category } & \multicolumn{3}{c|}{ Zone Category } \\
\hline High Organic/Very Acidic & High Impact & $\begin{array}{c}\text { Intermediate } \\
\text { Impact }\end{array}$ & Groundwater \\
\hline High Organic/Near Neutral & $\mathrm{A}$ & $\mathrm{B}$ & $\mathrm{F}$ \\
\hline Very High Salt/Very Basic & $\mathrm{B}$ & $\mathrm{B}$ & $\mathrm{F}$ \\
\hline Chelates/High Salts & $\mathrm{D}$ & $\mathrm{E}$ & $\mathrm{F}$ \\
\hline Low Organic/Low Salts/Acidic & $\mathrm{G}$ & $\mathrm{G}$ & $\mathrm{C}$ \\
\hline Low Organic/Low Salts/Near Neutral & $\mathrm{H}$ & $\mathrm{F}$ & $\mathrm{F}$ \\
\hline
\end{tabular}

(a) Categories with the same letters have similar background chemistries and, therefore, similar $K_{d}$ values. 
Table E.5. Distribution Coefficient $\left(K_{d}\right)$ Values for Source-Zone Category $A^{(a)}$

\begin{tabular}{|c|c|c|c|}
\hline Element & $\begin{array}{c}\text { Conservative } \\
\text { and ("Best") } K_{d} \\
\text { Estimate (mL/g) }\end{array}$ & $\begin{array}{c}\text { Range } K_{d} \\
\text { Estimate } \\
(\mathrm{mL} / \mathrm{g})\end{array}$ & Justification/References \\
\hline $\begin{array}{l}\text { Tritium, } \\
\text { Cl, Tc }\end{array}$ & $\begin{array}{c}0 \\
(0)\end{array}$ & 0 to 1 & $\begin{array}{l}\text { No adsorption studies pertinent to these conditions were found in the } \\
\text { literature. Tritium moves with water. A very slight degree of adsorption }\left(\mathrm{K}_{\mathrm{d}}\right. \\
\sim 0.1 \mathrm{~mL} / \mathrm{g} \text { ) has been reported resulting from } \mathrm{HO}^{\circ} \text { sorbing to iron-oxides or } \\
\text { tritiated water exchanging for regular water on clay surfaces } \\
\text { and tech). Chlorine } \\
\text { not to sorb to mineral surfaces. }\end{array}$ \\
\hline $\begin{array}{l}\text { Ac, Am, } \\
\mathrm{Ce}, \mathrm{Cm}, \\
\mathrm{Eu}\end{array}$ & $\begin{array}{c}0 \\
(0.3)\end{array}$ & 0 to 2 & 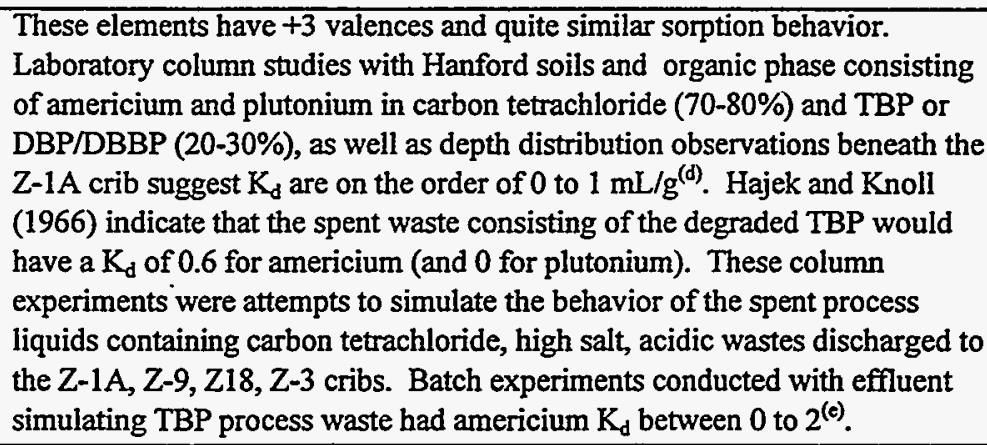 \\
\hline $\bar{C}$ & $\begin{array}{c}0 \\
0\end{array}$ & 0 to 0.4 & $\begin{array}{l}\text { Carbon-14 is introduced into the source in an inorganic form. The assumed } \\
\text { dominant species are } \mathrm{CO}_{2} \text { gas under these acidic conditions, and } \mathrm{C} \text {-Organic } \\
\text { compound, } \mathrm{HCO}_{3}^{-} \text {under neutral pH conditions. Carbon chemistry is very } \\
\text { complicated in such mixed systems. It can become complexed with } \\
\text { inorganic metals, enter into the structure of organic compounds, be } \\
\text { volatilized out of solutions as } \mathrm{CO}_{2} \text { gas, or (co)precipitate into natural calcite } \\
\text { minerals existing in the aquifer. The net effect of these conflicting processes } \\
\text { is difficult to quantify because of a lack of experimental data. In an } \\
\text { experiment conducted with Hanford groundwater spiked with } \mathrm{HCO}^{-} \text {(no } \\
\text { organics in liquid phase) and Hanford sediments in which the calcite } \\
\text { coatings were removed with acid, } \mathrm{K}_{d} \text { values were measured for carbon-14 } \\
\text { between } 0.27 \text { to } 0.38 \mathrm{~mL} / \mathrm{g}^{(\hat{)}} \text {. }\end{array}$ \\
\hline$\overline{\mathrm{Co}}$ & $\begin{array}{c}0 \\
(0)\end{array}$ & 0 to 2 & $\begin{array}{l}\text { Cobalt did not sorb to a Hanford sediment when it was.in the presence of } \\
\text { bismuth phosphate-uranium-recovery scavenged waste containing } \\
\text { ferrocyanide process effluent }(\mathrm{gb}) \text {. Cobalt is soluble in acid and readily } \\
\text { complexed. Tests conducted to simulate uranium-recovery scavenged waste } \\
\text { containing ferrocyanide process waste moving through soil columns showed } \\
\text { that cobaitt-60 was not removed by adsorption on the soil. The presence of } \\
\text { nonexchangeable cobalt-60 became a limiting factor in the disposal of some } \\
\text { wastes }(\mathrm{g}, \mathrm{h}) \text {. }\end{array}$ \\
\hline$\overline{C s}$ & $\begin{array}{c}5 \\
(7)\end{array}$ & 5 to 50 & $\begin{array}{l}\text { Cesium } \mathrm{K}_{d} \text { values generally decrease as pH decreases }{ }^{(0)} \cdot \mathrm{K}_{\mathrm{d}} \text { values of } 5 \text { to } 50 \\
\text { in Hanford sediments have been reported for cesium. }\end{array}$ \\
\hline$\overline{\mathrm{I}}$ & $\begin{array}{c}0 \\
0 \\
(0)\end{array}$ & 0 & Iodine is a soluble anion. $K_{d}$ values were estimated. ${ }^{(1)}$ \\
\hline $\begin{array}{l}\mathrm{Ni}, \mathrm{Sn}, \\
\mathrm{Nb}\end{array}$ & $\begin{array}{c}2 \\
(4)\end{array}$ & 0 to 10 & The $\mathrm{K}_{d}$ values were estimated. \\
\hline
\end{tabular}


Table E.5. (contd)

\begin{tabular}{|c|c|c|c|}
\hline Element & $\begin{array}{l}\text { Conservative } \\
\text { and ("Best") } K_{d} \\
\text { Estimate }(\mathrm{mL} / \mathrm{g})\end{array}$ & $\begin{array}{c}\text { Range } \mathbf{K}_{\mathbf{d}} \\
\text { Estimate } \\
(\mathrm{mL} / \mathrm{g})\end{array}$ & Justification/References \\
\hline $\mathrm{Np}, \mathrm{Pa}$ & $\begin{array}{c}0.1 \\
(0.2)\end{array}$ & 0.1 to 1 & $\begin{array}{l}\text { The effects of organic phase are unknown. They are assumed to behave } \\
\text { similar to plutonium. The assumed dominant protactinium species is } \mathrm{PaO}_{2}^{+} \\
\text {and } \mathrm{NpO}_{2}^{+} \text {is assumed to be a reasonable analog. }\end{array}$ \\
\hline$\overline{\mathrm{Pb}}$ & $\begin{array}{c}0 \\
(0)\end{array}$ & 0 to 10 & The $\mathrm{K}_{d}$ values were estimated. ${ }^{(1)}$ \\
\hline$\overline{\mathrm{Pu}}$ & $\begin{array}{c}0.1 \\
(0.4)\end{array}$ & 0.1 to 1 & $\begin{array}{l}\text { See discussion above for actinium, americium, cerium, curium, and } \\
\text { europium. The range for plutonium in Hanford sediments is } 0.1 \text { to } 1 \mathrm{~mL} / \mathrm{g} \\
\text { in liquid phases. }{ }^{(c k)}\end{array}$ \\
\hline $\mathrm{Ra}, \mathrm{Sr}$ & $\begin{array}{c}0.1 \\
(0.4)\end{array}$ & 0.1 to 5 & $\mathrm{~K}_{\mathrm{d}}$ values were based on strontium sorption experiments. ${ }^{\left({ }^{(}\right)}$ \\
\hline$\overline{\mathrm{Ru}}$ & $\begin{array}{l}0.1 \\
(0.4)\end{array}$ & 0 to 10 & $\begin{array}{l}\mathrm{K}_{\mathrm{d}} \text { values were estimated. } \\
\text { sorption. }\end{array}$ \\
\hline $\mathrm{Se}$ & $\begin{array}{c}0 \\
(0)\end{array}$ & 0 & $\mathrm{~K}_{\mathrm{d}}$ values were estimated. ${ }^{(\mathrm{j})}$ \\
\hline $\mathrm{Th}, \mathrm{Zr}$ & $\begin{array}{c}1 \\
(5)\end{array}$ & 1 to 20 & $\mathrm{~K}_{\mathrm{d}}$ values were estimated. (I,I) \\
\hline$\overline{\mathrm{U}}$ & $\begin{array}{c}0.1 \\
(0.2)\end{array}$ & 0.1 to 1 & $\begin{array}{l}\mathrm{K}_{\mathrm{d}} \text { values were estimated. } \\
\text { are assumed to behave similarly to plutonium. }\end{array}$ \\
\hline
\end{tabular}

(a) Category $A$ is defined in Table E.4.

(b) From Ames and Rai (1978).

(c) From Thibault, Sheppard, and Smith (1990).

(d) From Hajek and Knoll (1966).

(e) From Knoll (1969).

(f) From Martin (1996).

(g) From Haney (1957).

(h) From Rhodes and Nelson (1957).

(i) From Ames and Serne (1991).

(j) From Pourbaix (1966).

(k) From Benson (1960).

(l) From Prout (1959).

TBP $=$ Tributyl phosphate

$\mathrm{DBP}=$ Dibutyl phosphate

DBBP = Dibutyl butyl phosphate 
Table E.6. Distribution Coefficient $\left(\mathrm{K}_{d}\right)$ Values for Source-Zone Category B ${ }^{(a)}$

\begin{tabular}{|c|c|c|c|}
\hline Element & $\begin{array}{l}\text { Conservative } \\
\text { and ("Best") } K_{d} \\
\text { Estimate }(\mathrm{mL} / \mathrm{g})\end{array}$ & $\begin{array}{l}\text { Range } K_{d} \\
\text { Estimate } \\
(\mathrm{mL} / \mathrm{g})\end{array}$ & Justification/References \\
\hline $\begin{array}{l}\text { tritium, } \\
\mathrm{Cl}, \mathrm{Tc}\end{array}$ & $\begin{array}{c}0 \\
(0)\end{array}$ & 0 to 1 & $\begin{array}{l}\text { Tritium moves with water. A very slight degree of adsorption }\left(\mathrm{K}_{\mathrm{d}}-0.1 \mathrm{~mL} / \mathrm{g}\right) \\
\text { has been reported resulting from HO sorbing to iron oxides or tritiated water } \\
\text { exchanging for regular water on clay surfaces }{ }^{(b, c)} \text { Chlorine and technetium } \\
\text { exist in groundwater primarily as anions. }{ }^{(b, c)} \text { The } \mathrm{K}_{\mathrm{d}} \text { value for } \mathrm{TcO}_{4}{ }^{-} \mathrm{K}_{\mathrm{d}} \text { is }-0.6 \\
\text { to } 0.02 \text { in organic rich solid phase. }{ }^{(\mathrm{d})}\end{array}$ \\
\hline $\begin{array}{l}\text { Ac, Am, } \\
\mathrm{Ce}, \mathrm{Cm} \\
\mathrm{Eu}\end{array}$ & $\begin{array}{c}10 \\
(20)\end{array}$ & 20 to $>200$ & $\begin{array}{l}\text { Hajek and Knoll }{ }^{(\mathrm{d})} \text { conducted column breakthrough tests simulating the } \\
\text { behavior of the spent process liquids containing carbon tetrachloride, high salt, } \\
\text { acidic wastes discharged to the } Z-1 \mathrm{~A}, \mathrm{Z}-9, Z 18, Z-3 \text { cribs. When waste liquid } \\
\text { was neutralized, the americium } \mathrm{K}_{\mathrm{d}} \text { value went up to over } 200 \mathrm{~mL} / \mathrm{g} \text { (in acid } \\
\text { solutions the } \mathrm{K}_{\mathrm{d}} \text { for americium was } \sim 1 \mathrm{~mL} / \mathrm{g} \text { ). When neutralized waste mixed } \\
\text { with } 20 \% \text { by volume organics (carbon tetrachloride: TBP/DBBP mix), the } \mathrm{K}_{\mathrm{d}} \\
\text { for americium dropped to } 40 \text {. Once the americium (or plutonium) was } \\
\text { adsorbed on the soil column, the organic mixture was not effective in } \\
\text { removing it. The only PFP crib in this category is the Z-12 crib that received } \\
\text { low-salt, neutralized waste containing americium (and plutonium) and some } \\
\text { amounts of organic (carbon tetrachloride, TBP). Because of the } \\
\text { neutralization, the americium and plutonium had a high affinity for the soil } \\
\text { either resulting from sorption or resulting from filtering of particulate phases } \\
\text { that may have formed prior to disposal. }\end{array}$ \\
\hline $\mathrm{C}$ & $\overline{0}$ & 0 to 10 & $\begin{array}{l}\text { The assumed dominant species are } \mathrm{CO}_{2} \text { and } \mathrm{C}-\mathrm{Organic} \text { compound, } \mathrm{HCO}_{3}^{-} \text {. } \\
\text { Carbon chemistry is complex in these mixed systems. Carbon can become } \\
\text { complexed with inorganic metals, enter into the structure of organic } \\
\text { compounds, be volatilized out of solutions as } \mathrm{CO}_{2} \text { gas, or (co)precipitate into } \\
\text { natural calcite minerals existing in the aquifer. The net effect of these } \\
\text { conflicting processes are difficult to quantify because of the lack of } \\
\text { experimental data. The } \mathrm{K}_{d} \text { is an estimate. }{ }^{()^{2}}\end{array}$ \\
\hline Co & $\begin{array}{l}0.1 \\
(3)\end{array}$ & 0.1 to 10 & $(f, g, h)$ \\
\hline Cs & $\begin{array}{c}5 \\
(10)\end{array}$ & 3 to 300 & $\overline{(\mathrm{g}, \mathrm{h})}$ \\
\hline I & $\begin{array}{c}0 \\
(0.1)\end{array}$ & 0 to 1 & Iodine is a soluble anion. The $\mathrm{K}_{\mathrm{d}}$ value was estimated. ${ }^{(I)}$ \\
\hline $\begin{array}{l}\mathrm{Ni}, \mathrm{Sn} \\
\mathrm{Nb}\end{array}$ & $\begin{array}{c}3 \\
(4)\end{array}$ & 0 to 30 & The $\mathrm{K}_{\mathrm{d}}$ value was estimated. \\
\hline $\mathrm{Np}, \mathrm{Pa}$ & $\begin{array}{c}0.1 \\
(0.2)\end{array}$ & 0.1 to 5 & $\begin{array}{l}\text { Neptunium is assumed to behave like plutonium, for which more data are } \\
\text { available under these groundwater conditions. The dominant protactinium } \\
\text { species is } \mathrm{PaO}_{2}^{+} \text {and that } \mathrm{Np}_{2}{ }^{+} \text {is a reasonable analog. }{ }^{(k)}\end{array}$ \\
\hline $\mathrm{Pb}$ & $\begin{array}{c}0 \\
(4)\end{array}$ & 0 to 10 & $\begin{array}{l}\mathrm{Pb}^{2+} \text { forms stronger complexes with cyanide than } \mathrm{Co}^{2+} \cdot(1) \text { Cobalt mobility is } \\
\text { greatly increased in the subsurface when cyanide is present. }\end{array}$ \\
\hline
\end{tabular}


Table E.6. (contd)

\begin{tabular}{|c|c|c|c|}
\hline Element & $\begin{array}{c}\text { Conservative } \\
\text { and ("Best") } K_{d} \\
\text { Estimate }(\mathrm{mL} / \mathrm{g})\end{array}$ & $\begin{array}{c}\text { Range } \bar{K}_{d} \\
\text { Estimate } \\
(\mathrm{mL} / \mathrm{g})\end{array}$ & Justification/References \\
\hline$\overline{\mathrm{Pu}}$ & $\begin{array}{c}15 \\
(25)\end{array}$ & 15 to 50 & $\begin{array}{l}\text { See discussion above for actinium, americium, cerium, curium, and europium. } \\
\text { Depth distribution studies show that over } 95 \% \text { of the inventory is within the } \\
\text { upper } 2-3 \mathrm{~m}(6-10 \mathrm{ft} \text { ) beneath crib bottoms. Some has been found deeper, } \\
\text { which may reflect a small fraction present as colloids or complexed. Based on } \\
\text { simple one-dimensional unit gradient (steady-state flow) predictions of } \\
\text { migration depth and observed maximum depths, }{ }^{(\mathrm{m})} \text { a } \mathrm{K}_{\mathrm{d}} \text { of } 25 \text { for plutonium } \\
\text { would account for the most mobile (greatest depth of penetration) fraction of } \\
\text { the inventory. Based on the above, the maximum depth of inventory is } 0.25 \mathrm{~m} \\
\text { ( } 1 \% \text { of inventory), and }>90 \% \text { of the inventory is at } 0.01 \mathrm{~m} \text { depth. The } \mathrm{K}_{\mathrm{d}} \text { for } \\
\text { this intermediate-impact zone of } 25 \mathrm{~mL} / \mathrm{g} \text { is believed to be the best estimate. } \\
\text { A conservative estimate of } 15 \mathrm{~mL} / \mathrm{g} \text { is suggested.. Subsequent (groundwater } \\
\text { zone) are assumed to be the same because of natural pH of around } 8 \text { in soil } \\
\text { moisture and groundwater. }\end{array}$ \\
\hline $\mathrm{Ra}, \mathrm{Sr}$ & $\begin{array}{c}5 \\
(7)\end{array}$ & 5 to 20 & $(\mathrm{n}, \mathrm{o}, \mathrm{h})$ \\
\hline$\overline{\mathrm{Ru}}$ & $\begin{array}{l}0.1 \\
(2)\end{array}$ & 0 to 30 & $(p, q, h)$ \\
\hline$\overline{\mathrm{Se}}$ & $\begin{array}{c}0 \\
(0)\end{array}$ & 0 to 1 & Selenium is a soluble anion. The $K_{d}$ value was estimated. (n) $^{(n)}$ \\
\hline Th, $\mathrm{Zr}$ & $\begin{array}{c}20 \\
(40)\end{array}$ & 20 to 200 & These elements are strong absorbers. The $K_{d}$ values were estimated. ${ }^{(b)}$ \\
\hline$\overline{\mathrm{U}}$ & $\begin{array}{c}0.2 \\
(0.2)\end{array}$ & 0.2 to 10 & Carbonate complexes are anionic. The $\mathrm{K}_{d}$ value was estimated. ${ }^{(\mathrm{m}, \mathrm{T})}$ \\
\hline
\end{tabular}

(a) Category $B$ is defined in Table E.4.

(b) From Ames and Rai (1978).

(c) From Thibault, Sheppard, and Smith (1990).

(d) From Hajek and Knoll (1966).

(e) From Martin (1996).

(f) From Haney (1957).

(g) From Barney (1978).

(h) From Ames and Serne (1991).

(i) From Brown (1967).

(j) From Prout (1959).

(k) From Pourbaix (1966).

(l) From Smith and Martell (1976).

(m) From Johnson (1993).

(n) From Rhodes (1956).

(o) From Routson et al. (1981).

(p) From Raymond (1964).

(q) From Raymond (1965).

(r) From Kaplan and Serne (1995) and Kaplan, Serne, and Piepho (1995).

TBP $=$ Tributyl phosphate

$\mathrm{DBBP}=$ Dibutyl butyl phosphate

PFP = Plutonium Finishing Plant (Z Plant) 
Table E.7. Distribution Coefficient $\left(K_{d}\right)$ Values for Source-Zone Category $C^{(a, b)}$

\begin{tabular}{|c|c|c|c|}
\hline Element & $\begin{array}{c}\text { Conservative } \\
\text { and ("Best") } K_{d} \\
\text { Estimate (mL/g) }\end{array}$ & $\begin{array}{c}\text { Range } \mathrm{K}_{\mathrm{d}} \\
\text { Estimate } \\
(\mathrm{mL} / \mathrm{g})\end{array}$ & Justification/References \\
\hline Co & $\begin{array}{c}0 \\
(0)\end{array}$ & 0 to 3 & $\begin{array}{l}\text { Cobalt is likely complexed with EDTA and/or cyanide. Field data suggest that } \\
\text { the cobalt-chelate complexed species exists and moves rapidly. }\end{array}$ \\
\hline $\begin{array}{l}\mathrm{Sr}, \mathrm{Pb} \\
\mathrm{Ni}, \mathrm{Sn}\end{array}$ & $\begin{array}{c}2 \\
(4)\end{array}$ & 2 to 20 & $\begin{array}{l}\text { A strontium } \mathrm{K}_{\mathrm{d}} \text { of } 0.4 \mathrm{~mL} / \mathrm{g} \text { has been measured in one Hanford soil (soil } \mathrm{P} \text { ) and } \\
1.5 \mathrm{~mL} / \mathrm{g} \text { in another Hanford soil (soil } \mathrm{S} \text { ) in an aqueous system containing high } \\
\text { concentrations of salts and medium to high concentrations of complexing } \\
\text { agents, such as EDTA and.HEDTA. }{ }^{(c)} \text { A slightly higher } \mathrm{K}_{d} \text { value than these is } \\
\text { likely to exist in the Hanford Site because the complexing agent concentrations } \\
\text { will likely be appreciably lower. It is also anticipated that an appreciable } \\
\text { amount of microbial degradation will occur to the organic complexes during } \\
\text { their extended travel time to the far field. }{ }^{(d, e)}\end{array}$ \\
\hline $\mathrm{Pu}$ & $\begin{array}{c}20 \\
(40)\end{array}$ & $\begin{array}{c}20 \text { to } \\
>1980\end{array}$ & $\begin{array}{l}\text { A plutonium } K_{d} \text { of } 21 \mathrm{~mL} / \mathrm{g} \text { has been measured in one Hanford soil (soil P) and } \\
26 \mathrm{~mL} / \mathrm{g} \text { in another Hanford soil (soil } \mathrm{S} \text { ) in an aqueous system containing high } \\
\text { concentrations of salts and medium to high concentrations of complexing } \\
\text { agents, such as EDTA and HEDTA. }{ }^{\left({ }^{\circ}\right)} \text { A slightly higher } \mathrm{K}_{\mathrm{d}} \text { value than these is } \\
\text { likely because the complexing agent concentrations will likely be appreciably } \\
\text { lower and it is anticipated that an appreciable amount of microbial degradation } \\
\text { will occur to the organic complexes during their extended travel time to the far } \\
\text { field. }{ }^{(d, e)}\end{array}$ \\
\hline $\mathrm{Np}, \mathrm{Pa}$ & $\begin{array}{c}2 \\
(5)\end{array}$ & 2 to 15 & $\begin{array}{l}\mathrm{A} \mathrm{K}_{\mathrm{d}} \text { of } 8.7 \mathrm{~mL} / \mathrm{g} \text { has been measured for neptunium in one Hanford soil (soil P) } \\
\text { and } 12 \mathrm{~mL} / \mathrm{g} \text { in another Hanford soil (soil } \mathrm{S} \text { ) in an aqueous system containing } \\
\text { high concentrations of salts and medium to high concentrations of complexing } \\
\text { agents, such as EDTA and HEDTA. }{ }^{(c)} \text { Slightly higher } \mathrm{K}_{\mathrm{d}} \text { values than these are } \\
\text { likely to exist because the complexing agent concentrations will likely be } \\
\text { appreciably lower, and it is anticipated that an appreciable amount of microbial } \\
\text { degradation will occur to the organic complexes during their extended travel } \\
\text { time to the far field.(de) The assumed dominant protactinium species is } \mathrm{PaO}_{2}^{+} \\
\text {and that } \mathrm{NpO}_{2}^{+} \text {is a reasonable analog. }\end{array}$ \\
\hline $\begin{array}{l}\mathrm{Ac}, \mathrm{Am}, \\
\mathrm{Ce}, \mathrm{Cm}, \\
\mathrm{Eu}\end{array}$ & $\begin{array}{c}10 \\
(50)\end{array}$ & 10 to 500 & $\begin{array}{l}\text { A } \mathrm{K}_{\mathrm{d}} \text { of } 5.6 \mathrm{~mL} / \mathrm{g} \text { has been measured for americium in one Hanford soil (soil P) } \\
\text { and } 10 \mathrm{~mL} / \mathrm{g} \text { in another Hanford soil (soil } \mathrm{S} \text { ) in an aqueous system containing } \\
\text { high concentrations of salts and medium to high concentrations of complexing } \\
\text { agents, such as EDTA and HEDTA. }{ }^{\left({ }^{(}\right)} \text {Slightly higher } \mathrm{K}_{\mathrm{d}} \text { values than these are } \\
\text { likely to exist because the complexing agent concentration will likely be } \\
\text { appreciably lower and it is anticipated that an appreciable amount of microbial } \\
\text { degradation will occur to the organic complexes during their extended travel } \\
\text { time to the far field. Actinium, cerium, and curium also have }+3 \text { valance. } \text { (de) }^{\left({ }^{2}\right)}\end{array}$ \\
\hline
\end{tabular}

(a) All $\mathrm{K}_{d}$ values not reported in this table are identical to those in Table $\mathrm{E} .10$ for the far field groundwater.

(b) Category $\mathrm{C}$ is defined in Table E.4.

(c) From Delegard and Bamey (1983) (see Table 11, "Dilute complexed" data)

(d) From Serne et al. (1995).

(e) From Ames and Rai (1978).

(f) From Pourbaix (1966).

EDTA $=$ ethylenediaminetetraacetic acid

HEDTA $=\mathrm{N}$-(2-hydroxyethyl) ethylene diaminetetraacetic acid 
Table E.8. Distribution Coefficient $\left(\mathrm{K}_{\mathrm{d}}\right)$ Values for Source-Zone Category $\mathrm{D}^{(\mathrm{a})}$

\begin{tabular}{|c|c|c|c|}
\hline Element & $\begin{array}{c}\text { Conservative } \\
\text { and ("Best") } K_{d} \\
\text { Estimate (mL/g) }\end{array}$ & $\begin{array}{c}\text { Range } \mathrm{K}_{\mathrm{d}} \\
\text { Estimate } \\
\text { (mL/g) }\end{array}$ & Justification/References \\
\hline $\begin{array}{l}\text { Tritium, } \\
\text { Cl, Tc, I, } \\
\text { Se, Ru, } \\
\text { C }\end{array}$ & $\begin{array}{c}0 \\
(0)\end{array}$ & 0 to 0.2 & $\begin{array}{l}\text { Technetium, carbon, iodine, selenium, and chlorine are anionic. Tritium } \\
\text { will move with water. Ruthenium has often been suggested as being } \\
\text { coincident with water in tank-leak scenarios based on gamma borehole } \\
\text { logging. Carbon as carbonate in high-pH tank environments is insoluble } \\
\text { and combines with alkaline earth elements. To account for insolubility a } \mathrm{K}_{\mathrm{d}} \\
\text { value }>0 \text { is appropriate, but to keep carbon from getting stuck permanently } \\
\text { in this source (high impact) zone the value must be set at } 0 .{ }^{(0, c, d)}\end{array}$ \\
\hline $\begin{array}{l}\text { Ac, Am, } \\
\mathrm{Ce}, \mathrm{Cm}, \\
\mathrm{Eu},\end{array}$ & $\begin{array}{c}2 \\
(5)\end{array}$ & 2 to 10 & 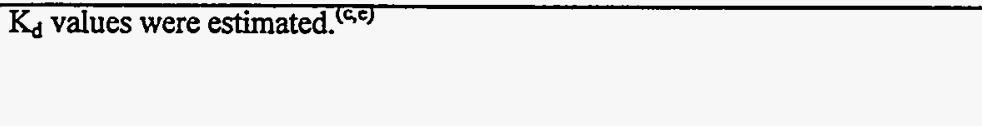 \\
\hline Cs & $\begin{array}{c}1 \\
(1.5)\end{array}$ & 1 to 25 & $\begin{array}{l}\text { Based on observations at Tank T-106, cesium- } 137 \text { seemed to peak at about } \\
3 \mathrm{~m}(10 \mathrm{ft}) \text { below the base (elevation) of the tank and nitrate seemed to peak } \\
\text { at about } 24 \mathrm{~m} \text { ( } 80 \mathrm{ft}) \text {. This implies an in situ retardation factor of about } 8 \text { or } \\
\mathrm{K}_{\mathrm{d}} \text { in the range of } 1-2 \text { during the initial tank leak. The lack of cesium in } \\
\text { groundwater beneath tanks suggests it may not have broken through and } \\
\text { more likely than not has a } \mathrm{K}_{\mathrm{d}} \text { that approaches the default value for neutral, } \\
\text { high salt at greater distances from the source. Serne and Burke }{ }^{(\mathrm{f})} \text { measured } \\
\text { a } \mathrm{K}_{\mathrm{d}} \text { of } 26 \mathrm{~mL} / \mathrm{g} \text { for a simulated REDOX tank liquor. But the results are not } \\
\text { consistent with inferred cesium migration using gamma borehole logging at } \\
\mathrm{SX} \text { tank farm. }{ }^{(\mathrm{g})}\end{array}$ \\
\hline $\begin{array}{l}\mathrm{Co}, \mathrm{Ni} \\
\mathrm{Nb}, \mathrm{Np} \\
\mathrm{Pa}, \mathrm{Sn}\end{array}$ & $\begin{array}{c}0.1 \\
(0.2)\end{array}$ & 0.1 to 4 & The $\mathrm{K}_{\mathrm{d}}$ values were estimated. ${ }^{(\sigma)}$ \\
\hline$\overline{\mathrm{Sr}, \mathrm{Ra}}$ & $\begin{array}{c}4 \\
(10)\end{array}$ & 4 to 20 & $\begin{array}{l}\text { Strontium is known to be rather insoluble in tank liquors and does not } \\
\text { migrate through soils in tank liquor as rapidly as other cations. }(e)\end{array}$ \\
\hline $\begin{array}{l}\text { Th, } \mathrm{Zr} \\
\mathrm{Pb}, \mathrm{Pu}\end{array}$ & $\begin{array}{c}5 \\
(10)\end{array}$ & 5 to 100 & The $\mathrm{K}_{d}$ values were estimated. (घ) \\
\hline$\overline{\mathrm{U}}$ & $\begin{array}{c}5 \\
(20)\end{array}$ & 10 to 800 & $\begin{array}{l}\text { Kaplan et al. }{ }^{(n)} \text { reported that uranium } \mathrm{K}_{\mathrm{d}} \text { values increased from } \sim 2 \text { to } \\
>400 \mathrm{~mL} / \mathrm{g} \text { when the } \mathrm{pH} \text { of a Hanford sediment/groundwater slurry } \\
\text { increased from } 8.3 \text { to }>10.5 \text {. The extremely high } \mathrm{K}_{\mathrm{d}} \text { was attributed to } \\
\text { uranium (co)precipitation either as uranium phases or as calcite phases. } \\
\text { Over a } 1000 \text {-year period, it is anticipated that the solutions } \mathrm{pH} \text { of any near } \\
\text { field would eventually decrease. Thus, over time, the } \mathrm{K}_{\mathrm{d}} \text { values would be } \\
\text { expected to decrease as the pH increased above } \sim 10.5 \text { and the uranium } \\
\text { dissolved from the solid phase. }\end{array}$ \\
\hline
\end{tabular}

(a) Category $\mathrm{D}$ is defined in Table E.4.

(b) From Ames and Rai (1978).

(c) From Thibault, Sheppard, and Smith (1990).

(d) From Martin (1996).

(e) From Ames and Seme (1991),

(f) From Serne and Burke (1997).

(g) From Hartman and Dresel (1997).

(h) From Kaplan et al. (1996). 
Table E.9. Distribution Coefficient $\left(\mathrm{K}_{\mathrm{d}}\right)$ Values for Source-Zone Category $\mathrm{E}^{(\mathrm{a})}$

\begin{tabular}{|c|c|c|c|}
\hline Element & $\begin{array}{c}\text { Conservative } \\
\text { and ("Best") } K_{d} \\
\text { Estimate }(\mathrm{mL} / \mathrm{g})\end{array}$ & $\begin{array}{l}\text { Range } \mathbf{K}_{\mathbf{d}} \\
\text { Estimate } \\
\text { (mL/g) }\end{array}$ & Justification/References \\
\hline $\begin{array}{l}\text { Tritium, } \mathrm{Cl} \text {, } \\
\text { Tc }\end{array}$ & $\begin{array}{c}0 \\
(0)\end{array}$ & 0 to 0.1 & Technetium and chlorine are anionic. Tritium will move with $\mathrm{H}_{2} \mathrm{O}$. \\
\hline $\begin{array}{l}\mathrm{Ac}, \mathrm{Am}, \mathrm{Ce} \\
\mathrm{Cm}, \mathrm{Eu}\end{array}$ & $\begin{array}{c}100 \\
(350)\end{array}$ & $\begin{array}{l}280 \text { to } \\
>1200\end{array}$ & $\begin{array}{l}\text { Americium in a calcium-dominated system has } \mathrm{K}_{\mathrm{d}} \text { values }>1200 \mathrm{~mL} / \mathrm{g} \text {. In } \\
\text { a sodium-dominated system, americium has a } \mathrm{K}_{\mathrm{d}} \text { value of } 280 \mathrm{~mL} / \mathrm{g} \text {. }{ }^{\mathrm{b}} \text { ) }\end{array}$ \\
\hline $\mathrm{C}$ & $0(0)$ & 0 to 10 & $\mathrm{~K}_{\mathrm{d}}$ values were estimated. ${ }^{(c)}$ \\
\hline Co & $\begin{array}{c}50 \\
(50)\end{array}$ & $\begin{array}{c}222 \text { to } \\
4760\end{array}$ & $\begin{array}{l}\text { In a sodium-dominated system, } \mathrm{K}_{\mathrm{d}} \text { values are } 1060 \text { to } 4760 \mathrm{~mL} / \mathrm{g} \text { (d) } \\
\text { In a calcium-dominated system, } \mathrm{K}_{\mathrm{d}} \text { values are } 222 \text { to } 640 \mathrm{~mL} / \mathrm{g} \text { (d) } \\
\text { Cobalt forms complexes, especially with organics. }\end{array}$ \\
\hline Cs & $\begin{array}{c}64 \\
(500)\end{array}$ & 64 to 1360 & $\begin{array}{l}\text { In a sodium-dominated system, } \mathrm{K}_{\mathrm{d}} \text { values are } 64 \text { to } 1170 \mathrm{~mL} / \mathrm{g}{ }^{(\mathrm{d})} \text {. } \\
\text { In a calcium-dominated system, } \mathrm{K}_{\mathrm{d}} \text { values are } 790 \text { to } 1360 \mathrm{~mL} / \mathrm{g}{ }^{(\mathrm{d})} \text {. } \\
\text { Cesium does not form complexes. }\end{array}$ \\
\hline $\mathrm{I}$ & $0(0)$ & 0 to 2 & Iodine is an anion. $\mathrm{K}_{\mathrm{d}}$ values were estimated.(Ct) \\
\hline $\mathrm{Ni}, \mathrm{Sn}, \mathrm{Nb}$ & $\begin{array}{c}30 \\
(50)\end{array}$ & 3 to 40 & $\begin{array}{l}\text { Nickel is similar to cobalt but adsorbs slightly less possibly because of } \\
\text { moderate complexing. } K_{d} \text { values were estimated. }{ }^{(\kappa)}\end{array}$ \\
\hline $\mathrm{Np}, \mathrm{Pa}$ & $\begin{array}{c}0.2 \\
(0.8)\end{array}$ & 0.4 to 4 & $\begin{array}{l}\mathrm{K}_{\mathrm{d}} \text { values range from } 0.4 \text { to } 4 \mathrm{~mL} / \mathrm{g} . \\
\text { is }\end{array}$ \\
\hline$\overline{\mathrm{Pb}}$ & $\begin{array}{c}20 \\
(100)\end{array}$ & 20 to 1000 & Lead is a good absorber, it is insoluble. The $\mathrm{K}_{\mathrm{d}}$ values were estimated. (") \\
\hline $\mathrm{Pu}$ & $\begin{array}{c}5 \\
(20)\end{array}$ & 5 to $>98$ & The $\mathrm{K}_{\mathrm{d}}$ value is $>98 \mathrm{~mL} / \mathrm{g}$. \\
\hline $\mathrm{Ra}, \mathrm{Sr}$ & $\begin{array}{c}0.2 \\
(0.5)\end{array}$ & 0.3 to 42 & $\begin{array}{l}\text { In a sodium-dominated system, } K_{d} \text { values range from } 1.7 \text { to } 42 \mathrm{~mL} / \mathrm{g} \text { for } \\
\text { strontium. } \\
\text { In a calcium-dominated system, } K_{d} \text { value range from } 0.3 \text { to } 1.6 \mathrm{~mL} / \mathrm{g} \text { for } \\
\text { strontium. }\end{array}$ \\
\hline $\mathrm{Ru}$ & $\begin{array}{c}0 \\
(1)\end{array}$ & 0 to 500 & $\begin{array}{l}\text { This element may form } \mathrm{RuO}_{4}{ }^{2-} \text { and/or anionic complexes with nitrates and } \\
\text { nitrites. The } \mathrm{K}_{\mathrm{d}} \text { values were estimated. }{ }^{(\mathrm{e}, \mathrm{i})}\end{array}$ \\
\hline $\mathrm{Se}$ & $\begin{array}{c}0 \\
(0)\end{array}$ & 0 to 4 & Selenium is anionic. The $\mathrm{K}_{d}$ values were estimated. ${ }^{(1)}$ \\
\hline Th, Zr & $\begin{array}{c}40 \\
(50)\end{array}$ & 40 to 470 & In sandy soil, thorium has $\mathrm{K}_{\mathrm{d}}$ values ranging from 40 to $470 \mathrm{~mL} / \mathrm{g}$. ${ }^{(\mathrm{k})}$ \\
\hline $\mathrm{U}$ & $\begin{array}{c}0 \\
(0.3)\end{array}$ & 0 to 3 & $\begin{array}{l}\text { Uranium is anionic and forms neutral carbonate and hydroxide species. } K_{d} \\
\text { values were estimated. }{ }^{(e, t)}\end{array}$ \\
\hline
\end{tabular}

(a) Category $E$ is defined in Table E.4.

(b) From Routson, Jansen, and Robinson (1976).

(c) From Martin (1996).

(d) From Routson, Barney, and Seil (1978).

(e) From Ames and Serne (1991).

(f) From Kaplan et al. (1996).

(g) From Pourbaix (1966).

(h) From Rhodes (1957b).

(i) From Ames and Rai (1978).

(j) From Barney (1978).

(k) From Sheppard, Kittrick, and Hardt (1976). 
Table E.10. Distribution Coefficient $\left(\mathrm{K}_{d}\right)$ Values for Source-Zone Category $\mathrm{F}^{(\mathrm{a})}$

\begin{tabular}{|c|c|c|c|}
\hline Element & $\begin{array}{c}\text { Conservative } \\
\text { and ("Best") } \mathbf{K}_{d} \\
\text { Estimate (mL/g) }\end{array}$ & $\begin{array}{c}\text { Range } K_{d} \\
\text { Estimate } \\
\text { (mL/g) }\end{array}$ & Justification/References \\
\hline $\begin{array}{l}\text { Tritium, } \mathrm{Cl} \text {, } \\
\text { Tc }\end{array}$ & $\begin{array}{c}0 \\
(0)\end{array}$ & -2.8 to 0.6 & $\begin{array}{l}\text { Technetium exists predominantly as } \mathrm{TcO}_{4}^{-} \cdot \mathrm{K}_{\mathrm{d}} \text { values have been reported } \\
\text { for technetium in Hanford sediments ranging from }-2.8 \text { to } 0.6 \mathrm{~mL} / \mathrm{g} \text { for } \\
15 \text { observations with a median of } 0.1 \mathrm{~mL} / \mathrm{g} .^{\left({ }^{())}\right.} \text {Later studies did not } \\
\text { change this range but did decrease the median slightly to }-0.1 \mathrm{~mL} / \mathrm{g} .{ }^{\left({ }^{(}\right)} \\
\text {Negative } \mathrm{K}_{\mathrm{d}} \text { values are physically possible and may not be an } \\
\text { experimental artifact }{ }^{(c)} \text { Tritium is expected to move along with water. } \\
\text { Chlorine is expected to behave as a dissolved anionic species. }\end{array}$ \\
\hline $\begin{array}{l}\text { Ac, Am, Ce, } \\
\mathrm{Cm}, \mathrm{Eu}\end{array}$ & $\begin{array}{c}100 \\
(300)\end{array}$ & 67 to 1330 & $\begin{array}{l}\text { Two ranges for } \mathrm{K}_{\mathrm{d}} \text { values for americium have been reported: } 67 \text { to } \\
>1200 \mathrm{~mL} / \mathrm{g} \cdot{ }^{\left({ }^{(}\right)} \text {and } 125 \text { to } 833 \mathrm{~mL} / \mathrm{g} .{ }^{\left({ }^{(}\right)}\end{array}$ \\
\hline $\bar{C}$ & $\begin{array}{c}0.5 \\
(5, \text { see } \\
\text { Justification })\end{array}$ & 0.5 to 1000 & 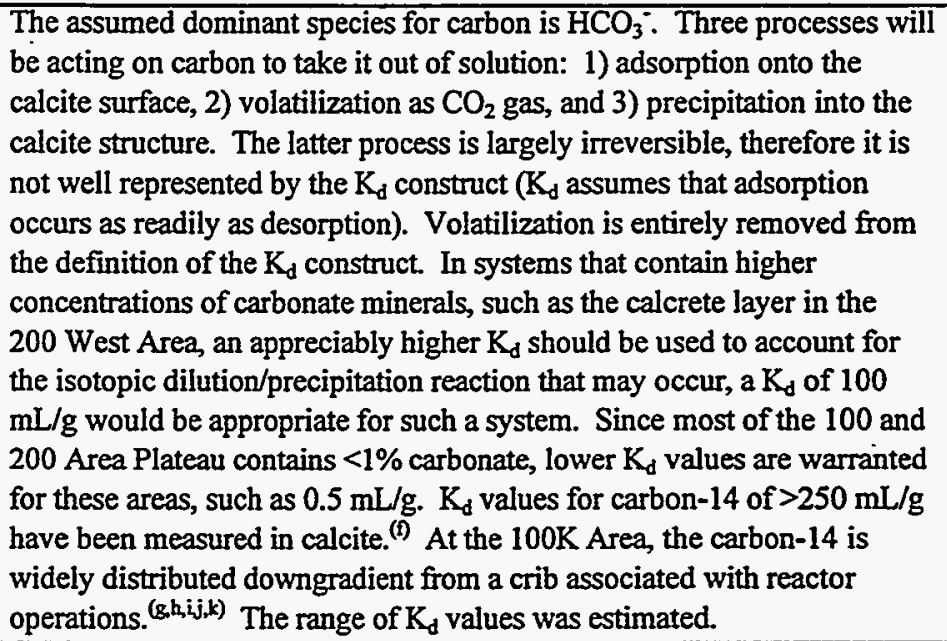 \\
\hline Co & $\begin{array}{c}1200 \\
(1200)\end{array}$ & $\begin{array}{l}1200 \text { to } \\
12500\end{array}$ & $\begin{array}{l}\text { In a sodium-dominated system, the } K_{d} \text { values range from } 1290 \text { to } \\
2120 \mathrm{~mL} / \mathrm{g}^{(1)} \\
\text { In a calcium-dominated system, the } \mathrm{K}_{d} \text { values range from } 2000 \text { to } \\
3870 \mathrm{~mL} / \mathrm{g}^{(1)} \\
\text { In the Hanford sediment/groundwater system, the } \mathrm{K}_{d} \text { values range from } \\
11600 \text { to } 12500 \mathrm{~mL} / \mathrm{g} \text { ( }^{(\mathrm{m})}\end{array}$ \\
\hline $\mathrm{Cs}$ & $\begin{array}{c}540 \\
(1500)\end{array}$ & 540 to 3180 & $\begin{array}{l}\text { In a sodium-dominated system, the } \mathrm{K}_{\mathrm{d}} \text { values range from } 1410 \text { to } \\
1590 \mathrm{~mL} / \mathrm{g}^{(\mathrm{l})} \\
\text { In the Hanford sediment/groundwater system, the } \mathrm{K}_{\mathrm{d}} \text { values range from } \\
540 \text { to } 3180 \mathrm{~mL} / \mathrm{g}^{(\mathrm{m})}\end{array}$ \\
\hline $\bar{I}$ & $\begin{array}{c}0.3 \\
(0.5)\end{array}$ & 0.2 to 15 & $\begin{array}{l}\text { A review of } \mathrm{K}_{\mathrm{d}} \text { values for iodine in Hanford sediments showed a range of } \\
0.7 \text { to } 15 \mathrm{~mL} / \mathrm{g} \text { for } 9 \text { observations; the median was } 0.7 \mathrm{~mL} / \mathrm{g}{ }^{\left({ }^{())}\right.} \mathrm{Later} \\
\text { studies increased this range to } 0.2 \text { to } 15 \mathrm{~mL} / \mathrm{g} \text {; the median was decreased } \\
\text { to } 0.3 \mathrm{~mL} / \mathrm{g} \text {. }{ }^{(c)}\end{array}$ \\
\hline $\mathrm{Ni}, \mathrm{Sn}, \mathrm{Nb}$ & $\begin{array}{c}50 \\
(300)\end{array}$ & 50 to 2350 & $\begin{array}{l}\text { In the Hanford sediment/groundwater system, } \mathrm{K}_{\mathrm{d}} \text { values for nickel ranged } \\
\text { from } 440 \text { to } 2350 \mathrm{~mL} / \mathrm{g}{ }^{(\mathrm{m})} \\
\text { In a broad range of sediments, including those from Hanford, } \mathrm{K}_{\mathrm{d}} \text { values } \\
\left.\text { for nickel ranged from } 50 \text { to } 340 \mathrm{~mL} / \mathrm{g}{ }^{(\mathrm{a})}\right)\end{array}$ \\
\hline
\end{tabular}


Table E.10. (contd)

\begin{tabular}{|c|c|c|c|}
\hline Element & $\begin{array}{l}\text { Conservative } \\
\text { and ("Best") } \mathbf{K}_{\mathrm{d}} \\
\text { Estimate (mL/g) }\end{array}$ & $\begin{array}{c}\text { Range } K_{d} \\
\text { Estimate } \\
(\mathrm{mL} / \mathrm{g})\end{array}$ & Justification/References \\
\hline $\mathrm{Np}, \mathrm{Pa}$ & $\begin{array}{c}10 \\
(15)\end{array}$ & 2.4 to 21.9 & $\begin{array}{l}\text { A review of neptunium } \mathrm{K}_{\mathrm{d}} \text { values for Hanford sediments showed range of } \\
2.4 \text { to } 21.7 \mathrm{~mL} / \mathrm{g} \text { for } 4 \text { observations; the median was } 17.8 \mathrm{~mL} / \mathrm{g} \text {. }{ }^{\left({ }^{\circ}\right)} \text { Later } \\
\text { studies increased the } \mathrm{K}_{\mathrm{d}} \text { values slightly to } 2.2 \text { to } 21.7 \mathrm{~mL} / \mathrm{g} \text {; the median } \\
\text { was slightly lowered, } 15 \mathrm{~mL} / \mathrm{g} \cdot{ }^{(c)} \text { The dominant protactinium species is } \\
\text { assumed to be } \mathrm{PaO}_{2}{ }^{+} \text {and } \mathrm{NpO}_{2}{ }^{+} \text {is assumed to be a reasonable analog. }{ }^{(i)}\end{array}$ \\
\hline$\overline{\mathrm{Pb}}$ & $\begin{array}{c}2,000 \\
(6,000)\end{array}$ & $\begin{array}{l}13,000 \text { to } \\
79,000\end{array}$ & $\begin{array}{l}\text { In a system where the } \mathrm{pH} \text { is } 6 \text { and there are no competing ions, the } \mathrm{K}_{\mathrm{d}} \\
\text { values range from } 13,000 \text { to } 79,000 \mathrm{~mL} / \mathrm{g} .{ }^{(0)}\end{array}$ \\
\hline $\mathrm{Pu}$ & $\begin{array}{c}80 \\
(200)\end{array}$ & 80 to $>1980$ & $\begin{array}{l}\text { For plutonium }(\mathrm{V}, \mathrm{VI}) \text { where the } \mathrm{pH} \text { is } 4 \text { to } 12 \text {, the } \mathrm{K}_{\mathrm{d}} \text { values range from } \\
80 \text { to }>1980 \mathrm{~mL} / \mathrm{g} .{ }^{(\mathrm{p})}\end{array}$ \\
\hline $\mathrm{Ra}, \mathrm{Sr}$ & $\begin{array}{c}8 \\
(20)\end{array}$ & 5 to 173 & 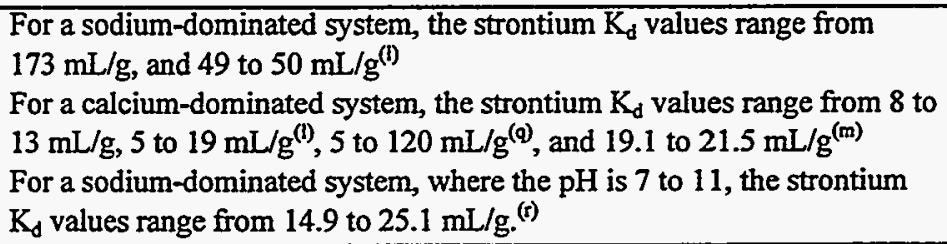 \\
\hline$\overline{\mathrm{Ru}}$ & $\begin{array}{c}10 \\
(20)\end{array}$ & 10 to 1,000 & $\mathrm{~K}_{\mathrm{d}}$ values were estimated. ${ }^{(p, 1)}$ \\
\hline$\overline{\mathrm{Se}}$ & $\begin{array}{c}0 \\
(0)\end{array}$ & $\begin{array}{c}-3.44 \text { to } \\
0.78\end{array}$ & $\begin{array}{l}\text { In the Hanford groundwater } / \text { sediment system, the } \mathrm{K}_{\mathrm{d}} \text { values range from } \\
-3.44 \text { to } 0.78 \mathrm{~mL} / \mathrm{g}^{(\mathrm{m})}\end{array}$ \\
\hline Th, $\mathrm{Zr}$ & $\begin{array}{c}40 \\
(1000)\end{array}$ & 40 to $>2000$ & $\begin{array}{l}\mathrm{K}_{d} \text { values were estimated. } \\
\text { For zirconium, when the } \mathrm{pH} \text { is } 6 \text { to } 12 \text {, the } \mathrm{K}_{\mathrm{d}} \text { values range from } 90 \text { to } \\
>2000 \mathrm{~mL} / \mathrm{g}^{(p)}\end{array}$ \\
\hline$\overline{\mathbf{U}}$ & $\begin{array}{l}0.6 \\
(3)\end{array}$ & 0.1 to 79.3 & $\begin{array}{l}\text { A review of Hanford sediment uranium } \mathrm{K}_{\mathrm{d}} \text { values showed range of } 0.1 \text { to } \\
79.3 \mathrm{~mL} / \mathrm{g} \text { for } 13 \text { observations; the median was } 0.6 \mathrm{~mL} / \mathrm{g} .{ }^{(\mathfrak{b})} \text { Results from } \\
\text { later studies support the range. }{ }^{(c)} \text { In all reported data, some uranium was } \\
\text { adsorbed by Hanford sediments and }>90 \% \text { of the values were between } \\
0.6 \text { and } 4 \mathrm{~mL} / \mathrm{g} \text {. }\end{array}$ \\
\hline
\end{tabular}

(a) Category $\mathrm{F}$ is defined in Table E.4.

(b) From Kaplan and Seme (1995).

(c) From Kaplan et al. (1996).

(d) From Routson, Jansen, and Robinson (1976)

(e) From Sheppard, Kittrick, and Hardt (1976)

(f) From Martin (1996).

(g) From Striegl and Armstrong (1990).

(h) From Garnier (1985).

(i) From Pourbaix (1966).

(j) From Mozeto, Fritz, and Reardon (1983).

(k) From Zhang, Quay, and Wibur (1995).

(l) From Routson, Bamey, and Seil (1978).

(m) From Seme et al. (1993).

(n) From Seme and Relyea (1983).

(o) From Rhodes et al. (1992)

(p) From Rhodes (1957b).

(q) From Rhodes (1957a).

(r) From Nelson (1959). 
Table E.11. Distribution Coefficients $\left(K_{d}\right)$ Values for Source-Zone Category $G^{(a)}$

\begin{tabular}{|c|c|c|c|}
\hline Element & $\begin{array}{l}\text { Conservative } \\
\text { and ("Best") } K_{d} \\
\text { Estimate }(\mathrm{mL} / \mathrm{g})\end{array}$ & $\begin{array}{c}\text { Range } K_{d} \\
\text { Estimate } \\
(\mathrm{mL} / \mathrm{g})\end{array}$ & Justification/References \\
\hline $\begin{array}{l}\text { Tritium, } \\
\text { Cl, Tc, C, } \\
\text { Co, I, Se }\end{array}$ & $\begin{array}{c}0 \\
(0)\end{array}$ & 0 to 0.5 & $\begin{array}{l}\text { Technetium, iodine, selenium, and chlorine are anions. Cobalt forms } \\
\text { an unusually strong complex with EDTA by virtue of unique chemical } \\
\text { reactions, namely the } \mathrm{Co}(\mathrm{II}) \text { converts to } \mathrm{Co}(\mathrm{III}) \text { through an auto- } \\
\text { oxidation process and the } \mathrm{Co}(\mathrm{III}) \text { forms very strong complexes with } \\
\text { the EDTA. Tritium is assumed to behave like water. The others do } \\
\text { not complex with chelators and their low } \mathrm{K}_{\mathrm{d}} \text { values are controlled by } \\
\text { virtue of their anionic nature. }\end{array}$ \\
\hline $\begin{array}{l}\text { Ac, Am, } \\
\text { Ce, Cm, } \\
\text { Eu }\end{array}$ & $\begin{array}{c}3 \\
(3)\end{array}$ & 3 to 50 & $\begin{array}{l}\mathrm{AK}_{\mathrm{d}} \text { for americium of } 5.6 \mathrm{~mL} / \mathrm{g} \text { has been measured in one Hanford } \\
\text { soil (soil } \mathrm{P} \text { ) and } 24 \mathrm{~mL} / \mathrm{g} \text { in another Hanford soil (soil } \mathrm{S} \text { ) in an } \\
\text { aqueous system containing high concentrations of salts and high } \\
\text { concentrations of complexing agents, such as EDTA and HEDTA. } \\
\text { Additionally, bore hole data beneath } 216-\mathrm{Z}-1 \mathrm{~A} \text { Crib suggest that } \\
\text { americium moves appreciably slower than carbon tetrachloride }{ }^{(\mathrm{d})} \text { If } \\
\text { carbon tetrachloride is considered a conservative tracer, then it would } \\
\text { appear that americium behaves as if it has a nonzero } \mathrm{K}_{d} \text { value, i.e., that } \\
\text { it is retarded. } \\
\text { Curium, cerium, and europium have a }+3 \text { valence and were assumed } \\
\text { to behave like americium. }\end{array}$ \\
\hline Cs & $\begin{array}{c}6 \\
(10)\end{array}$ & 6 to 18 & $\begin{array}{l}\text { These estimates are based on column breakthrough curves using actual } \\
\text { uranium recovery scavenged waste. }{ }^{(\mathfrak{)})} \text { The lack of cesium in the } \\
\text { groundwater beneath the cribs suggests it has not broken through and } \\
\text { more likely than not has a } \mathrm{K}_{\mathrm{d}} \text { value that approaches the default value } \\
\text { (Table E.10). }\end{array}$ \\
\hline $\mathrm{Np}, \mathrm{Pa}$ & $\begin{array}{c}2 \\
(5)\end{array}$ & 2 to 10 & $\begin{array}{l}\text { A } \mathrm{K}_{\mathrm{d}} \text { value of } 3.9 \mathrm{~mL} / \mathrm{g} \text { has been measured for neptunium in one } \\
\text { Hanford soil (soil } \mathrm{P} \text { ) and } 6.8 \mathrm{~mL} / \mathrm{g} \text { in another (soil } \mathrm{S} \text { ) using an } \\
\text { aqueous system containing high concentrations of salts and high } \\
\text { concentrations of complexing agents, such as EDTA and HEDTA }{ }^{(c)} \\
\text { It is also assumed that protactinium speciation is predominately } \mathrm{PaO}_{2}{ }^{+} \\
\left.\text {and that } \mathrm{NpO}_{2}{ }^{+} \text {is a reasonable analog. }{ }^{+}\right)\end{array}$ \\
\hline$\overline{\mathrm{U}}$ & $\begin{array}{c}0.2 \\
(0.4)\end{array}$ & 0.2 to 3 & $\mathrm{~K}_{\mathrm{d}}$ values were estimated. ${ }^{(0, a)}$ \\
\hline $\begin{array}{l}\mathrm{Ra}, \mathrm{Sr}, \mathrm{Pb}, \\
\mathrm{Ru}, \mathrm{Ni}, \\
\mathrm{Nb}, \mathrm{Sn}\end{array}$ & $\begin{array}{l}0.4 \\
(5)\end{array}$ & 0 to 30 & $\begin{array}{l}\mathrm{AK}_{\mathrm{d}} \text { value of } 0.02 \mathrm{~mL} / \mathrm{g} \text { has been measured for strontium in one } \\
\text { Hanford soil (soil P) and } 1.5 \mathrm{~mL} / \mathrm{g} \text { in another Hanford soil (soil S) in } \\
\text { an aqueous system containing high concentrations of salts and high } \\
\text { concentrations of complexing agents, such as EDTA and HEDTA }{ }^{(c)} \\
\text { These organic complexants are likely to be degraded by microbes over } \\
\text { time, thereby converting the radionuclides into a more adsorbing } \\
\text { species. } \\
\text { Strontium is used as an analogue because of its similar }+2 \text { valence. }\end{array}$ \\
\hline
\end{tabular}


Table E.11. (contd)

\begin{tabular}{|c|c|c|c|}
\hline Elẹment & $\begin{array}{c}\text { Conservative } \\
\text { and ("Best") } K_{d} \\
\text { Estimate }(\mathrm{mL} / \mathrm{g})\end{array}$ & $\begin{array}{c}\text { Range } \mathrm{K}_{\mathrm{d}} \\
\text { Estimate } \\
(\mathrm{mL} / \mathrm{g})\end{array}$ & Justification/References \\
\hline $\mathrm{Th}, \mathrm{Zr}, \mathrm{Pu}$ & $\begin{array}{l}0.5 \\
(3)\end{array}$ & 0.6 to 100 & $\begin{array}{l}\text { A K } \mathrm{K}_{\mathrm{d}} \text { of } 0.6 \mathrm{~mL} / \mathrm{g} \text { has been measured for plutonium in one Hanford } \\
\text { soil (soil } \mathrm{P} \text { ) and } 2.6 \mathrm{~mL} / \mathrm{g} \text { in another (soil } \mathrm{S} \text { ) with an aqueous system } \\
\text { containing high concentrations of salts and high concentrations of } \\
\text { complexing agents, such as EDTA and HEDTA. }{ }^{(c)} \text { Additionally, bore } \\
\text { hole data beneath } 216-Z-1 \mathrm{~A} \text { Crib suggest that plutonium and } \\
\text { americium move appreciably slower than carbon tetrachloride. } \\
\text { carbon tetrachloride is considered a conservative tracer, then it would } \\
\text { appear that both actinides behave as if they have nonzero } \mathrm{K}_{d} \text { values. }\end{array}$ \\
\hline
\end{tabular}

(a) Category $\mathrm{G}$ is defined in Table E.4.

(b) From Serne et al. (1995).

(c) From Delegard and Barney (1983) (see Table 1.1, "Highly complexed" data).

(d) From Price et al. (1979).

(e) From Delegard and Barney (1983).

(f) From Rhodes and Nelson (1957).

(g) From Pourbaix (1966).

(h) From Ames and Rai (1978).

EDTA $=$ ethylenediaminetetraacetic acid

HEDTA $=\mathrm{N}$-(2-hydroxyethyl) ethylenediaminetetraacetic acid 
Table E.12. Distribution Coefficients $\left(K_{d}\right)$ Values for Source-Zone Category $H^{(a)}$

\begin{tabular}{|c|c|c|c|}
\hline Element & $\begin{array}{l}\text { Conservative and } \\
\text { ("Best") } \mathbf{K}_{d} \\
\text { Estimate }(\mathrm{mL} / \mathrm{g})\end{array}$ & $\begin{array}{l}\text { Range } K_{d} \\
\text { Estimate } \\
(\mathrm{mL} / \mathrm{g})\end{array}$ & Justification/References \\
\hline Tritium & $0(0)$ & 0 & \\
\hline $\begin{array}{l}\mathrm{Ac}, \mathrm{Am}, \\
\mathrm{Ce}, \mathrm{Cm}, \mathrm{Eu}\end{array}$ & $\begin{array}{c}25 \\
(50)\end{array}$ & 50 to 200 & $\mathrm{~K}_{\mathrm{d}}$ values were estimated. (b,a,d) \\
\hline $\mathrm{C}$ & $\begin{array}{c}0.1 \\
(0.2)\end{array}$ & 0.1 to 5 & $\begin{array}{l}\text { In an experiment conducted with } \mathrm{HCO}_{3} \text { spiked Hanford } \\
\text { groundwater (no organics in liquid phase) and Hanford sediments in } \\
\text { which the calcite coatings were removed with acid, } \mathrm{K}_{\mathrm{d}} \text { values } \\
\text { between } 0.27 \text { to } 0.38 \mathrm{~mL} / \mathrm{g} \text { were measured for carbon. } \\
\text { conditions inorganic carbon is predominately } \mathrm{CO}_{2} \text { gas. The main } \\
\text { reason for the nonzero } \mathrm{K}_{\mathrm{d}} \text { value is that anions tend to sorb more to } \\
\text { sediments in acid environments than in basic environments. }\end{array}$ \\
\hline Co & $0.2(5)$ & 0.2 to 20 & $\mathrm{~K}_{d}$ values were estimated. ${ }^{(\mathrm{d})}$ \\
\hline Cs & $\begin{array}{c}10 \\
(30)\end{array}$ & 10 to 100 & $\mathrm{~K}_{d}$ values were estimated.(5,8,h) \\
\hline $\begin{array}{l}\mathrm{I}, \mathrm{Cl}, \mathrm{Tc}, \\
\mathrm{Se}\end{array}$ & $\begin{array}{c}0.1 \\
(0.2)\end{array}$ & 0.1 to 2 & Anions sorb to iron oxides and kaolinite at lower $\mathrm{pH}$ levels. (का) \\
\hline $\mathrm{Ni}, \mathrm{Sn}, \mathrm{Nb}$ & $\begin{array}{c}10 \\
(20)\end{array}$ & 10 to 1,000 & $\mathrm{~K}_{\mathrm{d}}$ values were estimated. ${ }^{(0)}$ \\
\hline$\overline{\mathrm{U}}$ & $\begin{array}{c}20 \\
(30)\end{array}$ & 20 to 200 & $\mathrm{~K}_{d}$ values were estimated. ${ }^{(\mathrm{C})}$ \\
\hline $\mathrm{Np}, \overline{\mathrm{Pa}}$ & $\begin{array}{c}3 \\
(5)\end{array}$ & - & $\begin{array}{l}\mathrm{K}_{\mathrm{d}} \text { values were estimated. }{ }^{(\mathrm{C})} \text { It was also assumed that the dominant } \\
\text { protectinium species is } \mathrm{PaO}_{2}^{+} \text {and that } \mathrm{NpO}_{2}^{+} \text {is a reasonable } \\
\text { analog. }{ }^{\left({ }^{\prime}\right)}\end{array}$ \\
\hline $\mathrm{Pb}$ & $\begin{array}{c}25 \\
(50)\end{array}$ & - & $\mathrm{K}_{d}$ values were estimated. ${ }^{(0)}$ \\
\hline$\overline{\mathrm{Pu}}$ & $\begin{array}{c}20 \\
(50)\end{array}$ & 20 to 200 & $\mathrm{~K}_{\mathrm{d}}$ values were estimated.(5x) \\
\hline $\mathrm{Ra}, \mathrm{Sr}$ & $\begin{array}{c}10 \\
(50)\end{array}$ & 50 to 200 & $\mathrm{~K}_{d}$ values were estimated. ${ }^{\text {(o) }}$ \\
\hline$\overline{\mathbf{R u}}$ & $\begin{array}{c}10 \\
(20)\end{array}$ & 10 to 1000 & $\mathrm{~K}_{\mathrm{d}}$ values were estimated. ${ }^{(\varsigma)}$ \\
\hline Th, Zr & $\begin{array}{c}30 \\
(100)\end{array}$ & 30 to 5000 & $\mathrm{~K}_{\mathrm{d}}$ values were estimated. \\
\hline \multicolumn{4}{|c|}{$\begin{array}{l}\text { (a) Category } \mathrm{H} \text { is defined in Table E.4. } \\
\text { (b) From Benson (1960). } \\
\text { (c) From Routson, Jansen, and Robinson (1976). } \\
\text { (d) From Sheppard, Kittrick, and Hardt (1976). } \\
\text { (e) From Ames and Serne (1991). } \\
\text { (f) From Martin (1996). } \\
\text { (g) From McHenry (1954). } \\
\text { (h) From Rhodes and Nelson (1957). } \\
\text { (i) From Ames and Rai (1978). } \\
\text { (j) From Pourbaix (1966). } \\
\text { (k) From Rhodes (1957b). } \\
\text { (l) From Rhodes (1957a). }\end{array}$} \\
\hline
\end{tabular}


Table E.13. Summary of Best-Estimate $\mathrm{K}_{d}$ Values Used in the Composite Analysis

\begin{tabular}{|c|c|c|c|c|c|c|c|c|c|c|c|c|c|c|c|c|c|c|c|c|c|c|c|c|c|c|c|}
\hline Sources & $\begin{array}{c}\text { Source } \\
\text { Catogory }\end{array}$ & \multicolumn{26}{|c|}{ Eloments } \\
\hline & & $\mathrm{H}$ & $\mathrm{Cl}$ & $T C$ & $A C$ & $\mathrm{Am}$ & Co & $\mathrm{Cm}$ & Eu & C & $\mathrm{Co}_{0}$ & Cs & 1 & NI & \begin{tabular}{|l|}
$\mathrm{Sn}$ \\
\end{tabular} & $\mathrm{Nb}$ & $\mathrm{Np}$ & $\mathrm{Pa}$ & $\mathrm{Pb}$ & $\mathrm{Pu}$ & $\mathrm{Ra}$ & Sr & $\mathrm{Ru}$ & So & Th & $2 r$ & $U$ \\
\hline $\begin{array}{l}\text { Hlgh Organke } \\
\text { Very Acldle }\end{array}$ & $\begin{array}{l}A \\
B \\
F\end{array}$ & $\begin{array}{l}0 \\
0 \\
0\end{array}$ & $\begin{array}{l}0 \\
0 \\
0\end{array}$ & $\begin{array}{l}0 \\
0 \\
0\end{array}$ & $\begin{array}{r}0.3 \\
20 \\
300\end{array}$ & $\begin{array}{r}0.3 \\
20 \\
300\end{array}$ & $\begin{array}{r}0.3 \\
20 \\
300\end{array}$ & \begin{tabular}{|r|}
0.3 \\
20 \\
300
\end{tabular} & $\begin{array}{r}0.3 \\
20 \\
300\end{array}$ & $\begin{array}{l}0 \\
0 \\
5\end{array}$ & $\begin{array}{r}0 \\
3 \\
1200\end{array}$ & $\begin{array}{r}7 \\
10 \\
1500\end{array}$ & $\begin{array}{r}0 \\
0.1 \\
0.5\end{array}$ & $\begin{array}{r}4 \\
4 \\
300\end{array}$ & $\begin{array}{r}4 \\
4 \\
300\end{array}$ & $\begin{array}{r}4 \\
4 \\
300\end{array}$ & $\begin{array}{r}0.2 \\
0.2 \\
15\end{array}$ & $\begin{array}{r}0.2 \\
0.2 \\
15\end{array}$ & $\begin{array}{r}0 \\
4 \\
6000 \\
\end{array}$ & $\begin{array}{r}0.4 \\
25 \\
200\end{array}$ & $\begin{array}{r}0.4 \\
7 \\
20\end{array}$ & $\begin{array}{r}0.4 \\
7 \\
20\end{array}$ & $\begin{array}{r}0.4 \\
2 \\
20\end{array}$ & $\begin{array}{l}0 \\
0 \\
0\end{array}$ & $\begin{array}{r}5 \\
40 \\
1000\end{array}$ & $\begin{array}{r}5 \\
40 \\
1000\end{array}$ & $\begin{array}{r}0.2 \\
0.2 \\
3\end{array}$ \\
\hline $\begin{array}{l}\text { Hlgh Organic } \\
\text { Near Neutral }\end{array}$ & $\begin{array}{l}B \\
B \\
F\end{array}$ & $\begin{array}{l}0 \\
0 \\
0\end{array}$ & $\begin{array}{l}0 \\
0 \\
0\end{array}$ & $\begin{array}{l}0 \\
0 \\
0\end{array}$ & $\begin{array}{r}20 \\
20 \\
300\end{array}$ & $\begin{array}{r}20 \\
20 \\
300\end{array}$ & $\begin{array}{r}20 \\
20 \\
300\end{array}$ & $\begin{array}{r}20 \\
20 \\
300 \\
\end{array}$ & $\begin{array}{r}20 \\
20 \\
300 \\
\end{array}$ & $\begin{array}{l}0 \\
0 \\
5\end{array}$ & $\begin{array}{r}3 \\
3 \\
1200\end{array}$ & $\begin{array}{r}10 \\
10 \\
1500\end{array}$ & $\begin{array}{l}0.1 \\
0.1 \\
0.5\end{array}$ & $\begin{array}{r}4 \\
4 \\
300\end{array}$ & $\begin{array}{r}4 \\
4 \\
300 \\
\end{array}$ & $\begin{array}{r}4 \\
4 \\
300\end{array}$ & $\begin{array}{c}0.2 \\
0.2 \\
15\end{array}$ & $\begin{array}{l}0.2 \\
0.2 \\
15\end{array}$ & $\begin{array}{r}4 \\
.4 \\
6000\end{array}$ & $\begin{array}{r}25 \\
25 \\
200\end{array}$ & $\begin{array}{r}7 \\
7 \\
20\end{array}$ & $\begin{array}{r}7 \\
7 \\
20\end{array}$ & $\begin{array}{r}2 \\
2 \\
20\end{array}$ & $\begin{array}{l}0 \\
0 \\
0\end{array}$ & $\begin{array}{r}40 \\
40 \\
1000\end{array}$ & $\begin{array}{r}40 \\
40 \\
1000\end{array}$ & $\begin{array}{r}0.2 \\
0.2 \\
3\end{array}$ \\
\hline $\begin{array}{l}\text { Very Hlgh Salts } \\
\text { Very Baste. }\end{array}$ & $\begin{array}{l}D \\
E \\
F\end{array}$ & $\begin{array}{l}0 \\
0 \\
0\end{array}$ & $\begin{array}{l}0 \\
0 \\
0\end{array}$ & $\begin{array}{l}0 \\
0 \\
0\end{array}$ & $\begin{array}{r}5 \\
350 \\
300 \\
\end{array}$ & $\begin{array}{r}5 \\
350 \\
300 \\
\end{array}$ & $\begin{array}{r}5 \\
350 \\
300 \\
\end{array}$ & $\begin{array}{r}5 \\
350 \\
300 \\
\end{array}$ & \begin{tabular}{r|}
5 \\
350 \\
300 \\
\end{tabular} & $\begin{array}{l}0 \\
0 \\
5 \\
\end{array}$ & $\begin{array}{r}0.2 \\
50 \\
1200 \\
\end{array}$ & $\begin{array}{r}1.5 \\
500 \\
1500 \\
\end{array}$ & $\begin{array}{r}0 \\
0 \\
0.5 \\
\end{array}$ & $\begin{array}{r}0.2 \\
50 \\
300 \\
\end{array}$ & $\begin{array}{r}0.2 \\
50 \\
300 \\
\end{array}$ & $\begin{array}{r}0.2 \\
50 \\
300 \\
\end{array}$ & \begin{tabular}{r|}
0.2 \\
0.8 \\
15 \\
\end{tabular} & $\begin{array}{r}0.2 \\
0.8 \\
15 \\
\end{array}$ & \begin{tabular}{|r|}
10 \\
100 \\
6000 \\
\end{tabular} & $\begin{array}{r}10 \\
20 \\
200 \\
\end{array}$ & \begin{tabular}{r|}
10 \\
0.5 \\
20
\end{tabular} & $\begin{array}{r}10 \\
0.5 \\
20\end{array}$ & $\begin{array}{r}1 \\
1 \\
20\end{array}$ & $\begin{array}{l}0 \\
0 \\
0\end{array}$ & $\begin{array}{r}10 \\
50 \\
1000 \\
\end{array}$ & $\begin{array}{r}10 \\
50 \\
1000\end{array}$ & $\begin{array}{r}20 \\
0.3 \\
3\end{array}$ \\
\hline $\begin{array}{l}\text { Chelates } \\
\text { High Salts }\end{array}$ & $\begin{array}{l}\mathbf{G} \\
\mathbf{G} \\
\mathbf{C}\end{array}$ & $\begin{array}{l}0 \\
0 \\
0\end{array}$ & $\begin{array}{l}0 \\
0 \\
0\end{array}$ & $\begin{array}{l}\overline{0} \\
0 \\
0\end{array}$ & $\begin{array}{r}3 \\
3 \\
50\end{array}$ & $\begin{array}{r}3 \\
3 \\
50 \\
\end{array}$ & $\begin{array}{r}3 \\
3 \\
50\end{array}$ & $\begin{array}{r}3 \\
3 \\
50\end{array}$ & \begin{tabular}{r|}
3 \\
3 \\
50
\end{tabular} & \begin{tabular}{l|}
0 \\
0 \\
5
\end{tabular} & $\begin{array}{l}0 \\
0 \\
0\end{array}$ & $\begin{array}{r}10 \\
10 \\
1500\end{array}$ & $\begin{array}{r}0 \\
0 \\
0.5\end{array}$ & $\begin{array}{l}5 \\
5 \\
4\end{array}$ & $\begin{array}{l}5 \\
5 \\
4\end{array}$ & $\begin{array}{r}5 \\
5 \\
300 \\
\end{array}$ & \begin{tabular}{l|}
5 \\
5 \\
5
\end{tabular} & $\begin{array}{l}5 \\
5 \\
5\end{array}$ & $\begin{array}{l}5 \\
5 \\
4 \\
\end{array}$ & \begin{tabular}{r|}
3 \\
3 \\
40
\end{tabular} & \begin{tabular}{r|}
5 \\
5 \\
20
\end{tabular} & $\begin{array}{l}5 \\
5 \\
4\end{array}$ & $\begin{array}{r}5 \\
5 \\
20\end{array}$ & $\begin{array}{l}0 \\
0 \\
0\end{array}$ & \begin{tabular}{|r|}
3 \\
3 \\
1000
\end{tabular} & $\begin{array}{r}3 \\
3 \\
3000\end{array}$ & $\begin{array}{r}0.4 \\
0.4 \\
3\end{array}$ \\
\hline $\begin{array}{l}\text { Low Organic } \\
\text { Low Salts }\end{array}$ & $\begin{array}{l}\mathrm{H} \\
\mathrm{F}\end{array}$ & 0 & $\begin{array}{r}0.2 \\
0\end{array}$ & $\begin{array}{r}0.2 \\
0\end{array}$ & $\begin{array}{r}50 \\
300\end{array}$ & $\begin{array}{r}50 \\
300 \\
\end{array}$ & $\begin{array}{r}50 \\
300 \\
\end{array}$ & $\begin{array}{r}50 \\
300 \\
\end{array}$ & $\begin{array}{r}50 \\
300 \\
\end{array}$ & $\begin{array}{r}0.2 \\
5\end{array}$ & $\begin{array}{r}5 \\
1200\end{array}$ & $\begin{array}{r}30 \\
1500\end{array}$ & $\begin{array}{l}0.2 \\
0.5\end{array}$ & $\begin{array}{r}20 \\
300\end{array}$ & \begin{tabular}{r|}
20 \\
300
\end{tabular} & $\begin{array}{r}20 \\
300 \\
\end{array}$ & $\begin{array}{r}5 \\
15\end{array}$ & $\begin{array}{r}5 \\
15\end{array}$ & \begin{tabular}{|r|}
50 \\
6000 \\
\end{tabular} & $\begin{array}{r}50 \\
200\end{array}$ & $\begin{array}{l}50 \\
20\end{array}$ & $\begin{array}{l}50 \\
20\end{array}$ & $\begin{array}{l}20 \\
20\end{array}$ & $\begin{array}{r}0.2 \\
0\end{array}$ & $\begin{array}{r}100 \\
1000 \\
\end{array}$ & $\begin{array}{r}100 \\
1000\end{array}$ & $\begin{array}{r}30 \\
3\end{array}$ \\
\hline Acidic & $F$ & 0 & 0 & 0 & 300 & 300 & 300 & 300 & 300 & 5) & 1200 & 1500 & 0.5 & 300 & 300 & 300 & 16 & 15 & 6000 & 200 & 20) & 20 & 20 & of & $\begin{array}{l}1000 \\
\end{array}$ & 1000 & 3 \\
\hline $\begin{array}{l}\text { Low Organle } \\
\text { Low Salts }\end{array}$ & $\begin{array}{l}F \\
F\end{array}$ & $\begin{array}{l}0 \\
0\end{array}$ & $\begin{array}{l}0 \\
0\end{array}$ & $\begin{array}{l}0 \\
0\end{array}$ & $\begin{array}{l}300 \\
300\end{array}$ & $\begin{array}{l}300 \\
300\end{array}$ & $\begin{array}{l}300 \\
300\end{array}$ & $\begin{array}{l}300 \\
300\end{array}$ & $\begin{array}{l}300 \\
300\end{array}$ & $\begin{array}{l}5 \\
5\end{array}$ & $\begin{array}{l}1200 \\
1200\end{array}$ & $\begin{array}{l}1500 \\
1500\end{array}$ & $\begin{array}{l}0.5 \\
0.5\end{array}$ & $\begin{array}{l}300 \\
300\end{array}$ & $\begin{array}{l}300 \\
300\end{array}$ & $\begin{array}{l}300 \\
300\end{array}$ & $\begin{array}{l}15 \\
15\end{array}$ & $\begin{array}{l}15 \\
15\end{array}$ & $\begin{array}{l}6000 \\
6000\end{array}$ & $\begin{array}{l}200 \\
200\end{array}$ & $\begin{array}{l}20 \\
20\end{array}$ & $\begin{array}{l}20 \\
20\end{array}$ & $\begin{array}{l}20 \\
20\end{array}$ & $\begin{array}{l}0 \\
0\end{array}$ & \begin{tabular}{|l|}
1000 \\
1000 \\
\end{tabular} & $\begin{array}{l}1000 \\
1000\end{array}$ & $\begin{array}{l}3 \\
3\end{array}$ \\
\hline Noar Noutral & $\mathbf{F}$ & 0 & 0 & 0 & 300 & 300 & 300 & 300 & 300 & 5 & 1200 & 1500 & 0.5 & 300 & 300 & 300 & 15 & 15 & 6000 & 200 & 20 & 20 & 20 & of & 1000 & 1000 & 3 \\
\hline
\end{tabular}


Table E.14. Summary of Best-Estimate $\mathrm{K}_{d}$ Values Used in the Composite Analysis, Adjusted to a Maximum of $40 \mathrm{~mL} / \mathrm{g}$

\begin{tabular}{|c|c|c|c|c|c|c|c|c|c|c|c|c|c|c|c|c|c|c|c|c|c|c|c|c|c|c|c|}
\hline Sources & $\begin{array}{c}\text { Source } \\
\text { Calegory }\end{array}$ & \multicolumn{26}{|c|}{ Eloments } \\
\hline & & H] & 11 & & $A C$ & $\mathrm{Am}$ & Co & $\mathrm{Cm}$ & Eu & C & Co & $\mathrm{CB}_{8}$ & 1 & NII & Sn & $\mathrm{Nb}$ & \begin{tabular}{|l|}
$\mathrm{Np}$ \\
\end{tabular} & \begin{tabular}{|l|}
$\mathrm{Pa}$ \\
\end{tabular} & $\mathrm{Pb}$ & Pu & $\mathrm{Ra}$ & $\mathrm{Sr}$ & \begin{tabular}{|l|l|} 
Ru \\
\end{tabular} & Se & Th & $\bar{z}$ & $\bar{U}$ \\
\hline $\begin{array}{l}\text { High Organic } \\
\text { Very Acidic }\end{array}$ & $\begin{array}{l}A \\
B \\
F\end{array}$ & $\begin{array}{l}0 \\
0 \\
0\end{array}$ & $\begin{array}{l}0 \\
0 \\
0\end{array}$ & $\begin{array}{l}0 \\
0 \\
0\end{array}$ & $\begin{array}{r}0.3 \\
20 \\
40\end{array}$ & $\begin{array}{r}0.3 \\
20 \\
40\end{array}$ & $\begin{array}{r}0.3 \\
20 \\
40\end{array}$ & $\begin{array}{r}0.3 \\
20 \\
40\end{array}$ & $\begin{array}{r}0.3 \\
20 \\
40\end{array}$ & $\begin{array}{l}0 \\
0 \\
5\end{array}$ & $\begin{array}{r}0 \\
3 \\
40\end{array}$ & $\begin{array}{r}7 \\
10 \\
40\end{array}$ & $\begin{array}{r}0 \\
0.1 \\
0.5\end{array}$ & $\begin{array}{r}4 \\
4 \\
40\end{array}$ & $\begin{array}{r}4 \\
4 \\
40\end{array}$ & $\begin{array}{r}4 \\
4 \\
40\end{array}$ & $\left|\begin{array}{c}0.2 \\
0.2 \\
15\end{array}\right|$ & \begin{tabular}{|c|}
0.2 \\
0.2 \\
15
\end{tabular} & $\begin{array}{r}0 \\
4 \\
40\end{array}$ & \begin{tabular}{r|}
0.4 \\
25 \\
40
\end{tabular} & $\begin{array}{r}0.4 \\
7 \\
20\end{array}$ & $\begin{array}{r}0.4 \\
7 \\
20\end{array}$ & $\begin{array}{r}0.4 \\
2 \\
20\end{array} \mid$ & $\begin{array}{l}0 \\
0 \\
0\end{array}$ & $\begin{array}{r}5 \\
40 \\
40\end{array}$ & $\begin{array}{r}5 \\
40 \\
40\end{array}$ & $\begin{array}{r}0.2 \\
0.2 \\
3\end{array}$ \\
\hline $\begin{array}{l}\text { High Organic } \\
\text { Near Neutral }\end{array}$ & $\begin{array}{l}B \\
B \\
F\end{array}$ & $\begin{array}{l}0 \\
0 \\
0 \\
0\end{array}$ & $\begin{array}{l}0 \\
0 \\
0 \\
0\end{array}$ & $\begin{array}{l}0 \\
0 \\
0 \\
0\end{array}$ & $\begin{array}{l}20 \\
20 \\
40\end{array}$ & $\begin{array}{l}20 \\
20 \\
40\end{array}$ & $\begin{array}{l}20 \\
20 \\
40\end{array}$ & $\begin{array}{l}20 \\
20 \\
40\end{array}$ & $\begin{array}{l}20 \\
20 \\
40\end{array}$ & $\begin{array}{l}0 \\
0 \\
5 \\
5\end{array}$ & $\begin{array}{r}3 \\
3 \\
40\end{array}$ & \begin{tabular}{c|}
10 \\
10 \\
40
\end{tabular} & $\begin{array}{l}0.1 \\
0.1 \\
0.5\end{array}$ & $\begin{array}{r}4 \\
4 \\
\end{array}$ & \begin{tabular}{r|}
4 \\
4 \\
\end{tabular} & \begin{tabular}{r|}
4 \\
4 \\
4
\end{tabular} & \begin{tabular}{|l|}
0.2 \\
0.2 \\
\end{tabular} & \begin{tabular}{|l|}
0.2 \\
0.2 \\
\end{tabular} & \begin{tabular}{r|}
4 \\
4 \\
\end{tabular} & $\begin{array}{l}25 \\
25 \\
\end{array}$ & $\begin{array}{r}7 \\
7 \\
20\end{array}$ & $\begin{array}{r}7 \\
7 \\
\end{array}$ & \begin{tabular}{r|}
2 \\
2 \\
\end{tabular} & 吘 & $\begin{array}{l}40 \\
40 \\
\end{array}$ & $\begin{array}{l}40 \\
40\end{array}$ & $\begin{array}{l}0.2 \\
0.2\end{array}$ \\
\hline $\begin{array}{l}\text { Very Hilgh Satts } \\
\text { Very Basic }\end{array}$ & $\begin{array}{l}D \\
E \\
F\end{array}$ & $\begin{array}{l}0 \\
0 \\
0\end{array}$ & $\begin{array}{l}0 \\
0 \\
0\end{array}$ & $\begin{array}{l}0 \\
0 \\
0\end{array}$ & $\begin{array}{r}\quad \\
40 \\
40\end{array}$ & $\begin{array}{r}5 \\
40 \\
40\end{array}$ & $\begin{array}{r}5 \\
40 \\
40\end{array}$ & $\begin{array}{r}5 \\
40 \\
40\end{array}$ & $\begin{array}{r}5 \\
40 \\
40\end{array}$ & $\begin{array}{l}0 \\
0 \\
5 \\
5\end{array}$ & \begin{tabular}{l|}
0.2 \\
40 \\
40
\end{tabular} & $\begin{array}{l}1.5 \\
40 \\
40\end{array}$ & $\begin{array}{r}0 \\
0 \\
0.5\end{array}$ & $\begin{array}{l}0.2 \\
40 \\
40\end{array}$ & \begin{tabular}{c|}
0.2 \\
40 \\
40
\end{tabular} & \begin{tabular}{l|}
0.2 \\
40 \\
40
\end{tabular} & \begin{tabular}{|c|}
0.2 \\
0.8 \\
15
\end{tabular} & \begin{tabular}{|c|}
0.2 \\
0.8 \\
15 \\
\end{tabular} & $\begin{array}{l}10 \\
40 \\
40\end{array}$ & \begin{tabular}{l|}
40 \\
20 \\
40
\end{tabular} & \begin{tabular}{l|}
10 \\
0.5 \\
20
\end{tabular} & $\begin{array}{r}10 \\
0.5 \\
20\end{array}$ & \begin{tabular}{r|}
$r$ \\
1 \\
20 \\
20
\end{tabular} & $\begin{array}{l}0 \\
0 \\
0 \\
0\end{array}$ & $\begin{array}{l}40 \\
10 \\
40 \\
40\end{array}$ & $\begin{array}{l}40 \\
40 \\
40 \\
40\end{array}$ & $\begin{array}{r}20 \\
0.3 \\
\end{array}$ \\
\hline $\begin{array}{l}\text { Chalales } \\
\text { High Sahts }\end{array}$ & $\begin{array}{l}G \\
G \\
c \\
\end{array}$ & $\begin{array}{l}0 \\
0 \\
0 \\
\end{array}$ & $\begin{array}{l}\overline{0} \\
0 \\
0 \\
\end{array}$ & $\begin{array}{l}0 \\
0 \\
0 \\
\end{array}$ & $\begin{array}{r}3 \\
3 \\
40 \\
\end{array}$ & $\begin{array}{r}3 \\
3 \\
40 \\
\end{array}$ & $\begin{array}{r}3 \\
3 \\
40 \\
\end{array}$ & $\begin{array}{r}3 \\
3 \\
40 \\
\end{array}$ & $\begin{array}{r}3 \\
3 \\
40 \\
\end{array}$ & $\begin{array}{l}0 \\
0 \\
5\end{array}$ & $\begin{array}{l}0 \\
0 \\
0\end{array}$ & $\begin{array}{l}10 \\
10 \\
40 \\
\end{array}$ & $\begin{array}{r}0 \\
0 \\
0.5 \\
\end{array}$ & $\begin{array}{l}5 \\
5 \\
4\end{array}$ & $\begin{array}{l}5 \\
5 \\
4\end{array}$ & $\begin{array}{r}5 \\
5 \\
40 \\
\end{array}$ & \begin{tabular}{|l|}
5 \\
5 \\
\end{tabular} & $\begin{array}{l}5 \\
5 \\
5\end{array}$ & \begin{tabular}{l|}
5 \\
5 \\
4
\end{tabular} & \begin{tabular}{r|r|}
3 \\
3 \\
40
\end{tabular} & $\begin{array}{r}5 \\
5 \\
20\end{array}$ & $\begin{array}{l}5 \\
5 \\
4\end{array}$ & $\begin{array}{r}5 \\
5 \\
20 \\
\end{array}$ & $\begin{array}{l}0 \\
0 \\
0\end{array}$ & \begin{tabular}{r|}
3 \\
3 \\
40
\end{tabular} & \begin{tabular}{r|}
3 \\
3 \\
40
\end{tabular} & $\begin{array}{r}0.4 \\
0.4 \\
3\end{array}$ \\
\hline $\begin{array}{l}\text { Low Organle } \\
\text { Low Salts } \\
\text { Acldic }\end{array}$ & $\begin{array}{l}H \\
F \\
F\end{array}$ & $\begin{array}{l}0 \\
0 \\
0 \\
0\end{array}$ & \begin{tabular}{r|r|}
1.2 \\
0 \\
0
\end{tabular} & $\begin{array}{r}0.2 \\
0 \\
0\end{array}$ & $\begin{array}{l}40 \\
40 \\
40\end{array}$ & $\begin{array}{l}40 \\
40 \\
40\end{array}$ & $\begin{array}{l}40 \\
40 \\
40\end{array}$ & $\begin{array}{l}40 \\
40 \\
40\end{array}$ & $\begin{array}{l}40 \\
40 \\
40\end{array}$ & $\begin{array}{r}0.2 \\
5 \\
5\end{array}$ & \begin{tabular}{r|}
5 \\
40 \\
40
\end{tabular} & $\begin{array}{l}30 \\
40 \\
40\end{array}$ & $\begin{array}{l}0.2 \\
0.5 \\
0.5\end{array}$ & $\begin{array}{l}20 \\
40 \\
40\end{array}$ & $\begin{array}{l}20 \\
40 \\
40\end{array}$ & $\begin{array}{l}20 \\
40 \\
40\end{array}$ & \begin{tabular}{r|}
5 \\
15 \\
15
\end{tabular} & \begin{tabular}{l|}
5 \\
15 \\
15
\end{tabular} & $\begin{array}{l}40 \\
40 \\
40\end{array}$ & $\begin{array}{l}40 \\
40 \\
40\end{array}$ & $\begin{array}{l}40 \\
20 \\
20\end{array}$ & $\begin{array}{l}40 \\
20 \\
20\end{array}$ & $\begin{array}{l}20 \\
20 \\
20\end{array}$ & \begin{tabular}{r|r|r}
0.2 \\
0 \\
0
\end{tabular} & \begin{tabular}{l|l|}
40 \\
40 \\
40
\end{tabular} & $\begin{array}{l}40 \\
40 \\
40 \\
40\end{array}$ & $\begin{array}{r}30 \\
3\end{array}$ \\
\hline $\begin{array}{l}\text { Low Organic } \\
\text { Low Salts } \\
\text { Near Neutral }\end{array}$ & $\begin{array}{l}F \\
F \\
F\end{array}$ & $\begin{array}{c}0 \\
0 \\
0\end{array}$ & 0 & $\begin{array}{l}0 \\
0 \\
0\end{array}$ & $\begin{array}{l}40 \\
40 \\
40\end{array}$ & $\begin{array}{l}40 \\
40 \\
40 \\
\end{array}$ & $\begin{array}{l}40 \\
40 \\
40\end{array}$ & $\begin{array}{l}40 \\
40 \\
40 \\
\end{array}$ & $\begin{array}{l}40 \\
40 \\
40\end{array}$ & $\begin{array}{l}5 \\
5 \\
5\end{array}$ & \begin{tabular}{l|}
40 \\
40 \\
40
\end{tabular} & $\begin{array}{l}40 \\
40 \\
40\end{array}$ & $\begin{array}{l}0.5 \\
0.5 \\
0.5\end{array}$ & \begin{tabular}{l|}
40 \\
40 \\
40
\end{tabular} & $\begin{array}{l}40 \\
40 \\
40\end{array}$ & $\begin{array}{l}80 \\
40 \\
40\end{array}$ & $\begin{array}{l}15 \\
15 \\
15\end{array}$ & \begin{tabular}{|l|}
15 \\
15 \\
15 \\
\end{tabular} & $\begin{array}{l}40 \\
40 \\
40\end{array}$ & $\begin{array}{l}40 \\
40 \\
40\end{array}$ & $\begin{array}{l}20 \\
20 \\
20\end{array}$ & $\begin{array}{l}20 \\
20 \\
20\end{array}$ & $\begin{array}{l}20 \\
20 \\
20\end{array}$ & $\begin{array}{l}0 \\
0 \\
0\end{array}$ & $\begin{array}{l}40 \\
40 \\
40\end{array}$ & $\begin{array}{l}40 \\
40 \\
40\end{array}$ & $\begin{array}{l}3 \\
3 \\
\end{array}$ \\
\hline
\end{tabular}


Table E.15. Summary of Conservative $\mathrm{K}_{d}$ Values Used in the Composite Analysis

\begin{tabular}{|c|c|c|c|c|c|c|c|c|c|c|c|c|c|c|c|c|c|c|c|c|c|c|c|c|c|c|c|}
\hline Sources & $\begin{array}{c}\text { Source } \\
\text { Catogory }\end{array}$ & \multicolumn{26}{|c|}{ Elements } \\
\hline & & $\mathrm{HI}$ & CI & Tc & $A C$ & $\mathrm{Am}$ & Ce & $\mathrm{Cm}$ & Eu & C & Co & $\mathrm{C}_{8}$ & $I$ & NI & Sn & $\mathrm{Nb}$ & $\mathrm{Np}$ & $\mathbf{P a}$ & $\mathrm{Pb}$ & Pu & $\mathbf{R a}$ & Sr & Ru & $S_{\theta}$ & Th & $2 r$ & U \\
\hline $\begin{array}{l}\text { High Organic } \\
\text { Very Acldlc }\end{array}$ & $\begin{array}{l}A \\
B \\
F\end{array}$ & $\begin{array}{l}0 \\
0 \\
0\end{array}$ & $\begin{array}{l}0 \\
0 \\
0\end{array}$ & $\begin{array}{l}0 \\
0 \\
0\end{array}$ & $\begin{array}{r}0 \\
10 \\
100\end{array}$ & $\begin{array}{r}0 \\
10 \\
100\end{array}$ & $\begin{array}{r}0 \\
10 \\
100\end{array}$ & $\begin{array}{r}0 \\
10 \\
100\end{array}$ & $\begin{array}{r}0 \\
10 \\
100\end{array}$ & $\begin{array}{r}0 \\
0 \\
0.5\end{array}$ & $\begin{array}{r}0 \\
0.1 \\
1200\end{array}$ & $\begin{array}{r}5 \\
5 \\
540\end{array}$ & $\begin{array}{r}0 \\
0 \\
0.3\end{array}$ & $\begin{array}{r}2 \\
3 \\
50\end{array}$ & $\begin{array}{r}2 \\
3 \\
50\end{array}$ & $\begin{array}{r}2 \\
3 \\
50\end{array}$ & $\begin{array}{r}0.1 \\
0.1 \\
10\end{array}$ & $\begin{array}{r}0.1 \\
0.1 \\
10\end{array}$ & $\begin{array}{r}0 \\
0 \\
2000\end{array}$ & $\begin{array}{r}0.1 \\
15 \\
80\end{array}$ & $\begin{array}{r}0.1 \\
5 \\
0\end{array}$ & $\begin{array}{r}0.1 \\
5 \\
8\end{array}$ & $\begin{array}{l}0.1 \\
0.1 \\
10\end{array}$ & $\begin{array}{l}0 \\
0 \\
0\end{array}$ & $\begin{array}{r}1 \\
20 \\
40\end{array}$ & $\begin{array}{r}1 \\
20 \\
40\end{array}$ & $\begin{array}{l}0.1 \\
0.2 \\
0.6\end{array}$ \\
\hline Hlgh Organlc & $\bar{B}$ & 0 & $\overline{0}$ & $\overline{0}$ & 70 & 10 & 10 & 10 & 10 & 0 & 0.1 & 5 & 0 & 3 & 3 & 3 & 0.1 & 0.1 & 0 & 15 & 5 & 5 & 0.1 & 0 & 20 & 20 & 0.2 \\
\hline Near Neutral & $B$ & of & 0 & 0 & 10 & 10 & 10 & 10 & 10 & 0 & 0.1 & 5 & 0 & 3 & 3 & 3 & 0.1 & 0.1 & 0 & 15 & 5 & 5 & 0.1 & 0 & 20 & 20 & 0.2 \\
\hline & $\mathbf{F}$ & of & 0 & 0 & 100 & 100 & 100 & 100 & 100 & 0.5 & 1200 & 540 & 0.3 & 50 & 50 & 50 & 10 & 10 & 2000 & 80 & 8 & 8 & 10 & 의 & 40 & 40 & 0.6 \\
\hline $\begin{array}{l}\text { Very Hlgh Salts } \\
\text { Very Basic }\end{array}$ & $\begin{array}{l}\bar{D} \\
\mathrm{E}\end{array}$ & $\begin{array}{l}0 \\
0 \\
0\end{array}$ & $\begin{array}{l}0 \\
0\end{array}$ & $\begin{array}{l}0 \\
0\end{array}$ & $\begin{array}{r}2 \\
100\end{array}$ & $\begin{array}{r}2 \\
100\end{array}$ & $\begin{array}{r}2 \\
100\end{array}$ & $\begin{array}{r}2 \\
100\end{array}$ & $\begin{array}{r}2 \\
100\end{array}$ & $\begin{array}{l}0 \\
0\end{array}$ & $\begin{array}{r}0.1 \\
50\end{array}$ & $\begin{array}{r}1 \\
84\end{array}$ & $\begin{array}{l}0 \\
0\end{array}$ & $\begin{array}{l}0.1 \\
30\end{array}$ & $\begin{array}{r}0.1 \\
30\end{array}$ & $\begin{array}{r}0.1 \\
30\end{array}$ & $\begin{array}{l}0.1 \\
0.2\end{array}$ & $\begin{array}{l}0.1 \\
0.2\end{array}$ & $\begin{array}{r}5 \\
20\end{array}$ & $\begin{array}{l}5 \\
5\end{array}$ & $\begin{array}{r}4 \\
0.2\end{array}$ & $\begin{array}{r}4 \\
0.2\end{array}$ & $\begin{array}{l}0 \\
0\end{array}$ & $\begin{array}{l}0 \\
0\end{array}$ & $\begin{array}{r}5 \\
40\end{array}$ & $\begin{array}{r}5 \\
40\end{array}$ & $\begin{array}{l}5 \\
0\end{array}$ \\
\hline & $F$ & 0 & 0 & 0 & $\begin{array}{l}100 \\
100\end{array}$ & $\begin{array}{l}100 \\
100\end{array}$ & 100 & 100 & 100 & 0.5 & 1200 & 540 & 0.3 & 50 & 50 & 50 & $\begin{array}{l}.4 \\
10\end{array}$ & $\begin{array}{r}.2 \\
10\end{array}$ & 2000 & 80 & $\begin{array}{r}0.2 \\
8\end{array}$ & $\begin{array}{r}0.4 \\
8\end{array}$ & 10 & 0 & 40 & $\begin{array}{l}40 \\
40\end{array}$ & 0.8 \\
\hline Chelates & G & 0 & $\overline{0}$ & $\overline{0}$ & 3 & 3 & 3 & 3 & 3 & 0 & 0 & 6 & 0 & 0.4 & 0.4 & $\begin{array}{l}0.4 \\
\end{array}$ & 2 & 2 & 0.4 & 0.5 & 0.4 & 0.4 & $\overline{0.4}$ & 0 & 0.5 & 0.5 & 0.2 \\
\hline Hilgh Salts & $\begin{array}{l}G \\
c\end{array}$ & ? & 0 & $\begin{array}{l}0 \\
0\end{array}$ & $\begin{array}{r}3 \\
10\end{array}$ & $\begin{array}{r}3 \\
10\end{array}$ & $\begin{array}{r}3 \\
10\end{array}$ & $\begin{array}{r}3 \\
10\end{array}$ & $\begin{array}{r}3 \\
10\end{array}$ & 0 & $\begin{array}{l}0 \\
0\end{array}$ & 6 & .0 & 0.4 & 0.4 & 0.4 & 2 & 2 & 0.4 & 0.5 & 0.4 & 0.4 & 0.4 & 0 & 0.5 & 0.5 & 0.2 \\
\hline Low Omanlc & $\frac{C}{H}$ & 0 & 0.1 & 0.1 & 25 & 25 & $\frac{10}{25}$ & $\frac{10}{25}$ & $\frac{10}{25}$ & $\frac{0.5}{0.1}$ & 0.2 & $\frac{540}{10}$ & 0.3 & $\frac{2}{10}$ & $\frac{2}{10}$ & $\frac{50}{10}$ & $\frac{2}{3}$ & $\frac{4}{3}$ & $\frac{2}{25}$ & $\frac{20}{20}$ & $\begin{array}{r}8 \\
10\end{array}$ & $\frac{2}{10}$ & $\frac{10}{10}$ & 0 & $\frac{40}{30}$ & $\frac{40}{30}$ & $\frac{0.6}{20}$ \\
\hline Low Salts & $\tilde{F}$ & 0 & 0 & 0 & 100 & 100 & 100 & 100 & 100 & 0.5 & 1200 & 540 & 0.3 & 50 & 50 & 50 & 10 & 10 & 2000 & 80 & 8 & 8 & 10 & 0 & 40 & 40 & 0.6 \\
\hline Acldic & $\mathbf{F}$ & of & 0 & 0 & 100 & 100 & 100 & 100 & 100 & 0.5 & 1200 & 540 & 0.3 & 50 & 50 & 50 & 10 & 10 & 2000 & 80 & 8 & 8 & 10 & 0 & 40 & 40 & 0.6 \\
\hline Low Organic & $F$ & of & 0 & $\overline{0}$ & 100 & 100 & 100 & 100 & 100 & 0.5 & 1200 & 540 & 0.3 & 50 & 50 & 50 & 10 & 10 & 2000 & 80 & 8 & $\overline{8}$ & 10 & 0 & 40 & 40 & 0.6 \\
\hline Low Salts & $\mathbf{F}$ & of & 0 & 0 & 100 & 100 & 100 & 100 & 100 & 0.5 & 1200 & 640 & 0.3 & 50 & 50 & 50 & 10 & 10| & 2000 & 80 & 8] & 8 & 10 & 0 & 40 & 40 & 0.6 \\
\hline Near Neutral & $F$ & 0 & 0 & 0 & 100 & 100 & 100 & 100 & 100 & 0.5 & 1200 & 540 & 0.3 & 50 & 50 & 50 & 10 & 10 & 2000 & 80 & 8 & 8 & 10 & 0 & 40 & 40 & 0.6 \\
\hline
\end{tabular}


Table E.16. Summary of Conservative $K_{d}$ Values for the Composite Analysis, Adjusted to a Maximum of $40 \mathrm{~mL} / \mathrm{g}$

\begin{tabular}{|c|c|c|c|c|c|c|c|c|c|c|c|c|c|c|c|c|c|c|c|c|c|c|c|c|c|c|c|}
\hline Sources & $\begin{array}{l}\text { Source } \\
\text { Category }\end{array}$ & \multicolumn{26}{|c|}{ Elements } \\
\hline \multirow{4}{*}{$\begin{array}{l}\text { High Organle } \\
\text { Vory Acldic }\end{array}$} & & HI & CI & $T E$ & $\overline{A C}$ & $\mathrm{Am}$ & $\mathrm{Ce}$ & $\mathrm{Cm}$ & Eu & C & $\mathrm{Co}$ & Cs & 1 & NI & Sn & $\mathrm{Nb}$ & $\mathrm{Np}$ & $\mathbf{P a}$ & $P_{b}$ & $\mathbf{P u}$ & $\mathbf{R e}$ & $\mathrm{Sr}$ & Ru & Se & Th & $\mathbf{Z r}$ & $\mathbf{U}$ \\
\hline & A & o & 이 & o) & of & 이 & o & 이 & 0 & of & 이 & 5 & 이 & 2 & 2 & 2) & 0.1 & 0.1 & 이 & 0.1 & 0.9 & 0.1 & 0.1 & 이 & 1 & 1 & 0.1 \\
\hline & B & of & o) & 0 & 10 & 10 & 10 & 10 & 10 & 0 & 0.1 & 5 & 0 & 3 & 3 & 3 & 0.1 & 0.1 & o) & 15 & 5 & 5 & 0.1 & o & 20 & 20 & 0.2 \\
\hline & $F$ & of & o) & 0 & 40 & 40 & 40 & 40 & 40 & 0.5 & 40 & 40 & 0.3 & 40 & 40 & 40 & 10 & 10 & 40 & 40 & a & 8 & 10 & o & 40 & 40 & 0.6 \\
\hline \multirow{3}{*}{$\begin{array}{l}\text { Hlgh Orgenle } \\
\text { Near Neutral }\end{array}$} & $\bar{B}$ & 0 & 0) & $\overline{0}$ & 10 & 10 & 10 & 10 & 10 & 0 & 0.1 & 5 & 0 & 3 & -3) & 3 & 0.1 & 0.1 & 0 & 15 & 5 & 5 & 0.1 & 0 & 20 & 20 & 0.2 \\
\hline & B & o & o) & 0 & 10] & 10 & 10 & 10) & 10 & 0 & 0.1 & 5 & 0 & 3 & 3 & 3 & 0.1 & 0.1 & 0 & 15 & 5 & 5 & 0.1 & of & 20 & 20 & 0.2 \\
\hline & $\mathbf{F}$ & of & of & 0 & 40 & 40 & 40 & 40 & 40 & 0.5 & 40 & 40 & 0.3 & 40 & 40 & 40 & 10 & 10 & 40 & 40 & 8) & 8 & 10 & of & 40 & 40 & 0.6 \\
\hline \multirow{3}{*}{$\begin{array}{l}\text { Vory High Salts } \\
\text { Very Baslc }\end{array}$} & D & 可 & 可 & $\overline{0}$ & 2 & 2 & 2 & 2 & 2 & 0 & 0.1 & 1 & 0 & 0.1 & 0.1 & 0.1 & 0.1 & 0.1 & 5 & 5 & 4 & 4 & 0 & 0 & 5 & 5 & 5 \\
\hline & E & of & o & o) & 40 & 40 & 40 & 40 & 10 & 0 & 40 & 40 & of & 30 & 30 & 30 & 0.2 & 0.2 & 20 & 5 & 0.2 & 0.2 & 0 & of & 40 & 40 & of \\
\hline & $F$ & o & 0 & 의 & 40 & 40 & 40 & 40 & 40 & 0.5 & 40 & 40 & 0.3 & 40 & 40 & 40 & 10 & 10 & 40 & 40 & 8 & 8 & 10 & of & 40 & 40 & 0.6 \\
\hline \multirow{3}{*}{$\begin{array}{l}\text { Chelales } \\
\text { High Salls }\end{array}$} & G & - & 0 & 0 & 3 & 3 & $\overline{3}$ & 3 & 3 & 0 & 0 & 6 & 0 & 0.4 & 0.4 & 0.4 & 2 & 2 & 0.4 & 0.5 & 0.4 & 0.4 & 0.4 & 0 & 0.5 & 0.5 & 0.2 \\
\hline & G & o & 0 & 0 & 3 & 3 & 3 & 3 & 3 & 0 & 0 & 8 & 0 & 0.4 & 0.4 & 0.4 & 2 & 2 & 0.4 & 0.5 & 0.4 & 0.4 & 0.4 & 이 & 0.5 & 0.5 & 0.2 \\
\hline & c & o & 0 & 0 & 10 & 10 & 10 & 10 & 10 & 0.5 & of & 40 & 0.3 & 2 & 2 & 40 & 2 & 2 & 2 & 20 & 8 & 2 & 10 & of & 40 & 40 & 0.6 \\
\hline Low Orgenlc & $\bar{H}$ & D & 0.1 & 0.1 & 25 & 25 & 25 & 25 & 25 & 0.1 & 0.2 & 10 & 0.1 & 10 & 10 & 10 & $\overline{3}$ & 3 & 25 & 20 & 10 & 10 & 10 & 0.1 & 30 & 30 & 20 \\
\hline Low Salls & $F$ & of & of & 0 & 40 & 40 & 40 & 40 & 40 & 0.5 & 40 & 40 & 0.3 & 40 & 40 & 40 & 10 & 10| & 40| & 40 & 8 & 8 & 10 & o) & 40 & 40 & 0.6 \\
\hline Aclalc & $F$ & of & of & 0 & 40 & 40 & 40 & 40 & 40 & 0.5 & 40. & 40. & 0.3 & 40 & 10 & 40 & 10) & 10 & 40 & 40 & 8 & 8) & 10 & of & 40 & 40 & 0.6 \\
\hline Low Organlc & $F$ & 이 & 0 & $\overline{0}$ & 40 & 40 & 40 & 40 & 40 & 0.5 & 40 & 40 & 0.3 & 40 & 40 & 40 & 10 & 10 & 40 & 40 & 8 & 8 & 10 & of & 40 & 40 & 0.8 \\
\hline Low Salts & $\mathbf{F}$ & 이 & o) & 0 & 40 & 40 & 40 & 40 & 40 & 0.5 & $40 \mid$ & 40) & 0.3 & 40 & 40 & 40 & 10) & 10 & 40 & 40) & 8 & 8 & 10) & 0 & 40 & 40 & 0.6 \\
\hline Near Neutral & $\boldsymbol{F}$ & 이 & 의 & 0 & 40 & 40 & 40 & 40] & 40 & 0.5 & 40) & 40I & 0.3 & 40 & 40 & 40 & 10 & 10 & 40 & 40 & 8] & 8 & 10 & of & 40 & 40 & 0.6 \\
\hline
\end{tabular}


Table E.17. Summary of Ranges of $K_{d}$ Values for the Composite Analysis

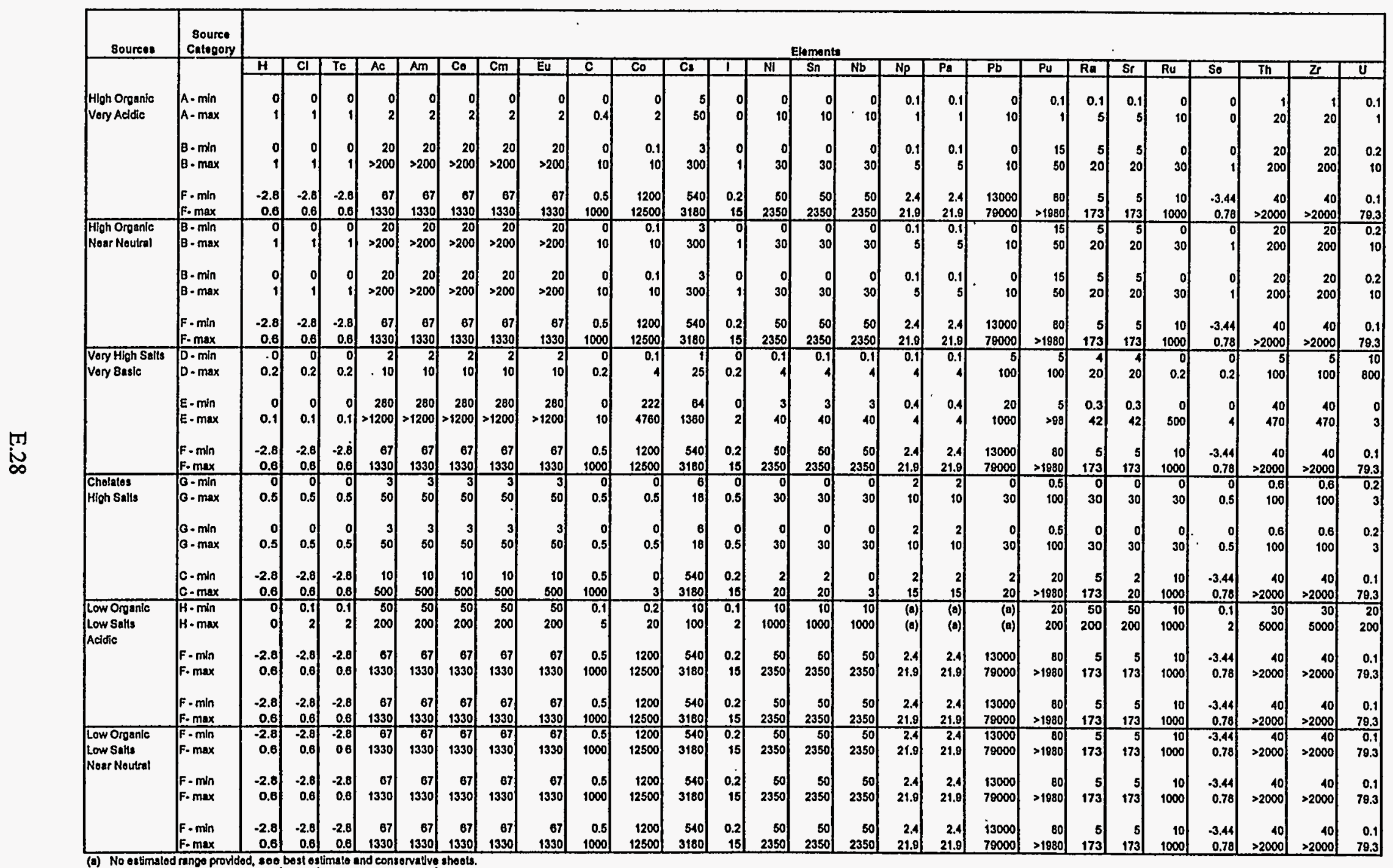




\section{Appendix F}

\section{Evaluation of Unit Dose Factors}




\title{
Appendix F
}

\section{Evaluation of Unit Dose Factors}

\author{
D. L. Strenge
}

This appendix provides a description of unit dose factors (UDFs) used for evaluation of radiation dose rate $^{(a)}$ and chemical health impacts for the Composite Analysis for Low-Level Waste Disposal in the 200 Area Plateau of the Hanford Site (Composite Analysis). The UDFs were used to provide an estimate of radiation dose rate or chemical health impact (in this case, from uranium) per unit concentration in a medium (e.g., groundwater). The dose rates were evaluated for an individual exposed via pathways associated with contact to that medium.

Exposure scenarios defined for the Hanford Site Risk Assessment Methodology (HSRAM) (DOE 1995) were used as the basis for determining the pathways and contact rates for each medium. The HSRAM scenarios were developed for the Hanford Site as a guide to performing evaluations of dose and risk related to the Comprehensive Environmental Response, Compensation, and Liability Act (CERCLA) remedial investigations and the Resource Conservation and Recovery Act (RCRA) facility investigations. The four HSRAM exposure scenarios are referred to as recreational, industrial, residential, and agricultural. These scenarios, associated parameters, and UDF values are described in this appendix. The general methods described in Strenge and Chamberlain (1994) were used in the Composite Analysis to evaluate the UDFs.

\section{F.1 Radionuclides and Chemicals of Interest}

The UDFs were evaluated for the radionuclides of interest and uranium (Table F.1). This list is based on the radionuclides that were considered to be the most likely to result in radiation or chemical exposure of individuals from releases to the environment. In developing this list of radionuclides, consideration was given to past analyses at the Hanford Site. The list includes the progeny radionuclides that are generated with time and are potentially present at exposure locations after release and transport. Dose results presented in this analysis are limited to a subset of those for which UDFs were developed. Those dose results reported include only the key mobile radionuclides, carbon-14, chlorine-36, selenium-79, technetium-99, iodine-129, and uranium.

(a) All dose rates in the Composite Analysis (except where noted) are in units of mrem effective dose equivalent (EDE) in a year. 


\section{F.2 Unit Dose Factors for Radionuclides}

The UDFs for radionuclides were used to provide an estimate of the annual radiation dose received by an individual exposed, as defined for the specific HSRAM scenarios. The dose is expressed in units of rem per year and represents the committed effective dose equivalent for one year of intake or exposure. The UDFs were evaluated for a unit concentration in a specific exposure medium. For example, when groundwater is the transport medium, the UDF is expressed per $\mathrm{pCi} / \mathrm{L}$ in the groundwater. When air is the transport medium, the UDF is expressed per $\mathrm{pCi} / \mathrm{m}^{3}$ in air. In all cases, the concentrations in the transport medium were assumed to be constant over the exposure duration. The concentrations were also assumed to be constant over a period of time prior to the exposure period during which the deposited contaminant (from irrigation or atmospheric deposition, if appropriate to the scenario) was allowed to reach equilibrium in the soil. Equilibrium was assumed when the deposition rate was equal to leaching and radioactive decay losses from the soil.

\section{F.3 Radiation Dosimetry Factors}

The evaluation of annual radiation dose was completed for the Composite Analysis based on radiation dose conversion factors published in Federal Guidance Reports No. 11 and 12 (Eckerman, Wolbarst, and Richardson 1988; Eckerman and Ryman 1993). These dose factors are based on recommendations of the International Commission on Radiological Protection (ICRP) as given in ICRP Publication 30 (ICRP 1979a, 1979b). The resulting doses represent the effective dose equivalent received over a commitment period of 50 years following intake in the first year.

The evaluation of annual radiation dose as the endpoint in the analysis is a deviation from the guidance in the HSRAM (DOE 1995) report. The HSRAM guidance is for evaluation of the lifetime cancer incidence risk from radionuclides using slope factors. The slope factors relate intake $(\mathrm{pCi})$ to the lifetime cancer incidence risk. However, the Composite Analysis required evaluation of annual radiation dose. Therefore, the use of radiation dose conversion factors replaced slope factors in the Composite Analysis.

\section{F.4 Unit Dose Factors for Uranium}

For chemicals, the unit dose factor is the hazard quotient defined by the U.S. Environmental Protection Agency (EPA) as the average daily intake of a chemical (in this case uranium) divided by the Reference Dose (RfD) for that chemical (EPA 1994). The hazard quotient was evaluated for both inhalation exposures and ingestion exposures with an $\mathrm{RfD}$ determined for each route.

\section{F.5 Uranium Reference Doses}

The EPA evaluates RfD values for selected chemicals and reports the values in the Integrated Risk Information System (IRIS) and the Health Effects Assessment Summary Tables (HEAST). At this time, neither IRIS nor HEAST provide estimates of RfD values for uranium. In order to generate an estimate of the health impact level from exposure to uranium (as a chemical toxicant), it was necessary to develop approximate values for the uranium RfDs (ingestion and inhalation intake routes). 
The ingestion reference dose value was taken from a previous version of the EPA HEAST report (EPA 1994). The previous report gave a value of $0.003 \mathrm{mg} /(\mathrm{kg}-\mathrm{d})$ average daily intake. Although this value was withdrawn by EPA, it was used in the Composite Analysis as the best available value for ingestion exposure.

The EPA has not presented a value for the inhalation reference dose for uranium. A value was estimated for the Composite Analysis based on the Threshold Limit Value (TLV) published by the American Conference of Governmental Industrial Hygienists (ACGIH 1991). The TLV value for occupational inhalation exposure to uranium (as soluble and insoluble compounds of natural uranium) is $0.2 \mathrm{mg} / \mathrm{m}^{3}$. This value was applied to workers exposed continuously during a 40-hour work week. In contrast, the $\mathrm{RfD}$ value was intended to represent safe levels for exposure of members of the public under continuous exposure ( 24 hours/day, 365 days/year). The TLV value can be converted to an inhalation RfD by consideration of relative inhalation rates, times of exposure, and sensitivity of members of the public relative to occupational workers. The conversion factor (Strenge, Peterson, and Sager 1989) used in the Composite Analysis was $0.007 \mathrm{mg} /(\mathrm{kg}-\mathrm{d})$ per $\mathrm{mg} / \mathrm{m}^{3}$, which includes a safety factor of 10 to account for sensitive members of the public. The resulting RfD was $0.0014 \mathrm{mg} /(\mathrm{kg}-\mathrm{d})$ for inhalation exposure to uranium compounds (with the intake expressed in $\mathrm{mg}$ of uranium).

\section{F.6 Evaluation of Unit Dose Factors}

Unit dose factors were calculated for radionuclides and chemicals with the exposure assessment component of the Multimedia Environmental Constituent Assessment System (MEPAS) code (Buck et al. 1995; Strenge and Chamberlain 1995; Droppo and Buck 1996). The evaluation was performed using equations and parameters for each exposure pathway as defined in the HSRAM report and modified for the Composite Analysis. The equations were structured to take advantage of the summary intake factor (SIF) concept presented in the HSRAM report. The concept of SIFs involved structuring the intake equations for each exposure pathway in such a way that constituent-independent parameters are separated from constituent-specific parameters and the initial media concentration. Each exposure pathway model was described as the product of three factors: a media concentration, an SIF independent of constituent, and a factor composed of all constituent-specific parameters. A general expression was used to calculate the dose or hazard quotient, as follows:

$$
\text { Dose or Hazard Quotient }=\mathrm{C}_{\mathrm{mi}} \mathrm{PF}_{\operatorname{mix}} \mathrm{SIF}_{\mathrm{smyx}}
$$

where $\quad$ Dose $=$ annual radiation dose from intake or exposure to a radionuclide (rem/yr)

Hazard Quotient $=$ hazard quotient from intake of a chemical toxicant based on the average daily intake over the one-year exposure period (dimensionless)

$\mathrm{C}_{\mathrm{mi}}=$ concentration of constituent $\mathrm{i}$ in medium $\mathrm{m}$ ( $\mathrm{mg}$ or pCi per unit quantity of medium $\mathrm{L}, \mathrm{kg}, \mathrm{m}^{3}$, or $\mathrm{m}^{2}$ )

$\mathrm{PF}_{\mathrm{mix}}=$ constituent specific factors for medium $\mathrm{m}$, constituent $\mathrm{i}$, and exposure pathway $x$ (units specific to analysis) 


$$
\begin{aligned}
\text { SIF }_{\text {smyx }}= & \text { summary intake factor for scenario } \mathrm{s} \text {, medium } \mathrm{m}, \text { constituent type } \mathrm{y}, \text { an } \\
& \text { exposure pathway } \mathrm{x} \text { (units specific to analysis). }
\end{aligned}
$$

The SIF values were evaluated for each toxicity type, radionuclides and noncarcinogenic chemicals. Carcinogenic effects were not included in the Composite Analysis because no carcinogenic chemicals were identified in the source inventory.

The MEPAS exposure component used in the present analysis allowed the user to provide SIF values as input. The SIF values were precalculated for each scenario, exposure pathway, constituent type (chemical and radionuclide) and medium (air and groundwater).

\section{F.7 Exposure Scenario Descriptions}

The four HSRAM exposure scenarios (DOE 1995), industrial, recreational, residential, and agricultural, were used as the basis for the UDF evaluations performed for the Composite Analysis. These exposure scenarios were adopted for the Composite Analysis because they are the current scenarios agreed upon by the U.S. Department of Energy (DOE), the State of Washington, and the EPA for Hanford Site evaluations of risk. They are routinely applied under the environmental restoration program. With one exception, the HSRAM scenarios and exposure parameter values were used as published. The HSRAM scenarios were defined for exposure over an extended duration (20 years for the industrial scenario and 30 years for other scenarios). The Composite Analysis required evaluation of the annual radiation dose received by potentially exposed individuals. The HSRAM scenarios were modified to reflect exposure for a one-year period, instead of a longer exposure duration.

As a result of this change, and because the total exposure to individuals from all exposure pathways was needed, the analysis did not include exposure of children. The HSRAM scenarios include exposures of children for a few pathways in which the child may receive a higher intake than adults. Some of the HSRAM scenarios involve exposure of a child for 6 years followed by exposure of an adult for 24 years. This example represents exposure for a 30-year period with partial intake as a child and partial intake as an adult. For the Composite Analysis, it was assumed to not be possible for an individual to be both a child and an adult during the one-year exposure period. The resulting dose estimates represent exposure of an individual as an adult, with contributions from all defined exposure pathways summed to give a total annual dose.

The first iteration Composite Analysis is an examination of radioactive waste disposal on the 200 Area Plateau. It is envisioned the DOE site boundary will shrink to include only the 200 Area Plateau as Hanford Site closure approaches. Historically agricultural land use and the groundwater have been the exposure scenario and environmental pathway yielding the maximum dose. Accordingly, no attempt was made in the Composite Analysis to model or estimate future contaminant concentrations in the Columbia River. Hence, the surface water medium and its associated exposure pathways (e.g., swimming and fish consumption) are omitted from this analysis.

Contaminant concentrations in groundwater prior to entering the river are greatly diluted in the Columbia River because of the mixing that occurs as water from the unconfined aquifer enters and is 
entrained into the river. Consequently, dose estimates based on groundwater use and consumption in the immediate vicinity of the shore of the Columbia River are higher than those based on use and consumption of surface water from the Columbia River. For this reason, the exclusion of surface water exposure pathways and use of groundwater-based exposure pathways is appropriate in the Composite Analysis.

\section{F.7.1 Industrial Scenario}

The industrial scenario is intended to represent potential exposures to workers in a commercial or industrial setting. The scenario involves mainly indoor activities. Exposure to radioactive contamination and uranium as.a chemical contaminant is limited to that originating with the groundwater, air, and soil (air deposition) transport media. The specific exposure pathways are listed in Table F.2 for the industrial scenario. Consistent with HSRAM (DOE 1995), the third pathway, dermal contact (e.g., bathing), was only applied in the chemical hazard analysis. The workers are assumed to wear no protective clothing. The scenario is not intended to represent exposure of remediation workers.

\section{F.7.2 Recreational Scenario}

The recreational scenario is intended to represent exposure to individuals engaging in seven days of recreational activity on the central portion of the Hanford Site. Exposure pathways included those associated with the groundwater, air, and soil (air deposition) transport media. The specific exposure pathways are listed in Table F.3 for the recreational scenario. Consistent with HSRAM (DOE 1995), the third exposure pathway, dermal contact through bathing, was only applied in the chemical hazard analysis. Elements of the HSRAM recreational scenario (DOE 1995) that involved surface water activities or sources of food (e.g., fish) were omitted from the scenario because of the exclusion of surface water exposure pathways from the Composite Analysis.

\section{F.7.3 Residential Scenario}

The residential scenario is intended to represent potential exposures to an individual who may take up residence of the Hanford Site in the future. The exposures are assumed to be continuous throughout the year, but limited to those originating with the groundwater, air, and soil (air deposition) transport media. Exposure pathways associated with residence on the Hanford Site and with both radionuclides and uranium as a chemical hazard are listed in Table F.4. Consistent with HSRAM (DOE 1995), the fourth exposure pathway, dermal contact through bathing, was only applied in the chemical hazard analysis. Surface water recreational activities are omitted from the scenario.

\section{F.7.4 Agricultural Scenario}

The agricultural scenario is intended to represent potential exposure to an individual who may reside on a small family farm on the Hanford Site in the future. The exposures are assumed to be continuous throughout the year. Exposures accrue from air, soil (air deposition), and groundwater transport media. The individual was assumed to take up residence on the Hanford Site and grow vegetables, fruit, and raise 
meat and milk animals. As in the other scenarios, surface water recreational activities and fish consumption are excluded. The specific exposure pathways included in the agricultural scenario are listed in Table F. 5 for radionuclides and chemicals. This scenario is unique because of the inclusion of contaminated meat and milk in the diet.

The only radionuclides identified in prior analyses as potentially significant in the air and soil (air transport) media were tritium, carbon-14, and radon-222. Of these, only tritium and carbon-14 were identified as potentially significant in a single source, i.e., the graphite cores of the surplus production reactors. Inclusion of the air and soil media will be seen to have negligible impact on overall.dose.

\section{F.8 Exposure Scenario Parameters}

Parameter values used to calculate unit dose factors are presented in Tables F.6, F.7, F.8, and F.9 for the industrial, recreational, residential, and agricultural exposure scenarios, respectively. These tables indicate the intake or exposure rate, exposure frequency (days/year), unit conversion factors, and other factors used for specific exposure pathways. The tables also include a list of the resulting values for summary intake factors for chemicals and radionuclides. The parameter values in Tables F.6 through F.9 are taken directly from the HSRAM report (DOE 1995).

\section{F.9 Exposure Pathways}

A total of 17 different exposure pathways were considered in the UDF analyses. The pathways included in a specific analysis depend on the transport medium, scenario, and constituent type (radionuclide or uranium treated as a chemical), as indicated in the previous section. Details of each exposure pathway by transport medium are described below. In general, the parameter values for a pathway were derived from the HSRAM report (DOE 1995).

\section{F.9.1 Soil (Air Deposition) Transport Medium}

Deposition of airborne activity to soil allows exposure to individuals who come in contact with the soil, breathe suspended particles from the soil, or eat foods grown in the soil. The contamination deposited onto soil was modeled as a concentration per unit area of soil. All UDF values were normalized to the area soil concentration in units of $\mathrm{mg} / \mathrm{m}^{2}$ or $\mathrm{pCi} / \mathrm{m}^{2}$. Some of the soil exposure pathways required that concentrations be expressed in units of soil mass ( $\mathrm{mg} / \mathrm{kg}$ or $\mathrm{pCi} / \mathrm{kg}$ dry soil). For these pathways, the conversion to soil mass was made using the conversion factor $60 \mathrm{~kg} / \mathrm{m}^{2}$, based on uniform distribution of the contaminant in the top $4 \mathrm{~cm}$ of soil having a density of $1.5 \mathrm{~g} / \mathrm{cm}^{3}$. This thickness is representative of the distribution of contaminants in residential soil (e.g., gardens or lawns) for deposition occurring over extended periods (several years).

The parameter values for each exposure pathway related to soil as a medium are summarized in Table F.9 for the agricultural exposure scenario. The assumptions for each of the exposure pathways are presented below. 


\section{F.9.1.1 Soil Ingestion}

The individual was assumed to inadvertently ingest contaminated soil as part of daily activities defined for the scenarios. Residential and agricultural individuals ingest soil at $100 \mathrm{mg} / \mathrm{d}$ for the entire year, while the industrial worker ingests $50 \mathrm{mg} / \mathrm{d}$ while on the job for 146 days per year. The worker is assumed to be exposed to soil for only 146 of the 250 work days per year. The recreational visitor is exposed for 7 days per year at $100 \mathrm{mg}$ per day.

\section{F.9.1.2 Soil External Exposure}

Radionuclides deposited onto soil may cause external radiation exposure to individuals near the contamination. The industrial worker is assumed to be exposed 8 hours per day for 146 days per year. The recreational visitor is exposed 8 hours per day for 7 days per year. The residential and agricultural scenario individuals are exposed 24 hours per day for 365 days per year.

\section{F.9.1.3 Soil Dermal Contact}

The dermal contact pathway was evaluated only for chemicals (as recommended in the HSRAM report). The individuals were assumed to have one contact event per day with soil adhering to the skin at a surface density of $0.2 \mathrm{mg} / \mathrm{cm}^{2}$ of skin. The area of skin contacted was assumed to be $5000 \mathrm{~cm}^{2}$. The industrial worker is exposed 146 days per year, the recreational visitor is exposed 7 days per year, and the residential and agricultural individuals are exposed 180 days per year.

\section{F.9.1.4 Soil Resuspension Inhalation}

Material deposited on the ground is assumed to be available for resuspension and inhalation by individuals close to the contamination. All exposure scenarios were based on the assumption that the individual inhales $20 \mathrm{~m}^{3}$ of contaminated air per day. The airborne concentration of soil was evaluated using a resuspension factor to give an air concentration equivalent to $50 \mu \mathrm{m} / \mathrm{m}^{3}$ of soil in air. The effective resuspension factor was assumed to be $8.33 \times 10^{-10} \mathrm{~m}^{-1}$, based on the soil density conversion factor of $60 \mathrm{~kg} / \mathrm{m}^{2}$ as described in Section F.9.1, "Soil (Air Deposition) Transport Medium."

\section{F.9.1.5 Food Crops}

Food crops are evaluated as fruits and vegetables. The crops were assumed to be contaminated when soil contamination (from airborne deposition) transfers to the edible parts of the plant by root uptake. Food crops were assumed to be eaten by the agricultural individual. The individual was assumed to consume $42 \mathrm{~g} / \mathrm{d}$ of fruit and $80 \mathrm{~g} / \mathrm{d}$ of vegetables throughout the year. The soil concentration was based on a soil mixing or plow depth of $15 \mathrm{~cm}$ and a soil density of $1.5 \mathrm{~g} / \mathrm{cm}^{3}$, which is equivalent to an areal soil density of $225 \mathrm{~kg} / \mathrm{m}^{2}$. 


\section{F.9.1.6 Game (Deer)}

For the recreational and agricultural scenarios the individual was assumed to hunt and kill one deer in the year. The deer becomes contaminated when foraging on plants grown in contaminated soil. The HSRAM scenario applies a hunter success rate of $19 \%$ for a season. This is appropriate when the exposure duration is many years ( 30 years for HSRAM), but was not appropriate for the one-year period considered in the Composite Analysis. The annual dose analysis was based on the assumption that the hunter is successful (success rate $=100 \%$ for the year of exposure). Also, the HSRAM intake rate for deer meat is based on the amount of animal fat in the consumed meat. While this may be appropriate for the organic chemical constituents that are lipophilic, it is not generally appropriate for radionuclides. Also, the exposure pathway models for radionuclides evaluate the activity in the edible meat, not fat. The intake rate for deer meat was adjusted to represent the amount of meat ingested. This value was assumed to be $15 \mathrm{~g} / \mathrm{d}$, as reported for the recreational scenario of the Columbia River Comprehensive Impact Assessment project (Napier et al. 1996).

\section{F.9.1.7 Meat and Milk Ingestion}

Individuals in the agricultural scenario were assumed to ingest $75 \mathrm{~g} / \mathrm{d}$ of meat (other than game), and $300 \mathrm{~g} / \mathrm{d}$ of dairy products (represented as milk). The animals were assumed to be exposed from eating feed crops contaminated by root uptake from contaminated soil.

\section{F.9.2 Air Transport Medium}

Airborne activity may result in inhalation exposure plus direct transfer to plant surfaces resulting in intake of contaminated food crops and animal products (from animals that eat contaminated feed crops). The UDFs for air transport were based on unit air concentrations expressed in units of $\mathrm{mg} / \mathrm{m}^{3}$ (chemicals) and $\mathrm{pCi} / \mathrm{m}^{3}$ (radionuclides).

The parameter values for each exposure pathway related to air as a medium are presented in Tables F.6 through F.9 for the exposure scenarios. The assumptions associated with each of the exposure pathways are presented below.

\section{F.9.2.1 Inhalation}

For all scenarios, the individual inhales $20 \mathrm{~m}^{3}$ of air during the time the individual is present. For the industrial worker and the recreational visitor, this volume of air is inhaled during an 8-hour period, during which the individuals are engaged in enhanced physical activity. For the residential and agricultural individuals, the air is inhaled during a 24-hour period at average daily inhalation rates. The industrial worker is exposed 250 days per year, the recreational visitor is exposed 7 days per year, and the residential and agricultural individuals are exposed 365 days per year. 


\section{F.9.2.2 Food Crops}

Food crops are evaluated as fruits and vegetables. The crops were assumed to be contaminated from transfer of airborne contamination directly to the plant surfaces and the edible portions of the plant. Food crops were assumed to be eaten by the residential and the agricultural individuals. The individuals were assumed to consume $42 \mathrm{~g} / \mathrm{d}$ of fruit and $80 \mathrm{~g} / \mathrm{d}$ of vegetables throughout the year.

\section{F.9.2.3 Game (Deer)}

The dose for this pathway was evaluated as described in Section F.9.1 "Soil (Air Deposition) Transport Medium." Deer are assumed to be contaminated from the air transport pathway when they eat plants contaminated by direct air deposition onto plant surfaces.

\section{F.9.2.4 Meat and Milk Ingestion}

Individuals in the agricultural scenario were assumed to ingest $75 \mathrm{~g} / \mathrm{d}$ of meat (other than game), and $300 \mathrm{~g} / \mathrm{d}$ of dairy products (represented as milk). The animals were assumed to be exposed from eating feed crops contaminated by direct air deposition.

\section{F.9.3 Groundwater Transport Medium}

Groundwater contamination may result in exposure from domestic uses of the water (drinking and showering), and from ingestion of food crops and animal products. Food crops were assumed to become contaminated when groundwater is used for irrigation. Animal products were assumed to become contaminated when animals are fed crops irrigated with groundwater or when animals drink groundwater. The UDFs were based on unit water concentrations expressed in units of $\mathrm{mg} / \mathrm{L}$ (chemicals) and $\mathrm{pCi} / \mathrm{L}$ (radionuclides).

Groundwater quality at a moment in time and point in space was defined by the maximum concentration observed for each radionuclide at any of the vertical nodes associated with a spatial surface point in the aquifer model. For example, assume a point in space represents the location of a groundwater well. The nodes aligned vertically below the land surface represent pints in the aquifer providing water to the well. The maximum concentration for one radionuclide (e.g., technetium-99) might be associated with an upper node in the profile of nodes representing the nine hydrostratigraphic units of the groundwater model. The maximum concentration of another radionuclide (e.g., iodine-129) might be associated with a lower node in the profile. The maximum concentration of each radionuclide, regardless of its nodal location in the vertical profile, is used to describe the water quality at that point in space and moment in time. This groundwater quality is then applied in the exposure scenarios.

The parameter values for each exposure pathway related to groundwater as a medium are presented in Tables F.6 through F.9 for the four exposure scenarios. The assumptions for each of the exposure pathways are described below. 


\section{F.9.3.1 Drinking Water Ingestion}

The industrial worker was assumed to drink $1 \mathrm{~L} / \mathrm{d}$ of water while on the job 250 days per year. The recreational visitor was assumed to drink $2 \mathrm{~L} / \mathrm{d}$ during 7 days per year. The residential and agricultural individuals were assumed to drink $2 \mathrm{~L} / \mathrm{d}$ for the entire year. In this exposure pathway scenario, no contaminants were assumed to be removed by a treatment process.

\section{F.9.3.2 Shower Dermal Contact}

Domestic use of groundwater for bathing was included for all scenarios. Individuals are assumed to take one 10-minute shower each day of exposure. The industrial workers were assumed to be exposed 250 days per year, the recreational visitor was assumed to be exposed 7 days per year, and the residential and agricultural individuals were assumed to be exposed 365 days per year. The skin surface area for exposure was set to $20,000 \mathrm{~cm}^{2}$. Dermal contact exposure was evaluated only for chemicals (as recommended in the HSRAM report).

\section{F.9.3.3 Indoor Air Inhalation}

Domestic use of water indoors (e.g., for showers, laundry, dishwashing, and cooking) was assumed to result in release of volatile chemicals and radionuclides into the indoor air. Individuals are then subject to inhalation exposure while indoors. This exposure pathway was considered for all exposure scenarios except the recreational visitor. (Even though the recreational visitor was assumed to use groundwater for showering at the recreational facilities, the visitor was not assumed to remain indoors for extended periods of time to allow significant inhalation exposure.) The air concentration was related to the water concentration using a volatilization factor, assumed to be $0.1 \mathrm{~L} / \mathrm{m}^{3}$ for radon-222, the only constituent considered to be volatile. The daily inhaled air volume for exposure to indoor contamination was assumed to be $15 \mathrm{~m}^{3}$ for the agricultural and residential individual and $20 \mathrm{~m}^{3}$ for the industrial worker (consistent with the daily inhalation rate for general air inhalation). The agricultural and residential individual inhalation rates were reduced slightly because the individual was not assumed to be indoors all day.

\section{F.9.3.4 Food Crop Ingestion}

Food crops are evaluated as fruits and vegetables. Food crops were assumed to become contaminated when groundwater is used for crop irrigation. The exposure pathway parameters are as described above for Section F.9.1 "Soil (Air Deposition) Transport Medium." For the groundwater irrigation route, irrigation water was assumed to be applied at a rate of $150 \mathrm{~L} / \mathrm{m}^{2} / \mathrm{month}$, which corresponds to an annual average application rate of $90 \mathrm{~cm} / \mathrm{yr}$.

\section{F.9.3.5 Game (Deer) Ingestion}

For the recreational and agricultural scenarios, the individual was assumed to hunt and kill one deer in the year. The dose for this pathway was evaluated as described in Section F.9.1 "Soil (Air Deposition) Transport Medium." Deer meat was assumed to become contaminated when the deer drink contaminated seep or spring water (groundwater). Deer were not assumed to eat irrigated crops. 


\section{F.9.3.6 Meat and Milk Ingestion}

Individuals in the agricultural scenario were assumed to ingest $75 \mathrm{~g} / \mathrm{d}$ of meat (other than game), and $300 \mathrm{~g} / \mathrm{d}$ of dairy products (represented as milk). The animals were assumed to be exposed to contaminants from drinking contaminated water and eating feed crops contaminated by irrigation deposition.

\section{F.10 Equilibrium Analysis}

Several pathways involved accumulation of radionuclides in soil over a period of time resulting from airborne deposition or from irrigation water applied on crops. Material deposited onto soil was subject to losses by leaching from the soil and radioactive decay. At equilibrium, these losses were assumed to equal the rate of deposition. The UDF values for such pathways were evaluated at a time when equilibrium had been attained.

An analysis was performed for the radionuclides of interest to determine the time necessary for equilibrium to be attained. In the analysis, two soil types were considered: agricultural soil and native soils. Properties of agricultural soil were used for the industrial, residential, and agricultural scenarios, while the native soil properties were used for recreational scenario and for game meat ingestion (agricultural scenario). The primary parameter affecting the time to equilibrium was the value used for the radionuclide distribution coefficient, $K_{d}$. Soil parameters (e.g., density) also affected the time to equilibrium, but were constant for all constituents. The agricultural soil was assumed to be represented by a $15-\mathrm{cm}$. thick layer with an average bulk density of $1.5 \mathrm{~g} / \mathrm{cm}^{3}$. The soil moisture content was assumed to be $10 \%$, and an average annual infiltration rate of $15 \mathrm{~cm} / \mathrm{yr}$ was used. The native soil properties were assumed to be the same except the infiltration rate is set to $0.2 \mathrm{~cm} / \mathrm{yr}$.

Losses from the surface soil layer were assumed to occur by radioactive decay and leaching. A liquid or water leaching rate constant i, i.e., $\lambda_{\text {wi }}$, was evaluated from the $K_{d}$ value and the parameters mentioned above as follows.

$$
\lambda_{w i}=\frac{i}{h \theta\left(1+\frac{\beta_{d}}{\theta} K_{d i}\right)}
$$

$$
\text { where } \begin{aligned}
\mathrm{i} & =\text { total infiltration rate }(\mathrm{cm} / \mathrm{yr}) \\
\mathrm{h} & =\text { thickness of the surface-soil layer }(\mathrm{cm}) \\
\theta & =\text { moisture content of the surface-soil layer (fraction) } \\
\beta_{\mathrm{d}} & =\text { bulk density of the surface-soil layer }\left(\mathrm{g} / \mathrm{cm}^{3}\right) \\
\mathrm{K}_{\mathrm{di}} & =\text { distribution coefficient for constituent } \mathrm{i}(\mathrm{mL} / \mathrm{g})
\end{aligned}
$$

Values used for the distribution coefficient were selected to give low leach rate constants that will result in long soil retention times. This selection resulted in a conservative (high) estimate of radiation dose for those exposure pathways that involve accumulation in soil. The parameters for agricultural soil were used for all exposure pathways (except recreational activities and game ingestion), as a simplification to the analysis and a further conservatism for the residential exposure pathways. Residential soil was 
expected to involve mixing in a smaller depth (represented in the above equation by parameter $h$ ). A smaller value for soil depth would result in a faster leach rate and lower equilibrium concentrations. Because of lawn irrigation, residential and industrial soils were assumed to be subject to the same infiltration rate as agricultural lands. For the recreational pathways, the infiltration rate was assumed to be $0.2 \mathrm{~cm} / \mathrm{yr}$. This value is in the midrange of reported values for Hanford (Kincaid et al. 1995). Other parameters were assumed to be unchanged with the $15-\mathrm{cm}$ depth representing a nominal depth for plant roots.

Results of the equilibrium analysis are presented in Table F.10. This table indicates the time to reach equilibrium ( $95 \%$ of the final value) when the radionuclide is deposited at a constant rate during prior years. The analysis is based on calculation of UDFs for each radionuclide. For radionuclides that have decay progeny, the dose from the progeny was included in the analysis. In some cases, the ingrowth of progeny (none are present at time zero or in the water) may extend the time to reach the equilibrium dose. Table F.10 also presents the ratio of the annual dose at 50 years to the equilibrium dose (or the dose at 1000 years, the time considered for this iteration of the Composite Analysis).

The UDFs generated (presented in Section F.11) were based on the dose received after the equilibrium time period. For those radionuclides that take more than 50 years to reach equilibrium, the dose was evaluated at 50 years. This simplification was necessary because the UDFs are based on constant deposition (i.e., constant air or water concentration) over the deposition period. The simplification was also needed in order to precalculate the UDF values. The precalculated UDF values were evaluated for a unit concentration or deposition rate and did not include consideration of parameter variation with time.

\section{F.11 Unit Dose Factors and Hazard Indices}

Unit dose factors were generated for the media contributing to each of the four exposure scenarios. The groundwater transport medium plays the dominant role. However, the air and soil transport media also contribute to the agricultural scenario where air and soil contaminant levels are estimated (i.e., atmospheric releases from the buried graphite cores of the production reactors). The UDFs are summed over all exposure pathways for a medium. Table F.11 presents the summed UDF results for all radionuclides for which doses were calculated. It also presents the summed UDF results for chemical health impacts of uranium for each exposure scenario. These latter values provide the hazard index for uranium exposures.

A hazard index of 1.0 indicates the exposure is just at the safe level. Hazard index values greater than 1.0 indicate higher exposures. The radionuclide UDF values provide annual radiation dose expressed in mrem.

\section{F.12 References}

American Conference of Governmental Industrial Hygienists (AGCIH). 1991. 1991 - 1992: Threshold Limit Values for Chemical Substances and Physical Agents and Biological Exposure Indices. Cincinnati, Ohio. 
Buck, J. W., G. Whelan, J. G. Droppo Jr., D. L. Strenge, K. J. Castleton, J. P. McDonald, C. Sato, and G. P Streile. 1995. Multimedia Environmental Pollutant Assessment System (MEPAS) Application Guidance. PNL-10395, Pacific Northwest Laboratory, Richland, Washington.

Comprehensive Environmental Response, Compensation, and Liability Act of 1980, as amended, Public Law 96-510, 94 Stat. 2767, 42 USC 9601 et seq.

Droppo, J. G., and J. W. Buck. 1996. The Multimedia Environmental Pollutant Assessment System (MEPAS): Atmospheric Pathway Formulations. PNNL-1 1080, Pacific Northwest National Laboratory, Richland, Washington.

Eckerman, K. F., A. B. Wolbarst, and A.C.B. Richardson. 1988. Limiting Values of Radionuclide Intake and Air Concentration and Dose Conversion Factors for Inhalation, Submersion, and Ingestion. Federal Guidance Report No. 11. EPA-520/1-88-202, U.S. Environmental Protection Agency, Office of Radiation Programs, Washington, DC.

Eckerman, K. F., and J. C. Ryman. 1993. External Exposure to Radionuclides in Air, Water, and Soil. Federal Guidance Report No. 12. EPA 402-R-93-081, U.S. Environmental Protection Agency, Office of Radiation Programs, Washington, DC.

International Commission on Radiological Protection (ICRP). 1979a. ICRP Publication 30, Part 1, Limits for Intakes of Radionuclides by Workers. Annals of the ICRP, Vol. 2, No. 3 and 4, Pergamon Press, Elmsford, New York.

International Commission on Radiological Protection (ICRP). 1979b. ICRP Publication 30, Supplement to Part 1, Limits for Intakes of Radionuclides by Workers. Annals of the ICRP, Vol. 3, No. 1-4, Pergamon Press, Elmsford, New York.

Kincaid, C. T., J. W. Shade, G. A. Whyatt, M. G. Piepho, K. Rhoads, J. A. Voogd, J. H. Westsik, Jr., M. D. Freshley, K. A. Blanchard, and B. G. Lauzon. 1995. Volume 1: Performance Assessment of Grouted Double-Shell Tank Waste Disposal at Hanford. WHC-SD-WM-EE-004, Rev. 1, Westinghouse Hanford Company, Richland, Washington.

Napier, B. A., B. L. Harper, N. K. Lane, D. L. Strenge, and R. B. Spivey. 1996. Human Scenarios for the Screening Assessment: Columbia River Comprehensive Impact Assessment. DOE/RL-96-16-a, U.S. Department of Energy, Richland, Washington.

Resource Conservation and Recovery Act of 1976, as amended, Public Law 94-580, 90 Stat. 3221, 42 USC 6901 et seq.

Strenge, D. L., and P. J. Chamberlain. 1994. Evaluation of Unit Risk Factors in Support of the Hanford Remedial Action Environmental Impact Statement. PNL-10190, Pacific Northwest Laboratory, Richland, Washington. 
Strenge, D. L., and P. J. Chamberlain. 1995. Multimedia Environmental Pollutant Assessment System (MEP AS): Exposure Pathway and Human Health Impact Assessment Models. PNL-10523, Pacific Northwest National Laboratory, Richland, Washington.

Strenge, D. L., S. R. Peterson, and S. Sager. 1989. Chemical Data Bases for the Multimedia Environmental Pollutant Assessment System (MEPAS): Version 1. PNL-7145, Pacific Northwest Laboratory, Richland, Washington.

U.S. Department of Energy (DOE). 1995. Hanford Site Risk Assessment Methodology. DOE/RL-91-45, Rev. 3, U.S. Department of Energy, Richland, Washington.

U.S. Environmental Protection Agency (EPA). 1994. Health Effects Summary Tables: 1994 Annual Report. EPA 540/R-94/020, Office of Solid Waste and Emergency Response, U.S. Environmental Protection Agency, Washington, D.C. 
Table F.1. Radionuclides and Chemicals of Interest in the Composite Analysis

\begin{tabular}{|c|c|}
\hline Radionuclides & Progeny \\
\hline${ }^{3} \mathrm{H}$ & \\
\hline${ }^{14} \mathrm{C}$ & \\
\hline${ }^{36} \mathrm{Cl}$ & \\
\hline${ }^{40} \mathrm{~K}$ & \\
\hline${ }^{63} \mathrm{Ni}$ & \\
\hline${ }^{79} \mathrm{Se}$ & \\
\hline${ }^{90} \mathrm{Sr}$ & \\
\hline${ }^{99} \mathrm{Tc}$ & \\
\hline${ }^{126} \mathrm{Sn}$ & \\
\hline${ }^{129} \mathrm{I}$ & \\
\hline${ }^{187} \mathrm{Re}$ & \\
\hline${ }^{234} \mathrm{U}$ & \\
\hline${ }^{235} \mathrm{U}$ & \\
\hline${ }^{238} \mathrm{U}$ & ${ }^{126} \mathrm{Sb}$ \\
\hline${ }^{244} \mathrm{Cm}$ & $\mathrm{Pa},{ }^{227} \mathrm{Ac},{ }^{227} \mathrm{Th},{ }^{223} \mathrm{Ra}$ \\
\hline & \\
\hline Chemicals & \\
\hline Uranium & \\
\hline & \\
\hline
\end{tabular}

Table F.2. Industrial Scenario Exposure Pathways

\begin{tabular}{|l|l|l|l|}
\hline \multicolumn{1}{|c|}{ Transport Medium } & \multicolumn{1}{|c|}{ Exposure Pathway } & \multicolumn{1}{c|}{ Chemical } & \multicolumn{1}{c|}{ Radioactive } \\
\hline \multirow{4}{*}{ Soil (air deposition) } & Ingestion & Yes & Yes \\
\cline { 2 - 5 } & External & No & Yes \\
\cline { 2 - 5 } & Dermal Contact & Yes & No \\
\cline { 2 - 5 } & Suspension - Inhalation & Yes & Yes \\
\hline Air & Inhalation & Yes & Yes \\
\hline Groundwater & Ingestion & Yes & Yes \\
\cline { 2 - 5 } & Dermal Contact & Yes & No \\
\hline
\end{tabular}


Table F.3. Recreational Scenario Exposure Pathways

\begin{tabular}{|l|l|l|l|}
\hline \multicolumn{1}{|c|}{ Transport Medium } & \multicolumn{1}{|c|}{ Exposure Pathway } & \multicolumn{1}{c|}{ Chemical } & \multicolumn{1}{c|}{ Radioactive } \\
\hline \multirow{4}{*}{ Soil (air deposition) } & Ingestion & Yes & Yes \\
\cline { 2 - 4 } & External & No & Yes \\
\cline { 2 - 5 } & Dermal Contact & Yes & No \\
\cline { 2 - 5 } & Suspension - Inhalation & Yes & Yes \\
\cline { 2 - 5 } & Biota - game (deer) & Yes & Yes \\
\hline \multirow{4}{*}{ Air } & Inhalation & Yes & Yes \\
\cline { 2 - 5 } & Biota - game (deer) & Yes & Yes \\
\hline \multirow{3}{*}{ Groundwater } & Ingestion & Yes & Yes \\
\cline { 2 - 5 } & Biota - game (deer) & Yes & Yes \\
\cline { 2 - 5 } & Dermal Contact (bathing) & Yes & No \\
\hline
\end{tabular}

Table F.4. Residential Scenario Exposure Pathways

\begin{tabular}{|l|l|l|l|}
\hline \multicolumn{1}{|c|}{ Transport Medium } & \multicolumn{1}{|c|}{ Exposure Pathway } & \multicolumn{1}{c|}{ Chemical } & \multicolumn{1}{c|}{ Radioactive } \\
\hline \multirow{5}{*}{ Soil (air deposition) } & Ingestion & Yes & Yes \\
\cline { 2 - 4 } & External & No & Yes \\
\cline { 2 - 4 } & Dermal Contact & Yes & No \\
\cline { 2 - 4 } & Biota - Fruit & Yes & Yes \\
\cline { 2 - 5 } & Biota - Vegetables & Yes & Yes \\
\cline { 2 - 5 } & Suspension - Inhalation & Yes & Yes \\
\hline \multirow{5}{*}{ Air } & Inhalation & Yes & Yes \\
\cline { 2 - 5 } & Biota - Fruit & Yes & Yes \\
\cline { 2 - 5 } & Biota - Vegetables & Yes & Yes \\
\hline \multirow{5}{*}{ Groundwater } & Ingestion & Yes & Yes \\
\cline { 2 - 5 } & Dermal Contact (bathing) & Yes & No \\
\cline { 2 - 5 } & Biota - Fruit & Yes & Yes \\
\cline { 2 - 5 } & Biota - Vegetables & Yes & Yes \\
\hline
\end{tabular}


Table F.5. Agricultural Scenario Exposure Pathways for Radionuclides and Chemicals

\begin{tabular}{|c|c|c|c|}
\hline Transport Medium & Exposure Pathway & Chemical & Radioactive \\
\hline \multirow[t]{9}{*}{ Soil (air deposition) } & Ingestion & yes & yes \\
\hline & External & no & yes \\
\hline & Dermal Contact & yes & no \\
\hline & Biota - Dairy & yes & yes \\
\hline & Biota - Meat & yes & yes \\
\hline & Biota - Game (deer) & yes & yes \\
\hline & Biota - Fruit & yes & yes \\
\hline & Biota - Vegetables & yes & yes \\
\hline & Suspension - Inhalation & yes & yes \\
\hline \multirow[t]{6}{*}{ Air } & Inhalation & yes & yes \\
\hline & Biota - Dairy & yes & yes \\
\hline & Biota - Meat & yes & yes \\
\hline & Biota - Game (deer) & yes & yes \\
\hline & Biota - Fruit & yes & yes \\
\hline & Biota - Vegetables & yes & yes \\
\hline \multirow[t]{8}{*}{ Groundwater } & Ingestion & yes & yes \\
\hline & Dermal Contact (bathing) & yes & no \\
\hline & Biota - Dairy & yes & yes \\
\hline & Biota-Meat & yes & yes \\
\hline & Biota - Game (deer) & yes & yes \\
\hline & Biota - Fruit & yes & yes \\
\hline & Biota - Vegetables & yes & yes \\
\hline & Inhalation indoor & yes & yes $(R n)$ \\
\hline
\end{tabular}


Table F.6. Industrial Scenario Exposure Factors

\begin{tabular}{|c|c|c|c|c|c|c|c|}
\hline \multicolumn{2}{|c|}{ Pathway } & \multicolumn{4}{|c|}{ Exposure Parameters $^{\left({ }^{(a)}\right.}$} & \multicolumn{2}{|c|}{ Summary Intake Factor } \\
\hline Media & $\begin{array}{c}\text { Exposure } \\
\text { Route }\end{array}$ & Intake Rate & $\begin{array}{c}\text { Exposure } \\
\text { Frequency } \\
\text { (d/yr) }\end{array}$ & $\begin{array}{c}\text { Conversion } \\
\text { Factors }\end{array}$ & Other Factors & $\begin{array}{c}\text { Chemical } \\
\text { Noncarcinogens }\end{array}$ & Radionuclides \\
\hline \multirow[t]{4}{*}{ Soil $^{(b)}$} & Ingestion & $50 \mathrm{mg} / \mathrm{d}$ & 146 & $\begin{array}{c}1 \mathrm{E}-06 \mathrm{~kg} / \mathrm{mg} \\
60 \mathrm{~kg} / \mathrm{m}^{2(\mathrm{c})}\end{array}$ & $-\cdots$ & $\begin{array}{c}4.76 \mathrm{E}-09 \mathrm{~m}^{2} \\
\text { soil//(kg-d) }\end{array}$ & $1.22 \mathrm{E}-04 \mathrm{~m}^{2}$ soil \\
\hline & External & $8 \mathrm{~h} / \mathrm{d}$ & 146 & -- & 0.8 & -- & $9.34 \mathrm{E}+02 \mathrm{~h}$ \\
\hline & Dermal & $0.2 \mathrm{mg} / \mathrm{cm}^{2}-\mathrm{d}$ & 146 & $\begin{array}{c}1 \mathrm{E}-06 \mathrm{~kg} / \mathrm{mg} \\
60 \mathrm{~kg} / \mathrm{m}^{2}\end{array}$ & $5000 \mathrm{~cm}^{2}$ & $\begin{array}{c}9.52 \mathrm{E}-08 \mathrm{~m}^{2} \\
\mathrm{soil} /(\mathrm{kg}-\mathrm{d})\end{array}$ & -- \\
\hline & Inhalation & $20 \mathrm{~m}^{3} / \mathrm{d}$ & 250 & $\begin{array}{c}1 \mathrm{E}-09 \mathrm{~kg} / \mu \mathrm{g} \\
60 \mathrm{~kg} / \mathrm{m}^{2}\end{array}$ & $50 \mu \mathrm{g} / \mathrm{m}^{3}$ & $\begin{array}{c}1.63 \mathrm{E}-10 \mathrm{~m}^{2} \\
\mathrm{soil} /(\mathrm{kg}-\mathrm{d})\end{array}$ & $4.16 \mathrm{E}-06 \mathrm{~m}^{2}$ soil \\
\hline $\mathrm{Air}^{(\mathrm{d})}$ & Inhalation & $20 \mathrm{~m}^{3} / \mathrm{d}$ & 250 & $-\cdots$ & -- & $\begin{array}{c}1.96 \mathrm{E}-01 \mathrm{~m}^{3} / \\
(\mathrm{kg}-\mathrm{d})\end{array}$ & $5.00 \mathrm{E}+03 \mathrm{~m}^{3}$ air \\
\hline \multirow[t]{3}{*}{ Groundwater $^{(e)}$} & Ingestion & $1 \mathrm{~L} / \mathrm{d}$ & 250 & -- & - & $\begin{array}{c}\text { 9.78E-03 L / } \\
(\mathrm{kg}-\mathrm{d})\end{array}$ & $2.50 \mathrm{E}+02 \mathrm{~L}$ \\
\hline & Inhalation & $20 \mathrm{~m}^{3} / \mathrm{d}$ & 250 & -- & $\begin{array}{c}0.5 \mathrm{~L} / \mathrm{m}^{3} \text { chemicals } \\
0.1 \mathrm{~L} / \mathrm{m}^{3} \text { radon }\end{array}$ & $\begin{array}{c}9.78 \mathrm{E}-02 \mathrm{~L} / \\
(\mathrm{kg}-\mathrm{d})\end{array}$ & $5.00 \mathrm{E}+02 \mathrm{~L}$ \\
\hline & Dermal & $0.17 \mathrm{~h} / \mathrm{d}$ & 250 & $1 \mathrm{E}-03 \mathrm{~L} / \mathrm{cm}^{3}$ & $20,000 \mathrm{~cm}^{2}$ & $\begin{array}{c}3.33 \mathrm{E}-02 \mathrm{~L} \mathrm{~h} / \\
(\mathrm{kg}-\mathrm{d}-\mathrm{cm})\end{array}$ & -- \\
\hline
\end{tabular}

(a) For all cases, the body weight is $70 \mathrm{~kg}$ and exposure is for 1 year.

(b) Units for soil concentration are $\mathrm{pCi} / \mathrm{kg}$ dry soil for radionuclides, and $\mathrm{mg} / \mathrm{kg}$ for chemicals.

(c) The factor $60 \mathrm{~kg} / \mathrm{m}^{2}$ is to convert soil concentration between mass $(\mathrm{kg})$ and area $\left(\mathrm{m}^{2}\right)$.

(d) Units for air concentration are $\mathrm{pCi} / \mathrm{m}^{3}$ for radionuclides, and $\mathrm{mg} / \mathrm{m}^{3}$ for chemicals.

(e) Units for water concentration are $\mathrm{pCi} / \mathrm{L}$ for radionuclides, and $\mathrm{mg} / \mathrm{L}$ for chemicals. 
Table F.7. Recreational Scenario Exposure Factors

\begin{tabular}{|c|c|c|c|c|c|c|c|}
\hline \multicolumn{2}{|c|}{ Pathway } & \multicolumn{4}{|c|}{ Exposure Parameters $^{(2)}$} & \multicolumn{2}{|c|}{ Summary Intake Factor } \\
\hline Media & $\begin{array}{c}\text { Exposure } \\
\text { Route }\end{array}$ & Intake Rate & $\begin{array}{c}\text { Exposure } \\
\text { Frequency } \\
\text { (d/yr) }\end{array}$ & $\begin{array}{c}\text { Conversion } \\
\text { Factors } \\
\end{array}$ & Other Factors & $\begin{array}{c}\text { Chemical } \\
\text { Noncarcinogens }\end{array}$ & Radionuclides \\
\hline \multirow[t]{4}{*}{ Soil $^{(b)}$} & Ingestion & $100 \mathrm{mg} / \mathrm{d}$ & 7 & $\begin{array}{c}1 \mathrm{E}-06 \mathrm{~kg} / \mathrm{mg} \\
60 \mathrm{~kg} / \mathrm{m}^{2(c)}\end{array}$ & -- & $\begin{array}{c}4.57 \mathrm{E}-10 \mathrm{~m}^{2} \\
\mathrm{soil} /(\mathrm{kg}-\mathrm{d})\end{array}$ & $1.17 \mathrm{E}-05 \mathrm{~m}^{2}$ soil \\
\hline & External & $8 \mathrm{~h} / \mathrm{d}$ & 7 & -- & 0.8 & $\ldots$ & $4.49 \mathrm{E}+01 \mathrm{~h}$ \\
\hline & Dermal & $0.2 \mathrm{mg} / \mathrm{cm}^{2}-\mathrm{d}$ & 7 & $\begin{array}{c}1 \mathrm{E}-06 \mathrm{~kg} / \mathrm{mg} \\
60 \mathrm{~kg} / \mathrm{m}^{2}\end{array}$ & $5000 \mathrm{~cm}^{2}$ & $\begin{array}{c}4.57 \mathrm{E}-09 \mathrm{~m}^{2} \\
\text { soil } /(\mathrm{kg}-\mathrm{d})\end{array}$ & $\ldots$ \\
\hline & Inhalation & $20 \mathrm{~m}^{3} / \mathrm{d}$ & 7 & $\begin{array}{c}1 \mathrm{E}-09 \mathrm{~kg} / \mu \mathrm{g} \\
60 \mathrm{~kg} / \mathrm{m}^{2}\end{array}$ & $50 \mu \mathrm{g} / \mathrm{m}^{3}$ & $\begin{array}{c}4.57 \mathrm{E}-12 \mathrm{~m}^{2} \\
\text { soil/(kg-d) }\end{array}$ & $1.17 \mathrm{E}-08 \mathrm{~m}^{2}$ soil \\
\hline $\mathrm{Air}^{(\mathrm{d})}$ & Inhalation & $20 \mathrm{~m}^{3} / \mathrm{d}$ & 7 & -- & -- & $\begin{array}{c}5.49 \mathrm{E}-03 \mathrm{~m}^{3} / \\
(\mathrm{kg}-\mathrm{d})\end{array}$ & $1.40 \mathrm{E}+02 \mathrm{~m}^{3}$ air \\
\hline \multirow[t]{2}{*}{ Groundwater $^{(c)}$} & Ingestion & $2 \mathrm{~L} / \mathrm{d}$ & 7 & $\cdots$ & -- & $\begin{array}{c}5.49 \mathrm{E}-04 \mathrm{~L} / \\
(\mathrm{kg}-\mathrm{d})\end{array}$ & $1.40 \mathrm{E}+01 \mathrm{~L}$ \\
\hline & Dermal & $0.17 \mathrm{~h} / \mathrm{d}$ & 7 & $1 \mathrm{E}-03 \mathrm{~L} / \mathrm{cm}^{3}$ & $20,000 \mathrm{~cm}^{2}$ & $\begin{array}{c}9.33 \mathrm{E}-04 \mathrm{~L} \mathrm{~h} / \\
(\mathrm{kg}-\mathrm{d}-\mathrm{cm})\end{array}$ & $\cdots$ \\
\hline Biota $^{(\mathfrak{l})}$ & Deer & $15 \mathrm{~g} / \mathrm{d}$ & 365 & $1 \mathrm{E}-03 \mathrm{~kg} / \mathrm{g}$ & -- & $\begin{array}{c}2.14 \mathrm{E}-04 \mathrm{~kg} \\
\text { deer } /(\mathrm{kg}-\mathrm{d})\end{array}$ & $5.48 \mathrm{~kg}$ deer $/(\mathrm{kg}-\mathrm{d})$ \\
\hline
\end{tabular}

(a) For all cases, the body weight is $70 \mathrm{~kg}$ and exposure is for 1 year.

(b) Units for soil concentration are $\mathrm{pCi} / \mathrm{kg}$ dry soil for radionuclides, and $\mathrm{mg} / \mathrm{kg}$ for chemicals.

(c) The factor $60 \mathrm{~kg} / \mathrm{m}^{2}$ is to convert soil concentration between mass $(\mathrm{kg})$ and area $\left(\mathrm{m}^{2}\right)$.

(d) Units for air concentration are $\mathrm{pCi} / \mathrm{m}^{3}$ for radionuclides, and $\mathrm{mg} / \mathrm{m}^{3}$ for chemicals.

(e) Units for water concentration are $\mathrm{pCi} / \mathrm{L}$ for radionuclides, and $\mathrm{mg} / \mathrm{L}$ for chemicals.

(f) Units for food concentration are pCi/kg wet food for radionuclides, and $\mathrm{mg} / \mathrm{kg}$ for chemicals. 
Table F.8. Residential Scenario Exposure Factors

\begin{tabular}{|c|c|c|c|c|c|c|c|}
\hline \multicolumn{2}{|c|}{ Pathway } & \multicolumn{4}{|c|}{ Exposure Parameters $^{\text {(a) }}$} & \multicolumn{2}{|c|}{ Summary Intake Factor } \\
\hline Media & $\begin{array}{l}\text { Exposure } \\
\text { Route }\end{array}$ & Intake Rate & $\begin{array}{c}\text { Exposure } \\
\text { Frequency } \\
\text { (d/yr) }\end{array}$ & $\begin{array}{c}\text { Conversion } \\
\text { Factors }\end{array}$ & Other Factors & $\begin{array}{c}\text { Chemical } \\
\text { Noncarcinogens }\end{array}$ & Radionuclides \\
\hline \multirow[t]{4}{*}{ Soil $^{(b)}$} & Ingestion & $100 \mathrm{mg} / \mathrm{d}$ & 365 & $\begin{array}{l}1 \mathrm{E}-06 \mathrm{~kg} / \mathrm{mg} \\
60 \mathrm{~kg} / \mathrm{m}^{2(\mathrm{c})}\end{array}$ & $\ldots$ & $\begin{array}{c}2.38 \mathrm{E}-08 \mathrm{~m}^{2} \\
\mathrm{soil} /(\mathrm{kg}-\mathrm{d})\end{array}$ & $6.09 \mathrm{E}-04 \mathrm{~m}^{2}$ soil \\
\hline & External & $24 \mathrm{~h} / \mathrm{d}$ & 365 & -- & 0.8 & - & $7.03 E+03 h$ \\
\hline & Dermal & $0.2 \mathrm{mg} / \mathrm{cm}^{2}-\mathrm{d}$ & 180 & $\begin{array}{c}1 \mathrm{E}-06 \mathrm{~kg} / \mathrm{mg} \\
60 \mathrm{~kg} / \mathrm{m}^{2}\end{array}$ & $5000 \mathrm{~cm}^{2}$ & $\begin{array}{l}1.17 \mathrm{E}-07 \mathrm{~m}^{2} \\
\text { soil/(kg-d) }\end{array}$ & -- \\
\hline & Inhalation & $20 \mathrm{~m}^{3} / \mathrm{d}$ & 365 & $\begin{array}{c}1 \mathrm{E}-09 \mathrm{~kg} / \mathrm{\mu g} \\
60 \mathrm{~kg} / \mathrm{m}^{2} \\
\end{array}$ & $50 \mu \mathrm{g} / \mathrm{m}^{3}$ & $\begin{array}{c}2.38 \mathrm{E}-10 \mathrm{~m}^{2} \\
\text { soil } /(\mathrm{kg}-\mathrm{d})\end{array}$ & $6.08 \mathrm{E}-06 \mathrm{~m}^{2}$ soil \\
\hline $\mathrm{Air}^{(\mathrm{d})}$ & Inhalation & $20 \mathrm{~m}^{3} / \mathrm{d}$ & 365 & -. & - & $\begin{array}{c}2.86 \mathrm{E}-01 \mathrm{~m}^{3} / \\
(\mathrm{kg}-\mathrm{d})\end{array}$ & $7.31 \mathrm{E}+03 \mathrm{~m}^{3}$ air \\
\hline \multirow[t]{3}{*}{ Groundwater $^{(e)}$} & Ingestion & $2 \mathrm{~L} / \mathrm{d}$ & 365 & -- & -- & $\begin{array}{c}2.86 \mathrm{E}-02 \mathrm{~L} / \\
(\mathrm{kg}-\mathrm{d})\end{array}$ & $7.31 \mathrm{E}+02 \mathrm{~L}$ \\
\hline & Inhalation & $15 \mathrm{~m}^{3} / \mathrm{d}$ & 365 & -- & $\begin{array}{c}0.5 \mathrm{~L} / \mathrm{m}^{3} \text { chemicals } \\
0.1 \mathrm{~L} / \mathrm{m}^{3} \text { radon }\end{array}$ & $\begin{array}{c}1.07 \mathrm{E}-01 \mathrm{~L} / \\
(\mathrm{kg}-\mathrm{d})\end{array}$ & $5.48 \mathrm{E}+02 \mathrm{~L}$ \\
\hline & Dermal & $0.17 \mathrm{~h} / \mathrm{d}$ & 365 & $1 \mathrm{E}-03 \mathrm{~L} / \mathrm{cm}^{3}$ & $20,000 \mathrm{~cm}^{2}$ & $\begin{array}{c}4.86 \mathrm{E}-02 \mathrm{~L} \mathrm{~h} / \\
(\mathrm{kg}-\mathrm{d}-\mathrm{cm})\end{array}$ & -- \\
\hline \multirow[t]{2}{*}{ Biota $^{(1)}$} & Fruit & $42 \mathrm{~g} / \mathrm{d}$ & 365 & $1 \mathrm{E}-03 \mathrm{~kg} / \mathrm{g}$ & -- & $\begin{array}{c}6.00 \mathrm{E}-04 \mathrm{~kg} \text { food } \\
/(\mathrm{kg}-\mathrm{d})\end{array}$ & $\begin{array}{c}1.53 \mathrm{E}+01 \mathrm{~kg} \\
\text { food }\end{array}$ \\
\hline & Vegetable & $80 \mathrm{~g} / \mathrm{d}$ & 365 & $1 \mathrm{E}-03 \mathrm{~kg} / \mathrm{g}$ & - & $\begin{array}{c}1.14 \mathrm{E}-03 \mathrm{~kg} \text { food } \\
/(\mathrm{kg}-\mathrm{d})\end{array}$ & $\begin{array}{l}2.92 \mathrm{E}+01 \mathrm{~kg} \\
\text { food }\end{array}$ \\
\hline
\end{tabular}

(a) For all cases, the body weight is $70 \mathrm{~kg}$ and exposure is for 1 year.

(b) Units for soil concentration are pCi/ $/ \mathrm{gg}$ dry soil for radionuclides, and $\mathrm{mg} / \mathrm{kg}$ for chemicals.

(c) The factor $60 \mathrm{~kg} / \mathrm{m}^{2}$ is to convert soil concentration between mass $(\mathrm{kg})$ and area $\left(\mathrm{m}^{2}\right)$.

(d) Units for air concentration are $\mathrm{pCi} / \mathrm{m}^{3}$ for radionuclides, and $\mathrm{mg} / \mathrm{m}^{3}$ for chemicals.

(e) Units for water concentration are $\mathrm{pCi} / \mathrm{L}$ for radionuclides, and $\mathrm{mg} / \mathrm{L}$ for chemicals.

(f) Units for food concentration are $\mathrm{pCi} / \mathrm{kg}$ wet food for radionuclides, and $\mathrm{mg} / \mathrm{kg}$ for chemicals. 
Table F.9. Agricultural Scenario Exposure Factors

\begin{tabular}{|c|c|c|c|c|c|c|c|}
\hline & Pathway & \multicolumn{4}{|c|}{ Exposure Parameters $^{(\mathbf{a})}$} & \multicolumn{2}{|c|}{ Summary Intake Factor } \\
\hline Media & $\begin{array}{c}\text { Exposure } \\
\text { Route }\end{array}$ & Intake Rate & $\begin{array}{c}\text { Exposure } \\
\text { Frequency } \\
\text { (d/yr) }\end{array}$ & $\begin{array}{l}\text { Conversion } \\
\text { Factors }\end{array}$ & Other Factors & $\begin{array}{c}\text { Chemical } \\
\text { Noncarcinogens }\end{array}$ & Radionuclides \\
\hline \multirow[t]{4}{*}{ Soil $^{(\mathrm{b})}$} & Ingestion & $100 \mathrm{mg} / \mathrm{d}$ & 365 & $\begin{array}{l}1 \mathrm{E}-06 \mathrm{~kg} / \mathrm{mg} \\
60 \mathrm{~kg} / \mathrm{m}^{2}(\mathrm{c})\end{array}$ & -- & $\begin{array}{c}2.38 \mathrm{E}-08 \mathrm{~m}^{2} \\
\mathrm{soil} /(\mathrm{kg}-\mathrm{d})\end{array}$ & $6.09 \mathrm{E}-04 \mathrm{~m}^{2}$ soil \\
\hline & External & $24 \mathrm{~h} / \mathrm{d}$ & 365 & -- & 0.8 & -- & $7.03 \mathrm{E}+03 \mathrm{~h}$ \\
\hline & Dermal & $0.2 \mathrm{mg} / \mathrm{cm}^{2}-\mathrm{d}$ & 180 & $\begin{array}{l}1 \mathrm{E}-06 \mathrm{~kg} / \mathrm{mg} \\
60 \mathrm{~kg} / \mathrm{m}^{2}\end{array}$ & $5000 \mathrm{~cm}^{2}$ & $\begin{array}{l}1.17 \mathrm{E}-07 \mathrm{~m}^{2} \\
\mathrm{soil} /(\mathrm{kg}-\mathrm{d})\end{array}$ & $-\infty$ \\
\hline & Inhalation & $20 \mathrm{~m}^{3} / \mathrm{d}$ & 365 & $\begin{array}{c}.1 \mathrm{E}-09 \mathrm{~kg} / \mu \mathrm{g} \\
60 \mathrm{~kg} / \mathrm{m}^{2}\end{array}$ & $50 \mu \mathrm{g} / \mathrm{m}^{3}$ & $\begin{array}{l}2.38 \mathrm{E}-10 \mathrm{~m}^{2} \\
\text { soil } /(\mathrm{kg}-\mathrm{d})\end{array}$ & $6.08 \mathrm{E}-06 \mathrm{~m}^{2}$ soil \\
\hline $\operatorname{Air}^{(d)}$ & Inhalation & $20 \mathrm{~m}^{3} / \mathrm{d}$ & 365 & -- & -- & $\begin{array}{c}2.86 \mathrm{E}-01 \mathrm{~m}^{3} \\
/(\mathrm{kg}-\mathrm{d})\end{array}$ & $7.31 \mathrm{E}+03 \mathrm{~m}^{3}$ air \\
\hline \multirow[t]{3}{*}{ Groundwater ${ }^{(e)}$} & Ingestion & $2 \mathrm{~L} / \mathrm{d}$ & 365 & -- & -- & $\begin{array}{c}2.86 \mathrm{E}-02 \mathrm{~L} \\
/(\mathrm{kg}-\mathrm{d})\end{array}$ & $7.31 \mathrm{E}+02 \mathrm{~L}$ \\
\hline & Inhalation & $15 \mathrm{~m}^{3} / \mathrm{d}$ & 365 & -- & $\begin{array}{c}0.5 \mathrm{~L} / \mathrm{m}^{3} \\
\text { chemicals } \\
0.1 \mathrm{~L} / \mathrm{m}^{3} \text { radon }\end{array}$ & $\begin{array}{c}1.07 \mathrm{E}-01 \mathrm{~L} \\
/(\mathrm{kg}-\mathrm{d})\end{array}$ & $5.48 \mathrm{E}+02 \mathrm{~L}$ \\
\hline & $\overline{\text { Dermal }}$ & $0.17 \mathrm{~h} / \mathrm{d}$ & 365 & $1 \mathrm{E}-03 \mathrm{~L} / \mathrm{cm}^{3}$ & $20,000 \mathrm{~cm}^{2}$ & $\begin{array}{c}4.86 \mathrm{E}-02 \mathrm{Lh} \\
/(\mathrm{kg}-\mathrm{d}-\mathrm{cm})\end{array}$ & -- \\
\hline \multirow[t]{5}{*}{ Biota $^{(\mathfrak{n})}$} & Dairy & $300 \mathrm{~g} / \mathrm{d}$ & 365 & $1 \mathrm{E}-03 \mathrm{~kg} / \mathrm{g}$ & - & $\begin{array}{c}4.29 \mathrm{E}-03 \mathrm{~kg} \text { food } \\
/(\mathrm{kg}-\mathrm{d})\end{array}$ & $1.10 \mathrm{E}+02 \mathrm{~kg}$ food \\
\hline & Beef & $75 \mathrm{~g} / \mathrm{d}$ & 365 & $1 \mathrm{E}-03 \mathrm{~kg} / \mathrm{g}$ & - & $\begin{array}{c}1.07 \mathrm{E}-03 \mathrm{~kg} \text { food } \\
/(\mathrm{kg}-\mathrm{d})\end{array}$ & $2.74 \mathrm{E}+01 \mathrm{~kg}$ food \\
\hline & Game & $15 \mathrm{~g} / \mathrm{d}$ & 365 & $1 \mathrm{E}-03 \mathrm{~kg} / \mathrm{g}$ & $-\overline{-}$ & $\begin{array}{c}2.14 \mathrm{E}-04 \mathrm{~kg} \text { food } \\
/(\mathrm{kg}-\mathrm{d})\end{array}$ & $5.48 \mathrm{E}+0 \mathrm{~kg}$ food \\
\hline & Fruit & $42 \mathrm{~g} / \mathrm{d}$ & 365 & $1 \mathrm{E}-03 \mathrm{~kg} / \mathrm{g}$ & -- & $\begin{array}{c}6.00 \mathrm{E}-04 \mathrm{~kg} \text { food } \\
/(\mathrm{kg}-\mathrm{d})\end{array}$ & $1.53 \mathrm{E}+01 \mathrm{~kg}$ food \\
\hline & Vegetable & $80 \mathrm{~g} / \mathrm{d}$ & 365 & $1 \mathrm{E}-03 \mathrm{~kg} / \mathrm{g}$ & - & $\begin{array}{c}1.14 \mathrm{E}-03 \mathrm{~kg} \text { food } \\
/(\mathrm{kg}-\mathrm{d})\end{array}$ & $2.92 \mathrm{E}+01 \mathrm{~kg}$ food \\
\hline
\end{tabular}

(a) For all cases, the body weight is $70 \mathrm{~kg}$ and exposure is for 1 year.

(b) Units for soil concentration are $\mathrm{pCi} / \mathrm{kg}$ dry soil for radionuclides, and $\mathrm{mg} / \mathrm{kg}$ for chemicals.

(c) The factor $60 \mathrm{~kg} / \mathrm{m}^{2}$ is to convert soil concentration between mass $(\mathrm{kg})$ and area $\left(\mathrm{m}^{2}\right)$.

(d) Units for air concentration are $\mathrm{pCi} / \mathrm{m}^{3}$ for radionuclides, and $\mathrm{mg} / \mathrm{m}^{3}$ for chemicals.

(e) Units for water concentration are $\mathrm{pCi} / \mathrm{L}$ for radionuclides, and $\mathrm{mg} / \mathrm{L}$ for chemicals.

(f) Units for food concentration are $\mathrm{pCi} / \mathrm{kg}$ wet food for radionuclides, and $\mathrm{mg} / \mathrm{kg}$ for chemicals. 
Table F.10. Time to Reach the Equilibrium Annual Dose

\begin{tabular}{|c|c|c|c|c|}
\hline \multirow[b]{3}{*}{ Constituent } & \multicolumn{4}{|c|}{ Time to Reach $95 \%$ of Equilibrium Dose, in years (Ratio of 50-Year Dose to Equilibrium Year Dose, or 1000-year dose) } \\
\hline & \multicolumn{2}{|c|}{\begin{tabular}{|c|} 
Agricultural Soil Retention \\
\end{tabular}} & \multicolumn{2}{|c|}{ Native Soil Retention } \\
\hline & Soil (air) Pathways & Agricultural (air) Pathways & Recreational Soil Pathways & Game Meat Ingestion \\
\hline Uranium & $16(1.0)$ & $31(0.99)$ & $>1000(0.13)$ & $>1000(0.088)$ \\
\hline${ }^{3} \mathrm{H}$ & $1(1.0)$ & zero dose & $27(1.0)$ & zero dose \\
\hline${ }^{14} \mathrm{C}$ & $31(0.99)$ & zero dose & $>1000(0.090)$ & zero dose \\
\hline${ }^{36} \mathrm{Cl}$ & $5(1.0)$ & $5(1.0)$ & $>1000(0.34)$ & $>1000(0.034)$ \\
\hline${ }^{40} \mathrm{~K}$ & $44(0.97)$ & $45(0.96)$ & $>1000(0.074)$ & $>1000(0.075)$ \\
\hline${ }^{79} \mathrm{Se}$ & $9(1.0)$ & $9(1.0)$ & $>1000(0.20)$ & $>1000(0.020)$ \\
\hline${ }^{99} \mathrm{TC}$ & $9(1.0)$ & $9(1.0)$ & $>1000(0.20)$ & $>1000(0.020)$ \\
\hline${ }^{129} \mathrm{I}$ & $70(0.89)$ & $70(0.89)$ & $>1000(0.066)$ & $>1000(0.066)$ \\
\hline${ }^{233} \mathrm{U}$ & $>1000(0.35)$ & $>1000(0.21)$ & $>1000(0.044)$ & $>1000(0.078)$ \\
\hline${ }^{234} U$ & $31(0.99)$ & $31(0.99)$ & $>1000(0.086)$ & $>1000(0.086)$ \\
\hline${ }^{233} U$ & $>1000(0.89)$ & $>1000(0.94)$ & $>1000(0.075)$ & $>1000(0.076)$ \\
\hline${ }^{236} \mathrm{U}$ & $31(0.99)$ & $32(0.99)$ & $>1000(0.086)$ & $>1000(0.086)$ \\
\hline${ }^{238} U$ & $32(0.99)$ & $31(0.99)$ & $>1000(0.086)$ & $>1000(0.086)$ \\
\hline${ }^{237} \mathrm{~Np}$ & $120(0.73)$ & $120(0.74)$ & $>1000(0.060)$ & $>1000(0.060)$ \\
\hline${ }^{234} \mathrm{Th}$ & $1(1.0)$ & $7(1.0)$ & $1(1.0)$ & $>1000(0.38)$ \\
\hline${ }^{231} \mathrm{Th}$ & $>1000(0.49)$ & $>1000(0.035)$ & $>1000(0.29)$ & $>1000(0.031)$ \\
\hline${ }^{230} \mathrm{Th}$ & $>1000(0.0095)$ & $>1000(0.0027)$ & $>1000(0.012)$ & $>1000(0.0017)$ \\
\hline${ }^{229} \mathrm{Th}$ & $>1000(0.056)$ & $>1000(0.056)$ & $>1000(0.053)$ & $>1000(0.053)$ \\
\hline${ }^{273} \mathrm{Th}$ & $1(1.0)$ & $1(1.0)$ & $1(1.0)$ & $1(1.0)$ \\
\hline${ }^{223} \mathrm{~Pa}$ & $1(1.0)$ & $>1000(0.49)$ & $1(1.0)$ & $>1000(0.18)$ \\
\hline${ }^{231} \mathrm{~Pa}$ & $>1000(0.034)$ & $>1000(0.035)$ & $>1000(0.032)$ & $>1000(0.031)$ \\
\hline${ }^{235} \mathrm{Ra}$ & $1(1.0)$ & $1(1.0)$ & $1(1.0)$ & $1(1.0)$ \\
\hline${ }^{227} \mathrm{Ac}$ & $100(0.80)$ & $100(0.80)$ & $100(0.80)$ & $100(0.80)$ \\
\hline${ }^{225} \mathrm{Ac}$ & $1(1.0)$ & $1(1.0)$ & $1(1.0)$ & $1(1.0)$ \\
\hline${ }^{226} \mathrm{Ra}$ & $>1000(0.10)$ & $>1000(0.059)$ & $>1000(0.060)$ & $>1000(0.036)$ \\
\hline${ }^{223} \mathrm{Ra}$ & $1(1.0)$ & $1(1.0)$ & $1(1.0)$ & $1(1.0)$ \\
\hline${ }^{210} \mathrm{~Pb}$ & $100(0.80)$ & $100(0.79)$ & $100(0.79)$ & $100(0.79)$ \\
\hline${ }^{210} \mathrm{Bi}$ & $2(1.0)$ & $2(1.0)$ & $2(1.0)$ & $2(1.0)$ \\
\hline${ }^{210} \mathrm{Po}$ & $2(1.0)$ & $2(1.0)$ & $2(1.0)$ & $2(1.0)$ \\
\hline
\end{tabular}


Table F.11. Unit Dose Factors for Radionuclides Contributing to Dose and Uranium as a Chemical Hazard

\begin{tabular}{|c|c|c|c|c|c|c|}
\hline & \multicolumn{6}{|c|}{ Scenario } \\
\hline & Residential & Industrial & Recreational & & Agricultural & \\
\hline Radionuclide & $\begin{array}{l}\text { Groundwater } \\
{[\mathrm{mrem} / \mathrm{pCi} / \mathrm{L}]}\end{array}$ & $\begin{array}{l}\text { Groundwater } \\
\text { [mrem/pCi/L] }\end{array}$ & $\begin{array}{l}\text { Groundwater } \\
{[\mathrm{mrem} / \mathrm{pCi} / \mathrm{L}]}\end{array}$ & $\begin{array}{l}\text { Groundwater } \\
{[\mathrm{mrem} / \mathrm{pCi} / \mathrm{L}]}\end{array}$ & $\begin{array}{c}\text { Air } \\
{\left[\mathrm{mrem} / \mathrm{pCi} / \mathrm{m}^{3}\right]}\end{array}$ & $\begin{array}{c}\text { Soil } \\
{\left[\mathrm{mrem} / \mathrm{pCi} / \mathrm{m}^{2}\right]}\end{array}$ \\
\hline Tritium & $4.85 \mathrm{E}-05$ & $1.57 \mathrm{E}-05$ & $1.05 \mathrm{E}-06$ & 5.69E-05 & $1.25 \mathrm{E}-03$ & $4.18 \mathrm{E}-11$ \\
\hline Carbon-14 & $1.52 \mathrm{E}-02$ & $5.22 \mathrm{E}-04$ & $2.99 \mathrm{E}-05$ & $4.09 \mathrm{E}-02$ & $2.53 \mathrm{E}-01$ & $2.93 \mathrm{E}-08$ \\
\hline Chlorine-36 & $1.76 \mathrm{E}-02$ & $7.58 \mathrm{E}-04$ & $5.29 \mathrm{E}-05$ & $1.08 \mathrm{E}-01$ & & \\
\hline Selenium-79 & $6.77 \mathrm{E}-03$ & $2.17 \mathrm{E}-03$ & $1.28 \mathrm{E}-04$ & $1.21 \mathrm{E}-02$ & & \\
\hline Strontium-90 & $2.53 \mathrm{E}-01$ & $3.58 \mathrm{E}-02$ & 2.01E-03 & $3.12 \mathrm{E}-01$ & & \\
\hline Technetium-99 & $1.36 \mathrm{E}-03$ & $3.65 \mathrm{E}-04$ & $2.10 \mathrm{E}-05$ & $3.66 \mathrm{E}-03$ & & \\
\hline Iodine-129 & $2.27 \mathrm{E}-01$ & $6.90 \mathrm{E}-02$ & $3.95 \mathrm{E}-03$ & $6.19 \mathrm{E}-01$ & & \\
\hline Uranium $^{(a)}$ & $\begin{array}{c}{[\mathrm{mrem} /(\mu \mathrm{g} / \mathrm{L})]} \\
1.69 \mathrm{E}-01\end{array}$ & $\begin{array}{c}{[\mathrm{mrem} /(\mu \mathrm{g} / \mathrm{L})]} \\
5.27 \mathrm{E}-02 \\
\end{array}$ & $\begin{array}{c}{[\mathrm{mrem} /(\mu \mathrm{g} / \mathrm{L})]} \\
2.96 \mathrm{E}-03 \\
\end{array}$ & $\begin{array}{c}{[\mathrm{mrem} /(\mu \mathrm{g} / \mathrm{L})]} \\
1.86 \mathrm{E}-01\end{array}$ & & \\
\hline Uranium $^{(0)}$ & $\begin{array}{c}\text { [hazard index/ } / \mu \mathrm{g} / \mathrm{L} \text { ] } \\
1.08 \mathrm{E}-02\end{array}$ & $\begin{array}{c}\text { [hazard index/ } \mu \mathrm{g} / \mathrm{L} \text { ] } \\
3.48 \mathrm{E}-03\end{array}$ & $\begin{array}{c}\text { [hazard index } / \mu \mathrm{g} / \mathrm{L} \text { ] } \\
1.89 \mathrm{E}-04\end{array}$ & $\begin{array}{c}\text { [hazard index } / \mu \mathrm{g} / \mathrm{L} \text { ] } \\
1.19 \mathrm{E}-02\end{array}$ & & \\
\hline
\end{tabular}

(a) Uranium modeled as contributing to radiation dose.

(b) Uranium modeled as contributing to chemical hazard. 


\section{Distribution}

No. of

Copies

\section{OFFSITE}

Wanapum Indian Band

P.O. Box 878

Ephrata, WA 98823

ATTN: R. Buck, Jr.

3 Washington State Department of Ecology

Nuclear Waste Program

1315 West $^{\text {th }}$

Kennewick, WA 99336-6018

ATTN: D.N. Goswami

D.P. Holland

S. Mohan

2 Yakima Indian Nation

P.O. Box 151

Toppenish, WA 98948

ATTN: R. Jim

W. Rigsbee

2 Confederated Tribes of the

Umatilla Indian Reservation

P.O. Box 638

Pendleton, OR 97801

ATTN: J.R. Wilkinson

S. Harris

2 Nez Perce Indian Tribe

P.O. Box 365

Lapwai, ID 83540-0365

ATTN: D.L. Powaukee

S.M. Sobczyk
No. of

Copies

2 Oregon Department of Energy

625 Marion St. N.E.

Salem, OR 97310

ATTN: R. Bennett

M.L. Blazek

U.S. Ecology

P.O. Box 638

Richland, WA 99352

ATTN: S. Bede

U.S. Department of Energy

Albuquerque Operations Office

Pennsylvania and $\mathrm{H}$ Street

Kirtland Air Force Base

Albuquerque, NM 87116

ATTN: R.J. Borders

3 U.S. Department of Energy

Savannah River Operations Office

Road 1

Aiken, SC 29801

ATTN: W.L. Noll

W. Smith

H.M. Crapse

2 U.S. Department of Energy, Headquarters

Forrestal Building

1000 Independence Avenue, S.W.

Washington, D.C. 20585

ATTN: J.T. Bachmaier.

A. Wallo

Distr.1 
No. of

Copies

2 Idaho National Engineering Laboratory

CF 90 MS 4148

Scoville, ID 83415

ATTN: J.T. Case

J.N. Perry

U.S. Department of Energy

Idaho Operations Office

765 Lindsay Blvd.

Idaho Falls, ID 83401

ATTN: G.J. Duggan

5 U.S. Department of Energy, Headquarters

Germantown

19901 Germantown Road

Germantown, MD 20874-1290

ATTN: M.K. Harmon

EM-44

K.T. Lang

EM-38

M.J. Letourneau

EM-35

V.W. Lowery

EM-35

J.E. Rhoderiek

EM-35

2 Idaho National Engineering Laboratory 850 Energy Drive Idaho Falls, ID 83401

ATTN: R.V. Curl

U.S. Department of Energy

Fernald Field Office

7400 Wiley Road

Cincinnati, $\mathrm{OH} 45030$

ATTN: R.C. Janke

2 U.S. Department of Energy

Nevada Operations Office

P.O. Box 98518

Las Vegas, NV 89193-8518

ATTN: B.A. Moore (2)
No. of

Copies

Westinghouse Savannah River Company

Savannah River Site

Aiken, SC 29808

ATTN: E. Wilhite

Los Alamos National Laboratory

P.O. Box 1663, MS-J595

Los Alamos, NM 87545

ATTN: Diana Hollis

2 Bechtel Nevada

Las Vegas, NV 89193

ATTN: G. Scott

V. Yucel

U.S. Department of Energy

Oak Ridge Operations Office

P.O. Box 2001

Oak Ridge, TN 37831

ATTN: W.G. McMillan

6 Department of Health

Division of Radiation Protection

Waste Management Section

P.O. Box 47827

Olympia, WA 98504-7827

ATTN: Maxine Dunkleman (6)

2 Oak Ridge National Laboratory

P.O. Box 2008

Oak Ridge, TN 37831

ATTN: D.W. Lee

R.J. Luxmoore 
No. of

Copies

ONSITE

20 DOE Richland Operations Office

L.K. Bauer

$\mathrm{H} 0-12$

N.R. Brown

P.F. Dunigan

B.L. Foley

J.D. Goodenough

R.X. Gonzalez

J.B. Hall

R.D. Hildebrand (10)

R.A. Holten

P.E. Lamont

R.W. Lober

K.M. Thompson

O. Robertson

G.H. Sanders

S.S. Seth

D.S. Shafer

G.L. Sinten

C.D. West

A.K. Wright

DOE Public Reading Room (2)

4 Fluor Daniel Northwest

M.C. Brown

R. Khaleel

F.M. Mann

J.D. Williams

International Technologies

D.A. Myers

G3-21

3 Lockheed Martin Hanford Corp.

E.A. Fredenburg

M.J. Kupfer

H6-12

H5-49

J.A. Voogd
No. of

Copies

3 Rust Federal Services of Hanford

F.M. Coony

H6-06

J.C. Sonnichsen

H6-26

M.I. Wood

H6-06

ICF Kaiser

P.J. Macbeth

R3-82

Jacobs Engineering

P.M. Rogers

B1-40

6 Bechtel Hanford

M.A. Buckmaster

H0-19

K.R. Fecht

$\mathrm{HO}-02$

M.J. Graham

H0-09

A.J. Knepp

$\mathrm{H} 0-19$

G.B. Mitchem

$\mathrm{H} 0-17$

F.V. Roech

$\mathrm{H} 0-17$

40 Pacific Northwest Laboratory

D.B. Barnett

K6-81

M.P. Bergeron

K9-36

C.R. Cole

K9-36

P.E. Dresel

K6-96

M.J. Fayer

K9-36

M.D. Freshley

K9-36

G.W. Gee

K9-33

N.L. Hassig

V.G. Johnson

K5-12

K6-96

D.I. Kaplan

K6-81

C.T. Kincaid (10)

K9-36

K.M. Krupka

K6-81

P.E. Long

K6-91

P.D. Meyer

K9-36

T.L Page

K9-18 
Pacific Northwest Laboratory (contd)

M.J. Quadrel

R.J. Serne

R.M. Smith

D.L. Strenge

G.P. Streile

P.D. Thorne

L.W. Vail

G.A. Whyatt

S.K. Wurstner

Information Release Office (7)
K9-70

K6-81

K6-96

K3-54

K9-36

K9-36

K9-36

P7-19

K9-36

2 U.S. Environmental Protection Agency

L.E. Gadbois

B5-01

D.R. Sherwood

B5-01 